\title{
Multiconfiguration Dirac-Hartree-Fock energy levels, weighted oscillator strengths, transitions probabilities, lifetimes, hyperfine constants, Landé $g$-factors and isotope shifts of Xe LIII
}

\author{
Dhia Elhak Salhi ${ }^{\mathrm{a}, \mathrm{b}, \mathrm{c}}$, Pascal Quinet ${ }^{\mathrm{d}, \mathrm{e}}$, Haikel Jelassi ${ }^{\mathrm{a}, \mathrm{b}, *}$ \\ a Laboratory on Energy and Matter for Nuclear Sciences Development, LR16CNS TN 02, Tunisia \\ b National Centre for Nuclear Sciences and Technologies, Sidi Thabet Technopark, 2020 Ariana, Tunisia \\ ${ }^{\mathrm{c}}$ Faculty of Sciences of Tunis, University Tunis El Manar Tunis, Tunisia \\ d Physique Atomique et Astrophysique, Université de Mons, 7000 Mons, Belgium \\ e IPNAS, Université de Liège, 4000 Liège, Belgium
}

\section{A R T I C L E I N F O}

\section{Article history:}

Received 1 March 2018

Received in revised form 4 April 2018

Accepted 19 April 2018

Available online 2 August 2018

\section{Keywords:}

Multiconfiguration Dirac-Hartree-Fock

Relativistic configuration interaction

Transition probability

Hyperfine constants

Landé g factors

Isotope shifts

\begin{abstract}
A B S T R A C T
Energy levels, weighted oscillator strengths and transition probabilities, lifetimes, hyperfine interaction constants, Landé $g_{J}$ factors and isotope shifts are calculated for all levels of $1 s^{2}$ and $1 \operatorname{snl}(n=2-7)$ configurations of He-like xenon ion (Xe LIII). Multiconfigurational Dirac-Hartree-Fock (MCDHF) method is adopted for calculating these spectroscopic data. Comparisons are made with similar data obtained with FAC (Flexible Atomic Code) to assess the accuracy of the results. Transition probabilities are reported for all $E 1, E 2, M 1$ and $M 2$ transitions from the ground level. Breit interactions and quantum electrodynamics effects are estimated in extensive Relativistic Configuration Interaction (RCI) calculations. Comparisons were made with the available data in the literature and good agreement was found which confirms the reliability of our results. The accuracy of the present calculations is high enough to facilitate identification of many observed spectral lines. Almost all atomic data of He-like xenon ion presented in this paper are calculated for the first time.
\end{abstract}

(c) 2018 Elsevier Inc. All rights reserved.

\footnotetext{
* Correspondence to: National Centre for Nuclear Sciences and Technologies, Sidi Thabet Technopark, 2020 Ariana, Tunisia.

E-mail address: haikel.jelassi@cnstn.rnrt.tn (H. Jelassi).
} 


\section{Contents}

1. Introduction

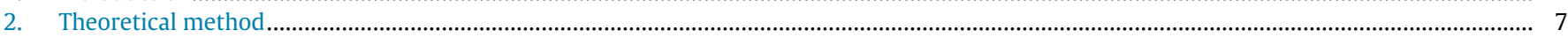

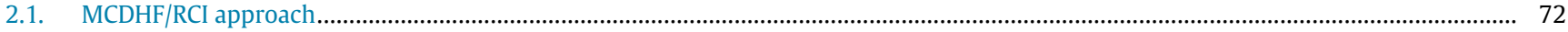

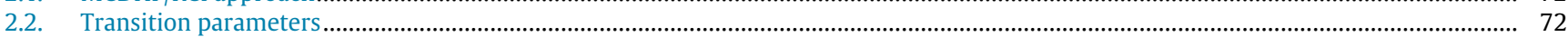

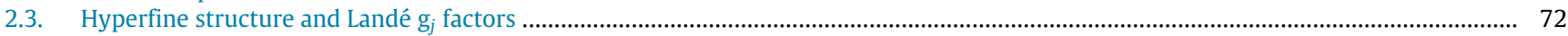

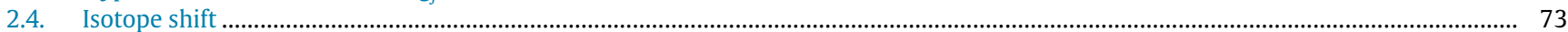

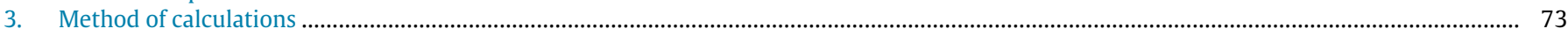

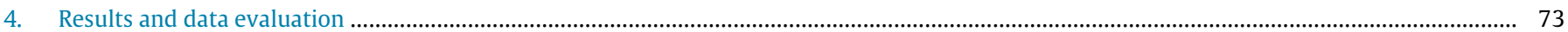

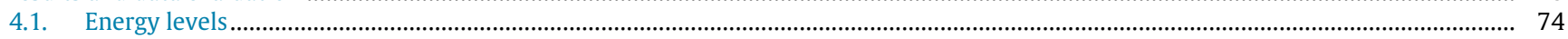

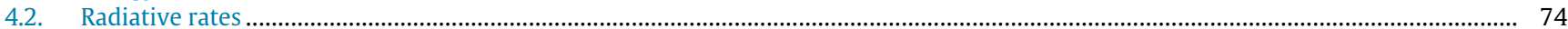

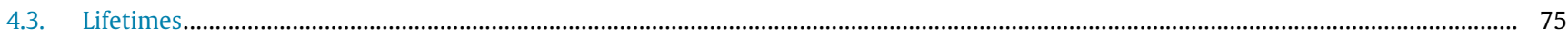

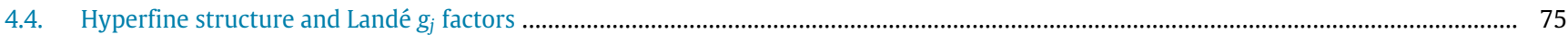

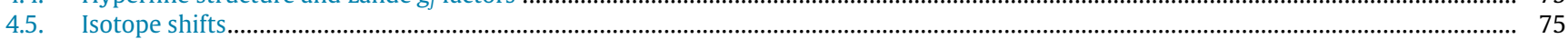

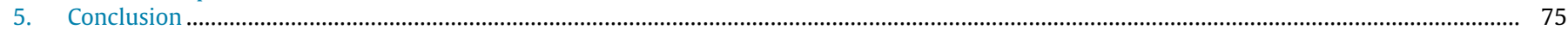

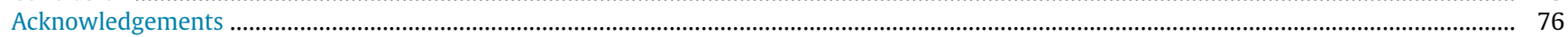

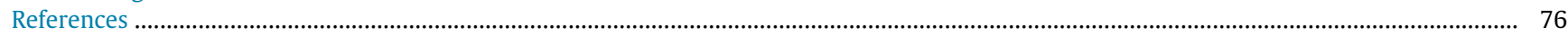

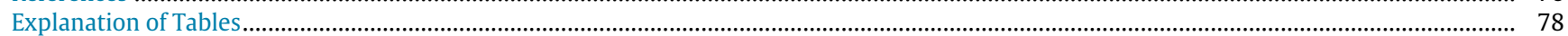

Table 1. Energies $\left(E\right.$ in $\mathrm{cm}^{-1}$ ) relative to the ground state for the lowest 97 levels arising from the $1 s^{2}$ and $1 \mathrm{~s} n l$ with $n=2-7$ configurations of Xe LIII. Two calculations are performed using FAC and GRASP2K and compared with data from NIST database. The GRASP1, GRASP2 and GRASP3 are determined respectively with increasing the active set (AS) up to $n=8, n=9$ and $n=10$. The last two columns denote the lifetimes (in $\mathrm{s}^{-1}$ ) obtained from our FAC and GRASP2K calculations......

Table 2. Calculated values using the GRASP2K code of the composition in LSJ coupling for all levels of interest with increasing the active set to $n=10$ (GRASP3).

Table 3. Electric dipole transitions $E 1$ calculated with GRASP2K and FAC for all $n=1-7$ configurations. For the GRASP2K calculation, the active set is expanded to $n=10$ (GRASP3) and only the length form is considered. A comparaison between the wavelengths, weighted oscillator strengths and transition probabilities is given. The relative difference between transition rates in the velocity and length gauge is given in the last column by $d T$.

Table 4. Electric quadrupole transitions E2 calculated with GRASP2K and FAC for all $n=1-7$ configurations. For the GRASP2K calculation, the active set is expanded to $n=10$ (GRASP3) and only the length form is considered. A comparaison between the wavelengths, weighted oscillator strengths and transition probabilities is given. The relative difference between transition probabilities in the velocity and length gauge is given in the last column by $d T$.

Table 5. Magnetic dipole transitions M1 calculated with GRASP2K and FAC for all $n=1-7$ configurations. For the GRASP2K calculation, the active set is expanded to $n=10$ (GRASP3) and only the length form is considered. A comparaison between the wavelengths, weighted oscillator strengths and transition probabilities is given...

Table 6. Magnetic quadrupole transitions M2 calculated with GRASP2K and FAC for all $n=1-7$ configurations. For the GRASP2K calculation, the active set is expanded to $n=10$ (GRASP3) and only the length form is considered. A comparaison between the wavelengths, weighted oscillator strengths and transition probabilities is given.

Table 7. Calculated values using the GRASP2K code of the magnetic dipole $A$, electric quadrupole $B$ and Landé $g$-factors for all levels of interest. For this calculation, the active set expanded to $n=10$ (GRASP3) has been considered.

Table 8. Calculated values using the GRASP2K code of normal, specific mass shifts and electron densities at the nucleus for the all singly excited levels up to $n=7$ with inceasing the active set to $n=10$ (GRASP3).......

\section{Introduction}

In recent years, there have been extensive spectroscopic studies, both experimental and theoretical, of helium isoelectronic sequence. An analysis of the spectra of solar, stellar and other astrophysical plasmas by many space missions, such as SOHO, Chandra and XMM Newton shows the presence of highly ionized atoms [1]. In addition, they are injected as impurities into tokamak plasmas [2,3]. In particular, high $Z$ elements of the fifth period are being increasingly used as injected impurities for tokamak fusion plasmas. Also, the inert gas atoms show a significant importance in the diagnostics of laboratory plasmas. Furthermore, atomic data (namely energy levels, weighted oscillator strengths, radiative rates, lifetimes, hyperfine structure and landé $\mathrm{g}_{j}$ factors and isotope shifts) are required for many ions in order to estimate the power loss from the impurities in the forthcoming fusion reactors, such as ITER project [4].

In the last few years, various theoretical and experimental research for providing atomic data for He-like ions have been carried out. But for He-like Xe, a limited number of studies has been carried out for providing atomic data for higher levels. In a recent review, Saloman [5] has listed energy levels for many ions of xenon, but unfortunately there is a paucity of data for this ion of interest.
These data are available at the National Institute of Standards and Technology (NIST) [6] database. Energy levels were calculated for the ground state and $n=2$ singlet states of heliumlike ions with nuclear charges in the range $4 \leq Z \leq 92$ by Cheng et al. [7]. Plante et al. [8] performed relativistic all-order manybody calculations on the He-isoelectronic sequence in the range $Z=5-100$ and included Breit energy and other higher order correction terms in their calculations. Drake [9] carried out extensive two-electron quantum electrodynamics (QED) calculations using a unified method. Indelicato [10] adopted the multi-configuration Dirac-Fock (MCDF) formalism to study the properties of He-like ions. Recently, energies and rates from $2 s 3 p-1 s 2 s$ transitions were calculated for 23 ions (He-like) in the range $Z=14-54$ using multi-configuration Dirac-Fock wave functions by L. Natarajan et al. [11].

For correct interpretation of the experimentally observed spectra in the stellar and laboratory induced high-temperature plasmas, accurate theoretical data regarding the atomic transitions of highly ionized atoms are needed [12].

As previously published [13-15], we continue to focus on He-like ions. We report in this work the hereabove mentioned parameters for a comparatively larger number of levels/transitions by performing a large-scale multiconfiguration Dirac-Hartree-Fock 
(MCDHF) [16] method to calculate the 97 lowest fine-structure levels for Xe LIII, in an effort to offer complete and consistent data sets of high accuracy. The calculations have been divided into two main groups, with even and odd parity. Also, wavelengths, weighted oscillator strengths, transition probabilities for the E1,E2,M1 and $M 2$ radiations have been computed. Accurate interpretation of stellar spectra requires the knowledge not only of weighted oscillator strengths, but also of isotope shifts and hyperfine structures, since they cause an asymmetry and splitting of atomic transitions. So, hyperfine structures, Landé $g_{j}$ factors and isotope shifts are also presented in this work.

We employ the FAC code [17] to compare some of these atomic data. The present MCDHF and FAC results are compared with each other and previous experimental and theoretical results, where available.

The configurations $1 s^{2}$ and $1 \operatorname{snl}(n=2-7, l \leq n-1)$ are included in this calculation. Breit interactions and quantum electrodynamics (QED) effects have been added. This computational approach enables us to present a consistent and improved data set of all important transitions of the Xe LIII spectrum, which are useful for identifying transition lines in further investigations.

\section{Theoretical method}

\section{1. $M C D H F / R C I$ approach}

Our calculations are performed by means of two numerical Codes. First, we employ the GRASP2K code. This is a fully relativistic code based on the Multiconfiguration Dirac-Hartree-Fock (MCDHF) method in jj-coupling. This method is fully described in detail by Grant [18]. Therefore, we only introduce the essential features here.

The atom is represented by an atomic state functions (ASF), which is a linear combination of configuration state functions (CSFs),

$\psi(\gamma P J M)=\sum_{j=1}^{N_{C S F}} c_{j} \Phi\left(\gamma_{j} P J M\right)$,

where $c_{j}$ are the mixing coefficients for the configuration $j, J$ and $M$ are the angular quantum numbers and $P$ is the parity. The label $\gamma_{j}$ denotes appropriate information about the configuration state function $j$, such as parity, orbital occupancy and angular coupling scheme.

For the CSF, as $\Phi\left(\gamma_{j} P J M\right)$, it is an antisymmetrized linear combination of products of relativistic orbitals. One relativistic orbital can be expressed as,

$\Phi(r)=\frac{1}{r}\left(\begin{array}{cc}P_{n \kappa}(r) & \chi_{\kappa m}(r) \\ i Q_{n \kappa}(r) & \chi_{-\kappa m}(r)\end{array}\right)$,

where $\kappa$ is the relativistic spherical symmetry quantum number, $m$ is the magnetic quantum number, $n$ is the principal quantum number, $P_{n \kappa}(r)$ and $Q_{n \kappa}(r)$ are the large and small component radial wavefunctions, and $\chi_{\kappa m}(r)$ is the spinor spherical harmonic in the lsj coupling scheme.

These relativistic orbitals can be generated by using an appropriate Hamiltonian. In our MCDHF calculations, the Dirac-Coulomb Hamiltonian can be described as,

$H_{D C}=\sum_{i=1}^{N}\left(c \alpha_{\mathbf{i}} \cdot \mathbf{p}_{\mathbf{i}}+\left(\beta_{i}-1\right) c^{2}+V\left(r_{i}\right)\right)+\sum_{i>j}^{N} \frac{1}{r_{i j}}$,

where $V\left(r_{i}\right)$ is the nuclear potential at radius $r, \alpha$ and $\beta$ are the $4 \times 4$ Dirac matrices, $\mathbf{p}_{\mathbf{i}}$ is the momentum operator of the electron $i$ and $c$ is the speed of light in atomic units. The first term describes the electron kinetic energy and the electron-nucleus interaction, and the second term is the two-body Coulomb interactions between the electrons.

Once a set of radial orbitals like $P_{n \kappa}(r)$ and $Q_{n \kappa}(r)$ has been obtained by self-consistent field procedure, they are used to determine the expansion coefficients of the CSFs by diagonalizing the Hamiltonian matrix.

The transverse interaction in the low-frequency limit, or the Breit interaction [19],

$H_{\text {Breit }}=-\sum_{i<j}^{N} \frac{1}{2 r_{i j}}\left[\alpha_{i} \cdot \alpha_{j}+\frac{\left(\alpha_{i} \cdot r_{i j}\right)\left(\alpha_{j} \cdot r_{i j}\right)}{r_{i j}^{2}}\right]$,

and leading quantum electrodynamic (QED) effects (vacuum polarization and self-energy) have been included in subsequent configuration interaction ( $\mathrm{RCI}$ ) calculations, where optimization is on a weighted sum of energies. At the end, a transformation from $j j$ - to $L S$-coupling [20] can be done for practical purposes.

\subsection{Transition parameters}

Transition parameters, such as transition rates or weighted oscillator strengths, between two states $\gamma P J$ and $\gamma^{\prime} P^{\prime} J^{\prime}$ can be described in terms of the reduced transition matrix element,

$\left\langle\psi(\gamma P J)\left\|Q_{k}^{(\lambda)}\right\| \psi\left(\gamma^{\prime} P^{\prime} J^{\prime}\right)\right\rangle$,

where $Q_{k}^{(\lambda)}$ is the electromagnetic multipole operator of order $k$ in Coulomb or Babushkin gauges [21]. The superscript designates the type of multipole: $\lambda=1$ for electric multipoles and $\lambda=0$ for magnetic multipoles. In cases where the wave functions of the two states $\gamma^{\prime} P^{\prime} J^{\prime}$ and $\gamma P J$ have been separately determined, the radial orbitals are not orthogonal. To deal with this complication, a transformation to a biorthonormal orbital basis was applied [22]. In the new representation, the evaluation of the matrix element has been done using standard Racah algebra techniques.

In order to ensure a rigorous accuracy assessment of results of energy levels and transition probabilities, we have performed another calculation with the widely used FAC code of Gu [17]. It is also a fully relativistic code which has higher efficiency. It enables user to carry out large-scale computations and allows finding various atomic parameters. It uses a modified Dirac-Fock-Slater central-field potential that includes an approximate treatment of the exchange interaction.

\subsection{Hyperfine structure and Landé $g_{j}$ factors}

The hyperfine interaction is caused by the interaction between the electrons and the electromagnetic multipole moments of the nucleus, and its Hamiltonian can be represented using the spherical tensor operators $\boldsymbol{T}^{(k)}$ and $\boldsymbol{M}^{(k)}$ [23],

$H_{\mathrm{hfs}}=\sum_{k \geq 1} \boldsymbol{T}^{(k)} \cdot \boldsymbol{M}^{(k)}$

Here, $k=1$ and $k=2$ represent the magnetic dipole and electric quadrupole interactions, respectively. For a $N$-electron atom, the electronic tensor operators $\boldsymbol{T}^{(1)}$ and $\boldsymbol{T}^{(2)}$ are the sums of the oneelectron operators $t^{(k)}$,

$\boldsymbol{T}^{(1)}=\sum_{j=1}^{N} \boldsymbol{t}^{(1)}(j)=\sum_{j=1}^{N}-i \alpha\left(\boldsymbol{\alpha}_{j} \cdot \mathbf{l}_{j} \boldsymbol{C}^{(1)}(j)\right) r_{j}^{-2}$

and

$\boldsymbol{T}^{(2)}=\sum_{j=1}^{N} \boldsymbol{t}^{(2)}(j)=\sum_{j=1}^{N}-\boldsymbol{C}^{(2)}(j) r_{j}^{-3}$. 
Here, $i$ is the imaginary unit, $\alpha$ is the fine-structure constant, $\boldsymbol{C}^{(1)}$ and $\boldsymbol{C}^{(2)}$ are spherical tensor operators, and $\mathbf{1}$ is the orbital angular momentum operator. The nuclear tensor operators $\boldsymbol{M}^{(1)}$ and $\boldsymbol{M}^{(2)}$ are related to magnetic dipole moment $\mu_{I}$ and electronic quadrupole moment $Q_{I}$ of the nucleus with spin $I$ through [24]

$\mu_{I}=\left\langle I M_{I}(=I)\left|\boldsymbol{M}_{0}^{(1)}\right| I M_{I}(=I)\right\rangle$

and

$Q_{I}=\left\langle M_{I}(=I)\left|\boldsymbol{M}_{0}^{(2)}\right| I M_{I}(=I)\right\rangle$.

The nuclear magnetic dipole moments $\mu_{I}$ and the nuclear quadrupole moments $Q_{I}$ for the different isotopes have been taken from a compilation by Stone [25].

According to perturbation theory, the magnetic dipole and the electric quadrupole $A_{J}$ and $B_{J}$ are [24]

$A_{J}=\frac{\mu_{I}}{I} \frac{1}{[J(J+1)]^{1 / 2}}\left\langle\Psi(P J)\left\|\boldsymbol{T}^{(1)}\right\| \Psi(P J)\right\rangle$

and

$B_{J}=2 Q\left[\frac{J(2 J-1)}{(J+1)(2 J+3)}\right]^{1 / 2}\left\langle\Psi(P J)\left\|\boldsymbol{T}^{(2)}\right\| \Psi(P J)\right\rangle$.

The Landé $\mathrm{g}_{j}$ factors are given by

$g_{J}=\frac{2}{\sqrt{J(J+1)}}\left\langle\gamma J\left\|\sum_{j=1}^{N}-i \frac{\sqrt{2}}{2 \alpha^{2}} r_{j}\left(\boldsymbol{\alpha}_{j} \mathbf{C}_{j}^{(1)}\right)^{(1)}+\frac{g_{s}-2}{2} \beta_{j} \Sigma_{j}\right\| \gamma J\right\rangle$,

where $i$ is the imaginary unit, $\Sigma$ the relativistic spin-matrix and $g_{s}=2.00232$ the $g$-factor of the electron spin corrected for QED effects. The Landé $g_{j}$ factors determine the splitting of magnetic sub-levels in external magnetic fields. In addition they give information about the coupling conditions in the system [26].

\subsection{Isotope shift}

The isotope shift (IS) for an atomic energy level is composed of the field shift (FS) and the mass shift (MS). The field shift, arising from the difference in the charge distribution between two isotopes with mass number $A$ and $A^{\prime}\left(A>A^{\prime}\right)$, is given in the approximation of the first-order perturbation theory by $[27,28]$

$\Delta E_{F S}^{A, A^{\prime}}=\left\langle\Psi(P J M)\left|\sum_{i} \delta V_{i}^{N, A A^{\prime}}\right| \Psi(P J M)\right\rangle$.

Here, $\delta V^{N, A A^{\prime}}=V^{N, A}-V^{N, A^{\prime}}$ and the nuclear potential $V^{N}$ for each isotope is produced by a two-parameter Fermi nuclear model [29,30]. Neglecting the higher-order nuclear moments [31], Eq. (14) is further simplified to

$\Delta E_{F S}^{A, A^{\prime}}=F \delta\left\langle r^{2}\right\rangle^{A, A^{\prime}}$,

where

$F_{k}=\frac{2 \pi}{3}\left(\frac{Z e^{2}}{4 \pi \epsilon_{0}}\right) \Delta|\Psi(\mathbf{0})|_{k}^{2}$

is the field-shift factor proportional to the total electron probability density at the origin between the levels $l$ and $u$,

$\Delta|\Psi(\mathbf{0})|_{k}^{2}=\Delta \rho_{k}^{e}(0)=\rho_{u}^{e}(0)-\rho_{l}^{e}(0)$

and $\delta\left\langle r^{2}\right\rangle^{A, A^{\prime}}$ is the difference of the nuclear charge mean square radius between these two isotopes.

The mass shift between two isotopes $A$ and $A^{\prime}$, caused by the motion of nucleus with the finite mass, is expressed as [32,33]

$\Delta E_{M S}^{A, A^{\prime}}=\frac{M^{\prime}-M}{M M^{\prime}} K_{M S}$.
Here, $M$ and $M^{\prime}$ are the nuclear masses for isotopes $A$ and $A^{\prime}$, respectively. The electronic factor $K_{M S}$ is defined by

$\frac{K_{M S}}{M} \equiv\left\langle\Psi(P J M)\left|H_{M S}\right| \Psi(P J M)\right\rangle$,

where

$H_{M S}=\frac{1}{2 M} \sum_{i, j} \boldsymbol{p}_{i} \cdot \boldsymbol{p}_{j}$.

The mass shift operator (Eq. (20)) can be split into two parts, that is, the one-body and the two-body mass shift operators

$H_{N M S}=\frac{1}{2 M} \sum_{i} p_{i}^{2}$

$H_{S M S}=\frac{1}{2 M} \sum_{i \neq j} \boldsymbol{p}_{i} \cdot \boldsymbol{p}_{j}$

which are also called the normal mass shift (NMS) and the specific mass shift (SMS) operator, respectively.

\section{Method of calculations}

The MCDHF/RCI calculations have been performed in the extended optimal level (EOL) scheme for each group of atomic states using configuration expansions including all lower states of the same parity and symmetry. For the optimization of the orbitals, Dirac-Coulomb version has been used, including Breit corrections in a final configuration interaction calculation.

To build a CSF expansion, the restrictive active space methods have been also used. The idea of the active space methods is to consider only electrons from the active space and to excite them from the occupied orbitals to unoccupied ones. The orbital has been increased systematically in order to monitor the convergence of the calculation. Since the orbitals with the same principal quantum number $\mathrm{n}$ often have similar energies, the active set is usually enlarged in steps of orbital layers. It is convenient to refer to the $\{1 s, 2 s, 2 p, 3 s, 3 p, 3 d, 4 s, 4 p, 4 d, 4 f\}$ set of orbitals as the $n=$ 4 orbital layer, $\{1 s, 2 s, 2 p, \ldots, 5 s, 5 p, 5 d, 5 f, 5 g\}$ as the $n=5$ layer, etc. We increase the AS in a systematic way to ensure the convergence of the atomic parameters under consideration. Only the outermost nl-orbitals are optimized while the inside ones are fixed. For example, the total number of CSF in the active set of $n=8$ is 3896 for even configurations and 3560 for odd configurations. For the active set of $n=9$, the number of CSF increases to 6104 and 5490 for even and odd, respectively. For the last active set of consideration $n=10$, the number will be 8824 and 7854 for even and odd, respectively. So, we can deduce that larger orbital sets can result in a considerable increase of computational time required for the problem, and appropriate restrictions may be necessary.

We have divided up the calculations into two parts, one where we optimized a set of orbitals for the even states and one for the odd states, i.e. the upper and lower states have been described by two independently optimized sets of orbitals. Because of this, we have to use biorthogonal transformation of the atomic state functions ASF to calculate the transition parameters. Radiative transition characteristics have been calculated for $E 1, M 1, E 2$ and M2 transitions, following the relativistic configuration-interaction $\mathrm{RCI}$ calculation in which both the Breit interaction and the other corrections to the DC Hamiltonian are included.

\section{Results and data evaluation}

In the following subsections, the results of the calculations are presented, and compared with values available from the literature. The identification of the levels is based on the LS composition obtained by transforming from $j j-$ to $L S$-coupling schemes using the $j j 2 l s j$ tool integrated in the new release of GRASP2K [16]. 


\subsection{Energy levels}

The calculated energy levels of $1 s^{2}, 1 s 2 l, 1 s 3 l, 1 s 4 l, 1 s 5 l, 1 s 6 l$ and $1 s 7 l$ configurations for the He-like xenon have been tabulated in Table 1, which generate up to 97 levels. These calculations are performed by means of two codes FAC and GRASP2K. The first column of results show the results from the FAC code. The other columns of GRASP1, GRASP2 and GRASP3 show the results from the GRASP2K code. The former results are calculated as function of increasing active sets with single excitations. All the calculated energy levels have been compared with experimental values. It is obvious that, when the active space is expanded to $n=8$ (GRASP1) and $n=9$ (GRASP2), the energy levels deviate very largely from experimental values taken from the National Institute of Standards and Technology NIST compilation [6], which is entirely based on the data listed by Ref [5]. When the active space set is expanded to $n=10$ (GRASP3), the calculated energy levels are substantially improved. So, we can clearly find that, with the increasing of active space set, the calculated energy levels are closer to experimental values.

The average difference between the GRASP3 values and NIST is $0.002 \%$ for the 8 states listed in the NIST database. The average difference between the calculated FAC and GRASP3 level energies is within $0.006 \%$ for all 97 states in Xe LIII while the average difference between the calculated FAC and NIST database level energies is $0.007 \%$. Small discrepancies can be observed in the GRASP and FAC energies mainly arise due to the different ways of calculations of electron wave-functions for radial orbitals and recoupling schemes of angular parts. Since some of energy levels are not available on NIST, our energy levels GRASP3 should be adopted in modelling applications. We may state that overall there is no discrepancy between theory and experiment and the results of GRASP3 are in good agreement with the experimental values for the energy levels of Xe LIII.

We compare also the lifetimes from the two calculations from the FAC and GRASP2K codes. Some large discrepancies have found for some levels like $1 s 2 s^{1} S_{0}$, $1 s 2 p^{3} P_{2}^{o}$, $1 s 2 p^{1} P_{1}^{o}$ when the differences are $14.54 \%, 6.62 \%$ and $7.00 \%$ respectively. For the others values the percentage do not exceed $2 \%$ for the almost levels.

We list in Table 2, the LSJ coupling percentage compositions for all levels of interest. The level composition in LSJ coupling values show that, the major contributions to the total wave function of a given level are those coming from the same configuration.

\subsection{Radiative rates}

The weighted oscillator strengths $g_{i} f_{i j}$ are related to the transition probabilities $A_{j i}$ for a transition from $i$ to $j$ by the following expression

$g_{i} f_{i j}=\frac{m c}{8 \pi^{2} e^{2}} \lambda_{j i}^{2} g_{j} A_{j i}=1.49 \times 10^{-16} \lambda_{j i}^{2} g_{j} A_{j i}$,

where $m$ and $e$ are the electron mass and charge, respectively, $c$ is the velocity of light, $\lambda_{j i}$ is the wavelength in $\AA, g_{i}$ and $g_{j}$ are the statistical weights of the lower $(i)$ and upper $(j)$ levels, respectively. Similarly, the weighted oscillator strength $g_{i} f_{i j}$ and the line strength $S$ are related by the standard equation for the electric dipole E1transitions,

$A_{j i}=\frac{2.0261 \times 10^{18}}{g_{j} \lambda_{j i}^{3}} S^{E 1} \quad$ and $\quad g_{i} f_{i j}=\frac{303.75}{\lambda_{j i}} S^{E 1}$

for the magnetic dipole $M 1$-transitions,

$A_{j i}=\frac{2.6974 \times 10^{13}}{g_{j} \lambda_{j i}^{3}} S^{M 1} \quad$ and $\quad g_{i} f_{i j}=\frac{4.044 \times 10^{-3}}{\lambda_{j i}} S^{M 1}$ for the electric quadrupole E2-transitions,

$A_{j i}=\frac{1.1199 \times 10^{18}}{g_{j} \lambda_{j i}^{5}} S^{E 2} \quad$ and $\quad g_{i} f_{i j}=\frac{167.89}{\lambda_{j i}^{3}} S^{E 2}$

and for the magnetic quadrupole $M 2$-transitions,

$A_{j i}=\frac{1.4910 \times 10^{13}}{g_{j} \lambda_{j i}^{5}} S^{M 2} \quad$ and $\quad g_{i} f_{i j}=\frac{2.236 \times 10^{-3}}{\lambda_{j i}^{3}} S^{M 2}$.

Below, we present and discuss our determined $g f$-values and $A$-values for transitions in Xe LIII for all types of transitions $E 1, E 2$, $M 1$ and M2 of all levels of energies in interest $1 s^{2}$ and $1 \mathrm{snl}(n=$ 2-7). The effects of Breit and QED corrections are assessed by our calculations. We should mention that for the GRASP2K calculation, the active set is expanded to $n=10$ (GRASP3) and only the length form for transition probabilities has tabulated in Tables 36 . We present in these tables the wavelengths, weighted oscillator strengths $g_{i} f_{i j}$ and radiative rates $A_{j i}$ for 1054 electric dipole $E 1$, 1389 electric quadrupole $E 2,1012$ magnetic dipole $M 1$ and 1430 magnetic quadrupole $M 2$ transitions among the 97 levels of Xe LIII calculated with GRASP2K and FAC codes. However, for these transitions only the $g f$-values and $A$-values are listed and the corresponding results for $S$-values can be easily obtained. We also present the uncertainty of the electric transitions $d T$ to provide an additional indicator of the accuracy of our GRAPS2K results in Tables 3 and 4.

We compare our calculated transition energy of the $1 s 2 s^{3} S_{1}$ $1 s 2 p^{3} P_{2}^{o}\left(3756964 \mathrm{~cm}^{-1}\right)$ transition with the experimental value measured by S. Martin [34] $\left(3750937 \mathrm{~cm}^{-1}\right)$ and other theoretical results elaborated by Indelicato [35] $\left(3752138 \mathrm{~cm}^{-1}\right)$ and Drake [9] (3751576 $\mathrm{cm}^{-1}$ ). Moreover, the calculated wavelength of the former transition is $26.617 \AA$ where the experimental value is $26.66 \AA$. We state that the differences are minor. Furthermore, our calculated wavelengths compared to the data from the NIST database shows a good agreement such as the case of transitions $1 s^{2}{ }^{1} S_{0}-1 s 2 p^{3} P_{1}^{o}(0.4104 \AA)$ and $1 s^{2}{ }^{1} S_{0}-1 s 2 p^{1} P_{1}^{o}(0.4047 \AA)$.

For almost, all strong transitions (those with $g f \geq 0.01$ ), the gf values and $A$-values from GRASP2K agree to better than $10 \%$ with those of FAC, which is satisfactory. Moreover, for many strong transitions, the difference between both velocity and length forms, does not exceed $2 \%$, giving its credit to the accuracy of calculations of wave functions from GRASP2K. To confirm that, we have plotted in Fig. 1, the uncertainty $d T$ as a function of transition rates of type E1.

We remind that the uncertainty $d T$ is generated by only the GRASP2K code and it is expressed by

$d T=\frac{\left|A_{l}-A_{\nu}\right|}{\max \left(A_{l}, A_{v}\right)}$,

where $A_{l}$ and $A_{v}$ are the transition rates calculated in length and velocity gauges, respectively.

However, for some weak transitions ( $g f<0.01$ ), the situation is more unclear such as the $1 s 2 s^{3} S_{1}-1 s 4 f{ }^{3} F_{2}^{o}$ transition ( $g f=$ $3.265 \times 10^{-09}$ ) for the transition of type $E 1$, the difference between two sets of $A$-values is large. We note also that we have large difference in some transitions because their transition energy is very small, such as $1 s 3 p^{3} P_{1}^{o}-1 s 3 s^{1} S_{0}, 1 s 4 p^{3} P_{1}^{o}-1 s 4 s^{1} S_{0}$ and $1 s 5 p^{3} P_{1}^{o}-1 s 5 s^{1} S_{0}$ for the type of $E 1$ transitions and the same case for $E 2, M 1$ and $M 2$ transitions. Hence, a slight variation in $\triangle E$ has a considerable effect on the values of $A$. Moreover, the contribution of the $A$-values for weak transitions that required in modelling applications is (generally) small in comparison to the stronger transitions.

Therefore, on the basis of these comparisons and the above discussion, we may state that for a majority of strong $E 1, E 2$, $M 1$ and $M 2$ transitions, our radiative rates are accurate to better than $10 \%$. However, for the weak transitions, this assessment of accuracy is not applied. 


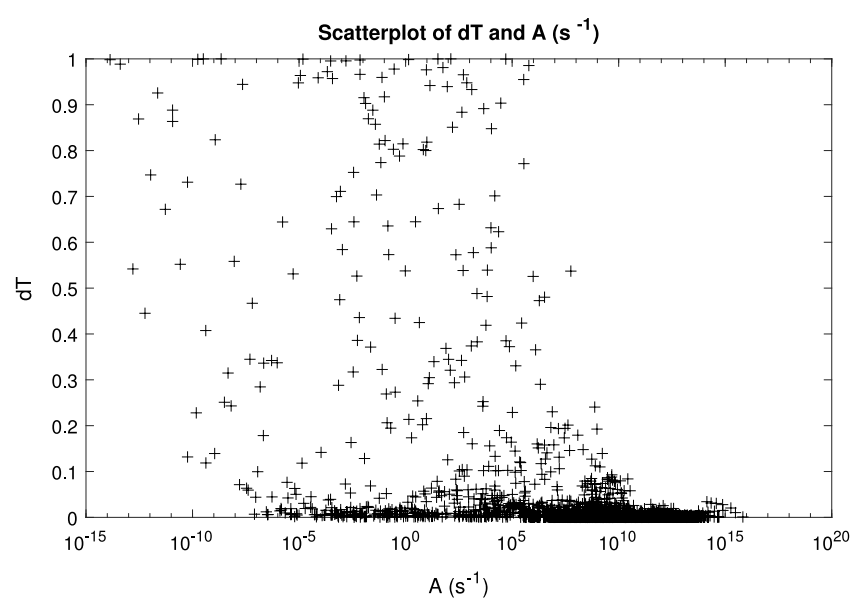

Fig. 1. The uncertainty $d T$ by the GRASP2K code between the velocity and length forms of E1 transition rates as function of E1 transition rates $A$.

\subsection{Lifetimes}

The lifetime $\tau$ for a level $j$ is defined as follows

$\tau_{j}=\frac{1}{\sum_{i} A_{j i}}$.

Since this is a measurable parameter. It provides a check on the accuracy of the calculations. Therefore, in Table 1 we have also listed our calculated lifetimes from the GRASP2K code, which include the contributions from four types of transitions: $E 1, E 2, M 1$ and $M 2$. To our knowledge, neither calculations nor measurements were determined for Xe LIII ion. We hope the present results will be useful for future comparisons and may encourage experimentalists to measure their lifetimes.

\subsection{Hyperfine structure and Landé $g_{j}$ factors}

The calculation of the hyperfine interaction constants and Landé $g_{j}$ factors have been performed only with the GRASP2K code. In the case of our ion Xe LIII, the constants for the spin I, magnetic dipole moments $\mu_{I}$ and the nuclear quadrupole moment $Q_{I}$ were taken $1.5,0.691 \times \mu_{N}$ and -0.1 barn, respectively. $\mu_{N}$ is the nuclear magneton.

The active set (AS) is considered up to $n=10$ and $l=7$ for all the configurations of interest $1 \mathrm{snl}(n=1-7)$. We list in Table A some of results of calculation of the magnetic dipole $A$ and electric quadrupole $B$ hyperfine interaction constants and Landé $g_{j}$ factors for the four lowest states $1 s 2 s^{3} S_{1}, 1 s 2 p^{3} P_{1}^{o}, 1 s 2 p^{3} P_{2}^{o}$ and $1 s 2 p^{1} P_{1}^{o}$ to study the effect of increasing the AS in the hyperfine structure and the Lande $g_{j}$ factors. We observe that the variation is minor. We can state that no influence of increasing set in the calculation of these hyperfine interaction constants.

In Table 7, we tabulate the hyperfine structure and Landé $g_{j}$ factors for all levels of interest.

We have been unable to compare our predicted results of the hyperfine structure and Landé $g_{j}$ factors with any other experimental or theoretical results because the required results are missing. However, our accurate values of Landé $g_{j}$ factors and hyperfine structure constants are of great interest for atomic spectroscopy researchers.

\subsection{Isotope shifts}

The isotope shift and the electron densities at the nucleus parameters are calculated in a first-order perturbation theory using
Table A

Calculated values using the GRASP2K code of magnetic dipole $A$, electric quadrupole $B$ and Landé $g$-factors with increasing the active set to $n=10$ (GRASP3).

\begin{tabular}{|c|c|c|c|c|}
\hline AS & $1 s 2 s^{3} S_{1}$ & $1 s 2 p^{3} P_{1}^{o}$ & $1 s 2 p^{3} P_{2}^{o}$ & $1 s 2 p^{1} P_{1}^{o}$ \\
\hline \multicolumn{5}{|c|}{ Magnetic dipole $A$ (in $\mathrm{MHz}$ ) } \\
\hline$n=3$ & $1.328 \mathrm{E} 7$ & $1.218 \mathrm{E} 7$ & $5.963 \mathrm{E} 6$ & $-5.633 \mathrm{E} 6$ \\
\hline$n=4$ & $1.328 \mathrm{E} 7$ & 1.218E7 & 5.963E6 & $-5.633 \mathrm{E} 6$ \\
\hline$n=5$ & $1.328 \mathrm{E} 7$ & $1.218 \mathrm{E} 7$ & 5.963E6 & $-5.633 \mathrm{E} 6$ \\
\hline$n=6$ & $1.328 \mathrm{E} 7$ & $1.218 \mathrm{E} 7$ & 5.963E6 & $-5.633 \mathrm{E} 6$ \\
\hline$n=7$ & $1.328 \mathrm{E} 7$ & $1.218 \mathrm{E} 7$ & 5.963E6 & $-5.633 \mathrm{E} 6$ \\
\hline$n=8$ & $1.328 \mathrm{E} 7$ & $1.218 \mathrm{E} 7$ & $5.964 \mathrm{E} 6$ & $-5.629 \mathrm{E} 6$ \\
\hline$n=9$ & $1.328 \mathrm{E} 7$ & $1.218 \mathrm{E} 7$ & $5.965 \mathrm{E} 6$ & $-5.633 \mathrm{E} 6$ \\
\hline$n=10$ & $1.329 \mathrm{E} 7$ & $1.218 \mathrm{E} 7$ & $5.965 \mathrm{E} 6$ & $-5.636 \mathrm{E} 6$ \\
\hline \multicolumn{5}{|c|}{ Electric quadrupole $B$ (in $\mathrm{MHz}$ ) } \\
\hline$n=3$ & -2.062 & 4603 & -64830 & -34880 \\
\hline$n=4$ & -1.8 & 4614 & -64830 & -34820 \\
\hline$n=5$ & -1.048 & 4615 & -64830 & -34810 \\
\hline$n=6$ & -0.7197 & 4550 & -64810 & -34820 \\
\hline$n=7$ & -0.5684 & 4561 & -64810 & -34820 \\
\hline$n=8$ & -0.4849 & 4475 & -65310 & -35650 \\
\hline$n=9$ & 5.223 & 4480 & -64910 & -35320 \\
\hline$n=10$ & 1.941 & 4527 & -64660 & -34880 \\
\hline \multicolumn{5}{|c|}{ Landé $g$-factors } \\
\hline$n=3$ & 1.93514 & 1.28539 & 1.459 & 1.15044 \\
\hline$n=4$ & 1.93515 & 1.28538 & 1.45899 & 1.15047 \\
\hline$n=5$ & 1.93515 & 1.28538 & 1.45899 & 1.15047 \\
\hline$n=6$ & 1.93515 & 1.28539 & 1.45899 & 1.15047 \\
\hline$n=7$ & 1.93515 & 1.28539 & 1.45899 & 1.15047 \\
\hline$n=8$ & 1.93515 & 1.28532 & 1.45904 & 1.15036 \\
\hline$n=9$ & 1.93516 & 1.28523 & 1.45905 & 1.15054 \\
\hline$n=10$ & 1.93517 & 1.28519 & 1.45897 & 1.15063 \\
\hline
\end{tabular}

the $\mathrm{MCDHF} / \mathrm{RCI}$ atomic state functions as the zero-order wavefunctions. In Table $B$, we start by studying the effect of increasing the active set (AS) up to $n=10$. For that, seven lowest levels $1 s^{2}{ }^{1} S_{0}$, $1 s 2 s^{3} S_{1}, 1 s 2 p^{3} P_{1}^{o}, 1 s 2 p^{3} P_{0}^{o}, 1 s 2 s^{1} S_{0}, 1 s 2 p^{3} P_{2}^{o}$ and $1 s 2 p^{1} P_{1}^{o}$ are chosen for consideration. No major effect is observed with increasing the active set. All calculations are performed with increasing the reference configurations up to $n=7$ when the active set AS is enlarged step by step up to $n=10$ and $l=7$. Moreover, all these parameters are displayed in Table 8 for all the known levels belonging to the configurations $1 s^{2}$ and 1 snl with $n=2-7, l \leq 6$.

We plot the calculated specific mass shifts (SMS) using the GRASP2K code, only for the ground state $1 s^{2}$ with increasing the active set AS up to $n=10$ in Fig. 2. The result is compared with previous works, MCDF [36], MCHF [36] and Drake [9]. Our result matches well with the result of the MCDF work which proves the reliability of results. The difference with the other results can be explained by the different ways of calculation of codes. The GRASP2K and MCDF are considered as a relativistic codes while MCHF is a non relativistic one. The difference between the results of the two relativistic codes does not exceed $2.2 \%$ but the differences with the MCHF and Drake are approximately $15 \%$.

\section{Conclusion}

In the present study, fine structure energy levels, wavelengths, weighted oscillator strengths, transition probabilities for $E 1, E 2$, $M 1$ and $M 2$ transitions among 97 energy levels belonging to He-like xenon are presented. Additionally, lifetimes, hyperfine structure constants, Landé $g_{j}$ factors and isotope shifts have been reported, although no measurements or other theoretical results are available for comparison except one work treating the calculation of only the ground state $1 s^{2}{ }^{1} S_{0}$. The self-consistent field approximation and the Breit interaction Hamiltonian as well as QED effects have been included in the calculations to improve the generated wave functions. The calculated energy levels, weighted 
Table B

Calculated values using the GRASP2K code of normal, specific mass shifts and electron densities at the nucleus for the seven lowest levels with increasing active set AS up to $n=10$.

\begin{tabular}{|c|c|c|c|c|c|c|c|}
\hline AS & $1 s^{2}{ }^{1} S_{0}$ & $1 s 2 s^{3} S_{1}$ & $1 s 2 p^{3} P_{1}^{o}$ & $1 s 2 p^{3} P_{0}^{o}$ & $1 s 2 s{ }^{1} S_{0}$ & $1 s 2 p^{3} P_{2}^{o}$ & $1 s 2 p^{1} P_{1}^{o}$ \\
\hline \multicolumn{8}{|c|}{ Normal mass shift NMS (a.u.) } \\
\hline$n=3$ & 2862.371 & 1818.908 & 1816.760 & 1815.086 & 1816.736 & 1803.102 & 1802.086 \\
\hline$n=4$ & 2862.365 & 1819.197 & 1816.720 & 1815.010 & 1815.793 & 1803.182 & 1801.819 \\
\hline$n=5$ & 2862.337 & 1819.261 & 1816.695 & 1814.981 & 1815.592 & 1803.179 & 1801.750 \\
\hline$n=6$ & 2862.332 & 1819.284 & 1816.689 & 1814.974 & 1815.522 & 1803.185 & 1801.726 \\
\hline$n=7$ & 2862.328 & 1819.295 & 1816.686 & 1814.971 & 1815.488 & 1803.186 & 1801.717 \\
\hline$n=8$ & 2862.349 & 1819.317 & 1818.194 & 1816.601 & 1815.483 & 1804.312 & 1803.566 \\
\hline$n=9$ & 2862.115 & 1819.47 & 1817.501 & 1815.864 & 1815.214 & 1803.816 & 1802.661 \\
\hline$n=10$ & 2862.115 & 1819.58 & 1817 & 1815.49 & 1815.192 & 1803.659 & 1802.099 \\
\hline \multicolumn{8}{|c|}{ Specific mass shift SMS (a.u.) } \\
\hline$n=3$ & 8.717903 & 0.5995701 & -80.16853 & -195.2675 & 2.357866 & -205.907 & 83.54837 \\
\hline$n=4$ & 8.70804 & 0.6207144 & -80.2113 & -195.2448 & 2.119602 & -205.9269 & 83.36892 \\
\hline$n=5$ & 8.680987 & 0.6271347 & -80.20777 & -195.2282 & 2.042898 & -205.9166 & 83.34515 \\
\hline$n=6$ & 8.663317 & 0.6302334 & -80.15193 & -195.1577 & 2.007405 & -205.89 & 83.36138 \\
\hline$n=7$ & 8.65289 & 0.6318741 & -80.15315 & -195.1561 & 1.988378 & -205.8906 & 83.35634 \\
\hline$n=8$ & 8.651809 & 0.6336825 & -80.89982 & -197.0734 & 1.980105 & -207.2639 & 84.04787 \\
\hline$n=9$ & 8.580615 & 0.65771 & -80.56291 & -196.2107 & 1.67668 & -206.4653 & 83.54352 \\
\hline$n=10$ & 8.37281 & 0.65445 & -80.2194 & -195.3285 & 1.61861 & -205.8238 & 83.21168 \\
\hline \multicolumn{8}{|c|}{ Electron densities at the nucleus (a.u.) } \\
\hline$n=3$ & 235656.3 & 136365.8 & 120103 & 119991.8 & 136038.1 & 119543.4 & 119715.1 \\
\hline$n=4$ & 235660.7 & 136377.1 & 120099.6 & 119989.3 & 136019.9 & 119540.7 & 119710.3 \\
\hline$n=5$ & 235658.2 & 136379.7 & 120096.3 & 119986.4 & 136016 & 119537.7 & 119706.4 \\
\hline$n=6$ & 235657.7 & 136380.8 & 120095.9 & 119986 & 136015.2 & 119537.4 & 119705.8 \\
\hline$n=7$ & 235657.2 & 136381.3 & 120095.5 & 119985.7 & 136014.9 & 119537 & 119705.2 \\
\hline$n=8$ & 235659.3 & 136382.9 & 120102.9 & 119998 & 136016.1 & 119534.3 & 119697.6 \\
\hline$n=9$ & 235598.7 & 136395.2 & 120130 & 120023.1 & 136025.1 & 119570.9 & 119733.3 \\
\hline$n=10$ & 235581.5 & 136426.2 & 120162.2 & 120117.3 & 136014.9 & 119664.2 & 119832.8 \\
\hline
\end{tabular}

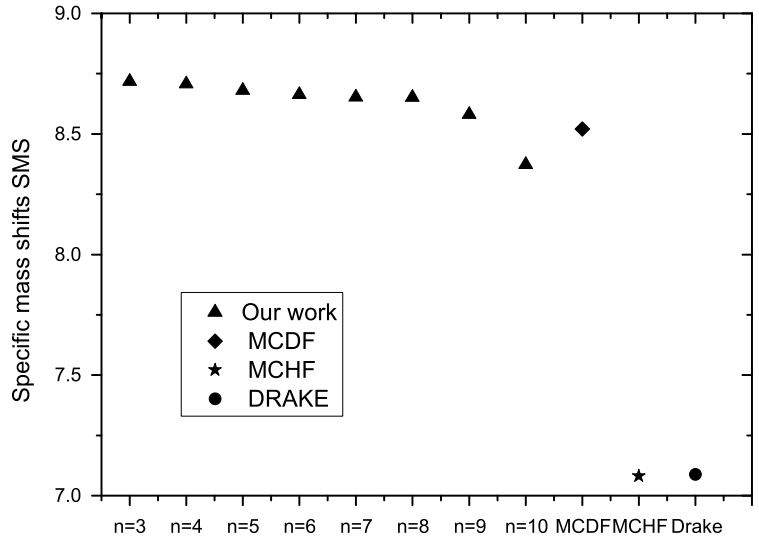

Fig. 2. Comparison of specific mass shifts (in a.u.) for the ground state $1 s^{2}{ }^{1} S_{0}$ with previous works MCDF [36], MCHF [36], Drake [9].

oscillator strengths and all radiative transitions show a good agreement with other published results from the literature. In addition, we have obtained some new data for the hyperfine structure constants, Landé $g_{j}$ factors and isotope shifts for this ion. The present data sets are believed to be the most comprehensive and accurate ones to date for Xe LIII. Our results are expected to be useful for many applications such as controlled thermonuclear fusion, laser and plasma physics as well as astrophysics.

\section{Acknowledgements}

PQ is Research Director of the Belgian National Fund for Scientific Research F.R.S.-FNRS (FC 49142). Financial support from this organization is gratefully acknowledged. Also, this work has been realized with the financial support of the Tunisian Ministry of Higher Education and Scientific Research (LR16CNSTN02).

\section{References}

[1] E. Landi, P.R. Young, K.P. Dere, G. Del Zanna, H.E. Mason, Astrophys. J. 109 (2013) 86

[2] F.P. Keenan, S.M. McCann, Solar Phys. 109 (1987) 01

[3] K.J.H. Phillips, F.P. Keenan, L.K. Harra, S.M. McCann, E. Rachlew-Kallne, J.E. Rice, M. Wilson, J. Phys. B: At. Mol. Opt. Phys. 27 (1994) 10.

[4] A.J.H. Donne, et al., Nucl. Fusion 47 (2007) S337.

[5] E.B. Saloman, J. Phys. Chem. Ref. Data 33 (2004) 765.

[6] A. Kramida, Yu. Ralchenko, J. Reader, NIST ASD Team, NIST Atomic Spectra Database (ver. 5.5.2), National Institute of Standards and Technology, Gaithersburg, MD, 2018. [Online]. Available: https://physics.nist.gov/asd [2018, February 20]

[7] K.T. Cheng, M.H. Chen, W.R. Johnson, J. Sapirstein, Phys. Rev. A 50 (1994) 247.

[8] D.R. Plante, W.R. Johnson, J. Sapiretein, Phys. Rev. A 49 (1994) 3519

[9] G.W.F. Drake, Can. J. Phys. 66 (1988) 586.

[10] P. Indelicato, Nucl. Instrum. Methods Phys. Res. B 31 (1988) 14.

[11] L. Natarajan, A. Natarajan, Phy. Rev. A 79 (2009) 062513.

[12] K.P. Dere, Astrophys. J. 221 (1978) 1062.

[13] D.E. Salhi, H. Jelassi, Procedings of the 13th Arab Conference on the Peaceful Uses of Atomic Energy, 18-22 December 2016, Hammamet, Tunisia.

[14] D.E. Salhi, H. Jelassi, Can. J. Phys. 96 (3) (2018) 343-352.

[15] D.E. Salhi, H. Jelassi, Orient. J. Phys. Sci. 2 (2) (2017).

[16] P. Jönsson, G. Gaigalas, J. Bieron, C. Froese Fischer, I.P. Grant, Comput. Phys Comm. 184 (2013) 2197

[17] M.F. Gu, Can. J. Phys. 86 (2008) 5.

[18] I.P. Grant, Relativistic Quantum Theory of Atoms and Molecules, Springer, New York, 2007.

[19] B.J. McKenzie, I.P. Grant, P.H. Norrington, Comput. Phys. Comm. 21 (1980) 02.

[20] G. Gaigalas, T. Zalandauskas, Z. Rudzikas, Data Nucl. Data Tables 84 (2003) 02

[21] I.P. Grant, J. Phys. B 7 (1974) 1458.

[22] J. Olsen, M.R. Godefroid, P. Jönsson, P.A. Malmqvist, C. Froese Fischer, Phys. Rev. E 52 (1995) 4499.

[23] C. Schwartz, Phys. Rev. 97 (1955) 380.

[24] P. Jönsson, F.A. Parpia, C.F. Fischer, Comput. Phys. Comm. 96 (1996) 301.

[25] N.J. Stone, At. Data Nucl. Data Tables 90 (2005) 75.

[26] C. Froese Fischer, P. Jönsson, J. Mol. Struct.: Theochem. 537 (2001) 55.

[27] S.A. Blundell, P.E.G. Baird, C.W.P. Palmer, D.N. Stacey, G.K. Woodgate, J. Phys, B: At. Mol. Phys. 20 (1987) 3663.

[28] G. Torbohm, B. Fricke, A. Rosén, Phys. Rev. A 31 (1985) 2038.

[29] F.A. Parpia, A.K. Mohanty, Phys. Rev. A 46 (1992) 3735. 
[30] J.G. Li, C. Nazé, M. Godefroid, S. Fritzsche, G. Gaigalas, P. Indelicato, P. Jönsson, Phys. Rev. A 86 (2012) 022518.

[31] E.C. Seltzer, Phys. Rev. 188 (1969) 1916

[32] C.W.P. Palmer, J. Phys. B: At. Mol. Phys. 20 (1987) 5987.

[33] E. Gaidamauskas, C. Nazé, P. Rynkun, G. Gaigalas, P. Jönsson, G. Gaigalas, J. Phys. B: At. Mol. Opt. Phys. 44 (2011) 175003.
[34] S. Martin, J.P. Buchet, M.C. Buchet-Poulizac, A. Denis, J. Desesquelles, M. Druetta, J.P. Grandin, D. Hennecart, X. Husson, D. Lecler, Europhys. Lett. 10 (7) (1989) 645-649.

[35] P. Indelicato, D. Gorceix, J.P. Desclaux, J. Phys. B 20 (1987) 651

[36] M.I. Bhatti, M. Bucardo, W.F. Perger, J. Phys. B: At. Mol. Opt. Phys. 34 (2001) $223-231$ 


\section{Explanation of Tables}

Table 1. Energies $\left(E\right.$ in $\left.\mathrm{cm}^{-1}\right)$ relative to the ground state for the lowest 97 levels arising from the $1 s^{2}$ and $1 s n l$ with $n=2-7$ configurations of Xe LIII. Two calculations are performed using FAC and GRASP2K and compared with data from NIST database. The GRASP1, GRASP2 and GRASP3 are determined respectively with increasing the active set (AS) up to $n=8, n=9$ and $n=10$. The last two columns denote the lifetimes (in $\mathrm{s}^{-1}$ ) obtained from our FAC and GRASP2K calculations.

Configuration Configuration of energy level

Level Configuration of energy level

FAC Energy levels calculated with the FAC code

GRASP1 Energy levels calculated with the GASP2K code up to $n=8$

GRASP2 Energy levels calculated with the GASP2K code up to $n=9$

GRASP3 Energy levels calculated with the GASP2K code up to $n=10$

NIST $\quad$ Energy levels compiled by the NIST database

Lifetimes (GRASP2K) compilation or from current literature

Lifetimes (FAC) Lifetimes calculated with the FAC code

Lifetimes (GRASP2K) Lifetimes calculated with the GRASP2K code

Table 2. Calculated values using the GRASP2K code of the composition in LSJ coupling for all levels of interest with increasing the active set to $n=10$ (GRASP3).

Leve

Configuration of the level of energy

Composition in LSJ coupling Composition in LSJ coupling for each level of energy

Table 3. Electric dipole transitions E1 calculated with GRASP2K and FAC for all $\boldsymbol{n}=1-7$ configurations. For the GRASP2K calculation, the active set is expanded to $n=10$ (GRASP3) and only the length form is considered. A comparaison between the wavelengths, weighted oscillator strengths and transition probabilities is given. The relative difference between transition rates in the velocity and length gauge is given in the last column by $d T$.

Lower Lower state of energy

Upper $\quad$ Upper state of energy

$\lambda(\AA) \quad$ Wavelengths calculated with the GRASP2K and FAC codes

gf Weighted oscillator strengths calculated with the GRASP2K and FAC codes

$A\left(\mathrm{~s}^{-1}\right) \quad$ Electric dipole transitions $E 1$ calculated with the GRASP2K and FAC codes

$d T \quad$ Uncertainty between the transition rates in the velocity and length gauge

Table 4. Electric quadrupole transitions $E 2$ calculated with GRASP2K and FAC for all $n=1-7$ configurations. For the GRASP2K calculation, the active set is expanded to $n=10$ (GRASP3) and only the length form is considered. A comparaison between the wavelengths, weighted oscillator strengths and transition probabilities is given. The relative difference between transition probabilities in the velocity and length gauge is given in the last column by $d T$.

Lower Lower state of energy

Upper $\quad$ Upper state of energy

$\lambda(\AA) \quad$ Wavelengths calculated with the GRASP2K and FAC codes

gf Weighted oscillator strengths calculated with the GRASP2K and FAC codes

$A\left(\mathrm{~s}^{-1}\right) \quad$ Electric dipole transitions $E 2$ calculated with the GRASP2K and FAC codes

$d T \quad$ Uncertainty between the transition rates in the velocity and length gauge

Table 5. Magnetic dipole transitions M1 calculated with GRASP2K and FAC for all $n=1-7$ configurations. For the GRASP2K calculation, the active set is expanded to $n=10$ (GRASP3) and only the length form is considered. A comparaison between the wavelengths, weighted oscillator strengths and transition probabilities is given.

Lower Lower state of energy

Upper Upper state of energy

$\lambda(\AA) \quad$ Wavelengths calculated with the GRASP2K and FAC codes

gf Weighted oscillator strengths calculated with the GRASP2K and FAC codes

$A\left(\mathrm{~s}^{-1}\right) \quad$ Electric dipole transitions $E 1$ calculated with the GRASP2K and FAC codes

Table 6. Magnetic quadrupole transitions $M 2$ calculated with GRASP2K and FAC for all $n=1-7$ configurations. For the GRASP2K calculation, the active set is expanded to $n=10$ (GRASP3) and only the length form is considered. A comparaison between the wavelengths, weighted oscillator strengths and transition probabilities is given.

Lower Lower state of energy

Upper Upper state of energy

$\lambda(\AA) \quad$ Wavelengths calculated with the GRASP2K and FAC codes

gf Weighted oscillator strengths calculated with the GRASP2K and FAC codes

$A\left(\mathrm{~s}^{-1}\right) \quad$ Electric dipole transitions $E 1$ calculated with the GRASP2K and FAC codes

Table 7. Calculated values using the GRASP2K code of the magnetic dipole $A$, electric quadrupole $B$ and Landé $g$-factors for all levels of interest. For this calculation, the active set expanded to $n=10$ (GRASP3) has been considered.

Configuration Configuration of the level of energy

$A(\mathrm{MHz}) \quad$ Magnetic dipole $A$

$B(\mathrm{MHz}) \quad$ Electric quadrupole $B$

g $\quad$ Landé $g$-factors

Table 8. Calculated values using the GRASP2K code of normal, specific mass shifts and electron densities at the nucleus for the all singly excited levels up to $n=7$ with inceasing the active set to $n=10$ (GRASP3).

Index

Configuration

$K_{N M S}$

$K_{S M S}$

Configuration of the level of energy

Normal mass shifts

Electron densities at the Electron densities at the nucleus

nucleus 
Table 1

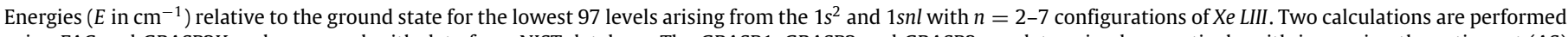

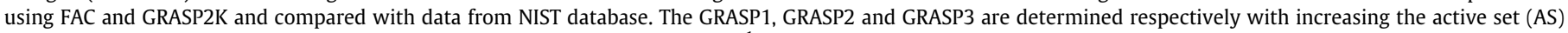
up to $n=8, n=9$ and $n=10$. The last two columns denote the lifetimes (in $\mathrm{s}^{-1}$ ) obtained from our FAC and GRASP2K calculations.

\begin{tabular}{|c|c|c|c|c|c|c|c|c|}
\hline Configuration & Level & FAC & GRASP1 & GRASP2 & GRASP3 & NIST & Lifetimes (FAC) & Lifetimes (GRASP2K) \\
\hline $1 s^{2}$ & ${ }^{1} S_{0}$ & 0 & 0 & 0 & 0 & 0 & & \\
\hline $1 s 2 s$ & ${ }^{3} S_{1}$ & 242981032 & 242992840 & 242996188 & 242999633 & 243004900 & $2.6569 \mathrm{E}-12$ & $2.6101 \mathrm{E}-12$ \\
\hline $1 s 2 p$ & ${ }^{3} P_{1}^{o}$ & 243610281 & 243619151 & 243620649 & 243622562 & 243626600 & $3.3975 \mathrm{E}-16$ & $3.3084 \mathrm{E}-16$ \\
\hline $1 s 2 p$ & ${ }^{3} P_{0}^{0}$ & 243656320 & 243667841 & 243669210 & 243670879 & 243674700 & $5.7419 \mathrm{E}-10$ & $5.8607 \mathrm{E}-10$ \\
\hline $1 s 2 s$ & ${ }^{1} S_{0}$ & 243677020 & 243680822 & 243683119 & 243685687 & 243691200 & $6.5427 \mathrm{E}-07$ & $7.6560 \mathrm{E}-07$ \\
\hline $1 s 2 p$ & ${ }^{3} P_{2}^{o}$ & 246740070 & 246751551 & 246753838 & 246756598 & 246756600 & $3.2404 \mathrm{E}-13$ & $3.4703 \mathrm{E}-13$ \\
\hline $1 s 2 p$ & ${ }^{1} P_{1}^{o}$ & 247026227 & 247036525 & 247037250 & 247038566 & 247044900 & $1.3777 \mathrm{E}-16$ & $1.4814 \mathrm{E}-16$ \\
\hline $1 s 3 s$ & ${ }^{3} S_{1}$ & 288924950 & 288935786 & 288939203 & 288942662 & & $1.4006 \mathrm{E}-14$ & $1.3954 \mathrm{E}-14$ \\
\hline $1 s 3 p$ & ${ }^{3} P_{1}^{o}$ & 289095210 & 289106563 & 289109120 & 289112287 & & $1.0612 \mathrm{E}-15$ & $1.0241 \mathrm{E}-15$ \\
\hline $1 s 3 s$ & ${ }^{1} S_{0}$ & 289108182 & 289117871 & 289120773 & 289123953 & & $1.4184 \mathrm{E}-14$ & $1.4470 \mathrm{E}-14$ \\
\hline $1 s 3 p$ & ${ }^{3} P_{0}^{o}$ & 289107415 & 289119367 & 289121868 & 289124969 & & $4.9927 \mathrm{E}-15$ & $5.1073 \mathrm{E}-15$ \\
\hline $1 s 3 p$ & ${ }^{3} P_{2}^{o}$ & 290026737 & 290038530 & 290041366 & 290044705 & & $5.7548 \mathrm{E}-15$ & $5.9395 \mathrm{E}-15$ \\
\hline $1 s 3 d$ & ${ }^{3} D_{2}$ & 290093250 & 290105217 & 290108667 & 290112135 & & $1.8090 \mathrm{E}-15$ & $1.8193 \mathrm{E}-15$ \\
\hline $1 s 3 d$ & ${ }^{3} D_{1}$ & 290105192 & 290117231 & 290120677 & 290124145 & & $1.8379 \mathrm{E}-15$ & $1.8516 \mathrm{E}-15$ \\
\hline $1 s 3 p$ & ${ }^{1} P_{1}^{o}$ & 290107569 & 290119473 & 290121577 & 290124581 & & $4.6643 \mathrm{E}-16$ & $4.9660 \mathrm{E}-16$ \\
\hline $1 s 3 d$ & ${ }^{3} D_{3}$ & 290385275 & 290397902 & 290401352 & 290404821 & 290413200 & $1.9104 \mathrm{E}-15$ & $1.9570 \mathrm{E}-15$ \\
\hline $1 s 3 d$ & ${ }^{1} D_{2}$ & 290396539 & 290409015 & 290412465 & 290415933 & & $1.8837 \mathrm{E}-15$ & $1.9162 \mathrm{E}-15$ \\
\hline $1 s 4 s$ & ${ }^{3} S_{1}$ & 304797684 & 304810015 & 304813454 & 304816918 & & $2.0732 \mathrm{E}-14$ & $2.0635 \mathrm{E}-14$ \\
\hline $1 s 4 p$ & ${ }^{3} P_{1}^{o}$ & 304866150 & 304879429 & 304882766 & 304886176 & & $2.4379 \mathrm{E}-15$ & $2.3464 \mathrm{E}-15$ \\
\hline $1 s 4 s$ & ${ }^{1} S_{0}$ & 304871588 & 304883666 & 304886805 & 304890134 & & $2.0958 \mathrm{E}-14$ & $2.1245 \mathrm{E}-14$ \\
\hline $1 s 4 p$ & ${ }^{3} P_{0}^{0}$ & 304871052 & 304884541 & 304887868 & 304891257 & & $8.6891 \mathrm{E}-15$ & $8.7915 \mathrm{E}-15$ \\
\hline $1 s 4 p$ & ${ }^{3} P_{2}^{o}$ & 305258024 & 305271468 & 305274839 & 305278274 & & $9.7044 \mathrm{E}-15$ & $9.9498 \mathrm{E}-15$ \\
\hline $1 s 4 d$ & ${ }^{3} D_{2}$ & 305287622 & 305299503 & 305302961 & 305306431 & & $4.1830 \mathrm{E}-15$ & $4.1928 \mathrm{E}-15$ \\
\hline $1 s 4 d$ & ${ }^{3} D_{1}$ & 305292454 & 305304374 & 305307831 & 305311301 & & $4.2748 \mathrm{E}-15$ & $4.2838 \mathrm{E}-15$ \\
\hline $1 s 4 p$ & ${ }^{1} P_{1}^{o}$ & 305291458 & 305304769 & 305307957 & 305311348 & & $1.1013 \mathrm{E}-15$ & $1.1689 \mathrm{E}-15$ \\
\hline $1 s 4 d$ & ${ }^{3} D_{3}$ & 305410833 & 305423127 & 305426585 & 305430056 & & $4.4651 \mathrm{E}-15$ & $4.5484 \mathrm{E}-15$ \\
\hline $1 s 4 f$ & ${ }^{3} F_{3}^{o}$ & 305411941 & 305425949 & 305428640 & 305431982 & & $8.8845 \mathrm{E}-15$ & $8.9144 \mathrm{E}-15$ \\
\hline $1 s 4 f$ & ${ }^{3} F_{2}^{o}$ & 305414511 & 305428553 & 305431218 & 305434552 & & $8.8976 \mathrm{E}-15$ & $8.9207 \mathrm{E}-15$ \\
\hline $1 s 4 d$ & ${ }^{1} D_{2}$ & 305415952 & 305428167 & 305431625 & 305435095 & & $4.3461 \mathrm{E}-15$ & $4.4234 \mathrm{E}-15$ \\
\hline $1 s 4 f$ & ${ }^{3} F_{4}^{o}$ & 305472935 & 305486462 & 305489675 & 305493125 & & $9.0070 \mathrm{E}-15$ & $9.0807 \mathrm{E}-15$ \\
\hline $1 s 4 f$ & ${ }^{1} F_{3}^{0}$ & 305474843 & 305488377 & 305491581 & 305495030 & & $9.0252 \mathrm{E}-15$ & $9.0870 \mathrm{E}-15$ \\
\hline $1 s 5 s$ & ${ }^{3} S_{1}$ & 312072452 & 312085468 & 312088918 & 312092385 & & $3.2951 \mathrm{E}-14$ & $3.2811 \mathrm{E}-14$ \\
\hline $1 s 5 p$ & ${ }^{3} P_{1}^{o}$ & 312106783 & 312120509 & 312123852 & 312127291 & & $4.6828 \mathrm{E}-15$ & $4.5019 \mathrm{E}-15$ \\
\hline $1 s 5 s$ & ${ }^{1} S_{0}$ & 312109421 & 312122408 & 312125671 & 312129058 & & $3.3281 \mathrm{E}-14$ & $3.3582 \mathrm{E}-14$ \\
\hline $1 s 5 p$ & ${ }^{3} P_{0}^{o}$ & 312109229 & 312123048 & 312126394 & 312129823 & & $1.4960 \mathrm{E}-14$ & $1.5067 \mathrm{E}-14$ \\
\hline $1 s 5 p$ & ${ }^{3} P_{2}^{o}$ & 312306662 & 312320496 & 312323875 & 312327320 & & $1.6453 \mathrm{E}-14$ & $1.6819 \mathrm{E}-14$ \\
\hline $1 s 5 d$ & ${ }^{3} D_{2}$ & 312322459 & 312334715 & 312338177 & 312341649 & & $8.0273 \mathrm{E}-15$ & $8.0351 \mathrm{E}-15$ \\
\hline $1 s 5 p$ & ${ }^{1} P_{1}^{o}$ & 312323602 & 312337225 & 312340644 & 312344087 & & $2.1439 \mathrm{E}-15$ & $2.2719 \mathrm{E}-15$ \\
\hline $1 s 5 d$ & ${ }^{3} D_{1}$ & 312324883 & 312337162 & 312340623 & 312344095 & & $8.2220 \mathrm{E}-15$ & $8.2189 \mathrm{E}-15$ \\
\hline $1 s 5 d$ & ${ }^{3} D_{3}$ & 312385482 & 312397978 & 312401440 & 312404912 & & $8.6074 \mathrm{E}-15$ & $8.7460 \mathrm{E}-15$ \\
\hline $1 s 5 f$ & ${ }^{3} F_{3}^{o}$ & 312385818 & 312400664 & 312402591 & 312405985 & & $1.7108 \mathrm{E}-14$ & $1.7138 \mathrm{E}-14$ \\
\hline $1 s 5 f$ & ${ }^{3} F_{2}^{o}$ & 312387134 & 312402027 & 312403912 & 312407300 & & $1.7127 \mathrm{E}-14$ & $1.7149 \mathrm{E}-14$ \\
\hline $1 s 5 d$ & ${ }^{1} D_{2}$ & 312388183 & 312400635 & 312404097 & 312407568 & & $8.3320 \mathrm{E}-15$ & $8.4797 \mathrm{E}-15$ \\
\hline $1 s 5 f$ & ${ }^{3} F_{4}^{o}$ & 312417093 & 312430652 & 312433874 & 312437344 & & $1.7355 \mathrm{E}-14$ & $1.7476 \mathrm{E}-14$ \\
\hline $1 s 5 g$ & ${ }^{3} G_{4}$ & 312417177 & 312430463 & 312433929 & 312437402 & & $2.9246 \mathrm{E}-14$ & $2.9310 \mathrm{E}-14$ \\
\hline $1 s 5 g$ & ${ }^{3} G_{3}$ & 312417930 & 312431215 & 312434682 & 312438155 & & $2.9267 \mathrm{E}-14$ & $2.9320 \mathrm{E}-14$ \\
\hline $1 s 5 f$ & ${ }^{1} F_{3}^{o}$ & 312418079 & 312431645 & 312434859 & 312438328 & & $1.7385 \mathrm{E}-14$ & $1.7489 \mathrm{E}-14$ \\
\hline $1 s 5 g$ & ${ }^{3} G_{5}$ & 312435809 & 312449135 & 312452602 & 312456074 & & $2.9431 \mathrm{E}-14$ & $2.9553 \mathrm{E}-14$ \\
\hline $1 s 5 g$ & ${ }^{1} G_{4}$ & 312436404 & 312449730 & 312453197 & 312456669 & & $2.9447 \mathrm{E}-14$ & $2.9560 \mathrm{E}-14$ \\
\hline $1 s 6 s$ & ${ }^{3} S_{1}$ & 315993781 & 316009270 & 316012725 & 316016194 & & $5.0842 \mathrm{E}-14$ & $5.0676 \mathrm{E}-14$ \\
\hline $1 s 6 p$ & ${ }^{3} P_{1}^{o}$ & 316015359 & 316030311 & 316032803 & 316036253 & & $8.2030 \mathrm{E}-15$ & $7.6967 \mathrm{E}-15$ \\
\hline $1 s 6 s$ & ${ }^{1} S_{0}$ & 316011643 & 316030412 & 316033745 & 316037162 & & $5.1360 \mathrm{E}-14$ & $5.1446 \mathrm{E}-14$ \\
\hline $1 s 6 p$ & ${ }^{3} P_{0}^{o}$ & 316011594 & 316031770 & 316034252 & 316037693 & & $2.4183 \mathrm{E}-14$ & $2.4286 \mathrm{E}-14$ \\
\hline $1 s 6 p$ & ${ }^{3} P_{2}^{o}$ & 316130593 & 316145514 & 316148194 & 316151654 & & $2.6341 \mathrm{E}-14$ & $2.6886 \mathrm{E}-14$ \\
\hline $1 s 6 d$ & ${ }^{3} D_{2}$ & 316140922 & 316152973 & 316156437 & 316159909 & & $1.4049 \mathrm{E}-14$ & $1.3705 \mathrm{E}-14$ \\
\hline $1 s 6 p$ & ${ }^{1} P_{1}^{o}$ & 316139182 & 316155139 & 316157859 & 316161294 & & $3.6568 \mathrm{E}-15$ & $3.9123 \mathrm{E}-15$ \\
\hline $1 s 6 d$ & ${ }^{3} D_{1}$ & 316140582 & 316154374 & 316157837 & 316161309 & & $1.3703 \mathrm{E}-14$ & $1.4021 \mathrm{E}-14$ \\
\hline $1 s 6 d$ & ${ }^{3} D_{3}$ & 316177189 & 316189550 & 316193014 & 316196486 & & $1.4721 \mathrm{E}-14$ & $1.4943 \mathrm{E}-14$ \\
\hline $1 s 6 f$ & ${ }^{3} F_{3}^{o}$ & 316177224 & 316192482 & 316193664 & 316197135 & & $2.9214 \mathrm{E}-14$ & $2.9210 \mathrm{E}-14$ \\
\hline $1 s 6 f$ & ${ }^{3} F_{2}^{o}$ & 316177216 & 316193297 & 316194426 & 316197896 & & $2.9180 \mathrm{E}-14$ & $2.9224 \mathrm{E}-14$ \\
\hline $1 s 6 d$ & ${ }^{1} D_{2}$ & 316177743 & 316191110 & 316194574 & 316198045 & & $1.4208 \mathrm{E}-14$ & $1.4457 \mathrm{E}-14$ \\
\hline $1 s 6 f$ & ${ }^{3} F_{4}^{o}$ & 316195254 & 316208405 & 316211819 & 316215291 & & $2.9614 \mathrm{E}-14$ & $2.9805 \mathrm{E}-14$ \\
\hline $1 s 6 g$ & ${ }^{3} G_{4}$ & 316195229 & 316208388 & 316211855 & 316215327 & & $5.0068 \mathrm{E}-14$ & $5.0144 \mathrm{E}-14$ \\
\hline $1 s 6 g$ & ${ }^{3} G_{3}$ & 316195229 & 316208825 & 316212291 & 316215764 & & $5.0109 \mathrm{E}-14$ & $5.0158 \mathrm{E}-14$ \\
\hline $1 s 6 f$ & ${ }^{1} F_{3}^{o}$ & 316195267 & 316208980 & 316212392 & 316215864 & & $2.9665 \mathrm{E}-14$ & $2.9824 \mathrm{E}-14$ \\
\hline $1 s 6 g$ & ${ }^{3} G_{5}$ & 316205987 & 316219204 & 316222671 & 316226143 & & $5.0397 \mathrm{E}-14$ & $5.0581 \mathrm{E}-14$ \\
\hline $1 s 6 h$ & ${ }^{3} \mathrm{H}_{5}^{0}$ & 316205987 & 316219251 & 316222682 & 316226155 & & $7.6145 \mathrm{E}-14$ & $7.6271 \mathrm{E}-14$ \\
\hline $1 s 6 h$ & ${ }^{3} \mathrm{H}_{4}^{0}$ & 316205987 & 316219534 & 316222964 & 316226437 & & $7.6189 \mathrm{E}-14$ & $7.6284 \mathrm{E}-14$ \\
\hline $1 s 6 g$ & ${ }^{1} G_{4}$ & 316205987 & 316219549 & 316223016 & 316226488 & & $5.0429 \mathrm{E}-14$ & $5.0591 \mathrm{E}-14$ \\
\hline $1 s 6 h$ & ${ }^{3} \mathrm{H}_{6}^{o}$ & 316213134 & 316226435 & 316229866 & 316233339 & & $7.6403 \mathrm{E}-14$ & $7.6606 \mathrm{E}-14$ \\
\hline
\end{tabular}


Table 1 (continued)

\begin{tabular}{|c|c|c|c|c|c|c|c|c|}
\hline Configuration & Level & FAC & GRASP1 & GRASP2 & GRASP3 & NIST & Lifetimes (FAC) & Lifetimes (GRASP2K) \\
\hline $1 s 6 h$ & ${ }^{1} H_{5}^{o}$ & 316213134 & 316226670 & 316230099 & 316233572 & & $7.6438 \mathrm{E}-14$ & $7.6616 \mathrm{E}-14$ \\
\hline $1 s 7 s$ & ${ }^{3} S_{1}$ & 318347824 & 318362488 & 318365947 & 318369417 & & $7.5196 \mathrm{E}-14$ & $7.5428 \mathrm{E}-14$ \\
\hline $1 s 7 p$ & ${ }^{3} P_{1}^{o}$ & 318361378 & 318379168 & 318379301 & 318382488 & & $1.2935 \mathrm{E}-14$ & $1.2034 \mathrm{E}-14$ \\
\hline $1 s 7 s$ & ${ }^{1} S_{0}$ & 318358961 & 318375742 & 318379117 & 318382551 & & $7.5936 \mathrm{E}-14$ & $7.4869 \mathrm{E}-14$ \\
\hline $1 s 7 p$ & ${ }^{3} P_{0}^{o}$ & 318359030 & 318380144 & 318380220 & 318383393 & & $3.6841 \mathrm{E}-14$ & $3.6655 \mathrm{E}-14$ \\
\hline $1 s 7 p$ & ${ }^{3} P_{2}^{0}$ & 318433740 & 318451061 & 318451711 & 318454952 & & $3.9905 \mathrm{E}-14$ & $4.0394 \mathrm{E}-14$ \\
\hline $1 s 7 d$ & ${ }^{3} D_{2}^{2}$ & 318440253 & 318452794 & 318456259 & 318459731 & & $2.1567 \mathrm{E}-14$ & $2.1568 \mathrm{E}-14$ \\
\hline $1 s 7 d$ & ${ }^{3} D_{1}$ & 318440035 & 318453670 & 318457135 & 318460607 & & $2.2119 \mathrm{E}-14$ & $2.2062 \mathrm{E}-14$ \\
\hline $1 s 7 p$ & ${ }^{1} P_{1}^{o}$ & 318439121 & 318457447 & 318457914 & 318461036 & & $5.7984 \mathrm{E}-15$ & $6.1365 \mathrm{E}-15$ \\
\hline $1 s 7 d$ & ${ }^{3} D_{3}$ & 318463080 & 318475804 & 318479269 & 318482741 & & $2.3193 \mathrm{E}-14$ & $2.3579 \mathrm{E}-14$ \\
\hline $1 s 7 f$ & ${ }^{3} F_{3}^{o}$ & 318463096 & 318479601 & 318479839 & 318483171 & & $4.5885 \mathrm{E}-14$ & $4.5872 \mathrm{E}-14$ \\
\hline $1 s 7 f$ & ${ }^{3} F_{2}^{o}$ & 318463090 & 318480145 & 318480317 & 318483651 & & $4.5848 \mathrm{E}-14$ & $4.5866 \mathrm{E}-14$ \\
\hline $1 s 7 d$ & ${ }^{1} D_{2}$ & 318463439 & 318476795 & 318480260 & 318483731 & & $2.2348 \mathrm{E}-14$ & $2.2705 \mathrm{E}-14$ \\
\hline $1 s 7 f$ & ${ }^{3} F_{4}^{0}$ & 318474448 & 318487999 & 318491154 & 318494599 & & $4.6540 \mathrm{E}-14$ & $4.6813 \mathrm{E}-14$ \\
\hline $1 s 7 g$ & ${ }^{3} G_{4}$ & 318474415 & 318487681 & 318491148 & 318494620 & & $7.8748 \mathrm{E}-14$ & $7.8818 \mathrm{E}-14$ \\
\hline $1 s 7 g$ & ${ }^{3} G_{3}$ & 318474415 & 318487956 & 318491423 & 318494895 & & $7.8784 \mathrm{E}-14$ & $7.8824 \mathrm{E}-14$ \\
\hline $1 s 7 f$ & ${ }^{1} F_{3}^{o}$ & 318474457 & 318488359 & 318491517 & 318494961 & & $4.6606 \mathrm{E}-14$ & $4.6830 \mathrm{E}-14$ \\
\hline $1 s 7 g$ & ${ }^{3} G_{5}$ & 318481193 & 318494494 & 318497961 & 318501433 & & $7.9274 \mathrm{E}-14$ & $7.9533 \mathrm{E}-14$ \\
\hline $1 s 7 h$ & ${ }^{3} \mathrm{H}_{5}^{o}$ & 318481193 & 318494511 & 318497969 & 318501442 & & $1.2015 \mathrm{E}-13$ & $1.2028 \mathrm{E}-13$ \\
\hline $1 s 7 \mathrm{~h}$ & ${ }^{3} H_{4}^{0}$ & 318481192 & 318494689 & 318498147 & 318501620 & & $1.2018 \mathrm{E}-13$ & $1.2030 \mathrm{E}-13$ \\
\hline $1 s 7 g$ & ${ }^{1} G_{4}$ & 318481192 & 318494712 & 318498178 & 318501651 & & $7.9303 \mathrm{E}-14$ & $7.9537 \mathrm{E}-14$ \\
\hline $1 s 7 h$ & ${ }^{3} H_{6}^{o}$ & 318485697 & 318499038 & 318502496 & 318505969 & & $1.2057 \mathrm{E}-13$ & $1.2083 \mathrm{E}-13$ \\
\hline $1 s 7 i$ & ${ }^{3} I_{6}$ & 318485696 & 318499030 & 318502497 & 318505969 & & $1.6971 \mathrm{E}-13$ & $1.6989 \mathrm{E}-13$ \\
\hline $1 s 7 i$ & ${ }^{3} I_{5}$ & 318485696 & 318499155 & 318502621 & 318506094 & & $1.6971 \mathrm{E}-13$ & $1.6990 \mathrm{E}-13$ \\
\hline $1 s 7 h$ & ${ }^{1} H_{5}^{o}$ & 318485697 & 318499185 & 318502643 & 318506116 & & $1.2059 \mathrm{E}-13$ & $1.2084 \mathrm{E}-13$ \\
\hline $1 s 7 i$ & ${ }^{3} I_{7}$ & 318488907 & 318502254 & 318505721 & 318509193 & & $1.7004 \mathrm{E}-13$ & $1.7033 \mathrm{E}-13$ \\
\hline $1 s 7 i$ & ${ }^{1} I_{6}$ & 318488907 & 318502361 & 318505827 & 318509300 & & $1.7004 \mathrm{E}-13$ & $1.7034 \mathrm{E}-13$ \\
\hline
\end{tabular}


Table 2

Calculated values using the GRASP2K code of the composition in LSJ coupling for all levels of interest with increasing the active set to $n=10$ (GRASP3).

\begin{tabular}{|c|c|}
\hline Level & Composition in LSJ coupling \\
\hline $1 s^{2}{ }^{1} S_{0}$ & $1 s^{2}{ }^{1} S_{0} 99.98 \%$ \\
\hline $1 s 2 s^{3} S_{1}$ & $1 s 2 s^{3} S_{1} 99.98 \%$ \\
\hline $1 s 2 s^{1} S_{0}$ & $1 s 2 s{ }^{1} S_{0} 99.94 \%$ \\
\hline $1 s 2 p^{3} P_{1}^{o}$ & $1 s 2 p^{3} P_{1}^{o} 70.31 \%+1 s 2 p^{1} P_{1}^{o} 29.64 \%$ \\
\hline $1 s 2 p^{3} P_{2}^{o}$ & $1 s 2 p^{3} P_{2}^{o} 99.97 \%$ \\
\hline $1 s 2 p^{3} P_{0}^{0}$ & $1 s 2 p^{3} P_{0}^{o} 99.96 \%$ \\
\hline $1 s 2 p^{1} P_{1}^{0}$ & $1 s 2 p^{1} P_{1}^{o} 70.29 \%+1 s 2 p^{3} P_{1}^{o} 29.65 \%$ \\
\hline $1 s 3 s^{3} S_{1}$ & $1 s 3 s^{3} S_{1} 99.95 \%$ \\
\hline $1 s 3 s{ }^{1} S_{0}$ & $1 s 3 s{ }^{1} S_{0} 99.88 \%$ \\
\hline $1 s 3 p^{3} P_{1}^{o}$ & $1 s 3 p^{3} P_{1}^{o} 70.04 \%+1 s 3 p^{1} P_{1}^{o} 29.84 \%$ \\
\hline $1 s 3 p^{3} P_{2}^{0}$ & $1 s 3 p^{3} P_{2}^{o} 99.90 \%$ \\
\hline $1 s 3 p^{3} P_{0}^{o}$ & $1 s 3 p^{3} P_{0}^{o} 99.88 \%$ \\
\hline $1 s 3 d^{3} D_{2}$ & $1 s 3 d^{3} D_{2} 60.30 \%+1 s 3 d^{1} D_{2} 39.67 \%$ \\
\hline $1 s 3 d^{3} D_{1}$ & $1 s 3 d^{3} D_{1} 99.97 \%$ \\
\hline $1 s 3 p{ }^{1} P_{1}^{o}$ & $1 s 3 p{ }^{1} P_{1}^{o} 70.01 \%+1 s 3 p^{3} P_{1}^{o} 29.85 \%$ \\
\hline $1 s 3 d^{3} D_{3}$ & $1 s 3 d^{3} D_{3} 99.90 \%$ \\
\hline $1 s 3 d^{1} D_{2}$ & $1 s 3 d^{1} D_{2} 60.26 \%+1 s 3 d^{3} D_{2} 39.64 \%$ \\
\hline $1 s 4 s^{3} S_{1}$ & $1 s 4 s^{3} S_{1} 99.90 \%$ \\
\hline $1 s 4 s^{1} S_{0}$ & $1 s 4 s{ }^{1} S_{0} 99.81 \%$ \\
\hline $1 s 4 p^{3} P_{1}^{o}$ & $1 s 4 p^{3} P_{1}^{o} 69.90 \%+1 s 4 p^{1} P_{1}^{o} 29.88 \%$ \\
\hline $1 s 4 p^{3} P_{0}^{0}$ & $1 s 4 p^{3} P_{0}^{o} 99.77 \%+1 s 5 p^{3} P_{0}^{0} 00.10 \%$ \\
\hline $1 s 4 p^{3} P_{2}^{o}$ & $1 s 4 p^{3} P_{2}^{o} 99.81 \%$ \\
\hline $1 s 4 d^{3} D_{2}$ & $1 s 4 d^{3} D_{2} 60.45 \%+1 s 4 d^{1} D_{2} 39.45 \%$ \\
\hline $1 s 4 d^{3} D_{1}$ & $1 s 4 d^{3} D_{1} 99.90 \%$ \\
\hline $1 s 4 p^{1} P_{1}^{o}$ & $1 s 4 p^{1} P_{1}^{o} 69.86 \%+1 s 4 p^{3} P_{1}^{o} 29.89 \%$ \\
\hline $1 s 4 d^{3} D_{3}$ & $1 s 4 d^{3} D_{3} 99.82 \%$ \\
\hline $1 s 4 d^{3} D_{1}$ & $1 s 4 d^{3} D_{1} 99.90 \%$ \\
\hline $1 s 4 f^{3} F_{3}^{o}$ & $1 s 4 f^{3} F_{3}^{o} 56.88 \%+1 s 4 f^{1} F_{3}^{o} 43.04 \%$ \\
\hline $1 s 4 f^{3} F_{2}^{o}$ & $1 s 4 f^{3} F_{2}^{0} 99.93 \%$ \\
\hline $1 s 4 d^{1} D_{2}$ & $1 s 4 d^{1} D_{2} 60.39 \%+1 s 4 d^{3} D_{2} 39.42 \%$ \\
\hline $1 s 4 f^{3} F_{4}^{o}$ & $1 s 4 f^{3} F_{4}^{o} 99.93 \%$ \\
\hline $1 s 4 f^{1} F_{3}^{0}$ & $1 s 4 f^{1} F_{3}^{0} 56.88 \%+1 s 4 f^{3} F_{3}^{o} 43.04 \%$ \\
\hline $1 s 5 s^{3} s_{1}^{3}$ & $1 s 5 s^{3} s_{1}^{3} 99.84 \%$ \\
\hline $1 s 5 s{ }^{1} S_{0}$ & $1 s 5 s{ }^{1} S_{0} 99.74 \%+1 s 6 s{ }^{1} S_{0}^{o} 00.13 \%$ \\
\hline $1 s 5 p^{3} P_{1}^{o}$ & $1 s 5 p^{3} P_{1}^{o} 69.79 \%+1 s 5 p{ }^{1} P_{1}^{o} 29.87 \%$ \\
\hline $1 s 5 p^{3} P_{2}^{0}$ & $1 s 5 p^{3} P_{2}^{o} 99.71 \%+1 s 6 p^{3} P_{2}^{o} 00.13 \%$ \\
\hline $1 s 5 d^{3} D_{2}$ & $1 s 5 d^{3} D_{2} 60.48 \%+1 s 5 d{ }^{1} D_{2} 39.34 \%$ \\
\hline $1 s 5 p^{1} P_{1}^{o}$ & $1 s 5 p^{1} P_{1}^{o} 69.74 \%+1 s 5 p^{3} P_{1}^{o} 29.89 \%+1 s 6 p{ }^{1} P_{1}^{o} 00.11 \%$ \\
\hline $1 s 5 p^{3} P_{0}^{0}$ & $1 s 5 p^{3} P_{0}^{o} 99.66 \%+1 s 6 p^{3} P_{0}^{o} 00.15 \%$ \\
\hline $1 s 5 d^{3} D_{1}$ & $1 s 5 d^{3} D_{1} 99.82 \%$ \\
\hline $1 s 5 d^{3} D_{3}$ & $1 s 5 d^{3} D_{3} 99.79 \%$ \\
\hline $1 s 5 f^{3} F_{3}^{o}$ & $1 s 5 f^{3} F_{3}^{o} 56.83 \%+1 s 5 f^{1} F_{3}^{o} 42.98 \%$ \\
\hline $1 s 5 f^{3} F_{2}^{0}$ & $1 s 5 f^{3} F_{2}^{0} 99.81 \%+1 s 6 f^{3} F_{2}^{0} 00.10 \%$ \\
\hline $1 s 5 d^{1} D_{2}$ & $1 s 5 d^{1} D_{2} 60.45 \%+1 s 5 d^{3} D_{2} 39.32 \%$ \\
\hline $1 s 5 f^{3} F_{4}^{o}$ & $1 s 5 f^{3} F_{4}^{o} 99.82 \%$ \\
\hline $1 s 5 g{ }^{3} G_{4}$ & $1 s 5 g^{3} G_{4} 55.34 \%+1 s 5 g{ }^{1} G_{4} 44.61 \%$ \\
\hline $1 s 5 g{ }^{3} G_{3}$ & $1 s 5 g{ }^{3} G_{3} 99.95 \%$ \\
\hline $1 s 5 f^{1} F_{3}^{o}$ & $1 s 5 f^{1} F_{3}^{o} 56.83 \%+1 s 5 f^{3} F_{3}^{o} 42.98 \%$ \\
\hline $1 s 5 g{ }^{3} G_{5}$ & $1 s 5 g{ }^{3} G_{5} 99.90 \%$ \\
\hline $1 s 5 g{ }^{1} G_{4}$ & $1 s 5 g{ }^{1} G_{4} 55.31 \%+1 s 5 g{ }^{3} G_{4} 44.59 \%$ \\
\hline $1 s 6 s^{3} s_{1}$ & $1 s 6 s^{3} S_{1} 99.79 \%+1 s 7 s^{3} S_{1} 00.11 \%$ \\
\hline $1 s 6 s{ }^{1} S_{0}$ & $1 s 6 s{ }^{1} S_{0} 99.68 \%+1 s 7 s{ }^{1} S_{0} 00.16 \%+1 s 5 s{ }^{1} S_{0} 00.12 \%$ \\
\hline $1 s 6 p^{3} P_{1}^{o}$ & $1 s 6 p^{3} P_{1}^{o} 69.70 \%+1 s 6 p^{1} P_{1}^{o} 29.85 \%+1 s 7 p^{3} P_{1}^{o} 00.12 \%$ \\
\hline $1 s 6 p^{3} P_{0}^{o}$ & $1 s 6 p^{3} P_{0}^{o} 99.54 \%+1 s 7 p^{3} P_{0}^{o} 00.19+1 s 5 p^{3} P_{0}^{o} 00.13 \%$ \\
\hline $1 s 6 p^{3} P_{2}^{o}$ & $1 s 6 p^{3} P_{2}^{o} 99.60 \%+1 s 7 p^{3} P_{2}^{o} 00.17 \%+1 s 5 p^{3} P_{2}^{o} 00.12 \%$ \\
\hline $1 s 6 d^{3} D_{2}$ & $1 s 6 d^{3} D_{2} 60.48 \%+1 s 6 d^{1} D_{2} 39.27 \%$ \\
\hline $1 s 6 p^{1} P_{1}^{o}$ & $1 s 6 p^{1} P_{1}^{o} 69.64 \%+1 s 6 p^{3} P_{1}^{o} 29.88 \%+1 s 7 p{ }^{1} P_{1}^{o} 00.15 \%+1 s 5 p{ }^{1} P_{1}^{o} 00.10 \%$ \\
\hline $1 s 6 d^{3} D_{1}$ & $1 s 6 d^{3} D_{1} 99.75 \%+1 s 7 d^{3} D_{1} 00.13 \%$ \\
\hline $1 s 6 d^{3} D_{3}$ & $1 s 6 d^{3} D_{3} 99.76 \%+1 s 7 d^{3} D_{3} 00.12 \%$ \\
\hline $1 s 6 f^{3} F_{3}^{o}$ & $1 s 6 f{ }^{3} F_{3}^{o} 56.77 \%+1 s 6 f{ }^{1} F_{3}^{o} 42.92 \%$ \\
\hline $1 s 6 f^{3} F_{2}^{0}$ & $1 s 6 f{ }^{3} F_{2}^{o} 99.69 \%+1 s 7 f^{3} F_{2}^{o} 00.14 \%$ \\
\hline $1 s 6 d^{1} D_{2}$ & $1 s 6 d^{1} D_{2} 60.48 \%+1 s 6 d^{3} D_{2} 39.27 \%$ \\
\hline $1 s 6 f^{3} F_{4}^{o}$ & $1 s 6 f^{3} F_{4}^{o} 99.70 \%+1 s 7 f^{3} F_{4}^{o} 00.14 \%$ \\
\hline $1 s 6 g{ }^{3} G_{4}$ & $1 s 6 g{ }^{3} G_{4} 55.29 \%+1 s 6 g{ }^{1} G_{4}^{4} 44.57 \%$ \\
\hline $1 s 6 g{ }^{3} G_{3}$ & $1 s 6 g{ }^{3} G_{3} 99.87 \%$ \\
\hline $1 s 6 f^{1} F_{3}^{o}$ & $1 s 6 f^{1} F_{3}^{o} 56.77 \%+1 s 6 f^{3} F_{3}^{o} 42.92 \%$ \\
\hline $1 s 6 g^{3} G_{5}$ & $1 s 6 g^{3} G_{5} 99.84 \%$ \\
\hline $1 s 6 h^{3} H_{5}^{o}$ & $1 s 6 h^{3} H_{5}^{o} 54.35 \%+1 s 6 h^{1} H_{5}^{o} 45.57 \%$ \\
\hline $1 s 6 h^{3} H_{4}^{o}$ & $1 s 6 h^{3} H_{4}^{o} 99.93 \%$ \\
\hline $1 s 6 g{ }^{1} G_{4}$ & $1 s 6 g{ }^{1} G_{4} 55.28 \%+1 s 6 g{ }^{3} G_{4} 44.56 \%$ \\
\hline $1 s 6 h^{3} H_{6}^{o}$ & $1 s 6 h^{3} H_{6}^{o} 99.93 \%$ \\
\hline
\end{tabular}


Table 2 (continued)

\begin{tabular}{|c|c|}
\hline Level & Composition in LSJ coupling \\
\hline $1 s 6 h^{1} H_{5}^{o}$ & $1 s 6 h^{1} H_{5}^{o} 54.35 \%+1 s 6 h^{3} H_{5}^{o} 45.57 \%$ \\
\hline $1 s 7 s^{3} s_{1}^{3}$ & $1 s 7 s^{3} s_{1} 99.86 \%+1 s 6 s^{3} S_{1} 00.10 \%$ \\
\hline $1 s 7 s^{1} S_{0}$ & $1 s 7 s{ }^{1} S_{0} 99.80 \%+1 s 6 s{ }^{1} S_{0} 00.15 \%$ \\
\hline $1 s 7 p^{3} P_{1}^{o}$ & $1 s 7 p^{3} P_{1}^{o} 69.68 \%+1 s 7 p{ }^{1} P_{1}^{o} 29.83 \%+1 s 6 p^{3} P_{1}^{o} 00.11 \%$ \\
\hline $1 s 7 p^{3} P_{2}^{0}$ & $1 s 7 p^{3} P_{2}^{0} 99.57 \%+1 s 6 p^{3} P_{2}^{o} 00.15 \%$ \\
\hline $1 s 7 p^{3} P_{0}^{o}$ & $1 s 7 p^{3} P_{0}^{o} 99.51 \%+1 s 6 p^{3} P_{0}^{o} 00.17 \%$ \\
\hline $1 s 7 d^{3} D_{2}$ & $1 s 7 d^{3} D_{2} 60.56 \%+1 s 7 d^{1} D_{2} 39.28 \%$ \\
\hline $1 s 7 d^{3} D_{1}$ & $1 s 7 d^{3} D_{1} 99.84 \%+1 s 6 d^{3} D_{1} 00.13 \%$ \\
\hline $1 s 7 p^{1} P_{1}^{o}$ & $1 s 7 p^{1} P_{1}^{o} 69.63 \%+1 s 7 p^{3} P_{1}^{o} 29.86 \%+1 s 6 p{ }^{1} P_{1}^{o} 00.13 \%$ \\
\hline $1 s 7 d^{3} D_{3}$ & $1 s 7 d^{3} D_{3} 99.84 \%+1 s 6 d^{3} D_{3} 00.12 \%$ \\
\hline $1 s 7 f^{3} F_{3}^{o}$ & $1 s 7 f^{3} F_{3}^{o} 56.72 \%+1 s 7 f^{1} F_{3}^{o} 42.87 \%$ \\
\hline $1 s 7 f^{3} F_{2}^{o}$ & $1 s 7 f^{3} F_{2}^{o} 99.58 \%+1 s 6 f^{3} F_{2}^{o} 00.13 \%$ \\
\hline $1 s 7 d{ }^{1} D_{2}$ & $1 s 7 d^{1} D_{2} 60.56 \%+1 s 7 d^{3} D_{2} 39.28 \%$ \\
\hline $1 s 7 f^{3} F_{4}^{o}$ & $1 s 7 f^{3} F_{4}^{o} 99.59 \%+1 s 6 f^{3} F_{4}^{o} 00.13 \%$ \\
\hline $1 s 7 g{ }^{3} G_{4}$ & $1 s 7 g{ }^{3} G_{4} 55.31 \%+1 s 7 g{ }^{1} G_{4} 44.59 \%$ \\
\hline $1 s 7 g{ }^{3} G_{3}$ & $1 s 7 g{ }^{3} G_{3} 99.91 \%$ \\
\hline $1 s 7 f^{1} F_{3}^{o}$ & $1 s 7 f^{1} F_{3}^{o} 56.72 \%+1 s 7 f^{3} F_{3}^{o} 42.87 \%$ \\
\hline $1 s 7 g{ }^{3} G_{5}$ & $1 s 7 g{ }^{3} G_{5} 99.91 \%$ \\
\hline $1 s 7 h^{3} H_{5}^{o}$ & $1 s 7 h^{3} H_{5}^{o} 54.29 \%+1 s 7 h^{1} H_{5}^{o} 45.52 \%$ \\
\hline $1 s 7 h^{3} H_{4}^{0}$ & $1 s 7 h^{3} H_{4}^{0} 99.81 \%$ \\
\hline $1 s 7 g{ }^{1} G_{4}$ & $1 s 7 g{ }^{1} G_{4} 55.32 \%+1 s 7 g{ }^{3} G_{4} 44.59 \%$ \\
\hline $1 s 7 h^{3} H_{6}^{o}$ & $1 s 7 h^{3} H_{6}^{o} 99.81 \%$ \\
\hline $1 s 7 i^{3} I_{6}$ & $1 s 7 i^{3} I_{6} 53.71 \%+1 s 7 i^{1} I_{6} 46.28 \%$ \\
\hline $1 s 7 i^{3} I_{5}$ & $1 s 7 i^{3} I_{5} 99.99 \%$ \\
\hline $1 s 7 h^{1} H_{5}^{o}$ & $1 s 7 h^{1} H_{5}^{o} 54.29 \%+1 s 7 h^{3} H_{5}^{o} 45.52 \%$ \\
\hline $1 s 7 i^{3} I_{7}{ }^{3}$ & $1 s 7 i^{3} I_{7} 99.99 \%$ \\
\hline $1 s 7 i^{1} I_{6}$ & $1 s 7 i^{1} I_{6} 53.71 \%+1 s 7 i^{3} I_{6} 46.28 \%$ \\
\hline
\end{tabular}


Table 3

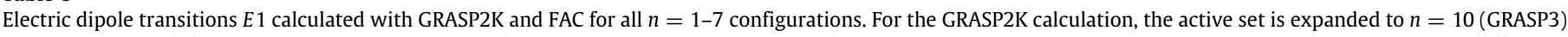

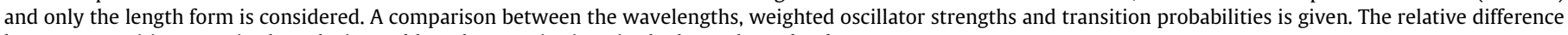
between transition rates in the velocity and length gauge is given in the last column by $d T$.

\begin{tabular}{|c|c|c|c|c|c|c|c|c|}
\hline \multirow[t]{2}{*}{ Lower } & \multirow[t]{2}{*}{ Upper } & \multicolumn{3}{|l|}{ GRASP2K } & \multicolumn{3}{|l|}{ FAC } & \multirow[t]{2}{*}{$d T$} \\
\hline & & $\lambda(\AA)$ & $g f$ & $A\left(\mathrm{~s}^{-1}\right)$ & $\lambda(\AA)$ & $g f$ & $A\left(\mathrm{~s}^{-1}\right)$ & \\
\hline $1 s^{2}{ }^{1} S_{0}$ & $1 s 2 p^{3} P_{1}^{o}$ & $4.1040 \mathrm{E}-01$ & $2.290 \mathrm{E}-01$ & $3.023 E+15$ & $4.0874 \mathrm{E}-01$ & $2.230 \mathrm{E}-01$ & $2.943 \mathrm{E}+15$ & 0.010 \\
\hline $1 s 2 s^{3} S_{1}$ & $1 s 2 p^{3} P_{1}^{o}$ & $1.6053 \mathrm{E}+02$ & $1.112 \mathrm{E}-02$ & $9.593 \mathrm{E}+08$ & $1.5824 \mathrm{E}+02$ & $1.129 \mathrm{E}-02$ & $9.944 \mathrm{E}+08$ & 0.193 \\
\hline $1 s 2 s^{3} S_{1}$ & $1 s 2 p^{3} P_{0}^{o}$ & $1.4898 \mathrm{E}+02$ & $5.677 \mathrm{E}-03$ & $1.706 \mathrm{E}+09$ & $1.4745 \mathrm{E}+02$ & $5.725 \mathrm{E}-03$ & $1.741 \mathrm{E}+09$ & 0.139 \\
\hline $1 s 2 p^{3} P_{1}^{o}$ & $1 s 2{ }^{1} S_{0}$ & $1.5842 \mathrm{E}+03$ & $4.752 \mathrm{E}-04$ & $1.263 \mathrm{E}+06$ & $1.4920 \mathrm{E}+03$ & $4.997 \mathrm{E}-04$ & $1.484 \mathrm{E}+06$ & 0.365 \\
\hline $1 s 2 s^{3} S_{1}$ & $1 s 2 p^{3} P_{2}^{o}$ & $2.6617 \mathrm{E}+01$ & $1.713 \mathrm{E}-01$ & $3.226 \mathrm{E}+11$ & $2.6489 \mathrm{E}+01$ & $1.725 \mathrm{E}-01$ & $3.252 \mathrm{E}+11$ & 0.028 \\
\hline $1 s^{2}{ }^{1} S_{0}$ & $1 s 2 p^{1} P_{1}^{o}$ & $4.0470 \mathrm{E}-01$ & $4.975 \mathrm{E}-01$ & $6.750 \mathrm{E}+15$ & $4.0309 \mathrm{E}-01$ & $5.349 \mathrm{E}-01$ & $7.257 \mathrm{E}+15$ & 0.001 \\
\hline $1 s 2 s^{3} S_{1}$ & $1 s 2 p^{1} P_{1}^{o}$ & $2.4759 \mathrm{E}+01$ & $3.299 \mathrm{E}-02$ & $1.196 \mathrm{E}+11$ & $2.4615 \mathrm{E}+01$ & $3.310 \mathrm{E}-02$ & $1.204 \mathrm{E}+11$ & 0.014 \\
\hline $1 s 2 s^{1} S_{0}$ & $1 s 2 p^{1} P_{1}^{o}$ & $2.9825 \mathrm{E}+01$ & $6.507 \mathrm{E}-02$ & $1.626 \mathrm{E}+11$ & $2.9731 \mathrm{E}+01$ & $6.480 \mathrm{E}-02$ & $1.616 \mathrm{E}+11$ & 0.019 \\
\hline $1 s 2 p^{3} P_{1}^{o}$ & $1 s 3 s^{3} s_{1}$ & $2.2065 \mathrm{E}+00$ & $3.037 \mathrm{E}-02$ & $1.387 \mathrm{E}+13$ & $2.1974 \mathrm{E}+00$ & $3.067 \mathrm{E}-02$ & $1.400 \mathrm{E}+13$ & 0.001 \\
\hline $1 s 2 p^{3} P_{0}^{o}$ & $1 s 3 s^{3} s_{1}$ & $2.2089 \mathrm{E}+00$ & $1.488 \mathrm{E}-02$ & $6.779 \mathrm{E}+12$ & $2.1996 \mathrm{E}+00$ & $1.466 \mathrm{E}-02$ & $6.681 \mathrm{E}+12$ & 0.001 \\
\hline $1 s 2 p^{3} P_{2}^{o}$ & $1 s 3 s^{3} S_{1}$ & $2.3705 \mathrm{E}+00$ & $1.089 \mathrm{E}-01$ & $4.309 \mathrm{E}+13$ & $2.3604 \mathrm{E}+00$ & $1.085 \mathrm{E}-01$ & $4.294 \mathrm{E}+13$ & 0.000 \\
\hline $1 s 2 p{ }^{1} P_{1}^{o}$ & $1 s 3 s^{3} s_{1}$ & $2.3864 \mathrm{E}+00$ & $1.988 \mathrm{E}-02$ & $7.761 \mathrm{E}+12$ & $2.3765 \mathrm{E}+00$ & $1.946 \mathrm{E}-02$ & $7.596 \mathrm{E}+12$ & 0.002 \\
\hline $1 s^{2}{ }^{1} S_{0}$ & $1 s 3 p^{3} P_{1}^{o}$ & $3.4580 \mathrm{E}-01$ & $4.196 \mathrm{E}-02$ & $7.798 E+14$ & $3.4443 \mathrm{E}-01$ & $4.008 \mathrm{E}-02$ & $7.449 \mathrm{E}+14$ & 0.015 \\
\hline $1 s 2 s^{3} S_{1}$ & $1 s 3 p^{3} P_{1}^{o}$ & $2.1686 \mathrm{E}+00$ & $2.902 \mathrm{E}-01$ & $1.372 \mathrm{E}+14$ & $2.1593 E+00$ & $2.946 \mathrm{E}-01$ & $1.393 \mathrm{E}+14$ & 0.003 \\
\hline $1 s 2 s{ }^{1} S_{0}$ & $1 s 3 p^{3} P_{1}^{o}$ & $2.2014 \mathrm{E}+00$ & $1.285 \mathrm{E}-01$ & $5.894 \mathrm{E}+13$ & $2.1924 \mathrm{E}+00$ & $1.253 \mathrm{E}-01$ & $5.750 \mathrm{E}+13$ & 0.006 \\
\hline $1 s 3 s^{3} s_{1}$ & $1 s 3 p^{3} P_{1}^{o}$ & $5.8954 \mathrm{E}+02$ & $1.889 \mathrm{E}-02$ & $1.208 \mathrm{E}+08$ & $5.8484 \mathrm{E}+02$ & $1.901 \mathrm{E}-02$ & $1.225 \mathrm{E}+08$ & 0.179 \\
\hline $1 s 2 p^{3} P_{1}^{o}$ & $1 s 3 s^{1} s_{0}$ & $2.1977 \mathrm{E}+00$ & $1.194 \mathrm{E}-02$ & $1.649 \mathrm{E}+13$ & $2.1885 \mathrm{E}+00$ & $1.243 \mathrm{E}-02$ & $1.717 \mathrm{E}+13$ & 0.005 \\
\hline $1 s 2 p{ }^{1} P_{1}^{o}$ & $1 s 3 s{ }^{1} S_{0}$ & $2.3761 \mathrm{E}+00$ & $4.454 \mathrm{E}-02$ & $5.262 \mathrm{E}+13$ & $2.3662 \mathrm{E}+00$ & $4.514 \mathrm{E}-02$ & $5.332 \mathrm{E}+13$ & 0.002 \\
\hline $1 s 3 p^{3} P_{1}^{o}$ & $1 s 3 s^{1} S_{0}$ & $8.5719 \mathrm{E}+03$ & $5.545 \mathrm{E}-04$ & $5.034 \mathrm{E}+04$ & $7.6765 E+03$ & $6.142 \mathrm{E}-04$ & $6.893 \mathrm{E}+04$ & 1.000 \\
\hline $1 s 2 s^{3} S_{1}$ & $1 s 3 p^{3} P_{0}^{o}$ & $2.1680 \mathrm{E}+00$ & $1.376 \mathrm{E}-01$ & $1.953 \mathrm{E}+14$ & $2.1587 \mathrm{E}+00$ & $1.407 \mathrm{E}-01$ & $1.997 \mathrm{E}+14$ & 0.003 \\
\hline $1 s 3 s^{3} S_{1}$ & $1 s 3 p^{3} P_{0}^{o}$ & $5.4853 \mathrm{E}+02$ & $9.695 \mathrm{E}-03$ & $2.149 \mathrm{E}+08$ & $5.4572 \mathrm{E}+02$ & $9.712 \mathrm{E}-03$ & $2.156 \mathrm{E}+08$ & 0.148 \\
\hline $1 s 2 s^{3} S_{1}$ & $1 s 3 p^{3} P_{2}^{o}$ & $2.1256 \mathrm{E}+00$ & $5.650 \mathrm{E}-01$ & $1.668 \mathrm{E}+14$ & $2.1165 E+00$ & $5.828 \mathrm{E}-01$ & $1.721 \mathrm{E}+14$ & 0.003 \\
\hline $1 s 3 s^{3} s_{1}$ & $1 s 3 p^{3} P_{2}^{o}$ & $9.0741 \mathrm{E}+01$ & $3.059 \mathrm{E}-01$ & $4.957 \mathrm{E}+10$ & $9.0375 \mathrm{E}+01$ & $3.069 \mathrm{E}-01$ & $4.970 \mathrm{E}+10$ & 0.026 \\
\hline $1 s 2 p^{3} P_{1}^{o}$ & $1 s 3 d^{3} D_{2}$ & $2.1510 \mathrm{E}+00$ & $1.556 \mathrm{E}+00$ & $4.485 \mathrm{E}+14$ & $2.1422 \mathrm{E}+00$ & $1.568 \mathrm{E}+00$ & $4.520 \mathrm{E}+14$ & 0.001 \\
\hline $1 s 2 p^{3} P_{2}^{o}$ & $1 s 3 d^{3} D_{2}$ & $2.3065 \mathrm{E}+00$ & $3.054 \mathrm{E}-01$ & $7.659 E+13$ & $2.2968 \mathrm{E}+00$ & $3.049 \mathrm{E}-01$ & $7.645 E+13$ & 0.001 \\
\hline $1 s 2 p{ }^{1} P_{1}^{o}$ & $1 s 3 d^{3} D_{2}$ & $2.3216 \mathrm{E}+00$ & $5.348 \mathrm{E}-02$ & $1.324 \mathrm{E}+13$ & $2.3121 \mathrm{E}+00$ & $5.146 \mathrm{E}-02$ & $1.273 \mathrm{E}+13$ & 0.002 \\
\hline $1 s 3 p^{3} P_{1}^{o}$ & $1 s 3 d^{3} D_{2}$ & $1.0002 \mathrm{E}+02$ & $1.760 \mathrm{E}-01$ & $2.348 \mathrm{E}+10$ & $9.9770 \mathrm{E}+01$ & $1.757 \mathrm{E}-01$ & $2.335 \mathrm{E}+10$ & 0.002 \\
\hline $1 s 3 p^{3} P_{2}^{0}$ & $1 s 3 d^{3} D_{2}$ & $1.4830 \mathrm{E}+03$ & $2.141 \mathrm{E}-03$ & $1.298 \mathrm{E}+06$ & & & & 0.003 \\
\hline $1 s 2 p^{3} P_{1}^{o}$ & $1 s 3 d^{3} D_{1}$ & $2.1505 \mathrm{E}+00$ & $3.325 \mathrm{E}-01$ & $1.598 \mathrm{E}+14$ & $2.1416 \mathrm{E}+00$ & $3.373 \mathrm{E}-01$ & $1.621 \mathrm{E}+14$ & 0.001 \\
\hline $1 s 2 p^{3} P_{0}^{o}$ & $1 s 3 d^{3} D_{1}$ & $2.1527 \mathrm{E}+00$ & $6.302 \mathrm{E}-01$ & $3.024 \mathrm{E}+14$ & $2.1437 \mathrm{E}+00$ & $6.350 \mathrm{E}-01$ & $3.046 \mathrm{E}+14$ & 0.001 \\
\hline $1 s 2 p^{3} P_{2}^{o}$ & $1 s 3 d^{3} D_{1}$ & $2.3059 \mathrm{E}+00$ & $3.362 \mathrm{E}-02$ & $1.406 \mathrm{E}+13$ & $2.2962 \mathrm{E}+00$ & $3.352 \mathrm{E}-02$ & $1.401 \mathrm{E}+13$ & 0.001 \\
\hline $1 s 2 p{ }^{1} P_{1}^{o}$ & $1 s 3 d^{3} D_{1}$ & $2.3210 \mathrm{E}+00$ & $1.511 \mathrm{E}-01$ & $6.236 \mathrm{E}+13$ & $2.3114 \mathrm{E}+00$ & $1.497 \mathrm{E}-01$ & $6.179 E+13$ & 0.002 \\
\hline $1 s 3 p^{3} P_{1}^{o}$ & $1 s 3 d^{3} D_{1}$ & $9.8828 \mathrm{E}+01$ & $3.812 \mathrm{E}-02$ & $8.679 \mathrm{E}+09$ & $9.8590 \mathrm{E}+01$ & $3.804 \mathrm{E}-02$ & $8.628 \mathrm{E}+09$ & 0.006 \\
\hline $1 s 3 p^{3} P_{0}^{o}$ & $1 s 3 d^{3} D_{1}$ & $1.0008 \mathrm{E}+02$ & $7.109 \mathrm{E}-02$ & $1.578 \mathrm{E}+10$ & $9.9796 \mathrm{E}+01$ & $7.095 \mathrm{E}-02$ & $1.570 \mathrm{E}+10$ & 0.006 \\
\hline $1 s 3 p^{3} P_{2}^{0}$ & $1 s 3 d^{3} D_{1}$ & $1.2588 \mathrm{E}+03$ & $2.725 \mathrm{E}-04$ & $3.823 \mathrm{E}+05$ & & & & 0.053 \\
\hline $1 s^{2}{ }^{1} S_{0}{ }^{2}$ & $1 s 3 p{ }^{1} P_{1}^{o}$ & $3.4460 \mathrm{E}-01$ & $9.869 \mathrm{E}-02$ & $1.847 \mathrm{E}+15$ & $3.4323 \mathrm{E}-01$ & $1.055 \mathrm{E}-01$ & $1.975 E+15$ & 0.020 \\
\hline $1 s 2 s^{3} S_{1}$ & $1 s 3 p^{1} P_{1}^{o}$ & $2.1220 \mathrm{E}+00$ & $9.943 \mathrm{E}-02$ & $4.910 \mathrm{E}+13$ & $2.1129 \mathrm{E}+00$ & $1.031 \mathrm{E}-01$ & $5.095 \mathrm{E}+13$ & 0.004 \\
\hline $1 s 2 s^{1} S_{0}$ & $1 s 3 p^{1} P_{1}^{o}$ & $2.1534 \mathrm{E}+00$ & $2.439 \mathrm{E}-01$ & $1.170 \mathrm{E}+14$ & $2.1446 \mathrm{E}+00$ & $2.435 \mathrm{E}-01$ & $1.167 \mathrm{E}+14$ & 0.004 \\
\hline $1 s 3 s^{3} s_{1}$ & $1 s 3 p^{1} P_{1}^{o}$ & $8.4608 \mathrm{E}+01$ & $5.893 \mathrm{E}-02$ & $1.830 \mathrm{E}+10$ & $8.4198 \mathrm{E}+01$ & $5.901 \mathrm{E}-02$ & $1.835 \mathrm{E}+10$ & 0.020 \\
\hline $1 s 3 s^{1} S_{0}$ & $1 s 3 p^{1} P_{1}^{o}$ & $9.9937 \mathrm{E}+01$ & $1.175 \mathrm{E}-01$ & $2.616 \mathrm{E}+10$ & $9.9635 \mathrm{E}+01$ & $1.170 \mathrm{E}-01$ & $2.599 \mathrm{E}+10$ & 0.026 \\
\hline $1 s 3 d^{3} D_{2}$ & $1 s 3 p^{1} P_{1}^{o}$ & $8.0349 \mathrm{E}+03$ & $6.680 \mathrm{E}-05$ & $2.301 \mathrm{E}+03$ & & & & 0.383 \\
\hline $1 s 3 d^{3} D_{1}$ & $1 s 3 p^{1} P_{1}^{o}$ & $2.2947 \mathrm{E}+05$ & $6.878 \mathrm{E}-06$ & $2.904 \mathrm{E}-01$ & & & & 0.978 \\
\hline $1 s 2 p^{3} P_{2}^{o}$ & $1 s 3 d^{3} D_{3}$ & $2.2910 \mathrm{E}+00$ & $2.807 \mathrm{E}+00$ & $5.095 \mathrm{E}+14$ & $2.2814 \mathrm{E}+00$ & $2.875 \mathrm{E}+00$ & $5.219 \mathrm{E}+14$ & 0.001 \\
\hline $1 s 3 p^{3} P_{2}^{o}$ & $1 s 3 d^{3} D_{3}$ & $2.7769 \mathrm{E}+02$ & $1.088 \mathrm{E}-01$ & $1.345 \mathrm{E}+09$ & $2.7772 \mathrm{E}+02$ & $1.082 \mathrm{E}-01$ & $1.326 \mathrm{E}+09$ & 0.004 \\
\hline $1 s 2 p^{3} P_{1}^{o}$ & $1 s 3 d^{1} D_{2}$ & $2.1371 \mathrm{E}+00$ & $2.237 \mathrm{E}-03$ & $6.534 \mathrm{E}+11$ & $2.1283 \mathrm{E}+00$ & $2.733 \mathrm{E}-03$ & $7.982 \mathrm{E}+11$ & 0.001 \\
\hline $1 s 2 p^{3} P_{2}^{o}$ & $1 s 3 d^{1} D_{2}$ & $2.2905 \mathrm{E}+00$ & $1.988 \mathrm{E}-01$ & $5.056 \mathrm{E}+13$ & $2.2809 \mathrm{E}+00$ & $2.036 \mathrm{E}-01$ & $5.177 \mathrm{E}+13$ & 0.001 \\
\hline $1 s 2 p^{1} P_{1}^{o}$ & $1 s 3 d^{1} D_{2}$ & $2.3053 \mathrm{E}+00$ & $1.814 \mathrm{E}+00$ & $4.553 \mathrm{E}+14$ & $2.2959 \mathrm{E}+00$ & $1.836 \mathrm{E}+00$ & $4.607 \mathrm{E}+14$ & 0.001 \\
\hline $1 s 3 p^{3} P_{1}^{o}$ & $1 s 3 d^{1} D_{2}$ & $7.6708 \mathrm{E}+01$ & $2.981 \mathrm{E}-04$ & $6.759 \mathrm{E}+07$ & $7.6517 \mathrm{E}+01$ & $2.967 \mathrm{E}-04$ & $6.704 \mathrm{E}+07$ & 0.000 \\
\hline $1 s 3 p^{3} P_{2}^{o}$ & $1 s 3 d^{1} D_{2}$ & $2.6938 \mathrm{E}+02$ & $7.955 \mathrm{E}-03$ & $1.463 \mathrm{E}+08$ & $2.6926 \mathrm{E}+02$ & $7.908 \mathrm{E}-03$ & $1.442 \mathrm{E}+08$ & 0.018 \\
\hline $1 s 3 p^{1} P_{1}^{o}$ & $1 s 3 d^{1} D_{2}$ & $3.4323 \mathrm{E}+02$ & $5.654 \mathrm{E}-02$ & $6.403 E+08$ & $3.4458 \mathrm{E}+02$ & $5.610 \mathrm{E}-02$ & $6.249 \mathrm{E}+08$ & 0.015 \\
\hline $1 s 2 p^{3} P_{1}^{o}$ & $1 s 4 s^{3} S_{1}$ & $1.6341 \mathrm{E}+00$ & $6.721 \mathrm{E}-03$ & $5.596 \mathrm{E}+12$ & $1.6274 \mathrm{E}+00$ & $6.812 \mathrm{E}-03$ & $5.671 \mathrm{E}+12$ & 0.003 \\
\hline $1 s 2 p^{3} P_{0}^{0}$ & $1 s 4 s^{3} S_{1}$ & $1.6354 \mathrm{E}+00$ & $3.290 \mathrm{E}-03$ & $2.735 \mathrm{E}+12$ & $1.6286 \mathrm{E}+00$ & $3.273 \mathrm{E}-03$ & $2.721 \mathrm{E}+12$ & 0.000 \\
\hline $1 s 2 p^{3} P_{2}^{o}$ & $1 s 4 s^{3} S_{1}$ & $1.7223 \mathrm{E}+00$ & $2.273 \mathrm{E}-02$ & $1.703 \mathrm{E}+13$ & $1.7151 \mathrm{E}+00$ & $2.261 \mathrm{E}-02$ & $1.694 \mathrm{E}+13$ & 0.001 \\
\hline $1 s 2 p^{1} P_{1}^{o}$ & $1 s 4 s^{3} S_{1}$ & $1.7308 \mathrm{E}+00$ & $4.129 \mathrm{E}-03$ & $3.065 \mathrm{E}+12$ & $1.7236 \mathrm{E}+00$ & $4.044 \mathrm{E}-03$ & $3.001 \mathrm{E}+12$ & 0.002 \\
\hline $1 s 3 p^{3} P_{1}^{o}$ & $1 s 4 s^{3} S_{1}$ & $6.3675 \mathrm{E}+00$ & $7.121 \mathrm{E}-02$ & $3.905 \mathrm{E}+12$ & $6.3413 E+00$ & $7.108 \mathrm{E}-02$ & $3.897 \mathrm{E}+12$ & 0.000 \\
\hline $1 s 3 p^{3} P_{0}^{o}$ & $1 s 4 s^{3} S_{1}$ & $6.3727 \mathrm{E}+00$ & $3.469 \mathrm{E}-02$ & $1.899 \mathrm{E}+12$ & $6.3462 \mathrm{E}+00$ & $3.391 \mathrm{E}-02$ & $1.856 \mathrm{E}+12$ & 0.001 \\
\hline $1 s 3 p^{3} P_{2}^{o}$ & $1 s 4 s^{3} S_{1}$ & $6.7695 \mathrm{E}+00$ & $2.464 \mathrm{E}-01$ & $1.195 \mathrm{E}+13$ & $6.7412 \mathrm{E}+00$ & $2.453 \mathrm{E}-01$ & $1.190 \mathrm{E}+13$ & 0.001 \\
\hline $1 s 3 p^{1} P_{1}^{o}$ & $1 s 4 s^{3} S_{1}$ & $6.8063 \mathrm{E}+00$ & $4.518 \mathrm{E}-02$ & $2.169 \mathrm{E}+12$ & $6.7783 E+00$ & $4.436 \mathrm{E}-02$ & $2.128 \mathrm{E}+12$ & 0.001 \\
\hline $1 s^{2}{ }^{1} S_{0}$ & $1 s 4 p^{3} P_{1}^{o}$ & $3.2790 \mathrm{E}-01$ & $1.510 \mathrm{E}-02$ & $3.121 \mathrm{E}+14$ & $3.2662 \mathrm{E}-01$ & $1.433 \mathrm{E}-02$ & $2.962 \mathrm{E}+14$ & 0.013 \\
\hline $1 s 2 s^{3} S_{1}$ & $1 s 4 p^{3} P_{1}^{o}$ & $1.6159 \mathrm{E}+00$ & $6.856 \mathrm{E}-02$ & $5.839 \mathrm{E}+13$ & $1.6090 \mathrm{E}+00$ & $6.888 \mathrm{E}-02$ & $5.866 \mathrm{E}+13$ & 0.002 \\
\hline $1 s 2 s^{1} S_{0}$ & $1 s 4 p^{3} P_{1}^{o}$ & $1.6340 \mathrm{E}+00$ & $2.993 \mathrm{E}-02$ & $2.492 \mathrm{E}+13$ & $1.6273 E+00$ & $2.929 \mathrm{E}-02$ & $2.438 \mathrm{E}+13$ & 0.006 \\
\hline $1 s 3 s^{3} S_{1}$ & $1 s 4 p^{3} P_{1}^{o}$ & $6.2721 \mathrm{E}+00$ & $3.224 \mathrm{E}-01$ & $1.822 \mathrm{E}+13$ & $6.2463 \mathrm{E}+00$ & $3.272 \mathrm{E}-01$ & $1.849 \mathrm{E}+13$ & 0.002 \\
\hline $1 s 3 s^{1} S_{0}$ & $1 s 4 p^{3} P_{1}^{o}$ & $6.3443 \mathrm{E}+00$ & $1.439 \mathrm{E}-01$ & $7.951 \mathrm{E}+12$ & $6.3190 \mathrm{E}+00$ & $1.408 \mathrm{E}-01$ & $7.778 \mathrm{E}+12$ & 0.003 \\
\hline $1 s 3 d^{3} D_{2}$ & $1 s 4 p^{3} P_{1}^{o}$ & $6.7686 \mathrm{E}+00$ & $7.309 \mathrm{E}-02$ & $3.547 \mathrm{E}+12$ & $6.7403 E+00$ & $7.330 \mathrm{E}-02$ & $3.557 \mathrm{E}+12$ & 0.000 \\
\hline $1 s 3 d^{3} D_{1}$ & $1 s 4 p^{3} P_{1}^{o}$ & $6.7741 \mathrm{E}+00$ & $1.585 \mathrm{E}-02$ & $7.680 \mathrm{E}+11$ & $6.7458 \mathrm{E}+00$ & $1.555 \mathrm{E}-02$ & $7.537 \mathrm{E}+11$ & 0.002 \\
\hline $1 s 3 d{ }^{1} D_{2}$ & $1 s 4 p^{3} P_{1}^{o}$ & $6.9107 \mathrm{E}+00$ & $1.013 \mathrm{E}-04$ & $4.717 \mathrm{E}+09$ & & & & 0.003 \\
\hline $1 s 4 s^{3} S_{1}$ & $1 s 4 p^{3} P_{1}^{o}$ & $1.4439 \mathrm{E}+03$ & $2.625 \mathrm{E}-02$ & $2.800 \mathrm{E}+07$ & $1.4544 \mathrm{E}+03$ & $2.601 \mathrm{E}-02$ & $2.711 \mathrm{E}+07$ & 0.173 \\
\hline $1 s 2 p^{3} P_{1}^{o}$ & $1 s 4 s{ }^{1} S_{0}$ & $1.6322 \mathrm{E}+00$ & $2.684 \mathrm{E}-03$ & $6.721 \mathrm{E}+12$ & $1.6254 \mathrm{E}+00$ & $2.783 \mathrm{E}-03$ & $6.967 \mathrm{E}+12$ & 0.005 \\
\hline
\end{tabular}


Table 3 (continued)

\begin{tabular}{|c|c|c|c|c|c|c|c|c|}
\hline \multirow[t]{2}{*}{ Lower } & \multirow[t]{2}{*}{ Upper } & \multicolumn{3}{|l|}{ GRASP2K } & \multicolumn{3}{|l|}{ FAC } & \multirow[t]{2}{*}{$d T$} \\
\hline & & $\lambda(\AA)$ & $g f$ & $A\left(\mathrm{~s}^{-1}\right)$ & $\lambda(\AA)$ & $g f$ & $A\left(\mathrm{~s}^{-1}\right)$ & \\
\hline $1 s 2 p^{1} P_{1}^{o}$ & $1 s 4 s^{1} S_{0}$ & $1.7286 \mathrm{E}+00$ & $9.390 \mathrm{E}-03$ & $2.096 \mathrm{E}+13$ & $1.7214 \mathrm{E}+00$ & $9.412 \mathrm{E}-03$ & $2.100 \mathrm{E}+13$ & 0.002 \\
\hline $1 s 3 p^{3} P_{1}^{o}$ & $1 s 4 s^{1} S_{0}$ & $6.3380 \mathrm{E}+00$ & $2.847 \mathrm{E}-02$ & $4.727 \mathrm{E}+12$ & $6.3116 \mathrm{E}+00$ & $2.931 \mathrm{E}-02$ & $4.867 \mathrm{E}+12$ & 0.002 \\
\hline $1 s 3 p{ }^{1} P_{1}^{o}$ & $1 s 4 s^{1} S_{0}$ & $6.7725 \mathrm{E}+00$ & $1.006 \mathrm{E}-01$ & $1.463 \mathrm{E}+13$ & $6.7444 \mathrm{E}+00$ & $1.020 \mathrm{E}-01$ & $1.484 \mathrm{E}+13$ & 0.001 \\
\hline $1 s 4 p^{3} P_{1}^{o}$ & $1 s 4 s^{1} S_{0}$ & $2.5265 \mathrm{E}+04$ & $6.429 \mathrm{E}-04$ & $6.718 E+03$ & $1.8312 \mathrm{E}+04$ & $8.806 \mathrm{E}-04$ & $1.736 \mathrm{E}+04$ & 0.482 \\
\hline $1 s 2 s^{3} S_{1}$ & $1 s 4 p^{3} P_{0}^{o}$ & $1.6157 \mathrm{E}+00$ & $3.257 \mathrm{E}-02$ & $8.322 E+13$ & $1.6089 \mathrm{E}+00$ & $3.284 \mathrm{E}-02$ & $8.391 E+13$ & 0.003 \\
\hline $1 s 3 s^{3} s_{1}$ & $1 s 4 p^{3} P_{0}^{o}$ & $6.2701 \mathrm{E}+00$ & $1.531 \mathrm{E}-01$ & $2.598 \mathrm{E}+13$ & $6.2444 \mathrm{E}+00$ & $1.569 \mathrm{E}-01$ & $2.661 \mathrm{E}+13$ & 0.003 \\
\hline $1 s 3 d^{3} D_{1}$ & $1 s 4 p^{3} P_{0}^{o}$ & $6.7718 \mathrm{E}+00$ & $2.941 \mathrm{E}-02$ & $4.278 \mathrm{E}+12$ & $6.7435 \mathrm{E}+00$ & $2.941 \mathrm{E}-02$ & $4.277 \mathrm{E}+12$ & 0.002 \\
\hline $1 s 4 s^{3} S_{1}$ & $1 s 4 p^{3} P_{0}^{o}$ & $1.3452 \mathrm{E}+03$ & $1.349 \mathrm{E}-02$ & $4.973 E+07$ & $1.3572 \mathrm{E}+03$ & $1.332 \mathrm{E}-02$ & $4.783 \mathrm{E}+07$ & 0.146 \\
\hline $1 s 2 s^{3} S_{1}$ & $1 s 4 p^{3} P_{2}^{o}$ & $1.6057 \mathrm{E}+00$ & $1.450 \mathrm{E}-01$ & $7.505 E+13$ & $1.5989 \mathrm{E}+00$ & $1.491 \mathrm{E}-01$ & $7.718 E+13$ & 0.003 \\
\hline $1 s 3 s^{3} S_{1}$ & $1 s 4 p^{3} P_{2}^{o}$ & $6.1216 \mathrm{E}+00$ & $6.028 \mathrm{E}-01$ & $2.146 \mathrm{E}+13$ & $6.0965 \mathrm{E}+00$ & $6.147 \mathrm{E}-01$ & $2.187 \mathrm{E}+13$ & 0.003 \\
\hline $1 s 3 d^{3} D_{2}$ & $1 s 4 p^{3} P_{2}^{o}$ & $6.5936 \mathrm{E}+00$ & $8.587 \mathrm{E}-03$ & $2.635 E+11$ & $6.5661 \mathrm{E}+00$ & $8.500 \mathrm{E}-03$ & $2.607 \mathrm{E}+11$ & 0.000 \\
\hline $1 s 3 d^{3} D_{1}$ & $1 s 4 p^{3} P_{2}^{o}$ & $6.5989 \mathrm{E}+00$ & $9.266 \mathrm{E}-04$ & $2.839 E+10$ & $6.5713 \mathrm{E}+00$ & $9.191 \mathrm{E}-04$ & $2.815 \mathrm{E}+10$ & 0.003 \\
\hline $1 s 3 d^{3} D_{3}$ & $1 s 4 p^{3} P_{2}^{o}$ & $6.7234 \mathrm{E}+00$ & $9.374 \mathrm{E}-02$ & $2.766 \mathrm{E}+12$ & $6.6951 \mathrm{E}+00$ & $9.195 \mathrm{E}-02$ & $2.713 \mathrm{E}+12$ & 0.001 \\
\hline $1 s 3 d{ }^{1} D_{2}$ & $1 s 4 p^{3} P_{2}^{o}$ & $6.7284 \mathrm{E}+00$ & $6.658 \mathrm{E}-03$ & $1.962 \mathrm{E}+11$ & $6.7001 \mathrm{E}+00$ & $6.480 \mathrm{E}-03$ & $1.909 \mathrm{E}+11$ & 0.003 \\
\hline $1 s 4 s^{3} S_{1}$ & $1 s 4 p^{3} P_{2}^{o}$ & $2.1675 \mathrm{E}+02$ & $4.308 \mathrm{E}-01$ & $1.223 \mathrm{E}+10$ & $2.1631 \mathrm{E}+02$ & $4.309 \mathrm{E}-01$ & $1.218 \mathrm{E}+10$ & 0.025 \\
\hline $1 s 2 p^{3} P_{1}^{o}$ & $1 s 4 d^{3} D_{2}$ & $1.6212 \mathrm{E}+00$ & $2.941 \mathrm{E}-01$ & $1.493 \mathrm{E}+14$ & $1.6144 \mathrm{E}+00$ & $2.954 \mathrm{E}-01$ & $1.499 \mathrm{E}+14$ & 0.001 \\
\hline $1 s 2 p^{3} P_{2}^{0}$ & $1 s 4 d^{3} D_{2}$ & $1.7079 \mathrm{E}+00$ & $5.276 \mathrm{E}-02$ & $2.413 \mathrm{E}+13$ & $1.7007 \mathrm{E}+00$ & $5.191 \mathrm{E}-02$ & $2.374 \mathrm{E}+13$ & 0.001 \\
\hline $1 s 2 p{ }^{1} P_{1}^{o}$ & $1 s 4 d^{3} D_{2}$ & $1.7162 \mathrm{E}+00$ & $8.902 \mathrm{E}-03$ & $4.032 \mathrm{E}+12$ & $1.7091 \mathrm{E}+00$ & $8.758 \mathrm{E}-03$ & $3.966 \mathrm{E}+12$ & 0.002 \\
\hline $1 s 3 p^{3} P_{1}^{o}$ & $1 s 4 d^{3} D_{2}$ & $6.1751 \mathrm{E}+00$ & $1.295 \mathrm{E}+00$ & $4.531 E+13$ & $6.1494 \mathrm{E}+00$ & $1.301 \mathrm{E}+00$ & $4.554 \mathrm{E}+13$ & 0.001 \\
\hline $1 s 3 p^{3} P_{2}^{o}$ & $1 s 4 d^{3} D_{2}$ & $6.5523 \mathrm{E}+00$ & $2.758 \mathrm{E}-01$ & $8.571 E+12$ & $6.5248 \mathrm{E}+00$ & $2.781 \mathrm{E}-01$ & $8.643 E+12$ & 0.001 \\
\hline $1 s 3 p^{1} P_{1}^{o}$ & $1 s 4 d^{3} D_{2}$ & $6.5868 \mathrm{E}+00$ & $4.612 \mathrm{E}-02$ & $1.418 \mathrm{E}+12$ & $6.5595 \mathrm{E}+00$ & $4.379 \mathrm{E}-02$ & $1.346 \mathrm{E}+12$ & 0.001 \\
\hline $1 s 4 p^{3} P_{1}^{o}$ & $1 s 4 d^{3} D_{2}$ & $2.3795 \mathrm{E}+02$ & $3.169 \mathrm{E}-01$ & $7.465 E+09$ & $2.3625 \mathrm{E}+02$ & $3.177 \mathrm{E}-01$ & $7.531 \mathrm{E}+09$ & 0.000 \\
\hline $1 s 4 p^{3} P_{2}^{o}$ & $1 s 4 d^{3} D_{2}$ & $3.5515 \mathrm{E}+03$ & $3.857 \mathrm{E}-03$ & $4.079 E+05$ & & & & 0.046 \\
\hline $1 s 2 p^{3} P_{1}^{o}$ & $1 s 4 d^{3} D_{1}$ & $1.6210 \mathrm{E}+00$ & $6.298 \mathrm{E}-02$ & $5.329 E+13$ & $1.6143 \mathrm{E}+00$ & $6.329 E-02$ & $5.354 \mathrm{E}+13$ & 0.001 \\
\hline $1 s 2 p^{3} P_{0}^{o}$ & $1 s 4 d^{3} D_{1}$ & $1.6223 \mathrm{E}+00$ & $1.192 \mathrm{E}-01$ & $1.007 \mathrm{E}+14$ & $1.6155 \mathrm{E}+00$ & $1.195 \mathrm{E}-01$ & $1.009 \mathrm{E}+14$ & 0.001 \\
\hline $1 s 2 p^{3} P_{2}^{o}$ & $1 s 4 d^{3} D_{1}$ & $1.7078 \mathrm{E}+00$ & $5.792 \mathrm{E}-03$ & $4.416 \mathrm{E}+12$ & $1.7006 \mathrm{E}+00$ & $5.679 \mathrm{E}-03$ & $4.329 \mathrm{E}+12$ & 0.001 \\
\hline $1 s 2 p^{1} P_{1}^{o}$ & $1 s 4 d^{3} D_{1}$ & $1.7161 \mathrm{E}+00$ & $2.583 \mathrm{E}-02$ & $1.950 \mathrm{E}+13$ & $1.7090 \mathrm{E}+00$ & $2.527 \mathrm{E}-02$ & $1.907 \mathrm{E}+13$ & 0.002 \\
\hline $1 s 3 p^{3} P_{1}^{o}$ & $1 s 4 d^{3} D_{1}$ & $6.1732 \mathrm{E}+00$ & $2.754 \mathrm{E}-01$ & $1.607 \mathrm{E}+13$ & $6.1476 \mathrm{E}+00$ & $2.797 \mathrm{E}-01$ & $1.631 \mathrm{E}+13$ & 0.001 \\
\hline $1 s 3 p^{3} P_{0}^{0}$ & $1 s 4 d^{3} D_{1}$ & $6.1781 \mathrm{E}+00$ & $5.239 \mathrm{E}-01$ & $3.052 E+13$ & $6.1522 \mathrm{E}+00$ & $5.268 \mathrm{E}-01$ & $3.068 \mathrm{E}+13$ & 0.001 \\
\hline $1 s 3 p^{3} P_{2}^{0}$ & $1 s 4 d^{3} D_{1}$ & $6.5502 \mathrm{E}+00$ & $3.014 \mathrm{E}-02$ & $1.562 \mathrm{E}+12$ & $6.5227 \mathrm{E}+00$ & $3.037 \mathrm{E}-02$ & $1.573 \mathrm{E}+12$ & 0.001 \\
\hline $1 s 3 p{ }^{1} P_{1}^{o}$ & $1 s 4 d^{3} D_{1}$ & $6.5847 \mathrm{E}+00$ & $1.379 \mathrm{E}-01$ & $7.069 \mathrm{E}+12$ & $6.5574 \mathrm{E}+00$ & $1.381 \mathrm{E}-01$ & $7.082 \mathrm{E}+12$ & 0.001 \\
\hline $1 s 4 p^{3} P_{1}^{o}$ & $1 s 4 d^{3} D_{1}$ & $2.3523 \mathrm{E}+02$ & $6.848 \mathrm{E}-02$ & $2.752 E+09$ & $2.3358 \mathrm{E}+02$ & $6.867 \mathrm{E}-02$ & $2.774 \mathrm{E}+09$ & 0.006 \\
\hline $1 s 4 p^{3} P_{0}^{o}$ & $1 s 4 d^{3} D_{1}$ & $2.3807 \mathrm{E}+02$ & $1.279 \mathrm{E}-01$ & $5.016 \mathrm{E}+09$ & $2.3629 \mathrm{E}+02$ & $1.282 \mathrm{E}-01$ & $5.063 \mathrm{E}+09$ & 0.005 \\
\hline $1 s 4 p^{3} P_{2}^{o}$ & $1 s 4 d^{3} D_{1}$ & $3.0279 \mathrm{E}+03$ & $4.889 \mathrm{E}-04$ & $1.186 \mathrm{E}+05$ & & & & 0.032 \\
\hline $1 s^{2}{ }^{1} S_{0}$ & $1 s 4 p^{1} P_{1}^{o}$ & $3.2750 \mathrm{E}-01$ & $3.647 \mathrm{E}-02$ & $7.559 E+14$ & $3.2616 \mathrm{E}-01$ & $3.896 \mathrm{E}-02$ & $8.074 \mathrm{E}+14$ & 0.028 \\
\hline $1 s 2 s^{3} S_{1}$ & $1 s 4 p^{1} P_{1}^{o}$ & $1.6048 \mathrm{E}+00$ & $2.578 \mathrm{E}-02$ & $2.226 \mathrm{E}+13$ & $1.5980 \mathrm{E}+00$ & $2.662 \mathrm{E}-02$ & $2.298 \mathrm{E}+13$ & 0.003 \\
\hline $1 s 2 s^{1} S_{0}$ & $1 s 4 p^{1} P_{1}^{o}$ & $1.6227 \mathrm{E}+00$ & $6.199 \mathrm{E}-02$ & $5.234 \mathrm{E}+13$ & $1.6161 \mathrm{E}+00$ & $6.223 \mathrm{E}-02$ & $5.253 E+13$ & 0.004 \\
\hline $1 s 3 s^{3} S_{1}$ & $1 s 4 p^{1} P_{1}^{o}$ & $6.1092 \mathrm{E}+00$ & $1.059 \mathrm{E}-01$ & $6.309 E+12$ & $6.0840 \mathrm{E}+00$ & $1.087 \mathrm{E}-01$ & $6.475 E+12$ & 0.003 \\
\hline $1 s 3 s^{1} S_{0}$ & $1 s 4 p^{1} P_{1}^{o}$ & $6.1776 \mathrm{E}+00$ & $2.605 \mathrm{E}-01$ & $1.518 \mathrm{E}+13$ & $6.1529 \mathrm{E}+00$ & $2.580 \mathrm{E}-01$ & $1.502 \mathrm{E}+13$ & 0.002 \\
\hline $1 s 3 d^{3} D_{2}$ & $1 s 4 p^{1} P_{1}^{o}$ & $6.5793 \mathrm{E}+00$ & $1.389 \mathrm{E}-03$ & $7.134 \mathrm{E}+10$ & $6.5517 \mathrm{E}+00$ & $1.599 \mathrm{E}-03$ & $8.215 E+10$ & 0.004 \\
\hline $1 s 3 d^{3} D_{1}$ & $1 s 4 p^{1} P_{1}^{o}$ & $6.5845 \mathrm{E}+00$ & $4.203 E-03$ & $2.156 \mathrm{E}+11$ & $6.5569 \mathrm{E}+00$ & $4.162 \mathrm{E}-03$ & $2.134 \mathrm{E}+11$ & 0.003 \\
\hline $1 s 3 d d^{1} D_{2}$ & $1 s 4 p^{1} P_{1}^{o}$ & $6.7135 \mathrm{E}+00$ & $5.832 \mathrm{E}-02$ & $2.877 \mathrm{E}+12$ & $6.6851 \mathrm{E}+00$ & $5.917 \mathrm{E}-02$ & $2.918 \mathrm{E}+12$ & 0.003 \\
\hline $1 s 4 s^{3} S_{1}$ & $1 s 4 p^{1} P_{1}^{o}$ & $2.0225 \mathrm{E}+02$ & $8.298 \mathrm{E}-02$ & $4.510 \mathrm{E}+09$ & $2.0166 \mathrm{E}+02$ & $8.289 \mathrm{E}-02$ & $4.493 \mathrm{E}+09$ & 0.022 \\
\hline $1 s 4 s^{1} S_{0}$ & $1 s 4 p^{1} P_{1}^{o}$ & $2.3741 \mathrm{E}+02$ & $1.660 \mathrm{E}-01$ & $6.547 \mathrm{E}+09$ & $2.3715 \mathrm{E}+02$ & $1.651 \mathrm{E}-01$ & $6.471 \mathrm{E}+09$ & 0.032 \\
\hline $1 s 4 d^{3} D_{2}$ & $1 s 4 p^{1} P_{1}^{o}$ & $2.0339 \mathrm{E}+04$ & $1.101 \mathrm{E}-04$ & $5.916 \mathrm{E}+02$ & & & & 0.306 \\
\hline $1 s 4 d^{3} D_{1}$ & $1 s 4 p{ }^{1} P_{1}^{o}$ & $2.1218 \mathrm{E}+06$ & $3.210 \mathrm{E}-06$ & $1.585 \mathrm{E}-03$ & & & & 0.996 \\
\hline $1 s 2 p^{3} P_{2}^{o}$ & $1 s 4 d^{3} D_{3}$ & $1.7043 \mathrm{E}+00$ & $5.008 \mathrm{E}-01$ & $1.643 \mathrm{E}+14$ & $1.6972 \mathrm{E}+00$ & $5.104 \mathrm{E}-01$ & $1.674 \mathrm{E}+14$ & 0.001 \\
\hline $1 s 3 p^{3} P_{2}^{o}$ & $1 s 4 d^{3} D_{3}$ & $6.4997 \mathrm{E}+00$ & $2.449 \mathrm{E}+00$ & $5.523 E+13$ & $6.4725 \mathrm{E}+00$ & $2.491 \mathrm{E}+00$ & $5.619 E+13$ & 0.001 \\
\hline $1 s 4 p^{3} P_{2}^{o}$ & $1 s 4 d^{3} D_{3}$ & $6.5884 \mathrm{E}+02$ & $1.960 \mathrm{E}-01$ & $4.302 E+08$ & $6.5162 \mathrm{E}+02$ & $1.972 \mathrm{E}-01$ & $4.388 \mathrm{E}+08$ & 0.008 \\
\hline $1 s 3 d^{3} D_{2}$ & $1 s 4 f^{3} F_{3}^{o}$ & $6.5275 \mathrm{E}+00$ & $4.625 \mathrm{E}+00$ & $1.034 \mathrm{E}+14$ & $6.5002 \mathrm{E}+00$ & $4.642 \mathrm{E}+00$ & $1.038 \mathrm{E}+14$ & 0.001 \\
\hline $1 s 3 d^{3} D_{3}$ & $1 s 4 f^{3} F_{3}^{o}$ & $6.6546 \mathrm{E}+00$ & $3.174 \mathrm{E}-01$ & $6.829 E+12$ & $6.6265 \mathrm{E}+00$ & $3.157 \mathrm{E}-01$ & $6.793 E+12$ & 0.001 \\
\hline $1 s 3 d d^{1} D_{2}$ & $1 s 4 f^{3} F_{3}^{o}$ & $6.6595 \mathrm{E}+00$ & $1.909 \mathrm{E}-02$ & $4.102 \mathrm{E}+11$ & $6.6315 \mathrm{E}+00$ & $1.914 \mathrm{E}-02$ & $4.113 \mathrm{E}+11$ & 0.001 \\
\hline $1 s 4 d^{3} D_{2}$ & $1 s 4 f^{3} F_{3}^{o}$ & $7.9649 \mathrm{E}+02$ & $9.484 \mathrm{E}-02$ & $1.425 \mathrm{E}+08$ & $8.0096 \mathrm{E}+02$ & $9.391 \mathrm{E}-02$ & $1.383 \mathrm{E}+08$ & 0.017 \\
\hline $1 s 4 d^{3} D_{3}$ & $1 s 4 f^{3} F_{3}^{o}$ & $5.1914 \mathrm{E}+04$ & $9.767 \mathrm{E}-05$ & $3.453 E+01$ & & & & 0.674 \\
\hline $1 s 2 s^{3} S_{1}$ & $1 s 4 f^{3} F_{2}^{0}$ & $1.6017 \mathrm{E}+00$ & $3.265 \mathrm{E}-09$ & $1.698 \mathrm{E}+06$ & $1.5949 \mathrm{E}+00$ & $1.924 \mathrm{E}-08$ & $1.000 \mathrm{E}+07$ & 0.151 \\
\hline $1 s 3 s^{3} S_{1}$ & $1 s 4 f^{3} F_{2}^{o}$ & $6.0636 \mathrm{E}+00$ & $3.445 \mathrm{E}-06$ & $1.250 \mathrm{E}+08$ & & & & 0.008 \\
\hline $1 s 3 d^{3} D_{2}$ & $1 s 4 f^{3} F_{2}^{o}$ & $6.5264 \mathrm{E}+00$ & $3.322 \mathrm{E}-01$ & $1.040 \mathrm{E}+13$ & $6.4991 \mathrm{E}+00$ & $3.338 \mathrm{E}-01$ & $1.045 \mathrm{E}+13$ & 0.000 \\
\hline $1 s 3 d^{3} D_{1}$ & $1 s 4 f^{3} F_{2}^{o}$ & $6.5315 \mathrm{E}+00$ & $2.975 \mathrm{E}+00$ & $9.304 \mathrm{E}+13$ & $6.5041 \mathrm{E}+00$ & $2.983 \mathrm{E}+00$ & $9.330 E+13$ & 0.000 \\
\hline $1 s 3 d^{3} D_{3}$ & $1 s 4 f^{3} F_{2}^{o}$ & $6.6535 \mathrm{E}+00$ & $1.590 \mathrm{E}-02$ & $4.793 E+11$ & $6.6254 \mathrm{E}+00$ & $1.582 \mathrm{E}-02$ & $4.767 \mathrm{E}+11$ & 0.000 \\
\hline $1 s 3 d^{1} D_{2}$ & $1 s 4 f^{3} F_{2}^{o}$ & $6.6584 \mathrm{E}+00$ & $2.213 \mathrm{E}-01$ & $6.660 \mathrm{E}+12$ & $6.6303 E+00$ & $2.198 \mathrm{E}-01$ & $6.613 E+12$ & 0.001 \\
\hline $1 s 4 s^{3} S_{1}$ & $1 s 4 f^{3} F_{2}^{o}$ & $1.6191 \mathrm{E}+02$ & $3.320 \mathrm{E}-07$ & $1.689 \mathrm{E}+04$ & $1.6143 \mathrm{E}+02$ & $3.195 \mathrm{E}-07$ & $1.622 \mathrm{E}+04$ & 0.102 \\
\hline $1 s 4 d^{3} D_{2}$ & $1 s 4 f^{3} F_{2}^{o}$ & $7.8052 \mathrm{E}+02$ & $6.988 \mathrm{E}-03$ & $1.530 \mathrm{E}+07$ & $7.8473 \mathrm{E}+02$ & $6.922 \mathrm{E}-03$ & $1.486 \mathrm{E}+07$ & 0.012 \\
\hline $1 s 4 d^{3} D_{1}$ & $1 s 4 f^{3} F_{2}^{o}$ & $8.1135 \mathrm{E}+02$ & $5.989 \mathrm{E}-02$ & $1.214 \mathrm{E}+08$ & $8.1580 \mathrm{E}+02$ & $5.930 \mathrm{E}-02$ & $1.178 \mathrm{E}+08$ & 0.014 \\
\hline $1 s 4 d^{3} D_{3}$ & $1 s 4 f^{3} F_{2}^{o}$ & $2.2242 \mathrm{E}+04$ & $1.126 \mathrm{E}-05$ & $3.037 \mathrm{E}+01$ & & & & 0.079 \\
\hline $1 s 2 p^{3} P_{1}^{o}$ & $1 s 4 d^{1} D_{2}$ & $1.6178 \mathrm{E}+00$ & $3.977 \mathrm{E}-04$ & $2.027 \mathrm{E}+11$ & $1.6111 \mathrm{E}+00$ & $4.270 \mathrm{E}-04$ & $2.176 \mathrm{E}+11$ & 0.002 \\
\hline $1 s 2 p^{3} P_{2}^{o}$ & $1 s 4 d^{1} D_{2}$ & $1.7042 \mathrm{E}+00$ & $3.537 \mathrm{E}-02$ & $1.625 \mathrm{E}+13$ & $1.6970 \mathrm{E}+00$ & $3.597 \mathrm{E}-02$ & $1.652 \mathrm{E}+13$ & 0.001 \\
\hline $1 s 2 p^{1} P_{1}^{o}$ & $1 s 4 d^{1} D_{2}$ & $1.7124 \mathrm{E}+00$ & $3.214 \mathrm{E}-01$ & $1.462 \mathrm{E}+14$ & $1.7053 \mathrm{E}+00$ & $3.266 \mathrm{E}-01$ & $1.485 \mathrm{E}+14$ & 0.001 \\
\hline $1 s 3 p^{3} P_{1}^{o}$ & $1 s 4 d^{1} D_{2}$ & $6.1264 \mathrm{E}+00$ & $1.393 \mathrm{E}-03$ & $4.950 \mathrm{E}+10$ & $6.1011 \mathrm{E}+00$ & $1.823 \mathrm{E}-03$ & $6.480 \mathrm{E}+10$ & 0.001 \\
\hline $1 s 3 p^{3} P_{2}^{o}$ & $1 s 4 d{ }^{1} D_{2}$ & $6.4976 \mathrm{E}+00$ & $1.724 \mathrm{E}-01$ & $5.448 \mathrm{E}+12$ & $6.4704 \mathrm{E}+00$ & $1.755 \mathrm{E}-01$ & $5.545 \mathrm{E}+12$ & 0.001 \\
\hline $1 s 3 p^{1} P_{1}^{o}$ & $1 s 4 d{ }^{1} D_{2}$ & $6.5315 \mathrm{E}+00$ & $1.593 \mathrm{E}+00$ & $4.980 \mathrm{E}+13$ & $6.5045 \mathrm{E}+00$ & $1.594 \mathrm{E}+00$ & $4.985 E+13$ & 0.001 \\
\hline $1 s 4 p^{3} P_{1}^{o}$ & $1 s 4 d{ }^{1} D_{2}$ & $1.8218 \mathrm{E}+02$ & $4.542 \mathrm{E}-04$ & $1.826 \mathrm{E}+07$ & $1.8111 \mathrm{E}+02$ & $4.521 \mathrm{E}-04$ & $1.823 \mathrm{E}+07$ & 0.010 \\
\hline
\end{tabular}


Table 3 (continued)

\begin{tabular}{|c|c|c|c|c|c|c|c|c|}
\hline \multirow[t]{2}{*}{ Lower } & \multirow[t]{2}{*}{ Upper } & \multicolumn{3}{|l|}{ GRASP2K } & \multicolumn{3}{|l|}{ FAC } & \multirow[t]{2}{*}{$d T$} \\
\hline & & $\lambda(\AA)$ & $g f$ & $A\left(\mathrm{~s}^{-1}\right)$ & $\lambda(\AA)$ & $g f$ & $A\left(\mathrm{~s}^{-1}\right)$ & \\
\hline $1 s 4 p^{3} P_{2}^{o}$ & $1 s 4 d^{1} D_{2}$ & $6.3767 \mathrm{E}+02$ & $1.428 \mathrm{E}-02$ & $4.685 \mathrm{E}+07$ & $6.3050 \mathrm{E}+02$ & $1.435 \mathrm{E}-02$ & $4.775 \mathrm{E}+07$ & 0.013 \\
\hline $1 s 4 p^{1} P_{1}^{o}$ & $1 s 4 d{ }^{1} D_{2}$ & $8.0810 \mathrm{E}+02$ & $1.027 \mathrm{E}-01$ & $2.098 \mathrm{E}+08$ & $7.9983 \mathrm{E}+02$ & $1.033 \mathrm{E}-01$ & $2.136 \mathrm{E}+08$ & 0.014 \\
\hline $1 s 4 f^{3} F_{3}^{o}$ & $1 s 4 d{ }^{1} D_{2}$ & $3.2125 \mathrm{E}+04$ & $1.005 \mathrm{E}-05$ & $1.300 \mathrm{E}+01$ & & & & 0.305 \\
\hline $1 s 4 f^{3} F_{2}^{o}$ & $1 s 4 d^{1} D_{2}$ & $1.8409 \mathrm{E}+05$ & $1.913 \mathrm{E}-05$ & $7.530 \mathrm{E}-01$ & & & & 0.815 \\
\hline $1 s 3 d^{3} D_{3}$ & $1 s 4 f^{3} F_{4}^{o}$ & $6.6276 \mathrm{E}+00$ & $6.440 \mathrm{E}+00$ & $1.087 \mathrm{E}+14$ & $6.5997 \mathrm{E}+00$ & $6.489 \mathrm{E}+00$ & $1.094 \mathrm{E}+14$ & 0.000 \\
\hline $1 s 4 d^{3} D_{3}$ & $1 s 4 f^{3} F_{4}^{0}$ & $1.5856 \mathrm{E}+03$ & $6.582 \mathrm{E}-02$ & $1.940 \mathrm{E}+07$ & $1.6034 \mathrm{E}+03$ & $6.481 \mathrm{E}-02$ & $1.852 \mathrm{E}+07$ & 0.023 \\
\hline $1 s 3 d^{3} D_{2}$ & $1 s 4 f^{1} F_{3}^{o}$ & $6.5007 \mathrm{E}+00$ & $1.376 \mathrm{E}-04$ & $3.102 E+09$ & & & & 0.001 \\
\hline $1 s 3 d^{3} D_{3}$ & $1 s 4 f^{1} F_{3}^{o}$ & $6.6268 \mathrm{E}+00$ & $2.397 \mathrm{E}-01$ & $5.202 E+12$ & $6.5989 \mathrm{E}+00$ & $2.415 \mathrm{E}-01$ & $5.241 \mathrm{E}+12$ & 0.000 \\
\hline $1 s 3 d^{1} D_{2}$ & $1 s 4 f^{1} F_{3}^{o}$ & $6.6317 \mathrm{E}+00$ & $4.771 E+00$ & $1.034 \mathrm{E}+14$ & $6.6038 \mathrm{E}+00$ & $4.802 \mathrm{E}+00$ & $1.040 \mathrm{E}+14$ & 0.000 \\
\hline $1 s 4 d^{3} D_{2}$ & $1 s 4 f^{1} F_{3}^{o}$ & $5.3023 \mathrm{E}+02$ & $7.960 \mathrm{E}-06$ & $2.698 \mathrm{E}+04$ & $5.3185 E+02$ & $8.835 \mathrm{E}-06$ & $2.951 \mathrm{E}+04$ & 0.047 \\
\hline $1 s 4 d^{3} D_{3}$ & $1 s 4 f^{1} F_{3}^{o}$ & $1.5391 \mathrm{E}+03$ & $2.525 \mathrm{E}-03$ & $1.016 \mathrm{E}+06$ & $1.5556 \mathrm{E}+03$ & $2.486 \mathrm{E}-03$ & $9.708 \mathrm{E}+05$ & 0.009 \\
\hline $1 s 4 d^{1} D_{2}$ & $1 s 4 f^{1} F_{3}^{o}$ & $1.6685 \mathrm{E}+03$ & $4.632 \mathrm{E}-02$ & $1.585 \mathrm{E}+07$ & $1.6908 \mathrm{E}+03$ & $4.551 \mathrm{E}-02$ & $1.504 \mathrm{E}+07$ & 0.015 \\
\hline $1 s 2 p^{3} P_{1}^{o}$ & $1 s 5 s^{3} s_{1}$ & $1.4605 \mathrm{E}+00$ & $2.662 \mathrm{E}-03$ & $2.774 \mathrm{E}+12$ & $1.4544 \mathrm{E}+00$ & $2.703 \mathrm{E}-03$ & $2.817 \mathrm{E}+12$ & 0.002 \\
\hline $1 s 2 p^{3} P_{0}^{o}$ & $1 s 5 s^{3} s_{1}$ & $1.4615 \mathrm{E}+00$ & $1.303 E-03$ & $1.356 \mathrm{E}+12$ & $1.4554 \mathrm{E}+00$ & $1.301 \mathrm{E}-03$ & $1.354 \mathrm{E}+12$ & 0.001 \\
\hline $1 s 2 p^{3} P_{2}^{o}$ & $1 s 5 s^{3} s_{1}$ & $1.5306 \mathrm{E}+00$ & $8.845 \mathrm{E}-03$ & $8.395 \mathrm{E}+12$ & $1.5241 \mathrm{E}+00$ & $8.802 \mathrm{E}-03$ & $8.353 \mathrm{E}+12$ & 0.002 \\
\hline $1 s 2 p{ }^{1} P_{1}^{o}$ & $1 s 5 s^{3} S_{1}$ & $1.5372 \mathrm{E}+00$ & $1.606 \mathrm{E}-03$ & $1.511 \mathrm{E}+12$ & $1.5308 \mathrm{E}+00$ & $1.572 \mathrm{E}-03$ & $1.479 \mathrm{E}+12$ & 0.002 \\
\hline $1 s 3 p^{3} P_{1}^{o}$ & $1 s 5 s^{3} s_{1}$ & $4.3516 \mathrm{E}+00$ & $1.619 \mathrm{E}-02$ & $1.901 \mathrm{E}+12$ & $4.3336 \mathrm{E}+00$ & $1.622 \mathrm{E}-02$ & $1.904 \mathrm{E}+12$ & 0.001 \\
\hline $1 s 3 p^{3} P_{0}^{o}$ & $1 s 5 s^{3} S_{1}$ & $4.3540 \mathrm{E}+00$ & $7.886 \mathrm{E}-03$ & $9.249 \mathrm{E}+11$ & $4.3359 \mathrm{E}+00$ & $7.777 \mathrm{E}-03$ & $9.119 \mathrm{E}+11$ & 0.001 \\
\hline $1 s 3 p^{3} P_{2}^{o}$ & $1 s 5 s^{3} S_{1}$ & $4.5356 \mathrm{E}+00$ & $5.271 \mathrm{E}-02$ & $5.697 \mathrm{E}+12$ & $4.5167 \mathrm{E}+00$ & $5.254 \mathrm{E}-02$ & $5.678 \mathrm{E}+12$ & 0.000 \\
\hline $1 s 3 p{ }^{1} P_{1}^{o}$ & $1 s 5 s^{3} s_{1}$ & $4.5521 \mathrm{E}+00$ & $9.615 \mathrm{E}-03$ & $1.032 \mathrm{E}+12$ & $4.5333 \mathrm{E}+00$ & $9.475 \mathrm{E}-03$ & $1.016 \mathrm{E}+12$ & 0.001 \\
\hline $1 s 4 p^{3} P_{1}^{o}$ & $1 s 5 s^{3} s_{1}$ & $1.3877 \mathrm{E}+01$ & $1.166 \mathrm{E}-01$ & $1.346 \mathrm{E}+12$ & $1.3818 \mathrm{E}+01$ & $1.159 \mathrm{E}-01$ & $1.339 \mathrm{E}+12$ & 0.001 \\
\hline $1 s 4 p^{3} P_{0}^{o}$ & $1 s 5 s^{3} s_{1}$ & $1.3887 \mathrm{E}+01$ & $5.665 \mathrm{E}-02$ & $6.532 \mathrm{E}+11$ & $1.3827 \mathrm{E}+01$ & $5.525 \mathrm{E}-02$ & $6.371 \mathrm{E}+11$ & 0.002 \\
\hline $1 s 4 p^{3} P_{2}^{o}$ & $1 s 5 s^{3} s_{1}$ & $1.4675 \mathrm{E}+01$ & $3.952 \mathrm{E}-01$ & $4.080 \mathrm{E}+12$ & $1.4612 \mathrm{E}+01$ & $3.932 \mathrm{E}-01$ & $4.060 \mathrm{E}+12$ & 0.001 \\
\hline $1 s 4 p{ }^{1} P_{1}^{o}$ & $1 s 5 s^{3} s_{1}$ & $1.4747 \mathrm{E}+01$ & $7.255 \mathrm{E}-02$ & $7.417 \mathrm{E}+11$ & $1.4684 \mathrm{E}+01$ & $7.130 \mathrm{E}-02$ & $7.289 \mathrm{E}+11$ & 0.001 \\
\hline $1 s 4 f^{3} F_{2}^{o}$ & $1 s 5 s^{3} S_{1}$ & $1.5020 \mathrm{E}+01$ & $4.244 \mathrm{E}-08$ & $4.183 \mathrm{E}+05$ & & & & 0.057 \\
\hline $1 s^{2}{ }^{1} s_{0}$ & $1 s 5 p^{3} P_{1}^{o}$ & $3.2030 \mathrm{E}-01$ & $7.183 E-03$ & $1.556 \mathrm{E}+14$ & $3.1904 \mathrm{E}-01$ & $6.799 \mathrm{E}-03$ & $1.472 \mathrm{E}+14$ & 0.011 \\
\hline $1 s 2 s^{3} S_{1}$ & $1 s 5 p^{3} P_{1}^{o}$ & $1.4466 \mathrm{E}+00$ & $2.787 \mathrm{E}-02$ & $2.961 \mathrm{E}+13$ & $1.4405 \mathrm{E}+00$ & $2.788 \mathrm{E}-02$ & $2.962 \mathrm{E}+13$ & 0.002 \\
\hline $1 s 2 s^{1} S_{0}$ & $1 s 5 p^{3} P_{1}^{o}$ & $1.4611 \mathrm{E}+00$ & $1.211 \mathrm{E}-02$ & $1.261 \mathrm{E}+13$ & $1.4551 \mathrm{E}+00$ & $1.185 \mathrm{E}-02$ & $1.234 \mathrm{E}+13$ & 0.006 \\
\hline $1 s 3 s^{3} S_{1}$ & $1 s 5 p^{3} P_{1}^{o}$ & $4.3132 \mathrm{E}+00$ & $8.123 E-02$ & $9.709 \mathrm{E}+12$ & $4.2953 E+00$ & $8.191 \mathrm{E}-02$ & $9.788 \mathrm{E}+12$ & 0.002 \\
\hline $1 s 3 s{ }^{1} S_{0}$ & $1 s 5 p^{3} P_{1}^{o}$ & $4.3472 \mathrm{E}+00$ & $3.579 \mathrm{E}-02$ & $4.210 \mathrm{E}+12$ & $4.3296 \mathrm{E}+00$ & $3.517 \mathrm{E}-02$ & $4.136 \mathrm{E}+12$ & 0.002 \\
\hline $1 s 3 d^{3} D_{2}$ & $1 s 5 p^{3} P_{1}^{o}$ & $4.5423 \mathrm{E}+00$ & $1.400 \mathrm{E}-02$ & $1.509 \mathrm{E}+12$ & $4.5233 \mathrm{E}+00$ & $1.405 \mathrm{E}-02$ & $1.514 \mathrm{E}+12$ & 0.001 \\
\hline $1 s 3 d^{3} D_{1}$ & $1 s 5 p^{3} P_{1}^{o}$ & $4.5448 \mathrm{E}+00$ & $3.044 \mathrm{E}-03$ & $3.276 \mathrm{E}+11$ & $4.5258 \mathrm{E}+00$ & $2.992 \mathrm{E}-03$ & $3.221 \mathrm{E}+11$ & 0.004 \\
\hline $1 s 3 d{ }^{1} D_{2}$ & $1 s 5 p^{3} P_{1}^{o}$ & $4.6059 \mathrm{E}+00$ & $1.856 \mathrm{E}-05$ & $1.945 \mathrm{E}+09$ & & & & 0.012 \\
\hline $1 s 4 s^{3} s_{1}$ & $1 s 5 p^{3} P_{1}^{o}$ & $1.3679 \mathrm{E}+01$ & $3.607 \mathrm{E}-01$ & $4.285 \mathrm{E}+12$ & $1.3623 \mathrm{E}+01$ & $3.658 \mathrm{E}-01$ & $4.346 \mathrm{E}+12$ & 0.002 \\
\hline $1 s 4 s^{1} S_{0}$ & $1 s 5 p^{3} P_{1}^{o}$ & $1.3818 \mathrm{E}+01$ & $1.618 \mathrm{E}-01$ & $1.884 \mathrm{E}+12$ & $1.3762 \mathrm{E}+01$ & $1.583 \mathrm{E}-01$ & $1.843 \mathrm{E}+12$ & 0.001 \\
\hline $1 s 4 d^{3} D_{2}$ & $1 s 5 p^{3} P_{1}^{o}$ & $1.4661 \mathrm{E}+01$ & $1.771 \mathrm{E}-01$ & $1.832 \mathrm{E}+12$ & $1.4602 \mathrm{E}+01$ & $1.772 \mathrm{E}-01$ & $1.832 \mathrm{E}+12$ & 0.000 \\
\hline $1 s 4 d^{3} D_{1}$ & $1 s 5 p^{3} P_{1}^{o}$ & $1.4671 \mathrm{E}+01$ & $3.825 \mathrm{E}-02$ & $3.951 \mathrm{E}+11$ & $1.4612 \mathrm{E}+01$ & $3.753 \mathrm{E}-02$ & $3.875 \mathrm{E}+11$ & 0.002 \\
\hline $1 s 4 d^{1} D_{2}$ & $1 s 5 p^{3} P_{1}^{o}$ & $1.4943 \mathrm{E}+01$ & $2.115 \mathrm{E}-04$ & $2.106 \mathrm{E}+09$ & $1.4882 \mathrm{E}+01$ & $1.298 \mathrm{E}-04$ & $1.292 \mathrm{E}+09$ & 0.007 \\
\hline $1 s 5 s^{3} S_{1}$ & $1 s 5 p^{3} P_{1}^{o}$ & $2.8648 \mathrm{E}+03$ & $3.352 \mathrm{E}-02$ & $9.081 \mathrm{E}+06$ & $2.9004 \mathrm{E}+03$ & $3.303 \mathrm{E}-02$ & $8.656 \mathrm{E}+06$ & 0.158 \\
\hline $1 s 2 p^{3} P_{1}^{o}$ & $1 s 5 s{ }^{1} s_{0}$ & $1.4597 \mathrm{E}+00$ & $1.072 \mathrm{E}-03$ & $3.357 \mathrm{E}+12$ & $1.4537 \mathrm{E}+00$ & $1.107 \mathrm{E}-03$ & $3.465 \mathrm{E}+12$ & 0.007 \\
\hline $1 s 2 p^{1} P_{1}^{o}$ & $1 s 5 s^{1} s_{0}$ & $1.5363 \mathrm{E}+00$ & $3.681 \mathrm{E}-03$ & $1.040 \mathrm{E}+13$ & $1.5300 \mathrm{E}+00$ & $3.664 \mathrm{E}-03$ & $1.035 \mathrm{E}+13$ & 0.003 \\
\hline $1 s 3 p^{3} P_{1}^{o}$ & $1 s 5 s{ }^{1} S_{0}$ & $4.3447 \mathrm{E}+00$ & $6.572 \mathrm{E}-03$ & $2.322 \mathrm{E}+12$ & $4.3266 \mathrm{E}+00$ & $6.732 \mathrm{E}-03$ & $2.378 \mathrm{E}+12$ & 0.001 \\
\hline $1 s 3 p{ }^{1} P_{1}^{o}$ & $1 s 5 s{ }^{1} S_{0}$ & $4.5445 \mathrm{E}+00$ & $2.173 \mathrm{E}-02$ & $7.020 \mathrm{E}+12$ & $4.5257 \mathrm{E}+00$ & $2.187 \mathrm{E}-02$ & $7.061 \mathrm{E}+12$ & 0.000 \\
\hline $1 s 4 p^{3} P_{1}^{o}$ & $1 s 5 s^{1} S_{0}$ & $1.3807 \mathrm{E}+01$ & $4.696 \mathrm{E}-02$ & $1.643 \mathrm{E}+12$ & $1.3747 \mathrm{E}+01$ & $4.812 \mathrm{E}-02$ & $1.684 \mathrm{E}+12$ & 0.000 \\
\hline $1 s 4 p{ }^{1} P_{1}^{o}$ & $1 s 5 s^{1} S_{0}$ & $1.4668 \mathrm{E}+01$ & $1.615 \mathrm{E}-01$ & $5.008 \mathrm{E}+12$ & $1.4605 \mathrm{E}+01$ & $1.637 \mathrm{E}-01$ & $5.077 \mathrm{E}+12$ & 0.000 \\
\hline $1 s 5 p^{3} P_{1}^{0}$ & $1 s 5 s{ }^{1} S_{0}$ & $5.6589 \mathrm{E}+04$ & $7.282 \mathrm{E}-04$ & $1.517 \mathrm{E}+03$ & $3.7737 \mathrm{E}+04$ & $1.084 \mathrm{E}-03$ & $5.038 \mathrm{E}+03$ & 0.577 \\
\hline $1 s 2 s^{3} S_{1}$ & $1 s 5 p^{3} P_{0}^{o}$ & $1.4465 \mathrm{E}+00$ & $1.324 \mathrm{E}-02$ & $4.222 \mathrm{E}+13$ & $1.4404 \mathrm{E}+00$ & $1.328 \mathrm{E}-02$ & $4.235 E+13$ & 0.003 \\
\hline $1 s 3 s^{3} S_{1}$ & $1 s 5 p^{3} P_{0}^{o}$ & $4.3127 \mathrm{E}+00$ & $3.863 \mathrm{E}-02$ & $1.385 \mathrm{E}+13$ & $4.2949 \mathrm{E}+00$ & $3.919 \mathrm{E}-02$ & $1.405 \mathrm{E}+13$ & 0.003 \\
\hline $1 s 3 d^{3} D_{1}$ & $1 s 5 p^{3} P_{0}^{o}$ & $4.5443 \mathrm{E}+00$ & $5.643 \mathrm{E}-03$ & $1.823 \mathrm{E}+12$ & $4.5253 \mathrm{E}+00$ & $5.635 \mathrm{E}-03$ & $1.819 \mathrm{E}+12$ & 0.004 \\
\hline $1 s 4 s^{3} S_{1}$ & $1 s 5 p^{3} P_{0}^{o}$ & $1.3675 \mathrm{E}+01$ & $1.715 \mathrm{E}-01$ & $6.118 \mathrm{E}+12$ & $1.3619 \mathrm{E}+01$ & $1.758 \mathrm{E}-01$ & $6.271 \mathrm{E}+12$ & 0.003 \\
\hline $1 s 4 d^{3} D_{1}$ & $1 s 5 p^{3} P_{0}^{o}$ & $1.4666 \mathrm{E}+01$ & $7.131 \mathrm{E}-02$ & $2.212 \mathrm{E}+12$ & $1.4607 \mathrm{E}+01$ & $7.119 \mathrm{E}-02$ & $2.206 \mathrm{E}+12$ & 0.002 \\
\hline $1 s 5 s^{3} S_{1}$ & $1 s 5 p^{3} P_{0}^{o}$ & $2.6711 \mathrm{E}+03$ & $1.723 \mathrm{E}-02$ & $1.611 \mathrm{E}+07$ & $2.7075 E+03$ & $1.693 \mathrm{E}-02$ & $1.527 \mathrm{E}+07$ & 0.131 \\
\hline $1 s 2 s^{3} s_{1}$ & $1 s 5 p^{3} P_{2}^{0}$ & $1.4424 \mathrm{E}+00$ & $6.070 \mathrm{E}-02$ & $3.892 \mathrm{E}+13$ & $1.4363 \mathrm{E}+00$ & $6.235 \mathrm{E}-02$ & $3.998 \mathrm{E}+13$ & 0.002 \\
\hline $1 s 3 s^{3} S_{1}$ & $1 s 5 p^{3} P_{2}^{o}$ & $4.2763 \mathrm{E}+00$ & $1.651 \mathrm{E}-01$ & $1.205 E+13$ & $4.2586 \mathrm{E}+00$ & $1.679 \mathrm{E}-01$ & $1.225 \mathrm{E}+13$ & 0.003 \\
\hline $1 s 3 d^{3} D_{2}$ & $1 s 5 p^{3} P_{2}^{o}$ & $4.5014 \mathrm{E}+00$ & $1.723 E-03$ & $1.135 \mathrm{E}+11$ & $4.4826 \mathrm{E}+00$ & $1.717 \mathrm{E}-03$ & $1.130 \mathrm{E}+11$ & 0.002 \\
\hline $1 s 3 d^{3} D_{1}$ & $1 s 5 p^{3} P_{2}^{o}$ & $4.5039 \mathrm{E}+00$ & $1.857 \mathrm{E}-04$ & $1.222 \mathrm{E}+10$ & $4.4850 \mathrm{E}+00$ & $1.847 \mathrm{E}-04$ & $1.214 \mathrm{E}+10$ & 0.005 \\
\hline $1 s 3 d^{3} D_{3}$ & $1 s 5 p^{3} P_{2}^{o}$ & $4.5615 \mathrm{E}+00$ & $1.853 \mathrm{E}-02$ & $1.188 \mathrm{E}+12$ & $4.5423 \mathrm{E}+00$ & $1.822 \mathrm{E}-02$ & $1.168 \mathrm{E}+12$ & 0.002 \\
\hline $1 s 3 d^{1} D_{2}$ & $1 s 5 p^{3} P_{2}^{o}$ & $4.5638 \mathrm{E}+00$ & $1.318 \mathrm{E}-03$ & $8.443 E+10$ & $4.5447 \mathrm{E}+00$ & $1.283 \mathrm{E}-03$ & $8.220 \mathrm{E}+10$ & 0.005 \\
\hline $1 s 4 s^{3} S_{1}$ & $1 s 5 p^{3} P_{2}^{o}$ & $1.3315 \mathrm{E}+01$ & $6.598 \mathrm{E}-01$ & $4.965 \mathrm{E}+12$ & $1.3261 \mathrm{E}+01$ & $6.702 \mathrm{E}-01$ & $5.041 \mathrm{E}+12$ & 0.003 \\
\hline $1 s 4 d^{3} D_{2}$ & $1 s 5 p^{3} P_{2}^{0}$ & $1.4243 \mathrm{E}+01$ & $2.163 \mathrm{E}-02$ & $1.422 \mathrm{E}+11$ & $1.4186 \mathrm{E}+01$ & $2.133 \mathrm{E}-02$ & $1.402 \mathrm{E}+11$ & 0.001 \\
\hline $1 s 4 d^{3} D_{1}$ & $1 s 5 p^{3} P_{2}^{o}$ & $1.4253 \mathrm{E}+01$ & $2.338 \mathrm{E}-03$ & $1.535 \mathrm{E}+10$ & $1.4196 \mathrm{E}+01$ & $2.306 \mathrm{E}-03$ & $1.513 \mathrm{E}+10$ & 0.002 \\
\hline $1 s 4 d^{3} D_{3}$ & $1 s 5 p^{3} P_{2}^{o}$ & $1.4499 \mathrm{E}+01$ & $2.318 \mathrm{E}-01$ & $1.471 \mathrm{E}+12$ & $1.4440 \mathrm{E}+01$ & $2.276 \mathrm{E}-01$ & $1.444 \mathrm{E}+12$ & 0.001 \\
\hline $1 s 4 d{ }^{1} D_{2}$ & $1 s 5 p^{3} P_{2}^{o}$ & $1.4509 \mathrm{E}+01$ & $1.640 \mathrm{E}-02$ & $1.039 \mathrm{E}+11$ & $1.4450 \mathrm{E}+01$ & $1.598 \mathrm{E}-02$ & $1.012 \mathrm{E}+11$ & 0.002 \\
\hline $1 s 5 s^{3} s_{1}$ & $1 s 5 p^{3} P_{2}^{o}$ & $4.2565 \mathrm{E}+02$ & $5.519 \mathrm{E}-01$ & $4.064 \mathrm{E}+09$ & $4.2515 \mathrm{E}+02$ & $5.512 \mathrm{E}-01$ & $4.033 \mathrm{E}+09$ & 0.022 \\
\hline $1 s 2 p^{3} P_{1}^{o}$ & $1 s 5 d^{3} D_{2}$ & $1.4552 \mathrm{E}+00$ & $1.097 \mathrm{E}-01$ & $6.914 \mathrm{E}+13$ & $1.4491 \mathrm{E}+00$ & $1.101 \mathrm{E}-01$ & $6.935 E+13$ & 0.001 \\
\hline $1 s 2 p^{3} P_{2}^{o}$ & $1 s 5 d^{3} D_{2}$ & $1.5247 \mathrm{E}+00$ & $1.907 \mathrm{E}-02$ & $1.094 \mathrm{E}+13$ & $1.5183 \mathrm{E}+00$ & $1.865 \mathrm{E}-02$ & $1.070 \mathrm{E}+13$ & 0.002 \\
\hline $1 s 2 p{ }^{1} P_{1}^{o}$ & $1 s 5 d^{3} D_{2}$ & $1.5313 \mathrm{E}+00$ & $3.170 \mathrm{E}-03$ & $1.803 \mathrm{E}+12$ & $1.5250 \mathrm{E}+00$ & $3.140 \mathrm{E}-03$ & $1.786 \mathrm{E}+12$ & 0.002 \\
\hline $1 s 3 p^{3} P_{1}^{o}$ & $1 s 5 d^{3} D_{2}$ & $4.3049 \mathrm{E}+00$ & $3.188 \mathrm{E}-01$ & $2.295 E+13$ & $4.2870 \mathrm{E}+00$ & $3.199 \mathrm{E}-01$ & $2.302 \mathrm{E}+13$ & 0.001 \\
\hline $1 s 3 p^{3} P_{2}^{o}$ & $1 s 5 d^{3} D_{2}$ & $4.4849 \mathrm{E}+00$ & $6.198 \mathrm{E}-02$ & $4.111 \mathrm{E}+12$ & $4.4661 \mathrm{E}+00$ & $6.194 \mathrm{E}-02$ & $4.107 \mathrm{E}+12$ & 0.001 \\
\hline $1 s 3 p^{1} P_{1}^{o}$ & $1 s 5 d^{3} D_{2}$ & $4.5010 \mathrm{E}+00$ & $1.016 \mathrm{E}-02$ & $6.693 \mathrm{E}+11$ & $4.4823 \mathrm{E}+00$ & $9.871 \mathrm{E}-03$ & $6.499 \mathrm{E}+11$ & 0.002 \\
\hline $1 s 4 p^{3} P_{1}^{o}$ & $1 s 5 d^{3} D_{2}$ & $1.3413 \mathrm{E}+01$ & $1.225 \mathrm{E}+00$ & $9.083 \mathrm{E}+12$ & $1.3354 \mathrm{E}+01$ & $1.230 \mathrm{E}+00$ & $9.125 \mathrm{E}+12$ & 0.000 \\
\hline
\end{tabular}


Table 3 (continued)

\begin{tabular}{|c|c|c|c|c|c|c|c|c|}
\hline \multirow[t]{2}{*}{ Lower } & \multirow[t]{2}{*}{ Upper } & \multicolumn{3}{|l|}{ GRASP2K } & \multicolumn{3}{|l|}{ FAC } & \multirow[t]{2}{*}{$d T$} \\
\hline & & $\lambda(\AA)$ & $g f$ & $A\left(\mathrm{~s}^{-1}\right)$ & $\lambda(\AA)$ & $g f$ & $A\left(\mathrm{~s}^{-1}\right)$ & \\
\hline $1 s 4 p^{3} P_{2}^{o}$ & $1 s 5 d^{3} D_{2}$ & $1.4158 \mathrm{E}+01$ & $2.724 \mathrm{E}-01$ & $1.813 \mathrm{E}+12$ & $1.4095 \mathrm{E}+01$ & $2.756 \mathrm{E}-01$ & $1.835 \mathrm{E}+12$ & 0.001 \\
\hline $1 s 4 p^{1} P_{1}^{o}$ & $1 s 5 d^{3} D_{2}$ & $1.4224 \mathrm{E}+01$ & $4.478 \mathrm{E}-02$ & $2.952 \mathrm{E}+11$ & $1.4162 \mathrm{E}+01$ & $4.221 \mathrm{E}-02$ & $2.784 \mathrm{E}+11$ & 0.001 \\
\hline $1 s 4 f^{3} F_{3}^{o}$ & $1 s 5 d^{3} D_{2}$ & $1.4473 \mathrm{E}+01$ & $6.876 \mathrm{E}-02$ & $4.380 \mathrm{E}+11$ & $1.4409 \mathrm{E}+01$ & $6.894 \mathrm{E}-02$ & $4.392 \mathrm{E}+11$ & 0.001 \\
\hline $1 s 4 f^{3} F_{2}^{o}$ & $1 s 5 d^{3} D_{2}$ & $1.4478 \mathrm{E}+01$ & $5.008 \mathrm{E}-03$ & $3.187 \mathrm{E}+10$ & $1.4414 \mathrm{E}+01$ & $5.000 \mathrm{E}-03$ & $3.183 E+10$ & 0.000 \\
\hline $1 s 4 f^{1} F_{3}^{o}$ & $1 s 5 d^{3} D_{2}$ & $1.4606 \mathrm{E}+01$ & $5.098 \mathrm{E}-06$ & $3.188 \mathrm{E}+07$ & & & & 0.008 \\
\hline $1 s 5 p^{3} P_{1}^{o}$ & $1 s 5 d^{3} D_{2}$ & $4.6651 \mathrm{E}+02$ & $4.431 \mathrm{E}-01$ & $2.716 \mathrm{E}+09$ & $4.6168 \mathrm{E}+02$ & $4.458 \mathrm{E}-01$ & $2.766 \mathrm{E}+09$ & 0.001 \\
\hline $1 s 5 p^{3} P_{2}^{o}$ & $1 s 5 d^{3} D_{2}$ & $6.9791 \mathrm{E}+03$ & $5.402 \mathrm{E}-03$ & $1.480 \mathrm{E}+05$ & & & & 0.074 \\
\hline $1 s^{2}{ }^{1} S_{0}$ & $1 s 5 p^{1} P_{1}^{o}$ & $3.2010 \mathrm{E}-01$ & $1.758 \mathrm{E}-02$ & $3.812 \mathrm{E}+14$ & $3.1882 \mathrm{E}-01$ & $1.876 \mathrm{E}-02$ & $4.069 \mathrm{E}+14$ & 0.030 \\
\hline $1 s 2 s^{3} S_{1}$ & $1 s 5 p^{1} P_{1}^{o}$ & $1.4421 \mathrm{E}+00$ & $1.083 \mathrm{E}-02$ & $1.158 \mathrm{E}+13$ & $1.4360 \mathrm{E}+00$ & $1.116 \mathrm{E}-02$ & $1.193 \mathrm{E}+13$ & 0.002 \\
\hline $1 s 2 s^{1} S_{0}$ & $1 s 5 p^{1} P_{1}^{o}$ & $1.4565 \mathrm{E}+00$ & $2.585 \mathrm{E}-02$ & $2.709 E+13$ & $1.4505 \mathrm{E}+00$ & $2.600 \mathrm{E}-02$ & $2.724 \mathrm{E}+13$ & 0.003 \\
\hline $1 s 3 s^{3} S_{1}$ & $1 s 5 p^{1} P_{1}^{o}$ & $4.2732 \mathrm{E}+00$ & $2.926 \mathrm{E}-02$ & $3.563 \mathrm{E}+12$ & $4.2555 \mathrm{E}+00$ & $2.990 \mathrm{E}-02$ & $3.640 \mathrm{E}+12$ & 0.003 \\
\hline $1 s 3 s^{1} S_{0}$ & $1 s 5 p^{1} P_{1}^{o}$ & $4.3066 \mathrm{E}+00$ & $7.083 \mathrm{E}-02$ & $8.491 \mathrm{E}+12$ & $4.2891 \mathrm{E}+00$ & $7.040 \mathrm{E}-02$ & $8.437 \mathrm{E}+12$ & 0.001 \\
\hline $1 s 3 d^{3} D_{2}$ & $1 s 5 p^{1} P_{1}^{o}$ & $4.4980 \mathrm{E}+00$ & $2.798 \mathrm{E}-04$ & $3.075 E+10$ & $4.4792 \mathrm{E}+00$ & $3.159 \mathrm{E}-04$ & $3.471 \mathrm{E}+10$ & 0.006 \\
\hline $1 s 3 d^{3} D_{1}$ & $1 s 5 p^{1} P_{1}^{o}$ & $4.5005 \mathrm{E}+00$ & $8.523 \mathrm{E}-04$ & $9.356 \mathrm{E}+10$ & $4.4816 \mathrm{E}+00$ & $8.478 \mathrm{E}-04$ & $9.306 \mathrm{E}+10$ & 0.006 \\
\hline $1 s 3 d{ }^{1} D_{2}$ & $1 s 5 p^{1} P_{1}^{o}$ & $4.5603 \mathrm{E}+00$ & $1.161 \mathrm{E}-02$ & $1.241 \mathrm{E}+12$ & $4.5411 \mathrm{E}+00$ & $1.171 \mathrm{E}-02$ & $1.251 \mathrm{E}+12$ & 0.006 \\
\hline $1 s 4 s^{3} S_{1}$ & $1 s 5 p^{1} P_{1}^{o}$ & $1.3285 \mathrm{E}+01$ & $1.158 \mathrm{E}-01$ & $1.458 \mathrm{E}+12$ & $1.3231 \mathrm{E}+01$ & $1.184 \mathrm{E}-01$ & $1.491 \mathrm{E}+12$ & 0.003 \\
\hline $1 s 4 s^{1} S_{0}$ & $1 s 5 p^{1} P_{1}^{o}$ & $1.3416 \mathrm{E}+01$ & $2.856 \mathrm{E}-01$ & $3.528 \mathrm{E}+12$ & $1.3362 \mathrm{E}+01$ & $2.818 \mathrm{E}-01$ & $3.479 E+12$ & 0.000 \\
\hline $1 s 4 d^{3} D_{2}$ & $1 s 5 p^{1} P_{1}^{o}$ & $1.4209 \mathrm{E}+01$ & $3.407 \mathrm{E}-03$ & $3.752 E+10$ & $1.4152 \mathrm{E}+01$ & $3.849 \mathrm{E}-03$ & $4.237 \mathrm{E}+10$ & 0.003 \\
\hline $1 s 4 d^{3} D_{1}$ & $1 s 5 p^{1} P_{1}^{o}$ & $1.4219 \mathrm{E}+01$ & $1.055 \mathrm{E}-02$ & $1.160 \mathrm{E}+11$ & $1.4162 \mathrm{E}+01$ & $1.040 \mathrm{E}-02$ & $1.144 \mathrm{E}+11$ & 0.003 \\
\hline $1 s 4 d{ }^{1} D_{2}$ & $1 s 5 p^{1} P_{1}^{o}$ & $1.4474 \mathrm{E}+01$ & $1.448 \mathrm{E}-01$ & $1.537 \mathrm{E}+12$ & $1.4415 \mathrm{E}+01$ & $1.466 \mathrm{E}-01$ & $1.555 \mathrm{E}+12$ & 0.003 \\
\hline $1 s 5 s^{3} S_{1}$ & $1 s 5 p^{1} P_{1}^{o}$ & $3.9729 \mathrm{E}+02$ & $1.063 \mathrm{E}-01$ & $1.497 \mathrm{E}+09$ & $3.9647 \mathrm{E}+02$ & $1.060 \mathrm{E}-01$ & $1.487 \mathrm{E}+09$ & 0.020 \\
\hline $1 s 5 s^{1} S_{0}$ & $1 s 5 p^{1} P_{1}^{o}$ & $4.6505 \mathrm{E}+02$ & $2.128 \mathrm{E}-01$ & $2.188 \mathrm{E}+09$ & $4.6491 \mathrm{E}+02$ & $2.116 \mathrm{E}-01$ & $2.159 \mathrm{E}+09$ & 0.040 \\
\hline $1 s 5 d^{3} D_{2}$ & $1 s 5 p^{1} P_{1}^{o}$ & $4.1006 \mathrm{E}+04$ & $1.481 \mathrm{E}-04$ & $1.958 \mathrm{E}+02$ & & & & 0.294 \\
\hline $1 s 2 p^{3} P_{1}^{o}$ & $1 s 5 d^{3} D_{1}$ & $1.4551 \mathrm{E}+00$ & $2.353 \mathrm{E}-02$ & $2.470 \mathrm{E}+13$ & $1.4491 \mathrm{E}+00$ & $2.355 \mathrm{E}-02$ & $2.472 \mathrm{E}+13$ & 0.001 \\
\hline $1 s 2 p^{3} P_{0}^{o}$ & $1 s 5 d^{3} D_{1}$ & $1.4562 \mathrm{E}+00$ & $4.450 \mathrm{E}-02$ & $4.666 \mathrm{E}+13$ & $1.4501 \mathrm{E}+00$ & $4.454 \mathrm{E}-02$ & $4.669 \mathrm{E}+13$ & 0.001 \\
\hline $1 s 2 p^{3} P_{2}^{o}$ & $1 s 5 d^{3} D_{1}$ & $1.5247 \mathrm{E}+00$ & $2.091 \mathrm{E}-03$ & $2.000 \mathrm{E}+12$ & $1.5182 \mathrm{E}+00$ & $2.036 \mathrm{E}-03$ & $1.947 \mathrm{E}+12$ & 0.002 \\
\hline $1 s 2 p^{1} P_{1}^{o}$ & $1 s 5 d^{3} D_{1}$ & $1.5313 \mathrm{E}+00$ & $9.303 E-03$ & $8.822 \mathrm{E}+12$ & $1.5249 \mathrm{E}+00$ & $9.050 \mathrm{E}-03$ & $8.580 \mathrm{E}+12$ & 0.002 \\
\hline $1 s 3 p^{3} P_{1}^{o}$ & $1 s 5 d^{3} D_{1}$ & $4.3044 \mathrm{E}+00$ & $6.792 \mathrm{E}-02$ & $8.151 E+12$ & $4.2865 \mathrm{E}+00$ & $6.846 \mathrm{E}-02$ & $8.213 E+12$ & 0.001 \\
\hline $1 s 3 p^{3} P_{0}^{0}$ & $1 s 5 d^{3} D_{1}$ & $4.3068 \mathrm{E}+00$ & $1.290 \mathrm{E}-01$ & $1.546 \mathrm{E}+13$ & $4.2888 \mathrm{E}+00$ & $1.293 \mathrm{E}-01$ & $1.550 \mathrm{E}+13$ & 0.001 \\
\hline $1 s 3 p^{3} P_{2}^{o}$ & $1 s 5 d^{3} D_{1}$ & $4.4844 \mathrm{E}+00$ & $6.769 \mathrm{E}-03$ & $7.484 \mathrm{E}+11$ & $4.4656 \mathrm{E}+00$ & $6.748 \mathrm{E}-03$ & $7.460 \mathrm{E}+11$ & 0.002 \\
\hline $1 s 3 p{ }^{1} P_{1}^{o}$ & $1 s 5 d^{3} D_{1}$ & $4.5005 \mathrm{E}+00$ & $3.073 \mathrm{E}-02$ & $3.374 \mathrm{E}+12$ & $4.4818 \mathrm{E}+00$ & $3.056 \mathrm{E}-02$ & $3.354 \mathrm{E}+12$ & 0.002 \\
\hline $1 s 4 p^{3} P_{1}^{o}$ & $1 s 5 d^{3} D_{1}$ & $1.3409 \mathrm{E}+01$ & $2.602 \mathrm{E}-01$ & $3.218 \mathrm{E}+12$ & $1.3350 \mathrm{E}+01$ & $2.644 \mathrm{E}-01$ & $3.271 \mathrm{E}+12$ & 0.001 \\
\hline $1 s 4 p^{3} P_{0}^{0}$ & $1 s 5 d^{3} D_{1}$ & $1.3418 \mathrm{E}+01$ & $4.953 \mathrm{E}-01$ & $6.116 \mathrm{E}+12$ & $1.3359 \mathrm{E}+01$ & $4.977 \mathrm{E}-01$ & $6.148 \mathrm{E}+12$ & 0.001 \\
\hline $1 s 4 p^{3} P_{2}^{o}$ & $1 s 5 d^{3} D_{1}$ & $1.4153 \mathrm{E}+01$ & $2.966 \mathrm{E}-02$ & $3.292 \mathrm{E}+11$ & $1.4090 \mathrm{E}+01$ & $2.999 \mathrm{E}-02$ & $3.330 \mathrm{E}+11$ & 0.001 \\
\hline $1 s 4 p^{1} P_{1}^{o}$ & $1 s 5 d^{3} D_{1}$ & $1.4219 \mathrm{E}+01$ & $1.367 \mathrm{E}-01$ & $1.504 \mathrm{E}+12$ & $1.4157 \mathrm{E}+01$ & $1.374 \mathrm{E}-01$ & $1.512 \mathrm{E}+12$ & 0.001 \\
\hline $1 s 4 f^{3} F_{2}^{o}$ & $1 s 5 d^{3} D_{1}$ & $1.4473 \mathrm{E}+01$ & $4.410 \mathrm{E}-02$ & $4.681 \mathrm{E}+11$ & $1.4409 \mathrm{E}+01$ & $4.432 \mathrm{E}-02$ & $4.706 \mathrm{E}+11$ & 0.000 \\
\hline $1 s 5 p^{3} P_{1}^{o}$ & $1 s 5 d^{3} D_{1}$ & $4.6125 \mathrm{E}+02$ & $9.567 \mathrm{E}-02$ & $9.998 \mathrm{E}+08$ & $4.5655 \mathrm{E}+02$ & $9.626 \mathrm{E}-02$ & $1.018 \mathrm{E}+09$ & 0.006 \\
\hline $1 s 5 p^{3} P_{0}^{o}$ & $1 s 5 d^{3} D_{1}$ & $4.6670 \mathrm{E}+02$ & $1.788 \mathrm{E}-01$ & $1.825 \mathrm{E}+09$ & $4.6173 \mathrm{E}+02$ & $1.798 \mathrm{E}-01$ & $1.860 \mathrm{E}+09$ & 0.005 \\
\hline $1 s 5 p^{3} P_{2}^{o}$ & $1 s 5 d^{3} D_{1}$ & $5.9615 \mathrm{E}+03$ & $6.837 \mathrm{E}-04$ & $4.277 \mathrm{E}+04$ & & & & 0.014 \\
\hline $1 s 5 p^{1} P_{1}^{o}$ & $1 s 5 d^{3} D_{1}$ & $1.4006 \mathrm{E}+07$ & $1.340 \mathrm{E}-06$ & $1.521 \mathrm{E}-05$ & & & & 0.999 \\
\hline $1 s 2 p^{3} P_{2}^{o}$ & $1 s 5 d^{3} D_{3}$ & $1.5233 \mathrm{E}+00$ & $1.832 \mathrm{E}-01$ & $7.522 \mathrm{E}+13$ & $1.5168 \mathrm{E}+00$ & $1.864 \mathrm{E}-01$ & $7.654 \mathrm{E}+13$ & 0.000 \\
\hline $1 s 3 p^{3} P_{2}^{o}$ & $1 s 5 d^{3} D_{3}$ & $4.4722 \mathrm{E}+00$ & $5.674 \mathrm{E}-01$ & $2.703 E+13$ & $4.4535 \mathrm{E}+00$ & $5.745 \mathrm{E}-01$ & $2.737 \mathrm{E}+13$ & 0.001 \\
\hline $1 s 4 p^{3} P_{2}^{o}$ & $1 s 5 d^{3} D_{3}$ & $1.4032 \mathrm{E}+01$ & $2.380 \mathrm{E}+00$ & $1.152 \mathrm{E}+13$ & $1.3970 \mathrm{E}+01$ & $2.417 \mathrm{E}+00$ & $1.170 \mathrm{E}+13$ & 0.000 \\
\hline $1 s 4 f^{3} F_{3}^{o}$ & $1 s 5 d^{3} D_{3}$ & $1.4341 \mathrm{E}+01$ & $3.955 \mathrm{E}-03$ & $1.833 \mathrm{E}+10$ & $1.4279 \mathrm{E}+01$ & $3.981 \mathrm{E}-03$ & $1.844 \mathrm{E}+10$ & 0.001 \\
\hline $1 s 4 f^{3} F_{2}^{o}$ & $1 s 5 d^{3} D_{3}$ & $1.4347 \mathrm{E}+01$ & $1.850 \mathrm{E}-04$ & $8.566 \mathrm{E}+08$ & & & & 0.000 \\
\hline $1 s 4 f^{3} F_{4}^{o}$ & $1 s 5 d^{3} D_{3}$ & $1.4468 \mathrm{E}+01$ & $8.764 \mathrm{E}-02$ & $3.990 \mathrm{E}+11$ & $1.4405 \mathrm{E}+01$ & $8.760 \mathrm{E}-02$ & $3.988 \mathrm{E}+11$ & 0.001 \\
\hline $1 s 4 f^{1} F_{3}^{o}$ & $1 s 5 d^{3} D_{3}$ & $1.4472 \mathrm{E}+01$ & $3.270 \mathrm{E}-03$ & $1.488 \mathrm{E}+10$ & $1.4409 \mathrm{E}+01$ & $3.258 \mathrm{E}-03$ & $1.482 \mathrm{E}+10$ & 0.000 \\
\hline $1 s 5 p^{3} P_{2}^{o}$ & $1 s 5 d^{3} D_{3}$ & $1.2888 \mathrm{E}+03$ & $2.743 \mathrm{E}-01$ & $1.574 \mathrm{E}+08$ & $1.2633 \mathrm{E}+03$ & $2.786 \mathrm{E}-01$ & $1.649 \mathrm{E}+08$ & 0.012 \\
\hline $1 s 3 d^{3} D_{2}$ & $1 s 5 f^{3} F_{3}^{o}$ & $4.4855 \mathrm{E}+00$ & $7.286 \mathrm{E}-01$ & $3.451 \mathrm{E}+13$ & $4.4667 \mathrm{E}+00$ & $7.301 \mathrm{E}-01$ & $3.457 \mathrm{E}+13$ & 0.001 \\
\hline $1 s 3 d^{3} D_{3}$ & $1 s 5 f^{3} F_{3}^{o}$ & $4.5452 \mathrm{E}+00$ & $4.817 \mathrm{E}-02$ & $2.222 \mathrm{E}+12$ & $4.5260 \mathrm{E}+00$ & $4.762 \mathrm{E}-02$ & $2.196 \mathrm{E}+12$ & 0.001 \\
\hline $1 s 3 d^{1} D_{2}$ & $1 s 5 f^{3} F_{3}^{o}$ & $4.5475 \mathrm{E}+00$ & $2.879 \mathrm{E}-03$ & $1.327 \mathrm{E}+11$ & $4.5283 \mathrm{E}+00$ & $2.884 \mathrm{E}-03$ & $1.329 \mathrm{E}+11$ & 0.001 \\
\hline $1 s 4 d^{3} D_{2}$ & $1 s 5 f^{3} F_{3}^{o}$ & $1.4085 \mathrm{E}+01$ & $3.989 \mathrm{E}+00$ & $1.916 \mathrm{E}+13$ & $1.4028 \mathrm{E}+01$ & $3.999 \mathrm{E}+00$ & $1.920 \mathrm{E}+13$ & 0.000 \\
\hline $1 s 4 d^{3} D_{3}$ & $1 s 5 f^{3} F_{3}^{o}$ & $1.4335 \mathrm{E}+01$ & $2.801 \mathrm{E}-01$ & $1.299 \mathrm{E}+12$ & $1.4276 \mathrm{E}+01$ & $2.797 \mathrm{E}-01$ & $1.296 \mathrm{E}+12$ & 0.001 \\
\hline $1 s 4 d{ }^{1} D_{2}$ & $1 s 5 f^{3} F_{3}^{o}$ & $1.4345 \mathrm{E}+01$ & $1.780 \mathrm{E}-02$ & $8.244 \mathrm{E}+10$ & $1.4286 \mathrm{E}+01$ & $1.789 \mathrm{E}-02$ & $8.283 E+10$ & 0.001 \\
\hline $1 s 5 d^{3} D_{2}$ & $1 s 5 f^{3} F_{3}^{0}$ & $1.5543 \mathrm{E}+03$ & $1.737 \mathrm{E}-01$ & $6.852 \mathrm{E}+07$ & $1.5716 \mathrm{E}+03$ & $1.710 \mathrm{E}-01$ & $6.544 \mathrm{E}+07$ & 0.010 \\
\hline $1 s 5 d^{3} D_{3}$ & $1 s 5 f^{3} F_{3}^{o}$ & $9.3195 \mathrm{E}+04$ & $1.950 \mathrm{E}-04$ & $2.140 \mathrm{E}+01$ & & & & 0.340 \\
\hline $1 s 2 s^{3} S_{1}$ & $1 s 5 f^{3} F_{2}^{o}$ & $1.4408 \mathrm{E}+00$ & $3.299 \mathrm{E}-09$ & $2.120 \mathrm{E}+06$ & $1.4347 \mathrm{E}+00$ & $1.307 \mathrm{E}-08$ & $8.404 \mathrm{E}+06$ & 0.290 \\
\hline $1 s 3 s^{3} S_{1}$ & $1 s 5 f^{3} F_{2}^{o}$ & $4.2617 \mathrm{E}+00$ & $5.278 \mathrm{E}-07$ & $3.876 \mathrm{E}+07$ & & & & 0.035 \\
\hline $1 s 3 d^{3} D_{2}$ & $1 s 5 f^{3} F_{2}^{o}$ & $4.4853 \mathrm{E}+00$ & $5.238 \mathrm{E}-02$ & $3.474 \mathrm{E}+12$ & $4.4664 \mathrm{E}+00$ & $5.249 \mathrm{E}-02$ & $3.480 \mathrm{E}+12$ & 0.000 \\
\hline $1 s 3 d^{3} D_{1}$ & $1 s 5 f^{3} F_{2}^{o}$ & $4.4877 \mathrm{E}+00$ & $4.685 \mathrm{E}-01$ & $3.103 E+13$ & $4.4688 \mathrm{E}+00$ & $4.693 \mathrm{E}-01$ & $3.108 E+13$ & 0.000 \\
\hline $1 s 3 d^{3} D_{3}$ & $1 s 5 f^{3} F_{2}^{o}$ & $4.5449 \mathrm{E}+00$ & $2.415 \mathrm{E}-03$ & $1.560 \mathrm{E}+11$ & $4.5257 \mathrm{E}+00$ & $2.384 \mathrm{E}-03$ & $1.539 \mathrm{E}+11$ & 0.001 \\
\hline $1 s 3 d{ }^{1} D_{2}$ & $1 s 5 f^{3} F_{2}^{o}$ & $4.5472 \mathrm{E}+00$ & $3.357 \mathrm{E}-02$ & $2.166 \mathrm{E}+12$ & $4.5280 \mathrm{E}+00$ & $3.314 \mathrm{E}-02$ & $2.138 \mathrm{E}+12$ & 0.001 \\
\hline $1 s 4 s^{3} S_{1}$ & $1 s 5 f^{3} F_{2}^{o}$ & $1.3175 \mathrm{E}+01$ & $3.531 \mathrm{E}-06$ & $2.714 \mathrm{E}+07$ & & & & 0.018 \\
\hline $1 s 4 d^{3} D_{2}$ & $1 s 5 f^{3} F_{2}^{o}$ & $1.4083 \mathrm{E}+01$ & $2.874 \mathrm{E}-01$ & $1.933 \mathrm{E}+12$ & $1.4025 \mathrm{E}+01$ & $2.887 \mathrm{E}-01$ & $1.941 \mathrm{E}+12$ & 0.000 \\
\hline $1 s 4 d^{3} D_{1}$ & $1 s 5 f^{3} F_{2}^{o}$ & $1.4092 \mathrm{E}+01$ & $2.568 \mathrm{E}+00$ & $1.725 \mathrm{E}+13$ & $1.4035 \mathrm{E}+01$ & $2.571 \mathrm{E}+00$ & $1.726 \mathrm{E}+13$ & 0.000 \\
\hline $1 s 4 d^{3} D_{3}$ & $1 s 5 f^{3} F_{2}^{o}$ & $1.4332 \mathrm{E}+01$ & $1.400 \mathrm{E}-02$ & $9.090 \mathrm{E}+10$ & $1.4273 \mathrm{E}+01$ & $1.398 \mathrm{E}-02$ & $9.080 \mathrm{E}+10$ & 0.000 \\
\hline $1 s 4 d^{1} D_{2}$ & $1 s 5 f^{3} F_{2}^{o}$ & $1.4343 \mathrm{E}+01$ & $1.944 \mathrm{E}-01$ & $1.261 \mathrm{E}+12$ & $1.4284 \mathrm{E}+01$ & $1.937 \mathrm{E}-01$ & $1.256 \mathrm{E}+12$ & 0.000 \\
\hline $1 s 5 s^{3} S_{1}$ & $1 s 5 f^{3} F_{2}^{o}$ & $3.1755 \mathrm{E}+02$ & $6.658 \mathrm{E}-07$ & $8.808 E+03$ & $3.1643 \mathrm{E}+02$ & $6.490 \mathrm{E}-07$ & $8.574 \mathrm{E}+03$ & 0.156 \\
\hline $1 s 5 d^{3} D_{2}$ & $1 s 5 f^{3} F_{2}^{o}$ & $1.5232 \mathrm{E}+03$ & $1.282 \mathrm{E}-02$ & $7.370 \mathrm{E}+06$ & $1.5396 \mathrm{E}+03$ & $1.263 \mathrm{E}-02$ & $7.049 E+06$ & 0.006 \\
\hline $1 s 5 d^{3} D_{1}$ & $1 s 5 f^{3} F_{2}^{o}$ & $1.5821 \mathrm{E}+03$ & $1.098 \mathrm{E}-01$ & $5.852 \mathrm{E}+07$ & $1.5996 \mathrm{E}+03$ & $1.081 \mathrm{E}-01$ & $5.590 \mathrm{E}+07$ & 0.005 \\
\hline $1 s 5 d^{3} D_{3}$ & $1 s 5 f^{3} F_{2}^{o}$ & $4.1865 \mathrm{E}+04$ & $2.141 \mathrm{E}-05$ & $1.630 \mathrm{E}+01$ & & & & 0.064 \\
\hline
\end{tabular}


Table 3 (continued)

\begin{tabular}{|c|c|c|c|c|c|c|c|c|}
\hline \multirow[t]{2}{*}{ Lower } & \multirow[t]{2}{*}{ Upper } & \multicolumn{3}{|l|}{ GRASP2K } & \multicolumn{3}{|l|}{ FAC } & \multirow[t]{2}{*}{$d T$} \\
\hline & & $\lambda(\AA)$ & $g f$ & $A\left(\mathrm{~s}^{-1}\right)$ & $\lambda(\AA)$ & $g f$ & $A\left(\mathrm{~s}^{-1}\right)$ & \\
\hline $1 s 2 p^{3} P_{1}^{o}$ & $1 s 5 d^{1} D_{2}$ & $1.4538 \mathrm{E}+00$ & $1.438 \mathrm{E}-04$ & $9.078 \mathrm{E}+10$ & $1.4478 \mathrm{E}+00$ & $1.474 \mathrm{E}-04$ & $9.306 \mathrm{E}+10$ & 0.001 \\
\hline $1 s 2 p^{3} P_{2}^{0}$ & $1 s 5 d^{1} D_{2}$ & $1.5232 \mathrm{E}+00$ & $1.293 \mathrm{E}-02$ & $7.432 \mathrm{E}+12$ & $1.5168 \mathrm{E}+00$ & $1.311 \mathrm{E}-02$ & $7.537 \mathrm{E}+12$ & 0.000 \\
\hline $1 s 2 p^{1} P_{1}^{o}$ & $1 s 5 d^{1} D_{2}$ & $1.5298 \mathrm{E}+00$ & $1.173 \mathrm{E}-01$ & $6.689 E+13$ & $1.5234 \mathrm{E}+00$ & $1.193 \mathrm{E}-01$ & $6.803 \mathrm{E}+13$ & 0.000 \\
\hline $1 s 3 p^{3} P_{1}^{o}$ & $1 s 5 d^{1} D_{2}$ & $4.2927 \mathrm{E}+00$ & $3.366 \mathrm{E}-04$ & $2.437 \mathrm{E}+10$ & $4.2749 \mathrm{E}+00$ & $3.884 \mathrm{E}-04$ & $2.811 \mathrm{E}+10$ & 0.001 \\
\hline $1 s 3 p^{3} P_{2}^{o}$ & $1 s 5 d^{1} D_{2}$ & $4.4717 \mathrm{E}+00$ & $3.994 \mathrm{E}-02$ & $2.665 \mathrm{E}+12$ & $4.4529 \mathrm{E}+00$ & $4.038 \mathrm{E}-02$ & $2.694 \mathrm{E}+12$ & 0.001 \\
\hline $1 s 3 p{ }^{1} P_{1}^{o}$ & $1 s 5 d^{1} D_{2}$ & $4.4877 \mathrm{E}+00$ & $3.668 \mathrm{E}-01$ & $2.429 \mathrm{E}+13$ & $4.4691 \mathrm{E}+00$ & $3.683 E-01$ & $2.439 \mathrm{E}+13$ & 0.001 \\
\hline $1 s 4 p^{3} P_{1}^{0}$ & $1 s 5 d^{1} D_{2}$ & $1.3295 \mathrm{E}+01$ & $1.171 \mathrm{E}-03$ & $8.835 \mathrm{E}+09$ & $1.3238 \mathrm{E}+01$ & $1.596 \mathrm{E}-03$ & $1.204 \mathrm{E}+10$ & 0.001 \\
\hline $1 s 4 p^{3} P_{2}^{o}$ & $1 s 5 d^{1} D_{2}$ & $1.4027 \mathrm{E}+01$ & $1.671 \mathrm{E}-01$ & $1.133 \mathrm{E}+12$ & $1.3965 \mathrm{E}+01$ & $1.698 \mathrm{E}-01$ & $1.152 \mathrm{E}+12$ & 0.001 \\
\hline $1 s 4 p{ }^{1} P_{1}^{o}$ & $1 s 5 d{ }^{1} D_{2}$ & $1.4092 \mathrm{E}+01$ & $1.553 \mathrm{E}+00$ & $1.043 E+13$ & $1.4031 \mathrm{E}+01$ & $1.548 \mathrm{E}+00$ & $1.040 \mathrm{E}+13$ & 0.001 \\
\hline $1 s 4 f^{3} F_{3}^{0}$ & $1 s 5 d^{1} D_{2}$ & $1.4336 \mathrm{E}+01$ & $2.568 \mathrm{E}-04$ & $1.667 \mathrm{E}+09$ & $1.4273 \mathrm{E}+01$ & $2.695 \mathrm{E}-04$ & $1.750 \mathrm{E}+09$ & 0.000 \\
\hline $1 s 4 f^{3} F_{2}^{o}$ & $1 s 5 d^{1} D_{2}$ & $1.4341 \mathrm{E}+01$ & $2.746 \mathrm{E}-03$ & $1.781 \mathrm{E}+10$ & $1.4279 \mathrm{E}+01$ & $2.764 \mathrm{E}-03$ & $1.793 \mathrm{E}+10$ & 0.001 \\
\hline $1 s 4 f^{1} F_{3}^{o}$ & $1 s 5 d^{1} D_{2}$ & $1.4467 \mathrm{E}+01$ & $6.463 \mathrm{E}-02$ & $4.120 \mathrm{E}+11$ & $1.4403 \mathrm{E}+01$ & $6.487 \mathrm{E}-02$ & $4.136 \mathrm{E}+11$ & 0.000 \\
\hline $1 s 5 p^{3} P_{1}^{o}$ & $1 s 5 d^{1} D_{2}$ & $3.5679 \mathrm{E}+02$ & $5.880 \mathrm{E}-04$ & $6.162 \mathrm{E}+06$ & $3.5385 \mathrm{E}+02$ & $5.862 \mathrm{E}-04$ & $6.193 E+06$ & 0.019 \\
\hline $1 s 5 p^{3} P_{2}^{o}$ & $1 s 5 d^{1} D_{2}$ & $1.2461 \mathrm{E}+03$ & $1.996 \mathrm{E}-02$ & $1.715 \mathrm{E}+07$ & $1.2214 \mathrm{E}+03$ & $2.024 \mathrm{E}-02$ & $1.794 \mathrm{E}+07$ & 0.011 \\
\hline $1 s 5 p^{1} P_{1}^{o}$ & $1 s 5 d^{1} D_{2}$ & $1.5753 \mathrm{E}+03$ & $1.443 \mathrm{E}-01$ & $7.759 \mathrm{E}+07$ & $1.5418 \mathrm{E}+03$ & $1.468 \mathrm{E}-01$ & $8.171 \mathrm{E}+07$ & 0.014 \\
\hline $1 s 5 f^{3} F_{3}^{o}$ & $1 s 5 d^{1} D_{2}$ & $6.3169 \mathrm{E}+04$ & $1.871 \mathrm{E}-05$ & $6.254 \mathrm{E}+00$ & & & & 0.202 \\
\hline $1 s 5 f^{3} F_{2}^{o}$ & $1 s 5 d^{1} D_{2}$ & $3.7392 \mathrm{E}+05$ & $3.369 \mathrm{E}-05$ & $3.214 \mathrm{E}-01$ & & & & 0.434 \\
\hline $1 s 3 d^{3} D_{3}$ & $1 s 5 f^{3} F_{4}^{o}$ & $4.5387 \mathrm{E}+00$ & $9.978 \mathrm{E}-01$ & $3.590 \mathrm{E}+13$ & $4.5196 \mathrm{E}+00$ & $1.005 E+00$ & $3.616 \mathrm{E}+13$ & 0.001 \\
\hline $1 s 4 d^{3} D_{3}$ & $1 s 5 f^{3} F_{4}^{o}$ & $1.4271 \mathrm{E}+01$ & $5.607 \mathrm{E}+00$ & $2.040 \mathrm{E}+13$ & $1.4212 \mathrm{E}+01$ & $5.633 \mathrm{E}+00$ & $2.049 \mathrm{E}+13$ & 0.000 \\
\hline $1 s 5 d^{3} D_{3}$ & $1 s 5 f^{3} F_{4}^{o}$ & $3.0834 \mathrm{E}+03$ & $1.209 \mathrm{E}-01$ & $9.428 \mathrm{E}+06$ & $3.1501 \mathrm{E}+03$ & $1.178 \mathrm{E}-01$ & $8.729 \mathrm{E}+06$ & 0.017 \\
\hline $1 s 4 f^{3} F_{3}^{o}$ & $1 s 5 g^{3} G_{4}$ & $1.4275 \mathrm{E}+01$ & $8.976 \mathrm{E}+00$ & $3.265 \mathrm{E}+13$ & $1.4214 \mathrm{E}+01$ & $8.998 \mathrm{E}+00$ & $3.272 \mathrm{E}+13$ & 0.000 \\
\hline $1 s 4 f^{3} F_{4}^{o}$ & $1 s 5 g^{3} G_{4}$ & $1.4400 \mathrm{E}+01$ & $3.232 \mathrm{E}-01$ & $1.155 \mathrm{E}+12$ & $1.4339 \mathrm{E}+01$ & $3.220 \mathrm{E}-01$ & $1.150 \mathrm{E}+12$ & 0.000 \\
\hline $1 s 4 f^{1} F_{3}^{o}$ & $1 s 5 g^{3} G_{4}$ & $1.4404 \mathrm{E}+01$ & $9.104 \mathrm{E}-03$ & $3.252 \mathrm{E}+10$ & $1.4343 \mathrm{E}+01$ & $9.083 \mathrm{E}-03$ & $3.244 \mathrm{E}+10$ & 0.000 \\
\hline $1 s 5 f^{3} F_{3}^{o}$ & $1 s 5 g^{3} G_{4}$ & $3.1830 \mathrm{E}+03$ & $6.601 \mathrm{E}-02$ & $4.829 \mathrm{E}+06$ & $3.1753 E+03$ & $6.588 \mathrm{E}-02$ & $4.802 \mathrm{E}+06$ & 0.007 \\
\hline $1 s 5 f^{3} F_{4}^{o}$ & $1 s 5 g{ }^{3} G_{4}$ & $1.7265 \mathrm{E}+06$ & $4.338 \mathrm{E}-06$ & $1.079 \mathrm{E}-03$ & & & & 0.584 \\
\hline $1 s 2 p^{3} P_{2}^{o}$ & $1 s 5 g{ }^{3} G_{3}$ & $1.5225 \mathrm{E}+00$ & $1.816 \mathrm{E}-10$ & $7.464 \mathrm{E}+04$ & $1.5161 \mathrm{E}+00$ & $8.545 \mathrm{E}-11$ & $3.512 \mathrm{E}+04$ & 0.372 \\
\hline $1 s 3 p^{3} P_{2}^{o}$ & $1 s 5 g^{3} G_{3}$ & $4.4656 \mathrm{E}+00$ & $2.805 \mathrm{E}-11$ & $1.340 \mathrm{E}+03$ & $4.4470 \mathrm{E}+00$ & $2.636 \mathrm{E}-10$ & $1.259 \mathrm{E}+04$ & 0.160 \\
\hline $1 s 4 p^{3} P_{2}^{o}$ & $1 s 5 g^{3} G_{3}$ & $1.3967 \mathrm{E}+01$ & $7.568 \mathrm{E}-07$ & $3.697 \mathrm{E}+06$ & & & & 0.002 \\
\hline $1 s 4 f^{3} F_{3}^{o}$ & $1 s 5 g^{3} G_{3}$ & $1.4273 \mathrm{E}+01$ & $3.312 \mathrm{E}-01$ & $1.549 \mathrm{E}+12$ & $1.4213 \mathrm{E}+01$ & $3.319 \mathrm{E}-01$ & $1.552 \mathrm{E}+12$ & 0.000 \\
\hline $1 s 4 f^{3} F_{2}^{o}$ & $1 s 5 g^{3} G_{3}$ & $1.4278 \mathrm{E}+01$ & $6.649 \mathrm{E}+00$ & $3.108 E+13$ & $1.4218 \mathrm{E}+01$ & $6.662 E+00$ & $3.113 E+13$ & 0.000 \\
\hline $1 s 4 f^{3} F_{4}^{0}$ & $1 s 5 g{ }^{3} G_{3}$ & $1.4399 \mathrm{E}+01$ & $9.264 \mathrm{E}-03$ & $4.258 \mathrm{E}+10$ & $1.4338 \mathrm{E}+01$ & $9.232 \mathrm{E}-03$ & $4.243 \mathrm{E}+10$ & 0.001 \\
\hline $1 s 4 f^{1} F_{3}^{o}$ & $1 s 5 g{ }^{3} G_{3}$ & $1.4403 \mathrm{E}+01$ & $2.515 \mathrm{E}-01$ & $1.155 \mathrm{E}+12$ & $1.4341 \mathrm{E}+01$ & $2.504 \mathrm{E}-01$ & $1.150 \mathrm{E}+12$ & 0.000 \\
\hline $1 s 5 p^{3} P_{2}^{o}$ & $1 s 5 g^{3} G_{3}$ & $9.0225 \mathrm{E}+02$ & $3.193 \mathrm{E}-08$ & $3.737 \mathrm{E}+01$ & & & & 0.058 \\
\hline $1 s 5 f^{3} F_{3}^{0}$ & $1 s 5 g^{3} G_{3}$ & $3.1085 E+03$ & $2.495 \mathrm{E}-03$ & $2.460 \mathrm{E}+05$ & $3.1008 \mathrm{E}+03$ & $2.490 \mathrm{E}-03$ & $2.446 \mathrm{E}+05$ & 0.029 \\
\hline $1 s 5 f^{3} F_{2}^{o}$ & $1 s 5 g^{3} G_{3}$ & $3.2410 \mathrm{E}+03$ & $4.801 \mathrm{E}-02$ & $4.356 \mathrm{E}+06$ & $3.2333 \mathrm{E}+03$ & $4.792 \mathrm{E}-02$ & $4.331 \mathrm{E}+06$ & 0.023 \\
\hline $1 s 5 f^{3} F_{4}^{0}$ & $1 s 5 g^{3} G_{3}$ & $1.2333 \mathrm{E}+05$ & $1.738 \mathrm{E}-06$ & $1.089 \mathrm{E}-01$ & & & & 0.822 \\
\hline $1 s 3 d^{3} D_{2}$ & $1 s 5 f^{1} F_{3}^{o}$ & $4.4790 \mathrm{E}+00$ & $2.109 \mathrm{E}-05$ & $1.002 \mathrm{E}+09$ & & & & 0.005 \\
\hline $1 s 3 d^{3} D_{3}$ & $1 s 5 f^{1} F_{3}^{o}$ & $4.5385 \mathrm{E}+00$ & $3.715 \mathrm{E}-02$ & $1.719 \mathrm{E}+12$ & $4.5194 \mathrm{E}+00$ & $3.740 \mathrm{E}-02$ & $1.730 \mathrm{E}+12$ & 0.000 \\
\hline $1 s 3 d^{1} D_{2}$ & $1 s 5 f^{1} F_{3}^{o}$ & $4.5408 \mathrm{E}+00$ & $7.388 \mathrm{E}-01$ & $3.414 \mathrm{E}+13$ & $4.5217 \mathrm{E}+00$ & $7.440 \mathrm{E}-01$ & $3.438 \mathrm{E}+13$ & 0.000 \\
\hline $1 s 4 d^{3} D_{2}$ & $1 s 5 f^{1} F_{3}^{o}$ & $1.4022 \mathrm{E}+01$ & $2.073 \mathrm{E}-04$ & $1.005 \mathrm{E}+09$ & & & & 0.003 \\
\hline $1 s 4 d^{3} D_{3}$ & $1 s 5 f^{1} F_{3}^{o}$ & $1.4269 \mathrm{E}+01$ & $2.086 \mathrm{E}-01$ & $9.761 \mathrm{E}+11$ & $1.4210 \mathrm{E}+01$ & $2.096 \mathrm{E}-01$ & $9.810 \mathrm{E}+11$ & 0.000 \\
\hline $1 s 4 d^{1} D_{2}$ & $1 s 5 f{ }^{1} F_{3}^{o}$ & $1.4279 \mathrm{E}+01$ & $4.156 \mathrm{E}+00$ & $1.943 E+13$ & $1.4221 \mathrm{E}+01$ & $4.169 \mathrm{E}+00$ & $1.948 \mathrm{E}+13$ & 0.000 \\
\hline $1 s 5 d^{3} D_{2}$ & $1 s 5 f^{1} F_{3}^{o}$ & $1.0343 \mathrm{E}+03$ & $1.740 \mathrm{E}-05$ & $1.550 \mathrm{E}+04$ & $1.0413 E+03$ & $1.923 \mathrm{E}-05$ & $1.675 \mathrm{E}+04$ & 0.068 \\
\hline $1 s 5 d^{3} D_{3}$ & $1 s 5 f^{1} F_{3}^{o}$ & $2.9925 \mathrm{E}+03$ & $4.638 \mathrm{E}-03$ & $4.936 \mathrm{E}+05$ & $3.0547 \mathrm{E}+03$ & $4.522 \mathrm{E}-03$ & $4.579 \mathrm{E}+05$ & 0.007 \\
\hline $1 s 5 d^{1} D_{2}$ & $1 s 5 f^{1} F_{3}^{o}$ & $3.2509 \mathrm{E}+03$ & $8.494 \mathrm{E}-02$ & $7.658 \mathrm{E}+06$ & $3.3306 \mathrm{E}+03$ & $8.255 \mathrm{E}-02$ & $7.031 \mathrm{E}+06$ & 0.005 \\
\hline $1 s 5 g^{3} G_{4}$ & $1 s 5 f^{1} F_{3}^{o}$ & $1.0795 \mathrm{E}+05$ & $1.971 \mathrm{E}-06$ & $1.612 \mathrm{E}-01$ & & & & 0.573 \\
\hline $1 s 5 g^{3} G_{3}$ & $1 s 5 f^{1} F_{3}^{o}$ & $5.7657 \mathrm{E}+05$ & $1.010 \mathrm{E}-05$ & $2.896 \mathrm{E}-02$ & & & & 0.888 \\
\hline $1 s 4 f^{3} F_{4}^{o}$ & $1 s 5 g^{3} G_{5}$ & $1.4362 \mathrm{E}+01$ & $1.142 \mathrm{E}+01$ & $3.356 \mathrm{E}+13$ & $1.4301 \mathrm{E}+01$ & $1.146 \mathrm{E}+01$ & $3.369 E+13$ & 0.000 \\
\hline $1 s 5 f^{3} F_{4}^{0}$ & $1 s 5 g^{3} G_{5}$ & $5.3388 \mathrm{E}+03$ & $4.992 \mathrm{E}-02$ & $1.062 \mathrm{E}+06$ & $5.3203 E+03$ & $4.988 \mathrm{E}-02$ & $1.059 \mathrm{E}+06$ & 0.007 \\
\hline $1 s 4 f^{3} F_{3}^{o}$ & $1 s 5 g^{1} G_{4}$ & $1.4236 \mathrm{E}+01$ & $8.057 \mathrm{E}-07$ & $2.947 \mathrm{E}+06$ & & & & 0.006 \\
\hline $1 s 4 f^{3} F_{4}^{0}$ & $1 s 5 g^{1} G_{4}$ & $1.4361 \mathrm{E}+01$ & $2.606 \mathrm{E}-01$ & $9.364 \mathrm{E}+11$ & $1.4299 \mathrm{E}+01$ & $2.616 \mathrm{E}-01$ & $9.402 \mathrm{E}+11$ & 0.000 \\
\hline $1 s 4 f^{1} F_{3}^{o}$ & $1 s 5 g{ }^{1} G_{4}$ & $1.4364 \mathrm{E}+01$ & $9.081 \mathrm{E}+00$ & $3.262 \mathrm{E}+13$ & $1.4303 \mathrm{E}+01$ & $9.114 \mathrm{E}+00$ & $3.273 \mathrm{E}+13$ & 0.000 \\
\hline $1 s 5 f^{3} F_{3}^{o}$ & $1 s 5 g^{1} G_{4}$ & $1.9730 \mathrm{E}+03$ & $2.721 \mathrm{E}-09$ & $5.180 \mathrm{E}-01$ & & & & 0.788 \\
\hline $1 s 5 f^{3} F_{4}^{o}$ & $1 s 5 g^{1} G_{4}$ & $5.1744 \mathrm{E}+03$ & $1.176 \mathrm{E}-03$ & $3.255 \mathrm{E}+04$ & $5.1563 \mathrm{E}+03$ & $1.174 \mathrm{E}-03$ & $3.246 \mathrm{E}+04$ & 0.043 \\
\hline $1 s 5 f^{1} F_{3}^{o}$ & $1 s 5 g^{1} G_{4}$ & $5.4521 \mathrm{E}+03$ & $3.888 \mathrm{E}-02$ & $9.694 \mathrm{E}+05$ & $5.4339 E+03$ & $3.884 \mathrm{E}-02$ & $9.667 \mathrm{E}+05$ & 0.028 \\
\hline $1 s 2 p^{3} P_{1}^{o}$ & $1 s 6 s^{3} S_{1}$ & $1.3813 \mathrm{E}+00$ & $1.347 \mathrm{E}-03$ & $1.569 \mathrm{E}+12$ & $1.3756 \mathrm{E}+00$ & $1.386 \mathrm{E}-03$ & $1.615 \mathrm{E}+12$ & 0.002 \\
\hline $1 s 2 p^{3} P_{0}^{o}$ & $1 s 6 s^{3} S_{1}$ & $1.3823 \mathrm{E}+00$ & $6.594 \mathrm{E}-04$ & $7.674 \mathrm{E}+11$ & $1.3765 \mathrm{E}+00$ & $6.489 \mathrm{E}-04$ & $7.550 \mathrm{E}+11$ & 0.002 \\
\hline $1 s 2 p^{3} P_{2}^{o}$ & $1 s 6 s^{3} S_{1}$ & $1.4438 \mathrm{E}+00$ & $4.439 \mathrm{E}-03$ & $4.734 \mathrm{E}+12$ & $1.4378 \mathrm{E}+00$ & $4.423 \mathrm{E}-03$ & $4.716 \mathrm{E}+12$ & 0.004 \\
\hline $1 s 2 p^{1} P_{1}^{o}$ & $1 s 6 s^{3} S_{1}$ & $1.4497 \mathrm{E}+00$ & $8.068 \mathrm{E}-04$ & $8.535 E+11$ & $1.4438 \mathrm{E}+00$ & $7.972 \mathrm{E}-04$ & $8.432 \mathrm{E}+11$ & 0.002 \\
\hline $1 s 3 p^{3} P_{1}^{0}$ & $1 s 6 s^{3} S_{1}$ & $3.7169 \mathrm{E}+00$ & $6.581 \mathrm{E}-03$ & $1.059 \mathrm{E}+12$ & $3.7018 \mathrm{E}+00$ & $6.645 \mathrm{E}-03$ & $1.069 \mathrm{E}+12$ & 0.001 \\
\hline $1 s 3 p^{3} P_{0}^{o}$ & $1 s 6 s^{3} S_{1}$ & $3.7187 \mathrm{E}+00$ & $3.203 \mathrm{E}-03$ & $5.150 \mathrm{E}+11$ & $3.7035 E+00$ & $3.126 \mathrm{E}-03$ & $5.024 \mathrm{E}+11$ & 0.002 \\
\hline $1 s 3 p^{3} P_{2}^{o}$ & $1 s 6 s^{3} S_{1}$ & $3.8504 \mathrm{E}+00$ & $2.103 \mathrm{E}-02$ & $3.154 \mathrm{E}+12$ & $3.8346 \mathrm{E}+00$ & $2.093 \mathrm{E}-02$ & $3.139 \mathrm{E}+12$ & 0.000 \\
\hline $1 s 3 p^{1} P_{1}^{o}$ & $1 s 6 s^{3} S_{1}$ & $3.8623 \mathrm{E}+00$ & $3.830 \mathrm{E}-03$ & $5.708 \mathrm{E}+11$ & $3.8466 \mathrm{E}+00$ & $3.799 \mathrm{E}-03$ & $5.660 \mathrm{E}+11$ & 0.001 \\
\hline $1 s 4 p^{3} P_{1}^{0}$ & $1 s 6 s^{3} S_{1}$ & $8.9847 \mathrm{E}+00$ & $2.684 \mathrm{E}-02$ & $7.392 \mathrm{E}+11$ & $8.9484 \mathrm{E}+00$ & $2.691 \mathrm{E}-02$ & $7.410 \mathrm{E}+11$ & 0.001 \\
\hline $1 s 4 p^{3} P_{0}^{o}$ & $1 s 6 s^{3} S_{1}$ & $8.9888 \mathrm{E}+00$ & $1.303 \mathrm{E}-02$ & $3.586 \mathrm{E}+11$ & $8.9523 E+00$ & $1.267 \mathrm{E}-02$ & $3.487 \mathrm{E}+11$ & 0.002 \\
\hline $1 s 4 p^{3} P_{2}^{o}$ & $1 s 6 s^{3} S_{1}$ & $9.3128 \mathrm{E}+00$ & $8.539 \mathrm{E}-02$ & $2.189 \mathrm{E}+12$ & $9.2750 \mathrm{E}+00$ & $8.504 \mathrm{E}-02$ & $2.179 \mathrm{E}+12$ & 0.001 \\
\hline $1 s 4 p^{1} P_{1}^{o}$ & $1 s 6 s^{3} S_{1}$ & $9.3416 \mathrm{E}+00$ & $1.559 \mathrm{E}-02$ & $3.972 \mathrm{E}+11$ & $9.3040 \mathrm{E}+00$ & $1.546 \mathrm{E}-02$ & $3.938 \mathrm{E}+11$ & 0.001 \\
\hline $1 s 4 f^{3} F_{2}^{o}$ & $1 s 6 s^{3} S_{1}$ & $9.4503 E+00$ & $5.582 \mathrm{E}-09$ & $1.390 \mathrm{E}+05$ & & & & 0.099 \\
\hline $1 s 5 p^{3} P_{1}^{o}$ & $1 s 6 s^{3} S_{1}$ & $2.5714 \mathrm{E}+01$ & $1.642 \mathrm{E}-01$ & $5.522 \mathrm{E}+11$ & $2.5617 \mathrm{E}+01$ & $1.636 \mathrm{E}-01$ & $5.499 \mathrm{E}+11$ & 0.001 \\
\hline $1 s 5 p^{3} P_{0}^{0}$ & $1 s 6 s^{3} S_{1}$ & $2.5731 \mathrm{E}+01$ & $7.964 \mathrm{E}-02$ & $2.675 E+11$ & $2.5633 E+01$ & $7.696 \mathrm{E}-02$ & $2.582 \mathrm{E}+11$ & 0.002 \\
\hline $1 s 5 p^{3} P_{2}^{o}$ & $1 s 6 s^{3} S_{1}$ & $2.7109 \mathrm{E}+01$ & $5.492 \mathrm{E}-01$ & $1.662 \mathrm{E}+12$ & $2.7006 \mathrm{E}+01$ & $5.457 \mathrm{E}-01$ & $1.649 \mathrm{E}+12$ & 0.002 \\
\hline
\end{tabular}


Table 3 (continued)

\begin{tabular}{|c|c|c|c|c|c|c|c|c|}
\hline \multirow[t]{2}{*}{ Lower } & \multirow[t]{2}{*}{ Upper } & \multicolumn{3}{|l|}{ GRASP2K } & \multicolumn{3}{|l|}{ FAC } & \multirow[t]{2}{*}{$d T$} \\
\hline & & $\lambda(\AA)$ & $g f$ & $A\left(\mathrm{~s}^{-1}\right)$ & $\lambda(\AA)$ & $g f$ & $A\left(\mathrm{~s}^{-1}\right)$ & \\
\hline $1 s 5 p^{1} P_{1}^{o}$ & $1 s 6 s^{3} S_{1}$ & $2.7232 \mathrm{E}+01$ & $1.009 \mathrm{E}-01$ & $3.024 \mathrm{E}+11$ & $2.7131 \mathrm{E}+01$ & $9.952 \mathrm{E}-02$ & $2.980 \mathrm{E}+11$ & 0.002 \\
\hline $1 s 5 f^{3} F_{2}^{o}$ & $1 s 6 s^{3} S_{1}$ & $2.7709 \mathrm{E}+01$ & $1.281 \mathrm{E}-07$ & $3.709 \mathrm{E}+05$ & & & & 0.078 \\
\hline $1 s^{2}{ }^{1} s_{0}$ & $1 s 6 p^{3} P_{1}^{o}$ & $3.1640 \mathrm{E}-01$ & $3.992 \mathrm{E}-03$ & $8.865 E+13$ & $3.1509 \mathrm{E}-01$ & $3.640 \mathrm{E}-03$ & $8.082 \mathrm{E}+13$ & 0.009 \\
\hline $1 s 2 s^{3} S_{1}$ & $1 s 6 p^{3} P_{1}^{o}$ & $1.3692 \mathrm{E}+00$ & $1.433 \mathrm{E}-02$ & $1.699 \mathrm{E}+13$ & $1.3634 \mathrm{E}+00$ & $1.449 \mathrm{E}-02$ & $1.718 \mathrm{E}+13$ & 0.002 \\
\hline $1 s 2 s^{1} S_{0}$ & $1 s 6 p^{3} P_{1}^{o}$ & $1.3822 \mathrm{E}+00$ & $6.212 \mathrm{E}-03$ & $7.230 \mathrm{E}+12$ & $1.3765 \mathrm{E}+00$ & $5.890 \mathrm{E}-03$ & $6.853 \mathrm{E}+12$ & 0.006 \\
\hline $1 s 3 s^{3} s_{1}$ & $1 s 6 p^{3} P_{1}^{o}$ & $3.6909 \mathrm{E}+00$ & $3.452 \mathrm{E}-02$ & $5.634 \mathrm{E}+12$ & $3.6756 \mathrm{E}+00$ & $3.517 \mathrm{E}-02$ & $5.739 \mathrm{E}+12$ & 0.002 \\
\hline $1 s 3 s{ }^{1} S_{0}$ & $1 s 6 p^{3} P_{1}^{o}$ & $3.7158 \mathrm{E}+00$ & $1.517 \mathrm{E}-02$ & $2.443 E+12$ & $3.7007 \mathrm{E}+00$ & $1.445 \mathrm{E}-02$ & $2.326 \mathrm{E}+12$ & 0.000 \\
\hline $1 s 3 d^{3} D_{2}$ & $1 s 6 p^{3} P_{1}^{o}$ & $3.8574 \mathrm{E}+00$ & $5.268 \mathrm{E}-03$ & $7.872 \mathrm{E}+11$ & $3.8413 \mathrm{E}+00$ & $5.279 \mathrm{E}-03$ & $7.887 \mathrm{E}+11$ & 0.003 \\
\hline $1 s 3 d^{3} D_{1}$ & $1 s 6 p^{3} P_{1}^{o}$ & $3.8592 \mathrm{E}+00$ & $1.151 \mathrm{E}-03$ & $1.719 \mathrm{E}+11$ & $3.8431 \mathrm{E}+00$ & $1.141 \mathrm{E}-03$ & $1.704 \mathrm{E}+11$ & 0.008 \\
\hline $1 s 3 d{ }^{1} D_{2}$ & $1 s 6 p^{3} P_{1}^{o}$ & $3.9032 E+00$ & $6.779 \mathrm{E}-06$ & $9.894 \mathrm{E}+08$ & $3.8868 \mathrm{E}+00$ & $8.128 \mathrm{E}-06$ & $1.186 \mathrm{E}+09$ & 0.029 \\
\hline $1 s 4 s^{3} S_{1}$ & $1 s 6 p^{3} P_{1}^{o}$ & $8.9132 \mathrm{E}+00$ & $9.266 \mathrm{E}-02$ & $2.593 E+12$ & $8.8765 \mathrm{E}+00$ & $9.472 \mathrm{E}-02$ & $2.650 \mathrm{E}+12$ & 0.003 \\
\hline $1 s 4 s^{1} S_{0}$ & $1 s 6 p^{3} P_{1}^{o}$ & $8.9717 \mathrm{E}+00$ & $4.111 \mathrm{E}-02$ & $1.136 \mathrm{E}+12$ & $8.9354 \mathrm{E}+00$ & $3.919 \mathrm{E}-02$ & $1.082 \mathrm{E}+12$ & 0.003 \\
\hline $1 s 4 d^{3} D_{2}$ & $1 s 6 p^{3} P_{1}^{o}$ & $9.3198 \mathrm{E}+00$ & $3.518 \mathrm{E}-02$ & $9.005 E+11$ & $9.2819 \mathrm{E}+00$ & $3.513 \mathrm{E}-02$ & $8.989 E+11$ & 0.001 \\
\hline $1 s 4 d^{3} D_{1}$ & $1 s 6 p^{3} P_{1}^{o}$ & $9.3241 \mathrm{E}+00$ & $7.616 \mathrm{E}-03$ & $1.948 \mathrm{E}+11$ & $9.2861 \mathrm{E}+00$ & $7.573 \mathrm{E}-03$ & $1.936 \mathrm{E}+11$ & 0.004 \\
\hline $1 s 4 d{ }^{1} D_{2}$ & $1 s 6 p^{3} P_{1}^{o}$ & $9.4329 \mathrm{E}+00$ & $4.037 \mathrm{E}-05$ & $1.009 E+09$ & $9.3943 \mathrm{E}+00$ & $4.947 \mathrm{E}-05$ & $1.235 \mathrm{E}+09$ & 0.020 \\
\hline $1 s 5 s^{3} S_{1}$ & $1 s 6 p^{3} P_{1}^{o}$ & $2.5356 \mathrm{E}+01$ & $4.018 \mathrm{E}-01$ & $1.390 \mathrm{E}+12$ & $2.5254 \mathrm{E}+01$ & $4.122 \mathrm{E}-01$ & $1.425 \mathrm{E}+12$ & 0.003 \\
\hline $1 s 5 s^{1} S_{0}$ & $1 s 6 p^{3} P_{1}^{o}$ & $2.5594 \mathrm{E}+01$ & $1.810 \mathrm{E}-01$ & $6.145 E+11$ & $2.5493 \mathrm{E}+01$ & $1.720 \mathrm{E}-01$ & $5.836 \mathrm{E}+11$ & 0.002 \\
\hline $1 s 5 d^{3} D_{2}$ & $1 s 6 p^{3} P_{1}^{o}$ & $2.7067 \mathrm{E}+01$ & $2.978 \mathrm{E}-01$ & $9.037 \mathrm{E}+11$ & $2.6964 \mathrm{E}+01$ & $2.966 \mathrm{E}-01$ & $8.996 \mathrm{E}+11$ & 0.001 \\
\hline $1 s 5 d^{3} D_{1}$ & $1 s 6 p^{3} P_{1}^{o}$ & $2.7084 \mathrm{E}+01$ & $6.418 \mathrm{E}-02$ & $1.945 \mathrm{E}+11$ & $2.6981 \mathrm{E}+01$ & $6.366 \mathrm{E}-02$ & $1.927 \mathrm{E}+11$ & 0.002 \\
\hline $1 s 5 d^{1} D_{2}$ & $1 s 6 p^{3} P_{1}^{o}$ & $2.7558 \mathrm{E}+01$ & $3.306 \mathrm{E}-04$ & $9.680 \mathrm{E}+08$ & $2.7452 \mathrm{E}+01$ & $3.795 \mathrm{E}-04$ & $1.110 \mathrm{E}+09$ & 0.010 \\
\hline $1 s 6 s^{3} s_{1}$ & $1 s 6 p^{3} P_{1}^{o}$ & $4.9854 \mathrm{E}+03$ & $4.082 \mathrm{E}-02$ & $3.652 E+06$ & $4.6146 \mathrm{E}+03$ & $4.469 \mathrm{E}-02$ & $4.627 \mathrm{E}+06$ & 0.127 \\
\hline $1 s 2 p^{3} P_{1}^{o}$ & $1 s 6 s^{1} S_{0}$ & $1.3809 \mathrm{E}+00$ & $5.496 \mathrm{E}-04$ & $1.922 \mathrm{E}+12$ & $1.3753 \mathrm{E}+00$ & $5.629 \mathrm{E}-04$ & $1.968 \mathrm{E}+12$ & 0.010 \\
\hline $1 s 2 p^{1} P_{1}^{o}$ & $1 s 6 s{ }^{1} s_{0}$ & $1.4493 \mathrm{E}+00$ & $1.870 \mathrm{E}-03$ & $5.937 \mathrm{E}+12$ & $1.4434 \mathrm{E}+00$ & $1.844 \mathrm{E}-03$ & $5.856 \mathrm{E}+12$ & 0.004 \\
\hline $1 s 3 p^{3} P_{1}^{o}$ & $1 s 6 s^{1} S_{0}$ & $3.7140 \mathrm{E}+00$ & $2.696 \mathrm{E}-03$ & $1.304 \mathrm{E}+12$ & $3.6994 \mathrm{E}+00$ & $2.744 \mathrm{E}-03$ & $1.326 \mathrm{E}+12$ & 0.003 \\
\hline $1 s 3 p^{1} P_{1}^{o}$ & $1 s 6 s^{1} S_{0}$ & $3.8591 \mathrm{E}+00$ & $8.737 \mathrm{E}-03$ & $3.913 E+12$ & $3.8440 \mathrm{E}+00$ & $8.725 \mathrm{E}-03$ & $3.905 E+12$ & 0.000 \\
\hline $1 s 4 p^{3} P_{1}^{o}$ & $1 s 6 s{ }^{1} s_{0}$ & $8.9678 \mathrm{E}+00$ & $1.099 \mathrm{E}-02$ & $9.117 E+11$ & $8.9340 \mathrm{E}+00$ & $1.117 \mathrm{E}-02$ & $9.258 \mathrm{E}+11$ & 0.001 \\
\hline $1 s 4 p^{1} P_{1}^{o}$ & $1 s 6 s^{1} s_{0}$ & $9.3233 \mathrm{E}+00$ & $3.529 \mathrm{E}-02$ & $2.708 \mathrm{E}+12$ & $9.2885 \mathrm{E}+00$ & $3.544 \mathrm{E}-02$ & $2.716 \mathrm{E}+12$ & 0.002 \\
\hline $1 s 5 p^{3} P_{1}^{o}$ & $1 s 6 s{ }^{1} S_{0}$ & $2.5576 \mathrm{E}+01$ & $6.646 \mathrm{E}-02$ & $6.777 \mathrm{E}+11$ & $2.5500 \mathrm{E}+01$ & $6.781 \mathrm{E}-02$ & $6.897 \mathrm{E}+11$ & 0.002 \\
\hline $1 s 5 p^{1} P_{1}^{o}$ & $1 s 6 s^{1} s_{0}$ & $2.7078 \mathrm{E}+01$ & $2.249 \mathrm{E}-01$ & $2.046 \mathrm{E}+12$ & $2.6999 \mathrm{E}+01$ & $2.273 \mathrm{E}-01$ & $2.063 E+12$ & 0.002 \\
\hline $1 s 6 p^{3} P_{1}^{o}$ & $1 s 6 s^{1} s_{0}$ & $1.0994 \mathrm{E}+05$ & $7.948 \mathrm{E}-04$ & $4.387 \mathrm{E}+02$ & & & & 0.884 \\
\hline $1 s 2 s^{3} S_{1}$ & $1 s 6 p^{3} P_{0}^{o}$ & $1.3691 \mathrm{E}+00$ & $6.808 \mathrm{E}-03$ & $2.423 \mathrm{E}+13$ & $1.3635 \mathrm{E}+00$ & $6.812 \mathrm{E}-03$ & $2.423 \mathrm{E}+13$ & 0.003 \\
\hline $1 s 3 s^{3} s_{1}$ & $1 s 6 p^{3} P_{0}^{o}$ & $3.6907 \mathrm{E}+00$ & $1.641 \mathrm{E}-02$ & $8.037 E+12$ & $3.6761 \mathrm{E}+00$ & $1.659 \mathrm{E}-02$ & $8.122 E+12$ & 0.004 \\
\hline $1 s 3 d^{3} D_{1}$ & $1 s 6 p^{3} P_{0}^{o}$ & $3.8590 \mathrm{E}+00$ & $2.133 E-03$ & $9.554 \mathrm{E}+11$ & $3.8436 \mathrm{E}+00$ & $2.122 \mathrm{E}-03$ & $9.501 \mathrm{E}+11$ & 0.008 \\
\hline $1 s 4 s^{3} S_{1}$ & $1 s 6 p^{3} P_{0}^{o}$ & $8.9120 \mathrm{E}+00$ & $4.409 \mathrm{E}-02$ & $3.703 E+12$ & $8.8795 \mathrm{E}+00$ & $4.483 \mathrm{E}-02$ & $3.760 \mathrm{E}+12$ & 0.004 \\
\hline $1 s 4 d^{3} D_{1}$ & $1 s 6 p^{3} P_{0}^{o}$ & $9.3228 \mathrm{E}+00$ & $1.419 \mathrm{E}-02$ & $1.089 \mathrm{E}+12$ & $9.2894 \mathrm{E}+00$ & $1.414 \mathrm{E}-02$ & $1.084 \mathrm{E}+12$ & 0.004 \\
\hline $1 s 5 s^{3} S_{1}$ & $1 s 6 p^{3} P_{0}^{o}$ & $2.5347 \mathrm{E}+01$ & $1.912 \mathrm{E}-01$ & $1.986 \mathrm{E}+12$ & $2.5278 \mathrm{E}+01$ & $1.959 \mathrm{E}-01$ & $2.028 \mathrm{E}+12$ & 0.004 \\
\hline $1 s 5 d^{3} D_{1}$ & $1 s 6 p^{3} P_{0}^{o}$ & $2.7074 \mathrm{E}+01$ & $1.200 \mathrm{E}-01$ & $1.092 \mathrm{E}+12$ & $2.7009 \mathrm{E}+01$ & $1.194 \mathrm{E}-01$ & $1.083 \mathrm{E}+12$ & 0.002 \\
\hline $1 s 6 s^{3} S_{1}$ & $1 s 6 p^{3} P_{0}^{o}$ & $4.6513 \mathrm{E}+03$ & $2.098 \mathrm{E}-02$ & $6.469 E+06$ & $5.5898 \mathrm{E}+03$ & $1.731 \mathrm{E}-02$ & $3.664 \mathrm{E}+06$ & 0.099 \\
\hline $1 s 2 s^{3} S_{1}$ & $1 s 6 p^{3} P_{2}^{o}$ & $1.3670 \mathrm{E}+00$ & $3.166 \mathrm{E}-02$ & $2.260 \mathrm{E}+13$ & $1.3612 \mathrm{E}+00$ & $3.251 \mathrm{E}-02$ & $2.320 \mathrm{E}+13$ & 0.002 \\
\hline $1 s 3 s^{3} S_{1}$ & $1 s 6 p^{3} P_{2}^{o}$ & $3.6753 \mathrm{E}+00$ & $7.231 \mathrm{E}-02$ & $7.141 \mathrm{E}+12$ & $3.6601 \mathrm{E}+00$ & $7.353 \mathrm{E}-02$ & $7.261 \mathrm{E}+12$ & 0.003 \\
\hline $1 s 3 d^{3} D_{2}$ & $1 s 6 p^{3} P_{2}^{o}$ & $3.8403 E+00$ & $6.550 \mathrm{E}-04$ & $5.925 E+10$ & $3.8243 \mathrm{E}+00$ & $6.596 \mathrm{E}-04$ & $5.965 E+10$ & 0.005 \\
\hline $1 s 3 d^{3} D_{1}$ & $1 s 6 p^{3} P_{2}^{o}$ & $3.8421 \mathrm{E}+00$ & $7.089 \mathrm{E}-05$ & $6.406 \mathrm{E}+09$ & $3.8260 \mathrm{E}+00$ & $6.699 \mathrm{E}-05$ & $6.053 E+09$ & 0.009 \\
\hline $1 s 3 d^{3} D_{3}$ & $1 s 6 p^{3} P_{2}^{o}$ & $3.8840 \mathrm{E}+00$ & $7.016 \mathrm{E}-03$ & $6.205 E+11$ & $3.8677 \mathrm{E}+00$ & $6.917 \mathrm{E}-03$ & $6.116 \mathrm{E}+11$ & 0.006 \\
\hline $1 s 3 d^{1} D_{2}$ & $1 s 6 p^{3} P_{2}^{o}$ & $3.8856 \mathrm{E}+00$ & $5.021 \mathrm{E}-04$ & $4.437 \mathrm{E}+10$ & $3.8694 \mathrm{E}+00$ & $4.897 \mathrm{E}-04$ & $4.326 \mathrm{E}+10$ & 0.008 \\
\hline $1 s 4 s^{3} S_{1}$ & $1 s 6 p^{3} P_{2}^{o}$ & $8.8224 \mathrm{E}+00$ & $1.843 \mathrm{E}-01$ & $3.158 \mathrm{E}+12$ & $8.7863 \mathrm{E}+00$ & $1.868 \mathrm{E}-01$ & $3.201 \mathrm{E}+12$ & 0.004 \\
\hline $1 s 4 d^{3} D_{2}$ & $1 s 6 p^{3} P_{2}^{o}$ & $9.2206 \mathrm{E}+00$ & $4.521 \mathrm{E}-03$ & $7.093 \mathrm{E}+10$ & $9.1833 \mathrm{E}+00$ & $4.502 \mathrm{E}-03$ & $7.061 \mathrm{E}+10$ & 0.003 \\
\hline $1 s 4 d^{3} D_{1}$ & $1 s 6 p^{3} P_{2}^{o}$ & $9.2248 \mathrm{E}+00$ & $4.885 \mathrm{E}-04$ & $7.658 E+09$ & $9.1874 \mathrm{E}+00$ & $4.667 \mathrm{E}-04$ & $7.314 \mathrm{E}+09$ & 0.004 \\
\hline $1 s 4 d^{3} D_{3}$ & $1 s 6 p^{3} P_{2}^{o}$ & $9.3270 \mathrm{E}+00$ & $4.766 \mathrm{E}-02$ & $7.308 \mathrm{E}+11$ & $9.2888 \mathrm{E}+00$ & $4.696 \mathrm{E}-02$ & $7.199 \mathrm{E}+11$ & 0.003 \\
\hline $1 s 4 d{ }^{1} D_{2}$ & $1 s 6 p^{3} P_{2}^{0}$ & $9.3314 \mathrm{E}+00$ & 3.377E-03 & $5.174 \mathrm{E}+10$ & $9.2933 \mathrm{E}+00$ & $3.309 \mathrm{E}-03$ & $5.068 \mathrm{E}+10$ & 0.004 \\
\hline $1 s 5 s^{3} s_{1}$ & $1 s 6 p^{3} P_{2}^{o}$ & $2.4635 \mathrm{E}+01$ & $7.249 \mathrm{E}-01$ & $1.594 \mathrm{E}+12$ & $2.4537 \mathrm{E}+01$ & $7.351 \mathrm{E}-01$ & $1.615 \mathrm{E}+12$ & 0.003 \\
\hline $1 s 5 d^{3} D_{2}$ & $1 s 6 p^{3} P_{2}^{o}$ & $2.6247 \mathrm{E}+01$ & $3.725 \mathrm{E}-02$ & $7.213 E+10$ & $2.6148 \mathrm{E}+01$ & $3.678 \mathrm{E}-02$ & $7.116 \mathrm{E}+10$ & 0.001 \\
\hline $1 s 5 d^{3} D_{1}$ & $1 s 6 p^{3} P_{2}^{o}$ & $2.6264 \mathrm{E}+01$ & $4.030 \mathrm{E}-03$ & $7.794 \mathrm{E}+09$ & $2.6164 \mathrm{E}+01$ & $3.868 \mathrm{E}-03$ & $7.475 \mathrm{E}+09$ & 0.002 \\
\hline $1 s 5 d^{3} D_{3}$ & $1 s 6 p^{3} P_{2}^{o}$ & $2.6690 \mathrm{E}+01$ & $3.948 \mathrm{E}-01$ & $7.394 \mathrm{E}+11$ & $2.6588 \mathrm{E}+01$ & $3.879 \mathrm{E}-01$ & $7.258 \mathrm{E}+11$ & 0.001 \\
\hline $1 s 5 d{ }^{1} D_{2}$ & $1 s 6 p^{3} P_{2}^{o}$ & $2.6709 \mathrm{E}+01$ & $2.789 \mathrm{E}-02$ & $5.215 E+10$ & $2.6607 \mathrm{E}+01$ & $2.727 \mathrm{E}-02$ & $5.096 \mathrm{E}+10$ & 0.002 \\
\hline $1 s 5 g{ }^{3} G_{3}$ & $1 s 6 p^{3} P_{2}^{o}$ & $2.6929 \mathrm{E}+01$ & $1.344 \mathrm{E}-08$ & $2.472 E+04$ & & & & 0.016 \\
\hline $1 s 6 s^{3} S_{1}$ & $1 s 6 p^{3} P_{2}^{o}$ & $7.3822 \mathrm{E}+02$ & $6.713 \mathrm{E}-01$ & $1.643 E+09$ & $7.2782 \mathrm{E}+02$ & $6.786 \mathrm{E}-01$ & $1.694 \mathrm{E}+09$ & 0.015 \\
\hline $1 s 2 p^{3} P_{1}^{o}$ & $1 s 6 d^{3} D_{2}$ & $1.3786 \mathrm{E}+00$ & $5.406 \mathrm{E}-02$ & $3.795 E+13$ & $1.3729 \mathrm{E}+00$ & $5.423 \mathrm{E}-02$ & $3.806 \mathrm{E}+13$ & 0.001 \\
\hline $1 s 2 p^{3} P_{2}^{o}$ & $1 s 6 d^{3} D_{2}$ & $1.4409 \mathrm{E}+00$ & $9.245 \mathrm{E}-03$ & $5.941 \mathrm{E}+12$ & $1.4348 \mathrm{E}+00$ & $9.097 \mathrm{E}-03$ & $5.845 \mathrm{E}+12$ & 0.003 \\
\hline $1 s 2 p^{1} P_{1}^{o}$ & $1 s 6 d^{3} D_{2}$ & $1.4467 \mathrm{E}+00$ & $1.528 \mathrm{E}-03$ & $9.741 \mathrm{E}+11$ & $1.4407 \mathrm{E}+00$ & $1.426 \mathrm{E}-03$ & $9.093 \mathrm{E}+11$ & 0.001 \\
\hline $1 s 3 p^{3} P_{1}^{o}$ & $1 s 6 d^{3} D_{2}$ & $3.6972 \mathrm{E}+00$ & $1.326 \mathrm{E}-01$ & $1.294 \mathrm{E}+13$ & $3.6817 \mathrm{E}+00$ & $1.329 \mathrm{E}-01$ & $1.297 \mathrm{E}+13$ & 0.000 \\
\hline $1 s 3 p^{3} P_{2}^{o}$ & $1 s 6 d^{3} D_{2}$ & $3.8292 \mathrm{E}+00$ & $2.492 \mathrm{E}-02$ & $2.268 \mathrm{E}+12$ & $3.8130 \mathrm{E}+00$ & $2.499 \mathrm{E}-02$ & $2.274 \mathrm{E}+12$ & 0.001 \\
\hline $1 s 3 p^{1} P_{1}^{o}$ & $1 s 6 d^{3} D_{2}$ & $3.8409 \mathrm{E}+00$ & $4.051 \mathrm{E}-03$ & $3.663 E+11$ & $3.8249 \mathrm{E}+00$ & $3.732 \mathrm{E}-03$ & $3.374 \mathrm{E}+11$ & 0.002 \\
\hline $1 s 4 p^{3} P_{1}^{o}$ & $1 s 6 d^{3} D_{2}$ & $8.8702 \mathrm{E}+00$ & $3.276 \mathrm{E}-01$ & $5.555 E+12$ & $8.8316 \mathrm{E}+00$ & $3.287 \mathrm{E}-01$ & $5.575 \mathrm{E}+12$ & 0.000 \\
\hline $1 s 4 p^{3} P_{2}^{o}$ & $1 s 6 d^{3} D_{2}$ & $9.1898 \mathrm{E}+00$ & $6.652 \mathrm{E}-02$ & $1.051 \mathrm{E}+12$ & $9.1496 \mathrm{E}+00$ & $6.733 \mathrm{E}-02$ & $1.063 \mathrm{E}+12$ & 0.000 \\
\hline $1 s 4 p^{1} P_{1}^{o}$ & $1 s 6 d^{3} D_{2}$ & $9.2178 \mathrm{E}+00$ & $1.079 \mathrm{E}-02$ & $1.693 \mathrm{E}+11$ & $9.1778 \mathrm{E}+00$ & $9.804 \mathrm{E}-03$ & $1.539 \mathrm{E}+11$ & 0.001 \\
\hline $1 s 4 f^{3} F_{3}^{o}$ & $1 s 6 d^{3} D_{2}$ & $9.3215 \mathrm{E}+00$ & $1.216 \mathrm{E}-02$ & $1.867 \mathrm{E}+11$ & $9.2809 \mathrm{E}+00$ & $1.219 \mathrm{E}-02$ & $1.871 \mathrm{E}+11$ & 0.001 \\
\hline $1 s 4 f^{3} F_{2}^{o}$ & $1 s 6 d^{3} D_{2}$ & $9.3237 \mathrm{E}+00$ & $8.868 \mathrm{E}-04$ & $1.361 \mathrm{E}+10$ & $9.2831 \mathrm{E}+00$ & $8.928 \mathrm{E}-04$ & $1.370 \mathrm{E}+10$ & 0.000 \\
\hline $1 s 4 f^{1} F_{3}^{o}$ & $1 s 6 d^{3} D_{2}$ & $9.3766 \mathrm{E}+00$ & $9.857 \mathrm{E}-07$ & $1.496 \mathrm{E}+07$ & & & & 0.011 \\
\hline $1 s 5 p^{3} P_{1}^{o}$ & $1 s 6 d^{3} D_{2}$ & $2.4798 \mathrm{E}+01$ & $1.222 \mathrm{E}+00$ & $2.651 \mathrm{E}+12$ & $2.4683 \mathrm{E}+01$ & $1.227 \mathrm{E}+00$ & $2.664 \mathrm{E}+12$ & 0.000 \\
\hline $1 s 5 p^{3} P_{2}^{o}$ & $1 s 6 d^{3} D_{2}$ & $2.6092 \mathrm{E}+01$ & $2.792 \mathrm{E}-01$ & $5.472 \mathrm{E}+11$ & $2.5970 \mathrm{E}+01$ & $2.851 \mathrm{E}-01$ & $5.591 \mathrm{E}+11$ & 0.000 \\
\hline $1 s 5 p^{1} P_{1}^{o}$ & $1 s 6 d^{3} D_{2}$ & $2.6207 \mathrm{E}+01$ & $4.553 \mathrm{E}-02$ & $8.843 \mathrm{E}+10$ & $2.6085 \mathrm{E}+01$ & $4.028 \mathrm{E}-02$ & $7.831 \mathrm{E}+10$ & 0.001 \\
\hline
\end{tabular}


Table 3 (continued)

\begin{tabular}{|c|c|c|c|c|c|c|c|c|}
\hline \multirow[t]{2}{*}{ Lower } & \multirow[t]{2}{*}{ Upper } & \multicolumn{3}{|l|}{ GRASP2K } & \multicolumn{3}{|l|}{ FAC } & \multirow[t]{2}{*}{$d T$} \\
\hline & & $\lambda(\AA)$ & $g f$ & $A\left(\mathrm{~s}^{-1}\right)$ & $\lambda(\AA)$ & $g f$ & $A\left(\mathrm{~s}^{-1}\right)$ & \\
\hline $1 s 5 f^{3} F_{3}^{o}$ & $1 s 6 d^{3} D_{2}$ & $2.6639 \mathrm{E}+01$ & $1.774 \mathrm{E}-01$ & $3.335 \mathrm{E}+11$ & $2.6517 \mathrm{E}+01$ & $1.775 \mathrm{E}-01$ & $3.339 E+11$ & 0.001 \\
\hline $1 s 5 f^{3} F_{2}^{0}$ & $1 s 6 d^{3} D_{2}$ & $2.6648 \mathrm{E}+01$ & $1.291 \mathrm{E}-02$ & $2.426 \mathrm{E}+10$ & $2.6526 \mathrm{E}+01$ & $1.297 \mathrm{E}-02$ & $2.439 \mathrm{E}+10$ & 0.000 \\
\hline $1 s 5 f^{1} F_{3}^{o}$ & $1 s 6 d^{3} D_{2}$ & $2.6870 \mathrm{E}+01$ & $1.399 \mathrm{E}-05$ & $2.585 \mathrm{E}+07$ & & & & 0.010 \\
\hline $1 s 6 p^{3} P_{1}^{o}$ & $1 s 6 d^{3} D_{2}$ & $8.0869 \mathrm{E}+02$ & $5.619 \mathrm{E}-01$ & $1.146 \mathrm{E}+09$ & $7.9302 \mathrm{E}+02$ & $5.686 \mathrm{E}-01$ & $1.196 \mathrm{E}+09$ & 0.003 \\
\hline $1 s 6 p^{3} P_{2}^{o}$ & $1 s 6 d^{3} D_{2}$ & $1.2110 \mathrm{E}-01$ & $6.859 \mathrm{E}-03$ & $6.234 \mathrm{E}+04$ & $9.6399 \mathrm{E}+03$ & $8.661 \mathrm{E}-03$ & $1.232 E+05$ & 0.102 \\
\hline $1 s^{2}{ }^{1} s_{0}$ & $1 s 6 p^{1} P_{1}^{o}$ & $3.1620 \mathrm{E}-01$ & $9.840 \mathrm{E}-03$ & $2.187 \mathrm{E}+14$ & $3.1497 \mathrm{E}-01$ & $1.063 \mathrm{E}-02$ & $2.362 \mathrm{E}+14$ & 0.031 \\
\hline $1 s 2 s^{3} S_{1}$ & $1 s 6 p^{1} P_{1}^{o}$ & $1.3668 \mathrm{E}+00$ & $5.663 \mathrm{E}-03$ & $6.739 E+12$ & $1.3611 \mathrm{E}+00$ & $5.637 \mathrm{E}-03$ & $6.708 \mathrm{E}+12$ & 0.001 \\
\hline $1 s 2 s{ }^{1} S_{0}$ & $1 s 6 p^{1} P_{1}^{o}$ & $1.3798 \mathrm{E}+00$ & $1.346 \mathrm{E}-02$ & $1.572 \mathrm{E}+13$ & $1.3742 \mathrm{E}+00$ & $1.374 \mathrm{E}-02$ & $1.604 \mathrm{E}+13$ & 0.003 \\
\hline $1 s 3 s^{3} S_{1}$ & $1 s 6 p^{1} P_{1}^{o}$ & $3.6740 \mathrm{E}+00$ & $1.286 \mathrm{E}-02$ & $2.118 \mathrm{E}+12$ & $3.6589 \mathrm{E}+00$ & $1.267 \mathrm{E}-02$ & $2.087 \mathrm{E}+12$ & 0.003 \\
\hline $1 s 3 s^{1} S_{0}$ & $1 s 6 p^{1} P_{1}^{o}$ & $3.6986 \mathrm{E}+00$ & $3.101 \mathrm{E}-02$ & $5.041 \mathrm{E}+12$ & $3.6837 \mathrm{E}+00$ & $3.125 \mathrm{E}-02$ & $5.077 \mathrm{E}+12$ & 0.002 \\
\hline $1 s 3 d^{3} D_{2}$ & $1 s 6 p^{1} P_{1}^{o}$ & $3.8389 \mathrm{E}+00$ & $1.069 \mathrm{E}-04$ & $1.613 \mathrm{E}+10$ & $3.8230 \mathrm{E}+00$ & $1.372 \mathrm{E}-04$ & $2.069 \mathrm{E}+10$ & 0.011 \\
\hline $1 s 3 d^{3} D_{1}$ & $1 s 6 p^{1} P_{1}^{o}$ & $3.8407 \mathrm{E}+00$ & $3.272 \mathrm{E}-04$ & $4.932 E+10$ & $3.8248 \mathrm{E}+00$ & $3.122 \mathrm{E}-04$ & $4.705 E+10$ & 0.011 \\
\hline $1 s 3 d^{1} D_{2}$ & $1 s 6 p^{1} P_{1}^{o}$ & $3.8842 \mathrm{E}+00$ & $4.432 \mathrm{E}-03$ & $6.532 \mathrm{E}+11$ & $3.8681 \mathrm{E}+00$ & $4.442 \mathrm{E}-03$ & $6.545 \mathrm{E}+11$ & 0.010 \\
\hline $1 s 4 s^{3} S_{1}$ & $1 s 6 p^{1} P_{1}^{o}$ & $8.8149 \mathrm{E}+00$ & $3.260 \mathrm{E}-02$ & $9.327 \mathrm{E}+11$ & $8.7796 \mathrm{E}+00$ & $3.204 \mathrm{E}-02$ & $9.164 \mathrm{E}+11$ & 0.003 \\
\hline $1 s 4 s^{1} S_{0}$ & $1 s 6 p^{1} P_{1}^{o}$ & $8.8722 \mathrm{E}+00$ & $7.942 \mathrm{E}-02$ & $2.243 \mathrm{E}+12$ & $8.8372 \mathrm{E}+00$ & $7.964 \mathrm{E}-02$ & $2.248 \mathrm{E}+12$ & 0.004 \\
\hline $1 s 4 d^{3} D_{2}$ & $1 s 6 p^{1} P_{1}^{o}$ & $9.2125 \mathrm{E}+00$ & $7.164 \mathrm{E}-04$ & $1.877 \mathrm{E}+10$ & $9.1760 \mathrm{E}+00$ & $9.080 \mathrm{E}-04$ & $2.377 \mathrm{E}+10$ & 0.007 \\
\hline $1 s 4 d^{3} D_{1}$ & $1 s 6 p^{1} P_{1}^{o}$ & $9.2166 \mathrm{E}+00$ & $2.227 \mathrm{E}-03$ & $5.828 \mathrm{E}+10$ & $9.1801 \mathrm{E}+00$ & $2.117 \mathrm{E}-03$ & $5.539 E+10$ & 0.006 \\
\hline $1 s 4 d{ }^{1} D_{2}$ & $1 s 6 p^{1} P_{1}^{o}$ & $9.3230 \mathrm{E}+00$ & $2.999 \mathrm{E}-02$ & $7.671 \mathrm{E}+11$ & $9.2858 \mathrm{E}+00$ & $3.019 \mathrm{E}-02$ & $7.720 \mathrm{E}+11$ & 0.005 \\
\hline $1 s 5 s^{3} S_{1}$ & $1 s 6 p^{1} P_{1}^{o}$ & $2.4577 \mathrm{E}+01$ & $1.271 \mathrm{E}-01$ & $4.677 \mathrm{E}+11$ & $2.4485 \mathrm{E}+01$ & $1.249 \mathrm{E}-01$ & $4.593 \mathrm{E}+11$ & 0.003 \\
\hline $1 s 5 s^{1} S_{0}$ & $1 s 6 p^{1} P_{1}^{o}$ & $2.4800 \mathrm{E}+01$ & $3.145 \mathrm{E}-01$ & $1.137 \mathrm{E}+12$ & $2.4710 \mathrm{E}+01$ & $3.143 \mathrm{E}-01$ & $1.134 \mathrm{E}+12$ & 0.004 \\
\hline $1 s 5 d^{3} D_{2}$ & $1 s 6 p^{1} P_{1}^{o}$ & $2.6180 \mathrm{E}+01$ & $5.800 \mathrm{E}-03$ & $1.881 \mathrm{E}+10$ & $2.6089 \mathrm{E}+01$ & $7.458 \mathrm{E}-03$ & $2.415 E+10$ & 0.003 \\
\hline $1 s 5 d^{3} D_{1}$ & $1 s 6 p^{1} P_{1}^{o}$ & $2.6197 \mathrm{E}+01$ & $1.815 \mathrm{E}-02$ & $5.882 \mathrm{E}+10$ & $2.6105 E+01$ & $1.715 \mathrm{E}-02$ & $5.550 \mathrm{E}+10$ & 0.003 \\
\hline $1 s 5 d{ }^{1} D_{2}$ & $1 s 6 p^{1} P_{1}^{o}$ & $2.6640 \mathrm{E}+01$ & $2.473 \mathrm{E}-01$ & $7.746 \mathrm{E}+11$ & $2.6546 \mathrm{E}+01$ & $2.497 \mathrm{E}-01$ & $7.814 \mathrm{E}+11$ & 0.003 \\
\hline $1 s 6 s^{3} S_{1}$ & $1 s 6 p^{1} P_{1}^{o}$ & $6.8918 \mathrm{E}+02$ & $1.292 \mathrm{E}-01$ & $6.050 \mathrm{E}+08$ & $6.8482 \mathrm{E}+02$ & $1.256 \mathrm{E}-01$ & $5.907 \mathrm{E}+08$ & 0.015 \\
\hline $1 s 6 s^{1} S_{0}$ & $1 s 6 p^{1} P_{1}^{o}$ & $8.0559 \mathrm{E}+02$ & $2.588 \mathrm{E}-01$ & $8.867 \mathrm{E}+08$ & $7.8073 \mathrm{E}+02$ & $2.693 \mathrm{E}-01$ & $9.741 E+08$ & 0.057 \\
\hline $1 s 6 d^{3} D_{2}$ & $1 s 6 p^{1} P_{1}^{o}$ & $7.2196 \mathrm{E}+04$ & $1.839 \mathrm{E}-04$ & $7.843 \mathrm{E}+01$ & & & & 0.369 \\
\hline $1 s 2 p^{3} P_{1}^{o}$ & $1 s 6 d^{3} D_{1}$ & $1.3786 \mathrm{E}+00$ & $1.160 \mathrm{E}-02$ & $1.357 \mathrm{E}+13$ & $1.3729 \mathrm{E}+00$ & $1.157 \mathrm{E}-02$ & $1.354 \mathrm{E}+13$ & 0.001 \\
\hline $1 s 2 p^{3} P_{0}^{0}$ & $1 s 6 d^{3} D_{1}$ & $1.3795 \mathrm{E}+00$ & $2.193 E-02$ & $2.563 \mathrm{E}+13$ & $1.3737 \mathrm{E}+00$ & $2.194 \mathrm{E}-02$ & $2.563 E+13$ & 0.001 \\
\hline $1 s 2 p^{3} P_{2}^{o}$ & $1 s 6 d^{3} D_{1}$ & $1.4408 \mathrm{E}+00$ & $1.013 \mathrm{E}-03$ & $1.085 \mathrm{E}+12$ & $1.4348 \mathrm{E}+00$ & $9.908 \mathrm{E}-04$ & $1.061 \mathrm{E}+12$ & 0.004 \\
\hline $1 s 2 p{ }^{1} P_{1}^{o}$ & $1 s 6 d^{3} D_{1}$ & $1.4467 \mathrm{E}+00$ & $4.506 \mathrm{E}-03$ & $4.787 \mathrm{E}+12$ & $1.4407 \mathrm{E}+00$ & $4.362 \mathrm{E}-03$ & $4.633 \mathrm{E}+12$ & 0.003 \\
\hline $1 s 3 p^{3} P_{1}^{o}$ & $1 s 6 d^{3} D_{1}$ & $3.6970 \mathrm{E}+00$ & $2.828 \mathrm{E}-02$ & $4.600 \mathrm{E}+12$ & $3.6817 \mathrm{E}+00$ & $2.835 \mathrm{E}-02$ & $4.611 \mathrm{E}+12$ & 0.001 \\
\hline $1 s 3 p^{3} P_{0}^{0}$ & $1 s 6 d^{3} D_{1}$ & $3.6987 \mathrm{E}+00$ & $5.368 \mathrm{E}-02$ & $8.725 E+12$ & $3.6834 \mathrm{E}+00$ & $5.377 \mathrm{E}-02$ & $8.738 E+12$ & 0.001 \\
\hline $1 s 3 p^{3} P_{2}^{o}$ & $1 s 6 d^{3} D_{1}$ & $3.8290 \mathrm{E}+00$ & $2.722 \mathrm{E}-03$ & $4.128 \mathrm{E}+11$ & $3.8131 \mathrm{E}+00$ & $2.724 \mathrm{E}-03$ & $4.131 E+11$ & 0.002 \\
\hline $1 s 3 p^{1} P_{1}^{o}$ & $1 s 6 d^{3} D_{1}$ & $3.8407 \mathrm{E}+00$ & $1.233 \mathrm{E}-02$ & $1.858 \mathrm{E}+12$ & $3.8249 \mathrm{E}+00$ & $1.218 \mathrm{E}-02$ & $1.836 \mathrm{E}+12$ & 0.002 \\
\hline $1 s 4 p^{3} P_{1}^{o}$ & $1 s 6 d^{3} D_{1}$ & $8.8691 \mathrm{E}+00$ & $6.971 \mathrm{E}-02$ & $1.970 \mathrm{E}+12$ & $8.8318 \mathrm{E}+00$ & $7.019 \mathrm{E}-02$ & $1.983 \mathrm{E}+12$ & 0.001 \\
\hline $1 s 4 p^{3} P_{0}^{o}$ & $1 s 6 d^{3} D_{1}$ & $8.8731 \mathrm{E}+00$ & $1.325 \mathrm{E}-01$ & $3.743 \mathrm{E}+12$ & $8.8357 \mathrm{E}+00$ & $1.329 \mathrm{E}-01$ & $3.755 E+12$ & 0.001 \\
\hline $1 s 4 p^{3} P_{2}^{o}$ & $1 s 6 d^{3} D_{1}$ & $9.1886 \mathrm{E}+00$ & $7.247 \mathrm{E}-03$ & $1.908 \mathrm{E}+11$ & $9.1499 \mathrm{E}+00$ & $7.347 \mathrm{E}-03$ & $1.934 \mathrm{E}+11$ & 0.001 \\
\hline $1 s 4 p{ }^{1} P_{1}^{o}$ & $1 s 6 d^{3} D_{1}$ & $9.2166 \mathrm{E}+00$ & $3.316 \mathrm{E}-02$ & $8.679 E+11$ & $9.1781 \mathrm{E}+00$ & $3.306 \mathrm{E}-02$ & $8.654 \mathrm{E}+11$ & 0.001 \\
\hline $1 s 4 f^{3} F_{2}^{o}$ & $1 s 6 d^{3} D_{1}$ & $9.3225 \mathrm{E}+00$ & $7.803 \mathrm{E}-03$ & $1.996 \mathrm{E}+11$ & $9.2834 \mathrm{E}+00$ & $7.840 \mathrm{E}-03$ & $2.005 E+11$ & 0.000 \\
\hline $1 s 5 p^{3} P_{1}^{o}$ & $1 s 6 d^{3} D_{1}$ & $2.4789 \mathrm{E}+01$ & $2.595 \mathrm{E}-01$ & $9.388 \mathrm{E}+11$ & $2.4685 \mathrm{E}+01$ & $2.631 \mathrm{E}-01$ & $9.519 \mathrm{E}+11$ & 0.001 \\
\hline $1 s 5 p^{3} P_{0}^{o}$ & $1 s 6 d^{3} D_{1}$ & $2.4805 \mathrm{E}+01$ & $4.939 \mathrm{E}-01$ & $1.785 \mathrm{E}+12$ & $2.4700 \mathrm{E}+01$ & $4.967 \mathrm{E}-01$ & $1.794 \mathrm{E}+12$ & 0.001 \\
\hline $1 s 5 p^{3} P_{2}^{0}$ & $1 s 6 d^{3} D_{1}$ & $2.6083 \mathrm{E}+01$ & $3.034 \mathrm{E}-02$ & $9.915 \mathrm{E}+10$ & $2.5972 \mathrm{E}+01$ & $3.115 \mathrm{E}-02$ & $1.018 \mathrm{E}+11$ & 0.001 \\
\hline $1 s 5 p{ }^{1} P_{1}^{o}$ & $1 s 6 d^{3} D_{1}$ & $2.6197 \mathrm{E}+01$ & $1.405 \mathrm{E}-01$ & $4.553 \mathrm{E}+11$ & $2.6087 \mathrm{E}+01$ & $1.410 \mathrm{E}-01$ & $4.568 \mathrm{E}+11$ & 0.001 \\
\hline $1 s 5 f^{3} F_{2}^{0}$ & $1 s 6 d^{3} D_{1}$ & $2.6638 \mathrm{E}+01$ & $1.138 \mathrm{E}-01$ & $3.567 \mathrm{E}+11$ & $2.6529 \mathrm{E}+01$ & $1.142 \mathrm{E}-01$ & $3.577 \mathrm{E}+11$ & 0.000 \\
\hline $1 s 6 p^{3} P_{1}^{o}$ & $1 s 6 d^{3} D_{1}$ & $7.9964 \mathrm{E}+02$ & $1.213 \mathrm{E}-01$ & $4.217 \mathrm{E}+08$ & $7.9517 \mathrm{E}+02$ & $1.225 \mathrm{E}-01$ & $4.272 \mathrm{E}+08$ & 0.006 \\
\hline $1 s 6 p^{3} P_{0}^{0}$ & $1 s 6 d^{3} D_{1}$ & $8.0896 \mathrm{E}+02$ & $2.267 \mathrm{E}-01$ & $7.701 \mathrm{E}+08$ & $7.7196 \mathrm{E}+02$ & $2.370 \mathrm{E}-01$ & $8.770 \mathrm{E}+08$ & 0.005 \\
\hline $1 s 6 p^{3} P_{2}^{o}$ & $1 s 6 d^{3} D_{1}$ & $1.0358 \mathrm{E}+04$ & $8.674 \mathrm{E}-04$ & $1.798 \mathrm{E}+04$ & & & & 0.007 \\
\hline $1 s 6 p^{1} P_{1}^{o}$ & $1 s 6 d^{3} D_{1}$ & $6.7159 \mathrm{E}+06$ & $6.153 \mathrm{E}-06$ & $3.033 \mathrm{E}-04$ & & & & 0.995 \\
\hline $1 s 2 p^{3} P_{2}^{o}$ & $1 s 6 d^{3} D_{3}$ & $1.4401 \mathrm{E}+00$ & $8.934 \mathrm{E}-02$ & $4.105 E+13$ & $1.4340 \mathrm{E}+00$ & $9.091 \mathrm{E}-02$ & $4.176 \mathrm{E}+13$ & 0.000 \\
\hline $1 s 3 p^{3} P_{2}^{o}$ & $1 s 6 d^{3} D_{3}$ & $3.8238 \mathrm{E}+00$ & $2.308 \mathrm{E}-01$ & $1.504 \mathrm{E}+13$ & $3.8077 \mathrm{E}+00$ & $2.333 \mathrm{E}-01$ & $1.520 \mathrm{E}+13$ & 0.000 \\
\hline $1 s 4 p^{3} P_{2}^{0}$ & $1 s 6 d^{3} D_{3}$ & $9.1590 \mathrm{E}+00$ & $5.989 \mathrm{E}-01$ & $6.802 \mathrm{E}+12$ & $9.1192 \mathrm{E}+00$ & $6.053 \mathrm{E}-01$ & $6.877 \mathrm{E}+12$ & 0.000 \\
\hline $1 s 4 f^{3} F_{3}^{o}$ & $1 s 6 d^{3} D_{3}$ & $9.2898 \mathrm{E}+00$ & $7.076 \mathrm{E}-04$ & $7.813 \mathrm{E}+09$ & $9.2496 \mathrm{E}+00$ & $7.143 \mathrm{E}-04$ & $7.889 \mathrm{E}+09$ & 0.001 \\
\hline $1 s 4 f^{3} F_{2}^{o}$ & $1 s 6 d^{3} D_{3}$ & $9.2920 \mathrm{E}+00$ & $3.269 \mathrm{E}-05$ & $3.608 \mathrm{E}+08$ & & & & 0.001 \\
\hline $1 s 4 f^{3} F_{4}^{o}$ & $1 s 6 d^{3} D_{3}$ & $9.3429 \mathrm{E}+00$ & $1.559 \mathrm{E}-02$ & $1.702 \mathrm{E}+11$ & $9.3023 \mathrm{E}+00$ & $1.557 \mathrm{E}-02$ & $1.700 \mathrm{E}+11$ & 0.001 \\
\hline $1 s 4 f^{1} F_{3}^{o}$ & $1 s 6 d^{3} D_{3}$ & $9.3445 \mathrm{E}+00$ & $5.815 \mathrm{E}-04$ & $6.346 \mathrm{E}+09$ & $9.3039 \mathrm{E}+00$ & $5.794 \mathrm{E}-04$ & $6.324 \mathrm{E}+09$ & 0.000 \\
\hline $1 s 5 p^{3} P_{2}^{o}$ & $1 s 6 d^{3} D_{3}$ & $2.5845 \mathrm{E}+01$ & $2.417 \mathrm{E}+00$ & $3.448 \mathrm{E}+12$ & $2.5726 \mathrm{E}+01$ & $2.453 \mathrm{E}+00$ & $3.503 E+12$ & 0.000 \\
\hline $1 s 5 f^{3} F_{3}^{o}$ & $1 s 6 d^{3} D_{3}$ & $2.6382 \mathrm{E}+01$ & $1.034 \mathrm{E}-02$ & $1.416 \mathrm{E}+10$ & $2.6263 \mathrm{E}+01$ & $1.037 \mathrm{E}-02$ & $1.421 \mathrm{E}+10$ & 0.001 \\
\hline $1 s 5 f^{3} F_{2}^{o}$ & $1 s 6 d^{3} D_{3}$ & $2.6391 \mathrm{E}+01$ & $4.907 \mathrm{E}-04$ & $6.713 E+08$ & $2.6272 \mathrm{E}+01$ & $4.885 \mathrm{E}-04$ & $6.687 \mathrm{E}+08$ & 0.000 \\
\hline $1 s 5 f^{3} F_{4}^{o}$ & $1 s 6 d^{3} D_{3}$ & $2.6602 \mathrm{E}+01$ & $2.275 \mathrm{E}-01$ & $3.063 \mathrm{E}+11$ & $2.6482 \mathrm{E}+01$ & $2.272 \mathrm{E}-01$ & $3.062 E+11$ & 0.001 \\
\hline $1 s 5 f^{1} F_{3}^{0}$ & $1 s 6 d^{3} D_{3}$ & $2.6609 \mathrm{E}+01$ & $8.482 \mathrm{E}-03$ & $1.142 \mathrm{E}+10$ & $2.6489 \mathrm{E}+01$ & $8.453 \mathrm{E}-03$ & $1.138 \mathrm{E}+10$ & 0.000 \\
\hline $1 s 6 p^{3} P_{2}^{o}$ & $1 s 6 d^{3} D_{3}$ & $2.2306 \mathrm{E}+03$ & $3.482 \mathrm{E}-01$ & $6.669 \mathrm{E}+07$ & $2.1370 \mathrm{E}+03$ & $3.618 \mathrm{E}-01$ & $7.486 \mathrm{E}+07$ & 0.018 \\
\hline $1 s 3 d^{3} D_{2}$ & $1 s 6 f^{3} F_{3}^{o}$ & $3.8336 \mathrm{E}+00$ & $2.524 \mathrm{E}-01$ & $1.637 \mathrm{E}+13$ & $3.8174 \mathrm{E}+00$ & $2.527 \mathrm{E}-01$ & $1.638 \mathrm{E}+13$ & 0.001 \\
\hline $1 s 3 d^{3} D_{3}$ & $1 s 6 f^{3} F_{3}^{o}$ & $3.8771 \mathrm{E}+00$ & $1.644 \mathrm{E}-02$ & $1.042 \mathrm{E}+12$ & $3.8607 \mathrm{E}+00$ & $1.627 \mathrm{E}-02$ & $1.031 \mathrm{E}+12$ & 0.002 \\
\hline $1 s 3 d d^{1} D_{2}$ & $1 s 6 f^{3} F_{3}^{o}$ & $3.8788 \mathrm{E}+00$ & $9.798 \mathrm{E}-04$ & $6.205 E+10$ & $3.8624 \mathrm{E}+00$ & $9.022 \mathrm{E}-04$ & $5.714 \mathrm{E}+10$ & 0.001 \\
\hline $1 s 4 d^{3} D_{2}$ & $1 s 6 f^{3} F_{3}^{o}$ & $9.1821 \mathrm{E}+00$ & $8.572 \mathrm{E}-01$ & $9.689 \mathrm{E}+12$ & $9.1440 \mathrm{E}+00$ & $8.586 \mathrm{E}-01$ & $9.702 \mathrm{E}+12$ & 0.001 \\
\hline $1 s 4 d^{3} D_{3}$ & $1 s 6 f^{3} F_{3}^{o}$ & $9.2876 \mathrm{E}+00$ & $5.814 \mathrm{E}-02$ & $6.423 \mathrm{E}+11$ & $9.2486 \mathrm{E}+00$ & $5.812 \mathrm{E}-02$ & $6.419 \mathrm{E}+11$ & 0.001 \\
\hline $1 s 4 d^{1} D_{2}$ & $1 s 6 f^{3} F_{3}^{o}$ & $9.2919 \mathrm{E}+00$ & $3.683 \mathrm{E}-03$ & $4.065 E+10$ & $9.2530 \mathrm{E}+00$ & $3.427 \mathrm{E}-03$ & $3.782 \mathrm{E}+10$ & 0.000 \\
\hline $1 s 5 d^{3} D_{2}$ & $1 s 6 f^{3} F_{3}^{o}$ & $2.5937 \mathrm{E}+01$ & $3.743 E+00$ & $5.302 \mathrm{E}+12$ & $2.5831 \mathrm{E}+01$ & $3.751 \mathrm{E}+00$ & $5.311 \mathrm{E}+12$ & 0.000 \\
\hline $1 s 5 d^{3} D_{3}$ & $1 s 6 f^{3} F_{3}^{o}$ & $2.6370 \mathrm{E}+01$ & $2.663 \mathrm{E}-01$ & $3.649 \mathrm{E}+11$ & $2.6261 \mathrm{E}+01$ & $2.675 \mathrm{E}-01$ & $3.665 \mathrm{E}+11$ & 0.001 \\
\hline $1 s 5 d{ }^{1} D_{2}$ & $1 s 6 f^{3} F_{3}^{o}$ & $2.6388 \mathrm{E}+01$ & $1.732 \mathrm{E}-02$ & $2.370 \mathrm{E}+10$ & $2.6279 \mathrm{E}+01$ & $1.612 \mathrm{E}-02$ & $2.205 \mathrm{E}+10$ & 0.000 \\
\hline $1 s 5 g^{3} G_{4}$ & $1 s 6 f^{3} F_{3}^{o}$ & $2.6598 \mathrm{E}+01$ & $7.014 \mathrm{E}-02$ & $9.448 \mathrm{E}+10$ & $2.6482 \mathrm{E}+01$ & $7.027 \mathrm{E}-02$ & $9.468 \mathrm{E}+10$ & 0.001 \\
\hline
\end{tabular}


Table 3 (continued)

\begin{tabular}{|c|c|c|c|c|c|c|c|c|}
\hline \multirow[t]{2}{*}{ Lower } & \multirow[t]{2}{*}{ Upper } & \multicolumn{3}{|l|}{ GRASP2K } & \multicolumn{3}{|l|}{ FAC } & \multirow[t]{2}{*}{$d T$} \\
\hline & & $\lambda(\AA)$ & $g f$ & $A\left(\mathrm{~s}^{-1}\right)$ & $\lambda(\AA)$ & $g f$ & $A\left(\mathrm{~s}^{-1}\right)$ & \\
\hline $1 s 5 g^{3} G_{3}$ & $1 s 6 f^{3} F_{3}^{o}$ & $2.6603 \mathrm{E}+01$ & $2.597 \mathrm{E}-03$ & $3.497 \mathrm{E}+09$ & $2.6487 \mathrm{E}+01$ & $2.606 \mathrm{E}-03$ & $3.509 E+09$ & 0.002 \\
\hline $1 s 5 g{ }^{1} G_{4}$ & $1 s 6 f^{3} F_{3}^{o}$ & $2.6735 \mathrm{E}+01$ & $2.453 \mathrm{E}-10$ & $3.270 \mathrm{E}+02$ & & & & 0.683 \\
\hline $1 s 6 d^{3} D_{2}$ & $1 s 6 f^{3} F_{3}^{o}$ & $2.6863 \mathrm{E}+03$ & $2.445 \mathrm{E}-01$ & $3.228 \mathrm{E}+07$ & $2.7429 E+03$ & $2.382 \mathrm{E}-01$ & $2.991 \mathrm{E}+07$ & 0.009 \\
\hline $1 s 6 d^{3} D_{3}$ & $1 s 6 f^{3} F_{3}^{o}$ & $1.5394 \mathrm{E}+05$ & $2.876 \mathrm{E}-04$ & $1.156 \mathrm{E}+01$ & & & & 0.292 \\
\hline $1 s 2 s^{3} S_{1}$ & $1 s 6 f^{3} F_{2}^{o}$ & $1.3662 \mathrm{E}+00$ & $2.099 \mathrm{E}-09$ & $1.500 \mathrm{E}+06$ & & & & 0.160 \\
\hline $1 s 3 s^{3} s_{1}$ & $1 s 6 f^{3} F_{2}^{o}$ & $3.6690 \mathrm{E}+00$ & $1.653 \mathrm{E}-07$ & $1.638 \mathrm{E}+07$ & & & & 0.106 \\
\hline $1 s 3 d^{3} D_{2}$ & $1 s 6 f^{3} F_{2}^{o}$ & $3.8335 \mathrm{E}+00$ & $1.815 \mathrm{E}-02$ & $1.648 \mathrm{E}+12$ & $3.8174 \mathrm{E}+00$ & $1.816 \mathrm{E}-02$ & $1.648 \mathrm{E}+12$ & 0.000 \\
\hline $1 s 3 d^{3} D_{1}$ & $1 s 6 f^{3} F_{2}^{o}$ & $3.8353 \mathrm{E}+00$ & $1.623 \mathrm{E}-01$ & $1.472 \mathrm{E}+13$ & $3.8192 \mathrm{E}+00$ & $1.624 \mathrm{E}-01$ & $1.473 E+13$ & 0.000 \\
\hline $1 s 3 d^{3} D_{3}$ & $1 s 6 f^{3} F_{2}^{o}$ & $3.8770 \mathrm{E}+00$ & $8.259 E-04$ & $7.330 \mathrm{E}+10$ & $3.8607 \mathrm{E}+00$ & $8.130 \mathrm{E}-04$ & $7.215 E+10$ & 0.004 \\
\hline $1 s 3 d d^{1} D_{2}$ & $1 s 6 f^{3} F_{2}^{o}$ & $3.8787 \mathrm{E}+00$ & $1.146 \mathrm{E}-02$ & $1.016 \mathrm{E}+12$ & $3.8624 \mathrm{E}+00$ & $1.127 \mathrm{E}-02$ & $9.996 \mathrm{E}+11$ & 0.001 \\
\hline $1 s 4 s^{3} S_{1}$ & $1 s 6 f^{3} F_{2}^{o}$ & $8.7866 \mathrm{E}+00$ & $7.760 \mathrm{E}-07$ & $1.341 \mathrm{E}+07$ & & & & 0.060 \\
\hline $1 s 4 d^{3} D_{2}$ & $1 s 6 f^{3} F_{2}^{o}$ & $9.1815 \mathrm{E}+00$ & $6.181 \mathrm{E}-02$ & $9.781 \mathrm{E}+11$ & $9.1440 \mathrm{E}+00$ & $6.194 \mathrm{E}-02$ & $9.799 \mathrm{E}+11$ & 0.000 \\
\hline $1 s 4 d^{3} D_{1}$ & $1 s 6 f^{3} F_{2}^{o}$ & $9.1856 \mathrm{E}+00$ & $5.516 \mathrm{E}-01$ & $8.722 \mathrm{E}+12$ & $9.1480 \mathrm{E}+00$ & $5.521 \mathrm{E}-01$ & $8.727 \mathrm{E}+12$ & 0.000 \\
\hline $1 s 4 d^{3} D_{3}$ & $1 s 6 f^{3} F_{2}^{o}$ & $9.2869 \mathrm{E}+00$ & $2.907 \mathrm{E}-03$ & $4.496 \mathrm{E}+10$ & $9.2486 \mathrm{E}+00$ & $2.903 \mathrm{E}-03$ & $4.489 \mathrm{E}+10$ & 0.000 \\
\hline $1 s 4 d{ }^{1} D_{2}$ & $1 s 6 f^{3} F_{2}^{o}$ & $9.2913 \mathrm{E}+00$ & $4.034 \mathrm{E}-02$ & $6.234 \mathrm{E}+11$ & $9.2530 \mathrm{E}+00$ & $4.006 \mathrm{E}-02$ & $6.189 \mathrm{E}+11$ & 0.001 \\
\hline $1 s 5 s^{3} S_{1}$ & $1 s 6 f^{3} F_{2}^{o}$ & $2.4358 \mathrm{E}+01$ & $3.345 \mathrm{E}-06$ & $7.522 \mathrm{E}+06$ & & & & 0.037 \\
\hline $1 s 5 d^{3} D_{2}$ & $1 s 6 f^{3} F_{2}^{o}$ & $2.5932 \mathrm{E}+01$ & $2.700 \mathrm{E}-01$ & $5.357 \mathrm{E}+11$ & $2.5831 \mathrm{E}+01$ & $2.711 \mathrm{E}-01$ & $5.374 \mathrm{E}+11$ & 0.000 \\
\hline $1 s 5 d^{3} D_{1}$ & $1 s 6 f^{3} F_{2}^{o}$ & $2.5948 \mathrm{E}+01$ & $2.411 \mathrm{E}+00$ & $4.776 \mathrm{E}+12$ & $2.5848 \mathrm{E}+01$ & $2.411 \mathrm{E}+00$ & $4.775 E+12$ & 0.000 \\
\hline $1 s 5 d^{3} D_{3}$ & $1 s 6 f^{3} F_{2}^{o}$ & $2.6365 \mathrm{E}+01$ & $1.328 \mathrm{E}-02$ & $2.549 \mathrm{E}+10$ & $2.6261 \mathrm{E}+01$ & $1.336 \mathrm{E}-02$ & $2.563 \mathrm{E}+10$ & 0.000 \\
\hline $1 s 5 d{ }^{1} D_{2}$ & $1 s 6 f^{3} F_{2}^{o}$ & $2.6383 \mathrm{E}+01$ & $1.846 \mathrm{E}-01$ & $3.537 \mathrm{E}+11$ & $2.6280 \mathrm{E}+01$ & $1.840 \mathrm{E}-01$ & $3.525 E+11$ & 0.000 \\
\hline $1 s 5 g^{3} G_{3}$ & $1 s 6 f^{3} F_{2}^{o}$ & $2.6598 \mathrm{E}+01$ & $5.191 \mathrm{E}-02$ & $9.789 \mathrm{E}+10$ & $2.6488 \mathrm{E}+01$ & $5.205 \mathrm{E}-02$ & $9.813 \mathrm{E}+10$ & 0.002 \\
\hline $1 s 6 s^{3} S_{1}$ & $1 s 6 f^{3} F_{2}^{o}$ & $5.5035 \mathrm{E}+02$ & $9.794 \mathrm{E}-07$ & $4.314 \mathrm{E}+03$ & & & & 0.252 \\
\hline $1 s 6 d^{3} D_{2}$ & $1 s 6 f^{3} F_{2}^{o}$ & $2.6325 \mathrm{E}+03$ & $1.805 \mathrm{E}-02$ & $3.475 \mathrm{E}+06$ & $2.7436 \mathrm{E}+03$ & $1.736 \mathrm{E}-02$ & $3.051 \mathrm{E}+06$ & 0.007 \\
\hline $1 s 6 d^{3} D_{1}$ & $1 s 6 f^{3} F_{2}^{o}$ & $2.7332 \mathrm{E}+03$ & $1.546 \mathrm{E}-01$ & $2.760 \mathrm{E}+07$ & $2.7182 \mathrm{E}+03$ & $1.547 \mathrm{E}-01$ & $2.770 \mathrm{E}+07$ & 0.001 \\
\hline $1 s 6 d^{3} D_{3}$ & $1 s 6 f^{3} F_{2}^{o}$ & $7.0900 \mathrm{E}+04$ & $3.077 \mathrm{E}-05$ & $8.167 E+00$ & & & & 0.051 \\
\hline $1 s 2 p^{3} P_{1}^{o}$ & $1 s 6 d^{1} D_{2}$ & $1.3779 \mathrm{E}+00$ & $6.889 \mathrm{E}-05$ & $4.840 \mathrm{E}+10$ & $1.3722 \mathrm{E}+00$ & $5.228 \mathrm{E}-05$ & $3.673 E+10$ & 0.008 \\
\hline $1 s 2 p^{3} P_{2}^{o}$ & $1 s 6 d^{1} D_{2}$ & $1.4401 \mathrm{E}+00$ & $6.303 E-03$ & $4.055 \mathrm{E}+12$ & $1.4340 \mathrm{E}+00$ & $6.314 \mathrm{E}-03$ & $4.061 \mathrm{E}+12$ & 0.000 \\
\hline $1 s 2 p{ }^{1} P_{1}^{o}$ & $1 s 6 d^{1} D_{2}$ & $1.4459 \mathrm{E}+00$ & $5.724 \mathrm{E}-02$ & $3.652 \mathrm{E}+13$ & $1.4399 \mathrm{E}+00$ & $5.833 \mathrm{E}-02$ & $3.721 \mathrm{E}+13$ & 0.000 \\
\hline $1 s 3 p^{3} P_{1}^{o}$ & $1 s 6 d^{1} D_{2}$ & $3.6920 \mathrm{E}+00$ & $1.380 \mathrm{E}-04$ & $1.350 \mathrm{E}+10$ & $3.6767 \mathrm{E}+00$ & $1.115 \mathrm{E}-04$ & $1.091 \mathrm{E}+10$ & 0.003 \\
\hline $1 s 3 p^{3} P_{2}^{o}$ & $1 s 6 d^{1} D_{2}$ & $3.8236 \mathrm{E}+00$ & $1.625 \mathrm{E}-02$ & $1.483 \mathrm{E}+12$ & $3.8077 \mathrm{E}+00$ & $1.618 \mathrm{E}-02$ & $1.476 \mathrm{E}+12$ & 0.001 \\
\hline $1 s 3 p{ }^{1} P_{1}^{o}$ & $1 s 6 d^{1} D_{2}$ & $3.8353 \mathrm{E}+00$ & $1.489 \mathrm{E}-01$ & $1.351 \mathrm{E}+13$ & $3.8195 \mathrm{E}+00$ & $1.498 \mathrm{E}-01$ & $1.359 \mathrm{E}+13$ & 0.001 \\
\hline $1 s 4 p^{3} P_{1}^{0}$ & $1 s 6 d^{1} D_{2}$ & $8.8403 \mathrm{E}+00$ & $3.141 \mathrm{E}-04$ & $5.361 \mathrm{E}+09$ & $8.8028 \mathrm{E}+00$ & $2.765 \mathrm{E}-04$ & $4.721 \mathrm{E}+09$ & 0.003 \\
\hline $1 s 4 p^{3} P_{2}^{o}$ & $1 s 6 d^{1} D_{2}$ & $9.1577 \mathrm{E}+00$ & $4.208 \mathrm{E}-02$ & $6.694 \mathrm{E}+11$ & $9.1187 \mathrm{E}+00$ & 4.197E-02 & $6.676 \mathrm{E}+11$ & 0.001 \\
\hline $1 s 4 p^{1} P_{1}^{o}$ & $1 s 6 d^{1} D_{2}$ & $9.1855 \mathrm{E}+00$ & $3.883 \mathrm{E}-01$ & $6.139 \mathrm{E}+12$ & $9.1467 \mathrm{E}+00$ & $3.889 \mathrm{E}-01$ & $6.149 \mathrm{E}+12$ & 0.001 \\
\hline $1 s 4 f^{3} F_{3}^{o}$ & $1 s 6 d^{1} D_{2}$ & $9.2884 \mathrm{E}+00$ & $4.663 \mathrm{E}-05$ & $7.211 \mathrm{E}+08$ & $9.2491 \mathrm{E}+00$ & $5.670 \mathrm{E}-05$ & $8.767 E+08$ & 0.000 \\
\hline $1 s 4 f^{3} F_{2}^{o}$ & $1 s 6 d^{1} D_{2}$ & $9.2907 \mathrm{E}+00$ & $4.915 \mathrm{E}-04$ & $7.596 \mathrm{E}+09$ & $9.2513 \mathrm{E}+00$ & $4.896 \mathrm{E}-04$ & $7.566 \mathrm{E}+09$ & 0.000 \\
\hline $1 s 4 f^{1} F_{3}^{o}$ & $1 s 6 d^{1} D_{2}$ & $9.3432 \mathrm{E}+00$ & $1.150 \mathrm{E}-02$ & $1.758 \mathrm{E}+11$ & $9.3035 \mathrm{E}+00$ & $1.152 \mathrm{E}-02$ & $1.761 \mathrm{E}+11$ & 0.000 \\
\hline $1 s 5 p^{3} P_{1}^{o}$ & $1 s 6 d^{1} D_{2}$ & $2.4566 \mathrm{E}+01$ & $1.099 \mathrm{E}-03$ & $2.430 \mathrm{E}+09$ & $2.4460 \mathrm{E}+01$ & $1.147 \mathrm{E}-03$ & $2.538 \mathrm{E}+09$ & 0.003 \\
\hline $1 s 5 p^{3} P_{2}^{o}$ & $1 s 6 d^{1} D_{2}$ & $2.5835 \mathrm{E}+01$ & $1.695 \mathrm{E}-01$ & $3.387 \mathrm{E}+11$ & $2.5723 \mathrm{E}+01$ & $1.700 \mathrm{E}-01$ & $3.399 E+11$ & 0.001 \\
\hline $1 s 5 p{ }^{1} P_{1}^{o}$ & $1 s 6 d^{1} D_{2}$ & $2.5947 \mathrm{E}+01$ & $1.579 \mathrm{E}+00$ & $3.130 \mathrm{E}+12$ & $2.5836 \mathrm{E}+01$ & $1.574 \mathrm{E}+00$ & $3.121 \mathrm{E}+12$ & 0.001 \\
\hline $1 s 5 f^{3} F_{3}^{o}$ & $1 s 6 d{ }^{1} D_{2}$ & $2.6371 \mathrm{E}+01$ & $6.778 \mathrm{E}-04$ & $1.300 \mathrm{E}+09$ & $2.6259 \mathrm{E}+01$ & $8.212 \mathrm{E}-04$ & $1.575 \mathrm{E}+09$ & 0.001 \\
\hline $1 s 5 f^{3} F_{2}^{o}$ & $1 s 6 d^{1} D_{2}$ & $2.6380 \mathrm{E}+01$ & $7.165 \mathrm{E}-03$ & $1.373 \mathrm{E}+10$ & $2.6269 \mathrm{E}+01$ & $7.091 \mathrm{E}-03$ & $1.359 \mathrm{E}+10$ & 0.000 \\
\hline $1 s 5 f^{1} F_{3}^{o}$ & $1 s 6 d^{1} D_{2}$ & $2.6598 \mathrm{E}+01$ & $1.678 \mathrm{E}-01$ & $3.164 \mathrm{E}+11$ & $2.6485 \mathrm{E}+01$ & $1.682 \mathrm{E}-01$ & $3.173 E+11$ & 0.000 \\
\hline $1 s 6 p^{3} P_{1}^{o}$ & $1 s 6 d^{1} D_{2}$ & $6.1807 \mathrm{E}+02$ & $7.142 \mathrm{E}-04$ & $2.494 \mathrm{E}+06$ & $6.1320 \mathrm{E}+02$ & $1.025 \mathrm{E}-03$ & $3.607 \mathrm{E}+06$ & 0.033 \\
\hline $1 s 6 p^{3} P_{2}^{o}$ & $1 s 6 d^{1} D_{2}$ & $2.1556 \mathrm{E}+03$ & $2.532 \mathrm{E}-02$ & $7.269 \mathrm{E}+06$ & $2.1118 \mathrm{E}+03$ & $2.540 \mathrm{E}-02$ & $7.534 \mathrm{E}+06$ & 0.009 \\
\hline $1 s 6 p^{1} P_{1}^{o}$ & $1 s 6 d^{1} D_{2}$ & $2.7210 \mathrm{E}+03$ & $1.836 \mathrm{E}-01$ & $3.308 \mathrm{E}+07$ & $2.5822 E+03$ & $1.928 \mathrm{E}-01$ & $3.826 \mathrm{E}+07$ & 0.017 \\
\hline $1 s 6 f^{3} F_{3}^{o}$ & $1 s 6 d^{1} D_{2}$ & $1.0989 \mathrm{E}+05$ & $2.647 \mathrm{E}-05$ & $2.924 \mathrm{E}+00$ & & & & 0.645 \\
\hline $1 s 6 f^{3} F_{2}^{o}$ & $1 s 6 d^{1} D_{2}$ & $6.7056 \mathrm{E}+05$ & $4.571 \mathrm{E}-05$ & $1.356 \mathrm{E}-01$ & & & & 0.206 \\
\hline $1 s 3 d^{3} D_{3}$ & $1 s 6 f^{3} F_{4}^{o}$ & $3.8744 \mathrm{E}+00$ & $3.435 \mathrm{E}-01$ & $1.696 \mathrm{E}+13$ & $3.8580 \mathrm{E}+00$ & $3.460 \mathrm{E}-01$ & $1.708 \mathrm{E}+13$ & 0.001 \\
\hline $1 s 4 d^{3} D_{3}$ & $1 s 6 f^{3} F_{4}^{o}$ & $9.2719 \mathrm{E}+00$ & $1.185 \mathrm{E}+00$ & $1.022 \mathrm{E}+13$ & $9.2331 \mathrm{E}+00$ & $1.190 \mathrm{E}+00$ & $1.026 \mathrm{E}+13$ & 0.001 \\
\hline $1 s 5 d^{3} D_{3}$ & $1 s 6 f^{3} F_{4}^{0}$ & $2.6244 \mathrm{E}+01$ & $5.292 \mathrm{E}+00$ & $5.695 \mathrm{E}+12$ & $2.6136 \mathrm{E}+01$ & $5.311 \mathrm{E}+00$ & $5.713 \mathrm{E}+12$ & 0.000 \\
\hline $1 s 5 g{ }^{3} G_{4}$ & $1 s 6 f^{3} F_{4}^{o}$ & $2.6470 \mathrm{E}+01$ & $2.307 \mathrm{E}-03$ & $2.440 \mathrm{E}+09$ & $2.6356 \mathrm{E}+01$ & $2.319 \mathrm{E}-03$ & $2.453 \mathrm{E}+09$ & 0.000 \\
\hline $1 s 5 g^{3} G_{3}$ & $1 s 6 f^{3} F_{4}^{0}$ & $2.6475 \mathrm{E}+01$ & $6.552 \mathrm{E}-05$ & $6.928 \mathrm{E}+07$ & & & & 0.002 \\
\hline $1 s 5 g^{3} G_{5}$ & $1 s 6 f^{3} F_{4}^{0}$ & $2.6601 \mathrm{E}+01$ & $8.595 \mathrm{E}-02$ & $9.002 \mathrm{E}+10$ & $2.6486 \mathrm{E}+01$ & $8.606 \mathrm{E}-02$ & $9.015 \mathrm{E}+10$ & 0.000 \\
\hline $1 s 5 g^{1} G_{4}$ & $1 s 6 f^{3} F_{4}^{0}$ & $2.6606 \mathrm{E}+01$ & $1.966 \mathrm{E}-03$ & $2.059 \mathrm{E}+09$ & $2.6491 \mathrm{E}+01$ & $1.963 \mathrm{E}-03$ & $2.056 \mathrm{E}+09$ & 0.002 \\
\hline $1 s 6 d^{3} D_{3}$ & $1 s 6 f^{3} F_{4}^{0}$ & $5.3175 \mathrm{E}+03$ & $1.705 \mathrm{E}-01$ & $4.469 \mathrm{E}+06$ & $5.5117 \mathrm{E}+03$ & $1.637 \mathrm{E}-01$ & $3.962 E+06$ & 0.017 \\
\hline $1 s 4 f^{3} F_{3}^{o}$ & $1 s 6 g^{3} G_{4}$ & $9.2736 \mathrm{E}+00$ & $1.229 \mathrm{E}+00$ & $1.060 \mathrm{E}+13$ & $9.2341 \mathrm{E}+00$ & $1.231 \mathrm{E}+00$ & $1.061 \mathrm{E}+13$ & 0.000 \\
\hline $1 s 4 f^{3} F_{4}^{o}$ & $1 s 6 g{ }^{3} G_{4}$ & $9.3264 \mathrm{E}+00$ & $4.335 \mathrm{E}-02$ & $3.694 \mathrm{E}+11$ & $9.2866 \mathrm{E}+00$ & $4.316 \mathrm{E}-02$ & $3.678 E+11$ & 0.000 \\
\hline $1 s 4 f^{1} F_{3}^{o}$ & $1 s 6 g{ }^{3} G_{4}$ & $9.3281 \mathrm{E}+00$ & $1.220 \mathrm{E}-03$ & $1.039 \mathrm{E}+10$ & $9.2883 \mathrm{E}+00$ & $1.070 \mathrm{E}-03$ & $9.114 \mathrm{E}+09$ & 0.000 \\
\hline 1s $5 f^{3} F_{3}^{0}$ & $1 s 6 g^{3} G_{4}$ & $2.6251 \mathrm{E}+01$ & $7.866 \mathrm{E}+00$ & $8.460 \mathrm{E}+12$ & $2.6139 \mathrm{E}+01$ & $7.880 \mathrm{E}+00$ & $8.475 E+12$ & 0.000 \\
\hline $1 s 5 f^{3} F_{4}^{o}$ & $1 s 6 g{ }^{3} G_{4}$ & $2.6469 \mathrm{E}+01$ & $2.860 \mathrm{E}-01$ & $3.026 \mathrm{E}+11$ & $2.6355 \mathrm{E}+01$ & $2.865 \mathrm{E}-01$ & $3.031 E+11$ & 0.000 \\
\hline $1 s 5 f^{1} F_{3}^{0}$ & $1 s 6 g^{3} G_{4}$ & $2.6476 \mathrm{E}+01$ & $8.129 \mathrm{E}-03$ & $8.594 \mathrm{E}+09$ & $2.6362 \mathrm{E}+01$ & $7.195 \mathrm{E}-03$ & $7.608 \mathrm{E}+09$ & 0.000 \\
\hline $1 s 6 f^{3} F_{3}^{o}$ & $1 s 6 g^{3} G_{4}$ & $5.4970 \mathrm{E}+03$ & $1.224 \mathrm{E}-01$ & $3.001 \mathrm{E}+06$ & $5.5303 \mathrm{E}+03$ & $1.210 \mathrm{E}-01$ & $2.909 \mathrm{E}+06$ & 0.000 \\
\hline $1 s 6 f^{3} F_{4}^{o}$ & $1 s 6 g^{3} G_{4}$ & $2.7996 \mathrm{E}+06$ & $8.576 \mathrm{E}-06$ & $8.111 \mathrm{E}-04$ & & & & 0.475 \\
\hline $1 s 2 p^{3} P_{2}^{o}$ & $1 s 6 g{ }^{3} G_{3}$ & $1.4397 \mathrm{E}+00$ & $2.240 \mathrm{E}-10$ & $1.030 \mathrm{E}+05$ & & & & 0.229 \\
\hline $1 s 3 p^{3} P_{2}^{o}$ & $1 s 6 g{ }^{3} G_{3}$ & $3.8210 \mathrm{E}+00$ & $1.594 \mathrm{E}-12$ & $1.041 \mathrm{E}+02$ & & & & 0.345 \\
\hline $1 s 4 p^{3} P_{2}^{0}$ & $1 s 6 g{ }^{3} G_{3}$ & $9.1429 \mathrm{E}+00$ & $1.067 \mathrm{E}-07$ & $1.217 \mathrm{E}+06$ & & & & 0.002 \\
\hline $1 s 4 f^{3} F_{3}^{o}$ & $1 s 6 g{ }^{3} G_{3}$ & $9.2732 \mathrm{E}+00$ & $4.539 \mathrm{E}-02$ & $5.029 \mathrm{E}+11$ & $9.2341 \mathrm{E}+00$ & $4.543 \mathrm{E}-02$ & $5.033 \mathrm{E}+11$ & 0.000 \\
\hline $1 s 4 f^{3} F_{2}^{o}$ & $1 s 6 g{ }^{3} G_{3}$ & $9.2754 \mathrm{E}+00$ & $9.106 \mathrm{E}-01$ & $1.009 \mathrm{E}+13$ & $9.2363 \mathrm{E}+00$ & $9.117 \mathrm{E}-01$ & $1.009 \mathrm{E}+13$ & 0.000 \\
\hline $1 s 4 f^{3} F_{4}^{o}$ & $1 s 6 g^{3} G_{3}$ & $9.3261 \mathrm{E}+00$ & $1.243 \mathrm{E}-03$ & $1.362 \mathrm{E}+10$ & $9.2866 \mathrm{E}+00$ & $1.233 \mathrm{E}-03$ & $1.351 \mathrm{E}+10$ & 0.001 \\
\hline $1 s 4 f^{1} F_{3}^{o}$ & $1 s 6 g{ }^{3} G_{3}$ & $9.3277 \mathrm{E}+00$ & $3.373 \mathrm{E}-02$ & $3.694 \mathrm{E}+11$ & $9.2883 \mathrm{E}+00$ & $3.346 \mathrm{E}-02$ & $3.664 \mathrm{E}+11$ & 0.001 \\
\hline
\end{tabular}


Table 3 (continued)

\begin{tabular}{|c|c|c|c|c|c|c|c|c|}
\hline \multirow[t]{2}{*}{ Lower } & \multirow[t]{2}{*}{ Upper } & \multicolumn{3}{|l|}{ GRASP2K } & \multicolumn{3}{|l|}{ FAC } & \multirow[t]{2}{*}{$d T$} \\
\hline & & $\lambda(\AA)$ & $g f$ & $A\left(\mathrm{~s}^{-1}\right)$ & $\lambda(\AA)$ & $g f$ & $A\left(\mathrm{~s}^{-1}\right)$ & \\
\hline $1 s 5 p^{3} P_{2}^{o}$ & $1 s 6 g^{3} G_{3}$ & $2.5717 \mathrm{E}+01$ & $1.070 \mathrm{E}-06$ & $1.542 \mathrm{E}+06$ & & & & 0.001 \\
\hline $1 s 5 f^{3} F_{3}^{0}$ & $1 s 6 g^{3} G_{3}$ & $2.6248 \mathrm{E}+01$ & $2.902 \mathrm{E}-01$ & $4.014 \mathrm{E}+11$ & $2.6139 \mathrm{E}+01$ & $2.908 \mathrm{E}-01$ & $4.021 \mathrm{E}+11$ & 0.000 \\
\hline $1 s 5 f^{3} F_{2}^{0}$ & $1 s 6 g^{3} G_{3}$ & $2.6257 \mathrm{E}+01$ & $5.828 \mathrm{E}+00$ & $8.055 \mathrm{E}+12$ & $2.6148 \mathrm{E}+01$ & $5.834 \mathrm{E}+00$ & $8.062 \mathrm{E}+12$ & 0.000 \\
\hline $1 s 5 f^{3} F_{4}^{0}$ & $1 s 6 g^{3} G_{3}$ & $2.6466 \mathrm{E}+01$ & $8.195 \mathrm{E}-03$ & $1.115 \mathrm{E}+10$ & $2.6355 \mathrm{E}+01$ & $8.187 \mathrm{E}-03$ & $1.113 \mathrm{E}+10$ & 0.001 \\
\hline $1 s 5 f^{1} F_{3}^{o}$ & $1 s 6 g^{3} G_{3}$ & $2.6473 \mathrm{E}+01$ & $2.225 \mathrm{E}-01$ & $3.025 E+11$ & $2.6362 \mathrm{E}+01$ & $2.220 \mathrm{E}-01$ & $3.018 \mathrm{E}+11$ & 0.000 \\
\hline $1 s 6 p^{3} P_{2}^{0}$ & $1 s 6 g{ }^{3} G_{3}$ & $1.5598 \mathrm{E}+03$ & $7.276 \mathrm{E}-08$ & $2.849 \mathrm{E}+01$ & & & & 0.044 \\
\hline $1 s 6 f^{3} F_{3}^{o}$ & $1 s 6 g^{3} G_{3}$ & $5.3682 \mathrm{E}+03$ & $4.626 \mathrm{E}-03$ & $1.530 \mathrm{E}+05$ & $5.5303 E+03$ & $4.489 \mathrm{E}-03$ & $1.386 \mathrm{E}+05$ & 0.028 \\
\hline $1 s 6 f^{3} F_{2}^{o}$ & $1 s 6 g^{3} G_{3}$ & $5.5968 \mathrm{E}+03$ & $8.901 \mathrm{E}-02$ & $2.708 \mathrm{E}+06$ & $5.5277 \mathrm{E}+03$ & $8.974 \mathrm{E}-02$ & $2.774 \mathrm{E}+06$ & 0.024 \\
\hline $1 s 6 f^{3} F_{4}^{0}$ & $1 s 6 g^{3} G_{3}$ & $2.1175 \mathrm{E}+05$ & $3.244 \mathrm{E}-06$ & $6.894 \mathrm{E}-02$ & & & & 0.774 \\
\hline $1 s 3 d^{3} D_{2}$ & $1 s 6 f^{1} F_{3}^{o}$ & $3.8309 \mathrm{E}+00$ & $7.118 \mathrm{E}-06$ & $4.622 \mathrm{E}+08$ & & & & 0.014 \\
\hline $1 s 3 d^{3} D_{3}$ & $1 s 6 f^{1} F_{3}^{o}$ & $3.8743 E+00$ & $1.279 \mathrm{E}-02$ & $8.119 E+11$ & $3.8580 \mathrm{E}+00$ & $1.279 \mathrm{E}-02$ & $8.125 E+11$ & 0.000 \\
\hline $1 s 3 d^{1} D_{2}$ & $1 s 6 f^{1} F_{3}^{o}$ & $3.8760 \mathrm{E}+00$ & $2.543 \mathrm{E}-01$ & $1.613 \mathrm{E}+13$ & $3.8597 \mathrm{E}+00$ & $2.562 \mathrm{E}-01$ & $1.625 \mathrm{E}+13$ & 0.000 \\
\hline $1 s 4 d^{3} D_{2}$ & $1 s 6 f^{1} F_{3}^{o}$ & $9.1664 \mathrm{E}+00$ & $4.419 \mathrm{E}-05$ & $5.012 \mathrm{E}+08$ & & & & 0.009 \\
\hline $1 s 4 d^{3} D_{3}$ & $1 s 6 f^{1} F_{3}^{o}$ & $9.2714 \mathrm{E}+00$ & $4.411 \mathrm{E}-02$ & $4.890 \mathrm{E}+11$ & $9.2331 \mathrm{E}+00$ & $4.404 \mathrm{E}-02$ & $4.880 \mathrm{E}+11$ & 0.000 \\
\hline $1 s 4 d^{1} D_{2}$ & $1 s 6 f^{1} F_{3}^{o}$ & $9.2758 \mathrm{E}+00$ & $8.782 \mathrm{E}-01$ & $9.726 \mathrm{E}+12$ & $9.2375 E+00$ & $8.816 \mathrm{E}-01$ & $9.761 \mathrm{E}+12$ & 0.000 \\
\hline $1 s 5 d^{3} D_{2}$ & $1 s 6 f^{1} F_{3}^{o}$ & $2.5812 \mathrm{E}+01$ & $2.352 \mathrm{E}-04$ & $3.364 \mathrm{E}+08$ & & & & 0.006 \\
\hline $1 s 5 d^{3} D_{3}$ & $1 s 6 f^{1} F_{3}^{o}$ & $2.6240 \mathrm{E}+01$ & $1.968 \mathrm{E}-01$ & $2.723 \mathrm{E}+11$ & $2.6136 \mathrm{E}+01$ & $1.964 \mathrm{E}-01$ & $2.716 \mathrm{E}+11$ & 0.000 \\
\hline $1 s 5 d^{1} D_{2}$ & $1 s 6 f^{1} F_{3}^{o}$ & $2.6259 \mathrm{E}+01$ & $3.925 \mathrm{E}+00$ & $5.424 \mathrm{E}+12$ & $2.6155 \mathrm{E}+01$ & $3.931 E+00$ & $5.429 \mathrm{E}+12$ & 0.000 \\
\hline $1 s 5 g{ }^{3} G_{4}$ & $1 s 6 f{ }^{1} F_{3}^{o}$ & $2.6466 \mathrm{E}+01$ & $6.562 \mathrm{E}-05$ & $8.927 E+07$ & & & & 0.004 \\
\hline $1 s 5 g^{3} G_{3}$ & $1 s 6 f^{1} F_{3}^{o}$ & $2.6471 \mathrm{E}+01$ & $1.795 \mathrm{E}-03$ & $2.440 \mathrm{E}+09$ & $2.6361 \mathrm{E}+01$ & $1.792 \mathrm{E}-03$ & $2.437 E+09$ & 0.002 \\
\hline $1 s 5 g^{1} G_{4}$ & $1 s 6 f^{1} F_{3}^{o}$ & $2.6601 \mathrm{E}+01$ & $6.833 \mathrm{E}-02$ & $9.201 \mathrm{E}+10$ & $2.6490 \mathrm{E}+01$ & $6.844 \mathrm{E}-02$ & $9.214 \mathrm{E}+10$ & 0.001 \\
\hline $1 s 6 d^{3} D_{2}$ & $1 s 6 f^{1} F_{3}^{o}$ & $1.7871 \mathrm{E}+03$ & $2.665 \mathrm{E}-05$ & $7.950 \mathrm{E}+03$ & $1.8323 \mathrm{E}+03$ & $4.615 \mathrm{E}-05$ & $1.298 \mathrm{E}+04$ & 0.111 \\
\hline $1 s 6 d^{3} D_{3}$ & $1 s 6 f^{1} F_{3}^{o}$ & $5.1604 \mathrm{E}+03$ & $6.538 \mathrm{E}-03$ & $2.340 \mathrm{E}+05$ & $5.5079 \mathrm{E}+03$ & $6.062 \mathrm{E}-03$ & $1.888 \mathrm{E}+05$ & 0.009 \\
\hline $1 s 6 d^{1} D_{2}$ & $1 s 6 f^{1} F_{3}^{o}$ & $5.6121 \mathrm{E}+03$ & $1.196 \mathrm{E}-01$ & $3.619 E+06$ & $5.6823 E+03$ & $1.176 \mathrm{E}-01$ & $3.443 \mathrm{E}+06$ & 0.002 \\
\hline $1 s 6 g{ }^{3} G_{4}$ & $1 s 6 f^{1} F_{3}^{o}$ & $1.8628 \mathrm{E}+05$ & $3.680 \mathrm{E}-06$ & $1.011 \mathrm{E}-01$ & & & & 0.917 \\
\hline $1 s 6 g^{3} G_{3}$ & $1 s 6 f^{1} F_{3}^{o}$ & $9.9701 \mathrm{E}+05$ & $1.872 \mathrm{E}-05$ & $1.795 \mathrm{E}-02$ & & & & 0.869 \\
\hline $1 s 4 f^{3} F_{4}^{o}$ & $1 s 6 g^{3} G_{5}$ & $9.3170 \mathrm{E}+00$ & $1.552 \mathrm{E}+00$ & $1.084 \mathrm{E}+13$ & $9.2773 \mathrm{E}+00$ & $1.558 \mathrm{E}+00$ & $1.088 \mathrm{E}+13$ & 0.000 \\
\hline $1 s 5 f^{3} F_{4}^{0}$ & $1 s 6 g^{3} G_{5}$ & $2.6394 \mathrm{E}+01$ & $1.003 \mathrm{E}+01$ & $8.734 \mathrm{E}+12$ & $2.6281 \mathrm{E}+01$ & $1.006 \mathrm{E}+01$ & $8.758 \mathrm{E}+12$ & 0.000 \\
\hline $1 s 6 f^{3} F_{4}^{0}$ & $1 s 6 g^{3} G_{5}$ & $9.2152 \mathrm{E}+03$ & $9.257 \mathrm{E}-02$ & $6.610 \mathrm{E}+05$ & $9.2775 E+03$ & $9.155 \mathrm{E}-02$ & $6.395 \mathrm{E}+05$ & 0.002 \\
\hline $1 s 5 g^{3} G_{4}$ & $1 s 6 h^{3} H_{5}^{o}$ & $2.6394 \mathrm{E}+01$ & $1.465 \mathrm{E}+01$ & $1.275 \mathrm{E}+13$ & $2.6281 \mathrm{E}+01$ & $1.467 \mathrm{E}+01$ & $1.277 \mathrm{E}+13$ & 0.000 \\
\hline $1 s 5 g^{3} G_{5}$ & $1 s 6 h^{3} H_{5}^{o}$ & $2.6525 E+01$ & $3.263 \mathrm{E}-01$ & $2.812 \mathrm{E}+11$ & $2.6411 \mathrm{E}+01$ & $3.264 \mathrm{E}-01$ & $2.813 \mathrm{E}+11$ & 0.000 \\
\hline $1 s 5 g^{1} G_{4}$ & $1 s 6 h^{3} H_{5}^{o}$ & $2.6529 \mathrm{E}+01$ & $5.862 \mathrm{E}-03$ & $5.051 \mathrm{E}+09$ & $2.6415 \mathrm{E}+01$ & $4.968 \mathrm{E}-03$ & $4.281 \mathrm{E}+09$ & 0.001 \\
\hline $1 s 6 g^{3} G_{4}$ & $1 s 6 h^{3} H_{5}^{o}$ & $9.2359 \mathrm{E}+03$ & $5.084 \mathrm{E}-02$ & $3.614 \mathrm{E}+05$ & $9.2564 \mathrm{E}+03$ & $5.050 \mathrm{E}-02$ & $3.544 \mathrm{E}+05$ & 0.018 \\
\hline $1 s 6 g^{3} G_{5}$ & $1 s 6 h^{3} H_{5}^{o}$ & $8.7873 \mathrm{E}+06$ & $1.183 \mathrm{E}-06$ & $9.290 \mathrm{E}-06$ & & & & 0.948 \\
\hline $1 s 3 d^{3} D_{3}$ & $1 s 6 h^{3} H_{4}^{o}$ & $3.8727 \mathrm{E}+00$ & $3.209 \mathrm{E}-12$ & $1.586 \mathrm{E}+02$ & & & & 0.851 \\
\hline $1 s 4 d^{3} D_{3}$ & $1 s 6 h^{3} H_{4}^{0}$ & $9.2624 \mathrm{E}+00$ & $6.140 \mathrm{E}-11$ & $5.305 E+02$ & & & & 0.185 \\
\hline $1 s 5 d^{3} D_{3}$ & $1 s 6 h^{3} H_{4}^{0}$ & $2.6168 \mathrm{E}+01$ & $4.788 \mathrm{E}-09$ & $5.183 \mathrm{E}+03$ & & & & 0.002 \\
\hline $1 s 5 g{ }^{3} G_{4}$ & $1 s 6 h^{3} H_{4}^{0}$ & $2.6392 \mathrm{E}+01$ & $3.318 \mathrm{E}-01$ & $3.530 \mathrm{E}+11$ & $2.6281 \mathrm{E}+01$ & $3.323 E-01$ & $3.536 \mathrm{E}+11$ & 0.000 \\
\hline $1 s 5 g^{3} G_{3}$ & $1 s 6 h^{3} H_{4}^{o}$ & $2.6397 \mathrm{E}+01$ & $1.165 \mathrm{E}+01$ & $1.239 \mathrm{E}+13$ & $2.6286 \mathrm{E}+01$ & $1.166 \mathrm{E}+01$ & $1.241 \mathrm{E}+13$ & 0.000 \\
\hline $1 s 5 g^{3} G_{5}$ & $1 s 6 h^{3} H_{4}^{0}$ & $2.6523 \mathrm{E}+01$ & $6.061 \mathrm{E}-03$ & $6.385 E+09$ & $2.6411 \mathrm{E}+01$ & $6.044 \mathrm{E}-03$ & $6.367 \mathrm{E}+09$ & 0.001 \\
\hline $1 s 5 g^{1} G_{4}$ & $1 s 6 h^{3} H_{4}^{0}$ & $2.6527 \mathrm{E}+01$ & $2.678 \mathrm{E}-01$ & $2.820 \mathrm{E}+11$ & $2.6415 E+01$ & $2.670 \mathrm{E}-01$ & $2.812 \mathrm{E}+11$ & 0.000 \\
\hline $1 s 6 d^{3} D_{3}$ & $1 s 6 h^{3} H_{4}^{0}$ & $3.3388 \mathrm{E}+03$ & $1.016 \mathrm{E}-10$ & $6.754 \mathrm{E}-03$ & & & & 0.436 \\
\hline $1 s 6 g{ }^{3} G_{4}$ & $1 s 6 h^{3} H_{4}^{0}$ & $9.0013 E+03$ & $1.182 \mathrm{E}-03$ & $1.081 \mathrm{E}+04$ & $9.2564 \mathrm{E}+03$ & $1.147 \mathrm{E}-03$ & $9.845 \mathrm{E}+03$ & 0.004 \\
\hline $1 s 6 g{ }^{3} G_{3}$ & $1 s 6 h^{3} H_{4}^{0}$ & $9.3695 \mathrm{E}+03$ & $3.986 \mathrm{E}-02$ & $3.365 E+05$ & $9.2564 \mathrm{E}+03$ & $4.017 \mathrm{E}-02$ & $3.445 \mathrm{E}+05$ & 0.012 \\
\hline $1 s 6 g^{3} G_{5}$ & $1 s 6 h^{3} H_{4}^{0}$ & $3.4069 \mathrm{E}+05$ & $5.670 \mathrm{E}-07$ & $3.620 \mathrm{E}-03$ & & & & 0.752 \\
\hline $1 s 4 f^{3} F_{3}^{o}$ & $1 s 6 g^{1} G_{4}$ & $9.2640 \mathrm{E}+00$ & $1.114 \mathrm{E}-07$ & $9.625 \mathrm{E}+05$ & & & & 0.014 \\
\hline $1 s 4 f^{3} F_{4}^{0}$ & $1 s 6 g^{1} G_{4}$ & $9.3167 \mathrm{E}+00$ & $3.545 \mathrm{E}-02$ & $3.027 \mathrm{E}+11$ & $9.2773 \mathrm{E}+00$ & $3.542 \mathrm{E}-02$ & $3.024 \mathrm{E}+11$ & 0.000 \\
\hline $1 s 4 f^{1} F_{3}^{0}$ & $1 s 6 g^{1} G_{4}$ & $9.3184 \mathrm{E}+00$ & $1.235 \mathrm{E}+00$ & $1.054 \mathrm{E}+13$ & $9.2790 \mathrm{E}+00$ & $1.239 \mathrm{E}+00$ & $1.057 \mathrm{E}+13$ & 0.000 \\
\hline $1 s 5 f^{3} F_{3}^{0}$ & $1 s 6 g^{1} G_{4}$ & $2.6175 E+01$ & $1.994 \mathrm{E}-07$ & $2.157 \mathrm{E}+05$ & & & & 0.031 \\
\hline $1 s 5 f^{3} F_{4}^{0}$ & $1 \mathrm{~s} 6 g^{1} G_{4}$ & $2.6391 \mathrm{E}+01$ & $2.290 \mathrm{E}-01$ & $2.437 \mathrm{E}+11$ & $2.6281 \mathrm{E}+01$ & $2.286 \mathrm{E}-01$ & $2.432 \mathrm{E}+11$ & 0.000 \\
\hline $1 s 5 f^{1} F_{3}^{0}$ & $1 s 6 g{ }^{1} G_{4}$ & $2.6398 \mathrm{E}+01$ & $7.983 \mathrm{E}+00$ & $8.490 \mathrm{E}+12$ & $2.6287 \mathrm{E}+01$ & $8.001 E+00$ & $8.508 \mathrm{E}+12$ & 0.000 \\
\hline $1 s 6 f{ }^{3} F_{3}^{o}$ & $1 s 6 g^{1} G_{4}$ & $3.4068 \mathrm{E}+03$ & $1.222 \mathrm{E}-09$ & $7.804 \mathrm{E}-02$ & & & & 0.960 \\
\hline $1 s 6 f^{3} F_{4}^{0}$ & $1 s 6 g^{1} G_{4}$ & $8.9311 \mathrm{E}+03$ & $2.180 \mathrm{E}-03$ & $2.026 \mathrm{E}+04$ & $9.2773 \mathrm{E}+03$ & $2.080 \mathrm{E}-03$ & $1.776 \mathrm{E}+04$ & 0.042 \\
\hline $1 s 6 f^{1} F_{3}^{o}$ & $1 \mathrm{~s} 6 g^{1} G_{4}$ & $9.4124 \mathrm{E}+03$ & $7.208 \mathrm{E}-02$ & $6.030 \mathrm{E}+05$ & $9.2882 \mathrm{E}+03$ & $7.274 \mathrm{E}-02$ & $6.196 \mathrm{E}+05$ & 0.031 \\
\hline $1 s 6 h^{3} H_{5}^{o}$ & $1 s 6 g^{1} G_{4}$ & $2.9962 \mathrm{E}+05$ & $6.236 \mathrm{E}-07$ & $5.149 \mathrm{E}-03$ & & & & 0.526 \\
\hline $1 s 6 h^{3} H_{4}^{0}$ & $1 s 6 g^{1} G_{4}$ & $1.9372 \mathrm{E}+06$ & $4.406 \mathrm{E}-06$ & $8.701 \mathrm{E}-04$ & & & & 0.711 \\
\hline $1 s 5 g^{3} G_{5}$ & $1 s 6 h^{3} H_{6}^{o}$ & $2.6474 \mathrm{E}+01$ & $1.773 \mathrm{E}+01$ & $1.298 \mathrm{E}+13$ & $2.6361 \mathrm{E}+01$ & $1.777 \mathrm{E}+01$ & $1.301 \mathrm{E}+13$ & 0.000 \\
\hline $1 s 6 g^{3} G_{5}$ & $1 s 6 h^{3} H_{6}^{o}$ & $1.3897 \mathrm{E}+04$ & $4.086 \mathrm{E}-02$ & $1.085 \mathrm{E}+05$ & $1.3933 \mathrm{E}+04$ & $4.057 \mathrm{E}-02$ & $1.063 \mathrm{E}+05$ & 0.026 \\
\hline $1 s 5 g^{3} G_{4}$ & $1 s 6 h^{1} H_{5}^{o}$ & $2.6342 \mathrm{E}+01$ & $1.711 \mathrm{E}-06$ & $1.496 \mathrm{E}+06$ & & & & 0.001 \\
\hline $1 s 5 g^{3} G_{5}$ & $1 s 6 h^{1} H_{5}^{o}$ & $2.6473 \mathrm{E}+01$ & $2.737 \mathrm{E}-01$ & $2.369 \mathrm{E}+11$ & $2.6361 \mathrm{E}+01$ & $2.735 \mathrm{E}-01$ & $2.366 \mathrm{E}+11$ & 0.000 \\
\hline $1 s 5 g^{1} G_{4}$ & $1 s 6 h^{1} H_{5}^{o}$ & $2.6477 \mathrm{E}+01$ & $1.473 \mathrm{E}+01$ & $1.274 \mathrm{E}+13$ & $2.6365 E+01$ & $1.476 \mathrm{E}+01$ & $1.277 \mathrm{E}+13$ & 0.000 \\
\hline $1 s 6 g^{3} G_{4}$ & $1 s 6 h^{1} H_{5}^{o}$ & $5.4809 \mathrm{E}+03$ & $9.930 \mathrm{E}-09$ & $2.004 \mathrm{E}-01$ & & & & 0.195 \\
\hline $1 s 6 g{ }^{3} G_{5}$ & $1 s 6 h{ }^{1} H_{5}^{o}$ & $1.3461 \mathrm{E}+04$ & $6.513 \mathrm{E}-04$ & $2.180 \mathrm{E}+03$ & $1.3933 \mathrm{E}+04$ & $6.242 \mathrm{E}-04$ & $1.933 \mathrm{E}+03$ & 0.002 \\
\hline $1 s 6 g^{1} G_{4}$ & $1 s 6 h^{1} H_{5}^{o}$ & $1.4116 \mathrm{E}+04$ & $3.341 \mathrm{E}-02$ & $1.017 \mathrm{E}+05$ & $1.3934 \mathrm{E}+04$ & $3.370 \mathrm{E}-02$ & $1.043 \mathrm{E}+05$ & 0.019 \\
\hline $1 s 2 p^{3} P_{1}^{o}$ & $1 s 7 s^{3} S_{1}$ & $1.3378 \mathrm{E}+00$ & $7.720 \mathrm{E}-04$ & $9.590 \mathrm{E}+11$ & $1.3323 \mathrm{E}+00$ & $8.087 \mathrm{E}-04$ & $1.004 \mathrm{E}+12$ & 0.015 \\
\hline $1 s 2 p^{3} P_{0}^{0}$ & $1 s 7 s^{3} S_{1}$ & $1.3387 \mathrm{E}+00$ & $3.810 \mathrm{E}-04$ & $4.727 \mathrm{E}+11$ & $1.3331 \mathrm{E}+00$ & $3.785 \mathrm{E}-04$ & $4.696 \mathrm{E}+11$ & 0.001 \\
\hline $1 s 2 p^{3} P_{2}^{0}$ & $1 s 7 s^{3} S_{1}$ & $1.3964 \mathrm{E}+00$ & $2.525 \mathrm{E}-03$ & $2.879 \mathrm{E}+12$ & $1.3905 \mathrm{E}+00$ & $2.566 \mathrm{E}-03$ & $2.926 \mathrm{E}+12$ & 0.019 \\
\hline $1 s 2 p{ }^{1} P_{1}^{o}$ & $1 s 7 s^{3} S_{1}$ & $1.4019 \mathrm{E}+00$ & $4.638 \mathrm{E}-04$ & $5.247 \mathrm{E}+11$ & $1.3961 \mathrm{E}+00$ & $4.626 \mathrm{E}-04$ & $5.232 \mathrm{E}+11$ & 0.004 \\
\hline $1 s 3 p^{3} P_{1}^{0}$ & $1 s 7 s^{3} S_{1}$ & $3.4180 \mathrm{E}+00$ & $3.432 \mathrm{E}-03$ & $6.531 \mathrm{E}+11$ & $3.4039 E+00$ & $3.453 E-03$ & $6.570 \mathrm{E}+11$ & 0.006 \\
\hline $1 s 3 p^{3} P_{0}^{0}$ & $1 s 7 s^{3} S_{1}$ & $3.4195 \mathrm{E}+00$ & $1.667 \mathrm{E}-03$ & $3.171 \mathrm{E}+11$ & $3.4054 \mathrm{E}+00$ & $1.625 \mathrm{E}-03$ & $3.089 \mathrm{E}+11$ & 0.006 \\
\hline $1 s 3 p^{3} P_{2}^{o}$ & $1 s 7 s^{3} S_{1}$ & $3.5305 \mathrm{E}+00$ & $1.085 \mathrm{E}-02$ & $1.935 E+12$ & $3.5159 \mathrm{E}+00$ & $1.077 \mathrm{E}-02$ & $1.922 \mathrm{E}+12$ & 0.004 \\
\hline
\end{tabular}


Table 3 (continued)

\begin{tabular}{|c|c|c|c|c|c|c|c|c|}
\hline \multirow[t]{2}{*}{ Lower } & \multirow[t]{2}{*}{ Upper } & \multicolumn{3}{|l|}{ GRASP2K } & \multicolumn{3}{|l|}{ FAC } & \multirow[t]{2}{*}{$d T$} \\
\hline & & $\lambda(\AA)$ & $g f$ & $A\left(\mathrm{~s}^{-1}\right)$ & $\lambda(\AA)$ & $g f$ & $A\left(\mathrm{~s}^{-1}\right)$ & \\
\hline $1 s 3 p^{1} P_{1}^{o}$ & $1 s 7 s^{3} S_{1}$ & $3.5405 E+00$ & $1.974 \mathrm{E}-03$ & $3.501 \mathrm{E}+11$ & $3.5260 \mathrm{E}+00$ & $1.955 \mathrm{E}-03$ & $3.467 \mathrm{E}+11$ & 0.005 \\
\hline $1 s 4 p^{3} P_{1}^{o}$ & $1 s 7 s^{3} S_{1}$ & $7.4166 \mathrm{E}+00$ & $1.104 \mathrm{E}-02$ & $4.464 \mathrm{E}+11$ & $7.3859 \mathrm{E}+00$ & $1.108 \mathrm{E}-02$ & $4.478 \mathrm{E}+11$ & 0.004 \\
\hline $1 s 4 p^{3} P_{0}^{o}$ & $1 s 7 s^{3} S_{1}$ & $7.4194 \mathrm{E}+00$ & $5.355 \mathrm{E}-03$ & $2.163 E+11$ & $7.3886 \mathrm{E}+00$ & $5.225 \mathrm{E}-03$ & $2.110 \mathrm{E}+11$ & 0.004 \\
\hline $1 s 4 p^{3} P_{2}^{o}$ & $1 s 7 s^{3} S_{1}$ & $7.6388 \mathrm{E}+00$ & $3.445 \mathrm{E}-02$ & $1.313 E+12$ & $7.6070 \mathrm{E}+00$ & $3.431 \mathrm{E}-02$ & $1.307 \mathrm{E}+12$ & 0.004 \\
\hline $1 s 4 p^{1} P_{1}^{o}$ & $1 s 7 s^{3} S_{1}$ & $7.6581 \mathrm{E}+00$ & $6.277 \mathrm{E}-03$ & $2.380 \mathrm{E}+11$ & $7.6265 \mathrm{E}+00$ & $6.235 \mathrm{E}-03$ & $2.363 E+11$ & 0.004 \\
\hline $1 s 4 f^{3} F_{2}^{o}$ & $1 s 7 s^{3} S_{1}$ & $7.7310 \mathrm{E}+00$ & $1.468 \mathrm{E}-09$ & $5.461 \mathrm{E}+04$ & & & & 0.174 \\
\hline $1 s 5 p^{3} P_{1}^{o}$ & $1 s 7 s^{3} S_{1}$ & $1.6020 \mathrm{E}+01$ & $3.789 \mathrm{E}-02$ & $3.283 E+11$ & $1.5955 \mathrm{E}+01$ & $3.806 \mathrm{E}-02$ & $3.296 \mathrm{E}+11$ & 0.001 \\
\hline $1 s 5 p^{3} P_{0}^{o}$ & $1 s 7 s^{3} S_{1}$ & $1.6027 \mathrm{E}+01$ & $1.835 \mathrm{E}-02$ & $1.589 \mathrm{E}+11$ & $1.5961 \mathrm{E}+01$ & $1.794 \mathrm{E}-02$ & $1.553 \mathrm{E}+11$ & 0.001 \\
\hline $1 s 5 p^{3} P_{2}^{o}$ & $1 s 7 s^{3} S_{1}$ & $1.6551 \mathrm{E}+01$ & $1.189 \mathrm{E}-01$ & $9.653 E+11$ & $1.6483 \mathrm{E}+01$ & $1.187 \mathrm{E}-01$ & $9.639 \mathrm{E}+11$ & 0.001 \\
\hline $1 s 5 p^{1} P_{1}^{o}$ & $1 s 7 s^{3} S_{1}$ & $1.6597 \mathrm{E}+01$ & $2.172 \mathrm{E}-02$ & $1.753 E+11$ & $1.6529 \mathrm{E}+01$ & $2.161 \mathrm{E}-02$ & $1.744 \mathrm{E}+11$ & 0.000 \\
\hline $1 s 5 f^{3} F_{2}^{o}$ & $1 s 7 s^{3} S_{1}$ & $1.6773 \mathrm{E}+01$ & $1.742 \mathrm{E}-08$ & $1.377 \mathrm{E}+05$ & & & & 0.145 \\
\hline $1 s 6 p^{3} P_{1}^{o}$ & $1 s 7 s^{3} S_{1}$ & $4.2860 \mathrm{E}+01$ & $2.134 \mathrm{E}-01$ & $2.582 \mathrm{E}+11$ & $4.2690 \mathrm{E}+01$ & $2.157 \mathrm{E}-01$ & $2.610 \mathrm{E}+11$ & 0.005 \\
\hline $1 s 6 p^{3} P_{0}^{o}$ & $1 s 7 s^{3} S_{1}$ & $4.2887 \mathrm{E}+01$ & $1.033 \mathrm{E}-01$ & $1.249 \mathrm{E}+11$ & $4.2622 \mathrm{E}+01$ & $1.000 \mathrm{E}-01$ & $1.214 \mathrm{E}+11$ & 0.006 \\
\hline $1 s 6 p^{3} P_{2}^{o}$ & $1 s 7 s^{3} S_{1}$ & $4.5091 \mathrm{E}+01$ & $7.068 \mathrm{E}-01$ & $7.730 \mathrm{E}+11$ & $4.4909 \mathrm{E}+01$ & $7.019 \mathrm{E}-01$ & $7.672 E+11$ & 0.005 \\
\hline $1 s 6 p^{1} P_{1}^{o}$ & $1 s 7 s^{3} S_{1}$ & $4.5287 \mathrm{E}+01$ & $1.298 \mathrm{E}-01$ & $1.408 \mathrm{E}+11$ & $4.5084 \mathrm{E}+01$ & $1.247 \mathrm{E}-01$ & $1.352 \mathrm{E}+11$ & 0.004 \\
\hline $1 s 6 f^{3} F_{2}^{o}$ & $1 s 7 s^{3} S_{1}$ & $4.6051 \mathrm{E}+01$ & $2.376 \mathrm{E}-07$ & $2.491 \mathrm{E}+05$ & & & & 0.119 \\
\hline $1 s^{2}{ }^{1} s_{0}$ & $1 s 7 p^{3} P_{1}^{o} 8$ & $3.1400 \mathrm{E}-01$ & $2.473 \mathrm{E}-03$ & $5.575 E+13$ & $3.1277 \mathrm{E}-01$ & $2.233 \mathrm{E}-03$ & $5.033 \mathrm{E}+13$ & 0.014 \\
\hline $1 s 2 s^{3} S_{1}$ & $1 s 7 p^{3} P_{1}^{o}$ & $1.3266 \mathrm{E}+00$ & $8.485 \mathrm{E}-03$ & $1.072 \mathrm{E}+13$ & $1.3210 \mathrm{E}+00$ & $8.499 \mathrm{E}-03$ & $1.073 \mathrm{E}+13$ & 0.001 \\
\hline $1 s 2 s{ }^{1} S_{0}$ & $1 s 7 p^{3} P_{1}^{o}$ & $1.3387 \mathrm{E}+00$ & $3.675 \mathrm{E}-03$ & $4.559 E+12$ & $1.3333 \mathrm{E}+00$ & $3.455 \mathrm{E}-03$ & $4.285 E+12$ & 0.005 \\
\hline $1 s 3 s^{3} s_{1}$ & $1 s 7 p^{3} P_{1}^{o}$ & $3.3968 \mathrm{E}+00$ & $1.851 \mathrm{E}-02$ & $3.568 \mathrm{E}+12$ & $3.3827 \mathrm{E}+00$ & $1.869 \mathrm{E}-02$ & $3.601 \mathrm{E}+12$ & 0.003 \\
\hline $1 s 3 s{ }^{1} S_{0}$ & $1 s 7 p^{3} P_{1}^{o}$ & $3.4178 \mathrm{E}+00$ & $8.150 \mathrm{E}-03$ & $1.551 \mathrm{E}+12$ & $3.4039 \mathrm{E}+00$ & $7.676 \mathrm{E}-03$ & $1.460 \mathrm{E}+12$ & 0.004 \\
\hline $1 s 3 d^{3} D_{2}$ & $1 s 7 p^{3} P_{1}^{o}$ & $3.5373 \mathrm{E}+00$ & $2.636 \mathrm{E}-03$ & $4.685 E+11$ & $3.5225 \mathrm{E}+00$ & $2.629 \mathrm{E}-03$ & $4.672 \mathrm{E}+11$ & 0.007 \\
\hline $1 s 3 d^{3} D_{1}$ & $1 s 7 p^{3} P_{1}^{o}$ & $3.5388 \mathrm{E}+00$ & $5.820 \mathrm{E}-04$ & $1.033 \mathrm{E}+11$ & $3.5240 \mathrm{E}+00$ & $5.690 \mathrm{E}-04$ & $1.010 \mathrm{E}+11$ & 0.014 \\
\hline $1 s 3 d{ }^{1} D_{2}$ & $1 s 7 p^{3} P_{1}^{o}$ & $3.5757 \mathrm{E}+00$ & $3.300 \mathrm{E}-06$ & $5.739 E+08$ & $3.5607 \mathrm{E}+00$ & $4.032 \mathrm{E}-06$ & $7.011 \mathrm{E}+08$ & 0.058 \\
\hline $1 s 4 s^{3} S_{1}$ & $1 s 7 p^{3} P_{1}^{o}$ & $7.3716 \mathrm{E}+00$ & $4.040 \mathrm{E}-02$ & $1.653 \mathrm{E}+12$ & $7.3412 \mathrm{E}+00$ & $4.095 \mathrm{E}-02$ & $1.675 \mathrm{E}+12$ & 0.005 \\
\hline $1 s 4 s^{1} S_{0}$ & $1 s 7 p^{3} P_{1}^{o}$ & $7.4116 \mathrm{E}+00$ & $1.804 \mathrm{E}-02$ & $7.300 \mathrm{E}+11$ & $7.3814 \mathrm{E}+00$ & $1.691 \mathrm{E}-02$ & $6.845 \mathrm{E}+11$ & 0.013 \\
\hline $1 s 4 d^{3} D_{2}$ & $1 s 7 p^{3} P_{1}^{o}$ & $7.6476 \mathrm{E}+00$ & $1.361 \mathrm{E}-02$ & $5.173 E+11$ & $7.6163 \mathrm{E}+00$ & $1.350 \mathrm{E}-02$ & $5.132 E+11$ & 0.004 \\
\hline $1 s 4 d^{3} D_{1}$ & $1 s 7 p^{3} P_{1}^{o}$ & $7.6504 \mathrm{E}+00$ & $2.969 \mathrm{E}-03$ & $1.128 \mathrm{E}+11$ & $7.6191 \mathrm{E}+00$ & $2.915 \mathrm{E}-03$ & $1.106 \mathrm{E}+11$ & 0.011 \\
\hline $1 s 4 d{ }^{1} D_{2}$ & $1 s 7 p^{3} P_{1}^{o}$ & $7.7236 \mathrm{E}+00$ & $1.514 \mathrm{E}-05$ & $5.643 \mathrm{E}+08$ & $7.6918 \mathrm{E}+00$ & $1.914 \mathrm{E}-05$ & $7.134 \mathrm{E}+08$ & 0.050 \\
\hline $1 s 5 s^{3} S_{1}$ & $1 s 7 p^{3} P_{1}^{o}$ & $1.5898 \mathrm{E}+01$ & $1.045 \mathrm{E}-01$ & $9.197 \mathrm{E}+11$ & $1.5833 \mathrm{E}+01$ & $1.061 \mathrm{E}-01$ & $9.332 \mathrm{E}+11$ & 0.005 \\
\hline $1 s 5 s{ }^{1} s_{0}$ & $1 s 7 p^{3} P_{1}^{o}$ & $1.5991 \mathrm{E}+01$ & $4.695 \mathrm{E}-02$ & $4.082 \mathrm{E}+11$ & $1.5927 \mathrm{E}+01$ & $4.406 \mathrm{E}-02$ & $3.829 \mathrm{E}+11$ & 0.014 \\
\hline $1 s 5 d^{3} D_{2}$ & $1 s 7 p^{3} P_{1}^{o}$ & $1.6554 \mathrm{E}+01$ & $6.096 \mathrm{E}-02$ & $4.946 \mathrm{E}+11$ & $1.6489 \mathrm{E}+01$ & $6.030 \mathrm{E}-02$ & $4.890 \mathrm{E}+11$ & 0.002 \\
\hline $1 s 5 d^{3} D_{1}$ & $1 s 7 p^{3} P_{1}^{o}$ & $1.6561 \mathrm{E}+01$ & $1.320 \mathrm{E}-02$ & $1.070 \mathrm{E}+11$ & $1.6495 \mathrm{E}+01$ & $1.298 \mathrm{E}-02$ & $1.052 \mathrm{E}+11$ & 0.007 \\
\hline $1 s 5 d{ }^{1} D_{2}$ & $1 s 7 p^{3} P_{1}^{o}$ & $1.6737 \mathrm{E}+01$ & $6.527 \mathrm{E}-05$ & $5.181 \mathrm{E}+08$ & $1.6670 \mathrm{E}+01$ & $8.178 \mathrm{E}-05$ & $6.487 \mathrm{E}+08$ & 0.033 \\
\hline $1 s 6 s^{3} S_{1}$ & $1 s 7 p^{3} P_{1}^{o}$ & $4.2260 \mathrm{E}+01$ & $4.475 \mathrm{E}-01$ & $5.571 E+11$ & $4.2057 \mathrm{E}+01$ & $4.571 \mathrm{E}-01$ & $5.697 \mathrm{E}+11$ & 0.006 \\
\hline $1 s 6 s{ }^{1} s_{0}$ & $1 s 7 p^{3} P_{1}^{o}$ & $4.2638 \mathrm{E}+01$ & $2.029 \mathrm{E}-01$ & $2.482 \mathrm{E}+11$ & $4.2377 \mathrm{E}+01$ & $1.912 \mathrm{E}-01$ & $2.347 \mathrm{E}+11$ & 0.009 \\
\hline $1 s 6 d^{3} D_{2}$ & $1 s 7 p^{3} P_{1}^{o}$ & $4.4993 \mathrm{E}+01$ & $4.312 \mathrm{E}-01$ & $4.736 E+11$ & $4.4844 \mathrm{E}+01$ & $4.266 \mathrm{E}-01$ & $4.677 \mathrm{E}+11$ & 0.002 \\
\hline $1 s 6 d^{3} D_{1}$ & $1 s 7 p^{3} P_{1}^{o}$ & $4.5021 \mathrm{E}+01$ & $9.291 \mathrm{E}-02$ & $1.019 \mathrm{E}+11$ & $4.4837 \mathrm{E}+01$ & $9.088 \mathrm{E}-02$ & $9.966 \mathrm{E}+10$ & 0.002 \\
\hline $1 s 6 d{ }^{1} D_{2}$ & $1 s 7 p^{3} P_{1}^{o}$ & $4.5778 \mathrm{E}+01$ & $4.619 \mathrm{E}-04$ & $4.901 \mathrm{E}+08$ & $4.5600 \mathrm{E}+01$ & $3.977 \mathrm{E}-04$ & $4.217 \mathrm{E}+08$ & 0.019 \\
\hline $1 s 7 s^{3} S_{1}$ & $1 s 7 p^{3} P_{1}^{o}$ & $7.6504 \mathrm{E}+03$ & $4.971 \mathrm{E}-02$ & $1.888 \mathrm{E}+06$ & $7.3461 \mathrm{E}+03$ & $5.274 \mathrm{E}-02$ & $2.154 \mathrm{E}+06$ & 0.473 \\
\hline $1 s 2 p^{3} P_{1}^{o}$ & $1 s 7 s^{1} S_{0}$ & $1.3376 \mathrm{E}+00$ & $3.292 \mathrm{E}-04$ & $1.227 \mathrm{E}+12$ & $1.3321 \mathrm{E}+00$ & $3.285 \mathrm{E}-04$ & $1.224 \mathrm{E}+12$ & 0.026 \\
\hline $1 s 2 p^{1} P_{1}^{o}$ & $1 s 7 s^{1} S_{0}$ & $1.4017 \mathrm{E}+00$ & $1.114 \mathrm{E}-03$ & $3.782 E+12$ & $1.3959 \mathrm{E}+00$ & $1.070 \mathrm{E}-03$ & $3.634 \mathrm{E}+12$ & 0.019 \\
\hline $1 s 3 p^{3} P_{1}^{o}$ & $1 s 7 s^{1} S_{0}$ & $3.4164 \mathrm{E}+00$ & $1.425 \mathrm{E}-03$ & $8.144 \mathrm{E}+11$ & $3.4026 \mathrm{E}+00$ & $1.427 \mathrm{E}-03$ & $8.155 E+11$ & 0.006 \\
\hline $1 s 3 p^{1} P_{1}^{o}$ & $1 s 7 s^{1} S_{0}$ & $3.5388 \mathrm{E}+00$ & $4.562 \mathrm{E}-03$ & $2.430 \mathrm{E}+12$ & $3.5246 \mathrm{E}+00$ & $4.493 \mathrm{E}-03$ & $2.392 \mathrm{E}+12$ & 0.001 \\
\hline $1 s 4 p^{3} P_{1}^{o}$ & $1 s 7 s^{1} S_{0}$ & $7.4094 \mathrm{E}+00$ & $4.589 \mathrm{E}-03$ & $5.575 E+11$ & $7.3798 \mathrm{E}+00$ & $4.609 \mathrm{E}-03$ & $5.597 \mathrm{E}+11$ & 0.001 \\
\hline $1 s 4 p^{1} P_{1}^{o}$ & $1 s 7 s^{1} S_{0}$ & $7.6504 \mathrm{E}+00$ & $1.442 \mathrm{E}-02$ & $1.643 \mathrm{E}+12$ & $7.6200 \mathrm{E}+00$ & $1.430 \mathrm{E}-02$ & $1.629 \mathrm{E}+12$ & 0.002 \\
\hline $1 s 5 p^{3} P_{1}^{o}$ & $1 s 7 s^{1} S_{0}$ & $1.5987 \mathrm{E}+01$ & $1.568 \mathrm{E}-02$ & $4.091 \mathrm{E}+11$ & $1.5926 \mathrm{E}+01$ & $1.585 \mathrm{E}-02$ & $4.135 E+11$ & 0.008 \\
\hline $1 s 5 p^{1} P_{1}^{o}$ & $1 s 7 s^{1} S_{0}$ & $1.6561 \mathrm{E}+01$ & $4.949 \mathrm{E}-02$ & $1.204 \mathrm{E}+12$ & $1.6498 \mathrm{E}+01$ & $4.952 \mathrm{E}-02$ & $1.203 \mathrm{E}+12$ & 0.007 \\
\hline $1 s 6 p^{3} P_{1}^{o}$ & $1 s 7 s^{1} S_{0}$ & $4.2620 \mathrm{E}+01$ & $8.694 \mathrm{E}-02$ & $3.193 E+11$ & $4.2488 \mathrm{E}+01$ & $8.468 \mathrm{E}-02$ & $3.102 E+11$ & 0.004 \\
\hline $1 s 6 p^{1} P_{1}^{o}$ & $1 s 7 s^{1} S_{0}$ & $4.5020 \mathrm{E}+01$ & $2.908 \mathrm{E}-01$ & $9.571 \mathrm{E}+11$ & $4.4858 \mathrm{E}+01$ & $2.959 \mathrm{E}-01$ & $9.728 \mathrm{E}+11$ & 0.004 \\
\hline $1 s 7 p^{3} P_{1}^{o}$ & $1 s 7 s^{1} S_{0}$ & $1.5939 \mathrm{E}+06$ & $1.021 \mathrm{E}-04$ & $2.681 \mathrm{E}-01$ & & & & 0.803 \\
\hline $1 s 2 s^{3} S_{1}$ & $1 s 7 p^{3} P_{0}^{o}$ & $1.3265 \mathrm{E}+00$ & $4.036 \mathrm{E}-03$ & $1.530 \mathrm{E}+13$ & $1.3210 \mathrm{E}+00$ & $3.995 \mathrm{E}-03$ & $1.514 \mathrm{E}+13$ & 0.003 \\
\hline $1 s 3 s^{3} s_{1}$ & $1 s 7 p^{3} P_{0}^{0}$ & $3.3967 \mathrm{E}+00$ & $8.794 \mathrm{E}-03$ & $5.084 \mathrm{E}+12$ & $3.3830 \mathrm{E}+00$ & $8.817 \mathrm{E}-03$ & $5.095 \mathrm{E}+12$ & 0.006 \\
\hline $1 s 3 d^{3} D_{1}$ & $1 s 7 p^{3} P_{0}^{o}$ & $3.5387 \mathrm{E}+00$ & $1.077 \mathrm{E}-03$ & $5.739 E+11$ & $3.5243 \mathrm{E}+00$ & $1.057 \mathrm{E}-03$ & $5.628 \mathrm{E}+11$ & 0.014 \\
\hline $1 s 4 s^{3} S_{1}$ & $1 s 7 p^{3} P_{0}^{o}$ & $7.3711 \mathrm{E}+00$ & $1.921 \mathrm{E}-02$ & $2.358 \mathrm{E}+12$ & $7.3425 \mathrm{E}+00$ & $1.936 \mathrm{E}-02$ & $2.376 \mathrm{E}+12$ & 0.007 \\
\hline $1 s 4 d^{3} D_{1}$ & $1 s 7 p^{3} P_{0}^{o}$ & $7.6499 \mathrm{E}+00$ & $5.527 \mathrm{E}-03$ & $6.300 \mathrm{E}+11$ & $7.6205 \mathrm{E}+00$ & $5.438 \mathrm{E}-03$ & $6.193 E+11$ & 0.010 \\
\hline $1 s 5 s^{3} s_{1}$ & $1 s 7 p^{3} P_{0}^{o}$ & $1.5896 \mathrm{E}+01$ & $4.976 \mathrm{E}-02$ & $1.314 \mathrm{E}+12$ & $1.5839 \mathrm{E}+01$ & $5.030 \mathrm{E}-02$ & $1.326 \mathrm{E}+12$ & 0.007 \\
\hline $1 s 5 d^{3} D_{1}$ & $1 s 7 p^{3} P_{0}^{o}$ & $1.6558 \mathrm{E}+01$ & $2.465 \mathrm{E}-02$ & $5.997 \mathrm{E}+11$ & $1.6502 \mathrm{E}+01$ & $2.430 \mathrm{E}-02$ & $5.902 \mathrm{E}+11$ & 0.006 \\
\hline $1 s 6 s^{3} S_{1}$ & $1 s 7 p^{3} P_{0}^{o}$ & $4.2244 \mathrm{E}+01$ & $2.130 \mathrm{E}-01$ & $7.963 \mathrm{E}+11$ & $4.2099 \mathrm{E}+01$ & $2.165 \mathrm{E}-01$ & $8.080 \mathrm{E}+11$ & 0.007 \\
\hline $1 s 6 d^{3} D_{1}$ & $1 s 7 p^{3} P_{0}^{o}$ & $4.5003 \mathrm{E}+01$ & $1.739 \mathrm{E}-01$ & $5.729 \mathrm{E}+11$ & $4.4885 \mathrm{E}+01$ & $1.725 \mathrm{E}-01$ & $5.665 \mathrm{E}+11$ & 0.001 \\
\hline $1 s 7 s^{3} S_{1}$ & $1 s 7 p^{3} P_{0}^{o}$ & $7.1550 \mathrm{E}+03$ & $2.549 \mathrm{E}-02$ & $3.321 E+06$ & $8.8861 \mathrm{E}+03$ & $2.046 \mathrm{E}-02$ & $1.714 \mathrm{E}+06$ & 0.480 \\
\hline $1 s 2 s^{3} S_{1}$ & $1 s 7 p^{3} P_{2}^{o}$ & $1.3253 \mathrm{E}+00$ & $1.891 \mathrm{E}-02$ & $1.437 \mathrm{E}+13$ & $1.3197 \mathrm{E}+00$ & $1.925 \mathrm{E}-02$ & $1.462 \mathrm{E}+13$ & 0.001 \\
\hline $1 s 3 s^{3} s_{1}$ & $1 s 7 p^{3} P_{2}^{o}$ & $3.3884 \mathrm{E}+00$ & $3.934 \mathrm{E}-02$ & $4.571 E+12$ & $3.3744 \mathrm{E}+00$ & $3.973 \mathrm{E}-02$ & $4.615 \mathrm{E}+12$ & 0.005 \\
\hline $1 s 3 d^{3} D_{2}$ & $1 s 7 p^{3} P_{2}^{o}$ & $3.5282 \mathrm{E}+00$ & $3.283 \mathrm{E}-04$ & $3.518 \mathrm{E}+10$ & $3.5135 \mathrm{E}+00$ & $3.304 \mathrm{E}-04$ & $3.541 \mathrm{E}+10$ & 0.010 \\
\hline $1 s 3 d^{3} D_{1}$ & $1 s 7 p^{3} P_{2}^{o}$ & $3.5297 \mathrm{E}+00$ & $3.595 \mathrm{E}-05$ & $3.849 E+09$ & $3.5150 \mathrm{E}+00$ & $3.345 \mathrm{E}-05$ & $3.581 \mathrm{E}+09$ & 0.017 \\
\hline $1 s 3 d^{3} D_{3}$ & $1 s 7 p^{3} P_{2}^{o}$ & $3.5650 \mathrm{E}+00$ & $3.513 \mathrm{E}-03$ & $3.687 \mathrm{E}+11$ & $3.5501 \mathrm{E}+00$ & $3.455 \mathrm{E}-03$ & $3.626 \mathrm{E}+11$ & 0.013 \\
\hline $1 s 3 d^{1} D_{2}$ & $1 s 7 p^{3} P_{2}^{o}$ & $3.5665 \mathrm{E}+00$ & $2.544 \mathrm{E}-04$ & $2.668 \mathrm{E}+10$ & $3.5515 \mathrm{E}+00$ & $2.446 \mathrm{E}-04$ & $2.565 \mathrm{E}+10$ & 0.013 \\
\hline $1 s 4 s^{3} s_{1}$ & $1 s 7 p^{3} P_{2}^{o}$ & $7.3324 \mathrm{E}+00$ & $8.272 \mathrm{E}-02$ & $2.052 \mathrm{E}+12$ & $7.3023 \mathrm{E}+00$ & $8.341 \mathrm{E}-02$ & $2.069 \mathrm{E}+12$ & 0.007 \\
\hline $1 s 4 d^{3} D_{2}$ & $1 s 7 p^{3} P_{2}^{o}$ & $7.6054 \mathrm{E}+00$ & $1.768 \mathrm{E}-03$ & $4.077 \mathrm{E}+10$ & $7.5744 \mathrm{E}+00$ & $1.757 \mathrm{E}-03$ & $4.050 \mathrm{E}+10$ & 0.006 \\
\hline $1 s 4 d^{3} D_{1}$ & $1 s 7 p^{3} P_{2}^{o}$ & $7.6082 \mathrm{E}+00$ & $1.921 \mathrm{E}-04$ & $4.428 \mathrm{E}+09$ & $7.5772 \mathrm{E}+00$ & $1.813 \mathrm{E}-04$ & $4.178 \mathrm{E}+09$ & 0.008 \\
\hline $1 s 4 d^{3} D_{3}$ & $1 s 7 p^{3} P_{2}^{o}$ & $7.6776 \mathrm{E}+00$ & $1.856 \mathrm{E}-02$ & $4.201 E+11$ & $7.6461 \mathrm{E}+00$ & $1.822 \mathrm{E}-02$ & $4.122 \mathrm{E}+11$ & 0.008 \\
\hline
\end{tabular}


Table 3 (continued)

\begin{tabular}{|c|c|c|c|c|c|c|c|c|}
\hline \multirow[t]{2}{*}{ Lower } & \multirow[t]{2}{*}{ Upper } & \multicolumn{3}{|l|}{ GRASP2K } & \multicolumn{3}{|l|}{ FAC } & \multirow[t]{2}{*}{$d T$} \\
\hline & & $\lambda(\AA)$ & $g f$ & $A\left(\mathrm{~s}^{-1}\right)$ & $\lambda(\AA)$ & $g f$ & $A\left(\mathrm{~s}^{-1}\right)$ & \\
\hline $1 s 4 d^{1} D_{2}$ & $1 s 7 p^{3} P_{2}^{o}$ & $7.6806 \mathrm{E}+00$ & $1.326 \mathrm{E}-03$ & $2.999 \mathrm{E}+10$ & $7.6491 \mathrm{E}+00$ & $1.284 \mathrm{E}-03$ & $2.903 E+10$ & 0.009 \\
\hline $1 s 5 s^{3} s_{1}$ & $1 s 7 p^{3} P_{2}^{o}$ & $1.5717 \mathrm{E}+01$ & $2.047 \mathrm{E}-01$ & $1.106 \mathrm{E}+12$ & $1.5653 \mathrm{E}+01$ & $2.060 \mathrm{E}-01$ & $1.112 \mathrm{E}+12$ & 0.007 \\
\hline $1 s 5 d^{3} D_{2}$ & $1 s 7 p^{3} P_{2}^{o}$ & $1.6358 \mathrm{E}+01$ & $8.043 E-03$ & $4.010 \mathrm{E}+10$ & $1.6293 \mathrm{E}+01$ & $7.930 \mathrm{E}-03$ & $3.951 \mathrm{E}+10$ & 0.003 \\
\hline $1 s 5 d^{3} D_{1}$ & $1 s 7 p^{3} P_{2}^{o}$ & $1.6364 \mathrm{E}+01$ & $8.712 \mathrm{E}-04$ & $4.340 \mathrm{E}+09$ & $1.6300 \mathrm{E}+01$ & $8.288 \mathrm{E}-04$ & $4.126 \mathrm{E}+09$ & 0.004 \\
\hline $1 s 5 d^{3} D_{3}$ & $1 s 7 p^{3} P_{2}^{o}$ & $1.6529 \mathrm{E}+01$ & $8.380 \mathrm{E}-02$ & $4.092 \mathrm{E}+11$ & $1.6463 \mathrm{E}+01$ & $8.203 \mathrm{E}-02$ & $4.003 E+11$ & 0.004 \\
\hline $1 s 5 d^{1} D_{2}$ & $1 s 7 p^{3} P_{2}^{o}$ & $1.6536 \mathrm{E}+01$ & $5.940 \mathrm{E}-03$ & $2.898 \mathrm{E}+10$ & $1.6471 \mathrm{E}+01$ & $5.769 \mathrm{E}-03$ & $2.812 \mathrm{E}+10$ & 0.005 \\
\hline $1 s 5 g{ }^{3} G_{3}$ & $1 s 7 p^{3} P_{2}^{o}$ & $1.6620 \mathrm{E}+01$ & $2.424 \mathrm{E}-09$ & $1.171 \mathrm{E}+04$ & & & & 0.041 \\
\hline $1 s 6 s^{3} S_{1}$ & $1 s 7 p^{3} P_{2}^{o}$ & $4.1005 \mathrm{E}+01$ & $7.989 \mathrm{E}-01$ & $6.339 \mathrm{E}+11$ & $4.0810 \mathrm{E}+01$ & $8.054 \mathrm{E}-01$ & $6.397 E+11$ & 0.007 \\
\hline $1 s 6 d^{3} D_{2}$ & $1 s 7 p^{3} P_{2}^{o}$ & $4.3572 \mathrm{E}+01$ & $5.481 \mathrm{E}-02$ & $3.851 \mathrm{E}+10$ & $4.3429 \mathrm{E}+01$ & $5.418 \mathrm{E}-02$ & $3.800 \mathrm{E}+10$ & 0.003 \\
\hline $1 s 6 d^{3} D_{1}$ & $1 s 7 p^{3} P_{2}^{o}$ & $4.3599 \mathrm{E}+01$ & $5.937 \mathrm{E}-03$ & $4.167 \mathrm{E}+09$ & $4.3422 \mathrm{E}+01$ & $5.899 \mathrm{E}-03$ & $4.138 \mathrm{E}+09$ & 0.000 \\
\hline $1 s 6 d^{3} D_{3}$ & $1 s 7 p^{3} P_{2}^{o}$ & $4.4278 \mathrm{E}+01$ & $5.768 \mathrm{E}-01$ & $3.925 \mathrm{E}+11$ & $4.4127 \mathrm{E}+01$ & $5.633 \mathrm{E}-01$ & $3.826 \mathrm{E}+11$ & 0.003 \\
\hline $1 s 6 d^{1} D_{2}$ & $1 s 7 p^{3} P_{2}^{o}$ & $4.4308 \mathrm{E}+01$ & $4.074 \mathrm{E}-02$ & $2.768 \mathrm{E}+10$ & $4.4138 \mathrm{E}+01$ & $3.914 \mathrm{E}-02$ & $2.657 \mathrm{E}+10$ & 0.001 \\
\hline $1 s 6 g{ }^{3} G_{3}$ & $1 s 7 p^{3} P_{2}^{o}$ & $4.4659 \mathrm{E}+01$ & $4.072 \mathrm{E}-08$ & $2.724 \mathrm{E}+04$ & & & & 0.013 \\
\hline $1 s 7 s^{3} S_{1}$ & $1 s 7 p^{3} P_{2}^{o}$ & $1.1691 \mathrm{E}+03$ & $7.901 \mathrm{E}-01$ & $7.712 \mathrm{E}+08$ & $1.1590 \mathrm{E}+03$ & $7.980 \mathrm{E}-01$ & $7.858 \mathrm{E}+08$ & 0.072 \\
\hline $1 s 2 p^{3} P_{1}^{o}$ & $1 s 7 d^{3} D_{2}$ & $1.3362 \mathrm{E}+00$ & $3.096 \mathrm{E}-02$ & $2.313 \mathrm{E}+13$ & $1.3307 \mathrm{E}+00$ & $3.108 \mathrm{E}-02$ & $2.321 \mathrm{E}+13$ & 0.001 \\
\hline $1 s 2 p^{3} P_{2}^{o}$ & $1 s 7 d^{3} D_{2}$ & $1.3946 \mathrm{E}+00$ & $5.230 \mathrm{E}-03$ & $3.587 \mathrm{E}+12$ & $1.3888 \mathrm{E}+00$ & $5.160 \mathrm{E}-03$ & $3.539 E+12$ & 0.008 \\
\hline $1 s 2 p{ }^{1} P_{1}^{o}$ & $1 s 7 d^{3} D_{2}$ & $1.4001 \mathrm{E}+00$ & $8.791 \mathrm{E}-04$ & $5.983 \mathrm{E}+11$ & $1.3943 E+00$ & $8.081 \mathrm{E}-04$ & $5.498 \mathrm{E}+11$ & 0.013 \\
\hline $1 s 3 p^{3} P_{1}^{o}$ & $1 s 7 d^{3} D_{2}$ & $3.4075 \mathrm{E}+00$ & $6.964 \mathrm{E}-02$ & $8.001 E+12$ & $3.3932 E+00$ & $6.971 \mathrm{E}-02$ & $8.008 E+12$ & 0.001 \\
\hline $1 s 3 p^{3} P_{2}^{o}$ & $1 s 7 d^{3} D_{2}$ & $3.5193 \mathrm{E}+00$ & $1.286 \mathrm{E}-02$ & $1.385 \mathrm{E}+12$ & $3.5045 \mathrm{E}+00$ & $1.286 \mathrm{E}-02$ & $1.385 \mathrm{E}+12$ & 0.001 \\
\hline $1 s 3 p{ }^{1} P_{1}^{o}$ & $1 s 7 d^{3} D_{2}$ & $3.5292 \mathrm{E}+00$ & $2.078 \mathrm{E}-03$ & $2.225 \mathrm{E}+11$ & $3.5145 E+00$ & $1.921 \mathrm{E}-03$ & $2.057 \mathrm{E}+11$ & 0.003 \\
\hline $1 s 4 p^{3} P_{1}^{o}$ & $1 s 7 d^{3} D_{2}$ & $7.3673 \mathrm{E}+00$ & $1.429 \mathrm{E}-01$ & $3.511 \mathrm{E}+12$ & $7.3356 \mathrm{E}+00$ & $1.431 \mathrm{E}-01$ & $3.519 E+12$ & 0.001 \\
\hline $1 s 4 p^{3} P_{2}^{o}$ & $1 s 7 d^{3} D_{2}$ & $7.5864 \mathrm{E}+00$ & $2.803 \mathrm{E}-02$ & $6.498 \mathrm{E}+11$ & $7.5537 \mathrm{E}+00$ & $2.826 \mathrm{E}-02$ & $6.552 E+11$ & 0.001 \\
\hline $1 s 4 p{ }^{1} P_{1}^{o}$ & $1 s 7 d^{3} D_{2}$ & $7.6055 \mathrm{E}+00$ & $4.508 \mathrm{E}-03$ & $1.040 \mathrm{E}+11$ & $7.5729 \mathrm{E}+00$ & $4.133 \mathrm{E}-03$ & $9.533 E+10$ & 0.003 \\
\hline $1 s 4 f^{3} F_{3}^{0}$ & $1 s 7 d^{3} D_{2}$ & $7.6759 \mathrm{E}+00$ & $4.363 \mathrm{E}-03$ & $9.879 \mathrm{E}+10$ & $7.6429 \mathrm{E}+00$ & $4.349 \mathrm{E}-03$ & $9.848 \mathrm{E}+10$ & 0.006 \\
\hline $1 s 4 f^{3} F_{2}^{o}$ & $1 s 7 d^{3} D_{2}$ & $7.6774 \mathrm{E}+00$ & $3.185 \mathrm{E}-04$ & $7.208 \mathrm{E}+09$ & $7.6444 \mathrm{E}+00$ & $3.188 \mathrm{E}-04$ & $7.218 \mathrm{E}+09$ & 0.006 \\
\hline $1 s 4 f^{1} F_{3}^{o}$ & $1 s 7 d^{3} D_{2}$ & $7.7133 \mathrm{E}+00$ & $3.659 E-07$ & $8.205 E+06$ & & & & 0.014 \\
\hline $1 s 5 p^{3} P_{1}^{o}$ & $1 s 7 d^{3} D_{2}$ & $1.5792 \mathrm{E}+01$ & $3.371 \mathrm{E}-01$ & $1.803 \mathrm{E}+12$ & $1.5722 \mathrm{E}+01$ & $3.385 \mathrm{E}-01$ & $1.811 \mathrm{E}+12$ & 0.001 \\
\hline $1 s 5 p^{3} P_{2}^{0}$ & $1 s 7 d^{3} D_{2}$ & $1.6307 \mathrm{E}+01$ & $7.040 \mathrm{E}-02$ & $3.532 \mathrm{E}+11$ & $1.6234 \mathrm{E}+01$ & $7.148 \mathrm{E}-02$ & $3.587 E+11$ & 0.001 \\
\hline $1 s 5 p^{1} P_{1}^{o}$ & $1 s 7 d^{3} D_{2}$ & $1.6352 \mathrm{E}+01$ & $1.134 \mathrm{E}-02$ & $5.658 \mathrm{E}+10$ & $1.6279 \mathrm{E}+01$ & $1.027 \mathrm{E}-02$ & $5.130 \mathrm{E}+10$ & 0.004 \\
\hline $1 s 5 f^{3} F_{3}^{0}$ & $1 s 7 d^{3} D_{2}$ & $1.6519 \mathrm{E}+01$ & $3.339 E-02$ & $1.633 \mathrm{E}+11$ & $1.6446 \mathrm{E}+01$ & $3.340 \mathrm{E}-02$ & $1.633 E+11$ & 0.001 \\
\hline $1 s 5 f^{3} F_{2}^{o}$ & $1 s 7 d^{3} D_{2}$ & $1.6522 \mathrm{E}+01$ & $2.432 \mathrm{E}-03$ & $1.189 \mathrm{E}+10$ & $1.6450 \mathrm{E}+01$ & $2.444 \mathrm{E}-03$ & $1.195 E+10$ & 0.001 \\
\hline $1 s 5 f^{1} F_{3}^{o}$ & $1 s 7 d^{3} D_{2}$ & $1.6607 \mathrm{E}+01$ & $2.729 E-06$ & $1.320 \mathrm{E}+07$ & & & & 0.014 \\
\hline $1 s 6 p^{3} P_{1}^{o}$ & $1 s 7 d^{3} D_{2}$ & $4.1263 \mathrm{E}+01$ & $1.251 \mathrm{E}+00$ & $9.802 \mathrm{E}+11$ & $4.1063 \mathrm{E}+01$ & $1.250 \mathrm{E}+00$ & $9.806 \mathrm{E}+11$ & 0.001 \\
\hline $1 s 6 p^{3} P_{2}^{o}$ & $1 s 7 d^{3} D_{2}$ & $4.3326 \mathrm{E}+01$ & $2.912 \mathrm{E}-01$ & $2.069 \mathrm{E}+11$ & $4.3112 \mathrm{E}+01$ & $2.974 \mathrm{E}-01$ & $2.116 E+11$ & 0.001 \\
\hline $1 s 6 p^{1} P_{1}^{o}$ & $1 s 7 d^{3} D_{2}$ & $4.3508 \mathrm{E}+01$ & $4.727 \mathrm{E}-02$ & $3.331 \mathrm{E}+10$ & $4.3273 \mathrm{E}+01$ & $4.648 \mathrm{E}-02$ & $3.283 E+10$ & 0.001 \\
\hline $1 s 6 f^{3} F_{3}^{0}$ & $1 s 7 d^{3} D_{2}$ & $4.4197 \mathrm{E}+01$ & $3.118 \mathrm{E}-01$ & $2.129 \mathrm{E}+11$ & $4.4000 \mathrm{E}+01$ & $3.118 \mathrm{E}-01$ & $2.130 \mathrm{E}+11$ & 0.000 \\
\hline $1 s 6 f^{3} F_{2}^{o}$ & $1 s 7 d^{3} D_{2}$ & $4.4212 \mathrm{E}+01$ & $2.269 \mathrm{E}-02$ & $1.548 \mathrm{E}+10$ & $4.4000 \mathrm{E}+01$ & $2.271 \mathrm{E}-02$ & $1.552 \mathrm{E}+10$ & 0.000 \\
\hline $1 s 6 f^{1} F_{3}^{o}$ & $1 s 7 d^{3} D_{2}$ & $4.4566 \mathrm{E}+01$ & $2.533 \mathrm{E}-05$ & $1.702 \mathrm{E}+07$ & & & & 0.015 \\
\hline $1 s 7 p^{3} P_{1}^{0}$ & $1 s 7 d^{3} D_{2}$ & $1.2946 \mathrm{E}+03$ & $6.694 \mathrm{E}-01$ & $5.328 \mathrm{E}+08$ & $1.2624 \mathrm{E}+03$ & $6.847 \mathrm{E}-01$ & $5.683 E+08$ & 0.085 \\
\hline $1 s 7 p^{3} P_{2}^{o}$ & $1 s 7 d^{3} D_{2}$ & $2.0924 \mathrm{E}+04$ & $7.595 \mathrm{E}-03$ & $2.314 \mathrm{E}+04$ & $1.5290 \mathrm{E}+04$ & $1.048 \mathrm{E}-02$ & $5.933 E+04$ & 0.623 \\
\hline $1 s 2 p^{3} P_{1}^{o}$ & $1 s 7 d^{3} D_{1}$ & $1.3362 \mathrm{E}+00$ & $6.637 \mathrm{E}-03$ & $8.265 \mathrm{E}+12$ & $1.3307 \mathrm{E}+00$ & $6.631 \mathrm{E}-03$ & $8.256 \mathrm{E}+12$ & 0.004 \\
\hline $1 s 2 p^{3} P_{0}^{o}$ & $1 s 7 d^{3} D_{1}$ & $1.3371 \mathrm{E}+00$ & $1.257 \mathrm{E}-02$ & $1.564 \mathrm{E}+13$ & $1.3315 \mathrm{E}+00$ & $1.257 \mathrm{E}-02$ & $1.563 E+13$ & 0.002 \\
\hline $1 s 2 p^{3} P_{2}^{o}$ & $1 s 7 d^{3} D_{1}$ & $1.3946 \mathrm{E}+00$ & $5.723 \mathrm{E}-04$ & $6.542 \mathrm{E}+11$ & $1.3888 \mathrm{E}+00$ & $5.618 \mathrm{E}-04$ & $6.421 \mathrm{E}+11$ & 0.010 \\
\hline $1 s 2 p{ }^{1} P_{1}^{o}$ & $1 s 7 d^{3} D_{1}$ & $1.4001 \mathrm{E}+00$ & $2.555 \mathrm{E}-03$ & $2.898 \mathrm{E}+12$ & $1.3943 \mathrm{E}+00$ & $2.472 \mathrm{E}-03$ & $2.803 E+12$ & 0.006 \\
\hline $1 s 3 p^{3} P_{1}^{o}$ & $1 s 7 d^{3} D_{1}$ & $3.4074 \mathrm{E}+00$ & $1.488 \mathrm{E}-02$ & $2.849 \mathrm{E}+12$ & $3.3932 E+00$ & $1.485 \mathrm{E}-02$ & $2.844 \mathrm{E}+12$ & 0.000 \\
\hline $1 s 3 p^{3} P_{0}^{0}$ & $1 s 7 d^{3} D_{1}$ & $3.4088 \mathrm{E}+00$ & $2.822 \mathrm{E}-02$ & $5.400 \mathrm{E}+12$ & $3.3947 \mathrm{E}+00$ & $2.819 \mathrm{E}-02$ & $5.393 \mathrm{E}+12$ & 0.000 \\
\hline $1 s 3 p^{3} P_{2}^{o}$ & $1 s 7 d^{3} D_{1}$ & $3.5192 \mathrm{E}+00$ & $1.406 \mathrm{E}-03$ & $2.523 \mathrm{E}+11$ & $3.5045 \mathrm{E}+00$ & $1.401 \mathrm{E}-03$ & $2.515 E+11$ & 0.000 \\
\hline $1 s 3 p^{1} P_{1}^{o}$ & $1 s 7 d^{3} D_{1}$ & $3.5291 \mathrm{E}+00$ & $6.358 \mathrm{E}-03$ & $1.135 \mathrm{E}+12$ & $3.5145 \mathrm{E}+00$ & $6.259 \mathrm{E}-03$ & $1.117 \mathrm{E}+12$ & 0.000 \\
\hline $1 s 4 p^{3} P_{1}^{o}$ & $1 s 7 d^{3} D_{1}$ & $7.3668 \mathrm{E}+00$ & $3.045 \mathrm{E}-02$ & $1.248 \mathrm{E}+12$ & $7.3357 \mathrm{E}+00$ & $3.052 \mathrm{E}-02$ & $1.250 \mathrm{E}+12$ & 0.000 \\
\hline $1 s 4 p^{3} P_{0}^{o}$ & $1 s 7 d^{3} D_{1}$ & $7.3695 \mathrm{E}+00$ & $5.785 \mathrm{E}-02$ & $2.368 \mathrm{E}+12$ & $7.3384 \mathrm{E}+00$ & $5.791 \mathrm{E}-02$ & $2.370 \mathrm{E}+12$ & 0.000 \\
\hline $1 s 4 p^{3} P_{2}^{o}$ & $1 s 7 d^{3} D_{1}$ & $7.5859 \mathrm{E}+00$ & $3.056 \mathrm{E}-03$ & $1.181 \mathrm{E}+11$ & $7.5538 \mathrm{E}+00$ & $3.082 \mathrm{E}-03$ & $1.190 \mathrm{E}+11$ & 0.000 \\
\hline $1 s 4 p{ }^{1} P_{1}^{o}$ & $1 s 7 d^{3} D_{1}$ & $7.6050 \mathrm{E}+00$ & $1.395 \mathrm{E}-02$ & $5.361 \mathrm{E}+11$ & $7.5730 \mathrm{E}+00$ & $1.384 \mathrm{E}-02$ & $5.323 E+11$ & 0.000 \\
\hline $1 s 4 f^{3} F_{2}^{0}$ & $1 s 7 d^{3} D_{1}$ & $7.6769 \mathrm{E}+00$ & $2.803 E-03$ & $1.058 \mathrm{E}+11$ & $7.6445 \mathrm{E}+00$ & $2.797 \mathrm{E}-03$ & $1.055 \mathrm{E}+11$ & 0.006 \\
\hline $1 s 5 p^{3} P_{1}^{o}$ & $1 s 7 d^{3} D_{1}$ & $1.5790 \mathrm{E}+01$ & $7.173 \mathrm{E}-02$ & $6.397 \mathrm{E}+11$ & $1.5722 \mathrm{E}+01$ & $7.230 \mathrm{E}-02$ & $6.448 \mathrm{E}+11$ & 0.002 \\
\hline $1 s 5 p^{3} P_{0}^{o}$ & $1 s 7 d^{3} D_{1}$ & $1.5796 \mathrm{E}+01$ & $1.364 \mathrm{E}-01$ & $1.215 \mathrm{E}+12$ & $1.5728 \mathrm{E}+01$ & $1.369 \mathrm{E}-01$ & $1.220 \mathrm{E}+12$ & 0.002 \\
\hline $1 s 5 p^{3} P_{2}^{o}$ & $1 s 7 d^{3} D_{1}$ & $1.6305 \mathrm{E}+01$ & $7.659 E-03$ & $6.406 \mathrm{E}+10$ & $1.6235 \mathrm{E}+01$ & $7.804 \mathrm{E}-03$ & $6.528 \mathrm{E}+10$ & 0.001 \\
\hline $1 s 5 p^{1} P_{1}^{o}$ & $1 s 7 d^{3} D_{1}$ & $1.6349 \mathrm{E}+01$ & $3.522 \mathrm{E}-02$ & $2.929 E+11$ & $1.6280 \mathrm{E}+01$ & $3.519 \mathrm{E}-02$ & $2.927 \mathrm{E}+11$ & 0.002 \\
\hline $1 s 5 f^{3} F_{2}^{o}$ & $1 s 7 d^{3} D_{1}$ & $1.6520 \mathrm{E}+01$ & $2.145 \mathrm{E}-02$ & $1.748 \mathrm{E}+11$ & $1.6451 \mathrm{E}+01$ & $2.148 \mathrm{E}-02$ & $1.750 \mathrm{E}+11$ & 0.001 \\
\hline $1 s 6 p^{3} P_{1}^{o}$ & $1 s 7 d^{3} D_{1}$ & $4.1248 \mathrm{E}+01$ & $2.657 \mathrm{E}-01$ & $3.472 \mathrm{E}+11$ & $4.1067 \mathrm{E}+01$ & $2.734 \mathrm{E}-01$ & $3.574 \mathrm{E}+11$ & 0.000 \\
\hline $1 s 6 p^{3} P_{0}^{0}$ & $1 s 7 d^{3} D_{1}$ & $4.1273 \mathrm{E}+01$ & $5.057 \mathrm{E}-01$ & $6.601 \mathrm{E}+11$ & $4.1003 \mathrm{E}+01$ & $5.090 \mathrm{E}-01$ & $6.675 E+11$ & 0.000 \\
\hline $1 s 6 p^{3} P_{2}^{o}$ & $1 s 7 d^{3} D_{1}$ & $4.3310 \mathrm{E}+01$ & $3.160 \mathrm{E}-02$ & $3.746 \mathrm{E}+10$ & $4.3116 \mathrm{E}+01$ & $3.243 \mathrm{E}-02$ & $3.846 \mathrm{E}+10$ & 0.000 \\
\hline $1 s 6 p^{1} P_{1}^{o}$ & $1 s 7 d^{3} D_{1}$ & $4.3491 \mathrm{E}+01$ & $1.469 \mathrm{E}-01$ & $1.726 \mathrm{E}+11$ & $4.3277 \mathrm{E}+01$ & $1.432 \mathrm{E}-01$ & $1.686 \mathrm{E}+11$ & 0.000 \\
\hline $1 s 6 f^{3} F_{2}^{o}$ & $1 s 7 d^{3} D_{1}$ & $4.4195 \mathrm{E}+01$ & $2.002 \mathrm{E}-01$ & $2.279 \mathrm{E}+11$ & $4.4004 \mathrm{E}+01$ & $2.007 \mathrm{E}-01$ & $2.285 E+11$ & 0.000 \\
\hline $1 s 7 p^{3} P_{1}^{o}$ & $1 s 7 d^{3} D_{1}$ & $1.2801 \mathrm{E}+03$ & $1.445 \mathrm{E}-01$ & $1.960 \mathrm{E}+08$ & $1.2659 \mathrm{E}+03$ & $1.475 \mathrm{E}-01$ & $2.029 E+08$ & 0.069 \\
\hline $1 s 7 p^{3} P_{0}^{o}$ & $1 s 7 d^{3} D_{1}$ & $1.2951 \mathrm{E}+03$ & $2.699 \mathrm{E}-01$ & $3.578 \mathrm{E}+08$ & $1.2292 \mathrm{E}+03$ & $2.853 \mathrm{E}-01$ & $4.163 E+08$ & 0.073 \\
\hline $1 s 7 p^{3} P_{2}^{o}$ & $1 s 7 d^{3} D_{1}$ & $1.7684 \mathrm{E}+04$ & $9.719 E-04$ & $6.910 \mathrm{E}+03$ & & & & 0.539 \\
\hline $1 s^{2}{ }^{1} S_{0}$ & $1 s 7 p^{1} P_{1}^{o}$ & $3.1400 \mathrm{E}-01$ & $6.133 \mathrm{E}-03$ & $1.383 \mathrm{E}+14$ & $3.1269 \mathrm{E}-01$ & $6.558 \mathrm{E}-03$ & $1.478 \mathrm{E}+14$ & 0.034 \\
\hline $1 s 2 s^{3} S_{1}$ & $1 s 7 p^{1} P_{1}^{o}$ & $1.3252 \mathrm{E}+00$ & $3.384 \mathrm{E}-03$ & $4.284 \mathrm{E}+12$ & $1.3196 \mathrm{E}+00$ & $3.343 \mathrm{E}-03$ & $4.232 \mathrm{E}+12$ & 0.001 \\
\hline $1 s 2 s^{1} S_{0}$ & $1 s 7 p^{1} P_{1}^{o}$ & $1.3373 \mathrm{E}+00$ & $8.044 \mathrm{E}-03$ & $1.000 \mathrm{E}+13$ & $1.3319 \mathrm{E}+00$ & $8.135 E-03$ & $1.011 \mathrm{E}+13$ & 0.002 \\
\hline $1 s 3 s^{3} S_{1}$ & $1 s 7 p^{1} P_{1}^{o}$ & $3.3877 \mathrm{E}+00$ & $7.007 \mathrm{E}-03$ & $1.358 \mathrm{E}+12$ & $3.3738 \mathrm{E}+00$ & $6.862 \mathrm{E}-03$ & $1.329 \mathrm{E}+12$ & 0.004 \\
\hline $1 s 3 s^{1} S_{0}$ & $1 s 7 p^{1} P_{1}^{o}$ & $3.4087 \mathrm{E}+00$ & $1.698 \mathrm{E}-02$ & $3.249 \mathrm{E}+12$ & $3.3948 \mathrm{E}+00$ & $1.687 \mathrm{E}-02$ & $3.228 \mathrm{E}+12$ & 0.007 \\
\hline
\end{tabular}


Table 3 (continued)

\begin{tabular}{|c|c|c|c|c|c|c|c|c|}
\hline \multirow[t]{2}{*}{ Lower } & \multirow[t]{2}{*}{ Upper } & \multicolumn{3}{|l|}{ GRASP2K } & \multicolumn{3}{|l|}{ FAC } & \multirow[t]{2}{*}{$d T$} \\
\hline & & $\lambda(\AA)$ & $g f$ & $A\left(\mathrm{~s}^{-1}\right)$ & $\lambda(\AA)$ & $g f$ & $A\left(\mathrm{~s}^{-1}\right)$ & \\
\hline $1 s 3 d^{3} D_{2}$ & $1 s 7 p^{1} P_{1}^{o}$ & $3.5275 \mathrm{E}+00$ & $5.455 \mathrm{E}-05$ & $9.748 \mathrm{E}+09$ & $3.5128 \mathrm{E}+00$ & $6.841 \mathrm{E}-05$ & $1.222 \mathrm{E}+10$ & 0.020 \\
\hline $1 s 3 d^{3} D_{1}$ & $1 s 7 p^{1} P_{1}^{o}$ & $3.5290 \mathrm{E}+00$ & $1.667 \mathrm{E}-04$ & $2.975 \mathrm{E}+10$ & $3.5143 \mathrm{E}+00$ & $1.567 \mathrm{E}-04$ & $2.797 \mathrm{E}+10$ & 0.023 \\
\hline $1 s 3 d^{1} D_{2}$ & $1 s 7 p^{1} P_{1}^{o}$ & $3.5657 \mathrm{E}+00$ & $2.254 \mathrm{E}-03$ & $3.941 \mathrm{E}+11$ & $3.5508 \mathrm{E}+00$ & $2.218 \mathrm{E}-03$ & $3.879 \mathrm{E}+11$ & 0.018 \\
\hline $1 s 4 s^{3} S_{1}$ & $1 s 7 p^{1} P_{1}^{o}$ & $7.3292 \mathrm{E}+00$ & $1.467 \mathrm{E}-02$ & $6.074 \mathrm{E}+11$ & $7.2994 \mathrm{E}+00$ & $1.435 \mathrm{E}-02$ & $5.938 \mathrm{E}+11$ & 0.006 \\
\hline $1 s 4 s^{1} s_{0}$ & $1 s 7 p^{1} P_{1}^{o}$ & $7.3687 \mathrm{E}+00$ & $3.615 \mathrm{E}-02$ & $1.480 \mathrm{E}+12$ & $7.3391 \mathrm{E}+00$ & $3.552 \mathrm{E}-02$ & $1.453 \mathrm{E}+12$ & 0.018 \\
\hline $1 s 4 d^{3} D_{2}$ & $1 s 7 p^{1} P_{1}^{o}$ & $7.6019 \mathrm{E}+00$ & $2.848 \mathrm{E}-04$ & $1.096 \mathrm{E}+10$ & $7.5713 \mathrm{E}+00$ & $3.513 \mathrm{E}-04$ & $1.351 \mathrm{E}+10$ & 0.015 \\
\hline $1 s 4 d^{3} D_{1}$ & $1 s 7 p^{1} P_{1}^{o}$ & $7.6047 \mathrm{E}+00$ & $8.821 \mathrm{E}-04$ & $3.391 \mathrm{E}+10$ & $7.5741 \mathrm{E}+00$ & $8.289 \mathrm{E}-04$ & $3.185 \mathrm{E}+10$ & 0.015 \\
\hline $1 s 4 d{ }^{1} D_{2}$ & $1 s 7 p^{1} P_{1}^{o}$ & $7.6770 \mathrm{E}+00$ & $1.182 \mathrm{E}-02$ & $4.460 \mathrm{E}+11$ & $7.6459 \mathrm{E}+00$ & $1.171 \mathrm{E}-02$ & $4.416 \mathrm{E}+11$ & 0.012 \\
\hline $1 s 5 s^{3} S_{1}$ & $1 s 7 p^{1} P_{1}^{o}$ & $1.5702 \mathrm{E}+01$ & $3.615 \mathrm{E}-02$ & $3.260 \mathrm{E}+11$ & $1.5640 \mathrm{E}+01$ & $3.530 \mathrm{E}-02$ & $3.181 E+11$ & 0.006 \\
\hline $1 s 5 s{ }^{1} S_{0}$ & $1 s 7 p^{1} P_{1}^{o}$ & $1.5793 \mathrm{E}+01$ & $8.962 \mathrm{E}-02$ & $7.989 \mathrm{E}+11$ & $1.5731 \mathrm{E}+01$ & $8.793 \mathrm{E}-02$ & $7.833 \mathrm{E}+11$ & 0.020 \\
\hline $1 s 5 d^{3} D_{2}$ & $1 s 7 p^{1} P_{1}^{o}$ & $1.6342 \mathrm{E}+01$ & $1.272 \mathrm{E}-03$ & $1.059 \mathrm{E}+10$ & $1.6279 \mathrm{E}+01$ & $1.567 \mathrm{E}-03$ & $1.303 E+10$ & 0.010 \\
\hline $1 s 5 d^{3} D_{1}$ & $1 s 7 p^{1} P_{1}^{o}$ & $1.6348 \mathrm{E}+01$ & $3.965 \mathrm{E}-03$ & $3.299 \mathrm{E}+10$ & $1.6286 \mathrm{E}+01$ & $3.728 \mathrm{E}-03$ & $3.099 E+10$ & 0.009 \\
\hline $1 s 5 d{ }^{1} D_{2}$ & $1 s 7 p^{1} P_{1}^{o}$ & $1.6520 \mathrm{E}+01$ & $5.302 \mathrm{E}-02$ & $4.320 \mathrm{E}+11$ & $1.6456 \mathrm{E}+01$ & $5.276 \mathrm{E}-02$ & $4.295 \mathrm{E}+11$ & 0.008 \\
\hline $1 s 6 s^{3} S_{1}$ & $1 s 7 p^{1} P_{1}^{o}$ & $4.0902 \mathrm{E}+01$ & $1.398 \mathrm{E}-01$ & $1.858 \mathrm{E}+11$ & $4.0720 \mathrm{E}+01$ & $1.370 \mathrm{E}-01$ & $1.821 \mathrm{E}+11$ & 0.007 \\
\hline $1 s 6 s{ }^{1} S_{0}$ & $1 s 7 p^{1} P_{1}^{o}$ & $4.1256 \mathrm{E}+01$ & $3.498 \mathrm{E}-01$ & $4.569 \mathrm{E}+11$ & $4.1020 \mathrm{E}+01$ & $3.451 \mathrm{E}-01$ & $4.522 \mathrm{E}+11$ & 0.014 \\
\hline $1 s 6 d^{3} D_{2}$ & $1 s 7 p^{1} P_{1}^{o}$ & $4.3457 \mathrm{E}+01$ & $8.540 \mathrm{E}-03$ & $1.005 \mathrm{E}+10$ & $4.3327 \mathrm{E}+01$ & $1.016 \mathrm{E}-02$ & $1.193 E+10$ & 0.003 \\
\hline $1 s 6 d^{3} D_{1}$ & $1 s 7 p^{1} P_{1}^{o}$ & $4.3483 \mathrm{E}+01$ & $2.672 \mathrm{E}-02$ & $3.142 \mathrm{E}+10$ & $4.3321 \mathrm{E}+01$ & $2.490 \mathrm{E}-02$ & $2.926 \mathrm{E}+10$ & 0.003 \\
\hline $1 s 6 d{ }^{1} D_{2}$ & $1 s 7 p^{1} P_{1}^{o}$ & $4.4189 \mathrm{E}+01$ & $3.623 \mathrm{E}-01$ & $4.126 \mathrm{E}+11$ & $4.4032 \mathrm{E}+01$ & $3.636 \mathrm{E}-01$ & $4.134 \mathrm{E}+11$ & 0.002 \\
\hline $1 s 7 s^{3} S_{1}$ & $1 s 7 p^{1} P_{1}^{o}$ & $1.0915 \mathrm{E}+03$ & $1.520 \mathrm{E}-01$ & $2.837 \mathrm{E}+08$ & $1.0907 \mathrm{E}+03$ & $1.478 \mathrm{E}-01$ & $2.739 \mathrm{E}+08$ & 0.066 \\
\hline $1 s 7 s^{1} S_{0}$ & $1 s 7 p^{1} P_{1}^{o}$ & $1.2741 \mathrm{E}+03$ & $3.038 \mathrm{E}-01$ & $4.162 \mathrm{E}+08$ & $1.2422 \mathrm{E}+03$ & $3.169 \mathrm{E}-01$ & $4.528 \mathrm{E}+08$ & 0.028 \\
\hline $1 s 7 d^{3} D_{2}$ & $1 s 7 p^{1} P_{1}^{o}$ & $7.6625 \mathrm{E}+04$ & $3.302 \mathrm{E}-04$ & $1.250 \mathrm{E}+02$ & & & & 0.321 \\
\hline $1 s 7 d^{3} D_{1}$ & $1 s 7 p^{1} P_{1}^{o}$ & $2.3287 \mathrm{E}+05$ & $3.390 \mathrm{E}-04$ & $1.390 \mathrm{E}+01$ & & & & 0.942 \\
\hline $1 s 2 p^{3} P_{2}^{o}$ & $1 s 7 d^{3} D_{3}$ & $1.3942 \mathrm{E}+00$ & $5.071 \mathrm{E}-02$ & $2.486 \mathrm{E}+13$ & $1.3883 \mathrm{E}+00$ & $5.182 \mathrm{E}-02$ & $2.540 \mathrm{E}+13$ & 0.003 \\
\hline $1 s 3 p^{3} P_{2}^{0}$ & $1 s 7 d^{3} D_{3}$ & $3.5164 \mathrm{E}+00$ & $1.199 \mathrm{E}-01$ & $9.238 \mathrm{E}+12$ & $3.5016 \mathrm{E}+00$ & $1.209 \mathrm{E}-01$ & $9.318 \mathrm{E}+12$ & 0.001 \\
\hline $1 s 4 p^{3} P_{2}^{o}$ & $1 s 7 d^{3} D_{3}$ & $7.5732 \mathrm{E}+00$ & $2.553 \mathrm{E}-01$ & $4.241 \mathrm{E}+12$ & $7.5406 \mathrm{E}+00$ & $2.573 \mathrm{E}-01$ & $4.276 \mathrm{E}+12$ & 0.001 \\
\hline $1 s 4 f^{3} F_{3}^{o}$ & $1 s 7 d^{3} D_{3}$ & $7.6624 \mathrm{E}+00$ & $2.543 \mathrm{E}-04$ & $4.128 \mathrm{E}+09$ & $7.6295 \mathrm{E}+00$ & $2.555 \mathrm{E}-04$ & $4.148 \mathrm{E}+09$ & 0.002 \\
\hline $1 s 4 f^{3} F_{2}^{o}$ & $1 s 7 d^{3} D_{3}$ & $7.6639 \mathrm{E}+00$ & $1.167 \mathrm{E}-05$ & $1.894 \mathrm{E}+08$ & & & & 0.001 \\
\hline $1 s 4 f^{3} F_{4}^{o}$ & $1 s 7 d^{3} D_{3}$ & $7.6985 \mathrm{E}+00$ & $5.558 \mathrm{E}-03$ & $8.936 \mathrm{E}+10$ & $7.6654 \mathrm{E}+00$ & $5.556 \mathrm{E}-03$ & $8.934 \mathrm{E}+10$ & 0.000 \\
\hline $1 s 4 f^{1} F_{3}^{o}$ & $1 s 7 d^{3} D_{3}$ & $7.6996 \mathrm{E}+00$ & $2.073 \mathrm{E}-04$ & $3.331 \mathrm{E}+09$ & $7.6665 \mathrm{E}+00$ & $2.067 \mathrm{E}-04$ & $3.323 E+09$ & 0.001 \\
\hline $1 s 5 p^{3} P_{2}^{o}$ & $1 s 7 d^{3} D_{3}$ & $1.6246 \mathrm{E}+01$ & $6.275 \mathrm{E}-01$ & $2.266 \mathrm{E}+12$ & $1.6174 \mathrm{E}+01$ & $6.344 \mathrm{E}-01$ & $2.291 \mathrm{E}+12$ & 0.001 \\
\hline $1 s 5 f^{3} F_{3}^{o}$ & $1 s 7 d^{3} D_{3}$ & $1.6456 \mathrm{E}+01$ & $1.973 \mathrm{E}-03$ & $6.943 \mathrm{E}+09$ & $1.6385 \mathrm{E}+01$ & $1.981 \mathrm{E}-03$ & $6.974 \mathrm{E}+09$ & 0.002 \\
\hline $1 s 5 f^{3} F_{2}^{0}$ & $1 s 7 d^{3} D_{3}$ & $1.6460 \mathrm{E}+01$ & $9.278 \mathrm{E}-05$ & $3.263 \mathrm{E}+08$ & $1.6388 \mathrm{E}+01$ & $9.234 \mathrm{E}-05$ & $3.248 \mathrm{E}+08$ & 0.001 \\
\hline $1 s 5 f^{3} F_{4}^{o}$ & $1 s 7 d^{3} D_{3}$ & $1.6542 \mathrm{E}+01$ & $4.307 \mathrm{E}-02$ & $1.500 \mathrm{E}+11$ & $1.6469 \mathrm{E}+01$ & $4.303 \mathrm{E}-02$ & $1.498 \mathrm{E}+11$ & 0.001 \\
\hline $1 s 5 f^{1} F_{3}^{o}$ & $1 s 7 d^{3} D_{3}$ & $1.6544 \mathrm{E}+01$ & $1.605 \mathrm{E}-03$ & $5.589 \mathrm{E}+09$ & $1.6472 \mathrm{E}+01$ & $1.600 \mathrm{E}-03$ & $5.573 E+09$ & 0.000 \\
\hline $1 s 6 p^{3} P_{2}^{o}$ & $1 s 7 d^{3} D_{3}$ & $4.2898 \mathrm{E}+01$ & $2.505 E+00$ & $1.297 \mathrm{E}+12$ & $4.2690 \mathrm{E}+01$ & $2.541 \mathrm{E}+00$ & $1.317 \mathrm{E}+12$ & 0.001 \\
\hline $1 s 6 f^{3} F_{3}^{o}$ & $1 s 7 d^{3} D_{3}$ & $4.3752 \mathrm{E}+01$ & $1.834 \mathrm{E}-02$ & $9.130 \mathrm{E}+09$ & $4.3561 \mathrm{E}+01$ & $1.845 \mathrm{E}-02$ & $9.187 \mathrm{E}+09$ & 0.001 \\
\hline $1 s 6 f^{3} F_{2}^{o}$ & $1 s 7 d^{3} D_{3}$ & $4.3767 \mathrm{E}+01$ & $8.773 \mathrm{E}-04$ & $4.364 \mathrm{E}+08$ & $4.3561 \mathrm{E}+01$ & $9.216 \mathrm{E}-04$ & $4.589 \mathrm{E}+08$ & 0.000 \\
\hline $1 s 6 f^{3} F_{4}^{o}$ & $1 s 7 d^{3} D_{3}$ & $4.4102 \mathrm{E}+01$ & $4.014 \mathrm{E}-01$ & $1.966 \mathrm{E}+11$ & $4.3907 \mathrm{E}+01$ & $4.009 \mathrm{E}-01$ & $1.965 \mathrm{E}+11$ & 0.001 \\
\hline $1 s 6 f^{1} F_{3}^{o}$ & $1 s 7 d^{3} D_{3}$ & $4.4114 \mathrm{E}+01$ & $1.496 \mathrm{E}-02$ & $7.327 \mathrm{E}+09$ & $4.3908 \mathrm{E}+01$ & $1.483 \mathrm{E}-02$ & $7.268 \mathrm{E}+09$ & 0.000 \\
\hline $1 s 6 h^{3} H_{4}^{o}$ & $1 s 7 d^{3} D_{3}$ & $4.4320 \mathrm{E}+01$ & $7.374 \mathrm{E}-11$ & $3.577 \mathrm{E}+01$ & & & & 0.055 \\
\hline $1 s 7 p^{3} P_{2}^{o}$ & $1 s 7 d^{3} D_{3}$ & $3.5985 \mathrm{E}+03$ & $4.118 \mathrm{E}-01$ & $3.031 \mathrm{E}+07$ & $3.3938 \mathrm{E}+03$ & $4.365 \mathrm{E}-01$ & $3.581 \mathrm{E}+07$ & 0.194 \\
\hline $1 s 3 d^{3} D_{2}$ & $1 s 7 f^{3} F_{3}^{o}$ & $3.5247 \mathrm{E}+00$ & $1.202 \mathrm{E}-01$ & $9.218 \mathrm{E}+12$ & $3.5099 \mathrm{E}+00$ & $1.203 \mathrm{E}-01$ & $9.226 \mathrm{E}+12$ & 0.001 \\
\hline $1 s 3 d^{3} D_{3}$ & $1 s 7 f^{3} F_{3}^{o}$ & $3.5615 \mathrm{E}+00$ & $7.764 \mathrm{E}-03$ & $5.832 \mathrm{E}+11$ & $3.5464 \mathrm{E}+00$ & $7.675 \mathrm{E}-03$ & $5.766 \mathrm{E}+11$ & 0.002 \\
\hline $1 s 3 d^{1} D_{2}$ & $1 s 7 f^{3} F_{3}^{o}$ & $3.5629 \mathrm{E}+00$ & $4.626 \mathrm{E}-04$ & $3.472 \mathrm{E}+10$ & $3.5478 \mathrm{E}+00$ & $4.247 \mathrm{E}-04$ & $3.188 \mathrm{E}+10$ & 0.001 \\
\hline $1 s 4 d^{3} D_{2}$ & $1 s 7 f^{3} F_{3}^{0}$ & $7.5891 \mathrm{E}+00$ & $3.358 \mathrm{E}-01$ & $5.555 \mathrm{E}+12$ & $7.5575 \mathrm{E}+00$ & $3.361 \mathrm{E}-01$ & $5.561 \mathrm{E}+12$ & 0.001 \\
\hline $1 s 4 d^{3} D_{3}$ & $1 s 7 f^{3} F_{3}^{o}$ & $7.6610 \mathrm{E}+00$ & $2.246 \mathrm{E}-02$ & $3.646 \mathrm{E}+11$ & $7.6289 \mathrm{E}+00$ & $2.240 \mathrm{E}-02$ & $3.637 \mathrm{E}+11$ & 0.002 \\
\hline $1 s 4 d{ }^{1} D_{2}$ & $1 s 7 f^{3} F_{3}^{o}$ & $7.6640 \mathrm{E}+00$ & $1.423 \mathrm{E}-03$ & $2.309 \mathrm{E}+10$ & $7.6319 \mathrm{E}+00$ & $1.320 \mathrm{E}-03$ & $2.142 E+10$ & 0.002 \\
\hline $1 s 5 d^{3} D_{2}$ & $1 s 7 f^{3} F_{3}^{o}$ & $1.6283 \mathrm{E}+01$ & $8.980 \mathrm{E}-01$ & $3.228 \mathrm{E}+12$ & $1.6216 \mathrm{E}+01$ & $8.992 \mathrm{E}-01$ & $3.231 \mathrm{E}+12$ & 0.001 \\
\hline $1 s 5 d^{3} D_{3}$ & $1 s 7 f^{3} F_{3}^{o}$ & $1.6452 \mathrm{E}+01$ & $6.182 \mathrm{E}-02$ & $2.176 \mathrm{E}+11$ & $1.6384 \mathrm{E}+01$ & $6.195 \mathrm{E}-02$ & $2.180 \mathrm{E}+11$ & 0.001 \\
\hline $1 s 5 d^{1} D_{2}$ & $1 s 7 f^{3} F_{3}^{o}$ & $1.6459 \mathrm{E}+01$ & $4.017 \mathrm{E}-03$ & $1.413 \mathrm{E}+10$ & $1.6391 \mathrm{E}+01$ & $3.740 \mathrm{E}-03$ & $1.315 \mathrm{E}+10$ & 0.002 \\
\hline $1 s 5 g{ }^{3} G_{4}$ & $1 s 7 f^{3} F_{3}^{o}$ & $1.6541 \mathrm{E}+01$ & $1.113 \mathrm{E}-02$ & $3.876 \mathrm{E}+10$ & $1.6470 \mathrm{E}+01$ & $1.117 \mathrm{E}-02$ & $3.890 \mathrm{E}+10$ & 0.003 \\
\hline $1 s 5 g^{3} G_{3}$ & $1 s 7 f^{3} F_{3}^{0}$ & $1.6543 \mathrm{E}+01$ & $4.140 \mathrm{E}-04$ & $1.442 \mathrm{E}+09$ & $1.6472 \mathrm{E}+01$ & $4.143 \mathrm{E}-04$ & $1.442 \mathrm{E}+09$ & 0.005 \\
\hline $1 s 5 g{ }^{1} G_{4}$ & $1 s 7 f^{3} F_{3}^{o}$ & $1.6593 \mathrm{E}+01$ & $1.212 \mathrm{E}-10$ & $4.195 \mathrm{E}+02$ & & & & 0.342 \\
\hline $1 s 6 d^{3} D_{2}$ & $1 s 7 f^{3} F_{3}^{o}$ & $4.3043 \mathrm{E}+01$ & $3.664 \mathrm{E}+00$ & $1.885 \mathrm{E}+12$ & $4.2880 \mathrm{E}+01$ & $3.666 \mathrm{E}+00$ & $1.884 \mathrm{E}+12$ & 0.000 \\
\hline $1 s 6 d^{3} D_{3}$ & $1 s 7 f^{3} F_{3}^{o}$ & $4.3731 \mathrm{E}+01$ & $2.630 \mathrm{E}-01$ & $1.310 \mathrm{E}+11$ & $4.3560 \mathrm{E}+01$ & $2.642 \mathrm{E}-01$ & $1.315 \mathrm{E}+11$ & 0.001 \\
\hline $1 s 6 d^{1} D_{2}$ & $1 s 7 f^{3} F_{3}^{0}$ & $4.3761 \mathrm{E}+01$ & $1.732 \mathrm{E}-02$ & $8.618 E+09$ & $4.3571 \mathrm{E}+01$ & $1.851 \mathrm{E}-02$ & $9.214 \mathrm{E}+09$ & 0.002 \\
\hline $1 s 6 g{ }^{3} G_{4}$ & $1 s 7 f^{3} F_{3}^{o}$ & $4.4095 \mathrm{E}+01$ & $1.866 \mathrm{E}-01$ & $9.147 \mathrm{E}+10$ & $4.3907 \mathrm{E}+01$ & $1.868 \mathrm{E}-01$ & $9.159 \mathrm{E}+10$ & 0.001 \\
\hline $1 s 6 g^{3} G_{3}$ & $1 s 7 f^{3} F_{3}^{o}$ & $4.4103 \mathrm{E}+01$ & $6.915 \mathrm{E}-03$ & $3.388 \mathrm{E}+09$ & $4.3907 \mathrm{E}+01$ & $6.929 \mathrm{E}-03$ & $3.396 \mathrm{E}+09$ & 0.002 \\
\hline $1 s 6 g{ }^{1} G_{4}$ & $1 s 7 f^{3} F_{3}^{0}$ & $4.4313 \mathrm{E}+01$ & $1.954 \mathrm{E}-11$ & $9.481 \mathrm{E}+00$ & & & & 0.976 \\
\hline $1 s 7 d^{3} D_{2}$ & $1 s 7 f^{3} F_{3}^{o}$ & $4.2661 \mathrm{E}+03$ & $3.105 \mathrm{E}-01$ & $1.626 \mathrm{E}+07$ & $4.3591 \mathrm{E}+03$ & $3.024 \mathrm{E}-01$ & $1.504 \mathrm{E}+07$ & 0.021 \\
\hline $1 s 7 d^{3} D_{3}$ & $1 s 7 f^{3} F_{3}^{o}$ & $2.3237 \mathrm{E}+05$ & $3.848 \mathrm{E}-04$ & $6.791 \mathrm{E}+00$ & & & & 0.802 \\
\hline $1 s 2 s^{3} S_{1}$ & $1 s 7 f^{3} F_{2}^{o}$ & $1.3248 \mathrm{E}+00$ & $4.328 \mathrm{E}-14$ & $3.290 \mathrm{E}+01$ & & & & 1.000 \\
\hline $1 s 3 s^{3} S_{1}$ & $1 s 7 f^{3} F_{2}^{o}$ & $3.3851 \mathrm{E}+00$ & $6.587 \mathrm{E}-08$ & $7.668 \mathrm{E}+06$ & & & & 0.230 \\
\hline $1 s 3 d^{3} D_{2}$ & $1 s 7 f^{3} F_{2}^{o}$ & $3.5247 \mathrm{E}+00$ & $8.645 \mathrm{E}-03$ & $9.283 \mathrm{E}+11$ & $3.5099 \mathrm{E}+00$ & $8.646 \mathrm{E}-03$ & $9.283 \mathrm{E}+11$ & 0.001 \\
\hline $1 s 3 d^{3} D_{1}$ & $1 s 7 f^{3} F_{2}^{o}$ & $3.5262 \mathrm{E}+00$ & $7.731 \mathrm{E}-02$ & $8.295 \mathrm{E}+12$ & $3.5113 \mathrm{E}+00$ & $7.733 \mathrm{E}-02$ & $8.296 \mathrm{E}+12$ & 0.000 \\
\hline $1 s 3 d^{3} D_{3}$ & $1 s 7 f^{3} F_{2}^{o}$ & $3.5614 \mathrm{E}+00$ & $3.920 \mathrm{E}-04$ & $4.123 \mathrm{E}+10$ & $3.5464 \mathrm{E}+00$ & $3.833 \mathrm{E}-04$ & $4.031 \mathrm{E}+10$ & 0.009 \\
\hline $1 s 3 d^{1} D_{2}$ & $1 s 7 f^{3} F_{2}^{o}$ & $3.5628 \mathrm{E}+00$ & $5.413 \mathrm{E}-03$ & $5.689 \mathrm{E}+11$ & $3.5478 \mathrm{E}+00$ & $5.315 \mathrm{E}-03$ & $5.586 \mathrm{E}+11$ & 0.001 \\
\hline $1 s 4 s^{3} S_{1}$ & $1 s 7 f^{3} F_{2}^{o}$ & $7.3170 \mathrm{E}+00$ & $2.637 \mathrm{E}-07$ & $6.571 \mathrm{E}+06$ & & & & 0.196 \\
\hline $1 s 4 d^{3} D_{2}$ & $1 s 7 f^{3} F_{2}^{o}$ & $7.5889 \mathrm{E}+00$ & $2.421 \mathrm{E}-02$ & $5.609 \mathrm{E}+11$ & $7.5575 \mathrm{E}+00$ & $2.425 \mathrm{E}-02$ & $5.616 \mathrm{E}+11$ & 0.001 \\
\hline $1 s 4 d^{3} D_{1}$ & $1 s 7 f^{3} F_{2}^{o}$ & $7.5917 \mathrm{E}+00$ & $2.162 \mathrm{E}-01$ & $5.004 \mathrm{E}+12$ & $7.5603 \mathrm{E}+00$ & $2.161 \mathrm{E}-01$ & $5.003 \mathrm{E}+12$ & 0.000 \\
\hline $1 s 4 d^{3} D_{3}$ & $1 s 7 f^{3} F_{2}^{o}$ & $7.6607 \mathrm{E}+00$ & $1.123 \mathrm{E}-03$ & $2.554 \mathrm{E}+10$ & $7.6289 \mathrm{E}+00$ & $1.119 \mathrm{E}-03$ & $2.543 E+10$ & 0.001 \\
\hline
\end{tabular}


Table 3 (continued)

\begin{tabular}{|c|c|c|c|c|c|c|c|c|}
\hline \multirow[t]{2}{*}{ Lower } & \multirow[t]{2}{*}{ Upper } & \multicolumn{3}{|l|}{ GRASP2K } & \multicolumn{3}{|l|}{ FAC } & \multirow[t]{2}{*}{$d T$} \\
\hline & & $\lambda(\AA)$ & $g f$ & $A\left(\mathrm{~s}^{-1}\right)$ & $\lambda(\AA)$ & $g f$ & $A\left(\mathrm{~s}^{-1}\right)$ & \\
\hline $1 s 4 d^{1} D_{2}$ & $1 s 7 f^{3} F_{2}^{o}$ & $7.6637 \mathrm{E}+00$ & $1.559 \mathrm{E}-02$ & $3.541 \mathrm{E}+11$ & $7.6319 E+00$ & $1.544 \mathrm{E}-02$ & $3.507 E+11$ & 0.000 \\
\hline $1 s 5 s^{3} s_{1}$ & $1 s 7 f^{3} F_{2}^{o}$ & $1.5646 \mathrm{E}+01$ & $7.887 \mathrm{E}-07$ & $4.298 \mathrm{E}+06$ & & & & 0.141 \\
\hline $1 s 5 d^{3} D_{2}$ & $1 s 7 f^{3} F_{2}^{o}$ & $1.6281 \mathrm{E}+01$ & $6.482 \mathrm{E}-02$ & $3.262 \mathrm{E}+11$ & $1.6216 \mathrm{E}+01$ & $6.497 \mathrm{E}-02$ & $3.268 \mathrm{E}+11$ & 0.001 \\
\hline $1 s 5 d^{3} D_{1}$ & $1 s 7 f^{3} F_{2}^{o}$ & $1.6288 \mathrm{E}+01$ & $5.783 \mathrm{E}-01$ & $2.908 \mathrm{E}+12$ & $1.6222 \mathrm{E}+01$ & $5.783 \mathrm{E}-01$ & $2.906 \mathrm{E}+12$ & 0.000 \\
\hline $1 s 5 d^{3} D_{3}$ & $1 s 7 f^{3} F_{2}^{0}$ & $1.6451 \mathrm{E}+01$ & $3.084 \mathrm{E}-03$ & $1.520 \mathrm{E}+10$ & $1.6384 \mathrm{E}+01$ & $3.094 \mathrm{E}-03$ & $1.524 \mathrm{E}+10$ & 0.001 \\
\hline $1 s 5 d^{1} D_{2}$ & $1 s 7 f^{3} F_{2}^{o}$ & $1.6458 \mathrm{E}+01$ & $4.283 \mathrm{E}-02$ & $2.110 \mathrm{E}+11$ & $1.6391 \mathrm{E}+01$ & $4.261 \mathrm{E}-02$ & $2.098 \mathrm{E}+11$ & 0.000 \\
\hline $1 s 5 g{ }^{3} G_{3}$ & $1 s 7 f^{3} F_{2}^{o}$ & $1.6541 \mathrm{E}+01$ & $8.275 E-03$ & $4.035 \mathrm{E}+10$ & $1.6472 \mathrm{E}+01$ & $8.273 E-03$ & $4.033 E+10$ & 0.005 \\
\hline $1 s 6 s^{3} s_{1}$ & $1 s 7 f^{3} F_{2}^{o}$ & $4.0528 \mathrm{E}+01$ & $3.074 \mathrm{E}-06$ & $2.497 \mathrm{E}+06$ & & & & 0.094 \\
\hline $1 s 6 d^{3} D_{2}$ & $1 s 7 f^{3} F_{2}^{o}$ & $4.3034 \mathrm{E}+01$ & $2.645 \mathrm{E}-01$ & $1.905 \mathrm{E}+11$ & $4.2880 \mathrm{E}+01$ & $2.675 \mathrm{E}-01$ & $1.924 \mathrm{E}+11$ & 0.000 \\
\hline $1 s 6 d^{3} D_{1}$ & $1 s 7 f^{3} F_{2}^{o}$ & $4.3060 \mathrm{E}+01$ & $2.361 \mathrm{E}+00$ & $1.698 \mathrm{E}+12$ & $4.2874 \mathrm{E}+01$ & $2.361 \mathrm{E}+00$ & $1.699 \mathrm{E}+12$ & 0.000 \\
\hline $1 s 6 d^{3} D_{3}$ & $1 s 7 f^{3} F_{2}^{o}$ & $4.3722 \mathrm{E}+01$ & $1.309 \mathrm{E}-02$ & $9.135 \mathrm{E}+09$ & $4.3560 \mathrm{E}+01$ & $1.319 \mathrm{E}-02$ & $9.200 E+09$ & 0.000 \\
\hline $1 s 6 d^{1} D_{2}$ & $1 s 7 f^{3} F_{2}^{o}$ & $4.3752 \mathrm{E}+01$ & $1.821 \mathrm{E}-01$ & $1.269 \mathrm{E}+11$ & $4.3571 \mathrm{E}+01$ & $1.795 \mathrm{E}-01$ & $1.251 \mathrm{E}+11$ & 0.000 \\
\hline $1 s 6 g{ }^{3} G_{3}$ & $1 s 7 f^{3} F_{2}^{o}$ & $4.4094 \mathrm{E}+01$ & $1.383 \mathrm{E}-01$ & $9.486 \mathrm{E}+10$ & $4.3907 \mathrm{E}+01$ & $1.384 \mathrm{E}-01$ & $9.499 E+10$ & 0.002 \\
\hline $1 s 7 s^{3} S_{1}$ & $1 s 7 f^{3} F_{2}^{o}$ & $8.7540 \mathrm{E}+02$ & $1.283 \mathrm{E}-06$ & $2.234 \mathrm{E}+03$ & & & & 0.488 \\
\hline $1 s 7 d^{3} D_{2}$ & $1 s 7 f^{3} F_{2}^{o}$ & $4.1806 \mathrm{E}+03$ & $2.294 \mathrm{E}-02$ & $1.751 \mathrm{E}+06$ & $4.3602 \mathrm{E}+03$ & $2.205 \mathrm{E}-02$ & $1.534 \mathrm{E}+06$ & 0.022 \\
\hline $1 s 7 d^{3} D_{1}$ & $1 s 7 f^{3} F_{2}^{o}$ & $4.3395 \mathrm{E}+03$ & $1.964 \mathrm{E}-01$ & $1.391 \mathrm{E}+07$ & $4.3189 \mathrm{E}+03$ & $1.965 \mathrm{E}-01$ & $1.393 \mathrm{E}+07$ & 0.003 \\
\hline $1 s 7 d^{3} D_{3}$ & $1 s 7 f^{3} F_{2}^{o}$ & $1.0992 \mathrm{E}+05$ & $4.006 \mathrm{E}-05$ & $4.423 \mathrm{E}+00$ & & & & 0.425 \\
\hline $1 s 2 p^{3} P_{1}^{o}$ & $1 s 7 d^{1} D_{2}$ & $1.3358 \mathrm{E}+00$ & $3.593 \mathrm{E}-05$ & $2.686 \mathrm{E}+10$ & $1.3303 \mathrm{E}+00$ & $2.913 \mathrm{E}-05$ & $2.177 \mathrm{E}+10$ & 0.084 \\
\hline $1 s 2 p^{3} P_{2}^{0}$ & $1 s 7 d^{1} D_{2}$ & $1.3942 \mathrm{E}+00$ & $3.581 \mathrm{E}-03$ & $2.458 \mathrm{E}+12$ & $1.3883 \mathrm{E}+00$ & $3.597 \mathrm{E}-03$ & $2.468 \mathrm{E}+12$ & 0.003 \\
\hline $1 s 2 p^{1} P_{1}^{o}$ & $1 s 7 d^{1} D_{2}$ & $1.3997 \mathrm{E}+00$ & $3.271 \mathrm{E}-02$ & $2.227 \mathrm{E}+13$ & $1.3939 \mathrm{E}+00$ & $3.326 \mathrm{E}-02$ & $2.264 \mathrm{E}+13$ & 0.003 \\
\hline $1 s 3 p^{3} P_{1}^{0}$ & $1 s 7 d^{1} D_{2}$ & $3.4047 \mathrm{E}+00$ & 7.199E-05 & $8.285 \mathrm{E}+09$ & $3.3905 \mathrm{E}+00$ & $5.619 \mathrm{E}-05$ & $6.465 E+09$ & 0.008 \\
\hline $1 s 3 p^{3} P_{2}^{o}$ & $1 s 7 d^{1} D_{2}$ & $3.5163 \mathrm{E}+00$ & $8.443 E-03$ & $9.110 \mathrm{E}+11$ & $3.5016 \mathrm{E}+00$ & $8.386 \mathrm{E}-03$ & $9.046 \mathrm{E}+11$ & 0.000 \\
\hline $1 s 3 p^{1} P_{1}^{o}$ & $1 s 7 d^{1} D_{2}$ & $3.5262 \mathrm{E}+00$ & $7.729 \mathrm{E}-02$ & $8.293 E+12$ & $3.5116 \mathrm{E}+00$ & $7.772 \mathrm{E}-02$ & $8.336 E+12$ & 0.000 \\
\hline $1 s 4 p^{3} P_{1}^{o}$ & $1 s 7 d^{1} D_{2}$ & $7.3543 \mathrm{E}+00$ & $1.374 \mathrm{E}-04$ & $3.390 \mathrm{E}+09$ & $7.3231 \mathrm{E}+00$ & $1.131 \mathrm{E}-04$ & $2.791 \mathrm{E}+09$ & 0.017 \\
\hline $1 s 4 p^{3} P_{2}^{o}$ & $1 s 7 d^{1} D_{2}$ & $7.5726 \mathrm{E}+00$ & $1.795 \mathrm{E}-02$ & $4.176 \mathrm{E}+11$ & $7.5404 \mathrm{E}+00$ & $1.783 \mathrm{E}-02$ & $4.149 \mathrm{E}+11$ & 0.000 \\
\hline $1 s 4 p^{1} P_{1}^{o}$ & $1 s 7 d^{1} D_{2}$ & $7.5916 \mathrm{E}+00$ & $1.652 \mathrm{E}-01$ & $3.823 \mathrm{E}+12$ & $7.5595 \mathrm{E}+00$ & $1.654 \mathrm{E}-01$ & $3.829 \mathrm{E}+12$ & 0.001 \\
\hline $1 s 4 f^{3} F_{3}^{o}$ & $1 s 7 d^{1} D_{2}$ & $7.6618 \mathrm{E}+00$ & $1.695 \mathrm{E}-05$ & $3.852 \mathrm{E}+08$ & $7.6293 \mathrm{E}+00$ & $2.044 \mathrm{E}-05$ & $4.644 \mathrm{E}+08$ & 0.001 \\
\hline $1 s 4 f^{3} F_{2}^{o}$ & $1 s 7 d^{1} D_{2}$ & $7.6633 \mathrm{E}+00$ & $1.769 \mathrm{E}-04$ & $4.018 \mathrm{E}+09$ & $7.6308 \mathrm{E}+00$ & $1.751 \mathrm{E}-04$ & $3.978 E+09$ & 0.001 \\
\hline $1 s 4 f^{1} F_{3}^{o}$ & $1 s 7 d^{1} D_{2}$ & $7.6990 \mathrm{E}+00$ & $4.105 \mathrm{E}-03$ & $9.239 \mathrm{E}+10$ & $7.6663 \mathrm{E}+00$ & $4.113 \mathrm{E}-03$ & $9.258 \mathrm{E}+10$ & 0.001 \\
\hline $1 s 5 p^{3} P_{1}^{o}$ & $1 s 7 d^{1} D_{2}$ & $1.5732 \mathrm{E}+01$ & $3.089 \mathrm{E}-04$ & $1.665 \mathrm{E}+09$ & $1.5665 \mathrm{E}+01$ & $2.767 \mathrm{E}-04$ & $1.492 \mathrm{E}+09$ & 0.013 \\
\hline $1 s 5 p^{3} P_{2}^{0}$ & $1 s 7 d^{1} D_{2}$ & $1.6243 \mathrm{E}+01$ & $4.406 \mathrm{E}-02$ & $2.228 \mathrm{E}+11$ & $1.6173 \mathrm{E}+01$ & $4.394 \mathrm{E}-02$ & $2.222 \mathrm{E}+11$ & 0.002 \\
\hline $1 s 5 p^{1} P_{1}^{o}$ & $1 s 7 d^{1} D_{2}$ & $1.6288 \mathrm{E}+01$ & $4.077 \mathrm{E}-01$ & $2.050 \mathrm{E}+12$ & $1.6218 \mathrm{E}+01$ & $4.078 \mathrm{E}-01$ & $2.051 \mathrm{E}+12$ & 0.002 \\
\hline $1 s 5 f^{3} F_{3}^{o}$ & $1 s 7 d^{1} D_{2}$ & $1.6454 \mathrm{E}+01$ & $1.308 \mathrm{E}-04$ & $6.446 \mathrm{E}+08$ & $1.6384 \mathrm{E}+01$ & $1.575 \mathrm{E}-04$ & $7.764 \mathrm{E}+08$ & 0.001 \\
\hline $1 s 5 f^{3} F_{2}^{0}$ & $1 s 7 d^{1} D_{2}$ & $1.6457 \mathrm{E}+01$ & $1.369 \mathrm{E}-03$ & $6.741 \mathrm{E}+09$ & $1.6387 \mathrm{E}+01$ & $1.355 \mathrm{E}-03$ & $6.674 \mathrm{E}+09$ & 0.001 \\
\hline $1 s 5 f^{1} F_{3}^{o}$ & $1 s 7 d^{1} D_{2}$ & $1.6542 \mathrm{E}+01$ & $3.181 \mathrm{E}-02$ & $1.551 \mathrm{E}+11$ & $1.6471 \mathrm{E}+01$ & $3.185 \mathrm{E}-02$ & $1.553 \mathrm{E}+11$ & 0.000 \\
\hline $1 s 6 p^{3} P_{1}^{0}$ & $1 s 7 d^{1} D_{2}$ & $4.0858 \mathrm{E}+01$ & $1.084 \mathrm{E}-03$ & $8.665 \mathrm{E}+08$ & $4.0674 \mathrm{E}+01$ & $2.276 \mathrm{E}-03$ & $1.819 \mathrm{E}+09$ & 0.010 \\
\hline $1 s 6 p^{3} P_{2}^{o}$ & $1 s 7 d^{1} D_{2}$ & $4.2880 \mathrm{E}+01$ & $1.755 \mathrm{E}-01$ & $1.273 \mathrm{E}+11$ & $4.2684 \mathrm{E}+01$ & $1.759 \mathrm{E}-01$ & $1.277 \mathrm{E}+11$ & 0.000 \\
\hline $1 s 6 p^{1} P_{1}^{o}$ & $1 s 7 d^{1} D_{2}$ & $4.3058 \mathrm{E}+01$ & $1.639 \mathrm{E}+00$ & $1.180 \mathrm{E}+12$ & $4.2841 \mathrm{E}+01$ & $1.631 \mathrm{E}+00$ & $1.175 \mathrm{E}+12$ & 0.000 \\
\hline $1 s 6 f{ }^{3} F_{3}^{o}$ & $1 s 7 d^{1} D_{2}$ & $4.3733 \mathrm{E}+01$ & $1.210 \mathrm{E}-03$ & $8.439 E+08$ & $4.3554 \mathrm{E}+01$ & $1.357 \mathrm{E}-03$ & $9.465 E+08$ & 0.002 \\
\hline $1 s 6 f^{3} F_{2}^{0}$ & $1 s 7 d^{1} D_{2}$ & $4.3748 \mathrm{E}+01$ & $1.269 \mathrm{E}-02$ & $8.846 \mathrm{E}+09$ & $4.3554 \mathrm{E}+01$ & $1.249 \mathrm{E}-02$ & $8.711 \mathrm{E}+09$ & 0.000 \\
\hline $1 s 6 f^{1} F_{3}^{o}$ & $1 s 7 d^{1} D_{2}$ & $4.4094 \mathrm{E}+01$ & $2.963 \mathrm{E}-01$ & $2.033 \mathrm{E}+11$ & $4.3901 \mathrm{E}+01$ & $2.970 \mathrm{E}-01$ & $2.038 \mathrm{E}+11$ & 0.000 \\
\hline $1 s 7 p^{3} P_{1}^{0}$ & $1 s 7 d^{1} D_{2}$ & $9.8772 \mathrm{E}+02$ & $8.471 \mathrm{E}-04$ & $1.158 \mathrm{E}+06$ & $9.7564 \mathrm{E}+02$ & $1.201 \mathrm{E}-03$ & $1.670 \mathrm{E}+06$ & 0.072 \\
\hline $1 s 7 p^{3} P_{2}^{o}$ & $1 s 7 d^{1} D_{2}$ & $3.4747 \mathrm{E}+03$ & $2.994 \mathrm{E}-02$ & $3.308 \mathrm{E}+06$ & $3.3529 E+03$ & $3.063 \mathrm{E}-02$ & $3.604 \mathrm{E}+06$ & 0.158 \\
\hline $1 s 7 p^{1} P_{1}^{o}$ & $1 s 7 d^{1} D_{2}$ & $4.4062 \mathrm{E}+03$ & $2.162 \mathrm{E}-01$ & $1.486 \mathrm{E}+07$ & $4.0947 \mathrm{E}+03$ & $2.330 \mathrm{E}-01$ & $1.838 \mathrm{E}+07$ & 0.194 \\
\hline $1 s 7 f^{3} F_{3}^{o}$ & $1 s 7 d^{1} D_{2}$ & $1.7858 \mathrm{E}+05$ & $3.308 \mathrm{E}-05$ & $1.384 \mathrm{E}+00$ & & & & 0.998 \\
\hline $1 s 7 f^{3} F_{2}^{o}$ & $1 s 7 d^{1} D_{2}$ & $1.2412 \mathrm{E}+06$ & $4.984 \mathrm{E}-05$ & $4.316 \mathrm{E}-02$ & & & & 0.703 \\
\hline $1 s 3 d^{3} D_{3}$ & $1 s 7 f^{3} F_{4}^{o}$ & $3.5600 \mathrm{E}+00$ & $1.630 \mathrm{E}-01$ & $9.533 \mathrm{E}+12$ & $3.5449 \mathrm{E}+00$ & $1.642 \mathrm{E}-01$ & $9.604 \mathrm{E}+12$ & 0.001 \\
\hline $1 s 4 d^{3} D_{3}$ & $1 s 7 f^{3} F_{4}^{o}$ & $7.6543 \mathrm{E}+00$ & $4.614 \mathrm{E}-01$ & $5.836 \mathrm{E}+12$ & $7.6222 \mathrm{E}+00$ & $4.634 \mathrm{E}-01$ & $5.861 \mathrm{E}+12$ & 0.001 \\
\hline $1 s 5 d^{3} D_{3}$ & $1 s 7 f^{3} F_{4}^{0}$ & $1.6421 \mathrm{E}+01$ & $1.249 \mathrm{E}+00$ & $3.434 \mathrm{E}+12$ & $1.6353 \mathrm{E}+01$ & $1.253 \mathrm{E}+00$ & $3.444 \mathrm{E}+12$ & 0.001 \\
\hline $1 s 5 g^{3} G_{4}$ & $1 s 7 f^{3} F_{4}^{o}$ & $1.6509 \mathrm{E}+01$ & $3.674 \mathrm{E}-04$ & $9.991 \mathrm{E}+08$ & $1.6439 \mathrm{E}+01$ & $3.709 E-04$ & $1.008 E+09$ & 0.002 \\
\hline $1 s 5 g{ }^{3} G_{3}$ & $1 s 7 f^{3} F_{4}^{o}$ & $1.6511 \mathrm{E}+01$ & $1.046 \mathrm{E}-05$ & $2.843 \mathrm{E}+07$ & & & & 0.007 \\
\hline $1 s 5 g^{3} G_{5}$ & $1 s 7 f^{3} F_{4}^{0}$ & $1.6560 \mathrm{E}+01$ & $1.366 \mathrm{E}-02$ & $3.691 \mathrm{E}+10$ & $1.6489 \mathrm{E}+01$ & $1.369 \mathrm{E}-02$ & $3.700 E+10$ & 0.003 \\
\hline $1 s 5 g^{1} G_{4}$ & $1 s 7 f^{3} F_{4}^{0}$ & $1.6562 \mathrm{E}+01$ & $3.137 \mathrm{E}-04$ & $8.476 \mathrm{E}+08$ & $1.6491 \mathrm{E}+01$ & $3.124 \mathrm{E}-04$ & $8.442 E+08$ & 0.005 \\
\hline $1 s 6 d^{3} D_{3}$ & $1 s 7 f^{3} F_{4}^{o}$ & $4.3514 \mathrm{E}+01$ & $5.201 \mathrm{E}+00$ & $2.036 \mathrm{E}+12$ & $4.3345 \mathrm{E}+01$ & $5.214 \mathrm{E}+00$ & $2.039 E+12$ & 0.001 \\
\hline $1 s 6 g{ }^{3} G_{4}$ & $1 s 7 f^{3} F_{4}^{o}$ & $4.3874 \mathrm{E}+01$ & $6.176 \mathrm{E}-03$ & $2.378 \mathrm{E}+09$ & $4.3688 \mathrm{E}+01$ & $6.221 \mathrm{E}-03$ & $2.395 E+09$ & 0.001 \\
\hline $1 s 6 g^{3} G_{3}$ & $1 s 7 f^{3} F_{4}^{0}$ & $4.3882 \mathrm{E}+01$ & $1.758 \mathrm{E}-04$ & $6.767 \mathrm{E}+07$ & & & & 0.003 \\
\hline $1 s 6 g^{3} G_{5}$ & $1 s 7 f^{3} F_{4}^{o}$ & $4.4083 \mathrm{E}+01$ & $2.292 \mathrm{E}-01$ & $8.743 E+10$ & $4.3895 \mathrm{E}+01$ & $2.295 \mathrm{E}-01$ & $8.752 E+10$ & 0.001 \\
\hline $1 s 6 g{ }^{1} G_{4}$ & $1 s 7 f^{3} F_{4}^{o}$ & $4.4090 \mathrm{E}+01$ & $5.247 \mathrm{E}-03$ & $2.001 \mathrm{E}+09$ & $4.3895 \mathrm{E}+01$ & $5.215 \mathrm{E}-03$ & $1.989 E+09$ & 0.002 \\
\hline $1 s 7 d^{3} D_{3}$ & $1 s 7 f^{3} F_{4}^{0}$ & $8.4332 \mathrm{E}+03$ & $2.169 \mathrm{E}-01$ & $2.260 \mathrm{E}+06$ & $8.7593 E+03$ & $2.079 \mathrm{E}-01$ & $1.991 \mathrm{E}+06$ & 0.031 \\
\hline $1 s 4 f^{3} F_{3}^{o}$ & $1 s 7 g{ }^{3} G_{4}$ & $7.6554 \mathrm{E}+00$ & $3.948 \mathrm{E}-01$ & $4.993 \mathrm{E}+12$ & $7.6229 \mathrm{E}+00$ & $3.951 \mathrm{E}-01$ & $4.997 \mathrm{E}+12$ & 0.000 \\
\hline $1 s 4 f^{3} F_{4}^{o}$ & $1 s 7 g{ }^{3} G_{4}$ & $7.6914 \mathrm{E}+00$ & $1.379 \mathrm{E}-02$ & $1.728 \mathrm{E}+11$ & $7.6587 \mathrm{E}+00$ & $1.370 \mathrm{E}-02$ & $1.716 \mathrm{E}+11$ & 0.001 \\
\hline $1 s 4 f^{1} F_{3}^{o}$ & $1 s 7 g{ }^{3} G_{4}$ & $7.6926 \mathrm{E}+00$ & $3.877 \mathrm{E}-04$ & $4.856 \mathrm{E}+09$ & $7.6598 \mathrm{E}+00$ & $3.390 \mathrm{E}-04$ & $4.246 E+09$ & 0.000 \\
\hline $1 s 5 f^{3} F_{3}^{0}$ & $1 s 7 g{ }^{3} G_{4}$ & $1.6424 \mathrm{E}+01$ & $1.538 \mathrm{E}+00$ & $4.224 \mathrm{E}+12$ & $1.6354 \mathrm{E}+01$ & $1.539 \mathrm{E}+00$ & $4.229 \mathrm{E}+12$ & 0.000 \\
\hline $1 s 5 f^{3} F_{4}^{o}$ & $1 s 7 g{ }^{3} G_{4}$ & $1.6509 \mathrm{E}+01$ & $5.485 \mathrm{E}-02$ & $1.492 \mathrm{E}+11$ & $1.6439 \mathrm{E}+01$ & $5.482 \mathrm{E}-02$ & $1.490 \mathrm{E}+11$ & 0.000 \\
\hline $1 s 5 f^{1} F_{3}^{o}$ & $1 s 7 g{ }^{3} G_{4}$ & $1.6512 \mathrm{E}+01$ & $1.557 \mathrm{E}-03$ & $4.233 \mathrm{E}+09$ & $1.6441 \mathrm{E}+01$ & $1.373 \mathrm{E}-03$ & $3.733 E+09$ & 0.000 \\
\hline $1 s 6 f^{3} F_{3}^{o}$ & $1 s 7 g{ }^{3} G_{4}$ & $4.3526 \mathrm{E}+01$ & $7.332 \mathrm{E}+00$ & $2.868 \mathrm{E}+12$ & $4.3346 \mathrm{E}+01$ & $7.341 \mathrm{E}+00$ & $2.871 \mathrm{E}+12$ & 0.000 \\
\hline $1 s 6 f^{3} F_{4}^{0}$ & $1 s 7 g{ }^{3} G_{4}$ & $4.3873 \mathrm{E}+01$ & $2.683 \mathrm{E}-01$ & $1.033 \mathrm{E}+11$ & $4.3689 \mathrm{E}+01$ & $2.690 \mathrm{E}-01$ & $1.035 \mathrm{E}+11$ & 0.000 \\
\hline $1 s 6 f^{1} F_{3}^{0}$ & $1 s 7 g{ }^{3} G_{4}$ & $4.3884 \mathrm{E}+01$ & $7.664 \mathrm{E}-03$ & $2.949 \mathrm{E}+09$ & $4.3689 \mathrm{E}+01$ & $7.956 \mathrm{E}-03$ & $3.063 E+09$ & 0.000 \\
\hline $1 s 6 h^{3} H_{5}^{o}$ & $1 s 7 g{ }^{3} G_{4}$ & $4.4083 \mathrm{E}+01$ & $7.172 \mathrm{E}-02$ & $2.735 \mathrm{E}+10$ & $4.3896 \mathrm{E}+01$ & $7.180 \mathrm{E}-02$ & $2.738 E+10$ & 0.001 \\
\hline $1 s 6 h^{3} H_{4}^{o}$ & $1 s 7 g{ }^{3} G_{4}$ & $4.4088 \mathrm{E}+01$ & $1.627 \mathrm{E}-03$ & $6.203 E+08$ & $4.3896 \mathrm{E}+01$ & $1.632 \mathrm{E}-03$ & $6.224 \mathrm{E}+08$ & 0.000 \\
\hline $1 s 6 h{ }^{1} H_{5}^{0}$ & $1 s 7 g{ }^{3} G_{4}$ & $4.4227 \mathrm{E}+01$ & $8.330 \mathrm{E}-09$ & $3.156 \mathrm{E}+03$ & & & & 0.017 \\
\hline
\end{tabular}


Table 3 (continued)

\begin{tabular}{|c|c|c|c|c|c|c|c|c|}
\hline \multirow[t]{2}{*}{ Lower } & \multirow[t]{2}{*}{ Upper } & \multicolumn{3}{|l|}{ GRASP2K } & \multicolumn{3}{|l|}{ FAC } & \multirow[t]{2}{*}{$d T$} \\
\hline & & $\lambda(\AA)$ & $g f$ & $A\left(\mathrm{~s}^{-1}\right)$ & $\lambda(\AA)$ & $g f$ & $A\left(\mathrm{~s}^{-1}\right)$ & \\
\hline $1 s 7 f^{3} F_{3}^{o}$ & $1 s 7 g{ }^{3} G_{4}$ & $8.7347 \mathrm{E}+03$ & $1.730 \mathrm{E}-01$ & $1.681 \mathrm{E}+06$ & $8.7974 \mathrm{E}+03$ & $1.710 \mathrm{E}-01$ & $1.623 \mathrm{E}+06$ & 0.020 \\
\hline $1 s 7 f^{3} F_{4}^{o}$ & $1 s 7 g{ }^{3} G_{4}$ & $4.7642 \mathrm{E}+06$ & $1.133 \mathrm{E}-05$ & $3.699 \mathrm{E}-04$ & & & & 0.957 \\
\hline $1 s 2 p^{3} P_{2}^{o}$ & $1 s 7 g{ }^{3} G_{3}$ & $1.3940 \mathrm{E}+00$ & $7.262 \mathrm{E}-10$ & $3.561 \mathrm{E}+05$ & & & & 0.771 \\
\hline $1 s 3 p^{3} P_{2}^{o}$ & $1 s 7 g{ }^{3} G_{3}$ & $3.5149 \mathrm{E}+00$ & $7.323 \mathrm{E}-13$ & $5.648 \mathrm{E}+01$ & & & & 0.981 \\
\hline $1 s 4 p^{3} P_{2}^{o}$ & $1 s 7 g{ }^{3} G_{3}$ & $7.5662 \mathrm{E}+00$ & $3.484 \mathrm{E}-08$ & $5.800 \mathrm{E}+05$ & & & & 0.013 \\
\hline $1 s 4 f^{3} F_{3}^{o}$ & $1 s 7 g{ }^{3} G_{3}$ & $7.6553 \mathrm{E}+00$ & $1.458 \mathrm{E}-02$ & $2.371 \mathrm{E}+11$ & $7.6229 \mathrm{E}+00$ & $1.457 \mathrm{E}-02$ & $2.370 \mathrm{E}+11$ & 0.000 \\
\hline $1 s 4 f^{3} F_{2}^{o}$ & $1 s 7 g{ }^{3} G_{3}$ & $7.6568 \mathrm{E}+00$ & $2.925 \mathrm{E}-01$ & $4.754 \mathrm{E}+12$ & $7.6244 \mathrm{E}+00$ & $2.926 \mathrm{E}-01$ & $4.755 \mathrm{E}+12$ & 0.000 \\
\hline $1 s 4 f^{3} F_{4}^{o}$ & $1 s 7 g{ }^{3} G_{3}$ & $7.6913 \mathrm{E}+00$ & $3.956 \mathrm{E}-04$ & $6.373 E+09$ & $7.6587 \mathrm{E}+00$ & $3.914 \mathrm{E}-04$ & $6.305 E+09$ & 0.001 \\
\hline $1 s 4 f^{1} F_{3}^{o}$ & $1 s 7 g{ }^{3} G_{3}$ & $7.6924 \mathrm{E}+00$ & $1.073 \mathrm{E}-02$ & $1.728 \mathrm{E}+11$ & $7.6598 \mathrm{E}+00$ & $1.062 \mathrm{E}-02$ & $1.710 \mathrm{E}+11$ & 0.001 \\
\hline $1 s 5 p^{3} P_{2}^{o}$ & $1 s 7 g{ }^{3} G_{3}$ & $1.6214 \mathrm{E}+01$ & $2.161 \mathrm{E}-07$ & $7.833 \mathrm{E}+05$ & & & & 0.004 \\
\hline $1 s 5 f^{3} F_{3}^{o}$ & $1 s 7 g{ }^{3} G_{3}$ & $1.6423 \mathrm{E}+01$ & $5.677 \mathrm{E}-02$ & $2.005 E+11$ & $1.6354 \mathrm{E}+01$ & $5.681 \mathrm{E}-02$ & $2.006 \mathrm{E}+11$ & 0.000 \\
\hline $1 s 5 f^{3} F_{2}^{o}$ & $1 s 7 g{ }^{3} G_{3}$ & $1.6427 \mathrm{E}+01$ & $1.139 \mathrm{E}+00$ & $4.023 \mathrm{E}+12$ & $1.6358 \mathrm{E}+01$ & $1.139 \mathrm{E}+00$ & $4.025 E+12$ & 0.000 \\
\hline $1 s 5 f^{3} F_{4}^{o}$ & $1 s 7 g{ }^{3} G_{3}$ & $1.6508 \mathrm{E}+01$ & $1.573 \mathrm{E}-03$ & $5.499 \mathrm{E}+09$ & $1.6439 \mathrm{E}+01$ & $1.566 \mathrm{E}-03$ & $5.476 \mathrm{E}+09$ & 0.001 \\
\hline $1 s 5 f^{1} F_{3}^{o}$ & $1 s 7 g{ }^{3} G_{3}$ & $1.6511 \mathrm{E}+01$ & $4.267 \mathrm{E}-02$ & $1.491 \mathrm{E}+11$ & $1.6441 \mathrm{E}+01$ & $4.248 \mathrm{E}-02$ & $1.484 \mathrm{E}+11$ & 0.001 \\
\hline $1 s 6 p^{3} P_{2}^{o}$ & $1 s 7 g{ }^{3} G_{3}$ & $4.2676 \mathrm{E}+01$ & $1.217 \mathrm{E}-06$ & $6.370 \mathrm{E}+05$ & & & & 0.001 \\
\hline $1 s 6 f^{3} F_{3}^{o}$ & $1 s 7 g{ }^{3} G_{3}$ & $4.3521 \mathrm{E}+01$ & $2.705 \mathrm{E}-01$ & $1.361 \mathrm{E}+11$ & $4.3346 \mathrm{E}+01$ & $2.721 \mathrm{E}-01$ & $1.368 \mathrm{E}+11$ & 0.000 \\
\hline $1 s 6 f^{3} F_{2}^{o}$ & $1 s 7 g{ }^{3} G_{3}$ & $4.3535 \mathrm{E}+01$ & $5.434 \mathrm{E}+00$ & $2.732 \mathrm{E}+12$ & $4.3346 \mathrm{E}+01$ & $5.438 \mathrm{E}+00$ & $2.734 \mathrm{E}+12$ & 0.000 \\
\hline $1 s 6 f^{3} F_{4}^{o}$ & $1 s 7 g{ }^{3} G_{3}$ & $4.3867 \mathrm{E}+01$ & $7.685 \mathrm{E}-03$ & $3.806 \mathrm{E}+09$ & $4.3689 \mathrm{E}+01$ & $7.686 \mathrm{E}-03$ & $3.804 \mathrm{E}+09$ & 0.000 \\
\hline $1 s 6 f{ }^{1} F_{3}^{o}$ & $1 s 7 g{ }^{3} G_{3}$ & $4.3878 \mathrm{E}+01$ & $2.087 \mathrm{E}-01$ & $1.033 \mathrm{E}+11$ & $4.3689 \mathrm{E}+01$ & $2.072 \mathrm{E}-01$ & $1.025 \mathrm{E}+11$ & 0.000 \\
\hline $1 s 6 h^{3} H_{4}^{o}$ & $1 s 7 g{ }^{3} G_{3}$ & $4.4083 \mathrm{E}+01$ & $5.701 \mathrm{E}-02$ & $2.796 \mathrm{E}+10$ & $4.3896 \mathrm{E}+01$ & $5.711 \mathrm{E}-02$ & $2.800 \mathrm{E}+10$ & 0.000 \\
\hline $1 s 7 p^{3} P_{2}^{o}$ & $1 s 7 g{ }^{3} G_{3}$ & $2.5035 \mathrm{E}+03$ & $1.166 \mathrm{E}-07$ & $1.772 \mathrm{E}+01$ & & & & 0.002 \\
\hline $1 s 7 f^{3} F_{3}^{o}$ & $1 s 7 g{ }^{3} G_{3}$ & $8.5297 \mathrm{E}+03$ & $6.540 \mathrm{E}-03$ & $8.565 E+04$ & $8.7975 E+03$ & $6.341 \mathrm{E}-03$ & $7.740 \mathrm{E}+04$ & 0.028 \\
\hline $1 s 7 f^{3} F_{2}^{o}$ & $1 s 7 g{ }^{3} G_{3}$ & $8.8934 \mathrm{E}+03$ & $1.258 \mathrm{E}-01$ & $1.516 \mathrm{E}+06$ & $8.7928 \mathrm{E}+03$ & $1.267 \mathrm{E}-01$ & $1.548 \mathrm{E}+06$ & 0.024 \\
\hline $1 s 7 f^{3} F_{4}^{o}$ & $1 s 7 g{ }^{3} G_{3}$ & $3.3768 \mathrm{E}+05$ & $4.572 \mathrm{E}-06$ & $3.820 \mathrm{E}-02$ & & & & 0.857 \\
\hline $1 s 3 d^{3} D_{2}$ & $1 s 7 f^{1} F_{3}^{o}$ & $3.5233 \mathrm{E}+00$ & $3.298 \mathrm{E}-06$ & $2.531 \mathrm{E}+08$ & & & & 0.031 \\
\hline $1 s 3 d^{3} D_{3}$ & $1 s 7 f^{1} F_{3}^{o}$ & $3.5600 \mathrm{E}+00$ & $6.068 \mathrm{E}-03$ & $4.563 \mathrm{E}+11$ & $3.5449 \mathrm{E}+00$ & $6.074 \mathrm{E}-03$ & $4.567 \mathrm{E}+11$ & 0.001 \\
\hline $1 s 3 d{ }^{1} D_{2}$ & $1 s 7 f^{1} F_{3}^{o}$ & $3.5614 \mathrm{E}+00$ & $1.207 \mathrm{E}-01$ & $9.071 \mathrm{E}+12$ & $3.5463 \mathrm{E}+00$ & $1.216 \mathrm{E}-01$ & $9.136 \mathrm{E}+12$ & 0.000 \\
\hline $1 s 4 d^{3} D_{2}$ & $1 s 7 f^{1} F_{3}^{o}$ & $7.5823 \mathrm{E}+00$ & $1.691 \mathrm{E}-05$ & $2.802 \mathrm{E}+08$ & & & & 0.029 \\
\hline $1 s 4 d^{3} D_{3}$ & $1 s 7 f^{1} F_{3}^{o}$ & $7.6541 \mathrm{E}+00$ & $1.717 \mathrm{E}-02$ & $2.792 \mathrm{E}+11$ & $7.6222 \mathrm{E}+00$ & $1.713 \mathrm{E}-02$ & $2.787 \mathrm{E}+11$ & 0.001 \\
\hline $1 s 4 d^{1} D_{2}$ & $1 s 7 f^{1} F_{3}^{o}$ & $7.6570 \mathrm{E}+00$ & $3.420 \mathrm{E}-01$ & $5.558 \mathrm{E}+12$ & $7.6252 \mathrm{E}+00$ & $3.431 \mathrm{E}-01$ & $5.576 \mathrm{E}+12$ & 0.001 \\
\hline $1 s 5 d^{3} D_{2}$ & $1 s 7 f^{1} F_{3}^{o}$ & $1.6251 \mathrm{E}+01$ & $5.598 \mathrm{E}-05$ & $2.020 \mathrm{E}+08$ & & & & 0.022 \\
\hline $1 s 5 d^{3} D_{3}$ & $1 s 7 f^{1} F_{3}^{o}$ & $1.6420 \mathrm{E}+01$ & $4.647 \mathrm{E}-02$ & $1.642 \mathrm{E}+11$ & $1.6353 \mathrm{E}+01$ & $4.635 \mathrm{E}-02$ & $1.637 \mathrm{E}+11$ & 0.000 \\
\hline $1 s 5 d{ }^{1} D_{2}$ & $1 s 7 f^{1} F_{3}^{o}$ & $1.6427 \mathrm{E}+01$ & $9.263 \mathrm{E}-01$ & $3.271 \mathrm{E}+12$ & $1.6360 \mathrm{E}+01$ & $9.280 \mathrm{E}-01$ & $3.275 \mathrm{E}+12$ & 0.000 \\
\hline $1 s 5 g{ }^{3} G_{4}$ & $1 s 7 f^{1} F_{3}^{o}$ & $1.6508 \mathrm{E}+01$ & $1.041 \mathrm{E}-05$ & $3.639 \mathrm{E}+07$ & & & & 0.015 \\
\hline $1 s 5 g{ }^{3} G_{3}$ & $1 s 7 f^{1} F_{3}^{o}$ & $1.6510 \mathrm{E}+01$ & $2.872 \mathrm{E}-04$ & $1.004 \mathrm{E}+09$ & $1.6441 \mathrm{E}+01$ & $2.866 \mathrm{E}-04$ & $1.002 \mathrm{E}+09$ & 0.006 \\
\hline $1 s 5 g^{1} G_{4}$ & $1 s 7 f^{1} F_{3}^{o}$ & $1.6561 \mathrm{E}+01$ & $1.090 \mathrm{E}-02$ & $3.787 \mathrm{E}+10$ & $1.6491 \mathrm{E}+01$ & $1.088 \mathrm{E}-02$ & $3.782 \mathrm{E}+10$ & 0.004 \\
\hline $1 s 6 d^{3} D_{2}$ & $1 s 7 f^{1} F_{3}^{o}$ & $4.2826 \mathrm{E}+01$ & $2.508 \mathrm{E}-04$ & $1.303 E+08$ & $4.2671 \mathrm{E}+01$ & $4.916 \mathrm{E}-04$ & $2.551 \mathrm{E}+08$ & 0.014 \\
\hline $1 s 6 d^{3} D_{3}$ & $1 s 7 f^{1} F_{3}^{o}$ & $4.3507 \mathrm{E}+01$ & $1.933 \mathrm{E}-01$ & $9.731 \mathrm{E}+10$ & $4.3345 \mathrm{E}+01$ & $1.928 \mathrm{E}-01$ & $9.698 \mathrm{E}+10$ & 0.000 \\
\hline $1 s 6 d{ }^{1} D_{2}$ & $1 s 7 f^{1} F_{3}^{o}$ & $4.3537 \mathrm{E}+01$ & $3.859 \mathrm{E}+00$ & $1.940 \mathrm{E}+12$ & $4.3355 \mathrm{E}+01$ & $3.861 \mathrm{E}+00$ & $1.941 \mathrm{E}+12$ & 0.000 \\
\hline $1 s 6 g^{3} G_{4}$ & $1 s 7 f^{1} F_{3}^{o}$ & $4.3867 \mathrm{E}+01$ & $1.761 \mathrm{E}-04$ & $8.718 \mathrm{E}+07$ & & & & 0.006 \\
\hline $1 s 6 g^{3} G_{3}$ & $1 s 7 f^{1} F_{3}^{o}$ & $4.3875 \mathrm{E}+01$ & $4.808 \mathrm{E}-03$ & $2.380 \mathrm{E}+09$ & $4.3688 \mathrm{E}+01$ & $4.791 \mathrm{E}-03$ & $2.372 \mathrm{E}+09$ & 0.003 \\
\hline $1 s 6 g{ }^{1} G_{4}$ & $1 s 7 f^{1} F_{3}^{o}$ & $4.4083 \mathrm{E}+01$ & $1.824 \mathrm{E}-01$ & $8.943 E+10$ & $4.3895 \mathrm{E}+01$ & $1.825 \mathrm{E}-01$ & $8.952 E+10$ & 0.002 \\
\hline $1 s 7 d^{3} D_{2}$ & $1 s 7 f^{1} F_{3}^{o}$ & $2.8385 \mathrm{E}+03$ & $3.561 \mathrm{E}-05$ & $4.211 \mathrm{E}+03$ & $2.9112 \mathrm{E}+03$ & $6.098 \mathrm{E}-05$ & $6.798 E+03$ & 0.242 \\
\hline $1 s 7 d^{3} D_{3}$ & $1 s 7 f^{1} F_{3}^{o}$ & $8.1837 \mathrm{E}+03$ & $8.316 \mathrm{E}-03$ & $1.183 \mathrm{E}+05$ & $8.7525 \mathrm{E}+03$ & $7.695 \mathrm{E}-03$ & $9.491 \mathrm{E}+04$ & 0.023 \\
\hline $1 s 7 d^{1} D_{2}$ & $1 s 7 f^{1} F_{3}^{o}$ & $8.9054 \mathrm{E}+03$ & $1.521 \mathrm{E}-01$ & $1.827 \mathrm{E}+06$ & $9.0373 \mathrm{E}+03$ & $1.492 \mathrm{E}-01$ & $1.726 \mathrm{E}+06$ & 0.011 \\
\hline $1 s 7 g{ }^{3} G_{4}$ & $1 s 7 f^{1} F_{3}^{o}$ & $2.9370 \mathrm{E}+05$ & $5.267 \mathrm{E}-06$ & $5.818 \mathrm{E}-02$ & & & & 0.814 \\
\hline $1 s 7 g^{3} G_{3}$ & $1 s 7 f^{1} F_{3}^{o}$ & $1.5307 \mathrm{E}+06$ & $2.742 \mathrm{E}-05$ & $1.115 \mathrm{E}-02$ & & & & 0.915 \\
\hline $1 s 4 f^{3} F_{4}^{o}$ & $1 s 7 g{ }^{3} G_{5}$ & $7.6874 \mathrm{E}+00$ & $4.969 \mathrm{E}-01$ & $5.098 \mathrm{E}+12$ & $7.6547 \mathrm{E}+00$ & $4.989 \mathrm{E}-01$ & $5.120 \mathrm{E}+12$ & 0.000 \\
\hline $1 s 5 f^{3} F_{4}^{0}$ & $1 s 7 g{ }^{3} G_{5}$ & $1.6491 \mathrm{E}+01$ & $1.948 \mathrm{E}+00$ & $4.343 \mathrm{E}+12$ & $1.6420 \mathrm{E}+01$ & $1.952 \mathrm{E}+00$ & $4.354 \mathrm{E}+12$ & 0.000 \\
\hline $1 s 6 f^{3} F_{4}^{o}$ & $1 s 7 g{ }^{3} G_{5}$ & $4.3742 \mathrm{E}+01$ & $9.373 E+00$ & $2.970 \mathrm{E}+12$ & $4.3559 \mathrm{E}+01$ & $9.390 \mathrm{E}+00$ & $2.975 \mathrm{E}+12$ & 0.000 \\
\hline $1 s 6 h^{3} H_{5}^{o}$ & $1 s 7 g{ }^{3} G_{5}$ & $4.3951 \mathrm{E}+01$ & $1.512 \mathrm{E}-03$ & $4.745 E+08$ & $4.3765 \mathrm{E}+01$ & $1.522 \mathrm{E}-03$ & $4.777 \mathrm{E}+08$ & 0.001 \\
\hline $1 s 6 h^{3} H_{4}^{o}$ & $1 s 7 g{ }^{3} G_{5}$ & $4.3956 \mathrm{E}+01$ & $2.810 \mathrm{E}-05$ & $8.818 \mathrm{E}+06$ & & & & 0.001 \\
\hline $1 s 6 h^{3} H_{6}^{0}$ & $1 s 7 g^{3} G_{5}$ & $4.4090 \mathrm{E}+01$ & $8.517 \mathrm{E}-02$ & $2.657 \mathrm{E}+10$ & $4.3903 \mathrm{E}+01$ & $8.525 \mathrm{E}-02$ & $2.659 \mathrm{E}+10$ & 0.000 \\
\hline $1 s 6 h^{1} H_{5}^{o}$ & $1 s 7 g{ }^{3} G_{5}$ & $4.4094 \mathrm{E}+01$ & $1.316 \mathrm{E}-03$ & $4.106 \mathrm{E}+08$ & $4.3903 \mathrm{E}+01$ & $1.311 \mathrm{E}-03$ & $4.091 \mathrm{E}+08$ & 0.000 \\
\hline $1 s 7 f^{3} F_{4}^{o}$ & $1 s 7 g{ }^{3} G_{5}$ & $1.4632 \mathrm{E}+04$ & $1.310 \mathrm{E}-01$ & $3.709 E+05$ & $1.4765 \mathrm{E}+04$ & $1.292 \mathrm{E}-01$ & $3.564 \mathrm{E}+05$ & 0.023 \\
\hline $1 s 5 g^{3} G_{4}$ & $1 s 7 h^{3} H_{5}^{o}$ & $1.6491 \mathrm{E}+01$ & $1.774 \mathrm{E}+00$ & $3.956 \mathrm{E}+12$ & $1.6420 \mathrm{E}+01$ & $1.776 \mathrm{E}+00$ & $3.960 \mathrm{E}+12$ & 0.000 \\
\hline $1 s 5 g^{3} G_{5}$ & $1 s 7 h^{3} H_{5}^{o}$ & $1.6542 \mathrm{E}+01$ & $3.898 \mathrm{E}-02$ & $8.638 \mathrm{E}+10$ & $1.6471 \mathrm{E}+01$ & $3.887 \mathrm{E}-02$ & $8.615 E+10$ & 0.001 \\
\hline $1 s 5 g{ }^{1} G_{4}$ & $1 s 7 h^{3} H_{5}^{o}$ & $1.6543 \mathrm{E}+01$ & $6.999 \mathrm{E}-04$ & $1.551 \mathrm{E}+09$ & $1.6473 \mathrm{E}+01$ & $5.907 \mathrm{E}-04$ & $1.308 \mathrm{E}+09$ & 0.001 \\
\hline $1 s 6 g^{3} G_{4}$ & $1 s 7 h^{3} H_{5}^{o}$ & $4.3742 \mathrm{E}+01$ & $1.301 \mathrm{E}+01$ & $4.123 \mathrm{E}+12$ & $4.3559 \mathrm{E}+01$ & $1.302 \mathrm{E}+01$ & $4.126 \mathrm{E}+12$ & 0.000 \\
\hline $1 s 6 g^{3} G_{5}$ & $1 s 7 h^{3} H_{5}^{o}$ & $4.3950 \mathrm{E}+01$ & $2.913 \mathrm{E}-01$ & $9.143 \mathrm{E}+10$ & $4.3765 \mathrm{E}+01$ & $2.917 \mathrm{E}-01$ & $9.156 \mathrm{E}+10$ & 0.000 \\
\hline $1 s 6 g{ }^{1} G_{4}$ & $1 s 7 h^{3} H_{5}^{o}$ & $4.3957 \mathrm{E}+01$ & $5.234 \mathrm{E}-03$ & $1.643 \mathrm{E}+09$ & $4.3765 \mathrm{E}+01$ & $5.401 \mathrm{E}-03$ & $1.695 \mathrm{E}+09$ & 0.001 \\
\hline $1 s 7 g^{3} G_{4}$ & $1 s 7 h^{3} H_{5}^{o}$ & $1.4659 \mathrm{E}+04$ & $9.514 \mathrm{E}-02$ & $2.685 \mathrm{E}+05$ & $1.4689 \mathrm{E}+04$ & $9.453 \mathrm{E}-02$ & $2.634 \mathrm{E}+05$ & 0.021 \\
\hline $1 s 7 g^{3} G_{5}$ & $1 s 7 h^{3} H_{5}^{o}$ & $1.1751 \mathrm{E}+07$ & $2.630 \mathrm{E}-06$ & $1.154 \mathrm{E}-05$ & & & & 0.964 \\
\hline $1 s 3 d^{3} D_{3}$ & $1 s 7 h^{3} H_{4}^{o}$ & $3.5591 \mathrm{E}+00$ & $1.547 \mathrm{E}-12$ & $9.050 \mathrm{E}+01$ & & & & 0.940 \\
\hline $1 s 4 d^{3} D_{3}$ & $1 s 7 h^{3} H_{4}^{o}$ & $7.6502 \mathrm{E}+00$ & $3.959 \mathrm{E}-11$ & $5.014 \mathrm{E}+02$ & & & & 0.538 \\
\hline $1 s 5 d^{3} D_{3}$ & $1 s 7 h^{3} H_{4}^{o}$ & $1.6402 \mathrm{E}+01$ & $7.418 \mathrm{E}-10$ & $2.043 E+03$ & & & & 0.061 \\
\hline $1 s 5 g{ }^{3} G_{4}$ & $1 s 7 h^{3} H_{4}^{0}$ & $1.6490 \mathrm{E}+01$ & $4.020 \mathrm{E}-02$ & $1.096 \mathrm{E}+11$ & $1.6420 \mathrm{E}+01$ & $4.022 \mathrm{E}-02$ & $1.096 \mathrm{E}+11$ & 0.000 \\
\hline $1 s 5 g{ }^{3} G_{3}$ & $1 s 7 h^{3} H_{4}^{o}$ & $1.6492 \mathrm{E}+01$ & $1.411 \mathrm{E}+00$ & $3.845 \mathrm{E}+12$ & $1.6423 \mathrm{E}+01$ & $1.412 \mathrm{E}+00$ & $3.848 \mathrm{E}+12$ & 0.000 \\
\hline $1 s 5 g^{3} G_{5}$ & $1 s 7 h^{3} H_{4}^{o}$ & $1.6541 \mathrm{E}+01$ & $7.259 \mathrm{E}-04$ & $1.966 \mathrm{E}+09$ & $1.6471 \mathrm{E}+01$ & $7.199 \mathrm{E}-04$ & $1.950 \mathrm{E}+09$ & 0.004 \\
\hline $1 s 5 g{ }^{1} G_{4}$ & $1 s 7 h^{3} H_{4}^{0}$ & $1.6543 \mathrm{E}+01$ & $3.199 \mathrm{E}-02$ & $8.663 \mathrm{E}+10$ & $1.6473 \mathrm{E}+01$ & $3.181 \mathrm{E}-02$ & $8.614 \mathrm{E}+10$ & 0.000 \\
\hline $1 s 6 d^{3} D_{3}$ & $1 s 7 h^{3} H_{4}^{o}$ & $4.3381 \mathrm{E}+01$ & 8.687E-09 & $3.421 \mathrm{E}+03$ & & & & 0.040 \\
\hline
\end{tabular}


Table 3 (continued)

\begin{tabular}{|c|c|c|c|c|c|c|c|c|}
\hline \multirow[t]{2}{*}{ Lower } & \multirow[t]{2}{*}{ Upper } & \multicolumn{3}{|l|}{ GRASP2K } & \multicolumn{3}{|l|}{ FAC } & \multirow[t]{2}{*}{$d T$} \\
\hline & & $\lambda(\AA)$ & $g f$ & $A\left(\mathrm{~s}^{-1}\right)$ & $\lambda(\AA)$ & $g f$ & $A\left(\mathrm{~s}^{-1}\right)$ & \\
\hline $1 s 6 g{ }^{3} G_{4}$ & $1 s 7 h^{3} H_{4}^{o}$ & $4.3739 \mathrm{E}+01$ & $2.946 \mathrm{E}-01$ & $1.141 \mathrm{E}+11$ & $4.3559 \mathrm{E}+01$ & $2.959 \mathrm{E}-01$ & $1.146 \mathrm{E}+11$ & 0.000 \\
\hline $1 s 6 g^{3} G_{3}$ & $1 s 7 h^{3} H_{4}^{o}$ & $4.3747 \mathrm{E}+01$ & $1.035 \mathrm{E}+01$ & $4.007 \mathrm{E}+12$ & $4.3559 \mathrm{E}+01$ & $1.035 \mathrm{E}+01$ & $4.012 \mathrm{E}+12$ & 0.000 \\
\hline $1 s 6 g{ }^{3} G_{5}$ & $1 s 7 h^{3} H_{4}^{0}$ & $4.3947 \mathrm{E}+01$ & $5.409 \mathrm{E}-03$ & $2.076 \mathrm{E}+09$ & $4.3765 \mathrm{E}+01$ & $5.401 \mathrm{E}-03$ & $2.072 \mathrm{E}+09$ & 0.001 \\
\hline $1 s 6 g^{1} G_{4}$ & $1 s 7 h^{3} H_{4}^{0}$ & $4.3954 \mathrm{E}+01$ & $2.390 \mathrm{E}-01$ & $9.169 \mathrm{E}+10$ & $4.3765 \mathrm{E}+01$ & $2.376 \mathrm{E}-01$ & $9.119 \mathrm{E}+10$ & 0.000 \\
\hline $1 s 7 d^{3} D_{3}$ & $1 s 7 h^{3} H_{4}^{o}$ & $5.2970 \mathrm{E}+03$ & $2.748 \mathrm{E}-10$ & $7.259 \mathrm{E}-03$ & & & & 0.966 \\
\hline $1 s 7 g{ }^{3} G_{4}$ & $1 s 7 h^{3} H_{4}^{o}$ & $1.4287 \mathrm{E}+04$ & $2.211 \mathrm{E}-03$ & $8.030 \mathrm{E}+03$ & $1.4689 \mathrm{E}+04$ & $2.148 \mathrm{E}-03$ & $7.317 \mathrm{E}+03$ & 0.009 \\
\hline $1 s 7 g^{3} G_{3}$ & $1 s 7 h^{3} H_{4}^{0}$ & $1.4871 \mathrm{E}+04$ & $7.459 \mathrm{E}-02$ & $2.500 \mathrm{E}+05$ & $1.4689 \mathrm{E}+04$ & $7.520 \mathrm{E}-02$ & $2.561 \mathrm{E}+05$ & 0.009 \\
\hline $1 s 7 g^{3} G_{5}$ & $1 s 7 h^{3} H_{4}^{0}$ & $5.3648 \mathrm{E}+05$ & $1.070 \mathrm{E}-06$ & $2.756 \mathrm{E}-03$ & & & & 0.163 \\
\hline $1 s 4 f^{3} F_{3}^{o}$ & $1 s 7 g^{1} G_{4}$ & $7.6513 \mathrm{E}+00$ & $3.467 \mathrm{E}-08$ & $4.389 \mathrm{E}+05$ & & & & 0.018 \\
\hline $1 s 4 f^{3} F_{4}^{o}$ & $1 s 7 g^{1} G_{4}$ & $7.6873 \mathrm{E}+00$ & $1.135 \mathrm{E}-02$ & $1.424 \mathrm{E}+11$ & $7.6547 \mathrm{E}+00$ & $1.134 \mathrm{E}-02$ & $1.422 \mathrm{E}+11$ & 0.000 \\
\hline $1 s 4 f^{1} F_{3}^{0}$ & $1 s 7 g^{1} G_{4}$ & $7.6884 \mathrm{E}+00$ & $3.952 \mathrm{E}-01$ & $4.955 \mathrm{E}+12$ & $7.6558 \mathrm{E}+00$ & $3.968 \mathrm{E}-01$ & $4.975 \mathrm{E}+12$ & 0.000 \\
\hline $1 s 5 f^{3} F_{3}^{o}$ & $1 s 7 g^{1} G_{4}$ & $1.6405 \mathrm{E}+01$ & $3.735 \mathrm{E}-08$ & $1.029 \mathrm{E}+05$ & & & & 0.050 \\
\hline $1 s 5 f^{3} F_{4}^{o}$ & $1 s 7 g^{1} G_{4}$ & $1.6490 \mathrm{E}+01$ & $4.447 \mathrm{E}-02$ & $1.212 \mathrm{E}+11$ & $1.6420 \mathrm{E}+01$ & $4.438 \mathrm{E}-02$ & $1.209 \mathrm{E}+11$ & 0.000 \\
\hline $1 s 5 f^{1} F_{3}^{0}$ & $1 s 7 g^{1} G_{4}$ & $1.6493 \mathrm{E}+01$ & $1.549 \mathrm{E}+00$ & $4.222 \mathrm{E}+12$ & $1.6423 \mathrm{E}+01$ & $1.553 \mathrm{E}+00$ & $4.231 \mathrm{E}+12$ & 0.000 \\
\hline $1 s 6 f^{3} F_{3}^{o}$ & $1 s 7 g^{1} G_{4}$ & $4.3393 \mathrm{E}+01$ & $4.042 \mathrm{E}-08$ & $1.591 \mathrm{E}+04$ & & & & 0.133 \\
\hline $1 s 6 f^{3} F_{4}^{o}$ & $1 s 7 g^{1} G_{4}$ & $4.3738 \mathrm{E}+01$ & $2.139 \mathrm{E}-01$ & $8.286 \mathrm{E}+10$ & $4.3559 \mathrm{E}+01$ & $2.134 \mathrm{E}-01$ & $8.265 E+10$ & 0.000 \\
\hline $1 s 6 f^{1} F_{3}^{0}$ & $1 s 7 g^{1} G_{4}$ & $4.3749 \mathrm{E}+01$ & $7.458 \mathrm{E}+00$ & $2.888 \mathrm{E}+12$ & $4.3560 \mathrm{E}+01$ & $7.469 \mathrm{E}+00$ & $2.892 \mathrm{E}+12$ & 0.000 \\
\hline $1 s 6 h^{3} H_{5}^{o}$ & $1 s 7 g{ }^{1} G_{4}$ & $4.3947 \mathrm{E}+01$ & $2.711 \mathrm{E}-05$ & $1.041 \mathrm{E}+07$ & & & & 0.001 \\
\hline $1 s 6 h^{3} H_{4}^{o}$ & $1 s 7 g^{1} G_{4}$ & $4.3952 \mathrm{E}+01$ & $1.240 \mathrm{E}-03$ & $4.758 \mathrm{E}+08$ & $4.3765 \mathrm{E}+01$ & $1.240 \mathrm{E}-03$ & $4.757 \mathrm{E}+08$ & 0.000 \\
\hline $1 s 6 h^{1} H_{5}^{o}$ & $1 s 7 g^{1} G_{4}$ & $4.4090 \mathrm{E}+01$ & $7.073 \mathrm{E}-02$ & $2.696 \mathrm{E}+10$ & $4.3903 \mathrm{E}+01$ & $7.082 \mathrm{E}-02$ & $2.700 \mathrm{E}+10$ & 0.000 \\
\hline $1 s 7 f^{3} F_{3}^{o}$ & $1 s 7 g^{1} G_{4}$ & $5.4114 \mathrm{E}+03$ & $2.878 \mathrm{E}-10$ & $7.284 \mathrm{E}-03$ & & & & 0.998 \\
\hline $1 s 7 f^{3} F_{4}^{o}$ & $1 s 7 g{ }^{1} G_{4}$ & $1.4181 \mathrm{E}+04$ & $3.084 \mathrm{E}-03$ & $1.137 \mathrm{E}+04$ & $1.4765 \mathrm{E}+04$ & $2.937 \mathrm{E}-03$ & $9.900 \mathrm{E}+03$ & 0.048 \\
\hline $1 s 7 f^{1} F_{3}^{0}$ & $1 s 7 g{ }^{1} G_{4}$ & $1.4947 \mathrm{E}+04$ & $1.020 \mathrm{E}-01$ & $3.382 \mathrm{E}+05$ & $1.4784 \mathrm{E}+04$ & $1.026 \mathrm{E}-01$ & $3.451 \mathrm{E}+05$ & 0.037 \\
\hline $1 s 7 h^{3} H_{5}^{o}$ & $1 s 7 g^{1} G_{4}$ & $4.7824 \mathrm{E}+05$ & $1.162 \mathrm{E}-06$ & $3.764 \mathrm{E}-03$ & & & & 0.645 \\
\hline $1 s 7 h^{3} H_{4}^{o}$ & $1 s 7 g{ }^{1} G_{4}$ & $3.2051 \mathrm{E}+06$ & $7.916 \mathrm{E}-06$ & $5.712 \mathrm{E}-04$ & & & & 0.700 \\
\hline $1 s 5 g{ }^{3} G_{5}$ & $1 s 7 h^{3} H_{6}^{o}$ & $1.6529 \mathrm{E}+01$ & $2.139 \mathrm{E}+00$ & $4.017 \mathrm{E}+12$ & $1.6459 \mathrm{E}+01$ & $2.144 \mathrm{E}+00$ & $4.027 \mathrm{E}+12$ & 0.000 \\
\hline $1 s 6 g{ }^{3} G_{5}$ & $1 s 7 h^{3} H_{6}^{o}$ & $4.3863 \mathrm{E}+01$ & $1.577 \mathrm{E}+01$ & $4.205 E+12$ & $4.3678 \mathrm{E}+01$ & $1.579 \mathrm{E}+01$ & $4.211 \mathrm{E}+12$ & 0.000 \\
\hline $1 s 7 g{ }^{3} G_{5}$ & $1 s 7 h^{3} H_{6}^{o}$ & $2.2048 \mathrm{E}+04$ & $7.648 \mathrm{E}-02$ & $8.073 \mathrm{E}+04$ & $2.2104 \mathrm{E}+04$ & $7.596 \mathrm{E}-02$ & $7.910 \mathrm{E}+04$ & 0.025 \\
\hline $1 s 6 h^{3} H_{5}^{o}$ & $1 s 7 i^{3} I_{6}{ }^{0}$ & $4.3863 E+01$ & $2.165 \mathrm{E}+01$ & $5.775 \mathrm{E}+12$ & $4.3678 \mathrm{E}+01$ & $2.167 \mathrm{E}+01$ & $5.780 \mathrm{E}+12$ & 0.000 \\
\hline $1 s 6 h^{3} H_{6}^{0}$ & $1 s 7 i^{3} I_{6}$ & $4.4002 \mathrm{E}+01$ & $3.281 \mathrm{E}-01$ & $8.695 \mathrm{E}+10$ & $4.3816 \mathrm{E}+01$ & $3.283 E-01$ & $8.699 E+10$ & 0.000 \\
\hline $1 s 6 h^{1} H_{5}^{o}$ & $1 s 7 i^{3} I_{6}$ & $4.4006 \mathrm{E}+01$ & $4.130 \mathrm{E}-03$ & $1.094 \mathrm{E}+09$ & $4.3816 \mathrm{E}+01$ & $4.263 \mathrm{E}-03$ & $1.129 \mathrm{E}+09$ & 0.000 \\
\hline $1 s 7 h^{3} H_{5}^{o}$ & $1 s 7 i^{3} I_{6}$ & $2.2088 \mathrm{E}+04$ & $4.138 \mathrm{E}-02$ & $4.352 \mathrm{E}+04$ & $2.2113 \mathrm{E}+04$ & $4.114 \mathrm{E}-02$ & $4.281 \mathrm{E}+04$ & 0.010 \\
\hline $1 s 7 h^{3} H_{6}^{o}$ & $1 s 7 i^{3} I_{6}$ & $2.7778 \mathrm{E}+08$ & $4.919 \mathrm{E}-08$ & $3.208 \mathrm{E}-10$ & & & & 1.000 \\
\hline $1 s 4 f^{3} F_{4}^{0}$ & $1 s 7 i^{3} I_{5}$ & $7.6846 \mathrm{E}+00$ & $6.513 \mathrm{E}-12$ & $6.688 \mathrm{E}+01$ & & & & 0.024 \\
\hline $1 s 5 f^{3} F_{4}^{0}$ & $1 s 7 i^{3} I_{5}$ & $1.6478 \mathrm{E}+01$ & $2.181 \mathrm{E}-11$ & $4.870 \mathrm{E}+01$ & & & & 0.047 \\
\hline $1 s 6 f^{3} F_{4}^{0}$ & $1 s 7 i^{3} I_{5}$ & $4.3653 \mathrm{E}+01$ & $1.093 \mathrm{E}-14$ & $3.479 \mathrm{E}-03$ & & & & 0.317 \\
\hline $1 s 6 h^{3} H_{5}^{o}$ & $1 s 7 i^{3} I_{5}$ & $4.3861 \mathrm{E}+01$ & $3.322 \mathrm{E}-01$ & $1.047 \mathrm{E}+11$ & $4.3678 \mathrm{E}+01$ & $3.335 \mathrm{E}-01$ & $1.051 \mathrm{E}+11$ & 0.000 \\
\hline $1 s 6 h^{3} H_{4}^{o}$ & $1 s 7 i^{3} I_{5}$ & $4.3866 \mathrm{E}+01$ & $1.799 \mathrm{E}+01$ & $5.669 \mathrm{E}+12$ & $4.3678 \mathrm{E}+01$ & $1.800 \mathrm{E}+01$ & $5.675 \mathrm{E}+12$ & 0.000 \\
\hline $1 s 6 h^{3} H_{6}^{o}$ & $1 s 7 i^{3} I_{5}$ & $4.4000 \mathrm{E}+01$ & $4.274 \mathrm{E}-03$ & $1.339 \mathrm{E}+09$ & $4.3816 \mathrm{E}+01$ & $4.263 \mathrm{E}-03$ & $1.335 \mathrm{E}+09$ & 0.001 \\
\hline $1 s 6 h^{1} H_{5}^{o}$ & $1 s 7 i^{3} I_{5}$ & $4.4004 \mathrm{E}+01$ & $2.786 \mathrm{E}-01$ & $8.725 E+10$ & $4.3816 \mathrm{E}+01$ & $2.771 \mathrm{E}-01$ & $8.679 E+10$ & 0.000 \\
\hline $1 s 7 f^{3} F_{4}^{0}$ & $1 s 7 i^{3} I_{5}$ & $8.6997 \mathrm{E}+03$ & $2.746 \mathrm{E}-16$ & $2.200 \mathrm{E}-09$ & & & & 1.000 \\
\hline $1 s 7 h^{3} H_{5}^{o}$ & $1 s 7 i^{3} I_{5}$ & $2.1496 \mathrm{E}+04$ & $6.522 \mathrm{E}-04$ & $8.558 \mathrm{E}+02$ & $2.2113 \mathrm{E}+04$ & $6.330 \mathrm{E}-04$ & $7.784 \mathrm{E}+02$ & 0.060 \\
\hline $1 s 7 h^{3} H_{4}^{0}$ & $1 s 7 i^{3} I_{5}$ & $2.2351 \mathrm{E}+04$ & $3.396 \mathrm{E}-02$ & $4.123 \mathrm{E}+04$ & $2.2113 \mathrm{E}+04$ & $3.418 \mathrm{E}-02$ & $4.203 E+04$ & 0.048 \\
\hline $1 s 7 h^{3} H_{6}^{0}$ & $1 s 7 i^{3} I_{5}$ & $8.0038 \mathrm{E}+05$ & $2.245 \mathrm{E}-07$ & $2.125 \mathrm{E}-04$ & & & & 0.972 \\
\hline $1 s 5 g^{3} G_{4}^{0}$ & $1 s 7 h{ }^{1} H_{5}^{o}$ & $1.6478 \mathrm{E}+01$ & $2.072 \mathrm{E}-07$ & $4.628 \mathrm{E}+05$ & & & & 0.011 \\
\hline $1 s 5 g^{3} G_{5}$ & $1 s 7 h^{1} H_{5}^{o}$ & $1.6529 \mathrm{E}+01$ & $3.303 \mathrm{E}-02$ & $7.332 \mathrm{E}+10$ & $1.6459 \mathrm{E}+01$ & $3.299 \mathrm{E}-02$ & $7.323 \mathrm{E}+10$ & 0.000 \\
\hline $1 s 5 g^{1} G_{4}$ & $1 s 7 h^{1} H_{5}^{o}$ & $1.6530 \mathrm{E}+01$ & $1.777 \mathrm{E}+00$ & $3.943 \mathrm{E}+12$ & $1.6460 \mathrm{E}+01$ & $1.781 \mathrm{E}+00$ & $3.953 \mathrm{E}+12$ & 0.000 \\
\hline $1 s 6 g^{3} G_{4}$ & $1 s 7 h^{1} H_{5}^{o}$ & $4.3653 \mathrm{E}+01$ & $1.484 \mathrm{E}-06$ & $4.723 \mathrm{E}+05$ & & & & 0.007 \\
\hline $1 s 6 g{ }^{3} G_{5}$ & $1 s 7 h^{1} H_{5}^{o}$ & $4.3860 \mathrm{E}+01$ & $2.434 \mathrm{E}-01$ & $7.671 \mathrm{E}+10$ & $4.3678 \mathrm{E}+01$ & $2.429 \mathrm{E}-01$ & $7.657 \mathrm{E}+10$ & 0.000 \\
\hline $1 s 6 g^{1} G_{4}$ & $1 s 7 h^{1} H_{5}^{o}$ & $4.3867 \mathrm{E}+01$ & $1.310 \mathrm{E}+01$ & $4.128 \mathrm{E}+12$ & $4.3678 \mathrm{E}+01$ & $1.312 \mathrm{E}+01$ & $4.135 \mathrm{E}+12$ & 0.000 \\
\hline $1 \mathrm{~s} 7 g^{3} G_{4}$ & $1 s 7 h{ }^{1} H_{5}^{o}$ & $8.6987 \mathrm{E}+03$ & $1.842 \mathrm{E}-08$ & $1.476 \mathrm{E}-01$ & & & & 0.636 \\
\hline $1 s 7 g{ }^{3} G_{5}$ & $1 s 7 h^{1} H_{5}^{o}$ & $2.1355 \mathrm{E}+04$ & $1.219 \mathrm{E}-03$ & $1.621 \mathrm{E}+03$ & $2.2104 \mathrm{E}+04$ & $1.168 \mathrm{E}-03$ & $1.438 \mathrm{E}+03$ & 0.005 \\
\hline $1 s 7 g^{1} G_{4}$ & $1 s 7 h^{1} H_{5}^{o}$ & $2.2395 \mathrm{E}+04$ & $6.255 \mathrm{E}-02$ & $7.562 \mathrm{E}+04$ & $2.2104 \mathrm{E}+04$ & $6.310 \mathrm{E}-02$ & $7.765 \mathrm{E}+04$ & 0.011 \\
\hline $1 s 7 i^{3} I_{6}$ & $1 s 7 h{ }^{1} H_{5}^{o}$ & $6.8074 \mathrm{E}+05$ & $2.552 \mathrm{E}-07$ & $3.340 \mathrm{E}-04$ & & & & 0.629 \\
\hline $1 s 7 i^{3} I_{5}$ & $1 s 7 h^{1} H_{5}^{o}$ & $4.4823 \mathrm{E}+06$ & $2.614 \mathrm{E}-06$ & $7.893 \mathrm{E}-05$ & & & & 0.959 \\
\hline $1 s 6 h^{3} H_{6}^{o}$ & $1 s 7 i^{3} I_{7}^{3}$ & $4.3940 \mathrm{E}+01$ & $2.539 \mathrm{E}+01$ & $5.847 \mathrm{E}+12$ & $4.3754 \mathrm{E}+01$ & $2.543 E+01$ & $5.857 \mathrm{E}+12$ & 0.000 \\
\hline $1 s 7 h^{3} H_{6}^{0}$ & $1 s 7 i^{3} I_{7}$ & $3.1010 \mathrm{E}+04$ & $3.454 \mathrm{E}-02$ & $1.597 \mathrm{E}+04$ & $3.1022 \mathrm{E}+04$ & $3.437 \mathrm{E}-02$ & $1.574 \mathrm{E}+04$ & 0.010 \\
\hline $1 s 6 h^{3} H_{5}^{o}$ & $1 s 7 i{ }^{1} I_{6}$ & $4.3799 \mathrm{E}+01$ & $1.223 \mathrm{E}-06$ & $3.271 \mathrm{E}+05$ & & & & 0.004 \\
\hline $1 s 6 h^{3} H_{6}^{o}$ & $1 s 7 i{ }^{1} I_{6}$ & $4.3938 \mathrm{E}+01$ & $2.829 \mathrm{E}-01$ & $7.519 \mathrm{E}+10$ & $4.3754 \mathrm{E}+01$ & $2.825 \mathrm{E}-01$ & $7.509 \mathrm{E}+10$ & 0.000 \\
\hline $1 s 6 h^{1} H_{5}^{o}$ & $1 s 7 i{ }^{1} I_{6}$ & $4.3942 \mathrm{E}+01$ & $2.172 \mathrm{E}+01$ & $5.772 \mathrm{E}+12$ & $4.3754 \mathrm{E}+01$ & $2.175 \mathrm{E}+01$ & $5.782 \mathrm{E}+12$ & 0.000 \\
\hline $1 s 7 h^{3} H_{5}^{o}$ & $1 s 7 i{ }^{1} I_{6}$ & $1.2726 \mathrm{E}+04$ & $4.183 \mathrm{E}-09$ & $1.325 \mathrm{E}-02$ & & & & 0.903 \\
\hline $1 s 7 h^{3} H_{6}^{0}$ & $1 s 7 i^{1} I_{6}$ & $3.0020 \mathrm{E}+04$ & $3.975 \mathrm{E}-04$ & $2.263 \mathrm{E}+02$ & $3.1022 \mathrm{E}+04$ & $3.819 \mathrm{E}-04$ & $2.019 E+02$ & 0.083 \\
\hline $1 s 7 h^{1} H_{5}^{o}$ & $1 s 7 i{ }^{1} I_{6}$ & $3.1409 E+04$ & $2.917 \mathrm{E}-02$ & $1.517 \mathrm{E}+04$ & $3.1022 \mathrm{E}+04$ & $2.940 \mathrm{E}-02$ & $1.554 \mathrm{E}+04$ & 0.060 \\
\hline
\end{tabular}


Table 4

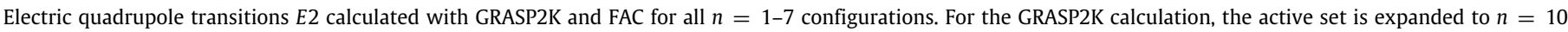

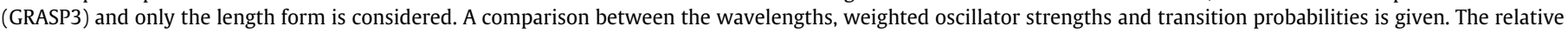
difference between transition probabilities in the velocity and length gauge is given in the last column by $d T$.

\begin{tabular}{|c|c|c|c|c|c|c|c|c|}
\hline \multirow[t]{2}{*}{ Lower } & \multirow[t]{2}{*}{ Upper } & \multicolumn{3}{|l|}{ GRASP2K } & \multicolumn{3}{|l|}{ FAC } & \multirow[t]{2}{*}{$d T$} \\
\hline & & $\lambda(\AA)$ & $g f$ & $A\left(\mathrm{~s}^{-1}\right)$ & $\lambda(\AA)$ & $g f$ & $A\left(\mathrm{~s}^{-1}\right)$ & \\
\hline $1 s 2 p^{3} P_{1}^{o}$ & $1 s 2 p^{3} P_{2}^{o}$ & $3.1908 \mathrm{E}+01$ & $3.112 \mathrm{E}-07$ & $4.078 \mathrm{E}+05$ & $3.1815 \mathrm{E}+01$ & $3.135 \mathrm{E}-07$ & $4.098 \mathrm{E}+05$ & 0.006 \\
\hline $1 s 2 p^{3} P_{0}^{o}$ & $1 s 2 p^{3} P_{2}^{o}$ & $3.2407 \mathrm{E}+01$ & $1.878 \mathrm{E}-07$ & $2.386 \mathrm{E}+05$ & $3.2290 \mathrm{E}+01$ & $1.887 \mathrm{E}-07$ & $2.394 \mathrm{E}+05$ & 0.019 \\
\hline $1 s 2 p^{3} P_{1}^{o}$ & $1 s 2 p^{1} P_{1}^{o}$ & $2.9274 \mathrm{E}+01$ & $3.608 \mathrm{E}-07$ & $9.361 \mathrm{E}+05$ & $2.9150 \mathrm{E}+01$ & $3.594 \mathrm{E}-07$ & $9.325 \mathrm{E}+05$ & 0.011 \\
\hline $1 s 2 p^{3} P_{2}^{o}$ & $1 s 2 p^{1} P_{1}^{o}$ & $3.5465 \mathrm{E}+02$ & $1.078 \mathrm{E}-10$ & $1.905 E+00$ & & & & 0.174 \\
\hline $1 s 2 s^{3} S_{1}$ & $1 s 3 s^{3} s_{1}$ & $2.1766 \mathrm{E}+00$ & $4.067 \mathrm{E}-09$ & $1.909 \mathrm{E}+06$ & $2.1673 \mathrm{E}+00$ & $4.604 \mathrm{E}-09$ & $2.161 \mathrm{E}+06$ & 0.026 \\
\hline $1 s 2 p^{3} P_{1}^{o}$ & $1 s 3 p^{3} P_{1}^{o}$ & $2.1983 E+00$ & $3.696 \mathrm{E}-06$ & $1.700 \mathrm{E}+09$ & $2.1892 \mathrm{E}+00$ & $4.175 \mathrm{E}-06$ & $1.920 \mathrm{E}+09$ & 0.009 \\
\hline $1 s 2 p^{3} P_{2}^{o}$ & $1 s 3 p^{3} P_{1}^{o}$ & $2.3610 \mathrm{E}+00$ & $7.133 \mathrm{E}-04$ & $2.845 \mathrm{E}+11$ & $2.3509 \mathrm{E}+00$ & $7.174 \mathrm{E}-04$ & $2.861 \mathrm{E}+11$ & 0.002 \\
\hline $1 s 2 p{ }^{1} P_{1}^{o}$ & $1 s 3 p^{3} P_{1}^{o}$ & $2.3768 \mathrm{E}+00$ & $6.407 \mathrm{E}-04$ & $2.522 \mathrm{E}+11$ & $2.3669 \mathrm{E}+00$ & $6.330 \mathrm{E}-04$ & $2.491 \mathrm{E}+11$ & 0.001 \\
\hline $1 s 2 p^{3} P_{2}^{o}$ & $1 s 3 p^{3} P_{0}^{o}$ & $2.3603 E+00$ & $4.510 \mathrm{E}-04$ & $5.400 \mathrm{E}+11$ & $2.3503 \mathrm{E}+00$ & $4.561 \mathrm{E}-04$ & $5.461 \mathrm{E}+11$ & 0.000 \\
\hline $1 s 2 p^{3} P_{1}^{o}$ & $1 s 3 p^{3} P_{2}^{o}$ & $2.1541 \mathrm{E}+00$ & $6.263 \mathrm{E}-04$ & $1.800 \mathrm{E}+11$ & $2.1452 \mathrm{E}+00$ & $6.425 \mathrm{E}-04$ & $1.846 \mathrm{E}+11$ & 0.002 \\
\hline $1 s 2 p^{3} P_{0}^{o}$ & $1 s 3 p^{3} P_{2}^{o}$ & $2.1564 \mathrm{E}+00$ & $3.953 \mathrm{E}-04$ & $1.134 \mathrm{E}+11$ & $2.1474 \mathrm{E}+00$ & $4.009 \mathrm{E}-04$ & $1.150 \mathrm{E}+11$ & 0.001 \\
\hline $1 s 2 p^{3} P_{2}^{o}$ & $1 s 3 p^{3} P_{2}^{o}$ & $2.3101 \mathrm{E}+00$ & $7.422 \mathrm{E}-04$ & $1.855 \mathrm{E}+11$ & $2.3003 \mathrm{E}+00$ & $7.550 \mathrm{E}-04$ & $1.887 \mathrm{E}+11$ & 0.000 \\
\hline $1 s 2 p{ }^{1} P_{1}^{o}$ & $1 s 3 p^{3} P_{2}^{o}$ & $2.3252 \mathrm{E}+00$ & $2.840 \mathrm{E}-04$ & $7.006 \mathrm{E}+10$ & $2.3156 \mathrm{E}+00$ & $2.859 \mathrm{E}-04$ & $7.054 \mathrm{E}+10$ & 0.002 \\
\hline $1 s 3 p^{3} P_{1}^{o}$ & $1 s 3 p^{3} P_{2}^{o}$ & $1.0725 \mathrm{E}+02$ & $3.088 \mathrm{E}-07$ & $3.581 \mathrm{E}+04$ & $1.0689 \mathrm{E}+02$ & $3.098 \mathrm{E}-07$ & $3.587 \mathrm{E}+04$ & 0.001 \\
\hline $1 s 3 p^{3} P_{0}^{o}$ & $1 s 3 p^{3} P_{2}^{o}$ & $1.0873 \mathrm{E}+02$ & $1.880 \mathrm{E}-07$ & $2.121 \mathrm{E}+04$ & $1.0831 \mathrm{E}+02$ & $1.883 \mathrm{E}-07$ & $2.123 \mathrm{E}+04$ & 0.002 \\
\hline $1 s^{2}{ }^{1} s_{0}$ & $1 s 3 d^{3} D_{2}$ & $3.4460 \mathrm{E}-01$ & $8.826 \mathrm{E}-04$ & $9.910 \mathrm{E}+12$ & $3.4325 \mathrm{E}-01$ & $9.055 \mathrm{E}-04$ & $1.016 \mathrm{E}+13$ & 0.003 \\
\hline $1 s 2 s^{3} S_{1}$ & $1 s 3 d^{3} D_{2}$ & $2.1226 \mathrm{E}+00$ & $2.875 \mathrm{E}-03$ & $8.513 E+11$ & $2.1136 \mathrm{E}+00$ & $2.919 \mathrm{E}-03$ & $8.644 \mathrm{E}+11$ & 0.003 \\
\hline $1 s 2 s{ }^{1} S_{0}$ & $1 s 3 d^{3} D_{2}$ & $2.1539 \mathrm{E}+00$ & $1.869 \mathrm{E}-03$ & $5.374 \mathrm{E}+11$ & $2.1452 \mathrm{E}+00$ & $1.833 \mathrm{E}-03$ & $5.270 \mathrm{E}+11$ & 0.003 \\
\hline $1 s 3 s^{3} S_{1}$ & $1 s 3 d^{3} D_{2}$ & $8.5509 E+01$ & $3.819 \mathrm{E}-07$ & $6.968 \mathrm{E}+04$ & $8.5230 \mathrm{E}+01$ & $3.815 \mathrm{E}-07$ & $6.947 \mathrm{E}+04$ & 0.030 \\
\hline $1 s 3 s^{1} S_{0}$ & $1 s 3 d^{3} D_{2}$ & $1.0120 \mathrm{E}+02$ & $1.521 \mathrm{E}-07$ & $1.982 E+04$ & $1.0108 \mathrm{E}+02$ & $1.504 \mathrm{E}-07$ & $1.947 \mathrm{E}+04$ & 0.037 \\
\hline $1 s 2 s^{3} S_{1}$ & $1 s 3 d^{3} D_{1}$ & $2.1220 \mathrm{E}+00$ & $2.860 \mathrm{E}-03$ & $1.412 \mathrm{E}+12$ & $2.1130 \mathrm{E}+00$ & $2.904 \mathrm{E}-03$ & $1.434 \mathrm{E}+12$ & 0.002 \\
\hline $1 s 3 s^{3} S_{1}$ & $1 s 3 d^{3} D_{1}$ & $8.4639 E+01$ & $3.934 \mathrm{E}-07$ & $1.221 \mathrm{E}+05$ & $8.4368 \mathrm{E}+01$ & $3.922 \mathrm{E}-07$ & $1.214 \mathrm{E}+05$ & 0.027 \\
\hline $1 s 3 d^{3} D_{2}$ & $1 s 3 d^{3} D_{1}$ & $8.3264 \mathrm{E}+03$ & $1.645 \mathrm{E}-13$ & $5.276 \mathrm{E}-06$ & & & & 0.531 \\
\hline $1 s 2 p^{3} P_{1}^{o}$ & $1 s 3 p^{1} P_{1}^{o}$ & $2.1504 \mathrm{E}+00$ & $5.523 \mathrm{E}-04$ & $2.655 E+11$ & $2.1415 \mathrm{E}+00$ & $5.632 \mathrm{E}-04$ & $2.707 \mathrm{E}+11$ & 0.000 \\
\hline $1 s 2 p^{3} P_{2}^{o}$ & $1 s 3 p^{1} P_{1}^{o}$ & $2.3058 \mathrm{E}+00$ & $2.829 \mathrm{E}-04$ & $1.183 \mathrm{E}+11$ & $2.2961 \mathrm{E}+00$ & $2.891 \mathrm{E}-04$ & $1.209 \mathrm{E}+11$ & 0.002 \\
\hline $1 s 2 p{ }^{1} P_{1}^{o}$ & $1 s 3 p^{1} P_{1}^{o}$ & $2.3209 E+00$ & $3.899 \mathrm{E}-04$ & $1.610 \mathrm{E}+11$ & $2.3113 \mathrm{E}+00$ & $3.893 \mathrm{E}-04$ & $1.606 \mathrm{E}+11$ & 0.000 \\
\hline $1 s 3 p^{3} P_{1}^{o}$ & $1 s 3 p^{1} P_{1}^{o}$ & $9.8786 \mathrm{E}+01$ & $3.556 \mathrm{E}-07$ & $8.103 E+04$ & $9.8358 \mathrm{E}+01$ & $3.546 \mathrm{E}-07$ & $8.082 E+04$ & 0.005 \\
\hline $1 s 3 p^{3} P_{2}^{0}$ & $1 s 3 p^{1} P_{1}^{o}$ & $1.2519 \mathrm{E}+03$ & $8.846 \mathrm{E}-11$ & $1.255 \mathrm{E}-01$ & & & & 0.052 \\
\hline $1 s 2 s^{3} S_{1}^{2}$ & $1 s 3 d^{3} D_{3}$ & $2.1095 \mathrm{E}+00$ & $6.734 \mathrm{E}-03$ & $1.442 \mathrm{E}+12$ & $2.1005 E+00$ & $6.967 \mathrm{E}-03$ & $1.492 \mathrm{E}+12$ & 0.003 \\
\hline $1 s 3 s^{3} S_{1}$ & $1 s 3 d^{3} D_{3}$ & $6.8392 \mathrm{E}+01$ & $1.793 \mathrm{E}-06$ & $3.652 \mathrm{E}+05$ & $6.8186 \mathrm{E}+01$ & $1.790 \mathrm{E}-06$ & $3.637 \mathrm{E}+05$ & 0.024 \\
\hline $1 s 3 d^{3} D_{2}$ & $1 s 3 d^{3} D_{3}$ & $3.4166 \mathrm{E}+02$ & $2.752 \mathrm{E}-09$ & $2.246 \mathrm{E}+01$ & & & & 0.004 \\
\hline $1 s 3 d^{3} D_{1}$ & $1 s 3 d^{3} D_{3}$ & $3.5628 \mathrm{E}+02$ & $3.967 \mathrm{E}-10$ & $2.978 \mathrm{E}+00$ & & & & 0.044 \\
\hline $1 s^{2}{ }^{1} S_{0}$ & $1 s 3 d^{1} D_{2}$ & $3.4430 \mathrm{E}-01$ & $1.235 \mathrm{E}-03$ & $1.389 \mathrm{E}+13$ & $3.4289 \mathrm{E}-01$ & $1.431 \mathrm{E}-03$ & $1.610 \mathrm{E}+13$ & 0.003 \\
\hline $1 s 2 s^{3} S_{1}$ & $1 s 3 d^{1} D_{2}$ & $2.1090 \mathrm{E}+00$ & $1.910 \mathrm{E}-03$ & $5.729 E+11$ & $2.1000 \mathrm{E}+00$ & $1.974 \mathrm{E}-03$ & $5.922 \mathrm{E}+11$ & 0.002 \\
\hline $1 s 2 s^{1} S_{0}$ & $1 s 3 d^{1} D_{2}$ & $2.1399 \mathrm{E}+00$ & $2.871 \mathrm{E}-03$ & $8.364 \mathrm{E}+11$ & $2.1313 \mathrm{E}+00$ & $2.875 \mathrm{E}-03$ & $8.373 \mathrm{E}+11$ & 0.002 \\
\hline $1 s 3 s^{3} S_{1}$ & $1 s 3 d^{1} D_{2}$ & $6.7876 \mathrm{E}+01$ & $5.194 \mathrm{E}-07$ & $1.504 \mathrm{E}+05$ & $6.7664 \mathrm{E}+01$ & $5.176 \mathrm{E}-07$ & $1.495 \mathrm{E}+05$ & 0.021 \\
\hline $1 s 3 s^{1} S_{0}$ & $1 s 3 d^{1} D_{2}$ & $7.7401 \mathrm{E}+01$ & $5.347 \mathrm{E}-07$ & $1.191 \mathrm{E}+05$ & $7.7288 \mathrm{E}+01$ & $5.296 \mathrm{E}-07$ & $1.172 \mathrm{E}+05$ & 0.025 \\
\hline $1 s 3 d^{3} D_{2}$ & $1 s 3 d^{1} D_{2}$ & $3.2917 \mathrm{E}+02$ & $7.638 \mathrm{E}-10$ & $9.405 E+00$ & & & & 0.034 \\
\hline $1 s 3 d^{3} D_{1}$ & $1 s 3 d^{1} D_{2}$ & $3.4272 \mathrm{E}+02$ & $1.578 \mathrm{E}-09$ & $1.792 \mathrm{E}+01$ & & & & 0.011 \\
\hline $1 s 3 d^{3} D_{3}$ & $1 s 3 d^{1} D_{2}$ & $8.9990 \mathrm{E}+03$ & $1.023 \mathrm{E}-13$ & $1.685 \mathrm{E}-06$ & & & & 0.644 \\
\hline $1 s 2 s^{3} S_{1}$ & $1 s 4 s^{3} S_{1}$ & $1.6177 \mathrm{E}+00$ & $1.741 \mathrm{E}-10$ & $1.480 \mathrm{E}+05$ & $1.6108 \mathrm{E}+00$ & $4.748 \mathrm{E}-10$ & $4.034 \mathrm{E}+05$ & 0.331 \\
\hline $1 s 3 s^{3} S_{1}$ & $1 s 4 s^{3} S_{1}$ & $6.2995 \mathrm{E}+00$ & $5.359 \mathrm{E}-09$ & $3.003 E+05$ & & & & 0.020 \\
\hline $1 s 3 d^{3} D_{2}$ & $1 s 4 s^{3} S_{1}$ & $6.8005 E+00$ & $1.094 \mathrm{E}-04$ & $5.261 \mathrm{E}+09$ & $6.7717 \mathrm{E}+00$ & $1.086 \mathrm{E}-04$ & $5.221 \mathrm{E}+09$ & 0.001 \\
\hline $1 s 3 d^{3} D_{1}$ & $1 s 4 s^{3} s_{1}$ & $6.8061 \mathrm{E}+00$ & $1.100 \mathrm{E}-04$ & $5.277 \mathrm{E}+09$ & $6.7772 \mathrm{E}+00$ & $1.084 \mathrm{E}-04$ & $5.207 \mathrm{E}+09$ & 0.002 \\
\hline $1 s 3 d^{3} D_{3}$ & $1 s 4 s^{3} S_{1}$ & $6.9386 \mathrm{E}+00$ & $2.664 \mathrm{E}-04$ & $1.230 \mathrm{E}+10$ & $6.9089 \mathrm{E}+00$ & $2.627 \mathrm{E}-04$ & $1.213 \mathrm{E}+10$ & 0.001 \\
\hline $1 s 3 d{ }^{1} D_{2}$ & $1 s 4 s^{3} S_{1}$ & $6.9440 \mathrm{E}+00$ & $7.527 \mathrm{E}-05$ & $3.471 \mathrm{E}+09$ & $6.9143 \mathrm{E}+00$ & $7.389 \mathrm{E}-05$ & $3.407 \mathrm{E}+09$ & 0.001 \\
\hline $1 s 2 p^{3} P_{1}^{o}$ & $1 s 4 p^{3} P_{1}^{o}$ & $1.6323 \mathrm{E}+00$ & $8.176 \mathrm{E}-07$ & $6.823 E+08$ & $1.6255 \mathrm{E}+00$ & $9.043 \mathrm{E}-07$ & $7.545 \mathrm{E}+08$ & 0.004 \\
\hline $1 s 2 p^{3} P_{2}^{o}$ & $1 s 4 p^{3} P_{1}^{o}$ & $1.7203 \mathrm{E}+00$ & $1.481 \mathrm{E}-04$ & $1.113 \mathrm{E}+11$ & $1.7131 \mathrm{E}+00$ & $1.462 \mathrm{E}-04$ & $1.099 \mathrm{E}+11$ & 0.004 \\
\hline $1 s 2 p^{1} P_{1}^{o}$ & $1 s 4 p^{3} P_{1}^{o}$ & $1.7287 \mathrm{E}+00$ & $1.320 \mathrm{E}-04$ & $9.819 \mathrm{E}+10$ & $1.7215 \mathrm{E}+00$ & $1.289 \mathrm{E}-04$ & $9.594 \mathrm{E}+10$ & 0.002 \\
\hline $1 s 3 p^{3} P_{1}^{o}$ & $1 s 4 p^{3} P_{1}^{o}$ & $6.3396 \mathrm{E}+00$ & $2.991 \mathrm{E}-06$ & $1.655 E+08$ & $6.3138 \mathrm{E}+00$ & $3.265 \mathrm{E}-06$ & $1.806 \mathrm{E}+08$ & 0.000 \\
\hline $1 s 3 p^{3} P_{2}^{o}$ & $1 s 4 p^{3} P_{1}^{o}$ & $6.7379 E+00$ & $6.333 \mathrm{E}-04$ & $3.101 E+10$ & $6.7101 \mathrm{E}+00$ & $6.376 \mathrm{E}-04$ & $3.122 \mathrm{E}+10$ & 0.000 \\
\hline $1 s 3 p^{1} P_{1}^{o}$ & $1 s 4 p^{3} P_{1}^{o}$ & $6.7743 \mathrm{E}+00$ & $5.713 \mathrm{E}-04$ & $2.768 \mathrm{E}+10$ & $6.7469 \mathrm{E}+00$ & $5.671 \mathrm{E}-04$ & $2.746 \mathrm{E}+10$ & 0.000 \\
\hline $1 s 3 d^{3} D_{2}$ & $1 s 4 s{ }^{1} S_{0}$ & $6.7668 \mathrm{E}+00$ & $7.055 \mathrm{E}-05$ & $1.028 \mathrm{E}+10$ & $6.7378 \mathrm{E}+00$ & $7.263 \mathrm{E}-05$ & $1.058 \mathrm{E}+10$ & 0.001 \\
\hline $1 s 3 d d^{1} D_{2}$ & $1 s 4 s^{1} S_{0}$ & $6.9088 \mathrm{E}+00$ & $1.123 \mathrm{E}-04$ & $1.569 \mathrm{E}+10$ & $6.8790 \mathrm{E}+00$ & $1.145 \mathrm{E}-04$ & $1.601 \mathrm{E}+10$ & 0.001 \\
\hline $1 s 2 p^{3} P_{2}^{o}$ & $1 s 4 p^{3} P_{0}^{o}$ & $1.7201 \mathrm{E}+00$ & $9.380 \mathrm{E}-05$ & $2.115 E+11$ & $1.7129 \mathrm{E}+00$ & $9.268 \mathrm{E}-05$ & $2.089 \mathrm{E}+11$ & 0.000 \\
\hline $1 s 3 p^{3} P_{2}^{o}$ & $1 s 4 p^{3} P_{0}^{o}$ & $6.7356 \mathrm{E}+00$ & $4.014 \mathrm{E}-04$ & $5.902 \mathrm{E}+10$ & $6.7079 \mathrm{E}+00$ & $4.061 \mathrm{E}-04$ & $5.970 \mathrm{E}+10$ & 0.000 \\
\hline $1 s 2 p^{3} P_{1}^{o}$ & $1 s 4 p^{3} P_{2}^{o}$ & $1.6219 \mathrm{E}+00$ & $1.580 \mathrm{E}-04$ & $8.014 \mathrm{E}+10$ & $1.6152 \mathrm{E}+00$ & $1.612 \mathrm{E}-04$ & $8.177 \mathrm{E}+10$ & 0.002 \\
\hline $1 s 2 p^{3} P_{0}^{0}$ & $1 s 4 p^{3} P_{2}^{o}$ & $1.6232 \mathrm{E}+00$ & $9.919 \mathrm{E}-05$ & $5.022 E+10$ & $1.6164 \mathrm{E}+00$ & $1.007 \mathrm{E}-04$ & $5.101 \mathrm{E}+10$ & 0.001 \\
\hline $1 s 2 p^{3} P_{2}^{o}$ & $1 s 4 p^{3} P_{2}^{o}$ & $1.7088 \mathrm{E}+00$ & $1.710 \mathrm{E}-04$ & $7.811 \mathrm{E}+10$ & $1.7016 \mathrm{E}+00$ & $1.722 \mathrm{E}-04$ & $7.870 \mathrm{E}+10$ & 0.001 \\
\hline $1 s 2 p{ }^{1} P_{1}^{o}$ & $1 s 4 p^{3} P_{2}^{o}$ & $1.7170 \mathrm{E}+00$ & $6.489 \mathrm{E}-05$ & $2.936 \mathrm{E}+10$ & $1.7100 \mathrm{E}+00$ & $6.523 \mathrm{E}-05$ & $2.950 \mathrm{E}+10$ & 0.004 \\
\hline $1 s 3 p^{3} P_{1}^{o}$ & $1 s 4 p^{3} P_{2}^{o}$ & $6.1858 \mathrm{E}+00$ & $5.474 \mathrm{E}-04$ & $1.909 \mathrm{E}+10$ & $6.1607 \mathrm{E}+00$ & $5.538 \mathrm{E}-04$ & $1.930 \mathrm{E}+10$ & 0.000 \\
\hline $1 s 3 p^{3} P_{0}^{o}$ & $1 s 4 p^{3} P_{2}^{o}$ & $6.1907 \mathrm{E}+00$ & $3.483 \mathrm{E}-04$ & $1.212 \mathrm{E}+10$ & $6.1653 \mathrm{E}+00$ & $3.485 \mathrm{E}-04$ & $1.212 \mathrm{E}+10$ & 0.000 \\
\hline $1 s 3 p^{3} P_{2}^{o}$ & $1 s 4 p^{3} P_{2}^{o}$ & $6.5644 \mathrm{E}+00$ & $6.573 \mathrm{E}-04$ & $2.035 E+10$ & $6.5375 \mathrm{E}+00$ & $6.633 \mathrm{E}-04$ & $2.052 \mathrm{E}+10$ & 0.000 \\
\hline $1 s 3 p{ }^{1} P_{1}^{o}$ & $1 s 4 p^{3} P_{2}^{o}$ & $6.5991 \mathrm{E}+00$ & $2.535 \mathrm{E}-04$ & $7.766 \mathrm{E}+09$ & $6.5723 \mathrm{E}+00$ & $2.534 \mathrm{E}-04$ & $7.759 \mathrm{E}+09$ & 0.000 \\
\hline $1 s 4 p^{3} P_{1}^{o}$ & $1 s 4 p^{3} P_{2}^{o}$ & $2.5504 \mathrm{E}+02$ & $2.619 \mathrm{E}-07$ & $5.371 \mathrm{E}+03$ & $2.5410 \mathrm{E}+02$ & $2.626 \mathrm{E}-07$ & $5.380 \mathrm{E}+03$ & 0.002 \\
\hline $1 s 4 p^{3} P_{0}^{o}$ & $1 s 4 p^{3} P_{2}^{o}$ & $2.5839 \mathrm{E}+02$ & $1.600 \mathrm{E}-07$ & $3.197 \mathrm{E}+03$ & $2.5732 \mathrm{E}+02$ & $1.603 \mathrm{E}-07$ & $3.202 \mathrm{E}+03$ & 0.001 \\
\hline $1 s^{2}{ }^{1} S_{0}$ & $1 s 4 d^{3} D_{2}$ & $3.2750 \mathrm{E}-01$ & $4.379 \mathrm{E}-04$ & $5.445 \mathrm{E}+12$ & $3.2616 \mathrm{E}-01$ & $4.466 \mathrm{E}-04$ & $5.553 \mathrm{E}+12$ & 0.003 \\
\hline $1 s 2 s^{3} S_{1}$ & $1 s 4 d^{3} D_{2}$ & $1.6050 \mathrm{E}+00$ & $2.179 \mathrm{E}-04$ & $1.128 \mathrm{E}+11$ & $1.5981 \mathrm{E}+00$ & $2.096 \mathrm{E}-04$ & $1.085 \mathrm{E}+11$ & 0.002 \\
\hline
\end{tabular}


Table 4 (continued)

\begin{tabular}{|c|c|c|c|c|c|c|c|c|}
\hline \multirow[t]{2}{*}{ Lower } & \multirow[t]{2}{*}{ Upper } & \multicolumn{3}{|l|}{ GRASP2K } & \multicolumn{3}{|l|}{ FAC } & \multirow[t]{2}{*}{$d T$} \\
\hline & & $\lambda(\AA)$ & $g f$ & $A\left(\mathrm{~s}^{-1}\right)$ & $\lambda(\AA)$ & $g f$ & $A\left(\mathrm{~s}^{-1}\right)$ & \\
\hline $1 s 2 s^{1} S_{0}$ & $1 s 4 d^{3} D_{2}$ & $1.6228 \mathrm{E}+00$ & $1.321 \mathrm{E}-04$ & $6.689 \mathrm{E}+10$ & $1.6162 \mathrm{E}+00$ & $1.318 \mathrm{E}-04$ & $6.677 \mathrm{E}+10$ & 0.003 \\
\hline $1 s 3 s^{3} s_{1}$ & $1 s 4 d^{3} D_{2}$ & $6.1111 \mathrm{E}+00$ & $1.685 \mathrm{E}-03$ & $6.018 \mathrm{E}+10$ & $6.0854 \mathrm{E}+00$ & $1.711 \mathrm{E}-03$ & $6.113 \mathrm{E}+10$ & 0.002 \\
\hline $1 s 3 s^{1} s_{0}$ & $1 s 4 d^{3} D_{2}$ & $6.1795 E+00$ & $1.100 \mathrm{E}-03$ & $3.842 \mathrm{E}+10$ & $6.1544 \mathrm{E}+00$ & $1.079 \mathrm{E}-03$ & $3.768 \mathrm{E}+10$ & 0.002 \\
\hline $1 s 3 d^{3} D_{2}$ & $1 s 4 d^{3} D_{2}$ & $6.5814 \mathrm{E}+00$ & $4.239 \mathrm{E}-04$ & $1.306 \mathrm{E}+10$ & $6.5534 \mathrm{E}+00$ & $4.253 \mathrm{E}-04$ & $1.310 \mathrm{E}+10$ & 0.000 \\
\hline $1 s 3 d^{3} D_{1}$ & $1 s 4 d^{3} D_{2}$ & $6.5866 \mathrm{E}+00$ & $1.859 \mathrm{E}-04$ & $5.715 E+09$ & $6.5585 \mathrm{E}+00$ & $1.860 \mathrm{E}-04$ & $5.720 \mathrm{E}+09$ & 0.000 \\
\hline $1 s 3 d^{3} D_{3}$ & $1 s 4 d^{3} D_{2}$ & $6.7107 \mathrm{E}+00$ & $2.138 \mathrm{E}-04$ & $6.333 E+09$ & $6.6818 \mathrm{E}+00$ & $2.135 \mathrm{E}-04$ & $6.326 \mathrm{E}+09$ & 0.000 \\
\hline $1 s 3 d{ }^{1} D_{2}$ & $1 s 4 d^{3} D_{2}$ & $6.7157 \mathrm{E}+00$ & $5.225 \mathrm{E}-05$ & $1.545 \mathrm{E}+09$ & $6.6868 \mathrm{E}+00$ & $5.192 \mathrm{E}-05$ & $1.536 \mathrm{E}+09$ & 0.000 \\
\hline $1 s 4 s^{3} S_{1}$ & $1 s 4 d^{3} D_{2}$ & $2.0428 \mathrm{E}+02$ & $4.053 \mathrm{E}-07$ & $1.296 \mathrm{E}+04$ & $2.0324 \mathrm{E}+02$ & $4.072 \mathrm{E}-07$ & $1.304 \mathrm{E}+04$ & 0.029 \\
\hline $1 s 4 s^{1} S_{0}$ & $1 s 4 d^{3} D_{2}$ & $2.4021 \mathrm{E}+02$ & $1.633 \mathrm{E}-07$ & $3.776 \mathrm{E}+03$ & $2.3934 \mathrm{E}+02$ & $1.627 \mathrm{E}-07$ & $3.756 \mathrm{E}+03$ & 0.039 \\
\hline $1 s 2 s^{3} S_{1}$ & $1 s 4 d^{3} D_{1}$ & $1.6048 \mathrm{E}+00$ & $2.169 \mathrm{E}-04$ & $1.872 \mathrm{E}+11$ & $1.5980 \mathrm{E}+00$ & $2.076 \mathrm{E}-04$ & $1.792 \mathrm{E}+11$ & 0.002 \\
\hline $1 s 3 s^{3} S_{1}$ & $1 s 4 d^{3} D_{1}$ & $6.1092 E+00$ & $1.673 \mathrm{E}-03$ & $9.967 \mathrm{E}+10$ & $6.0836 \mathrm{E}+00$ & $1.700 \mathrm{E}-03$ & $1.013 \mathrm{E}+11$ & 0.002 \\
\hline $1 s 3 d^{3} D_{2}$ & $1 s 4 d^{3} D_{1}$ & $6.5793 E+00$ & $1.840 \mathrm{E}-04$ & $9.451 \mathrm{E}+09$ & $6.5513 \mathrm{E}+00$ & $1.852 \mathrm{E}-04$ & $9.515 \mathrm{E}+09$ & 0.000 \\
\hline $1 s 3 d^{3} D_{1}$ & $1 s 4 d^{3} D_{1}$ & $6.5845 E+00$ & $1.810 \mathrm{E}-04$ & $9.280 \mathrm{E}+09$ & $6.5564 \mathrm{E}+00$ & $1.814 \mathrm{E}-04$ & $9.306 \mathrm{E}+09$ & 0.000 \\
\hline $1 s 3 d^{3} D_{3}$ & $1 s 4 d^{3} D_{1}$ & $6.7085 E+00$ & $3.503 \mathrm{E}-05$ & $1.731 \mathrm{E}+09$ & $6.6796 \mathrm{E}+00$ & $3.499 \mathrm{E}-05$ & $1.729 \mathrm{E}+09$ & 0.001 \\
\hline $1 s 3 d{ }^{1} D_{2}$ & $1 s 4 d^{3} D_{1}$ & $6.7135 E+00$ & $1.229 \mathrm{E}-04$ & $6.065 E+09$ & $6.6847 \mathrm{E}+00$ & $1.225 \mathrm{E}-04$ & $6.044 \mathrm{E}+09$ & 0.000 \\
\hline $1 s 4 s^{3} S_{1}$ & $1 s 4 d^{3} D_{1}$ & $2.0227 \mathrm{E}+02$ & $4.160 \mathrm{E}-07$ & $2.261 \mathrm{E}+04$ & $2.0125 \mathrm{E}+02$ & $4.171 \mathrm{E}-07$ & $2.270 \mathrm{E}+04$ & 0.026 \\
\hline $1 s 4 d^{3} D_{2}$ & $1 s 4 d^{3} D_{1}$ & $2.0536 \mathrm{E}+04$ & $1.788 \mathrm{E}-13$ & $9.425 \mathrm{E}-07$ & & & & 0.337 \\
\hline $1 s 2 p^{3} P_{1}^{o}$ & $1 s 4 p^{1} P_{1}^{o}$ & $1.6210 \mathrm{E}+00$ & $1.402 \mathrm{E}-04$ & $1.186 \mathrm{E}+11$ & $1.6143 E+00$ & $1.421 \mathrm{E}-04$ & $1.202 \mathrm{E}+11$ & 0.001 \\
\hline $1 s 2 p^{3} P_{2}^{o}$ & $1 s 4 p^{1} P_{1}^{o}$ & $1.7078 \mathrm{E}+00$ & $6.574 \mathrm{E}-05$ & $5.012 \mathrm{E}+10$ & $1.7006 \mathrm{E}+00$ & $6.655 \mathrm{E}-05$ & $5.072 \mathrm{E}+10$ & 0.006 \\
\hline $1 s 2 p^{1} P_{1}^{o}$ & $1 s 4 p^{1} P_{1}^{o}$ & $1.7161 \mathrm{E}+00$ & $8.979 \mathrm{E}-05$ & $6.779 E+10$ & $1.7090 \mathrm{E}+00$ & $8.874 \mathrm{E}-05$ & $6.699 \mathrm{E}+10$ & 0.000 \\
\hline $1 s 3 p^{3} P_{1}^{0}$ & $1 s 4 p^{1} P_{1}^{o}$ & $6.1732 E+00$ & $4.872 \mathrm{E}-04$ & $2.843 \mathrm{E}+10$ & $6.1480 \mathrm{E}+00$ & $4.916 \mathrm{E}-04$ & $2.867 \mathrm{E}+10$ & 0.000 \\
\hline $1 s 3 p^{3} P_{2}^{o}$ & $1 s 4 p^{1} P_{1}^{o}$ & $6.5502 \mathrm{E}+00$ & $2.512 \mathrm{E}-04$ & $1.302 \mathrm{E}+10$ & $6.5232 \mathrm{E}+00$ & $2.544 \mathrm{E}-04$ & $1.318 \mathrm{E}+10$ & 0.001 \\
\hline $1 s 3 p^{1} P_{1}^{o}$ & $1 s 4 p^{1} P_{1}^{o}$ & $6.5847 \mathrm{E}+00$ & $3.422 \mathrm{E}-04$ & $1.755 \mathrm{E}+10$ & $6.5579 \mathrm{E}+00$ & $3.415 \mathrm{E}-04$ & $1.750 \mathrm{E}+10$ & 0.000 \\
\hline $1 s 4 p^{3} P_{1}^{o}$ & $1 s 4 p^{1} P_{1}^{o}$ & $2.3520 \mathrm{E}+02$ & $3.010 \mathrm{E}-07$ & $1.210 \mathrm{E}+04$ & $2.3412 \mathrm{E}+02$ & $3.005 \mathrm{E}-07$ & $1.208 \mathrm{E}+04$ & 0.004 \\
\hline $1 s 4 p^{3} P_{2}^{o}$ & $1 s 4 p^{1} P_{1}^{o}$ & $3.0236 \mathrm{E}+03$ & $7.051 \mathrm{E}-11$ & $1.715 \mathrm{E}-02$ & & & & 0.024 \\
\hline $1 s 2 s^{3} S_{1}^{2}$ & $1 s 4 d^{3} D_{3}$ & $1.6018 \mathrm{E}+00$ & $5.612 \mathrm{E}-04$ & $2.084 \mathrm{E}+11$ & $1.5950 \mathrm{E}+00$ & $5.556 \mathrm{E}-04$ & $2.063 \mathrm{E}+11$ & 0.002 \\
\hline $1 s 3 s^{3} S_{1}$ & $1 s 4 d^{3} D_{3}$ & $6.0652 \mathrm{E}+00$ & $3.882 \mathrm{E}-03$ & $1.005 \mathrm{E}+11$ & $6.0400 \mathrm{E}+00$ & $3.968 \mathrm{E}-03$ & $1.027 \mathrm{E}+11$ & 0.002 \\
\hline $1 s 3 d^{3} D_{2}$ & $1 s 4 d^{3} D_{3}$ & $6.5283 E+00$ & $2.062 \mathrm{E}-04$ & $4.610 \mathrm{E}+09$ & $6.5006 \mathrm{E}+00$ & $2.084 \mathrm{E}-04$ & $4.660 \mathrm{E}+09$ & 0.000 \\
\hline $1 s 3 d^{3} D_{1}$ & $1 s 4 d^{3} D_{3}$ & $6.5334 \mathrm{E}+00$ & $3.327 \mathrm{E}-05$ & $7.427 \mathrm{E}+08$ & $6.5057 \mathrm{E}+00$ & $3.358 \mathrm{E}-05$ & $7.497 \mathrm{E}+08$ & 0.001 \\
\hline $1 s 3 d^{3} D_{3}$ & $1 s 4 d^{3} D_{3}$ & $6.6555 E+00$ & $8.341 \mathrm{E}-04$ & $1.794 \mathrm{E}+10$ & $6.6270 \mathrm{E}+00$ & $8.375 \mathrm{E}-04$ & $1.801 \mathrm{E}+10$ & 0.000 \\
\hline $1 s 3 d^{1} D_{2}$ & $1 s 4 d^{3} D_{3}$ & $6.6604 \mathrm{E}+00$ & $1.380 \mathrm{E}-04$ & $2.964 \mathrm{E}+09$ & $6.6320 \mathrm{E}+00$ & $1.380 \mathrm{E}-04$ & $2.966 \mathrm{E}+09$ & 0.000 \\
\hline $1 s 4 s^{3} S_{1}$ & $1 s 4 d^{3} D_{3}$ & $1.6310 \mathrm{E}+02$ & $1.890 \mathrm{E}-06$ & $6.771 \mathrm{E}+04$ & $1.6240 \mathrm{E}+02$ & $1.893 \mathrm{E}-06$ & $6.783 \mathrm{E}+04$ & 0.022 \\
\hline $1 s 4 d^{3} D_{2}$ & $1 s 4 d^{3} D_{3}$ & $8.0890 \mathrm{E}+02$ & $3.369 \mathrm{E}-09$ & $4.906 \mathrm{E}+00$ & & & & 0.003 \\
\hline $1 s 4 d^{3} D_{1}$ & $1 s 4 d^{3} D_{3}$ & $8.4207 \mathrm{E}+02$ & $4.858 \mathrm{E}-10$ & $6.529 \mathrm{E}-01$ & & & & 0.015 \\
\hline $1 s 2 p^{3} P_{1}^{o}$ & $1 s 4 f^{3} F_{3}^{o}$ & $1.6179 \mathrm{E}+00$ & $2.847 \mathrm{E}-03$ & $1.036 \mathrm{E}+12$ & $1.6112 \mathrm{E}+00$ & $2.908 \mathrm{E}-03$ & $1.058 \mathrm{E}+12$ & 0.001 \\
\hline $1 s 2 p^{3} P_{2}^{0}$ & $1 s 4 f^{3} F_{3}^{0}$ & $1.7043 \mathrm{E}+00$ & $7.811 \mathrm{E}-04$ & $2.562 \mathrm{E}+11$ & $1.6971 \mathrm{E}+00$ & $7.937 \mathrm{E}-04$ & $2.603 \mathrm{E}+11$ & 0.001 \\
\hline $1 s 2 p^{1} P_{1}^{o}$ & $1 s 4 f^{3} F_{3}^{o}$ & $1.7125 \mathrm{E}+00$ & $1.912 \mathrm{E}-04$ & $6.213 E+10$ & $1.7055 \mathrm{E}+00$ & $1.844 \mathrm{E}-04$ & $5.990 \mathrm{E}+10$ & 0.003 \\
\hline $1 s 3 p^{3} P_{1}^{o}$ & $1 s 4 f^{3} F_{3}^{o}$ & $6.1276 \mathrm{E}+00$ & $4.973 \mathrm{E}-03$ & $1.262 \mathrm{E}+11$ & $6.1026 \mathrm{E}+00$ & $4.989 \mathrm{E}-03$ & $1.265 \mathrm{E}+11$ & 0.000 \\
\hline $1 s 3 p^{3} P_{2}^{o}$ & $1 s 4 f^{3} F_{3}^{o}$ & $6.4989 \mathrm{E}+00$ & $1.148 \mathrm{E}-03$ & $2.591 \mathrm{E}+10$ & $6.4721 \mathrm{E}+00$ & $1.153 \mathrm{E}-03$ & $2.601 \mathrm{E}+10$ & 0.000 \\
\hline $1 s 3 p^{1} P_{1}^{o}$ & $1 s 4 f^{3} F_{3}^{0}$ & $6.5328 \mathrm{E}+00$ & $2.725 \mathrm{E}-04$ & $6.084 \mathrm{E}+09$ & $6.5062 \mathrm{E}+00$ & $2.680 \mathrm{E}-04$ & $5.982 \mathrm{E}+09$ & 0.000 \\
\hline $1 s 4 p^{3} P_{1}^{o}$ & $1 s 4 f^{3} F_{3}^{o}$ & $1.8322 \mathrm{E}+02$ & $6.325 \mathrm{E}-07$ & $1.795 \mathrm{E}+04$ & $1.8244 \mathrm{E}+02$ & $6.324 \mathrm{E}-07$ & $1.795 E+04$ & 0.003 \\
\hline $1 s 4 p^{3} P_{2}^{0}$ & $1 s 4 f^{3} F_{3}^{o}$ & $6.5059 \mathrm{E}+02$ & $3.452 \mathrm{E}-09$ & $7.771 \mathrm{E}+00$ & $6.4694 \mathrm{E}+02$ & $3.463 \mathrm{E}-09$ & $7.818 \mathrm{E}+00$ & 0.016 \\
\hline $1 s 4 p^{1} P_{1}^{o}$ & $1 s 4 f^{3} F_{3}^{o}$ & $8.2895 E+02$ & $3.954 \mathrm{E}-10$ & $5.482 \mathrm{E}-01$ & & & & 0.020 \\
\hline $1 s 2 p^{3} P_{1}^{o}$ & $1 s 4 f^{3} F_{2}^{o}$ & $1.6178 \mathrm{E}+00$ & $8.729 \mathrm{E}-04$ & $4.449 \mathrm{E}+11$ & $1.6111 \mathrm{E}+00$ & $9.021 \mathrm{E}-04$ & $4.597 \mathrm{E}+11$ & 0.001 \\
\hline $1 s 2 p^{3} P_{0}^{o}$ & $1 s 4 f^{3} F_{2}^{o}$ & $1.6191 \mathrm{E}+00$ & $1.244 \mathrm{E}-03$ & $6.329 E+11$ & $1.6123 \mathrm{E}+00$ & $1.269 \mathrm{E}-03$ & $6.460 \mathrm{E}+11$ & 0.000 \\
\hline $1 s 2 p^{3} P_{2}^{o}$ & $1 s 4 f^{3} F_{2}^{o}$ & $1.7042 \mathrm{E}+00$ & $1.959 \mathrm{E}-04$ & $8.998 E+10$ & $1.6971 \mathrm{E}+00$ & $1.993 \mathrm{E}-04$ & $9.155 \mathrm{E}+10$ & 0.000 \\
\hline $1 s 2 p^{1} P_{1}^{o}$ & $1 s 4 f^{3} F_{2}^{o}$ & $1.7124 \mathrm{E}+00$ & $4.103 \mathrm{E}-04$ & $1.867 \mathrm{E}+11$ & $1.7054 \mathrm{E}+00$ & $4.121 \mathrm{E}-04$ & $1.874 \mathrm{E}+11$ & 0.002 \\
\hline $1 s 3 p^{3} P_{1}^{0}$ & $1 s 4 f^{3} F_{2}^{o}$ & $6.1266 \mathrm{E}+00$ & $1.521 \mathrm{E}-03$ & $5.406 \mathrm{E}+10$ & $6.1016 \mathrm{E}+00$ & $1.533 \mathrm{E}-03$ & $5.447 \mathrm{E}+10$ & 0.000 \\
\hline $1 s 3 p^{3} P_{0}^{o}$ & $1 s 4 f^{3} F_{2}^{o}$ & $6.1314 \mathrm{E}+00$ & $2.165 \mathrm{E}-03$ & $7.684 \mathrm{E}+10$ & $6.1062 \mathrm{E}+00$ & $2.172 \mathrm{E}-03$ & $7.706 \mathrm{E}+10$ & 0.000 \\
\hline $1 s 3 p^{3} P_{2}^{o}$ & $1 s 4 f^{3} F_{2}^{o}$ & $6.4978 \mathrm{E}+00$ & $2.880 \mathrm{E}-04$ & $9.101 \mathrm{E}+09$ & $6.4710 \mathrm{E}+00$ & $2.892 \mathrm{E}-04$ & $9.137 \mathrm{E}+09$ & 0.000 \\
\hline $1 s 3 p^{1} P_{1}^{o}$ & $1 s 4 f^{3} F_{2}^{o}$ & $6.5317 \mathrm{E}+00$ & $5.991 \mathrm{E}-04$ & $1.874 \mathrm{E}+10$ & $6.5052 \mathrm{E}+00$ & $5.988 \mathrm{E}-04$ & $1.871 \mathrm{E}+10$ & 0.000 \\
\hline $1 s 4 p^{3} P_{1}^{0}$ & $1 s 4 f^{3} F_{2}^{o}$ & $1.8236 \mathrm{E}+02$ & $1.962 \mathrm{E}-07$ & $7.869 \mathrm{E}+03$ & $1.8158 \mathrm{E}+02$ & $1.960 \mathrm{E}-07$ & $7.865 \mathrm{E}+03$ & 0.002 \\
\hline $1 s 4 p^{3} P_{0}^{0}$ & $1 s 4 f^{3} F_{2}^{o}$ & $1.8406 \mathrm{E}+02$ & $2.716 \mathrm{E}-07$ & $1.070 \mathrm{E}+04$ & $1.8322 \mathrm{E}+02$ & $2.717 \mathrm{E}-07$ & $1.070 \mathrm{E}+04$ & 0.003 \\
\hline $1 s 4 p^{3} P_{2}^{o}$ & $1 s 4 f^{3} F_{2}^{o}$ & $6.3989 E+02$ & $9.105 \mathrm{E}-10$ & $2.967 \mathrm{E}+00$ & $6.3631 \mathrm{E}+02$ & $9.130 \mathrm{E}-10$ & $2.982 \mathrm{E}+00$ & 0.014 \\
\hline $1 s 4 p^{1} P_{1}^{o}$ & $1 s 4 f^{3} F_{2}^{o}$ & $8.1166 \mathrm{E}+02$ & $9.363 \mathrm{E}-10$ & $1.896 \mathrm{E}+00$ & $8.0920 \mathrm{E}+02$ & $9.307 \mathrm{E}-10$ & $1.880 \mathrm{E}+00$ & 0.020 \\
\hline $1 s 4 f^{3} F_{3}^{o}$ & $1 s 4 f^{3} F_{2}^{o}$ & $3.8916 \mathrm{E}+04$ & $1.030 \mathrm{E}-14$ & $9.073 \mathrm{E}-09$ & & & & 0.558 \\
\hline $1 s^{2}{ }^{1} S_{0}$ & $1 s 4 d^{1} D_{2}$ & $3.2740 \mathrm{E}-01$ & $6.231 \mathrm{E}-04$ & $7.755 E+12$ & $3.2603 \mathrm{E}-01$ & $7.226 \mathrm{E}-04$ & $8.992 \mathrm{E}+12$ & 0.002 \\
\hline $1 s 2 s^{3} S_{1}$ & $1 s 4 d{ }^{1} D_{2}$ & $1.6017 \mathrm{E}+00$ & $1.591 \mathrm{E}-04$ & $8.273 E+10$ & $1.5948 \mathrm{E}+00$ & $1.567 \mathrm{E}-04$ & $8.150 \mathrm{E}+10$ & 0.001 \\
\hline $1 s 2 s{ }^{1} S_{0}$ & $1 s 4 d^{1} D_{2}$ & $1.6194 \mathrm{E}+00$ & $2.277 \mathrm{E}-04$ & $1.158 \mathrm{E}+11$ & $1.6128 \mathrm{E}+00$ & $2.323 \mathrm{E}-04$ & $1.181 \mathrm{E}+11$ & 0.002 \\
\hline $1 s 3 s^{3} s_{1}$ & $1 s 4 d^{1} D_{2}$ & $6.0634 \mathrm{E}+00$ & $1.094 \mathrm{E}-03$ & $3.969 \mathrm{E}+10$ & $6.0381 \mathrm{E}+00$ & $1.117 \mathrm{E}-03$ & $4.054 \mathrm{E}+10$ & 0.002 \\
\hline $1 s 3 s^{1} S_{0}$ & $1 s 4 d{ }^{1} D_{2}$ & $6.1308 \mathrm{E}+00$ & $1.677 \mathrm{E}-03$ & $5.953 \mathrm{E}+10$ & $6.1059 \mathrm{E}+00$ & $1.661 \mathrm{E}-03$ & $5.893 \mathrm{E}+10$ & 0.002 \\
\hline $1 s 3 d^{3} D_{2}$ & $1 s 4 d{ }^{1} D_{2}$ & $6.5262 \mathrm{E}+00$ & $5.168 \mathrm{E}-05$ & $1.619 \mathrm{E}+09$ & $6.4985 \mathrm{E}+00$ & $5.227 \mathrm{E}-05$ & $1.637 \mathrm{E}+09$ & 0.000 \\
\hline $1 s 3 d^{3} D_{1}$ & $1 s 4 d^{1} D_{2}$ & $6.5313 \mathrm{E}+00$ & $1.191 \mathrm{E}-04$ & $3.724 \mathrm{E}+09$ & $6.5035 \mathrm{E}+00$ & $1.198 \mathrm{E}-04$ & $3.747 \mathrm{E}+09$ & 0.000 \\
\hline $1 s 3 d^{3} D_{3}$ & $1 s 4 d^{1} D_{2}$ & $6.6532 E+00$ & $1.372 \mathrm{E}-04$ & $4.134 \mathrm{E}+09$ & $6.6247 \mathrm{E}+00$ & $1.377 \mathrm{E}-04$ & $4.152 \mathrm{E}+09$ & 0.000 \\
\hline $1 s 3 d^{1} D_{2}$ & $1 s 4 d{ }^{1} D_{2}$ & $6.6582 \mathrm{E}+00$ & $5.591 \mathrm{E}-04$ & $1.683 \mathrm{E}+10$ & $6.6297 \mathrm{E}+00$ & $5.608 \mathrm{E}-04$ & $1.687 \mathrm{E}+10$ & 0.000 \\
\hline $1 s 4 s^{3} S_{1}$ & $1 s 4 d^{1} D_{2}$ & $1.6177 \mathrm{E}+02$ & $5.455 \mathrm{E}-07$ & $2.781 \mathrm{E}+04$ & $1.6105 \mathrm{E}+02$ & $5.455 \mathrm{E}-07$ & $2.782 \mathrm{E}+04$ & 0.020 \\
\hline $1 s 4 s{ }^{1} S_{0}$ & $1 s 4 d^{1} D_{2}$ & $1.8350 \mathrm{E}+02$ & $5.750 \mathrm{E}-07$ & $2.278 \mathrm{E}+04$ & $1.8292 \mathrm{E}+02$ & $5.727 \mathrm{E}-07$ & $2.264 \mathrm{E}+04$ & 0.027 \\
\hline $1 s 4 d^{3} D_{2}$ & $1 s 4 d^{1} D_{2}$ & $7.7722 \mathrm{E}+02$ & $9.379 \mathrm{E}-10$ & $2.071 \mathrm{E}+00$ & & & & 0.018 \\
\hline $1 s 4 d^{3} D_{1}$ & $1 s 4 d^{1} D_{2}$ & $8.0779 E+02$ & $1.943 \mathrm{E}-09$ & $3.972 \mathrm{E}+00$ & & & & 0.005 \\
\hline $1 s 4 d^{3} D_{3}$ & $1 s 4 d^{1} D_{2}$ & $1.9845 \mathrm{E}+04$ & $1.515 \mathrm{E}-13$ & $5.133 \mathrm{E}-07$ & & & & 0.342 \\
\hline $1 s 2 p^{3} P_{2}^{o}$ & $1 s 4 f^{3} F_{4}^{o}$ & $1.7025 \mathrm{E}+00$ & $5.177 \mathrm{E}-03$ & $1.324 \mathrm{E}+12$ & $1.6954 \mathrm{E}+00$ & $5.446 \mathrm{E}-03$ & $1.392 \mathrm{E}+12$ & 0.000 \\
\hline
\end{tabular}


Table 4 (continued)

\begin{tabular}{|c|c|c|c|c|c|c|c|c|}
\hline \multirow[t]{2}{*}{ Lower } & \multirow[t]{2}{*}{ Upper } & \multicolumn{3}{|l|}{ GRASP2K } & \multicolumn{3}{|l|}{ FAC } & \multirow[t]{2}{*}{$d T$} \\
\hline & & $\lambda(\AA)$ & $g f$ & $A\left(\mathrm{~s}^{-1}\right)$ & $\lambda(\AA)$ & $g f$ & $A\left(\mathrm{~s}^{-1}\right)$ & \\
\hline $1 s 3 p^{3} P_{2}^{o}$ & $1 s 4 f^{3} F_{4}^{o}$ & $6.4732 \mathrm{E}+00$ & $7.864 \mathrm{E}-03$ & $1.391 \mathrm{E}+11$ & $6.4465 \mathrm{E}+00$ & $7.967 \mathrm{E}-03$ & $1.408 \mathrm{E}+11$ & 0.000 \\
\hline $1 s 4 p^{3} P_{2}^{o}$ & $1 s 4 f^{3} F_{4}^{o}$ & $4.6544 \mathrm{E}+02$ & $6.500 \mathrm{E}-08$ & $2.224 \mathrm{E}+02$ & $4.6333 \mathrm{E}+02$ & $6.501 \mathrm{E}-08$ & $2.225 \mathrm{E}+02$ & 0.009 \\
\hline $1 s 4 f^{3} F_{3}^{o}$ & $1 s 4 f^{3} F_{4}^{0}$ & $1.6355 \mathrm{E}+03$ & $1.450 \mathrm{E}-10$ & $4.017 \mathrm{E}-02$ & $1.6325 \mathrm{E}+03$ & $1.439 \mathrm{E}-10$ & $3.970 \mathrm{E}-02$ & 0.016 \\
\hline $1 s 4 f^{3} F_{2}^{o}$ & $1 s 4 f^{3} F_{4}^{o}$ & $1.7073 \mathrm{E}+03$ & $1.015 \mathrm{E}-11$ & $2.582 \mathrm{E}-03$ & & & & 0.053 \\
\hline $1 s 2 p^{3} P_{1}^{o}$ & $1 s 4 f^{1} F_{3}^{o}$ & $1.6162 \mathrm{E}+00$ & $5.484 \mathrm{E}-06$ & $2.001 \mathrm{E}+09$ & $1.6095 \mathrm{E}+00$ & $7.555 \mathrm{E}-06$ & $2.755 E+09$ & 0.014 \\
\hline $1 s 2 p^{3} P_{2}^{o}$ & $1 s 4 f^{1} F_{3}^{o}$ & $1.7025 \mathrm{E}+00$ & $5.783 \mathrm{E}-04$ & $1.901 \mathrm{E}+11$ & $1.6953 \mathrm{E}+00$ & $6.082 \mathrm{E}-04$ & $1.999 \mathrm{E}+11$ & 0.000 \\
\hline $1 s 2 p{ }^{1} P_{1}^{o}$ & $1 s 4 f^{1} F_{3}^{o}$ & $1.7107 \mathrm{E}+00$ & $3.470 \mathrm{E}-03$ & $1.130 \mathrm{E}+12$ & $1.7036 \mathrm{E}+00$ & $3.569 \mathrm{E}-03$ & $1.161 \mathrm{E}+12$ & 0.001 \\
\hline $1 s 3 p^{3} P_{1}^{o}$ & $1 s 4 f^{1} F_{3}^{o}$ & $6.1040 \mathrm{E}+00$ & $8.647 \mathrm{E}-06$ & $2.211 \mathrm{E}+08$ & $6.0791 \mathrm{E}+00$ & $9.972 \mathrm{E}-06$ & $2.549 \mathrm{E}+08$ & 0.003 \\
\hline $1 s 3 p^{3} P_{2}^{o}$ & $1 s 4 f^{1} F_{3}^{o}$ & $6.4724 \mathrm{E}+00$ & $8.785 \mathrm{E}-04$ & $1.998 \mathrm{E}+10$ & $6.4457 \mathrm{E}+00$ & $8.900 \mathrm{E}-04$ & $2.023 \mathrm{E}+10$ & 0.000 \\
\hline $1 s 3 p{ }^{1} P_{1}^{o}$ & $1 s 4 f^{1} F_{3}^{o}$ & $6.5060 \mathrm{E}+00$ & $5.198 \mathrm{E}-03$ & $1.170 \mathrm{E}+11$ & $6.4796 \mathrm{E}+00$ & $5.218 \mathrm{E}-03$ & $1.174 \mathrm{E}+11$ & 0.000 \\
\hline $1 s 4 p^{3} P_{1}^{o}$ & $1 s 4 f^{1} F_{3}^{o}$ & $1.6424 \mathrm{E}+02$ & $1.482 \mathrm{E}-09$ & $5.234 \mathrm{E}+01$ & $1.6359 \mathrm{E}+02$ & $1.492 \mathrm{E}-09$ & $5.269 \mathrm{E}+01$ & 0.001 \\
\hline $1 s 4 p^{3} P_{2}^{o}$ & $1 s 4 f^{1} F_{3}^{o}$ & $4.6135 \mathrm{E}+02$ & $7.452 \mathrm{E}-09$ & $3.336 \mathrm{E}+01$ & $4.5925 \mathrm{E}+02$ & $7.450 \mathrm{E}-09$ & $3.337 \mathrm{E}+01$ & 0.006 \\
\hline $1 s 4 p^{1} P_{1}^{o}$ & $1 s 4 f^{1} F_{3}^{o}$ & $5.4442 \mathrm{E}+02$ & $2.699 \mathrm{E}-08$ & $8.676 \mathrm{E}+01$ & $5.4298 \mathrm{E}+02$ & $2.686 \mathrm{E}-08$ & $8.609 \mathrm{E}+01$ & 0.009 \\
\hline $1 s 4 f^{3} F_{3}^{o}$ & $1 s 4 f^{1} F_{3}^{o}$ & $1.5861 \mathrm{E}+03$ & $1.917 \mathrm{E}-11$ & $7.263 \mathrm{E}-03$ & & & & 0.019 \\
\hline $1 s 4 f^{3} F_{2}^{o}$ & $1 s 4 f^{1} F_{3}^{o}$ & $1.6535 \mathrm{E}+03$ & $1.021 \mathrm{E}-10$ & $3.557 \mathrm{E}-02$ & $1.6504 \mathrm{E}+03$ & $1.013 \mathrm{E}-10$ & $3.513 \mathrm{E}-02$ & 0.007 \\
\hline $1 s 4 f^{3} F_{4}^{o}$ & $1 s 4 f^{1} F_{3}^{o}$ & $5.2510 \mathrm{E}+04$ & $3.365 \mathrm{E}-15$ & $1.163 \mathrm{E}-09$ & & & & 0.824 \\
\hline $1 s 2 s^{3} S_{1}$ & $1 s 5 s^{3} S_{1}$ & $1.4473 \mathrm{E}+00$ & $4.378 \mathrm{E}-12$ & $4.647 \mathrm{E}+03$ & $1.4412 \mathrm{E}+00$ & $1.023 E-10$ & $1.086 \mathrm{E}+05$ & 0.891 \\
\hline $1 s 3 s^{3} S_{1}$ & $1 s 5 s^{3} S_{1}$ & $4.3197 \mathrm{E}+00$ & $7.655 \mathrm{E}-10$ & $9.121 \mathrm{E}+04$ & & & & 0.164 \\
\hline $1 s 3 d^{3} D_{2}$ & $1 s 5 s^{3} S_{1}$ & $4.5495 \mathrm{E}+00$ & $2.933 \mathrm{E}-05$ & $3.150 \mathrm{E}+09$ & $4.5304 \mathrm{E}+00$ & $2.931 \mathrm{E}-05$ & $3.148 \mathrm{E}+09$ & 0.001 \\
\hline $1 s 3 d^{3} D_{1}$ & $1 s 5 s^{3} S_{1}$ & $4.5520 \mathrm{E}+00$ & $2.949 \mathrm{E}-05$ & $3.165 E+09$ & $4.5328 \mathrm{E}+00$ & $2.930 \mathrm{E}-05$ & $3.144 \mathrm{E}+09$ & 0.003 \\
\hline $1 s 3 d^{3} D_{3}$ & $1 s 5 s^{3} S_{1}$ & $4.6109 \mathrm{E}+00$ & $7.001 \mathrm{E}-05$ & $7.322 E+09$ & $4.5914 \mathrm{E}+00$ & $6.937 \mathrm{E}-05$ & $7.254 \mathrm{E}+09$ & 0.001 \\
\hline $1 s 3 d{ }^{1} D_{2}$ & $1 s 5 s^{3} S_{1}$ & $4.6133 \mathrm{E}+00$ & $1.982 \mathrm{E}-05$ & $2.071 \mathrm{E}+09$ & $4.5938 \mathrm{E}+00$ & $1.952 \mathrm{E}-05$ & $2.039 \mathrm{E}+09$ & 0.003 \\
\hline $1 s 4 s^{3} S_{1}$ & $1 s 5 s^{3} S_{1}$ & $1.3745 \mathrm{E}+01$ & $5.045 \mathrm{E}-09$ & $5.937 E+04$ & & & & 0.022 \\
\hline $1 s 4 d^{3} D_{2}$ & $1 s 5 s^{3} S_{1}$ & $1.4736 \mathrm{E}+01$ & $1.591 \mathrm{E}-04$ & $1.629 E+09$ & $1.4676 \mathrm{E}+01$ & $1.575 \mathrm{E}-04$ & $1.612 \mathrm{E}+09$ & 0.001 \\
\hline $1 s 4 d^{3} D_{1}$ & $1 s 5 s^{3} S_{1}$ & $1.4747 \mathrm{E}+01$ & $1.592 \mathrm{E}-04$ & $1.628 \mathrm{E}+09$ & $1.4686 \mathrm{E}+01$ & $1.567 \mathrm{E}-04$ & $1.602 \mathrm{E}+09$ & 0.002 \\
\hline $1 s 4 d^{3} D_{3}$ & $1 s 5 s^{3} S_{1}$ & $1.5010 \mathrm{E}+01$ & $3.835 \mathrm{E}-04$ & $3.785 E+09$ & $1.4947 \mathrm{E}+01$ & $3.784 \mathrm{E}-04$ & $3.734 \mathrm{E}+09$ & 0.001 \\
\hline $1 s 4 d{ }^{1} D_{2}$ & $1 s 5 s^{3} S_{1}$ & $1.5021 \mathrm{E}+01$ & $1.079 \mathrm{E}-04$ & $1.063 \mathrm{E}+09$ & $1.4959 \mathrm{E}+01$ & $1.059 \mathrm{E}-04$ & $1.044 \mathrm{E}+09$ & 0.002 \\
\hline $1 s 2 p^{3} P_{1}^{o}$ & $1 s 5 p^{3} P_{1}^{o}$ & $1.4598 \mathrm{E}+00$ & $3.294 \mathrm{E}-07$ & $3.437 \mathrm{E}+08$ & $1.4537 \mathrm{E}+00$ & $3.558 \mathrm{E}-07$ & $3.711 \mathrm{E}+08$ & 0.007 \\
\hline $1 s 2 p^{3} P_{2}^{o}$ & $1 s 5 p^{3} P_{1}^{o}$ & $1.5297 \mathrm{E}+00$ & $5.743 \mathrm{E}-05$ & $5.456 \mathrm{E}+10$ & $1.5233 \mathrm{E}+00$ & $5.632 \mathrm{E}-05$ & $5.350 \mathrm{E}+10$ & 0.003 \\
\hline $1 s 2 p{ }^{1} P_{1}^{o}$ & $1 s 5 p^{3} P_{1}^{o}$ & $1.5364 \mathrm{E}+00$ & $5.106 \mathrm{E}-05$ & $4.809 \mathrm{E}+10$ & $1.5300 \mathrm{E}+00$ & $4.964 \mathrm{E}-05$ & $4.674 \mathrm{E}+10$ & 0.002 \\
\hline $1 s 3 p^{3} P_{1}^{o}$ & $1 s 5 p^{3} P_{1}^{o}$ & $4.3450 \mathrm{E}+00$ & $7.585 \mathrm{E}-07$ & $8.933 E+07$ & $4.3271 \mathrm{E}+00$ & $8.116 \mathrm{E}-07$ & $9.556 \mathrm{E}+07$ & 0.006 \\
\hline $1 s 3 p^{3} P_{2}^{o}$ & $1 s 5 p^{3} P_{1}^{o}$ & $4.5285 \mathrm{E}+00$ & $1.479 \mathrm{E}-04$ & $1.603 \mathrm{E}+10$ & $4.5097 \mathrm{E}+00$ & $1.475 \mathrm{E}-04$ & $1.598 \mathrm{E}+10$ & 0.000 \\
\hline $1 s 3 p{ }^{1} P_{1}^{o}$ & $1 s 5 p^{3} P_{1}^{o}$ & $4.5449 \mathrm{E}+00$ & $1.323 \mathrm{E}-04$ & $1.424 \mathrm{E}+10$ & $4.5263 \mathrm{E}+00$ & $1.309 \mathrm{E}-04$ & $1.408 \mathrm{E}+10$ & 0.000 \\
\hline $1 s 4 p^{3} P_{1}^{o}$ & $1 s 5 p^{3} P_{1}^{o}$ & $1.3810 \mathrm{E}+01$ & $2.420 \mathrm{E}-06$ & $2.821 \mathrm{E}+07$ & $1.3752 \mathrm{E}+01$ & $2.598 \mathrm{E}-06$ & $3.028 \mathrm{E}+07$ & 0.001 \\
\hline $1 s 4 p^{3} P_{2}^{o}$ & $1 s 5 p^{3} P_{1}^{o}$ & $1.4601 \mathrm{E}+01$ & $5.296 \mathrm{E}-04$ & $5.524 \mathrm{E}+09$ & $1.4539 \mathrm{E}+01$ & $5.330 \mathrm{E}-04$ & $5.559 \mathrm{E}+09$ & 0.000 \\
\hline $1 s 4 p{ }^{1} P_{1}^{o}$ & $1 s 5 p^{3} P_{1}^{o}$ & $1.4672 \mathrm{E}+01$ & $4.785 \mathrm{E}-04$ & $4.943 E+09$ & $1.4610 \mathrm{E}+01$ & $4.758 \mathrm{E}-04$ & $4.914 \mathrm{E}+09$ & 0.000 \\
\hline $1 s 4 f^{3} F_{3}^{o}$ & $1 s 5 p^{3} P_{1}^{o}$ & $1.4936 \mathrm{E}+01$ & $9.431 \mathrm{E}-05$ & $9.400 E+08$ & $1.4873 \mathrm{E}+01$ & $9.437 \mathrm{E}-05$ & $9.404 \mathrm{E}+08$ & 0.000 \\
\hline $1 s 4 f^{3} F_{2}^{o}$ & $1 s 5 p^{3} P_{1}^{o}$ & $1.4942 \mathrm{E}+01$ & $2.888 \mathrm{E}-05$ & $2.876 \mathrm{E}+08$ & $1.4879 \mathrm{E}+01$ & $2.862 \mathrm{E}-05$ & $2.850 \mathrm{E}+08$ & 0.001 \\
\hline $1 s 4 f^{1} F_{3}^{o}$ & $1 s 5 p^{3} P_{1}^{o}$ & $1.5078 \mathrm{E}+01$ & $1.573 \mathrm{E}-07$ & $1.538 \mathrm{E}+06$ & $1.5014 \mathrm{E}+01$ & $1.253 \mathrm{E}-07$ & $1.225 \mathrm{E}+06$ & 0.002 \\
\hline $1 s 3 d^{3} D_{2}$ & $1 s 5 s^{1} S_{0}$ & $4.5420 \mathrm{E}+00$ & $1.917 \mathrm{E}-05$ & $6.199 E+09$ & $4.5228 \mathrm{E}+00$ & $1.941 \mathrm{E}-05$ & $6.277 \mathrm{E}+09$ & 0.001 \\
\hline $1 s 3 d d^{1} D_{2}$ & $1 s 5 s^{1} s_{0}$ & $4.6055 \mathrm{E}+00$ & $2.999 \mathrm{E}-05$ & $9.431 \mathrm{E}+09$ & $4.5859 \mathrm{E}+00$ & $2.997 \mathrm{E}-05$ & $9.427 \mathrm{E}+09$ & 0.002 \\
\hline $1 s 4 d^{3} D_{2}$ & $1 s 5 s{ }^{1} S_{0}$ & $1.4657 \mathrm{E}+01$ & $1.021 \mathrm{E}-04$ & $3.171 E+09$ & $1.4596 \mathrm{E}+01$ & $1.045 \mathrm{E}-04$ & $3.246 \mathrm{E}+09$ & 0.000 \\
\hline $1 s 4 d{ }^{1} D_{2}$ & $1 s 5 s{ }^{1} S_{0}$ & $1.4939 \mathrm{E}+01$ & $1.627 \mathrm{E}-04$ & $4.864 \mathrm{E}+09$ & $1.4876 \mathrm{E}+01$ & $1.658 \mathrm{E}-04$ & $4.956 \mathrm{E}+09$ & 0.001 \\
\hline $1 s 2 p^{3} P_{2}^{o}$ & $1 s 5 p^{3} P_{0}^{o}$ & $1.5297 \mathrm{E}+00$ & $3.640 \mathrm{E}-05$ & $1.038 \mathrm{E}+11$ & $1.5233 \mathrm{E}+00$ & $3.564 \mathrm{E}-05$ & $1.015 \mathrm{E}+11$ & 0.000 \\
\hline $1 s 3 p^{3} P_{2}^{o}$ & $1 s 5 p^{3} P_{0}^{o}$ & $4.5279 \mathrm{E}+00$ & $9.388 \mathrm{E}-05$ & $3.054 \mathrm{E}+10$ & $4.5092 \mathrm{E}+00$ & $9.356 \mathrm{E}-05$ & $3.043 E+10$ & 0.000 \\
\hline $1 s 4 p^{3} P_{2}^{o}$ & $1 s 5 p^{3} P_{0}^{o}$ & $1.4595 \mathrm{E}+01$ & $3.360 \mathrm{E}-04$ & $1.052 \mathrm{E}+10$ & $1.4534 \mathrm{E}+01$ & $3.399 \mathrm{E}-04$ & $1.064 \mathrm{E}+10$ & 0.000 \\
\hline $1 s 4 f^{3} F_{2}^{o}$ & $1 s 5 p^{3} P_{0}^{o}$ & $1.4936 \mathrm{E}+01$ & $4.095 \mathrm{E}-05$ & $1.225 \mathrm{E}+09$ & $1.4874 \mathrm{E}+01$ & $4.095 \mathrm{E}-05$ & $1.224 \mathrm{E}+09$ & 0.001 \\
\hline $1 s 2 p^{3} P_{1}^{o}$ & $1 s 5 p^{3} P_{2}^{o}$ & $1.4555 \mathrm{E}+00$ & $6.570 \mathrm{E}-05$ & $4.138 \mathrm{E}+10$ & $1.4495 \mathrm{E}+00$ & $6.694 \mathrm{E}-05$ & $4.214 \mathrm{E}+10$ & 0.002 \\
\hline $1 s 2 p^{3} P_{0}^{o}$ & $1 s 5 p^{3} P_{2}^{o}$ & $1.4565 \mathrm{E}+00$ & $4.114 \mathrm{E}-05$ & $2.587 \mathrm{E}+10$ & $1.4505 \mathrm{E}+00$ & $4.181 \mathrm{E}-05$ & $2.628 \mathrm{E}+10$ & 0.001 \\
\hline $1 s 2 p^{3} P_{2}^{o}$ & $1 s 5 p^{3} P_{2}^{o}$ & $1.5251 \mathrm{E}+00$ & $6.889 \mathrm{E}-05$ & $3.951 E+10$ & $1.5187 \mathrm{E}+00$ & $6.910 \mathrm{E}-05$ & $3.963 \mathrm{E}+10$ & 0.001 \\
\hline $1 s 2 p{ }^{1} P_{1}^{o}$ & $1 s 5 p^{3} P_{2}^{o}$ & $1.5317 \mathrm{E}+00$ & $2.611 \mathrm{E}-05$ & $1.485 \mathrm{E}+10$ & $1.5253 \mathrm{E}+00$ & $2.616 \mathrm{E}-05$ & $1.487 \mathrm{E}+10$ & 0.004 \\
\hline $1 s 3 p^{3} P_{1}^{o}$ & $1 s 5 p^{3} P_{2}^{o}$ & $4.3076 \mathrm{E}+00$ & $1.546 \mathrm{E}-04$ & $1.112 \mathrm{E}+10$ & $4.2899 \mathrm{E}+00$ & $1.560 \mathrm{E}-04$ & $1.121 \mathrm{E}+10$ & 0.000 \\
\hline $1 s 3 p^{3} P_{0}^{o}$ & $1 s 5 p^{3} P_{2}^{o}$ & $4.3099 \mathrm{E}+00$ & $9.802 \mathrm{E}-05$ & $7.040 E+09$ & $4.2921 \mathrm{E}+00$ & $9.847 \mathrm{E}-05$ & $7.070 \mathrm{E}+09$ & 0.000 \\
\hline $1 s 3 p^{3} P_{2}^{o}$ & $1 s 5 p^{3} P_{2}^{o}$ & $4.4878 \mathrm{E}+00$ & $1.697 \mathrm{E}-04$ & $1.124 \mathrm{E}+10$ & $4.4692 \mathrm{E}+00$ & $1.704 \mathrm{E}-04$ & $1.128 \mathrm{E}+10$ & 0.000 \\
\hline $1 s 3 p^{1} P_{1}^{o}$ & $1 s 5 p^{3} P_{2}^{o}$ & $4.5039 \mathrm{E}+00$ & $6.500 \mathrm{E}-05$ & $4.275 E+09$ & $4.4855 \mathrm{E}+00$ & $6.502 \mathrm{E}-05$ & $4.274 \mathrm{E}+09$ & 0.001 \\
\hline $1 s 4 p^{3} P_{1}^{o}$ & $1 s 5 p^{3} P_{2}^{o}$ & $1.3439 \mathrm{E}+01$ & $4.552 \mathrm{E}-04$ & $3.363 E+09$ & $1.3383 \mathrm{E}+01$ & $4.582 \mathrm{E}-04$ & $3.384 \mathrm{E}+09$ & 0.000 \\
\hline $1 s 4 p^{3} P_{0}^{o}$ & $1 s 5 p^{3} P_{2}^{o}$ & $1.3448 \mathrm{E}+01$ & $2.902 \mathrm{E}-04$ & $2.140 E+09$ & $1.3392 \mathrm{E}+01$ & $2.890 \mathrm{E}-04$ & $2.131 \mathrm{E}+09$ & 0.000 \\
\hline $1 s 4 p^{3} P_{2}^{o}$ & $1 s 5 p^{3} P_{2}^{o}$ & $1.4186 \mathrm{E}+01$ & $5.493 \mathrm{E}-04$ & $3.641 \mathrm{E}+09$ & $1.4127 \mathrm{E}+01$ & $5.527 \mathrm{E}-04$ & $3.663 \mathrm{E}+09$ & 0.000 \\
\hline $1 s 4 p{ }^{1} P_{1}^{o}$ & $1 s 5 p^{3} P_{2}^{o}$ & $1.4253 \mathrm{E}+01$ & $2.123 \mathrm{E}-04$ & $1.394 \mathrm{E}+09$ & $1.4194 \mathrm{E}+01$ & $2.117 \mathrm{E}-04$ & $1.390 \mathrm{E}+09$ & 0.000 \\
\hline $1 s 4 f^{3} F_{3}^{o}$ & $1 s 5 p^{3} P_{2}^{0}$ & $1.4503 \mathrm{E}+01$ & $1.980 \mathrm{E}-05$ & $1.256 \mathrm{E}+08$ & $1.4442 \mathrm{E}+01$ & $1.963 \mathrm{E}-05$ & $1.245 \mathrm{E}+08$ & 0.001 \\
\hline $1 s 4 f^{3} F_{2}^{o}$ & $1 s 5 p^{3} P_{2}^{o}$ & $1.4508 \mathrm{E}+01$ & $4.987 \mathrm{E}-06$ & $3.161 \mathrm{E}+07$ & $1.4447 \mathrm{E}+01$ & $4.933 \mathrm{E}-06$ & $3.126 \mathrm{E}+07$ & 0.000 \\
\hline $1 s 4 f^{3} F_{4}^{o}$ & $1 s 5 p^{3} P_{2}^{o}$ & $1.4632 \mathrm{E}+01$ & $1.385 \mathrm{E}-04$ & $8.628 E+08$ & $1.4571 \mathrm{E}+01$ & $1.367 \mathrm{E}-04$ & $8.519 E+08$ & 0.000 \\
\hline $1 s 4 f^{1} F_{3}^{0}$ & $1 s 5 p^{3} P_{2}^{o}$ & $1.4636 \mathrm{E}+01$ & $1.545 \mathrm{E}-05$ & $9.618 \mathrm{E}+07$ & $1.4575 \mathrm{E}+01$ & $1.522 \mathrm{E}-05$ & $9.479 \mathrm{E}+07$ & 0.000 \\
\hline $1 s 5 p^{3} P_{1}^{o}$ & $1 s 5 p^{3} P_{2}^{o}$ & $4.9993 \mathrm{E}+02$ & $2.211 \mathrm{E}-07$ & $1.180 \mathrm{E}+03$ & $4.9817 \mathrm{E}+02$ & $2.213 \mathrm{E}-07$ & $1.179 \mathrm{E}+03$ & 0.003 \\
\hline $1 s 5 p^{3} P_{0}^{o}$ & $1 s 5 p^{3} P_{2}^{o}$ & $5.0634 \mathrm{E}+02$ & $1.353 \mathrm{E}-07$ & $7.042 \mathrm{E}+02$ & $5.0434 \mathrm{E}+02$ & $1.354 \mathrm{E}-07$ & $7.042 \mathrm{E}+02$ & 0.002 \\
\hline $1 s^{2}{ }^{1} S_{0}$ & $1 s 5 d^{3} D_{2}$ & $3.2010 \mathrm{E}-01$ & $2.358 \mathrm{E}-04$ & $3.069 \mathrm{E}+12$ & $3.1882 \mathrm{E}-01$ & $2.397 \mathrm{E}-04$ & $3.120 \mathrm{E}+12$ & 0.002 \\
\hline $1 s 2 s^{3} S_{1}$ & $1 s 5 d^{3} D_{2}$ & $1.4421 \mathrm{E}+00$ & $4.328 \mathrm{E}-05$ & $2.776 \mathrm{E}+10$ & $1.4360 \mathrm{E}+00$ & $3.977 \mathrm{E}-05$ & $2.551 \mathrm{E}+10$ & 0.000 \\
\hline $1 s 2 s^{1} S_{0}$ & $1 s 5 d^{3} D_{2}$ & $1.4565 \mathrm{E}+00$ & $2.475 \mathrm{E}-05$ & $1.556 \mathrm{E}+10$ & $1.4506 \mathrm{E}+00$ & $2.497 \mathrm{E}-05$ & $1.569 \mathrm{E}+10$ & 0.003 \\
\hline $1 s 3 s^{3} S_{1}$ & $1 s 5 d^{3} D_{2}$ & $4.2737 \mathrm{E}+00$ & $2.788 \mathrm{E}-04$ & $2.036 \mathrm{E}+10$ & $4.2558 \mathrm{E}+00$ & $2.762 \mathrm{E}-04$ & $2.017 \mathrm{E}+10$ & 0.002 \\
\hline $1 s 3 s^{1} S_{0}$ & $1 s 5 d^{3} D_{2}$ & $4.3071 \mathrm{E}+00$ & $1.759 \mathrm{E}-04$ & $1.265 \mathrm{E}+10$ & $4.2893 \mathrm{E}+00$ & $1.752 \mathrm{E}-04$ & $1.259 \mathrm{E}+10$ & 0.001 \\
\hline $1 s 3 d^{3} D_{2}$ & $1 s 5 d^{3} D_{2}$ & $4.4985 \mathrm{E}+00$ & $9.377 \mathrm{E}-05$ & $6.182 \mathrm{E}+09$ & $4.4794 \mathrm{E}+00$ & $9.361 \mathrm{E}-05$ & $6.171 \mathrm{E}+09$ & 0.001 \\
\hline
\end{tabular}


Table 4 (continued)

\begin{tabular}{|c|c|c|c|c|c|c|c|c|}
\hline \multirow[t]{2}{*}{ Lower } & \multirow[t]{2}{*}{ Upper } & \multicolumn{3}{|l|}{ GRASP2K } & \multicolumn{3}{|l|}{ FAC } & \multirow[t]{2}{*}{$d T$} \\
\hline & & $\lambda(\AA)$ & $g f$ & $A\left(\mathrm{~s}^{-1}\right)$ & $\lambda(\AA)$ & $g f$ & $A\left(\mathrm{~s}^{-1}\right)$ & \\
\hline $1 s 3 d^{3} D_{1}$ & $1 s 5 d^{3} D_{2}$ & $4.5010 \mathrm{E}+00$ & $4.110 \mathrm{E}-05$ & $2.707 \mathrm{E}+09$ & $4.4818 \mathrm{E}+00$ & $4.103 \mathrm{E}-05$ & $2.702 E+09$ & 0.002 \\
\hline $1 s 3 d^{3} D_{3}$ & $1 s 5 d^{3} D_{2}$ & $4.5585 \mathrm{E}+00$ & $4.574 \mathrm{E}-05$ & $2.936 \mathrm{E}+09$ & $4.5391 \mathrm{E}+00$ & $4.533 \mathrm{E}-05$ & $2.910 \mathrm{E}+09$ & 0.001 \\
\hline $1 s 3 d^{1} D_{2}$ & $1 s 5 d^{3} D_{2}$ & $4.5609 \mathrm{E}+00$ & $1.111 \mathrm{E}-05$ & $7.124 \mathrm{E}+08$ & $4.5414 \mathrm{E}+00$ & $1.094 \mathrm{E}-05$ & $7.020 \mathrm{E}+08$ & 0.002 \\
\hline $1 s 4 s^{3} S_{1}$ & $1 s 5 d^{3} D_{2}$ & $1.3290 \mathrm{E}+01$ & $1.104 \mathrm{E}-03$ & $8.339 E+09$ & $1.3233 \mathrm{E}+01$ & $1.121 \mathrm{E}-03$ & $8.472 \mathrm{E}+09$ & 0.002 \\
\hline $1 s 4 s^{1} S_{0}$ & $1 s 5 d^{3} D_{2}$ & $1.3420 \mathrm{E}+01$ & $7.221 \mathrm{E}-04$ & $5.349 E+09$ & $1.3364 \mathrm{E}+01$ & $7.080 \mathrm{E}-04$ & $5.244 \mathrm{E}+09$ & 0.001 \\
\hline $1 s 4 d^{3} D_{2}$ & $1 s 5 d^{3} D_{2}$ & $1.4214 \mathrm{E}+01$ & $4.446 \mathrm{E}-04$ & $2.936 \mathrm{E}+09$ & $1.4154 \mathrm{E}+01$ & $4.454 \mathrm{E}-04$ & $2.940 \mathrm{E}+09$ & 0.000 \\
\hline $1 s 4 d^{3} D_{1}$ & $1 s 5 d^{3} D_{2}$ & $1.4224 \mathrm{E}+01$ & $1.955 \mathrm{E}-04$ & $1.289 \mathrm{E}+09$ & $1.4164 \mathrm{E}+01$ & $1.954 \mathrm{E}-04$ & $1.288 \mathrm{E}+09$ & 0.000 \\
\hline $1 s 4 d^{3} D_{3}$ & $1 s 5 d^{3} D_{2}$ & $1.4468 \mathrm{E}+01$ & $2.257 \mathrm{E}-04$ & $1.438 \mathrm{E}+09$ & $1.4407 \mathrm{E}+01$ & $2.258 \mathrm{E}-04$ & $1.439 E+09$ & 0.000 \\
\hline $1 s 4 d{ }^{1} D_{2}$ & $1 s 5 d^{3} D_{2}$ & $1.4479 \mathrm{E}+01$ & $5.540 \mathrm{E}-05$ & $3.525 \mathrm{E}+08$ & $1.4417 \mathrm{E}+01$ & $5.517 \mathrm{E}-05$ & $3.511 \mathrm{E}+08$ & 0.000 \\
\hline $1 s 5 s^{3} s_{1}$ & $1 s 5 d^{3} D_{2}$ & $4.0118 \mathrm{E}+02$ & $3.701 \mathrm{E}-07$ & $3.067 \mathrm{E}+03$ & $3.9828 \mathrm{E}+02$ & $3.741 \mathrm{E}-07$ & $3.119 \mathrm{E}+03$ & 0.027 \\
\hline $1 s 5 s{ }^{1} S_{0}$ & $1 s 5 d^{3} D_{2}$ & $4.7039 \mathrm{E}+02$ & $1.499 \mathrm{E}-07$ & $9.037 \mathrm{E}+02$ & $4.6740 \mathrm{E}+02$ & $1.505 \mathrm{E}-07$ & $9.114 \mathrm{E}+02$ & 0.045 \\
\hline $1 s 2 p^{3} P_{1}^{o}$ & $1 s 5 p^{1} P_{1}^{o}$ & $1.4551 \mathrm{E}+00$ & $5.848 \mathrm{E}-05$ & $6.140 \mathrm{E}+10$ & $1.4491 \mathrm{E}+00$ & $5.907 \mathrm{E}-05$ & $6.201 \mathrm{E}+10$ & 0.000 \\
\hline $1 s 2 p^{3} P_{2}^{o}$ & $1 s 5 p^{1} P_{1}^{o}$ & $1.5247 \mathrm{E}+00$ & $2.653 \mathrm{E}-05$ & $2.538 \mathrm{E}+10$ & $1.5183 \mathrm{E}+00$ & $2.678 \mathrm{E}-05$ & $2.561 \mathrm{E}+10$ & 0.008 \\
\hline $1 s 2 p^{1} P_{1}^{o}$ & $1 s 5 p^{1} P_{1}^{o}$ & $1.5313 \mathrm{E}+00$ & $3.621 \mathrm{E}-05$ & $3.434 \mathrm{E}+10$ & $1.5249 \mathrm{E}+00$ & $3.560 \mathrm{E}-05$ & $3.375 E+10$ & 0.002 \\
\hline $1 s 3 p^{3} P_{1}^{o}$ & $1 s 5 p^{1} P_{1}^{o}$ & $4.3044 \mathrm{E}+00$ & $1.385 \mathrm{E}-04$ & $1.662 \mathrm{E}+10$ & $4.2867 \mathrm{E}+00$ & $1.391 \mathrm{E}-04$ & $1.669 \mathrm{E}+10$ & 0.000 \\
\hline $1 s 3 p^{3} P_{2}^{o}$ & $1 s 5 p^{1} P_{1}^{o}$ & $4.4844 \mathrm{E}+00$ & $6.547 \mathrm{E}-05$ & $7.239 \mathrm{E}+09$ & $4.4658 \mathrm{E}+00$ & $6.579 \mathrm{E}-05$ & $7.272 \mathrm{E}+09$ & 0.001 \\
\hline $1 s 3 p^{1} P_{1}^{o}$ & $1 s 5 p^{1} P_{1}^{o}$ & $4.5006 \mathrm{E}+00$ & $8.834 \mathrm{E}-05$ & $9.697 \mathrm{E}+09$ & $4.4821 \mathrm{E}+00$ & $8.767 E-05$ & $9.621 \mathrm{E}+09$ & 0.000 \\
\hline $1 s 4 p^{3} P_{1}^{o}$ & $1 s 5 p^{1} P_{1}^{o}$ & $1.3409 \mathrm{E}+01$ & $4.062 \mathrm{E}-04$ & $5.023 \mathrm{E}+09$ & $1.3352 \mathrm{E}+01$ & $4.085 \mathrm{E}-04$ & $5.051 \mathrm{E}+09$ & 0.000 \\
\hline $1 s 4 p^{3} P_{2}^{o}$ & $1 s 5 p^{1} P_{1}^{o}$ & $1.4153 \mathrm{E}+01$ & $2.101 \mathrm{E}-04$ & $2.332 \mathrm{E}+09$ & $1.4093 \mathrm{E}+01$ & $2.121 \mathrm{E}-04$ & $2.355 \mathrm{E}+09$ & 0.000 \\
\hline $1 s 4 p^{1} P_{1}^{o}$ & $1 s 5 p^{1} P_{1}^{o}$ & $1.4219 \mathrm{E}+01$ & $2.851 \mathrm{E}-04$ & $3.135 E+09$ & $1.4160 \mathrm{E}+01$ & $2.846 \mathrm{E}-04$ & $3.130 \mathrm{E}+09$ & 0.000 \\
\hline $1 s 4 f^{3} F_{3}^{o}$ & $1 s 5 p^{1} P_{1}^{o}$ & $1.4467 \mathrm{E}+01$ & $4.625 \mathrm{E}-06$ & $4.913 \mathrm{E}+07$ & $1.4407 \mathrm{E}+01$ & $4.908 \mathrm{E}-06$ & $5.213 \mathrm{E}+07$ & 0.000 \\
\hline $1 s 4 f^{3} F_{2}^{o}$ & $1 s 5 p^{1} P_{1}^{o}$ & $1.4473 \mathrm{E}+01$ & $1.034 \mathrm{E}-05$ & $1.097 \mathrm{E}+08$ & $1.4412 \mathrm{E}+01$ & $1.028 \mathrm{E}-05$ & $1.091 \mathrm{E}+08$ & 0.001 \\
\hline $1 s 4 f^{1} F_{3}^{o}$ & $1 s 5 p^{1} P_{1}^{o}$ & $1.4601 \mathrm{E}+01$ & $9.082 \mathrm{E}-05$ & $9.472 \mathrm{E}+08$ & $1.4539 \mathrm{E}+01$ & $9.153 \mathrm{E}-05$ & $9.546 \mathrm{E}+08$ & 0.000 \\
\hline $1 s 5 p^{3} P_{1}^{0}$ & $1 s 5 p^{1} P_{1}^{o}$ & $4.6126 \mathrm{E}+02$ & $2.539 \mathrm{E}-07$ & $2.653 \mathrm{E}+03$ & $4.5925 \mathrm{E}+02$ & $2.534 \mathrm{E}-07$ & $2.649 \mathrm{E}+03$ & 0.004 \\
\hline $1 s 5 p^{3} P_{2}^{o}$ & $1 s 5 p^{1} P_{1}^{o}$ & $5.9640 \mathrm{E}+03$ & $5.793 \mathrm{E}-11$ & $3.621 \mathrm{E}-03$ & & & & 0.013 \\
\hline $1 s 2 s^{3} S_{1}^{2}$ & $1 s 5 d^{3} D_{1}$ & $1.4421 \mathrm{E}+00$ & $4.306 \mathrm{E}-05$ & $4.604 \mathrm{E}+10$ & $1.4359 \mathrm{E}+00$ & $3.931 \mathrm{E}-05$ & $4.203 \mathrm{E}+10$ & 0.002 \\
\hline $1 s 3 s^{3} S_{1}$ & $1 s 5 d^{3} D_{1}$ & $4.2732 \mathrm{E}+00$ & $2.771 \mathrm{E}-04$ & $3.375 \mathrm{E}+10$ & $4.2553 \mathrm{E}+00$ & $2.739 \mathrm{E}-04$ & $3.335 \mathrm{E}+10$ & 0.002 \\
\hline $1 s 3 d^{3} D_{2}$ & $1 s 5 d^{3} D_{1}$ & $4.4980 \mathrm{E}+00$ & $4.080 \mathrm{E}-05$ & $4.484 \mathrm{E}+09$ & $4.4789 \mathrm{E}+00$ & $4.078 \mathrm{E}-05$ & $4.481 \mathrm{E}+09$ & 0.001 \\
\hline $1 s 3 d^{3} D_{1}$ & $1 s 5 d^{3} D_{1}$ & $4.5005 \mathrm{E}+00$ & $4.016 \mathrm{E}-05$ & $4.408 \mathrm{E}+09$ & $4.4813 \mathrm{E}+00$ & $3.999 \mathrm{E}-05$ & $4.390 \mathrm{E}+09$ & 0.001 \\
\hline $1 s 3 d^{3} D_{3}$ & $1 s 5 d^{3} D_{1}$ & $4.5580 \mathrm{E}+00$ & $7.478 \mathrm{E}-06$ & $8.003 E+08$ & $4.5386 \mathrm{E}+00$ & $7.403 \mathrm{E}-06$ & $7.923 \mathrm{E}+08$ & 0.003 \\
\hline $1 s 3 d^{1} D_{2}$ & $1 s 5 d^{3} D_{1}$ & $4.5603 \mathrm{E}+00$ & $2.632 \mathrm{E}-05$ & $2.814 \mathrm{E}+09$ & $4.5409 \mathrm{E}+00$ & $2.597 \mathrm{E}-05$ & $2.777 \mathrm{E}+09$ & 0.001 \\
\hline $1 s 4 s^{3} S_{1}$ & $1 s 5 d^{3} D_{1}$ & $1.3285 \mathrm{E}+01$ & $1.095 \mathrm{E}-03$ & $1.380 \mathrm{E}+10$ & $1.3229 \mathrm{E}+01$ & $1.114 \mathrm{E}-03$ & $1.403 \mathrm{E}+10$ & 0.002 \\
\hline $1 s 4 d^{3} D_{2}$ & $1 s 5 d^{3} D_{1}$ & $1.4209 \mathrm{E}+01$ & $1.940 \mathrm{E}-04$ & $2.137 \mathrm{E}+09$ & $1.4150 \mathrm{E}+01$ & $1.951 \mathrm{E}-04$ & $2.149 E+09$ & 0.000 \\
\hline $1 s 4 d^{3} D_{1}$ & $1 s 5 d^{3} D_{1}$ & $1.4219 \mathrm{E}+01$ & $1.902 \mathrm{E}-04$ & $2.091 \mathrm{E}+09$ & $1.4159 \mathrm{E}+01$ & $1.904 \mathrm{E}-04$ & $2.094 \mathrm{E}+09$ & 0.000 \\
\hline $1 s 4 d^{3} D_{3}$ & $1 s 5 d^{3} D_{1}$ & $1.4463 \mathrm{E}+01$ & $3.687 \mathrm{E}-05$ & $3.919 E+08$ & $1.4402 \mathrm{E}+01$ & $3.689 \mathrm{E}-05$ & $3.921 \mathrm{E}+08$ & 0.001 \\
\hline $1 s 4 d{ }^{1} D_{2}$ & $1 s 5 d^{3} D_{1}$ & $1.4474 \mathrm{E}+01$ & $1.291 \mathrm{E}-04$ & $1.370 \mathrm{E}+09$ & $1.4412 \mathrm{E}+01$ & $1.289 \mathrm{E}-04$ & $1.368 \mathrm{E}+09$ & 0.000 \\
\hline $1 s 5 s^{3} s_{1}$ & $1 s 5 d^{3} D_{1}$ & $3.9728 \mathrm{E}+02$ & $3.792 \mathrm{E}-07$ & $5.342 \mathrm{E}+03$ & $3.9446 \mathrm{E}+02$ & $3.826 \mathrm{E}-07$ & $5.421 \mathrm{E}+03$ & 0.023 \\
\hline $1 s 5 d^{3} D_{2}$ & $1 s 5 d^{3} D_{1}$ & $4.0886 \mathrm{E}+04$ & $1.644 \mathrm{E}-13$ & $2.186 \mathrm{E}-07$ & & & & 0.336 \\
\hline $1 s 2 s^{3} S_{1}$ & $1 s 5 d^{3} D_{3}$ & $1.4408 \mathrm{E}+00$ & $1.204 \mathrm{E}-04$ & $5.525 \mathrm{E}+10$ & $1.4347 \mathrm{E}+00$ & $1.149 \mathrm{E}-04$ & $5.277 \mathrm{E}+10$ & 0.002 \\
\hline $1 s 3 s^{3} S_{1}$ & $1 s 5 d^{3} D_{3}$ & $4.2622 \mathrm{E}+00$ & $6.786 \mathrm{E}-04$ & $3.560 \mathrm{E}+10$ & $4.2443 \mathrm{E}+00$ & $6.795 \mathrm{E}-04$ & $3.563 \mathrm{E}+10$ & 0.002 \\
\hline $1 s 3 d^{3} D_{2}$ & $1 s 5 d^{3} D_{3}$ & $4.4858 \mathrm{E}+00$ & $4.726 \mathrm{E}-05$ & $2.238 \mathrm{E}+09$ & $4.4668 \mathrm{E}+00$ & $4.769 \mathrm{E}-05$ & $2.258 \mathrm{E}+09$ & 0.001 \\
\hline $1 s 3 d^{3} D_{1}$ & $1 s 5 d^{3} D_{3}$ & $4.4882 \mathrm{E}+00$ & $7.661 \mathrm{E}-06$ & $3.624 \mathrm{E}+08$ & $4.4692 \mathrm{E}+00$ & $7.705 \mathrm{E}-06$ & $3.644 \mathrm{E}+08$ & 0.002 \\
\hline $1 s 3 d^{3} D_{3}$ & $1 s 5 d^{3} D_{3}$ & $4.5454 \mathrm{E}+00$ & $1.848 \mathrm{E}-04$ & $8.523 E+09$ & $4.5261 \mathrm{E}+00$ & $1.847 \mathrm{E}-04$ & $8.521 \mathrm{E}+09$ & 0.001 \\
\hline $1 s 3 d^{1} D_{2}$ & $1 s 5 d^{3} D_{3}$ & $4.5477 \mathrm{E}+00$ & $3.058 \mathrm{E}-05$ & $1.409 \mathrm{E}+09$ & $4.5284 \mathrm{E}+00$ & $3.047 \mathrm{E}-05$ & $1.404 \mathrm{E}+09$ & 0.001 \\
\hline $1 s 4 s^{3} S_{1}$ & $1 s 5 d^{3} D_{3}$ & $1.3179 \mathrm{E}+01$ & $2.524 \mathrm{E}-03$ & $1.385 \mathrm{E}+10$ & $1.3123 \mathrm{E}+01$ & $2.570 \mathrm{E}-03$ & $1.410 \mathrm{E}+10$ & 0.002 \\
\hline $1 s 4 d^{3} D_{2}$ & $1 s 5 d^{3} D_{3}$ & $1.4088 \mathrm{E}+01$ & $2.165 \mathrm{E}-04$ & $1.040 \mathrm{E}+09$ & $1.4029 \mathrm{E}+01$ & $2.178 \mathrm{E}-04$ & $1.046 \mathrm{E}+09$ & 0.000 \\
\hline $1 s 4 d^{3} D_{1}$ & $1 s 5 d^{3} D_{3}$ & $1.4097 \mathrm{E}+01$ & $3.491 \mathrm{E}-05$ & $1.674 \mathrm{E}+08$ & $1.4038 \mathrm{E}+01$ & $3.507 \mathrm{E}-05$ & $1.681 \mathrm{E}+08$ & 0.000 \\
\hline $1 s 4 d^{3} D_{3}$ & $1 s 5 d^{3} D_{3}$ & $1.4337 \mathrm{E}+01$ & $8.763 \mathrm{E}-04$ & $4.062 \mathrm{E}+09$ & $1.4277 \mathrm{E}+01$ & $8.786 \mathrm{E}-04$ & $4.072 E+09$ & 0.000 \\
\hline $1 s 4 d{ }^{1} D_{2}$ & $1 s 5 d^{3} D_{3}$ & $1.4348 \mathrm{E}+01$ & $1.443 \mathrm{E}-04$ & $6.680 \mathrm{E}+08$ & $1.4287 \mathrm{E}+01$ & $1.441 \mathrm{E}-04$ & $6.672 E+08$ & 0.000 \\
\hline $1 s 5 s^{3} S_{1}$ & $1 s 5 d^{3} D_{3}$ & $3.1997 \mathrm{E}+02$ & $1.720 \mathrm{E}-06$ & $1.600 \mathrm{E}+04$ & $3.1810 \mathrm{E}+02$ & $1.730 \mathrm{E}-06$ & $1.615 \mathrm{E}+04$ & 0.020 \\
\hline $1 s 5 d^{3} D_{2}$ & $1 s 5 d^{3} D_{3}$ & $1.5807 \mathrm{E}+03$ & $3.267 \mathrm{E}-09$ & $1.246 \mathrm{E}+00$ & & & & 0.003 \\
\hline $1 s 5 d^{3} D_{1}$ & $1 s 5 d^{3} D_{3}$ & $1.6443 \mathrm{E}+03$ & $4.714 \mathrm{E}-10$ & $1.661 \mathrm{E}-01$ & & & & 0.008 \\
\hline $1 s 2 p^{3} P_{1}^{o}$ & $1 s 5 f^{3} F_{3}^{o}$ & $1.4538 \mathrm{E}+00$ & $1.563 \mathrm{E}-03$ & $7.049 \mathrm{E}+11$ & $1.4478 \mathrm{E}+00$ & $1.594 \mathrm{E}-03$ & $7.186 \mathrm{E}+11$ & 0.001 \\
\hline $1 s 2 p^{3} P_{2}^{o}$ & $1 s 5 f^{3} F_{3}^{o}$ & $1.5232 \mathrm{E}+00$ & $4.174 \mathrm{E}-04$ & $1.714 \mathrm{E}+11$ & $1.5168 \mathrm{E}+00$ & $4.215 E-04$ & $1.730 \mathrm{E}+11$ & 0.001 \\
\hline $1 s 2 p^{1} P_{1}^{o}$ & $1 s 5 f^{3} F_{3}^{o}$ & $1.5298 \mathrm{E}+00$ & $1.017 \mathrm{E}-04$ & $4.140 \mathrm{E}+10$ & $1.5235 \mathrm{E}+00$ & $9.866 \mathrm{E}-05$ & $4.016 \mathrm{E}+10$ & 0.003 \\
\hline $1 s 3 p^{3} P_{1}^{0}$ & $1 s 5 f^{3} F_{3}^{0}$ & $4.2930 \mathrm{E}+00$ & $6.727 \mathrm{E}-05$ & $3.478 \mathrm{E}+09$ & $4.2753 \mathrm{E}+00$ & $6.478 \mathrm{E}-05$ & $3.348 \mathrm{E}+09$ & 0.001 \\
\hline $1 s 3 p^{3} P_{2}^{o}$ & $1 s 5 f^{3} F_{3}^{o}$ & $4.4720 \mathrm{E}+00$ & $4.140 \mathrm{E}-06$ & $1.972 \mathrm{E}+08$ & $4.4534 \mathrm{E}+00$ & $3.472 \mathrm{E}-06$ & $1.654 \mathrm{E}+08$ & 0.002 \\
\hline $1 s 3 p^{1} P_{1}^{o}$ & $1 s 5 f^{3} F_{3}^{o}$ & $4.4880 \mathrm{E}+00$ & $8.259 \mathrm{E}-07$ & $3.907 \mathrm{E}+07$ & $4.4696 \mathrm{E}+00$ & $1.108 \mathrm{E}-06$ & $5.240 \mathrm{E}+07$ & 0.012 \\
\hline $1 s 4 p^{3} P_{1}^{0}$ & $1 s 5 f^{3} F_{3}^{0}$ & $1.3298 \mathrm{E}+01$ & $3.606 \mathrm{E}-03$ & $1.943 \mathrm{E}+10$ & $1.3242 \mathrm{E}+01$ & $3.616 \mathrm{E}-03$ & $1.948 \mathrm{E}+10$ & 0.000 \\
\hline $1 s 4 p^{3} P_{2}^{o}$ & $1 s 5 f^{3} F_{3}^{o}$ & $1.4030 \mathrm{E}+01$ & $8.664 \mathrm{E}-04$ & $4.194 \mathrm{E}+09$ & $1.3970 \mathrm{E}+01$ & $8.723 E-04$ & $4.223 \mathrm{E}+09$ & 0.000 \\
\hline $1 s 4 p^{1} P_{1}^{o}$ & $1 s 5 f^{3} F_{3}^{o}$ & $1.4095 \mathrm{E}+01$ & $2.047 \mathrm{E}-04$ & $9.816 \mathrm{E}+08$ & $1.4036 \mathrm{E}+01$ & $2.005 E-04$ & $9.620 \mathrm{E}+08$ & 0.000 \\
\hline $1 s 4 f^{3} F_{3}^{o}$ & $1 s 5 f^{3} F_{3}^{o}$ & $1.4339 \mathrm{E}+01$ & $5.397 \mathrm{E}-04$ & $2.501 \mathrm{E}+09$ & $1.4278 \mathrm{E}+01$ & $5.406 \mathrm{E}-04$ & $2.505 E+09$ & 0.000 \\
\hline 1s $4 f^{3} F_{2}^{0}$ & $1 s 5 f^{3} F_{3}^{0}$ & $1.4344 \mathrm{E}+01$ & $8.972 \mathrm{E}-05$ & $4.155 \mathrm{E}+08$ & $1.4283 \mathrm{E}+01$ & $8.978 \mathrm{E}-05$ & $4.157 \mathrm{E}+08$ & 0.000 \\
\hline $1 s 4 f^{3} F_{4}^{o}$ & $1 s 5 f^{3} F_{3}^{o}$ & $1.4466 \mathrm{E}+01$ & $9.385 \mathrm{E}-05$ & $4.274 \mathrm{E}+08$ & $1.4404 \mathrm{E}+01$ & $9.364 \mathrm{E}-05$ & $4.264 \mathrm{E}+08$ & 0.000 \\
\hline $1 s 4 f^{1} F_{3}^{o}$ & $1 s 5 f^{3} F_{3}^{o}$ & $1.4470 \mathrm{E}+01$ & $1.130 \mathrm{E}-05$ & $5.141 \mathrm{E}+07$ & $1.4408 \mathrm{E}+01$ & $1.125 \mathrm{E}-05$ & $5.124 \mathrm{E}+07$ & 0.000 \\
\hline $1 s 5 p^{3} P_{1}^{0}$ & $1 s 5 f^{3} F_{3}^{o}$ & $3.5882 \mathrm{E}+02$ & $8.216 \mathrm{E}-07$ & $6.081 \mathrm{E}+03$ & $3.5685 \mathrm{E}+02$ & $8.246 \mathrm{E}-07$ & $6.118 \mathrm{E}+03$ & 0.001 \\
\hline $1 s 5 p^{3} P_{2}^{o}$ & $1 s 5 f^{3} F_{3}^{o}$ & $1.2712 \mathrm{E}+03$ & $4.533 \mathrm{E}-09$ & $2.673 \mathrm{E}+00$ & $1.2579 \mathrm{E}+03$ & $4.615 \mathrm{E}-09$ & $2.756 \mathrm{E}+00$ & 0.010 \\
\hline $1 s 5 p^{1} P_{1}^{o}$ & $1 s 5 f^{3} F_{3}^{o}$ & $1.6156 \mathrm{E}+03$ & $5.213 \mathrm{E}-10$ & $1.903 \mathrm{E}-01$ & & & & 0.012 \\
\hline $1 s 2 p^{3} P_{1}^{o}$ & $1 s 5 f^{3} F_{2}^{o}$ & $1.4538 \mathrm{E}+00$ & $4.796 \mathrm{E}-04$ & $3.027 \mathrm{E}+11$ & $1.4478 \mathrm{E}+00$ & $4.936 \mathrm{E}-04$ & $3.115 \mathrm{E}+11$ & 0.001 \\
\hline $1 s 2 p^{3} P_{0}^{o}$ & $1 s 5 f^{3} F_{2}^{o}$ & $1.4548 \mathrm{E}+00$ & $6.831 \mathrm{E}-04$ & $4.305 \mathrm{E}+11$ & $1.4488 \mathrm{E}+00$ & $6.959 \mathrm{E}-04$ & $4.385 \mathrm{E}+11$ & 0.000 \\
\hline $1 s 2 p^{3} P_{2}^{o}$ & $1 s 5 f^{3} F_{2}^{o}$ & $1.5232 \mathrm{E}+00$ & $1.045 \mathrm{E}-04$ & $6.006 \mathrm{E}+10$ & $1.5168 \mathrm{E}+00$ & $1.058 \mathrm{E}-04$ & $6.085 \mathrm{E}+10$ & 0.001 \\
\hline
\end{tabular}


Table 4 (continued)

\begin{tabular}{|c|c|c|c|c|c|c|c|c|}
\hline \multirow[t]{2}{*}{ Lower } & \multirow[t]{2}{*}{ Upper } & \multicolumn{3}{|l|}{ GRASP2K } & \multicolumn{3}{|l|}{ FAC } & \multirow[t]{2}{*}{$d T$} \\
\hline & & $\lambda(\AA)$ & $g f$ & $A\left(\mathrm{~s}^{-1}\right)$ & $\lambda(\AA)$ & $g f$ & $A\left(\mathrm{~s}^{-1}\right)$ & \\
\hline $1 s 2 p{ }^{1} P_{1}^{o}$ & $1 s 5 f^{3} F_{2}^{o}$ & $1.5298 \mathrm{E}+00$ & $2.187 \mathrm{E}-04$ & $1.247 \mathrm{E}+11$ & $1.5234 \mathrm{E}+00$ & $2.185 \mathrm{E}-04$ & $1.245 \mathrm{E}+11$ & 0.002 \\
\hline $1 s 3 p^{3} P_{1}^{o}$ & $1 s 5 f^{3} F_{2}^{o}$ & $4.2928 \mathrm{E}+00$ & $2.075 \mathrm{E}-05$ & $1.502 \mathrm{E}+09$ & $4.2750 \mathrm{E}+00$ & $1.920 \mathrm{E}-05$ & $1.389 \mathrm{E}+09$ & 0.002 \\
\hline $1 s 3 p^{3} P_{0}^{o}$ & $1 s 5 f^{3} F_{2}^{o}$ & $4.2951 \mathrm{E}+00$ & $2.908 \mathrm{E}-05$ & $2.103 E+09$ & $4.2773 \mathrm{E}+00$ & $2.793 \mathrm{E}-05$ & $2.019 \mathrm{E}+09$ & 0.001 \\
\hline $1 s 3 p^{3} P_{2}^{o}$ & $1 s 5 f^{3} F_{2}^{o}$ & $4.4718 \mathrm{E}+00$ & $1.047 \mathrm{E}-06$ & $6.984 \mathrm{E}+07$ & $4.4531 \mathrm{E}+00$ & $8.606 \mathrm{E}-07$ & $5.740 \mathrm{E}+07$ & 0.005 \\
\hline $1 s 3 p{ }^{1} P_{1}^{o}$ & $1 s 5 f^{3} F_{2}^{o}$ & $4.4878 \mathrm{E}+00$ & $1.836 \mathrm{E}-06$ & $1.216 \mathrm{E}+08$ & $4.4693 \mathrm{E}+00$ & $1.609 \mathrm{E}-06$ & $1.065 \mathrm{E}+08$ & 0.004 \\
\hline $1 s 4 p^{3} P_{1}^{o}$ & $1 s 5 f^{3} F_{2}^{o}$ & $1.3296 \mathrm{E}+01$ & $1.101 \mathrm{E}-03$ & $8.310 E+09$ & $1.3240 \mathrm{E}+01$ & $1.110 \mathrm{E}-03$ & $8.382 E+09$ & 0.000 \\
\hline $1 s 4 p^{3} P_{0}^{o}$ & $1 s 5 f^{3} F_{2}^{o}$ & $1.3305 \mathrm{E}+01$ & $1.570 \mathrm{E}-03$ & $1.183 \mathrm{E}+10$ & $1.3248 \mathrm{E}+01$ & $1.574 \mathrm{E}-03$ & $1.186 \mathrm{E}+10$ & 0.000 \\
\hline $1 s 4 p^{3} P_{2}^{o}$ & $1 s 5 f^{3} F_{2}^{o}$ & $1.4027 \mathrm{E}+01$ & $2.174 \mathrm{E}-04$ & $1.474 \mathrm{E}+09$ & $1.3967 \mathrm{E}+01$ & $2.189 \mathrm{E}-04$ & $1.484 \mathrm{E}+09$ & 0.000 \\
\hline $1 s 4 p{ }^{1} P_{1}^{o}$ & $1 s 5 f^{3} F_{2}^{o}$ & $1.4093 \mathrm{E}+01$ & $4.547 \mathrm{E}-04$ & $3.054 \mathrm{E}+09$ & $1.4033 \mathrm{E}+01$ & $4.555 \mathrm{E}-04$ & $3.059 \mathrm{E}+09$ & 0.000 \\
\hline $1 s 4 f^{3} F_{3}^{o}$ & $1 s 5 f^{3} F_{2}^{o}$ & $1.4336 \mathrm{E}+01$ & $8.950 \mathrm{E}-05$ & $5.809 E+08$ & $1.4275 \mathrm{E}+01$ & $8.974 \mathrm{E}-05$ & $5.825 \mathrm{E}+08$ & 0.000 \\
\hline $1 s 4 f^{3} F_{2}^{o}$ & $1 s 5 f^{3} F_{2}^{o}$ & $1.4342 \mathrm{E}+01$ & $3.591 \mathrm{E}-04$ & $2.329 E+09$ & $1.4281 \mathrm{E}+01$ & $3.595 \mathrm{E}-04$ & $2.332 \mathrm{E}+09$ & 0.000 \\
\hline $1 s 4 f^{3} F_{4}^{o}$ & $1 s 5 f^{3} F_{2}^{o}$ & $1.4463 \mathrm{E}+01$ & $7.505 \mathrm{E}-06$ & $4.786 \mathrm{E}+07$ & $1.4401 \mathrm{E}+01$ & $7.492 \mathrm{E}-06$ & $4.778 \mathrm{E}+07$ & 0.000 \\
\hline $1 s 4 f^{1} F_{3}^{o}$ & $1 s 5 f^{3} F_{2}^{o}$ & $1.4467 \mathrm{E}+01$ & $6.818 \mathrm{E}-05$ & $4.346 \mathrm{E}+08$ & $1.4405 \mathrm{E}+01$ & $6.800 \mathrm{E}-05$ & $4.334 \mathrm{E}+08$ & 0.000 \\
\hline $1 s 5 p^{3} P_{1}^{o}$ & $1 s 5 f^{3} F_{2}^{o}$ & $3.5713 \mathrm{E}+02$ & $2.546 \mathrm{E}-07$ & $2.663 E+03$ & $3.5518 \mathrm{E}+02$ & $2.555 \mathrm{E}-07$ & $2.679 \mathrm{E}+03$ & 0.001 \\
\hline $1 s 5 p^{3} P_{0}^{o}$ & $1 s 5 f^{3} F_{2}^{o}$ & $3.6039 \mathrm{E}+02$ & $3.530 \mathrm{E}-07$ & $3.626 E+03$ & $3.5830 \mathrm{E}+02$ & $3.545 \mathrm{E}-07$ & $3.652 \mathrm{E}+03$ & 0.001 \\
\hline $1 s 5 p^{3} P_{2}^{o}$ & $1 s 5 f^{3} F_{2}^{o}$ & $1.2503 \mathrm{E}+03$ & $1.196 \mathrm{E}-09$ & $1.021 \mathrm{E}+00$ & $1.2374 \mathrm{E}+03$ & $1.217 \mathrm{E}-09$ & $1.051 \mathrm{E}+00$ & 0.009 \\
\hline $1 s 5 p^{1} P_{1}^{o}$ & $1 s 5 f^{3} F_{2}^{o}$ & $1.5820 \mathrm{E}+03$ & $1.240 \mathrm{E}-09$ & $6.612 \mathrm{E}-01$ & $1.5673 \mathrm{E}+03$ & $1.256 \mathrm{E}-09$ & $6.767 \mathrm{E}-01$ & 0.013 \\
\hline $1 s 5 f^{3} F_{3}^{o}$ & $1 s 5 f^{3} F_{2}^{o}$ & $7.6009 \mathrm{E}+04$ & $1.358 \mathrm{E}-14$ & $3.135 \mathrm{E}-09$ & & & & 0.251 \\
\hline $1 s^{2}{ }^{1} S_{0}$ & $1 s 5 d^{1} D_{2}$ & $3.2000 \mathrm{E}-01$ & $3.384 \mathrm{E}-04$ & $4.406 \mathrm{E}+12$ & $3.1875 \mathrm{E}-01$ & $3.920 \mathrm{E}-04$ & $5.103 E+12$ & 0.001 \\
\hline $1 s 2 s^{3} S_{1}$ & $1 s 5 d^{1} D_{2}$ & $1.4408 \mathrm{E}+00$ & $3.421 \mathrm{E}-05$ & $2.199 \mathrm{E}+10$ & $1.4346 \mathrm{E}+00$ & $3.238 \mathrm{E}-05$ & $2.081 \mathrm{E}+10$ & 0.001 \\
\hline $1 s 2 s{ }^{1} S_{0}$ & $1 s 5 d^{1} D_{2}$ & $1.4551 \mathrm{E}+00$ & $4.690 \mathrm{E}-05$ & $2.955 E+10$ & $1.4492 \mathrm{E}+00$ & $4.826 \mathrm{E}-05$ & $3.040 \mathrm{E}+10$ & 0.002 \\
\hline $1 s 3 s^{3} S_{1}$ & $1 s 5 d^{1} D_{2}$ & $4.2617 \mathrm{E}+00$ & $1.913 \mathrm{E}-04$ & $1.405 \mathrm{E}+10$ & $4.2438 \mathrm{E}+00$ & $1.909 \mathrm{E}-04$ & $1.402 \mathrm{E}+10$ & 0.002 \\
\hline $1 s 3 s{ }^{1} S_{0}$ & $1 s 5 d^{1} D_{2}$ & $4.2949 \mathrm{E}+00$ & $2.860 \mathrm{E}-04$ & $2.068 \mathrm{E}+10$ & $4.2772 \mathrm{E}+00$ & $2.874 \mathrm{E}-04$ & $2.078 \mathrm{E}+10$ & 0.001 \\
\hline $1 s 3 d^{3} D_{2}$ & $1 s 5 d^{1} D_{2}$ & $4.4852 \mathrm{E}+00$ & $1.193 \mathrm{E}-05$ & $7.912 \mathrm{E}+08$ & $4.4662 \mathrm{E}+00$ & $1.201 \mathrm{E}-05$ & $7.964 \mathrm{E}+08$ & 0.000 \\
\hline $1 s 3 d^{3} D_{1}$ & $1 s 5 d^{1} D_{2}$ & $4.4876 \mathrm{E}+00$ & $2.723 \mathrm{E}-05$ & $1.804 \mathrm{E}+09$ & $4.4686 \mathrm{E}+00$ & $2.736 \mathrm{E}-05$ & $1.812 \mathrm{E}+09$ & 0.001 \\
\hline $1 s 3 d^{3} D_{3}$ & $1 s 5 d^{1} D_{2}$ & $4.5449 \mathrm{E}+00$ & $3.039 \mathrm{E}-05$ & $1.963 \mathrm{E}+09$ & $4.5255 \mathrm{E}+00$ & $3.031 \mathrm{E}-05$ & $1.958 \mathrm{E}+09$ & 0.001 \\
\hline $1 s 3 d^{1} D_{2}$ & $1 s 5 d^{1} D_{2}$ & $4.5472 \mathrm{E}+00$ & $1.242 \mathrm{E}-04$ & $8.013 E+09$ & $4.5278 \mathrm{E}+00$ & $1.237 \mathrm{E}-04$ & $7.986 \mathrm{E}+09$ & 0.001 \\
\hline $1 s 4 s^{3} S_{1}$ & $1 s 5 d^{1} D_{2}$ & $1.3174 \mathrm{E}+01$ & $7.090 \mathrm{E}-04$ & $5.450 \mathrm{E}+09$ & $1.3118 \mathrm{E}+01$ & $7.220 \mathrm{E}-04$ & $5.550 \mathrm{E}+09$ & 0.002 \\
\hline $1 s 4 s^{1} S_{0}$ & $1 s 5 d^{1} D_{2}$ & $1.3302 \mathrm{E}+01$ & $1.097 \mathrm{E}-03$ & $8.271 E+09$ & $1.3247 \mathrm{E}+01$ & $1.082 \mathrm{E}-03$ & $8.157 \mathrm{E}+09$ & 0.001 \\
\hline $1 s 4 d^{3} D_{2}$ & $1 s 5 d^{1} D_{2}$ & $1.4082 \mathrm{E}+01$ & $5.367 \mathrm{E}-05$ & $3.610 \mathrm{E}+08$ & $1.4023 \mathrm{E}+01$ & $5.400 \mathrm{E}-05$ & $3.632 \mathrm{E}+08$ & 0.000 \\
\hline $1 s 4 d^{3} D_{1}$ & $1 s 5 d^{1} D_{2}$ & $1.4092 \mathrm{E}+01$ & $1.243 \mathrm{E}-04$ & $8.352 \mathrm{E}+08$ & $1.4033 \mathrm{E}+01$ & $1.245 \mathrm{E}-04$ & $8.366 \mathrm{E}+08$ & 0.000 \\
\hline $1 s 4 d^{3} D_{3}$ & $1 s 5 d^{1} D_{2}$ & $1.4332 \mathrm{E}+01$ & $1.438 \mathrm{E}-04$ & $9.338 \mathrm{E}+08$ & $1.4271 \mathrm{E}+01$ & $1.442 \mathrm{E}-04$ & $9.366 \mathrm{E}+08$ & 0.000 \\
\hline $1 s 4 d{ }^{1} D_{2}$ & $1 s 5 d{ }^{1} D_{2}$ & $1.4342 \mathrm{E}+01$ & $5.883 \mathrm{E}-04$ & $3.816 \mathrm{E}+09$ & $1.4282 \mathrm{E}+01$ & $5.895 \mathrm{E}-04$ & $3.823 \mathrm{E}+09$ & 0.000 \\
\hline $1 s 5 s^{3} S_{1}$ & $1 s 5 d{ }^{1} D_{2}$ & $3.1728 \mathrm{E}+02$ & $4.955 \mathrm{E}-07$ & $6.566 \mathrm{E}+03$ & $3.1538 \mathrm{E}+02$ & $4.978 \mathrm{E}-07$ & $6.620 \mathrm{E}+03$ & 0.018 \\
\hline $1 s 5 s^{1} S_{0}$ & $1 s 5 d^{1} D_{2}$ & $3.5905 \mathrm{E}+02$ & $5.277 \mathrm{E}-07$ & $5.460 \mathrm{E}+03$ & $3.5720 \mathrm{E}+02$ & $5.289 \mathrm{E}-07$ & $5.482 \mathrm{E}+03$ & 0.031 \\
\hline $1 s 5 d^{3} D_{2}$ & $1 s 5 d^{1} D_{2}$ & $1.5170 \mathrm{E}+03$ & $9.109 \mathrm{E}-10$ & $5.280 \mathrm{E}-01$ & & & & 0.016 \\
\hline $1 s 5 d^{3} D_{1}$ & $1 s 5 d^{1} D_{2}$ & $1.5755 \mathrm{E}+03$ & $1.889 \mathrm{E}-09$ & $1.015 \mathrm{E}+00$ & & & & 0.003 \\
\hline $1 s 5 d^{3} D_{3}$ & $1 s 5 d^{1} D_{2}$ & $3.7649 \mathrm{E}+04$ & $1.593 \mathrm{E}-13$ & $1.499 \mathrm{E}-07$ & & & & 0.285 \\
\hline $1 s 2 p^{3} P_{2}^{o}$ & $1 s 5 f^{3} F_{4}^{o}$ & $1.5225 \mathrm{E}+00$ & $2.780 \mathrm{E}-03$ & $8.890 \mathrm{E}+11$ & $1.5161 \mathrm{E}+00$ & $2.919 \mathrm{E}-03$ & $9.332 \mathrm{E}+11$ & 0.000 \\
\hline $1 s 3 p^{3} P_{2}^{o}$ & $1 s 5 f^{3} F_{4}^{0}$ & $4.4658 \mathrm{E}+00$ & $3.882 \mathrm{E}-05$ & $1.443 E+09$ & $4.4472 \mathrm{E}+00$ & $3.385 \mathrm{E}-05$ & $1.257 \mathrm{E}+09$ & 0.001 \\
\hline $1 s 4 p^{3} P_{2}^{o}$ & $1 s 5 f^{3} F_{4}^{o}$ & $1.3968 \mathrm{E}+01$ & $5.895 \mathrm{E}-03$ & $2.239 \mathrm{E}+10$ & $1.3909 \mathrm{E}+01$ & $5.959 \mathrm{E}-03$ & $2.263 \mathrm{E}+10$ & 0.000 \\
\hline $1 s 4 f^{3} F_{3}^{o}$ & $1 s 5 f^{3} F_{4}^{o}$ & $1.4275 \mathrm{E}+01$ & $9.254 \mathrm{E}-05$ & $3.366 \mathrm{E}+08$ & $1.4214 \mathrm{E}+01$ & $9.308 \mathrm{E}-05$ & $3.385 E+08$ & 0.000 \\
\hline $1 s 4 f^{3} F_{2}^{o}$ & $1 s 5 f^{3} F_{4}^{o}$ & $1.4280 \mathrm{E}+01$ & $7.287 \mathrm{E}-06$ & $2.648 \mathrm{E}+07$ & $1.4220 \mathrm{E}+01$ & $7.321 \mathrm{E}-06$ & $2.660 \mathrm{E}+07$ & 0.000 \\
\hline $1 s 4 f^{3} F_{4}^{o}$ & $1 s 5 f^{3} F_{4}^{o}$ & $1.4401 \mathrm{E}+01$ & $7.716 \mathrm{E}-04$ & $2.758 \mathrm{E}+09$ & $1.4339 \mathrm{E}+01$ & $7.730 \mathrm{E}-04$ & $2.762 \mathrm{E}+09$ & 0.000 \\
\hline $1 s 4 f^{1} F_{3}^{o}$ & $1 s 5 f^{3} F_{4}^{0}$ & $1.4404 \mathrm{E}+01$ & $7.051 \mathrm{E}-05$ & $2.519 E+08$ & $1.4343 \mathrm{E}+01$ & $7.056 \mathrm{E}-05$ & $2.520 \mathrm{E}+08$ & 0.000 \\
\hline $1 s 5 p^{3} P_{2}^{o}$ & $1 s 5 f^{3} F_{4}^{o}$ & $9.0890 \mathrm{E}+02$ & $8.517 \mathrm{E}-08$ & $7.641 \mathrm{E}+01$ & $9.0168 \mathrm{E}+02$ & $8.608 \mathrm{E}-08$ & $7.780 \mathrm{E}+01$ & 0.007 \\
\hline $1 s 5 f^{3} F_{3}^{o}$ & $1 s 5 f^{3} F_{4}^{o}$ & $3.1889 \mathrm{E}+03$ & $1.921 \mathrm{E}-10$ & $1.400 \mathrm{E}-02$ & $3.1838 \mathrm{E}+03$ & $1.906 \mathrm{E}-10$ & $1.382 \mathrm{E}-02$ & 0.001 \\
\hline $1 s 5 f^{3} F_{2}^{o}$ & $1 s 5 f^{3} F_{4}^{o}$ & $3.3285 \mathrm{E}+03$ & $1.343 \mathrm{E}-11$ & $8.982 \mathrm{E}-04$ & & & & 0.017 \\
\hline $1 s 3 d^{3} D_{2}$ & $1 s 5 g{ }^{3} G_{4}$ & $4.4792 \mathrm{E}+00$ & $6.254 \mathrm{E}-03$ & $2.310 \mathrm{E}+11$ & $4.4604 \mathrm{E}+00$ & $6.315 \mathrm{E}-03$ & $2.332 \mathrm{E}+11$ & 0.000 \\
\hline $1 s 3 d^{3} D_{3}$ & $1 s 5 g{ }^{3} G_{4}$ & $4.5387 \mathrm{E}+00$ & $6.548 \mathrm{E}-04$ & $2.356 \mathrm{E}+10$ & $4.5195 \mathrm{E}+00$ & $6.553 \mathrm{E}-04$ & $2.357 \mathrm{E}+10$ & 0.000 \\
\hline $1 s 3 d{ }^{1} D_{2}$ & $1 s 5 g{ }^{3} G_{4}$ & $4.5410 \mathrm{E}+00$ & $5.882 \mathrm{E}-05$ & $2.114 \mathrm{E}+09$ & $4.5219 \mathrm{E}+00$ & $5.907 \mathrm{E}-05$ & $2.122 \mathrm{E}+09$ & 0.000 \\
\hline $1 s 4 d^{3} D_{2}$ & $1 s 5 g{ }^{3} G_{4}$ & $1.4023 \mathrm{E}+01$ & $5.755 \mathrm{E}-03$ & $2.169 \mathrm{E}+10$ & $1.3966 \mathrm{E}+01$ & $5.765 \mathrm{E}-03$ & $2.172 \mathrm{E}+10$ & 0.000 \\
\hline $1 s 4 d^{3} D_{3}$ & $1 s 5 g{ }^{3} G_{4}$ & $1.4271 \mathrm{E}+01$ & $5.740 \mathrm{E}-04$ & $2.089 E+09$ & $1.4212 \mathrm{E}+01$ & $5.735 \mathrm{E}-04$ & $2.086 \mathrm{E}+09$ & 0.000 \\
\hline $1 s 4 d{ }^{1} D_{2}$ & $1 s 5 g^{3} G_{4}$ & $1.4281 \mathrm{E}+01$ & $5.352 \mathrm{E}-05$ & $1.945 E+08$ & $1.4222 \mathrm{E}+01$ & $5.388 \mathrm{E}-05$ & $1.957 \mathrm{E}+08$ & 0.000 \\
\hline $1 s 5 d^{3} D_{2}$ & $1 s 5 g^{3} G_{4}$ & $1.0444 \mathrm{E}+03$ & $2.420 \mathrm{E}-08$ & $1.645 \mathrm{E}+01$ & $1.0513 \mathrm{E}+03$ & $2.342 \mathrm{E}-08$ & $1.557 \mathrm{E}+01$ & 0.002 \\
\hline $1 s 5 d^{3} D_{3}$ & $1 s 5 g{ }^{3} G_{4}$ & $3.0779 \mathrm{E}+03$ & $9.602 \mathrm{E}-11$ & $7.512 \mathrm{E}-03$ & $3.1417 \mathrm{E}+03$ & $8.912 \mathrm{E}-11$ & $6.635 \mathrm{E}-03$ & 0.001 \\
\hline $1 s 5 d{ }^{1} D_{2}$ & $1 s 5 g^{3} G_{4}$ & $3.3519 \mathrm{E}+03$ & $7.054 \mathrm{E}-12$ & $4.653 \mathrm{E}-04$ & & & & 0.014 \\
\hline $1 s 2 s^{3} S_{1}$ & $1 s 5 g{ }^{3} G_{3}$ & $1.4401 \mathrm{E}+00$ & $2.205 \mathrm{E}-11$ & $1.013 \mathrm{E}+04$ & $1.4340 \mathrm{E}+00$ & $5.275 \mathrm{E}-14$ & $2.423 \mathrm{E}+01$ & 0.632 \\
\hline $1 s 3 s^{3} S_{1}$ & $1 s 5 g^{3} G_{3}$ & $4.2561 \mathrm{E}+00$ & $6.131 \mathrm{E}-09$ & $3.225 E+05$ & & & & 0.019 \\
\hline $1 s 3 d^{3} D_{2}$ & $1 s 5 g{ }^{3} G_{3}$ & $4.4791 \mathrm{E}+00$ & $6.989 \mathrm{E}-04$ & $3.319 \mathrm{E}+10$ & $4.4603 \mathrm{E}+00$ & $7.065 \mathrm{E}-04$ & $3.355 E+10$ & 0.000 \\
\hline $1 s 3 d^{3} D_{1}$ & $1 s 5 g^{3} G_{3}$ & $4.4815 \mathrm{E}+00$ & $4.174 \mathrm{E}-03$ & $1.980 \mathrm{E}+11$ & $4.4627 \mathrm{E}+00$ & $4.206 \mathrm{E}-03$ & $1.995 \mathrm{E}+11$ & 0.000 \\
\hline $1 s 3 d^{3} D_{3}$ & $1 s 5 g^{3} G_{3}$ & $4.5386 \mathrm{E}+00$ & $7.882 \mathrm{E}-05$ & $3.646 E+09$ & $4.5194 \mathrm{E}+00$ & $7.892 \mathrm{E}-05$ & $3.651 \mathrm{E}+09$ & 0.000 \\
\hline $1 s 3 d^{1} D_{2}$ & $1 s 5 g^{3} G_{3}$ & $4.5409 \mathrm{E}+00$ & $4.695 \mathrm{E}-04$ & $2.170 \mathrm{E}+10$ & $4.5217 \mathrm{E}+00$ & $4.687 \mathrm{E}-04$ & $2.165 \mathrm{E}+10$ & 0.000 \\
\hline $1 s 4 s^{3} S_{1}$ & $1 s 5 g{ }^{3} G_{3}$ & $1.3121 \mathrm{E}+01$ & $9.510 \mathrm{E}-09$ & $5.263 \mathrm{E}+04$ & & & & 0.006 \\
\hline $1 s 4 d^{3} D_{2}$ & $1 s 5 g{ }^{3} G_{3}$ & $1.4022 \mathrm{E}+01$ & $6.457 \mathrm{E}-04$ & $3.129 E+09$ & $1.3965 \mathrm{E}+01$ & $6.475 \mathrm{E}-04$ & $3.137 \mathrm{E}+09$ & 0.000 \\
\hline $1 s 4 d^{3} D_{1}$ & $1 s 5 g{ }^{3} G_{3}$ & $1.4031 \mathrm{E}+01$ & $3.837 \mathrm{E}-03$ & $1.857 \mathrm{E}+10$ & $1.3974 \mathrm{E}+01$ & $3.841 \mathrm{E}-03$ & $1.858 \mathrm{E}+10$ & 0.000 \\
\hline $1 s 4 d^{3} D_{3}$ & $1 s 5 g{ }^{3} G_{3}$ & $1.4269 \mathrm{E}+01$ & $6.912 \mathrm{E}-05$ & $3.235 E+08$ & $1.4210 \mathrm{E}+01$ & $6.907 \mathrm{E}-05$ & $3.231 \mathrm{E}+08$ & 0.000 \\
\hline $1 s 4 d{ }^{1} D_{2}$ & $1 s 5 g{ }^{3} G_{3}$ & $1.4280 \mathrm{E}+01$ & $4.090 \mathrm{E}-04$ & $1.912 \mathrm{E}+09$ & $1.4221 \mathrm{E}+01$ & $4.079 \mathrm{E}-04$ & $1.905 \mathrm{E}+09$ & 0.000 \\
\hline $1 s 5 s^{3} S_{1}$ & $1 s 5 g{ }^{3} G_{3}$ & $2.8921 \mathrm{E}+02$ & $1.975 \mathrm{E}-12$ & $2.250 \mathrm{E}-02$ & $2.8822 \mathrm{E}+02$ & $1.905 \mathrm{E}-12$ & $2.167 \mathrm{E}-02$ & 0.069 \\
\hline $1 s 5 d^{3} D_{2}$ & $1 s 5 g{ }^{3} G_{3}$ & $1.0362 \mathrm{E}+03$ & $2.785 \mathrm{E}-09$ & $2.471 \mathrm{E}+00$ & $1.0430 \mathrm{E}+03$ & $2.697 \mathrm{E}-09$ & $2.342 \mathrm{E}+00$ & 0.001 \\
\hline $1 s 5 d^{3} D_{1}$ & $1 s 5 g{ }^{3} G_{3}$ & $1.0632 \mathrm{E}+03$ & $1.531 \mathrm{E}-08$ & $1.291 \mathrm{E}+01$ & $1.0701 \mathrm{E}+03$ & $1.482 \mathrm{E}-08$ & $1.222 \mathrm{E}+01$ & 0.002 \\
\hline
\end{tabular}


Table 4 (continued)

\begin{tabular}{|c|c|c|c|c|c|c|c|c|}
\hline \multirow[t]{2}{*}{ Lower } & \multirow[t]{2}{*}{ Upper } & \multicolumn{3}{|l|}{ GRASP2K } & \multicolumn{3}{|l|}{ FAC } & \multirow[t]{2}{*}{$d T$} \\
\hline & & $\lambda(\AA)$ & $g f$ & $A\left(\mathrm{~s}^{-1}\right)$ & $\lambda(\AA)$ & $g f$ & $A\left(\mathrm{~s}^{-1}\right)$ & \\
\hline $1 s 5 d^{3} D_{3}$ & $1 s 5 g^{3} G_{3}$ & $3.0082 \mathrm{E}+03$ & $1.239 \mathrm{E}-11$ & $1.304 \mathrm{E}-03$ & & & & 0.004 \\
\hline $1 s 5 d^{1} D_{2}$ & $1 s 5 g^{3} G_{3}$ & $3.2694 \mathrm{E}+03$ & $5.703 \mathrm{E}-11$ & $5.084 \mathrm{E}-03$ & $3.3474 \mathrm{E}+03$ & $5.238 \mathrm{E}-11$ & $4.417 \mathrm{E}-03$ & 0.014 \\
\hline $1 s 5 g^{3} G_{4}$ & $1 s 5 g^{3} G_{3}$ & $1.3281 \mathrm{E}+05$ & $1.079 \mathrm{E}-15$ & $5.826 \mathrm{E}-11$ & & & & 0.731 \\
\hline $1 s 2 p^{3} P_{1}^{o}$ & $1 s 5 f^{1} F_{3}^{o}$ & $1.4532 \mathrm{E}+00$ & $3.021 \mathrm{E}-06$ & $1.363 \mathrm{E}+09$ & $1.4471 \mathrm{E}+00$ & $3.966 \mathrm{E}-06$ & $1.789 \mathrm{E}+09$ & 0.017 \\
\hline $1 s 2 p^{3} P_{2}^{o}$ & $1 s 5 f^{1} F_{3}^{o}$ & $1.5225 \mathrm{E}+00$ & $3.106 \mathrm{E}-04$ & $1.277 \mathrm{E}+11$ & $1.5161 \mathrm{E}+00$ & $3.258 \mathrm{E}-04$ & $1.339 \mathrm{E}+11$ & 0.001 \\
\hline $1 s 2 p^{1} P_{1}^{o}$ & $1 s 5 f^{1} F_{3}^{o}$ & $1.5291 \mathrm{E}+00$ & $1.859 \mathrm{E}-03$ & $7.576 \mathrm{E}+11$ & $1.5227 \mathrm{E}+00$ & $1.916 \mathrm{E}-03$ & $7.807 \mathrm{E}+11$ & 0.001 \\
\hline $1 s 3 p^{3} P_{1}^{o}$ & $1 s 5 f^{1} F_{3}^{o}$ & $4.2871 \mathrm{E}+00$ & $1.345 \mathrm{E}-07$ & $6.974 \mathrm{E}+06$ & $4.2694 \mathrm{E}+00$ & $4.281 \mathrm{E}-08$ & $2.219 \mathrm{E}+06$ & 0.026 \\
\hline $1 s 3 p^{3} P_{2}^{o}$ & $1 s 5 f^{1} F_{3}^{o}$ & $4.4656 \mathrm{E}+00$ & $4.376 \mathrm{E}-06$ & $2.091 \mathrm{E}+08$ & $4.4470 \mathrm{E}+00$ & $3.777 \mathrm{E}-06$ & $1.804 \mathrm{E}+08$ & 0.001 \\
\hline $1 s 3 p^{1} P_{1}^{o}$ & $1 s 5 f^{1} F_{3}^{o}$ & $4.4815 \mathrm{E}+00$ & $2.232 \mathrm{E}-05$ & $1.059 \mathrm{E}+09$ & $4.4631 \mathrm{E}+00$ & $2.227 \mathrm{E}-05$ & $1.056 \mathrm{E}+09$ & 0.002 \\
\hline $1 s 4 p^{3} P_{1}^{o}$ & $1 s 5 f^{1} F_{3}^{o}$ & $1.3241 \mathrm{E}+01$ & $5.944 \mathrm{E}-06$ & $3.231 \mathrm{E}+07$ & $1.3185 \mathrm{E}+01$ & $6.974 \mathrm{E}-06$ & $3.790 \mathrm{E}+07$ & 0.001 \\
\hline $1 s 4 p^{3} P_{2}^{o}$ & $1 s 5 f^{1} F_{3}^{o}$ & $1.3966 \mathrm{E}+01$ & $6.580 \mathrm{E}-04$ & $3.215 E+09$ & $1.3907 \mathrm{E}+01$ & $6.653 E-04$ & $3.250 \mathrm{E}+09$ & 0.000 \\
\hline $1 s 4 p^{1} P_{1}^{o}$ & $1 s 5 f^{1} F_{3}^{o}$ & $1.4031 \mathrm{E}+01$ & $3.910 \mathrm{E}-03$ & $1.892 \mathrm{E}+10$ & $1.3972 \mathrm{E}+01$ & $3.910 \mathrm{E}-03$ & $1.892 \mathrm{E}+10$ & 0.000 \\
\hline $1 s 4 f^{3} F_{3}^{o}$ & $1 s 5 f^{1} F_{3}^{o}$ & $1.4273 \mathrm{E}+01$ & $1.118 \mathrm{E}-05$ & $5.231 \mathrm{E}+07$ & $1.4212 \mathrm{E}+01$ & $1.125 \mathrm{E}-05$ & $5.265 \mathrm{E}+07$ & 0.000 \\
\hline $1 s 4 f^{3} F_{2}^{o}$ & $1 s 5 f^{1} F_{3}^{o}$ & $1.4278 \mathrm{E}+01$ & $6.735 \mathrm{E}-05$ & $3.148 \mathrm{E}+08$ & $1.4218 \mathrm{E}+01$ & $6.769 \mathrm{E}-05$ & $3.163 \mathrm{E}+08$ & 0.000 \\
\hline $1 s 4 f^{3} F_{4}^{o}$ & $1 s 5 f^{1} F_{3}^{o}$ & $1.4398 \mathrm{E}+01$ & $7.046 \mathrm{E}-05$ & $3.239 \mathrm{E}+08$ & $1.4337 \mathrm{E}+01$ & $7.063 \mathrm{E}-05$ & $3.246 \mathrm{E}+08$ & 0.000 \\
\hline $1 s 4 f^{1} F_{3}^{o}$ & $1 s 5 f^{1} F_{3}^{o}$ & $1.4402 \mathrm{E}+01$ & $5.838 \mathrm{E}-04$ & $2.682 \mathrm{E}+09$ & $1.4341 \mathrm{E}+01$ & $5.847 \mathrm{E}-04$ & $2.686 \mathrm{E}+09$ & 0.000 \\
\hline $1 s 5 p^{3} P_{1}^{0}$ & $1 s 5 f^{1} F_{3}^{o}$ & $3.2151 \mathrm{E}+02$ & $1.870 \mathrm{E}-09$ & $1.724 \mathrm{E}+01$ & $3.1987 \mathrm{E}+02$ & $1.897 \mathrm{E}-09$ & $1.752 \mathrm{E}+01$ & 0.002 \\
\hline $1 s 5 p^{3} P_{2}^{o}$ & $1 s 5 f^{1} F_{3}^{o}$ & $9.0084 \mathrm{E}+02$ & $9.762 \mathrm{E}-09$ & $1.146 \mathrm{E}+01$ & $8.9370 \mathrm{E}+02$ & $9.861 \mathrm{E}-09$ & $1.166 \mathrm{E}+01$ & 0.005 \\
\hline $1 s 5 p^{1} P_{1}^{o}$ & $1 s 5 f^{1} F_{3}^{o}$ & $1.0611 \mathrm{E}+03$ & $3.558 \mathrm{E}-08$ & $3.011 \mathrm{E}+01$ & $1.0539 \mathrm{E}+03$ & $3.585 \mathrm{E}-08$ & $3.049 \mathrm{E}+01$ & 0.007 \\
\hline $1 s 5 f^{3} F_{3}^{o}$ & $1 s 5 f^{1} F_{3}^{o}$ & $3.0918 \mathrm{E}+03$ & $2.542 \mathrm{E}-11$ & $2.534 \mathrm{E}-03$ & & & & 0.013 \\
\hline $1 s 5 f^{3} F_{2}^{o}$ & $1 s 5 f^{1} F_{3}^{o}$ & $3.2229 \mathrm{E}+03$ & $1.353 \mathrm{E}-10$ & $1.241 \mathrm{E}-02$ & $3.2177 \mathrm{E}+03$ & $1.342 \mathrm{E}-10$ & $1.224 \mathrm{E}-02$ & 0.002 \\
\hline $1 s 5 f^{3} F_{4}^{o}$ & $1 s 5 f^{1} F_{3}^{o}$ & $1.0160 \mathrm{E}+05$ & $4.530 \mathrm{E}-15$ & $4.182 \mathrm{E}-10$ & & & & 0.407 \\
\hline $1 s 3 d^{3} D_{3}$ & $1 s 5 g^{3} G_{5}$ & $4.5349 \mathrm{E}+00$ & $8.603 E-03$ & $2.537 \mathrm{E}+11$ & $4.5157 \mathrm{E}+00$ & $8.751 E-03$ & $2.580 \mathrm{E}+11$ & 0.000 \\
\hline $1 s 4 d^{3} D_{3}$ & $1 s 5 g^{3} G_{5}$ & $1.4233 \mathrm{E}+01$ & $7.663 \mathrm{E}-03$ & $2.294 \mathrm{E}+10$ & $1.4174 \mathrm{E}+01$ & $7.697 \mathrm{E}-03$ & $2.303 \mathrm{E}+10$ & 0.000 \\
\hline $1 s 5 d^{3} D_{3}$ & $1 s 5 g{ }^{3} G_{5}$ & $1.9545 \mathrm{E}+03$ & $5.017 \mathrm{E}-09$ & $7.964 \mathrm{E}-01$ & $1.9786 \mathrm{E}+03$ & $4.774 \mathrm{E}-09$ & $7.332 \mathrm{E}-01$ & 0.001 \\
\hline $1 s 5 g^{3} G_{4}$ & $1 s 5 g^{3} G_{5}$ & $5.3554 \mathrm{E}+03$ & $1.676 \mathrm{E}-11$ & $3.544 \mathrm{E}-04$ & $5.3442 \mathrm{E}+03$ & $1.665 \mathrm{E}-11$ & $3.506 \mathrm{E}-04$ & 0.005 \\
\hline $1 s 5 g^{3} G_{3}$ & $1 s 5 g^{3} G_{5}$ & $5.5804 \mathrm{E}+03$ & $7.076 \mathrm{E}-13$ & $1.378 \mathrm{E}-05$ & & & & 0.118 \\
\hline $1 s 3 d^{3} D_{2}$ & $1 s 5 g^{1} G_{4}$ & $4.4754 \mathrm{E}+00$ & $1.669 \mathrm{E}-07$ & $6.178 \mathrm{E}+06$ & & & & 0.002 \\
\hline $1 s 3 d^{3} D_{3}$ & $1 s 5 g^{1} G_{4}$ & $4.5348 \mathrm{E}+00$ & $5.236 \mathrm{E}-04$ & $1.887 \mathrm{E}+10$ & $4.5156 \mathrm{E}+00$ & $5.326 \mathrm{E}-04$ & $1.919 \mathrm{E}+10$ & 0.000 \\
\hline $1 s 3 d^{1} D_{2}$ & $1 s 5 g^{1} G_{4}$ & $4.5371 \mathrm{E}+00$ & $6.519 \mathrm{E}-03$ & $2.347 \mathrm{E}+11$ & $4.5179 \mathrm{E}+00$ & $6.617 \mathrm{E}-03$ & $2.382 \mathrm{E}+11$ & 0.000 \\
\hline $1 s 4 d^{3} D_{2}$ & $1 s 5 g^{1} G_{4}$ & $1.3986 \mathrm{E}+01$ & $2.967 \mathrm{E}-07$ & $1.124 \mathrm{E}+06$ & & & & 0.001 \\
\hline $1 s 4 d^{3} D_{3}$ & $1 s 5 g^{1} G_{4}$ & $1.4232 \mathrm{E}+01$ & $4.665 \mathrm{E}-04$ & $1.707 \mathrm{E}+09$ & $1.4173 \mathrm{E}+01$ & $4.686 \mathrm{E}-04$ & $1.714 \mathrm{E}+09$ & 0.000 \\
\hline $1 s 4 d^{1} D_{2}$ & $1 s 5 g{ }^{1} G_{4}$ & $1.4242 \mathrm{E}+01$ & $5.798 \mathrm{E}-03$ & $2.119 \mathrm{E}+10$ & $1.4183 \mathrm{E}+01$ & $5.817 \mathrm{E}-03$ & $2.125 \mathrm{E}+10$ & 0.000 \\
\hline $1 s 5 d^{3} D_{2}$ & $1 s 5 g{ }^{1} G_{4}$ & $8.6941 \mathrm{E}+02$ & $2.714 \mathrm{E}-12$ & $2.661 \mathrm{E}-03$ & $8.7388 \mathrm{E}+02$ & $2.967 \mathrm{E}-12$ & $2.855 \mathrm{E}-03$ & 0.022 \\
\hline $1 s 5 d^{3} D_{3}$ & $1 s 5 g^{1} G_{4}$ & $1.9321 \mathrm{E}+03$ & $3.162 \mathrm{E}-10$ & $6.278 \mathrm{E}-02$ & $1.9554 \mathrm{E}+03$ & $3.010 \mathrm{E}-10$ & $5.784 \mathrm{E}-02$ & 0.001 \\
\hline $1 s 5 d{ }^{1} D_{2}$ & $1 s 5 g^{1} G_{4}$ & $2.0366 \mathrm{E}+03$ & $3.358 \mathrm{E}-09$ & $6.000 \mathrm{E}-01$ & $2.0649 \mathrm{E}+03$ & $3.180 \mathrm{E}-09$ & $5.481 \mathrm{E}-01$ & 0.007 \\
\hline $1 s 5 g^{3} G_{4}$ & $1 s 5 g^{1} G_{4}$ & $5.1900 \mathrm{E}+03$ & $1.321 \mathrm{E}-12$ & $3.636 \mathrm{E}-05$ & & & & 0.045 \\
\hline $1 s 5 g^{3} G_{3}$ & $1 s 5 g^{1} G_{4}$ & $5.4011 \mathrm{E}+03$ & $1.294 \mathrm{E}-11$ & $3.288 \mathrm{E}-04$ & $5.3899 E+03$ & $1.285 \mathrm{E}-11$ & $3.251 \mathrm{E}-04$ & 0.008 \\
\hline $1 s 5 g^{3} G_{5}$ & $1 s 5 g{ }^{1} G_{4}$ & $1.6804 \mathrm{E}+05$ & $4.412 \mathrm{E}-16$ & $1.158 \mathrm{E}-11$ & & & & 0.888 \\
\hline $1 s 2 s^{3} s_{1}$ & $1 s 6 s^{3} s_{1}$ & $1.3696 \mathrm{E}+00$ & $2.953 \mathrm{E}-10$ & $3.501 \mathrm{E}+05$ & & & & 0.955 \\
\hline $1 s 3 s^{3} S_{1}$ & $1 s 6 s^{3} S_{1}$ & $3.6936 \mathrm{E}+00$ & $9.398 \mathrm{E}-11$ & $1.532 \mathrm{E}+04$ & & & & 0.701 \\
\hline $1 s 3 d^{3} D_{2}$ & $1 s 6 s^{3} S_{1}$ & $3.8604 \mathrm{E}+00$ & $1.286 \mathrm{E}-05$ & $1.919 \mathrm{E}+09$ & $3.8445 \mathrm{E}+00$ & $1.300 \mathrm{E}-05$ & $1.939 \mathrm{E}+09$ & 0.008 \\
\hline $1 s 3 d^{3} D_{1}$ & $1 s 6 s^{3} s_{1}$ & $3.8622 \mathrm{E}+00$ & $1.310 \mathrm{E}-05$ & $1.952 \mathrm{E}+09$ & $3.8463 \mathrm{E}+00$ & $1.290 \mathrm{E}-05$ & $1.923 \mathrm{E}+09$ & 0.012 \\
\hline $1 s 3 d^{3} D_{3}$ & $1 s 6 s^{3} s_{1}$ & $3.9045 \mathrm{E}+00$ & $3.054 \mathrm{E}-05$ & $4.453 \mathrm{E}+09$ & $3.8883 \mathrm{E}+00$ & $3.041 \mathrm{E}-05$ & $4.435 \mathrm{E}+09$ & 0.008 \\
\hline $1 s 3 d{ }^{1} D_{2}$ & $1 s 6 s^{3} s_{1}$ & $3.9062 \mathrm{E}+00$ & $8.750 \mathrm{E}-06$ & $1.275 \mathrm{E}+09$ & $3.8900 \mathrm{E}+00$ & $8.603 E-06$ & $1.253 \mathrm{E}+09$ & 0.010 \\
\hline $1 s 4 s^{3} S_{1}$ & $1 s 6 s^{3} s_{1}$ & $8.9291 \mathrm{E}+00$ & $9.105 \mathrm{E}-10$ & $2.539 \mathrm{E}+04$ & & & & 0.189 \\
\hline $1 s 4 d^{3} D_{2}$ & $1 s 6 s^{3} S_{1}$ & $9.3373 \mathrm{E}+00$ & $4.394 \mathrm{E}-05$ & $1.121 \mathrm{E}+09$ & $9.3006 \mathrm{E}+00$ & $4.402 \mathrm{E}-05$ & $1.122 \mathrm{E}+09$ & 0.001 \\
\hline $1 s 4 d^{3} D_{1}$ & $1 s 6 s^{3} S_{1}$ & $9.3415 \mathrm{E}+00$ & $4.405 \mathrm{E}-05$ & $1.122 \mathrm{E}+09$ & $9.3048 \mathrm{E}+00$ & $4.355 \mathrm{E}-05$ & $1.109 \mathrm{E}+09$ & 0.004 \\
\hline $1 s 4 d^{3} D_{3}$ & $1 s 6 s^{3} S_{1}$ & $9.4463 \mathrm{E}+00$ & $1.037 \mathrm{E}-04$ & $2.583 \mathrm{E}+09$ & $9.4089 \mathrm{E}+00$ & $1.029 \mathrm{E}-04$ & $2.563 \mathrm{E}+09$ & 0.002 \\
\hline $1 s 4 d^{1} D_{2}$ & $1 s 6 s^{3} S_{1}$ & $9.4508 \mathrm{E}+00$ & $2.923 \mathrm{E}-05$ & $7.276 \mathrm{E}+08$ & $9.4135 \mathrm{E}+00$ & $2.897 \mathrm{E}-05$ & $7.208 E+08$ & 0.003 \\
\hline $1 s 5 s^{3} S_{1}$ & $1 s 6 s^{3} s_{1}$ & $2.5485 \mathrm{E}+01$ & $4.451 \mathrm{E}-09$ & $1.524 \mathrm{E}+04$ & & & & 0.034 \\
\hline $1 s 5 d^{3} D_{2}$ & $1 s 6 s^{3} s_{1}$ & $2.7214 \mathrm{E}+01$ & $1.771 \mathrm{E}-04$ & $5.316 \mathrm{E}+08$ & $2.7122 \mathrm{E}+01$ & $1.754 \mathrm{E}-04$ & $5.256 \mathrm{E}+08$ & 0.001 \\
\hline $1 s 5 d^{3} D_{1}$ & $1 s 6 s^{3} s_{1}$ & $2.7232 \mathrm{E}+01$ & $1.769 \mathrm{E}-04$ & $5.303 \mathrm{E}+08$ & $2.7140 \mathrm{E}+01$ & $1.731 \mathrm{E}-04$ & $5.181 \mathrm{E}+08$ & 0.002 \\
\hline $1 s 5 d^{3} D_{3}$ & $1 s 6 s^{3} S_{1}$ & $2.7691 \mathrm{E}+01$ & $4.244 \mathrm{E}-04$ & $1.231 \mathrm{E}+09$ & $2.7596 \mathrm{E}+01$ & $4.178 \mathrm{E}-04$ & $1.209 \mathrm{E}+09$ & 0.001 \\
\hline $1 s 5 d{ }^{1} D_{2}$ & $1 s 6 s^{3} S_{1}$ & $2.7711 \mathrm{E}+01$ & $1.192 \mathrm{E}-04$ & $3.450 \mathrm{E}+08$ & $2.7617 \mathrm{E}+01$ & $1.172 \mathrm{E}-04$ & $3.389 E+08$ & 0.002 \\
\hline $1 s 5 g{ }^{3} G_{3}$ & $1 s 6 s^{3} S_{1}$ & $2.7948 \mathrm{E}+01$ & $1.280 \mathrm{E}-10$ & $3.644 \mathrm{E}+02$ & & & & 0.022 \\
\hline $1 s 2 p^{3} P_{1}^{o}$ & $1 s 6 p^{3} P_{1}^{o}$ & $1.3810 \mathrm{E}+00$ & $1.717 \mathrm{E}-07$ & $2.002 \mathrm{E}+08$ & $1.3752 \mathrm{E}+00$ & $2.325 \mathrm{E}-07$ & $2.710 \mathrm{E}+08$ & 0.030 \\
\hline $1 s 2 p^{3} P_{2}^{o}$ & $1 s 6 p^{3} P_{1}^{o}$ & $1.4434 \mathrm{E}+00$ & $2.896 \mathrm{E}-05$ & $3.090 \mathrm{E}+10$ & $1.4374 \mathrm{E}+00$ & $2.858 \mathrm{E}-05$ & $3.050 \mathrm{E}+10$ & 0.007 \\
\hline $1 s 2 p^{1} P_{1}^{o}$ & $1 s 6 p^{3} P_{1}^{o}$ & $1.4493 \mathrm{E}+00$ & $2.557 \mathrm{E}-05$ & $2.706 \mathrm{E}+10$ & $1.4433 \mathrm{E}+00$ & $2.438 \mathrm{E}-05$ & $2.580 \mathrm{E}+10$ & 0.000 \\
\hline $1 s 3 p^{3} P_{1}^{o}$ & $1 s 6 p^{3} P_{1}^{o}$ & $3.7142 \mathrm{E}+00$ & $3.248 \mathrm{E}-07$ & $5.236 \mathrm{E}+07$ & $3.6989 E+00$ & $4.474 \mathrm{E}-07$ & $7.210 \mathrm{E}+07$ & 0.004 \\
\hline $1 s 3 p^{3} P_{2}^{o}$ & $1 s 6 p^{3} P_{1}^{o}$ & $3.8474 \mathrm{E}+00$ & $6.170 \mathrm{E}-05$ & $9.267 \mathrm{E}+09$ & $3.8314 \mathrm{E}+00$ & $6.176 \mathrm{E}-05$ & $9.275 E+09$ & 0.006 \\
\hline $1 s 3 p^{1} P_{1}^{o}$ & $1 s 6 p^{3} P_{1}^{o}$ & $3.8593 \mathrm{E}+00$ & $5.498 \mathrm{E}-05$ & $8.208 \mathrm{E}+09$ & $3.8434 \mathrm{E}+00$ & $5.307 \mathrm{E}-05$ & $7.920 \mathrm{E}+09$ & 0.005 \\
\hline $1 s 4 p^{3} P_{1}^{o}$ & $1 s 6 p^{3} P_{1}^{o}$ & $8.9685 \mathrm{E}+00$ & $6.588 \mathrm{E}-07$ & $1.821 \mathrm{E}+07$ & $8.9310 E+00$ & $8.937 \mathrm{E}-07$ & $2.470 \mathrm{E}+07$ & 0.001 \\
\hline $1 s 4 p^{3} P_{2}^{0}$ & $1 s 6 p^{3} P_{1}^{o}$ & $9.2954 \mathrm{E}+00$ & $1.314 \mathrm{E}-04$ & $3.381 \mathrm{E}+09$ & $9.2564 \mathrm{E}+00$ & $1.331 \mathrm{E}-04$ & $3.424 \mathrm{E}+09$ & 0.001 \\
\hline $1 s 4 p^{1} P_{1}^{o}$ & $1 s 6 p^{3} P_{1}^{o}$ & $9.3241 \mathrm{E}+00$ & $1.177 \mathrm{E}-04$ & $3.010 \mathrm{E}+09$ & $9.2853 \mathrm{E}+00$ & $1.149 \mathrm{E}-04$ & $2.939 \mathrm{E}+09$ & 0.000 \\
\hline $1 s 4 f^{3} F_{3}^{o}$ & $1 s 6 p^{3} P_{1}^{o}$ & $9.4302 \mathrm{E}+00$ & $2.483 \mathrm{E}-05$ & $6.209 E+08$ & $9.3908 \mathrm{E}+00$ & $2.464 \mathrm{E}-05$ & $6.161 \mathrm{E}+08$ & 0.001 \\
\hline $1 s 4 f^{3} F_{2}^{o}$ & $1 s 6 p^{3} P_{1}^{o}$ & $9.4324 \mathrm{E}+00$ & $7.599 \mathrm{E}-06$ & $1.899 \mathrm{E}+08$ & $9.3930 \mathrm{E}+00$ & $7.633 \mathrm{E}-06$ & $1.907 \mathrm{E}+08$ & 0.001 \\
\hline $1 s 4 f^{1} F_{3}^{o}$ & $1 s 6 p^{3} P_{1}^{o}$ & $9.4866 \mathrm{E}+00$ & $4.042 \mathrm{E}-08$ & $9.986 \mathrm{E}+05$ & $9.4468 \mathrm{E}+00$ & $5.810 \mathrm{E}-08$ & $1.435 \mathrm{E}+06$ & 0.005 \\
\hline $1 s 5 p^{3} P_{1}^{o}$ & $1 s 6 p^{3} P_{1}^{o}$ & $2.5582 \mathrm{E}+01$ & $2.016 \mathrm{E}-06$ & $6.850 \mathrm{E}+06$ & $2.5476 \mathrm{E}+01$ & $2.721 \mathrm{E}-06$ & $9.243 \mathrm{E}+06$ & 0.000 \\
\hline $1 s 5 p^{3} P_{2}^{0}$ & $1 s 6 p^{3} P_{1}^{o}$ & $2.6962 \mathrm{E}+01$ & $4.480 \mathrm{E}-04$ & $1.370 \mathrm{E}+09$ & $2.6849 \mathrm{E}+01$ & $4.561 \mathrm{E}-04$ & $1.395 \mathrm{E}+09$ & 0.000 \\
\hline $1 s 5 p^{1} P_{1}^{o}$ & $1 s 6 p^{3} P_{1}^{0}$ & $2.7084 \mathrm{E}+01$ & $4.051 \mathrm{E}-04$ & $1.228 \mathrm{E}+09$ & $2.6972 \mathrm{E}+01$ & $3.966 \mathrm{E}-04$ & $1.202 \mathrm{E}+09$ & 0.000 \\
\hline
\end{tabular}


Table 4 (continued)

\begin{tabular}{|c|c|c|c|c|c|c|c|c|}
\hline \multirow[t]{2}{*}{ Lower } & \multirow[t]{2}{*}{ Upper } & \multicolumn{3}{|l|}{ GRASP2K } & \multicolumn{3}{|l|}{ FAC } & \multirow[t]{2}{*}{$d T$} \\
\hline & & $\lambda(\AA)$ & $g f$ & $A\left(\mathrm{~s}^{-1}\right)$ & $\lambda(\AA)$ & $g f$ & $A\left(\mathrm{~s}^{-1}\right)$ & \\
\hline $1 s 5 f^{3} F_{3}^{o}$ & $1 s 6 p^{3} P_{1}^{o}$ & $2.7546 \mathrm{E}+01$ & $1.673 \mathrm{E}-04$ & $4.903 \mathrm{E}+08$ & $2.7434 \mathrm{E}+01$ & $1.664 \mathrm{E}-04$ & $4.875 \mathrm{E}+08$ & 0.000 \\
\hline $1 s 5 f^{3} F_{2}^{o}$ & $1 s 6 p^{3} P_{1}^{0}$ & $2.7556 \mathrm{E}+01$ & $5.120 \mathrm{E}-05$ & $1.499 \mathrm{E}+08$ & $2.7444 \mathrm{E}+01$ & $5.132 \mathrm{E}-05$ & $1.502 \mathrm{E}+08$ & 0.000 \\
\hline $1 s 5 f^{1} F_{3}^{o}$ & $1 s 6 p^{3} P_{1}^{o}$ & $2.7794 \mathrm{E}+01$ & $2.736 \mathrm{E}-07$ & $7.876 \mathrm{E}+05$ & $2.7680 \mathrm{E}+01$ & $3.570 \mathrm{E}-07$ & $1.027 \mathrm{E}+06$ & 0.000 \\
\hline $1 s 3 d^{3} D_{2}$ & $1 s 6 s^{1} S_{0}$ & $3.8573 \mathrm{E}+00$ & $8.512 \mathrm{E}-06$ & $3.816 \mathrm{E}+09$ & $3.8418 \mathrm{E}+00$ & $8.554 \mathrm{E}-06$ & $3.833 E+09$ & 0.004 \\
\hline $1 s 3 d d^{1} D_{2}$ & $1 s 6 s^{1} S_{0}$ & $3.9030 \mathrm{E}+00$ & $1.339 \mathrm{E}-05$ & $5.863 E+09$ & $3.8873 \mathrm{E}+00$ & $1.312 \mathrm{E}-05$ & $5.742 \mathrm{E}+09$ & 0.012 \\
\hline $1 s 4 d^{3} D_{2}$ & $1 s 6 s{ }^{1} S_{0}$ & $9.3190 \mathrm{E}+00$ & $2.867 \mathrm{E}-05$ & $2.202 E+09$ & $9.2851 \mathrm{E}+00$ & $2.880 \mathrm{E}-05$ & $2.209 \mathrm{E}+09$ & 0.002 \\
\hline $1 s 4 d{ }^{1} D_{2}$ & $1 s 6 s^{1} S_{0}$ & $9.4321 \mathrm{E}+00$ & $4.483 \mathrm{E}-05$ & $3.361 \mathrm{E}+09$ & $9.3976 \mathrm{E}+00$ & $4.468 \mathrm{E}-05$ & $3.346 \mathrm{E}+09$ & 0.002 \\
\hline $1 s 5 d^{3} D_{2}$ & $1 s 6 s{ }^{1} S_{0}$ & $2.7060 \mathrm{E}+01$ & $1.136 \mathrm{E}-04$ & $1.034 \mathrm{E}+09$ & $2.6991 \mathrm{E}+01$ & $1.153 \mathrm{E}-04$ & $1.047 \mathrm{E}+09$ & 0.001 \\
\hline $1 s 5 d{ }^{1} D_{2}$ & $1 s 6 s^{1} S_{0}$ & $2.7551 \mathrm{E}+01$ & $1.808 \mathrm{E}-04$ & $1.589 \mathrm{E}+09$ & $2.7480 \mathrm{E}+01$ & $1.831 \mathrm{E}-04$ & $1.604 \mathrm{E}+09$ & 0.000 \\
\hline $1 s 2 p^{3} P_{2}^{o}$ & $1 s 6 p^{3} P_{0}^{o}$ & $1.4434 \mathrm{E}+00$ & $1.839 \mathrm{E}-05$ & $5.887 \mathrm{E}+10$ & $1.4374 \mathrm{E}+00$ & $1.782 \mathrm{E}-05$ & $5.703 E+10$ & 0.005 \\
\hline $1 s 3 p^{3} P_{2}^{o}$ & $1 s 6 p^{3} P_{0}^{o}$ & $3.8472 \mathrm{E}+00$ & $3.921 \mathrm{E}-05$ & $1.767 \mathrm{E}+10$ & $3.8320 \mathrm{E}+00$ & $3.856 \mathrm{E}-05$ & $1.736 \mathrm{E}+10$ & 0.006 \\
\hline $1 s 4 p^{3} P_{2}^{o}$ & $1 s 6 p^{3} P_{0}^{o}$ & $9.2942 \mathrm{E}+00$ & $8.350 \mathrm{E}-05$ & $6.448 \mathrm{E}+09$ & $9.2596 \mathrm{E}+00$ & $8.326 \mathrm{E}-05$ & $6.422 \mathrm{E}+09$ & 0.001 \\
\hline $1 s 4 f^{3} F_{2}^{o}$ & $1 s 6 p^{3} P_{0}^{o}$ & $9.4312 \mathrm{E}+00$ & $1.079 \mathrm{E}-05$ & $8.090 E+08$ & $9.3964 \mathrm{E}+00$ & $1.073 \mathrm{E}-05$ & $8.042 \mathrm{E}+08$ & 0.001 \\
\hline $1 s 5 p^{3} P_{2}^{o}$ & $1 s 6 p^{3} P_{0}^{o}$ & $2.6952 \mathrm{E}+01$ & $2.844 \mathrm{E}-04$ & $2.612 E+09$ & $2.6876 \mathrm{E}+01$ & $2.863 \mathrm{E}-04$ & $2.621 \mathrm{E}+09$ & 0.000 \\
\hline $1 s 5 f^{3} F_{2}^{o}$ & $1 s 6 p^{3} P_{0}^{o}$ & $2.7545 \mathrm{E}+01$ & $7.268 \mathrm{E}-05$ & $6.390 E+08$ & $2.7473 \mathrm{E}+01$ & $7.228 \mathrm{E}-05$ & $6.334 \mathrm{E}+08$ & 0.000 \\
\hline $1 s 2 p^{3} P_{1}^{0}$ & $1 s 6 p^{3} P_{2}^{o}$ & $1.3788 \mathrm{E}+00$ & $3.430 \mathrm{E}-05$ & $2.407 \mathrm{E}+10$ & $1.3731 \mathrm{E}+00$ & $3.466 \mathrm{E}-05$ & $2.432 \mathrm{E}+10$ & 0.006 \\
\hline $1 s 2 p^{3} P_{0}^{o}$ & $1 s 6 p^{3} P_{2}^{o}$ & $1.3797 \mathrm{E}+00$ & $2.139 \mathrm{E}-05$ & $1.499 \mathrm{E}+10$ & $1.3739 \mathrm{E}+00$ & $2.180 \mathrm{E}-05$ & $1.528 \mathrm{E}+10$ & 0.002 \\
\hline $1 s 2 p^{3} P_{2}^{o}$ & $1 s 6 p^{3} P_{2}^{o}$ & $1.4410 \mathrm{E}+00$ & $3.551 \mathrm{E}-05$ & $2.281 \mathrm{E}+10$ & $1.4350 \mathrm{E}+00$ & $3.534 \mathrm{E}-05$ & $2.270 \mathrm{E}+10$ & 0.006 \\
\hline $1 s 2 p{ }^{1} P_{1}^{o}$ & $1 s 6 p^{3} P_{2}^{o}$ & $1.4469 \mathrm{E}+00$ & $1.342 \mathrm{E}-05$ & $8.551 E+09$ & $1.4409 \mathrm{E}+00$ & $1.332 \mathrm{E}-05$ & $8.486 E+09$ & 0.000 \\
\hline $1 s 3 p^{3} P_{1}^{o}$ & $1 s 6 p^{3} P_{2}^{o}$ & $3.6983 \mathrm{E}+00$ & $6.924 \mathrm{E}-05$ & $6.753 E+09$ & $3.6831 \mathrm{E}+00$ & $6.933 \mathrm{E}-05$ & $6.760 \mathrm{E}+09$ & 0.007 \\
\hline $1 s 3 p^{3} P_{0}^{0}$ & $1 s 6 p^{3} P_{2}^{o}$ & $3.7000 \mathrm{E}+00$ & $4.378 \mathrm{E}-05$ & $4.266 \mathrm{E}+09$ & $3.6848 \mathrm{E}+00$ & $4.378 \mathrm{E}-05$ & $4.265 \mathrm{E}+09$ & 0.006 \\
\hline $1 s 3 p^{3} P_{2}^{o}$ & $1 s 6 p^{3} P_{2}^{o}$ & $3.8304 \mathrm{E}+00$ & $7.355 \mathrm{E}-05$ & $6.688 E+09$ & $3.8145 \mathrm{E}+00$ & $7.319 \mathrm{E}-05$ & $6.653 \mathrm{E}+09$ & 0.007 \\
\hline $1 s 3 p{ }^{1} P_{1}^{o}$ & $1 s 6 p^{3} P_{2}^{o}$ & $3.8422 \mathrm{E}+00$ & $2.807 \mathrm{E}-05$ & $2.536 \mathrm{E}+09$ & $3.8264 \mathrm{E}+00$ & $2.789 \mathrm{E}-05$ & $2.520 \mathrm{E}+09$ & 0.005 \\
\hline $1 s 4 p^{3} P_{1}^{o}$ & $1 s 6 p^{3} P_{2}^{o}$ & $8.8767 \mathrm{E}+00$ & $1.362 \mathrm{E}-04$ & $2.306 \mathrm{E}+09$ & $8.8397 \mathrm{E}+00$ & $1.369 \mathrm{E}-04$ & $2.318 \mathrm{E}+09$ & 0.001 \\
\hline $1 s 4 p^{3} P_{0}^{o}$ & $1 s 6 p^{3} P_{2}^{o}$ & $8.8807 \mathrm{E}+00$ & $8.658 \mathrm{E}-05$ & $1.465 \mathrm{E}+09$ & $8.8435 \mathrm{E}+00$ & $8.652 \mathrm{E}-05$ & $1.463 \mathrm{E}+09$ & 0.001 \\
\hline $1 s 4 p^{3} P_{2}^{o}$ & $1 s 6 p^{3} P_{2}^{o}$ & $9.1968 \mathrm{E}+00$ & $1.504 \mathrm{E}-04$ & $2.372 E+09$ & $9.1583 \mathrm{E}+00$ & $1.507 \mathrm{E}-04$ & $2.376 \mathrm{E}+09$ & 0.001 \\
\hline $1 s 4 p{ }^{1} P_{1}^{o}$ & $1 s 6 p^{3} P_{2}^{o}$ & $9.2248 \mathrm{E}+00$ & $5.772 \mathrm{E}-05$ & $9.049 E+08$ & $9.1865 \mathrm{E}+00$ & $5.772 \mathrm{E}-05$ & $9.047 \mathrm{E}+08$ & 0.000 \\
\hline $1 s 4 f^{3} F_{3}^{o}$ & $1 s 6 p^{3} P_{2}^{o}$ & $9.3286 \mathrm{E}+00$ & $5.477 \mathrm{E}-06$ & $8.396 E+07$ & $9.2898 \mathrm{E}+00$ & $5.452 \mathrm{E}-06$ & $8.357 \mathrm{E}+07$ & 0.001 \\
\hline $1 s 4 f^{3} F_{2}^{o}$ & $1 s 6 p^{3} P_{2}^{o}$ & $9.3309 \mathrm{E}+00$ & $1.379 \mathrm{E}-06$ & $2.113 E+07$ & $9.2920 \mathrm{E}+00$ & $1.357 \mathrm{E}-06$ & $2.079 \mathrm{E}+07$ & 0.002 \\
\hline $1 s 4 f^{3} F_{4}^{o}$ & $1 s 6 p^{3} P_{2}^{o}$ & $9.3822 \mathrm{E}+00$ & $3.795 \mathrm{E}-05$ & $5.752 E+08$ & $9.3430 \mathrm{E}+00$ & $3.754 \mathrm{E}-05$ & $5.688 \mathrm{E}+08$ & 0.000 \\
\hline $1 s 4 f^{1} F_{3}^{o}$ & $1 s 6 p^{3} P_{2}^{o}$ & $9.3838 \mathrm{E}+00$ & $4.233 \mathrm{E}-06$ & $6.413 E+07$ & $9.3446 \mathrm{E}+00$ & $4.189 \mathrm{E}-06$ & $6.346 \mathrm{E}+07$ & 0.000 \\
\hline $1 s 5 p^{3} P_{1}^{o}$ & $1 s 6 p^{3} P_{2}^{o}$ & $2.4849 \mathrm{E}+01$ & $3.840 \mathrm{E}-04$ & $8.296 E+08$ & $2.4746 \mathrm{E}+01$ & $3.860 \mathrm{E}-04$ & $8.339 \mathrm{E}+08$ & 0.000 \\
\hline $1 s 5 p^{3} P_{0}^{o}$ & $1 s 6 p^{3} P_{2}^{o}$ & $2.4864 \mathrm{E}+01$ & $2.449 \mathrm{E}-04$ & $5.284 \mathrm{E}+08$ & $2.4761 \mathrm{E}+01$ & $2.428 \mathrm{E}-04$ & $5.240 \mathrm{E}+08$ & 0.000 \\
\hline $1 s 5 p^{3} P_{2}^{o}$ & $1 s 6 p^{3} P_{2}^{o}$ & $2.6148 \mathrm{E}+01$ & $4.645 \mathrm{E}-04$ & $9.063 \mathrm{E}+08$ & $2.6040 \mathrm{E}+01$ & $4.663 \mathrm{E}-04$ & $9.096 \mathrm{E}+08$ & 0.000 \\
\hline $1 s 5 p{ }^{1} P_{1}^{o}$ & $1 s 6 p^{3} P_{2}^{o}$ & $2.6264 \mathrm{E}+01$ & $1.798 \mathrm{E}-04$ & $3.477 \mathrm{E}+08$ & $2.6156 \mathrm{E}+01$ & $1.793 \mathrm{E}-04$ & $3.466 \mathrm{E}+08$ & 0.000 \\
\hline $1 s 5 f^{3} F_{3}^{0}$ & $1 s 6 p^{3} P_{2}^{o}$ & $2.6698 \mathrm{E}+01$ & $3.580 \mathrm{E}-05$ & $6.700 \mathrm{E}+07$ & $2.6590 \mathrm{E}+01$ & $3.549 \mathrm{E}-05$ & $6.640 \mathrm{E}+07$ & 0.000 \\
\hline $1 s 5 f^{3} F_{2}^{o}$ & $1 s 6 p^{3} P_{2}^{o}$ & $2.6707 \mathrm{E}+01$ & $9.011 \mathrm{E}-06$ & $1.685 \mathrm{E}+07$ & $2.6599 E+01$ & $8.838 \mathrm{E}-06$ & $1.652 \mathrm{E}+07$ & 0.000 \\
\hline $1 s 5 f^{3} F_{4}^{o}$ & $1 s 6 p^{3} P_{2}^{o}$ & $2.6923 \mathrm{E}+01$ & $2.493 \mathrm{E}-04$ & $4.588 \mathrm{E}+08$ & $2.6814 \mathrm{E}+01$ & $2.461 \mathrm{E}-04$ & $4.528 \mathrm{E}+08$ & 0.000 \\
\hline $1 s 5 f^{1} F_{3}^{o}$ & $1 s 6 p^{3} P_{2}^{o}$ & $2.6930 \mathrm{E}+01$ & $2.780 \mathrm{E}-05$ & $5.113 \mathrm{E}+07$ & $2.6821 \mathrm{E}+01$ & $2.745 \mathrm{E}-05$ & $5.048 \mathrm{E}+07$ & 0.000 \\
\hline $1 s 6 p^{3} P_{1}^{o}$ & $1 s 6 p^{3} P_{2}^{o}$ & $8.6654 \mathrm{E}+02$ & $1.895 \mathrm{E}-07$ & $3.367 \mathrm{E}+02$ & $8.6410 \mathrm{E}+02$ & $1.920 \mathrm{E}-07$ & $3.402 E+02$ & 0.003 \\
\hline $1 s 6 p^{3} P_{0}^{o}$ & $1 s 6 p^{3} P_{2}^{o}$ & $8.7749 \mathrm{E}+02$ & $1.161 \mathrm{E}-07$ & $2.012 \mathrm{E}+02$ & $8.3677 \mathrm{E}+02$ & $1.322 \mathrm{E}-07$ & $2.498 \mathrm{E}+02$ & 0.003 \\
\hline $1 s^{2}{ }^{1} S_{0}$ & $1 s 6 d^{3} D_{2}$ & $3.1620 \mathrm{E}-01$ & $1.394 \mathrm{E}-04$ & $1.859 \mathrm{E}+12$ & $3.1497 \mathrm{E}-01$ & $1.398 \mathrm{E}-04$ & $1.864 \mathrm{E}+12$ & 0.002 \\
\hline $1 s 2 s^{3} S_{1}$ & $1 s 6 d^{3} D_{2}$ & $1.3669 \mathrm{E}+00$ & $1.366 \mathrm{E}-05$ & $9.753 \mathrm{E}+09$ & $1.3610 \mathrm{E}+00$ & $1.209 \mathrm{E}-05$ & $8.637 \mathrm{E}+09$ & 0.007 \\
\hline $1 s 2 s^{1} S_{0}$ & $1 s 6 d^{3} D_{2}$ & $1.3798 \mathrm{E}+00$ & $7.380 \mathrm{E}-06$ & $5.171 \mathrm{E}+09$ & $1.3741 \mathrm{E}+00$ & $7.407 \mathrm{E}-06$ & $5.189 \mathrm{E}+09$ & 0.004 \\
\hline $1 s 3 s^{3} S_{1}$ & $1 s 6 d^{3} D_{2}$ & $3.6741 \mathrm{E}+00$ & $8.920 \mathrm{E}-05$ & $8.815 E+09$ & $3.6587 \mathrm{E}+00$ & $8.805 \mathrm{E}-05$ & $8.701 E+09$ & 0.004 \\
\hline $1 s 3 s{ }^{1} S_{0}$ & $1 s 6 d^{3} D_{2}$ & $3.6988 \mathrm{E}+00$ & $5.586 \mathrm{E}-05$ & $5.447 \mathrm{E}+09$ & $3.6835 \mathrm{E}+00$ & $5.475 \mathrm{E}-05$ & $5.337 \mathrm{E}+09$ & 0.013 \\
\hline $1 s 3 d^{3} D_{2}$ & $1 s 6 d^{3} D_{2}$ & $3.8391 \mathrm{E}+00$ & $3.737 \mathrm{E}-05$ & $3.382 E+09$ & $3.8228 \mathrm{E}+00$ & $3.713 \mathrm{E}-05$ & $3.361 \mathrm{E}+09$ & 0.003 \\
\hline $1 s 3 d^{3} D_{1}$ & $1 s 6 d^{3} D_{2}$ & $3.8409 \mathrm{E}+00$ & $1.647 \mathrm{E}-05$ & $1.489 \mathrm{E}+09$ & $3.8245 \mathrm{E}+00$ & $1.647 \mathrm{E}-05$ & $1.490 \mathrm{E}+09$ & 0.007 \\
\hline $1 s 3 d^{3} D_{3}$ & $1 s 6 d^{3} D_{2}$ & $3.8827 \mathrm{E}+00$ & $1.797 \mathrm{E}-05$ & $1.590 \mathrm{E}+09$ & $3.8661 \mathrm{E}+00$ & $1.793 \mathrm{E}-05$ & $1.587 \mathrm{E}+09$ & 0.004 \\
\hline $1 s 3 d^{1} D_{2}$ & $1 s 6 d^{3} D_{2}$ & $3.8844 \mathrm{E}+00$ & $4.392 \mathrm{E}-06$ & $3.883 \mathrm{E}+08$ & $3.8678 \mathrm{E}+00$ & $4.140 \mathrm{E}-06$ & $3.661 \mathrm{E}+08$ & 0.010 \\
\hline $1 s 4 s^{3} S_{1}$ & $1 s 6 d^{3} D_{2}$ & $8.8160 \mathrm{E}+00$ & $2.394 \mathrm{E}-04$ & $4.109 \mathrm{E}+09$ & $8.7783 \mathrm{E}+00$ & $2.410 \mathrm{E}-04$ & $4.138 \mathrm{E}+09$ & 0.003 \\
\hline $1 s 4 s{ }^{1} S_{0}$ & $1 s 6 d^{3} D_{2}$ & $8.8733 \mathrm{E}+00$ & $1.533 \mathrm{E}-04$ & $2.598 \mathrm{E}+09$ & $8.8358 \mathrm{E}+00$ & $1.502 \mathrm{E}-04$ & $2.545 \mathrm{E}+09$ & 0.007 \\
\hline $1 s 4 d^{3} D_{2}$ & $1 s 6 d^{3} D_{2}$ & $9.2136 \mathrm{E}+00$ & $1.112 \mathrm{E}-04$ & $1.748 \mathrm{E}+09$ & $9.1745 \mathrm{E}+00$ & $1.107 \mathrm{E}-04$ & $1.740 \mathrm{E}+09$ & 0.001 \\
\hline $1 s 4 d^{3} D_{1}$ & $1 s 6 d^{3} D_{2}$ & $9.2178 \mathrm{E}+00$ & $4.892 \mathrm{E}-05$ & $7.682 E+08$ & $9.1786 \mathrm{E}+00$ & $4.923 \mathrm{E}-05$ & $7.730 \mathrm{E}+08$ & 0.002 \\
\hline $1 s 4 d^{3} D_{3}$ & $1 s 6 d^{3} D_{2}$ & $9.3198 \mathrm{E}+00$ & $5.465 \mathrm{E}-05$ & $8.393 E+08$ & $9.2799 \mathrm{E}+00$ & $5.491 \mathrm{E}-05$ & $8.435 E+08$ & 0.001 \\
\hline $1 s 4 d{ }^{1} D_{2}$ & $1 s 6 d^{3} D_{2}$ & $9.3242 \mathrm{E}+00$ & $1.339 \mathrm{E}-05$ & $2.054 \mathrm{E}+08$ & $9.2843 \mathrm{E}+00$ & $1.284 \mathrm{E}-05$ & $1.970 \mathrm{E}+08$ & 0.003 \\
\hline $1 s 5 s^{3} S_{1}$ & $1 s 6 d^{3} D_{2}$ & $2.4585 \mathrm{E}+01$ & $7.948 \mathrm{E}-04$ & $1.754 \mathrm{E}+09$ & $2.4475 \mathrm{E}+01$ & $8.142 \mathrm{E}-04$ & $1.798 \mathrm{E}+09$ & 0.002 \\
\hline $1 s 5 s{ }^{1} S_{0}$ & $1 s 6 d^{3} D_{2}$ & $2.4809 \mathrm{E}+01$ & $5.211 \mathrm{E}-04$ & $1.130 \mathrm{E}+09$ & $2.4699 \mathrm{E}+01$ & $5.045 \mathrm{E}-04$ & $1.094 \mathrm{E}+09$ & 0.001 \\
\hline $1 s 5 d^{3} D_{2}$ & $1 s 6 d^{3} D_{2}$ & $2.6190 \mathrm{E}+01$ & $4.075 \mathrm{E}-04$ & $7.925 \mathrm{E}+08$ & $2.6077 \mathrm{E}+01$ & $4.066 \mathrm{E}-04$ & $7.910 \mathrm{E}+08$ & 0.000 \\
\hline $1 s 5 d^{3} D_{1}$ & $1 s 6 d^{3} D_{2}$ & $2.6207 \mathrm{E}+01$ & $1.795 \mathrm{E}-04$ & $3.486 \mathrm{E}+08$ & $2.6094 \mathrm{E}+01$ & $1.806 \mathrm{E}-04$ & $3.509 E+08$ & 0.001 \\
\hline $1 s 5 d^{3} D_{3}$ & $1 s 6 d^{3} D_{2}$ & $2.6631 \mathrm{E}+01$ & $2.076 \mathrm{E}-04$ & $3.904 \mathrm{E}+08$ & $2.6515 \mathrm{E}+01$ & $2.095 \mathrm{E}-04$ & $3.941 \mathrm{E}+08$ & 0.000 \\
\hline $1 s 5 d{ }^{1} D_{2}$ & $1 s 6 d^{3} D_{2}$ & $2.6650 \mathrm{E}+01$ & $5.102 \mathrm{E}-05$ & $9.582 \mathrm{E}+07$ & $2.6534 \mathrm{E}+01$ & $4.929 \mathrm{E}-05$ & $9.260 \mathrm{E}+07$ & 0.001 \\
\hline $1 s 5 g^{3} G_{4}$ & $1 s 6 d^{3} D_{2}$ & $2.6864 \mathrm{E}+01$ & $6.067 \mathrm{E}-05$ & $1.121 \mathrm{E}+08$ & $2.6740 \mathrm{E}+01$ & $6.071 \mathrm{E}-05$ & $1.123 \mathrm{E}+08$ & 0.000 \\
\hline $1 s 5 g^{3} G_{3}$ & $1 s 6 d^{3} D_{2}$ & $2.6869 \mathrm{E}+01$ & $6.827 \mathrm{E}-06$ & $1.262 \mathrm{E}+07$ & $2.6746 \mathrm{E}+01$ & $6.882 \mathrm{E}-06$ & $1.272 \mathrm{E}+07$ & 0.000 \\
\hline $1 s 5 g{ }^{1} G_{4}$ & $1 s 6 d^{3} D_{2}$ & $2.7003 \mathrm{E}+01$ & $4.401 \mathrm{E}-09$ & $8.052 E+03$ & & & & 0.005 \\
\hline $1 s 6 s^{3} S_{1}$ & $1 s 6 d^{3} D_{2}$ & $6.9582 \mathrm{E}+02$ & $3.295 E-07$ & $9.080 \mathrm{E}+02$ & $6.7673 E+02$ & $3.578 \mathrm{E}-07$ & $1.033 \mathrm{E}+03$ & 0.023 \\
\hline $1 s 6 s{ }^{1} S_{0}$ & $1 s 6 d^{3} D_{2}$ & $8.1469 \mathrm{E}+02$ & $1.337 \mathrm{E}-07$ & $2.688 \mathrm{E}+02$ & $7.7023 \mathrm{E}+02$ & $1.541 \mathrm{E}-07$ & $3.436 \mathrm{E}+02$ & 0.058 \\
\hline $1 s 2 p^{3} P_{1}^{o}$ & $1 s 6 p^{1} P_{1}^{o}$ & $1.3786 \mathrm{E}+00$ & $3.042 \mathrm{E}-05$ & $3.559 E+10$ & $1.3729 \mathrm{E}+00$ & $3.065 \mathrm{E}-05$ & $3.585 E+10$ & 0.000 \\
\hline $1 s 2 p^{3} P_{2}^{o}$ & $1 s 6 p^{1} P_{1}^{o}$ & $1.4408 \mathrm{E}+00$ & $1.370 \mathrm{E}-05$ & $1.468 \mathrm{E}+10$ & $1.4348 \mathrm{E}+00$ & $1.329 \mathrm{E}-05$ & $1.423 \mathrm{E}+10$ & 0.002 \\
\hline $1 s 2 p{ }^{1} P_{1}^{o}$ & $1 s 6 p^{1} P_{1}^{o}$ & $1.4467 \mathrm{E}+00$ & $1.838 \mathrm{E}-05$ & $1.952 E+10$ & $1.4407 \mathrm{E}+00$ & $1.863 \mathrm{E}-05$ & $1.979 \mathrm{E}+10$ & 0.007 \\
\hline $1 s 3 p^{3} P_{1}^{o}$ & $1 s 6 p^{1} P_{1}^{o}$ & $3.6970 \mathrm{E}+00$ & $6.212 \mathrm{E}-05$ & $1.010 \mathrm{E}+10$ & $3.6819 \mathrm{E}+00$ & $6.180 \mathrm{E}-05$ & $1.005 \mathrm{E}+10$ & 0.006 \\
\hline
\end{tabular}


Table 4 (continued)

\begin{tabular}{|c|c|c|c|c|c|c|c|c|}
\hline \multirow[t]{2}{*}{ Lower } & \multirow[t]{2}{*}{ Upper } & \multicolumn{3}{|l|}{ GRASP2K } & \multicolumn{3}{|l|}{ FAC } & \multirow[t]{2}{*}{$d T$} \\
\hline & & $\lambda(\AA)$ & $g f$ & $A\left(\mathrm{~s}^{-1}\right)$ & $\lambda(\AA)$ & $g f$ & $A\left(\mathrm{~s}^{-1}\right)$ & \\
\hline $1 s 3 p^{3} P_{2}^{o}$ & $1 s 6 p^{1} P_{1}^{o}$ & $3.8290 \mathrm{E}+00$ & $2.847 \mathrm{E}-05$ & $4.318 E+09$ & $3.8133 E+00$ & $2.744 \mathrm{E}-05$ & $4.160 \mathrm{E}+09$ & 0.005 \\
\hline $1 s 3 p^{1} P_{1}^{o}$ & $1 s 6 p^{1} P_{1}^{o}$ & $3.8407 \mathrm{E}+00$ & $3.813 \mathrm{E}-05$ & $5.747 \mathrm{E}+09$ & $3.8251 \mathrm{E}+00$ & $3.860 \mathrm{E}-05$ & $5.816 \mathrm{E}+09$ & 0.003 \\
\hline $1 s 4 p^{3} P_{1}^{o}$ & $1 s 6 p^{1} P_{1}^{o}$ & $8.8691 \mathrm{E}+00$ & $1.223 \mathrm{E}-04$ & $3.458 \mathrm{E}+09$ & $8.8329 \mathrm{E}+00$ & $1.223 \mathrm{E}-04$ & $3.455 E+09$ & 0.001 \\
\hline $1 s 4 p^{3} P_{2}^{o}$ & $1 s 6 p^{1} P_{1}^{o}$ & $9.1886 \mathrm{E}+00$ & $5.804 \mathrm{E}-05$ & $1.529 \mathrm{E}+09$ & $9.1511 \mathrm{E}+00$ & $5.631 \mathrm{E}-05$ & $1.482 \mathrm{E}+09$ & 0.001 \\
\hline $1 s 4 p^{1} P_{1}^{o}$ & $1 s 6 p^{1} P_{1}^{o}$ & $9.2166 \mathrm{E}+00$ & $7.802 \mathrm{E}-05$ & $2.042 \mathrm{E}+09$ & $9.1793 \mathrm{E}+00$ & $7.959 \mathrm{E}-05$ & $2.082 E+09$ & 0.000 \\
\hline $1 s 4 f^{3} F_{3}^{o}$ & $1 s 6 p^{1} P_{1}^{o}$ & $9.3203 \mathrm{E}+00$ & $1.284 \mathrm{E}-06$ & $3.286 \mathrm{E}+07$ & $9.2824 \mathrm{E}+00$ & $1.452 \mathrm{E}-06$ & $3.715 E+07$ & 0.003 \\
\hline $1 s 4 f^{3} F_{2}^{o}$ & $1 s 6 p^{1} P_{1}^{o}$ & $9.3225 \mathrm{E}+00$ & $2.872 \mathrm{E}-06$ & $7.347 \mathrm{E}+07$ & $9.2846 \mathrm{E}+00$ & $2.760 \mathrm{E}-06$ & $7.058 \mathrm{E}+07$ & 0.001 \\
\hline $1 s 4 f^{1} F_{3}^{o}$ & $1 s 6 p^{1} P_{1}^{o}$ & $9.3754 \mathrm{E}+00$ & $2.499 \mathrm{E}-05$ & $6.322 \mathrm{E}+08$ & $9.3371 \mathrm{E}+00$ & $2.499 \mathrm{E}-05$ & $6.319 E+08$ & 0.001 \\
\hline $1 s 5 p^{3} P_{1}^{0}$ & $1 s 6 p^{1} P_{1}^{o}$ & $2.4789 \mathrm{E}+01$ & $3.429 \mathrm{E}-04$ & $1.241 \mathrm{E}+09$ & $2.4694 \mathrm{E}+01$ & $3.435 \mathrm{E}-04$ & $1.241 \mathrm{E}+09$ & 0.000 \\
\hline $1 s 5 p^{3} P_{2}^{o}$ & $1 s 6 p^{1} P_{1}^{o}$ & $2.6083 \mathrm{E}+01$ & $1.778 \mathrm{E}-04$ & $5.810 \mathrm{E}+08$ & $2.5981 \mathrm{E}+01$ & $1.727 \mathrm{E}-04$ & $5.643 E+08$ & 0.000 \\
\hline $1 s 5 p^{1} P_{1}^{o}$ & $1 s 6 p^{1} P_{1}^{o}$ & $2.6197 \mathrm{E}+01$ & $2.407 \mathrm{E}-04$ & $7.798 E+08$ & $2.6097 \mathrm{E}+01$ & $2.471 \mathrm{E}-04$ & $8.000 E+08$ & 0.000 \\
\hline $1 s 5 f^{3} F_{3}^{o}$ & $1 s 6 p^{1} P_{1}^{o}$ & $2.6629 \mathrm{E}+01$ & $8.342 \mathrm{E}-06$ & $2.616 \mathrm{E}+07$ & $2.6529 \mathrm{E}+01$ & $9.671 \mathrm{E}-06$ & $3.029 E+07$ & 0.000 \\
\hline $1 s 5 f^{3} F_{2}^{o}$ & $1 s 6 p^{1} P_{1}^{o}$ & $2.6638 \mathrm{E}+01$ & $1.871 \mathrm{E}-05$ & $5.863 \mathrm{E}+07$ & $2.6539 \mathrm{E}+01$ & $1.789 \mathrm{E}-05$ & $5.600 \mathrm{E}+07$ & 0.000 \\
\hline $1 s 5 f^{1} F_{3}^{o}$ & $1 s 6 p^{1} P_{1}^{o}$ & $2.6860 \mathrm{E}+01$ & $1.637 \mathrm{E}-04$ & $5.046 \mathrm{E}+08$ & $2.6759 \mathrm{E}+01$ & $1.646 \mathrm{E}-04$ & $5.067 \mathrm{E}+08$ & 0.000 \\
\hline $1 s 6 p^{3} P_{1}^{0}$ & $1 s 6 p^{1} P_{1}^{o}$ & $7.9973 \mathrm{E}+02$ & $2.175 \mathrm{E}-07$ & $7.560 \mathrm{E}+02$ & $8.0416 \mathrm{E}+02$ & $2.068 \mathrm{E}-07$ & $7.051 \mathrm{E}+02$ & 0.003 \\
\hline $1 s 6 p^{3} P_{2}^{o}$ & $1 s 6 p^{1} P_{1}^{o}$ & $1.0374 \mathrm{E}+04$ & $4.887 \mathrm{E}-11$ & $1.010 \mathrm{E}-03$ & & & & 0.005 \\
\hline $1 s 2 s^{3} S_{1}^{2}$ & $1 s 6 d^{3} D_{1}$ & $1.3668 \mathrm{E}+00$ & $1.350 \mathrm{E}-05$ & $1.607 \mathrm{E}+10$ & $1.3611 \mathrm{E}+00$ & $1.183 \mathrm{E}-05$ & $1.408 \mathrm{E}+10$ & 0.004 \\
\hline $1 s 3 s^{3} S_{1}$ & $1 s 6 d^{3} D_{1}$ & $3.6740 \mathrm{E}+00$ & $8.868 \mathrm{E}-05$ & $1.461 \mathrm{E}+10$ & $3.6587 \mathrm{E}+00$ & $8.652 \mathrm{E}-05$ & $1.424 \mathrm{E}+10$ & 0.006 \\
\hline $1 s 3 d^{3} D_{2}$ & $1 s 6 d^{3} D_{1}$ & $3.8389 \mathrm{E}+00$ & $1.627 \mathrm{E}-05$ & $2.454 \mathrm{E}+09$ & $3.8228 \mathrm{E}+00$ & $1.618 \mathrm{E}-05$ & $2.441 \mathrm{E}+09$ & 0.005 \\
\hline $1 s 3 d^{3} D_{1}$ & $1 s 6 d^{3} D_{1}$ & $3.8407 \mathrm{E}+00$ & $1.611 \mathrm{E}-05$ & $2.429 \mathrm{E}+09$ & $3.8246 \mathrm{E}+00$ & $1.597 \mathrm{E}-05$ & $2.408 E+09$ & 0.005 \\
\hline $1 s 3 d^{3} D_{3}$ & $1 s 6 d^{3} D_{1}$ & $3.8825 \mathrm{E}+00$ & $2.940 \mathrm{E}-06$ & $4.336 \mathrm{E}+08$ & $3.8662 \mathrm{E}+00$ & $2.932 \mathrm{E}-06$ & $4.324 \mathrm{E}+08$ & 0.006 \\
\hline $1 s 3 d^{1} D_{2}$ & $1 s 6 d^{3} D_{1}$ & $3.8842 \mathrm{E}+00$ & $1.041 \mathrm{E}-05$ & $1.534 \mathrm{E}+09$ & $3.8678 \mathrm{E}+00$ & $1.015 \mathrm{E}-05$ & $1.496 \mathrm{E}+09$ & 0.005 \\
\hline $1 s 4 s^{3} S_{1}$ & $1 s 6 d^{3} D_{1}$ & $8.8149 \mathrm{E}+00$ & $2.379 \mathrm{E}-04$ & $6.807 \mathrm{E}+09$ & $8.7785 \mathrm{E}+00$ & $2.371 \mathrm{E}-04$ & $6.783 E+09$ & 0.004 \\
\hline $1 s 4 d^{3} D_{2}$ & $1 s 6 d^{3} D_{1}$ & $9.2124 \mathrm{E}+00$ & $4.864 \mathrm{E}-05$ & $1.274 \mathrm{E}+09$ & $9.1748 \mathrm{E}+00$ & $4.850 \mathrm{E}-05$ & $1.270 \mathrm{E}+09$ & 0.002 \\
\hline $1 s 4 d^{3} D_{1}$ & $1 s 6 d^{3} D_{1}$ & $9.2166 \mathrm{E}+00$ & $4.770 \mathrm{E}-05$ & $1.249 \mathrm{E}+09$ & $9.1789 \mathrm{E}+00$ & $4.769 \mathrm{E}-05$ & $1.247 \mathrm{E}+09$ & 0.002 \\
\hline $1 s 4 d^{3} D_{3}$ & $1 s 6 d^{3} D_{1}$ & $9.3186 \mathrm{E}+00$ & $8.922 \mathrm{E}-06$ & $2.284 \mathrm{E}+08$ & $9.2802 \mathrm{E}+00$ & $8.978 \mathrm{E}-06$ & $2.298 \mathrm{E}+08$ & 0.002 \\
\hline $1 s 4 d{ }^{1} D_{2}$ & $1 s 6 d^{3} D_{1}$ & $9.3230 \mathrm{E}+00$ & $3.131 \mathrm{E}-05$ & $8.010 E+08$ & $9.2846 \mathrm{E}+00$ & $3.094 \mathrm{E}-05$ & $7.914 \mathrm{E}+08$ & 0.001 \\
\hline $1 s 5 s^{3} S_{1}$ & $1 s 6 d^{3} D_{1}$ & $2.4577 \mathrm{E}+01$ & $7.884 \mathrm{E}-04$ & $2.902 \mathrm{E}+09$ & $2.4477 \mathrm{E}+01$ & $8.016 \mathrm{E}-04$ & $2.949 E+09$ & 0.003 \\
\hline $1 s 5 d^{3} D_{2}$ & $1 s 6 d^{3} D_{1}$ & $2.6180 \mathrm{E}+01$ & $1.783 \mathrm{E}-04$ & $5.784 \mathrm{E}+08$ & $2.6079 \mathrm{E}+01$ & $1.784 \mathrm{E}-04$ & $5.784 \mathrm{E}+08$ & 0.001 \\
\hline $1 s 5 d^{3} D_{1}$ & $1 s 6 d^{3} D_{1}$ & $2.6197 \mathrm{E}+01$ & $1.744 \mathrm{E}-04$ & $5.652 \mathrm{E}+08$ & $2.6096 \mathrm{E}+01$ & $1.750 \mathrm{E}-04$ & $5.667 \mathrm{E}+08$ & 0.000 \\
\hline $1 s 5 d^{3} D_{3}$ & $1 s 6 d^{3} D_{1}$ & $2.6621 \mathrm{E}+01$ & $3.384 \mathrm{E}-05$ & $1.062 \mathrm{E}+08$ & $2.6517 \mathrm{E}+01$ & $3.426 \mathrm{E}-05$ & $1.074 \mathrm{E}+08$ & 0.001 \\
\hline $1 s 5 d^{1} D_{2}$ & $1 s 6 d^{3} D_{1}$ & $2.6640 \mathrm{E}+01$ & $1.185 \mathrm{E}-04$ & $3.712 \mathrm{E}+08$ & $2.6536 \mathrm{E}+01$ & $1.178 \mathrm{E}-04$ & $3.688 \mathrm{E}+08$ & 0.000 \\
\hline $1 s 5 g^{3} G_{3}$ & $1 s 6 d^{3} D_{1}$ & $2.6859 \mathrm{E}+01$ & $4.044 \mathrm{E}-05$ & $1.246 \mathrm{E}+08$ & $2.6748 \mathrm{E}+01$ & $4.053 \mathrm{E}-05$ & $1.248 \mathrm{E}+08$ & 0.000 \\
\hline $1 s 6 s^{3} s_{1}$ & $1 s 6 d^{3} D_{1}$ & $6.8911 \mathrm{E}+02$ & $3.374 \mathrm{E}-07$ & $1.580 \mathrm{E}+03$ & $6.7829 \mathrm{E}+02$ & $3.486 \mathrm{E}-07$ & $1.670 \mathrm{E}+03$ & 0.016 \\
\hline $1 s 6 d^{3} D_{2}$ & $1 s 6 d^{3} D_{1}$ & $7.1428 \mathrm{E}+04$ & $1.468 \mathrm{E}-13$ & $6.396 \mathrm{E}-08$ & & & & 0.467 \\
\hline $1 s 2 s^{3} S_{1}$ & $1 s 6 d^{3} D_{3}$ & $1.3662 \mathrm{E}+00$ & $4.040 \mathrm{E}-05$ & $2.062 \mathrm{E}+10$ & $1.3604 \mathrm{E}+00$ & $3.740 \mathrm{E}-05$ & $1.909 \mathrm{E}+10$ & 0.001 \\
\hline $1 s 3 s^{3} S_{1}$ & $1 s 6 d^{3} D_{3}$ & $3.6692 \mathrm{E}+00$ & $2.239 \mathrm{E}-04$ & $1.585 \mathrm{E}+10$ & $3.6538 \mathrm{E}+00$ & $2.222 \mathrm{E}-04$ & $1.572 \mathrm{E}+10$ & 0.005 \\
\hline $1 s 3 d^{3} D_{2}$ & $1 s 6 d^{3} D_{3}$ & $3.8337 \mathrm{E}+00$ & $1.908 \mathrm{E}-05$ & $1.237 \mathrm{E}+09$ & $3.8174 \mathrm{E}+00$ & $1.928 \mathrm{E}-05$ & $1.250 \mathrm{E}+09$ & 0.004 \\
\hline $1 s 3 d^{3} D_{1}$ & $1 s 6 d^{3} D_{3}$ & $3.8355 \mathrm{E}+00$ & $3.117 \mathrm{E}-06$ & $2.019 \mathrm{E}+08$ & $3.8192 \mathrm{E}+00$ & $3.126 \mathrm{E}-06$ & $2.025 \mathrm{E}+08$ & 0.005 \\
\hline $1 s 3 d^{3} D_{3}$ & $1 s 6 d^{3} D_{3}$ & $3.8772 \mathrm{E}+00$ & $7.363 \mathrm{E}-05$ & $4.667 \mathrm{E}+09$ & $3.8607 \mathrm{E}+00$ & $7.364 \mathrm{E}-05$ & $4.668 \mathrm{E}+09$ & 0.004 \\
\hline $1 s 3 d{ }^{1} D_{2}$ & $1 s 6 d^{3} D_{3}$ & $3.8789 \mathrm{E}+00$ & $1.225 \mathrm{E}-05$ & $7.761 \mathrm{E}+08$ & $3.8624 \mathrm{E}+00$ & $1.214 \mathrm{E}-05$ & $7.694 \mathrm{E}+08$ & 0.005 \\
\hline $1 s 4 s^{3} S_{1}$ & $1 s 6 d^{3} D_{3}$ & $8.7877 \mathrm{E}+00$ & $5.723 \mathrm{E}-04$ & $7.062 \mathrm{E}+09$ & $8.7503 E+00$ & $5.746 \mathrm{E}-04$ & $7.090 \mathrm{E}+09$ & 0.004 \\
\hline $1 s 4 d^{3} D_{2}$ & $1 s 6 d^{3} D_{3}$ & $9.1827 \mathrm{E}+00$ & $5.597 \mathrm{E}-05$ & $6.325 \mathrm{E}+08$ & $9.1440 \mathrm{E}+00$ & $5.630 \mathrm{E}-05$ & $6.362 E+08$ & 0.001 \\
\hline $1 s 4 d^{3} D_{1}$ & $1 s 6 d^{3} D_{3}$ & $9.1868 \mathrm{E}+00$ & $9.054 \mathrm{E}-06$ & $1.022 \mathrm{E}+08$ & $9.1480 \mathrm{E}+00$ & $9.077 \mathrm{E}-06$ & $1.024 \mathrm{E}+08$ & 0.001 \\
\hline $1 s 4 d^{3} D_{3}$ & $1 s 6 d^{3} D_{3}$ & $9.2881 \mathrm{E}+00$ & $2.193 \mathrm{E}-04$ & $2.423 \mathrm{E}+09$ & $9.2486 \mathrm{E}+00$ & $2.195 \mathrm{E}-04$ & $2.424 \mathrm{E}+09$ & 0.001 \\
\hline $1 s 4 d^{1} D_{2}$ & $1 s 6 d^{3} D_{3}$ & $9.2925 \mathrm{E}+00$ & $3.614 \mathrm{E}-05$ & $3.988 \mathrm{E}+08$ & $9.2530 \mathrm{E}+00$ & $3.603 \mathrm{E}-05$ & $3.976 \mathrm{E}+08$ & 0.002 \\
\hline $1 s 5 s^{3} S_{1}$ & $1 s 6 d^{3} D_{3}$ & $2.4366 \mathrm{E}+01$ & $1.808 \mathrm{E}-03$ & $2.903 \mathrm{E}+09$ & $2.4258 \mathrm{E}+01$ & $1.840 \mathrm{E}-03$ & $2.955 E+09$ & 0.002 \\
\hline $1 s 5 d^{3} D_{2}$ & $1 s 6 d^{3} D_{3}$ & $2.5941 \mathrm{E}+01$ & $1.985 \mathrm{E}-04$ & $2.811 \mathrm{E}+08$ & $2.5832 \mathrm{E}+01$ & $1.994 \mathrm{E}-04$ & $2.823 E+08$ & 0.000 \\
\hline $1 s 5 d^{3} D_{1}$ & $1 s 6 d^{3} D_{3}$ & $2.5958 \mathrm{E}+01$ & $3.202 \mathrm{E}-05$ & $4.528 \mathrm{E}+07$ & $2.5848 \mathrm{E}+01$ & $3.209 E-05$ & $4.538 \mathrm{E}+07$ & 0.000 \\
\hline $1 s 5 d^{3} D_{3}$ & $1 s 6 d^{3} D_{3}$ & $2.6374 \mathrm{E}+01$ & $8.038 \mathrm{E}-04$ & $1.101 \mathrm{E}+09$ & $2.6261 \mathrm{E}+01$ & $8.056 \mathrm{E}-04$ & $1.103 E+09$ & 0.000 \\
\hline $1 s 5 d^{1} D_{2}$ & $1 s 6 d^{3} D_{3}$ & $2.6393 \mathrm{E}+01$ & $1.321 \mathrm{E}-04$ & $1.808 \mathrm{E}+08$ & $2.6280 \mathrm{E}+01$ & $1.319 \mathrm{E}-04$ & $1.804 \mathrm{E}+08$ & 0.000 \\
\hline $1 s 5 g{ }^{3} G_{4}$ & $1 s 6 d^{3} D_{3}$ & $2.6602 \mathrm{E}+01$ & $5.844 \mathrm{E}-06$ & $7.869 \mathrm{E}+06$ & $2.6482 \mathrm{E}+01$ & $5.860 \mathrm{E}-06$ & $7.894 \mathrm{E}+06$ & 0.000 \\
\hline $1 s 5 g^{3} G_{3}$ & $1 s 6 d^{3} D_{3}$ & $2.6608 \mathrm{E}+01$ & $7.044 \mathrm{E}-07$ & $9.481 \mathrm{E}+05$ & $2.6488 \mathrm{E}+01$ & $7.042 \mathrm{E}-07$ & $9.483 \mathrm{E}+05$ & 0.000 \\
\hline $1 s 5 g^{3} G_{5}$ & $1 s 6 d^{3} D_{3}$ & $2.6735 \mathrm{E}+01$ & $7.898 \mathrm{E}-05$ & $1.053 \mathrm{E}+08$ & $2.6614 \mathrm{E}+01$ & $7.896 \mathrm{E}-05$ & $1.053 \mathrm{E}+08$ & 0.000 \\
\hline $1 s 5 g{ }^{1} G_{4}$ & $1 s 6 d^{3} D_{3}$ & $2.6739 \mathrm{E}+01$ & $4.809 \mathrm{E}-06$ & $6.409 E+06$ & $2.6619 \mathrm{E}+01$ & $4.803 \mathrm{E}-06$ & $6.404 \mathrm{E}+06$ & 0.000 \\
\hline $1 s 6 s^{3} S_{1}$ & $1 s 6 d^{3} D_{3}$ & $5.5466 \mathrm{E}+02$ & $1.528 \mathrm{E}-06$ & $4.732 \mathrm{E}+03$ & $5.4291 \mathrm{E}+02$ & $1.608 \mathrm{E}-06$ & $5.157 \mathrm{E}+03$ & 0.017 \\
\hline $1 s 6 d^{3} D_{2}$ & $1 s 6 d^{3} D_{3}$ & $2.7340 \mathrm{E}+03$ & $3.001 \mathrm{E}-09$ & $3.826 \mathrm{E}-01$ & & & & 0.003 \\
\hline $1 s 6 d^{3} D_{1}$ & $1 s 6 d^{3} D_{3}$ & $2.8428 \mathrm{E}+03$ & $4.330 \mathrm{E}-10$ & $5.106 \mathrm{E}-02$ & & & & 0.008 \\
\hline $1 s 2 p^{3} P_{1}^{o}$ & $1 s 6 f^{3} F_{3}^{o}$ & $1.3779 \mathrm{E}+00$ & $9.054 \mathrm{E}-04$ & $4.544 \mathrm{E}+11$ & $1.3722 \mathrm{E}+00$ & $9.227 \mathrm{E}-04$ & $4.630 \mathrm{E}+11$ & 0.001 \\
\hline $1 s 2 p^{3} P_{2}^{o}$ & $1 s 6 f^{3} F_{3}^{0}$ & $1.4401 \mathrm{E}+00$ & $2.384 \mathrm{E}-04$ & $1.095 \mathrm{E}+11$ & $1.4340 \mathrm{E}+00$ & $2.412 \mathrm{E}-04$ & $1.108 \mathrm{E}+11$ & 0.001 \\
\hline $1 s 2 p^{1} P_{1}^{o}$ & $1 s 6 f^{3} F_{3}^{o}$ & $1.4460 \mathrm{E}+00$ & $5.807 \mathrm{E}-05$ & $2.646 \mathrm{E}+10$ & $1.4400 \mathrm{E}+00$ & $5.515 \mathrm{E}-05$ & $2.513 E+10$ & 0.001 \\
\hline $1 s 3 p^{3} P_{1}^{o}$ & $1 s 6 f^{3} F_{3}^{o}$ & $3.6921 \mathrm{E}+00$ & $3.684 \mathrm{E}-06$ & $2.575 \mathrm{E}+08$ & $3.6768 \mathrm{E}+00$ & $4.342 \mathrm{E}-06$ & $3.035 \mathrm{E}+08$ & 0.024 \\
\hline $1 s 3 p^{3} P_{2}^{0}$ & $1 s 6 f^{3} F_{3}^{o}$ & $3.8237 \mathrm{E}+00$ & $6.010 \mathrm{E}-06$ & $3.917 \mathrm{E}+08$ & $3.8077 \mathrm{E}+00$ & $6.772 \mathrm{E}-06$ & $4.413 \mathrm{E}+08$ & 0.007 \\
\hline $1 s 3 p^{1} P_{1}^{o}$ & $1 s 6 f^{3} F_{3}^{o}$ & $3.8355 \mathrm{E}+00$ & $1.590 \mathrm{E}-06$ & $1.030 \mathrm{E}+08$ & $3.8195 \mathrm{E}+00$ & $1.275 \mathrm{E}-06$ & $8.258 E+07$ & 0.008 \\
\hline $1 s 4 p^{3} P_{1}^{o}$ & $1 s 6 f^{3} F_{3}^{o}$ & $8.8410 \mathrm{E}+00$ & $3.538 \mathrm{E}-04$ & $4.314 \mathrm{E}+09$ & $8.8032 \mathrm{E}+00$ & $3.517 \mathrm{E}-04$ & $4.288 \mathrm{E}+09$ & 0.000 \\
\hline $1 s 4 p^{3} P_{2}^{o}$ & $1 s 6 f^{3} F_{3}^{o}$ & $9.1585 \mathrm{E}+00$ & $6.227 \mathrm{E}-05$ & $7.074 \mathrm{E}+08$ & $9.1192 \mathrm{E}+00$ & $6.110 \mathrm{E}-05$ & $6.942 \mathrm{E}+08$ & 0.000 \\
\hline $1 s 4 p^{1} P_{1}^{o}$ & $1 s 6 f^{3} F_{3}^{o}$ & $9.1863 \mathrm{E}+00$ & $1.425 \mathrm{E}-05$ & $1.609 \mathrm{E}+08$ & $9.1472 \mathrm{E}+00$ & $1.460 \mathrm{E}-05$ & $1.649 \mathrm{E}+08$ & 0.000 \\
\hline $1 s 4 f^{3} F_{3}^{o}$ & $1 s 6 f^{3} F_{3}^{o}$ & $9.2892 \mathrm{E}+00$ & $1.116 \mathrm{E}-04$ & $1.233 \mathrm{E}+09$ & $9.2496 \mathrm{E}+00$ & $1.113 \mathrm{E}-04$ & $1.229 \mathrm{E}+09$ & 0.001 \\
\hline $1 s 4 f^{3} F_{2}^{o}$ & $1 s 6 f^{3} F_{3}^{o}$ & $9.2914 \mathrm{E}+00$ & $1.854 \mathrm{E}-05$ & $2.047 \mathrm{E}+08$ & $9.2518 \mathrm{E}+00$ & $1.857 \mathrm{E}-05$ & $2.050 \mathrm{E}+08$ & 0.001 \\
\hline $1 s 4 f^{3} F_{4}^{o}$ & $1 s 6 f^{3} F_{3}^{o}$ & $9.3423 \mathrm{E}+00$ & $1.905 \mathrm{E}-05$ & $2.080 \mathrm{E}+08$ & $9.3023 \mathrm{E}+00$ & $1.898 \mathrm{E}-05$ & $2.072 \mathrm{E}+08$ & 0.001 \\
\hline $1 s 4 f^{1} F_{3}^{o}$ & $1 s 6 f^{3} F_{3}^{o}$ & $9.3440 \mathrm{E}+00$ & $2.289 \mathrm{E}-06$ & $2.499 \mathrm{E}+07$ & $9.3039 E+00$ & $2.187 \mathrm{E}-06$ & $2.387 \mathrm{E}+07$ & 0.001 \\
\hline
\end{tabular}


Table 4 (continued)

\begin{tabular}{|c|c|c|c|c|c|c|c|c|}
\hline \multirow[t]{2}{*}{ Lower } & \multirow[t]{2}{*}{ Upper } & \multicolumn{3}{|l|}{ GRASP2K } & \multicolumn{3}{|l|}{ FAC } & \multirow[t]{2}{*}{$d T$} \\
\hline & & $\lambda(\AA)$ & $g f$ & $A\left(\mathrm{~s}^{-1}\right)$ & $\lambda(\AA)$ & $g f$ & $A\left(\mathrm{~s}^{-1}\right)$ & \\
\hline $1 s 5 p^{3} P_{1}^{o}$ & $1 s 6 f^{3} F_{3}^{o}$ & $2.4571 \mathrm{E}+01$ & $2.580 \mathrm{E}-03$ & $4.072 \mathrm{E}+09$ & $2.4463 \mathrm{E}+01$ & $2.587 \mathrm{E}-03$ & $4.085 \mathrm{E}+09$ & 0.000 \\
\hline $1 s 5 p^{3} P_{2}^{o}$ & $1 s 6 f^{3} F_{3}^{o}$ & $2.5841 \mathrm{E}+01$ & $6.351 \mathrm{E}-04$ & $9.063 \mathrm{E}+08$ & $2.5726 \mathrm{E}+01$ & $6.431 \mathrm{E}-04$ & $9.181 \mathrm{E}+08$ & 0.000 \\
\hline $1 s 5 p^{1} P_{1}^{o}$ & $1 s 6 f^{3} F_{3}^{o}$ & $2.5954 \mathrm{E}+01$ & $1.498 \mathrm{E}-04$ & $2.119 \mathrm{E}+08$ & $2.5839 \mathrm{E}+01$ & $1.432 \mathrm{E}-04$ & $2.027 \mathrm{E}+08$ & 0.000 \\
\hline $1 s 5 f^{3} F_{3}^{o}$ & $1 s 6 f^{3} F_{3}^{o}$ & $2.6377 \mathrm{E}+01$ & $6.346 \mathrm{E}-04$ & $8.692 \mathrm{E}+08$ & $2.6263 \mathrm{E}+01$ & $6.351 \mathrm{E}-04$ & $8.700 \mathrm{E}+08$ & 0.000 \\
\hline $1 s 5 f^{3} F_{2}^{o}$ & $1 s 6 f^{3} F_{3}^{o}$ & $2.6386 \mathrm{E}+01$ & $1.056 \mathrm{E}-04$ & $1.445 E+08$ & $2.6272 \mathrm{E}+01$ & $1.060 \mathrm{E}-04$ & $1.451 \mathrm{E}+08$ & 0.000 \\
\hline $1 s 5 f^{3} F_{4}^{o}$ & $1 s 6 f^{3} F_{3}^{0}$ & $2.6597 \mathrm{E}+01$ & $1.106 \mathrm{E}-04$ & $1.490 \mathrm{E}+08$ & $2.6482 \mathrm{E}+01$ & $1.110 \mathrm{E}-04$ & $1.495 \mathrm{E}+08$ & 0.000 \\
\hline $1 s 5 f^{1} F_{3}^{o}$ & $1 s 6 f^{3} F_{3}^{o}$ & $2.6604 \mathrm{E}+01$ & $1.332 \mathrm{E}-05$ & $1.793 E+07$ & $2.6488 \mathrm{E}+01$ & $1.282 \mathrm{E}-05$ & $1.727 \mathrm{E}+07$ & 0.000 \\
\hline $1 s 6 p^{3} P_{1}^{o}$ & $1 s 6 f^{3} F_{3}^{o}$ & $6.2157 \mathrm{E}+02$ & $8.428 \mathrm{E}-07$ & $2.079 E+03$ & $6.1517 \mathrm{E}+02$ & $8.540 \mathrm{E}-07$ & $2.132 \mathrm{E}+03$ & 0.002 \\
\hline $1 s 6 p^{3} P_{2}^{o}$ & $1 s 6 f^{3} F_{3}^{o}$ & $2.1987 \mathrm{E}+03$ & $4.683 \mathrm{E}-09$ & $9.231 \mathrm{E}-01$ & $2.1353 \mathrm{E}+03$ & $5.072 \mathrm{E}-09$ & $1.051 \mathrm{E}+00$ & 0.014 \\
\hline $1 s 6 p{ }^{1} P_{1}^{o}$ & $1 s 6 f^{3} F_{3}^{o}$ & $2.7901 \mathrm{E}+03$ & $5.400 \mathrm{E}-10$ & $6.610 \mathrm{E}-02$ & & & & 0.014 \\
\hline $1 s 2 p^{3} P_{1}^{o}$ & $1 s 6 f^{3} F_{2}^{o}$ & $1.3779 \mathrm{E}+00$ & $2.777 \mathrm{E}-04$ & $1.951 \mathrm{E}+11$ & $1.3722 \mathrm{E}+00$ & $2.854 \mathrm{E}-04$ & $2.005 E+11$ & 0.001 \\
\hline $1 s 2 p^{3} P_{0}^{o}$ & $1 s 6 f^{3} F_{2}^{o}$ & $1.3788 \mathrm{E}+00$ & $3.955 \mathrm{E}-04$ & $2.775 E+11$ & $1.3730 \mathrm{E}+00$ & $4.024 \mathrm{E}-04$ & $2.823 \mathrm{E}+11$ & 0.000 \\
\hline $1 s 2 p^{3} P_{2}^{o}$ & $1 s 6 f^{3} F_{2}^{o}$ & $1.4401 \mathrm{E}+00$ & $5.965 \mathrm{E}-05$ & $3.838 \mathrm{E}+10$ & $1.4340 \mathrm{E}+00$ & $6.023 \mathrm{E}-05$ & $3.874 \mathrm{E}+10$ & 0.002 \\
\hline $1 s 2 p^{1} P_{1}^{o}$ & $1 s 6 f^{3} F_{2}^{o}$ & $1.4459 \mathrm{E}+00$ & $1.248 \mathrm{E}-04$ & $7.961 \mathrm{E}+10$ & $1.4400 \mathrm{E}+00$ & $1.244 \mathrm{E}-04$ & $7.940 \mathrm{E}+10$ & 0.002 \\
\hline $1 s 3 p^{3} P_{1}^{o}$ & $1 s 6 f^{3} F_{2}^{o}$ & $3.6920 \mathrm{E}+00$ & $1.087 \mathrm{E}-06$ & $1.064 \mathrm{E}+08$ & $3.6768 \mathrm{E}+00$ & $1.477 \mathrm{E}-06$ & $1.445 \mathrm{E}+08$ & 0.029 \\
\hline $1 s 3 p^{3} P_{0}^{o}$ & $1 s 6 f^{3} F_{2}^{o}$ & $3.6937 \mathrm{E}+00$ & $1.645 \mathrm{E}-06$ & $1.609 \mathrm{E}+08$ & $3.6784 \mathrm{E}+00$ & $1.937 \mathrm{E}-06$ & $1.893 \mathrm{E}+08$ & 0.023 \\
\hline $1 s 3 p^{3} P_{2}^{o}$ & $1 s 6 f^{3} F_{2}^{o}$ & $3.8236 \mathrm{E}+00$ & $1.498 \mathrm{E}-06$ & $1.367 \mathrm{E}+08$ & $3.8077 \mathrm{E}+00$ & $1.691 \mathrm{E}-06$ & $1.543 \mathrm{E}+08$ & 0.012 \\
\hline $1 s 3 p{ }^{1} P_{1}^{o}$ & $1 s 6 f^{3} F_{2}^{o}$ & $3.8353 \mathrm{E}+00$ & $3.469 \mathrm{E}-06$ & $3.146 E+08$ & $3.8195 \mathrm{E}+00$ & $3.746 \mathrm{E}-06$ & $3.396 \mathrm{E}+08$ & 0.003 \\
\hline $1 s 4 p^{3} P_{1}^{o}$ & $1 s 6 f^{3} F_{2}^{o}$ & $8.8404 \mathrm{E}+00$ & $1.083 \mathrm{E}-04$ & $1.849 \mathrm{E}+09$ & $8.8032 \mathrm{E}+00$ & $1.065 \mathrm{E}-04$ & $1.819 \mathrm{E}+09$ & 0.001 \\
\hline $1 s 4 p^{3} P_{0}^{o}$ & $1 s 6 f^{3} F_{2}^{o}$ & $8.8444 \mathrm{E}+00$ & $1.537 \mathrm{E}-04$ & $2.622 E+09$ & $8.8071 \mathrm{E}+00$ & $1.527 \mathrm{E}-04$ & $2.605 \mathrm{E}+09$ & 0.000 \\
\hline $1 s 4 p^{3} P_{2}^{o}$ & $1 s 6 f^{3} F_{2}^{o}$ & $9.1578 \mathrm{E}+00$ & $1.565 \mathrm{E}-05$ & $2.489 E+08$ & $9.1192 \mathrm{E}+00$ & $1.529 \mathrm{E}-05$ & $2.432 \mathrm{E}+08$ & 0.001 \\
\hline $1 s 4 p^{1} P_{1}^{o}$ & $1 s 6 f^{3} F_{2}^{o}$ & $9.1856 \mathrm{E}+00$ & $3.180 \mathrm{E}-05$ & $5.028 \mathrm{E}+08$ & $9.1472 \mathrm{E}+00$ & $3.119 \mathrm{E}-05$ & $4.931 \mathrm{E}+08$ & 0.000 \\
\hline $1 s 4 f^{3} F_{3}^{o}$ & $1 s 6 f^{3} F_{2}^{o}$ & $9.2886 \mathrm{E}+00$ & $1.855 \mathrm{E}-05$ & $2.868 \mathrm{E}+08$ & $9.2496 \mathrm{E}+00$ & $1.847 \mathrm{E}-05$ & $2.856 \mathrm{E}+08$ & 0.001 \\
\hline $1 s 4 f^{3} F_{2}^{o}$ & $1 s 6 f^{3} F_{2}^{o}$ & $9.2908 \mathrm{E}+00$ & $7.429 \mathrm{E}-05$ & $1.148 \mathrm{E}+09$ & $9.2518 \mathrm{E}+00$ & $7.409 \mathrm{E}-05$ & $1.145 \mathrm{E}+09$ & 0.000 \\
\hline $1 s 4 f^{3} F_{4}^{o}$ & $1 s 6 f^{3} F_{2}^{o}$ & $9.3416 \mathrm{E}+00$ & $1.524 \mathrm{E}-06$ & $2.329 \mathrm{E}+07$ & $9.3023 \mathrm{E}+00$ & $1.517 \mathrm{E}-06$ & $2.318 \mathrm{E}+07$ & 0.001 \\
\hline $1 s 4 f^{1} F_{3}^{o}$ & $1 s 6 f^{3} F_{2}^{o}$ & $9.3433 \mathrm{E}+00$ & $1.384 \mathrm{E}-05$ & $2.116 E+08$ & $9.3039 \mathrm{E}+00$ & $1.371 \mathrm{E}-05$ & $2.095 \mathrm{E}+08$ & 0.001 \\
\hline $1 s 5 p^{3} P_{1}^{o}$ & $1 s 6 f^{3} F_{2}^{o}$ & $2.4566 \mathrm{E}+01$ & $7.873 \mathrm{E}-04$ & $1.740 \mathrm{E}+09$ & $2.4463 \mathrm{E}+01$ & $7.936 \mathrm{E}-04$ & $1.754 \mathrm{E}+09$ & 0.000 \\
\hline $1 s 5 p^{3} P_{0}^{o}$ & $1 s 6 f^{3} F_{2}^{o}$ & $2.4582 \mathrm{E}+01$ & $1.123 \mathrm{E}-03$ & $2.479 \mathrm{E}+09$ & $2.4477 \mathrm{E}+01$ & $1.126 \mathrm{E}-03$ & $2.486 \mathrm{E}+09$ & 0.000 \\
\hline $1 s 5 p^{3} P_{2}^{o}$ & $1 s 6 f^{3} F_{2}^{o}$ & $2.5836 \mathrm{E}+01$ & $1.593 \mathrm{E}-04$ & $3.185 E+08$ & $2.5726 \mathrm{E}+01$ & $1.609 \mathrm{E}-04$ & $3.216 \mathrm{E}+08$ & 0.000 \\
\hline $1 s 5 p^{1} P_{1}^{o}$ & $1 s 6 f^{3} F_{2}^{o}$ & $2.5948 \mathrm{E}+01$ & $3.343 \mathrm{E}-04$ & $6.624 \mathrm{E}+08$ & $2.5839 \mathrm{E}+01$ & $3.349 \mathrm{E}-04$ & $6.635 \mathrm{E}+08$ & 0.000 \\
\hline $1 s 5 f^{3} F_{3}^{o}$ & $1 s 6 f^{3} F_{2}^{o}$ & $2.6372 \mathrm{E}+01$ & $1.053 \mathrm{E}-04$ & $2.020 \mathrm{E}+08$ & $2.6263 \mathrm{E}+01$ & $1.054 \mathrm{E}-04$ & $2.022 \mathrm{E}+08$ & 0.000 \\
\hline $1 s 5 f^{3} F_{2}^{o}$ & $1 s 6 f^{3} F_{2}^{o}$ & $2.6381 \mathrm{E}+01$ & $4.223 \mathrm{E}-04$ & $8.094 \mathrm{E}+08$ & $2.6272 \mathrm{E}+01$ & $4.226 \mathrm{E}-04$ & $8.098 \mathrm{E}+08$ & 0.000 \\
\hline $1 s 5 f^{3} F_{4}^{o}$ & $1 s 6 f^{3} F_{2}^{o}$ & $2.6592 \mathrm{E}+01$ & $8.828 \mathrm{E}-06$ & $1.665 \mathrm{E}+07$ & $2.6482 \mathrm{E}+01$ & $8.872 \mathrm{E}-06$ & $1.673 \mathrm{E}+07$ & 0.000 \\
\hline $1 s 5 f^{1} F_{3}^{o}$ & $1 s 6 f^{3} F_{2}^{o}$ & $2.6599 \mathrm{E}+01$ & $8.031 \mathrm{E}-05$ & $1.514 \mathrm{E}+08$ & $2.6489 \mathrm{E}+01$ & $8.015 \mathrm{E}-05$ & $1.511 \mathrm{E}+08$ & 0.000 \\
\hline $1 s 6 p^{3} P_{1}^{o}$ & $1 s 6 f^{3} F_{2}^{o}$ & $6.1864 \mathrm{E}+02$ & $2.611 \mathrm{E}-07$ & $9.100 \mathrm{E}+02$ & $6.1520 \mathrm{E}+02$ & $2.653 \mathrm{E}-07$ & $9.272 \mathrm{E}+02$ & 0.001 \\
\hline $1 s 6 p^{3} P_{0}^{0}$ & $1 s 6 f^{3} F_{2}^{o}$ & $6.2421 \mathrm{E}+02$ & $3.621 \mathrm{E}-07$ & $1.240 \mathrm{E}+03$ & $6.0122 \mathrm{E}+02$ & $4.004 \mathrm{E}-07$ & $1.465 \mathrm{E}+03$ & 0.001 \\
\hline $1 s 6 p^{3} P_{2}^{o}$ & $1 s 6 f^{3} F_{2}^{o}$ & $2.1625 \mathrm{E}+03$ & $1.236 \mathrm{E}-09$ & $3.526 \mathrm{E}-01$ & $2.1357 \mathrm{E}+03$ & $1.266 \mathrm{E}-09$ & $3.671 \mathrm{E}-01$ & 0.013 \\
\hline $1 s 6 p{ }^{1} P_{1}^{o}$ & $1 s 6 f^{3} F_{2}^{o}$ & $2.7321 \mathrm{E}+03$ & $1.288 \mathrm{E}-09$ & $2.302 \mathrm{E}-01$ & $2.6181 \mathrm{E}+03$ & $1.394 \mathrm{E}-09$ & $2.690 \mathrm{E}-01$ & 0.017 \\
\hline $1 s 6 f^{3} F_{3}^{o}$ & $1 s 6 f^{3} F_{2}^{o}$ & $1.3144 \mathrm{E}+05$ & $1.419 \mathrm{E}-14$ & $1.096 \mathrm{E}-09$ & & & & 0.139 \\
\hline $1 s^{2}{ }^{1} S_{0}$ & $1 s 6 d^{1} D_{2}$ & $3.1620 \mathrm{E}-01$ & $2.010 \mathrm{E}-04$ & $2.681 \mathrm{E}+12$ & $3.1493 \mathrm{E}-01$ & $2.346 \mathrm{E}-04$ & $3.129 E+12$ & 0.002 \\
\hline $1 s 2 s^{3} S_{1}$ & $1 s 6 d^{1} D_{2}$ & $1.3661 \mathrm{E}+00$ & $1.156 \mathrm{E}-05$ & $8.265 E+09$ & $1.3604 \mathrm{E}+00$ & $1.042 \mathrm{E}-05$ & $7.448 \mathrm{E}+09$ & 0.008 \\
\hline $1 s 2 s{ }^{1} S_{0}$ & $1 s 6 d^{1} D_{2}$ & $1.3791 \mathrm{E}+00$ & $1.520 \mathrm{E}-05$ & $1.066 \mathrm{E}+10$ & $1.3734 \mathrm{E}+00$ & $1.583 \mathrm{E}-05$ & $1.110 \mathrm{E}+10$ & 0.002 \\
\hline $1 s 3 s^{3} s_{1}$ & $1 s 6 d^{1} D_{2}$ & $3.6690 \mathrm{E}+00$ & $6.320 \mathrm{E}-05$ & $6.263 \mathrm{E}+09$ & $3.6537 \mathrm{E}+00$ & $6.164 \mathrm{E}-05$ & $6.108 \mathrm{E}+09$ & 0.004 \\
\hline $1 s 3 s^{1} S_{0}$ & $1 s 6 d^{1} D_{2}$ & $3.6936 \mathrm{E}+00$ & $9.420 \mathrm{E}-05$ & $9.212 \mathrm{E}+09$ & $3.6785 \mathrm{E}+00$ & $9.501 \mathrm{E}-05$ & $9.288 \mathrm{E}+09$ & 0.013 \\
\hline $1 s 3 d^{3} D_{2}$ & $1 s 6 d^{1} D_{2}$ & $3.8335 \mathrm{E}+00$ & $4.853 \mathrm{E}-06$ & $4.406 \mathrm{E}+08$ & $3.8174 \mathrm{E}+00$ & $4.999 \mathrm{E}-06$ & $4.537 \mathrm{E}+08$ & 0.001 \\
\hline $1 s 3 d^{3} D_{1}$ & $1 s 6 d^{1} D_{2}$ & $3.8353 \mathrm{E}+00$ & $1.105 \mathrm{E}-05$ & $1.002 \mathrm{E}+09$ & $3.8191 \mathrm{E}+00$ & $1.091 \mathrm{E}-05$ & $9.901 \mathrm{E}+08$ & 0.006 \\
\hline $1 s 3 d^{3} D_{3}$ & $1 s 6 d^{1} D_{2}$ & $3.8770 \mathrm{E}+00$ & $1.211 \mathrm{E}-05$ & $1.075 \mathrm{E}+09$ & $3.8606 \mathrm{E}+00$ & $1.192 \mathrm{E}-05$ & $1.058 \mathrm{E}+09$ & 0.005 \\
\hline $1 s 3 d^{1} D_{2}$ & $1 s 6 d^{1} D_{2}$ & $3.8787 \mathrm{E}+00$ & $4.990 \mathrm{E}-05$ & $4.425 \mathrm{E}+09$ & $3.8623 \mathrm{E}+00$ & $4.949 \mathrm{E}-05$ & $4.388 \mathrm{E}+09$ & 0.006 \\
\hline $1 s 4 s^{3} S_{1}$ & $1 s 6 d^{1} D_{2}$ & $8.7865 \mathrm{E}+00$ & $1.610 \mathrm{E}-04$ & $2.782 E+09$ & $8.7499 \mathrm{E}+00$ & $1.592 \mathrm{E}-04$ & $2.751 \mathrm{E}+09$ & 0.003 \\
\hline $1 s 4 s{ }^{1} S_{0}$ & $1 s 6 d^{1} D_{2}$ & $8.8434 \mathrm{E}+00$ & $2.451 \mathrm{E}-04$ & $4.180 \mathrm{E}+09$ & $8.8071 \mathrm{E}+00$ & $2.460 \mathrm{E}-04$ & $4.195 \mathrm{E}+09$ & 0.007 \\
\hline $1 s 4 d^{3} D_{2}$ & $1 s 6 d^{1} D_{2}$ & $9.1814 \mathrm{E}+00$ & $1.395 \mathrm{E}-05$ & $2.208 \mathrm{E}+08$ & $9.1435 \mathrm{E}+00$ & $1.436 \mathrm{E}-05$ & $2.272 \mathrm{E}+08$ & 0.000 \\
\hline $1 s 4 d^{3} D_{1}$ & $1 s 6 d^{1} D_{2}$ & $9.1855 \mathrm{E}+00$ & $3.215 \mathrm{E}-05$ & $5.084 \mathrm{E}+08$ & $9.1476 \mathrm{E}+00$ & $3.177 \mathrm{E}-05$ & $5.021 \mathrm{E}+08$ & 0.002 \\
\hline $1 s 4 d^{3} D_{3}$ & $1 s 6 d^{1} D_{2}$ & $9.2868 \mathrm{E}+00$ & $3.602 \mathrm{E}-05$ & $5.571 \mathrm{E}+08$ & $9.2482 \mathrm{E}+00$ & $3.553 \mathrm{E}-05$ & $5.496 \mathrm{E}+08$ & 0.002 \\
\hline $1 s 4 d{ }^{1} D_{2}$ & $1 s 6 d^{1} D_{2}$ & $9.2911 \mathrm{E}+00$ & $1.477 \mathrm{E}-04$ & $2.282 E+09$ & $9.2526 \mathrm{E}+00$ & $1.477 \mathrm{E}-04$ & $2.282 \mathrm{E}+09$ & 0.002 \\
\hline $1 s 5 s^{3} S_{1}$ & $1 s 6 d^{1} D_{2}$ & $2.4357 \mathrm{E}+01$ & $5.073 \mathrm{E}-04$ & $1.141 \mathrm{E}+09$ & $2.4255 \mathrm{E}+01$ & $5.096 \mathrm{E}-04$ & $1.145 \mathrm{E}+09$ & 0.003 \\
\hline $1 s 5 s{ }^{1} S_{0}$ & $1 s 6 d^{1} D_{2}$ & $2.4576 \mathrm{E}+01$ & $7.897 \mathrm{E}-04$ & $1.744 \mathrm{E}+09$ & $2.4475 \mathrm{E}+01$ & $7.829 \mathrm{E}-04$ & $1.728 \mathrm{E}+09$ & 0.001 \\
\hline $1 s 5 d^{3} D_{2}$ & $1 s 6 d^{1} D_{2}$ & $2.5931 \mathrm{E}+01$ & $4.900 \mathrm{E}-05$ & $9.721 \mathrm{E}+07$ & $2.5828 \mathrm{E}+01$ & $5.056 \mathrm{E}-05$ & $1.002 \mathrm{E}+08$ & 0.000 \\
\hline $1 s 5 d^{3} D_{1}$ & $1 s 6 d^{1} D_{2}$ & $2.5947 \mathrm{E}+01$ & $1.137 \mathrm{E}-04$ & $2.253 \mathrm{E}+08$ & $2.5844 \mathrm{E}+01$ & $1.121 \mathrm{E}-04$ & $2.221 \mathrm{E}+08$ & 0.000 \\
\hline $1 s 5 d^{3} D_{3}$ & $1 s 6 d^{1} D_{2}$ & $2.6363 \mathrm{E}+01$ & $1.317 \mathrm{E}-04$ & $2.529 E+08$ & $2.6257 \mathrm{E}+01$ & $1.303 \mathrm{E}-04$ & $2.501 \mathrm{E}+08$ & 0.000 \\
\hline $1 s 5 d{ }^{1} D_{2}$ & $1 s 6 d^{1} D_{2}$ & $2.6382 \mathrm{E}+01$ & $5.402 \mathrm{E}-04$ & $1.035 E+09$ & $2.6276 \mathrm{E}+01$ & $5.422 \mathrm{E}-04$ & $1.038 \mathrm{E}+09$ & 0.000 \\
\hline $1 s 5 g^{3} G_{4}$ & $1 s 6 d^{1} D_{2}$ & $2.6591 \mathrm{E}+01$ & $5.584 \mathrm{E}-07$ & $1.054 \mathrm{E}+06$ & $2.6478 \mathrm{E}+01$ & $6.309 \mathrm{E}-07$ & $1.190 \mathrm{E}+06$ & 0.000 \\
\hline $1 s 5 g^{3} G_{3}$ & $1 s 6 d^{1} D_{2}$ & $2.6597 \mathrm{E}+01$ & $4.150 \mathrm{E}-06$ & $7.826 \mathrm{E}+06$ & $2.6484 \mathrm{E}+01$ & $4.105 \mathrm{E}-06$ & $7.742 \mathrm{E}+06$ & 0.000 \\
\hline $1 s 5 g{ }^{1} G_{4}$ & $1 s 6 d^{1} D_{2}$ & $2.6728 \mathrm{E}+01$ & $5.969 \mathrm{E}-05$ & $1.115 \mathrm{E}+08$ & $2.6615 \mathrm{E}+01$ & $5.978 \mathrm{E}-05$ & $1.116 \mathrm{E}+08$ & 0.000 \\
\hline $1 s 6 s^{3} S_{1}$ & $1 s 6 d^{1} D_{2}$ & $5.4990 \mathrm{E}+02$ & $4.398 \mathrm{E}-07$ & $1.940 E+03$ & $5.4127 \mathrm{E}+02$ & $4.505 \mathrm{E}-07$ & $2.034 \mathrm{E}+03$ & 0.014 \\
\hline $1 s 6 s{ }^{1} S_{0}$ & $1 s 6 d^{1} D_{2}$ & $6.2157 \mathrm{E}+02$ & $4.706 \mathrm{E}-07$ & $1.625 \mathrm{E}+03$ & $5.9948 \mathrm{E}+02$ & $5.219 \mathrm{E}-07$ & $1.921 \mathrm{E}+03$ & 0.040 \\
\hline $1 s 6 d^{3} D_{2}$ & $1 s 6 d^{1} D_{2}$ & $2.6222 \mathrm{E}+03$ & $8.371 \mathrm{E}-10$ & $1.624 \mathrm{E}-01$ & & & & 0.019 \\
\hline $1 s 6 d^{3} D_{1}$ & $1 s 6 d^{1} D_{2}$ & $2.7221 \mathrm{E}+03$ & $1.737 \mathrm{E}-09$ & $3.127 \mathrm{E}-01$ & & & & 0.001 \\
\hline $1 s 6 d^{3} D_{3}$ & $1 s 6 d^{1} D_{2}$ & $6.4120 \mathrm{E}+04$ & $1.525 \mathrm{E}-13$ & $4.950 \mathrm{E}-08$ & & & & 0.345 \\
\hline $1 s 2 p^{3} P_{2}^{o}$ & $1 s 6 f^{3} F_{4}^{o}$ & $1.4397 \mathrm{E}+00$ & $1.592 \mathrm{E}-03$ & $5.693 \mathrm{E}+11$ & $1.4336 \mathrm{E}+00$ & $1.671 \mathrm{E}-03$ & $5.974 \mathrm{E}+11$ & 0.001 \\
\hline $1 s 3 p^{3} P_{2}^{o}$ & $1 s 6 f^{3} F_{4}^{0}$ & $3.8211 \mathrm{E}+00$ & $3.236 \mathrm{E}-05$ & $1.643 \mathrm{E}+09$ & $3.8051 \mathrm{E}+00$ & $3.745 \mathrm{E}-05$ & $1.901 \mathrm{E}+09$ & 0.010 \\
\hline $1 s 4 p^{3} P_{2}^{o}$ & $1 s 6 f^{3} F_{4}^{0}$ & $9.1433 \mathrm{E}+00$ & $4.491 \mathrm{E}-04$ & $3.981 \mathrm{E}+09$ & $9.1041 \mathrm{E}+00$ & $4.417 \mathrm{E}-04$ & $3.916 \mathrm{E}+09$ & 0.000 \\
\hline
\end{tabular}


Table 4 (continued)

\begin{tabular}{|c|c|c|c|c|c|c|c|c|}
\hline \multirow[t]{2}{*}{ Lower } & \multirow[t]{2}{*}{ Upper } & \multicolumn{3}{|l|}{ GRASP2K } & \multicolumn{3}{|l|}{ FAC } & \multirow[t]{2}{*}{$d T$} \\
\hline & & $\lambda(\AA)$ & $g f$ & $A\left(\mathrm{~s}^{-1}\right)$ & $\lambda(\AA)$ & $g f$ & $A\left(\mathrm{~s}^{-1}\right)$ & \\
\hline $1 s 4 f^{3} F_{3}^{o}$ & $1 s 6 f^{3} F_{4}^{o}$ & $9.2736 \mathrm{E}+00$ & $1.951 \mathrm{E}-05$ & $1.681 \mathrm{E}+08$ & $9.2341 \mathrm{E}+00$ & $1.958 \mathrm{E}-05$ & $1.688 \mathrm{E}+08$ & 0.001 \\
\hline $1 s 4 f^{3} F_{2}^{o}$ & $1 s 6 f^{3} F_{4}^{0}$ & $9.2758 \mathrm{E}+00$ & $1.549 \mathrm{E}-06$ & $1.335 \mathrm{E}+07$ & $9.2363 \mathrm{E}+00$ & $1.553 \mathrm{E}-06$ & $1.338 \mathrm{E}+07$ & 0.001 \\
\hline $1 s 4 f^{3} F_{4}^{o}$ & $1 s 6 f^{3} F_{4}^{0}$ & $9.3265 \mathrm{E}+00$ & $1.596 \mathrm{E}-04$ & $1.360 \mathrm{E}+09$ & $9.2866 \mathrm{E}+00$ & $1.593 \mathrm{E}-04$ & $1.358 \mathrm{E}+09$ & 0.000 \\
\hline $1 s 4 f^{1} F_{3}^{o}$ & $1 s 6 f^{3} F_{4}^{o}$ & $9.3281 \mathrm{E}+00$ & $1.458 \mathrm{E}-05$ & $1.242 \mathrm{E}+08$ & $9.2883 \mathrm{E}+00$ & $1.455 \mathrm{E}-05$ & $1.239 \mathrm{E}+08$ & 0.000 \\
\hline $1 s 5 p^{3} P_{2}^{o}$ & $1 s 6 f^{3} F_{4}^{0}$ & $2.5720 \mathrm{E}+01$ & $4.305 \mathrm{E}-03$ & $4.823 E+09$ & $2.5607 \mathrm{E}+01$ & $4.350 \mathrm{E}-03$ & $4.875 E+09$ & 0.000 \\
\hline $1 s 5 f^{3} F_{3}^{o}$ & $1 s 6 f^{3} F_{4}^{o}$ & $2.6252 \mathrm{E}+01$ & $1.086 \mathrm{E}-04$ & $1.168 \mathrm{E}+08$ & $2.6139 \mathrm{E}+01$ & $1.090 \mathrm{E}-04$ & $1.172 \mathrm{E}+08$ & 0.000 \\
\hline $1 s 5 f^{3} F_{2}^{o}$ & $1 s 6 f^{3} F_{4}^{0}$ & $2.6261 \mathrm{E}+01$ & $8.548 \mathrm{E}-06$ & $9.187 \mathrm{E}+06$ & $2.6148 \mathrm{E}+01$ & $8.573 E-06$ & $9.214 \mathrm{E}+06$ & 0.000 \\
\hline $1 s 5 f^{3} F_{4}^{o}$ & $1 s 6 f^{3} F_{4}^{o}$ & $2.6469 \mathrm{E}+01$ & $9.072 \mathrm{E}-04$ & $9.597 \mathrm{E}+08$ & $2.6355 E+01$ & $9.087 \mathrm{E}-04$ & $9.613 E+08$ & 0.000 \\
\hline $1 s 5 f^{1} F_{3}^{o}$ & $1 s 6 f^{3} F_{4}^{0}$ & $2.6476 \mathrm{E}+01$ & $8.289 \mathrm{E}-05$ & $8.763 E+07$ & $2.6362 \mathrm{E}+01$ & $8.292 \mathrm{E}-05$ & $8.768 E+07$ & 0.000 \\
\hline $1 s 6 p^{3} P_{2}^{o}$ & $1 s 6 f^{3} F_{4}^{o}$ & $1.5714 \mathrm{E}+03$ & $8.788 \mathrm{E}-08$ & $2.638 \mathrm{E}+01$ & $1.5399 \mathrm{E}+03$ & $9.216 \mathrm{E}-08$ & $2.856 \mathrm{E}+01$ & 0.009 \\
\hline $1 s 6 f^{3} F_{3}^{o}$ & $1 s 6 f^{3} F_{4}^{o}$ & $5.5078 \mathrm{E}+03$ & $2.014 \mathrm{E}-10$ & $4.921 \mathrm{E}-03$ & $5.5226 \mathrm{E}+03$ & $1.981 \mathrm{E}-10$ & $4.774 \mathrm{E}-03$ & 0.002 \\
\hline $1 s 6 f^{3} F_{2}^{o}$ & $1 s 6 f^{3} F_{4}^{0}$ & $5.7487 \mathrm{E}+03$ & $1.406 \mathrm{E}-11$ & $3.153 E-04$ & & & & 0.005 \\
\hline $1 s 3 d^{3} D_{2}$ & $1 s 6 g^{3} G_{4}$ & $3.8309 \mathrm{E}+00$ & $3.357 \mathrm{E}-03$ & $1.695 E+11$ & $3.8148 \mathrm{E}+00$ & $3.386 \mathrm{E}-03$ & $1.709 \mathrm{E}+11$ & 0.000 \\
\hline $1 s 3 d^{3} D_{3}$ & $1 s 6 g^{3} G_{4}$ & $3.8744 \mathrm{E}+00$ & $3.474 \mathrm{E}-04$ & $1.715 E+10$ & $3.8580 \mathrm{E}+00$ & $3.479 \mathrm{E}-04$ & $1.717 \mathrm{E}+10$ & 0.000 \\
\hline $1 s 3 d{ }^{1} D_{2}$ & $1 s 6 g^{3} G_{4}$ & $3.8761 \mathrm{E}+00$ & $3.116 \mathrm{E}-05$ & $1.537 \mathrm{E}+09$ & $3.8597 \mathrm{E}+00$ & $3.004 \mathrm{E}-05$ & $1.481 \mathrm{E}+09$ & 0.001 \\
\hline $1 s 4 d^{3} D_{2}$ & $1 s 6 g^{3} G_{4}$ & $9.1668 \mathrm{E}+00$ & $7.908 \mathrm{E}-05$ & $6.975 E+08$ & $9.1289 \mathrm{E}+00$ & $8.154 \mathrm{E}-05$ & $7.190 \mathrm{E}+08$ & 0.001 \\
\hline $1 s 4 d^{3} D_{3}$ & $1 s 6 g{ }^{3} G_{4}$ & $9.2719 \mathrm{E}+00$ & $1.137 \mathrm{E}-05$ & $9.800 \mathrm{E}+07$ & $9.2332 \mathrm{E}+00$ & $1.167 \mathrm{E}-05$ & $1.006 \mathrm{E}+08$ & 0.001 \\
\hline $1 s 4 d{ }^{1} D_{2}$ & $1 s 6 g^{3} G_{4}$ & $9.2762 \mathrm{E}+00$ & $1.073 \mathrm{E}-06$ & $9.238 \mathrm{E}+06$ & $9.2375 E+00$ & $1.032 \mathrm{E}-06$ & $8.892 E+06$ & 0.003 \\
\hline $1 s 5 d^{3} D_{2}$ & $1 s 6 g^{3} G_{4}$ & $2.5815 \mathrm{E}+01$ & $5.070 \mathrm{E}-03$ & $5.639 \mathrm{E}+09$ & $2.5711 \mathrm{E}+01$ & $5.076 \mathrm{E}-03$ & $5.642 E+09$ & 0.000 \\
\hline $1 s 5 d^{3} D_{3}$ & $1 s 6 g^{3} G_{4}$ & $2.6244 \mathrm{E}+01$ & $5.114 \mathrm{E}-04$ & $5.504 \mathrm{E}+08$ & $2.6137 \mathrm{E}+01$ & $5.132 \mathrm{E}-04$ & $5.520 \mathrm{E}+08$ & 0.000 \\
\hline $1 s 5 d{ }^{1} D_{2}$ & $1 s 6 g^{3} G_{4}$ & $2.6262 \mathrm{E}+01$ & $4.852 \mathrm{E}-05$ & $5.214 \mathrm{E}+07$ & $2.6155 \mathrm{E}+01$ & $4.698 \mathrm{E}-05$ & $5.047 \mathrm{E}+07$ & 0.000 \\
\hline $1 s 5 g^{3} G_{4}$ & $1 s 6 g^{3} G_{4}$ & $2.6470 \mathrm{E}+01$ & $5.388 \mathrm{E}-04$ & $5.699 \mathrm{E}+08$ & $2.6356 \mathrm{E}+01$ & $5.393 E-04$ & $5.705 E+08$ & 0.000 \\
\hline $1 s 5 g^{3} G_{3}$ & $1 s 6 g^{3} G_{4}$ & $2.6475 E+01$ & $4.880 \mathrm{E}-05$ & $5.160 \mathrm{E}+07$ & $2.6361 \mathrm{E}+01$ & $4.899 \mathrm{E}-05$ & $5.180 \mathrm{E}+07$ & 0.000 \\
\hline $1 s 5 g^{3} G_{5}$ & $1 s 6 g^{3} G_{4}$ & $2.6601 \mathrm{E}+01$ & $4.984 \mathrm{E}-05$ & $5.220 \mathrm{E}+07$ & $2.6487 \mathrm{E}+01$ & $4.993 \mathrm{E}-05$ & $5.231 E+07$ & 0.000 \\
\hline $1 s 5 g{ }^{1} G_{4}$ & $1 s 6 g^{3} G_{4}$ & $2.6605 E+01$ & $3.575 \mathrm{E}-06$ & $3.744 \mathrm{E}+06$ & $2.6491 \mathrm{E}+01$ & $3.402 \mathrm{E}-06$ & $3.562 E+06$ & 0.000 \\
\hline $1 s 6 d^{3} D_{2}$ & $1 s 6 g^{3} G_{4}$ & $1.8045 \mathrm{E}+03$ & $3.645 \mathrm{E}-08$ & $8.298 E+00$ & $1.8335 \mathrm{E}+03$ & $3.427 \mathrm{E}-08$ & $7.491 \mathrm{E}+00$ & 0.002 \\
\hline $1 s 6 d^{3} D_{3}$ & $1 s 6 g^{3} G_{4}$ & $5.3075 \mathrm{E}+03$ & $1.458 \mathrm{E}-10$ & $3.836 \mathrm{E}-03$ & $5.5194 \mathrm{E}+03$ & $1.284 \mathrm{E}-10$ & $3.097 \mathrm{E}-03$ & 0.000 \\
\hline $1 s 6 d^{1} D_{2}$ & $1 s 6 g^{3} G_{4}$ & $5.7864 \mathrm{E}+03$ & $1.077 \mathrm{E}-11$ & $2.384 \mathrm{E}-04$ & & & & 0.038 \\
\hline $1 s 2 s^{3} S_{1}$ & $1 s 6 g{ }^{3} G_{3}$ & $1.3658 \mathrm{E}+00$ & $5.739 \mathrm{E}-11$ & $2.931 \mathrm{E}+04$ & & & & 0.903 \\
\hline $1 s 3 s^{3} S_{1}$ & $1 s 6 g^{3} G_{3}$ & $3.6666 \mathrm{E}+00$ & $3.747 \mathrm{E}-09$ & $2.656 \mathrm{E}+05$ & & & & 0.102 \\
\hline $1 s 3 d^{3} D_{2}$ & $1 s 6 g{ }^{3} G_{3}$ & $3.8309 \mathrm{E}+00$ & $3.753 \mathrm{E}-04$ & $2.437 \mathrm{E}+10$ & $3.8148 \mathrm{E}+00$ & $3.787 E-04$ & $2.459 \mathrm{E}+10$ & 0.000 \\
\hline $1 s 3 d^{3} D_{1}$ & $1 s 6 g^{3} G_{3}$ & $3.8326 \mathrm{E}+00$ & $2.240 \mathrm{E}-03$ & $1.453 \mathrm{E}+11$ & $3.8166 \mathrm{E}+00$ & $2.255 \mathrm{E}-03$ & $1.463 \mathrm{E}+11$ & 0.000 \\
\hline $1 s 3 d^{3} D_{3}$ & $1 s 6 g^{3} G_{3}$ & $3.8743 \mathrm{E}+00$ & $4.181 \mathrm{E}-05$ & $2.654 \mathrm{E}+09$ & $3.8580 \mathrm{E}+00$ & $4.175 \mathrm{E}-05$ & $2.650 \mathrm{E}+09$ & 0.000 \\
\hline $1 s 3 d^{1} D_{2}$ & $1 s 6 g^{3} G_{3}$ & $3.8760 \mathrm{E}+00$ & $2.490 \mathrm{E}-04$ & $1.579 \mathrm{E}+10$ & $3.8597 \mathrm{E}+00$ & $2.479 \mathrm{E}-04$ & $1.572 \mathrm{E}+10$ & 0.000 \\
\hline $1 s 4 s^{3} S_{1}$ & $1 s 6 g^{3} G_{3}$ & $8.7728 \mathrm{E}+00$ & $1.855 \mathrm{E}-11$ & $2.297 \mathrm{E}+02$ & & & & 0.573 \\
\hline $1 s 4 d^{3} D_{2}$ & $1 s 6 g^{3} G_{3}$ & $9.1665 E+00$ & $8.833 \mathrm{E}-06$ & $1.002 E+08$ & $9.1289 \mathrm{E}+00$ & $9.173 E-06$ & $1.039 \mathrm{E}+08$ & 0.000 \\
\hline $1 s 4 d^{3} D_{1}$ & $1 s 6 g^{3} G_{3}$ & $9.1706 \mathrm{E}+00$ & $5.325 \mathrm{E}-05$ & $6.034 \mathrm{E}+08$ & $9.1329 \mathrm{E}+00$ & $5.436 \mathrm{E}-05$ & $6.157 E+08$ & 0.002 \\
\hline $1 s 4 d^{3} D_{3}$ & $1 s 6 g^{3} G_{3}$ & $9.2715 \mathrm{E}+00$ & $1.365 \mathrm{E}-06$ & $1.513 \mathrm{E}+07$ & $9.2332 \mathrm{E}+00$ & $1.401 \mathrm{E}-06$ & $1.553 \mathrm{E}+07$ & 0.000 \\
\hline $1 s 4 d^{1} D_{2}$ & $1 s 6 g{ }^{3} G_{3}$ & $9.2759 \mathrm{E}+00$ & $8.174 \mathrm{E}-06$ & $9.052 E+07$ & $9.2375 E+00$ & $8.299 \mathrm{E}-06$ & $9.189 E+07$ & 0.001 \\
\hline $1 s 5 s^{3} s_{1}$ & $1 s 6 g^{3} G_{3}$ & $2.4252 \mathrm{E}+01$ & $9.019 \mathrm{E}-09$ & $1.461 \mathrm{E}+04$ & & & & 0.014 \\
\hline $1 s 5 d^{3} D_{2}$ & $1 s 6 g^{3} G_{3}$ & $2.5812 \mathrm{E}+01$ & $5.697 \mathrm{E}-04$ & $8.147 E+08$ & $2.5711 \mathrm{E}+01$ & $5.708 \mathrm{E}-04$ & $8.158 E+08$ & 0.000 \\
\hline $1 s 5 d^{3} D_{1}$ & $1 s 6 g^{3} G_{3}$ & $2.5829 \mathrm{E}+01$ & $3.383 \mathrm{E}-03$ & $4.832 E+09$ & $2.5727 \mathrm{E}+01$ & $3.382 \mathrm{E}-03$ & $4.827 E+09$ & 0.000 \\
\hline $1 s 5 d^{3} D_{3}$ & $1 s 6 g{ }^{3} G_{3}$ & $2.6241 \mathrm{E}+01$ & $6.159 \mathrm{E}-05$ & $8.523 E+07$ & $2.6137 \mathrm{E}+01$ & $6.159 \mathrm{E}-05$ & $8.518 E+07$ & 0.000 \\
\hline $1 s 5 d{ }^{1} D_{2}$ & $1 s 6 g^{3} G_{3}$ & $2.6259 \mathrm{E}+01$ & $3.639 \mathrm{E}-04$ & $5.028 \mathrm{E}+08$ & $2.6155 \mathrm{E}+01$ & $3.629 \mathrm{E}-04$ & $5.012 \mathrm{E}+08$ & 0.000 \\
\hline $1 s 5 g^{3} G_{4}$ & $1 s 6 g{ }^{3} G_{3}$ & $2.6467 \mathrm{E}+01$ & $4.878 \mathrm{E}-05$ & $6.636 \mathrm{E}+07$ & $2.6356 \mathrm{E}+01$ & $4.884 \mathrm{E}-05$ & $6.644 \mathrm{E}+07$ & 0.000 \\
\hline $1 s 5 g^{3} G_{3}$ & $1 s 6 g^{3} G_{3}$ & $2.6472 \mathrm{E}+01$ & $4.079 \mathrm{E}-04$ & $5.547 \mathrm{E}+08$ & $2.6361 \mathrm{E}+01$ & $4.082 \mathrm{E}-04$ & $5.550 \mathrm{E}+08$ & 0.000 \\
\hline $1 s 5 g^{3} G_{5}$ & $1 s 6 g^{3} G_{3}$ & $2.6598 \mathrm{E}+01$ & $2.379 \mathrm{E}-06$ & $3.205 E+06$ & $2.6487 \mathrm{E}+01$ & $2.378 \mathrm{E}-06$ & $3.202 E+06$ & 0.001 \\
\hline $1 s 5 g{ }^{1} G_{4}$ & $1 s 6 g^{3} G_{3}$ & $2.6602 \mathrm{E}+01$ & $3.946 \mathrm{E}-05$ & $5.314 \mathrm{E}+07$ & $2.6491 \mathrm{E}+01$ & $3.938 \mathrm{E}-05$ & $5.302 E+07$ & 0.000 \\
\hline $1 s 6 s^{3} S_{1}$ & $1 s 6 g^{3} G_{3}$ & $5.0108 \mathrm{E}+02$ & $3.106 \mathrm{E}-12$ & $1.179 \mathrm{E}-02$ & & & & 0.128 \\
\hline $1 s 6 d^{3} D_{2}$ & $1 s 6 g{ }^{3} G_{3}$ & $1.7904 \mathrm{E}+03$ & $4.198 \mathrm{E}-09$ & $1.248 \mathrm{E}+00$ & $1.8335 \mathrm{E}+03$ & $3.889 E-09$ & $1.093 E+00$ & 0.003 \\
\hline $1 s 6 d^{3} D_{1}$ & $1 s 6 g^{3} G_{3}$ & $1.8364 \mathrm{E}+03$ & $2.308 \mathrm{E}-08$ & $6.523 E+00$ & $1.8221 \mathrm{E}+03$ & $2.333 \mathrm{E}-08$ & $6.639 E+00$ & 0.002 \\
\hline $1 s 6 d^{3} D_{3}$ & $1 s 6 g{ }^{3} G_{3}$ & $5.1873 E+03$ & $1.881 \mathrm{E}-11$ & $6.661 \mathrm{E}-04$ & & & & 0.003 \\
\hline $1 s 6 d^{1} D_{2}$ & $1 s 6 g^{3} G_{3}$ & $5.6439 \mathrm{E}+03$ & $8.622 \mathrm{E}-11$ & $2.579 \mathrm{E}-03$ & $5.6946 \mathrm{E}+03$ & $8.174 \mathrm{E}-11$ & $2.381 \mathrm{E}-03$ & 0.011 \\
\hline $1 s 6 g{ }^{3} G_{4}$ & $1 s 6 g{ }^{3} G_{3}$ & $2.2908 \mathrm{E}+05$ & $1.468 \mathrm{E}-15$ & $2.666 \mathrm{E}-11$ & & & & 0.552 \\
\hline $1 s 2 p^{3} P_{1}^{o}$ & $1 s 6 f^{1} F_{3}^{o}$ & $1.3775 \mathrm{E}+00$ & $1.744 \mathrm{E}-06$ & $8.756 E+08$ & $1.3718 \mathrm{E}+00$ & $2.023 \mathrm{E}-06$ & $1.016 \mathrm{E}+09$ & 0.014 \\
\hline $1 s 2 p^{3} P_{2}^{o}$ & $1 s 6 f^{1} F_{3}^{o}$ & $1.4397 \mathrm{E}+00$ & $1.779 \mathrm{E}-04$ & $8.177 E+10$ & $1.4336 \mathrm{E}+00$ & $1.854 \mathrm{E}-04$ & $8.523 E+10$ & 0.000 \\
\hline $1 s 2 p^{1} P_{1}^{o}$ & $1 s 6 f{ }^{1} F_{3}^{o}$ & $1.4456 \mathrm{E}+00$ & $1.064 \mathrm{E}-03$ & $4.851 \mathrm{E}+11$ & $1.4396 \mathrm{E}+00$ & $1.098 \mathrm{E}-03$ & $5.009 E+11$ & 0.001 \\
\hline $1 s 3 p^{3} P_{1}^{o}$ & $1 s 6 f^{1} F_{3}^{o}$ & $3.6895 \mathrm{E}+00$ & $3.092 \mathrm{E}-09$ & $2.165 \mathrm{E}+05$ & $3.6743 \mathrm{E}+00$ & $3.901 \mathrm{E}-08$ & $2.730 \mathrm{E}+06$ & 0.011 \\
\hline $1 s 3 p^{3} P_{2}^{o}$ & $1 s 6 f^{1} F_{3}^{o}$ & $3.8210 \mathrm{E}+00$ & $3.586 \mathrm{E}-06$ & $2.340 \mathrm{E}+08$ & $3.8051 \mathrm{E}+00$ & $4.156 \mathrm{E}-06$ & $2.711 E+08$ & 0.011 \\
\hline $1 s 3 p{ }^{1} P_{1}^{o}$ & $1 s 6 f^{1} F_{3}^{o}$ & $3.8327 \mathrm{E}+00$ & $2.406 \mathrm{E}-05$ & $1.561 E+09$ & $3.8169 \mathrm{E}+00$ & $2.471 \mathrm{E}-05$ & $1.602 E+09$ & 0.008 \\
\hline $1 s 4 p^{3} P_{1}^{o}$ & $1 s 6 f^{1} F_{3}^{o}$ & $8.8264 \mathrm{E}+00$ & $6.111 \mathrm{E}-07$ & $7.475 \mathrm{E}+06$ & $8.7892 \mathrm{E}+00$ & $4.307 \mathrm{E}-07$ & $5.268 \mathrm{E}+06$ & 0.001 \\
\hline $1 s 4 p^{3} P_{2}^{0}$ & $1 s 6 f^{1} F_{3}^{o}$ & $9.1428 \mathrm{E}+00$ & $5.020 \mathrm{E}-05$ & $5.723 E+08$ & $9.1041 \mathrm{E}+00$ & $4.900 \mathrm{E}-05$ & $5.585 E+08$ & 0.001 \\
\hline $1 s 4 p^{1} P_{1}^{o}$ & $1 s 6 f^{1} F_{3}^{o}$ & $9.1705 E+00$ & $2.897 \mathrm{E}-04$ & $3.283 \mathrm{E}+09$ & $9.1320 \mathrm{E}+00$ & $2.914 \mathrm{E}-04$ & $3.302 E+09$ & 0.000 \\
\hline $1 s 4 f^{3} F_{3}^{o}$ & $1 s 6 f{ }^{1} F_{3}^{o}$ & $9.2731 \mathrm{E}+00$ & $2.362 \mathrm{E}-06$ & $2.618 E+07$ & $9.2341 \mathrm{E}+00$ & $2.453 \mathrm{E}-06$ & $2.718 \mathrm{E}+07$ & 0.001 \\
\hline $1 s 4 f^{3} F_{2}^{o}$ & $1 s 6 f^{1} F_{3}^{o}$ & $9.2753 \mathrm{E}+00$ & $1.418 \mathrm{E}-05$ & $1.571 \mathrm{E}+08$ & $9.2363 \mathrm{E}+00$ & $1.414 \mathrm{E}-05$ & $1.566 \mathrm{E}+08$ & 0.001 \\
\hline $1 s 4 f^{3} F_{4}^{o}$ & $1 s 6 f^{1} F_{3}^{o}$ & $9.3260 \mathrm{E}+00$ & $1.458 \mathrm{E}-05$ & $1.598 \mathrm{E}+08$ & $9.2866 \mathrm{E}+00$ & $1.446 \mathrm{E}-05$ & $1.585 E+08$ & 0.000 \\
\hline $1 s 4 f^{1} F_{3}^{o}$ & $1 s 6 f^{1} F_{3}^{o}$ & $9.3276 \mathrm{E}+00$ & $1.208 \mathrm{E}-04$ & $1.323 E+09$ & $9.2883 E+00$ & $1.206 \mathrm{E}-04$ & $1.320 \mathrm{E}+09$ & 0.000 \\
\hline $1 s 5 p^{3} P_{1}^{o}$ & $1 s 6 f^{1} F_{3}^{o}$ & $2.4458 \mathrm{E}+01$ & $4.144 \mathrm{E}-06$ & $6.601 E+06$ & $2.4355 \mathrm{E}+01$ & $4.367 \mathrm{E}-06$ & $6.957 E+06$ & 0.000 \\
\hline $1 s 5 p^{3} P_{2}^{o}$ & $1 s 6 f^{1} F_{3}^{o}$ & $2.5717 \mathrm{E}+01$ & $4.804 \mathrm{E}-04$ & $6.921 E+08$ & $2.5607 \mathrm{E}+01$ & $4.825 \mathrm{E}-04$ & $6.952 E+08$ & 0.000 \\
\hline $1 s 5 p{ }^{1} P_{1}^{o}$ & $1 s 6 f^{1} F_{3}^{o}$ & $2.5828 \mathrm{E}+01$ & $2.861 \mathrm{E}-03$ & $4.087 E+09$ & $2.5719 \mathrm{E}+01$ & $2.859 \mathrm{E}-03$ & $4.084 \mathrm{E}+09$ & 0.000 \\
\hline $1 s 5 f^{3} F_{3}^{o}$ & $1 s 6 f^{1} F_{3}^{o}$ & $2.6248 \mathrm{E}+01$ & $1.311 \mathrm{E}-05$ & $1.813 \mathrm{E}+07$ & $2.6139 \mathrm{E}+01$ & $1.363 \mathrm{E}-05$ & $1.885 \mathrm{E}+07$ & 0.000 \\
\hline
\end{tabular}


Table 4 (continued)

\begin{tabular}{|c|c|c|c|c|c|c|c|c|}
\hline \multirow[t]{2}{*}{ Lower } & \multirow[t]{2}{*}{ Upper } & \multicolumn{3}{|l|}{ GRASP2K } & \multicolumn{3}{|l|}{ FAC } & \multirow[t]{2}{*}{$d T$} \\
\hline & & $\lambda(\AA)$ & $g f$ & $A\left(\mathrm{~s}^{-1}\right)$ & $\lambda(\AA)$ & $g f$ & $A\left(\mathrm{~s}^{-1}\right)$ & \\
\hline $1 s 5 f^{3} F_{2}^{o}$ & $1 s 6 f^{1} F_{3}^{o}$ & $2.6257 \mathrm{E}+01$ & $7.903 \mathrm{E}-05$ & $1.092 \mathrm{E}+08$ & $2.6148 \mathrm{E}+01$ & $7.877 \mathrm{E}-05$ & $1.088 \mathrm{E}+08$ & 0.000 \\
\hline 1s $5 f^{3} F_{4}^{o}$ & $1 s 6 f^{1} F_{3}^{o}$ & $2.6465 \mathrm{E}+01$ & $8.283 \mathrm{E}-05$ & $1.127 \mathrm{E}+08$ & $2.6355 \mathrm{E}+01$ & $8.250 \mathrm{E}-05$ & $1.122 \mathrm{E}+08$ & 0.000 \\
\hline $1 s 5 f^{1} F_{3}^{o}$ & $1 s 6 f^{1} F_{3}^{o}$ & $2.6472 \mathrm{E}+01$ & $6.865 \mathrm{E}-04$ & $9.335 E+08$ & $2.6362 \mathrm{E}+01$ & $6.876 \mathrm{E}-04$ & $9.348 \mathrm{E}+08$ & 0.000 \\
\hline $1 s 6 p^{3} P_{1}^{o}$ & $1 s 6 f^{1} F_{3}^{o}$ & $5.5676 \mathrm{E}+02$ & $1.886 \mathrm{E}-09$ & $5.797 \mathrm{E}+00$ & $5.5347 \mathrm{E}+02$ & $2.833 \mathrm{E}-09$ & $8.739 E+00$ & 0.006 \\
\hline $1 s 6 p^{3} P_{2}^{o}$ & $1 s 6 f^{1} F_{3}^{o}$ & $1.5574 \mathrm{E}+03$ & $1.007 \mathrm{E}-08$ & $3.956 \mathrm{E}+00$ & $1.5396 \mathrm{E}+03$ & $1.023 \mathrm{E}-08$ & $4.078 \mathrm{E}+00$ & 0.008 \\
\hline $1 s 6 p{ }^{1} P_{1}^{o}$ & $1 s 6 f^{1} F_{3}^{o}$ & $1.8325 \mathrm{E}+03$ & $3.684 \mathrm{E}-08$ & $1.045 \mathrm{E}+01$ & $1.7754 \mathrm{E}+03$ & $4.001 \mathrm{E}-08$ & $1.199 \mathrm{E}+01$ & 0.009 \\
\hline $1 s 6 f^{3} F_{3}^{o}$ & $1 s 6 f^{1} F_{3}^{o}$ & $5.3394 \mathrm{E}+03$ & $2.665 \mathrm{E}-11$ & $8.908 E-04$ & & & & 0.009 \\
\hline $1 s 6 f^{3} F_{2}^{o}$ & $1 s 6 f^{1} F_{3}^{o}$ & $5.5655 \mathrm{E}+03$ & $1.419 \mathrm{E}-10$ & $4.365 \mathrm{E}-03$ & $5.5161 \mathrm{E}+03$ & $1.428 \mathrm{E}-10$ & $4.435 \mathrm{E}-03$ & 0.004 \\
\hline $1 s 6 f^{3} F_{4}^{o}$ & $1 s 6 f^{1} F_{3}^{o}$ & $1.7466 \mathrm{E}+05$ & $4.802 \mathrm{E}-15$ & $1.500 \mathrm{E}-10$ & & & & 0.228 \\
\hline $1 s 3 d^{3} D_{3}$ & $1 s 6 g^{3} G_{5}$ & $3.8728 \mathrm{E}+00$ & $4.582 \mathrm{E}-03$ & $1.853 \mathrm{E}+11$ & $3.8564 \mathrm{E}+00$ & $4.660 \mathrm{E}-03$ & $1.884 \mathrm{E}+11$ & 0.000 \\
\hline $1 s 4 d^{3} D_{3}$ & $1 s 6 g{ }^{3} G_{5}$ & $9.2626 \mathrm{E}+00$ & $1.359 \mathrm{E}-04$ & $9.604 \mathrm{E}+08$ & $9.2240 \mathrm{E}+00$ & $1.404 \mathrm{E}-04$ & $9.926 \mathrm{E}+08$ & 0.001 \\
\hline $1 s 5 d^{3} D_{3}$ & $1 s 6 g{ }^{3} G_{5}$ & $2.6170 \mathrm{E}+01$ & $6.805 \mathrm{E}-03$ & $6.026 \mathrm{E}+09$ & $2.6063 \mathrm{E}+01$ & $6.824 \mathrm{E}-03$ & $6.040 \mathrm{E}+09$ & 0.000 \\
\hline $1 s 5 g{ }^{3} G_{4}$ & $1 s 6 g{ }^{3} G_{5}$ & $2.6394 \mathrm{E}+01$ & $4.948 \mathrm{E}-05$ & $4.307 \mathrm{E}+07$ & $2.6281 \mathrm{E}+01$ & $4.968 \mathrm{E}-05$ & $4.325 E+07$ & 0.000 \\
\hline $1 s 5 g^{3} G_{3}$ & $1 s 6 g{ }^{3} G_{5}$ & $2.6399 \mathrm{E}+01$ & $2.359 \mathrm{E}-06$ & $2.053 E+06$ & $2.6286 \mathrm{E}+01$ & $2.367 \mathrm{E}-06$ & $2.059 E+06$ & 0.000 \\
\hline $1 s 5 g{ }^{3} G_{5}$ & $1 s 6 g{ }^{3} G_{5}$ & $2.6525 \mathrm{E}+01$ & $6.910 \mathrm{E}-04$ & $5.955 E+08$ & $2.6411 \mathrm{E}+01$ & $6.918 \mathrm{E}-04$ & $5.963 E+08$ & 0.000 \\
\hline $1 s 5 g^{1} G_{4}$ & $1 s 6 g{ }^{3} G_{5}$ & $2.6529 \mathrm{E}+01$ & $4.004 \mathrm{E}-05$ & $3.450 \mathrm{E}+07$ & $2.6415 \mathrm{E}+01$ & $4.006 \mathrm{E}-05$ & $3.452 E+07$ & 0.000 \\
\hline $1 s 6 d^{3} D_{3}$ & $1 s 6 g{ }^{3} G_{5}$ & $3.3718 \mathrm{E}+03$ & $7.594 \mathrm{E}-09$ & $4.050 \mathrm{E}-01$ & $3.4576 \mathrm{E}+03$ & $6.952 \mathrm{E}-09$ & $3.496 \mathrm{E}-01$ & 0.001 \\
\hline $1 s 6 g{ }^{3} G_{4}$ & $1 s 6 g{ }^{3} G_{5}$ & $9.2456 \mathrm{E}+03$ & $2.278 \mathrm{E}-11$ & $1.616 \mathrm{E}-04$ & $9.2559 \mathrm{E}+03$ & $2.249 \mathrm{E}-11$ & $1.578 \mathrm{E}-04$ & 0.004 \\
\hline $1 s 6 g{ }^{3} G_{3}$ & $1 s 6 g{ }^{3} G_{5}$ & $9.6344 \mathrm{E}+03$ & $9.609 \mathrm{E}-13$ & $6.277 \mathrm{E}-06$ & & & & 0.063 \\
\hline $1 s 4 f^{3} F_{3}^{o}$ & $1 s 6 h^{3} H_{5}^{o}$ & $9.2643 \mathrm{E}+00$ & $9.005 \mathrm{E}-03$ & $6.362 \mathrm{E}+10$ & $9.2249 \mathrm{E}+00$ & $9.057 \mathrm{E}-03$ & $6.399 E+10$ & 0.000 \\
\hline $1 s 4 f^{3} F_{4}^{o}$ & $1 s 6 h^{3} H_{5}^{o}$ & $9.3170 \mathrm{E}+00$ & $5.230 \mathrm{E}-04$ & $3.653 E+09$ & $9.2773 \mathrm{E}+00$ & $5.243 \mathrm{E}-04$ & $3.662 E+09$ & 0.000 \\
\hline $1 s 4 f^{1} F_{3}^{o}$ & $1 s 6 h^{3} H_{5}^{o}$ & $9.3187 \mathrm{E}+00$ & $2.439 \mathrm{E}-05$ & $1.703 E+08$ & $9.2790 \mathrm{E}+00$ & $2.295 \mathrm{E}-05$ & $1.602 \mathrm{E}+08$ & 0.000 \\
\hline $1 s 5 f^{3} F_{3}^{o}$ & $1 s 6 h^{3} H_{5}^{o}$ & $2.6177 \mathrm{E}+01$ & $5.783 \mathrm{E}-03$ & $5.118 \mathrm{E}+09$ & $2.6065 \mathrm{E}+01$ & $5.792 \mathrm{E}-03$ & $5.126 \mathrm{E}+09$ & 0.000 \\
\hline $1 s 5 f^{3} F_{4}^{o}$ & $1 s 6 h^{3} H_{5}^{o}$ & $2.6394 \mathrm{E}+01$ & $3.283 \mathrm{E}-04$ & $2.858 \mathrm{E}+08$ & $2.6281 \mathrm{E}+01$ & $3.291 \mathrm{E}-04$ & $2.864 \mathrm{E}+08$ & 0.000 \\
\hline $1 s 5 f^{1} F_{3}^{o}$ & $1 s 6 h^{3} H_{5}^{0}$ & $2.6400 \mathrm{E}+01$ & $1.539 \mathrm{E}-05$ & $1.339 \mathrm{E}+07$ & $2.6287 \mathrm{E}+01$ & $1.448 \mathrm{E}-05$ & $1.259 \mathrm{E}+07$ & 0.000 \\
\hline $1 s 6 f^{3} F_{3}^{o}$ & $1 s 6 h^{3} H_{5}^{o}$ & $3.4460 \mathrm{E}+03$ & $2.908 \mathrm{E}-09$ & $1.485 \mathrm{E}-01$ & $3.4619 \mathrm{E}+03$ & $2.830 \mathrm{E}-09$ & $1.420 \mathrm{E}-01$ & 0.000 \\
\hline $1 s 6 f^{3} F_{4}^{o}$ & $1 s 6 h^{3} H_{5}^{o}$ & $9.2055 \mathrm{E}+03$ & $8.735 \mathrm{E}-12$ & $6.251 \mathrm{E}-05$ & $9.2780 \mathrm{E}+03$ & $8.447 \mathrm{E}-12$ & $5.900 \mathrm{E}-05$ & 0.006 \\
\hline $1 s 6 f^{1} F_{3}^{o}$ & $1 s 6 h^{3} H_{5}^{o}$ & $9.7177 \mathrm{E}+03$ & $3.492 \mathrm{E}-13$ & $2.242 \mathrm{E}-06$ & & & & 0.010 \\
\hline $1 s 2 p^{3} P_{2}^{o}$ & $1 s 6 h^{3} H_{4}^{o}$ & $1.4395 \mathrm{E}+00$ & $2.079 \mathrm{E}-12$ & $7.436 \mathrm{E}+02$ & & & & 0.948 \\
\hline $1 s 3 p^{3} P_{2}^{o}$ & $1 s 6 h^{3} H_{4}^{0}$ & $3.8195 \mathrm{E}+00$ & $1.741 \mathrm{E}-13$ & $8.847 \mathrm{E}+00$ & & & & 0.800 \\
\hline $1 s 4 p^{3} P_{2}^{o}$ & $1 s 6 h^{3} H_{4}^{o}$ & $9.1340 \mathrm{E}+00$ & $7.955 \mathrm{E}-10$ & $7.067 \mathrm{E}+03$ & & & & 0.008 \\
\hline $1 s 4 f^{3} F_{3}^{o}$ & $1 s 6 h^{3} H_{4}^{0}$ & $9.2640 \mathrm{E}+00$ & $5.435 \mathrm{E}-04$ & $4.694 \mathrm{E}+09$ & $9.2249 \mathrm{E}+00$ & $5.467 \mathrm{E}-04$ & $4.721 \mathrm{E}+09$ & 0.000 \\
\hline $1 s 4 f^{3} F_{2}^{o}$ & $1 s 6 h^{3} H_{4}^{0}$ & $9.2662 \mathrm{E}+00$ & $6.821 \mathrm{E}-03$ & $5.888 \mathrm{E}+10$ & $9.2271 \mathrm{E}+00$ & $6.855 \mathrm{E}-03$ & $5.917 \mathrm{E}+10$ & 0.000 \\
\hline $1 s 4 f^{3} F_{4}^{o}$ & $1 s 6 h^{3} H_{4}^{0}$ & $9.3168 \mathrm{E}+00$ & $3.746 \mathrm{E}-05$ & $3.198 E+08$ & $9.2773 \mathrm{E}+00$ & $3.745 \mathrm{E}-05$ & $3.197 E+08$ & 0.000 \\
\hline $1 s 4 f^{1} F_{3}^{o}$ & $1 s 6 h^{3} H_{4}^{o}$ & $9.3184 \mathrm{E}+00$ & $4.142 \mathrm{E}-04$ & $3.535 E+09$ & $9.2790 \mathrm{E}+00$ & $4.138 \mathrm{E}-04$ & $3.531 \mathrm{E}+09$ & 0.000 \\
\hline $1 s 5 p^{3} P_{2}^{o}$ & $1 s 6 h^{3} H_{4}^{0}$ & $2.5647 \mathrm{E}+01$ & $9.502 \mathrm{E}-10$ & $1.071 \mathrm{E}+03$ & & & & 0.001 \\
\hline $1 s 5 f^{3} F_{3}^{o}$ & $1 s 6 h^{3} H_{4}^{0}$ & $2.6175 \mathrm{E}+01$ & $3.493 \mathrm{E}-04$ & $3.778 E+08$ & $2.6065 \mathrm{E}+01$ & $3.497 \mathrm{E}-04$ & $3.783 E+08$ & 0.000 \\
\hline $1 s 5 f^{3} F_{2}^{o}$ & $1 s 6 h^{3} H_{4}^{o}$ & $2.6184 \mathrm{E}+01$ & $4.378 \mathrm{E}-03$ & $4.733 E+09$ & $2.6074 \mathrm{E}+01$ & $4.383 \mathrm{E}-03$ & $4.737 \mathrm{E}+09$ & 0.000 \\
\hline $1 s 5 f^{3} F_{4}^{o}$ & $1 s 6 h^{3} H_{4}^{0}$ & $2.6392 \mathrm{E}+01$ & $2.352 \mathrm{E}-05$ & $2.503 E+07$ & $2.6281 \mathrm{E}+01$ & $2.350 \mathrm{E}-05$ & $2.501 \mathrm{E}+07$ & 0.000 \\
\hline $1 s 5 f^{1} F_{3}^{o}$ & $1 s 6 h^{3} H_{4}^{0}$ & $2.6398 \mathrm{E}+01$ & $2.598 \mathrm{E}-04$ & $2.763 E+08$ & $2.6287 \mathrm{E}+01$ & $2.595 \mathrm{E}-04$ & $2.760 \mathrm{E}+08$ & 0.000 \\
\hline $1 s 6 p^{3} P_{2}^{o}$ & $1 s 6 h^{3} H_{4}^{o}$ & $1.3372 \mathrm{E}+03$ & $1.003 \mathrm{E}-14$ & $4.157 \mathrm{E}-06$ & & & & 0.006 \\
\hline $1 s 6 f^{3} F_{3}^{o}$ & $1 s 6 h^{3} H_{4}^{o}$ & $3.4128 \mathrm{E}+03$ & $1.808 \mathrm{E}-10$ & $1.151 \mathrm{E}-02$ & $3.4619 \mathrm{E}+03$ & $1.717 \mathrm{E}-10$ & $1.053 \mathrm{E}-02$ & 0.001 \\
\hline $1 s 6 f^{3} F_{2}^{o}$ & $1 s 6 h^{3} H_{4}^{o}$ & $3.5038 \mathrm{E}+03$ & $2.095 \mathrm{E}-09$ & $1.265 \mathrm{E}-01$ & $3.4609 \mathrm{E}+03$ & $2.146 \mathrm{E}-09$ & $1.317 \mathrm{E}-01$ & 0.000 \\
\hline $1 s 6 f^{3} F_{4}^{o}$ & $1 s 6 h^{3} H_{4}^{o}$ & $8.9725 \mathrm{E}+03$ & $6.759 \mathrm{E}-13$ & $6.222 \mathrm{E}-06$ & & & & 0.002 \\
\hline $1 s 6 f^{1} F_{3}^{o}$ & $1 s 6 h^{3} H_{4}^{o}$ & $9.4584 \mathrm{E}+03$ & $6.374 \mathrm{E}-12$ & $5.281 \mathrm{E}-05$ & $9.2889 \mathrm{E}+03$ & $6.605 \mathrm{E}-12$ & $5.625 \mathrm{E}-05$ & 0.006 \\
\hline $1 s 6 h^{3} H_{5}^{o}$ & $1 s 6 h^{3} H_{4}^{0}$ & $3.5443 \mathrm{E}+05$ & $1.836 \mathrm{E}-16$ & $1.083 \mathrm{E}-12$ & & & & 0.747 \\
\hline $1 s 3 d^{3} D_{2}$ & $1 s 6 g{ }^{1} G_{4}^{4}$ & $3.8293 \mathrm{E}+00$ & $9.113 \mathrm{E}-08$ & $4.606 \mathrm{E}+06$ & & & & 0.015 \\
\hline $1 s 3 d^{3} D_{3}$ & $1 s 6 g{ }^{1} G_{4}$ & $3.8727 \mathrm{E}+00$ & $2.790 \mathrm{E}-04$ & $1.379 \mathrm{E}+10$ & $3.8564 \mathrm{E}+00$ & $2.824 \mathrm{E}-04$ & $1.395 \mathrm{E}+10$ & 0.000 \\
\hline $1 s 3 d^{1} D_{2}$ & $1 s 6 g{ }^{1} G_{4}$ & $3.8744 \mathrm{E}+00$ & $3.470 \mathrm{E}-03$ & $1.713 \mathrm{E}+11$ & $3.8580 \mathrm{E}+00$ & $3.526 \mathrm{E}-03$ & $1.740 \mathrm{E}+11$ & 0.000 \\
\hline $1 s 4 d^{3} D_{2}$ & $1 s 6 g{ }^{1} G_{4}$ & $9.1575 \mathrm{E}+00$ & $3.631 \mathrm{E}-09$ & $3.209 \mathrm{E}+04$ & & & & 0.043 \\
\hline $1 s 4 d^{3} D_{3}$ & $1 s 6 g{ }^{1} G_{4}$ & $9.2623 \mathrm{E}+00$ & $8.247 \mathrm{E}-06$ & $7.125 \mathrm{E}+07$ & $9.2240 \mathrm{E}+00$ & $8.512 \mathrm{E}-06$ & $7.352 \mathrm{E}+07$ & 0.000 \\
\hline $1 s 4 d{ }^{1} D_{2}$ & $1 s 6 g{ }^{1} G_{4}$ & $9.2666 \mathrm{E}+00$ & $1.039 \mathrm{E}-04$ & $8.965 E+08$ & $9.2283 \mathrm{E}+00$ & $1.062 \mathrm{E}-04$ & $9.168 \mathrm{E}+08$ & 0.001 \\
\hline $1 s 5 d^{3} D_{2}$ & $1 s 6 g{ }^{1} G_{4}$ & $2.5741 \mathrm{E}+01$ & $3.250 \mathrm{E}-07$ & $3.635 \mathrm{E}+05$ & & & & 0.002 \\
\hline $1 s 5 d^{3} D_{3}$ & $1 s 6 g{ }^{1} G_{4}$ & $2.6167 \mathrm{E}+01$ & $4.142 \mathrm{E}-04$ & $4.484 \mathrm{E}+08$ & $2.6063 \mathrm{E}+01$ & $4.135 \mathrm{E}-04$ & $4.474 \mathrm{E}+08$ & 0.000 \\
\hline $1 s 5 d^{1} D_{2}$ & $1 s 6 g{ }^{1} G_{4}$ & $2.6185 \mathrm{E}+01$ & $5.151 \mathrm{E}-03$ & $5.567 \mathrm{E}+09$ & $2.6082 \mathrm{E}+01$ & $5.158 \mathrm{E}-03$ & $5.572 \mathrm{E}+09$ & 0.000 \\
\hline $1 s 5 g^{3} G_{4}$ & $1 s 6 g{ }^{1} G_{4}$ & $2.6392 \mathrm{E}+01$ & $3.549 \mathrm{E}-06$ & $3.777 \mathrm{E}+06$ & $2.6281 \mathrm{E}+01$ & $3.733 \mathrm{E}-06$ & $3.972 E+06$ & 0.000 \\
\hline $1 s 5 g^{3} G_{3}$ & $1 s 6 g{ }^{1} G_{4}$ & $2.6397 \mathrm{E}+01$ & $3.919 \mathrm{E}-05$ & $4.168 \mathrm{E}+07$ & $2.6286 \mathrm{E}+01$ & $3.914 \mathrm{E}-05$ & $4.163 E+07$ & 0.000 \\
\hline $1 s 5 g^{3} G_{5}$ & $1 s 6 g{ }^{1} G_{4}$ & $2.6522 \mathrm{E}+01$ & $4.003 E-05$ & $4.218 \mathrm{E}+07$ & $2.6411 \mathrm{E}+01$ & $3.991 \mathrm{E}-05$ & $4.205 E+07$ & 0.000 \\
\hline $1 s 5 g{ }^{1} G_{4}$ & $1 s 6 g{ }^{1} G_{4}$ & $2.6527 \mathrm{E}+01$ & $5.578 \mathrm{E}-04$ & $5.875 E+08$ & $2.6415 \mathrm{E}+01$ & $5.583 \mathrm{E}-04$ & $5.880 \mathrm{E}+08$ & 0.000 \\
\hline $1 s 6 d^{3} D_{2}$ & $1 s 6 g{ }^{1} G_{4}$ & $1.5020 \mathrm{E}+03$ & $4.543 \mathrm{E}-12$ & $1.492 \mathrm{E}-03$ & $1.5304 \mathrm{E}+03$ & $8.482 \mathrm{E}-12$ & $2.661 \mathrm{E}-03$ & 0.073 \\
\hline $1 s 6 d^{3} D_{3}$ & $1 s 6 g{ }^{1} G_{4}$ & $3.3331 \mathrm{E}+03$ & $4.786 \mathrm{E}-10$ & $3.193 E-02$ & $3.4576 \mathrm{E}+03$ & $4.213 \mathrm{E}-10$ & $2.590 \mathrm{E}-02$ & 0.003 \\
\hline $1 s 6 d{ }^{1} D_{2}$ & $1 s 6 g{ }^{1} G_{4}$ & $3.5158 \mathrm{E}+03$ & $5.072 \mathrm{E}-09$ & $3.041 \mathrm{E}-01$ & $3.5255 \mathrm{E}+03$ & $4.967 \mathrm{E}-09$ & $2.936 \mathrm{E}-01$ & 0.007 \\
\hline $1 s 6 g{ }^{3} G_{4}$ & $1 s 6 g{ }^{1} G_{4}$ & $8.9597 \mathrm{E}+03$ & $1.796 \mathrm{E}-12$ & $1.658 \mathrm{E}-05$ & & & & 0.031 \\
\hline $1 s 6 g^{3} G_{3}$ & $1 s 6 g{ }^{1} G_{4}$ & $9.3244 \mathrm{E}+03$ & $1.758 \mathrm{E}-11$ & $1.499 \mathrm{E}-04$ & $9.2557 \mathrm{E}+03$ & $1.767 \mathrm{E}-11$ & $1.515 \mathrm{E}-04$ & 0.005 \\
\hline $1 s 6 g{ }^{3} G_{5}$ & $1 s 6 g{ }^{1} G_{4}$ & $2.8974 \mathrm{E}+05$ & $5.995 \mathrm{E}-16$ & $5.293 \mathrm{E}-12$ & & & & 0.672 \\
\hline $1 s 4 f^{3} F_{4}^{o}$ & $1 s 6 h^{3} H_{6}^{o}$ & $9.3108 \mathrm{E}+00$ & $1.132 \mathrm{E}-02$ & $6.701 \mathrm{E}+10$ & $9.2712 \mathrm{E}+00$ & $1.142 \mathrm{E}-02$ & $6.763 E+10$ & 0.000 \\
\hline $1 s 5 f^{3} F_{4}^{0}$ & $1 s 6 h^{3} H_{6}^{o}$ & $2.6344 \mathrm{E}+01$ & $7.177 \mathrm{E}-03$ & $5.306 \mathrm{E}+09$ & $2.6231 \mathrm{E}+01$ & $7.197 \mathrm{E}-03$ & $5.321 E+09$ & 0.000 \\
\hline $1 s 6 f^{3} F_{4}^{o}$ & $1 s 6 h^{3} H_{6}^{o}$ & $5.5410 \mathrm{E}+03$ & $8.762 \mathrm{E}-10$ & $1.464 \mathrm{E}-02$ & $5.5692 \mathrm{E}+03$ & $8.520 \mathrm{E}-10$ & $1.397 \mathrm{E}-02$ & 0.003 \\
\hline $1 s 6 h^{3} H_{5}^{o}$ & $1 s 6 h^{3} H_{6}^{o}$ & $1.3919 \mathrm{E}+04$ & $3.060 \mathrm{E}-12$ & $8.104 \mathrm{E}-06$ & $1.3932 \mathrm{E}+04$ & $3.021 \mathrm{E}-12$ & $7.919 \mathrm{E}-06$ & 0.005 \\
\hline $1 s 6 h^{3} H_{4}^{o}$ & $1 s 6 h^{3} H_{6}^{o}$ & $1.4488 \mathrm{E}+04$ & $8.641 \mathrm{E}-14$ & $2.112 \mathrm{E}-07$ & & & & 0.178 \\
\hline $1 s 4 f^{3} F_{3}^{o}$ & $1 s 6 h{ }^{1} H_{5}^{o}$ & $9.2579 \mathrm{E}+00$ & $3.678 \mathrm{E}-09$ & $2.602 \mathrm{E}+04$ & & & & 0.007 \\
\hline
\end{tabular}


Table 4 (continued)

\begin{tabular}{|c|c|c|c|c|c|c|c|c|}
\hline \multirow[t]{2}{*}{ Lower } & \multirow[t]{2}{*}{ Upper } & \multicolumn{3}{|l|}{ GRASP2K } & \multicolumn{3}{|l|}{ FAC } & \multirow[t]{2}{*}{$d T$} \\
\hline & & $\lambda(\AA)$ & $g f$ & $A\left(\mathrm{~s}^{-1}\right)$ & $\lambda(\AA)$ & $g f$ & $A\left(\mathrm{~s}^{-1}\right)$ & \\
\hline $1 s 4 f^{3} F_{4}^{o}$ & $1 s 6 h^{1} H_{5}^{o}$ & $9.3106 \mathrm{E}+00$ & $4.369 \mathrm{E}-04$ & $3.056 \mathrm{E}+09$ & $9.2712 \mathrm{E}+00$ & $4.395 \mathrm{E}-04$ & $3.074 \mathrm{E}+09$ & 0.000 \\
\hline $1 s 4 f^{1} F_{3}^{o}$ & $1 s 6 h^{1} H_{5}^{o}$ & $9.3123 \mathrm{E}+00$ & $9.144 \mathrm{E}-03$ & $6.394 \mathrm{E}+10$ & $9.2728 \mathrm{E}+00$ & $9.224 \mathrm{E}-03$ & $6.450 \mathrm{E}+10$ & 0.000 \\
\hline $1 s 5 f^{3} F_{3}^{o}$ & $1 s 6 h^{1} H_{5}^{o}$ & $2.6126 \mathrm{E}+01$ & $1.462 \mathrm{E}-09$ & $1.299 \mathrm{E}+03$ & & & & 0.002 \\
\hline $1 s 5 f^{3} F_{4}^{o}$ & $1 s 6 h{ }^{1} H_{5}^{o}$ & $2.6342 \mathrm{E}+01$ & $2.770 \mathrm{E}-04$ & $2.421 \mathrm{E}+08$ & $2.6231 \mathrm{E}+01$ & $2.768 \mathrm{E}-04$ & $2.419 \mathrm{E}+08$ & 0.000 \\
\hline $1 s 5 f^{1} F_{3}^{o}$ & $1 s 6 h^{1} H_{5}^{o}$ & $2.6349 \mathrm{E}+01$ & $5.794 \mathrm{E}-03$ & $5.061 \mathrm{E}+09$ & $2.6238 \mathrm{E}+01$ & $5.809 \mathrm{E}-03$ & $5.073 E+09$ & 0.000 \\
\hline $1 s 6 f^{3} F_{3}^{o}$ & $1 s 6 h{ }^{1} H_{5}^{o}$ & $2.7445 E+03$ & $1.003 \mathrm{E}-15$ & $8.072 \mathrm{E}-08$ & & & & 0.007 \\
\hline $1 s 6 f^{3} F_{4}^{o}$ & $1 s 6 h{ }^{1} H_{5}^{o}$ & $5.4702 \mathrm{E}+03$ & $3.515 \mathrm{E}-11$ & $7.124 \mathrm{E}-04$ & $5.5692 \mathrm{E}+03$ & $3.276 \mathrm{E}-11$ & $6.352 \mathrm{E}-04$ & 0.001 \\
\hline $1 s 6 f^{1} F_{3}^{o}$ & $1 s 6 h^{1} H_{5}^{o}$ & $5.6471 \mathrm{E}+03$ & $6.685 \mathrm{E}-10$ & $1.271 \mathrm{E}-02$ & $5.5731 \mathrm{E}+03$ & $6.867 \mathrm{E}-10$ & $1.329 \mathrm{E}-02$ & 0.002 \\
\hline $1 s 6 h^{3} H_{5}^{o}$ & $1 s 6 h{ }^{1} H_{5}^{o}$ & $1.3481 \mathrm{E}+04$ & $1.610 \mathrm{E}-13$ & $5.370 \mathrm{E}-07$ & & & & 0.045 \\
\hline $1 s 6 h^{3} H_{4}^{o}$ & $1 s 6 h^{1} H_{5}^{0}$ & $1.4014 \mathrm{E}+04$ & $2.491 \mathrm{E}-12$ & $7.690 \mathrm{E}-06$ & $1.3932 \mathrm{E}+04$ & $2.494 \mathrm{E}-12$ & $7.725 \mathrm{E}-06$ & 0.008 \\
\hline $1 s 6 h^{3} H_{6}^{o}$ & $1 s 6 h{ }^{1} H_{5}^{o}$ & $4.2819 \mathrm{E}+05$ & $8.864 \mathrm{E}-17$ & $2.932 E-13$ & & & & 0.869 \\
\hline $1 s 2 s^{3} S_{1}^{0}$ & $1 s 7 s^{3} S_{1}^{5}$ & $1.3268 \mathrm{E}+00$ & $4.817 \mathrm{E}-10$ & $6.084 \mathrm{E}+05$ & & & & 0.985 \\
\hline $1 s 3 s^{3} S_{1}$ & $1 s 7 s^{3} S_{1}$ & $3.3983 E+00$ & $2.685 \mathrm{E}-10$ & $5.170 \mathrm{E}+04$ & & & & 0.385 \\
\hline $1 s 3 d^{3} D_{2}$ & $1 s 7 s^{3} S_{1}$ & $3.5389 \mathrm{E}+00$ & $6.769 \mathrm{E}-06$ & $1.202 \mathrm{E}+09$ & $3.5242 \mathrm{E}+00$ & $7.076 \mathrm{E}-06$ & $1.256 \mathrm{E}+09$ & 0.040 \\
\hline $1 s 3 d^{3} D_{1}$ & $1 s 7 s^{3} s_{1}$ & $3.5404 \mathrm{E}+00$ & $7.411 \mathrm{E}-06$ & $1.315 E+09$ & $3.5257 \mathrm{E}+00$ & $7.024 \mathrm{E}-06$ & $1.245 \mathrm{E}+09$ & 0.056 \\
\hline $1 s 3 d^{3} D_{3}$ & $1 s 7 s^{3} S_{1}$ & $3.5760 \mathrm{E}+00$ & $1.602 \mathrm{E}-05$ & $2.785 E+09$ & $3.5610 \mathrm{E}+00$ & $1.650 \mathrm{E}-05$ & $2.868 \mathrm{E}+09$ & 0.040 \\
\hline $1 s 3 d^{1} D_{2}$ & $1 s 7 s^{3} S_{1}$ & $3.5774 \mathrm{E}+00$ & $4.893 \mathrm{E}-06$ & $8.502 E+08$ & $3.5624 \mathrm{E}+00$ & $4.667 \mathrm{E}-06$ & $8.108 E+08$ & 0.044 \\
\hline $1 s 4 s^{3} S_{1}$ & $1 s 7 s^{3} S_{1}$ & $7.3787 \mathrm{E}+00$ & $3.123 E-11$ & $1.275 \mathrm{E}+03$ & & & & 0.933 \\
\hline $1 s 4 d^{3} D_{2}$ & $1 s 7 s^{3} S_{1}$ & $7.6552 \mathrm{E}+00$ & $1.969 \mathrm{E}-05$ & $7.471 \mathrm{E}+08$ & $7.6242 \mathrm{E}+00$ & $2.005 E-05$ & $7.606 \mathrm{E}+08$ & 0.015 \\
\hline $1 s 4 d^{3} D_{1}$ & $1 s 7 s^{3} S_{1}$ & $7.6581 \mathrm{E}+00$ & $2.030 \mathrm{E}-05$ & $7.698 E+08$ & $7.6271 \mathrm{E}+00$ & $1.984 \mathrm{E}-05$ & $7.521 \mathrm{E}+08$ & 0.023 \\
\hline $1 s 4 d^{3} D_{3}$ & $1 s 7 s^{3} S_{1}$ & $7.7284 \mathrm{E}+00$ & $4.617 \mathrm{E}-05$ & $1.719 E+09$ & $7.6969 \mathrm{E}+00$ & $4.658 \mathrm{E}-05$ & $1.733 \mathrm{E}+09$ & 0.015 \\
\hline $1 s 4 d{ }^{1} D_{2}$ & $1 s 7 s^{3} S_{1}$ & $7.7314 \mathrm{E}+00$ & $1.334 \mathrm{E}-05$ & $4.961 \mathrm{E}+08$ & $7.6999 \mathrm{E}+00$ & $1.311 \mathrm{E}-05$ & $4.876 \mathrm{E}+08$ & 0.017 \\
\hline $1 s 5 s^{3} s_{1}$ & $1 s 7 s^{3} S_{1}$ & $1.5931 \mathrm{E}+01$ & $6.722 \mathrm{E}-10$ & $5.889 E+03$ & & & & 0.419 \\
\hline $1 s 5 d^{3} D_{2}$ & $1 s 7 s^{3} S_{1}$ & $1.6590 \mathrm{E}+01$ & $4.953 \mathrm{E}-05$ & $4.001 \mathrm{E}+08$ & $1.6526 \mathrm{E}+01$ & $4.980 \mathrm{E}-05$ & $4.020 \mathrm{E}+08$ & 0.004 \\
\hline $1 s 5 d^{3} D_{1}$ & $1 s 7 s^{3} S_{1}$ & $1.6597 \mathrm{E}+01$ & $4.987 \mathrm{E}-05$ & $4.025 E+08$ & $1.6532 \mathrm{E}+01$ & $4.920 \mathrm{E}-05$ & $3.968 E+08$ & 0.010 \\
\hline $1 s 5 d^{3} D_{3}$ & $1 s 7 s^{3} S_{1}$ & $1.6766 \mathrm{E}+01$ & $1.160 \mathrm{E}-04$ & $9.178 E+08$ & $1.6700 \mathrm{E}+01$ & $1.158 \mathrm{E}-04$ & $9.160 \mathrm{E}+08$ & 0.004 \\
\hline $1 s 5 d{ }^{1} D_{2}$ & $1 s 7 s^{3} S_{1}$ & $1.6773 \mathrm{E}+01$ & $3.281 \mathrm{E}-05$ & $2.593 E+08$ & $1.6708 \mathrm{E}+01$ & $3.254 \mathrm{E}-05$ & $2.570 \mathrm{E}+08$ & 0.007 \\
\hline $1 s 5 g^{3} G_{3}$ & $1 s 7 s^{3} S_{1}$ & $1.6860 \mathrm{E}+01$ & $3.065 \mathrm{E}-11$ & $2.398 \mathrm{E}+02$ & & & & 0.060 \\
\hline $1 s 6 s^{3} s_{1}$ & $1 s 7 s^{3} S_{1}$ & $4.2495 \mathrm{E}+01$ & $3.689 \mathrm{E}-09$ & $4.543 E+03$ & & & & 0.102 \\
\hline $1 s 6 d^{3} D_{2}$ & $1 s 7 s^{3} S_{1}$ & $4.5259 \mathrm{E}+01$ & $1.803 \mathrm{E}-04$ & $1.957 \mathrm{E}+08$ & $4.5119 \mathrm{E}+01$ & $1.799 E-04$ & $1.949 \mathrm{E}+08$ & 0.001 \\
\hline $1 s 6 d^{3} D_{1}$ & $1 s 7 s^{3} S_{1}$ & $4.5288 \mathrm{E}+01$ & $1.801 \mathrm{E}-04$ & $1.952 E+08$ & $4.5112 \mathrm{E}+01$ & $1.765 \mathrm{E}-04$ & $1.912 \mathrm{E}+08$ & 0.004 \\
\hline $1 s 6 d^{3} D_{3}$ & $1 s 7 s^{3} S_{1}$ & $4.6021 \mathrm{E}+01$ & $4.306 \mathrm{E}-04$ & $4.520 \mathrm{E}+08$ & $4.5873 E+01$ & $4.241 \mathrm{E}-04$ & $4.443 E+08$ & 0.001 \\
\hline $1 s 6 d{ }^{1} D_{2}$ & $1 s 7 s^{3} S_{1}$ & $4.6054 \mathrm{E}+01$ & $1.209 \mathrm{E}-04$ & $1.267 \mathrm{E}+08$ & $4.5885 E+01$ & $1.177 \mathrm{E}-04$ & $1.232 \mathrm{E}+08$ & 0.003 \\
\hline $1 s 6 g^{3} G_{3}$ & $1 s 7 s^{3} S_{1}$ & $4.6433 \mathrm{E}+01$ & $2.618 \mathrm{E}-10$ & $2.700 \mathrm{E}+02$ & & & & 0.039 \\
\hline $1 s 2 p^{3} P_{1}^{o}$ & $1 s 7 p^{3} P_{1}^{o}$ & $1.3376 \mathrm{E}+00$ & $4.582 \mathrm{E}-08$ & $5.693 \mathrm{E}+07$ & $1.3321 \mathrm{E}+00$ & $1.353 \mathrm{E}-07$ & $1.681 \mathrm{E}+08$ & 0.537 \\
\hline $1 s 2 p^{3} P_{2}^{o}$ & $1 s 7 p^{3} P_{1}^{o}$ & $1.3961 \mathrm{E}+00$ & $1.583 \mathrm{E}-05$ & $1.805 \mathrm{E}+10$ & $1.3903 \mathrm{E}+00$ & $1.652 \mathrm{E}-05$ & $1.885 \mathrm{E}+10$ & 0.058 \\
\hline $1 s 2 p^{1} P_{1}^{o}$ & $1 s 7 p^{3} P_{1}^{o}$ & $1.4017 \mathrm{E}+00$ & $1.535 \mathrm{E}-05$ & $1.738 \mathrm{E}+10$ & $1.3959 \mathrm{E}+00$ & $1.409 \mathrm{E}-05$ & $1.595 \mathrm{E}+10$ & 0.029 \\
\hline $1 s 3 p^{3} P_{1}^{o}$ & $1 s 7 p^{3} P_{1}^{o}$ & $3.4164 \mathrm{E}+00$ & $2.188 \mathrm{E}-07$ & $4.169 \mathrm{E}+07$ & $3.4024 \mathrm{E}+00$ & $2.384 \mathrm{E}-07$ & $4.540 \mathrm{E}+07$ & 0.201 \\
\hline $1 s 3 p^{3} P_{2}^{o}$ & $1 s 7 p^{3} P_{1}^{o}$ & $3.5289 \mathrm{E}+00$ & $3.518 \mathrm{E}-05$ & $6.282 E+09$ & $3.5142 \mathrm{E}+00$ & $3.240 \mathrm{E}-05$ & $5.784 \mathrm{E}+09$ & 0.079 \\
\hline $1 s 3 p{ }^{1} P_{1}^{o}$ & $1 s 7 p^{3} P_{1}^{o}$ & $3.5388 \mathrm{E}+00$ & $3.051 \mathrm{E}-05$ & $5.417 \mathrm{E}+09$ & $3.5243 E+00$ & $2.782 \mathrm{E}-05$ & $4.939 E+09$ & 0.053 \\
\hline $1 s 4 p^{3} P_{1}^{0}$ & $1 s 7 p^{3} P_{1}^{0}$ & $7.4094 \mathrm{E}+00$ & $3.166 \mathrm{E}-07$ & $1.282 \mathrm{E}+07$ & $7.3785 \mathrm{E}+00$ & $3.980 \mathrm{E}-07$ & $1.611 \mathrm{E}+07$ & 0.067 \\
\hline $1 s 4 p^{3} P_{2}^{o}$ & $1 s 7 p^{3} P_{1}^{o}$ & $7.6311 \mathrm{E}+00$ & $5.802 \mathrm{E}-05$ & $2.215 E+09$ & $7.5991 \mathrm{E}+00$ & $5.725 \mathrm{E}-05$ & $2.185 E+09$ & 0.017 \\
\hline $1 s 4 p^{1} P_{1}^{o}$ & $1 s 7 p^{3} P_{1}^{o}$ & $7.6504 \mathrm{E}+00$ & $5.135 \mathrm{E}-05$ & $1.951 \mathrm{E}+09$ & $7.6186 \mathrm{E}+00$ & $4.936 \mathrm{E}-05$ & $1.874 \mathrm{E}+09$ & 0.007 \\
\hline $1 s 4 f^{3} F_{3}^{o}$ & $1 s 7 p^{3} P_{1}^{o}$ & $7.7217 \mathrm{E}+00$ & $1.128 \mathrm{E}-05$ & $4.205 E+08$ & $7.6895 \mathrm{E}+00$ & $1.078 \mathrm{E}-05$ & $4.020 E+08$ & 0.032 \\
\hline $1 s 4 f^{3} F_{2}^{o}$ & $1 s 7 p^{3} P_{1}^{o}$ & $7.7232 \mathrm{E}+00$ & $3.454 \mathrm{E}-06$ & $1.288 \mathrm{E}+08$ & $7.6910 \mathrm{E}+00$ & $3.342 \mathrm{E}-06$ & $1.245 \mathrm{E}+08$ & 0.033 \\
\hline $1 s 4 f^{1} F_{3}^{o}$ & $1 s 7 p^{3} P_{1}^{0}$ & $7.7595 \mathrm{E}+00$ & $1.761 \mathrm{E}-08$ & $6.504 \mathrm{E}+05$ & $7.7270 \mathrm{E}+00$ & $2.587 \mathrm{E}-08$ & $9.555 \mathrm{E}+05$ & 0.017 \\
\hline $1 s 5 p^{3} P_{1}^{o}$ & $1 s 7 p^{3} P_{1}^{0}$ & $1.5987 \mathrm{E}+01$ & $5.833 \mathrm{E}-07$ & $5.075 E+06$ & $1.5920 \mathrm{E}+01$ & $7.664 \mathrm{E}-07$ & $6.666 \mathrm{E}+06$ & 0.015 \\
\hline $1 s 5 p^{3} P_{2}^{o}$ & $1 s 7 p^{3} P_{1}^{o}$ & $1.6515 \mathrm{E}+01$ & $1.159 \mathrm{E}-04$ & $9.447 \mathrm{E}+08$ & $1.6446 \mathrm{E}+01$ & $1.167 \mathrm{E}-04$ & $9.520 \mathrm{E}+08$ & 0.003 \\
\hline $1 s 5 p^{1} P_{1}^{o}$ & $1 s 7 p^{3} P_{1}^{o}$ & $1.6561 \mathrm{E}+01$ & $1.036 \mathrm{E}-04$ & $8.397 E+08$ & $1.6492 \mathrm{E}+01$ & $1.010 \mathrm{E}-04$ & $8.193 E+08$ & 0.006 \\
\hline $1 s 5 f^{3} F_{3}^{o}$ & $1 s 7 p^{3} P_{1}^{o}$ & $1.6732 \mathrm{E}+01$ & $4.609 \mathrm{E}-05$ & $3.661 \mathrm{E}+08$ & $1.6664 \mathrm{E}+01$ & $4.535 \mathrm{E}-05$ & $3.601 E+08$ & 0.002 \\
\hline $1 s 5 f^{3} F_{2}^{o}$ & $1 s 7 p^{3} P_{1}^{0}$ & $1.6736 \mathrm{E}+01$ & $1.410 \mathrm{E}-05$ & $1.119 \mathrm{E}+08$ & $1.6667 \mathrm{E}+01$ & $1.404 \mathrm{E}-05$ & $1.114 \mathrm{E}+08$ & 0.002 \\
\hline $1 s 5 f^{1} F_{3}^{o}$ & $1 s 7 p^{3} P_{1}^{0}$ & $1.6823 \mathrm{E}+01$ & $7.450 \mathrm{E}-08$ & $5.853 E+05$ & $1.6754 \mathrm{E}+01$ & $1.071 \mathrm{E}-07$ & $8.412 E+05$ & 0.002 \\
\hline $1 s 6 p^{3} P_{1}^{0}$ & $1 s 7 p^{3} P_{1}^{o}$ & $4.2622 \mathrm{E}+01$ & $1.744 \mathrm{E}-06$ & $2.135 E+06$ & $4.2444 \mathrm{E}+01$ & $2.994 \mathrm{E}-06$ & $3.664 \mathrm{E}+06$ & 0.005 \\
\hline $1 s 6 p^{3} P_{2}^{o}$ & $1 s 7 p^{3} P_{1}^{o}$ & $4.4826 \mathrm{E}+01$ & $3.888 \mathrm{E}-04$ & $4.302 E+08$ & $4.4636 \mathrm{E}+01$ & $3.931 \mathrm{E}-04$ & $4.350 \mathrm{E}+08$ & 0.001 \\
\hline $1 s 6 p^{1} P_{1}^{o}$ & $1 s 7 p^{3} P_{1}^{0}$ & $4.5021 \mathrm{E}+01$ & $3.515 \mathrm{E}-04$ & $3.856 \mathrm{E}+08$ & $4.4809 \mathrm{E}+01$ & $3.422 \mathrm{E}-04$ & $3.757 \mathrm{E}+08$ & 0.000 \\
\hline $1 s 6 f^{3} F_{3}^{o}$ & $1 s 7 p^{3} P_{1}^{o}$ & $4.5759 \mathrm{E}+01$ & $2.138 \mathrm{E}-04$ & $2.270 \mathrm{E}+08$ & $4.5589 \mathrm{E}+01$ & $2.114 \mathrm{E}-04$ & $2.242 E+08$ & 0.002 \\
\hline $1 s 6 f^{3} F_{2}^{o}$ & $1 s 7 p^{3} P_{1}^{o}$ & $4.5775 \mathrm{E}+01$ & $6.539 \mathrm{E}-05$ & $6.939 E+07$ & $4.5589 \mathrm{E}+01$ & $6.507 \mathrm{E}-05$ & $6.902 E+07$ & 0.002 \\
\hline $1 s 6 f^{1} F_{3}^{o}$ & $1 s 7 p^{3} P_{1}^{o}$ & $4.6155 \mathrm{E}+01$ & $3.481 \mathrm{E}-07$ & $3.633 \mathrm{E}+05$ & $4.5969 \mathrm{E}+01$ & $4.043 \mathrm{E}-07$ & $4.218 \mathrm{E}+05$ & 0.001 \\
\hline $1 s 3 d^{3} D_{2}^{3}$ & $1 s 7 s^{1} S_{0}$ & $3.5373 \mathrm{E}+00$ & $4.635 \mathrm{E}-06$ & $2.471 E+09$ & $3.5228 \mathrm{E}+00$ & $4.652 \mathrm{E}-06$ & $2.479 E+09$ & 0.016 \\
\hline $1 s 3 d d^{1} D_{2}$ & $1 s 7 s^{1} S_{0}$ & $3.5757 \mathrm{E}+00$ & $7.727 \mathrm{E}-06$ & $4.031 \mathrm{E}+09$ & $3.5610 \mathrm{E}+00$ & $7.113 E-06$ & $3.709 E+09$ & 0.063 \\
\hline $1 s 4 d^{3} D_{2}$ & $1 s 7 s^{1} S_{0}$ & $7.6475 \mathrm{E}+00$ & $1.316 \mathrm{E}-05$ & $1.501 \mathrm{E}+09$ & $7.6177 \mathrm{E}+00$ & $1.309 \mathrm{E}-05$ & $1.492 \mathrm{E}+09$ & 0.007 \\
\hline $1 s 4 d{ }^{1} D_{2}$ & $1 s 7 s^{1} S_{0}$ & $7.7235 \mathrm{E}+00$ & $2.094 \mathrm{E}-05$ & $2.341 \mathrm{E}+09$ & $7.6933 \mathrm{E}+00$ & $2.017 \mathrm{E}-05$ & $2.254 \mathrm{E}+09$ & 0.024 \\
\hline $1 s 5 d^{3} D_{2}$ & $1 s 7 s^{1} S_{0}$ & $1.6554 \mathrm{E}+01$ & $3.255 \mathrm{E}-05$ & $7.924 \mathrm{E}+08$ & $1.6495 E+01$ & $3.248 \mathrm{E}-05$ & $7.896 \mathrm{E}+08$ & 0.005 \\
\hline $1 s 5 d^{1} D_{2}$ & $1 s 7 s^{1} S_{0}$ & $1.6736 \mathrm{E}+01$ & $5.105 \mathrm{E}-05$ & $1.216 \mathrm{E}+09$ & $1.6677 \mathrm{E}+01$ & $5.040 \mathrm{E}-05$ & $1.198 \mathrm{E}+09$ & 0.006 \\
\hline $1 s 6 d^{3} D_{2}$ & $1 s 7 s^{1} S_{0}$ & $4.4992 \mathrm{E}+01$ & $1.160 \mathrm{E}-04$ & $3.821 \mathrm{E}+08$ & $4.4893 \mathrm{E}+01$ & $1.158 \mathrm{E}-04$ & $3.801 E+08$ & 0.003 \\
\hline $1 s 6 d^{1} D_{2}$ & $1 s 7 s^{1} S_{0}$ & $4.5777 \mathrm{E}+01$ & $1.847 \mathrm{E}-04$ & $5.878 E+08$ & $4.5651 \mathrm{E}+01$ & $1.878 \mathrm{E}-04$ & $5.962 E+08$ & 0.000 \\
\hline $1 s 2 p^{3} P_{2}^{o}$ & $1 s 7 p^{3} P_{0}^{o}$ & $1.3961 \mathrm{E}+00$ & $1.007 \mathrm{E}-05$ & $3.447 \mathrm{E}+10$ & $1.3903 \mathrm{E}+00$ & $1.030 \mathrm{E}-05$ & $3.524 \mathrm{E}+10$ & 0.058 \\
\hline $1 s 3 p^{3} P_{2}^{o}$ & $1 s 7 p^{3} P_{0}^{o}$ & $3.5287 \mathrm{E}+00$ & $2.240 \mathrm{E}-05$ & $1.200 \mathrm{E}+10$ & $3.5145 \mathrm{E}+00$ & $2.021 \mathrm{E}-05$ & $1.082 \mathrm{E}+10$ & 0.080 \\
\hline $1 s 4 p^{3} P_{2}^{o}$ & $1 s 7 p^{3} P_{0}^{o}$ & $7.6306 \mathrm{E}+00$ & $3.690 \mathrm{E}-05$ & $4.228 \mathrm{E}+09$ & $7.6005 E+00$ & $3.577 \mathrm{E}-05$ & $4.096 E+09$ & 0.017 \\
\hline $1 s 4 f^{3} F_{2}^{o}$ & $1 s 7 p^{3} P_{0}^{o}$ & $7.7227 \mathrm{E}+00$ & $4.908 \mathrm{E}-06$ & $5.489 E+08$ & $7.6924 \mathrm{E}+00$ & $4.701 \mathrm{E}-06$ & $5.254 \mathrm{E}+08$ & 0.033 \\
\hline $1 s 5 p^{3} P_{2}^{o}$ & $1 s 7 p^{3} P_{0}^{o}$ & $1.6512 \mathrm{E}+01$ & $7.368 \mathrm{E}-05$ & $1.802 \mathrm{E}+09$ & $1.6452 \mathrm{E}+01$ & $7.311 \mathrm{E}-05$ & $1.786 \mathrm{E}+09$ & 0.003 \\
\hline
\end{tabular}


Table 4 (continued)

\begin{tabular}{|c|c|c|c|c|c|c|c|c|}
\hline \multirow[t]{2}{*}{ Lower } & \multirow[t]{2}{*}{ Upper } & \multicolumn{3}{|l|}{ GRASP2K } & \multicolumn{3}{|l|}{ FAC } & \multirow[t]{2}{*}{$d T$} \\
\hline & & $\lambda(\AA)$ & $g f$ & $A\left(\mathrm{~s}^{-1}\right)$ & $\lambda(\AA)$ & $g f$ & $A\left(\mathrm{~s}^{-1}\right)$ & \\
\hline $1 s 5 f^{3} F_{2}^{o}$ & $1 s 7 p^{3} P_{0}^{o}$ & $1.6733 \mathrm{E}+01$ & $2.004 \mathrm{E}-05$ & $4.773 \mathrm{E}+08$ & $1.6674 \mathrm{E}+01$ & $1.976 \mathrm{E}-05$ & $4.701 \mathrm{E}+08$ & 0.002 \\
\hline $1 s 6 p^{3} P_{2}^{o}$ & $1 s 7 p^{3} P_{0}^{0}$ & $4.4808 \mathrm{E}+01$ & $2.469 \mathrm{E}-04$ & $8.203 E+08$ & $4.4683 \mathrm{E}+01$ & $2.466 \mathrm{E}-04$ & $8.170 E+08$ & 0.001 \\
\hline $1 s 6 f^{3} F_{2}^{o}$ & $1 s 7 p^{3} P_{0}^{o}$ & $4.5756 \mathrm{E}+01$ & $9.289 \mathrm{E}-05$ & $2.959 E+08$ & $4.5638 \mathrm{E}+01$ & $9.204 \mathrm{E}-05$ & $2.922 \mathrm{E}+08$ & 0.002 \\
\hline $1 s 2 p^{3} P_{1}^{o}$ & $1 s 7 p^{3} P_{2}^{o}$ & $1.3363 \mathrm{E}+00$ & $1.951 \mathrm{E}-05$ & $1.457 \mathrm{E}+10$ & $1.3308 \mathrm{E}+00$ & $2.048 \mathrm{E}-05$ & $1.529 \mathrm{E}+10$ & 0.042 \\
\hline $1 s 2 p^{3} P_{0}^{o}$ & $1 s 7 p^{3} P_{2}^{o}$ & $1.3372 \mathrm{E}+00$ & $1.256 \mathrm{E}-05$ & $9.370 \mathrm{E}+09$ & $1.3316 \mathrm{E}+00$ & $1.289 \mathrm{E}-05$ & $9.615 E+09$ & 0.014 \\
\hline $1 s 2 p^{3} P_{2}^{o}$ & $1 s 7 p^{3} P_{2}^{o}$ & $1.3947 \mathrm{E}+00$ & $1.980 \mathrm{E}-05$ & $1.358 \mathrm{E}+10$ & $1.3889 \mathrm{E}+00$ & $2.069 \mathrm{E}-05$ & $1.419 \mathrm{E}+10$ & 0.052 \\
\hline $1 s 2 p^{1} P_{1}^{o}$ & $1 s 7 p^{3} P_{2}^{o}$ & $1.4002 \mathrm{E}+00$ & $7.809 \mathrm{E}-06$ & $5.313 E+09$ & $1.3944 \mathrm{E}+00$ & $7.795 \mathrm{E}-06$ & $5.303 E+09$ & 0.015 \\
\hline $1 s 3 p^{3} P_{1}^{o}$ & $1 s 7 p^{3} P_{2}^{o}$ & $3.4080 \mathrm{E}+00$ & $4.148 \mathrm{E}-05$ & $4.764 \mathrm{E}+09$ & $3.3940 \mathrm{E}+00$ & $3.776 \mathrm{E}-05$ & $4.336 \mathrm{E}+09$ & 0.093 \\
\hline $1 s 3 p^{3} P_{0}^{o}$ & $1 s 7 p^{3} P_{2}^{o}$ & $3.4095 \mathrm{E}+00$ & $2.597 \mathrm{E}-05$ & $2.980 E+09$ & $3.3954 \mathrm{E}+00$ & $2.386 \mathrm{E}-05$ & $2.738 \mathrm{E}+09$ & 0.083 \\
\hline $1 s 3 p^{3} P_{2}^{o}$ & $1 s 7 p^{3} P_{2}^{o}$ & $3.5199 \mathrm{E}+00$ & $4.316 \mathrm{E}-05$ & $4.648 \mathrm{E}+09$ & $3.5053 \mathrm{E}+00$ & $3.919 \mathrm{E}-05$ & $4.219 E+09$ & 0.088 \\
\hline $1 s 3 p{ }^{1} P_{1}^{o}$ & $1 s 7 p^{3} P_{2}^{o}$ & $3.5298 \mathrm{E}+00$ & $1.624 \mathrm{E}-05$ & $1.739 E+09$ & $3.5153 \mathrm{E}+00$ & $1.492 \mathrm{E}-05$ & $1.597 \mathrm{E}+09$ & 0.074 \\
\hline $1 s 4 p^{3} P_{1}^{o}$ & $1 s 7 p^{3} P_{2}^{o}$ & $7.3699 \mathrm{E}+00$ & $6.450 \mathrm{E}-05$ & $1.584 \mathrm{E}+09$ & $7.3391 \mathrm{E}+00$ & $6.330 \mathrm{E}-05$ & $1.554 \mathrm{E}+09$ & 0.017 \\
\hline $1 s 4 p^{3} P_{0}^{0}$ & $1 s 7 p^{3} P_{2}^{o}$ & $7.3726 \mathrm{E}+00$ & $4.081 \mathrm{E}-05$ & $1.002 \mathrm{E}+09$ & $7.3418 \mathrm{E}+00$ & $4.004 \mathrm{E}-05$ & $9.826 \mathrm{E}+08$ & 0.013 \\
\hline $1 s 4 p^{3} P_{2}^{o}$ & $1 s 7 p^{3} P_{2}^{o}$ & $7.5892 \mathrm{E}+00$ & $6.902 \mathrm{E}-05$ & $1.599 \mathrm{E}+09$ & $7.5574 \mathrm{E}+00$ & $6.734 \mathrm{E}-05$ & $1.559 \mathrm{E}+09$ & 0.019 \\
\hline $1 s 4 p^{1} P_{1}^{o}$ & $1 s 7 p^{3} P_{2}^{0}$ & $7.6083 \mathrm{E}+00$ & $2.631 \mathrm{E}-05$ & $6.063 E+08$ & $7.5766 \mathrm{E}+00$ & $2.576 \mathrm{E}-05$ & $5.936 \mathrm{E}+08$ & 0.014 \\
\hline $1 s 4 f^{3} F_{3}^{o}$ & $1 s 7 p^{3} P_{2}^{o}$ & $7.6787 \mathrm{E}+00$ & $2.525 \mathrm{E}-06$ & $5.713 E+07$ & $7.6467 \mathrm{E}+00$ & $2.419 \mathrm{E}-06$ & $5.473 E+07$ & 0.035 \\
\hline $1 s 4 f^{3} F_{2}^{0}$ & $1 s 7 p^{3} P_{2}^{o}$ & $7.6803 \mathrm{E}+00$ & $6.368 \mathrm{E}-07$ & $1.440 \mathrm{E}+07$ & $7.6482 \mathrm{E}+00$ & $6.023 \mathrm{E}-07$ & $1.362 \mathrm{E}+07$ & 0.038 \\
\hline $1 s 4 f^{3} F_{4}^{o}$ & $1 s 7 p^{3} P_{2}^{o}$ & $7.7150 \mathrm{E}+00$ & $1.674 \mathrm{E}-05$ & $3.753 E+08$ & $7.6827 \mathrm{E}+00$ & $1.660 \mathrm{E}-05$ & $3.721 E+08$ & 0.011 \\
\hline $1 s 4 f^{1} F_{3}^{o}$ & $1 s 7 p^{3} P_{2}^{o}$ & $7.7161 \mathrm{E}+00$ & $1.866 \mathrm{E}-06$ & $4.181 \mathrm{E}+07$ & $7.6838 \mathrm{E}+00$ & $1.853 \mathrm{E}-06$ & $4.152 \mathrm{E}+07$ & 0.012 \\
\hline $1 s 5 p^{3} P_{1}^{o}$ & $1 s 7 p^{3} P_{2}^{o}$ & $1.5804 \mathrm{E}+01$ & $1.192 \mathrm{E}-04$ & $6.370 \mathrm{E}+08$ & $1.5738 \mathrm{E}+01$ & $1.193 \mathrm{E}-04$ & $6.376 \mathrm{E}+08$ & 0.006 \\
\hline $1 s 5 p^{3} P_{0}^{o}$ & $1 s 7 p^{3} P_{2}^{o}$ & $1.5810 \mathrm{E}+01$ & $7.580 \mathrm{E}-05$ & $4.046 \mathrm{E}+08$ & $1.5744 \mathrm{E}+01$ & $7.546 \mathrm{E}-05$ & $4.027 E+08$ & 0.007 \\
\hline $1 s 5 p^{3} P_{2}^{o}$ & $1 s 7 p^{3} P_{2}^{o}$ & $1.6320 \mathrm{E}+01$ & $1.324 \mathrm{E}-04$ & $6.632 E+08$ & $1.6251 \mathrm{E}+01$ & $1.318 \mathrm{E}-04$ & $6.605 E+08$ & 0.003 \\
\hline $1 s 5 p^{1} P_{1}^{o}$ & $1 s 7 p^{3} P_{2}^{o}$ & $1.6364 \mathrm{E}+01$ & $5.081 \mathrm{E}-05$ & $2.531 \mathrm{E}+08$ & $1.6297 \mathrm{E}+01$ & $5.059 \mathrm{E}-05$ & $2.519 E+08$ & 0.005 \\
\hline $1 s 5 f^{3} F_{3}^{o}$ & $1 s 7 p^{3} P_{2}^{o}$ & $1.6532 \mathrm{E}+01$ & $1.040 \mathrm{E}-05$ & $5.079 E+07$ & $1.6464 \mathrm{E}+01$ & $1.026 \mathrm{E}-05$ & $5.006 \mathrm{E}+07$ & 0.002 \\
\hline $1 s 5 f^{3} F_{2}^{o}$ & $1 s 7 p^{3} P_{2}^{o}$ & $1.6535 \mathrm{E}+01$ & $2.619 \mathrm{E}-06$ & $1.278 \mathrm{E}+07$ & $1.6468 \mathrm{E}+01$ & $2.556 \mathrm{E}-06$ & $1.246 \mathrm{E}+07$ & 0.003 \\
\hline $1 s 5 f^{3} F_{4}^{0}$ & $1 s 7 p^{3} P_{2}^{o}$ & $1.6618 \mathrm{E}+01$ & $7.173 \mathrm{E}-05$ & $3.465 \mathrm{E}+08$ & $1.6550 \mathrm{E}+01$ & $7.050 \mathrm{E}-05$ & $3.404 \mathrm{E}+08$ & 0.000 \\
\hline $1 s 5 f^{1} F_{3}^{o}$ & $1 s 7 p^{3} P_{2}^{o}$ & $1.6621 \mathrm{E}+01$ & $7.995 \mathrm{E}-06$ & $3.861 \mathrm{E}+07$ & $1.6552 \mathrm{E}+01$ & $7.865 \mathrm{E}-06$ & $3.797 E+07$ & 0.000 \\
\hline $1 s 6 p^{3} P_{1}^{o}$ & $1 s 7 p^{3} P_{2}^{o}$ & $4.1345 \mathrm{E}+01$ & $3.330 \mathrm{E}-04$ & $2.599 \mathrm{E}+08$ & $4.1174 \mathrm{E}+01$ & $3.369 \mathrm{E}-04$ & $2.629 E+08$ & 0.002 \\
\hline $1 s 6 p^{3} P_{0}^{0}$ & $1 s 7 p^{3} P_{2}^{o}$ & $4.1369 \mathrm{E}+01$ & $2.124 \mathrm{E}-04$ & $1.656 \mathrm{E}+08$ & $4.1110 \mathrm{E}+01$ & $2.102 \mathrm{E}-04$ & $1.645 \mathrm{E}+08$ & 0.002 \\
\hline $1 s 6 p^{3} P_{2}^{o}$ & $1 s 7 p^{3} P_{2}^{o}$ & $4.3416 \mathrm{E}+01$ & $4.033 \mathrm{E}-04$ & $2.854 \mathrm{E}+08$ & $4.3234 \mathrm{E}+01$ & $4.015 \mathrm{E}-04$ & $2.841 \mathrm{E}+08$ & 0.002 \\
\hline $1 s 6 p^{1} P_{1}^{o}$ & $1 s 7 p^{3} P_{2}^{o}$ & $4.3599 \mathrm{E}+01$ & $1.561 \mathrm{E}-04$ & $1.096 \mathrm{E}+08$ & $4.3396 \mathrm{E}+01$ & $1.504 \mathrm{E}-04$ & $1.056 \mathrm{E}+08$ & 0.001 \\
\hline $1 s 6 f^{3} F_{3}^{0}$ & $1 s 7 p^{3} P_{2}^{o}$ & $4.4291 \mathrm{E}+01$ & $4.633 \mathrm{E}-05$ & $3.151 \mathrm{E}+07$ & $4.4127 \mathrm{E}+01$ & $4.585 \mathrm{E}-05$ & $3.114 \mathrm{E}+07$ & 0.002 \\
\hline $1 s 6 f^{3} F_{2}^{0}$ & $1 s 7 p^{3} P_{2}^{o}$ & $4.4306 \mathrm{E}+01$ & $1.166 \mathrm{E}-05$ & $7.924 \mathrm{E}+06$ & $4.4127 \mathrm{E}+01$ & $1.145 \mathrm{E}-05$ & $7.778 \mathrm{E}+06$ & 0.002 \\
\hline $1 s 6 f^{3} F_{4}^{o}$ & $1 s 7 p^{3} P_{2}^{o}$ & $4.4650 \mathrm{E}+01$ & $3.218 \mathrm{E}-04$ & $2.154 \mathrm{E}+08$ & $4.4483 \mathrm{E}+01$ & $3.160 \mathrm{E}-04$ & $2.112 \mathrm{E}+08$ & 0.002 \\
\hline $1 s 6 f^{1} F_{3}^{o}$ & $1 s 7 p^{3} P_{2}^{o}$ & $4.4661 \mathrm{E}+01$ & $3.588 \mathrm{E}-05$ & $2.400 \mathrm{E}+07$ & $4.4483 \mathrm{E}+01$ & $3.507 \mathrm{E}-05$ & $2.344 \mathrm{E}+07$ & 0.002 \\
\hline $1 s 6 h^{3} H_{4}^{0}$ & $1 s 7 p^{3} P_{2}^{0}$ & $4.4873 \mathrm{E}+01$ & $1.000 \mathrm{E}-11$ & $6.626 \mathrm{E}+00$ & & & & 0.004 \\
\hline $1 s 7 p^{3} P_{1}^{o}$ & $1 s 7 p^{3} P_{2}^{o}$ & $1.3800 \mathrm{E}+03$ & $1.615 \mathrm{E}-07$ & $1.131 \mathrm{E}+02$ & $1.3760 \mathrm{E}+03$ & $1.671 \mathrm{E}-07$ & $1.167 \mathrm{E}+02$ & 0.050 \\
\hline $1 s 7 p^{3} P_{0}^{0}$ & $1 s 7 p^{3} P_{2}^{o}$ & $1.3975 \mathrm{E}+03$ & $9.892 \mathrm{E}-08$ & $6.757 \mathrm{E}+01$ & $1.3328 \mathrm{E}+03$ & $1.150 \mathrm{E}-07$ & $8.569 \mathrm{E}+01$ & 0.056 \\
\hline $1 s^{2}{ }^{1} s_{0}$ & $1 s 7 d^{3} D_{2}$ & $3.1400 \mathrm{E}-01$ & $8.874 \mathrm{E}-05$ & $1.201 \mathrm{E}+12$ & $3.1269 \mathrm{E}-01$ & $8.899 \mathrm{E}-05$ & $1.203 E+12$ & 0.003 \\
\hline $1 s 2 s^{3} S_{1}$ & $1 s 7 d^{3} D_{2}$ & $1.3252 \mathrm{E}+00$ & $5.634 \mathrm{E}-06$ & $4.280 \mathrm{E}+09$ & $1.3196 \mathrm{E}+00$ & $4.807 \mathrm{E}-06$ & $3.652 E+09$ & 0.011 \\
\hline $1 s 2 s{ }^{1} S_{0}$ & $1 s 7 d^{3} D_{2}$ & $1.3374 \mathrm{E}+00$ & $2.905 \mathrm{E}-06$ & $2.166 \mathrm{E}+09$ & $1.3319 \mathrm{E}+00$ & $2.936 \mathrm{E}-06$ & $2.189 E+09$ & 0.005 \\
\hline $1 s 3 s^{3} s_{1}$ & $1 s 7 d^{3} D_{2}$ & $3.3879 \mathrm{E}+00$ & $3.868 \mathrm{E}-05$ & $4.496 \mathrm{E}+09$ & $3.3736 \mathrm{E}+00$ & $3.838 \mathrm{E}-05$ & $4.460 \mathrm{E}+09$ & 0.019 \\
\hline $1 s 3 s^{1} S_{0}$ & $1 s 7 d^{3} D_{2}$ & $3.4088 \mathrm{E}+00$ & $2.598 \mathrm{E}-05$ & $2.982 E+09$ & $3.3947 \mathrm{E}+00$ & $2.385 \mathrm{E}-05$ & $2.738 \mathrm{E}+09$ & 0.083 \\
\hline $1 s 3 d^{3} D_{2}$ & $1 s 7 d^{3} D_{2}$ & $3.5276 \mathrm{E}+00$ & $1.899 \mathrm{E}-05$ & $2.036 \mathrm{E}+09$ & $3.5127 \mathrm{E}+00$ & $1.903 \mathrm{E}-05$ & $2.040 \mathrm{E}+09$ & 0.014 \\
\hline $1 s 3 d^{3} D_{1}$ & $1 s 7 d^{3} D_{2}$ & $3.5291 \mathrm{E}+00$ & $8.666 \mathrm{E}-06$ & $9.282 E+08$ & $3.5142 \mathrm{E}+00$ & $8.452 \mathrm{E}-06$ & $9.053 E+08$ & 0.033 \\
\hline $1 s 3 d^{3} D_{3}$ & $1 s 7 d^{3} D_{2}$ & $3.5644 \mathrm{E}+00$ & $8.974 \mathrm{E}-06$ & $9.422 \mathrm{E}+08$ & $3.5492 \mathrm{E}+00$ & $9.129 \mathrm{E}-06$ & $9.585 E+08$ & 0.026 \\
\hline $1 s 3 d d^{1} D_{2}$ & $1 s 7 d^{3} D_{2}$ & $3.5659 \mathrm{E}+00$ & $2.321 \mathrm{E}-06$ & $2.435 \mathrm{E}+08$ & $3.5507 \mathrm{E}+00$ & $2.101 \mathrm{E}-06$ & $2.204 E+08$ & 0.049 \\
\hline $1 s 4 s^{3} S_{1}$ & $1 s 7 d^{3} D_{2}$ & $7.3299 \mathrm{E}+00$ & $8.891 \mathrm{E}-05$ & $2.208 E+09$ & $7.2988 \mathrm{E}+00$ & $8.993 E-05$ & $2.233 E+09$ & 0.018 \\
\hline $1 s 4 s{ }^{1} S_{0}$ & $1 s 7 d^{3} D_{2}$ & $7.3694 \mathrm{E}+00$ & $6.004 \mathrm{E}-05$ & $1.475 \mathrm{E}+09$ & $7.3385 \mathrm{E}+00$ & $5.614 \mathrm{E}-05$ & $1.378 \mathrm{E}+09$ & 0.065 \\
\hline $1 s 4 d^{3} D_{2}$ & $1 s 7 d^{3} D_{2}$ & $7.6027 \mathrm{E}+00$ & $4.717 \mathrm{E}-05$ & $1.089 \mathrm{E}+09$ & $7.5707 \mathrm{E}+00$ & $4.709 \mathrm{E}-05$ & $1.086 \mathrm{E}+09$ & 0.005 \\
\hline $1 s 4 d^{3} D_{1}$ & $1 s 7 d^{3} D_{2}$ & $7.6055 \mathrm{E}+00$ & $2.106 \mathrm{E}-05$ & $4.857 \mathrm{E}+08$ & $7.5734 \mathrm{E}+00$ & $2.095 \mathrm{E}-05$ & $4.831 \mathrm{E}+08$ & 0.015 \\
\hline $1 s 4 d^{3} D_{3}$ & $1 s 7 d^{3} D_{2}$ & $7.6748 \mathrm{E}+00$ & $2.282 \mathrm{E}-05$ & $5.168 E+08$ & $7.6422 \mathrm{E}+00$ & $2.304 \mathrm{E}-05$ & $5.219 E+08$ & 0.009 \\
\hline $1 s 4 d^{1} D_{2}$ & $1 s 7 d^{3} D_{2}$ & $7.6778 \mathrm{E}+00$ & $5.714 \mathrm{E}-06$ & $1.293 \mathrm{E}+08$ & $7.6453 \mathrm{E}+00$ & $5.373 \mathrm{E}-06$ & $1.216 \mathrm{E}+08$ & 0.022 \\
\hline $1 s 5 s^{3} S_{1}$ & $1 s 7 d^{3} D_{2}$ & $1.5705 \mathrm{E}+01$ & $1.967 \mathrm{E}-04$ & $1.064 \mathrm{E}+09$ & $1.5637 \mathrm{E}+01$ & $1.998 \mathrm{E}-04$ & $1.080 \mathrm{E}+09$ & 0.010 \\
\hline $1 s 5 s{ }^{1} S_{0}$ & $1 s 7 d^{3} D_{2}$ & $1.5796 \mathrm{E}+01$ & $1.300 \mathrm{E}-04$ & $6.949 E+08$ & $1.5728 \mathrm{E}+01$ & $1.247 \mathrm{E}-04$ & $6.669 E+08$ & 0.032 \\
\hline $1 s 5 d^{3} D_{2}$ & $1 s 7 d^{3} D_{2}$ & $1.6345 \mathrm{E}+01$ & $1.087 \mathrm{E}-04$ & $5.426 \mathrm{E}+08$ & $1.6276 \mathrm{E}+01$ & $1.083 \mathrm{E}-04$ & $5.410 \mathrm{E}+08$ & 0.002 \\
\hline $1 s 5 d^{3} D_{1}$ & $1 s 7 d^{3} D_{2}$ & $1.6352 \mathrm{E}+01$ & $4.805 \mathrm{E}-05$ & $2.397 \mathrm{E}+08$ & $1.6283 \mathrm{E}+01$ & $4.822 \mathrm{E}-05$ & $2.406 \mathrm{E}+08$ & 0.006 \\
\hline $1 s 5 d^{3} D_{3}$ & $1 s 7 d^{3} D_{2}$ & $1.6516 \mathrm{E}+01$ & $5.357 \mathrm{E}-05$ & $2.620 \mathrm{E}+08$ & $1.6446 \mathrm{E}+01$ & $5.404 \mathrm{E}-05$ & $2.643 E+08$ & 0.003 \\
\hline $1 s 5 d{ }^{1} D_{2}$ & $1 s 7 d^{3} D_{2}$ & $1.6523 \mathrm{E}+01$ & $1.324 \mathrm{E}-05$ & $6.468 \mathrm{E}+07$ & $1.6453 \mathrm{E}+01$ & $1.267 \mathrm{E}-05$ & $6.194 E+07$ & 0.008 \\
\hline $1 s 5 g^{3} G_{4}$ & $1 s 7 d^{3} D_{2}$ & $1.6605 \mathrm{E}+01$ & $1.575 \mathrm{E}-05$ & $7.622 \mathrm{E}+07$ & $1.6532 \mathrm{E}+01$ & $1.579 \mathrm{E}-05$ & $7.646 \mathrm{E}+07$ & 0.004 \\
\hline $1 s 5 g{ }^{3} G_{3}$ & $1 s 7 d^{3} D_{2}$ & $1.6607 \mathrm{E}+01$ & $1.787 \mathrm{E}-06$ & $8.644 \mathrm{E}+06$ & $1.6534 \mathrm{E}+01$ & $1.793 \mathrm{E}-06$ & $8.675 E+06$ & 0.006 \\
\hline $1 s 5 g{ }^{1} G_{4}$ & $1 s 7 d^{3} D_{2}$ & $1.6658 \mathrm{E}+01$ & $1.251 \mathrm{E}-09$ & $6.016 \mathrm{E}+03$ & & & & 0.062 \\
\hline $1 s 6 s^{3} S_{1}$ & $1 s 7 d^{3} D_{2}$ & $4.0924 \mathrm{E}+01$ & $6.099 \mathrm{E}-04$ & $4.858 \mathrm{E}+08$ & $4.0701 \mathrm{E}+01$ & $6.286 \mathrm{E}-04$ & $5.019 E+08$ & 0.005 \\
\hline $1 s 6 s{ }^{1} S_{0}$ & $1 s 7 d^{3} D_{2}$ & $4.1279 \mathrm{E}+01$ & $4.030 \mathrm{E}-04$ & $3.155 E+08$ & $4.1000 \mathrm{E}+01$ & $3.906 \mathrm{E}-04$ & $3.073 E+08$ & 0.010 \\
\hline $1 s 6 d^{3} D_{2}$ & $1 s 7 d^{3} D_{2}$ & $4.3482 \mathrm{E}+01$ & $3.650 \mathrm{E}-04$ & $2.575 E+08$ & $4.3306 \mathrm{E}+01$ & $3.626 \mathrm{E}-04$ & $2.557 \mathrm{E}+08$ & 0.000 \\
\hline $1 s 6 d^{3} D_{1}$ & $1 s 7 d^{3} D_{2}$ & $4.3508 \mathrm{E}+01$ & $1.610 \mathrm{E}-04$ & $1.134 \mathrm{E}+08$ & $4.3299 \mathrm{E}+01$ & $1.611 \mathrm{E}-04$ & $1.136 \mathrm{E}+08$ & 0.002 \\
\hline $1 s 6 d^{3} D_{3}$ & $1 s 7 d^{3} D_{2}$ & $4.4184 \mathrm{E}+01$ & $1.862 \mathrm{E}-04$ & $1.273 \mathrm{E}+08$ & $4.4000 \mathrm{E}+01$ & $1.879 \mathrm{E}-04$ & $1.284 \mathrm{E}+08$ & 0.001 \\
\hline $1 s 6 d^{1} D_{2}$ & $1 s 7 d^{3} D_{2}$ & $4.4215 \mathrm{E}+01$ & $4.585 \mathrm{E}-05$ & $3.129 \mathrm{E}+07$ & $4.4010 \mathrm{E}+01$ & $4.550 \mathrm{E}-05$ & $3.107 \mathrm{E}+07$ & 0.002 \\
\hline $1 s 6 g{ }^{3} G_{4}$ & $1 s 7 d^{3} D_{2}$ & $4.4555 \mathrm{E}+01$ & $1.252 \mathrm{E}-04$ & $8.413 E+07$ & $4.4353 \mathrm{E}+01$ & $1.252 \mathrm{E}-04$ & $8.424 \mathrm{E}+07$ & 0.001 \\
\hline $1 s 6 g{ }^{3} G_{3}$ & $1 s 7 d^{3} D_{2}$ & $4.4564 \mathrm{E}+01$ & $1.411 \mathrm{E}-05$ & $9.479 \mathrm{E}+06$ & $4.4353 \mathrm{E}+01$ & $1.421 \mathrm{E}-05$ & $9.558 \mathrm{E}+06$ & 0.001 \\
\hline $1 s 6 g{ }^{1} G_{4}$ & $1 s 7 d^{3} D_{2}$ & $4.4778 \mathrm{E}+01$ & $9.656 \mathrm{E}-09$ & $6.424 \mathrm{E}+03$ & & & & 0.020 \\
\hline
\end{tabular}


Table 4 (continued)

\begin{tabular}{|c|c|c|c|c|c|c|c|c|}
\hline \multirow[t]{2}{*}{ Lower } & \multirow[t]{2}{*}{ Upper } & \multicolumn{3}{|l|}{ GRASP2K } & \multicolumn{3}{|l|}{ FAC } & \multirow[t]{2}{*}{$d T$} \\
\hline & & $\lambda(\AA)$ & $g f$ & $A\left(\mathrm{~s}^{-1}\right)$ & $\lambda(\AA)$ & $g f$ & $A\left(\mathrm{~s}^{-1}\right)$ & \\
\hline $1 s 7 s^{3} S_{1}$ & $1 s 7 d^{3} D_{2}$ & $1.1072 \mathrm{E}+03$ & $2.944 \mathrm{E}-07$ & $3.203 E+02$ & $1.0773 \mathrm{E}+03$ & $3.188 \mathrm{E}-07$ & $3.634 \mathrm{E}+02$ & 0.006 \\
\hline $1 s 7 s{ }^{1} S_{0}$ & $1 s 7 d^{3} D_{2}$ & $1.2957 \mathrm{E}+03$ & $1.189 \mathrm{E}-07$ & $9.449 \mathrm{E}+01$ & $1.2249 \mathrm{E}+03$ & $1.375 \mathrm{E}-07$ & $1.212 \mathrm{E}+02$ & 0.126 \\
\hline $1 s 2 s^{3} S_{1}$ & $1 s 7 d^{3} D_{1}$ & $1.3252 \mathrm{E}+00$ & $5.552 \mathrm{E}-06$ & $7.029 \mathrm{E}+09$ & $1.3196 \mathrm{E}+00$ & $4.696 \mathrm{E}-06$ & $5.945 E+09$ & 0.006 \\
\hline $1 s 3 s^{3} S_{1}$ & $1 s 7 d^{3} D_{1}$ & $3.3878 \mathrm{E}+00$ & $3.820 \mathrm{E}-05$ & $7.401 \mathrm{E}+09$ & $3.3737 \mathrm{E}+00$ & $3.769 \mathrm{E}-05$ & $7.300 \mathrm{E}+09$ & 0.030 \\
\hline $1 s 3 d^{3} D_{2}$ & $1 s 7 d^{3} D_{1}$ & $3.5275 \mathrm{E}+00$ & $8.231 \mathrm{E}-06$ & $1.471 \mathrm{E}+09$ & $3.5127 \mathrm{E}+00$ & $8.300 \mathrm{E}-06$ & $1.483 \mathrm{E}+09$ & 0.022 \\
\hline $1 s 3 d^{3} D_{1}$ & $1 s 7 d^{3} D_{1}$ & $3.5290 \mathrm{E}+00$ & $8.464 \mathrm{E}-06$ & $1.511 \mathrm{E}+09$ & $3.5142 \mathrm{E}+00$ & $8.198 \mathrm{E}-06$ & $1.463 \mathrm{E}+09$ & 0.028 \\
\hline $1 s 3 d^{3} D_{3}$ & $1 s 7 d^{3} D_{1}$ & $3.5643 \mathrm{E}+00$ & $1.509 \mathrm{E}-06$ & $2.641 \mathrm{E}+08$ & $3.5493 \mathrm{E}+00$ & $1.491 \mathrm{E}-06$ & $2.609 \mathrm{E}+08$ & 0.001 \\
\hline $1 s 3 d^{1} D_{2}$ & $1 s 7 d^{3} D_{1}$ & $3.5657 \mathrm{E}+00$ & $5.413 \mathrm{E}-06$ & $9.465 \mathrm{E}+08$ & $3.5507 \mathrm{E}+00$ & $5.166 \mathrm{E}-06$ & $9.033 E+08$ & 0.024 \\
\hline $1 s 4 s^{3} S_{1}$ & $1 s 7 d^{3} D_{1}$ & $7.3294 \mathrm{E}+00$ & $8.823 \mathrm{E}-05$ & $3.652 \mathrm{E}+09$ & $7.2989 \mathrm{E}+00$ & $8.841 \mathrm{E}-05$ & $3.658 \mathrm{E}+09$ & 0.022 \\
\hline $1 s 4 d^{3} D_{2}$ & $1 s 7 d^{3} D_{1}$ & $7.6021 \mathrm{E}+00$ & $2.061 \mathrm{E}-05$ & $7.929 \mathrm{E}+08$ & $7.5708 \mathrm{E}+00$ & $2.062 \mathrm{E}-05$ & $7.933 E+08$ & 0.010 \\
\hline $1 s 4 d^{3} D_{1}$ & $1 s 7 d^{3} D_{1}$ & $7.6050 \mathrm{E}+00$ & $2.052 \mathrm{E}-05$ & $7.888 \mathrm{E}+08$ & $7.5736 \mathrm{E}+00$ & $2.029 \mathrm{E}-05$ & $7.799 E+08$ & 0.011 \\
\hline $1 s 4 d^{3} D_{3}$ & $1 s 7 d^{3} D_{1}$ & $7.6743 \mathrm{E}+00$ & $3.729 \mathrm{E}-06$ & $1.408 \mathrm{E}+08$ & $7.6424 \mathrm{E}+00$ & $3.765 \mathrm{E}-06$ & $1.421 \mathrm{E}+08$ & 0.009 \\
\hline $1 s 4 d^{1} D_{2}$ & $1 s 7 d^{3} D_{1}$ & $7.6772 \mathrm{E}+00$ & $1.328 \mathrm{E}-05$ & $5.009 \mathrm{E}+08$ & $7.6454 \mathrm{E}+00$ & $1.298 \mathrm{E}-05$ & $4.895 \mathrm{E}+08$ & 0.009 \\
\hline $1 s 5 s^{3} S_{1}$ & $1 s 7 d^{3} D_{1}$ & $1.5703 \mathrm{E}+01$ & $1.954 \mathrm{E}-04$ & $1.762 \mathrm{E}+09$ & $1.5638 \mathrm{E}+01$ & $1.965 \mathrm{E}-04$ & $1.771 \mathrm{E}+09$ & 0.012 \\
\hline $1 s 5 d^{3} D_{2}$ & $1 s 7 d^{3} D_{1}$ & $1.6343 \mathrm{E}+01$ & $4.762 \mathrm{E}-05$ & $3.964 \mathrm{E}+08$ & $1.6277 \mathrm{E}+01$ & $4.755 \mathrm{E}-05$ & $3.957 \mathrm{E}+08$ & 0.004 \\
\hline $1 s 5 d^{3} D_{1}$ & $1 s 7 d^{3} D_{1}$ & $1.6349 \mathrm{E}+01$ & $4.677 \mathrm{E}-05$ & $3.891 \mathrm{E}+08$ & $1.6283 \mathrm{E}+01$ & $4.670 \mathrm{E}-05$ & $3.883 E+08$ & 0.004 \\
\hline $1 s 5 d^{3} D_{3}$ & $1 s 7 d^{3} D_{1}$ & $1.6513 \mathrm{E}+01$ & $8.732 \mathrm{E}-06$ & $7.120 \mathrm{E}+07$ & $1.6446 \mathrm{E}+01$ & $8.833 \mathrm{E}-06$ & $7.199 E+07$ & 0.004 \\
\hline $1 s 5 d^{1} D_{2}$ & $1 s 7 d^{3} D_{1}$ & $1.6521 \mathrm{E}+01$ & $3.074 \mathrm{E}-05$ & $2.504 \mathrm{E}+08$ & $1.6453 \mathrm{E}+01$ & $3.038 \mathrm{E}-05$ & $2.474 \mathrm{E}+08$ & 0.003 \\
\hline $1 s 5 g{ }^{3} G_{3}$ & $1 s 7 d^{3} D_{1}$ & $1.6605 \mathrm{E}+01$ & $1.059 \mathrm{E}-05$ & $8.544 \mathrm{E}+07$ & $1.6535 \mathrm{E}+01$ & $1.055 \mathrm{E}-05$ & $8.509 E+07$ & 0.005 \\
\hline $1 s 6 s^{3} S_{1}$ & $1 s 7 d^{3} D_{1}$ & $4.0910 \mathrm{E}+01$ & $6.049 \mathrm{E}-04$ & $8.036 \mathrm{E}+08$ & $4.0705 \mathrm{E}+01$ & $6.166 \mathrm{E}-04$ & $8.204 E+08$ & 0.005 \\
\hline $1 s 6 d^{3} D_{2}$ & $1 s 7 d^{3} D_{1}$ & $4.3465 \mathrm{E}+01$ & $1.599 \mathrm{E}-04$ & $1.882 \mathrm{E}+08$ & $4.3310 \mathrm{E}+01$ & $1.611 \mathrm{E}-04$ & $1.893 \mathrm{E}+08$ & 0.001 \\
\hline $1 s 6 d^{3} D_{1}$ & $1 s 7 d^{3} D_{1}$ & $4.3492 \mathrm{E}+01$ & $1.564 \mathrm{E}-04$ & $1.839 \mathrm{E}+08$ & $4.3303 \mathrm{E}+01$ & $1.580 \mathrm{E}-04$ & $1.858 \mathrm{E}+08$ & 0.001 \\
\hline $1 s 6 d^{3} D_{3}$ & $1 s 7 d^{3} D_{1}$ & $4.4167 \mathrm{E}+01$ & $3.032 \mathrm{E}-05$ & $3.456 \mathrm{E}+07$ & $4.4004 \mathrm{E}+01$ & $3.073 \mathrm{E}-05$ & $3.498 \mathrm{E}+07$ & 0.001 \\
\hline $1 s 6 d^{1} D_{2}$ & $1 s 7 d^{3} D_{1}$ & $4.4198 \mathrm{E}+01$ & $1.063 \mathrm{E}-04$ & $1.210 \mathrm{E}+08$ & $4.4015 \mathrm{E}+01$ & $1.044 \mathrm{E}-04$ & $1.188 \mathrm{E}+08$ & 0.001 \\
\hline $1 s 6 g{ }^{3} G_{3}$ & $1 s 7 d^{3} D_{1}$ & $4.4547 \mathrm{E}+01$ & $8.358 \mathrm{E}-05$ & $9.365 \mathrm{E}+07$ & $4.4358 \mathrm{E}+01$ & $8.369 \mathrm{E}-05$ & $9.377 \mathrm{E}+07$ & 0.001 \\
\hline $1 s 7 s^{3} S_{1}$ & $1 s 7 d^{3} D_{1}$ & $1.0966 \mathrm{E}+03$ & $3.011 \mathrm{E}-07$ & $5.566 \mathrm{E}+02$ & $1.0799 \mathrm{E}+03$ & $3.104 \mathrm{E}-07$ & $5.869 \mathrm{E}+02$ & 0.012 \\
\hline $1 s 7 d^{3} D_{2}$ & $1 s 7 d^{3} D_{1}$ & $1.1420 \mathrm{E}+05$ & $1.309 \mathrm{E}-13$ & $2.231 \mathrm{E}-08$ & & & & 0.944 \\
\hline $1 s 2 p^{3} P_{1}^{o}$ & $1 s 7 p^{1} P_{1}^{o}$ & $1.3362 \mathrm{E}+00$ & $1.852 \mathrm{E}-05$ & $2.307 \mathrm{E}+10$ & $1.3307 \mathrm{E}+00$ & $1.812 \mathrm{E}-05$ & $2.256 \mathrm{E}+10$ & 0.019 \\
\hline $1 s 2 p^{3} P_{2}^{0}$ & $1 s 7 p^{1} P_{1}^{o}$ & $1.3946 \mathrm{E}+00$ & $7.635 \mathrm{E}-06$ & $8.728 \mathrm{E}+09$ & $1.3888 \mathrm{E}+00$ & $7.793 E-06$ & $8.907 \mathrm{E}+09$ & 0.061 \\
\hline $1 s 2 p^{1} P_{1}^{o}$ & $1 s 7 p{ }^{1} P_{1}^{o}$ & $1.4001 \mathrm{E}+00$ & $1.156 \mathrm{E}-05$ & $1.311 \mathrm{E}+10$ & $1.3943 \mathrm{E}+00$ & $1.090 \mathrm{E}-05$ & $1.237 \mathrm{E}+10$ & 0.054 \\
\hline $1 s 3 p^{3} P_{1}^{o}$ & $1 s 7 p^{1} P_{1}^{o}$ & $3.4073 \mathrm{E}+00$ & $3.665 \mathrm{E}-05$ & $7.020 \mathrm{E}+09$ & $3.3933 E+00$ & $3.370 \mathrm{E}-05$ & $6.452 E+09$ & 0.076 \\
\hline $1 s 3 p^{3} P_{2}^{o}$ & $1 s 7 p^{1} P_{1}^{o}$ & $3.5191 \mathrm{E}+00$ & $1.673 \mathrm{E}-05$ & $3.003 E+09$ & $3.5046 \mathrm{E}+00$ & $1.472 \mathrm{E}-05$ & $2.642 \mathrm{E}+09$ & 0.087 \\
\hline $1 s 3 p{ }^{1} P_{1}^{o}$ & $1 s 7 p{ }^{1} P_{1}^{o}$ & $3.5290 \mathrm{E}+00$ & $2.143 \mathrm{E}-05$ & $3.826 \mathrm{E}+09$ & $3.5146 \mathrm{E}+00$ & $2.066 \mathrm{E}-05$ & $3.687 \mathrm{E}+09$ & 0.042 \\
\hline $1 s 4 p^{3} P_{1}^{o}$ & $1 s 7 p{ }^{1} P_{1}^{o}$ & $7.3666 \mathrm{E}+00$ & $5.772 \mathrm{E}-05$ & $2.365 \mathrm{E}+09$ & $7.3362 \mathrm{E}+00$ & $5.662 \mathrm{E}-05$ & $2.319 \mathrm{E}+09$ & 0.010 \\
\hline $1 s 4 p^{3} P_{2}^{o}$ & $1 s 7 p^{1} P_{1}^{o}$ & $7.5857 \mathrm{E}+00$ & $2.671 \mathrm{E}-05$ & $1.032 \mathrm{E}+09$ & $7.5543 \mathrm{E}+00$ & $2.524 \mathrm{E}-05$ & $9.753 \mathrm{E}+08$ & 0.018 \\
\hline $1 s 4 p{ }^{1} P_{1}^{o}$ & $1 s 7 p^{1} P_{1}^{o}$ & $7.6047 \mathrm{E}+00$ & $3.529 \mathrm{E}-05$ & $1.357 \mathrm{E}+09$ & $7.5735 \mathrm{E}+00$ & $3.553 E-05$ & $1.365 \mathrm{E}+09$ & 0.001 \\
\hline $1 s 4 f^{3} F_{3}^{0}$ & $1 s 7 p{ }^{1} P_{1}^{o}$ & $7.6752 \mathrm{E}+00$ & $5.944 \mathrm{E}-07$ & $2.244 \mathrm{E}+07$ & $7.6436 \mathrm{E}+00$ & $6.387 \mathrm{E}-07$ & $2.410 \mathrm{E}+07$ & 0.038 \\
\hline $1 s 4 f^{3} F_{2}^{o}$ & $1 s 7 p{ }^{1} P_{1}^{o}$ & $7.6767 \mathrm{E}+00$ & $1.327 \mathrm{E}-06$ & $5.007 \mathrm{E}+07$ & $7.6451 \mathrm{E}+00$ & $1.227 \mathrm{E}-06$ & $4.628 \mathrm{E}+07$ & 0.037 \\
\hline $1 s 4 f^{1} F_{3}^{o}$ & $1 s 7 p^{1} P_{1}^{o}$ & $7.7125 \mathrm{E}+00$ & $1.103 \mathrm{E}-05$ & $4.124 \mathrm{E}+08$ & $7.6807 \mathrm{E}+00$ & $1.104 \mathrm{E}-05$ & $4.127 \mathrm{E}+08$ & 0.012 \\
\hline $1 s 5 p^{3} P_{1}^{o}$ & $1 s 7 p{ }^{1} P_{1}^{o}$ & $1.5788 \mathrm{E}+01$ & $1.070 \mathrm{E}-04$ & $9.547 \mathrm{E}+08$ & $1.5725 \mathrm{E}+01$ & $1.067 \mathrm{E}-04$ & $9.520 \mathrm{E}+08$ & 0.009 \\
\hline $1 s 5 p^{3} P_{2}^{0}$ & $1 s 7 p^{1} P_{1}^{o}$ & $1.6303 \mathrm{E}+01$ & $5.105 \mathrm{E}-05$ & $4.271 \mathrm{E}+08$ & $1.6237 \mathrm{E}+01$ & $4.929 \mathrm{E}-05$ & $4.121 \mathrm{E}+08$ & 0.003 \\
\hline $1 s 5 p^{1} P_{1}^{o}$ & $1 s 7 p^{1} P_{1}^{o}$ & $1.6348 \mathrm{E}+01$ & $6.835 \mathrm{E}-05$ & $5.686 \mathrm{E}+08$ & $1.6282 \mathrm{E}+01$ & $6.968 \mathrm{E}-05$ & $5.795 E+08$ & 0.009 \\
\hline $1 s 5 f^{3} F_{3}^{o}$ & $1 s 7 p^{1} P_{1}^{o}$ & $1.6515 \mathrm{E}+01$ & $2.442 \mathrm{E}-06$ & $1.991 \mathrm{E}+07$ & $1.6450 \mathrm{E}+01$ & $2.714 \mathrm{E}-06$ & $2.211 \mathrm{E}+07$ & 0.003 \\
\hline $1 s 5 f^{3} F_{2}^{o}$ & $1 s 7 p^{1} P_{1}^{0}$ & $1.6519 \mathrm{E}+01$ & $5.460 \mathrm{E}-06$ & $4.449 \mathrm{E}+07$ & $1.6453 \mathrm{E}+01$ & $5.203 \mathrm{E}-06$ & $4.237 \mathrm{E}+07$ & 0.003 \\
\hline $1 s 5 f^{1} F_{3}^{o}$ & $1 s 7 p{ }^{1} P_{1}^{o}$ & $1.6604 \mathrm{E}+01$ & $4.732 \mathrm{E}-05$ & $3.817 \mathrm{E}+08$ & $1.6538 \mathrm{E}+01$ & $4.695 \mathrm{E}-05$ & $3.785 E+08$ & 0.000 \\
\hline $1 s 6 p^{3} P_{1}^{o}$ & $1 s 7 p{ }^{1} P_{1}^{o}$ & $4.1241 \mathrm{E}+01$ & $2.976 \mathrm{E}-04$ & $3.891 \mathrm{E}+08$ & $4.1082 \mathrm{E}+01$ & $2.898 \mathrm{E}-04$ & $3.786 \mathrm{E}+08$ & 0.001 \\
\hline $1 s 6 p^{3} P_{2}^{o}$ & $1 s 7 p^{1} P_{1}^{o}$ & $4.3302 \mathrm{E}+01$ & $1.543 \mathrm{E}-04$ & $1.829 \mathrm{E}+08$ & $4.3133 \mathrm{E}+01$ & $1.489 \mathrm{E}-04$ & $1.764 \mathrm{E}+08$ & 0.002 \\
\hline $1 s 6 p^{1} P_{1}^{o}$ & $1 s 7 p^{1} P_{1}^{0}$ & $4.3483 \mathrm{E}+01$ & $2.090 \mathrm{E}-04$ & $2.457 \mathrm{E}+08$ & $4.3294 \mathrm{E}+01$ & $2.181 \mathrm{E}-04$ & $2.565 E+08$ & 0.001 \\
\hline $1 s 6 f^{3} F_{3}^{o}$ & $1 s 7 p{ }^{1} P_{1}^{o}$ & $4.4172 \mathrm{E}+01$ & $1.082 \mathrm{E}-05$ & $1.233 \mathrm{E}+07$ & $4.4022 \mathrm{E}+01$ & $1.212 \mathrm{E}-05$ & $1.379 \mathrm{E}+07$ & 0.002 \\
\hline $1 s 6 f^{3} F_{2}^{o}$ & $1 s 7 p^{1} P_{1}^{o}$ & $4.4186 \mathrm{E}+01$ & $2.423 \mathrm{E}-05$ & $2.759 \mathrm{E}+07$ & $4.4022 \mathrm{E}+01$ & $2.300 \mathrm{E}-05$ & $2.617 \mathrm{E}+07$ & 0.002 \\
\hline $1 s 6 f^{1} F_{3}^{o}$ & $1 s 7 p^{1} P_{1}^{o}$ & $4.4540 \mathrm{E}+01$ & $2.117 \mathrm{E}-04$ & $2.373 \mathrm{E}+08$ & $4.4376 \mathrm{E}+01$ & $2.118 \mathrm{E}-04$ & $2.371 \mathrm{E}+08$ & 0.002 \\
\hline $1 s 7 p^{3} P_{1}^{o}$ & $1 s 7 p{ }^{1} P_{1}^{o}$ & $1.2731 \mathrm{E}+03$ & $1.853 \mathrm{E}-07$ & $2.541 \mathrm{E}+02$ & $1.2808 \mathrm{E}+03$ & $1.800 \mathrm{E}-07$ & $2.420 \mathrm{E}+02$ & 0.047 \\
\hline $1 s 7 p^{3} P_{2}^{o}$ & $1 s 7 p^{1} P_{1}^{o}$ & $1.6436 \mathrm{E}+04$ & $4.209 \mathrm{E}-11$ & $3.465 \mathrm{E}-04$ & & & & 0.021 \\
\hline $1 s 2 s^{3} S_{1}$ & $1 s 7 d^{3} D_{3}$ & $1.3248 \mathrm{E}+00$ & $1.758 \mathrm{E}-05$ & $9.543 \mathrm{E}+09$ & $1.3192 \mathrm{E}+00$ & $1.588 \mathrm{E}-05$ & $8.622 \mathrm{E}+09$ & 0.002 \\
\hline $1 s 3 s^{3} S_{1}$ & $1 s 7 d^{3} D_{3}$ & $3.3852 E+00$ & $9.868 \mathrm{E}-05$ & $8.205 E+09$ & $3.3710 \mathrm{E}+00$ & $9.907 \mathrm{E}-05$ & $8.237 E+09$ & 0.026 \\
\hline $1 s 3 d^{3} D_{2}$ & $1 s 7 d^{3} D_{3}$ & $3.5248 \mathrm{E}+00$ & $9.707 \mathrm{E}-06$ & $7.445 \mathrm{E}+08$ & $3.5099 \mathrm{E}+00$ & $9.973 \mathrm{E}-06$ & $7.649 E+08$ & 0.021 \\
\hline $1 s 3 d^{3} D_{1}$ & $1 s 7 d^{3} D_{3}$ & $3.5263 E+00$ & $1.642 \mathrm{E}-06$ & $1.258 \mathrm{E}+08$ & $3.5113 \mathrm{E}+00$ & $1.619 \mathrm{E}-06$ & $1.240 \mathrm{E}+08$ & 0.023 \\
\hline $1 s 3 d^{3} D_{3}$ & $1 s 7 d^{3} D_{3}$ & $3.5615 \mathrm{E}+00$ & $3.721 \mathrm{E}-05$ & $2.795 \mathrm{E}+09$ & $3.5464 \mathrm{E}+00$ & $3.778 \mathrm{E}-05$ & $2.838 \mathrm{E}+09$ & 0.022 \\
\hline $1 s 3 d^{1} D_{2}$ & $1 s 7 d^{3} D_{3}$ & $3.5629 \mathrm{E}+00$ & $6.409 \mathrm{E}-06$ & $4.811 \mathrm{E}+08$ & $3.5478 \mathrm{E}+00$ & $6.234 \mathrm{E}-06$ & $4.679 \mathrm{E}+08$ & 0.024 \\
\hline $1 s 4 s^{3} S_{1}$ & $1 s 7 d^{3} D_{3}$ & $7.3175 \mathrm{E}+00$ & $2.171 \mathrm{E}-04$ & $3.863 \mathrm{E}+09$ & $7.2866 \mathrm{E}+00$ & $2.196 \mathrm{E}-04$ & $3.908 \mathrm{E}+09$ & 0.021 \\
\hline $1 s 4 d^{3} D_{2}$ & $1 s 7 d^{3} D_{3}$ & $7.5894 \mathrm{E}+00$ & $2.398 \mathrm{E}-05$ & $3.967 \mathrm{E}+08$ & $7.5575 \mathrm{E}+00$ & $2.428 \mathrm{E}-05$ & $4.016 \mathrm{E}+08$ & 0.009 \\
\hline $1 s 4 d^{3} D_{1}$ & $1 s 7 d^{3} D_{3}$ & $7.5922 \mathrm{E}+00$ & $3.931 \mathrm{E}-06$ & $6.498 \mathrm{E}+07$ & $7.5603 \mathrm{E}+00$ & $3.920 \mathrm{E}-06$ & $6.479 E+07$ & 0.007 \\
\hline $1 s 4 d^{3} D_{3}$ & $1 s 7 d^{3} D_{3}$ & $7.6613 \mathrm{E}+00$ & $9.279 \mathrm{E}-05$ & $1.506 \mathrm{E}+09$ & $7.6289 \mathrm{E}+00$ & $9.340 \mathrm{E}-05$ & $1.516 \mathrm{E}+09$ & 0.009 \\
\hline $1 s 4 d^{1} D_{2}$ & $1 s 7 d^{3} D_{3}$ & $7.6642 \mathrm{E}+00$ & $1.550 \mathrm{E}-05$ & $2.515 \mathrm{E}+08$ & $7.6319 \mathrm{E}+00$ & $1.533 \mathrm{E}-05$ & $2.487 \mathrm{E}+08$ & 0.010 \\
\hline $1 s 5 s^{3} S_{1}$ & $1 s 7 d^{3} D_{3}$ & $1.5649 \mathrm{E}+01$ & $4.656 \mathrm{E}-04$ & $1.812 \mathrm{E}+09$ & $1.5581 \mathrm{E}+01$ & $4.708 \mathrm{E}-04$ & $1.832 \mathrm{E}+09$ & 0.011 \\
\hline $1 s 5 d^{3} D_{2}$ & $1 s 7 d^{3} D_{3}$ & $1.6284 \mathrm{E}+01$ & $5.459 \mathrm{E}-05$ & $1.962 \mathrm{E}+08$ & $1.6216 \mathrm{E}+01$ & $5.492 \mathrm{E}-05$ & $1.973 \mathrm{E}+08$ & 0.003 \\
\hline $1 s 5 d^{3} D_{1}$ & $1 s 7 d^{3} D_{3}$ & $1.6290 \mathrm{E}+01$ & $8.844 \mathrm{E}-06$ & $3.176 \mathrm{E}+07$ & $1.6222 \mathrm{E}+01$ & $8.848 \mathrm{E}-06$ & $3.176 \mathrm{E}+07$ & 0.002 \\
\hline $1 s 5 d^{3} D_{3}$ & $1 s 7 d^{3} D_{3}$ & $1.6453 \mathrm{E}+01$ & $2.142 \mathrm{E}-04$ & $7.541 \mathrm{E}+08$ & $1.6384 \mathrm{E}+01$ & $2.148 \mathrm{E}-04$ & $7.562 E+08$ & 0.003 \\
\hline $1 s 5 d^{1} D_{2}$ & $1 s 7 d^{3} D_{3}$ & $1.6460 \mathrm{E}+01$ & $3.535 \mathrm{E}-05$ & $1.243 \mathrm{E}+08$ & $1.6391 \mathrm{E}+01$ & $3.519 \mathrm{E}-05$ & $1.237 \mathrm{E}+08$ & 0.004 \\
\hline $1 s 5 g^{3} G_{4}$ & $1 s 7 d^{3} D_{3}$ & $1.6542 \mathrm{E}+01$ & $1.538 \mathrm{E}-06$ & $5.355 \mathrm{E}+06$ & $1.6470 \mathrm{E}+01$ & $1.547 \mathrm{E}-06$ & $5.389 E+06$ & 0.004 \\
\hline $1 s 5 g{ }^{3} G_{3}$ & $1 s 7 d^{3} D_{3}$ & $1.6544 \mathrm{E}+01$ & $1.868 \mathrm{E}-07$ & $6.503 \mathrm{E}+05$ & $1.6472 \mathrm{E}+01$ & $1.859 \mathrm{E}-07$ & $6.476 \mathrm{E}+05$ & 0.006 \\
\hline
\end{tabular}


Table 4 (continued)

\begin{tabular}{|c|c|c|c|c|c|c|c|c|}
\hline \multirow[t]{2}{*}{ Lower } & \multirow[t]{2}{*}{ Upper } & \multicolumn{3}{|l|}{ GRASP2K } & \multicolumn{3}{|l|}{ FAC } & \multirow[t]{2}{*}{$d T$} \\
\hline & & $\lambda(\AA)$ & $g f$ & $A\left(\mathrm{~s}^{-1}\right)$ & $\lambda(\AA)$ & $g f$ & $A\left(\mathrm{~s}^{-1}\right)$ & \\
\hline $1 s 5 g{ }^{3} G_{5}$ & $1 s 7 d^{3} D_{3}$ & $1.6593 \mathrm{E}+01$ & $2.071 \mathrm{E}-05$ & $7.169 \mathrm{E}+07$ & $1.6521 \mathrm{E}+01$ & $2.073 \mathrm{E}-05$ & $7.178 \mathrm{E}+07$ & 0.003 \\
\hline $1 s 5 g^{1} G_{4}$ & $1 s 7 d^{3} D_{3}$ & $1.6595 \mathrm{E}+01$ & $1.270 \mathrm{E}-06$ & $4.394 \mathrm{E}+06$ & $1.6522 \mathrm{E}+01$ & $1.261 \mathrm{E}-06$ & $4.366 \mathrm{E}+06$ & 0.005 \\
\hline $1 s 6 s^{3} s_{1}$ & $1 s 7 d^{3} D_{3}$ & $4.0543 \mathrm{E}+01$ & $1.383 \mathrm{E}-03$ & $8.018 E+08$ & $4.0325 \mathrm{E}+01$ & $1.412 \mathrm{E}-03$ & $8.208 E+08$ & 0.005 \\
\hline $1 s 6 d^{3} D_{2}$ & $1 s 7 d^{3} D_{3}$ & $4.3051 \mathrm{E}+01$ & $1.778 \mathrm{E}-04$ & $9.140 \mathrm{E}+07$ & $4.2880 \mathrm{E}+01$ & $1.798 \mathrm{E}-04$ & $9.239 \mathrm{E}+07$ & 0.001 \\
\hline $1 s 6 d^{3} D_{1}$ & $1 s 7 d^{3} D_{3}$ & $4.3077 \mathrm{E}+01$ & $2.870 \mathrm{E}-05$ & $1.474 \mathrm{E}+07$ & $4.2874 \mathrm{E}+01$ & $2.939 \mathrm{E}-05$ & $1.510 \mathrm{E}+07$ & 0.001 \\
\hline $1 s 6 d^{3} D_{3}$ & $1 s 7 d^{3} D_{3}$ & $4.3740 \mathrm{E}+01$ & $7.202 \mathrm{E}-04$ & $3.587 \mathrm{E}+08$ & $4.3560 \mathrm{E}+01$ & $7.214 \mathrm{E}-04$ & $3.592 \mathrm{E}+08$ & 0.001 \\
\hline $1 s 6 d{ }^{1} D_{2}$ & $1 s 7 d^{3} D_{3}$ & $4.3770 \mathrm{E}+01$ & $1.184 \mathrm{E}-04$ & $5.887 \mathrm{E}+07$ & $4.3571 \mathrm{E}+01$ & $1.168 \mathrm{E}-04$ & $5.813 \mathrm{E}+07$ & 0.001 \\
\hline $1 s 6 g{ }^{3} G_{4}$ & $1 s 7 d^{3} D_{3}$ & $4.4103 \mathrm{E}+01$ & $1.214 \mathrm{E}-05$ & $5.945 E+06$ & $4.3907 \mathrm{E}+01$ & $1.220 \mathrm{E}-05$ & $5.980 \mathrm{E}+06$ & 0.001 \\
\hline $1 s 6 g{ }^{3} G_{3}$ & $1 s 7 d^{3} D_{3}$ & $4.4112 \mathrm{E}+01$ & $1.464 \mathrm{E}-06$ & $7.171 \mathrm{E}+05$ & $4.3907 \mathrm{E}+01$ & $1.464 \mathrm{E}-06$ & $7.176 \mathrm{E}+05$ & 0.001 \\
\hline $1 s 6 g^{3} G_{5}$ & $1 s 7 d^{3} D_{3}$ & $4.4315 \mathrm{E}+01$ & $1.637 \mathrm{E}-04$ & $7.941 \mathrm{E}+07$ & $4.4116 \mathrm{E}+01$ & $1.636 \mathrm{E}-04$ & $7.946 \mathrm{E}+07$ & 0.001 \\
\hline $1 s 6 g{ }^{1} G_{4}$ & $1 s 7 d^{3} D_{3}$ & $4.4321 \mathrm{E}+01$ & $9.975 \mathrm{E}-06$ & $4.839 \mathrm{E}+06$ & $4.4116 \mathrm{E}+01$ & $9.920 \mathrm{E}-06$ & $4.815 \mathrm{E}+06$ & 0.001 \\
\hline $1 s 7 s^{3} S_{1}$ & $1 s 7 d^{3} D_{3}$ & $8.8243 \mathrm{E}+02$ & $1.362 \mathrm{E}-06$ & $1.667 \mathrm{E}+03$ & $8.6393 \mathrm{E}+02$ & $1.431 \mathrm{E}-06$ & $1.811 \mathrm{E}+03$ & 0.002 \\
\hline $1 s 7 d^{3} D_{2}$ & $1 s 7 d^{3} D_{3}$ & $4.3459 \mathrm{E}+03$ & $2.721 \mathrm{E}-09$ & $1.373 \mathrm{E}-01$ & & & & 0.004 \\
\hline $1 s 7 d^{3} D_{1}$ & $1 s 7 d^{3} D_{3}$ & $4.5178 \mathrm{E}+03$ & $3.927 \mathrm{E}-10$ & $1.833 \mathrm{E}-02$ & & & & 0.021 \\
\hline $1 s 2 p^{3} P_{1}^{o}$ & $1 s 7 f^{3} F_{3}^{o}$ & $1.3358 \mathrm{E}+00$ & $5.669 \mathrm{E}-04$ & $3.027 \mathrm{E}+11$ & $1.3303 \mathrm{E}+00$ & $5.764 \mathrm{E}-04$ & $3.077 \mathrm{E}+11$ & 0.000 \\
\hline $1 s 2 p^{3} P_{2}^{o}$ & $1 s 7 f^{3} F_{3}^{o}$ & $1.3942 \mathrm{E}+00$ & $1.500 \mathrm{E}-04$ & $7.354 \mathrm{E}+10$ & $1.3883 \mathrm{E}+00$ & $1.493 \mathrm{E}-04$ & $7.322 \mathrm{E}+10$ & 0.014 \\
\hline $1 s 2 p^{1} P_{1}^{o}$ & $1 s 7 f^{3} F_{3}^{0}$ & $1.3997 \mathrm{E}+00$ & $3.473 \mathrm{E}-05$ & $1.689 \mathrm{E}+10$ & $1.3939 \mathrm{E}+00$ & $3.421 \mathrm{E}-05$ & $1.663 \mathrm{E}+10$ & 0.036 \\
\hline $1 s 3 p^{3} P_{1}^{o}$ & $1 s 7 f^{3} F_{3}^{o}$ & $3.4047 \mathrm{E}+00$ & $1.597 \mathrm{E}-05$ & $1.313 E+09$ & $3.3906 \mathrm{E}+00$ & $1.883 \mathrm{E}-05$ & $1.547 \mathrm{E}+09$ & 0.110 \\
\hline $1 s 3 p^{3} P_{2}^{o}$ & $1 s 7 f^{3} F_{3}^{o}$ & $3.5164 \mathrm{E}+00$ & $9.649 \mathrm{E}-06$ & $7.436 \mathrm{E}+08$ & $3.5016 \mathrm{E}+00$ & $1.109 \mathrm{E}-05$ & $8.551 \mathrm{E}+08$ & 0.073 \\
\hline $1 s 3 p^{1} P_{1}^{o}$ & $1 s 7 f^{3} F_{3}^{o}$ & $3.5263 \mathrm{E}+00$ & $2.608 \mathrm{E}-06$ & $1.999 \mathrm{E}+08$ & $3.5116 \mathrm{E}+00$ & $2.267 \mathrm{E}-06$ & $1.737 \mathrm{E}+08$ & 0.007 \\
\hline $1 s 4 p^{3} P_{1}^{0}$ & $1 s 7 f^{3} F_{3}^{0}$ & $7.3546 \mathrm{E}+00$ & $6.464 \mathrm{E}-05$ & $1.139 \mathrm{E}+09$ & $7.3233 \mathrm{E}+00$ & $6.291 \mathrm{E}-05$ & $1.108 \mathrm{E}+09$ & 0.012 \\
\hline $1 s 4 p^{3} P_{2}^{o}$ & $1 s 7 f^{3} F_{3}^{o}$ & $7.5729 \mathrm{E}+00$ & $7.761 \mathrm{E}-06$ & $1.290 \mathrm{E}+08$ & $7.5406 \mathrm{E}+00$ & $7.141 \mathrm{E}-06$ & $1.186 \mathrm{E}+08$ & 0.023 \\
\hline $1 s 4 p^{1} P_{1}^{o}$ & $1 s 7 f^{3} F_{3}^{0}$ & $7.5920 \mathrm{E}+00$ & $1.634 \mathrm{E}-06$ & $2.702 \mathrm{E}+07$ & $7.5597 \mathrm{E}+00$ & $1.856 \mathrm{E}-06$ & $3.069 \mathrm{E}+07$ & 0.016 \\
\hline $1 s 4 f^{3} F_{3}^{o}$ & $1 s 7 f^{3} F_{3}^{o}$ & $7.6621 \mathrm{E}+00$ & $4.344 \mathrm{E}-05$ & $7.051 \mathrm{E}+08$ & $7.6295 \mathrm{E}+00$ & $4.280 \mathrm{E}-05$ & $6.948 \mathrm{E}+08$ & 0.011 \\
\hline $1 s 4 f^{3} F_{2}^{o}$ & $1 s 7 f^{3} F_{3}^{o}$ & $7.6636 \mathrm{E}+00$ & $7.207 \mathrm{E}-06$ & $1.169 \mathrm{E}+08$ & $7.6310 \mathrm{E}+00$ & $7.140 \mathrm{E}-06$ & $1.158 \mathrm{E}+08$ & 0.011 \\
\hline $1 s 4 f^{3} F_{4}^{o}$ & $1 s 7 f^{3} F_{3}^{o}$ & $7.6982 \mathrm{E}+00$ & $7.258 \mathrm{E}-06$ & $1.167 \mathrm{E}+08$ & $7.6653 \mathrm{E}+00$ & $7.235 \mathrm{E}-06$ & $1.163 \mathrm{E}+08$ & 0.004 \\
\hline $1 s 4 f^{1} F_{3}^{o}$ & $1 s 7 f^{3} F_{3}^{o}$ & $7.6993 \mathrm{E}+00$ & $8.713 \mathrm{E}-07$ & $1.401 \mathrm{E}+07$ & $7.6665 \mathrm{E}+00$ & $8.328 \mathrm{E}-07$ & $1.338 \mathrm{E}+07$ & 0.003 \\
\hline $1 s 5 p^{3} P_{1}^{0}$ & $1 s 7 f^{3} F_{3}^{0}$ & $1.5734 \mathrm{E}+01$ & $4.148 \mathrm{E}-04$ & $1.597 \mathrm{E}+09$ & $1.5665 \mathrm{E}+01$ & $4.152 \mathrm{E}-04$ & $1.598 \mathrm{E}+09$ & 0.004 \\
\hline $1 s 5 p^{3} P_{2}^{0}$ & $1 s 7 f^{3} F_{3}^{o}$ & $1.6245 \mathrm{E}+01$ & $8.271 \mathrm{E}-05$ & $2.987 \mathrm{E}+08$ & $1.6174 \mathrm{E}+01$ & $8.226 \mathrm{E}-05$ & $2.971 \mathrm{E}+08$ & 0.002 \\
\hline $1 s 5 p^{1} P_{1}^{o}$ & $1 s 7 f^{3} F_{3}^{o}$ & $1.6289 \mathrm{E}+01$ & $1.901 \mathrm{E}-05$ & $6.828 \mathrm{E}+07$ & $1.6219 \mathrm{E}+01$ & $1.921 \mathrm{E}-05$ & $6.900 \mathrm{E}+07$ & 0.008 \\
\hline $1 s 5 f^{3} F_{3}^{o}$ & $1 s 7 f^{3} F_{3}^{o}$ & $1.6455 \mathrm{E}+01$ & $1.515 \mathrm{E}-04$ & $5.333 \mathrm{E}+08$ & $1.6385 \mathrm{E}+01$ & $1.511 \mathrm{E}-04$ & $5.319 \mathrm{E}+08$ & 0.001 \\
\hline $1 s 5 f^{3} F_{2}^{o}$ & $1 s 7 f^{3} F_{3}^{o}$ & $1.6459 \mathrm{E}+01$ & $2.517 \mathrm{E}-05$ & $8.855 E+07$ & $1.6388 \mathrm{E}+01$ & $2.523 \mathrm{E}-05$ & $8.877 \mathrm{E}+07$ & 0.001 \\
\hline $1 s 5 f^{3} F_{4}^{o}$ & $1 s 7 f^{3} F_{3}^{o}$ & $1.6540 \mathrm{E}+01$ & $2.593 \mathrm{E}-05$ & $9.030 \mathrm{E}+07$ & $1.6469 \mathrm{E}+01$ & $2.592 \mathrm{E}-05$ & $9.031 \mathrm{E}+07$ & 0.000 \\
\hline $1 s 5 f^{1} F_{3}^{o}$ & $1 s 7 f^{3} F_{3}^{o}$ & $1.6543 \mathrm{E}+01$ & $3.118 \mathrm{E}-06$ & $1.086 \mathrm{E}+07$ & $1.6472 \mathrm{E}+01$ & $2.991 \mathrm{E}-06$ & $1.041 \mathrm{E}+07$ & 0.000 \\
\hline $1 s 6 p^{3} P_{1}^{o}$ & $1 s 7 f^{3} F_{3}^{0}$ & $4.0868 \mathrm{E}+01$ & $1.936 \mathrm{E}-03$ & $1.105 E+09$ & $4.0680 \mathrm{E}+01$ & $1.929 \mathrm{E}-03$ & $1.101 \mathrm{E}+09$ & 0.001 \\
\hline $1 s 6 p^{3} P_{2}^{o}$ & $1 s 7 f^{3} F_{3}^{o}$ & $4.2891 \mathrm{E}+01$ & $4.845 \mathrm{E}-04$ & $2.510 \mathrm{E}+08$ & $4.2690 \mathrm{E}+01$ & $4.909 \mathrm{E}-04$ & $2.545 \mathrm{E}+08$ & 0.001 \\
\hline $1 s 6 p{ }^{1} P_{1}^{o}$ & $1 s 7 f^{3} F_{3}^{o}$ & $4.3069 \mathrm{E}+01$ & $1.141 \mathrm{E}-04$ & $5.864 \mathrm{E}+07$ & $4.2848 \mathrm{E}+01$ & $1.182 \mathrm{E}-04$ & $6.086 \mathrm{E}+07$ & 0.000 \\
\hline $1 s 6 f^{3} F_{3}^{o}$ & $1 s 7 f^{3} F_{3}^{o}$ & $4.3744 \mathrm{E}+01$ & $6.234 \mathrm{E}-04$ & $3.104 \mathrm{E}+08$ & $4.3561 \mathrm{E}+01$ & $6.229 \mathrm{E}-04$ & $3.101 \mathrm{E}+08$ & 0.000 \\
\hline $1 s 6 f^{3} F_{2}^{o}$ & $1 s 7 f^{3} F_{3}^{o}$ & $4.3758 \mathrm{E}+01$ & $1.037 \mathrm{E}-04$ & $5.161 \mathrm{E}+07$ & $4.3560 \mathrm{E}+01$ & $1.039 \mathrm{E}-04$ & $5.177 \mathrm{E}+07$ & 0.000 \\
\hline $1 s 6 f^{3} F_{4}^{o}$ & $1 s 7 f^{3} F_{3}^{o}$ & $4.4094 \mathrm{E}+01$ & $1.088 \mathrm{E}-04$ & $5.331 \mathrm{E}+07$ & $4.3907 \mathrm{E}+01$ & $1.091 \mathrm{E}-04$ & $5.351 \mathrm{E}+07$ & 0.000 \\
\hline $1 s 6 f^{1} F_{3}^{o}$ & $1 s 7 f^{3} F_{3}^{0}$ & $4.4105 \mathrm{E}+01$ & $1.310 \mathrm{E}-05$ & $6.419 E+06$ & $4.3907 \mathrm{E}+01$ & $1.307 \mathrm{E}-05$ & $6.406 \mathrm{E}+06$ & 0.000 \\
\hline $1 s 6 h^{3} H_{5}^{o}$ & $1 s 7 f^{3} F_{3}^{0}$ & $4.4306 \mathrm{E}+01$ & $4.016 \mathrm{E}-05$ & $1.949 \mathrm{E}+07$ & $4.4116 \mathrm{E}+01$ & $4.014 \mathrm{E}-05$ & $1.949 \mathrm{E}+07$ & 0.001 \\
\hline $1 s 6 h^{3} H_{4}^{o}$ & $1 s 7 f^{3} F_{3}^{o}$ & $4.4312 \mathrm{E}+01$ & $2.427 \mathrm{E}-06$ & $1.178 \mathrm{E}+06$ & $4.4116 \mathrm{E}+01$ & $2.436 \mathrm{E}-06$ & $1.182 \mathrm{E}+06$ & 0.001 \\
\hline $1 s 6 h^{1} H_{5}^{o}$ & $1 s 7 f^{3} F_{3}^{o}$ & $4.4452 \mathrm{E}+01$ & $5.514 \mathrm{E}-12$ & $2.659 \mathrm{E}+00$ & & & & 0.017 \\
\hline $1 s 7 p^{3} P_{1}^{o}$ & $1 s 7 f^{3} F_{3}^{o}$ & $9.9322 \mathrm{E}+02$ & $7.907 \mathrm{E}-07$ & $7.638 \mathrm{E}+02$ & $9.7893 \mathrm{E}+02$ & $8.183 \mathrm{E}-07$ & $8.067 \mathrm{E}+02$ & 0.104 \\
\hline $1 s 7 p^{3} P_{2}^{o}$ & $1 s 7 f^{3} F_{3}^{o}$ & $3.5436 \mathrm{E}+03$ & $4.295 \mathrm{E}-09$ & $3.259 \mathrm{E}-01$ & $3.3920 \mathrm{E}+03$ & $4.895 \mathrm{E}-09$ & $4.019 \mathrm{E}-01$ & 0.273 \\
\hline $1 s 7 p^{1} P_{1}^{o}$ & $1 s 7 f^{3} F_{3}^{0}$ & $4.5177 \mathrm{E}+03$ & $4.880 \mathrm{E}-10$ & $2.279 \mathrm{E}-02$ & & & & 0.371 \\
\hline $1 s 2 p^{3} P_{1}^{o}$ & $1 s 7 f^{3} F_{2}^{0}$ & $1.3358 \mathrm{E}+00$ & $1.757 \mathrm{E}-04$ & $1.314 \mathrm{E}+11$ & $1.3303 \mathrm{E}+00$ & $1.782 \mathrm{E}-04$ & $1.332 \mathrm{E}+11$ & 0.010 \\
\hline $1 s 2 p^{3} P_{0}^{o}$ & $1 s 7 f^{3} F_{2}^{0}$ & $1.3367 \mathrm{E}+00$ & $2.481 \mathrm{E}-04$ & $1.852 \mathrm{E}+11$ & $1.3311 \mathrm{E}+00$ & $2.514 \mathrm{E}-04$ & $1.876 \mathrm{E}+11$ & 0.002 \\
\hline $1 s 2 p^{3} P_{2}^{o}$ & $1 s 7 f^{3} F_{2}^{o}$ & $1.3942 \mathrm{E}+00$ & $3.754 \mathrm{E}-05$ & $2.577 \mathrm{E}+10$ & $1.3883 \mathrm{E}+00$ & $3.730 \mathrm{E}-05$ & $2.559 \mathrm{E}+10$ & 0.012 \\
\hline $1 s 2 p^{1} P_{1}^{o}$ & $1 s 7 f^{3} F_{2}^{o}$ & $1.3997 \mathrm{E}+00$ & $7.771 \mathrm{E}-05$ & $5.292 \mathrm{E}+10$ & $1.3939 \mathrm{E}+00$ & $7.705 \mathrm{E}-05$ & $5.245 \mathrm{E}+10$ & 0.006 \\
\hline $1 s 3 p^{3} P_{1}^{o}$ & $1 s 7 f^{3} F_{2}^{o}$ & $3.4047 \mathrm{E}+00$ & $4.739 \mathrm{E}-06$ & $5.454 \mathrm{E}+08$ & $3.3906 \mathrm{E}+00$ & $5.997 \mathrm{E}-06$ & $6.900 \mathrm{E}+08$ & 0.127 \\
\hline $1 s 3 p^{3} P_{0}^{o}$ & $1 s 7 f^{3} F_{2}^{o}$ & $3.4061 \mathrm{E}+00$ & $6.996 \mathrm{E}-06$ & $8.044 \mathrm{E}+08$ & $3.3920 \mathrm{E}+00$ & $8.272 \mathrm{E}-06$ & $9.510 \mathrm{E}+08$ & 0.112 \\
\hline $1 s 3 p^{3} P_{2}^{o}$ & $1 s 7 f^{3} F_{2}^{o}$ & $3.5163 \mathrm{E}+00$ & $2.405 \mathrm{E}-06$ & $2.595 E+08$ & $3.5016 \mathrm{E}+00$ & $2.771 \mathrm{E}-06$ & $2.990 \mathrm{E}+08$ & 0.079 \\
\hline $1 s 3 p^{1} P_{1}^{o}$ & $1 s 7 f^{3} F_{2}^{o}$ & $3.5262 \mathrm{E}+00$ & $5.409 \mathrm{E}-06$ & $5.803 E+08$ & $3.5116 \mathrm{E}+00$ & $5.984 \mathrm{E}-06$ & $6.419 \mathrm{E}+08$ & 0.060 \\
\hline $1 s 4 p^{3} P_{1}^{o}$ & $1 s 7 f^{3} F_{2}^{o}$ & $7.3543 \mathrm{E}+00$ & $1.993 \mathrm{E}-05$ & $4.916 \mathrm{E}+08$ & $7.3233 \mathrm{E}+00$ & $1.884 \mathrm{E}-05$ & $4.647 \mathrm{E}+08$ & 0.016 \\
\hline $1 s 4 p^{3} P_{0}^{o}$ & $1 s 7 f^{3} F_{2}^{o}$ & $7.3571 \mathrm{E}+00$ & $2.804 \mathrm{E}-05$ & $6.912 \mathrm{E}+08$ & $7.3259 \mathrm{E}+00$ & $2.725 \mathrm{E}-05$ & $6.717 \mathrm{E}+08$ & 0.013 \\
\hline $1 s 4 p^{3} P_{2}^{o}$ & $1 s 7 f^{3} F_{2}^{o}$ & $7.5727 \mathrm{E}+00$ & $1.956 \mathrm{E}-06$ & $4.550 \mathrm{E}+07$ & $7.5406 \mathrm{E}+00$ & $1.789 \mathrm{E}-06$ & $4.163 \mathrm{E}+07$ & 0.025 \\
\hline $1 s 4 p^{1} P_{1}^{o}$ & $1 s 7 f^{3} F_{2}^{o}$ & $7.5917 \mathrm{E}+00$ & $3.790 \mathrm{E}-06$ & $8.772 E+07$ & $7.5597 \mathrm{E}+00$ & $3.539 \mathrm{E}-06$ & $8.193 \mathrm{E}+07$ & 0.018 \\
\hline $1 s 4 f^{3} F_{3}^{o}$ & $1 s 7 f^{3} F_{2}^{o}$ & $7.6619 \mathrm{E}+00$ & $7.214 \mathrm{E}-06$ & $1.639 E+08$ & $7.6295 \mathrm{E}+00$ & $7.104 \mathrm{E}-06$ & $1.614 \mathrm{E}+08$ & 0.010 \\
\hline $1 s 4 f^{3} F_{2}^{o}$ & $1 s 7 f^{3} F_{2}^{o}$ & $7.6634 \mathrm{E}+00$ & $2.888 \mathrm{E}-05$ & $6.561 \mathrm{E}+08$ & $7.6310 \mathrm{E}+00$ & $2.850 \mathrm{E}-05$ & $6.474 \mathrm{E}+08$ & 0.010 \\
\hline $1 s 4 f^{3} F_{4}^{o}$ & $1 s 7 f^{3} F_{2}^{o}$ & $7.6979 \mathrm{E}+00$ & $5.832 \mathrm{E}-07$ & $1.313 E+07$ & $7.6653 \mathrm{E}+00$ & $5.781 \mathrm{E}-07$ & $1.301 \mathrm{E}+07$ & 0.001 \\
\hline $1 s 4 f^{1} F_{3}^{0}$ & $1 s 7 f^{3} F_{2}^{0}$ & $7.6990 \mathrm{E}+00$ & $5.275 \mathrm{E}-06$ & $1.187 \mathrm{E}+08$ & $7.6665 \mathrm{E}+00$ & $5.227 \mathrm{E}-06$ & $1.176 \mathrm{E}+08$ & 0.004 \\
\hline $1 s 5 p^{3} P_{1}^{o}$ & $1 s 7 f^{3} F_{2}^{o}$ & $1.5732 \mathrm{E}+01$ & $1.269 \mathrm{E}-04$ & $6.840 \mathrm{E}+08$ & $1.5665 \mathrm{E}+01$ & $1.261 \mathrm{E}-04$ & $6.801 \mathrm{E}+08$ & 0.003 \\
\hline $1 s 5 p^{3} P_{0}^{o}$ & $1 s 7 f^{3} F_{2}^{o}$ & $1.5739 \mathrm{E}+01$ & $1.803 \mathrm{E}-04$ & $9.713 E+08$ & $1.5671 \mathrm{E}+01$ & $1.804 \mathrm{E}-04$ & $9.720 \mathrm{E}+08$ & 0.004 \\
\hline $1 s 5 p^{3} P_{2}^{o}$ & $1 s 7 f^{3} F_{2}^{o}$ & $1.6243 \mathrm{E}+01$ & $2.078 \mathrm{E}-05$ & $1.051 \mathrm{E}+08$ & $1.6174 \mathrm{E}+01$ & $2.059 \mathrm{E}-05$ & $1.041 \mathrm{E}+08$ & 0.002 \\
\hline $1 s 5 p^{1} P_{1}^{o}$ & $1 s 7 f^{3} F_{2}^{o}$ & $1.6288 \mathrm{E}+01$ & $4.275 \mathrm{E}-05$ & $2.150 E+08$ & $1.6219 \mathrm{E}+01$ & $4.240 \mathrm{E}-05$ & $2.132 \mathrm{E}+08$ & 0.003 \\
\hline $1 s 5 f^{3} F_{3}^{o}$ & $1 s 7 f^{3} F_{2}^{o}$ & $1.6454 \mathrm{E}+01$ & $2.516 \mathrm{E}-05$ & $1.240 \mathrm{E}+08$ & $1.6385 \mathrm{E}+01$ & $2.509 \mathrm{E}-05$ & $1.236 \mathrm{E}+08$ & 0.001 \\
\hline $1 s 5 f^{3} F_{2}^{o}$ & $1 s 7 f^{3} F_{2}^{o}$ & $1.6457 \mathrm{E}+01$ & $1.008 \mathrm{E}-04$ & $4.967 \mathrm{E}+08$ & $1.6388 \mathrm{E}+01$ & $1.006 \mathrm{E}-04$ & $4.955 \mathrm{E}+08$ & 0.001 \\
\hline $1 s 5 f^{3} F_{4}^{o}$ & $1 s 7 f^{3} F_{2}^{o}$ & $1.6539 \mathrm{E}+01$ & $2.071 \mathrm{E}-06$ & $1.010 \mathrm{E}+07$ & $1.6469 \mathrm{E}+01$ & $2.072 \mathrm{E}-06$ & $1.010 \mathrm{E}+07$ & 0.001 \\
\hline $1 s 5 f^{1} F_{3}^{o}$ & $1 s 7 f^{3} F_{2}^{o}$ & $1.6542 \mathrm{E}+01$ & $1.883 \mathrm{E}-05$ & $9.182 \mathrm{E}+07$ & $1.6472 \mathrm{E}+01$ & $1.872 \mathrm{E}-05$ & $9.128 \mathrm{E}+07$ & 0.000 \\
\hline
\end{tabular}


Table 4 (continued)

\begin{tabular}{|c|c|c|c|c|c|c|c|c|}
\hline \multirow[t]{2}{*}{ Lower } & \multirow[t]{2}{*}{ Upper } & \multicolumn{3}{|l|}{ GRASP2K } & \multicolumn{3}{|l|}{ FAC } & \multirow[t]{2}{*}{$d T$} \\
\hline & & $\lambda(\AA)$ & $g f$ & $A\left(\mathrm{~s}^{-1}\right)$ & $\lambda(\AA)$ & $g f$ & $A\left(\mathrm{~s}^{-1}\right)$ & \\
\hline $1 s 6 p^{3} P_{1}^{o}$ & $1 s 7 f^{3} F_{2}^{o}$ & $4.0860 \mathrm{E}+01$ & $5.906 \mathrm{E}-04$ & $4.719 \mathrm{E}+08$ & $4.0680 \mathrm{E}+01$ & $6.038 \mathrm{E}-04$ & $4.826 \mathrm{E}+08$ & 0.001 \\
\hline $1 s 6 p^{3} P_{0}^{o}$ & $1 s 7 f^{3} F_{2}^{o}$ & $4.0884 \mathrm{E}+01$ & $8.426 \mathrm{E}-04$ & $6.725 E+08$ & $4.0618 \mathrm{E}+01$ & $8.498 \mathrm{E}-04$ & $6.814 \mathrm{E}+08$ & 0.001 \\
\hline $1 s 6 p^{3} P_{2}^{o}$ & $1 s 7 f^{3} F_{2}^{o}$ & $4.2882 \mathrm{E}+01$ & $1.216 \mathrm{E}-04$ & $8.820 \mathrm{E}+07$ & $4.2690 \mathrm{E}+01$ & $1.225 \mathrm{E}-04$ & $8.897 \mathrm{E}+07$ & 0.001 \\
\hline $1 s 6 p^{1} P_{1}^{o}$ & $1 s 7 f^{3} F_{2}^{o}$ & $4.3060 \mathrm{E}+01$ & $2.556 \mathrm{E}-04$ & $1.839 \mathrm{E}+08$ & $4.2848 \mathrm{E}+01$ & $2.487 \mathrm{E}-04$ & $1.792 E+08$ & 0.001 \\
\hline $1 s 6 f^{3} F_{3}^{o}$ & $1 s 7 f^{3} F_{2}^{o}$ & $4.3735 E+01$ & $1.035 \mathrm{E}-04$ & $7.216 \mathrm{E}+07$ & $4.3561 \mathrm{E}+01$ & $1.039 \mathrm{E}-04$ & $7.247 \mathrm{E}+07$ & 0.000 \\
\hline $1 s 6 f^{3} F_{2}^{o}$ & $1 s 7 f^{3} F_{2}^{o}$ & $4.3749 \mathrm{E}+01$ & $4.148 \mathrm{E}-04$ & $2.891 \mathrm{E}+08$ & $4.3561 \mathrm{E}+01$ & $4.154 \mathrm{E}-04$ & $2.896 \mathrm{E}+08$ & 0.000 \\
\hline $1 s 6 f^{3} F_{4}^{o}$ & $1 s 7 f^{3} F_{2}^{o}$ & $4.4085 \mathrm{E}+01$ & $8.672 \mathrm{E}-06$ & $5.953 E+06$ & $4.3907 \mathrm{E}+01$ & $8.726 \mathrm{E}-06$ & $5.987 E+06$ & 0.000 \\
\hline $1 s 6 f^{1} F_{3}^{o}$ & $1 s 7 f^{3} F_{2}^{o}$ & $4.4096 \mathrm{E}+01$ & $7.898 \mathrm{E}-05$ & $5.418 E+07$ & $4.3907 \mathrm{E}+01$ & $7.843 \mathrm{E}-05$ & $5.381 \mathrm{E}+07$ & 0.000 \\
\hline $1 s 6 h^{3} H_{4}^{o}$ & $1 s 7 f^{3} F_{2}^{o}$ & $4.4302 \mathrm{E}+01$ & $3.039 \mathrm{E}-05$ & $2.066 \mathrm{E}+07$ & $4.4116 \mathrm{E}+01$ & $3.041 \mathrm{E}-05$ & $2.067 \mathrm{E}+07$ & 0.001 \\
\hline $1 s 7 p^{3} P_{1}^{o}$ & $1 s 7 f^{3} F_{2}^{o}$ & $9.8851 \mathrm{E}+02$ & $2.450 \mathrm{E}-07$ & $3.345 \mathrm{E}+02$ & $9.7899 \mathrm{E}+02$ & $2.541 \mathrm{E}-07$ & $3.507 \mathrm{E}+02$ & 0.101 \\
\hline $1 s 7 p^{3} P_{0}^{o}$ & $1 s 7 f^{3} F_{2}^{o}$ & $9.9743 \mathrm{E}+02$ & $3.397 \mathrm{E}-07$ & $4.556 \mathrm{E}+02$ & $9.5689 \mathrm{E}+02$ & $3.834 \mathrm{E}-07$ & $5.539 E+02$ & 0.104 \\
\hline $1 s 7 p^{3} P_{2}^{o}$ & $1 s 7 f^{3} F_{2}^{o}$ & $3.4844 \mathrm{E}+03$ & $1.135 \mathrm{E}-09$ & $1.247 \mathrm{E}-01$ & $3.3927 E+03$ & $1.221 \mathrm{E}-09$ & $1.403 \mathrm{E}-01$ & 0.269 \\
\hline $1 s 7 p^{1} P_{1}^{o}$ & $1 s 7 f^{3} F_{2}^{o}$ & $4.4219 \mathrm{E}+03$ & $1.166 \mathrm{E}-09$ & $7.956 \mathrm{E}-02$ & $4.1543 E+03$ & $1.350 \mathrm{E}-09$ & $1.035 \mathrm{E}-01$ & 0.323 \\
\hline $1 s 7 f^{3} F_{3}^{o}$ & $1 s 7 f^{3} F_{2}^{o}$ & $2.0860 \mathrm{E}+05$ & $1.383 \mathrm{E}-14$ & $4.240 \mathrm{E}-10$ & & & & 0.119 \\
\hline $1 s^{2}{ }^{1} S_{0}$ & $1 s 7 d^{1} D_{2}$ & $3.1390 \mathrm{E}-01$ & $1.285 \mathrm{E}-04$ & $1.738 \mathrm{E}+12$ & $3.1267 \mathrm{E}-01$ & $1.498 \mathrm{E}-04$ & $2.027 \mathrm{E}+12$ & 0.002 \\
\hline $1 s 2 s^{3} S_{1}$ & $1 s 7 d^{1} D_{2}$ & $1.3248 \mathrm{E}+00$ & $5.060 \mathrm{E}-06$ & $3.847 \mathrm{E}+09$ & $1.3192 \mathrm{E}+00$ & $4.425 \mathrm{E}-06$ & $3.363 E+09$ & 0.012 \\
\hline $1 s 2 s^{1} S_{0}$ & $1 s 7 d^{1} D_{2}$ & $1.3369 \mathrm{E}+00$ & $6.434 \mathrm{E}-06$ & $4.802 E+09$ & $1.3314 \mathrm{E}+00$ & $6.727 \mathrm{E}-06$ & $5.019 E+09$ & 0.002 \\
\hline $1 s 3 s^{3} S_{1}$ & $1 s 7 d^{1} D_{2}$ & $3.3851 \mathrm{E}+00$ & $2.803 \mathrm{E}-05$ & $3.263 E+09$ & $3.3710 \mathrm{E}+00$ & $2.747 \mathrm{E}-05$ & $3.197 E+09$ & 0.019 \\
\hline $1 s 3 s^{1} S_{0}$ & $1 s 7 d^{1} D_{2}$ & $3.4060 \mathrm{E}+00$ & $4.485 \mathrm{E}-05$ & $5.158 E+09$ & $3.3920 \mathrm{E}+00$ & $4.243 E-05$ & $4.878 E+09$ & 0.081 \\
\hline $1 s 3 d^{3} D_{2}$ & $1 s 7 d^{1} D_{2}$ & $3.5247 \mathrm{E}+00$ & $2.531 \mathrm{E}-06$ & $2.717 \mathrm{E}+08$ & $3.5098 \mathrm{E}+00$ & $2.588 \mathrm{E}-06$ & $2.779 E+08$ & 0.006 \\
\hline $1 s 3 d^{3} D_{1}$ & $1 s 7 d^{1} D_{2}$ & $3.5261 \mathrm{E}+00$ & $5.861 \mathrm{E}-06$ & $6.288 E+08$ & $3.5113 \mathrm{E}+00$ & $5.640 \mathrm{E}-06$ & $6.051 E+08$ & 0.033 \\
\hline $1 s 3 d^{3} D_{3}$ & $1 s 7 d^{1} D_{2}$ & $3.5614 \mathrm{E}+00$ & $6.094 \mathrm{E}-06$ & $6.409 E+08$ & $3.5463 \mathrm{E}+00$ & $6.116 \mathrm{E}-06$ & $6.433 E+08$ & 0.027 \\
\hline $1 s 3 d^{1} D_{2}$ & $1 s 7 d^{1} D_{2}$ & $3.5628 \mathrm{E}+00$ & $2.633 \mathrm{E}-05$ & $2.767 E+09$ & $3.5477 \mathrm{E}+00$ & $2.540 \mathrm{E}-05$ & $2.669 E+09$ & 0.033 \\
\hline $1 s 4 s^{3} S_{1}$ & $1 s 7 d^{1} D_{2}$ & $7.3170 \mathrm{E}+00$ & $6.124 \mathrm{E}-05$ & $1.526 \mathrm{E}+09$ & $7.2864 \mathrm{E}+00$ & $6.084 \mathrm{E}-05$ & $1.515 E+09$ & 0.018 \\
\hline $1 s 4 s{ }^{1} S_{0}$ & $1 s 7 d^{1} D_{2}$ & $7.3564 \mathrm{E}+00$ & $9.856 \mathrm{E}-05$ & $2.430 \mathrm{E}+09$ & $7.3260 \mathrm{E}+00$ & $9.436 \mathrm{E}-05$ & $2.325 E+09$ & 0.064 \\
\hline $1 s 4 d^{3} D_{2}$ & $1 s 7 d^{1} D_{2}$ & $7.5888 \mathrm{E}+00$ & $6.059 \mathrm{E}-06$ & $1.403 E+08$ & $7.5573 E+00$ & $6.203 E-06$ & $1.436 E+08$ & 0.003 \\
\hline $1 s 4 d^{3} D_{1}$ & $1 s 7 d^{1} D_{2}$ & $7.5916 \mathrm{E}+00$ & $1.403 \mathrm{E}-05$ & $3.247 \mathrm{E}+08$ & $7.5601 \mathrm{E}+00$ & $1.369 \mathrm{E}-05$ & $3.169 E+08$ & 0.015 \\
\hline $1 s 4 d^{3} D_{3}$ & $1 s 7 d^{1} D_{2}$ & $7.6607 \mathrm{E}+00$ & $1.525 \mathrm{E}-05$ & $3.466 \mathrm{E}+08$ & $7.6287 \mathrm{E}+00$ & $1.511 \mathrm{E}-05$ & $3.435 E+08$ & 0.010 \\
\hline $1 s 4 d{ }^{1} D_{2}$ & $1 s 7 d^{1} D_{2}$ & $7.6636 \mathrm{E}+00$ & $6.371 \mathrm{E}-05$ & $1.447 \mathrm{E}+09$ & $7.6317 \mathrm{E}+00$ & $6.287 \mathrm{E}-05$ & $1.427 \mathrm{E}+09$ & 0.014 \\
\hline $1 s 5 s^{3} S_{1}$ & $1 s 7 d^{1} D_{2}$ & $1.5646 \mathrm{E}+01$ & $1.309 \mathrm{E}-04$ & $7.133 E+08$ & $1.5580 \mathrm{E}+01$ & $1.303 \mathrm{E}-04$ & $7.100 \mathrm{E}+08$ & 0.010 \\
\hline $1 s 5 s{ }^{1} S_{0}$ & $1 s 7 d^{1} D_{2}$ & $1.5737 \mathrm{E}+01$ & $2.061 \mathrm{E}-04$ & $1.110 \mathrm{E}+09$ & $1.5671 \mathrm{E}+01$ & $2.021 \mathrm{E}-04$ & $1.088 \mathrm{E}+09$ & 0.033 \\
\hline $1 s 5 d^{3} D_{2}$ & $1 s 7 d^{1} D_{2}$ & $1.6281 \mathrm{E}+01$ & $1.358 \mathrm{E}-05$ & $6.833 \mathrm{E}+07$ & $1.6215 \mathrm{E}+01$ & $1.394 \mathrm{E}-05$ & $7.013 E+07$ & 0.001 \\
\hline $1 s 5 d^{3} D_{1}$ & $1 s 7 d^{1} D_{2}$ & $1.6288 \mathrm{E}+01$ & $3.146 \mathrm{E}-05$ & $1.582 \mathrm{E}+08$ & $1.6221 \mathrm{E}+01$ & $3.092 \mathrm{E}-05$ & $1.554 \mathrm{E}+08$ & 0.006 \\
\hline $1 s 5 d^{3} D_{3}$ & $1 s 7 d^{1} D_{2}$ & $1.6451 \mathrm{E}+01$ & $3.517 \mathrm{E}-05$ & $1.734 \mathrm{E}+08$ & $1.6383 \mathrm{E}+01$ & $3.475 \mathrm{E}-05$ & $1.712 \mathrm{E}+08$ & 0.004 \\
\hline $1 s 5 d^{1} D_{2}$ & $1 s 7 d^{1} D_{2}$ & $1.6458 \mathrm{E}+01$ & $1.450 \mathrm{E}-04$ & $7.144 \mathrm{E}+08$ & $1.6390 \mathrm{E}+01$ & $1.447 \mathrm{E}-04$ & $7.124 \mathrm{E}+08$ & 0.005 \\
\hline $1 s 5 g^{3} G_{4}$ & $1 s 7 d^{1} D_{2}$ & $1.6539 \mathrm{E}+01$ & $1.475 \mathrm{E}-07$ & $7.192 E+05$ & $1.6469 \mathrm{E}+01$ & $1.668 \mathrm{E}-07$ & $8.136 E+05$ & 0.010 \\
\hline $1 s 5 g^{3} G_{3}$ & $1 s 7 d^{1} D_{2}$ & $1.6541 \mathrm{E}+01$ & $1.101 \mathrm{E}-06$ & $5.368 \mathrm{E}+06$ & $1.6471 \mathrm{E}+01$ & $1.083 \mathrm{E}-06$ & $5.283 E+06$ & 0.005 \\
\hline $1 s 5 g{ }^{1} G_{4}$ & $1 s 7 d^{1} D_{2}$ & $1.6592 \mathrm{E}+01$ & $1.578 \mathrm{E}-05$ & $7.645 \mathrm{E}+07$ & $1.6521 \mathrm{E}+01$ & $1.569 \mathrm{E}-05$ & $7.608 E+07$ & 0.004 \\
\hline $1 s 6 s^{3} S_{1}$ & $1 s 7 d^{1} D_{2}$ & $4.0526 \mathrm{E}+01$ & $3.877 \mathrm{E}-04$ & $3.150 \mathrm{E}+08$ & $4.0319 \mathrm{E}+01$ & $3.915 \mathrm{E}-04$ & $3.186 E+08$ & 0.005 \\
\hline $1 s 6 s{ }^{1} S_{0}$ & $1 s 7 d^{1} D_{2}$ & $4.0874 \mathrm{E}+01$ & $6.098 \mathrm{E}-04$ & $4.870 \mathrm{E}+08$ & $4.0613 \mathrm{E}+01$ & $6.046 \mathrm{E}-04$ & $4.848 \mathrm{E}+08$ & 0.010 \\
\hline $1 s 6 d^{3} D_{2}$ & $1 s 7 d^{1} D_{2}$ & $4.3033 \mathrm{E}+01$ & $4.383 \mathrm{E}-05$ & $3.157 E+07$ & $4.2873 \mathrm{E}+01$ & $4.367 \mathrm{E}-05$ & $3.143 E+07$ & 0.000 \\
\hline $1 s 6 d^{3} D_{1}$ & $1 s 7 d^{1} D_{2}$ & $4.3059 \mathrm{E}+01$ & $1.018 \mathrm{E}-04$ & $7.325 \mathrm{E}+07$ & $4.2867 \mathrm{E}+01$ & $9.979 \mathrm{E}-05$ & $7.183 E+07$ & 0.002 \\
\hline $1 s 6 d^{3} D_{3}$ & $1 s 7 d^{1} D_{2}$ & $4.3721 \mathrm{E}+01$ & $1.180 \mathrm{E}-04$ & $8.234 \mathrm{E}+07$ & $4.3553 \mathrm{E}+01$ & $1.166 \mathrm{E}-04$ & $8.133 E+07$ & 0.001 \\
\hline $1 s 6 d^{1} D_{2}$ & $1 s 7 d^{1} D_{2}$ & $4.3751 \mathrm{E}+01$ & $4.848 \mathrm{E}-04$ & $3.379 E+08$ & $4.3564 \mathrm{E}+01$ & $4.878 \mathrm{E}-04$ & $3.400 \mathrm{E}+08$ & 0.001 \\
\hline $1 s 6 g{ }^{3} G_{4}$ & $1 s 7 d^{1} D_{2}$ & $4.4084 \mathrm{E}+01$ & $1.166 \mathrm{E}-06$ & $8.003 E+05$ & $4.3900 \mathrm{E}+01$ & $1.267 \mathrm{E}-06$ & $8.701 \mathrm{E}+05$ & 0.003 \\
\hline $1 s 6 g^{3} G_{3}$ & $1 s 7 d^{1} D_{2}$ & $4.4092 \mathrm{E}+01$ & $8.625 \mathrm{E}-06$ & $5.918 E+06$ & $4.3900 \mathrm{E}+01$ & $8.518 \mathrm{E}-06$ & $5.846 \mathrm{E}+06$ & 0.001 \\
\hline $1 s 6 g^{1} G_{4}$ & $1 s 7 d^{1} D_{2}$ & $4.4302 \mathrm{E}+01$ & $1.239 \mathrm{E}-04$ & $8.420 E+07$ & $4.4109 \mathrm{E}+01$ & $1.240 \mathrm{E}-04$ & $8.433 E+07$ & 0.001 \\
\hline $1 s 7 s^{3} S_{1}$ & $1 s 7 d^{1} D_{2}$ & $8.7478 E+02$ & $3.916 \mathrm{E}-07$ & $6.827 \mathrm{E}+02$ & $8.6126 \mathrm{E}+02$ & $4.005 \mathrm{E}-07$ & $7.142 E+02$ & 0.006 \\
\hline $1 s 7 s^{1} S_{0}$ & $1 s 7 d^{1} D_{2}$ & $9.8833 \mathrm{E}+02$ & $4.178 \mathrm{E}-07$ & $5.706 \mathrm{E}+02$ & $9.5307 \mathrm{E}+02$ & $4.657 \mathrm{E}-07$ & $6.782 E+02$ & 0.090 \\
\hline $1 s 7 d^{3} D_{2}$ & $1 s 7 d^{1} D_{2}$ & $4.1666 \mathrm{E}+03$ & $7.586 \mathrm{E}-10$ & $5.829 \mathrm{E}-02$ & & & & 0.042 \\
\hline $1 s 7 d^{3} D_{1}$ & $1 s 7 d^{1} D_{2}$ & $4.3244 \mathrm{E}+03$ & $1.574 \mathrm{E}-09$ & $1.123 \mathrm{E}-01$ & & & & 0.014 \\
\hline $1 s 7 d^{3} D_{3}$ & $1 s 7 d^{1} D_{2}$ & $1.0098 \mathrm{E}+05$ & $1.418 \mathrm{E}-13$ & $1.855 \mathrm{E}-08$ & & & & 0.727 \\
\hline $1 s 2 p^{3} P_{2}^{o}$ & $1 s 7 f^{3} F_{4}^{o}$ & $1.3940 \mathrm{E}+00$ & $1.003 \mathrm{E}-03$ & $3.825 E+11$ & $1.3881 \mathrm{E}+00$ & $1.037 \mathrm{E}-03$ & $3.957 E+11$ & 0.013 \\
\hline $1 s 3 p^{3} P_{2}^{o}$ & $1 s 7 f^{3} F_{4}^{o}$ & $3.5150 \mathrm{E}+00$ & $5.714 \mathrm{E}-05$ & $3.428 \mathrm{E}+09$ & $3.5002 \mathrm{E}+00$ & $6.732 \mathrm{E}-05$ & $4.038 E+09$ & 0.079 \\
\hline $1 s 4 p^{3} P_{2}^{o}$ & $1 s 7 f^{3} F_{4}^{0}$ & $7.5664 \mathrm{E}+00$ & $6.079 \mathrm{E}-05$ & $7.869 \mathrm{E}+08$ & $7.5341 \mathrm{E}+00$ & $5.644 \mathrm{E}-05$ & $7.306 \mathrm{E}+08$ & 0.021 \\
\hline $1 s 4 f^{3} F_{3}^{o}$ & $1 s 7 f^{3} F_{4}^{0}$ & $7.6554 \mathrm{E}+00$ & $7.647 \mathrm{E}-06$ & $9.670 \mathrm{E}+07$ & $7.6229 \mathrm{E}+00$ & $7.602 \mathrm{E}-06$ & $9.614 \mathrm{E}+07$ & 0.010 \\
\hline $1 s 4 f^{3} F_{2}^{o}$ & $1 s 7 f^{3} F_{4}^{o}$ & $7.6569 \mathrm{E}+00$ & $6.110 \mathrm{E}-07$ & $7.724 \mathrm{E}+06$ & $7.6244 \mathrm{E}+00$ & $6.060 \mathrm{E}-07$ & $7.660 \mathrm{E}+06$ & 0.012 \\
\hline $1 s 4 f^{3} F_{4}^{o}$ & $1 s 7 f^{3} F_{4}^{o}$ & $7.6914 \mathrm{E}+00$ & $6.132 \mathrm{E}-05$ & $7.682 E+08$ & $7.6587 \mathrm{E}+00$ & $6.131 \mathrm{E}-05$ & $7.682 E+08$ & 0.004 \\
\hline $1 s 4 f^{1} F_{3}^{o}$ & $1 s 7 f^{3} F_{4}^{0}$ & $7.6926 \mathrm{E}+00$ & $5.597 \mathrm{E}-06$ & $7.009 E+07$ & $7.6598 \mathrm{E}+00$ & $5.600 \mathrm{E}-06$ & $7.013 E+07$ & 0.004 \\
\hline $1 s 5 p^{3} P_{2}^{0}$ & $1 s 7 f^{3} F_{4}^{0}$ & $1.6215 \mathrm{E}+01$ & $5.823 \mathrm{E}-04$ & $1.642 \mathrm{E}+09$ & $1.6144 \mathrm{E}+01$ & $5.789 \mathrm{E}-04$ & $1.632 E+09$ & 0.002 \\
\hline $1 s 5 f^{3} F_{3}^{o}$ & $1 s 7 f^{3} F_{4}^{o}$ & $1.6424 \mathrm{E}+01$ & $2.639 \mathrm{E}-05$ & $7.251 \mathrm{E}+07$ & $1.6354 \mathrm{E}+01$ & $2.644 \mathrm{E}-05$ & $7.266 \mathrm{E}+07$ & 0.001 \\
\hline $1 s 5 f^{3} F_{2}^{o}$ & $1 s 7 f^{3} F_{4}^{0}$ & $1.6428 \mathrm{E}+01$ & $2.088 \mathrm{E}-06$ & $5.735 \mathrm{E}+06$ & $1.6358 \mathrm{E}+01$ & $2.090 \mathrm{E}-06$ & $5.741 \mathrm{E}+06$ & 0.001 \\
\hline $1 s 5 f^{3} F_{4}^{o}$ & $1 s 7 f^{3} F_{4}^{0}$ & $1.6509 \mathrm{E}+01$ & $2.165 \mathrm{E}-04$ & $5.887 E+08$ & $1.6439 \mathrm{E}+01$ & $2.163 \mathrm{E}-04$ & $5.883 E+08$ & 0.000 \\
\hline $1 s 5 f^{1} F_{3}^{o}$ & $1 s 7 f^{3} F_{4}^{0}$ & $1.6512 \mathrm{E}+01$ & $1.977 \mathrm{E}-05$ & $5.373 E+07$ & $1.6441 \mathrm{E}+01$ & $1.975 \mathrm{E}-05$ & $5.369 E+07$ & 0.000 \\
\hline $1 s 6 p^{3} P_{2}^{o}$ & $1 s 7 f^{3} F_{4}^{o}$ & $4.2681 \mathrm{E}+01$ & $3.276 \mathrm{E}-03$ & $1.333 \mathrm{E}+09$ & $4.2483 \mathrm{E}+01$ & $3.308 \mathrm{E}-03$ & $1.346 E+09$ & 0.001 \\
\hline $1 s 6 f^{3} F_{3}^{o}$ & $1 s 7 f^{3} F_{4}^{0}$ & $4.3526 \mathrm{E}+01$ & $1.066 \mathrm{E}-04$ & $4.170 \mathrm{E}+07$ & $4.3345 E+01$ & $1.073 \mathrm{E}-04$ & $4.197 \mathrm{E}+07$ & 0.000 \\
\hline $1 s 6 f^{3} F_{2}^{o}$ & $1 s 7 f^{3} F_{4}^{0}$ & $4.3541 \mathrm{E}+01$ & $8.390 \mathrm{E}-06$ & $3.280 E+06$ & $4.3345 \mathrm{E}+01$ & $8.576 \mathrm{E}-06$ & $3.354 \mathrm{E}+06$ & 0.000 \\
\hline $1 s 6 f^{3} F_{4}^{o}$ & $1 s 7 f^{3} F_{4}^{0}$ & $4.3873 \mathrm{E}+01$ & $8.912 \mathrm{E}-04$ & $3.432 E+08$ & $4.3688 \mathrm{E}+01$ & $8.920 \mathrm{E}-04$ & $3.434 \mathrm{E}+08$ & 0.000 \\
\hline $1 s 6 f^{1} F_{3}^{o}$ & $1 s 7 f^{3} F_{4}^{o}$ & $4.3884 \mathrm{E}+01$ & $8.141 \mathrm{E}-05$ & $3.133 E+07$ & $4.3689 \mathrm{E}+01$ & $8.098 \mathrm{E}-05$ & $3.118 E+07$ & 0.000 \\
\hline $1 s 6 h^{3} H_{5}^{o}$ & $1 s 7 f^{3} F_{4}^{o}$ & $4.4083 \mathrm{E}+01$ & $2.238 \mathrm{E}-06$ & $8.534 \mathrm{E}+05$ & $4.3895 \mathrm{E}+01$ & $2.247 \mathrm{E}-06$ & $8.571 E+05$ & 0.001 \\
\hline $1 s 6 h^{3} H_{4}^{o}$ & $1 s 7 f^{3} F_{4}^{o}$ & $4.4089 \mathrm{E}+01$ & $1.604 \mathrm{E}-07$ & $6.116 E+04$ & & & & 0.001 \\
\hline
\end{tabular}


Table 4 (continued)

\begin{tabular}{|c|c|c|c|c|c|c|c|c|}
\hline \multirow[t]{2}{*}{ Lower } & \multirow[t]{2}{*}{ Upper } & \multicolumn{3}{|l|}{ GRASP2K } & \multicolumn{3}{|l|}{ FAC } & \multirow[t]{2}{*}{$d T$} \\
\hline & & $\lambda(\AA)$ & $g f$ & $A\left(\mathrm{~s}^{-1}\right)$ & $\lambda(\AA)$ & $g f$ & $A\left(\mathrm{~s}^{-1}\right)$ & \\
\hline $1 s 6 h^{3} H_{6}^{o}$ & $1 s 7 f^{3} F_{4}^{o}$ & $4.4223 \mathrm{E}+01$ & $4.928 \mathrm{E}-05$ & $1.868 \mathrm{E}+07$ & $4.4034 \mathrm{E}+01$ & $4.929 \mathrm{E}-05$ & $1.868 \mathrm{E}+07$ & 0.000 \\
\hline $1 s 6 h^{1} H_{5}^{o}$ & $1 s 7 f^{3} F_{4}^{0}$ & $4.4228 \mathrm{E}+01$ & $1.903 \mathrm{E}-06$ & $7.210 \mathrm{E}+05$ & $4.4034 \mathrm{E}+01$ & $1.895 \mathrm{E}-06$ & $7.184 \mathrm{E}+05$ & 0.000 \\
\hline $1 s 7 p^{3} P_{2}^{o}$ & $1 s 7 f^{3} F_{4}^{o}$ & $2.5222 \mathrm{E}+03$ & $8.144 \mathrm{E}-08$ & $9.488 \mathrm{E}+00$ & $2.4461 \mathrm{E}+03$ & $8.879 \mathrm{E}-08$ & $1.090 \mathrm{E}+01$ & 0.215 \\
\hline $1 s 7 f^{3} F_{3}^{o}$ & $1 s 7 f^{3} F_{4}^{o}$ & $8.7508 \mathrm{E}+03$ & $1.956 \mathrm{E}-10$ & $1.893 \mathrm{E}-03$ & $8.7713 \mathrm{E}+03$ & $1.926 \mathrm{E}-10$ & $1.840 \mathrm{E}-03$ & 0.012 \\
\hline $1 s 7 f^{3} F_{2}^{0}$ & $1 s 7 f^{3} F_{4}^{0}$ & $9.1339 \mathrm{E}+03$ & $1.364 \mathrm{E}-11$ & $1.211 \mathrm{E}-04$ & & & & 0.004 \\
\hline $1 s 3 d^{3} D_{2}$ & $1 s 7 g{ }^{3} G_{4}$ & $3.5233 \mathrm{E}+00$ & $1.943 \mathrm{E}-03$ & $1.160 \mathrm{E}+11$ & $3.5085 \mathrm{E}+00$ & $1.957 \mathrm{E}-03$ & $1.168 \mathrm{E}+11$ & 0.001 \\
\hline $1 s 3 d^{3} D_{3}$ & $1 s 7 g{ }^{3} G_{4}$ & $3.5600 \mathrm{E}+00$ & $2.000 \mathrm{E}-04$ & $1.170 \mathrm{E}+10$ & $3.5449 \mathrm{E}+00$ & $1.996 \mathrm{E}-04$ & $1.167 \mathrm{E}+10$ & 0.002 \\
\hline $1 s 3 d{ }^{1} D_{2}$ & $1 s 7 g{ }^{3} G_{4}$ & $3.5614 \mathrm{E}+00$ & $1.781 \mathrm{E}-05$ & $1.041 \mathrm{E}+09$ & $3.5464 \mathrm{E}+00$ & $1.724 \mathrm{E}-05$ & $1.007 \mathrm{E}+09$ & 0.006 \\
\hline $1 s 4 d^{3} D_{2}$ & $1 s 7 g{ }^{3} G_{4}$ & $7.5825 \mathrm{E}+00$ & $3.212 \mathrm{E}-04$ & $4.141 \mathrm{E}+09$ & $7.5510 \mathrm{E}+00$ & $3.252 \mathrm{E}-04$ & $4.191 E+09$ & 0.002 \\
\hline $1 s 4 d^{3} D_{3}$ & $1 s 7 g{ }^{3} G_{4}$ & $7.6543 \mathrm{E}+00$ & $3.711 \mathrm{E}-05$ & $4.695 \mathrm{E}+08$ & $7.6223 \mathrm{E}+00$ & $3.748 \mathrm{E}-05$ & $4.741 E+08$ & 0.003 \\
\hline $1 s 4 d^{1} D_{2}$ & $1 s 7 g{ }^{3} G_{4}$ & $7.6572 \mathrm{E}+00$ & $3.439 \mathrm{E}-06$ & $4.347 \mathrm{E}+07$ & $7.6253 \mathrm{E}+00$ & $3.353 \mathrm{E}-06$ & $4.237 \mathrm{E}+07$ & 0.011 \\
\hline $1 s 5 d^{3} D_{2}$ & $1 s 7 g{ }^{3} G_{4}$ & $1.6252 \mathrm{E}+01$ & $1.272 \mathrm{E}-04$ & $3.569 E+08$ & $1.6186 \mathrm{E}+01$ & $1.258 \mathrm{E}-04$ & $3.529 E+08$ & 0.002 \\
\hline $1 s 5 d^{3} D_{3}$ & $1 s 7 g{ }^{3} G_{4}$ & $1.6421 \mathrm{E}+01$ & $1.005 \mathrm{E}-05$ & $2.763 \mathrm{E}+07$ & $1.6353 \mathrm{E}+01$ & $9.930 \mathrm{E}-06$ & $2.728 \mathrm{E}+07$ & 0.003 \\
\hline $1 s 5 d{ }^{1} D_{2}$ & $1 s 7 g{ }^{3} G_{4}$ & $1.6428 \mathrm{E}+01$ & $9.544 \mathrm{E}-07$ & $2.621 \mathrm{E}+06$ & $1.6361 \mathrm{E}+01$ & $9.268 \mathrm{E}-07$ & $2.544 \mathrm{E}+06$ & 0.013 \\
\hline $1 s 5 g^{3} G_{4}$ & $1 s 7 g{ }^{3} G_{4}$ & $1.6509 \mathrm{E}+01$ & $1.030 \mathrm{E}-04$ & $2.801 \mathrm{E}+08$ & $1.6439 \mathrm{E}+01$ & $1.029 \mathrm{E}-04$ & $2.799 E+08$ & 0.001 \\
\hline $1 s 5 g^{3} G_{3}$ & $1 s 7 g{ }^{3} G_{4}$ & $1.6511 \mathrm{E}+01$ & $9.340 \mathrm{E}-06$ & $2.539 E+07$ & $1.6441 \mathrm{E}+01$ & $9.352 \mathrm{E}-06$ & $2.542 \mathrm{E}+07$ & 0.001 \\
\hline $1 s 5 g^{3} G_{5}$ & $1 s 7 g{ }^{3} G_{4}$ & $1.6560 \mathrm{E}+01$ & $9.418 \mathrm{E}-06$ & $2.545 \mathrm{E}+07$ & $1.6490 \mathrm{E}+01$ & $9.404 \mathrm{E}-06$ & $2.541 \mathrm{E}+07$ & 0.001 \\
\hline $1 s 5 g{ }^{1} G_{4}$ & $1 s 7 g{ }^{3} G_{4}$ & $1.6562 \mathrm{E}+01$ & $6.755 \mathrm{E}-07$ & $1.825 \mathrm{E}+06$ & $1.6491 \mathrm{E}+01$ & $6.402 \mathrm{E}-07$ & $1.730 \mathrm{E}+06$ & 0.000 \\
\hline $1 s 6 d^{3} D_{2}$ & $1 s 7 g{ }^{3} G_{4}$ & $4.2832 \mathrm{E}+01$ & $4.032 \mathrm{E}-03$ & $1.629 \mathrm{E}+09$ & $4.2672 \mathrm{E}+01$ & $4.028 \mathrm{E}-03$ & $1.625 E+09$ & 0.000 \\
\hline $1 s 6 d^{3} D_{3}$ & $1 s 7 g{ }^{3} G_{4}$ & $4.3514 \mathrm{E}+01$ & $4.098 \mathrm{E}-04$ & $1.604 \mathrm{E}+08$ & $4.3345 \mathrm{E}+01$ & $4.110 \mathrm{E}-04$ & $1.607 \mathrm{E}+08$ & 0.000 \\
\hline $1 s 6 d{ }^{1} D_{2}$ & $1 s 7 g{ }^{3} G_{4}$ & $4.3543 \mathrm{E}+01$ & $3.925 \mathrm{E}-05$ & $1.534 \mathrm{E}+07$ & $4.3356 \mathrm{E}+01$ & $4.179 \mathrm{E}-05$ & $1.633 E+07$ & 0.001 \\
\hline $1 s 6 g{ }^{3} G_{4}$ & $1 s 7 g{ }^{3} G_{4}$ & $4.3873 \mathrm{E}+01$ & $6.859 \mathrm{E}-04$ & $2.641 \mathrm{E}+08$ & $4.3688 \mathrm{E}+01$ & $6.861 \mathrm{E}-04$ & $2.641 \mathrm{E}+08$ & 0.000 \\
\hline $1 s 6 g{ }^{3} G_{3}$ & $1 s 7 g{ }^{3} G_{4}$ & $4.3882 \mathrm{E}+01$ & $6.214 \mathrm{E}-05$ & $2.392 \mathrm{E}+07$ & $4.3688 \mathrm{E}+01$ & $6.237 \mathrm{E}-05$ & $2.401 E+07$ & 0.000 \\
\hline $1 s 6 g^{3} G_{5}$ & $1 s 7 g{ }^{3} G_{4}$ & $4.4082 \mathrm{E}+01$ & $6.352 \mathrm{E}-05$ & $2.423 E+07$ & $4.3896 \mathrm{E}+01$ & $6.369 E-05$ & $2.429 E+07$ & 0.000 \\
\hline $1 s 6 g{ }^{1} G_{4}$ & $1 s 7 g{ }^{3} G_{4}$ & $4.4089 \mathrm{E}+01$ & $4.557 \mathrm{E}-06$ & $1.737 \mathrm{E}+06$ & $4.3896 \mathrm{E}+01$ & $4.548 \mathrm{E}-06$ & $1.734 \mathrm{E}+06$ & 0.000 \\
\hline $1 s 7 d^{3} D_{2}$ & $1 s 7 g{ }^{3} G_{4}$ & $2.8662 \mathrm{E}+03$ & $4.119 \mathrm{E}-08$ & $3.716 \mathrm{E}+00$ & $2.9148 \mathrm{E}+03$ & $3.861 \mathrm{E}-08$ & $3.339 \mathrm{E}+00$ & 0.001 \\
\hline $1 s 7 d^{3} D_{3}$ & $1 s 7 g{ }^{3} G_{4}$ & $8.4183 \mathrm{E}+03$ & $1.657 \mathrm{E}-10$ & $1.733 \mathrm{E}-03$ & $8.7854 \mathrm{E}+03$ & $1.443 \mathrm{E}-10$ & $1.374 \mathrm{E}-03$ & 0.001 \\
\hline $1 s 7 d{ }^{1} D_{2}$ & $1 s 7 g{ }^{3} G_{4}$ & $9.1839 \mathrm{E}+03$ & $1.227 \mathrm{E}-11$ & $1.078 \mathrm{E}-04$ & & & & 0.142 \\
\hline $1 s 2 s^{3} S_{1}$ & $1 s 7 g{ }^{3} G_{3}$ & $1.3246 \mathrm{E}+00$ & $1.973 \mathrm{E}-11$ & $1.072 \mathrm{E}+04$ & & & & 0.848 \\
\hline $1 s 3 s^{3} S_{1}$ & $1 s 7 g^{3} G_{3}$ & $3.3838 \mathrm{E}+00$ & $3.349 \mathrm{E}-09$ & $2.787 \mathrm{E}+05$ & & & & 0.424 \\
\hline $1 s 3 d^{3} D_{2}$ & $1 s 7 g{ }^{3} G_{3}$ & $3.5233 \mathrm{E}+00$ & $2.174 \mathrm{E}-04$ & $1.669 \mathrm{E}+10$ & $3.5085 E+00$ & $2.189 \mathrm{E}-04$ & $1.680 \mathrm{E}+10$ & 0.002 \\
\hline $1 s 3 d^{3} D_{1}$ & $1 s 7 g{ }^{3} G_{3}$ & $3.5248 \mathrm{E}+00$ & $1.293 \mathrm{E}-03$ & $9.919 E+10$ & $3.5099 \mathrm{E}+00$ & $1.304 \mathrm{E}-03$ & $1.000 \mathrm{E}+11$ & 0.002 \\
\hline $1 s 3 d^{3} D_{3}$ & $1 s 7 g{ }^{3} G_{3}$ & $3.5600 \mathrm{E}+00$ & $2.399 \mathrm{E}-05$ & $1.804 \mathrm{E}+09$ & $3.5449 \mathrm{E}+00$ & $2.396 \mathrm{E}-05$ & $1.801 \mathrm{E}+09$ & 0.003 \\
\hline $1 s 3 d{ }^{1} D_{2}$ & $1 s 7 g{ }^{3} G_{3}$ & $3.5614 \mathrm{E}+00$ & $1.430 \mathrm{E}-04$ & $1.074 \mathrm{E}+10$ & $3.5464 \mathrm{E}+00$ & $1.423 \mathrm{E}-04$ & $1.069 \mathrm{E}+10$ & 0.002 \\
\hline $1 s 4 s^{3} S_{1}$ & $1 s 7 g{ }^{3} G_{3}$ & $7.3110 \mathrm{E}+00$ & $5.849 \mathrm{E}-10$ & $1.043 E+04$ & & & & 0.588 \\
\hline $1 s 4 d^{3} D_{2}$ & $1 s 7 g{ }^{3} G_{3}$ & $7.5824 \mathrm{E}+00$ & $3.602 \mathrm{E}-05$ & $5.970 E+08$ & $7.5510 \mathrm{E}+00$ & $3.653 \mathrm{E}-05$ & $6.054 \mathrm{E}+08$ & 0.002 \\
\hline $1 s 4 d^{3} D_{1}$ & $1 s 7 g{ }^{3} G_{3}$ & $7.5852 \mathrm{E}+00$ & $2.142 \mathrm{E}-04$ & $3.547 \mathrm{E}+09$ & $7.5538 \mathrm{E}+00$ & $2.168 \mathrm{E}-04$ & $3.590 \mathrm{E}+09$ & 0.004 \\
\hline $1 s 4 d^{3} D_{3}$ & $1 s 7 g{ }^{3} G_{3}$ & $7.6541 \mathrm{E}+00$ & $4.463 \mathrm{E}-06$ & $7.260 \mathrm{E}+07$ & $7.6223 \mathrm{E}+00$ & $4.498 \mathrm{E}-06$ & $7.314 \mathrm{E}+07$ & 0.001 \\
\hline $1 s 4 d^{1} D_{2}$ & $1 s 7 g{ }^{3} G_{3}$ & $7.6571 \mathrm{E}+00$ & $2.646 \mathrm{E}-05$ & $4.300 \mathrm{E}+08$ & $7.6253 \mathrm{E}+00$ & $2.660 \mathrm{E}-05$ & $4.323 E+08$ & 0.003 \\
\hline $1 s 5 s^{3} S_{1}$ & $1 s 7 g{ }^{3} G_{3}$ & $1.5619 \mathrm{E}+01$ & $3.106 \mathrm{E}-10$ & $1.213 E+03$ & & & & 0.374 \\
\hline $1 s 5 d^{3} D_{2}$ & $1 s 7 g{ }^{3} G_{3}$ & $1.6252 \mathrm{E}+01$ & $1.432 \mathrm{E}-05$ & $5.167 \mathrm{E}+07$ & $1.6186 \mathrm{E}+01$ & $1.412 \mathrm{E}-05$ & $5.095 \mathrm{E}+07$ & 0.002 \\
\hline $1 s 5 d^{3} D_{1}$ & $1 s 7 g{ }^{3} G_{3}$ & $1.6258 \mathrm{E}+01$ & $8.465 \mathrm{E}-05$ & $3.052 E+08$ & $1.6192 \mathrm{E}+01$ & $8.387 \mathrm{E}-05$ & $3.022 \mathrm{E}+08$ & 0.004 \\
\hline $1 s 5 d^{3} D_{3}$ & $1 s 7 g{ }^{3} G_{3}$ & $1.6420 \mathrm{E}+01$ & $1.213 \mathrm{E}-06$ & $4.288 \mathrm{E}+06$ & $1.6353 \mathrm{E}+01$ & $1.192 \mathrm{E}-06$ & $4.211 \mathrm{E}+06$ & 0.003 \\
\hline $1 s 5 d{ }^{1} D_{2}$ & $1 s 7 g{ }^{3} G_{3}$ & $1.6428 \mathrm{E}+01$ & $7.124 \mathrm{E}-06$ & $2.515 E+07$ & $1.6361 \mathrm{E}+01$ & $7.012 \mathrm{E}-06$ & $2.475 E+07$ & 0.003 \\
\hline $1 s 5 g^{3} G_{4}$ & $1 s 7 g{ }^{3} G_{3}$ & $1.6509 \mathrm{E}+01$ & $9.333 \mathrm{E}-06$ & $3.263 \mathrm{E}+07$ & $1.6439 \mathrm{E}+01$ & $9.323 \mathrm{E}-06$ & $3.259 E+07$ & 0.001 \\
\hline $1 s 5 g{ }^{3} G_{3}$ & $1 s 7 g{ }^{3} G_{3}$ & $1.6511 \mathrm{E}+01$ & $7.814 \mathrm{E}-05$ & $2.732 E+08$ & $1.6441 \mathrm{E}+01$ & $7.792 \mathrm{E}-05$ & $2.723 E+08$ & 0.001 \\
\hline $1 s 5 g^{3} G_{5}$ & $1 s 7 g{ }^{3} G_{3}$ & $1.6560 \mathrm{E}+01$ & $4.513 \mathrm{E}-07$ & $1.568 \mathrm{E}+06$ & $1.6490 \mathrm{E}+01$ & $4.478 \mathrm{E}-07$ & $1.556 \mathrm{E}+06$ & 0.001 \\
\hline $1 s 5 g{ }^{1} G_{4}$ & $1 s 7 g{ }^{3} G_{3}$ & $1.6561 \mathrm{E}+01$ & $7.471 \mathrm{E}-06$ & $2.595 \mathrm{E}+07$ & $1.6491 \mathrm{E}+01$ & $7.418 \mathrm{E}-06$ & $2.577 \mathrm{E}+07$ & 0.001 \\
\hline $1 s 6 s^{3} S_{1}$ & $1 s 7 g{ }^{3} G_{3}$ & $4.0344 \mathrm{E}+01$ & $7.208 \mathrm{E}-09$ & $4.220 \mathrm{E}+03$ & & & & 0.052 \\
\hline $1 s 6 d^{3} D_{2}$ & $1 s 7 g{ }^{3} G_{3}$ & $4.2827 \mathrm{E}+01$ & $4.534 \mathrm{E}-04$ & $2.355 \mathrm{E}+08$ & $4.2672 \mathrm{E}+01$ & $4.574 \mathrm{E}-04$ & $2.373 E+08$ & 0.000 \\
\hline $1 s 6 d^{3} D_{1}$ & $1 s 7 g{ }^{3} G_{3}$ & $4.2853 \mathrm{E}+01$ & $2.691 \mathrm{E}-03$ & $1.397 \mathrm{E}+09$ & $4.2665 \mathrm{E}+01$ & $2.693 \mathrm{E}-03$ & $1.397 \mathrm{E}+09$ & 0.000 \\
\hline $1 s 6 d^{3} D_{3}$ & $1 s 7 g{ }^{3} G_{3}$ & $4.3508 \mathrm{E}+01$ & $4.934 \mathrm{E}-05$ & $2.484 \mathrm{E}+07$ & $4.3345 \mathrm{E}+01$ & $4.933 \mathrm{E}-05$ & $2.480 \mathrm{E}+07$ & 0.000 \\
\hline $1 s 6 d{ }^{1} D_{2}$ & $1 s 7 g{ }^{3} G_{3}$ & $4.3538 \mathrm{E}+01$ & $2.913 \mathrm{E}-04$ & $1.465 \mathrm{E}+08$ & $4.3356 \mathrm{E}+01$ & $2.873 \mathrm{E}-04$ & $1.444 \mathrm{E}+08$ & 0.000 \\
\hline $1 s 6 g^{3} G_{4}$ & $1 s 7 g{ }^{3} G_{3}$ & $4.3868 \mathrm{E}+01$ & $6.210 \mathrm{E}-05$ & $3.075 E+07$ & $4.3688 \mathrm{E}+01$ & $6.237 \mathrm{E}-05$ & $3.087 E+07$ & 0.000 \\
\hline $1 s 6 g^{3} G_{3}$ & $1 s 7 g{ }^{3} G_{3}$ & $4.3876 \mathrm{E}+01$ & $5.195 \mathrm{E}-04$ & $2.571 \mathrm{E}+08$ & $4.3688 \mathrm{E}+01$ & $5.197 \mathrm{E}-04$ & $2.573 E+08$ & 0.000 \\
\hline $1 s 6 g^{3} G_{5}$ & $1 s 7 g{ }^{3} G_{3}$ & $4.4077 \mathrm{E}+01$ & $3.033 \mathrm{E}-06$ & $1.488 \mathrm{E}+06$ & $4.3896 \mathrm{E}+01$ & $3.033 \mathrm{E}-06$ & $1.487 \mathrm{E}+06$ & 0.001 \\
\hline $1 s 6 g{ }^{1} G_{4}$ & $1 s 7 g{ }^{3} G_{3}$ & $4.4084 \mathrm{E}+01$ & $5.031 \mathrm{E}-05$ & $2.467 \mathrm{E}+07$ & $4.3896 \mathrm{E}+01$ & $5.004 \mathrm{E}-05$ & $2.453 E+07$ & 0.000 \\
\hline $1 s 7 s^{3} S_{1}$ & $1 s 7 g{ }^{3} G_{3}$ & $7.9695 \mathrm{E}+02$ & $3.661 \mathrm{E}-12$ & $5.493 \mathrm{E}-03$ & & & & 0.386 \\
\hline $1 s 7 d^{3} D_{2}$ & $1 s 7 g{ }^{3} G_{3}$ & $2.8438 \mathrm{E}+03$ & $4.743 \mathrm{E}-09$ & $5.589 \mathrm{E}-01$ & $2.9148 \mathrm{E}+03$ & $4.385 \mathrm{E}-09$ & $4.876 \mathrm{E}-01$ & 0.015 \\
\hline $1 s 7 d^{3} D_{1}$ & $1 s 7 g{ }^{3} G_{3}$ & $2.9164 \mathrm{E}+03$ & $2.608 \mathrm{E}-08$ & $2.922 \mathrm{E}+00$ & $2.8963 \mathrm{E}+03$ & $2.630 \mathrm{E}-08$ & $2.962 E+00$ & 0.002 \\
\hline $1 s 7 d^{3} D_{3}$ & $1 s 7 g{ }^{3} G_{3}$ & $8.2277 \mathrm{E}+03$ & $2.137 \mathrm{E}-11$ & $3.008 \mathrm{E}-04$ & & & & 0.039 \\
\hline $1 s 7 d^{1} D_{2}$ & $1 s 7 g{ }^{3} G_{3}$ & $8.9575 \mathrm{E}+03$ & $9.768 \mathrm{E}-11$ & $1.160 \mathrm{E}-03$ & $9.0724 \mathrm{E}+03$ & $9.158 \mathrm{E}-11$ & $1.051 \mathrm{E}-03$ & 0.002 \\
\hline $1 s 7 g{ }^{3} G_{4}$ & $1 s 7 g{ }^{3} G_{3}$ & $3.6344 \mathrm{E}+05$ & $1.613 \mathrm{E}-15$ & $1.163 \mathrm{E}-11$ & & & & 0.864 \\
\hline $1 s 2 p^{3} P_{1}^{o}$ & $1 s 7 f^{1} F_{3}^{o}$ & $1.3356 \mathrm{E}+00$ & $1.408 \mathrm{E}-06$ & $7.519 E+08$ & $1.3301 \mathrm{E}+00$ & $1.248 \mathrm{E}-06$ & $6.667 E+08$ & 0.241 \\
\hline $1 s 2 p^{3} P_{2}^{o}$ & $1 s 7 f^{1} F_{3}^{o}$ & $1.3940 \mathrm{E}+00$ & $1.120 \mathrm{E}-04$ & $5.494 \mathrm{E}+10$ & $1.3881 \mathrm{E}+00$ & $1.151 \mathrm{E}-04$ & $5.645 E+10$ & 0.014 \\
\hline $1 s 2 p^{1} P_{1}^{o}$ & $1 s 7 f^{1} F_{3}^{o}$ & $1.3995 \mathrm{E}+00$ & $6.566 \mathrm{E}-04$ & $3.195 E+11$ & $1.3937 \mathrm{E}+00$ & $6.826 \mathrm{E}-04$ & $3.320 \mathrm{E}+11$ & 0.007 \\
\hline $1 s 3 p^{3} P_{1}^{o}$ & $1 s 7 f^{1} F_{3}^{o}$ & $3.4034 \mathrm{E}+00$ & $1.180 \mathrm{E}-08$ & $9.704 \mathrm{E}+05$ & $3.3893 \mathrm{E}+00$ & $6.892 \mathrm{E}-08$ & $5.669 \mathrm{E}+06$ & 0.526 \\
\hline $1 s 3 p^{3} P_{2}^{o}$ & $1 s 7 f^{1} F_{3}^{o}$ & $3.5149 \mathrm{E}+00$ & $6.355 \mathrm{E}-06$ & $4.902 \mathrm{E}+08$ & $3.5002 \mathrm{E}+00$ & $7.469 \mathrm{E}-06$ & $5.759 E+08$ & 0.079 \\
\hline $1 s 3 p{ }^{1} P_{1}^{o}$ & $1 s 7 f^{1} F_{3}^{o}$ & $3.5248 \mathrm{E}+00$ & $4.161 \mathrm{E}-05$ & $3.192 \mathrm{E}+09$ & $3.5102 \mathrm{E}+00$ & $4.445 \mathrm{E}-05$ & $3.408 E+09$ & 0.049 \\
\hline $1 s 4 p^{3} P_{1}^{o}$ & $1 s 7 f^{1} F_{3}^{o}$ & $7.3482 \mathrm{E}+00$ & $1.302 \mathrm{E}-07$ & $2.298 \mathrm{E}+06$ & $7.3172 \mathrm{E}+00$ & $5.346 \mathrm{E}-08$ & $9.435 E+05$ & 0.117 \\
\hline $1 s 4 p^{3} P_{2}^{o}$ & $1 s 7 f^{1} F_{3}^{o}$ & $7.5662 \mathrm{E}+00$ & $6.809 \mathrm{E}-06$ & $1.133 \mathrm{E}+08$ & $7.5341 \mathrm{E}+00$ & $6.257 \mathrm{E}-06$ & $1.041 \mathrm{E}+08$ & 0.022 \\
\hline
\end{tabular}


Table 4 (continued)

\begin{tabular}{|c|c|c|c|c|c|c|c|c|}
\hline \multirow[t]{2}{*}{ Lower } & \multirow[t]{2}{*}{ Upper } & \multicolumn{3}{|l|}{ GRASP2K } & \multicolumn{3}{|l|}{ FAC } & \multirow[t]{2}{*}{$d T$} \\
\hline & & $\lambda(\AA)$ & $g f$ & $A\left(\mathrm{~s}^{-1}\right)$ & $\lambda(\AA)$ & $g f$ & $A\left(\mathrm{~s}^{-1}\right)$ & \\
\hline $1 s 4 p^{1} P_{1}^{o}$ & $1 s 7 f^{1} F_{3}^{o}$ & $7.5852 \mathrm{E}+00$ & $3.726 \mathrm{E}-05$ & $6.171 \mathrm{E}+08$ & $7.5532 \mathrm{E}+00$ & $3.730 \mathrm{E}-05$ & $6.177 \mathrm{E}+08$ & 0.008 \\
\hline $1 s 4 f^{3} F_{3}^{o}$ & $1 s 7 f^{1} F_{3}^{o}$ & $7.6552 \mathrm{E}+00$ & $9.269 \mathrm{E}-07$ & $1.507 \mathrm{E}+07$ & $7.6229 \mathrm{E}+00$ & $9.527 \mathrm{E}-07$ & $1.549 \mathrm{E}+07$ & 0.010 \\
\hline $1 s 4 f^{3} F_{2}^{o}$ & $1 s 7 f^{1} F_{3}^{o}$ & $7.6567 \mathrm{E}+00$ & $5.558 \mathrm{E}-06$ & $9.034 \mathrm{E}+07$ & $7.6244 \mathrm{E}+00$ & $5.487 \mathrm{E}-06$ & $8.918 \mathrm{E}+07$ & 0.011 \\
\hline $1 s 4 f^{3} F_{4}^{o}$ & $1 s 7 f^{1} F_{3}^{o}$ & $7.6912 \mathrm{E}+00$ & $5.600 \mathrm{E}-06$ & $9.020 \mathrm{E}+07$ & $7.6586 \mathrm{E}+00$ & $5.566 \mathrm{E}-06$ & $8.966 \mathrm{E}+07$ & 0.005 \\
\hline $1 s 4 f^{1} F_{3}^{o}$ & $1 s 7 f^{1} F_{3}^{o}$ & $7.6923 \mathrm{E}+00$ & $4.638 \mathrm{E}-05$ & $7.469 \mathrm{E}+08$ & $7.6598 \mathrm{E}+00$ & $4.641 \mathrm{E}-05$ & $7.473 \mathrm{E}+08$ & 0.004 \\
\hline $1 s 5 p^{3} P_{1}^{o}$ & $1 s 7 f^{1} F_{3}^{o}$ & $1.5704 \mathrm{E}+01$ & $7.014 \mathrm{E}-07$ & $2.710 \mathrm{E}+06$ & $1.5637 \mathrm{E}+01$ & $5.464 \mathrm{E}-07$ & $2.111 \mathrm{E}+06$ & 0.020 \\
\hline $1 s 5 p^{3} P_{2}^{o}$ & $1 s 7 f^{1} F_{3}^{o}$ & $1.6214 \mathrm{E}+01$ & $6.503 \mathrm{E}-05$ & $2.357 \mathrm{E}+08$ & $1.6144 \mathrm{E}+01$ & $6.420 \mathrm{E}-05$ & $2.327 \mathrm{E}+08$ & 0.002 \\
\hline $1 s 5 p^{1} P_{1}^{o}$ & $1 s 7 f^{1} F_{3}^{o}$ & $1.6258 \mathrm{E}+01$ & $3.793 \mathrm{E}-04$ & $1.367 \mathrm{E}+09$ & $1.6189 \mathrm{E}+01$ & $3.824 \mathrm{E}-04$ & $1.378 \mathrm{E}+09$ & 0.004 \\
\hline $1 s 5 f^{3} F_{3}^{o}$ & $1 s 7 f^{1} F_{3}^{o}$ & $1.6423 \mathrm{E}+01$ & $3.189 \mathrm{E}-06$ & $1.127 \mathrm{E}+07$ & $1.6354 \mathrm{E}+01$ & $3.307 \mathrm{E}-06$ & $1.168 \mathrm{E}+07$ & 0.001 \\
\hline $1 s 5 f^{3} F_{2}^{o}$ & $1 s 7 f^{1} F_{3}^{o}$ & $1.6427 \mathrm{E}+01$ & $1.919 \mathrm{E}-05$ & $6.775 E+07$ & $1.6358 \mathrm{E}+01$ & $1.910 \mathrm{E}-05$ & $6.744 \mathrm{E}+07$ & 0.001 \\
\hline $1 s 5 f^{3} F_{4}^{o}$ & $1 s 7 f^{1} F_{3}^{o}$ & $1.6508 \mathrm{E}+01$ & $1.977 \mathrm{E}-05$ & $6.914 \mathrm{E}+07$ & $1.6439 \mathrm{E}+01$ & $1.963 \mathrm{E}-05$ & $6.866 \mathrm{E}+07$ & 0.000 \\
\hline $1 s 5 f^{1} F_{3}^{o}$ & $1 s 7 f^{1} F_{3}^{o}$ & $1.6511 \mathrm{E}+01$ & $1.638 \mathrm{E}-04$ & $5.726 \mathrm{E}+08$ & $1.6441 \mathrm{E}+01$ & $1.637 \mathrm{E}-04$ & $5.723 E+08$ & 0.000 \\
\hline $1 s 6 p^{3} P_{1}^{o}$ & $1 s 7 f^{1} F_{3}^{o}$ & $4.0672 \mathrm{E}+01$ & $3.065 \mathrm{E}-06$ & $1.766 \mathrm{E}+06$ & $4.0492 \mathrm{E}+01$ & $5.449 \mathrm{E}-06$ & $3.139 \mathrm{E}+06$ & 0.004 \\
\hline $1 s 6 p^{3} P_{2}^{o}$ & $1 s 7 f^{1} F_{3}^{o}$ & $4.2675 \mathrm{E}+01$ & $3.654 \mathrm{E}-04$ & $1.912 \mathrm{E}+08$ & $4.2483 \mathrm{E}+01$ & $3.670 \mathrm{E}-04$ & $1.921 \mathrm{E}+08$ & 0.001 \\
\hline $1 s 6 p^{1} P_{1}^{o}$ & $1 s 7 f^{1} F_{3}^{o}$ & $4.2851 \mathrm{E}+01$ & $2.180 \mathrm{E}-03$ & $1.131 \mathrm{E}+09$ & $4.2639 \mathrm{E}+01$ & $2.177 \mathrm{E}-03$ & $1.131 \mathrm{E}+09$ & 0.001 \\
\hline $1 s 6 f^{3} F_{3}^{o}$ & $1 s 7 f^{1} F_{3}^{o}$ & $4.3519 \mathrm{E}+01$ & $1.285 \mathrm{E}-05$ & $6.468 \mathrm{E}+06$ & $4.3345 \mathrm{E}+01$ & $1.287 \mathrm{E}-05$ & $6.472 \mathrm{E}+06$ & 0.000 \\
\hline $1 s 6 f^{3} F_{2}^{o}$ & $1 s 7 f^{1} F_{3}^{o}$ & $4.3534 \mathrm{E}+01$ & $7.753 \mathrm{E}-05$ & $3.898 \mathrm{E}+07$ & $4.3345 \mathrm{E}+01$ & 7.707E-05 & $3.875 \mathrm{E}+07$ & 0.000 \\
\hline $1 s 6 f^{3} F_{4}^{o}$ & $1 s 7 f^{1} F_{3}^{o}$ & $4.3866 \mathrm{E}+01$ & $8.135 \mathrm{E}-05$ & $4.029 \mathrm{E}+07$ & $4.3688 \mathrm{E}+01$ & $8.097 \mathrm{E}-05$ & $4.008 \mathrm{E}+07$ & 0.000 \\
\hline $1 s 6 f^{1} F_{3}^{o}$ & $1 s 7 f^{1} F_{3}^{o}$ & $4.3877 \mathrm{E}+01$ & $6.744 \mathrm{E}-04$ & $3.338 \mathrm{E}+08$ & $4.3688 \mathrm{E}+01$ & $6.760 \mathrm{E}-04$ & $3.346 \mathrm{E}+08$ & 0.000 \\
\hline $1 s 6 h^{3} H_{5}^{o}$ & $1 s 7 f^{1} F_{3}^{o}$ & $4.4076 \mathrm{E}+01$ & $1.054 \mathrm{E}-07$ & $5.168 \mathrm{E}+04$ & & & & 0.001 \\
\hline $1 s 6 h^{3} H_{4}^{o}$ & $1 s 7 f^{1} F_{3}^{o}$ & $4.4082 \mathrm{E}+01$ & $1.770 \mathrm{E}-06$ & $8.681 \mathrm{E}+05$ & $4.3895 \mathrm{E}+01$ & $1.763 \mathrm{E}-06$ & $8.646 \mathrm{E}+05$ & 0.001 \\
\hline $1 s 6 h{ }^{1} H_{5}^{o}$ & $1 s 7 f^{1} F_{3}^{o}$ & $4.4221 \mathrm{E}+01$ & $3.978 \mathrm{E}-05$ & $1.938 \mathrm{E}+07$ & $4.4034 \mathrm{E}+01$ & $3.981 \mathrm{E}-05$ & $1.939 \mathrm{E}+07$ & 0.000 \\
\hline $1 s 7 p^{3} P_{1}^{o}$ & $1 s 7 f^{1} F_{3}^{o}$ & $8.8911 \mathrm{E}+02$ & $1.783 \mathrm{E}-09$ & $2.149 \mathrm{E}+00$ & $8.8057 \mathrm{E}+02$ & $2.686 \mathrm{E}-09$ & $3.273 \mathrm{E}+00$ & 0.046 \\
\hline $1 s 7 p^{3} P_{2}^{o}$ & $1 s 7 f^{1} F_{3}^{o}$ & $2.4995 \mathrm{E}+03$ & $9.333 \mathrm{E}-09$ & $1.424 \mathrm{E}+00$ & $2.4456 \mathrm{E}+03$ & $9.858 \mathrm{E}-09$ & $1.557 \mathrm{E}+00$ & 0.214 \\
\hline $1 s 7 p^{1} P_{1}^{o}$ & $1 s 7 f^{1} F_{3}^{o}$ & $2.9477 \mathrm{E}+03$ & $3.391 \mathrm{E}-08$ & $3.719 \mathrm{E}+00$ & $2.8179 \mathrm{E}+03$ & $3.864 \mathrm{E}-08$ & $4.598 \mathrm{E}+00$ & 0.254 \\
\hline $1 s 7 f^{3} F_{3}^{o}$ & $1 s 7 f^{1} F_{3}^{o}$ & $8.4824 \mathrm{E}+03$ & $2.588 \mathrm{E}-11$ & $3.428 \mathrm{E}-04$ & & & & 0.007 \\
\hline $1 s 7 f^{3} F_{2}^{o}$ & $1 s 7 f^{1} F_{3}^{o}$ & $8.8420 \mathrm{E}+03$ & $1.378 \mathrm{E}-10$ & $1.679 \mathrm{E}-03$ & $8.7598 E+03$ & $1.389 \mathrm{E}-10$ & $1.710 \mathrm{E}-03$ & 0.012 \\
\hline $1 s 7 f^{3} F_{4}^{o}$ & $1 s 7 f^{1} F_{3}^{o}$ & $2.7665 \mathrm{E}+05$ & $4.698 \mathrm{E}-15$ & $5.850 \mathrm{E}-11$ & & & & 0.132 \\
\hline $1 s 3 d^{3} D_{3}$ & $1 s 7 g^{3} G_{5}$ & $3.5591 \mathrm{E}+00$ & $2.642 \mathrm{E}-03$ & $1.265 \mathrm{E}+11$ & $3.5441 \mathrm{E}+00$ & $2.685 E-03$ & $1.285 \mathrm{E}+11$ & 0.001 \\
\hline $1 s 4 d^{3} D_{3}$ & $1 s 7 g{ }^{3} G_{5}$ & $7.6503 \mathrm{E}+00$ & $4.732 \mathrm{E}-04$ & $4.903 \mathrm{E}+09$ & $7.6183 \mathrm{E}+00$ & $4.810 \mathrm{E}-04$ & $4.983 \mathrm{E}+09$ & 0.003 \\
\hline $1 s 5 d^{3} D_{3}$ & $1 s 7 g{ }^{3} G_{5}$ & $1.6403 \mathrm{E}+01$ & $1.449 \mathrm{E}-04$ & $3.266 \mathrm{E}+08$ & $1.6335 \mathrm{E}+01$ & $1.433 \mathrm{E}-04$ & $3.230 \mathrm{E}+08$ & 0.003 \\
\hline $1 s 5 g^{3} G_{4}$ & $1 s 7 g{ }^{3} G_{5}$ & $1.6491 \mathrm{E}+01$ & $9.575 \mathrm{E}-06$ & $2.135 \mathrm{E}+07$ & $1.6420 \mathrm{E}+01$ & $9.612 \mathrm{E}-06$ & $2.143 \mathrm{E}+07$ & 0.001 \\
\hline $1 s 5 g^{3} G_{3}$ & $1 s 7 g{ }^{3} G_{5}$ & $1.6493 \mathrm{E}+01$ & $4.578 \mathrm{E}-07$ & $1.021 \mathrm{E}+06$ & $1.6423 \mathrm{E}+01$ & $4.587 \mathrm{E}-07$ & $1.022 \mathrm{E}+06$ & 0.002 \\
\hline $1 s 5 g^{3} G_{5}$ & $1 s 7 g^{3} G_{5}$ & $1.6542 \mathrm{E}+01$ & $1.322 \mathrm{E}-04$ & $2.929 \mathrm{E}+08$ & $1.6471 \mathrm{E}+01$ & $1.320 \mathrm{E}-04$ & $2.927 \mathrm{E}+08$ & 0.001 \\
\hline $1 s 5 g{ }^{1} G_{4}$ & $1 s 7 g{ }^{3} G_{5}$ & $1.6543 \mathrm{E}+01$ & $7.667 \mathrm{E}-06$ & $1.699 \mathrm{E}+07$ & $1.6473 \mathrm{E}+01$ & $7.651 \mathrm{E}-06$ & $1.695 \mathrm{E}+07$ & 0.001 \\
\hline $1 s 6 d^{3} D_{3}$ & $1 s 7 g{ }^{3} G_{5}$ & $4.3385 \mathrm{E}+01$ & $5.440 \mathrm{E}-03$ & $1.753 \mathrm{E}+09$ & $4.3218 \mathrm{E}+01$ & $5.448 \mathrm{E}-03$ & $1.753 \mathrm{E}+09$ & 0.000 \\
\hline $1 s 6 g{ }^{3} G_{4}$ & $1 s 7 g{ }^{3} G_{5}$ & $4.3743 \mathrm{E}+01$ & $6.292 \mathrm{E}-05$ & $1.994 \mathrm{E}+07$ & $4.3559 \mathrm{E}+01$ & $6.329 \mathrm{E}-05$ & $2.005 \mathrm{E}+07$ & 0.000 \\
\hline $1 s 6 g^{3} G_{3}$ & $1 s 7 g{ }^{3} G_{5}$ & $4.3751 \mathrm{E}+01$ & $3.000 \mathrm{E}-06$ & $9.504 \mathrm{E}+05$ & $4.3559 \mathrm{E}+01$ & $3.014 \mathrm{E}-06$ & $9.551 \mathrm{E}+05$ & 0.001 \\
\hline $1 s 6 g{ }^{3} G_{5}$ & $1 s 7 g{ }^{3} G_{5}$ & $4.3950 \mathrm{E}+01$ & $8.795 \mathrm{E}-04$ & $2.761 \mathrm{E}+08$ & $4.3765 \mathrm{E}+01$ & $8.804 \mathrm{E}-04$ & $2.763 \mathrm{E}+08$ & 0.000 \\
\hline $1 s 6 g{ }^{1} G_{4}$ & $1 s 7 g{ }^{3} G_{5}$ & $4.3957 \mathrm{E}+01$ & $5.098 \mathrm{E}-05$ & $1.600 \mathrm{E}+07$ & $4.3765 \mathrm{E}+01$ & $5.079 \mathrm{E}-05$ & $1.594 \mathrm{E}+07$ & 0.000 \\
\hline $1 s 7 d^{3} D_{3}$ & $1 s 7 g{ }^{3} G_{5}$ & $5.3499 \mathrm{E}+03$ & $8.610 \mathrm{E}-09$ & $1.824 \mathrm{E}-01$ & $5.4978 \mathrm{E}+03$ & $7.830 \mathrm{E}-09$ & $1.557 \mathrm{E}-01$ & 0.002 \\
\hline $1 s 7 g{ }^{3} G_{4}$ & $1 s 7 g{ }^{3} G_{5}$ & $1.4677 \mathrm{E}+04$ & $2.497 \mathrm{E}-11$ & $7.029 \mathrm{E}-05$ & $1.4692 \mathrm{E}+04$ & $2.466 \mathrm{E}-11$ & $6.870 \mathrm{E}-05$ & 0.006 \\
\hline $1 s 7 g^{3} G_{3}$ & $1 s 7 g{ }^{3} G_{5}$ & $1.5295 \mathrm{E}+04$ & $1.053 \mathrm{E}-12$ & $2.729 \mathrm{E}-06$ & & & & 0.076 \\
\hline $1 s 4 f^{3} F_{3}^{o}$ & $1 s 7 h^{3} H_{5}^{o}$ & $7.6514 \mathrm{E}+00$ & $4.645 \mathrm{E}-03$ & $4.811 \mathrm{E}+10$ & $7.6190 \mathrm{E}+00$ & $4.671 \mathrm{E}-03$ & $4.838 \mathrm{E}+10$ & 0.000 \\
\hline $1 s 4 f^{3} F_{4}^{o}$ & $1 s 7 h^{3} H_{5}^{o}$ & $7.6874 \mathrm{E}+00$ & $2.681 \mathrm{E}-04$ & $2.751 \mathrm{E}+09$ & $7.6547 \mathrm{E}+00$ & $2.682 \mathrm{E}-04$ & $2.752 \mathrm{E}+09$ & 0.000 \\
\hline $1 s 4 f^{1} F_{3}^{o}$ & $1 s 7 h^{3} H_{5}^{o}$ & $7.6885 \mathrm{E}+00$ & $1.250 \mathrm{E}-05$ & $1.282 \mathrm{E}+08$ & $7.6558 \mathrm{E}+00$ & $1.173 \mathrm{E}-05$ & $1.203 \mathrm{E}+08$ & 0.000 \\
\hline $1 s 5 f^{3} F_{3}^{o}$ & $1 s 7 h^{3} H_{5}^{o}$ & $1.6406 \mathrm{E}+01$ & $5.665 \mathrm{E}-04$ & $1.276 \mathrm{E}+09$ & $1.6336 \mathrm{E}+01$ & $5.720 \mathrm{E}-04$ & $1.288 \mathrm{E}+09$ & 0.000 \\
\hline 1s $5 f^{3} F_{4}^{o}$ & $1 s 7 h^{3} H_{5}^{o}$ & $1.6491 \mathrm{E}+01$ & $3.505 \mathrm{E}-05$ & $7.816 \mathrm{E}+07$ & $1.6420 \mathrm{E}+01$ & $3.535 \mathrm{E}-05$ & $7.882 \mathrm{E}+07$ & 0.000 \\
\hline $1 s 5 f^{1} F_{3}^{o}$ & $1 s 7 h^{3} H_{5}^{o}$ & $1.6493 \mathrm{E}+01$ & $1.647 \mathrm{E}-06$ & $3.671 \mathrm{E}+06$ & $1.6423 \mathrm{E}+01$ & $1.560 \mathrm{E}-06$ & $3.478 \mathrm{E}+06$ & 0.000 \\
\hline $1 s 6 f^{3} F_{3}^{o}$ & $1 s 7 h^{3} H_{5}^{o}$ & $4.3397 \mathrm{E}+01$ & $5.768 \mathrm{E}-03$ & $1.857 \mathrm{E}+09$ & $4.3218 \mathrm{E}+01$ & $5.771 \mathrm{E}-03$ & $1.857 \mathrm{E}+09$ & 0.000 \\
\hline $1 s 6 f^{3} F_{4}^{o}$ & $1 s 7 h^{3} H_{5}^{o}$ & $4.3742 \mathrm{E}+01$ & $3.291 \mathrm{E}-04$ & $1.043 \mathrm{E}+08$ & $4.3559 \mathrm{E}+01$ & $3.298 \mathrm{E}-04$ & $1.045 \mathrm{E}+08$ & 0.000 \\
\hline $1 s 6 f^{1} F_{3}^{o}$ & $1 s 7 h^{3} H_{5}^{o}$ & $4.3753 \mathrm{E}+01$ & $1.547 \mathrm{E}-05$ & $4.902 \mathrm{E}+06$ & $4.3560 \mathrm{E}+01$ & $1.605 \mathrm{E}-05$ & $5.087 \mathrm{E}+06$ & 0.000 \\
\hline $1 s 6 h^{3} H_{5}^{o}$ & $1 s 7 h^{3} H_{5}^{o}$ & $4.3951 \mathrm{E}+01$ & $5.094 \mathrm{E}-04$ & $1.599 \mathrm{E}+08$ & $4.3765 \mathrm{E}+01$ & $5.095 \mathrm{E}-04$ & $1.599 \mathrm{E}+08$ & 0.000 \\
\hline $1 s 6 h^{3} H_{4}^{o}$ & $1 s 7 h^{3} H_{5}^{o}$ & $4.3956 \mathrm{E}+01$ & $2.930 \mathrm{E}-05$ & $9.194 \mathrm{E}+06$ & $4.3765 \mathrm{E}+01$ & $2.939 \mathrm{E}-05$ & $9.227 \mathrm{E}+06$ & 0.000 \\
\hline $1 s 6 h^{3} H_{6}^{0}$ & $1 s 7 h^{3} H_{5}^{o}$ & $4.4090 \mathrm{E}+01$ & $2.964 \mathrm{E}-05$ & $9.245 \mathrm{E}+06$ & $4.3903 \mathrm{E}+01$ & $2.968 \mathrm{E}-05$ & $9.258 \mathrm{E}+06$ & 0.000 \\
\hline $1 s 6 h{ }^{1} H_{5}^{o}$ & $1 s 7 h^{3} H_{5}^{0}$ & $4.4094 \mathrm{E}+01$ & $1.416 \mathrm{E}-06$ & $4.417 \mathrm{E}+05$ & $4.3903 \mathrm{E}+01$ & $1.413 \mathrm{E}-06$ & $4.408 \mathrm{E}+05$ & 0.000 \\
\hline $1 s 7 f^{3} F_{3}^{o}$ & $1 s 7 h^{3} H_{5}^{o}$ & $5.4734 \mathrm{E}+03$ & $4.836 \mathrm{E}-09$ & $9.789 \mathrm{E}-02$ & $5.5022 \mathrm{E}+03$ & $4.700 \mathrm{E}-09$ & $9.334 \mathrm{E}-02$ & 0.005 \\
\hline $1 s 7 f^{3} F_{4}^{o}$ & $1 s 7 h^{3} H_{5}^{o}$ & $1.4614 \mathrm{E}+04$ & $1.456 \mathrm{E}-11$ & $4.135 \mathrm{E}-05$ & $1.4763 E+04$ & $1.399 \mathrm{E}-11$ & $3.860 \mathrm{E}-05$ & 0.019 \\
\hline $1 s 7 f^{1} F_{3}^{o}$ & $1 s 7 h^{3} H_{5}^{o}$ & $1.5429 \mathrm{E}+04$ & $5.832 \mathrm{E}-13$ & $1.485 \mathrm{E}-06$ & & & & 0.016 \\
\hline $1 s 2 p^{3} P_{2}^{o}$ & $1 s 7 h^{3} H_{4}^{o}$ & $1.3938 \mathrm{E}+00$ & $1.355 \mathrm{E}-12$ & $5.171 \mathrm{E}+02$ & & & & 0.965 \\
\hline $1 s 3 p^{3} P_{2}^{o}$ & $1 s 7 h^{3} H_{4}^{0}$ & $3.5141 \mathrm{E}+00$ & $1.656 \mathrm{E}-13$ & $9.938 \mathrm{E}+00$ & & & & 0.818 \\
\hline $1 s 4 p^{3} P_{2}^{o}$ & $1 s 7 h^{3} H_{4}^{o}$ & $7.5624 \mathrm{E}+00$ & $4.126 \mathrm{E}-10$ & $5.348 \mathrm{E}+03$ & & & & 0.039 \\
\hline $1 s 4 f^{3} F_{3}^{o}$ & $1 s 7 h^{3} H_{4}^{0}$ & $7.6513 \mathrm{E}+00$ & $2.804 \mathrm{E}-04$ & $3.550 \mathrm{E}+09$ & $7.6190 \mathrm{E}+00$ & $2.819 \mathrm{E}-04$ & $3.569 \mathrm{E}+09$ & 0.000 \\
\hline $1 s 4 f^{3} F_{2}^{o}$ & $1 s 7 h^{3} H_{4}^{0}$ & $7.6528 \mathrm{E}+00$ & $3.518 \mathrm{E}-03$ & $4.452 \mathrm{E}+10$ & $7.6205 \mathrm{E}+00$ & $3.536 \mathrm{E}-03$ & $4.474 \mathrm{E}+10$ & 0.000 \\
\hline $1 s 4 f^{3} F_{4}^{o}$ & $1 s 7 h^{3} H_{4}^{0}$ & $7.6873 \mathrm{E}+00$ & $1.920 \mathrm{E}-05$ & $2.408 \mathrm{E}+08$ & $7.6547 \mathrm{E}+00$ & $1.916 \mathrm{E}-05$ & $2.403 E+08$ & 0.000 \\
\hline $1 s 4 f^{1} F_{3}^{o}$ & $1 s 7 h^{3} H_{4}^{o}$ & $7.6884 \mathrm{E}+00$ & $2.123 \mathrm{E}-04$ & $2.662 \mathrm{E}+09$ & $7.6558 \mathrm{E}+00$ & $2.117 \mathrm{E}-04$ & $2.654 \mathrm{E}+09$ & 0.000 \\
\hline $1 s 5 p^{3} P_{2}^{o}$ & $1 s 7 h^{3} H_{4}^{o}$ & $1.6196 \mathrm{E}+01$ & $6.804 \mathrm{E}-11$ & $1.922 \mathrm{E}+02$ & & & & 0.031 \\
\hline $1 s 5 f^{3} F_{3}^{o}$ & $1 s 7 h^{3} H_{4}^{o}$ & $1.6405 \mathrm{E}+01$ & $3.416 \mathrm{E}-05$ & $9.407 \mathrm{E}+07$ & $1.6336 \mathrm{E}+01$ & $3.453 \mathrm{E}-05$ & $9.509 \mathrm{E}+07$ & 0.000 \\
\hline $1 s 5 f^{3} F_{2}^{o}$ & $1 s 7 h^{3} H_{4}^{0}$ & $1.6409 \mathrm{E}+01$ & $4.298 \mathrm{E}-04$ & $1.183 \mathrm{E}+09$ & $1.6340 \mathrm{E}+01$ & $4.329 \mathrm{E}-04$ & $1.191 \mathrm{E}+09$ & 0.000 \\
\hline $1 s 5 f^{3} F_{4}^{o}$ & $1 s 7 h^{3} H_{4}^{0}$ & $1.6490 \mathrm{E}+01$ & $2.507 \mathrm{E}-06$ & $6.833 \mathrm{E}+06$ & $1.6420 \mathrm{E}+01$ & $2.525 E-06$ & $6.881 \mathrm{E}+06$ & 0.001 \\
\hline $1 s 5 f^{1} F_{3}^{o}$ & $1 s 7 h^{3} H_{4}^{0}$ & $1.6493 \mathrm{E}+01$ & $2.778 \mathrm{E}-05$ & $7.568 \mathrm{E}+07$ & $1.6423 \mathrm{E}+01$ & $2.788 \mathrm{E}-05$ & $7.597 \mathrm{E}+07$ & 0.000 \\
\hline
\end{tabular}


Table 4 (continued)

\begin{tabular}{|c|c|c|c|c|c|c|c|c|}
\hline \multirow[t]{2}{*}{ Lower } & \multirow[t]{2}{*}{ Upper } & \multicolumn{3}{|l|}{ GRASP2K } & \multicolumn{3}{|l|}{ FAC } & \multirow[t]{2}{*}{$d T$} \\
\hline & & $\lambda(\AA)$ & $g f$ & $A\left(\mathrm{~s}^{-1}\right)$ & $\lambda(\AA)$ & $g f$ & $A\left(\mathrm{~s}^{-1}\right)$ & \\
\hline $1 s 6 p^{3} P_{2}^{o}$ & $1 s 7 h^{3} H_{4}^{0}$ & $4.2554 \mathrm{E}+01$ & $1.171 \mathrm{E}-09$ & $4.791 \mathrm{E}+02$ & & & & 0.003 \\
\hline $1 s 6 f^{3} F_{3}^{o}$ & $1 s 7 h^{3} H_{4}^{0}$ & $4.3394 \mathrm{E}+01$ & $3.484 \mathrm{E}-04$ & $1.371 \mathrm{E}+08$ & $4.3218 \mathrm{E}+01$ & $3.501 \mathrm{E}-04$ & $1.377 \mathrm{E}+08$ & 0.000 \\
\hline $1 s 6 f^{3} F_{2}^{o}$ & $1 s 7 h^{3} H_{4}^{0}$ & $4.3408 \mathrm{E}+01$ & $4.367 \mathrm{E}-03$ & $1.718 \mathrm{E}+09$ & $4.3218 \mathrm{E}+01$ & $4.372 \mathrm{E}-03$ & $1.720 \mathrm{E}+09$ & 0.000 \\
\hline $1 s 6 f^{3} F_{4}^{o}$ & $1 s 7 h^{3} H_{4}^{o}$ & $4.3738 \mathrm{E}+01$ & $2.358 \mathrm{E}-05$ & $9.134 \mathrm{E}+06$ & $4.3559 \mathrm{E}+01$ & $2.356 \mathrm{E}-05$ & $9.125 E+06$ & 0.000 \\
\hline $1 s 6 f^{1} F_{3}^{o}$ & $1 s 7 h^{3} H_{4}^{o}$ & $4.3749 \mathrm{E}+01$ & $2.604 \mathrm{E}-04$ & $1.008 \mathrm{E}+08$ & $4.3560 \mathrm{E}+01$ & $2.588 \mathrm{E}-04$ & $1.002 \mathrm{E}+08$ & 0.000 \\
\hline $1 s 6 h^{3} H_{5}^{o}$ & $1 s 7 h^{3} H_{4}^{o}$ & $4.3947 \mathrm{E}+01$ & $2.929 \mathrm{E}-05$ & $1.124 \mathrm{E}+07$ & $4.3765 \mathrm{E}+01$ & $2.939 \mathrm{E}-05$ & $1.127 \mathrm{E}+07$ & 0.000 \\
\hline $1 s 6 h^{3} H_{4}^{o}$ & $1 s 7 h^{3} H_{4}^{o}$ & $4.3953 \mathrm{E}+01$ & $4.113 \mathrm{E}-04$ & $1.578 \mathrm{E}+08$ & $4.3765 \mathrm{E}+01$ & $4.115 \mathrm{E}-04$ & $1.578 \mathrm{E}+08$ & 0.000 \\
\hline $1 s 6 h^{3} H_{6}^{o}$ & $1 s 7 h^{3} H_{4}^{0}$ & $4.4086 \mathrm{E}+01$ & $9.436 \mathrm{E}-07$ & $3.598 \mathrm{E}+05$ & $4.3903 \mathrm{E}+01$ & $9.422 \mathrm{E}-07$ & $3.592 \mathrm{E}+05$ & 0.000 \\
\hline $1 s 6 h{ }^{1} H_{5}^{o}$ & $1 s 7 h^{3} H_{4}^{0}$ & $4.4091 \mathrm{E}+01$ & $2.461 \mathrm{E}-05$ & $9.384 \mathrm{E}+06$ & $4.3903 \mathrm{E}+01$ & $2.449 \mathrm{E}-05$ & $9.340 \mathrm{E}+06$ & 0.000 \\
\hline $1 s 7 p^{3} P_{2}^{o}$ & $1 s 7 h^{3} H_{4}^{o}$ & $2.1428 \mathrm{E}+03$ & $1.866 \mathrm{E}-14$ & $3.013 E-06$ & & & & 0.042 \\
\hline $1 s 7 f^{3} F_{3}^{o}$ & $1 s 7 h^{3} H_{4}^{o}$ & $5.4206 \mathrm{E}+03$ & $3.007 \mathrm{E}-10$ & $7.586 \mathrm{E}-03$ & $5.5022 \mathrm{E}+03$ & $2.852 \mathrm{E}-10$ & $6.922 \mathrm{E}-03$ & 0.007 \\
\hline $1 s 7 f^{3} F_{2}^{o}$ & $1 s 7 h^{3} H_{4}^{o}$ & $5.5652 \mathrm{E}+03$ & $3.484 \mathrm{E}-09$ & $8.338 \mathrm{E}-02$ & $5.5003 \mathrm{E}+03$ & $3.564 \mathrm{E}-09$ & $8.658 \mathrm{E}-02$ & 0.006 \\
\hline $1 s 7 f^{3} F_{4}^{o}$ & $1 s 7 h^{3} H_{4}^{0}$ & $1.4244 \mathrm{E}+04$ & $1.127 \mathrm{E}-12$ & $4.117 \mathrm{E}-06$ & & & & 0.019 \\
\hline $1 s 7 f^{1} F_{3}^{o}$ & $1 s 7 h^{3} H_{4}^{o}$ & $1.5017 \mathrm{E}+04$ & $1.062 \mathrm{E}-11$ & $3.491 \mathrm{E}-05$ & $1.4782 \mathrm{E}+04$ & $1.093 \mathrm{E}-11$ & $3.677 \mathrm{E}-05$ & 0.022 \\
\hline $1 s 7 h^{3} H_{5}^{o}$ & $1 s 7 h^{3} H_{4}^{0}$ & $5.6215 E+05$ & $2.508 \mathrm{E}-16$ & $5.883 \mathrm{E}-13$ & & & & 0.445 \\
\hline $1 s 3 d^{3} D_{2}$ & $1 s 7 g{ }^{1} G_{4}$ & $3.5224 \mathrm{E}+00$ & $5.663 \mathrm{E}-08$ & $3.382 E+06$ & & & & 0.083 \\
\hline $1 s 3 d^{3} D_{3}$ & $1 s 7 g{ }^{1} G_{4}$ & $3.5591 \mathrm{E}+00$ & $1.610 \mathrm{E}-04$ & $9.419 E+09$ & $3.5441 \mathrm{E}+00$ & $1.627 \mathrm{E}-04$ & $9.520 E+09$ & 0.002 \\
\hline $1 s 3 d{ }^{1} D_{2}$ & $1 s 7 g{ }^{1} G_{4}$ & $3.5605 \mathrm{E}+00$ & $1.996 \mathrm{E}-03$ & $1.167 \mathrm{E}+11$ & $3.5455 \mathrm{E}+00$ & $2.031 \mathrm{E}-03$ & $1.187 \mathrm{E}+11$ & 0.002 \\
\hline $1 s 4 d^{3} D_{2}$ & $1 s 7 g{ }^{1} G_{4}$ & $7.5785 \mathrm{E}+00$ & $1.751 \mathrm{E}-08$ & $2.260 \mathrm{E}+05$ & & & & 0.121 \\
\hline $1 s 4 d^{3} D_{3}$ & $1 s 7 g{ }^{1} G_{4}$ & $7.6502 \mathrm{E}+00$ & $2.879 \mathrm{E}-05$ & $3.646 \mathrm{E}+08$ & $7.6183 \mathrm{E}+00$ & $2.915 E-05$ & $3.691 E+08$ & 0.002 \\
\hline $1 s 4 d^{1} D_{2}$ & $1 s 7 g{ }^{1} G_{4}$ & $7.6531 \mathrm{E}+00$ & $3.580 \mathrm{E}-04$ & $4.530 \mathrm{E}+09$ & $7.6213 \mathrm{E}+00$ & $3.640 \mathrm{E}-04$ & $4.605 E+09$ & 0.004 \\
\hline $1 s 5 d^{3} D_{2}$ & $1 s 7 g{ }^{1} G_{4}$ & $1.6234 \mathrm{E}+01$ & $8.108 \mathrm{E}-09$ & $2.280 \mathrm{E}+04$ & & & & 0.102 \\
\hline $1 s 5 d^{3} D_{3}$ & $1 s 7 g{ }^{1} G_{4}$ & $1.6402 \mathrm{E}+01$ & $8.842 \mathrm{E}-06$ & $2.436 \mathrm{E}+07$ & $1.6335 \mathrm{E}+01$ & $8.687 \mathrm{E}-06$ & $2.392 \mathrm{E}+07$ & 0.002 \\
\hline $1 s 5 d^{1} D_{2}$ & $1 s 7 g{ }^{1} G_{4}$ & $1.6409 \mathrm{E}+01$ & $1.093 \mathrm{E}-04$ & $3.008 E+08$ & $1.6342 \mathrm{E}+01$ & $1.084 \mathrm{E}-04$ & $2.984 \mathrm{E}+08$ & 0.004 \\
\hline $1 s 5 g{ }^{3} G_{4}$ & $1 s 7 g{ }^{1} G_{4}$ & $1.6490 \mathrm{E}+01$ & $6.864 \mathrm{E}-07$ & $1.871 \mathrm{E}+06$ & $1.6420 \mathrm{E}+01$ & $7.222 \mathrm{E}-07$ & $1.968 \mathrm{E}+06$ & 0.003 \\
\hline $1 s 5 g{ }^{3} G_{3}$ & $1 s 7 g{ }^{1} G_{4}$ & $1.6492 \mathrm{E}+01$ & $7.595 \mathrm{E}-06$ & $2.069 \mathrm{E}+07$ & $1.6423 \mathrm{E}+01$ & $7.575 \mathrm{E}-06$ & $2.064 \mathrm{E}+07$ & 0.001 \\
\hline $1 s 5 g{ }^{3} G_{5}$ & $1 s 7 g{ }^{1} G_{4}$ & $1.6541 \mathrm{E}+01$ & $7.660 \mathrm{E}-06$ & $2.075 E+07$ & $1.6471 \mathrm{E}+01$ & $7.620 \mathrm{E}-06$ & $2.064 \mathrm{E}+07$ & 0.001 \\
\hline $1 s 5 g{ }^{1} G_{4}$ & $1 s 7 g{ }^{1} G_{4}$ & $1.6543 \mathrm{E}+01$ & $1.069 \mathrm{E}-04$ & $2.894 \mathrm{E}+08$ & $1.6473 \mathrm{E}+01$ & $1.066 \mathrm{E}-04$ & $2.887 \mathrm{E}+08$ & 0.001 \\
\hline $1 s 6 d^{3} D_{2}$ & $1 s 7 g{ }^{1} G_{4}$ & $4.2703 \mathrm{E}+01$ & $2.860 \mathrm{E}-07$ & $1.162 \mathrm{E}+05$ & $4.2548 \mathrm{E}+01$ & $6.028 \mathrm{E}-07$ & $2.447 \mathrm{E}+05$ & 0.010 \\
\hline $1 s 6 d^{3} D_{3}$ & $1 s 7 g{ }^{1} G_{4}$ & $4.3381 \mathrm{E}+01$ & $3.312 \mathrm{E}-04$ & $1.304 \mathrm{E}+08$ & $4.3218 \mathrm{E}+01$ & $3.302 \mathrm{E}-04$ & $1.299 \mathrm{E}+08$ & 0.000 \\
\hline $1 s 6 d{ }^{1} D_{2}$ & $1 s 7 g{ }^{1} G_{4}$ & $4.3410 \mathrm{E}+01$ & $4.120 \mathrm{E}-03$ & $1.620 \mathrm{E}+09$ & $4.3228 \mathrm{E}+01$ & $4.124 \mathrm{E}-03$ & $1.621 \mathrm{E}+09$ & 0.000 \\
\hline $1 s 6 g{ }^{3} G_{4}$ & $1 s 7 g{ }^{1} G_{4}$ & $4.3738 \mathrm{E}+01$ & $4.512 \mathrm{E}-06$ & $1.748 \mathrm{E}+06$ & $4.3559 \mathrm{E}+01$ & $4.522 \mathrm{E}-06$ & $1.751 \mathrm{E}+06$ & 0.001 \\
\hline $1 s 6 g{ }^{3} G_{3}$ & $1 s 7 g{ }^{1} G_{4}$ & $4.3747 \mathrm{E}+01$ & $4.984 \mathrm{E}-05$ & $1.930 \mathrm{E}+07$ & $4.3559 \mathrm{E}+01$ & $4.973 \mathrm{E}-05$ & $1.926 \mathrm{E}+07$ & 0.000 \\
\hline $1 s 6 g{ }^{3} G_{5}$ & $1 s 7 g{ }^{1} G_{4}$ & $4.3946 \mathrm{E}+01$ & $5.095 \mathrm{E}-05$ & $1.955 \mathrm{E}+07$ & $4.3765 \mathrm{E}+01$ & $5.079 \mathrm{E}-05$ & $1.948 \mathrm{E}+07$ & 0.000 \\
\hline $1 s 6 g{ }^{1} G_{4}$ & $1 s 7 g{ }^{1} G_{4}$ & $4.3953 \mathrm{E}+01$ & $7.102 \mathrm{E}-04$ & $2.725 E+08$ & $4.3765 \mathrm{E}+01$ & $7.111 \mathrm{E}-04$ & $2.728 \mathrm{E}+08$ & 0.000 \\
\hline $1 s 7 d^{3} D_{2}$ & $1 s 7 g{ }^{1} G_{4}$ & $2.3855 \mathrm{E}+03$ & $5.465 \mathrm{E}-12$ & $7.117 \mathrm{E}-04$ & $2.4322 \mathrm{E}+03$ & $1.002 \mathrm{E}-11$ & $1.245 \mathrm{E}-03$ & 0.288 \\
\hline $1 s 7 d^{3} D_{3}$ & $1 s 7 g{ }^{1} G_{4}$ & $5.2883 \mathrm{E}+03$ & $5.425 \mathrm{E}-10$ & $1.438 \mathrm{E}-02$ & $5.4978 \mathrm{E}+03$ & $4.745 \mathrm{E}-10$ & $1.153 \mathrm{E}-02$ & 0.023 \\
\hline $1 s 7 d^{1} D_{2}$ & $1 s 7 g{ }^{1} G_{4}$ & $5.5805 \mathrm{E}+03$ & $5.741 \mathrm{E}-09$ & $1.366 \mathrm{E}-01$ & $5.6088 \mathrm{E}+03$ & $5.585 \mathrm{E}-09$ & $1.304 \mathrm{E}-01$ & 0.009 \\
\hline $1 s 7 g{ }^{3} G_{4}$ & $1 s 7 g{ }^{1} G_{4}$ & $1.4223 \mathrm{E}+04$ & $1.969 \mathrm{E}-12$ & $7.213 \mathrm{E}-06$ & & & & 0.050 \\
\hline $1 s 7 g{ }^{3} G_{3}$ & $1 s 7 g{ }^{1} G_{4}$ & $1.4802 \mathrm{E}+04$ & $1.927 \mathrm{E}-11$ & $6.519 \mathrm{E}-05$ & $1.4691 \mathrm{E}+04$ & $1.938 \mathrm{E}-11$ & $6.599 \mathrm{E}-05$ & 0.001 \\
\hline $1 s 7 g{ }^{3} G_{5}$ & $1 s 7 g{ }^{1} G_{4}$ & $4.5954 \mathrm{E}+05$ & $6.580 \mathrm{E}-16$ & $2.309 \mathrm{E}-12$ & & & & 0.926 \\
\hline $1 s 4 f^{3} F_{4}^{o}$ & $1 s 7 h^{3} H_{6}^{o}$ & $7.6847 \mathrm{E}+00$ & $5.821 \mathrm{E}-03$ & $5.058 \mathrm{E}+10$ & $7.6520 \mathrm{E}+00$ & $5.875 \mathrm{E}-03$ & $5.104 \mathrm{E}+10$ & 0.000 \\
\hline $1 s 5 f^{3} F_{4}^{0}$ & $1 s 7 h^{3} H_{6}^{o}$ & $1.6478 \mathrm{E}+01$ & $7.392 \mathrm{E}-04$ & $1.397 \mathrm{E}+09$ & $1.6408 \mathrm{E}+01$ & $7.475 \mathrm{E}-04$ & $1.412 \mathrm{E}+09$ & 0.000 \\
\hline $1 s 6 f^{3} F_{4}^{o}$ & $1 s 7 h^{3} H_{6}^{o}$ & $4.3655 \mathrm{E}+01$ & $7.178 \mathrm{E}-03$ & $1.933 \mathrm{E}+09$ & $4.3474 \mathrm{E}+01$ & $7.190 \mathrm{E}-03$ & $1.935 \mathrm{E}+09$ & 0.000 \\
\hline $1 s 6 h^{3} H_{5}^{o}$ & $1 s 7 h^{3} H_{6}^{o}$ & $4.3863 \mathrm{E}+01$ & $2.951 \mathrm{E}-05$ & $7.870 \mathrm{E}+06$ & $4.3678 \mathrm{E}+01$ & $2.967 \mathrm{E}-05$ & $7.912 E+06$ & 0.000 \\
\hline $1 s 6 h^{3} H_{4}^{o}$ & $1 s 7 h^{3} H_{6}^{o}$ & $4.3869 \mathrm{E}+01$ & $9.395 \mathrm{E}-07$ & $2.505 E+05$ & $4.3678 \mathrm{E}+01$ & $9.419 \mathrm{E}-07$ & $2.511 \mathrm{E}+05$ & 0.000 \\
\hline $1 s 6 h^{3} H_{6}^{o}$ & $1 s 7 h^{3} H_{6}^{o}$ & $4.4002 \mathrm{E}+01$ & $6.178 \mathrm{E}-04$ & $1.637 \mathrm{E}+08$ & $4.3816 \mathrm{E}+01$ & $6.182 \mathrm{E}-04$ & $1.638 \mathrm{E}+08$ & 0.000 \\
\hline $1 s 6 h{ }^{1} H_{5}^{o}$ & $1 s 7 h^{3} H_{6}^{0}$ & $4.4006 \mathrm{E}+01$ & $2.480 \mathrm{E}-05$ & $6.570 \mathrm{E}+06$ & $4.3816 \mathrm{E}+01$ & $2.473 \mathrm{E}-05$ & $6.553 E+06$ & 0.000 \\
\hline $1 s 7 f^{3} F_{4}^{o}$ & $1 s 7 h^{3} H_{6}^{o}$ & $8.7953 \mathrm{E}+03$ & $1.460 \mathrm{E}-09$ & $9.686 \mathrm{E}-03$ & $8.8520 \mathrm{E}+03$ & $1.414 \mathrm{E}-09$ & $9.182 \mathrm{E}-03$ & 0.009 \\
\hline $1 s 7 h^{3} H_{5}^{o}$ & $1 s 7 h^{3} H_{6}^{o}$ & $2.2090 \mathrm{E}+04$ & $4.176 \mathrm{E}-12$ & $4.392 \mathrm{E}-06$ & $2.2109 \mathrm{E}+04$ & $4.124 \mathrm{E}-12$ & $4.292 \mathrm{E}-06$ & 0.008 \\
\hline $1 s 7 h^{3} H_{4}^{o}$ & $1 s 7 h^{3} H_{6}^{o}$ & $2.2993 \mathrm{E}+04$ & $1.179 \mathrm{E}-13$ & $1.144 \mathrm{E}-07$ & & & & 0.100 \\
\hline $1 s 5 g{ }^{3} G_{4}$ & $1 s 7 i^{3} I_{6}$ & $1.6478 \mathrm{E}+01$ & $1.124 \mathrm{E}-02$ & $2.123 E+10$ & $1.6408 \mathrm{E}+01$ & $1.128 \mathrm{E}-02$ & $2.131 \mathrm{E}+10$ & 0.000 \\
\hline $1 s 5 g{ }^{3} G_{5}$ & $1 s 7 i^{3} I_{6}$ & $1.6529 \mathrm{E}+01$ & $4.191 \mathrm{E}-04$ & $7.871 \mathrm{E}+08$ & $1.6459 \mathrm{E}+01$ & $4.199 \mathrm{E}-04$ & $7.886 \mathrm{E}+08$ & 0.000 \\
\hline $1 s 5 g{ }^{1} G_{4}$ & $1 s 7 i^{3} I_{6}$ & $1.6531 \mathrm{E}+01$ & $1.290 \mathrm{E}-05$ & $2.422 \mathrm{E}+07$ & $1.6460 \mathrm{E}+01$ & $1.190 \mathrm{E}-05$ & $2.234 \mathrm{E}+07$ & 0.000 \\
\hline $1 s 6 g{ }^{3} G_{4}$ & $1 s 7 i^{3} I_{6}$ & $4.3656 \mathrm{E}+01$ & $5.574 \mathrm{E}-03$ & $1.501 \mathrm{E}+09$ & $4.3473 \mathrm{E}+01$ & $5.578 \mathrm{E}-03$ & $1.501 \mathrm{E}+09$ & 0.000 \\
\hline $1 s 6 g{ }^{3} G_{5}$ & $1 s 7 i^{3} I_{6}$ & $4.3863 \mathrm{E}+01$ & $2.052 \mathrm{E}-04$ & $5.471 \mathrm{E}+07$ & $4.3678 \mathrm{E}+01$ & $2.055 \mathrm{E}-04$ & $5.481 E+07$ & 0.000 \\
\hline $1 s 6 g{ }^{1} G_{4}$ & $1 s 7 i^{3} I_{6}$ & $4.3870 \mathrm{E}+01$ & $6.313 \mathrm{E}-06$ & $1.683 \mathrm{E}+06$ & $4.3678 \mathrm{E}+01$ & $6.525 \mathrm{E}-06$ & $1.740 \mathrm{E}+06$ & 0.000 \\
\hline $1 s 7 g{ }^{3} G_{4}$ & $1 s 7 i^{3} I_{6}$ & $8.8113 \mathrm{E}+03$ & $5.733 \mathrm{E}-10$ & $3.789 \mathrm{E}-03$ & $8.8262 \mathrm{E}+03$ & $5.630 \mathrm{E}-10$ & $3.677 \mathrm{E}-03$ & 0.006 \\
\hline $1 s 7 g{ }^{3} G_{5}$ & $1 s 7 i^{3} I_{6}$ & $2.2046 \mathrm{E}+04$ & $1.354 \mathrm{E}-12$ & $1.429 \mathrm{E}-06$ & $2.2108 \mathrm{E}+04$ & $1.328 \mathrm{E}-12$ & $1.383 \mathrm{E}-06$ & 0.019 \\
\hline $1 s 7 g{ }^{1} G_{4}$ & $1 s 7 i^{3} I_{6}$ & $2.3157 \mathrm{E}+04$ & $3.597 \mathrm{E}-14$ & $3.442 \mathrm{E}-08$ & & & & 0.064 \\
\hline $1 s 3 d^{3} D_{3}$ & $1 s 7 i^{3} I_{5}$ & $3.5586 \mathrm{E}+00$ & $2.773 \mathrm{E}-12$ & $1.328 \mathrm{E}+02$ & & & & 1.000 \\
\hline $1 s 4 d^{3} D_{3}$ & $1 s 7 i^{3} I_{5}$ & $7.6476 \mathrm{E}+00$ & $9.356 \mathrm{E}-14$ & $9.701 \mathrm{E}-01$ & & & & 0.538 \\
\hline $1 s 5 d^{3} D_{3}$ & $1 s 7 i^{3} I_{5}$ & $1.6390 \mathrm{E}+01$ & $3.142 \mathrm{E}-12$ & $7.092 \mathrm{E}+00$ & & & & 0.057 \\
\hline $1 s 5 g{ }^{3} G_{4}$ & $1 s 7 i^{3} I_{5}$ & $1.6478 \mathrm{E}+01$ & $4.307 \mathrm{E}-04$ & $9.619 \mathrm{E}+08$ & $1.6408 \mathrm{E}+01$ & $4.324 \mathrm{E}-04$ & $9.656 \mathrm{E}+08$ & 0.000 \\
\hline $1 s 5 g{ }^{3} G_{3}$ & $1 s 7 i^{3} I_{5}$ & $1.6480 \mathrm{E}+01$ & $9.076 \mathrm{E}-03$ & $2.026 \mathrm{E}+10$ & $1.6410 \mathrm{E}+01$ & $9.108 \mathrm{E}-03$ & $2.033 \mathrm{E}+10$ & 0.000 \\
\hline $1 s 5 g{ }^{3} G_{5}$ & $1 s 7 i^{3} I_{5}$ & $1.6529 \mathrm{E}+01$ & $2.000 \mathrm{E}-05$ & $4.439 \mathrm{E}+07$ & $1.6459 \mathrm{E}+01$ & $1.999 \mathrm{E}-05$ & $4.438 \mathrm{E}+07$ & 0.000 \\
\hline $1 s 5 g{ }^{1} G_{4}$ & $1 s 7 i^{3} I_{5}$ & $1.6531 \mathrm{E}+01$ & $3.483 \mathrm{E}-04$ & $7.728 \mathrm{E}+08$ & $1.6460 \mathrm{E}+01$ & $3.479 \mathrm{E}-04$ & $7.721 \mathrm{E}+08$ & 0.000 \\
\hline $1 s 6 d^{3} D_{3}$ & $1 s 7 i^{3} I_{5}$ & $4.3297 \mathrm{E}+01$ & $3.945 \mathrm{E}-12$ & $1.276 \mathrm{E}+00$ & & & & 0.033 \\
\hline $1 s 6 g{ }^{3} G_{4}$ & $1 s 7 i^{3} I_{5}$ & $4.3654 \mathrm{E}+01$ & $2.137 \mathrm{E}-04$ & $6.799 \mathrm{E}+07$ & $4.3473 E+01$ & $2.145 \mathrm{E}-04$ & $6.825 E+07$ & 0.000 \\
\hline $1 s 6 g^{3} G_{3}$ & $1 s 7 i^{3} I_{5}$ & $4.3662 \mathrm{E}+01$ & $4.500 \mathrm{E}-03$ & $1.431 \mathrm{E}+09$ & $4.3473 \mathrm{E}+01$ & $4.505 \mathrm{E}-03$ & $1.433 \mathrm{E}+09$ & 0.000 \\
\hline $1 s 6 g{ }^{3} G_{5}$ & $1 s 7 i^{3} I_{5}$ & $4.3861 \mathrm{E}+01$ & $9.795 \mathrm{E}-06$ & $3.088 \mathrm{E}+06$ & $4.3678 \mathrm{E}+01$ & $9.787 \mathrm{E}-06$ & $3.084 \mathrm{E}+06$ & 0.000 \\
\hline
\end{tabular}


Table 4 (continued)

\begin{tabular}{|c|c|c|c|c|c|c|c|c|}
\hline \multirow[t]{2}{*}{ Lower } & \multirow[t]{2}{*}{ Upper } & \multicolumn{3}{|l|}{ GRASP2K } & \multicolumn{3}{|l|}{ FAC } & \multirow[t]{2}{*}{$d T$} \\
\hline & & $\lambda(\AA)$ & $g f$ & $A\left(\mathrm{~s}^{-1}\right)$ & $\lambda(\AA)$ & $g f$ & $A\left(\mathrm{~s}^{-1}\right)$ & \\
\hline $1 s 6 g^{1} G_{4}$ & $1 s 7 i^{3} I_{5}$ & $4.3867 \mathrm{E}+01$ & $1.704 \mathrm{E}-04$ & $5.371 \mathrm{E}+07$ & $4.3678 \mathrm{E}+01$ & $1.696 \mathrm{E}-04$ & $5.346 \mathrm{E}+07$ & 0.000 \\
\hline $1 s 7 d^{3} D_{3}$ & $1 s 7 i^{3} I_{5}$ & $4.2822 \mathrm{E}+03$ & $5.348 \mathrm{E}-18$ & $1.769 \mathrm{E}-10$ & & & & 0.999 \\
\hline $1 s 7 g{ }^{3} G_{4}$ & $1 s 7 i^{3} I_{5}$ & $8.7156 \mathrm{E}+03$ & $2.271 \mathrm{E}-11$ & $1.813 \mathrm{E}-04$ & $8.8262 \mathrm{E}+03$ & $2.165 E-11$ & $1.671 \mathrm{E}-04$ & 0.016 \\
\hline $1 s 7 g{ }^{3} G_{3}$ & $1 s 7 i^{3} I_{5}$ & $8.9298 \mathrm{E}+03$ & $4.447 \mathrm{E}-10$ & $3.382 \mathrm{E}-03$ & $8.8261 \mathrm{E}+03$ & $4.547 \mathrm{E}-10$ & $3.510 \mathrm{E}-03$ & 0.007 \\
\hline $1 s 7 g{ }^{3} G_{5}$ & $1 s 7 i^{3} I_{5}$ & $2.1457 \mathrm{E}+04$ & $7.011 \mathrm{E}-14$ & $9.234 \mathrm{E}-08$ & & & & 0.044 \\
\hline $1 s 7 g{ }^{1} G_{4}$ & $1 s 7 i^{3} I_{5}$ & $2.2508 \mathrm{E}+04$ & $1.057 \mathrm{E}-12$ & $1.265 \mathrm{E}-06$ & $2.2108 \mathrm{E}+04$ & $1.096 \mathrm{E}-12$ & $1.349 \mathrm{E}-06$ & 0.014 \\
\hline $1 s 7 i^{3} I_{6}$ & $1 s 7 i^{3} I_{5}$ & $8.0263 E+05$ & $4.303 \mathrm{E}-17$ & $4.051 \mathrm{E}-14$ & & & & 0.989 \\
\hline $1 s 4 f^{3} F_{3}^{o}$ & $1 s 7 h^{1} H_{5}^{o}$ & $7.6487 \mathrm{E}+00$ & $1.890 \mathrm{E}-09$ & $1.959 \mathrm{E}+04$ & & & & 0.012 \\
\hline $1 s 4 f^{3} F_{4}^{o}$ & $1 s 7 h{ }^{1} H_{5}^{o}$ & $7.6846 \mathrm{E}+00$ & $2.247 \mathrm{E}-04$ & $2.307 \mathrm{E}+09$ & $7.6520 \mathrm{E}+00$ & $2.259 \mathrm{E}-04$ & $2.320 \mathrm{E}+09$ & 0.000 \\
\hline $1 s 4 f^{1} F_{3}^{0}$ & $1 s 7 h^{1} H_{5}^{o}$ & $7.6858 \mathrm{E}+00$ & $4.701 \mathrm{E}-03$ & $4.826 \mathrm{E}+10$ & $7.6532 \mathrm{E}+00$ & $4.743 E-03$ & $4.868 \mathrm{E}+10$ & 0.000 \\
\hline $1 s 5 f^{3} F_{3}^{o}$ & $1 s 7 h^{1} H_{5}^{o}$ & $1.6393 \mathrm{E}+01$ & $1.359 \mathrm{E}-10$ & $3.067 \mathrm{E}+02$ & & & & 0.012 \\
\hline $1 s 5 f^{3} F_{4}^{o}$ & $1 s 7 h^{1} H_{5}^{o}$ & $1.6478 \mathrm{E}+01$ & $2.850 \mathrm{E}-05$ & $6.365 \mathrm{E}+07$ & $1.6408 \mathrm{E}+01$ & $2.875 \mathrm{E}-05$ & $6.420 \mathrm{E}+07$ & 0.000 \\
\hline $1 s 5 f^{1} F_{3}^{o}$ & $1 s 7 h^{1} H_{5}^{o}$ & $1.6481 \mathrm{E}+01$ & $5.977 \mathrm{E}-04$ & $1.334 \mathrm{E}+09$ & $1.6411 \mathrm{E}+01$ & $6.034 \mathrm{E}-04$ & $1.347 \mathrm{E}+09$ & 0.000 \\
\hline $1 s 6 f^{3} F_{3}^{o}$ & $1 s 7 h^{1} H_{5}^{o}$ & $4.3309 \mathrm{E}+01$ & $1.004 \mathrm{E}-09$ & $3.246 \mathrm{E}+02$ & & & & 0.001 \\
\hline $1 s 6 f^{3} F_{4}^{0}$ & $1 s 7 h{ }^{1} H_{5}^{o}$ & $4.3652 \mathrm{E}+01$ & $2.771 \mathrm{E}-04$ & $8.817 E+07$ & $4.3474 \mathrm{E}+01$ & $2.765 \mathrm{E}-04$ & $8.798 E+07$ & 0.000 \\
\hline $1 s 6 f^{1} F_{3}^{o}$ & $1 s 7 h^{1} H_{5}^{o}$ & $4.3663 \mathrm{E}+01$ & $5.796 \mathrm{E}-03$ & $1.843 \mathrm{E}+09$ & $4.3474 \mathrm{E}+01$ & $5.807 \mathrm{E}-03$ & $1.847 \mathrm{E}+09$ & 0.000 \\
\hline $1 s 6 h^{3} H_{5}^{o}$ & $1 s 7 h^{1} H_{5}^{o}$ & $4.3860 \mathrm{E}+01$ & $1.410 \mathrm{E}-06$ & $4.444 \mathrm{E}+05$ & $4.3678 \mathrm{E}+01$ & $1.412 \mathrm{E}-06$ & $4.452 \mathrm{E}+05$ & 0.000 \\
\hline $1 s 6 h^{3} H_{4}^{o}$ & $1 s 7 h^{1} H_{5}^{o}$ & $4.3866 \mathrm{E}+01$ & $2.451 \mathrm{E}-05$ & $7.724 \mathrm{E}+06$ & $4.3678 \mathrm{E}+01$ & $2.449 \mathrm{E}-05$ & $7.718 \mathrm{E}+06$ & 0.000 \\
\hline $1 s 6 h^{3} H_{6}^{o}$ & $1 s 7 h^{1} H_{5}^{o}$ & $4.3999 \mathrm{E}+01$ & $2.480 \mathrm{E}-05$ & $7.767 \mathrm{E}+06$ & $4.3816 \mathrm{E}+01$ & $2.473 \mathrm{E}-05$ & $7.744 \mathrm{E}+06$ & 0.000 \\
\hline $1 s 6 h^{1} H_{5}^{o}$ & $1 s 7 h^{1} H_{5}^{o}$ & $4.4004 \mathrm{E}+01$ & $5.188 \mathrm{E}-04$ & $1.625 E+08$ & $4.3816 \mathrm{E}+01$ & $5.193 \mathrm{E}-04$ & $1.626 \mathrm{E}+08$ & 0.000 \\
\hline $1 s 7 f^{3} F_{3}^{o}$ & $1 s 7 h^{1} H_{5}^{o}$ & $4.3583 \mathrm{E}+03$ & $1.278 \mathrm{E}-15$ & $4.080 \mathrm{E}-08$ & & & & 0.059 \\
\hline $1 s 7 f^{3} F_{4}^{o}$ & $1 s 7 h^{1} H_{5}^{o}$ & $8.6829 E+03$ & $5.859 \mathrm{E}-11$ & $4.712 \mathrm{E}-04$ & $8.8520 \mathrm{E}+03$ & $5.439 \mathrm{E}-11$ & $4.174 \mathrm{E}-04$ & 0.007 \\
\hline $1 s 7 f^{1} F_{3}^{o}$ & $1 s 7 h^{1} H_{5}^{o}$ & $8.9642 \mathrm{E}+03$ & $1.114 \mathrm{E}-09$ & $8.405 E-03$ & $8.8589 \mathrm{E}+03$ & $1.139 \mathrm{E}-09$ & $8.731 \mathrm{E}-03$ & 0.008 \\
\hline $1 s 7 h^{3} H_{5}^{o}$ & $1 s 7 h{ }^{1} H_{5}^{o}$ & $2.1394 \mathrm{E}+04$ & $2.197 \mathrm{E}-13$ & $2.911 \mathrm{E}-07$ & & & & 0.012 \\
\hline $1 s 7 h^{3} H_{4}^{o}$ & $1 s 7 h^{1} H_{5}^{o}$ & $2.2240 \mathrm{E}+04$ & $3.399 \mathrm{E}-12$ & $4.167 \mathrm{E}-06$ & $2.2109 \mathrm{E}+04$ & $3.404 \mathrm{E}-12$ & $4.187 \mathrm{E}-06$ & 0.007 \\
\hline $1 s 7 h^{3} H_{6}^{0}$ & $1 s 7 h^{1} H_{5}^{o}$ & $6.7907 \mathrm{E}+05$ & $1.209 \mathrm{E}-16$ & $1.590 \mathrm{E}-13$ & & & & 0.542 \\
\hline $1 s 5 g^{3} G_{5}$ & $1 s 7 i^{3} I_{7}$ & $1.6520 \mathrm{E}+01$ & $1.348 \mathrm{E}-02$ & $2.196 \mathrm{E}+10$ & $1.6450 \mathrm{E}+01$ & $1.355 \mathrm{E}-02$ & $2.208 \mathrm{E}+10$ & 0.000 \\
\hline $1 s 6 g{ }^{3} G_{5}$ & $1 s 7 i^{3} I_{7}$ & $4.3801 \mathrm{E}+01$ & $6.642 \mathrm{E}-03$ & $1.539 \mathrm{E}+09$ & $4.3617 \mathrm{E}+01$ & $6.653 \mathrm{E}-03$ & $1.541 \mathrm{E}+09$ & 0.000 \\
\hline $1 s 7 g^{3} G_{5}$ & $1 s 7 i^{3} I_{7}$ & $1.2886 \mathrm{E}+04$ & $2.195 \mathrm{E}-10$ & $5.879 \mathrm{E}-04$ & $1.2907 \mathrm{E}+04$ & $2.156 \mathrm{E}-10$ & $5.708 \mathrm{E}-04$ & 0.009 \\
\hline $1 s 7 i^{3} I_{6}$ & $1 s 7 i^{3} I_{7}$ & $3.1014 \mathrm{E}+04$ & $7.499 \mathrm{E}-13$ & $3.467 \mathrm{E}-07$ & $3.1015 \mathrm{E}+04$ & $7.421 \mathrm{E}-13$ & $3.401 \mathrm{E}-07$ & 0.006 \\
\hline $1 s 7 i^{3} I_{5}$ & $1 s 7 i^{3} I_{7}$ & $3.2260 \mathrm{E}+04$ & $1.518 \mathrm{E}-14$ & $6.488 \mathrm{E}-09$ & & & & 0.243 \\
\hline $1 s 5 g{ }^{3} G_{4}$ & $1 s 7 i^{1} I_{6}$ & $1.6469 \mathrm{E}+01$ & $3.770 \mathrm{E}-09$ & $7.132 \mathrm{E}+03$ & & & & 0.005 \\
\hline $1 s 5 g{ }^{3} G_{5}$ & $1 s 7 i^{1} I_{6}$ & $1.6520 \mathrm{E}+01$ & $3.605 \mathrm{E}-04$ & $6.777 \mathrm{E}+08$ & $1.6450 \mathrm{E}+01$ & $3.615 E-04$ & $6.796 \mathrm{E}+08$ & 0.000 \\
\hline $1 s 5 g{ }^{1} G_{4}$ & $1 s 7 i{ }^{1} I_{6}$ & $1.6522 \mathrm{E}+01$ & $1.132 \mathrm{E}-02$ & $2.128 \mathrm{E}+10$ & $1.6452 \mathrm{E}+01$ & $1.138 \mathrm{E}-02$ & $2.139 \mathrm{E}+10$ & 0.000 \\
\hline $1 s 6 g^{3} G_{4}$ & $1 s 7 i^{1} I_{6}$ & $4.3593 \mathrm{E}+01$ & $1.872 \mathrm{E}-09$ & $5.054 \mathrm{E}+02$ & & & & 0.006 \\
\hline $1 s 6 g^{3} G_{5}$ & $1 s 7 i^{1} I_{6}$ & $4.3799 \mathrm{E}+01$ & $1.776 \mathrm{E}-04$ & $4.751 E+07$ & $4.3617 \mathrm{E}+01$ & $1.774 \mathrm{E}-04$ & $4.744 \mathrm{E}+07$ & 0.000 \\
\hline $1 s 6 g{ }^{1} G_{4}$ & $1 s 7 i^{1} I_{6}$ & $4.3806 \mathrm{E}+01$ & $5.577 \mathrm{E}-03$ & $1.491 \mathrm{E}+09$ & $4.3617 \mathrm{E}+01$ & $5.588 \mathrm{E}-03$ & $1.494 \mathrm{E}+09$ & 0.000 \\
\hline $1 s 7 g{ }^{3} G_{4}$ & $1 s 7 i^{1} I_{6}$ & $6.8121 \mathrm{E}+03$ & $4.202 \mathrm{E}-16$ & $4.646 \mathrm{E}-09$ & & & & 0.315 \\
\hline $1 s 7 g{ }^{3} G_{5}$ & $1 s 7 i^{1} I_{6}$ & $1.2712 \mathrm{E}+04$ & $6.116 \mathrm{E}-12$ & $1.942 \mathrm{E}-05$ & $1.2907 \mathrm{E}+04$ & $5.751 \mathrm{E}-12$ & $1.756 \mathrm{E}-05$ & 0.019 \\
\hline $1 s 7 g^{1} G_{4}$ & $1 s 7 i{ }^{1} I_{6}$ & $1.3074 \mathrm{E}+04$ & $1.765 \mathrm{E}-10$ & $5.300 \mathrm{E}-04$ & $1.2907 \mathrm{E}+04$ & $1.811 \mathrm{E}-10$ & $5.532 \mathrm{E}-04$ & 0.008 \\
\hline $1 s 7 i^{3} I_{6}$ & $1 s 7 i{ }^{1} I_{6}$ & $3.0023 E+04$ & $2.826 \mathrm{E}-14$ & $1.609 \mathrm{E}-08$ & & & & 0.071 \\
\hline $1 s 7 i^{3} I_{5}$ & $1 s 7 i{ }^{1} I_{6}$ & $3.1190 \mathrm{E}+04$ & $6.317 \mathrm{E}-13$ & $3.332 \mathrm{E}-07$ & $3.1015 E+04$ & $6.325 E-13$ & $3.345 \mathrm{E}-07$ & 0.006 \\
\hline $1 s 7 i^{3} I_{7}$ & $1 s 7 i^{1} I_{6}$ & $9.4020 \mathrm{E}+05$ & $2.328 \mathrm{E}-17$ & $1.351 \mathrm{E}-14$ & & & 0.998 & \\
\hline
\end{tabular}


Table 5

Magnetic dipole transitions M 1 calculated with GRASP2K and FAC for all $n=1-7$ configurations. For the GRASP2K calculation, the active set is expanded to $n=10$ (GRASP3) and only the length form is considered. A comparison between the wavelengths, weighted oscillator strengths and transition probabilities is given.

\begin{tabular}{|c|c|c|c|c|c|c|c|}
\hline \multirow[t]{2}{*}{ Lower } & \multirow[t]{2}{*}{ Upper } & \multicolumn{3}{|l|}{ GRASP2K } & \multicolumn{3}{|l|}{ FAC } \\
\hline & & $\lambda(\AA)$ & $g f$ & $A\left(\mathrm{~s}^{-1}\right)$ & $\lambda(\AA)$ & $g f$ & $A\left(\mathrm{~s}^{-1}\right)$ \\
\hline $1 s^{2}{ }^{1} S_{0}$ & $1 s 2 s^{3} S_{1}$ & $4.1150 \mathrm{E}-01$ & $2.918 \mathrm{E}-05$ & $3.831 \mathrm{E}+11$ & $4.0980 \mathrm{E}-01$ & $2.867 \mathrm{E}-05$ & $3.763 \mathrm{E}+11$ \\
\hline $1 s 2 p^{3} P_{1}^{o}$ & $1 s 2 p^{3} P_{0}^{o}$ & $2.0697 \mathrm{E}+03$ & $2.433 \mathrm{E}-06$ & $3.788 \mathrm{E}+03$ & $2.1628 \mathrm{E}+03$ & $2.322 \mathrm{E}-06$ & $3.283 \mathrm{E}+03$ \\
\hline $1 s 2 s^{3} S_{1}$ & $1 s 2 s{ }^{1} S_{0}$ & $1.4576 \mathrm{E}+02$ & $1.373 \mathrm{E}-07$ & $4.310 \mathrm{E}+04$ & $1.4307 \mathrm{E}+02$ & $1.353 \mathrm{E}-07$ & $4.373 E+04$ \\
\hline $1 s 2 p^{3} P_{1}^{o}$ & $1 s 2 p^{3} P_{2}^{o}$ & $3.1908 \mathrm{E}+01$ & $2.163 \mathrm{E}-04$ & $2.834 \mathrm{E}+08$ & $3.1815 \mathrm{E}+01$ & $2.161 \mathrm{E}-04$ & $2.824 \mathrm{E}+08$ \\
\hline $1 s 2 p^{3} P_{1}^{o}$ & $1 s 2 p^{1} P_{1}^{o}$ & $2.9274 \mathrm{E}+01$ & $4.310 \mathrm{E}-05$ & $1.118 \mathrm{E}+08$ & $2.9150 \mathrm{E}+01$ & $4.314 \mathrm{E}-05$ & $1.119 \mathrm{E}+08$ \\
\hline $1 s 2 p^{3} P_{0}^{o}$ & $1 s 2 p{ }^{1} P_{1}^{o}$ & $2.9694 \mathrm{E}+01$ & $7.934 \mathrm{E}-05$ & $2.001 \mathrm{E}+08$ & $2.9548 \mathrm{E}+01$ & $7.919 \mathrm{E}-05$ & $1.999 \mathrm{E}+08$ \\
\hline $1 s 2 p^{3} P_{2}^{o}$ & $1 s 2 p{ }^{1} P_{1}^{o}$ & $3.5465 \mathrm{E}+02$ & $6.279 \mathrm{E}-06$ & $1.110 \mathrm{E}+05$ & $3.4797 \mathrm{E}+02$ & $6.377 \mathrm{E}-06$ & $1.161 \mathrm{E}+05$ \\
\hline $1 s^{2}{ }^{1} S_{0}$ & $1 s 3 s^{3} S_{1}$ & $3.4600 \mathrm{E}-01$ & $8.922 \mathrm{E}-06$ & $1.656 \mathrm{E}+11$ & $3.4464 \mathrm{E}-01$ & $8.819 \mathrm{E}-06$ & $1.637 \mathrm{E}+11$ \\
\hline $1 s 2 s^{3} S_{1}$ & $1 s 3 s^{3} S_{1}$ & $2.1766 \mathrm{E}+00$ & $6.344 \mathrm{E}-07$ & $2.977 \mathrm{E}+08$ & $2.1673 E+00$ & $6.310 \mathrm{E}-07$ & $2.961 \mathrm{E}+08$ \\
\hline $1 s 2 s{ }^{1} S_{0}$ & $1 s 3 s^{3} S_{1}$ & $2.2096 \mathrm{E}+00$ & $3.489 \mathrm{E}-07$ & $1.589 \mathrm{E}+08$ & $2.2006 \mathrm{E}+00$ & $3.195 \mathrm{E}-07$ & $1.454 \mathrm{E}+08$ \\
\hline $1 s 2 p^{3} P_{1}^{o}$ & $1 s 3 p^{3} P_{1}^{o}$ & $2.1983 \mathrm{E}+00$ & $1.490 \mathrm{E}-07$ & $6.856 \mathrm{E}+07$ & $2.1892 \mathrm{E}+00$ & $1.782 \mathrm{E}-07$ & $8.199 \mathrm{E}+07$ \\
\hline $1 s 2 p^{3} P_{0}^{o}$ & $1 s 3 p^{3} P_{1}^{o}$ & $2.2006 \mathrm{E}+00$ & $7.444 \mathrm{E}-08$ & $3.417 \mathrm{E}+07$ & $2.1914 \mathrm{E}+00$ & $1.063 \mathrm{E}-07$ & $4.884 \mathrm{E}+07$ \\
\hline $1 s 2 p^{3} P_{2}^{o}$ & $1 s 3 p^{3} P_{1}^{o}$ & $2.3610 \mathrm{E}+00$ & $5.406 \mathrm{E}-06$ & $2.156 \mathrm{E}+09$ & $2.3509 \mathrm{E}+00$ & $5.520 \mathrm{E}-06$ & $2.202 \mathrm{E}+09$ \\
\hline $1 s 2 p{ }^{1} P_{1}^{o}$ & $1 s 3 p^{3} P_{1}^{o}$ & $2.3768 \mathrm{E}+00$ & $1.276 \mathrm{E}-06$ & $5.023 \mathrm{E}+08$ & $2.3669 \mathrm{E}+00$ & $1.177 \mathrm{E}-06$ & $4.633 \mathrm{E}+08$ \\
\hline $1 s 2 s^{3} S_{1}$ & $1 s 3 s{ }^{1} S_{0}$ & $2.1681 \mathrm{E}+00$ & $2.934 \mathrm{E}-07$ & $4.163 \mathrm{E}+08$ & $2.1587 \mathrm{E}+00$ & $3.141 \mathrm{E}-07$ & $4.459 \mathrm{E}+08$ \\
\hline $1 s 3 s^{3} S_{1}$ & $1 s 3 s{ }^{1} S_{0}$ & $5.5160 \mathrm{E}+02$ & $5.089 \mathrm{E}-08$ & $1.116 \mathrm{E}+03$ & $5.4343 \mathrm{E}+02$ & $5.012 \mathrm{E}-08$ & $1.122 \mathrm{E}+03$ \\
\hline $1 s 2 p^{3} P_{1}^{o}$ & $1 s 3 p^{3} P_{0}^{o}$ & $2.1977 \mathrm{E}+00$ & $1.005 \mathrm{E}-07$ & $1.389 \mathrm{E}+08$ & $2.1886 \mathrm{E}+00$ & $5.046 \mathrm{E}-08$ & $6.967 \mathrm{E}+07$ \\
\hline $1 s 2 p{ }^{1} P_{1}^{o}$ & $1 s 3 p^{3} P_{0}^{o}$ & $2.3761 \mathrm{E}+00$ & $2.226 \mathrm{E}-06$ & $2.630 \mathrm{E}+09$ & $2.3662 \mathrm{E}+00$ & $2.267 \mathrm{E}-06$ & $2.678 \mathrm{E}+09$ \\
\hline $1 s 3 p^{3} P_{1}^{o}$ & $1 s 3 p^{3} P_{0}^{o}$ & $7.8853 \mathrm{E}+03$ & $6.217 \mathrm{E}-07$ & $6.669 \mathrm{E}+01$ & $8.1583 E+03$ & $5.996 \mathrm{E}-07$ & $5.958 \mathrm{E}+01$ \\
\hline $1 s 2 p^{3} P_{1}^{o}$ & $1 s 3 p^{3} P_{2}^{o}$ & $2.1541 \mathrm{E}+00$ & $2.836 \mathrm{E}-06$ & $8.154 \mathrm{E}+08$ & $2.1452 \mathrm{E}+00$ & $2.941 \mathrm{E}-06$ & $8.454 \mathrm{E}+08$ \\
\hline $1 s 2 p^{3} P_{2}^{o}$ & $1 s 3 p^{3} P_{2}^{o}$ & $2.3101 \mathrm{E}+00$ & $2.316 \mathrm{E}-06$ & $5.790 \mathrm{E}+08$ & $2.3003 \mathrm{E}+00$ & $2.323 \mathrm{E}-06$ & $5.808 \mathrm{E}+08$ \\
\hline $1 s 2 p{ }^{1} P_{1}^{o}$ & $1 s 3 p^{3} P_{2}^{o}$ & $2.3252 \mathrm{E}+00$ & $2.020 \mathrm{E}-07$ & $4.984 \mathrm{E}+07$ & $2.3156 \mathrm{E}+00$ & $1.970 \mathrm{E}-07$ & $4.859 \mathrm{E}+07$ \\
\hline $1 s 3 p{ }^{3} P_{1}^{o}$ & $1 s 3 p^{3} P_{2}^{o}$ & $1.0725 \mathrm{E}+02$ & $6.475 \mathrm{E}-05$ & $7.509 \mathrm{E}+06$ & $1.0689 \mathrm{E}+02$ & $6.471 \mathrm{E}-05$ & $7.491 \mathrm{E}+06$ \\
\hline $1 s 2 s^{3} S_{1}$ & $1 s 3 d^{3} D_{2}$ & $2.1226 \mathrm{E}+00$ & $8.050 \mathrm{E}-08$ & $2.384 \mathrm{E}+07$ & $2.1136 \mathrm{E}+00$ & $8.285 \mathrm{E}-08$ & $2.453 \mathrm{E}+07$ \\
\hline $1 s 3 s^{3} S_{1}$ & $1 s 3 d^{3} D_{2}$ & $8.5509 \mathrm{E}+01$ & $7.271 \mathrm{E}-10$ & $1.327 \mathrm{E}+02$ & $8.5230 \mathrm{E}+01$ & $7.265 \mathrm{E}-10$ & $1.322 \mathrm{E}+02$ \\
\hline $1 s^{21} S_{0}$ & $1 s 3 d^{3} D_{1}$ & $3.4460 \mathrm{E}-01$ & $9.108 \mathrm{E}-08$ & $1.705 \mathrm{E}+09$ & $3.4323 \mathrm{E}-01$ & $9.156 \mathrm{E}-08$ & $1.713 \mathrm{E}+09$ \\
\hline $1 s 2 s^{3} S_{1}$ & $1 s 3 d^{3} D_{1}$ & $2.1220 \mathrm{E}+00$ & $2.165 \mathrm{E}-08$ & $1.069 \mathrm{E}+07$ & $2.1130 \mathrm{E}+00$ & $1.630 \mathrm{E}-08$ & $8.048 \mathrm{E}+06$ \\
\hline $1 s 2 s{ }^{1} S_{0}$ & $1 s 3 d^{3} D_{1}$ & $2.1534 \mathrm{E}+00$ & $3.345 \mathrm{E}-08$ & $1.604 \mathrm{E}+07$ & $2.1447 \mathrm{E}+00$ & $3.438 \mathrm{E}-08$ & $1.647 \mathrm{E}+07$ \\
\hline $1 s 3 s^{3} S_{1}$ & $1 s 3 d^{3} D_{1}$ & $8.4639 \mathrm{E}+01$ & $6.335 \mathrm{E}-10$ & $1.966 \mathrm{E}+02$ & $8.4368 \mathrm{E}+01$ & $6.521 \mathrm{E}-10$ & $2.019 \mathrm{E}+02$ \\
\hline $1 s 3 s{ }^{1} S_{0}$ & $1 s 3 d^{3} D_{1}$ & $9.9981 \mathrm{E}+01$ & $7.121 \mathrm{E}-11$ & $1.584 \mathrm{E}+01$ & $9.9873 \mathrm{E}+01$ & $7.246 \mathrm{E}-11$ & $1.601 \mathrm{E}+01$ \\
\hline $1 s 3 d^{3} D_{2}$ & $1 s 3 d^{3} D_{1}$ & $8.3264 \mathrm{E}+03$ & $1.110 \mathrm{E}-06$ & $3.561 \mathrm{E}+01$ & $8.3383 E+03$ & $1.107 \mathrm{E}-06$ & $3.511 \mathrm{E}+01$ \\
\hline $1 s 2 p^{3} P_{1}^{o}$ & $1 s 3 p{ }^{1} P_{1}^{o}$ & $2.1504 \mathrm{E}+00$ & $5.477 \mathrm{E}-07$ & $2.633 \mathrm{E}+08$ & $2.1415 \mathrm{E}+00$ & $5.343 \mathrm{E}-07$ & $2.568 \mathrm{E}+08$ \\
\hline $1 s 2 p^{3} P_{0}^{o}$ & $1 s 3 p{ }^{1} P_{1}^{o}$ & $2.1527 \mathrm{E}+00$ & $1.076 \mathrm{E}-06$ & $5.162 \mathrm{E}+08$ & $2.1436 \mathrm{E}+00$ & $1.127 \mathrm{E}-06$ & $5.408 \mathrm{E}+08$ \\
\hline $1 s 2 p^{3} P_{2}^{o}$ & $1 s 3 p{ }^{1} P_{1}^{o}$ & $2.3058 \mathrm{E}+00$ & $4.193 \mathrm{E}-07$ & $1.754 \mathrm{E}+08$ & $2.2961 \mathrm{E}+00$ & $3.571 \mathrm{E}-07$ & $1.493 \mathrm{E}+08$ \\
\hline $1 s 2 p{ }^{1} P_{1}^{o}$ & $1 s 3 p{ }^{1} P_{1}^{o}$ & $2.3209 \mathrm{E}+00$ & $1.288 \mathrm{E}-06$ & $5.315 \mathrm{E}+08$ & $2.3113 \mathrm{E}+00$ & $1.266 \mathrm{E}-06$ & $5.227 \mathrm{E}+08$ \\
\hline $1 s 3 p^{3} P_{1}^{o}$ & $1 s 3 p{ }^{1} P_{1}^{o}$ & $9.8786 \mathrm{E}+01$ & $1.299 \mathrm{E}-05$ & $2.960 \mathrm{E}+06$ & $9.8358 \mathrm{E}+01$ & $1.298 \mathrm{E}-05$ & $2.959 E+06$ \\
\hline $1 s 3 p^{3} P_{0}^{0}$ & $1 s 3 p^{1} P_{1}^{o}$ & $1.0004 \mathrm{E}+02$ & $2.400 \mathrm{E}-05$ & $5.332 \mathrm{E}+06$ & $9.9559 \mathrm{E}+01$ & $2.397 \mathrm{E}-05$ & $5.331 \mathrm{E}+06$ \\
\hline $1 s 3 p^{3} P_{2}^{o}$ & $1 s 3 p{ }^{1} P_{1}^{o}$ & $1.2519 \mathrm{E}+03$ & $1.718 \mathrm{E}-06$ & $2.437 \mathrm{E}+03$ & $1.2319 \mathrm{E}+03$ & $1.743 \mathrm{E}-06$ & $2.532 \mathrm{E}+03$ \\
\hline $1 s 3 d^{3} D_{2}$ & $1 s 3 d^{3} D_{3}$ & $3.4166 \mathrm{E}+02$ & $3.300 \mathrm{E}-05$ & $2.694 \mathrm{E}+05$ & $3.4098 \mathrm{E}+02$ & $3.294 \mathrm{E}-05$ & $2.677 \mathrm{E}+05$ \\
\hline $1 s 2 s^{3} S_{1}$ & $1 s 3 d^{1} D_{2}$ & $2.1090 \mathrm{E}+00$ & $5.714 \mathrm{E}-11$ & $1.714 \mathrm{E}+04$ & $2.1000 \mathrm{E}+00$ & $1.086 \mathrm{E}-12$ & $3.258 \mathrm{E}+02$ \\
\hline $1 s 3 s^{3} S_{1}$ & $1 s 3 d{ }^{1} D_{2}$ & $6.7876 \mathrm{E}+01$ & $1.745 \mathrm{E}-10$ & $5.052 \mathrm{E}+01$ & $6.7664 \mathrm{E}+01$ & $1.801 \mathrm{E}-10$ & $5.205 E+01$ \\
\hline $1 s 3 d^{3} D_{2}$ & $1 s 3 d{ }^{1} D_{2}$ & $3.2917 \mathrm{E}+02$ & $2.437 \mathrm{E}-06$ & $3.000 \mathrm{E}+04$ & $3.2831 \mathrm{E}+02$ & $2.433 \mathrm{E}-06$ & $2.985 E+04$ \\
\hline $1 s 3 d^{3} D_{1}$ & $1 s 3 d d^{1} D_{2}$ & $3.4272 \mathrm{E}+02$ & $2.090 \mathrm{E}-05$ & $2.374 \mathrm{E}+05$ & $3.4177 \mathrm{E}+02$ & $2.084 \mathrm{E}-05$ & $2.360 \mathrm{E}+05$ \\
\hline $1 s 3 d^{3} D_{3}$ & $1 s 3 d d^{1} D_{2}$ & $8.9990 \mathrm{E}+03$ & $6.377 \mathrm{E}-07$ & $1.050 \mathrm{E}+01$ & & & \\
\hline $1 s^{2}{ }^{1} S_{0}$ & $1 s 4 s^{3} S_{1}$ & $3.2800 \mathrm{E}-01$ & $3.766 \mathrm{E}-06$ & $7.780 \mathrm{E}+10$ & $3.2669 \mathrm{E}-01$ & $3.731 \mathrm{E}-06$ & $7.706 \mathrm{E}+10$ \\
\hline $1 s 2 s{ }^{3} S_{1}$ & $1 s 4 s{ }^{3} S_{1}$ & $1.6177 \mathrm{E}+00$ & $2.937 \mathrm{E}-07$ & $2.495 \mathrm{E}+08$ & $1.6108 \mathrm{E}+00$ & $2.937 \mathrm{E}-07$ & $2.495 \mathrm{E}+08$ \\
\hline $1 s 2 s{ }^{1} S_{0}$ & $1 s 4 s^{3} S_{1}$ & $1.6358 \mathrm{E}+00$ & $1.581 \mathrm{E}-07$ & $1.313 \mathrm{E}+08$ & $1.6291 \mathrm{E}+00$ & $1.481 \mathrm{E}-07$ & $1.230 \mathrm{E}+08$ \\
\hline $1 s 3 s^{3} S_{1}$ & $1 s 4 s^{3} S_{1}$ & $6.2995 E+00$ & $5.467 \mathrm{E}-08$ & $3.063 \mathrm{E}+06$ & $6.2733 \mathrm{E}+00$ & $5.451 \mathrm{E}-08$ & $3.053 \mathrm{E}+06$ \\
\hline $1 s 3 s{ }^{1} S_{0}$ & $1 s 4 s^{3} S_{1}$ & $6.3723 \mathrm{E}+00$ & $3.304 \mathrm{E}-08$ & $1.809 \mathrm{E}+06$ & $6.3465 E+00$ & $2.752 \mathrm{E}-08$ & $1.506 \mathrm{E}+06$ \\
\hline $1 s 3 d^{3} D_{2}$ & $1 s 4 s^{3} S_{1}$ & $6.8005 E+00$ & $7.832 \mathrm{E}-12$ & $3.766 \mathrm{E}+02$ & $6.7717 \mathrm{E}+00$ & $2.642 \mathrm{E}-11$ & $1.270 \mathrm{E}+03$ \\
\hline $1 s 3 d^{3} D_{1}$ & $1 s 4 s^{3} S_{1}$ & $6.8061 \mathrm{E}+00$ & $5.861 \mathrm{E}-11$ & $2.813 \mathrm{E}+03$ & & & \\
\hline $1 s 3 d^{1} D_{2}$ & $1 s 4 s^{3} S_{1}$ & $6.9440 \mathrm{E}+00$ & $7.996 \mathrm{E}-12$ & $3.687 \mathrm{E}+02$ & & & \\
\hline $1 s 2 p^{3} P_{1}^{o}$ & $1 s 4 p^{3} P_{1}^{o}$ & $1.6323 \mathrm{E}+00$ & $6.280 \mathrm{E}-08$ & $5.241 \mathrm{E}+07$ & $1.6255 \mathrm{E}+00$ & $7.524 \mathrm{E}-08$ & $6.277 \mathrm{E}+07$ \\
\hline $1 s 2 p^{3} P_{0}^{o}$ & $1 s 4 p^{3} P_{1}^{o}$ & $1.6336 \mathrm{E}+00$ & $3.333 \mathrm{E}-08$ & $2.777 \mathrm{E}+07$ & $1.6268 \mathrm{E}+00$ & $4.319 \mathrm{E}-08$ & $3.598 \mathrm{E}+07$ \\
\hline $1 s 2 p^{3} P_{2}^{o}$ & $1 s 4 p^{3} P_{1}^{o}$ & $1.7203 \mathrm{E}+00$ & $1.671 \mathrm{E}-06$ & $1.255 \mathrm{E}+09$ & $1.7131 \mathrm{E}+00$ & $1.700 \mathrm{E}-06$ & $1.277 \mathrm{E}+09$ \\
\hline $1 s 2 p^{1} P_{1}^{o}$ & $1 s 4 p^{3} P_{1}^{0}$ & $1.7287 \mathrm{E}+00$ & $3.958 \mathrm{E}-07$ & $2.945 \mathrm{E}+08$ & $1.7215 \mathrm{E}+00$ & $3.662 \mathrm{E}-07$ & $2.724 \mathrm{E}+08$ \\
\hline $1 s 3 p^{3} P_{1}^{o}$ & $1 s 4 p{ }^{3} P_{1}^{o}$ & $6.3396 \mathrm{E}+00$ & $1.616 \mathrm{E}-08$ & $8.938 \mathrm{E}+05$ & $6.3138 \mathrm{E}+00$ & $1.862 \mathrm{E}-08$ & $1.029 \mathrm{E}+06$ \\
\hline $1 s 3 p^{3} P_{0}^{o}$ & $1 s 4 p^{3} P_{1}^{o}$ & $6.3447 \mathrm{E}+00$ & $6.591 \mathrm{E}-09$ & $3.640 \mathrm{E}+05$ & $6.3187 \mathrm{E}+00$ & $1.409 \mathrm{E}-08$ & $7.784 \mathrm{E}+05$ \\
\hline $1 s 3 p^{3} P_{2}^{o}$ & $1 s 4 p^{3} P_{1}^{o}$ & $6.7379 \mathrm{E}+00$ & $1.885 \mathrm{E}-06$ & $9.233 \mathrm{E}+07$ & $6.7101 \mathrm{E}+00$ & $1.933 \mathrm{E}-06$ & $9.466 \mathrm{E}+07$ \\
\hline $1 s 3 p{ }^{1} P_{1}^{o}$ & $1 s 4 p^{3} P_{1}^{o}$ & $6.7743 \mathrm{E}+00$ & $4.274 \mathrm{E}-07$ & $2.071 \mathrm{E}+07$ & $6.7469 \mathrm{E}+00$ & $4.002 \mathrm{E}-07$ & $1.938 \mathrm{E}+07$ \\
\hline $1 s 2 s^{3} S_{1}$ & $1 s 4 s{ }^{1} S_{0}$ & $1.6158 \mathrm{E}+00$ & $1.404 \mathrm{E}-07$ & $3.587 \mathrm{E}+08$ & $1.6089 \mathrm{E}+00$ & $1.466 \mathrm{E}-07$ & $3.746 \mathrm{E}+08$ \\
\hline $1 s 3 s^{3} S_{1}$ & $1 s 4 s{ }^{1} S_{0}$ & $6.2706 \mathrm{E}+00$ & $2.269 \mathrm{E}-08$ & $3.850 \mathrm{E}+06$ & $6.2442 \mathrm{E}+00$ & $2.713 \mathrm{E}-08$ & $4.602 \mathrm{E}+06$ \\
\hline $1 s 3 d^{3} D_{1}$ & $1 s 4 s{ }^{1} S_{0}$ & $6.7723 \mathrm{E}+00$ & $8.192 \mathrm{E}-12$ & $1.191 \mathrm{E}+03$ & $6.7433 \mathrm{E}+00$ & $9.074 \mathrm{E}-12$ & $1.319 \mathrm{E}+03$ \\
\hline $1 s 4 s^{3} S_{1}$ & $1 s 4 s{ }^{1} S_{0}$ & $1.3658 \mathrm{E}+03$ & $2.282 \mathrm{E}-08$ & $8.159 \mathrm{E}+01$ & $1.3474 \mathrm{E}+03$ & $2.246 \mathrm{E}-08$ & $8.184 \mathrm{E}+01$ \\
\hline $1 s 2 p^{3} P_{1}^{o}$ & $1 s 4 p^{3} P_{0}^{o}$ & $1.6322 \mathrm{E}+00$ & $4.224 \mathrm{E}-08$ & $1.058 \mathrm{E}+08$ & $1.6254 \mathrm{E}+00$ & $2.329 \mathrm{E}-08$ & $5.831 \mathrm{E}+07$ \\
\hline $1 s 2 p^{1} P_{1}^{o}$ & $1 s 4 p^{3} P_{0}^{o}$ & $1.7285 \mathrm{E}+00$ & $6.899 \mathrm{E}-07$ & $1.540 \mathrm{E}+09$ & $1.7214 \mathrm{E}+00$ & $7.028 \mathrm{E}-07$ & $1.568 \mathrm{E}+09$ \\
\hline $1 s 3 p^{3} P_{1}^{0}$ & $1 s 4 p^{3} P_{0}^{o}$ & $6.3375 E+00$ & $1.172 \mathrm{E}-08$ & $1.946 \mathrm{E}+06$ & $6.3118 \mathrm{E}+00$ & $3.384 \mathrm{E}-09$ & $5.619 \mathrm{E}+05$ \\
\hline $1 s 3 p{ }^{1} P_{1}^{o}$ & $1 s 4 p^{3} P_{0}^{o}$ & $6.7720 \mathrm{E}+00$ & $7.666 \mathrm{E}-07$ & $1.115 \mathrm{E}+08$ & $6.7446 \mathrm{E}+00$ & $7.828 \mathrm{E}-07$ & $1.138 \mathrm{E}+08$ \\
\hline $1 s 4 p^{3} P_{1}^{o}$ & $1 s 4 p^{3} P_{0}^{o}$ & $1.9682 \mathrm{E}+04$ & $2.467 \mathrm{E}-07$ & $4.247 \mathrm{E}+00$ & $2.0312 \mathrm{E}+04$ & $2.386 \mathrm{E}-07$ & $3.825 \mathrm{E}+00$ \\
\hline
\end{tabular}


Table 5 (continued)

\begin{tabular}{|c|c|c|c|c|c|c|c|}
\hline \multirow[t]{2}{*}{ Lower } & \multirow[t]{2}{*}{ Upper } & \multicolumn{3}{|l|}{ GRASP2K } & \multicolumn{3}{|l|}{ FAC } \\
\hline & & $\lambda(\AA)$ & $g f$ & $A\left(\mathrm{~s}^{-1}\right)$ & $\lambda(\AA)$ & $g f$ & $A\left(\mathrm{~s}^{-1}\right)$ \\
\hline $1 s 2 p^{3} P_{1}^{o}$ & $1 s 4 p^{3} P_{2}^{o}$ & $1.6219 \mathrm{E}+00$ & $8.188 \mathrm{E}-07$ & $4.153 \mathrm{E}+08$ & $1.6152 \mathrm{E}+00$ & $8.517 \mathrm{E}-07$ & $4.318 \mathrm{E}+08$ \\
\hline $1 s 2 p^{3} P_{2}^{o}$ & $1 s 4 p^{3} P_{2}^{o}$ & $1.7088 \mathrm{E}+00$ & $9.864 \mathrm{E}-07$ & $4.507 \mathrm{E}+08$ & $1.7016 \mathrm{E}+00$ & $9.961 \mathrm{E}-07$ & $4.550 \mathrm{E}+08$ \\
\hline $1 s 2 p^{1} P_{1}^{o}$ & $1 s 4 p^{3} P_{2}^{o}$ & $1.7170 \mathrm{E}+00$ & $9.347 \mathrm{E}-08$ & $4.230 \mathrm{E}+07$ & $1.7100 \mathrm{E}+00$ & $8.866 \mathrm{E}-08$ & $4.010 \mathrm{E}+07$ \\
\hline $1 s 3 p^{3} P_{1}^{o}$ & $1 s 4 p^{3} P_{2}^{o}$ & $6.1858 \mathrm{E}+00$ & $1.301 \mathrm{E}-06$ & $4.535 \mathrm{E}+07$ & $6.1607 \mathrm{E}+00$ & $1.338 \mathrm{E}-06$ & $4.665 \mathrm{E}+07$ \\
\hline $1 s 3 p^{3} P_{2}^{o}$ & $1 s 4 p^{3} P_{2}^{o}$ & $6.5644 \mathrm{E}+00$ & $2.489 \mathrm{E}-07$ & $7.705 E+06$ & $6.5375 \mathrm{E}+00$ & $2.511 \mathrm{E}-07$ & $7.773 E+06$ \\
\hline $1 s 3 p^{1} P_{1}^{o}$ & $1 s 4 p^{3} P_{2}^{o}$ & $6.5991 \mathrm{E}+00$ & $1.418 \mathrm{E}-08$ & $4.343 E+05$ & $6.5723 \mathrm{E}+00$ & $1.571 \mathrm{E}-08$ & $4.812 \mathrm{E}+05$ \\
\hline $1 s 4 p^{3} P_{1}^{o}$ & $1 s 4 p^{3} P_{2}^{o}$ & $2.5504 \mathrm{E}+02$ & $2.729 \mathrm{E}-05$ & $5.597 \mathrm{E}+05$ & $2.5410 \mathrm{E}+02$ & $2.728 \mathrm{E}-05$ & $5.589 \mathrm{E}+05$ \\
\hline $1 s 2 s^{3} S_{1}$ & $1 s 4 d^{3} D_{2}$ & $1.6050 \mathrm{E}+00$ & $2.666 \mathrm{E}-08$ & $1.380 \mathrm{E}+07$ & $1.5981 \mathrm{E}+00$ & $2.759 \mathrm{E}-08$ & $1.429 \mathrm{E}+07$ \\
\hline $1 s 3 s^{3} S_{1}$ & $1 s 4 d^{3} D_{2}$ & $6.1111 \mathrm{E}+00$ & $2.618 \mathrm{E}-09$ & $9.352 \mathrm{E}+04$ & $6.0854 \mathrm{E}+00$ & $2.952 \mathrm{E}-09$ & $1.054 \mathrm{E}+05$ \\
\hline $1 s 3 d^{3} D_{2}$ & $1 s 4 d^{3} D_{2}$ & $6.5814 \mathrm{E}+00$ & $9.907 \mathrm{E}-08$ & $3.051 \mathrm{E}+06$ & $6.5534 \mathrm{E}+00$ & $9.901 \mathrm{E}-08$ & $3.049 \mathrm{E}+06$ \\
\hline $1 s 3 d^{3} D_{1}$ & $1 s 4 d^{3} D_{2}$ & $6.5866 \mathrm{E}+00$ & $7.839 \mathrm{E}-09$ & $2.410 \mathrm{E}+05$ & $6.5585 \mathrm{E}+00$ & $1.134 \mathrm{E}-08$ & $3.487 \mathrm{E}+05$ \\
\hline $1 s 3 d^{3} D_{3}$ & $1 s 4 d^{3} D_{2}$ & $6.7107 \mathrm{E}+00$ & $3.091 \mathrm{E}-07$ & $9.158 \mathrm{E}+06$ & $6.6818 \mathrm{E}+00$ & $3.090 \mathrm{E}-07$ & $9.155 \mathrm{E}+06$ \\
\hline $1 s 3 d^{1} D_{2}$ & $1 s 4 d^{3} D_{2}$ & $6.7157 \mathrm{E}+00$ & $2.412 \mathrm{E}-08$ & $7.136 \mathrm{E}+05$ & $6.6868 \mathrm{E}+00$ & $2.277 \mathrm{E}-08$ & $6.736 \mathrm{E}+05$ \\
\hline $1 s 4 s^{3} S_{1}$ & $1 s 4 d^{3} D_{2}$ & $2.0428 \mathrm{E}+02$ & $2.882 \mathrm{E}-10$ & $9.211 \mathrm{E}+00$ & $2.0324 \mathrm{E}+02$ & $2.883 \mathrm{E}-10$ & $9.234 \mathrm{E}+00$ \\
\hline $1 s^{2}{ }^{1} S_{0}$ & $1 s 4 d^{3} D_{1}$ & $3.2750 \mathrm{E}-01$ & $5.115 \mathrm{E}-08$ & $1.060 \mathrm{E}+09$ & $3.2616 \mathrm{E}-01$ & $5.140 \mathrm{E}-08$ & $1.065 \mathrm{E}+09$ \\
\hline $1 s 2 s^{3} S_{1}$ & $1 s 4 d^{3} D_{1}$ & $1.6048 \mathrm{E}+00$ & $7.191 \mathrm{E}-09$ & $6.208 E+06$ & $1.5980 \mathrm{E}+00$ & $5.396 \mathrm{E}-09$ & $4.658 \mathrm{E}+06$ \\
\hline $1 s 2 s^{1} S_{0}$ & $1 s 4 d^{3} D_{1}$ & $1.6227 \mathrm{E}+00$ & $1.101 \mathrm{E}-08$ & $9.298 \mathrm{E}+06$ & $1.6161 \mathrm{E}+00$ & $1.127 \mathrm{E}-08$ & $9.515 E+06$ \\
\hline $1 s 3 s^{3} S_{1}$ & $1 s 4 d^{3} D_{1}$ & $6.1092 \mathrm{E}+00$ & $1.076 \mathrm{E}-09$ & $6.410 \mathrm{E}+04$ & $6.0836 \mathrm{E}+00$ & $5.912 \mathrm{E}-10$ & $3.521 \mathrm{E}+04$ \\
\hline $1 s 3 s^{1} S_{0}$ & $1 s 4 d^{3} D_{1}$ & $6.1777 \mathrm{E}+00$ & $1.224 \mathrm{E}-09$ & $7.130 \mathrm{E}+04$ & $6.1525 \mathrm{E}+00$ & $1.246 \mathrm{E}-09$ & $7.260 \mathrm{E}+04$ \\
\hline $1 s 3 d^{3} D_{2}$ & $1 s 4 d^{3} D_{1}$ & $6.5793 \mathrm{E}+00$ & $1.479 \mathrm{E}-08$ & $7.599 \mathrm{E}+05$ & $6.5513 \mathrm{E}+00$ & $1.065 \mathrm{E}-08$ & $5.475 E+05$ \\
\hline $1 s 3 d^{3} D_{1}$ & $1 s 4 d^{3} D_{1}$ & $6.5845 \mathrm{E}+00$ & $5.503 \mathrm{E}-08$ & $2.822 \mathrm{E}+06$ & $6.5564 \mathrm{E}+00$ & $5.496 \mathrm{E}-08$ & $2.818 \mathrm{E}+06$ \\
\hline $1 s 3 d{ }^{1} D_{2}$ & $1 s 4 d^{3} D_{1}$ & $6.7135 \mathrm{E}+00$ & $1.969 \mathrm{E}-07$ & $9.715 E+06$ & $6.6847 \mathrm{E}+00$ & $1.995 \mathrm{E}-07$ & $9.846 \mathrm{E}+06$ \\
\hline $1 s 4 s^{3} S_{1}$ & $1 s 4 d^{3} D_{1}$ & $2.0227 \mathrm{E}+02$ & $4.243 \mathrm{E}-10$ & $2.306 \mathrm{E}+01$ & $2.0125 \mathrm{E}+02$ & $4.340 \mathrm{E}-10$ & $2.362 \mathrm{E}+01$ \\
\hline $1 s 4 s^{1} S_{0}$ & $1 s 4 d^{3} D_{1}$ & $2.3744 \mathrm{E}+02$ & $2.003 \mathrm{E}-11$ & $7.899 \mathrm{E}-01$ & $2.3659 \mathrm{E}+02$ & $2.025 \mathrm{E}-11$ & $7.976 \mathrm{E}-01$ \\
\hline $1 s 4 d^{3} D_{2}$ & $1 s 4 d^{3} D_{1}$ & $2.0536 \mathrm{E}+04$ & $4.486 \mathrm{E}-07$ & $2.365 \mathrm{E}+00$ & $2.0610 \mathrm{E}+04$ & $4.464 \mathrm{E}-07$ & $2.317 \mathrm{E}+00$ \\
\hline $1 s 2 p^{3} P_{1}^{o}$ & $1 s 4 p^{1} P_{1}^{o}$ & $1.6210 \mathrm{E}+00$ & $1.525 \mathrm{E}-07$ & $1.290 \mathrm{E}+08$ & $1.6143 \mathrm{E}+00$ & $1.504 \mathrm{E}-07$ & $1.272 \mathrm{E}+08$ \\
\hline $1 s 2 p^{3} P_{0}^{o}$ & $1 s 4 p^{1} P_{1}^{o}$ & $1.6223 \mathrm{E}+00$ & $3.076 \mathrm{E}-07$ & $2.598 \mathrm{E}+08$ & $1.6155 \mathrm{E}+00$ & $3.230 \mathrm{E}-07$ & $2.728 \mathrm{E}+08$ \\
\hline $1 s 2 p^{3} P_{2}^{0}$ & $1 s 4 p^{1} P_{1}^{o}$ & $1.7078 \mathrm{E}+00$ & $1.729 \mathrm{E}-07$ & $1.318 \mathrm{E}+08$ & $1.7006 \mathrm{E}+00$ & $1.452 \mathrm{E}-07$ & $1.107 \mathrm{E}+08$ \\
\hline $1 s 2 p^{1} P_{1}^{o}$ & $1 s 4 p^{1} P_{1}^{o}$ & $1.7161 \mathrm{E}+00$ & $5.523 \mathrm{E}-07$ & $4.170 \mathrm{E}+08$ & $1.7090 \mathrm{E}+00$ & $5.447 \mathrm{E}-07$ & $4.112 \mathrm{E}+08$ \\
\hline $1 s 3 p^{3} P_{1}^{o}$ & $1 s 4 p^{1} P_{1}^{o}$ & $6.1732 \mathrm{E}+00$ & $2.703 \mathrm{E}-07$ & $1.577 \mathrm{E}+07$ & $6.1480 \mathrm{E}+00$ & $2.570 \mathrm{E}-07$ & $1.499 \mathrm{E}+07$ \\
\hline $1 s 3 p^{3} P_{0}^{o}$ & $1 s 4 p{ }^{1} P_{1}^{o}$ & $6.1780 \mathrm{E}+00$ & $5.068 \mathrm{E}-07$ & $2.952 E+07$ & $6.1526 \mathrm{E}+00$ & $5.255 \mathrm{E}-07$ & $3.060 \mathrm{E}+07$ \\
\hline $1 s 3 p^{3} P_{2}^{0}$ & $1 s 4 p^{1} P_{1}^{o}$ & $6.5502 \mathrm{E}+00$ & $5.703 \mathrm{E}-08$ & $2.955 \mathrm{E}+06$ & $6.5232 \mathrm{E}+00$ & $4.794 \mathrm{E}-08$ & $2.483 \mathrm{E}+06$ \\
\hline $1 s 3 p^{1} P_{1}^{o}$ & $1 s 4 p^{1} P_{1}^{o}$ & $6.5847 \mathrm{E}+00$ & $1.399 \mathrm{E}-07$ & $7.175 E+06$ & $6.5579 \mathrm{E}+00$ & $1.361 \mathrm{E}-07$ & $6.978 \mathrm{E}+06$ \\
\hline $1 s 4 p^{3} P_{1}^{o}$ & $1 s 4 p^{1} P_{1}^{o}$ & $2.3520 \mathrm{E}+02$ & $5.486 \mathrm{E}-06$ & $2.205 E+05$ & $2.3412 \mathrm{E}+02$ & $5.485 \mathrm{E}-06$ & $2.206 \mathrm{E}+05$ \\
\hline $1 s 4 p^{3} P_{0}^{o}$ & $1 s 4 p{ }^{1} P_{1}^{o}$ & $2.3804 \mathrm{E}+02$ & $1.015 \mathrm{E}-05$ & $3.983 E+05$ & $2.3685 \mathrm{E}+02$ & $1.014 \mathrm{E}-05$ & $3.986 \mathrm{E}+05$ \\
\hline $1 s 4 p^{3} P_{2}^{o}$ & $1 s 4 p^{1} P_{1}^{o}$ & $3.0236 \mathrm{E}+03$ & $7.020 \mathrm{E}-07$ & $1.707 \mathrm{E}+02$ & $2.9782 \mathrm{E}+03$ & $7.124 \mathrm{E}-07$ & $1.770 \mathrm{E}+02$ \\
\hline $1 s 3 d^{3} D_{2}$ & $1 s 4 d^{3} D_{3}$ & $6.5283 \mathrm{E}+00$ & $1.218 \mathrm{E}-07$ & $2.724 \mathrm{E}+06$ & $6.5006 \mathrm{E}+00$ & $1.221 \mathrm{E}-07$ & $2.731 \mathrm{E}+06$ \\
\hline $1 s 3 d^{3} D_{3}$ & $1 s 4 d^{3} D_{3}$ & $6.6555 \mathrm{E}+00$ & $4.750 \mathrm{E}-07$ & $1.022 \mathrm{E}+07$ & $6.6270 \mathrm{E}+00$ & $4.735 \mathrm{E}-07$ & $1.018 \mathrm{E}+07$ \\
\hline $1 s 3 d^{1} D_{2}$ & $1 s 4 d^{3} D_{3}$ & $6.6604 \mathrm{E}+00$ & $2.024 \mathrm{E}-08$ & $4.348 \mathrm{E}+05$ & $6.6320 \mathrm{E}+00$ & $2.330 \mathrm{E}-08$ & $5.005 E+05$ \\
\hline $1 s 4 d^{3} D_{2}$ & $1 s 4 d^{3} D_{3}$ & $8.0890 \mathrm{E}+02$ & $1.403 \mathrm{E}-05$ & $2.043 \mathrm{E}+04$ & $8.0816 \mathrm{E}+02$ & $1.399 \mathrm{E}-05$ & $2.023 E+04$ \\
\hline $1 s 2 p^{3} P_{2}^{o}$ & $1 s 4 f^{3} F_{3}^{o}$ & $1.7043 \mathrm{E}+00$ & $2.513 \mathrm{E}-08$ & $8.245 E+06$ & $1.6971 \mathrm{E}+00$ & $2.356 \mathrm{E}-08$ & $7.728 \mathrm{E}+06$ \\
\hline $1 s 3 p^{3} P_{2}^{0}$ & $1 s 4 f^{3} F_{3}^{o}$ & $6.4989 \mathrm{E}+00$ & $1.254 \mathrm{E}-08$ & $2.829 \mathrm{E}+05$ & $6.4721 \mathrm{E}+00$ & $1.209 \mathrm{E}-08$ & $2.729 \mathrm{E}+05$ \\
\hline $1 s 4 p^{3} P_{2}^{o}$ & $1 s 4 f^{3} F_{3}^{o}$ & $6.5059 \mathrm{E}+02$ & $1.536 \mathrm{E}-11$ & $3.458 \mathrm{E}-02$ & $6.4694 \mathrm{E}+02$ & $1.460 \mathrm{E}-11$ & $3.297 \mathrm{E}-02$ \\
\hline $1 s 2 p^{3} P_{1}^{o}$ & $1 s 4 f^{3} F_{2}^{o}$ & $1.6178 \mathrm{E}+00$ & $3.126 \mathrm{E}-11$ & $1.593 \mathrm{E}+04$ & $1.6111 \mathrm{E}+00$ & $1.478 \mathrm{E}-11$ & $7.535 E+03$ \\
\hline $1 s 2 p^{3} P_{2}^{o}$ & $1 s 4 f^{3} F_{2}^{o}$ & $1.7042 \mathrm{E}+00$ & $1.963 \mathrm{E}-09$ & $9.016 \mathrm{E}+05$ & $1.6971 \mathrm{E}+00$ & $1.712 \mathrm{E}-09$ & $7.863 \mathrm{E}+05$ \\
\hline $1 s 2 p{ }^{1} P_{1}^{o}$ & $1 s 4 f^{3} F_{2}^{o}$ & $1.7124 \mathrm{E}+00$ & $1.619 \mathrm{E}-08$ & $7.364 \mathrm{E}+06$ & $1.7054 \mathrm{E}+00$ & $1.552 \mathrm{E}-08$ & $7.060 \mathrm{E}+06$ \\
\hline $1 s 3 p^{3} P_{1}^{o}$ & $1 s 4 f^{3} F_{2}^{o}$ & $6.1266 \mathrm{E}+00$ & $2.174 \mathrm{E}-11$ & $7.726 \mathrm{E}+02$ & $6.1016 \mathrm{E}+00$ & $1.249 \mathrm{E}-11$ & $4.437 \mathrm{E}+02$ \\
\hline $1 s 3 p^{3} P_{2}^{0}$ & $1 s 4 f^{3} F_{2}^{o}$ & $6.4978 \mathrm{E}+00$ & $1.077 \mathrm{E}-09$ & $3.402 E+04$ & $6.4710 \mathrm{E}+00$ & $8.534 \mathrm{E}-10$ & $2.696 \mathrm{E}+04$ \\
\hline $1 s 3 p{ }^{1} P_{1}^{o}$ & $1 s 4 f^{3} F_{2}^{o}$ & $6.5317 \mathrm{E}+00$ & $8.213 \mathrm{E}-09$ & $2.568 \mathrm{E}+05$ & $6.5052 \mathrm{E}+00$ & $7.853 \mathrm{E}-09$ & $2.454 \mathrm{E}+05$ \\
\hline $1 s 4 p^{3} P_{1}^{o}$ & $1 s 4 f^{3} F_{2}^{o}$ & $1.8236 \mathrm{E}+02$ & $4.207 \mathrm{E}-12$ & $1.688 \mathrm{E}-01$ & $1.8158 \mathrm{E}+02$ & $4.868 \mathrm{E}-12$ & $1.953 \mathrm{E}-01$ \\
\hline $1 s 4 p^{3} P_{2}^{o}$ & $1 s 4 f^{3} F_{2}^{o}$ & $6.3989 \mathrm{E}+02$ & $1.075 \mathrm{E}-11$ & $3.504 \mathrm{E}-02$ & $6.3631 \mathrm{E}+02$ & $1.094 \mathrm{E}-11$ & $3.574 \mathrm{E}-02$ \\
\hline $1 s 4 p{ }^{1} P_{1}^{o}$ & $1 s 4 f^{3} F_{2}^{o}$ & $8.1166 \mathrm{E}+02$ & $1.204 \mathrm{E}-12$ & $2.438 \mathrm{E}-03$ & $8.0920 \mathrm{E}+02$ & $1.035 \mathrm{E}-12$ & $2.090 \mathrm{E}-03$ \\
\hline $1 s 4 f^{3} F_{3}^{0}$ & $1 s 4 f^{3} F_{2}^{o}$ & $3.8916 \mathrm{E}+04$ & $3.280 \mathrm{E}-07$ & $2.889 \mathrm{E}-01$ & $3.8737 \mathrm{E}+04$ & $3.272 \mathrm{E}-07$ & $2.884 \mathrm{E}-01$ \\
\hline $1 s 2 s^{3} S_{1}$ & $1 s 4 d^{1} D_{2}$ & $1.6017 \mathrm{E}+00$ & $2.260 \mathrm{E}-11$ & $1.175 \mathrm{E}+04$ & $1.5948 \mathrm{E}+00$ & $8.840 \mathrm{E}-13$ & $4.597 \mathrm{E}+02$ \\
\hline $1 s 3 s^{3} s_{1}$ & $1 s 4 d{ }^{1} D_{2}$ & $6.0634 \mathrm{E}+00$ & $1.398 \mathrm{E}-11$ & $5.074 \mathrm{E}+02$ & & & \\
\hline $1 s 3 d^{3} D_{2}$ & $1 s 4 d{ }^{1} D_{2}$ & $6.5262 \mathrm{E}+00$ & $9.491 \mathrm{E}-09$ & $2.973 E+05$ & $6.4985 \mathrm{E}+00$ & $8.647 \mathrm{E}-09$ & $2.708 \mathrm{E}+05$ \\
\hline $1 s 3 d^{3} D_{1}$ & $1 s 4 d{ }^{1} D_{2}$ & $6.5313 \mathrm{E}+00$ & $7.615 \mathrm{E}-08$ & $2.382 E+06$ & $6.5035 \mathrm{E}+00$ & $7.788 \mathrm{E}-08$ & $2.435 E+06$ \\
\hline $1 s 3 d^{3} D_{3}$ & $1 s 4 d^{1} D_{2}$ & $6.6532 \mathrm{E}+00$ & $2.818 \mathrm{E}-08$ & $8.493 \mathrm{E}+05$ & $6.6247 \mathrm{E}+00$ & $2.465 \mathrm{E}-08$ & $7.431 \mathrm{E}+05$ \\
\hline $1 s 3 d^{1} D_{2}$ & $1 s 4 d^{1} D_{2}$ & $6.6582 \mathrm{E}+00$ & $3.320 \mathrm{E}-07$ & $9.989 E+06$ & $6.6297 \mathrm{E}+00$ & $3.309 \mathrm{E}-07$ & $9.959 \mathrm{E}+06$ \\
\hline $1 s 4 s^{3} S_{1}$ & $1 s 4 d^{1} D_{2}$ & $1.6177 \mathrm{E}+02$ & $1.007 \mathrm{E}-10$ & $5.133 E+00$ & $1.6105 \mathrm{E}+02$ & $1.039 \mathrm{E}-10$ & $5.302 \mathrm{E}+00$ \\
\hline $1 s 4 d^{3} D_{2}$ & $1 s 4 d{ }^{1} D_{2}$ & $7.7722 \mathrm{E}+02$ & $1.036 \mathrm{E}-06$ & $2.289 \mathrm{E}+03$ & $7.7592 \mathrm{E}+02$ & $1.033 \mathrm{E}-06$ & $2.271 \mathrm{E}+03$ \\
\hline $1 s 4 d^{3} D_{1}$ & $1 s 4 d{ }^{1} D_{2}$ & $8.0779 \mathrm{E}+02$ & $8.860 \mathrm{E}-06$ & $1.811 \mathrm{E}+04$ & $8.0628 \mathrm{E}+02$ & $8.829 \mathrm{E}-06$ & $1.796 \mathrm{E}+04$ \\
\hline $1 s 4 d^{3} D_{3}$ & $1 s 4 d{ }^{1} D_{2}$ & $1.9845 \mathrm{E}+04$ & $2.845 \mathrm{E}-07$ & $9.638 \mathrm{E}-01$ & & & \\
\hline $1 s 4 f^{3} F_{3}^{o}$ & $1 s 4 f^{3} F_{4}^{o}$ & $1.6355 \mathrm{E}+03$ & $9.461 \mathrm{E}-06$ & $2.621 \mathrm{E}+03$ & $1.6325 E+03$ & $9.436 \mathrm{E}-06$ & $2.602 E+03$ \\
\hline $1 s 2 p^{3} P_{2}^{o}$ & $1 s 4 f^{1} F_{3}^{o}$ & $1.7025 \mathrm{E}+00$ & $1.542 \mathrm{E}-12$ & $5.069 \mathrm{E}+02$ & $1.6953 \mathrm{E}+00$ & $1.104 \mathrm{E}-13$ & $3.631 \mathrm{E}+01$ \\
\hline $1 s 3 p^{3} P_{2}^{o}$ & $1 s 4 f^{1} F_{3}^{o}$ & $6.4724 \mathrm{E}+00$ & $5.423 \mathrm{E}-13$ & $1.234 \mathrm{E}+01$ & $6.4457 \mathrm{E}+00$ & $5.663 \mathrm{E}-14$ & $1.287 \mathrm{E}+00$ \\
\hline $1 s 4 p^{3} P_{2}^{o}$ & $1 s 4 f^{1} F_{3}^{o}$ & $4.6135 \mathrm{E}+02$ & $3.233 \mathrm{E}-12$ & $1.447 \mathrm{E}-02$ & & & \\
\hline $1 s 4 f^{3} F_{3}^{o}$ & $1 s 4 f^{1} F_{3}^{o}$ & $1.5861 \mathrm{E}+03$ & $3.618 \mathrm{E}-07$ & $1.371 \mathrm{E}+02$ & $1.5830 \mathrm{E}+03$ & $3.609 \mathrm{E}-07$ & $1.360 \mathrm{E}+02$ \\
\hline $1 s 4 f^{3} F_{2}^{o}$ & $1 s 4 f^{1} F_{3}^{o}$ & $1.6535 \mathrm{E}+03$ & $6.987 \mathrm{E}-06$ & $2.435 \mathrm{E}+03$ & $1.6504 \mathrm{E}+03$ & $6.969 \mathrm{E}-06$ & $2.417 \mathrm{E}+03$ \\
\hline $1 s 4 f^{3} F_{4}^{o}$ & $1 s 4 f^{1} F_{3}^{o}$ & $5.2510 \mathrm{E}+04$ & $1.734 \mathrm{E}-07$ & $5.991 \mathrm{E}-02$ & & & \\
\hline $1 s^{2}{ }^{1} S_{0}$ & $1 s 5 s^{3} S_{1}^{3}$ & $3.2040 \mathrm{E}-01$ & $1.922 \mathrm{E}-06$ & $4.161 \mathrm{E}+10$ & $3.1907 \mathrm{E}-01$ & $1.905 \mathrm{E}-06$ & $4.127 \mathrm{E}+10$ \\
\hline
\end{tabular}


Table 5 (continued)

\begin{tabular}{|c|c|c|c|c|c|c|c|}
\hline \multirow[t]{2}{*}{ Lower } & \multirow[t]{2}{*}{ Upper } & \multicolumn{3}{|l|}{ GRASP2K } & \multicolumn{3}{|l|}{ FAC } \\
\hline & & $\lambda(\AA)$ & $g f$ & $A\left(\mathrm{~s}^{-1}\right)$ & $\lambda(\AA)$ & $g f$ & $A\left(\mathrm{~s}^{-1}\right)$ \\
\hline $1 s 2 s^{3} S_{1}$ & $1 s 5 s^{3} S_{1}$ & $1.4473 \mathrm{E}+00$ & $1.544 \mathrm{E}-07$ & $1.639 E+08$ & $1.4412 \mathrm{E}+00$ & $1.548 \mathrm{E}-07$ & $1.643 E+08$ \\
\hline $1 s 2 s{ }^{1} S_{0}$ & $1 s 5 s^{3} S_{1}$ & $1.4618 \mathrm{E}+00$ & $8.255 E-08$ & $8.589 E+07$ & $1.4559 \mathrm{E}+00$ & $7.804 \mathrm{E}-08$ & $8.117 \mathrm{E}+07$ \\
\hline $1 s 3 s^{3} s_{1}$ & $1 s 5 s^{3} s_{1}$ & $4.3197 \mathrm{E}+00$ & $3.183 E-08$ & $3.792 E+06$ & $4.3017 \mathrm{E}+00$ & $3.191 \mathrm{E}-08$ & $3.802 \mathrm{E}+06$ \\
\hline $1 s 3 s^{1} S_{0}$ & $1 s 5 s^{3} s_{1}$ & $4.3538 \mathrm{E}+00$ & $1.834 \mathrm{E}-08$ & $2.152 \mathrm{E}+06$ & $4.3360 \mathrm{E}+00$ & $1.605 \mathrm{E}-08$ & $1.882 \mathrm{E}+06$ \\
\hline $1 s 3 d^{3} D_{2}$ & $1 s 5 s^{3} s_{1}$ & $4.5495 \mathrm{E}+00$ & $1.900 \mathrm{E}-12$ & $2.041 \mathrm{E}+02$ & $4.5304 \mathrm{E}+00$ & $7.521 \mathrm{E}-12$ & $8.078 \mathrm{E}+02$ \\
\hline $1 s 3 d^{3} D_{1}$ & $1 s 5 s^{3} S_{1}$ & $4.5520 \mathrm{E}+00$ & $1.815 \mathrm{E}-11$ & $1.948 \mathrm{E}+03$ & & & \\
\hline $1 s 3 d^{1} D_{2}$ & $1 s 5 s^{3} s_{1}$ & $4.6133 \mathrm{E}+00$ & $2.661 \mathrm{E}-12$ & $2.780 \mathrm{E}+02$ & & & \\
\hline $1 s 4 s^{3} S_{1}$ & $1 s 5 s^{3} S_{1}$ & $1.3745 \mathrm{E}+01$ & $8.945 E-09$ & $1.053 \mathrm{E}+05$ & $1.3688 \mathrm{E}+01$ & $8.938 E-09$ & $1.051 \mathrm{E}+05$ \\
\hline $1 s 4 s{ }^{1} S_{0}$ & $1 s 5 s^{3} s_{1}$ & $1.3885 \mathrm{E}+01$ & $6.095 \mathrm{E}-09$ & $7.030 \mathrm{E}+04$ & $1.3828 \mathrm{E}+01$ & $4.509 \mathrm{E}-09$ & $5.198 \mathrm{E}+04$ \\
\hline $1 s 4 d^{3} D_{2}$ & $1 s 5 s^{3} s_{1}$ & $1.4736 \mathrm{E}+01$ & $2.859 \mathrm{E}-12$ & $2.927 \mathrm{E}+01$ & $1.4676 \mathrm{E}+01$ & $1.277 \mathrm{E}-11$ & $1.308 \mathrm{E}+02$ \\
\hline $1 s 4 d^{3} D_{1}$ & $1 s 5 s^{3} s_{1}$ & $1.4747 \mathrm{E}+01$ & $3.595 E-11$ & $3.675 \mathrm{E}+02$ & $1.4686 \mathrm{E}+01$ & $2.338 \mathrm{E}-12$ & $2.389 \mathrm{E}+01$ \\
\hline $1 s 4 d{ }^{1} D_{2}$ & $1 s 5 s^{3} S_{1}$ & $1.5021 \mathrm{E}+01$ & $4.998 \mathrm{E}-12$ & $4.925 \mathrm{E}+01$ & & & \\
\hline $1 s 2 p^{3} P_{1}^{o}$ & $1 s 5 p^{3} P_{1}^{o}$ & $1.4598 \mathrm{E}+00$ & $3.325 E-08$ & $3.469 \mathrm{E}+07$ & $1.4537 \mathrm{E}+00$ & $3.812 \mathrm{E}-08$ & $3.977 \mathrm{E}+07$ \\
\hline $1 s 2 p^{3} P_{0}^{o}$ & $1 s 5 p^{3} P_{1}^{o}$ & $1.4608 \mathrm{E}+00$ & $1.660 \mathrm{E}-08$ & $1.729 \mathrm{E}+07$ & $1.4547 \mathrm{E}+00$ & $2.159 \mathrm{E}-08$ & $2.249 \mathrm{E}+07$ \\
\hline $1 s 2 p^{3} P_{2}^{o}$ & $1 s 5 p^{3} P_{1}^{0}$ & $1.5297 \mathrm{E}+00$ & $7.583 \mathrm{E}-07$ & $7.205 E+08$ & $1.5233 \mathrm{E}+00$ & $7.714 \mathrm{E}-07$ & $7.329 \mathrm{E}+08$ \\
\hline $1 s 2 p^{1} P_{1}^{o}$ & $1 s 5 p^{3} P_{1}^{o}$ & $1.5364 \mathrm{E}+00$ & $1.790 \mathrm{E}-07$ & $1.686 \mathrm{E}+08$ & $1.5300 \mathrm{E}+00$ & $1.667 \mathrm{E}-07$ & $1.570 \mathrm{E}+08$ \\
\hline $1 s 3 p^{3} P_{1}^{o}$ & $1 s 5 p^{3} P_{1}^{o}$ & $4.3450 \mathrm{E}+00$ & $9.303 E-09$ & $1.096 \mathrm{E}+06$ & $4.3271 \mathrm{E}+00$ & $1.018 \mathrm{E}-08$ & $1.198 \mathrm{E}+06$ \\
\hline $1 s 3 p^{3} P_{0}^{o}$ & $1 s 5 p^{3} P_{1}^{o}$ & $4.3474 \mathrm{E}+00$ & $3.783 E-09$ & $4.450 \mathrm{E}+05$ & $4.3294 \mathrm{E}+00$ & $7.068 \mathrm{E}-09$ & $8.313 \mathrm{E}+05$ \\
\hline $1 s 3 p^{3} P_{2}^{o}$ & $1 s 5 p^{3} P_{1}^{o}$ & $4.5285 \mathrm{E}+00$ & $6.596 \mathrm{E}-07$ & $7.151 \mathrm{E}+07$ & $4.5097 \mathrm{E}+00$ & $6.741 \mathrm{E}-07$ & $7.307 \mathrm{E}+07$ \\
\hline $1 s 3 p^{1} P_{1}^{o}$ & $1 s 5 p^{3} P_{1}^{o}$ & $4.5449 \mathrm{E}+00$ & $1.499 \mathrm{E}-07$ & $1.613 \mathrm{E}+07$ & $4.5263 \mathrm{E}+00$ & $1.410 \mathrm{E}-07$ & $1.517 \mathrm{E}+07$ \\
\hline $1 s 4 p^{3} P_{1}^{o}$ & $1 s 5 p^{3} P_{1}^{0}$ & $1.3810 \mathrm{E}+01$ & $3.141 \mathrm{E}-09$ & $3.662 \mathrm{E}+04$ & $1.3752 \mathrm{E}+01$ & $3.286 \mathrm{E}-09$ & $3.831 \mathrm{E}+04$ \\
\hline $1 s 4 p^{3} P_{0}^{o}$ & $1 s 5 p^{3} P_{1}^{o}$ & $1.3820 \mathrm{E}+01$ & $8.034 \mathrm{E}-10$ & $9.353 E+03$ & $1.3761 \mathrm{E}+01$ & $3.266 \mathrm{E}-09$ & $3.803 \mathrm{E}+04$ \\
\hline $1 s 4 p^{3} P_{2}^{o}$ & $1 s 5 p^{3} P_{1}^{o}$ & $1.4601 \mathrm{E}+01$ & $8.508 \mathrm{E}-07$ & $8.874 \mathrm{E}+06$ & $1.4539 \mathrm{E}+01$ & $8.744 \mathrm{E}-07$ & $9.120 \mathrm{E}+06$ \\
\hline $1 s 4 p^{1} P_{1}^{o}$ & $1 s 5 p^{3} P_{1}^{o}$ & $1.4672 \mathrm{E}+01$ & $1.898 \mathrm{E}-07$ & $1.961 \mathrm{E}+06$ & $1.4610 \mathrm{E}+01$ & $1.785 \mathrm{E}-07$ & $1.843 \mathrm{E}+06$ \\
\hline $1 s 4 f^{3} F_{2}^{o}$ & $1 s 5 p^{3} P_{1}^{o}$ & $1.4942 \mathrm{E}+01$ & $2.377 \mathrm{E}-13$ & $2.367 \mathrm{E}+00$ & & & \\
\hline $1 s 2 s^{3} S_{1}$ & $1 s 5 s^{1} s_{0}$ & $1.4466 \mathrm{E}+00$ & $7.497 \mathrm{E}-08$ & $2.390 \mathrm{E}+08$ & $1.4404 \mathrm{E}+00$ & $7.736 \mathrm{E}-08$ & $2.466 \mathrm{E}+08$ \\
\hline $1 s 3 s^{3} S_{1}$ & $1 s 5 s^{1} s_{0}$ & $4.3129 \mathrm{E}+00$ & $1.408 \mathrm{E}-08$ & $5.050 \mathrm{E}+06$ & $4.2949 \mathrm{E}+00$ & $1.592 \mathrm{E}-08$ & $5.711 \mathrm{E}+06$ \\
\hline $1 s 3 d^{3} D_{1}$ & $1 s 5 s^{1} s_{0}$ & $4.5444 \mathrm{E}+00$ & $2.226 \mathrm{E}-12$ & $7.191 \mathrm{E}+02$ & $4.5252 \mathrm{E}+00$ & $2.275 \mathrm{E}-12$ & $7.347 \mathrm{E}+02$ \\
\hline $1 s 4 s^{3} S_{1}$ & $1 s 5 s^{1} s_{0}$ & $1.3676 \mathrm{E}+01$ & $3.196 \mathrm{E}-09$ & $1.140 \mathrm{E}+05$ & $1.3618 \mathrm{E}+01$ & $4.447 \mathrm{E}-09$ & $1.585 \mathrm{E}+05$ \\
\hline $1 s 4 d^{3} D_{1}$ & $1 s 5 s^{1} s_{0}$ & $1.4668 \mathrm{E}+01$ & $4.200 \mathrm{E}-12$ & $1.302 \mathrm{E}+02$ & $1.4607 \mathrm{E}+01$ & $4.661 \mathrm{E}-12$ & $1.445 \mathrm{E}+02$ \\
\hline $1 s 5 s^{3} S_{1}$ & $1 s 5 s^{1} S_{0}$ & $2.7268 \mathrm{E}+03$ & $1.197 \mathrm{E}-08$ & $1.074 \mathrm{E}+01$ & $2.6934 \mathrm{E}+03$ & $1.177 \mathrm{E}-08$ & $1.073 \mathrm{E}+01$ \\
\hline $1 s 2 p^{3} P_{1}^{o}$ & $1 s 5 p^{3} P_{0}^{o}$ & $1.4597 \mathrm{E}+00$ & $2.071 \mathrm{E}-08$ & $6.482 \mathrm{E}+07$ & $1.4537 \mathrm{E}+00$ & $1.212 \mathrm{E}-08$ & $3.793 \mathrm{E}+07$ \\
\hline $1 s 2 p^{1} P_{1}^{o}$ & $1 s 5 p^{3} P_{0}^{o}$ & $1.5363 \mathrm{E}+00$ & $3.127 \mathrm{E}-07$ & $8.836 \mathrm{E}+08$ & $1.5300 \mathrm{E}+00$ & $3.196 \mathrm{E}-07$ & $9.030 \mathrm{E}+08$ \\
\hline $1 s 3 p^{3} P_{1}^{o}$ & $1 s 5 p^{3} P_{0}^{0}$ & $4.3445 \mathrm{E}+00$ & $5.943 \mathrm{E}-09$ & $2.100 \mathrm{E}+06$ & $4.3267 \mathrm{E}+00$ & $2.377 \mathrm{E}-09$ & $8.397 \mathrm{E}+05$ \\
\hline $1 s 3 p^{1} P_{1}^{o}$ & $1 s 5 p^{3} P_{0}^{o}$ & $4.5444 \mathrm{E}+00$ & $2.684 \mathrm{E}-07$ & $8.670 \mathrm{E}+07$ & $4.5258 \mathrm{E}+00$ & $2.745 \mathrm{E}-07$ & $8.864 \mathrm{E}+07$ \\
\hline $1 s 4 p^{3} P_{1}^{o}$ & $1 s 5 p^{3} P_{0}^{0}$ & $1.3805 \mathrm{E}+01$ & $2.173 E-09$ & $7.604 \mathrm{E}+04$ & $1.3747 \mathrm{E}+01$ & $2.595 \mathrm{E}-10$ & $9.084 \mathrm{E}+03$ \\
\hline $1 s 4 p^{1} P_{1}^{0}$ & $1 s 5 p^{3} P_{0}^{o}$ & $1.4666 \mathrm{E}+01$ & $3.440 \mathrm{E}-07$ & $1.067 \mathrm{E}+07$ & $1.4605 \mathrm{E}+01$ & $3.519 \mathrm{E}-07$ & $1.091 \mathrm{E}+07$ \\
\hline $1 s 5 p^{3} P_{1}^{o}$ & $1 s 5 p^{3} P_{0}^{o}$ & $3.9500 \mathrm{E}+04$ & $1.224 \mathrm{E}-07$ & $5.231 \mathrm{E}-01$ & $4.0718 \mathrm{E}+04$ & $1.185 \mathrm{E}-07$ & $4.729 \mathrm{E}-01$ \\
\hline $1 s 2 p^{3} P_{1}^{o}$ & $1 s 5 p^{3} P_{2}^{o}$ & $1.4555 \mathrm{E}+00$ & $3.625 \mathrm{E}-07$ & $2.283 \mathrm{E}+08$ & $1.4495 \mathrm{E}+00$ & $3.760 \mathrm{E}-07$ & $2.367 \mathrm{E}+08$ \\
\hline $1 s 2 p^{3} P_{2}^{0}$ & $1 s 5 p^{3} P_{2}^{o}$ & $1.5251 \mathrm{E}+00$ & $5.039 \mathrm{E}-07$ & $2.890 \mathrm{E}+08$ & $1.5187 \mathrm{E}+00$ & $5.078 \mathrm{E}-07$ & $2.912 \mathrm{E}+08$ \\
\hline $1 s 2 p^{1} P_{1}^{o}$ & $1 s 5 p^{3} P_{2}^{o}$ & $1.5317 \mathrm{E}+00$ & $4.698 \mathrm{E}-08$ & $2.672 \mathrm{E}+07$ & $1.5253 \mathrm{E}+00$ & $4.591 \mathrm{E}-08$ & $2.610 \mathrm{E}+07$ \\
\hline $1 s 3 p^{3} P_{1}^{o}$ & $1 s 5 p^{3} P_{2}^{o}$ & $4.3076 \mathrm{E}+00$ & $4.374 \mathrm{E}-07$ & $3.145 \mathrm{E}+07$ & $4.2899 \mathrm{E}+00$ & $4.510 \mathrm{E}-07$ & $3.242 \mathrm{E}+07$ \\
\hline $1 s 3 p^{3} P_{2}^{o}$ & $1 s 5 p^{3} P_{2}^{o}$ & $4.4878 \mathrm{E}+00$ & $1.396 \mathrm{E}-07$ & $9.247 \mathrm{E}+06$ & $4.4692 \mathrm{E}+00$ & $1.403 \mathrm{E}-07$ & $9.293 \mathrm{E}+06$ \\
\hline $1 s 3 p^{1} P_{1}^{o}$ & $1 s 5 p^{3} P_{2}^{o}$ & $4.5039 \mathrm{E}+00$ & $9.365 \mathrm{E}-09$ & $6.158 \mathrm{E}+05$ & $4.4855 \mathrm{E}+00$ & $1.008 \mathrm{E}-08$ & $6.628 \mathrm{E}+05$ \\
\hline $1 s 4 p^{3} P_{1}^{o}$ & $1 s 5 p^{3} P_{2}^{o}$ & $1.3439 \mathrm{E}+01$ & $6.586 \mathrm{E}-07$ & $4.865 E+06$ & $1.3383 \mathrm{E}+01$ & $6.762 \mathrm{E}-07$ & $4.994 \mathrm{E}+06$ \\
\hline $1 s 4 p^{3} P_{2}^{o}$ & $1 s 5 p^{3} P_{2}^{o}$ & $1.4186 \mathrm{E}+01$ & $4.513 E-08$ & $2.991 \mathrm{E}+05$ & $1.4127 \mathrm{E}+01$ & $4.470 \mathrm{E}-08$ & $2.963 \mathrm{E}+05$ \\
\hline $1 s 4 p^{1} P_{1}^{o}$ & $1 s 5 p^{3} P_{2}^{o}$ & $1.4253 \mathrm{E}+01$ & $1.024 \mathrm{E}-09$ & $6.726 \mathrm{E}+03$ & $1.4194 \mathrm{E}+01$ & $1.700 \mathrm{E}-09$ & $1.116 \mathrm{E}+04$ \\
\hline $1 s 4 f^{3} F_{3}^{o}$ & $1 s 5 p^{3} P_{2}^{o}$ & $1.4503 \mathrm{E}+01$ & $1.154 \mathrm{E}-12$ & $7.318 \mathrm{E}+00$ & & & \\
\hline $1 s 4 f^{3} F_{2}^{o}$ & $1 s 5 p^{3} P_{2}^{o}$ & $1.4508 \mathrm{E}+01$ & $3.204 \mathrm{E}-13$ & $2.031 \mathrm{E}+00$ & & & \\
\hline $1 s 4 f^{1} F_{3}^{o}$ & $1 s 5 p^{3} P_{2}^{o}$ & $1.4636 \mathrm{E}+01$ & $4.259 \mathrm{E}-14$ & $2.652 \mathrm{E}-01$ & & & \\
\hline $1 s 5 p^{3} P_{1}^{o}$ & $1 s 5 p^{3} P_{2}^{o}$ & $4.9993 \mathrm{E}+02$ & $1.394 \mathrm{E}-05$ & $7.439 \mathrm{E}+04$ & $4.9817 \mathrm{E}+02$ & $1.393 \mathrm{E}-05$ & $7.425 \mathrm{E}+04$ \\
\hline $1 s 2 s^{3} S_{1}$ & $1 s 5 d^{3} D_{2}$ & $1.4421 \mathrm{E}+00$ & $1.207 \mathrm{E}-08$ & $7.745 \mathrm{E}+06$ & $1.4360 \mathrm{E}+00$ & $1.251 \mathrm{E}-08$ & $8.030 \mathrm{E}+06$ \\
\hline $1 s 3 s^{3} S_{1}$ & $1 s 5 d^{3} D_{2}$ & $4.2737 \mathrm{E}+00$ & $1.741 \mathrm{E}-09$ & $1.272 \mathrm{E}+05$ & $4.2558 \mathrm{E}+00$ & $1.922 \mathrm{E}-09$ & $1.403 E+05$ \\
\hline $1 s 3 d^{3} D_{2}$ & $1 s 5 d^{3} D_{2}$ & $4.4985 \mathrm{E}+00$ & $4.750 \mathrm{E}-08$ & $3.132 \mathrm{E}+06$ & $4.4794 \mathrm{E}+00$ & $4.750 \mathrm{E}-08$ & $3.131 \mathrm{E}+06$ \\
\hline $1 s 3 d^{3} D_{1}$ & $1 s 5 d^{3} D_{2}$ & $4.5010 \mathrm{E}+00$ & $4.030 \mathrm{E}-09$ & $2.654 \mathrm{E}+05$ & $4.4818 \mathrm{E}+00$ & $5.426 \mathrm{E}-09$ & $3.573 \mathrm{E}+05$ \\
\hline $1 s 3 d^{3} D_{3}$ & $1 s 5 d^{3} D_{2}$ & $4.5585 \mathrm{E}+00$ & $1.063 \mathrm{E}-07$ & $6.826 \mathrm{E}+06$ & $4.5391 \mathrm{E}+00$ & $1.063 \mathrm{E}-07$ & $6.830 \mathrm{E}+06$ \\
\hline $1 s 3 d^{1} D_{2}$ & $1 s 5 d^{3} D_{2}$ & $4.5609 \mathrm{E}+00$ & $8.379 E-09$ & $5.374 \mathrm{E}+05$ & $4.5414 \mathrm{E}+00$ & $7.951 \mathrm{E}-09$ & $5.099 \mathrm{E}+05$ \\
\hline $1 s 4 s^{3} S_{1}$ & $1 s 5 d^{3} D_{2}$ & $1.3290 \mathrm{E}+01$ & $1.420 \mathrm{E}-10$ & $1.073 \mathrm{E}+03$ & $1.3233 \mathrm{E}+01$ & $1.983 \mathrm{E}-10$ & $1.498 \mathrm{E}+03$ \\
\hline $1 s 4 d^{3} D_{2}$ & $1 s 5 d^{3} D_{2}$ & $1.4214 \mathrm{E}+01$ & $2.217 \mathrm{E}-08$ & $1.464 \mathrm{E}+05$ & $1.4154 \mathrm{E}+01$ & $2.214 \mathrm{E}-08$ & $1.462 \mathrm{E}+05$ \\
\hline $1 s 4 d^{3} D_{1}$ & $1 s 5 d^{3} D_{2}$ & $1.4224 \mathrm{E}+01$ & $1.417 \mathrm{E}-09$ & $9.343 E+03$ & $1.4164 \mathrm{E}+01$ & $2.610 \mathrm{E}-09$ & $1.720 \mathrm{E}+04$ \\
\hline $1 s 4 d^{3} D_{3}$ & $1 s 5 d^{3} D_{2}$ & $1.4468 \mathrm{E}+01$ & $1.506 \mathrm{E}-07$ & $9.600 \mathrm{E}+05$ & $1.4407 \mathrm{E}+01$ & $1.507 \mathrm{E}-07$ & $9.606 \mathrm{E}+05$ \\
\hline $1 s 4 d{ }^{1} D_{2}$ & $1 s 5 d^{3} D_{2}$ & $1.4479 \mathrm{E}+01$ & $1.171 \mathrm{E}-08$ & $7.449 \mathrm{E}+04$ & $1.4417 \mathrm{E}+01$ & $1.098 \mathrm{E}-08$ & $6.991 \mathrm{E}+04$ \\
\hline $1 s 5 s^{3} S_{1}$ & $1 s 5 d^{3} D_{2}$ & $4.0118 \mathrm{E}+02$ & $1.290 \mathrm{E}-10$ & $1.070 \mathrm{E}+00$ & $3.9828 \mathrm{E}+02$ & $1.291 \mathrm{E}-10$ & $1.076 \mathrm{E}+00$ \\
\hline $1 s 2 p^{3} P_{1}^{o}$ & $1 s 5 p^{1} P_{1}^{o}$ & $1.4551 \mathrm{E}+00$ & $6.636 \mathrm{E}-08$ & $6.969 \mathrm{E}+07$ & $1.4491 \mathrm{E}+00$ & $6.566 \mathrm{E}-08$ & $6.893 \mathrm{E}+07$ \\
\hline $1 s 2 p^{3} P_{0}^{o}$ & $1 s 5 p^{1} P_{1}^{o}$ & $1.4562 \mathrm{E}+00$ & $1.354 \mathrm{E}-07$ & $1.420 \mathrm{E}+08$ & $1.4501 \mathrm{E}+00$ & $1.420 \mathrm{E}-07$ & $1.489 \mathrm{E}+08$ \\
\hline $1 s 2 p^{3} P_{2}^{o}$ & $1 s 5 p^{1} P_{1}^{o}$ & $1.5247 \mathrm{E}+00$ & $8.556 \mathrm{E}-08$ & $8.183 E+07$ & $1.5183 \mathrm{E}+00$ & $7.279 \mathrm{E}-08$ & $6.962 \mathrm{E}+07$ \\
\hline $1 s 2 p^{1} P_{1}^{o}$ & 1s $5 p^{1} P_{1}^{o}$ & $1.5313 E+00$ & $2.795 \mathrm{E}-07$ & $2.651 \mathrm{E}+08$ & $1.5249 \mathrm{E}+00$ & $2.779 \mathrm{E}-07$ & $2.635 \mathrm{E}+08$ \\
\hline $1 s 3 p^{3} P_{1}^{o}$ & $1 s 5 p^{1} P_{1}^{o}$ & $4.3044 \mathrm{E}+00$ & $8.940 \mathrm{E}-08$ & $1.073 \mathrm{E}+07$ & $4.2867 \mathrm{E}+00$ & $8.521 \mathrm{E}-08$ & $1.022 \mathrm{E}+07$ \\
\hline $1 s 3 p^{3} P_{0}^{o}$ & $1 s 5 p^{1} P_{1}^{o}$ & $4.3068 \mathrm{E}+00$ & $1.695 \mathrm{E}-07$ & $2.032 \mathrm{E}+07$ & $4.2890 \mathrm{E}+00$ & $1.758 \mathrm{E}-07$ & $2.107 \mathrm{E}+07$ \\
\hline $1 s 3 p^{3} P_{2}^{o}$ & 1s $5 p^{1} P_{1}^{o}$ & $4.4844 \mathrm{E}+00$ & $2.833 \mathrm{E}-08$ & $3.132 \mathrm{E}+06$ & $4.4658 \mathrm{E}+00$ & $2.403 \mathrm{E}-08$ & $2.656 \mathrm{E}+06$ \\
\hline $1 s 3 p^{1} P_{1}^{o}$ & $1 s 5 p^{1} P_{1}^{o}$ & $4.5006 \mathrm{E}+00$ & $7.769 \mathrm{E}-08$ & $8.528 \mathrm{E}+06$ & $4.4821 \mathrm{E}+00$ & $7.651 \mathrm{E}-08$ & $8.396 \mathrm{E}+06$ \\
\hline
\end{tabular}


Table 5 (continued)

\begin{tabular}{|c|c|c|c|c|c|c|c|}
\hline \multirow[t]{2}{*}{ Lower } & \multirow[t]{2}{*}{ Upper } & \multicolumn{3}{|l|}{ GRASP2K } & \multicolumn{3}{|l|}{ FAC } \\
\hline & & $\lambda(\AA)$ & $g f$ & $A\left(\mathrm{~s}^{-1}\right)$ & $\lambda(\AA)$ & $g f$ & $A\left(\mathrm{~s}^{-1}\right)$ \\
\hline $1 s 4 p^{3} P_{1}^{o}$ & $1 s 5 p^{1} P_{1}^{o}$ & $1.3409 \mathrm{E}+01$ & $1.400 \mathrm{E}-07$ & $1.732 \mathrm{E}+06$ & $1.3352 \mathrm{E}+01$ & $1.323 \mathrm{E}-07$ & $1.636 \mathrm{E}+06$ \\
\hline $1 s 4 p^{3} P_{0}^{o}$ & $1 s 5 p^{1} P_{1}^{o}$ & $1.3418 \mathrm{E}+01$ & $2.593 \mathrm{E}-07$ & $3.202 E+06$ & $1.3361 \mathrm{E}+01$ & $2.678 \mathrm{E}-07$ & $3.307 \mathrm{E}+06$ \\
\hline $1 s 4 p^{3} P_{2}^{o}$ & $1 s 5 p^{1} P_{1}^{o}$ & $1.4153 \mathrm{E}+01$ & $1.282 \mathrm{E}-08$ & $1.423 \mathrm{E}+05$ & $1.4093 \mathrm{E}+01$ & $1.104 \mathrm{E}-08$ & $1.226 \mathrm{E}+05$ \\
\hline $1 s 4 p^{1} P_{1}^{o}$ & $1 s 5 p^{1} P_{1}^{o}$ & $1.4219 \mathrm{E}+01$ & $2.450 \mathrm{E}-08$ & $2.695 \mathrm{E}+05$ & $1.4160 \mathrm{E}+01$ & $2.407 \mathrm{E}-08$ & $2.647 \mathrm{E}+05$ \\
\hline $1 s 4 f^{3} F_{2}^{o}$ & $1 s 5 p^{1} P_{1}^{o}$ & $1.4473 \mathrm{E}+01$ & $1.066 \mathrm{E}-12$ & $1.131 \mathrm{E}+01$ & & & \\
\hline $1 s 5 p^{3} P_{1}^{0}$ & $1 s 5 p^{1} P_{1}^{o}$ & $4.6126 \mathrm{E}+02$ & $2.804 \mathrm{E}-06$ & $2.931 \mathrm{E}+04$ & $4.5925 \mathrm{E}+02$ & $2.803 \mathrm{E}-06$ & $2.930 \mathrm{E}+04$ \\
\hline $1 s 5 p^{3} P_{0}^{0}$ & $1 s 5 p^{1} P_{1}^{o}$ & $4.6671 \mathrm{E}+02$ & $5.192 \mathrm{E}-06$ & $5.300 \mathrm{E}+04$ & $4.6449 \mathrm{E}+02$ & $5.187 \mathrm{E}-06$ & $5.301 \mathrm{E}+04$ \\
\hline $1 s 5 p^{3} P_{2}^{o}$ & $1 s 5 p^{1} P_{1}^{o}$ & $5.9640 \mathrm{E}+03$ & $3.535 \mathrm{E}-07$ & $2.210 \mathrm{E}+01$ & $5.8780 \mathrm{E}+03$ & $3.589 \mathrm{E}-07$ & $2.290 \mathrm{E}+01$ \\
\hline $1 s^{2}{ }^{1} s_{0}$ & $1 s 5 d^{3} D_{1}$ & $3.2010 \mathrm{E}-01$ & $2.906 \mathrm{E}-08$ & $6.303 E+08$ & $3.1882 \mathrm{E}-01$ & $2.923 \mathrm{E}-08$ & $6.341 \mathrm{E}+08$ \\
\hline $1 s 2 s^{3} S_{1}$ & $1 s 5 d^{3} D_{1}$ & $1.4421 \mathrm{E}+00$ & $3.269 \mathrm{E}-09$ & $3.496 \mathrm{E}+06$ & $1.4359 \mathrm{E}+00$ & $2.440 \mathrm{E}-09$ & $2.609 E+06$ \\
\hline $1 s 2 s{ }^{1} S_{0}$ & $1 s 5 d^{3} D_{1}$ & $1.4565 \mathrm{E}+00$ & $4.982 \mathrm{E}-09$ & $5.221 \mathrm{E}+06$ & $1.4505 \mathrm{E}+00$ & $5.091 \mathrm{E}-09$ & $5.335 \mathrm{E}+06$ \\
\hline $1 s 3 s^{3} S_{1}$ & $1 s 5 d^{3} D_{1}$ & $4.2732 \mathrm{E}+00$ & $6.295 \mathrm{E}-10$ & $7.664 \mathrm{E}+04$ & $4.2553 \mathrm{E}+00$ & $3.832 \mathrm{E}-10$ & $4.666 \mathrm{E}+04$ \\
\hline $1 s 3 s^{1} S_{0}$ & $1 s 5 d^{3} D_{1}$ & $4.3066 \mathrm{E}+00$ & $7.848 \mathrm{E}-10$ & $9.408 \mathrm{E}+04$ & $4.2889 \mathrm{E}+00$ & $7.962 \mathrm{E}-10$ & $9.543 \mathrm{E}+04$ \\
\hline $1 s 3 d^{3} D_{2}$ & $1 s 5 d^{3} D_{1}$ & $4.4980 \mathrm{E}+00$ & $6.754 \mathrm{E}-09$ & $7.423 \mathrm{E}+05$ & $4.4789 \mathrm{E}+00$ & $5.140 \mathrm{E}-09$ & $5.648 \mathrm{E}+05$ \\
\hline $1 s 3 d^{3} D_{1}$ & $1 s 5 d^{3} D_{1}$ & $4.5005 \mathrm{E}+00$ & $2.642 \mathrm{E}-08$ & $2.900 \mathrm{E}+06$ & $4.4813 \mathrm{E}+00$ & $2.637 \mathrm{E}-08$ & $2.895 \mathrm{E}+06$ \\
\hline $1 s 3 d^{1} D_{2}$ & $1 s 5 d^{3} D_{1}$ & $4.5603 \mathrm{E}+00$ & $6.789 \mathrm{E}-08$ & $7.258 \mathrm{E}+06$ & $4.5409 \mathrm{E}+00$ & $6.881 \mathrm{E}-08$ & $7.357 \mathrm{E}+06$ \\
\hline $1 s 4 s^{3} S_{1}$ & $1 s 5 d^{3} D_{1}$ & $1.3285 \mathrm{E}+01$ & $1.345 \mathrm{E}-10$ & $1.694 \mathrm{E}+03$ & $1.3229 \mathrm{E}+01$ & $4.034 \mathrm{E}-11$ & $5.082 \mathrm{E}+02$ \\
\hline $1 s 4 s^{1} S_{0}$ & $1 s 5 d^{3} D_{1}$ & $1.3416 \mathrm{E}+01$ & $8.406 \mathrm{E}-11$ & $1.038 \mathrm{E}+03$ & $1.3360 \mathrm{E}+01$ & $8.608 \mathrm{E}-11$ & $1.063 \mathrm{E}+03$ \\
\hline $1 s 4 d^{3} D_{2}$ & $1 s 5 d^{3} D_{1}$ & $1.4209 \mathrm{E}+01$ & $3.779 \mathrm{E}-09$ & $4.162 \mathrm{E}+04$ & $1.4150 \mathrm{E}+01$ & $2.284 \mathrm{E}-09$ & $2.515 \mathrm{E}+04$ \\
\hline $1 s 4 d^{3} D_{1}$ & $1 s 5 d^{3} D_{1}$ & $1.4219 \mathrm{E}+01$ & $1.231 \mathrm{E}-08$ & $1.354 \mathrm{E}+05$ & $1.4159 \mathrm{E}+01$ & $1.228 \mathrm{E}-08$ & $1.350 \mathrm{E}+05$ \\
\hline $1 s 4 d{ }^{1} D_{2}$ & $1 s 5 d^{3} D_{1}$ & $1.4474 \mathrm{E}+01$ & $9.593 \mathrm{E}-08$ & $1.018 \mathrm{E}+06$ & $1.4412 \mathrm{E}+01$ & $9.728 \mathrm{E}-08$ & $1.032 \mathrm{E}+06$ \\
\hline $1 s 5 s^{3} S_{1}$ & $1 s 5 d^{3} D_{1}$ & $3.9728 \mathrm{E}+02$ & $2.647 \mathrm{E}-10$ & $3.728 \mathrm{E}+00$ & $3.9446 \mathrm{E}+02$ & $2.698 \mathrm{E}-10$ & $3.823 E+00$ \\
\hline $1 s 5 s{ }^{1} S_{0}$ & $1 s 5 d^{3} D_{1}$ & $4.6504 \mathrm{E}+02$ & $6.464 \mathrm{E}-12$ & $6.646 \mathrm{E}-02$ & $4.6214 \mathrm{E}+02$ & $6.512 \mathrm{E}-12$ & $6.722 \mathrm{E}-02$ \\
\hline $1 s 5 d^{3} D_{2}$ & $1 s 5 d^{3} D_{1}$ & $4.0886 \mathrm{E}+04$ & $2.249 \mathrm{E}-07$ & $2.991 \mathrm{E}-01$ & $4.1075 \mathrm{E}+04$ & $2.236 \mathrm{E}-07$ & $2.922 \mathrm{E}-01$ \\
\hline $1 s 3 d^{3} D_{2}$ & $1 s 5 d^{3} D_{3}$ & $4.4858 \mathrm{E}+00$ & $3.427 \mathrm{E}-08$ & $1.623 \mathrm{E}+06$ & $4.4668 \mathrm{E}+00$ & $3.435 \mathrm{E}-08$ & $1.627 \mathrm{E}+06$ \\
\hline $1 s 3 d^{3} D_{3}$ & $1 s 5 d^{3} D_{3}$ & $4.5454 \mathrm{E}+00$ & $2.279 \mathrm{E}-07$ & $1.051 \mathrm{E}+07$ & $4.5261 \mathrm{E}+00$ & $2.277 \mathrm{E}-07$ & $1.050 \mathrm{E}+07$ \\
\hline $1 s 3 d^{1} D_{2}$ & $1 s 5 d^{3} D_{3}$ & $4.5477 \mathrm{E}+00$ & $1.002 \mathrm{E}-08$ & $4.616 \mathrm{E}+05$ & $4.5284 \mathrm{E}+00$ & $1.125 \mathrm{E}-08$ & $5.183 E+05$ \\
\hline $1 s 4 d^{3} D_{2}$ & $1 s 5 d^{3} D_{3}$ & $1.4088 \mathrm{E}+01$ & $8.337 \mathrm{E}-08$ & $4.003 \mathrm{E}+05$ & $1.4029 \mathrm{E}+01$ & $8.359 E-08$ & $4.013 E+05$ \\
\hline $1 s 4 d^{3} D_{3}$ & $1 s 5 d^{3} D_{3}$ & $1.4337 \mathrm{E}+01$ & $1.068 \mathrm{E}-07$ & $4.952 \mathrm{E}+05$ & $1.4277 \mathrm{E}+01$ & $1.064 \mathrm{E}-07$ & $4.936 \mathrm{E}+05$ \\
\hline $1 s 4 d^{1} D_{2}$ & $1 s 5 d^{3} D_{3}$ & $1.4348 \mathrm{E}+01$ & $4.050 \mathrm{E}-09$ & $1.875 \mathrm{E}+04$ & $1.4287 \mathrm{E}+01$ & $5.092 \mathrm{E}-09$ & $2.357 \mathrm{E}+04$ \\
\hline $1 s 5 d^{3} D_{2}$ & $1 s 5 d^{3} D_{3}$ & $1.5807 \mathrm{E}+03$ & $7.199 \mathrm{E}-06$ & $2.745 E+03$ & $1.5799 \mathrm{E}+03$ & $7.177 \mathrm{E}-06$ & $2.716 \mathrm{E}+03$ \\
\hline $1 s 2 p^{3} P_{2}^{o}$ & $1 s 5 f^{3} F_{3}^{o}$ & $1.5232 \mathrm{E}+00$ & $1.777 \mathrm{E}-08$ & $7.298 E+06$ & $1.5168 \mathrm{E}+00$ & $1.651 \mathrm{E}-08$ & $6.783 E+06$ \\
\hline $1 s 3 p^{3} P_{2}^{o}$ & $1 s 5 f^{3} F_{3}^{o}$ & $4.4720 \mathrm{E}+00$ & $2.760 \mathrm{E}-09$ & $1.315 \mathrm{E}+05$ & $4.4534 \mathrm{E}+00$ & $2.752 \mathrm{E}-09$ & $1.311 \mathrm{E}+05$ \\
\hline $1 s 4 p^{3} P_{2}^{o}$ & $1 s 5 f^{3} F_{3}^{o}$ & $1.4030 \mathrm{E}+01$ & $1.450 \mathrm{E}-09$ & $7.019 E+03$ & $1.3970 \mathrm{E}+01$ & $1.413 \mathrm{E}-09$ & $6.842 \mathrm{E}+03$ \\
\hline $1 s 4 f^{3} F_{3}^{o}$ & 1s $5 f^{3} F_{3}^{o}$ & $1.4339 \mathrm{E}+01$ & $1.073 \mathrm{E}-08$ & $4.974 \mathrm{E}+04$ & $1.4278 \mathrm{E}+01$ & $4.705 \mathrm{E}-08$ & $2.180 \mathrm{E}+05$ \\
\hline $1 s 4 f^{3} F_{2}^{o}$ & $1 s 5 f^{3} F_{3}^{o}$ & $1.4344 \mathrm{E}+01$ & $2.972 \mathrm{E}-08$ & $1.376 \mathrm{E}+05$ & $1.4283 \mathrm{E}+01$ & $2.334 \mathrm{E}-09$ & $1.081 \mathrm{E}+04$ \\
\hline $1 s 4 f^{3} F_{4}^{o}$ & $1 s 5 f^{3} F_{3}^{0}$ & $1.4466 \mathrm{E}+01$ & $4.502 \mathrm{E}-08$ & $2.050 \mathrm{E}+05$ & $1.4404 \mathrm{E}+01$ & $4.477 \mathrm{E}-08$ & $2.039 \mathrm{E}+05$ \\
\hline $1 s 4 f^{1} F_{3}^{o}$ & $1 s 5 f^{3} F_{3}^{o}$ & $1.4470 \mathrm{E}+01$ & $1.697 \mathrm{E}-09$ & $7.722 \mathrm{E}+03$ & $1.4408 \mathrm{E}+01$ & $1.639 \mathrm{E}-09$ & $7.462 \mathrm{E}+03$ \\
\hline $1 s 5 p^{3} P_{2}^{o}$ & $1 s 5 f^{3} F_{3}^{o}$ & $1.2712 \mathrm{E}+03$ & $9.159 \mathrm{E}-12$ & $5.401 \mathrm{E}-03$ & $1.2579 \mathrm{E}+03$ & $8.880 \mathrm{E}-12$ & $5.302 \mathrm{E}-03$ \\
\hline $1 s 2 p^{3} P_{1}^{o}$ & $1 s 5 f^{3} F_{2}^{o}$ & $1.4538 \mathrm{E}+00$ & $2.242 \mathrm{E}-11$ & $1.415 \mathrm{E}+04$ & $1.4478 \mathrm{E}+00$ & $1.093 \mathrm{E}-11$ & $6.903 E+03$ \\
\hline $1 s 2 p^{3} P_{2}^{o}$ & $1 s 5 f^{3} F_{2}^{o}$ & $1.5232 \mathrm{E}+00$ & $1.262 \mathrm{E}-09$ & $7.255 \mathrm{E}+05$ & $1.5168 \mathrm{E}+00$ & $1.202 \mathrm{E}-09$ & $6.912 \mathrm{E}+05$ \\
\hline $1 s 2 p{ }^{1} P_{1}^{o}$ & $1 s 5 f^{3} F_{2}^{o}$ & $1.5298 \mathrm{E}+00$ & $1.114 \mathrm{E}-08$ & $6.351 \mathrm{E}+06$ & $1.5234 \mathrm{E}+00$ & $1.084 \mathrm{E}-08$ & $6.179 \mathrm{E}+06$ \\
\hline $1 s 3 p^{3} P_{1}^{o}$ & $1 s 5 f^{3} F_{2}^{o}$ & $4.2928 \mathrm{E}+00$ & $5.686 \mathrm{E}-12$ & $4.116 \mathrm{E}+02$ & $4.2750 \mathrm{E}+00$ & $3.642 \mathrm{E}-12$ & $2.636 \mathrm{E}+02$ \\
\hline $1 s 3 p^{3} P_{2}^{o}$ & $1 s 5 f^{3} F_{2}^{o}$ & $4.4718 \mathrm{E}+00$ & $2.443 \mathrm{E}-10$ & $1.630 \mathrm{E}+04$ & $4.4531 \mathrm{E}+00$ & $1.918 \mathrm{E}-10$ & $1.279 \mathrm{E}+04$ \\
\hline $1 s 3 p^{1} P_{1}^{o}$ & $1 s 5 f^{3} F_{2}^{o}$ & $4.4878 \mathrm{E}+00$ & $1.807 \mathrm{E}-09$ & $1.197 \mathrm{E}+05$ & $4.4693 \mathrm{E}+00$ & $1.767 \mathrm{E}-09$ & $1.170 \mathrm{E}+05$ \\
\hline $1 s 4 p^{3} P_{1}^{o}$ & $1 s 5 f^{3} F_{2}^{o}$ & $1.3296 \mathrm{E}+01$ & $3.412 \mathrm{E}-12$ & $2.575 \mathrm{E}+01$ & & & \\
\hline $1 s 4 p^{3} P_{2}^{o}$ & $1 s 5 f^{3} F_{2}^{o}$ & $1.4027 \mathrm{E}+01$ & $1.361 \mathrm{E}-10$ & $9.228 \mathrm{E}+02$ & $1.3967 \mathrm{E}+01$ & $1.003 \mathrm{E}-10$ & $6.806 \mathrm{E}+02$ \\
\hline $1 s 4 p{ }^{1} P_{1}^{o}$ & $1 s 5 f^{3} F_{2}^{o}$ & $1.4093 \mathrm{E}+01$ & $9.657 \mathrm{E}-10$ & $6.487 \mathrm{E}+03$ & $1.4033 \mathrm{E}+01$ & $9.194 \mathrm{E}-10$ & $6.175 E+03$ \\
\hline $1 s 4 f^{3} F_{3}^{o}$ & $1 s 5 f^{3} F_{2}^{o}$ & $1.4336 \mathrm{E}+01$ & $3.412 \mathrm{E}-08$ & $2.215 \mathrm{E}+05$ & $1.4275 \mathrm{E}+01$ & $2.392 \mathrm{E}-09$ & $1.552 \mathrm{E}+04$ \\
\hline $1 s 4 f^{3} F_{2}^{o}$ & $1 s 5 f^{3} F_{2}^{o}$ & $1.4342 \mathrm{E}+01$ & $6.360 \mathrm{E}-08$ & $4.125 E+05$ & $1.4281 \mathrm{E}+01$ & $3.295 E-08$ & $2.137 \mathrm{E}+05$ \\
\hline $1 s 4 f^{1} F_{3}^{o}$ & $1 s 5 f^{3} F_{2}^{o}$ & $1.4467 \mathrm{E}+01$ & $3.281 \mathrm{E}-08$ & $2.091 \mathrm{E}+05$ & $1.4405 \mathrm{E}+01$ & $3.308 \mathrm{E}-08$ & $2.109 \mathrm{E}+05$ \\
\hline $1 s 5 p^{3} P_{1}^{0}$ & $1 s 5 f^{3} F_{2}^{o}$ & $3.5713 \mathrm{E}+02$ & $3.711 \mathrm{E}-12$ & $3.882 \mathrm{E}-02$ & $3.5518 \mathrm{E}+02$ & $4.170 \mathrm{E}-12$ & $4.372 \mathrm{E}-02$ \\
\hline $1 s 5 p^{3} P_{2}^{o}$ & $1 s 5 f^{3} F_{2}^{o}$ & $1.2503 \mathrm{E}+03$ & $9.975 \mathrm{E}-12$ & $8.513 E-03$ & $1.2374 \mathrm{E}+03$ & $9.996 \mathrm{E}-12$ & $8.635 \mathrm{E}-03$ \\
\hline $1 s 5 p^{1} P_{1}^{o}$ & $1 s 5 f^{3} F_{2}^{o}$ & $1.5820 \mathrm{E}+03$ & $3.550 \mathrm{E}-13$ & $1.892 \mathrm{E}-04$ & $1.5673 \mathrm{E}+03$ & $3.191 \mathrm{E}-13$ & $1.718 \mathrm{E}-04$ \\
\hline $1 s 5 f^{3} F_{3}^{0}$ & $1 s 5 f^{3} F_{2}^{o}$ & $7.6009 \mathrm{E}+04$ & $1.688 \mathrm{E}-07$ & $3.897 \mathrm{E}-02$ & $7.5649 \mathrm{E}+04$ & $1.670 \mathrm{E}-07$ & $3.861 \mathrm{E}-02$ \\
\hline $1 s 2 s^{3} s_{1}^{3}$ & $1 s 5 d^{1} D_{2}$ & $1.4408 \mathrm{E}+00$ & $1.086 \mathrm{E}-11$ & $6.980 \mathrm{E}+03$ & $1.4346 \mathrm{E}+00$ & $5.345 \mathrm{E}-13$ & $3.435 \mathrm{E}+02$ \\
\hline $1 s 3 s^{3} s_{1}$ & $1 s 5 d^{1} D_{2}$ & $4.2617 \mathrm{E}+00$ & $6.144 \mathrm{E}-12$ & $4.513 \mathrm{E}+02$ & & & \\
\hline $1 s 3 d^{3} D_{2}$ & $1 s 5 d^{1} D_{2}$ & $4.4852 \mathrm{E}+00$ & $2.704 \mathrm{E}-09$ & $1.793 \mathrm{E}+05$ & $4.4662 \mathrm{E}+00$ & $2.441 \mathrm{E}-09$ & $1.619 \mathrm{E}+05$ \\
\hline $1 s 3 d^{3} D_{1}$ & $1 s 5 d^{1} D_{2}$ & $4.4876 \mathrm{E}+00$ & $2.134 \mathrm{E}-08$ & $1.413 \mathrm{E}+06$ & $4.4686 \mathrm{E}+00$ & $2.183 \mathrm{E}-08$ & $1.446 \mathrm{E}+06$ \\
\hline $1 s 3 d^{3} D_{3}$ & $1 s 5 d{ }^{1} D_{2}$ & $4.5449 \mathrm{E}+00$ & $1.323 \mathrm{E}-08$ & $8.542 \mathrm{E}+05$ & $4.5255 \mathrm{E}+00$ & $1.184 \mathrm{E}-08$ & $7.652 \mathrm{E}+05$ \\
\hline $1 s 3 d^{1} D_{2}$ & $1 s 5 d{ }^{1} D_{2}$ & $4.5472 \mathrm{E}+00$ & $1.594 \mathrm{E}-07$ & $1.028 \mathrm{E}+07$ & $4.5278 \mathrm{E}+00$ & $1.591 \mathrm{E}-07$ & $1.026 \mathrm{E}+07$ \\
\hline $1 s 4 s^{3} S_{1}$ & $1 s 5 d{ }^{1} D_{2}$ & $1.3174 \mathrm{E}+01$ & $5.744 \mathrm{E}-12$ & $4.415 \mathrm{E}+01$ & & & \\
\hline $1 s 4 d^{3} D_{2}$ & $1 s 5 d^{1} D_{2}$ & $1.4082 \mathrm{E}+01$ & $6.400 \mathrm{E}-09$ & $4.306 \mathrm{E}+04$ & $1.4023 \mathrm{E}+01$ & $5.870 \mathrm{E}-09$ & $3.948 \mathrm{E}+04$ \\
\hline $1 s 4 d^{3} D_{1}$ & $1 s 5 d{ }^{1} D_{2}$ & $1.4092 \mathrm{E}+01$ & $5.236 \mathrm{E}-08$ & $3.517 \mathrm{E}+05$ & $1.4033 \mathrm{E}+01$ & $5.341 \mathrm{E}-08$ & $3.587 \mathrm{E}+05$ \\
\hline $1 s 4 d^{3} D_{3}$ & $1 s 5 d{ }^{1} D_{2}$ & $1.4332 \mathrm{E}+01$ & $6.968 \mathrm{E}-09$ & $4.525 \mathrm{E}+04$ & $1.4271 \mathrm{E}+01$ & $5.701 \mathrm{E}-09$ & $3.703 E+04$ \\
\hline $1 s 4 d{ }^{1} D_{2}$ & $1 s 5 d{ }^{1} D_{2}$ & $1.4342 \mathrm{E}+01$ & $7.473 \mathrm{E}-08$ & $4.847 \mathrm{E}+05$ & $1.4282 \mathrm{E}+01$ & $7.441 \mathrm{E}-08$ & $4.825 E+05$ \\
\hline $1 s 5 s^{3} S_{1}$ & $1 s 5 d{ }^{1} D_{2}$ & $3.1728 \mathrm{E}+02$ & $5.803 \mathrm{E}-11$ & $7.691 \mathrm{E}-01$ & $3.1538 \mathrm{E}+02$ & $5.834 \mathrm{E}-11$ & $7.758 \mathrm{E}-01$ \\
\hline $1 s 5 d^{3} D_{2}$ & $1 s 5 d^{1} D_{2}$ & $1.5170 \mathrm{E}+03$ & $5.320 \mathrm{E}-07$ & $3.084 \mathrm{E}+02$ & $1.5150 \mathrm{E}+03$ & $5.304 \mathrm{E}-07$ & $3.056 \mathrm{E}+02$ \\
\hline $1 s 5 d^{3} D_{1}$ & $1 s 5 d^{1} D_{2}$ & $1.5755 \mathrm{E}+03$ & $4.542 \mathrm{E}-06$ & $2.441 \mathrm{E}+03$ & $1.5730 \mathrm{E}+03$ & $4.524 \mathrm{E}-06$ & $2.418 \mathrm{E}+03$ \\
\hline $1 s 5 d^{3} D_{3}$ & $1 s 5 d^{1} D_{2}$ & $3.7649 \mathrm{E}+04$ & $1.489 \mathrm{E}-07$ & $1.401 \mathrm{E}-01$ & & & \\
\hline $1 s 4 f^{3} F_{3}^{o}$ & $1 s 5 f^{3} F_{4}^{o}$ & $1.4275 \mathrm{E}+01$ & $1.498 \mathrm{E}-08$ & $5.450 \mathrm{E}+04$ & $1.4214 \mathrm{E}+01$ & $1.496 \mathrm{E}-08$ & $5.443 E+04$ \\
\hline
\end{tabular}


Table 5 (continued)

\begin{tabular}{|c|c|c|c|c|c|c|c|}
\hline \multirow[t]{2}{*}{ Lower } & \multirow[t]{2}{*}{ Upper } & \multicolumn{3}{|l|}{ GRASP2K } & \multicolumn{3}{|l|}{ FAC } \\
\hline & & $\lambda(\AA)$ & $g f$ & $A\left(\mathrm{~s}^{-1}\right)$ & $\lambda(\AA)$ & $g f$ & $A\left(\mathrm{~s}^{-1}\right)$ \\
\hline $1 s 4 f^{3} F_{4}^{o}$ & $1 s 5 f^{3} F_{4}^{o}$ & $1.4401 \mathrm{E}+01$ & $1.395 \mathrm{E}-07$ & $4.986 \mathrm{E}+05$ & $1.4339 \mathrm{E}+01$ & $1.440 \mathrm{E}-07$ & $5.146 \mathrm{E}+05$ \\
\hline $1 s 4 f^{1} F_{3}^{0}$ & $1 s 5 f^{3} F_{4}^{o}$ & $1.4404 \mathrm{E}+01$ & $4.779 \mathrm{E}-09$ & $1.707 \mathrm{E}+04$ & $1.4343 \mathrm{E}+01$ & $4.148 \mathrm{E}-09$ & $1.481 \mathrm{E}+04$ \\
\hline $1 s 5 f^{3} F_{3}^{o}$ & $1 s 5 f^{3} F_{4}^{o}$ & $3.1889 \mathrm{E}+03$ & $4.861 \mathrm{E}-06$ & $3.543 \mathrm{E}+02$ & $3.1838 \mathrm{E}+03$ & $4.848 \mathrm{E}-06$ & $3.514 \mathrm{E}+02$ \\
\hline $1 s 3 d^{3} D_{3}$ & $1 s 5 g^{3} G_{4}$ & $4.5387 \mathrm{E}+00$ & $6.705 \mathrm{E}-09$ & $2.412 \mathrm{E}+05$ & $4.5195 \mathrm{E}+00$ & $6.765 \mathrm{E}-09$ & $2.433 \mathrm{E}+05$ \\
\hline $1 s 4 d^{3} D_{3}$ & $1 s 5 g^{3} G_{4}$ & $1.4271 \mathrm{E}+01$ & $2.645 \mathrm{E}-09$ & $9.626 \mathrm{E}+03$ & $1.4212 \mathrm{E}+01$ & $2.645 \mathrm{E}-09$ & $9.624 \mathrm{E}+03$ \\
\hline $1 s 5 d^{3} D_{3}$ & $1 s 5 g^{3} G_{4}$ & $3.0779 \mathrm{E}+03$ & $3.885 \mathrm{E}-13$ & $3.040 \mathrm{E}-05$ & $3.1417 \mathrm{E}+03$ & $4.330 \mathrm{E}-13$ & $3.223 \mathrm{E}-05$ \\
\hline $1 s 3 d^{3} D_{2}$ & $1 s 5 g^{3} G_{3}$ & $4.4791 \mathrm{E}+00$ & $4.386 \mathrm{E}-13$ & $2.083 \mathrm{E}+01$ & & & \\
\hline $1 s 3 d^{3} D_{3}$ & $1 s 5 g^{3} G_{3}$ & $4.5386 \mathrm{E}+00$ & $2.962 \mathrm{E}-10$ & $1.370 \mathrm{E}+04$ & $4.5194 \mathrm{E}+00$ & $2.508 \mathrm{E}-10$ & $1.160 \mathrm{E}+04$ \\
\hline $1 s 3 d^{1} D_{2}$ & $1 s 5 g^{3} G_{3}$ & $4.5409 \mathrm{E}+00$ & $5.076 \mathrm{E}-09$ & $2.346 \mathrm{E}+05$ & $4.5217 \mathrm{E}+00$ & $5.023 \mathrm{E}-09$ & $2.321 \mathrm{E}+05$ \\
\hline $1 s 4 d^{3} D_{2}$ & $1 s 5 g^{3} G_{3}$ & $1.4022 \mathrm{E}+01$ & $1.928 \mathrm{E}-13$ & $9.345 \mathrm{E}-01$ & & & \\
\hline $1 s 4 d^{3} D_{3}$ & $1 s 5 g^{3} G_{3}$ & $1.4269 \mathrm{E}+01$ & $1.108 \mathrm{E}-10$ & $5.186 \mathrm{E}+02$ & $1.4210 \mathrm{E}+01$ & $9.782 \mathrm{E}-11$ & $4.576 \mathrm{E}+02$ \\
\hline $1 s 4 d{ }^{1} D_{2}$ & $1 s 5 g^{3} G_{3}$ & $1.4280 \mathrm{E}+01$ & $1.987 \mathrm{E}-09$ & $9.285 \mathrm{E}+03$ & $1.4221 \mathrm{E}+01$ & $1.961 \mathrm{E}-09$ & $9.165 E+03$ \\
\hline $1 s 5 d^{3} D_{2}$ & $1 s 5 g^{3} G_{3}$ & $1.0362 \mathrm{E}+03$ & $4.213 \mathrm{E}-15$ & $3.739 \mathrm{E}-06$ & $1.0430 \mathrm{E}+03$ & $4.189 \mathrm{E}-15$ & $3.638 \mathrm{E}-06$ \\
\hline $1 s 5 d^{3} D_{3}$ & $1 s 5 g^{3} G_{3}$ & $3.0082 \mathrm{E}+03$ & $1.694 \mathrm{E}-16$ & $1.784 \mathrm{E}-08$ & & & \\
\hline $1 s 5 d{ }^{1} D_{2}$ & $1 s 5 g^{3} G_{3}$ & $3.2694 \mathrm{E}+03$ & $2.036 \mathrm{E}-13$ & $1.815 \mathrm{E}-05$ & $3.3474 \mathrm{E}+03$ & $1.574 \mathrm{E}-13$ & $1.327 \mathrm{E}-05$ \\
\hline $1 s 5 g^{3} G_{4}$ & $1 s 5 g^{3} G_{3}$ & $1.3281 \mathrm{E}+05$ & $1.209 \mathrm{E}-07$ & $6.534 \mathrm{E}-03$ & $1.3215 \mathrm{E}+05$ & $1.213 \mathrm{E}-07$ & $6.564 \mathrm{E}-03$ \\
\hline $1 s 2 p^{3} P_{2}^{o}$ & $1 s 5 f^{1} F_{3}^{o}$ & $1.5225 \mathrm{E}+00$ & $1.031 \mathrm{E}-12$ & $4.240 \mathrm{E}+02$ & $1.5161 \mathrm{E}+00$ & $6.722 \mathrm{E}-14$ & $2.763 \mathrm{E}+01$ \\
\hline $1 s 3 p^{3} P_{2}^{o}$ & $1 s 5 f^{1} F_{3}^{o}$ & $4.4656 \mathrm{E}+00$ & $1.185 \mathrm{E}-13$ & $5.664 \mathrm{E}+00$ & $4.4470 \mathrm{E}+00$ & $1.124 \mathrm{E}-14$ & $5.370 \mathrm{E}-01$ \\
\hline $1 s 4 p^{3} P_{2}^{o}$ & $1 s 5 f^{1} F_{3}^{o}$ & $1.3966 \mathrm{E}+01$ & $2.140 \mathrm{E}-13$ & $1.045 \mathrm{E}+00$ & & & \\
\hline $1 s 4 f^{3} F_{3}^{o}$ & $1 s 5 f^{1} F_{3}^{o}$ & $1.4273 \mathrm{E}+01$ & $6.420 \mathrm{E}-10$ & $3.003 E+03$ & $1.4212 \mathrm{E}+01$ & $5.692 \mathrm{E}-10$ & $2.662 \mathrm{E}+03$ \\
\hline $1 s 4 f^{3} F_{2}^{o}$ & $1 s 5 f^{1} F_{3}^{o}$ & $1.4278 \mathrm{E}+01$ & $1.100 \mathrm{E}-08$ & $5.141 \mathrm{E}+04$ & $1.4218 \mathrm{E}+01$ & $1.112 \mathrm{E}-08$ & $5.201 \mathrm{E}+04$ \\
\hline $1 s 4 f^{3} F_{4}^{o}$ & $1 s 5 f^{1} F_{3}^{o}$ & $1.4398 \mathrm{E}+01$ & $5.942 \mathrm{E}-09$ & $2.731 \mathrm{E}+04$ & $1.4337 \mathrm{E}+01$ & $4.059 \mathrm{E}-09$ & $1.866 \mathrm{E}+04$ \\
\hline $1 s 4 f^{1} F_{3}^{o}$ & $1 s 5 f^{1} F_{3}^{o}$ & $1.4402 \mathrm{E}+01$ & $1.154 \mathrm{E}-07$ & $5.300 \mathrm{E}+05$ & $1.4341 \mathrm{E}+01$ & $1.111 \mathrm{E}-07$ & $5.104 \mathrm{E}+05$ \\
\hline $1 s 5 p^{3} P_{2}^{o}$ & $1 s 5 f^{1} F_{3}^{o}$ & $9.0084 \mathrm{E}+02$ & $2.726 \mathrm{E}-12$ & $3.201 \mathrm{E}-03$ & & & \\
\hline $1 s 5 f^{3} F_{3}^{o}$ & $1 s 5 f^{1} F_{3}^{o}$ & $3.0918 \mathrm{E}+03$ & $1.860 \mathrm{E}-07$ & $1.854 \mathrm{E}+01$ & $3.0864 \mathrm{E}+03$ & $1.854 \mathrm{E}-07$ & $1.839 \mathrm{E}+01$ \\
\hline 1s $5 f^{3} F_{2}^{0}$ & $1 s 5 f^{1} F_{3}^{o}$ & $3.2229 \mathrm{E}+03$ & $3.589 \mathrm{E}-06$ & $3.293 E+02$ & $3.2177 \mathrm{E}+03$ & $3.579 \mathrm{E}-06$ & $3.266 \mathrm{E}+02$ \\
\hline $1 s 5 f^{3} F_{4}^{o}$ & $1 s 5 f^{1} F_{3}^{o}$ & $1.0160 \mathrm{E}+05$ & $8.920 \mathrm{E}-08$ & $8.235 \mathrm{E}-03$ & & & \\
\hline $1 s 5 g{ }^{3} G_{4}$ & $1 s 5 g^{3} G_{5}$ & $5.3554 \mathrm{E}+03$ & $3.669 \mathrm{E}-06$ & $7.758 \mathrm{E}+01$ & $5.3442 E+03$ & $3.661 \mathrm{E}-06$ & $7.707 \mathrm{E}+01$ \\
\hline $1 s 3 d^{3} D_{3}$ & $1 s 5 g^{1} G_{4}$ & $4.5348 \mathrm{E}+00$ & $1.337 \mathrm{E}-13$ & $4.817 \mathrm{E}+00$ & $4.5156 \mathrm{E}+00$ & $2.425 \mathrm{E}-14$ & $8.741 \mathrm{E}-01$ \\
\hline $1 s 4 d^{3} D_{3}$ & $1 s 5 g^{1} G_{4}$ & $1.4232 \mathrm{E}+01$ & $1.748 \mathrm{E}-14$ & $6.398 \mathrm{E}-02$ & $1.4173 \mathrm{E}+01$ & $9.478 \mathrm{E}-15$ & $3.467 \mathrm{E}-02$ \\
\hline $1 s 5 d^{3} D_{3}$ & $1 s 5 g^{1} G_{4}$ & $1.9321 \mathrm{E}+03$ & $3.849 \mathrm{E}-15$ & $7.643 \mathrm{E}-07$ & & & \\
\hline $1 s 5 g{ }^{3} G_{4}$ & $1 s 5 g^{1} G_{4}$ & $5.1900 \mathrm{E}+03$ & $8.607 \mathrm{E}-08$ & $2.368 \mathrm{E}+00$ & $5.1787 \mathrm{E}+03$ & $8.589 \mathrm{E}-08$ & $2.353 \mathrm{E}+00$ \\
\hline $1 s 5 g^{3} G_{3}$ & $1 s 5 g^{1} G_{4}$ & $5.4011 \mathrm{E}+03$ & $2.914 \mathrm{E}-06$ & $7.403 \mathrm{E}+01$ & $5.3899 \mathrm{E}+03$ & $2.907 \mathrm{E}-06$ & $7.354 \mathrm{E}+01$ \\
\hline $1 s 5 g{ }^{3} G_{5}$ & $1 s 5 g{ }^{1} G_{4}$ & $1.6804 \mathrm{E}+05$ & $7.357 \mathrm{E}-08$ & $1.931 \mathrm{E}-03$ & & & \\
\hline $1 s^{21} S_{0}$ & $1 s 6 s^{3} S_{1}$ & $3.1640 \mathrm{E}-01$ & $1.107 \mathrm{E}-06$ & $2.457 \mathrm{E}+10$ & $3.1511 \mathrm{E}-01$ & $1.098 \mathrm{E}-06$ & $2.438 \mathrm{E}+10$ \\
\hline $1 s 2 s^{3} S_{1}$ & $1 s 6 s^{3} S_{1}$ & $1.3696 \mathrm{E}+00$ & $9.015 \mathrm{E}-08$ & $1.069 \mathrm{E}+08$ & $1.3638 \mathrm{E}+00$ & $9.066 \mathrm{E}-08$ & $1.074 \mathrm{E}+08$ \\
\hline $1 s 2 s{ }^{1} S_{0}$ & $1 s 6 s^{3} S_{1}$ & $1.3825 \mathrm{E}+00$ & $4.807 \mathrm{E}-08$ & $5.592 \mathrm{E}+07$ & $1.3769 \mathrm{E}+00$ & $4.572 \mathrm{E}-08$ & $5.317 \mathrm{E}+07$ \\
\hline $1 s 3 s^{3} S_{1}$ & $1 s 6 s^{3} S_{1}$ & $3.6936 \mathrm{E}+00$ & $1.931 \mathrm{E}-08$ & $3.147 \mathrm{E}+06$ & $3.6786 \mathrm{E}+00$ & $1.938 \mathrm{E}-08$ & $3.157 \mathrm{E}+06$ \\
\hline $1 s 3 s^{1} S_{0}$ & $1 s 6 s^{3} s_{1}$ & $3.7185 \mathrm{E}+00$ & $1.086 \mathrm{E}-08$ & $1.747 \mathrm{E}+06$ & $3.7036 \mathrm{E}+00$ & $9.751 \mathrm{E}-09$ & $1.567 \mathrm{E}+06$ \\
\hline $1 s 3 d^{3} D_{2}$ & $1 s 6 s^{3} s_{1}$ & $3.8604 \mathrm{E}+00$ & $5.613 \mathrm{E}-13$ & $8.374 \mathrm{E}+01$ & & & \\
\hline $1 s 3 d^{3} D_{1}$ & $1 s 6 s^{3} S_{1}$ & $3.8622 \mathrm{E}+00$ & $8.232 \mathrm{E}-12$ & $1.227 \mathrm{E}+03$ & & & \\
\hline $1 s 3 d{ }^{1} D_{2}$ & $1 s 6 s^{3} s_{1}$ & $3.9062 \mathrm{E}+00$ & $1.282 \mathrm{E}-12$ & $1.868 \mathrm{E}+02$ & & & \\
\hline $1 s 4 s^{3} S_{1}$ & $1 s 6 s^{3} s_{1}$ & $8.9291 \mathrm{E}+00$ & $6.023 \mathrm{E}-09$ & $1.680 \mathrm{E}+05$ & $8.8936 \mathrm{E}+00$ & $6.051 \mathrm{E}-09$ & $1.686 \mathrm{E}+05$ \\
\hline $1 s 4 s^{1} S_{0}$ & $1 s 6 s^{3} s_{1}$ & $8.9879 \mathrm{E}+00$ & $3.778 \mathrm{E}-09$ & $1.040 \mathrm{E}+05$ & $8.9527 \mathrm{E}+00$ & $3.044 \mathrm{E}-09$ & $8.374 \mathrm{E}+04$ \\
\hline $1 s 4 d^{3} D_{2}$ & $1 s 6 s^{3} S_{1}$ & $9.3373 \mathrm{E}+00$ & $1.297 \mathrm{E}-12$ & $3.306 \mathrm{E}+01$ & $9.3006 \mathrm{E}+00$ & $4.837 \mathrm{E}-12$ & $1.232 \mathrm{E}+02$ \\
\hline $1 s 4 d^{3} D_{1}$ & $1 s 6 s^{3} s_{1}$ & $9.3415 \mathrm{E}+00$ & $1.313 \mathrm{E}-11$ & $3.344 \mathrm{E}+02$ & $9.3048 \mathrm{E}+00$ & $1.214 \mathrm{E}-12$ & $3.092 \mathrm{E}+01$ \\
\hline $1 s 4 d{ }^{1} D_{2}$ & $1 s 6 s^{3} S_{1}$ & $9.4508 \mathrm{E}+00$ & $1.784 \mathrm{E}-12$ & $4.442 \mathrm{E}+01$ & & & \\
\hline $1 s 5 s^{3} S_{1}$ & $1 s 6 s^{3} S_{1}$ & $2.5485 \mathrm{E}+01$ & $2.125 \mathrm{E}-09$ & $7.273 \mathrm{E}+03$ & $2.5393 E+01$ & $2.131 \mathrm{E}-09$ & $7.285 E+03$ \\
\hline $1 s 5 s{ }^{1} S_{0}$ & $1 s 6 s^{3} S_{1}$ & $2.5726 \mathrm{E}+01$ & $1.670 \mathrm{E}-09$ & $5.611 \mathrm{E}+03$ & $2.5635 \mathrm{E}+01$ & $1.075 E-09$ & $3.606 \mathrm{E}+03$ \\
\hline $1 s 5 d^{3} D_{2}$ & $1 s 6 s^{3} S_{1}$ & $2.7214 \mathrm{E}+01$ & $7.250 \mathrm{E}-13$ & $2.176 \mathrm{E}+00$ & $2.7122 \mathrm{E}+01$ & $5.172 \mathrm{E}-12$ & $1.550 \mathrm{E}+01$ \\
\hline $1 s 5 d^{3} D_{1}$ & $1 s 6 s^{3} S_{1}$ & $2.7232 \mathrm{E}+01$ & $2.052 \mathrm{E}-11$ & $6.152 \mathrm{E}+01$ & $2.7140 \mathrm{E}+01$ & $1.190 \mathrm{E}-12$ & $3.562 \mathrm{E}+00$ \\
\hline $1 s 5 d^{1} D_{2}$ & $1 s 6 s^{3} s_{1}$ & $2.7711 \mathrm{E}+01$ & $3.013 \mathrm{E}-12$ & $8.724 \mathrm{E}+00$ & & & \\
\hline $1 s 2 p^{3} P_{1}^{o}$ & $1 s 6 p^{3} P_{1}^{o}$ & $1.3810 \mathrm{E}+00$ & $2.067 \mathrm{E}-08$ & $2.410 \mathrm{E}+07$ & $1.3752 \mathrm{E}+00$ & $2.129 \mathrm{E}-08$ & $2.482 \mathrm{E}+07$ \\
\hline $1 s 2 p^{3} P_{0}^{o}$ & $1 s 6 p^{3} P_{1}^{o}$ & $1.3819 \mathrm{E}+00$ & $8.740 \mathrm{E}-09$ & $1.018 \mathrm{E}+07$ & $1.3761 \mathrm{E}+00$ & $1.295 \mathrm{E}-08$ & $1.508 \mathrm{E}+07$ \\
\hline $1 s 2 p^{3} P_{2}^{o}$ & $1 s 6 p^{3} P_{1}^{o}$ & $1.4434 \mathrm{E}+00$ & $4.128 \mathrm{E}-07$ & $4.405 E+08$ & $1.4374 \mathrm{E}+00$ & $4.168 \mathrm{E}-07$ & $4.448 \mathrm{E}+08$ \\
\hline $1 s 2 p^{1} P_{1}^{o}$ & $1 s 6 p^{3} P_{1}^{o}$ & $1.4493 \mathrm{E}+00$ & $9.654 \mathrm{E}-08$ & $1.022 \mathrm{E}+08$ & $1.4433 \mathrm{E}+00$ & $9.341 \mathrm{E}-08$ & $9.885 \mathrm{E}+07$ \\
\hline $1 s 3 p^{3} P_{1}^{o}$ & $1 s 6 p^{3} P_{1}^{o}$ & $3.7142 \mathrm{E}+00$ & $6.133 \mathrm{E}-09$ & $9.884 \mathrm{E}+05$ & $3.6989 \mathrm{E}+00$ & $5.685 \mathrm{E}-09$ & $9.160 \mathrm{E}+05$ \\
\hline $1 s 3 p^{3} P_{0}^{o}$ & $1 s 6 p^{3} P_{1}^{o}$ & $3.7159 \mathrm{E}+00$ & $2.025 \mathrm{E}-09$ & $3.261 \mathrm{E}+05$ & $3.7005 E+00$ & $4.431 \mathrm{E}-09$ & $7.134 \mathrm{E}+05$ \\
\hline $1 s 3 p^{3} P_{2}^{o}$ & $1 s 6 p^{3} P_{1}^{o}$ & $3.8474 \mathrm{E}+00$ & $3.258 \mathrm{E}-07$ & $4.893 \mathrm{E}+07$ & $3.8314 \mathrm{E}+00$ & $3.312 \mathrm{E}-07$ & $4.974 \mathrm{E}+07$ \\
\hline $1 s 3 p^{1} P_{1}^{o}$ & $1 s 6 p^{3} P_{1}^{o}$ & $3.8593 \mathrm{E}+00$ & $7.372 \mathrm{E}-08$ & $1.101 \mathrm{E}+07$ & $3.8434 \mathrm{E}+00$ & $7.108 \mathrm{E}-08$ & $1.060 \mathrm{E}+07$ \\
\hline $1 s 4 p^{3} P_{1}^{o}$ & $1 s 6 p^{3} P_{1}^{o}$ & $8.9685 E+00$ & $2.399 \mathrm{E}-09$ & $6.631 \mathrm{E}+04$ & $8.9310 \mathrm{E}+00$ & $1.888 \mathrm{E}-09$ & $5.218 \mathrm{E}+04$ \\
\hline $1 s 4 p^{3} P_{0}^{o}$ & $1 s 6 p^{3} P_{1}^{o}$ & $8.9726 \mathrm{E}+00$ & $4.830 \mathrm{E}-10$ & $1.334 \mathrm{E}+04$ & $8.9350 \mathrm{E}+00$ & $2.091 \mathrm{E}-09$ & $5.775 E+04$ \\
\hline $1 s 4 p^{3} P_{2}^{0}$ & $1 s 6 p^{3} P_{1}^{o}$ & $9.2954 \mathrm{E}+00$ & $3.183 \mathrm{E}-07$ & $8.190 \mathrm{E}+06$ & $9.2564 \mathrm{E}+00$ & $3.253 \mathrm{E}-07$ & $8.369 \mathrm{E}+06$ \\
\hline $1 s 4 p^{1} P_{1}^{o}$ & $1 s 6 p^{3} P_{1}^{o}$ & $9.3241 \mathrm{E}+00$ & $7.090 \mathrm{E}-08$ & $1.813 \mathrm{E}+06$ & $9.2853 \mathrm{E}+00$ & $6.788 \mathrm{E}-08$ & $1.735 \mathrm{E}+06$ \\
\hline $1 s 4 f^{3} F_{2}^{o}$ & $1 s 6 p^{3} P_{1}^{o}$ & $9.4324 \mathrm{E}+00$ & $7.883 \mathrm{E}-14$ & $1.970 \mathrm{E}+00$ & & & \\
\hline $1 s 5 p^{3} P_{1}^{o}$ & $1 s 6 p^{3} P_{1}^{o}$ & $2.5582 \mathrm{E}+01$ & $1.080 \mathrm{E}-09$ & $3.667 \mathrm{E}+03$ & $2.5476 \mathrm{E}+01$ & $6.650 \mathrm{E}-10$ & $2.258 \mathrm{E}+03$ \\
\hline $1 s 5 p^{3} P_{0}^{0}$ & $1 s 6 p^{3} P_{1}^{0}$ & $2.5599 \mathrm{E}+01$ & $6.529 \mathrm{E}-11$ & $2.215 \mathrm{E}+02$ & $2.5492 \mathrm{E}+01$ & $1.370 \mathrm{E}-09$ & $4.650 \mathrm{E}+03$ \\
\hline $1 s 5 p^{3} P_{2}^{o}$ & $1 s 6 p^{3} P_{1}^{o}$ & $2.6962 \mathrm{E}+01$ & $4.537 \mathrm{E}-07$ & $1.388 \mathrm{E}+06$ & $2.6849 \mathrm{E}+01$ & $4.659 \mathrm{E}-07$ & $1.424 \mathrm{E}+06$ \\
\hline $1 s 5 p^{1} P_{1}^{o}$ & $1 s 6 p^{3} P_{1}^{o}$ & $2.7084 \mathrm{E}+01$ & $1.005 \mathrm{E}-07$ & $3.045 \mathrm{E}+05$ & $2.6972 \mathrm{E}+01$ & $9.510 \mathrm{E}-08$ & $2.881 \mathrm{E}+05$ \\
\hline $1 s 5 f^{3} F_{2}^{o}$ & $1 s 6 p^{3} P_{1}^{0}$ & $2.7556 \mathrm{E}+01$ & $2.189 \mathrm{E}-13$ & $6.408 \mathrm{E}-01$ & & & \\
\hline $1 s 2 s^{3} S_{1}$ & $1 s 6 s^{1} S_{0}^{1}$ & $1.3692 \mathrm{E}+00$ & $4.431 \mathrm{E}-08$ & $1.577 \mathrm{E}+08$ & $1.3635 \mathrm{E}+00$ & $4.532 \mathrm{E}-08$ & $1.612 \mathrm{E}+08$ \\
\hline
\end{tabular}


Table 5 (continued)

\begin{tabular}{|c|c|c|c|c|c|c|c|}
\hline \multirow[t]{2}{*}{ Lower } & \multirow[t]{2}{*}{ Upper } & \multicolumn{3}{|l|}{ GRASP2K } & \multicolumn{3}{|l|}{ FAC } \\
\hline & & $\lambda(\AA)$ & $g f$ & $A\left(\mathrm{~s}^{-1}\right)$ & $\lambda(\AA)$ & $g f$ & $A\left(\mathrm{~s}^{-1}\right)$ \\
\hline $1 s 3 s^{3} S_{1}$ & $1 s 6 s^{1} S_{0}$ & $3.6908 \mathrm{E}+00$ & $8.788 \mathrm{E}-09$ & $4.303 E+06$ & $3.6761 \mathrm{E}+00$ & $9.684 \mathrm{E}-09$ & $4.739 \mathrm{E}+06$ \\
\hline $1 s 3 d^{3} D_{1}$ & $1 s 6 s{ }^{1} S_{0}$ & $3.8591 \mathrm{E}+00$ & $9.086 \mathrm{E}-13$ & $4.070 \mathrm{E}+02$ & & & \\
\hline $1 s 4 s^{3} S_{1}$ & $1 s 6 s^{1} S_{0}$ & $8.9125 \mathrm{E}+00$ & $2.416 \mathrm{E}-09$ & $2.029 \mathrm{E}+05$ & $8.8795 \mathrm{E}+00$ & $3.021 \mathrm{E}-09$ & $2.534 \mathrm{E}+05$ \\
\hline $1 s 4 d^{3} D_{1}$ & $1 s 6 s^{1} S_{0}$ & $9.3233 \mathrm{E}+00$ & $1.668 \mathrm{E}-12$ & $1.280 \mathrm{E}+02$ & $9.2893 \mathrm{E}+00$ & $1.732 \mathrm{E}-12$ & $1.327 \mathrm{E}+02$ \\
\hline $1 s 5 s^{3} S_{1}$ & $1 s 6 s^{1} S_{0}$ & $2.5350 \mathrm{E}+01$ & $6.194 \mathrm{E}-10$ & $6.429 E+03$ & $2.5278 \mathrm{E}+01$ & $1.061 \mathrm{E}-09$ & $1.098 \mathrm{E}+04$ \\
\hline $1 s 5 d^{3} D_{1}$ & $1 s 6 s{ }^{1} S_{0}$ & $2.7078 \mathrm{E}+01$ & $1.807 \mathrm{E}-12$ & $1.644 \mathrm{E}+01$ & $2.7009 \mathrm{E}+01$ & $1.992 \mathrm{E}-12$ & $1.806 \mathrm{E}+01$ \\
\hline $1 s 6 s^{3} S_{1}$ & $1 s 6 s^{1} S_{0}$ & $4.7691 \mathrm{E}+03$ & $7.013 \mathrm{E}-09$ & $2.057 \mathrm{E}+00$ & $5.5745 E+03$ & $5.830 \mathrm{E}-09$ & $1.240 \mathrm{E}+00$ \\
\hline $1 s 2 p^{3} P_{1}^{o}$ & $1 s 6 p^{3} P_{0}^{o}$ & $1.3809 \mathrm{E}+00$ & $1.094 \mathrm{E}-08$ & $3.828 \mathrm{E}+07$ & $1.3753 \mathrm{E}+00$ & $7.037 \mathrm{E}-09$ & $2.460 \mathrm{E}+07$ \\
\hline $1 s 2 p^{1} P_{1}^{o}$ & $1 s 6 p^{3} P_{0}^{o}$ & $1.4493 \mathrm{E}+00$ & $1.695 \mathrm{E}-07$ & $5.381 \mathrm{E}+08$ & $1.4434 \mathrm{E}+00$ & $1.740 \mathrm{E}-07$ & $5.525 E+08$ \\
\hline $1 s 3 p^{3} P_{1}^{o}$ & $1 s 6 p^{3} P_{0}^{o}$ & $3.7140 \mathrm{E}+00$ & $3.120 \mathrm{E}-09$ & $1.509 \mathrm{E}+06$ & $3.6994 \mathrm{E}+00$ & $1.511 \mathrm{E}-09$ & $7.306 \mathrm{E}+05$ \\
\hline $1 s 3 p{ }^{1} P_{1}^{o}$ & $1 s 6 p^{3} P_{0}^{o}$ & $3.8591 \mathrm{E}+00$ & $1.323 \mathrm{E}-07$ & $5.925 \mathrm{E}+07$ & $3.8440 \mathrm{E}+00$ & $1.357 \mathrm{E}-07$ & $6.077 \mathrm{E}+07$ \\
\hline $1 s 4 p^{3} P_{1}^{o}$ & $1 s 6 p^{3} P_{0}^{o}$ & $8.9674 \mathrm{E}+00$ & $1.092 \mathrm{E}-09$ & $9.058 \mathrm{E}+04$ & $8.9341 \mathrm{E}+00$ & $3.075 \mathrm{E}-10$ & $2.548 \mathrm{E}+04$ \\
\hline $1 s 4 p{ }^{1} P_{1}^{o}$ & $1 s 6 p^{3} P_{0}^{o}$ & $9.3228 \mathrm{E}+00$ & $1.285 \mathrm{E}-07$ & $9.865 \mathrm{E}+06$ & $9.2885 E+00$ & $1.318 \mathrm{E}-07$ & $1.010 \mathrm{E}+07$ \\
\hline $1 s 5 p^{3} P_{1}^{o}$ & $1 s 6 p^{3} P_{0}^{o}$ & $2.5573 \mathrm{E}+01$ & $4.594 \mathrm{E}-10$ & $4.685 E+03$ & & & \\
\hline $1 s 5 p^{1} P_{1}^{o}$ & $1 s 6 p^{3} P_{0}^{o}$ & $2.7074 \mathrm{E}+01$ & $1.828 \mathrm{E}-07$ & $1.663 \mathrm{E}+06$ & $2.7000 \mathrm{E}+01$ & $1.870 \mathrm{E}-07$ & $1.697 \mathrm{E}+06$ \\
\hline $1 s 6 p^{3} P_{1}^{o}$ & $1 s 6 p^{3} P_{0}^{o}$ & $6.9398 \mathrm{E}+04$ & $6.942 \mathrm{E}-08$ & $9.614 \mathrm{E}-02$ & & & \\
\hline $1 s 2 p^{3} P_{1}^{o}$ & $1 s 6 p^{3} P_{2}^{o}$ & $1.3788 \mathrm{E}+00$ & $1.951 \mathrm{E}-07$ & $1.369 \mathrm{E}+08$ & $1.3731 \mathrm{E}+00$ & $2.017 \mathrm{E}-07$ & $1.415 \mathrm{E}+08$ \\
\hline $1 s 2 p^{3} P_{2}^{o}$ & $1 s 6 p^{3} P_{2}^{o}$ & $1.4410 \mathrm{E}+00$ & $2.930 \mathrm{E}-07$ & $1.883 \mathrm{E}+08$ & $1.4350 \mathrm{E}+00$ & $2.925 \mathrm{E}-07$ & $1.878 \mathrm{E}+08$ \\
\hline $1 s 2 p{ }^{1} P_{1}^{o}$ & $1 s 6 p^{3} P_{2}^{o}$ & $1.4469 \mathrm{E}+00$ & $2.548 \mathrm{E}-08$ & $1.624 \mathrm{E}+07$ & $1.4409 \mathrm{E}+00$ & $2.662 \mathrm{E}-08$ & $1.696 \mathrm{E}+07$ \\
\hline $1 s 3 p^{3} P_{1}^{o}$ & $1 s 6 p^{3} P_{2}^{o}$ & $3.6983 \mathrm{E}+00$ & $2.115 \mathrm{E}-07$ & $2.063 \mathrm{E}+07$ & $3.6831 \mathrm{E}+00$ & $2.178 \mathrm{E}-07$ & $2.123 E+07$ \\
\hline $1 s 3 p^{3} P_{2}^{o}$ & $1 s 6 p^{3} P_{2}^{o}$ & $3.8304 \mathrm{E}+00$ & $8.438 \mathrm{E}-08$ & $7.672 \mathrm{E}+06$ & $3.8145 \mathrm{E}+00$ & $8.336 \mathrm{E}-08$ & $7.578 \mathrm{E}+06$ \\
\hline $1 s 3 p^{1} P_{1}^{o}$ & $1 s 6 p^{3} P_{2}^{o}$ & $3.8422 \mathrm{E}+00$ & $5.285 \mathrm{E}-09$ & $4.776 \mathrm{E}+05$ & $3.8264 \mathrm{E}+00$ & $6.273 \mathrm{E}-09$ & $5.667 \mathrm{E}+05$ \\
\hline $1 s 4 p^{3} P_{1}^{o}$ & $1 s 6 p^{3} P_{2}^{o}$ & $8.8767 \mathrm{E}+00$ & $2.421 \mathrm{E}-07$ & $4.098 \mathrm{E}+06$ & $8.8397 \mathrm{E}+00$ & $2.488 \mathrm{E}-07$ & $4.212 \mathrm{E}+06$ \\
\hline $1 s 4 p^{3} P_{2}^{o}$ & $1 s 6 p^{3} P_{2}^{o}$ & $9.1968 \mathrm{E}+00$ & $3.073 \mathrm{E}-08$ & $4.846 \mathrm{E}+05$ & $9.1583 E+00$ & $2.935 \mathrm{E}-08$ & $4.630 \mathrm{E}+05$ \\
\hline $1 s 4 p^{1} P_{1}^{o}$ & $1 s 6 p^{3} P_{2}^{o}$ & $9.2248 \mathrm{E}+00$ & $8.915 \mathrm{E}-10$ & $1.398 \mathrm{E}+04$ & $9.1865 \mathrm{E}+00$ & $1.536 \mathrm{E}-09$ & $2.408 \mathrm{E}+04$ \\
\hline $1 s 4 f^{3} F_{3}^{o}$ & $1 s 6 p^{3} P_{2}^{o}$ & $9.3286 \mathrm{E}+00$ & $6.451 \mathrm{E}-14$ & $9.889 \mathrm{E}-01$ & & & \\
\hline $1 s 4 f^{3} F_{2}^{o}$ & $1 s 6 p^{3} P_{2}^{o}$ & $9.3309 \mathrm{E}+00$ & $3.163 \mathrm{E}-14$ & $4.846 \mathrm{E}-01$ & & & \\
\hline $1 s 4 f^{1} F_{3}^{o}$ & $1 s 6 p^{3} P_{2}^{o}$ & $9.3838 \mathrm{E}+00$ & $1.153 \mathrm{E}-14$ & $1.747 \mathrm{E}-01$ & & & \\
\hline $1 s 5 p^{3} P_{1}^{0}$ & $1 s 6 p^{3} P_{2}^{o}$ & $2.4849 \mathrm{E}+01$ & $3.725 \mathrm{E}-07$ & $8.048 E+05$ & $2.4746 \mathrm{E}+01$ & $3.819 \mathrm{E}-07$ & $8.251 \mathrm{E}+05$ \\
\hline $1 s 5 p^{3} P_{2}^{o}$ & $1 s 6 p^{3} P_{2}^{o}$ & $2.6148 \mathrm{E}+01$ & $1.229 \mathrm{E}-08$ & $2.397 \mathrm{E}+04$ & $2.6040 \mathrm{E}+01$ & $1.110 \mathrm{E}-08$ & $2.166 \mathrm{E}+04$ \\
\hline $1 s 5 p{ }^{1} P_{1}^{o}$ & $1 s 6 p^{3} P_{2}^{o}$ & $2.6264 \mathrm{E}+01$ & $4.017 \mathrm{E}-13$ & $7.770 \mathrm{E}-01$ & $2.6156 \mathrm{E}+01$ & $1.763 \mathrm{E}-10$ & $3.409 \mathrm{E}+02$ \\
\hline $1 s 5 f^{3} F_{3}^{o}$ & $1 s 6 p^{3} P_{2}^{o}$ & $2.6698 \mathrm{E}+01$ & $1.051 \mathrm{E}-12$ & $1.966 \mathrm{E}+00$ & & & \\
\hline $1 s 5 f^{3} F_{2}^{o}$ & $1 s 6 p^{3} P_{2}^{o}$ & $2.6707 \mathrm{E}+01$ & $3.579 \mathrm{E}-13$ & $6.695 \mathrm{E}-01$ & & & \\
\hline $1 s 5 f{ }^{1} F_{3}^{o}$ & $1 s 6 p^{3} P_{2}^{o}$ & $2.6930 \mathrm{E}+01$ & $4.012 \mathrm{E}-14$ & $7.380 \mathrm{E}-02$ & & & \\
\hline $1 s 6 p^{3} P_{1}^{o}$ & $1 s 6 p^{3} P_{2}^{o}$ & $8.6654 \mathrm{E}+02$ & $8.046 \mathrm{E}-06$ & $1.429 \mathrm{E}+04$ & $8.6410 \mathrm{E}+02$ & $8.128 \mathrm{E}-06$ & $1.440 \mathrm{E}+04$ \\
\hline $1 s 2 s^{3} S_{1}$ & $1 s 6 d^{3} D_{2}$ & $1.3669 \mathrm{E}+00$ & $6.514 \mathrm{E}-09$ & $4.651 \mathrm{E}+06$ & $1.3610 \mathrm{E}+00$ & $6.766 \mathrm{E}-09$ & $4.831 \mathrm{E}+06$ \\
\hline $1 s 3 s^{3} S_{1}$ & $1 s 6 d^{3} D_{2}$ & $3.6741 \mathrm{E}+00$ & $1.083 \mathrm{E}-09$ & $1.070 \mathrm{E}+05$ & $3.6587 \mathrm{E}+00$ & $1.183 \mathrm{E}-09$ & $1.169 \mathrm{E}+05$ \\
\hline $1 s 3 d^{3} D_{2}$ & $1 s 6 d^{3} D_{2}$ & $3.8391 \mathrm{E}+00$ & $2.622 \mathrm{E}-08$ & $2.373 E+06$ & $3.8228 \mathrm{E}+00$ & $2.617 \mathrm{E}-08$ & $2.369 \mathrm{E}+06$ \\
\hline $1 s 3 d^{3} D_{1}$ & $1 s 6 d^{3} D_{2}$ & $3.8409 \mathrm{E}+00$ & $2.274 \mathrm{E}-09$ & $2.057 \mathrm{E}+05$ & $3.8245 \mathrm{E}+00$ & $3.045 \mathrm{E}-09$ & $2.753 \mathrm{E}+05$ \\
\hline $1 s 3 d^{3} D_{3}$ & $1 s 6 d^{3} D_{2}$ & $3.8827 \mathrm{E}+00$ & $5.188 \mathrm{E}-08$ & $4.591 \mathrm{E}+06$ & $3.8661 \mathrm{E}+00$ & $5.171 \mathrm{E}-08$ & $4.576 \mathrm{E}+06$ \\
\hline $1 s 3 d^{1} D_{2}$ & $1 s 6 d^{3} D_{2}$ & $3.8844 \mathrm{E}+00$ & $4.104 \mathrm{E}-09$ & $3.629 E+05$ & $3.8678 \mathrm{E}+00$ & $4.089 \mathrm{E}-09$ & $3.615 \mathrm{E}+05$ \\
\hline $1 s 4 s^{3} S_{1}$ & $1 s 6 d^{3} D_{2}$ & $8.8160 \mathrm{E}+00$ & $1.556 \mathrm{E}-10$ & $2.671 E+03$ & $8.7783 \mathrm{E}+00$ & $1.935 \mathrm{E}-10$ & $3.322 \mathrm{E}+03$ \\
\hline $1 s 4 d^{3} D_{2}$ & $1 s 6 d^{3} D_{2}$ & $9.2136 \mathrm{E}+00$ & $1.330 \mathrm{E}-08$ & $2.090 \mathrm{E}+05$ & $9.1745 E+00$ & $1.325 \mathrm{E}-08$ & $2.082 \mathrm{E}+05$ \\
\hline $1 s 4 d^{3} D_{1}$ & $1 s 6 d^{3} D_{2}$ & $9.2178 \mathrm{E}+00$ & $9.779 \mathrm{E}-10$ & $1.535 \mathrm{E}+04$ & $9.1786 \mathrm{E}+00$ & $1.599 \mathrm{E}-09$ & $2.511 \mathrm{E}+04$ \\
\hline $1 s 4 d^{3} D_{3}$ & $1 s 6 d^{3} D_{2}$ & $9.3198 \mathrm{E}+00$ & $5.775 \mathrm{E}-08$ & $8.870 \mathrm{E}+05$ & $9.2799 \mathrm{E}+00$ & $5.767 \mathrm{E}-08$ & $8.859 \mathrm{E}+05$ \\
\hline $1 s 4 d{ }^{1} D_{2}$ & $1 s 6 d^{3} D_{2}$ & $9.3242 \mathrm{E}+00$ & $4.505 \mathrm{E}-09$ & $6.913 E+04$ & $9.2843 \mathrm{E}+00$ & $4.384 \mathrm{E}-09$ & $6.727 \mathrm{E}+04$ \\
\hline $1 s 5 s^{3} s_{1}$ & $1 s 6 d^{3} D_{2}$ & $2.4585 \mathrm{E}+01$ & $7.247 \mathrm{E}-12$ & $1.600 \mathrm{E}+01$ & $2.4475 E+01$ & $1.833 \mathrm{E}-11$ & $4.048 \mathrm{E}+01$ \\
\hline $1 s 5 d^{3} D_{2}$ & $1 s 6 d^{3} D_{2}$ & $2.6190 \mathrm{E}+01$ & $5.971 \mathrm{E}-09$ & $1.161 \mathrm{E}+04$ & $2.6077 \mathrm{E}+01$ & $5.919 \mathrm{E}-09$ & $1.151 \mathrm{E}+04$ \\
\hline $1 s 5 d^{3} D_{1}$ & $1 s 6 d^{3} D_{2}$ & $2.6207 \mathrm{E}+01$ & $2.824 \mathrm{E}-10$ & $5.485 \mathrm{E}+02$ & $2.6094 \mathrm{E}+01$ & $7.777 \mathrm{E}-10$ & $1.510 \mathrm{E}+03$ \\
\hline $1 s 5 d^{3} D_{3}$ & $1 s 6 d^{3} D_{2}$ & $2.6631 \mathrm{E}+01$ & $7.999 \mathrm{E}-08$ & $1.505 \mathrm{E}+05$ & $2.6515 E+01$ & $8.000 \mathrm{E}-08$ & $1.505 \mathrm{E}+05$ \\
\hline $1 s 5 d{ }^{1} D_{2}$ & $1 s 6 d^{3} D_{2}$ & $2.6650 \mathrm{E}+01$ & $6.203 \mathrm{E}-09$ & $1.165 \mathrm{E}+04$ & $2.6534 \mathrm{E}+01$ & $5.909 \mathrm{E}-09$ & $1.110 \mathrm{E}+04$ \\
\hline $1 s 5 g{ }^{3} G_{3}$ & $1 s 6 d^{3} D_{2}$ & $2.6869 \mathrm{E}+01$ & $6.611 \mathrm{E}-17$ & $1.222 \mathrm{E}-04$ & & & \\
\hline $1 s 6 s^{3} S_{1}$ & $1 s 6 d^{3} D_{2}$ & $6.9582 \mathrm{E}+02$ & $6.652 \mathrm{E}-11$ & $1.833 \mathrm{E}-01$ & $6.7673 \mathrm{E}+02$ & $4.523 \mathrm{E}-12$ & $1.306 \mathrm{E}-02$ \\
\hline $1 s 2 p^{3} P_{1}^{o}$ & $1 s 6 p^{1} P_{1}^{o}$ & $1.3786 \mathrm{E}+00$ & $3.538 \mathrm{E}-08$ & $4.139 \mathrm{E}+07$ & $1.3729 \mathrm{E}+00$ & $3.559 \mathrm{E}-08$ & $4.163 E+07$ \\
\hline $1 s 2 p^{3} P_{0}^{0}$ & $1 s 6 p^{1} P_{1}^{o}$ & $1.3795 \mathrm{E}+00$ & $7.258 \mathrm{E}-08$ & $8.481 E+07$ & $1.3738 \mathrm{E}+00$ & $7.541 \mathrm{E}-08$ & $8.809 \mathrm{E}+07$ \\
\hline $1 s 2 p^{3} P_{2}^{o}$ & $1 s 6 p^{1} P_{1}^{o}$ & $1.4408 \mathrm{E}+00$ & $4.726 \mathrm{E}-08$ & $5.062 E+07$ & $1.4348 \mathrm{E}+00$ & $4.441 \mathrm{E}-08$ & $4.756 \mathrm{E}+07$ \\
\hline $1 s 2 p^{1} P_{1}^{o}$ & $1 s 6 p^{1} P_{1}^{o}$ & $1.4467 \mathrm{E}+00$ & $1.592 \mathrm{E}-07$ & $1.691 \mathrm{E}+08$ & $1.4407 \mathrm{E}+00$ & $1.576 \mathrm{E}-07$ & $1.674 \mathrm{E}+08$ \\
\hline $1 s 3 p^{3} P_{1}^{0}$ & $1 s 6 p^{1} P_{1}^{0}$ & $3.6970 \mathrm{E}+00$ & $4.301 \mathrm{E}-08$ & $6.997 \mathrm{E}+06$ & $3.6819 \mathrm{E}+00$ & $4.116 \mathrm{E}-08$ & $6.693 \mathrm{E}+06$ \\
\hline $1 s 3 p^{3} P_{0}^{0}$ & $1 s 6 p^{1} P_{1}^{o}$ & $3.6987 \mathrm{E}+00$ & $8.169 \mathrm{E}-08$ & $1.328 \mathrm{E}+07$ & $3.6836 \mathrm{E}+00$ & $8.426 \mathrm{E}-08$ & $1.368 \mathrm{E}+07$ \\
\hline $1 s 3 p^{3} P_{2}^{o}$ & $1 s 6 p^{1} P_{1}^{o}$ & $3.8290 \mathrm{E}+00$ & $1.541 \mathrm{E}-08$ & $2.336 \mathrm{E}+06$ & $3.8133 \mathrm{E}+00$ & $1.521 \mathrm{E}-08$ & $2.307 \mathrm{E}+06$ \\
\hline $1 s 3 p^{1} P_{1}^{o}$ & $1 s 6 p^{1} P_{1}^{o}$ & $3.8407 \mathrm{E}+00$ & $4.522 \mathrm{E}-08$ & $6.817 \mathrm{E}+06$ & $3.8251 \mathrm{E}+00$ & $4.434 \mathrm{E}-08$ & $6.680 \mathrm{E}+06$ \\
\hline $1 s 4 p^{3} P_{1}^{o}$ & $1 s 6 p^{1} P_{1}^{o}$ & $8.8691 \mathrm{E}+00$ & $5.110 \mathrm{E}-08$ & $1.444 \mathrm{E}+06$ & $8.8329 E+00$ & $4.835 \mathrm{E}-08$ & $1.366 \mathrm{E}+06$ \\
\hline $1 s 4 p^{3} P_{0}^{o}$ & $1 s 6 p^{1} P_{1}^{o}$ & $8.8731 \mathrm{E}+00$ & $9.506 \mathrm{E}-08$ & $2.685 \mathrm{E}+06$ & $8.8368 \mathrm{E}+00$ & $9.774 \mathrm{E}-08$ & $2.759 \mathrm{E}+06$ \\
\hline $1 s 4 p^{3} P_{2}^{o}$ & $1 s 6 p^{1} P_{1}^{o}$ & $9.1886 \mathrm{E}+00$ & $6.437 \mathrm{E}-09$ & $1.695 \mathrm{E}+05$ & $9.1511 \mathrm{E}+00$ & $7.060 \mathrm{E}-09$ & $1.858 \mathrm{E}+05$ \\
\hline $1 s 4 p^{1} P_{1}^{o}$ & $1 s 6 p^{1} P_{1}^{o}$ & $9.2166 \mathrm{E}+00$ & $1.567 \mathrm{E}-08$ & $4.102 E+05$ & $9.1793 \mathrm{E}+00$ & $1.527 \mathrm{E}-08$ & $3.997 \mathrm{E}+05$ \\
\hline $1 s 4 f^{3} F_{2}^{o}$ & $1 s 6 p^{1} P_{1}^{o}$ & $9.3225 \mathrm{E}+00$ & $8.951 \mathrm{E}-14$ & $2.290 \mathrm{E}+00$ & & & \\
\hline $1 s 5 p^{3} P_{1}^{0}$ & $1 s 6 p^{1} P_{1}^{o}$ & $2.4789 \mathrm{E}+01$ & $7.996 \mathrm{E}-08$ & $2.893 \mathrm{E}+05$ & $2.4694 \mathrm{E}+01$ & $7.555 \mathrm{E}-08$ & $2.731 \mathrm{E}+05$ \\
\hline $1 s 5 p^{3} P_{0}^{o}$ & $1 s 6 p^{1} P_{1}^{o}$ & $2.4805 \mathrm{E}+01$ & $1.475 \mathrm{E}-07$ & $5.332 E+05$ & $2.4708 \mathrm{E}+01$ & $1.515 \mathrm{E}-07$ & $5.473 \mathrm{E}+05$ \\
\hline $1 s 5 p^{3} P_{2}^{o}$ & $1 s 6 p^{1} P_{1}^{o}$ & $2.6083 \mathrm{E}+01$ & $3.633 \mathrm{E}-09$ & $1.187 \mathrm{E}+04$ & $2.5981 \mathrm{E}+01$ & $4.572 \mathrm{E}-09$ & $1.493 \mathrm{E}+04$ \\
\hline $1 s 5 p^{1} P_{1}^{0}$ & $1 s 6 p^{1} P_{1}^{o}$ & $2.6197 \mathrm{E}+01$ & $5.654 \mathrm{E}-09$ & $1.832 \mathrm{E}+04$ & $2.6097 \mathrm{E}+01$ & $5.441 \mathrm{E}-09$ & $1.761 \mathrm{E}+04$ \\
\hline $1 s 5 f^{3} F_{2}^{o}$ & $1 s 6 p^{1} P_{1}^{o}$ & $2.6638 \mathrm{E}+01$ & $9.179 E-13$ & $2.876 \mathrm{E}+00$ & & & \\
\hline
\end{tabular}


Table 5 (continued)

\begin{tabular}{|c|c|c|c|c|c|c|c|}
\hline \multirow[t]{2}{*}{ Lower } & \multirow[t]{2}{*}{ Upper } & \multicolumn{3}{|l|}{ GRASP2K } & \multicolumn{3}{|l|}{ FAC } \\
\hline & & $\lambda(\AA)$ & $g f$ & $A\left(\mathrm{~s}^{-1}\right)$ & $\lambda(\AA)$ & $g f$ & $A\left(\mathrm{~s}^{-1}\right)$ \\
\hline $1 s 6 p^{3} P_{1}^{o}$ & $1 s 6 p^{1} P_{1}^{o}$ & $7.9973 \mathrm{E}+02$ & $1.620 \mathrm{E}-06$ & $5.631 \mathrm{E}+03$ & $8.0416 \mathrm{E}+02$ & $1.584 \mathrm{E}-06$ & $5.402 \mathrm{E}+03$ \\
\hline $1 s 6 p^{3} P_{0}^{0}$ & $1 s 6 p^{1} P_{1}^{o}$ & $8.0906 \mathrm{E}+02$ & $3.000 \mathrm{E}-06$ & $1.019 \mathrm{E}+04$ & $7.8043 \mathrm{E}+02$ & $3.001 \mathrm{E}-06$ & $1.086 \mathrm{E}+04$ \\
\hline $1 s 6 p^{3} P_{2}^{o}$ & $1 s 6 p^{1} P_{1}^{o}$ & $1.0374 \mathrm{E}+04$ & $2.022 \mathrm{E}-07$ & $4.177 \mathrm{E}+00$ & $1.1592 \mathrm{E}+04$ & $1.745 \mathrm{E}-07$ & $2.864 \mathrm{E}+00$ \\
\hline $1 s^{2}{ }^{1} S_{0}$ & $1 s 6 d^{3} D_{1}$ & $3.1620 \mathrm{E}-01$ & $1.772 \mathrm{E}-08$ & $3.939 E+08$ & $3.1497 \mathrm{E}-01$ & $1.829 \mathrm{E}-08$ & $4.066 \mathrm{E}+08$ \\
\hline $1 s 2 s^{3} S_{1}$ & $1 s 6 d^{3} D_{1}$ & $1.3668 \mathrm{E}+00$ & $1.773 \mathrm{E}-09$ & $2.110 \mathrm{E}+06$ & $1.3611 \mathrm{E}+00$ & $1.353 \mathrm{E}-09$ & $1.610 \mathrm{E}+06$ \\
\hline $1 s 2 s^{1} S_{0}$ & $1 s 6 d^{3} D_{1}$ & $1.3798 \mathrm{E}+00$ & $2.691 \mathrm{E}-09$ & $3.142 \mathrm{E}+06$ & $1.3741 \mathrm{E}+00$ & $2.709 \mathrm{E}-09$ & $3.163 E+06$ \\
\hline $1 s 3 s^{3} S_{1}$ & $1 s 6 d^{3} D_{1}$ & $3.6740 \mathrm{E}+00$ & $3.764 \mathrm{E}-10$ & $6.199 E+04$ & $3.6587 \mathrm{E}+00$ & $2.428 \mathrm{E}-10$ & $3.998 \mathrm{E}+04$ \\
\hline $1 s 3 s^{1} S_{0}$ & $1 s 6 d^{3} D_{1}$ & $3.6986 \mathrm{E}+00$ & $4.789 \mathrm{E}-10$ & $7.785 E+04$ & $3.6835 \mathrm{E}+00$ & $4.807 \mathrm{E}-10$ & $7.811 \mathrm{E}+04$ \\
\hline $1 s 3 d^{3} D_{2}$ & $1 s 6 d^{3} D_{1}$ & $3.8389 \mathrm{E}+00$ & $3.667 \mathrm{E}-09$ & $5.532 E+05$ & $3.8228 \mathrm{E}+00$ & $2.842 \mathrm{E}-09$ & $4.287 \mathrm{E}+05$ \\
\hline $1 s 3 d^{3} D_{1}$ & $1 s 6 d^{3} D_{1}$ & $3.8407 \mathrm{E}+00$ & $1.459 \mathrm{E}-08$ & $2.199 E+06$ & $3.8246 \mathrm{E}+00$ & $1.456 \mathrm{E}-08$ & $2.194 \mathrm{E}+06$ \\
\hline $1 s 3 d^{1} D_{2}$ & $1 s 6 d^{3} D_{1}$ & $3.8842 \mathrm{E}+00$ & $3.313 \mathrm{E}-08$ & $4.882 \mathrm{E}+06$ & $3.8678 \mathrm{E}+00$ & $3.359 E-08$ & $4.950 \mathrm{E}+06$ \\
\hline $1 s 4 s^{3} S_{1}$ & $1 s 6 d^{3} D_{1}$ & $8.8149 \mathrm{E}+00$ & $9.426 \mathrm{E}-11$ & $2.697 \mathrm{E}+03$ & $8.7785 E+00$ & $4.075 \mathrm{E}-11$ & $1.165 \mathrm{E}+03$ \\
\hline $1 s 4 s^{1} S_{0}$ & $1 s 6 d^{3} D_{1}$ & $8.8722 \mathrm{E}+00$ & $8.005 E-11$ & $2.261 \mathrm{E}+03$ & $8.8361 \mathrm{E}+00$ & $7.985 \mathrm{E}-11$ & $2.254 \mathrm{E}+03$ \\
\hline $1 s 4 d^{3} D_{2}$ & $1 s 6 d^{3} D_{1}$ & $9.2124 \mathrm{E}+00$ & $2.076 \mathrm{E}-09$ & $5.438 \mathrm{E}+04$ & $9.1748 \mathrm{E}+00$ & $1.393 \mathrm{E}-09$ & $3.648 \mathrm{E}+04$ \\
\hline $1 s 4 d^{3} D_{1}$ & $1 s 6 d^{3} D_{1}$ & $9.2166 \mathrm{E}+00$ & $7.395 \mathrm{E}-09$ & $1.936 \mathrm{E}+05$ & $9.1789 \mathrm{E}+00$ & $7.377 \mathrm{E}-09$ & $1.930 \mathrm{E}+05$ \\
\hline $1 s 4 d^{1} D_{2}$ & $1 s 6 d^{3} D_{1}$ & $9.3230 \mathrm{E}+00$ & $3.684 \mathrm{E}-08$ & $9.425 E+05$ & $9.2846 \mathrm{E}+00$ & $3.736 \mathrm{E}-08$ & $9.555 \mathrm{E}+05$ \\
\hline $1 s 5 s^{3} S_{1}$ & $1 s 6 d^{3} D_{1}$ & $2.4577 \mathrm{E}+01$ & $3.235 \mathrm{E}-11$ & $1.191 \mathrm{E}+02$ & & & \\
\hline $1 s 5 s^{1} S_{0}$ & $1 s 6 d^{3} D_{1}$ & $2.4800 \mathrm{E}+01$ & $7.939 \mathrm{E}-12$ & $2.870 \mathrm{E}+01$ & $2.4701 \mathrm{E}+01$ & $8.057 \mathrm{E}-12$ & $2.911 \mathrm{E}+01$ \\
\hline $1 s 5 d^{3} D_{2}$ & $1 s 6 d^{3} D_{1}$ & $2.6180 \mathrm{E}+01$ & $1.194 \mathrm{E}-09$ & $3.875 E+03$ & $2.6079 \mathrm{E}+01$ & $5.867 \mathrm{E}-10$ & $1.901 \mathrm{E}+03$ \\
\hline $1 s 5 d^{3} D_{1}$ & $1 s 6 d^{3} D_{1}$ & $2.6197 \mathrm{E}+01$ & $3.318 \mathrm{E}-09$ & $1.075 E+04$ & $2.6096 \mathrm{E}+01$ & $3.305 E-09$ & $1.069 \mathrm{E}+04$ \\
\hline $1 s 5 d{ }^{1} D_{2}$ & $1 s 6 d^{3} D_{1}$ & $2.6640 \mathrm{E}+01$ & $5.090 \mathrm{E}-08$ & $1.595 \mathrm{E}+05$ & $2.6536 \mathrm{E}+01$ & $5.164 \mathrm{E}-08$ & $1.616 \mathrm{E}+05$ \\
\hline $1 s 6 s^{3} S_{1}$ & $1 s 6 d^{3} D_{1}$ & $6.8911 \mathrm{E}+02$ & $1.701 \mathrm{E}-10$ & $7.963 \mathrm{E}-01$ & $6.7829 \mathrm{E}+02$ & $9.027 \mathrm{E}-13$ & $4.325 \mathrm{E}-03$ \\
\hline $1 s 6 s^{1} S_{0}$ & $1 s 6 d^{3} D_{1}$ & $8.0550 \mathrm{E}+02$ & $2.477 \mathrm{E}-12$ & $8.489 \mathrm{E}-03$ & $7.7225 \mathrm{E}+02$ & $1.588 \mathrm{E}-12$ & $5.871 \mathrm{E}-03$ \\
\hline $1 s 6 d^{3} D_{2}$ & $1 s 6 d^{3} D_{1}$ & $7.1428 \mathrm{E}+04$ & $1.286 \mathrm{E}-07$ & $5.604 \mathrm{E}-02$ & & & \\
\hline $1 s 3 d^{3} D_{2}$ & $1 s 6 d^{3} D_{3}$ & $3.8337 \mathrm{E}+00$ & $1.524 \mathrm{E}-08$ & $9.880 \mathrm{E}+05$ & $3.8174 \mathrm{E}+00$ & $1.528 \mathrm{E}-08$ & $9.910 \mathrm{E}+05$ \\
\hline $1 s 3 d^{3} D_{3}$ & $1 s 6 d^{3} D_{3}$ & $3.8772 \mathrm{E}+00$ & $1.259 \mathrm{E}-07$ & $7.984 \mathrm{E}+06$ & $3.8607 \mathrm{E}+00$ & $1.258 \mathrm{E}-07$ & $7.977 \mathrm{E}+06$ \\
\hline $1 s 3 d{ }^{1} D_{2}$ & $1 s 6 d^{3} D_{3}$ & $3.8789 \mathrm{E}+00$ & $5.579 \mathrm{E}-09$ & $3.533 E+05$ & $3.8624 \mathrm{E}+00$ & $6.223 E-09$ & $3.941 \mathrm{E}+05$ \\
\hline $1 s 4 d^{3} D_{2}$ & $1 s 6 d^{3} D_{3}$ & $9.1827 \mathrm{E}+00$ & $2.734 \mathrm{E}-08$ & $3.090 \mathrm{E}+05$ & $9.1440 \mathrm{E}+00$ & $2.741 \mathrm{E}-08$ & $3.097 \mathrm{E}+05$ \\
\hline $1 s 4 d^{3} D_{3}$ & $1 s 6 d^{3} D_{3}$ & $9.2881 \mathrm{E}+00$ & $6.413 \mathrm{E}-08$ & $7.083 E+05$ & $9.2486 \mathrm{E}+00$ & $6.411 \mathrm{E}-08$ & $7.081 \mathrm{E}+05$ \\
\hline $1 s 4 d^{1} D_{2}$ & $1 s 6 d^{3} D_{3}$ & $9.2925 \mathrm{E}+00$ & $2.601 \mathrm{E}-09$ & $2.870 \mathrm{E}+04$ & $9.2530 \mathrm{E}+00$ & $3.101 \mathrm{E}-09$ & $3.422 \mathrm{E}+04$ \\
\hline $1 s 5 d^{3} D_{2}$ & $1 s 6 d^{3} D_{3}$ & $2.5941 \mathrm{E}+01$ & $5.300 \mathrm{E}-08$ & $7.505 E+04$ & $2.5832 \mathrm{E}+01$ & $5.314 \mathrm{E}-08$ & $7.524 \mathrm{E}+04$ \\
\hline $1 s 5 d^{3} D_{3}$ & $1 s 6 d^{3} D_{3}$ & $2.6374 \mathrm{E}+01$ & $2.886 \mathrm{E}-08$ & $3.954 \mathrm{E}+04$ & $2.6261 \mathrm{E}+01$ & $2.878 \mathrm{E}-08$ & $3.944 \mathrm{E}+04$ \\
\hline $1 s 5 d{ }^{1} D_{2}$ & $1 s 6 d^{3} D_{3}$ & $2.6393 \mathrm{E}+01$ & $9.368 \mathrm{E}-10$ & $1.281 \mathrm{E}+03$ & $2.6280 \mathrm{E}+01$ & $1.329 \mathrm{E}-09$ & $1.818 \mathrm{E}+03$ \\
\hline $1 s 5 g{ }^{3} G_{4}$ & $1 s 6 d^{3} D_{3}$ & $2.6602 \mathrm{E}+01$ & $1.073 \mathrm{E}-13$ & $1.445 \mathrm{E}-01$ & & & \\
\hline $1 s 5 g^{3} G_{3}$ & $1 s 6 d^{3} D_{3}$ & $2.6608 \mathrm{E}+01$ & $3.697 \mathrm{E}-15$ & $4.976 \mathrm{E}-03$ & & & \\
\hline $1 s 5 g^{1} G_{4}$ & $1 s 6 d^{3} D_{3}$ & $2.6739 \mathrm{E}+01$ & $2.636 \mathrm{E}-18$ & $3.513 \mathrm{E}-06$ & & & \\
\hline $1 s 6 d^{3} D_{2}$ & $1 s 6 d^{3} D_{3}$ & $2.7340 \mathrm{E}+03$ & $4.169 \mathrm{E}-06$ & $5.314 \mathrm{E}+02$ & $2.7456 \mathrm{E}+03$ & $4.164 \mathrm{E}-06$ & $5.219 \mathrm{E}+02$ \\
\hline $1 s 2 p^{3} P_{2}^{o}$ & $1 s 6 f^{3} F_{3}^{o}$ & $1.4401 \mathrm{E}+00$ & $1.195 \mathrm{E}-08$ & $5.491 E+06$ & $1.4340 \mathrm{E}+00$ & $1.080 \mathrm{E}-08$ & $4.965 \mathrm{E}+06$ \\
\hline $1 s 3 p^{3} P_{2}^{o}$ & $1 s 6 f^{3} F_{3}^{o}$ & $3.8237 \mathrm{E}+00$ & $9.083 \mathrm{E}-10$ & $5.920 \mathrm{E}+04$ & $3.8077 \mathrm{E}+00$ & $9.348 \mathrm{E}-10$ & $6.092 \mathrm{E}+04$ \\
\hline $1 s 4 p^{3} P_{2}^{o}$ & $1 s 6 f^{3} F_{3}^{o}$ & $9.1585 \mathrm{E}+00$ & $7.725 \mathrm{E}-10$ & $8.776 E+03$ & $9.1192 \mathrm{E}+00$ & $7.636 \mathrm{E}-10$ & $8.676 E+03$ \\
\hline $1 s 4 f^{3} F_{3}^{o}$ & $1 s 6 f^{3} F_{3}^{o}$ & $9.2892 \mathrm{E}+00$ & $1.742 \mathrm{E}-09$ & $1.924 \mathrm{E}+04$ & $9.2496 \mathrm{E}+00$ & $2.338 \mathrm{E}-08$ & $2.582 \mathrm{E}+05$ \\
\hline $1 s 4 f^{3} F_{2}^{o}$ & $1 s 6 f^{3} F_{3}^{o}$ & $9.2914 \mathrm{E}+00$ & $6.431 \mathrm{E}-08$ & $7.099 \mathrm{E}+05$ & $9.2518 \mathrm{E}+00$ & $1.172 \mathrm{E}-09$ & $1.294 \mathrm{E}+04$ \\
\hline $1 s 4 f^{3} F_{4}^{o}$ & $1 s 6 f^{3} F_{3}^{o}$ & $9.3423 \mathrm{E}+00$ & $1.585 \mathrm{E}-08$ & $1.731 E+05$ & $9.3023 \mathrm{E}+00$ & $1.577 \mathrm{E}-08$ & $1.722 \mathrm{E}+05$ \\
\hline $1 s 4 f^{1} F_{3}^{o}$ & $1 s 6 f^{3} F_{3}^{o}$ & $9.3440 \mathrm{E}+00$ & $5.753 \mathrm{E}-10$ & $6.279 E+03$ & $9.3039 \mathrm{E}+00$ & $6.071 \mathrm{E}-10$ & $6.627 \mathrm{E}+03$ \\
\hline $1 s 5 p^{3} P_{2}^{o}$ & $1 s 6 f^{3} F_{3}^{o}$ & $2.5841 \mathrm{E}+01$ & $2.188 \mathrm{E}-10$ & $3.122 E+02$ & $2.5726 \mathrm{E}+01$ & $2.180 \mathrm{E}-10$ & $3.112 \mathrm{E}+02$ \\
\hline $1 s 5 f^{3} F_{3}^{o}$ & $1 s 6 f^{3} F_{3}^{o}$ & $2.6377 \mathrm{E}+01$ & $2.923 \mathrm{E}-09$ & $4.004 \mathrm{E}+03$ & $2.6263 \mathrm{E}+01$ & $1.627 \mathrm{E}-08$ & $2.228 \mathrm{E}+04$ \\
\hline $1 s 5 f^{3} F_{2}^{o}$ & $1 s 6 f^{3} F_{3}^{o}$ & $2.6386 \mathrm{E}+01$ & $5.326 \mathrm{E}-08$ & $7.290 \mathrm{E}+04$ & $2.6272 \mathrm{E}+01$ & $8.180 \mathrm{E}-10$ & $1.119 \mathrm{E}+03$ \\
\hline $1 s 5 f^{3} F_{4}^{o}$ & $1 s 6 f^{3} F_{3}^{o}$ & $2.6597 \mathrm{E}+01$ & $2.772 \mathrm{E}-08$ & $3.733 \mathrm{E}+04$ & $2.6482 \mathrm{E}+01$ & $2.755 \mathrm{E}-08$ & $3.712 \mathrm{E}+04$ \\
\hline $1 s 5 f^{1} F_{3}^{o}$ & $1 s 6 f^{3} F_{3}^{o}$ & $2.6604 \mathrm{E}+01$ & $1.038 \mathrm{E}-09$ & $1.397 \mathrm{E}+03$ & $2.6488 \mathrm{E}+01$ & $1.044 \mathrm{E}-09$ & $1.406 \mathrm{E}+03$ \\
\hline $1 s 6 p^{3} P_{2}^{o}$ & $1 s 6 f^{3} F_{3}^{o}$ & $2.1987 \mathrm{E}+03$ & $5.085 \mathrm{E}-12$ & $1.002 \mathrm{E}-03$ & $2.1353 \mathrm{E}+03$ & $1.018 \mathrm{E}-12$ & $2.110 \mathrm{E}-04$ \\
\hline $1 s 2 p^{3} P_{1}^{o}$ & $1 s 6 f^{3} F_{2}^{o}$ & $1.3779 \mathrm{E}+00$ & $1.521 \mathrm{E}-11$ & $1.069 \mathrm{E}+04$ & $1.3722 \mathrm{E}+00$ & $8.594 \mathrm{E}-12$ & $6.038 E+03$ \\
\hline $1 s 2 p^{3} P_{2}^{o}$ & $1 s 6 f^{3} F_{2}^{o}$ & $1.4401 \mathrm{E}+00$ & $7.687 \mathrm{E}-10$ & $4.945 \mathrm{E}+05$ & $1.4340 \mathrm{E}+00$ & $7.719 \mathrm{E}-10$ & $4.965 \mathrm{E}+05$ \\
\hline $1 s 2 p^{1} P_{1}^{o}$ & $1 s 6 f^{3} F_{2}^{o}$ & $1.4459 \mathrm{E}+00$ & $7.214 \mathrm{E}-09$ & $4.603 E+06$ & $1.4400 \mathrm{E}+00$ & $7.095 \mathrm{E}-09$ & $4.526 \mathrm{E}+06$ \\
\hline $1 s 3 p^{3} P_{1}^{o}$ & $1 s 6 f^{3} F_{2}^{o}$ & $3.6920 \mathrm{E}+00$ & $2.140 \mathrm{E}-12$ & $2.094 \mathrm{E}+02$ & $3.6768 \mathrm{E}+00$ & $9.298 \mathrm{E}-13$ & $9.098 \mathrm{E}+01$ \\
\hline $1 s 3 p^{3} P_{2}^{o}$ & $1 s 6 f^{3} F_{2}^{o}$ & $3.8236 \mathrm{E}+00$ & $8.615 E-11$ & $7.861 \mathrm{E}+03$ & $3.8077 \mathrm{E}+00$ & $6.677 \mathrm{E}-11$ & $6.092 E+03$ \\
\hline $1 s 3 p{ }^{1} P_{1}^{o}$ & $1 s 6 f^{3} F_{2}^{o}$ & $3.8353 \mathrm{E}+00$ & $6.057 \mathrm{E}-10$ & $5.493 E+04$ & $3.8195 \mathrm{E}+00$ & $5.957 \mathrm{E}-10$ & $5.401 \mathrm{E}+04$ \\
\hline $1 s 4 p^{3} P_{1}^{o}$ & $1 s 6 f^{3} F_{2}^{o}$ & $8.8404 \mathrm{E}+00$ & $1.764 \mathrm{E}-12$ & $3.011 \mathrm{E}+01$ & & & \\
\hline $1 s 4 p^{3} P_{2}^{o}$ & $1 s 6 f^{3} F_{2}^{o}$ & $9.1578 \mathrm{E}+00$ & $7.183 \mathrm{E}-11$ & $1.143 E+03$ & $9.1192 \mathrm{E}+00$ & $5.527 \mathrm{E}-11$ & $8.792 \mathrm{E}+02$ \\
\hline $1 s 4 p{ }^{1} P_{1}^{o}$ & $1 s 6 f^{3} F_{2}^{o}$ & $9.1856 \mathrm{E}+00$ & $5.112 \mathrm{E}-10$ & $8.083 E+03$ & $9.1472 \mathrm{E}+00$ & $4.920 \mathrm{E}-10$ & 7.779E +03 \\
\hline $1 s 4 f^{3} F_{3}^{o}$ & $1 s 6 f^{3} F_{2}^{o}$ & $9.2886 \mathrm{E}+00$ & $6.731 \mathrm{E}-08$ & $1.041 \mathrm{E}+06$ & $9.2496 \mathrm{E}+00$ & $1.186 \mathrm{E}-09$ & $1.833 \mathrm{E}+04$ \\
\hline $1 s 4 f^{3} F_{2}^{o}$ & $1 s 6 f^{3} F_{2}^{o}$ & $9.2908 \mathrm{E}+00$ & $6.173 \mathrm{E}-08$ & $9.540 \mathrm{E}+05$ & $9.2518 \mathrm{E}+00$ & $1.637 \mathrm{E}-08$ & $2.530 \mathrm{E}+05$ \\
\hline $1 s 4 f^{1} F_{3}^{o}$ & $1 s 6 f^{3} F_{2}^{o}$ & $9.3433 \mathrm{E}+00$ & $1.148 \mathrm{E}-08$ & $1.754 \mathrm{E}+05$ & $9.3039 \mathrm{E}+00$ & $1.167 \mathrm{E}-08$ & $1.783 \mathrm{E}+05$ \\
\hline $1 s 5 p^{3} P_{1}^{o}$ & $1 s 6 f^{3} F_{2}^{o}$ & $2.4566 \mathrm{E}+01$ & $8.810 \mathrm{E}-13$ & $1.948 \mathrm{E}+00$ & & & \\
\hline $1 s 5 p^{3} P_{2}^{o}$ & $1 s 6 f^{3} F_{2}^{o}$ & $2.5836 \mathrm{E}+01$ & $2.337 E-11$ & $4.672 \mathrm{E}+01$ & $2.5726 \mathrm{E}+01$ & $1.599 \mathrm{E}-11$ & $3.195 \mathrm{E}+01$ \\
\hline $1 s 5 p{ }^{1} P_{1}^{o}$ & $1 s 6 f^{3} F_{2}^{o}$ & $2.5948 \mathrm{E}+01$ & $1.502 \mathrm{E}-10$ & $2.975 \mathrm{E}+02$ & $2.5839 \mathrm{E}+01$ & $1.421 \mathrm{E}-10$ & $2.815 \mathrm{E}+02$ \\
\hline $1 s 5 f^{3} F_{3}^{o}$ & $1 s 6 f^{3} F_{2}^{o}$ & $2.6372 \mathrm{E}+01$ & $5.803 \mathrm{E}-08$ & $1.113 E+05$ & $2.6263 \mathrm{E}+01$ & $8.311 \mathrm{E}-10$ & $1.593 \mathrm{E}+03$ \\
\hline $1 s 5 f^{3} F_{2}^{o}$ & $1 s 6 f^{3} F_{2}^{o}$ & $2.6381 \mathrm{E}+01$ & $4.801 \mathrm{E}-08$ & $9.203 E+04$ & $2.6272 E+01$ & $1.140 \mathrm{E}-08$ & $2.184 \mathrm{E}+04$ \\
\hline $1 s 5 f^{1} F_{3}^{o}$ & $1 s 6 f^{3} F_{2}^{o}$ & $2.6599 \mathrm{E}+01$ & $2.011 \mathrm{E}-08$ & $3.793 E+04$ & $2.6489 \mathrm{E}+01$ & $2.040 \mathrm{E}-08$ & $3.845 E+04$ \\
\hline $1 s 6 p^{3} P_{1}^{o}$ & $1 s 6 f^{3} F_{2}^{o}$ & $6.1864 \mathrm{E}+02$ & $2.732 \mathrm{E}-12$ & $9.524 \mathrm{E}-03$ & $6.1520 \mathrm{E}+02$ & $4.924 \mathrm{E}-15$ & $1.721 \mathrm{E}-05$ \\
\hline $1 s 6 p^{3} P_{2}^{o}$ & $1 s 6 f^{3} F_{2}^{o}$ & $2.1625 E+03$ & $7.609 \mathrm{E}-12$ & $2.171 \mathrm{E}-03$ & $2.1357 \mathrm{E}+03$ & $7.272 \mathrm{E}-14$ & $2.109 \mathrm{E}-05$ \\
\hline $1 s 6 p{ }^{1} P_{1}^{o}$ & $1 s 6 f^{3} F_{2}^{o}$ & $2.7321 \mathrm{E}+03$ & $7.961 \mathrm{E}-14$ & $1.423 \mathrm{E}-05$ & $2.6181 \mathrm{E}+03$ & $5.329 E-13$ & $1.028 \mathrm{E}-04$ \\
\hline
\end{tabular}


Table 5 (continued)

\begin{tabular}{|c|c|c|c|c|c|c|c|}
\hline \multirow[t]{2}{*}{ Lower } & \multirow[t]{2}{*}{ Upper } & \multicolumn{3}{|l|}{ GRASP2K } & \multicolumn{3}{|l|}{ FAC } \\
\hline & & $\lambda(\AA)$ & $g f$ & $A\left(\mathrm{~s}^{-1}\right)$ & $\lambda(\AA)$ & $g f$ & $A\left(\mathrm{~s}^{-1}\right)$ \\
\hline $1 s 6 f^{3} F_{3}^{o}$ & $1 s 6 f^{3} F_{2}^{o}$ & $1.3144 \mathrm{E}+05$ & $9.863 \mathrm{E}-08$ & $7.617 \mathrm{E}-03$ & & & \\
\hline $1 s 2 s^{3} S_{1}$ & $1 s 6 d^{1} D_{2}$ & $1.3661 \mathrm{E}+00$ & $5.469 \mathrm{E}-12$ & $3.909 \mathrm{E}+03$ & $1.3604 \mathrm{E}+00$ & $9.449 \mathrm{E}-13$ & $6.754 \mathrm{E}+02$ \\
\hline $1 s 3 s^{3} S_{1}$ & $1 s 6 d{ }^{1} D_{2}$ & $3.6690 \mathrm{E}+00$ & $3.296 \mathrm{E}-12$ & $3.266 \mathrm{E}+02$ & & & \\
\hline $1 s 3 d^{3} D_{2}$ & $1 s 6 d^{1} D_{2}$ & $3.8335 \mathrm{E}+00$ & $1.210 \mathrm{E}-09$ & $1.098 \mathrm{E}+05$ & $3.8174 \mathrm{E}+00$ & $1.142 \mathrm{E}-09$ & $1.037 \mathrm{E}+05$ \\
\hline $1 s 3 d^{3} D_{1}$ & $1 s 6 d^{1} D_{2}$ & $3.8353 \mathrm{E}+00$ & $9.479 \mathrm{E}-09$ & $8.597 \mathrm{E}+05$ & $3.8191 \mathrm{E}+00$ & $9.644 \mathrm{E}-09$ & $8.746 \mathrm{E}+05$ \\
\hline $1 s 3 d^{3} D_{3}$ & $1 s 6 d^{1} D_{2}$ & $3.8770 \mathrm{E}+00$ & $7.265 \mathrm{E}-09$ & $6.447 \mathrm{E}+05$ & $3.8606 \mathrm{E}+00$ & $6.726 \mathrm{E}-09$ & $5.969 \mathrm{E}+05$ \\
\hline $1 s 3 d^{1} D_{2}$ & $1 s 6 d^{1} D_{2}$ & $3.8787 \mathrm{E}+00$ & $8.796 \mathrm{E}-08$ & $7.800 \mathrm{E}+06$ & $3.8623 \mathrm{E}+00$ & $8.773 \mathrm{E}-08$ & $7.779 \mathrm{E}+06$ \\
\hline $1 s 4 s^{3} S_{1}$ & $1 s 6 d^{1} D_{2}$ & $8.7865 \mathrm{E}+00$ & $2.542 \mathrm{E}-12$ & $4.392 \mathrm{E}+01$ & & & \\
\hline $1 s 4 d^{3} D_{2}$ & $1 s 6 d^{1} D_{2}$ & $9.1814 \mathrm{E}+00$ & $2.104 \mathrm{E}-09$ & $3.330 \mathrm{E}+04$ & $9.1435 \mathrm{E}+00$ & $1.965 \mathrm{E}-09$ & $3.109 E+04$ \\
\hline $1 s 4 d^{3} D_{1}$ & $1 s 6 d^{1} D_{2}$ & $9.1855 \mathrm{E}+00$ & $1.712 \mathrm{E}-08$ & $2.707 \mathrm{E}+05$ & $9.1476 \mathrm{E}+00$ & $1.742 \mathrm{E}-08$ & $2.753 \mathrm{E}+05$ \\
\hline $1 s 4 d^{3} D_{3}$ & $1 s 6 d^{1} D_{2}$ & $9.2868 \mathrm{E}+00$ & $3.981 \mathrm{E}-09$ & $6.158 E+04$ & $9.2482 \mathrm{E}+00$ & $3.535 \mathrm{E}-09$ & $5.468 \mathrm{E}+04$ \\
\hline $1 s 4 d^{1} D_{2}$ & $1 s 6 d^{1} D_{2}$ & $9.2911 \mathrm{E}+00$ & $4.489 \mathrm{E}-08$ & $6.938 \mathrm{E}+05$ & $9.2526 \mathrm{E}+00$ & $4.466 \mathrm{E}-08$ & $6.900 \mathrm{E}+05$ \\
\hline $1 s 5 s^{3} S_{1}$ & $1 s 6 d^{1} D_{2}$ & $2.4357 \mathrm{E}+01$ & $3.021 \mathrm{E}-12$ & $6.793 \mathrm{E}+00$ & & & \\
\hline $1 s 5 d^{3} D_{2}$ & $1 s 6 d^{1} D_{2}$ & $2.5931 \mathrm{E}+01$ & $4.067 \mathrm{E}-09$ & $8.068 E+03$ & $2.5828 \mathrm{E}+01$ & $3.787 \mathrm{E}-09$ & $7.509 E+03$ \\
\hline $1 s 5 d^{3} D_{1}$ & $1 s 6 d{ }^{1} D_{2}$ & $2.5947 \mathrm{E}+01$ & $3.338 \mathrm{E}-08$ & $6.614 \mathrm{E}+04$ & $2.5844 \mathrm{E}+01$ & $3.395 \mathrm{E}-08$ & $6.723 E+04$ \\
\hline $1 s 5 d^{3} D_{3}$ & $1 s 6 d^{1} D_{2}$ & $2.6363 \mathrm{E}+01$ & $2.106 \mathrm{E}-09$ & $4.043 E+03$ & $2.6257 \mathrm{E}+01$ & $1.705 \mathrm{E}-09$ & $3.271 \mathrm{E}+03$ \\
\hline $1 s 5 d^{1} D_{2}$ & $1 s 6 d^{1} D_{2}$ & $2.6382 \mathrm{E}+01$ & $2.022 \mathrm{E}-08$ & $3.875 E+04$ & $2.6276 \mathrm{E}+01$ & $2.001 \mathrm{E}-08$ & $3.834 \mathrm{E}+04$ \\
\hline $1 s 5 g{ }^{3} G_{3}$ & $1 s 6 d^{1} D_{2}$ & $2.6597 \mathrm{E}+01$ & $7.626 \mathrm{E}-14$ & $1.438 \mathrm{E}-01$ & & & \\
\hline $1 s 6 s^{3} S_{1}$ & $1 s 6 d^{1} D_{2}$ & $5.4990 \mathrm{E}+02$ & $3.558 \mathrm{E}-11$ & $1.570 \mathrm{E}-01$ & & & \\
\hline $1 s 6 d^{3} D_{2}$ & $1 s 6 d^{1} D_{2}$ & $2.6222 \mathrm{E}+03$ & $3.081 \mathrm{E}-07$ & $5.978 \mathrm{E}+01$ & $2.7042 \mathrm{E}+03$ & $2.977 \mathrm{E}-07$ & $5.385 E+01$ \\
\hline $1 s 6 d^{3} D_{1}$ & $1 s 6 d^{1} D_{2}$ & $2.7221 \mathrm{E}+03$ & $2.629 \mathrm{E}-06$ & $4.733 E+02$ & $2.6795 \mathrm{E}+03$ & $2.626 \mathrm{E}-06$ & $4.838 \mathrm{E}+02$ \\
\hline $1 s 6 d^{3} D_{3}$ & $1 s 6 d{ }^{1} D_{2}$ & $6.4120 \mathrm{E}+04$ & $8.705 E-08$ & $2.825 \mathrm{E}-02$ & & & \\
\hline $1 s 4 f^{3} F_{3}^{o}$ & $1 s 6 f^{3} F_{4}^{o}$ & $9.2736 \mathrm{E}+00$ & $3.992 \mathrm{E}-09$ & $3.440 \mathrm{E}+04$ & $9.2341 \mathrm{E}+00$ & $3.977 \mathrm{E}-09$ & $3.427 \mathrm{E}+04$ \\
\hline $1 s 4 f^{3} F_{4}^{o}$ & $1 s 6 f^{3} F_{4}^{0}$ & $9.3265 \mathrm{E}+00$ & $6.690 \mathrm{E}-08$ & $5.701 \mathrm{E}+05$ & $9.2866 \mathrm{E}+00$ & $7.168 \mathrm{E}-08$ & $6.107 \mathrm{E}+05$ \\
\hline $1 s 4 f^{1} F_{3}^{o}$ & $1 s 6 f^{3} F_{4}^{o}$ & $9.3281 \mathrm{E}+00$ & $2.991 \mathrm{E}-09$ & $2.548 \mathrm{E}+04$ & $9.2883 \mathrm{E}+00$ & $2.060 \mathrm{E}-09$ & $1.755 \mathrm{E}+04$ \\
\hline $1 s 5 f^{3} F_{3}^{o}$ & $1 s 6 f^{3} F_{4}^{o}$ & $2.6252 \mathrm{E}+01$ & $1.288 \mathrm{E}-08$ & $1.385 \mathrm{E}+04$ & $2.6139 \mathrm{E}+01$ & $1.283 \mathrm{E}-08$ & $1.380 \mathrm{E}+04$ \\
\hline $1 s 5 f^{3} F_{4}^{o}$ & $1 s 6 f^{3} F_{4}^{o}$ & $2.6469 \mathrm{E}+01$ & $4.726 \mathrm{E}-08$ & $4.999 \mathrm{E}+04$ & $2.6355 \mathrm{E}+01$ & $4.994 \mathrm{E}-08$ & $5.283 E+04$ \\
\hline $1 s 5 f^{1} F_{3}^{o}$ & $1 s 6 f^{3} F_{4}^{o}$ & $2.6476 \mathrm{E}+01$ & $1.811 \mathrm{E}-09$ & $1.915 \mathrm{E}+03$ & $2.6362 \mathrm{E}+01$ & $1.444 \mathrm{E}-09$ & $1.527 \mathrm{E}+03$ \\
\hline $1 s 6 f^{3} F_{3}^{o}$ & $1 s 6 f^{3} F_{4}^{0}$ & $5.5078 \mathrm{E}+03$ & $2.817 \mathrm{E}-06$ & $6.883 \mathrm{E}+01$ & $5.5226 \mathrm{E}+03$ & $2.808 \mathrm{E}-06$ & $6.766 \mathrm{E}+01$ \\
\hline $1 s 3 d^{3} D_{3}$ & $1 s 6 g{ }^{3} G_{4}$ & $3.8744 \mathrm{E}+00$ & $5.198 \mathrm{E}-09$ & $2.567 \mathrm{E}+05$ & $3.8580 \mathrm{E}+00$ & $5.256 \mathrm{E}-09$ & $2.595 \mathrm{E}+05$ \\
\hline $1 s 4 d^{3} D_{3}$ & $1 s 6 g{ }^{3} G_{4}$ & $9.2719 \mathrm{E}+00$ & $2.896 \mathrm{E}-10$ & $2.497 \mathrm{E}+03$ & $9.2332 \mathrm{E}+00$ & $2.894 \mathrm{E}-10$ & $2.494 \mathrm{E}+03$ \\
\hline $1 s 5 d^{3} D_{3}$ & $1 s 6 g{ }^{3} G_{4}$ & $2.6244 \mathrm{E}+01$ & $5.638 \mathrm{E}-10$ & $6.067 \mathrm{E}+02$ & $2.6137 \mathrm{E}+01$ & $5.631 \mathrm{E}-10$ & $6.057 \mathrm{E}+02$ \\
\hline $1 s 5 g{ }^{3} G_{4}$ & $1 s 6 g{ }^{3} G_{4}$ & $2.6470 \mathrm{E}+01$ & $2.319 \mathrm{E}-08$ & $2.453 \mathrm{E}+04$ & $2.6356 \mathrm{E}+01$ & $2.313 \mathrm{E}-08$ & $2.447 \mathrm{E}+04$ \\
\hline $1 s 5 g^{3} G_{3}$ & $1 s 6 g{ }^{3} G_{4}$ & $2.6475 \mathrm{E}+01$ & $5.258 \mathrm{E}-10$ & $5.560 \mathrm{E}+02$ & $2.6361 \mathrm{E}+01$ & $6.612 \mathrm{E}-10$ & $6.992 \mathrm{E}+02$ \\
\hline $1 s 5 g{ }^{3} G_{5}$ & $1 s 6 g{ }^{3} G_{4}$ & $2.6601 \mathrm{E}+01$ & $1.013 \mathrm{E}-08$ & $1.061 \mathrm{E}+04$ & $2.6487 \mathrm{E}+01$ & $1.007 \mathrm{E}-08$ & $1.054 \mathrm{E}+04$ \\
\hline $1 s 5 g^{1} G_{4}$ & $1 s 6 g{ }^{3} G_{4}$ & $2.6605 \mathrm{E}+01$ & $2.379 \mathrm{E}-10$ & $2.491 \mathrm{E}+02$ & $2.6491 \mathrm{E}+01$ & $2.376 \mathrm{E}-10$ & $2.489 \mathrm{E}+02$ \\
\hline $1 s 6 d^{3} D_{3}$ & $1 s 6 g{ }^{3} G_{4}$ & $5.3075 \mathrm{E}+03$ & $2.734 \mathrm{E}-13$ & $7.194 \mathrm{E}-06$ & $5.5194 \mathrm{E}+03$ & $2.107 \mathrm{E}-13$ & $5.082 \mathrm{E}-06$ \\
\hline $1 s 3 d^{3} D_{2}$ & $1 s 6 g{ }^{3} G_{3}$ & $3.8309 \mathrm{E}+00$ & $3.305 \mathrm{E}-13$ & $2.146 \mathrm{E}+01$ & & & \\
\hline $1 s 3 d^{3} D_{3}$ & $1 s 6 g{ }^{3} G_{3}$ & $3.8743 \mathrm{E}+00$ & $2.294 \mathrm{E}-10$ & $1.456 \mathrm{E}+04$ & $3.8580 \mathrm{E}+00$ & $1.946 \mathrm{E}-10$ & $1.235 \mathrm{E}+04$ \\
\hline $1 s 3 d^{1} D_{2}$ & $1 s 6 g{ }^{3} G_{3}$ & $3.8760 \mathrm{E}+00$ & 3.937E-09 & $2.497 \mathrm{E}+05$ & $3.8597 \mathrm{E}+00$ & $3.902 \mathrm{E}-09$ & $2.474 \mathrm{E}+05$ \\
\hline $1 s 4 d^{3} D_{2}$ & $1 s 6 g{ }^{3} G_{3}$ & $9.1665 \mathrm{E}+00$ & $1.291 \mathrm{E}-14$ & $1.464 \mathrm{E}-01$ & & & \\
\hline $1 s 4 d^{3} D_{3}$ & $1 s 6 g{ }^{3} G_{3}$ & $9.2715 \mathrm{E}+00$ & $1.119 \mathrm{E}-11$ & $1.241 \mathrm{E}+02$ & $9.2332 \mathrm{E}+00$ & $1.071 \mathrm{E}-11$ & $1.187 \mathrm{E}+02$ \\
\hline $1 s 4 d^{1} D_{2}$ & $1 s 6 g{ }^{3} G_{3}$ & $9.2759 \mathrm{E}+00$ & $2.148 \mathrm{E}-10$ & $2.379 \mathrm{E}+03$ & $9.2375 \mathrm{E}+00$ & $2.140 \mathrm{E}-10$ & $2.370 \mathrm{E}+03$ \\
\hline $1 s 5 d^{3} D_{2}$ & $1 s 6 g{ }^{3} G_{3}$ & $2.5812 \mathrm{E}+01$ & $5.221 \mathrm{E}-14$ & $7.467 \mathrm{E}-02$ & & & \\
\hline $1 s 5 d^{3} D_{3}$ & $1 s 6 g{ }^{3} G_{3}$ & $2.6241 \mathrm{E}+01$ & $2.382 \mathrm{E}-11$ & $3.297 \mathrm{E}+01$ & $2.6137 \mathrm{E}+01$ & $2.088 \mathrm{E}-11$ & $2.888 \mathrm{E}+01$ \\
\hline $1 s 5 d^{1} D_{2}$ & $1 s 6 g{ }^{3} G_{3}$ & $2.6259 \mathrm{E}+01$ & $4.243 \mathrm{E}-10$ & $5.863 \mathrm{E}+02$ & $2.6155 \mathrm{E}+01$ & $4.177 \mathrm{E}-10$ & $5.769 \mathrm{E}+02$ \\
\hline $1 s 5 g^{3} G_{4}$ & $1 s 6 g{ }^{3} G_{3}$ & $2.6467 \mathrm{E}+01$ & $8.195 \mathrm{E}-10$ & $1.115 \mathrm{E}+03$ & $2.6356 \mathrm{E}+01$ & $6.700 \mathrm{E}-10$ & $9.112 \mathrm{E}+02$ \\
\hline $1 s 5 g^{3} G_{3}$ & $1 s 6 g{ }^{3} G_{3}$ & $2.6472 \mathrm{E}+01$ & $1.789 \mathrm{E}-08$ & $2.432 \mathrm{E}+04$ & $2.6361 \mathrm{E}+01$ & $1.785 \mathrm{E}-08$ & $2.427 \mathrm{E}+04$ \\
\hline $1 s 5 g^{1} G_{4}$ & $1 s 6 g{ }^{3} G_{3}$ & $2.6602 \mathrm{E}+01$ & $7.974 \mathrm{E}-09$ & $1.074 \mathrm{E}+04$ & $2.6491 \mathrm{E}+01$ & $8.002 \mathrm{E}-09$ & $1.077 \mathrm{E}+04$ \\
\hline $1 s 6 d^{3} D_{2}$ & $1 s 6 g{ }^{3} G_{3}$ & $1.7904 \mathrm{E}+03$ & $5.103 \mathrm{E}-15$ & $1.517 \mathrm{E}-06$ & $1.8335 \mathrm{E}+03$ & $6.550 \mathrm{E}-17$ & $1.841 \mathrm{E}-08$ \\
\hline $1 s 6 d^{3} D_{3}$ & $1 s 6 g{ }^{3} G_{3}$ & $5.1873 \mathrm{E}+03$ & $1.899 \mathrm{E}-15$ & $6.726 \mathrm{E}-08$ & $5.5194 \mathrm{E}+03$ & $7.803 \mathrm{E}-15$ & $2.420 \mathrm{E}-07$ \\
\hline $1 s 6 d^{1} D_{2}$ & $1 s 6 g{ }^{3} G_{3}$ & $5.6439 \mathrm{E}+03$ & $1.309 \mathrm{E}-13$ & $3.915 \mathrm{E}-06$ & $5.6946 \mathrm{E}+03$ & $1.512 \mathrm{E}-13$ & $4.406 \mathrm{E}-06$ \\
\hline $1 s 6 g{ }^{3} G_{4}$ & $1 s 6 g{ }^{3} G_{3}$ & $2.2908 \mathrm{E}+05$ & $6.999 \mathrm{E}-08$ & $1.271 \mathrm{E}-03$ & & & \\
\hline $1 s 2 p^{3} P_{2}^{o}$ & $1 s 6 f^{1} F_{3}^{o}$ & $1.4397 \mathrm{E}+00$ & $7.159 \mathrm{E}-13$ & $3.291 \mathrm{E}+02$ & $1.4336 \mathrm{E}+00$ & $3.610 \mathrm{E}-15$ & $1.659 \mathrm{E}+00$ \\
\hline $1 s 3 p^{3} P_{2}^{o}$ & $1 s 6 f^{1} F_{3}^{o}$ & $3.8210 \mathrm{E}+00$ & $4.243 \mathrm{E}-14$ & $2.769 \mathrm{E}+00$ & $3.8051 \mathrm{E}+00$ & $3.132 \mathrm{E}-16$ & $2.044 \mathrm{E}-02$ \\
\hline $1 s 4 p^{3} P_{2}^{o}$ & $1 s 6 f^{1} F_{3}^{o}$ & $9.1428 \mathrm{E}+00$ & $9.110 \mathrm{E}-14$ & $1.038 \mathrm{E}+00$ & & & \\
\hline $1 s 4 f^{3} F_{3}^{o}$ & $1 s 6 f^{1} F_{3}^{o}$ & $9.2731 \mathrm{E}+00$ & $1.854 \mathrm{E}-10$ & $2.055 \mathrm{E}+03$ & $9.2341 \mathrm{E}+00$ & $1.626 \mathrm{E}-10$ & $1.802 \mathrm{E}+03$ \\
\hline $1 s 4 f^{3} F_{2}^{o}$ & $1 s 6 f^{1} F_{3}^{o}$ & $9.2753 \mathrm{E}+00$ & $2.969 \mathrm{E}-09$ & $3.289 \mathrm{E}+04$ & $9.2363 \mathrm{E}+00$ & $2.951 \mathrm{E}-09$ & $3.269 \mathrm{E}+04$ \\
\hline $1 s 4 f^{3} F_{4}^{o}$ & $1 s 6 f^{1} F_{3}^{o}$ & $9.3260 \mathrm{E}+00$ & $3.495 \mathrm{E}-09$ & $3.829 E+04$ & $9.2866 \mathrm{E}+00$ & $2.054 \mathrm{E}-09$ & $2.250 \mathrm{E}+04$ \\
\hline $1 s 4 f^{1} F_{3}^{o}$ & $1 s 6 f{ }^{1} F_{3}^{o}$ & $9.3276 \mathrm{E}+00$ & $5.878 \mathrm{E}-08$ & $6.437 \mathrm{E}+05$ & $9.2883 \mathrm{E}+00$ & $5.528 \mathrm{E}-08$ & $6.054 \mathrm{E}+05$ \\
\hline $1 s 5 p^{3} P_{2}^{o}$ & $1 s 6 f^{1} F_{3}^{o}$ & $2.5717 \mathrm{E}+01$ & $9.470 \mathrm{E}-14$ & $1.365 \mathrm{E}-01$ & & & \\
\hline $1 s 5 f^{3} F_{3}^{o}$ & $1 s 6 f^{1} F_{3}^{o}$ & $2.6248 \mathrm{E}+01$ & $5.478 \mathrm{E}-10$ & $7.577 \mathrm{E}+02$ & $2.6139 \mathrm{E}+01$ & $4.968 \mathrm{E}-10$ & $6.870 \mathrm{E}+02$ \\
\hline 1s $5 f^{3} F_{2}^{o}$ & $1 s 6 f^{1} F_{3}^{o}$ & $2.6257 \mathrm{E}+01$ & $9.502 \mathrm{E}-09$ & $1.313 E+04$ & $2.6148 \mathrm{E}+01$ & $9.514 \mathrm{E}-09$ & $1.314 \mathrm{E}+04$ \\
\hline 1s $5 f^{3} F_{4}^{o}$ & $1 s 6 f^{1} F_{3}^{o}$ & $2.6465 \mathrm{E}+01$ & $2.429 \mathrm{E}-09$ & $3.305 E+03$ & $2.6355 \mathrm{E}+01$ & $1.434 \mathrm{E}-09$ & $1.950 \mathrm{E}+03$ \\
\hline $1 s 5 f^{1} F_{3}^{o}$ & $1 s 6 f^{1} F_{3}^{o}$ & $2.6472 \mathrm{E}+01$ & $4.062 \mathrm{E}-08$ & $5.523 E+04$ & $2.6362 \mathrm{E}+01$ & $3.850 \mathrm{E}-08$ & $5.234 \mathrm{E}+04$ \\
\hline $1 s 6 p^{3} P_{2}^{o}$ & $1 s 6 f^{1} F_{3}^{o}$ & $1.5574 \mathrm{E}+03$ & $1.960 \mathrm{E}-12$ & $7.699 \mathrm{E}-04$ & & & \\
\hline $1 s 6 f^{3} F_{3}^{o}$ & $1 s 6 f{ }^{1} F_{3}^{o}$ & $5.3394 \mathrm{E}+03$ & $1.078 \mathrm{E}-07$ & $3.603 E+00$ & $5.5188 \mathrm{E}+03$ & $1.040 \mathrm{E}-07$ & $3.227 \mathrm{E}+00$ \\
\hline $1 s 6 f^{3} F_{2}^{o}$ & $1 s 6 f^{1} F_{3}^{o}$ & $5.5655 \mathrm{E}+03$ & $2.080 \mathrm{E}-06$ & $6.399 \mathrm{E}+01$ & $5.5161 \mathrm{E}+03$ & $2.078 \mathrm{E}-06$ & $6.453 \mathrm{E}+01$ \\
\hline $1 s 6 f^{3} F_{4}^{o}$ & $1 s 6 f^{1} F_{3}^{o}$ & $1.7466 \mathrm{E}+05$ & $5.173 \mathrm{E}-08$ & $1.616 \mathrm{E}-03$ & & & \\
\hline $1 s 5 g^{3} G_{4}$ & $1 s 6 g{ }^{3} G_{5}$ & $2.6394 \mathrm{E}+01$ & $3.048 \mathrm{E}-09$ & $2.653 \mathrm{E}+03$ & $2.6281 \mathrm{E}+01$ & $3.041 \mathrm{E}-09$ & $2.647 \mathrm{E}+03$ \\
\hline $1 s 5 g{ }^{3} G_{5}$ & $1 s 6 g{ }^{3} G_{5}$ & $2.6525 \mathrm{E}+01$ & $5.550 \mathrm{E}-08$ & $4.783 \mathrm{E}+04$ & $2.6411 \mathrm{E}+01$ & $5.531 \mathrm{E}-08$ & $4.767 \mathrm{E}+04$ \\
\hline
\end{tabular}


Table 5 (continued)

\begin{tabular}{|c|c|c|c|c|c|c|c|}
\hline \multirow[t]{2}{*}{ Lower } & \multirow[t]{2}{*}{ Upper } & \multicolumn{3}{|l|}{ GRASP2K } & \multicolumn{3}{|l|}{ FAC } \\
\hline & & $\lambda(\AA)$ & $g f$ & $A\left(\mathrm{~s}^{-1}\right)$ & $\lambda(\AA)$ & $g f$ & $A\left(\mathrm{~s}^{-1}\right)$ \\
\hline $1 s 5 g{ }^{1} G_{4}$ & $1 s 6 g^{3} G_{5}$ & $2.6529 \mathrm{E}+01$ & $9.023 \mathrm{E}-10$ & $7.774 \mathrm{E}+02$ & $2.6415 \mathrm{E}+01$ & $1.031 \mathrm{E}-09$ & $8.883 \mathrm{E}+02$ \\
\hline $1 s 6 g{ }^{3} G_{4}$ & $1 s 6 g^{3} G_{5}$ & $9.2456 \mathrm{E}+03$ & $2.127 \mathrm{E}-06$ & $1.509 \mathrm{E}+01$ & $9.2559 \mathrm{E}+03$ & $2.122 \mathrm{E}-06$ & $1.489 \mathrm{E}+01$ \\
\hline $1 s 4 f^{3} F_{4}^{o}$ & $1 s 6 h^{3} H_{5}^{o}$ & $9.3170 \mathrm{E}+00$ & $2.422 \mathrm{E}-09$ & $1.692 \mathrm{E}+04$ & $9.2773 \mathrm{E}+00$ & $2.373 \mathrm{E}-09$ & $1.658 \mathrm{E}+04$ \\
\hline $1 s 5 f^{3} F_{4}^{o}$ & $1 s 6 h^{3} H_{5}^{o}$ & $2.6394 \mathrm{E}+01$ & $7.696 \mathrm{E}-10$ & $6.699 \mathrm{E}+02$ & $2.6281 \mathrm{E}+01$ & $7.530 \mathrm{E}-10$ & $6.555 \mathrm{E}+02$ \\
\hline $1 s 6 f^{3} F_{4}^{0}$ & $1 s 6 h^{3} H_{5}^{o}$ & $9.2055 \mathrm{E}+03$ & $4.621 \mathrm{E}-14$ & $3.307 \mathrm{E}-07$ & $9.2780 \mathrm{E}+03$ & $4.284 \mathrm{E}-14$ & $2.992 \mathrm{E}-07$ \\
\hline $1 s 4 f^{3} F_{3}^{o}$ & $1 s 6 h^{3} H_{4}^{o}$ & $9.2640 \mathrm{E}+00$ & $8.889 \mathrm{E}-15$ & $7.676 \mathrm{E}-02$ & & & \\
\hline $1 s 4 f^{3} F_{4}^{o}$ & $1 s 6 h^{3} H_{4}^{o}$ & $9.3168 \mathrm{E}+00$ & $6.293 \mathrm{E}-11$ & $5.373 \mathrm{E}+02$ & $9.2773 \mathrm{E}+00$ & $5.395 \mathrm{E}-11$ & $4.606 \mathrm{E}+02$ \\
\hline $1 s 4 f^{1} F_{3}^{o}$ & $1 s 6 h^{3} H_{4}^{0}$ & $9.3184 \mathrm{E}+00$ & $1.950 \mathrm{E}-09$ & $1.664 \mathrm{E}+04$ & $9.2790 \mathrm{E}+00$ & $1.890 \mathrm{E}-09$ & $1.613 E+04$ \\
\hline $1 s 5 f^{3} F_{3}^{o}$ & $1 s 6 h^{3} H_{4}^{0}$ & $2.6175 \mathrm{E}+01$ & $1.242 \mathrm{E}-15$ & $1.343 \mathrm{E}-03$ & & & \\
\hline $1 s 5 f^{3} F_{4}^{o}$ & $1 s 6 h^{3} H_{4}^{0}$ & $2.6392 \mathrm{E}+01$ & $2.026 \mathrm{E}-11$ & $2.156 \mathrm{E}+01$ & $2.6281 \mathrm{E}+01$ & $1.711 \mathrm{E}-11$ & $1.820 \mathrm{E}+01$ \\
\hline $1 s 5 f^{1} F_{3}^{o}$ & $1 s 6 h^{3} H_{4}^{o}$ & $2.6398 \mathrm{E}+01$ & $6.215 \mathrm{E}-10$ & $6.610 \mathrm{E}+02$ & $2.6287 \mathrm{E}+01$ & $5.992 \mathrm{E}-10$ & $6.372 \mathrm{E}+02$ \\
\hline $1 s 6 f^{3} F_{3}^{o}$ & $1 s 6 h^{3} H_{4}^{0}$ & $3.4128 \mathrm{E}+03$ & $1.701 \mathrm{E}-18$ & $1.082 \mathrm{E}-10$ & & & \\
\hline $1 s 6 f^{3} F_{4}^{o}$ & $1 s 6 h^{3} H_{4}^{0}$ & $8.9725 E+03$ & $8.514 \mathrm{E}-16$ & $7.838 \mathrm{E}-09$ & $9.2780 \mathrm{E}+03$ & $9.738 \mathrm{E}-16$ & $8.313 E-09$ \\
\hline $1 s 6 f^{1} F_{3}^{o}$ & $1 s 6 h^{3} H_{4}^{0}$ & $9.4584 \mathrm{E}+03$ & $3.479 \mathrm{E}-14$ & $2.882 \mathrm{E}-07$ & $9.2889 \mathrm{E}+03$ & $3.404 \mathrm{E}-14$ & $2.899 \mathrm{E}-07$ \\
\hline $1 s 6 h^{3} H_{5}^{o}$ & $1 s 6 h^{3} H_{4}^{0}$ & $3.5443 \mathrm{E}+05$ & $5.462 \mathrm{E}-08$ & $3.222 \mathrm{E}-04$ & & & \\
\hline $1 s 3 d^{3} D_{3}$ & $1 s 6 g^{1} G_{4}$ & $3.8727 \mathrm{E}+00$ & $9.494 \mathrm{E}-14$ & $4.692 \mathrm{E}+00$ & & & \\
\hline $1 s 4 d^{3} D_{3}$ & $1 s 6 g^{1} G_{4}$ & $9.2623 \mathrm{E}+00$ & $1.894 \mathrm{E}-17$ & $1.636 \mathrm{E}-04$ & & & \\
\hline $1 s 5 d^{3} D_{3}$ & $1 s 6 g{ }^{1} G_{4}$ & $2.6167 \mathrm{E}+01$ & $4.856 \mathrm{E}-15$ & $5.256 \mathrm{E}-03$ & & & \\
\hline $1 s 5 g{ }^{3} G_{4}$ & $1 s 6 g{ }^{1} G_{4}$ & $2.6392 \mathrm{E}+01$ & $7.790 \mathrm{E}-11$ & $8.290 \mathrm{E}+01$ & $2.6281 \mathrm{E}+01$ & $7.613 \mathrm{E}-11$ & $8.100 \mathrm{E}+01$ \\
\hline $1 s 5 g{ }^{3} G_{3}$ & $1 s 6 g^{1} G_{4}$ & $2.6397 \mathrm{E}+01$ & $2.401 \mathrm{E}-09$ & $2.553 \mathrm{E}+03$ & $2.6286 \mathrm{E}+01$ & $2.423 \mathrm{E}-09$ & $2.577 \mathrm{E}+03$ \\
\hline $1 s 5 g{ }^{3} G_{5}$ & $1 s 6 g^{1} G_{4}$ & $2.6522 \mathrm{E}+01$ & $1.155 \mathrm{E}-09$ & $1.217 \mathrm{E}+03$ & $2.6411 \mathrm{E}+01$ & $1.024 \mathrm{E}-09$ & $1.079 \mathrm{E}+03$ \\
\hline $1 s 5 g{ }^{1} G_{4}$ & $1 s 6 g^{1} G_{4}$ & $2.6527 \mathrm{E}+01$ & $4.522 \mathrm{E}-08$ & $4.763 \mathrm{E}+04$ & $2.6415 \mathrm{E}+01$ & $4.506 \mathrm{E}-08$ & $4.745 E+04$ \\
\hline $1 s 6 d^{3} D_{3}$ & $1 s 6 g^{1} G_{4}$ & $3.3331 \mathrm{E}+03$ & $4.467 \mathrm{E}-15$ & $2.980 \mathrm{E}-07$ & & & \\
\hline $1 s 6 g{ }^{3} G_{4}$ & $1 s 6 g^{1} G_{4}$ & $8.9597 \mathrm{E}+03$ & $4.990 \mathrm{E}-08$ & $4.607 \mathrm{E}-01$ & $9.2558 \mathrm{E}+03$ & $4.823 \mathrm{E}-08$ & $4.137 \mathrm{E}-01$ \\
\hline $1 s 6 g{ }^{3} G_{3}$ & $1 s 6 g{ }^{1} G_{4}$ & $9.3244 \mathrm{E}+03$ & $1.689 \mathrm{E}-06$ & $1.440 \mathrm{E}+01$ & $9.2557 \mathrm{E}+03$ & $1.688 \mathrm{E}-06$ & $1.448 \mathrm{E}+01$ \\
\hline $1 s 6 g{ }^{3} G_{5}$ & $1 s 6 g^{1} G_{4}$ & $2.8974 \mathrm{E}+05$ & $4.257 \mathrm{E}-08$ & $3.758 \mathrm{E}-04$ & & & \\
\hline $1 s 6 h^{3} H_{5}^{o}$ & $1 s 6 h^{3} H_{6}^{o}$ & $1.3919 \mathrm{E}+04$ & $1.709 \mathrm{E}-06$ & $4.525 \mathrm{E}+00$ & $1.3932 \mathrm{E}+04$ & $1.704 \mathrm{E}-06$ & $4.466 \mathrm{E}+00$ \\
\hline $1 s 4 f^{3} F_{4}^{o}$ & $1 s 6 h^{1} H_{5}^{o}$ & $9.3106 \mathrm{E}+00$ & $2.276 \mathrm{E}-14$ & $1.592 \mathrm{E}-01$ & & & \\
\hline $1 s 5 f^{3} F_{4}^{0}$ & $1 s 6 h^{1} H_{5}^{o}$ & $2.6342 \mathrm{E}+01$ & $3.217 \mathrm{E}-15$ & $2.811 \mathrm{E}-03$ & & & \\
\hline $1 s 6 f^{3} F_{4}^{0}$ & $1 s 6 h^{1} H_{5}^{o}$ & $5.4702 \mathrm{E}+03$ & $1.230 \mathrm{E}-18$ & $2.493 \mathrm{E}-11$ & & & \\
\hline $1 s 6 h^{3} H_{5}^{o}$ & $1 s 6 h^{1} H_{5}^{o}$ & $1.3481 \mathrm{E}+04$ & $2.713 \mathrm{E}-08$ & $9.052 \mathrm{E}-02$ & $1.3932 \mathrm{E}+04$ & $2.621 \mathrm{E}-08$ & $8.121 \mathrm{E}-02$ \\
\hline $1 s 6 h^{3} H_{4}^{o}$ & $1 s 6 h^{1} H_{5}^{o}$ & $1.4014 \mathrm{E}+04$ & $1.418 \mathrm{E}-06$ & $4.378 \mathrm{E}+00$ & $1.3932 \mathrm{E}+04$ & $1.415 \mathrm{E}-06$ & $4.385 \mathrm{E}+00$ \\
\hline $1 s 6 h^{3} H_{6}^{0}$ & $1 s 6 h^{1} H_{5}^{o}$ & $4.2819 \mathrm{E}+05$ & $3.644 \mathrm{E}-08$ & $1.205 \mathrm{E}-04$ & & & \\
\hline $1 s^{2}{ }^{1} S_{0}$ & $1 s 7 s^{3} s_{1}$ & $3.1410 \mathrm{E}-01$ & $6.926 \mathrm{E}-07$ & $1.561 \mathrm{E}+10$ & $3.1278 \mathrm{E}-01$ & $6.900 \mathrm{E}-07$ & $1.554 \mathrm{E}+10$ \\
\hline $1 s 2 s^{3} S_{1}$ & $1 s 7 s^{3} S_{1}$ & $1.3268 \mathrm{E}+00$ & $5.679 \mathrm{E}-08$ & $7.173 \mathrm{E}+07$ & $1.3212 \mathrm{E}+00$ & $5.741 \mathrm{E}-08$ & $7.251 \mathrm{E}+07$ \\
\hline $1 s 2 s{ }^{1} S_{0}$ & $1 s 7 s^{3} S_{1}$ & $1.3390 \mathrm{E}+00$ & $3.028 \mathrm{E}-08$ & $3.755 \mathrm{E}+07$ & $1.3335 \mathrm{E}+00$ & $2.894 \mathrm{E}-08$ & $3.588 \mathrm{E}+07$ \\
\hline $1 s 3 s^{3} S_{1}$ & $1 s 7 s^{3} S_{1}$ & $3.3983 \mathrm{E}+00$ & $1.255 \mathrm{E}-08$ & $2.417 \mathrm{E}+06$ & $3.3842 \mathrm{E}+00$ & $1.249 \mathrm{E}-08$ & $2.405 E+06$ \\
\hline $1 s 3 s{ }^{1} S_{0}$ & $1 s 7 s^{3} S_{1}$ & $3.4193 \mathrm{E}+00$ & $6.643 \mathrm{E}-09$ & $1.263 \mathrm{E}+06$ & $3.4054 \mathrm{E}+00$ & $6.283 \mathrm{E}-09$ & $1.194 \mathrm{E}+06$ \\
\hline $1 s 3 d^{3} D_{2}$ & $1 s 7 s^{3} S_{1}$ & $3.5389 \mathrm{E}+00$ & $1.439 \mathrm{E}-13$ & $2.554 \mathrm{E}+01$ & & & \\
\hline $1 s 3 d^{3} D_{1}$ & $1 s 7 s^{3} S_{1}$ & $3.5404 \mathrm{E}+00$ & $4.644 \mathrm{E}-12$ & $8.237 \mathrm{E}+02$ & & & \\
\hline $1 s 3 d{ }^{1} D_{2}$ & $1 s 7 s^{3} S_{1}$ & $3.5774 \mathrm{E}+00$ & $7.601 \mathrm{E}-13$ & $1.321 \mathrm{E}+02$ & & & \\
\hline $1 s 4 s^{3} S_{1}$ & $1 s 7 s^{3} S_{1}$ & $7.3787 \mathrm{E}+00$ & $4.062 \mathrm{E}-09$ & $1.659 \mathrm{E}+05$ & $7.3486 \mathrm{E}+00$ & $4.055 \mathrm{E}-09$ & $1.655 \mathrm{E}+05$ \\
\hline $1 s 4 s^{1} S_{0}$ & $1 s 7 s^{3} S_{1}$ & $7.4188 \mathrm{E}+00$ & $2.370 \mathrm{E}-09$ & $9.573 \mathrm{E}+04$ & $7.3889 E+00$ & $2.038 \mathrm{E}-09$ & $8.229 E+04$ \\
\hline $1 s 4 d^{3} D_{2}$ & $1 s 7 s^{3} S_{1}$ & $7.6552 \mathrm{E}+00$ & $5.626 \mathrm{E}-13$ & $2.135 \mathrm{E}+01$ & $7.6242 \mathrm{E}+00$ & $2.363 \mathrm{E}-12$ & $8.964 \mathrm{E}+01$ \\
\hline $1 s 4 d^{3} D_{1}$ & $1 s 7 s^{3} S_{1}$ & $7.6581 \mathrm{E}+00$ & $6.580 \mathrm{E}-12$ & $2.495 \mathrm{E}+02$ & & & \\
\hline $1 s 4 d{ }^{1} D_{2}$ & $1 s 7 s^{3} S_{1}$ & $7.7314 \mathrm{E}+00$ & $9.050 \mathrm{E}-13$ & $3.366 \mathrm{E}+01$ & & & \\
\hline $1 s 5 s^{3} S_{1}$ & $1 s 7 s^{3} S_{1}$ & $1.5931 \mathrm{E}+01$ & $1.585 \mathrm{E}-09$ & $1.389 \mathrm{E}+04$ & $1.5867 \mathrm{E}+01$ & $1.594 \mathrm{E}-09$ & $1.396 \mathrm{E}+04$ \\
\hline $1 s 5 s{ }^{1} S_{0}$ & $1 s 7 s^{3} S_{1}$ & $1.6025 \mathrm{E}+01$ & $1.087 \mathrm{E}-09$ & $9.413 \mathrm{E}+03$ & $1.5961 \mathrm{E}+01$ & $8.018 \mathrm{E}-10$ & $6.938 \mathrm{E}+03$ \\
\hline $1 s 5 d^{3} D_{2}$ & $1 s 7 s^{3} S_{1}$ & $1.6590 \mathrm{E}+01$ & $5.370 \mathrm{E}-13$ & $4.338 \mathrm{E}+00$ & $1.6526 \mathrm{E}+01$ & $2.554 \mathrm{E}-12$ & $2.062 \mathrm{E}+01$ \\
\hline $1 s 5 d^{3} D_{1}$ & $1 s 7 s^{3} S_{1}$ & $1.6597 \mathrm{E}+01$ & $8.216 \mathrm{E}-12$ & $6.632 \mathrm{E}+01$ & $1.6532 \mathrm{E}+01$ & $6.071 \mathrm{E}-13$ & $4.897 \mathrm{E}+00$ \\
\hline $1 s 5 d^{1} D_{2}$ & $1 s 7 s^{3} S_{1}$ & $1.6773 \mathrm{E}+01$ & $1.120 \mathrm{E}-12$ & $8.850 \mathrm{E}+00$ & & & \\
\hline $1 s 6 s^{3} S_{1}$ & $1 s 7 s^{3} S_{1}$ & $4.2495 \mathrm{E}+01$ & $6.431 \mathrm{E}-10$ & $7.918 \mathrm{E}+02$ & $4.2299 \mathrm{E}+01$ & $6.487 \mathrm{E}-10$ & $7.993 \mathrm{E}+02$ \\
\hline $1 s 6 s^{1} S_{0}$ & $1 s 7 s^{3} S_{1}$ & $4.2877 \mathrm{E}+01$ & $5.937 \mathrm{E}-10$ & $7.180 \mathrm{E}+02$ & $4.2623 \mathrm{E}+01$ & $3.267 \mathrm{E}-10$ & $3.965 \mathrm{E}+02$ \\
\hline $1 s 6 d^{3} D_{2}$ & $1 s 7 s^{3} S_{1}$ & $4.5259 \mathrm{E}+01$ & $1.365 \mathrm{E}-13$ & $1.482 \mathrm{E}-01$ & $4.5119 \mathrm{E}+01$ & $2.252 \mathrm{E}-12$ & $2.439 \mathrm{E}+00$ \\
\hline $1 s 6 d^{3} D_{1}$ & $1 s 7 s^{3} S_{1}$ & $4.5288 \mathrm{E}+01$ & $1.227 \mathrm{E}-11$ & $1.330 \mathrm{E}+01$ & $4.5112 \mathrm{E}+01$ & $4.512 \mathrm{E}-13$ & $4.887 \mathrm{E}-01$ \\
\hline $1 s 6 d{ }^{1} D_{2}$ & $1 s 7 s^{3} S_{1}$ & $4.6054 \mathrm{E}+01$ & $1.888 \mathrm{E}-12$ & $1.980 \mathrm{E}+00$ & & & \\
\hline $1 s 2 p^{3} P_{1}^{o}$ & $1 s 7 p^{3} P_{1}^{o}$ & $1.3376 \mathrm{E}+00$ & $1.508 \mathrm{E}-08$ & $1.873 \mathrm{E}+07$ & $1.3321 \mathrm{E}+00$ & $1.333 \mathrm{E}-08$ & $1.656 \mathrm{E}+07$ \\
\hline $1 s 2 p^{3} P_{0}^{o}$ & $1 s 7 p^{3} P_{1}^{o}$ & $1.3385 E+00$ & $4.191 \mathrm{E}-09$ & $5.202 \mathrm{E}+06$ & $1.3329 E+00$ & $8.068 \mathrm{E}-09$ & $1.001 \mathrm{E}+07$ \\
\hline $1 s 2 p^{3} P_{2}^{o}$ & $1 s 7 p^{3} P_{1}^{o}$ & $1.3961 \mathrm{E}+00$ & $2.546 \mathrm{E}-07$ & $2.904 \mathrm{E}+08$ & $1.3903 \mathrm{E}+00$ & $2.530 \mathrm{E}-07$ & $2.886 \mathrm{E}+08$ \\
\hline $1 s 2 p^{1} P_{1}^{o}$ & $1 s 7 p^{3} P_{1}^{o}$ & $1.4017 \mathrm{E}+00$ & $5.769 \mathrm{E}-08$ & $6.529 \mathrm{E}+07$ & $1.3959 \mathrm{E}+00$ & $5.678 \mathrm{E}-08$ & $6.424 \mathrm{E}+07$ \\
\hline $1 s 3 p^{3} P_{1}^{o}$ & $1 s 7 p^{3} P_{1}^{o}$ & $3.4164 \mathrm{E}+00$ & $5.090 \mathrm{E}-09$ & $9.696 \mathrm{E}+05$ & $3.4024 \mathrm{E}+00$ & $3.619 \mathrm{E}-09$ & $6.892 \mathrm{E}+05$ \\
\hline $1 s 3 p^{3} P_{0}^{o}$ & $1 s 7 p^{3} P_{1}^{o}$ & $3.4179 \mathrm{E}+00$ & $9.304 \mathrm{E}-10$ & $1.771 \mathrm{E}+05$ & $3.4038 \mathrm{E}+00$ & $2.752 \mathrm{E}-09$ & $5.236 \mathrm{E}+05$ \\
\hline $1 s 3 p^{3} P_{2}^{o}$ & $1 s 7 p^{3} P_{1}^{o}$ & $3.5289 \mathrm{E}+00$ & $1.888 \mathrm{E}-07$ & $3.370 \mathrm{E}+07$ & $3.5142 \mathrm{E}+00$ & $1.914 \mathrm{E}-07$ & $3.417 \mathrm{E}+07$ \\
\hline $1 s 3 p{ }^{1} P_{1}^{o}$ & $1 s 7 p^{3} P_{1}^{o}$ & $3.5388 \mathrm{E}+00$ & $4.200 \mathrm{E}-08$ & $7.456 \mathrm{E}+06$ & $3.5243 E+00$ & $4.122 \mathrm{E}-08$ & $7.316 \mathrm{E}+06$ \\
\hline $1 s 4 p^{3} P_{1}^{o}$ & $1 s 7 p^{3} P_{1}^{o}$ & $7.4094 \mathrm{E}+00$ & $2.361 \mathrm{E}-09$ & $9.563 \mathrm{E}+04$ & $7.3785 \mathrm{E}+00$ & $1.251 \mathrm{E}-09$ & $5.067 \mathrm{E}+04$ \\
\hline $1 s 4 p^{3} P_{0}^{o}$ & $1 s 7 p^{3} P_{1}^{o}$ & $7.4122 \mathrm{E}+00$ & $1.525 \mathrm{E}-10$ & $6.170 \mathrm{E}+03$ & $7.3811 \mathrm{E}+00$ & $1.273 \mathrm{E}-09$ & $5.152 \mathrm{E}+04$ \\
\hline $1 s 4 p^{3} P_{2}^{o}$ & $1 s 7 p^{3} P_{1}^{0}$ & $7.6311 \mathrm{E}+00$ & $1.667 \mathrm{E}-07$ & $6.363 \mathrm{E}+06$ & $7.5991 \mathrm{E}+00$ & $1.690 \mathrm{E}-07$ & $6.451 \mathrm{E}+06$ \\
\hline $1 s 4 p{ }^{1} P_{1}^{o}$ & $1 s 7 p^{3} P_{1}^{o}$ & $7.6504 \mathrm{E}+00$ & $3.684 \mathrm{E}-08$ & $1.400 \mathrm{E}+06$ & $7.6186 \mathrm{E}+00$ & $3.546 \mathrm{E}-08$ & $1.347 \mathrm{E}+06$ \\
\hline $1 s 4 f^{3} F_{2}^{o}$ & $1 s 7 p^{3} P_{1}^{o}$ & $7.7232 \mathrm{E}+00$ & $3.851 \mathrm{E}-14$ & $1.436 \mathrm{E}+00$ & & & \\
\hline $1 s 5 p^{3} P_{1}^{o}$ & $1 s 7 p^{3} P_{1}^{o}$ & $1.5987 \mathrm{E}+01$ & $1.514 \mathrm{E}-09$ & $1.317 \mathrm{E}+04$ & $1.5920 \mathrm{E}+01$ & $4.931 \mathrm{E}-10$ & $4.289 \mathrm{E}+03$ \\
\hline $1 s 5 p^{3} P_{0}^{o}$ & $1 s 7 p^{3} P_{1}^{o}$ & $1.5993 \mathrm{E}+01$ & $6.465 \mathrm{E}-13$ & $5.620 \mathrm{E}+00$ & $1.5926 \mathrm{E}+01$ & $7.581 \mathrm{E}-10$ & $6.589 \mathrm{E}+03$ \\
\hline
\end{tabular}


Table 5 (continued)

\begin{tabular}{|c|c|c|c|c|c|c|c|}
\hline \multirow[t]{2}{*}{ Lower } & \multirow[t]{2}{*}{ Upper } & \multicolumn{3}{|l|}{ GRASP2K } & \multicolumn{3}{|l|}{ FAC } \\
\hline & & $\lambda(\AA)$ & $g f$ & $A\left(\mathrm{~s}^{-1}\right)$ & $\lambda(\AA)$ & $g f$ & $A\left(\mathrm{~s}^{-1}\right)$ \\
\hline $1 s 5 p^{3} P_{2}^{o}$ & $1 s 7 p^{3} P_{1}^{o}$ & $1.6515 \mathrm{E}+01$ & $1.788 \mathrm{E}-07$ & $1.458 \mathrm{E}+06$ & $1.6446 \mathrm{E}+01$ & $1.815 \mathrm{E}-07$ & $1.480 \mathrm{E}+06$ \\
\hline $1 s 5 p^{1} P_{1}^{o}$ & $1 s 7 p^{3} P_{1}^{o}$ & $1.6561 \mathrm{E}+01$ & $3.921 \mathrm{E}-08$ & $3.179 \mathrm{E}+05$ & $1.6492 \mathrm{E}+01$ & $3.741 \mathrm{E}-08$ & $3.033 E+05$ \\
\hline $1 s 5 f^{3} F_{2}^{o}$ & $1 s 7 p^{3} P_{1}^{o}$ & $1.6736 \mathrm{E}+01$ & $7.663 \mathrm{E}-14$ & $6.083 \mathrm{E}-01$ & & & \\
\hline $1 s 6 p^{3} P_{1}^{o}$ & $1 s 7 p^{3} P_{1}^{o}$ & $4.2622 \mathrm{E}+01$ & $1.096 \mathrm{E}-09$ & $1.342 \mathrm{E}+03$ & $4.2444 \mathrm{E}+01$ & $2.699 E-10$ & $3.304 \mathrm{E}+02$ \\
\hline $1 s 6 p^{3} P_{0}^{o}$ & $1 s 7 p^{3} P_{1}^{o}$ & $4.2648 \mathrm{E}+01$ & $5.644 \mathrm{E}-11$ & $6.899 \mathrm{E}+01$ & $4.2376 \mathrm{E}+01$ & $6.084 \mathrm{E}-10$ & $7.470 \mathrm{E}+02$ \\
\hline $1 s 6 p^{3} P_{2}^{o}$ & $1 s 7 p^{3} P_{1}^{o}$ & $4.4826 \mathrm{E}+01$ & $2.721 \mathrm{E}-07$ & $3.011 \mathrm{E}+05$ & $4.4636 \mathrm{E}+01$ & $2.777 \mathrm{E}-07$ & $3.073 \mathrm{E}+05$ \\
\hline $1 s 6 p^{1} P_{1}^{o}$ & $1 s 7 p^{3} P_{1}^{0}$ & $4.5021 \mathrm{E}+01$ & $5.950 \mathrm{E}-08$ & $6.527 \mathrm{E}+04$ & $4.4809 \mathrm{E}+01$ & $5.628 \mathrm{E}-08$ & $6.180 \mathrm{E}+04$ \\
\hline $1 s 6 f^{3} F_{2}^{o}$ & $1 s 7 p^{3} P_{1}^{o}$ & $4.5775 \mathrm{E}+01$ & $1.568 \mathrm{E}-13$ & $1.663 \mathrm{E}-01$ & & & \\
\hline $1 s 2 s^{3} S_{1}$ & $1 s 7 s^{1} S_{0}$ & $1.3266 \mathrm{E}+00$ & $2.842 \mathrm{E}-08$ & $1.077 \mathrm{E}+08$ & $1.3210 \mathrm{E}+00$ & $2.870 \mathrm{E}-08$ & $1.087 E+08$ \\
\hline $1 s 3 s^{3} S_{1}$ & $1 s 7 s^{1} S_{0}$ & $3.3968 \mathrm{E}+00$ & $5.855 \mathrm{E}-09$ & $3.385 \mathrm{E}+06$ & $3.3830 \mathrm{E}+00$ & $6.245 \mathrm{E}-09$ & $3.609 E+06$ \\
\hline $1 s 3 d^{3} D_{1}$ & $1 s 7 s{ }^{1} S_{0}$ & $3.5388 \mathrm{E}+00$ & $6.161 \mathrm{E}-13$ & $3.281 \mathrm{E}+02$ & & & \\
\hline $1 s 4 s^{3} S_{1}$ & $1 s 7 s^{1} S_{0}$ & $7.3716 \mathrm{E}+00$ & $1.719 \mathrm{E}-09$ & $2.110 \mathrm{E}+05$ & $7.3425 \mathrm{E}+00$ & $2.026 \mathrm{E}-09$ & $2.485 E+05$ \\
\hline $1 s 4 d^{3} D_{1}$ & $1 s 7 s^{1} S_{0}$ & $7.6504 \mathrm{E}+00$ & $8.796 \mathrm{E}-13$ & $1.002 \mathrm{E}+02$ & $7.6206 \mathrm{E}+00$ & $8.205 E-13$ & $9.344 \mathrm{E}+01$ \\
\hline $1 s 5 s^{3} S_{1}$ & $1 s 7 s^{1} S_{0}$ & $1.5898 \mathrm{E}+01$ & $5.646 \mathrm{E}-10$ & $1.490 \mathrm{E}+04$ & $1.5839 \mathrm{E}+01$ & $7.961 \mathrm{E}-10$ & $2.098 \mathrm{E}+04$ \\
\hline $1 s 5 d^{3} D_{1}$ & $1 s 7 s^{1} S_{0}$ & $1.6561 \mathrm{E}+01$ & $9.135 E-13$ & $2.222 \mathrm{E}+01$ & $1.6502 \mathrm{E}+01$ & $9.451 \mathrm{E}-13$ & $2.295 \mathrm{E}+01$ \\
\hline $1 s 6 s^{3} S_{1}$ & $1 s 7 s^{1} S_{0}$ & $4.2259 \mathrm{E}+01$ & $1.431 \mathrm{E}-10$ & $5.345 \mathrm{E}+02$ & $4.2100 \mathrm{E}+01$ & $3.228 \mathrm{E}-10$ & $1.204 \mathrm{E}+03$ \\
\hline $1 s 6 d^{3} D_{1}$ & $1 s 7 s^{1} S_{0}$ & $4.5020 \mathrm{E}+01$ & $8.016 \mathrm{E}-13$ & $2.638 \mathrm{E}+00$ & $4.4886 \mathrm{E}+01$ & $9.411 \mathrm{E}-13$ & $3.089 \mathrm{E}+00$ \\
\hline $1 s 7 s^{3} S_{1}$ & $1 s 7 s{ }^{1} S_{0}$ & $7.6139 \mathrm{E}+03$ & $4.456 \mathrm{E}-09$ & $5.128 \mathrm{E}-01$ & $8.9405 E+03$ & $3.689 \mathrm{E}-09$ & $3.052 \mathrm{E}-01$ \\
\hline $1 s 2 p^{3} P_{1}^{o}$ & $1 s 7 p^{3} P_{0}^{o}$ & $1.3376 \mathrm{E}+00$ & $5.365 \mathrm{E}-09$ & $2.000 \mathrm{E}+07$ & $1.3321 \mathrm{E}+00$ & $4.432 \mathrm{E}-09$ & $1.652 \mathrm{E}+07$ \\
\hline $1 s 2 p^{1} P_{1}^{o}$ & $1 s 7 p^{3} P_{0}^{o}$ & $1.4016 \mathrm{E}+00$ & $1.035 \mathrm{E}-07$ & $3.514 \mathrm{E}+08$ & $1.3959 \mathrm{E}+00$ & $1.057 \mathrm{E}-07$ & $3.589 \mathrm{E}+08$ \\
\hline $1 s 3 p^{3} P_{1}^{0}$ & $1 s 7 p^{3} P_{0}^{o}$ & $3.4163 \mathrm{E}+00$ & $1.518 \mathrm{E}-09$ & $8.674 \mathrm{E}+05$ & $3.4026 \mathrm{E}+00$ & $9.937 \mathrm{E}-10$ & $5.676 E+05$ \\
\hline $1 s 3 p^{1} P_{1}^{o}$ & $1 s 7 p^{3} P_{0}^{o}$ & $3.5387 \mathrm{E}+00$ & $7.620 \mathrm{E}-08$ & $4.059 \mathrm{E}+07$ & $3.5246 \mathrm{E}+00$ & $7.860 \mathrm{E}-08$ & $4.184 \mathrm{E}+07$ \\
\hline $1 s 4 p^{3} P_{1}^{o}$ & $1 s 7 p^{3} P_{0}^{o}$ & $7.4089 \mathrm{E}+00$ & $4.150 \mathrm{E}-10$ & $5.042 \mathrm{E}+04$ & $7.3797 \mathrm{E}+00$ & $2.397 \mathrm{E}-10$ & $2.912 \mathrm{E}+04$ \\
\hline $1 s 4 p^{1} P_{1}^{o}$ & $1 s 7 p^{3} P_{0}^{0}$ & $7.6499 \mathrm{E}+00$ & $6.694 \mathrm{E}-08$ & $7.630 \mathrm{E}+06$ & $7.6199 \mathrm{E}+00$ & $6.869 \mathrm{E}-08$ & $7.824 \mathrm{E}+06$ \\
\hline $1 s 5 p^{3} P_{1}^{o}$ & $1 s 7 p^{3} P_{0}^{o}$ & $1.5984 \mathrm{E}+01$ & $8.383 \mathrm{E}-11$ & $2.189 \mathrm{E}+03$ & $1.5926 \mathrm{E}+01$ & $3.891 \mathrm{E}-11$ & $1.014 \mathrm{E}+03$ \\
\hline $1 s 5 p^{1} P_{1}^{o}$ & $1 s 7 p^{3} P_{0}^{o}$ & $1.6558 \mathrm{E}+01$ & $7.163 \mathrm{E}-08$ & $1.743 \mathrm{E}+06$ & $1.6498 \mathrm{E}+01$ & $7.327 \mathrm{E}-08$ & $1.780 \mathrm{E}+06$ \\
\hline $1 s 6 p^{3} P_{1}^{o}$ & $1 s 7 p^{3} P_{0}^{o}$ & $4.2605 \mathrm{E}+01$ & $7.779 \mathrm{E}-12$ & $2.859 \mathrm{E}+01$ & $4.2486 \mathrm{E}+01$ & $2.961 \mathrm{E}-11$ & $1.085 \mathrm{E}+02$ \\
\hline $1 s 6 p^{1} P_{1}^{o}$ & $1 s 7 p^{3} P_{0}^{0}$ & $4.5003 \mathrm{E}+01$ & $1.090 \mathrm{E}-07$ & $3.590 \mathrm{E}+05$ & $4.4856 \mathrm{E}+01$ & $1.112 \mathrm{E}-07$ & $3.657 \mathrm{E}+05$ \\
\hline $1 s 7 p^{3} P_{1}^{o}$ & $1 s 7 p^{3} P_{0}^{o}$ & $1.1050 \mathrm{E}+05$ & $4.339 \mathrm{E}-08$ & $2.370 \mathrm{E}-02$ & & & \\
\hline $1 s 2 p^{3} P_{1}^{o}$ & $1 s 7 p^{3} P_{2}^{o}$ & $1.3363 \mathrm{E}+00$ & $1.183 \mathrm{E}-07$ & $8.840 \mathrm{E}+07$ & $1.3308 \mathrm{E}+00$ & $1.215 \mathrm{E}-07$ & $9.078 E+07$ \\
\hline $1 s 2 p^{3} P_{2}^{o}$ & $1 s 7 p^{3} P_{2}^{o}$ & $1.3947 \mathrm{E}+00$ & $1.912 \mathrm{E}-07$ & $1.311 \mathrm{E}+08$ & $1.3889 \mathrm{E}+00$ & $1.835 \mathrm{E}-07$ & $1.258 \mathrm{E}+08$ \\
\hline $1 s 2 p^{1} P_{1}^{o}$ & $1 s 7 p^{3} P_{2}^{o}$ & $1.4002 \mathrm{E}+00$ & $1.400 \mathrm{E}-08$ & $9.523 \mathrm{E}+06$ & $1.3944 \mathrm{E}+00$ & $1.676 \mathrm{E}-08$ & $1.140 \mathrm{E}+07$ \\
\hline $1 s 3 p^{3} P_{1}^{o}$ & $1 s 7 p^{3} P_{2}^{o}$ & $3.4080 \mathrm{E}+00$ & $1.229 \mathrm{E}-07$ & $1.412 \mathrm{E}+07$ & $3.3940 \mathrm{E}+00$ & $1.242 \mathrm{E}-07$ & $1.427 \mathrm{E}+07$ \\
\hline $1 s 3 p^{3} P_{2}^{o}$ & $1 s 7 p^{3} P_{2}^{o}$ & $3.5199 \mathrm{E}+00$ & $5.498 \mathrm{E}-08$ & $5.921 \mathrm{E}+06$ & $3.5053 \mathrm{E}+00$ & $5.309 \mathrm{E}-08$ & $5.715 E+06$ \\
\hline $1 s 3 p{ }^{1} P_{1}^{o}$ & $1 s 7 p^{3} P_{2}^{o}$ & $3.5298 \mathrm{E}+00$ & $2.478 \mathrm{E}-09$ & $2.654 \mathrm{E}+05$ & $3.5153 \mathrm{E}+00$ & $4.084 \mathrm{E}-09$ & $4.372 \mathrm{E}+05$ \\
\hline $1 s 4 p^{3} P_{1}^{0}$ & $1 s 7 p^{3} P_{2}^{0}$ & $7.3699 \mathrm{E}+00$ & $1.253 \mathrm{E}-07$ & $3.077 \mathrm{E}+06$ & $7.3391 \mathrm{E}+00$ & $1.273 \mathrm{E}-07$ & $3.128 \mathrm{E}+06$ \\
\hline $1 s 4 p^{3} P_{2}^{o}$ & $1 s 7 p^{3} P_{2}^{o}$ & $7.5892 \mathrm{E}+00$ & $2.251 \mathrm{E}-08$ & $5.215 \mathrm{E}+05$ & $7.5574 \mathrm{E}+00$ & $1.936 \mathrm{E}-08$ & $4.484 \mathrm{E}+05$ \\
\hline $1 s 4 p^{1} P_{1}^{o}$ & $1 s 7 p^{3} P_{2}^{o}$ & $7.6083 \mathrm{E}+00$ & $2.879 \mathrm{E}-10$ & $6.635 E+03$ & $7.5766 \mathrm{E}+00$ & $1.124 \mathrm{E}-09$ & $2.590 \mathrm{E}+04$ \\
\hline $1 s 4 f^{3} F_{3}^{o}$ & $1 s 7 p^{3} P_{2}^{o}$ & $7.6787 \mathrm{E}+00$ & $4.986 \mathrm{E}-18$ & $1.128 \mathrm{E}-04$ & & & \\
\hline $1 s 4 f^{3} F_{2}^{o}$ & $1 s 7 p^{3} P_{2}^{o}$ & $7.6803 \mathrm{E}+00$ & $4.502 \mathrm{E}-16$ & $1.018 \mathrm{E}-02$ & & & \\
\hline $1 s 4 f^{1} F_{3}^{o}$ & $1 s 7 p^{3} P_{2}^{o}$ & $7.7161 \mathrm{E}+00$ & $5.132 \mathrm{E}-15$ & $1.150 \mathrm{E}-01$ & & & \\
\hline $1 s 5 p^{3} P_{1}^{0}$ & $1 s 7 p^{3} P_{2}^{o}$ & $1.5804 \mathrm{E}+01$ & $1.465 \mathrm{E}-07$ & $7.824 \mathrm{E}+05$ & $1.5738 \mathrm{E}+01$ & $1.489 \mathrm{E}-07$ & $7.952 E+05$ \\
\hline $1 s 5 p^{3} P_{2}^{o}$ & $1 s 7 p^{3} P_{2}^{o}$ & $1.6320 \mathrm{E}+01$ & $1.116 \mathrm{E}-08$ & $5.589 \mathrm{E}+04$ & $1.6251 \mathrm{E}+01$ & $8.133 E-09$ & $4.073 \mathrm{E}+04$ \\
\hline $1 s 5 p^{1} P_{1}^{o}$ & $1 s 7 p^{3} P_{2}^{o}$ & $1.6364 \mathrm{E}+01$ & $2.116 \mathrm{E}-11$ & $1.054 \mathrm{E}+02$ & $1.6297 \mathrm{E}+01$ & $2.649 \mathrm{E}-10$ & $1.319 \mathrm{E}+03$ \\
\hline $1 s 5 f^{3} F_{3}^{o}$ & $1 s 7 p^{3} P_{2}^{o}$ & $1.6532 \mathrm{E}+01$ & $1.343 \mathrm{E}-13$ & $6.555 \mathrm{E}-01$ & & & \\
\hline $1 s 5 f^{3} F_{2}^{o}$ & $1 s 7 p^{3} P_{2}^{o}$ & $1.6535 \mathrm{E}+01$ & $4.833 \mathrm{E}-14$ & $2.358 \mathrm{E}-01$ & & & \\
\hline $1 s 5 f^{1} F_{3}^{o}$ & $1 s 7 p^{3} P_{2}^{o}$ & $1.6621 \mathrm{E}+01$ & $1.386 \mathrm{E}-14$ & $6.691 \mathrm{E}-02$ & & & \\
\hline $1 s 6 p^{3} P_{1}^{0}$ & $1 s 7 p^{3} P_{2}^{o}$ & $4.1345 \mathrm{E}+01$ & $2.311 \mathrm{E}-07$ & $1.803 \mathrm{E}+05$ & $4.1174 \mathrm{E}+01$ & $2.351 \mathrm{E}-07$ & $1.834 \mathrm{E}+05$ \\
\hline $1 s 6 p^{3} P_{2}^{o}$ & $1 s 7 p^{3} P_{2}^{o}$ & $4.3416 \mathrm{E}+01$ & $5.959 \mathrm{E}-09$ & $4.217 \mathrm{E}+03$ & $4.3234 \mathrm{E}+01$ & $3.466 \mathrm{E}-09$ & $2.452 \mathrm{E}+03$ \\
\hline $1 s 6 p^{1} P_{1}^{o}$ & $1 s 7 p^{3} P_{2}^{o}$ & $4.3599 \mathrm{E}+01$ & $5.062 \mathrm{E}-10$ & $3.553 \mathrm{E}+02$ & & & \\
\hline $1 s 6 f^{3} F_{3}^{o}$ & $1 s 7 p^{3} P_{2}^{o}$ & $4.4291 \mathrm{E}+01$ & $5.946 \mathrm{E}-13$ & $4.044 \mathrm{E}-01$ & & & \\
\hline $1 s 6 f^{3} F_{2}^{o}$ & $1 s 7 p^{3} P_{2}^{o}$ & $4.4306 \mathrm{E}+01$ & $2.356 \mathrm{E}-13$ & $1.601 \mathrm{E}-01$ & & & \\
\hline $1 s 6 f^{1} F_{3}^{o}$ & $1 s 7 p^{3} P_{2}^{o}$ & $4.4661 \mathrm{E}+01$ & $3.239 \mathrm{E}-14$ & $2.166 \mathrm{E}-02$ & & & \\
\hline $1 s 7 p^{3} P_{1}^{o}$ & $1 s 7 p^{3} P_{2}^{o}$ & $1.3800 \mathrm{E}+03$ & $5.054 \mathrm{E}-06$ & $3.541 \mathrm{E}+03$ & $1.3760 \mathrm{E}+03$ & $5.105 \mathrm{E}-06$ & $3.566 \mathrm{E}+03$ \\
\hline $1 s 2 s^{3} S_{1}$ & $1 s 7 d^{3} D_{2}$ & $1.3252 \mathrm{E}+00$ & $3.914 \mathrm{E}-09$ & $2.974 \mathrm{E}+06$ & $1.3196 \mathrm{E}+00$ & $4.084 \mathrm{E}-09$ & $3.102 E+06$ \\
\hline $1 s 3 s^{3} S_{1}$ & $1 s 7 d^{3} D_{2}$ & $3.3879 \mathrm{E}+00$ & $7.078 \mathrm{E}-10$ & $8.227 \mathrm{E}+04$ & $3.3736 \mathrm{E}+00$ & $7.633 \mathrm{E}-10$ & $8.872 \mathrm{E}+04$ \\
\hline $1 s 3 d^{3} D_{2}$ & $1 s 7 d^{3} D_{2}$ & $3.5276 \mathrm{E}+00$ & $1.603 \mathrm{E}-08$ & $1.718 \mathrm{E}+06$ & $3.5127 \mathrm{E}+00$ & $1.599 \mathrm{E}-08$ & $1.714 \mathrm{E}+06$ \\
\hline $1 s 3 d^{3} D_{1}$ & $1 s 7 d^{3} D_{2}$ & $3.5291 \mathrm{E}+00$ & $1.400 \mathrm{E}-09$ & $1.499 \mathrm{E}+05$ & $3.5142 \mathrm{E}+00$ & $1.857 \mathrm{E}-09$ & $1.989 \mathrm{E}+05$ \\
\hline $1 s 3 d^{3} D_{3}$ & $1 s 7 d^{3} D_{2}$ & $3.5644 \mathrm{E}+00$ & $2.985 \mathrm{E}-08$ & $3.134 \mathrm{E}+06$ & $3.5492 \mathrm{E}+00$ & $2.966 \mathrm{E}-08$ & $3.115 E+06$ \\
\hline $1 s 3 d^{1} D_{2}$ & $1 s 7 d^{3} D_{2}$ & $3.5659 \mathrm{E}+00$ & $2.352 \mathrm{E}-09$ & $2.468 \mathrm{E}+05$ & $3.5507 \mathrm{E}+00$ & $2.360 \mathrm{E}-09$ & $2.476 \mathrm{E}+05$ \\
\hline $1 s 4 s^{3} S_{1}$ & $1 s 7 d^{3} D_{2}$ & $7.3299 \mathrm{E}+00$ & $1.241 \mathrm{E}-10$ & $3.081 \mathrm{E}+03$ & $7.2988 \mathrm{E}+00$ & $1.467 \mathrm{E}-10$ & $3.644 \mathrm{E}+03$ \\
\hline $1 s 4 d^{3} D_{2}$ & $1 s 7 d^{3} D_{2}$ & $7.6027 \mathrm{E}+00$ & $8.357 \mathrm{E}-09$ & $1.929 \mathrm{E}+05$ & $7.5707 \mathrm{E}+00$ & $8.334 \mathrm{E}-09$ & $1.923 E+05$ \\
\hline $1 s 4 d^{3} D_{1}$ & $1 s 7 d^{3} D_{2}$ & $7.6055 \mathrm{E}+00$ & $6.426 \mathrm{E}-10$ & $1.482 \mathrm{E}+04$ & $7.5734 \mathrm{E}+00$ & $9.979 \mathrm{E}-10$ & $2.301 \mathrm{E}+04$ \\
\hline $1 s 4 d^{3} D_{3}$ & $1 s 7 d^{3} D_{2}$ & $7.6748 \mathrm{E}+00$ & $3.028 \mathrm{E}-08$ & $6.858 \mathrm{E}+05$ & $7.6422 \mathrm{E}+00$ & $3.021 \mathrm{E}-08$ & $6.842 \mathrm{E}+05$ \\
\hline $1 s 4 d{ }^{1} D_{2}$ & $1 s 7 d^{3} D_{2}$ & $7.6778 \mathrm{E}+00$ & $2.362 \mathrm{E}-09$ & $5.346 \mathrm{E}+04$ & $7.6453 \mathrm{E}+00$ & $2.315 \mathrm{E}-09$ & $5.239 E+04$ \\
\hline $1 s 5 s^{3} s_{1}$ & $1 s 7 d^{3} D_{2}$ & $1.5705 \mathrm{E}+01$ & $1.638 \mathrm{E}-11$ & $8.860 \mathrm{E}+01$ & $1.5637 \mathrm{E}+01$ & $2.578 \mathrm{E}-11$ & $1.395 E+02$ \\
\hline $1 s 5 d^{3} D_{2}$ & $1 s 7 d^{3} D_{2}$ & $1.6345 \mathrm{E}+01$ & $4.113 \mathrm{E}-09$ & $2.054 \mathrm{E}+04$ & $1.6276 \mathrm{E}+01$ & $4.090 \mathrm{E}-09$ & $2.042 \mathrm{E}+04$ \\
\hline $1 s 5 d^{3} D_{1}$ & $1 s 7 d^{3} D_{2}$ & $1.6352 \mathrm{E}+01$ & $2.503 E-10$ & $1.249 \mathrm{E}+03$ & $1.6283 \mathrm{E}+01$ & $5.152 \mathrm{E}-10$ & $2.570 \mathrm{E}+03$ \\
\hline $1 s 5 d^{3} D_{3}$ & $1 s 7 d^{3} D_{2}$ & $1.6516 \mathrm{E}+01$ & $3.236 \mathrm{E}-08$ & $1.583 \mathrm{E}+05$ & $1.6446 \mathrm{E}+01$ & $3.234 \mathrm{E}-08$ & $1.582 \mathrm{E}+05$ \\
\hline $1 s 5 d{ }^{1} D_{2}$ & $1 s 7 d^{3} D_{2}$ & $1.6523 \mathrm{E}+01$ & $2.512 \mathrm{E}-09$ & $1.227 \mathrm{E}+04$ & $1.6453 \mathrm{E}+01$ & $2.418 \mathrm{E}-09$ & $1.181 \mathrm{E}+04$ \\
\hline $1 s 5 g{ }^{3} G_{3}$ & $1 s 7 d^{3} D_{2}$ & $1.6607 \mathrm{E}+01$ & $9.716 \mathrm{E}-18$ & $4.700 \mathrm{E}-05$ & & & \\
\hline
\end{tabular}


Table 5 (continued)

\begin{tabular}{|c|c|c|c|c|c|c|c|}
\hline \multirow[t]{2}{*}{ Lower } & \multirow[t]{2}{*}{ Upper } & \multicolumn{3}{|l|}{ GRASP2K } & \multicolumn{3}{|l|}{ FAC } \\
\hline & & $\lambda(\AA)$ & $g f$ & $A\left(\mathrm{~s}^{-1}\right)$ & $\lambda(\AA)$ & $g f$ & $A\left(\mathrm{~s}^{-1}\right)$ \\
\hline $1 s 6 s^{3} S_{1}$ & $1 s 7 d^{3} D_{2}$ & $4.0924 \mathrm{E}+01$ & $1.212 \mathrm{E}-14$ & $9.653 \mathrm{E}-03$ & & & \\
\hline $1 s 6 d^{3} D_{2}$ & $1 s 7 d^{3} D_{2}$ & $4.3482 \mathrm{E}+01$ & $1.940 \mathrm{E}-09$ & $1.369 \mathrm{E}+03$ & $4.3306 \mathrm{E}+01$ & $1.938 \mathrm{E}-09$ & $1.367 \mathrm{E}+03$ \\
\hline $1 s 6 d^{3} D_{1}$ & $1 s 7 d^{3} D_{2}$ & $4.3508 \mathrm{E}+01$ & $5.943 \mathrm{E}-11$ & $4.188 \mathrm{E}+01$ & $4.3299 \mathrm{E}+01$ & $2.702 \mathrm{E}-10$ & $1.906 \mathrm{E}+02$ \\
\hline $1 s 6 d^{3} D_{3}$ & $1 s 7 d^{3} D_{2}$ & $4.4184 \mathrm{E}+01$ & $4.703 \mathrm{E}-08$ & $3.214 \mathrm{E}+04$ & $4.4000 \mathrm{E}+01$ & $4.706 \mathrm{E}-08$ & $3.215 \mathrm{E}+04$ \\
\hline $1 s 6 d{ }^{1} D_{2}$ & $1 s 7 d^{3} D_{2}$ & $4.4215 \mathrm{E}+01$ & $3.640 \mathrm{E}-09$ & $2.484 \mathrm{E}+03$ & $4.4010 \mathrm{E}+01$ & $3.423 \mathrm{E}-09$ & $2.338 \mathrm{E}+03$ \\
\hline $1 s 6 g{ }^{3} G_{3}$ & $1 s 7 d^{3} D_{2}$ & $4.4564 \mathrm{E}+01$ & $1.069 \mathrm{E}-16$ & $7.182 \mathrm{E}-05$ & & & \\
\hline $1 s 7 s^{3} S_{1}$ & $1 s 7 d^{3} D_{2}$ & $1.1072 \mathrm{E}+03$ & $3.819 \mathrm{E}-11$ & $4.155 \mathrm{E}-02$ & $1.0773 \mathrm{E}+03$ & $1.749 \mathrm{E}-12$ & $1.994 \mathrm{E}-03$ \\
\hline $1 s^{2}{ }^{1} S_{0}$ & $1 s 7 d^{3} D_{1}$ & $3.1400 \mathrm{E}-01$ & $1.149 \mathrm{E}-08$ & $2.591 \mathrm{E}+08$ & $3.1269 \mathrm{E}-01$ & $1.185 \mathrm{E}-08$ & $2.673 \mathrm{E}+08$ \\
\hline $1 s 2 s^{3} S_{1}$ & $1 s 7 d^{3} D_{1}$ & $1.3252 \mathrm{E}+00$ & $1.091 \mathrm{E}-09$ & $1.381 \mathrm{E}+06$ & $1.3196 \mathrm{E}+00$ & $8.170 \mathrm{E}-10$ & $1.034 \mathrm{E}+06$ \\
\hline $1 s 2 s{ }^{1} S_{0}$ & $1 s 7 d^{3} D_{1}$ & $1.3373 \mathrm{E}+00$ & $1.626 \mathrm{E}-09$ & $2.021 \mathrm{E}+06$ & $1.3319 \mathrm{E}+00$ & $1.633 \mathrm{E}-09$ & $2.029 \mathrm{E}+06$ \\
\hline $1 s 3 s^{3} S_{1}$ & $1 s 7 d^{3} D_{1}$ & $3.3878 \mathrm{E}+00$ & $2.425 \mathrm{E}-10$ & $4.698 E+04$ & $3.3737 \mathrm{E}+00$ & $1.564 \mathrm{E}-10$ & $3.030 \mathrm{E}+04$ \\
\hline $1 s 3 s^{1} S_{0}$ & $1 s 7 d^{3} D_{1}$ & $3.4087 \mathrm{E}+00$ & $2.964 \mathrm{E}-10$ & $5.672 \mathrm{E}+04$ & $3.3947 \mathrm{E}+00$ & $3.091 \mathrm{E}-10$ & $5.914 \mathrm{E}+04$ \\
\hline $1 s 3 d^{3} D_{2}$ & $1 s 7 d^{3} D_{1}$ & $3.5275 \mathrm{E}+00$ & $2.219 \mathrm{E}-09$ & $3.966 \mathrm{E}+05$ & $3.5127 \mathrm{E}+00$ & $1.737 \mathrm{E}-09$ & $3.104 \mathrm{E}+05$ \\
\hline $1 s 3 d^{3} D_{1}$ & $1 s 7 d^{3} D_{1}$ & $3.5290 \mathrm{E}+00$ & $8.898 \mathrm{E}-09$ & $1.589 \mathrm{E}+06$ & $3.5142 \mathrm{E}+00$ & $8.895 \mathrm{E}-09$ & $1.587 \mathrm{E}+06$ \\
\hline $1 s 3 d d^{1} D_{2}$ & $1 s 7 d^{3} D_{1}$ & $3.5657 \mathrm{E}+00$ & $1.895 \mathrm{E}-08$ & $3.314 \mathrm{E}+06$ & $3.5507 \mathrm{E}+00$ & $1.928 \mathrm{E}-08$ & $3.371 \mathrm{E}+06$ \\
\hline $1 s 4 s^{3} S_{1}$ & $1 s 7 d^{3} D_{1}$ & $7.3294 \mathrm{E}+00$ & $6.510 \mathrm{E}-11$ & $2.694 \mathrm{E}+03$ & $7.2989 \mathrm{E}+00$ & $3.075 E-11$ & $1.272 \mathrm{E}+03$ \\
\hline $1 s 4 s^{1} S_{0}$ & $1 s 7 d^{3} D_{1}$ & $7.3689 \mathrm{E}+00$ & $5.760 \mathrm{E}-11$ & $2.358 \mathrm{E}+03$ & $7.3387 \mathrm{E}+00$ & $6.006 \mathrm{E}-11$ & $2.458 \mathrm{E}+03$ \\
\hline $1 s 4 d^{3} D_{2}$ & $1 s 7 d^{3} D_{1}$ & $7.6021 \mathrm{E}+00$ & $1.263 \mathrm{E}-09$ & $4.859 E+04$ & $7.5708 \mathrm{E}+00$ & $8.803 E-10$ & $3.385 \mathrm{E}+04$ \\
\hline $1 s 4 d^{3} D_{1}$ & $1 s 7 d^{3} D_{1}$ & $7.6050 \mathrm{E}+00$ & $4.648 \mathrm{E}-09$ & $1.787 \mathrm{E}+05$ & $7.5736 \mathrm{E}+00$ & $4.638 \mathrm{E}-09$ & $1.782 \mathrm{E}+05$ \\
\hline $1 s 4 d{ }^{1} D_{2}$ & $1 s 7 d^{3} D_{1}$ & $7.6772 \mathrm{E}+00$ & $1.930 \mathrm{E}-08$ & $7.282 \mathrm{E}+05$ & $7.6454 \mathrm{E}+00$ & $1.958 \mathrm{E}-08$ & $7.388 \mathrm{E}+05$ \\
\hline $1 s 5 s^{3} S_{1}$ & $1 s 7 d^{3} D_{1}$ & $1.5703 \mathrm{E}+01$ & $2.284 \mathrm{E}-11$ & $2.060 \mathrm{E}+02$ & $1.5638 \mathrm{E}+01$ & $5.601 \mathrm{E}-12$ & $5.049 \mathrm{E}+01$ \\
\hline $1 s 5 s^{1} s_{0}$ & $1 s 7 d^{3} D_{1}$ & $1.5794 \mathrm{E}+01$ & $1.050 \mathrm{E}-11$ & $9.360 \mathrm{E}+01$ & $1.5729 \mathrm{E}+01$ & $1.078 \mathrm{E}-11$ & $9.608 \mathrm{E}+01$ \\
\hline $1 s 5 d^{3} D_{2}$ & $1 s 7 d^{3} D_{1}$ & $1.6343 \mathrm{E}+01$ & $7.197 \mathrm{E}-10$ & $5.991 \mathrm{E}+03$ & $1.6277 \mathrm{E}+01$ & $4.166 \mathrm{E}-10$ & $3.466 \mathrm{E}+03$ \\
\hline $1 s 5 d^{3} D_{1}$ & $1 s 7 d^{3} D_{1}$ & $1.6349 \mathrm{E}+01$ & $2.289 \mathrm{E}-09$ & $1.904 \mathrm{E}+04$ & $1.6283 \mathrm{E}+01$ & $2.280 \mathrm{E}-09$ & $1.895 \mathrm{E}+04$ \\
\hline $1 s 5 d^{1} D_{2}$ & $1 s 7 d^{3} D_{1}$ & $1.6521 \mathrm{E}+01$ & $2.062 \mathrm{E}-08$ & $1.679 E+05$ & $1.6453 \mathrm{E}+01$ & $2.091 \mathrm{E}-08$ & $1.703 E+05$ \\
\hline $1 s 6 s^{3} S_{1}$ & $1 s 7 d^{3} D_{1}$ & $4.0910 \mathrm{E}+01$ & $1.214 \mathrm{E}-11$ & $1.613 \mathrm{E}+01$ & & & \\
\hline $1 s 6 s^{1} S_{0}$ & $1 s 7 d^{3} D_{1}$ & $4.1264 \mathrm{E}+01$ & $7.813 \mathrm{E}-13$ & $1.020 \mathrm{E}+00$ & & & \\
\hline $1 s 6 d^{3} D_{2}$ & $1 s 7 d^{3} D_{1}$ & $4.3465 \mathrm{E}+01$ & $4.636 \mathrm{E}-10$ & $5.456 \mathrm{E}+02$ & $4.3310 \mathrm{E}+01$ & $1.580 \mathrm{E}-10$ & $1.857 \mathrm{E}+02$ \\
\hline $1 s 6 d^{3} D_{1}$ & $1 s 7 d^{3} D_{1}$ & $4.3492 \mathrm{E}+01$ & $1.079 \mathrm{E}-09$ & $1.268 \mathrm{E}+03$ & $4.3303 \mathrm{E}+01$ & $1.072 \mathrm{E}-09$ & $1.260 \mathrm{E}+03$ \\
\hline $1 s 6 d^{1} D_{2}$ & $1 s 7 d^{3} D_{1}$ & $4.4198 \mathrm{E}+01$ & $2.990 \mathrm{E}-08$ & $3.404 \mathrm{E}+04$ & $4.4015 \mathrm{E}+01$ & $3.037 \mathrm{E}-08$ & $3.456 \mathrm{E}+04$ \\
\hline $1 s 7 s^{3} S_{1}$ & $1 s 7 d^{3} D_{1}$ & $1.0966 \mathrm{E}+03$ & $1.138 \mathrm{E}-10$ & $2.103 E-01$ & $1.0799 \mathrm{E}+03$ & $3.492 \mathrm{E}-13$ & $6.601 \mathrm{E}-04$ \\
\hline $1 s 7 s^{1} S_{0}$ & $1 s 7 d^{3} D_{1}$ & $1.2811 \mathrm{E}+03$ & $1.093 \mathrm{E}-12$ & $1.480 \mathrm{E}-03$ & $1.2282 \mathrm{E}+03$ & $6.150 \mathrm{E}-13$ & $8.988 \mathrm{E}-04$ \\
\hline $1 s 7 d^{3} D_{2}$ & $1 s 7 d^{3} D_{1}$ & $1.1420 \mathrm{E}+05$ & $8.038 \mathrm{E}-08$ & $1.370 \mathrm{E}-02$ & & & \\
\hline $1 s 2 p^{3} P_{1}^{o}$ & $1 s 7 p{ }^{1} P_{1}^{o}$ & $1.3362 \mathrm{E}+00$ & $2.129 \mathrm{E}-08$ & $2.651 \mathrm{E}+07$ & $1.3307 \mathrm{E}+00$ & $2.137 \mathrm{E}-08$ & $2.661 \mathrm{E}+07$ \\
\hline $1 s 2 p^{3} P_{0}^{o}$ & $1 s 7 p^{1} P_{1}^{o}$ & $1.3371 \mathrm{E}+00$ & $4.382 \mathrm{E}-08$ & $5.450 \mathrm{E}+07$ & $1.3315 \mathrm{E}+00$ & $4.537 \mathrm{E}-08$ & $5.642 \mathrm{E}+07$ \\
\hline $1 s 2 p^{3} P_{2}^{o}$ & $1 s 7 p^{1} P_{1}^{o}$ & $1.3946 \mathrm{E}+00$ & $2.779 \mathrm{E}-08$ & $3.177 \mathrm{E}+07$ & $1.3888 \mathrm{E}+00$ & $2.769 \mathrm{E}-08$ & $3.166 \mathrm{E}+07$ \\
\hline $1 s 2 p^{1} P_{1}^{o}$ & $1 s 7 p^{1} P_{1}^{o}$ & $1.4001 \mathrm{E}+00$ & $9.952 \mathrm{E}-08$ & $1.129 \mathrm{E}+08$ & $1.3943 \mathrm{E}+00$ & $9.895 \mathrm{E}-08$ & $1.122 \mathrm{E}+08$ \\
\hline $1 s 3 p^{3} P_{1}^{o}$ & $1 s 7 p^{1} P_{1}^{o}$ & $3.4073 \mathrm{E}+00$ & $2.514 \mathrm{E}-08$ & $4.814 \mathrm{E}+06$ & $3.3933 \mathrm{E}+00$ & $2.339 \mathrm{E}-08$ & $4.479 \mathrm{E}+06$ \\
\hline $1 s 3 p^{3} P_{0}^{0}$ & $1 s 7 p^{1} P_{1}^{o}$ & $3.4088 \mathrm{E}+00$ & $4.744 \mathrm{E}-08$ & $9.077 \mathrm{E}+06$ & $3.3948 \mathrm{E}+00$ & $4.799 \mathrm{E}-08$ & $9.180 \mathrm{E}+06$ \\
\hline $1 s 3 p^{3} P_{2}^{o}$ & $1 s 7 p^{1} P_{1}^{o}$ & $3.5191 \mathrm{E}+00$ & 8.199E-09 & $1.472 \mathrm{E}+06$ & $3.5046 \mathrm{E}+00$ & $9.472 \mathrm{E}-09$ & $1.700 \mathrm{E}+06$ \\
\hline $1 s 3 p{ }^{1} P_{1}^{o}$ & $1 s 7 p^{1} P_{1}^{o}$ & $3.5290 \mathrm{E}+00$ & $2.701 \mathrm{E}-08$ & $4.823 E+06$ & $3.5146 \mathrm{E}+00$ & $2.829 \mathrm{E}-08$ & $5.050 \mathrm{E}+06$ \\
\hline $1 s 4 p^{3} P_{1}^{o}$ & $1 s 7 p^{1} P_{1}^{o}$ & $7.3666 \mathrm{E}+00$ & $2.634 \mathrm{E}-08$ & $1.079 E+06$ & $7.3362 \mathrm{E}+00$ & $2.462 \mathrm{E}-08$ & $1.008 \mathrm{E}+06$ \\
\hline $1 s 4 p^{3} P_{0}^{0}$ & $1 s 7 p^{1} P_{1}^{o}$ & $7.3693 \mathrm{E}+00$ & $4.921 \mathrm{E}-08$ & $2.015 E+06$ & $7.3389 \mathrm{E}+00$ & $4.990 \mathrm{E}-08$ & $2.042 \mathrm{E}+06$ \\
\hline $1 s 4 p^{3} P_{2}^{o}$ & $1 s 7 p^{1} P_{1}^{o}$ & $7.5857 \mathrm{E}+00$ & $2.994 \mathrm{E}-09$ & $1.157 \mathrm{E}+05$ & $7.5543 \mathrm{E}+00$ & $4.311 \mathrm{E}-09$ & $1.665 \mathrm{E}+05$ \\
\hline $1 s 4 p^{1} P_{1}^{o}$ & $1 s 7 p^{1} P_{1}^{o}$ & $7.6047 \mathrm{E}+00$ & $9.460 \mathrm{E}-09$ & $3.637 \mathrm{E}+05$ & $7.5735 \mathrm{E}+00$ & $1.015 \mathrm{E}-08$ & $3.903 \mathrm{E}+05$ \\
\hline $1 s 4 f^{3} F_{2}^{o}$ & $1 s 7 p^{1} P_{1}^{o}$ & $7.6767 \mathrm{E}+00$ & $6.581 \mathrm{E}-16$ & $2.483 \mathrm{E}-02$ & & & \\
\hline $1 s 5 p^{3} P_{1}^{o}$ & $1 s 7 p^{1} P_{1}^{o}$ & $1.5788 \mathrm{E}+01$ & $3.150 \mathrm{E}-08$ & $2.810 \mathrm{E}+05$ & $1.5725 \mathrm{E}+01$ & $2.923 \mathrm{E}-08$ & $2.606 \mathrm{E}+05$ \\
\hline $1 s 5 p^{3} P_{0}^{0}$ & $1 s 7 p^{1} P_{1}^{0}$ & $1.5795 \mathrm{E}+01$ & $5.816 \mathrm{E}-08$ & $5.183 \mathrm{E}+05$ & $1.5731 \mathrm{E}+01$ & $5.883 \mathrm{E}-08$ & $5.241 \mathrm{E}+05$ \\
\hline $1 s 5 p^{3} P_{2}^{o}$ & $1 s 7 p^{1} P_{1}^{o}$ & $1.6303 \mathrm{E}+01$ & $1.149 \mathrm{E}-09$ & $9.614 \mathrm{E}+03$ & $1.6237 \mathrm{E}+01$ & $2.508 \mathrm{E}-09$ & $2.097 \mathrm{E}+04$ \\
\hline $1 s 5 p^{1} P_{1}^{o}$ & $1 s 7 p^{1} P_{1}^{o}$ & $1.6348 \mathrm{E}+01$ & $3.464 \mathrm{E}-09$ & $2.882 \mathrm{E}+04$ & $1.6282 \mathrm{E}+01$ & $4.145 \mathrm{E}-09$ & $3.447 \mathrm{E}+04$ \\
\hline $1 s 5 f^{3} F_{2}^{0}$ & $1 s 7 p^{1} P_{1}^{o}$ & $1.6519 \mathrm{E}+01$ & $1.011 \mathrm{E}-13$ & $8.235 \mathrm{E}-01$ & & & \\
\hline $1 s 6 p^{3} P_{1}^{o}$ & $1 s 7 p^{1} P_{1}^{o}$ & $4.1241 \mathrm{E}+01$ & $5.029 \mathrm{E}-08$ & $6.574 \mathrm{E}+04$ & $4.1082 \mathrm{E}+01$ & $4.653 \mathrm{E}-08$ & $6.078 \mathrm{E}+04$ \\
\hline $1 s 6 p^{3} P_{0}^{o}$ & $1 s 7 p^{1} P_{1}^{o}$ & $4.1265 \mathrm{E}+01$ & $9.222 \mathrm{E}-08$ & $1.204 \mathrm{E}+05$ & $4.1019 \mathrm{E}+01$ & $9.378 \mathrm{E}-08$ & $1.228 \mathrm{E}+05$ \\
\hline $1 s 6 p^{3} P_{2}^{o}$ & $1 s 7 p^{1} P_{1}^{o}$ & $4.3302 \mathrm{E}+01$ & $5.836 \mathrm{E}-10$ & $6.920 \mathrm{E}+02$ & $4.3133 \mathrm{E}+01$ & $1.991 \mathrm{E}-09$ & $2.359 \mathrm{E}+03$ \\
\hline $1 s 6 p^{1} P_{1}^{o}$ & $1 s 7 p^{1} P_{1}^{o}$ & $4.3483 \mathrm{E}+01$ & $1.175 \mathrm{E}-09$ & $1.382 \mathrm{E}+03$ & $4.3294 \mathrm{E}+01$ & $1.809 \mathrm{E}-09$ & $2.128 \mathrm{E}+03$ \\
\hline $1 s 6 f^{3} F_{2}^{o}$ & $1 s 7 p^{1} P_{1}^{o}$ & $4.4186 \mathrm{E}+01$ & $4.893 \mathrm{E}-13$ & $5.572 \mathrm{E}-01$ & & & \\
\hline $1 s 7 p^{3} P_{1}^{o}$ & $1 s 7 p^{1} P_{1}^{o}$ & $1.2731 \mathrm{E}+03$ & $1.019 \mathrm{E}-06$ & $1.398 \mathrm{E}+03$ & $1.2808 \mathrm{E}+03$ & $9.958 \mathrm{E}-07$ & $1.338 \mathrm{E}+03$ \\
\hline $1 s 7 p^{3} P_{0}^{o}$ & $1 s 7 p^{1} P_{1}^{o}$ & $1.2879 \mathrm{E}+03$ & $1.886 \mathrm{E}-06$ & $2.528 \mathrm{E}+03$ & $1.2433 \mathrm{E}+03$ & $1.886 \mathrm{E}-06$ & $2.690 \mathrm{E}+03$ \\
\hline $1 s 7 p^{3} P_{2}^{o}$ & $1 s 7 p^{1} P_{1}^{o}$ & $1.6436 \mathrm{E}+04$ & $1.261 \mathrm{E}-07$ & $1.038 \mathrm{E}+00$ & $1.8507 \mathrm{E}+04$ & $1.091 \mathrm{E}-07$ & $7.026 \mathrm{E}-01$ \\
\hline $1 s 3 d^{3} D_{2}$ & $1 s 7 d^{3} D_{3}$ & $3.5248 \mathrm{E}+00$ & $8.286 \mathrm{E}-09$ & $6.355 E+05$ & $3.5099 \mathrm{E}+00$ & $8.335 E-09$ & $6.392 \mathrm{E}+05$ \\
\hline $1 s 3 d^{3} D_{3}$ & $1 s 7 d^{3} D_{3}$ & $3.5615 \mathrm{E}+00$ & $7.741 \mathrm{E}-08$ & $5.816 \mathrm{E}+06$ & $3.5464 \mathrm{E}+00$ & $7.691 \mathrm{E}-08$ & $5.777 \mathrm{E}+06$ \\
\hline $1 s 3 d^{1} D_{2}$ & $1 s 7 d^{3} D_{3}$ & $3.5629 \mathrm{E}+00$ & $3.385 \mathrm{E}-09$ & $2.541 \mathrm{E}+05$ & $3.5478 \mathrm{E}+00$ & $3.805 \mathrm{E}-09$ & $2.856 \mathrm{E}+05$ \\
\hline $1 s 4 d^{3} D_{2}$ & $1 s 7 d^{3} D_{3}$ & $7.5894 \mathrm{E}+00$ & $1.320 \mathrm{E}-08$ & $2.183 E+05$ & $7.5575 \mathrm{E}+00$ & $1.324 \mathrm{E}-08$ & $2.190 \mathrm{E}+05$ \\
\hline $1 s 4 d^{3} D_{3}$ & $1 s 7 d^{3} D_{3}$ & $7.6613 \mathrm{E}+00$ & $4.040 \mathrm{E}-08$ & $6.558 \mathrm{E}+05$ & $7.6289 \mathrm{E}+00$ & $4.032 \mathrm{E}-08$ & $6.546 \mathrm{E}+05$ \\
\hline $1 s 4 d{ }^{1} D_{2}$ & $1 s 7 d^{3} D_{3}$ & $7.6642 \mathrm{E}+00$ & $1.665 \mathrm{E}-09$ & $2.702 E+04$ & $7.6319 \mathrm{E}+00$ & $1.958 \mathrm{E}-09$ & $3.177 \mathrm{E}+04$ \\
\hline $1 s 5 d^{3} D_{2}$ & $1 s 7 d^{3} D_{3}$ & $1.6284 \mathrm{E}+01$ & $1.901 \mathrm{E}-08$ & $6.830 \mathrm{E}+04$ & $1.6216 \mathrm{E}+01$ & $1.905 \mathrm{E}-08$ & $6.847 \mathrm{E}+04$ \\
\hline $1 s 5 d^{3} D_{3}$ & $1 s 7 d^{3} D_{3}$ & $1.6453 \mathrm{E}+01$ & $1.990 \mathrm{E}-08$ & $7.005 E+04$ & $1.6384 \mathrm{E}+01$ & $1.990 \mathrm{E}-08$ & $7.005 \mathrm{E}+04$ \\
\hline $1 s 5 d{ }^{1} D_{2}$ & $1 s 7 d^{3} D_{3}$ & $1.6460 \mathrm{E}+01$ & $7.283 \mathrm{E}-10$ & $2.561 \mathrm{E}+03$ & $1.6391 \mathrm{E}+01$ & $9.397 \mathrm{E}-10$ & $3.304 \mathrm{E}+03$ \\
\hline $1 s 5 g^{3} G_{4}$ & $1 s 7 d^{3} D_{3}$ & $1.6542 \mathrm{E}+01$ & $1.731 \mathrm{E}-16$ & $6.029 E-04$ & & & \\
\hline $1 s 5 g^{3} G_{3}$ & $1 s 7 d^{3} D_{3}$ & $1.6544 \mathrm{E}+01$ & $3.024 \mathrm{E}-18$ & $1.053 \mathrm{E}-05$ & & & \\
\hline $1 s 5 g{ }^{1} G_{4}$ & $1 s 7 d^{3} D_{3}$ & $1.6595 \mathrm{E}+01$ & $6.733 \mathrm{E}-20$ & $2.330 \mathrm{E}-07$ & & & \\
\hline
\end{tabular}


Table 5 (continued)

\begin{tabular}{|c|c|c|c|c|c|c|c|}
\hline \multirow[t]{2}{*}{ Lower } & \multirow[t]{2}{*}{ Upper } & \multicolumn{3}{|l|}{ GRASP2K } & \multicolumn{3}{|l|}{ FAC } \\
\hline & & $\lambda(\AA)$ & $g f$ & $A\left(\mathrm{~s}^{-1}\right)$ & $\lambda(\AA)$ & $g f$ & $A\left(\mathrm{~s}^{-1}\right)$ \\
\hline $1 s 6 d^{3} D_{2}$ & $1 s 7 d^{3} D_{3}$ & $4.3051 \mathrm{E}+01$ & $3.466 \mathrm{E}-08$ & $1.782 \mathrm{E}+04$ & $4.2880 \mathrm{E}+01$ & $3.476 \mathrm{E}-08$ & $1.786 \mathrm{E}+04$ \\
\hline $1 s 6 d^{3} D_{3}$ & $1 s 7 d^{3} D_{3}$ & $4.3740 \mathrm{E}+01$ & $9.385 \mathrm{E}-09$ & $4.675 \mathrm{E}+03$ & $4.3560 \mathrm{E}+01$ & $9.372 \mathrm{E}-09$ & $4.666 \mathrm{E}+03$ \\
\hline $1 s 6 d^{1} D_{2}$ & $1 s 7 d^{3} D_{3}$ & $4.3770 \mathrm{E}+01$ & $2.487 \mathrm{E}-10$ & $1.237 \mathrm{E}+02$ & $4.3571 \mathrm{E}+01$ & $3.789 \mathrm{E}-10$ & $1.886 \mathrm{E}+02$ \\
\hline $1 s 6 g{ }^{3} G_{4}$ & $1 s 7 d^{3} D_{3}$ & $4.4103 \mathrm{E}+01$ & $1.879 \mathrm{E}-13$ & $9.204 \mathrm{E}-02$ & & & \\
\hline $1 s 6 g^{3} G_{3}$ & $1 s 7 d^{3} D_{3}$ & $4.4112 \mathrm{E}+01$ & $7.209 \mathrm{E}-15$ & $3.530 \mathrm{E}-03$ & & & \\
\hline $1 s 6 g{ }^{1} G_{4}$ & $1 s 7 d^{3} D_{3}$ & $4.4321 \mathrm{E}+01$ & $6.432 \mathrm{E}-18$ & $3.120 \mathrm{E}-06$ & & & \\
\hline $1 s 7 d^{3} D_{2}$ & $1 s 7 d^{3} D_{3}$ & $4.3459 \mathrm{E}+03$ & $2.625 \mathrm{E}-06$ & $1.324 \mathrm{E}+02$ & $4.3620 \mathrm{E}+03$ & $2.623 \mathrm{E}-06$ & $1.302 \mathrm{E}+02$ \\
\hline $1 s 2 p^{3} P_{2}^{o}$ & $1 s 7 f^{3} F_{3}^{o}$ & $1.3942 \mathrm{E}+00$ & $8.521 \mathrm{E}-09$ & $4.177 \mathrm{E}+06$ & $1.3883 \mathrm{E}+00$ & $7.247 \mathrm{E}-09$ & $3.552 E+06$ \\
\hline $1 s 3 p^{3} P_{2}^{o}$ & $1 s 7 f^{3} F_{3}^{o}$ & $3.5164 \mathrm{E}+00$ & $3.762 \mathrm{E}-10$ & $2.899 \mathrm{E}+04$ & $3.5016 \mathrm{E}+00$ & $4.070 \mathrm{E}-10$ & $3.136 \mathrm{E}+04$ \\
\hline $1 s 4 p^{3} P_{2}^{0}$ & $1 s 7 f^{3} F_{3}^{o}$ & $7.5729 \mathrm{E}+00$ & $4.137 \mathrm{E}-10$ & $6.874 \mathrm{E}+03$ & $7.5406 \mathrm{E}+00$ & $4.246 \mathrm{E}-10$ & $7.056 \mathrm{E}+03$ \\
\hline $1 s 4 f^{3} F_{3}^{o}$ & $1 s 7 f^{3} F_{3}^{o}$ & $7.6621 \mathrm{E}+00$ & $4.484 \mathrm{E}-08$ & $7.279 \mathrm{E}+05$ & $7.6295 \mathrm{E}+00$ & $1.331 \mathrm{E}-08$ & $2.161 \mathrm{E}+05$ \\
\hline $1 s 4 f^{3} F_{2}^{o}$ & $1 s 7 f^{3} F_{3}^{o}$ & $7.6636 \mathrm{E}+00$ & $1.593 \mathrm{E}-07$ & $2.584 \mathrm{E}+06$ & $7.6310 \mathrm{E}+00$ & $6.677 \mathrm{E}-10$ & $1.083 E+04$ \\
\hline $1 s 4 f^{3} F_{4}^{0}$ & $1 s 7 f^{3} F_{3}^{o}$ & $7.6982 \mathrm{E}+00$ & $7.787 \mathrm{E}-09$ & $1.252 \mathrm{E}+05$ & $7.6653 \mathrm{E}+00$ & $7.865 \mathrm{E}-09$ & $1.264 \mathrm{E}+05$ \\
\hline $1 s 4 f^{1} F_{3}^{o}$ & $1 s 7 f^{3} F_{3}^{o}$ & $7.6993 \mathrm{E}+00$ & $2.637 \mathrm{E}-10$ & $4.239 \mathrm{E}+03$ & $7.6665 \mathrm{E}+00$ & $3.040 \mathrm{E}-10$ & $4.887 \mathrm{E}+03$ \\
\hline $1 s 5 p^{3} P_{2}^{0}$ & $1 s 7 f^{3} F_{3}^{o}$ & $1.6245 \mathrm{E}+01$ & $1.679 \mathrm{E}-10$ & $6.064 \mathrm{E}+02$ & $1.6174 \mathrm{E}+01$ & $1.729 \mathrm{E}-10$ & $6.247 \mathrm{E}+02$ \\
\hline $1 s 5 f^{3} F_{3}^{o}$ & $1 s 7 f^{3} F_{3}^{o}$ & $1.6455 \mathrm{E}+01$ & $6.347 \mathrm{E}-08$ & $2.234 \mathrm{E}+05$ & $1.6385 \mathrm{E}+01$ & $1.004 \mathrm{E}-08$ & $3.533 E+04$ \\
\hline $1 s 5 f^{3} F_{2}^{o}$ & $1 s 7 f^{3} F_{3}^{o}$ & $1.6459 \mathrm{E}+01$ & $1.769 \mathrm{E}-07$ & $6.224 \mathrm{E}+05$ & $1.6388 \mathrm{E}+01$ & $5.043 \mathrm{E}-10$ & $1.774 \mathrm{E}+03$ \\
\hline $1 s 5 f^{3} F_{4}^{o}$ & $1 s 7 f^{3} F_{3}^{o}$ & $1.6540 \mathrm{E}+01$ & $1.096 \mathrm{E}-08$ & $3.816 \mathrm{E}+04$ & $1.6469 \mathrm{E}+01$ & $1.094 \mathrm{E}-08$ & $3.811 \mathrm{E}+04$ \\
\hline $1 s 5 f^{1} F_{3}^{o}$ & $1 s 7 f^{3} F_{3}^{o}$ & $1.6543 \mathrm{E}+01$ & $3.832 \mathrm{E}-10$ & $1.334 \mathrm{E}+03$ & $1.6472 \mathrm{E}+01$ & $4.176 \mathrm{E}-10$ & $1.454 \mathrm{E}+03$ \\
\hline $1 s 6 p^{3} P_{2}^{o}$ & $1 s 7 f^{3} F_{3}^{o}$ & $4.2891 \mathrm{E}+01$ & $4.093 \mathrm{E}-11$ & $2.120 \mathrm{E}+01$ & $4.2690 \mathrm{E}+01$ & $4.269 \mathrm{E}-11$ & $2.213 \mathrm{E}+01$ \\
\hline $1 s 6 f^{3} F_{3}^{o}$ & $1 s 7 f^{3} F_{3}^{o}$ & $4.3744 \mathrm{E}+01$ & $4.845 \mathrm{E}-08$ & $2.412 \mathrm{E}+04$ & $4.3561 \mathrm{E}+01$ & $5.803 \mathrm{E}-09$ & $2.889 \mathrm{E}+03$ \\
\hline $1 s 6 f^{3} F_{2}^{o}$ & $1 s 7 f^{3} F_{3}^{o}$ & $4.3758 \mathrm{E}+01$ & $1.217 \mathrm{E}-07$ & $6.059 \mathrm{E}+04$ & $4.3560 \mathrm{E}+01$ & $2.919 \mathrm{E}-10$ & $1.453 \mathrm{E}+02$ \\
\hline $1 s 6 f^{3} F_{4}^{o}$ & $1 s 7 f^{3} F_{3}^{o}$ & $4.4094 \mathrm{E}+01$ & $1.673 \mathrm{E}-08$ & $8.197 E+03$ & $4.3907 \mathrm{E}+01$ & $1.663 \mathrm{E}-08$ & $8.152 E+03$ \\
\hline $1 s 6 f^{1} F_{3}^{o}$ & $1 s 7 f^{3} F_{3}^{o}$ & $4.4105 \mathrm{E}+01$ & $6.132 \mathrm{E}-10$ & $3.004 \mathrm{E}+02$ & $4.3907 \mathrm{E}+01$ & $6.178 \mathrm{E}-10$ & $3.027 \mathrm{E}+02$ \\
\hline $1 s 6 h^{3} H_{4}^{o}$ & $1 s 7 f^{3} F_{3}^{o}$ & $4.4312 \mathrm{E}+01$ & $1.635 \mathrm{E}-17$ & $7.936 \mathrm{E}-06$ & & & \\
\hline $1 s 7 p^{3} P_{2}^{o}$ & 1s $7 f^{3} F_{3}^{o}$ & $3.5436 \mathrm{E}+03$ & $2.931 \mathrm{E}-12$ & $2.224 \mathrm{E}-04$ & $3.3920 \mathrm{E}+03$ & $4.564 \mathrm{E}-13$ & $3.748 \mathrm{E}-05$ \\
\hline $1 s 2 p^{3} P_{1}^{o}$ & $1 s 7 f^{3} F_{2}^{o}$ & $1.3358 \mathrm{E}+00$ & $7.084 \mathrm{E}-12$ & $5.296 \mathrm{E}+03$ & $1.3303 \mathrm{E}+00$ & $5.834 \mathrm{E}-12$ & $4.360 \mathrm{E}+03$ \\
\hline $1 s 2 p^{3} P_{2}^{0}$ & $1 s 7 f^{3} F_{2}^{o}$ & $1.3942 \mathrm{E}+00$ & $4.476 \mathrm{E}-10$ & $3.072 \mathrm{E}+05$ & $1.3883 \mathrm{E}+00$ & $5.176 \mathrm{E}-10$ & $3.552 \mathrm{E}+05$ \\
\hline $1 s 2 p{ }^{1} P_{1}^{o}$ & $1 s 7 f^{3} F_{2}^{o}$ & $1.3997 \mathrm{E}+00$ & $4.948 \mathrm{E}-09$ & $3.370 \mathrm{E}+06$ & $1.3939 \mathrm{E}+00$ & $4.754 \mathrm{E}-09$ & $3.236 \mathrm{E}+06$ \\
\hline $1 s 3 p^{3} P_{1}^{o}$ & $1 s 7 f^{3} F_{2}^{o}$ & $3.4047 \mathrm{E}+00$ & $9.120 \mathrm{E}-13$ & $1.050 \mathrm{E}+02$ & $3.3906 \mathrm{E}+00$ & $4.222 \mathrm{E}-13$ & $4.858 \mathrm{E}+01$ \\
\hline $1 s 3 p^{3} P_{2}^{o}$ & $1 s 7 f^{3} F_{2}^{o}$ & $3.5163 \mathrm{E}+00$ & $4.173 \mathrm{E}-11$ & $4.503 E+03$ & $3.5016 \mathrm{E}+00$ & $2.907 \mathrm{E}-11$ & $3.136 \mathrm{E}+03$ \\
\hline $1 s 3 p{ }^{1} P_{1}^{o}$ & $1 s 7 f^{3} F_{2}^{o}$ & $3.5262 \mathrm{E}+00$ & $2.694 \mathrm{E}-10$ & $2.890 \mathrm{E}+04$ & $3.5116 \mathrm{E}+00$ & $2.584 \mathrm{E}-10$ & $2.771 \mathrm{E}+04$ \\
\hline $1 s 4 p^{3} P_{1}^{o}$ & $1 s 7 f^{3} F_{2}^{o}$ & $7.3543 E+00$ & $9.639 \mathrm{E}-13$ & $2.378 \mathrm{E}+01$ & & & \\
\hline $1 s 4 p^{3} P_{2}^{o}$ & $1 s 7 f^{3} F_{2}^{o}$ & $7.5727 \mathrm{E}+00$ & $3.962 \mathrm{E}-11$ & $9.216 \mathrm{E}+02$ & $7.5406 \mathrm{E}+00$ & $3.074 \mathrm{E}-11$ & $7.151 \mathrm{E}+02$ \\
\hline $1 s 4 p{ }^{1} P_{1}^{o}$ & $1 s 7 f^{3} F_{2}^{o}$ & $7.5917 \mathrm{E}+00$ & $2.769 \mathrm{E}-10$ & $6.409 \mathrm{E}+03$ & $7.5597 \mathrm{E}+00$ & $2.729 \mathrm{E}-10$ & $6.316 \mathrm{E}+03$ \\
\hline $1 s 4 f^{3} F_{3}^{o}$ & $1 s 7 f^{3} F_{2}^{o}$ & $7.6619 \mathrm{E}+00$ & $1.605 \mathrm{E}-07$ & $3.648 \mathrm{E}+06$ & $7.6295 \mathrm{E}+00$ & $6.748 \mathrm{E}-10$ & $1.533 \mathrm{E}+04$ \\
\hline $1 s 4 f^{3} F_{2}^{o}$ & $1 s 7 f^{3} F_{2}^{o}$ & $7.6634 \mathrm{E}+00$ & $8.990 \mathrm{E}-08$ & $2.042 E+06$ & $7.6310 \mathrm{E}+00$ & $9.326 \mathrm{E}-09$ & $2.118 \mathrm{E}+05$ \\
\hline $1 s 4 f^{1} F_{3}^{o}$ & $1 s 7 f^{3} F_{2}^{o}$ & $7.6990 \mathrm{E}+00$ & $5.551 \mathrm{E}-09$ & $1.249 \mathrm{E}+05$ & $7.6665 \mathrm{E}+00$ & $5.820 \mathrm{E}-09$ & $1.309 E+05$ \\
\hline $1 s 5 p^{3} P_{1}^{o}$ & $1 s 7 f^{3} F_{2}^{o}$ & $1.5732 \mathrm{E}+01$ & $5.384 \mathrm{E}-13$ & $2.902 \mathrm{E}+00$ & & & \\
\hline $1 s 5 p^{3} P_{2}^{o}$ & $1 s 7 f^{3} F_{2}^{o}$ & $1.6243 \mathrm{E}+01$ & $1.702 \mathrm{E}-11$ & $8.607 \mathrm{E}+01$ & $1.6174 \mathrm{E}+01$ & $1.264 \mathrm{E}-11$ & $6.394 \mathrm{E}+01$ \\
\hline $1 s 5 p^{1} P_{1}^{o}$ & $1 s 7 f^{3} F_{2}^{o}$ & $1.6288 \mathrm{E}+01$ & $1.139 \mathrm{E}-10$ & $5.729 \mathrm{E}+02$ & $1.6219 \mathrm{E}+01$ & $1.117 \mathrm{E}-10$ & $5.618 \mathrm{E}+02$ \\
\hline $1 s 5 f^{3} F_{3}^{o}$ & $1 s 7 f^{3} F_{2}^{o}$ & $1.6454 \mathrm{E}+01$ & $1.805 \mathrm{E}-07$ & $8.896 \mathrm{E}+05$ & $1.6385 \mathrm{E}+01$ & $5.107 \mathrm{E}-10$ & $2.516 \mathrm{E}+03$ \\
\hline $1 s 5 f^{3} F_{2}^{o}$ & $1 s 7 f^{3} F_{2}^{o}$ & $1.6457 \mathrm{E}+01$ & $9.042 \mathrm{E}-08$ & $4.454 \mathrm{E}+05$ & $1.6388 \mathrm{E}+01$ & $7.034 \mathrm{E}-09$ & $3.464 \mathrm{E}+04$ \\
\hline $1 s 5 f^{1} F_{3}^{o}$ & $1 s 7 f^{3} F_{2}^{o}$ & $1.6542 \mathrm{E}+01$ & $7.829 \mathrm{E}-09$ & $3.817 \mathrm{E}+04$ & $1.6472 \mathrm{E}+01$ & $8.100 \mathrm{E}-09$ & $3.948 E+04$ \\
\hline $1 s 6 p^{3} P_{1}^{o}$ & $1 s 7 f^{3} F_{2}^{o}$ & $4.0860 \mathrm{E}+01$ & $3.480 \mathrm{E}-13$ & $2.781 \mathrm{E}-01$ & & & \\
\hline $1 s 6 p^{3} P_{2}^{o}$ & $1 s 7 f^{3} F_{2}^{o}$ & $4.2882 \mathrm{E}+01$ & $5.412 \mathrm{E}-12$ & $3.926 \mathrm{E}+00$ & $4.2690 \mathrm{E}+01$ & $3.049 \mathrm{E}-12$ & $2.213 E+00$ \\
\hline $1 s 6 p^{1} P_{1}^{o}$ & $1 s 7 f^{3} F_{2}^{o}$ & $4.3060 \mathrm{E}+01$ & $2.950 \mathrm{E}-11$ & $2.123 \mathrm{E}+01$ & $4.2848 \mathrm{E}+01$ & $2.777 \mathrm{E}-11$ & $2.001 \mathrm{E}+01$ \\
\hline $1 s 6 f^{3} F_{3}^{0}$ & $1 s 7 f^{3} F_{2}^{o}$ & $4.3735 \mathrm{E}+01$ & $1.272 \mathrm{E}-07$ & $8.872 \mathrm{E}+04$ & $4.3561 \mathrm{E}+01$ & $2.879 \mathrm{E}-10$ & $2.007 \mathrm{E}+02$ \\
\hline $1 s 6 f^{3} F_{2}^{o}$ & $1 s 7 f^{3} F_{2}^{o}$ & $4.3749 \mathrm{E}+01$ & $6.015 \mathrm{E}-08$ & $4.193 \mathrm{E}+04$ & $4.3561 \mathrm{E}+01$ & $4.062 \mathrm{E}-09$ & $2.831 \mathrm{E}+03$ \\
\hline $1 s 6 f^{1} F_{3}^{o}$ & $1 s 7 f^{3} F_{2}^{o}$ & $4.4096 \mathrm{E}+01$ & $1.205 \mathrm{E}-08$ & $8.267 \mathrm{E}+03$ & $4.3907 \mathrm{E}+01$ & $1.232 \mathrm{E}-08$ & $8.457 E+03$ \\
\hline $1 s 7 p^{3} P_{1}^{o}$ & $1 s 7 f^{3} F_{2}^{o}$ & $9.8851 \mathrm{E}+02$ & $2.009 \mathrm{E}-12$ & $2.742 \mathrm{E}-03$ & $9.7899 \mathrm{E}+02$ & $2.186 \mathrm{E}-15$ & $3.018 \mathrm{E}-06$ \\
\hline $1 s 7 p^{3} P_{2}^{o}$ & $1 s 7 f^{3} F_{2}^{o}$ & $3.4844 \mathrm{E}+03$ & $5.719 \mathrm{E}-12$ & $6.284 \mathrm{E}-04$ & $3.3927 \mathrm{E}+03$ & $3.259 \mathrm{E}-14$ & $3.745 \mathrm{E}-06$ \\
\hline $1 s 7 p^{1} P_{1}^{o}$ & $1 s 7 f^{3} F_{2}^{o}$ & $4.4219 \mathrm{E}+03$ & $9.314 \mathrm{E}-15$ & $6.355 \mathrm{E}-07$ & $4.1543 E+03$ & $2.391 \mathrm{E}-13$ & $1.833 \mathrm{E}-05$ \\
\hline $1 s 7 f^{3} F_{3}^{o}$ & $1 s 7 f^{3} F_{2}^{o}$ & $2.0860 \mathrm{E}+05$ & $6.413 \mathrm{E}-08$ & $1.966 \mathrm{E}-03$ & & & \\
\hline $1 s 2 s^{3} s_{1}^{3}$ & $1 s 7 d^{1} D_{2}$ & $1.3248 \mathrm{E}+00$ & $2.700 \mathrm{E}-12$ & $2.052 \mathrm{E}+03$ & $1.3192 \mathrm{E}+00$ & $5.989 E-13$ & $4.552 \mathrm{E}+02$ \\
\hline $1 s 3 s^{3} S_{1}$ & $1 s 7 d^{1} D_{2}$ & $3.3851 \mathrm{E}+00$ & $1.746 \mathrm{E}-12$ & $2.033 \mathrm{E}+02$ & & & \\
\hline $1 s 3 d^{3} D_{2}$ & $1 s 7 d^{1} D_{2}$ & $3.5247 \mathrm{E}+00$ & $6.496 \mathrm{E}-10$ & $6.976 \mathrm{E}+04$ & $3.5098 \mathrm{E}+00$ & $6.265 \mathrm{E}-10$ & $6.727 \mathrm{E}+04$ \\
\hline $1 s 3 d^{3} D_{1}$ & $1 s 7 d^{1} D_{2}$ & $3.5261 \mathrm{E}+00$ & $5.200 \mathrm{E}-09$ & $5.579 \mathrm{E}+05$ & $3.5113 \mathrm{E}+00$ & $5.252 \mathrm{E}-09$ & $5.634 \mathrm{E}+05$ \\
\hline $1 s 3 d^{3} D_{3}$ & $1 s 7 d^{1} D_{2}$ & $3.5614 \mathrm{E}+00$ & $4.462 \mathrm{E}-09$ & $4.693 \mathrm{E}+05$ & $3.5463 \mathrm{E}+00$ & $4.109 \mathrm{E}-09$ & $4.321 \mathrm{E}+05$ \\
\hline $1 s 3 d^{1} D_{2}$ & $1 s 7 d{ }^{1} D_{2}$ & $3.5628 \mathrm{E}+00$ & $5.333 \mathrm{E}-08$ & $5.604 \mathrm{E}+06$ & $3.5477 \mathrm{E}+00$ & $5.362 \mathrm{E}-08$ & $5.635 \mathrm{E}+06$ \\
\hline $1 s 4 s^{3} S_{1}$ & $1 s 7 d^{1} D_{2}$ & $7.3170 \mathrm{E}+00$ & $1.395 \mathrm{E}-12$ & $3.475 \mathrm{E}+01$ & & & \\
\hline $1 s 4 d^{3} D_{2}$ & $1 s 7 d^{1} D_{2}$ & $7.5888 \mathrm{E}+00$ & $1.014 \mathrm{E}-09$ & $2.350 \mathrm{E}+04$ & $7.5573 \mathrm{E}+00$ & $9.516 \mathrm{E}-10$ & $2.204 \mathrm{E}+04$ \\
\hline $1 s 4 d^{3} D_{1}$ & $1 s 7 d^{1} D_{2}$ & $7.5916 \mathrm{E}+00$ & $8.270 \mathrm{E}-09$ & $1.914 \mathrm{E}+05$ & $7.5601 \mathrm{E}+00$ & $8.403 E-09$ & $1.944 \mathrm{E}+05$ \\
\hline $1 s 4 d^{3} D_{3}$ & $1 s 7 d^{1} D_{2}$ & $7.6607 \mathrm{E}+00$ & $2.469 \mathrm{E}-09$ & $5.613 \mathrm{E}+04$ & $7.6287 \mathrm{E}+00$ & $2.210 \mathrm{E}-09$ & $5.023 E+04$ \\
\hline $1 s 4 d{ }^{1} D_{2}$ & $1 s 7 d{ }^{1} D_{2}$ & $7.6636 \mathrm{E}+00$ & $2.816 \mathrm{E}-08$ & $6.396 \mathrm{E}+05$ & $7.6317 \mathrm{E}+00$ & $2.809 \mathrm{E}-08$ & $6.380 \mathrm{E}+05$ \\
\hline $1 s 5 s^{3} S_{1}$ & $1 s 7 d^{1} D_{2}$ & $1.5646 \mathrm{E}+01$ & $1.343 \mathrm{E}-12$ & $7.317 \mathrm{E}+00$ & & & \\
\hline $1 s 5 d^{3} D_{2}$ & $1 s 7 d^{1} D_{2}$ & $1.6281 \mathrm{E}+01$ & $1.459 \mathrm{E}-09$ & $7.342 \mathrm{E}+03$ & $1.6215 \mathrm{E}+01$ & $1.357 \mathrm{E}-09$ & $6.831 \mathrm{E}+03$ \\
\hline $1 s 5 d^{3} D_{1}$ & $1 s 7 d^{1} D_{2}$ & $1.6288 \mathrm{E}+01$ & $1.195 \mathrm{E}-08$ & $6.009 \mathrm{E}+04$ & $1.6221 \mathrm{E}+01$ & $1.215 \mathrm{E}-08$ & $6.108 E+04$ \\
\hline $1 s 5 d^{3} D_{3}$ & $1 s 7 d^{1} D_{2}$ & $1.6451 \mathrm{E}+01$ & $1.339 \mathrm{E}-09$ & $6.601 \mathrm{E}+03$ & $1.6383 \mathrm{E}+01$ & $1.137 \mathrm{E}-09$ & $5.606 \mathrm{E}+03$ \\
\hline $1 s 5 d{ }^{1} D_{2}$ & $1 s 7 d^{1} D_{2}$ & $1.6458 \mathrm{E}+01$ & $1.394 \mathrm{E}-08$ & $6.865 \mathrm{E}+04$ & $1.6390 \mathrm{E}+01$ & $1.385 \mathrm{E}-08$ & $6.820 \mathrm{E}+04$ \\
\hline $1 s 5 g{ }^{3} G_{3}$ & $1 s 7 d{ }^{1} D_{2}$ & $1.6541 \mathrm{E}+01$ & $8.041 \mathrm{E}-17$ & $3.921 \mathrm{E}-04$ & & & \\
\hline
\end{tabular}


Table 5 (continued)

\begin{tabular}{|c|c|c|c|c|c|c|c|}
\hline \multirow[t]{2}{*}{ Lower } & \multirow[t]{2}{*}{ Upper } & \multicolumn{3}{|l|}{ GRASP2K } & \multicolumn{3}{|l|}{ FAC } \\
\hline & & $\lambda(\AA)$ & $g f$ & $A\left(\mathrm{~s}^{-1}\right)$ & $\lambda(\AA)$ & $g f$ & $A\left(\mathrm{~s}^{-1}\right)$ \\
\hline $1 s 6 s^{3} S_{1}$ & $1 s 7 d^{1} D_{2}$ & $4.0526 \mathrm{E}+01$ & $1.824 \mathrm{E}-12$ & $1.481 \mathrm{E}+00$ & & & \\
\hline $1 s 6 d^{3} D_{2}$ & $1 s 7 d^{1} D_{2}$ & $4.3033 \mathrm{E}+01$ & $2.664 \mathrm{E}-09$ & $1.919 E+03$ & $4.2873 \mathrm{E}+01$ & $2.435 \mathrm{E}-09$ & $1.752 E+03$ \\
\hline $1 s 6 d^{3} D_{1}$ & $1 s 7 d^{1} D_{2}$ & $4.3059 \mathrm{E}+01$ & $2.187 \mathrm{E}-08$ & $1.574 \mathrm{E}+04$ & $4.2867 \mathrm{E}+01$ & $2.224 \mathrm{E}-08$ & $1.601 \mathrm{E}+04$ \\
\hline $1 s 6 d^{3} D_{3}$ & $1 s 7 d^{1} D_{2}$ & $4.3721 \mathrm{E}+01$ & $7.782 \mathrm{E}-10$ & $5.431 \mathrm{E}+02$ & $4.3553 \mathrm{E}+01$ & $5.898 \mathrm{E}-10$ & $4.112 \mathrm{E}+02$ \\
\hline $1 s 6 d^{1} D_{2}$ & $1 s 7 d^{1} D_{2}$ & $4.3751 \mathrm{E}+01$ & $6.584 \mathrm{E}-09$ & $4.589 \mathrm{E}+03$ & $4.3564 \mathrm{E}+01$ & $6.539 \mathrm{E}-09$ & $4.558 \mathrm{E}+03$ \\
\hline $1 s 6 g{ }^{3} G_{3}$ & $1 s 7 d^{1} D_{2}$ & $4.4092 \mathrm{E}+01$ & $1.366 \mathrm{E}-13$ & $9.376 \mathrm{E}-02$ & & & \\
\hline $1 s 7 s^{3} S_{1}$ & $1 s 7 d^{1} D_{2}$ & $8.7478 \mathrm{E}+02$ & $2.312 \mathrm{E}-11$ & $4.030 \mathrm{E}-02$ & & & \\
\hline $1 s 7 d^{3} D_{2}$ & $1 s 7 d^{1} D_{2}$ & $4.1666 \mathrm{E}+03$ & $1.940 \mathrm{E}-07$ & $1.491 \mathrm{E}+01$ & $4.2946 \mathrm{E}+03$ & $1.876 \mathrm{E}-07$ & $1.345 \mathrm{E}+01$ \\
\hline $1 s 7 d^{3} D_{1}$ & $1 s 7 d^{1} D_{2}$ & $4.3244 \mathrm{E}+03$ & $1.655 \mathrm{E}-06$ & $1.180 \mathrm{E}+02$ & $4.2545 \mathrm{E}+03$ & $1.654 \mathrm{E}-06$ & $1.208 \mathrm{E}+02$ \\
\hline $1 s 7 d^{3} D_{3}$ & $1 s 7 d^{1} D_{2}$ & $1.0098 \mathrm{E}+05$ & $5.515 \mathrm{E}-08$ & $7.215 \mathrm{E}-03$ & & & \\
\hline $1 s 4 f^{3} F_{3}^{o}$ & $1 s 7 f^{3} F_{4}^{o}$ & $7.6554 \mathrm{E}+00$ & $1.719 \mathrm{E}-09$ & $2.174 \mathrm{E}+04$ & $7.6229 \mathrm{E}+00$ & $1.729 E-09$ & $2.187 E+04$ \\
\hline $1 s 4 f^{3} F_{4}^{o}$ & $1 s 7 f^{3} F_{4}^{0}$ & $7.6914 \mathrm{E}+00$ & $3.672 \mathrm{E}-08$ & $4.601 E+05$ & $7.6587 \mathrm{E}+00$ & $4.082 \mathrm{E}-08$ & $5.114 \mathrm{E}+05$ \\
\hline $1 s 4 f^{1} F_{3}^{o}$ & $1 s 7 f^{3} F_{4}^{0}$ & $7.6926 \mathrm{E}+00$ & $2.148 \mathrm{E}-09$ & $2.690 \mathrm{E}+04$ & $7.6598 \mathrm{E}+00$ & $1.172 \mathrm{E}-09$ & $1.468 \mathrm{E}+04$ \\
\hline $1 s 5 f^{3} F_{3}^{o}$ & $1 s 7 f^{3} F_{4}^{0}$ & $1.6424 \mathrm{E}+01$ & $4.060 \mathrm{E}-09$ & $1.116 \mathrm{E}+04$ & $1.6354 \mathrm{E}+01$ & $4.039 E-09$ & $1.109 \mathrm{E}+04$ \\
\hline $1 s 5 f^{3} F_{4}^{o}$ & $1 s 7 f^{3} F_{4}^{0}$ & $1.6509 \mathrm{E}+01$ & $2.875 \mathrm{E}-08$ & $7.819 E+04$ & $1.6439 \mathrm{E}+01$ & $3.085 E-08$ & $8.390 E+04$ \\
\hline $1 s 5 f^{1} F_{3}^{o}$ & $1 s 7 f^{3} F_{4}^{o}$ & $1.6512 \mathrm{E}+01$ & $1.249 \mathrm{E}-09$ & $3.394 \mathrm{E}+03$ & $1.6441 \mathrm{E}+01$ & $8.891 \mathrm{E}-10$ & $2.417 \mathrm{E}+03$ \\
\hline $1 s 6 f^{3} F_{3}^{o}$ & $1 s 7 f^{3} F_{4}^{0}$ & $4.3526 \mathrm{E}+01$ & $9.513 \mathrm{E}-09$ & $3.722 \mathrm{E}+03$ & $4.3345 \mathrm{E}+01$ & $9.476 \mathrm{E}-09$ & $3.706 \mathrm{E}+03$ \\
\hline $1 s 6 f^{3} F_{4}^{o}$ & $1 s 7 f^{3} F_{4}^{o}$ & $4.3873 \mathrm{E}+01$ & $1.853 \mathrm{E}-08$ & $7.134 \mathrm{E}+03$ & $4.3688 \mathrm{E}+01$ & $1.782 \mathrm{E}-08$ & $6.863 \mathrm{E}+03$ \\
\hline $1 s 6 f^{1} F_{3}^{o}$ & $1 s 7 f^{3} F_{4}^{o}$ & $4.3884 \mathrm{E}+01$ & $2.962 \mathrm{E}-10$ & $1.140 \mathrm{E}+02$ & $4.3689 \mathrm{E}+01$ & $5.068 \mathrm{E}-10$ & $1.951 \mathrm{E}+02$ \\
\hline $1 s 6 h^{3} H_{5}^{o}$ & $1 s 7 f^{3} F_{4}^{o}$ & $4.4083 \mathrm{E}+01$ & $8.911 \mathrm{E}-15$ & $3.399 E-03$ & & & \\
\hline $1 s 6 h^{3} H_{4}^{0}$ & $1 s 7 f^{3} F_{4}^{0}$ & $4.4089 \mathrm{E}+01$ & $6.179 E-16$ & $2.356 \mathrm{E}-04$ & & & \\
\hline $1 s 6 h{ }^{1} H_{5}^{o}$ & $1 s 7 f^{3} F_{4}^{0}$ & $4.4228 \mathrm{E}+01$ & $2.315 \mathrm{E}-17$ & $8.771 \mathrm{E}-06$ & & & \\
\hline $1 s 7 f^{3} F_{3}^{o}$ & $1 s 7 f^{3} F_{4}^{0}$ & $8.7508 \mathrm{E}+03$ & $1.774 \mathrm{E}-06$ & $1.717 \mathrm{E}+01$ & $8.7713 E+03$ & $1.769 \mathrm{E}-06$ & $1.690 \mathrm{E}+01$ \\
\hline $1 s 3 d^{3} D_{3}$ & $1 s 7 g{ }^{3} G_{4}$ & $3.5600 \mathrm{E}+00$ & $3.651 \mathrm{E}-09$ & $2.135 E+05$ & $3.5449 \mathrm{E}+00$ & $3.702 \mathrm{E}-09$ & $2.164 \mathrm{E}+05$ \\
\hline $1 s 4 d^{3} D_{3}$ & $1 s 7 g{ }^{3} G_{4}$ & $7.6543 \mathrm{E}+00$ & $2.963 \mathrm{E}-11$ & $3.748 \mathrm{E}+02$ & $7.6223 \mathrm{E}+00$ & $2.880 \mathrm{E}-11$ & $3.642 \mathrm{E}+02$ \\
\hline $1 s 5 d^{3} D_{3}$ & $1 s 7 g{ }^{3} G_{4}$ & $1.6421 \mathrm{E}+01$ & $2.342 \mathrm{E}-10$ & $6.438 \mathrm{E}+02$ & $1.6353 \mathrm{E}+01$ & $2.341 \mathrm{E}-10$ & $6.434 \mathrm{E}+02$ \\
\hline $1 s 5 g{ }^{3} G_{4}$ & $1 s 7 g{ }^{3} G_{4}$ & $1.6509 \mathrm{E}+01$ & $1.147 \mathrm{E}-08$ & $3.119 E+04$ & $1.6439 \mathrm{E}+01$ & $1.146 \mathrm{E}-08$ & $3.116 \mathrm{E}+04$ \\
\hline $1 s 5 g{ }^{3} G_{3}$ & $1 s 7 g{ }^{3} G_{4}$ & $1.6511 \mathrm{E}+01$ & $2.735 \mathrm{E}-10$ & $7.434 \mathrm{E}+02$ & $1.6441 \mathrm{E}+01$ & $3.276 \mathrm{E}-10$ & $8.906 \mathrm{E}+02$ \\
\hline $1 s 5 g{ }^{3} G_{5}$ & $1 s 7 g{ }^{3} G_{4}$ & $1.6560 \mathrm{E}+01$ & $3.529 \mathrm{E}-09$ & $9.536 \mathrm{E}+03$ & $1.6490 \mathrm{E}+01$ & $3.511 \mathrm{E}-09$ & $9.489 \mathrm{E}+03$ \\
\hline $1 s 5 g{ }^{1} G_{4}$ & $1 s 7 g{ }^{3} G_{4}$ & $1.6562 \mathrm{E}+01$ & $8.238 \mathrm{E}-11$ & $2.226 \mathrm{E}+02$ & $1.6491 \mathrm{E}+01$ & $8.350 \mathrm{E}-11$ & $2.256 \mathrm{E}+02$ \\
\hline $1 s 6 d^{3} D_{3}$ & $1 s 7 g{ }^{3} G_{4}$ & $4.3514 \mathrm{E}+01$ & $1.318 \mathrm{E}-10$ & $5.160 \mathrm{E}+01$ & $4.3345 \mathrm{E}+01$ & $1.315 \mathrm{E}-10$ & $5.144 \mathrm{E}+01$ \\
\hline $1 s 6 g{ }^{3} G_{4}$ & $1 s 7 g{ }^{3} G_{4}$ & $4.3873 \mathrm{E}+01$ & $1.072 \mathrm{E}-08$ & $4.127 \mathrm{E}+03$ & $4.3688 \mathrm{E}+01$ & $1.069 \mathrm{E}-08$ & $4.117 \mathrm{E}+03$ \\
\hline $1 s 6 g^{3} G_{3}$ & $1 s 7 g{ }^{3} G_{4}$ & $4.3882 \mathrm{E}+01$ & $2.236 \mathrm{E}-10$ & $8.608 E+01$ & $4.3688 \mathrm{E}+01$ & $3.056 \mathrm{E}-10$ & $1.176 \mathrm{E}+02$ \\
\hline $1 s 6 g{ }^{3} G_{5}$ & $1 s 7 g{ }^{3} G_{4}$ & $4.4082 \mathrm{E}+01$ & $7.394 \mathrm{E}-09$ & $2.820 \mathrm{E}+03$ & $4.3896 \mathrm{E}+01$ & $7.356 \mathrm{E}-09$ & $2.805 \mathrm{E}+03$ \\
\hline $1 s 6 g{ }^{1} G_{4}$ & $1 s 7 g{ }^{3} G_{4}$ & $4.4089 \mathrm{E}+01$ & $1.748 \mathrm{E}-10$ & $6.664 \mathrm{E}+01$ & $4.3896 \mathrm{E}+01$ & $1.672 \mathrm{E}-10$ & $6.377 \mathrm{E}+01$ \\
\hline $1 s 7 d^{3} D_{3}$ & $1 s 7 g{ }^{3} G_{4}$ & $8.4183 E+03$ & $1.604 \mathrm{E}-13$ & $1.677 \mathrm{E}-06$ & $8.7854 \mathrm{E}+03$ & $1.162 \mathrm{E}-13$ & $1.106 \mathrm{E}-06$ \\
\hline $1 s 3 d^{3} D_{2}$ & $1 s 7 g{ }^{3} G_{3}$ & $3.5233 \mathrm{E}+00$ & $2.179 E-13$ & $1.673 \mathrm{E}+01$ & & & \\
\hline $1 s 3 d^{3} D_{3}$ & $1 s 7 g{ }^{3} G_{3}$ & $3.5600 \mathrm{E}+00$ & $1.630 \mathrm{E}-10$ & $1.225 \mathrm{E}+04$ & $3.5449 \mathrm{E}+00$ & $1.371 \mathrm{E}-10$ & $1.030 \mathrm{E}+04$ \\
\hline $1 s 3 d{ }^{1} D_{2}$ & $1 s 7 g{ }^{3} G_{3}$ & $3.5614 \mathrm{E}+00$ & $2.777 \mathrm{E}-09$ & $2.086 \mathrm{E}+05$ & $3.5464 \mathrm{E}+00$ & $2.747 \mathrm{E}-09$ & $2.064 \mathrm{E}+05$ \\
\hline $1 s 4 d^{3} D_{2}$ & $1 s 7 g{ }^{3} G_{3}$ & $7.5824 \mathrm{E}+00$ & $3.207 \mathrm{E}-16$ & $5.316 \mathrm{E}-03$ & & & \\
\hline $1 s 4 d^{3} D_{3}$ & $1 s 7 g{ }^{3} G_{3}$ & $7.6541 \mathrm{E}+00$ & $9.324 \mathrm{E}-13$ & $1.517 \mathrm{E}+01$ & $7.6223 \mathrm{E}+00$ & $1.066 \mathrm{E}-12$ & $1.734 \mathrm{E}+01$ \\
\hline $1 s 4 d{ }^{1} D_{2}$ & $1 s 7 g{ }^{3} G_{3}$ & $7.6571 \mathrm{E}+00$ & $2.102 \mathrm{E}-11$ & $3.417 \mathrm{E}+02$ & $7.6253 \mathrm{E}+00$ & $2.121 \mathrm{E}-11$ & $3.447 \mathrm{E}+02$ \\
\hline $1 s 5 d^{3} D_{2}$ & $1 s 7 g{ }^{3} G_{3}$ & $1.6252 \mathrm{E}+01$ & $1.814 \mathrm{E}-14$ & $6.543 \mathrm{E}-02$ & & & \\
\hline $1 s 5 d^{3} D_{3}$ & $1 s 7 g{ }^{3} G_{3}$ & $1.6420 \mathrm{E}+01$ & $9.558 \mathrm{E}-12$ & $3.378 E+01$ & $1.6353 \mathrm{E}+01$ & $8.684 \mathrm{E}-12$ & $3.068 \mathrm{E}+01$ \\
\hline $1 s 5 d{ }^{1} D_{2}$ & $1 s 7 g{ }^{3} G_{3}$ & $1.6428 \mathrm{E}+01$ & $1.752 \mathrm{E}-10$ & $6.185 E+02$ & $1.6361 \mathrm{E}+01$ & $1.734 \mathrm{E}-10$ & $6.122 \mathrm{E}+02$ \\
\hline $1 s 5 g{ }^{3} G_{4}$ & $1 s 7 g{ }^{3} G_{3}$ & $1.6509 \mathrm{E}+01$ & $3.892 \mathrm{E}-10$ & $1.361 \mathrm{E}+03$ & $1.6439 \mathrm{E}+01$ & $3.312 \mathrm{E}-10$ & $1.158 \mathrm{E}+03$ \\
\hline $1 s 5 g{ }^{3} G_{3}$ & $1 s 7 g{ }^{3} G_{3}$ & $1.6511 \mathrm{E}+01$ & $8.849 \mathrm{E}-09$ & $3.093 E+04$ & $1.6441 \mathrm{E}+01$ & $8.844 \mathrm{E}-09$ & $3.091 \mathrm{E}+04$ \\
\hline $1 s 5 g{ }^{1} G_{4}$ & $1 s 7 g{ }^{3} G_{3}$ & $1.6561 \mathrm{E}+01$ & $2.778 \mathrm{E}-09$ & $9.652 E+03$ & $1.6491 \mathrm{E}+01$ & $2.789 \mathrm{E}-09$ & $9.690 \mathrm{E}+03$ \\
\hline $1 s 6 d^{3} D_{2}$ & $1 s 7 g{ }^{3} G_{3}$ & $4.2827 \mathrm{E}+01$ & $1.476 \mathrm{E}-14$ & $7.669 \mathrm{E}-03$ & & & \\
\hline $1 s 6 d^{3} D_{3}$ & $1 s 7 g{ }^{3} G_{3}$ & $4.3508 \mathrm{E}+01$ & $5.636 \mathrm{E}-12$ & $2.837 \mathrm{E}+00$ & $4.3345 \mathrm{E}+01$ & $4.871 \mathrm{E}-12$ & $2.449 \mathrm{E}+00$ \\
\hline $1 s 6 d^{1} D_{2}$ & $1 s 7 g{ }^{3} G_{3}$ & $4.3538 \mathrm{E}+01$ & $9.946 \mathrm{E}-11$ & $5.000 \mathrm{E}+01$ & $4.3356 \mathrm{E}+01$ & $9.747 \mathrm{E}-11$ & $4.899 \mathrm{E}+01$ \\
\hline $1 s 6 g{ }^{3} G_{4}$ & $1 s 7 g{ }^{3} G_{3}$ & $4.3868 \mathrm{E}+01$ & $4.042 \mathrm{E}-10$ & $2.002 \mathrm{E}+02$ & $4.3688 \mathrm{E}+01$ & $3.055 \mathrm{E}-10$ & $1.512 \mathrm{E}+02$ \\
\hline $1 s 6 g{ }^{3} G_{3}$ & $1 s 7 g{ }^{3} G_{3}$ & $4.3876 \mathrm{E}+01$ & $8.273 \mathrm{E}-09$ & $4.095 \mathrm{E}+03$ & $4.3688 \mathrm{E}+01$ & $8.251 \mathrm{E}-09$ & $4.084 \mathrm{E}+03$ \\
\hline $1 s 6 g{ }^{1} G_{4}$ & $1 s 7 g{ }^{3} G_{3}$ & $4.4084 \mathrm{E}+01$ & $5.822 \mathrm{E}-09$ & $2.855 \mathrm{E}+03$ & $4.3896 \mathrm{E}+01$ & $5.851 \mathrm{E}-09$ & $2.869 \mathrm{E}+03$ \\
\hline $1 s 7 d^{3} D_{2}$ & $1 s 7 g{ }^{3} G_{3}$ & $2.8438 \mathrm{E}+03$ & $4.640 \mathrm{E}-15$ & $5.467 \mathrm{E}-07$ & $2.9148 \mathrm{E}+03$ & $3.797 \mathrm{E}-17$ & $4.222 \mathrm{E}-09$ \\
\hline $1 s 7 d^{3} D_{3}$ & $1 s 7 g{ }^{3} G_{3}$ & $8.2277 \mathrm{E}+03$ & $3.480 \mathrm{E}-15$ & $4.899 \mathrm{E}-08$ & $8.7854 \mathrm{E}+03$ & $4.304 \mathrm{E}-15$ & $5.269 \mathrm{E}-08$ \\
\hline $1 s 7 d^{1} D_{2}$ & $1 s 7 g{ }^{3} G_{3}$ & $8.9575 E+03$ & $6.963 \mathrm{E}-14$ & $8.269 \mathrm{E}-07$ & $9.0724 \mathrm{E}+03$ & $8.336 \mathrm{E}-14$ & $9.568 \mathrm{E}-07$ \\
\hline $1 s 7 g{ }^{3} G_{4}$ & $1 s 7 g{ }^{3} G_{3}$ & $3.6344 \mathrm{E}+05$ & $4.407 \mathrm{E}-08$ & $3.179 \mathrm{E}-04$ & & & \\
\hline $1 s 2 p^{3} P_{2}^{o}$ & $1 s 7 f^{1} F_{3}^{o}$ & $1.3940 \mathrm{E}+00$ & $4.032 \mathrm{E}-13$ & $1.977 \mathrm{E}+02$ & $1.3881 \mathrm{E}+00$ & $2.999 \mathrm{E}-15$ & $1.470 \mathrm{E}+00$ \\
\hline $1 s 3 p^{3} P_{2}^{o}$ & $1 s 7 f^{1} F_{3}^{o}$ & $3.5149 \mathrm{E}+00$ & $1.959 \mathrm{E}-14$ & $1.511 \mathrm{E}+00$ & $3.5002 \mathrm{E}+00$ & $1.688 \mathrm{E}-16$ & $1.302 \mathrm{E}-02$ \\
\hline $1 s 4 p^{3} P_{2}^{o}$ & $1 s 7 f^{1} F_{3}^{o}$ & $7.5662 \mathrm{E}+00$ & $4.169 \mathrm{E}-14$ & $6.939 \mathrm{E}-01$ & & & \\
\hline $1 s 4 f^{3} F_{3}^{o}$ & $1 s 7 f^{1} F_{3}^{o}$ & $7.6552 \mathrm{E}+00$ & $8.991 \mathrm{E}-11$ & $1.462 \mathrm{E}+03$ & $7.6229 \mathrm{E}+00$ & $7.186 \mathrm{E}-11$ & $1.168 \mathrm{E}+03$ \\
\hline $1 s 4 f^{3} F_{2}^{0}$ & $1 s 7 f^{1} F_{3}^{0}$ & $7.6567 \mathrm{E}+00$ & $1.318 \mathrm{E}-09$ & $2.141 \mathrm{E}+04$ & $7.6244 \mathrm{E}+00$ & $1.283 \mathrm{E}-09$ & $2.086 \mathrm{E}+04$ \\
\hline $1 s 4 f^{3} F_{4}^{o}$ & $1 s 7 f^{1} F_{3}^{o}$ & $7.6912 \mathrm{E}+00$ & $2.427 \mathrm{E}-09$ & $3.910 \mathrm{E}+04$ & $7.6586 \mathrm{E}+00$ & $1.170 \mathrm{E}-09$ & $1.884 \mathrm{E}+04$ \\
\hline $1 s 4 f^{1} F_{3}^{o}$ & $1 s 7 f^{1} F_{3}^{o}$ & $7.6923 \mathrm{E}+00$ & $3.464 \mathrm{E}-08$ & $5.579 E+05$ & $7.6598 \mathrm{E}+00$ & $3.148 \mathrm{E}-08$ & $5.069 \mathrm{E}+05$ \\
\hline $1 s 5 p^{3} P_{2}^{o}$ & $1 s 7 f^{1} F_{3}^{o}$ & $1.6214 \mathrm{E}+01$ & $4.380 \mathrm{E}-14$ & $1.588 \mathrm{E}-01$ & & & \\
\hline $1 s 5 f^{3} F_{3}^{o}$ & $1 s 7 f^{1} F_{3}^{o}$ & $1.6423 \mathrm{E}+01$ & $1.913 \mathrm{E}-10$ & $6.757 \mathrm{E}+02$ & $1.6354 \mathrm{E}+01$ & $1.592 \mathrm{E}-10$ & $5.624 \mathrm{E}+02$ \\
\hline $1 s 5 f^{3} F_{2}^{o}$ & $1 s 7 f^{1} F_{3}^{o}$ & $1.6427 \mathrm{E}+01$ & $3.067 \mathrm{E}-09$ & $1.083 E+04$ & $1.6358 \mathrm{E}+01$ & $2.994 \mathrm{E}-09$ & $1.057 \mathrm{E}+04$ \\
\hline $1 s 5 f^{3} F_{4}^{o}$ & $1 s 7 f^{1} F_{3}^{o}$ & $1.6508 \mathrm{E}+01$ & $1.547 \mathrm{E}-09$ & $5.408 \mathrm{E}+03$ & $1.6439 \mathrm{E}+01$ & $8.855 \mathrm{E}-10$ & $3.096 \mathrm{E}+03$ \\
\hline $1 s 5 f^{1} F_{3}^{o}$ & $1 s 7 f^{1} F_{3}^{o}$ & $1.6511 \mathrm{E}+01$ & $2.526 \mathrm{E}-08$ & $8.829 E+04$ & $1.6441 \mathrm{E}+01$ & $2.379 \mathrm{E}-08$ & $8.315 E+04$ \\
\hline $1 s 6 p^{3} P_{2}^{o}$ & $1 s 7 f^{1} F_{3}^{o}$ & $4.2675 \mathrm{E}+01$ & $5.245 \mathrm{E}-14$ & $2.744 \mathrm{E}-02$ & & & \\
\hline
\end{tabular}


Table 5 (continued)

\begin{tabular}{|c|c|c|c|c|c|c|c|}
\hline \multirow[t]{2}{*}{ Lower } & \multirow[t]{2}{*}{ Upper } & \multicolumn{3}{|l|}{ GRASP2K } & \multicolumn{3}{|l|}{ FAC } \\
\hline & & $\lambda(\AA)$ & $g f$ & $A\left(\mathrm{~s}^{-1}\right)$ & $\lambda(\AA)$ & $g f$ & $A\left(\mathrm{~s}^{-1}\right)$ \\
\hline $1 s 6 f^{3} F_{3}^{o}$ & $1 s 7 f^{1} F_{3}^{o}$ & $4.3519 \mathrm{E}+01$ & $4.115 \mathrm{E}-10$ & $2.070 \mathrm{E}+02$ & $4.3345 \mathrm{E}+01$ & $3.501 \mathrm{E}-10$ & $1.760 \mathrm{E}+02$ \\
\hline $1 s 6 f^{3} F_{2}^{0}$ & $1 s 7 f^{1} F_{3}^{o}$ & $4.3534 \mathrm{E}+01$ & $7.076 \mathrm{E}-09$ & $3.558 \mathrm{E}+03$ & $4.3345 \mathrm{E}+01$ & $7.015 \mathrm{E}-09$ & $3.528 \mathrm{E}+03$ \\
\hline $1 s 6 f^{3} F_{4}^{o}$ & $1 s 7 f^{1} F_{3}^{o}$ & $4.3866 \mathrm{E}+01$ & $5.137 \mathrm{E}-10$ & $2.544 \mathrm{E}+02$ & $4.3688 \mathrm{E}+01$ & $5.131 \mathrm{E}-10$ & $2.540 \mathrm{E}+02$ \\
\hline $1 s 6 f^{1} F_{3}^{o}$ & $1 s 7 f^{1} F_{3}^{o}$ & $4.3877 \mathrm{E}+01$ & $1.345 \mathrm{E}-08$ & $6.659 \mathrm{E}+03$ & $4.3688 \mathrm{E}+01$ & $1.375 \mathrm{E}-08$ & $6.807 \mathrm{E}+03$ \\
\hline $1 s 6 h^{3} H_{4}^{o}$ & $1 s 7 f^{1} F_{3}^{o}$ & $4.4082 \mathrm{E}+01$ & $3.010 \mathrm{E}-15$ & $1.476 \mathrm{E}-03$ & & & \\
\hline $1 s 7 p^{3} P_{2}^{0}$ & $1 s 7 f^{1} F_{3}^{o}$ & $2.4995 \mathrm{E}+03$ & $1.402 \mathrm{E}-12$ & $2.139 \mathrm{E}-04$ & & & \\
\hline $1 s 7 f^{3} F_{3}^{o}$ & $1 s 7 f^{1} F_{3}^{o}$ & $8.4824 \mathrm{E}+03$ & $6.791 \mathrm{E}-08$ & $8.993 \mathrm{E}-01$ & $8.7645 E+03$ & $6.556 \mathrm{E}-08$ & $8.063 \mathrm{E}-01$ \\
\hline $1 s 7 f^{3} F_{2}^{o}$ & $1 s 7 f^{1} F_{3}^{o}$ & $8.8420 \mathrm{E}+03$ & $1.310 \mathrm{E}-06$ & $1.597 \mathrm{E}+01$ & $8.7598 \mathrm{E}+03$ & $1.309 \mathrm{E}-06$ & $1.612 \mathrm{E}+01$ \\
\hline $1 s 7 f^{3} F_{4}^{o}$ & $1 s 7 f^{1} F_{3}^{o}$ & $2.7665 \mathrm{E}+05$ & $3.244 \mathrm{E}-08$ & $4.039 \mathrm{E}-04$ & & & \\
\hline $1 s 5 g^{3} G_{4}$ & $1 s 7 g^{3} G_{5}^{3}$ & $1.6491 \mathrm{E}+01$ & $7.591 \mathrm{E}-10$ & $1.693 \mathrm{E}+03$ & $1.6420 \mathrm{E}+01$ & $7.556 \mathrm{E}-10$ & $1.685 \mathrm{E}+03$ \\
\hline $1 s 5 g^{3} G_{5}$ & $1 s 7 g{ }^{3} G_{5}$ & $1.6542 \mathrm{E}+01$ & $2.746 \mathrm{E}-08$ & $6.085 \mathrm{E}+04$ & $1.6471 \mathrm{E}+01$ & $2.743 \mathrm{E}-08$ & $6.079 \mathrm{E}+04$ \\
\hline $1 s 5 g{ }^{1} G_{4}$ & $1 s 7 g{ }^{3} G_{5}$ & $1.6543 \mathrm{E}+01$ & $4.584 \mathrm{E}-10$ & $1.016 \mathrm{E}+03$ & $1.6473 \mathrm{E}+01$ & $5.103 \mathrm{E}-10$ & $1.130 \mathrm{E}+03$ \\
\hline $1 s 6 g{ }^{3} G_{4}$ & $1 s 7 g{ }^{3} G_{5}$ & $4.3743 \mathrm{E}+01$ & $3.029 \mathrm{E}-09$ & $9.600 \mathrm{E}+02$ & $4.3559 \mathrm{E}+01$ & $3.023 \mathrm{E}-09$ & $9.580 \mathrm{E}+02$ \\
\hline $1 s 6 g{ }^{3} G_{5}$ & $1 s 7 g{ }^{3} G_{5}$ & $4.3950 \mathrm{E}+01$ & $2.568 \mathrm{E}-08$ & $8.060 \mathrm{E}+03$ & $4.3765 \mathrm{E}+01$ & $2.559 \mathrm{E}-08$ & $8.033 E+03$ \\
\hline $1 s 6 g{ }^{1} G_{4}$ & $1 s 7 g{ }^{3} G_{5}$ & $4.3957 \mathrm{E}+01$ & $3.996 \mathrm{E}-10$ & $1.254 \mathrm{E}+02$ & $4.3765 \mathrm{E}+01$ & $4.739 \mathrm{E}-10$ & $1.487 \mathrm{E}+02$ \\
\hline $1 s 7 g{ }^{3} G_{4}$ & $1 s 7 g{ }^{3} G_{5}$ & $1.4677 \mathrm{E}+04$ & $1.341 \mathrm{E}-06$ & $3.774 \mathrm{E}+00$ & $1.4692 \mathrm{E}+04$ & $1.337 \mathrm{E}-06$ & $3.726 \mathrm{E}+00$ \\
\hline $1 s 4 f^{3} F_{4}^{o}$ & $1 s 7 h^{3} H_{5}^{o}$ & $7.6874 \mathrm{E}+00$ & $1.958 \mathrm{E}-09$ & $2.009 \mathrm{E}+04$ & $7.6547 \mathrm{E}+00$ & $1.933 \mathrm{E}-09$ & $1.984 \mathrm{E}+04$ \\
\hline $1 s 5 f^{3} F_{4}^{0}$ & $1 s 7 h^{3} H_{5}^{o}$ & $1.6491 \mathrm{E}+01$ & $2.258 \mathrm{E}-11$ & $5.035 \mathrm{E}+01$ & $1.6420 \mathrm{E}+01$ & $2.098 \mathrm{E}-11$ & $4.678 \mathrm{E}+01$ \\
\hline $1 s 6 f^{3} F_{4}^{o}$ & $1 s 7 h^{3} H_{5}^{o}$ & $4.3742 \mathrm{E}+01$ & $2.390 \mathrm{E}-10$ & $7.574 \mathrm{E}+01$ & $4.3559 \mathrm{E}+01$ & $2.347 \mathrm{E}-10$ & $7.437 \mathrm{E}+01$ \\
\hline $1 s 6 h^{3} H_{5}^{o}$ & $1 s 7 h^{3} H_{5}^{o}$ & $4.3951 \mathrm{E}+01$ & $1.167 \mathrm{E}-08$ & $3.663 \mathrm{E}+03$ & $4.3765 \mathrm{E}+01$ & $1.215 \mathrm{E}-08$ & $3.814 \mathrm{E}+03$ \\
\hline $1 s 6 h^{3} H_{4}^{o}$ & $1 s 7 h^{3} H_{5}^{o}$ & $4.3956 \mathrm{E}+01$ & $2.729 \mathrm{E}-10$ & $8.564 \mathrm{E}+01$ & $4.3765 \mathrm{E}+01$ & $2.250 \mathrm{E}-10$ & $7.063 \mathrm{E}+01$ \\
\hline $1 s 6 h^{3} H_{6}^{o}$ & $1 s 7 h^{3} H_{5}^{o}$ & $4.4090 \mathrm{E}+01$ & $2.985 \mathrm{E}-09$ & $9.311 \mathrm{E}+02$ & $4.3903 \mathrm{E}+01$ & $2.974 \mathrm{E}-09$ & $9.277 \mathrm{E}+02$ \\
\hline $1 s 6 h{ }^{1} H_{5}^{o}$ & $1 s 7 h^{3} H_{5}^{o}$ & $4.4094 \mathrm{E}+01$ & $4.732 \mathrm{E}-11$ & $1.476 \mathrm{E}+01$ & $4.3903 \mathrm{E}+01$ & $4.575 \mathrm{E}-11$ & $1.427 \mathrm{E}+01$ \\
\hline $1 s 7 f^{3} F_{4}^{o}$ & $1 s 7 h^{3} H_{5}^{o}$ & $1.4614 \mathrm{E}+04$ & $3.511 \mathrm{E}-14$ & $9.969 \mathrm{E}-08$ & $1.4763 \mathrm{E}+04$ & $3.624 \mathrm{E}-14$ & $1.000 \mathrm{E}-07$ \\
\hline $1 s 4 f^{3} F_{3}^{0}$ & $1 s 7 h^{3} H_{4}^{o}$ & $7.6513 \mathrm{E}+00$ & $4.508 \mathrm{E}-15$ & $5.707 \mathrm{E}-02$ & & & \\
\hline $1 s 4 f^{3} F_{4}^{o}$ & $1 s 7 h^{3} H_{4}^{0}$ & $7.6873 \mathrm{E}+00$ & $5.158 \mathrm{E}-11$ & $6.470 \mathrm{E}+02$ & $7.6547 \mathrm{E}+00$ & $4.394 \mathrm{E}-11$ & $5.511 \mathrm{E}+02$ \\
\hline $1 s 4 f^{1} F_{3}^{o}$ & $1 s 7 h^{3} H_{4}^{0}$ & $7.6884 \mathrm{E}+00$ & $1.583 \mathrm{E}-09$ & $1.985 \mathrm{E}+04$ & $7.6558 \mathrm{E}+00$ & $1.539 \mathrm{E}-09$ & $1.929 \mathrm{E}+04$ \\
\hline $1 s 5 f^{3} F_{3}^{o}$ & $1 s 7 h^{3} H_{4}^{0}$ & $1.6405 \mathrm{E}+01$ & $8.772 \mathrm{E}-18$ & $2.416 \mathrm{E}-05$ & & & \\
\hline $1 s 5 f^{3} F_{4}^{o}$ & $1 s 7 h^{3} H_{4}^{0}$ & $1.6490 \mathrm{E}+01$ & $5.160 \mathrm{E}-13$ & $1.406 \mathrm{E}+00$ & $1.6420 \mathrm{E}+01$ & $4.768 \mathrm{E}-13$ & $1.299 \mathrm{E}+00$ \\
\hline $1 s 5 f^{1} F_{3}^{o}$ & $1 s 7 h^{3} H_{4}^{0}$ & $1.6493 \mathrm{E}+01$ & $1.767 \mathrm{E}-11$ & $4.816 \mathrm{E}+01$ & $1.6423 \mathrm{E}+01$ & $1.666 \mathrm{E}-11$ & $4.540 \mathrm{E}+01$ \\
\hline $1 s 6 f^{3} F_{3}^{o}$ & $1 s 7 h^{3} H_{4}^{o}$ & $4.3394 \mathrm{E}+01$ & $6.981 \mathrm{E}-16$ & $2.748 \mathrm{E}-04$ & & & \\
\hline $1 s 6 f^{3} F_{4}^{o}$ & $1 s 7 h^{3} H_{4}^{0}$ & $4.3738 \mathrm{E}+01$ & $6.216 \mathrm{E}-12$ & $2.408 \mathrm{E}+00$ & $4.3559 \mathrm{E}+01$ & $5.334 \mathrm{E}-12$ & $2.065 \mathrm{E}+00$ \\
\hline $1 s 6 f^{1} F_{3}^{0}$ & $1 s 7 h^{3} H_{4}^{0}$ & $4.3749 \mathrm{E}+01$ & $1.922 \mathrm{E}-10$ & $7.442 \mathrm{E}+01$ & $4.3560 \mathrm{E}+01$ & $1.866 \mathrm{E}-10$ & $7.230 \mathrm{E}+01$ \\
\hline $1 s 6 h^{3} H_{5}^{o}$ & $1 s 7 h^{3} H_{4}^{0}$ & $4.3947 \mathrm{E}+01$ & $3.798 \mathrm{E}-10$ & $1.458 \mathrm{E}+02$ & $4.3765 \mathrm{E}+01$ & $2.250 \mathrm{E}-10$ & $8.632 \mathrm{E}+01$ \\
\hline $1 s 6 h^{3} H_{4}^{o}$ & $1 s 7 h^{3} H_{4}^{0}$ & $4.3953 \mathrm{E}+01$ & $1.029 \mathrm{E}-08$ & $3.949 \mathrm{E}+03$ & $4.3765 \mathrm{E}+01$ & $9.900 \mathrm{E}-09$ & $3.798 E+03$ \\
\hline $1 s 6 h{ }^{1} H_{5}^{o}$ & $1 s 7 h^{3} H_{4}^{0}$ & $4.4091 \mathrm{E}+01$ & $2.459 \mathrm{E}-09$ & $9.374 \mathrm{E}+02$ & $4.3903 \mathrm{E}+01$ & $2.470 \mathrm{E}-09$ & $9.420 \mathrm{E}+02$ \\
\hline $1 s 7 f^{3} F_{3}^{o}$ & $1 s 7 h^{3} H_{4}^{o}$ & $5.4206 \mathrm{E}+03$ & $1.109 \mathrm{E}-18$ & $2.798 \mathrm{E}-11$ & & & \\
\hline $1 s 7 f^{3} F_{4}^{o}$ & $1 s 7 h^{3} H_{4}^{0}$ & $1.4244 \mathrm{E}+04$ & $7.671 \mathrm{E}-16$ & $2.802 \mathrm{E}-09$ & $1.4763 \mathrm{E}+04$ & $8.238 \mathrm{E}-16$ & $2.777 \mathrm{E}-09$ \\
\hline $1 s 7 f^{1} F_{3}^{o}$ & $1 s 7 h^{3} H_{4}^{o}$ & $1.5017 \mathrm{E}+04$ & $2.719 \mathrm{E}-14$ & $8.935 \mathrm{E}-08$ & $1.4782 \mathrm{E}+04$ & $2.879 \mathrm{E}-14$ & $9.684 \mathrm{E}-08$ \\
\hline $1 s 7 h^{3} H_{5}^{o}$ & $1 s 7 h^{3} H_{4}^{0}$ & $5.6215 \mathrm{E}+05$ & $3.438 \mathrm{E}-08$ & $8.064 \mathrm{E}-05$ & & & \\
\hline $1 s 3 d^{3} D_{3}^{3}$ & $1 s 7 g{ }^{1} G_{4}$ & $3.5591 \mathrm{E}+00$ & $4.551 \mathrm{E}-14$ & $2.663 \mathrm{E}+00$ & $3.5441 \mathrm{E}+00$ & $5.680 \mathrm{E}-19$ & $3.323 E-05$ \\
\hline $1 s 4 d^{3} D_{3}$ & $1 s 7 g{ }^{1} G_{4}$ & $7.6502 \mathrm{E}+00$ & $1.289 \mathrm{E}-15$ & $1.633 \mathrm{E}-02$ & $7.6183 \mathrm{E}+00$ & $4.456 \mathrm{E}-21$ & $5.642 \mathrm{E}-08$ \\
\hline $1 s 5 d^{3} D_{3}$ & $1 s 7 g{ }^{1} G_{4}$ & $1.6402 \mathrm{E}+01$ & $6.373 \mathrm{E}-16$ & $1.756 \mathrm{E}-03$ & & & \\
\hline $1 s 5 g{ }^{3} G_{4}$ & $1 s 7 g^{1} G_{4}$ & $1.6490 \mathrm{E}+01$ & $1.984 \mathrm{E}-11$ & $5.409 \mathrm{E}+01$ & $1.6420 \mathrm{E}+01$ & $1.967 \mathrm{E}-11$ & $5.362 \mathrm{E}+01$ \\
\hline $1 s 5 g{ }^{3} G_{3}$ & $1 s 7 g{ }^{1} G_{4}$ & $1.6492 \mathrm{E}+01$ & $5.980 \mathrm{E}-10$ & $1.629 \mathrm{E}+03$ & $1.6423 \mathrm{E}+01$ & $6.027 \mathrm{E}-10$ & $1.642 \mathrm{E}+03$ \\
\hline $1 s 5 g^{3} G_{5}$ & $1 s 7 g{ }^{1} G_{4}$ & $1.6541 \mathrm{E}+01$ & $5.583 \mathrm{E}-10$ & $1.512 \mathrm{E}+03$ & $1.6471 \mathrm{E}+01$ & $5.080 \mathrm{E}-10$ & $1.376 \mathrm{E}+03$ \\
\hline $1 s 5 g{ }^{1} G_{4}$ & $1 s 7 g{ }^{1} G_{4}$ & $1.6543 \mathrm{E}+01$ & $2.237 \mathrm{E}-08$ & $6.059 \mathrm{E}+04$ & $1.6473 \mathrm{E}+01$ & $2.234 \mathrm{E}-08$ & $6.052 \mathrm{E}+04$ \\
\hline $1 s 6 d^{3} D_{3}$ & $1 s 7 g{ }^{1} G_{4}$ & $4.3381 \mathrm{E}+01$ & $1.570 \mathrm{E}-15$ & $6.182 \mathrm{E}-04$ & & & \\
\hline $1 s 6 g{ }^{3} G_{4}$ & $1 s 7 g{ }^{1} G_{4}$ & $4.3738 \mathrm{E}+01$ & $7.608 \mathrm{E}-11$ & $2.948 \mathrm{E}+01$ & $4.3559 \mathrm{E}+01$ & $6.873 \mathrm{E}-11$ & $2.662 \mathrm{E}+01$ \\
\hline $1 s 6 g{ }^{3} G_{3}$ & $1 s 7 g{ }^{1} G_{4}$ & $4.3747 \mathrm{E}+01$ & $2.385 \mathrm{E}-09$ & $9.236 \mathrm{E}+02$ & $4.3559 \mathrm{E}+01$ & $2.405 \mathrm{E}-09$ & $9.314 \mathrm{E}+02$ \\
\hline $1 s 6 g{ }^{3} G_{5}$ & $1 s 7 g{ }^{1} G_{4}$ & $4.3946 \mathrm{E}+01$ & $5.553 \mathrm{E}-10$ & $2.131 \mathrm{E}+02$ & $4.3765 \mathrm{E}+01$ & $4.739 \mathrm{E}-10$ & $1.818 \mathrm{E}+02$ \\
\hline $1 s 6 g{ }^{1} G_{4}$ & $1 s 7 g^{1} G_{4}$ & $4.3953 \mathrm{E}+01$ & $2.093 \mathrm{E}-08$ & $8.029 E+03$ & $4.3765 \mathrm{E}+01$ & $2.085 \mathrm{E}-08$ & $8.000 \mathrm{E}+03$ \\
\hline $1 s 7 d^{3} D_{3}$ & $1 s 7 g{ }^{1} G_{4}$ & $5.2883 \mathrm{E}+03$ & $3.941 \mathrm{E}-15$ & $1.044 \mathrm{E}-07$ & & & \\
\hline $1 s 7 g{ }^{3} G_{4}$ & $1 s 7 g{ }^{1} G_{4}$ & $1.4223 \mathrm{E}+04$ & $3.145 \mathrm{E}-08$ & $1.152 \mathrm{E}-01$ & $1.4691 \mathrm{E}+04$ & $3.040 \mathrm{E}-08$ & $1.035 \mathrm{E}-01$ \\
\hline $1 s 7 g{ }^{3} G_{3}$ & $1 s 7 g{ }^{1} G_{4}$ & $1.4802 \mathrm{E}+04$ & $1.065 \mathrm{E}-06$ & $3.602 \mathrm{E}+00$ & $1.4691 \mathrm{E}+04$ & $1.064 \mathrm{E}-06$ & $3.623 E+00$ \\
\hline $1 s 7 g{ }^{3} G_{5}$ & $1 s 7 g{ }^{1} G_{4}$ & $4.5954 \mathrm{E}+05$ & $2.680 \mathrm{E}-08$ & $9.406 \mathrm{E}-05$ & & & \\
\hline $1 s 6 h^{3} H_{5}^{o}$ & $1 s 7 h^{3} H_{6}^{o}$ & $4.3863 \mathrm{E}+01$ & $8.357 \mathrm{E}-10$ & $2.229 \mathrm{E}+02$ & $4.3678 \mathrm{E}+01$ & $8.370 \mathrm{E}-10$ & $2.232 \mathrm{E}+02$ \\
\hline $1 s 6 h^{3} H_{6}^{o}$ & $1 s 7 h^{3} H_{6}^{o}$ & $4.4002 \mathrm{E}+01$ & $2.409 \mathrm{E}-08$ & $6.384 \mathrm{E}+03$ & $4.3816 \mathrm{E}+01$ & $2.482 \mathrm{E}-08$ & $6.577 \mathrm{E}+03$ \\
\hline $1 s 6 h{ }^{1} H_{5}^{o}$ & $1 s 7 h^{3} H_{6}^{o}$ & $4.4006 \mathrm{E}+01$ & $4.069 \mathrm{E}-10$ & $1.078 \mathrm{E}+02$ & $4.3816 \mathrm{E}+01$ & $3.223 \mathrm{E}-10$ & $8.541 \mathrm{E}+01$ \\
\hline $1 s 7 h^{3} H_{5}^{o}$ & $1 s 7 h^{3} H_{6}^{o}$ & $2.2090 \mathrm{E}+04$ & $1.077 \mathrm{E}-06$ & $1.133 \mathrm{E}+00$ & $2.2109 \mathrm{E}+04$ & $1.074 \mathrm{E}-06$ & $1.118 \mathrm{E}+00$ \\
\hline $1 s 5 g^{3} G_{5}$ & $1 s 7 i^{3} I_{6}$ & $1.6529 \mathrm{E}+01$ & $9.680 \mathrm{E}-10$ & $1.818 \mathrm{E}+03$ & $1.6459 \mathrm{E}+01$ & $9.718 \mathrm{E}-10$ & $1.825 \mathrm{E}+03$ \\
\hline $1 s 6 g^{3} G_{5}$ & $1 s 7 i^{3} I_{6}$ & $4.3863 \mathrm{E}+01$ & $2.601 \mathrm{E}-10$ & $6.937 \mathrm{E}+01$ & $4.3678 \mathrm{E}+01$ & $2.590 \mathrm{E}-10$ & $6.907 \mathrm{E}+01$ \\
\hline $1 s 7 g{ }^{3} G_{5}$ & $1 s 7 i^{3} I_{6}$ & $2.2046 \mathrm{E}+04$ & $8.252 \mathrm{E}-15$ & $8.712 \mathrm{E}-09$ & $2.2108 \mathrm{E}+04$ & $8.218 \mathrm{E}-15$ & $8.555 \mathrm{E}-09$ \\
\hline $1 s 5 g^{3} G_{4}$ & $1 s 7 i^{3} I_{5}$ & $1.6478 \mathrm{E}+01$ & $2.959 \mathrm{E}-15$ & $6.609 \mathrm{E}-03$ & & & \\
\hline $1 s 5 g^{3} G_{5}$ & $1 s 7 i^{3} I_{5}$ & $1.6529 \mathrm{E}+01$ & $1.752 \mathrm{E}-11$ & $3.888 \mathrm{E}+01$ & $1.6459 \mathrm{E}+01$ & $1.495 \mathrm{E}-11$ & $3.318 \mathrm{E}+01$ \\
\hline $1 s 5 g{ }^{1} G_{4}$ & $1 s 7 i^{3} I_{5}$ & $1.6531 \mathrm{E}+01$ & $8.153 \mathrm{E}-10$ & $1.809 \mathrm{E}+03$ & $1.6460 \mathrm{E}+01$ & $8.077 \mathrm{E}-10$ & $1.792 \mathrm{E}+03$ \\
\hline $1 s 6 g^{3} G_{4}$ & $1 s 7 i^{3} I_{5}$ & $4.3654 \mathrm{E}+01$ & $1.569 \mathrm{E}-16$ & $4.993 \mathrm{E}-05$ & & & \\
\hline $1 s 6 g^{3} G_{5}$ & $1 s 7 i^{3} I_{5}$ & $4.3861 \mathrm{E}+01$ & $4.500 \mathrm{E}-12$ & $1.418 \mathrm{E}+00$ & $4.3678 \mathrm{E}+01$ & $3.985 \mathrm{E}-12$ & $1.255 \mathrm{E}+00$ \\
\hline $1 s 6 g{ }^{1} G_{4}$ & $1 s 7 i^{3} I_{5}$ & $4.3867 \mathrm{E}+01$ & $2.181 \mathrm{E}-10$ & $6.873 \mathrm{E}+01$ & $4.3678 \mathrm{E}+01$ & $2.152 \mathrm{E}-10$ & $6.782 \mathrm{E}+01$ \\
\hline $1 s 7 g{ }^{3} G_{4}$ & $1 s 7 i^{3} I_{5}$ & $8.7156 \mathrm{E}+03$ & $3.038 \mathrm{E}-20$ & $2.425 \mathrm{E}-13$ & & & \\
\hline $1 s 7 g{ }^{3} G_{5}$ & $1 s 7 i^{3} I_{5}$ & $2.1457 \mathrm{E}+04$ & $1.337 \mathrm{E}-16$ & $1.761 \mathrm{E}-10$ & $2.2108 \mathrm{E}+04$ & $1.264 \mathrm{E}-16$ & $1.555 \mathrm{E}-10$ \\
\hline
\end{tabular}


Table 5 (continued)

\begin{tabular}{|c|c|c|c|c|c|c|c|}
\hline \multirow[t]{2}{*}{ Lower } & \multirow[t]{2}{*}{ Upper } & \multicolumn{3}{|l|}{ GRASP2K } & \multicolumn{3}{|l|}{ FAC } \\
\hline & & $\lambda(\AA)$ & $g f$ & $A\left(\mathrm{~s}^{-1}\right)$ & $\lambda(\AA)$ & $g f$ & $A\left(\mathrm{~s}^{-1}\right)$ \\
\hline $1 s 7 g{ }^{1} G_{4}$ & $1 s 7 i^{3} I_{5}$ & $2.2508 \mathrm{E}+04$ & $6.722 \mathrm{E}-15$ & $8.045 \mathrm{E}-09$ & $2.2108 \mathrm{E}+04$ & $6.827 \mathrm{E}-15$ & $8.399 \mathrm{E}-09$ \\
\hline $1 s 7 i^{3} I_{6}$ & $1 s 7 i^{3} I_{5}$ & $8.0263 \mathrm{E}+05$ & $2.820 \mathrm{E}-08$ & $2.655 \mathrm{E}-05$ & & & \\
\hline $1 s 4 f^{3} F_{4}^{o}$ & $1 s 7 h^{1} H_{5}^{o}$ & $7.6846 \mathrm{E}+00$ & $1.967 \mathrm{E}-14$ & $2.020 \mathrm{E}-01$ & & & \\
\hline $1 s 5 f^{3} F_{4}^{o}$ & $1 s 7 h^{1} H_{5}^{o}$ & $1.6478 \mathrm{E}+01$ & $2.893 E-16$ & $6.462 \mathrm{E}-04$ & & & \\
\hline $1 s 6 f^{3} F_{4}^{0}$ & $1 s 7 h{ }^{1} H_{5}^{o}$ & $4.3652 \mathrm{E}+01$ & $9.188 \mathrm{E}-16$ & $2.924 \mathrm{E}-04$ & & & \\
\hline $1 s 6 h^{3} H_{5}^{0}$ & $1 s 7 h^{1} H_{5}^{o}$ & $4.3860 \mathrm{E}+01$ & $1.459 \mathrm{E}-11$ & $4.600 \mathrm{E}+00$ & $4.3678 \mathrm{E}+01$ & $1.287 \mathrm{E}-11$ & $4.058 \mathrm{E}+00$ \\
\hline $1 s 6 h^{3} H_{4}^{o}$ & $1 s 7 h^{1} H_{5}^{o}$ & $4.3866 \mathrm{E}+01$ & $6.893 \mathrm{E}-10$ & $2.172 \mathrm{E}+02$ & $4.3678 \mathrm{E}+01$ & $6.953 \mathrm{E}-10$ & $2.191 \mathrm{E}+02$ \\
\hline $1 s 6 h^{3} H_{6}^{o}$ & $1 s 7 h^{1} H_{5}^{o}$ & $4.3999 \mathrm{E}+01$ & $5.006 \mathrm{E}-10$ & $1.568 \mathrm{E}+02$ & $4.3816 \mathrm{E}+01$ & $3.223 \mathrm{E}-10$ & $1.009 E+02$ \\
\hline $1 s 6 h^{1} H_{5}^{o}$ & $1 s 7 h^{1} H_{5}^{o}$ & $4.4004 \mathrm{E}+01$ & $2.163 E-08$ & $6.773 E+03$ & $4.3816 \mathrm{E}+01$ & $2.095 \mathrm{E}-08$ & $6.561 \mathrm{E}+03$ \\
\hline $1 s 7 f^{3} F_{4}^{o}$ & $1 s 7 h^{1} H_{5}^{o}$ & $8.6829 E+03$ & $2.049 \mathrm{E}-19$ & $1.648 \mathrm{E}-12$ & & & \\
\hline $1 s 7 h^{3} H_{5}^{0}$ & $1 s 7 h{ }^{1} H_{5}^{o}$ & $2.1394 \mathrm{E}+04$ & $1.710 \mathrm{E}-08$ & $2.266 \mathrm{E}-02$ & $2.2109 \mathrm{E}+04$ & $1.653 \mathrm{E}-08$ & $2.033 \mathrm{E}-02$ \\
\hline $1 s 7 h^{3} H_{4}^{o}$ & $1 s 7 h^{1} H_{5}^{o}$ & $2.2240 \mathrm{E}+04$ & $8.940 \mathrm{E}-07$ & $1.096 \mathrm{E}+00$ & $2.2109 \mathrm{E}+04$ & $8.927 \mathrm{E}-07$ & $1.098 \mathrm{E}+00$ \\
\hline $1 s 7 h^{3} H_{6}^{o}$ & $1 s 7 h^{1} H_{5}^{o}$ & $6.7907 \mathrm{E}+05$ & $2.293 E-08$ & $3.015 \mathrm{E}-05$ & & & \\
\hline $1 s 7 i^{3} I_{6}$ & $1 s 7 i^{3} I_{7}^{5}$ & $3.1014 \mathrm{E}+04$ & $8.994 \mathrm{E}-07$ & $4.158 \mathrm{E}-01$ & $3.1015 \mathrm{E}+04$ & $8.974 \mathrm{E}-07$ & $4.113 \mathrm{E}-01$ \\
\hline $1 s 5 g{ }^{3} G_{5}$ & $1 s 7 i^{1} I_{6}$ & $1.6520 \mathrm{E}+01$ & $6.150 \mathrm{E}-15$ & $1.156 \mathrm{E}-02$ & & & \\
\hline $1 s 6 g^{3} G_{5}$ & $1 s 7 i^{1} I_{6}$ & $4.3799 \mathrm{E}+01$ & $5.374 \mathrm{E}-16$ & $1.437 \mathrm{E}-04$ & & & \\
\hline $1 s 7 g{ }^{3} G_{5}$ & $1 s 7 i^{1} I_{6}$ & $1.2712 \mathrm{E}+04$ & $7.915 \mathrm{E}-21$ & $2.513 E-14$ & & & \\
\hline $1 s 7 i^{3} I_{6}$ & $1 s 7 i^{1} I_{6}$ & $3.0023 E+04$ & $1.031 \mathrm{E}-08$ & $5.869 \mathrm{E}-03$ & $3.1015 \mathrm{E}+04$ & $9.971 \mathrm{E}-09$ & $5.273 \mathrm{E}-03$ \\
\hline $1 s 7 i^{3} I_{5}$ & $1 s 7 i^{1} I_{6}$ & $3.1190 \mathrm{E}+04$ & $7.687 \mathrm{E}-07$ & $4.055 \mathrm{E}-01$ & $3.1015 E+04$ & $7.678 \mathrm{E}-07$ & $4.060 \mathrm{E}-01$ \\
\hline $1 s 7 i^{3} I_{7}$ & $1 s 7 i^{1} I_{6}$ & $9.4020 \mathrm{E}+05$ & $2.004 \mathrm{E}-08$ & $1.163 \mathrm{E}-05$ & & & \\
\hline
\end{tabular}


Table 6

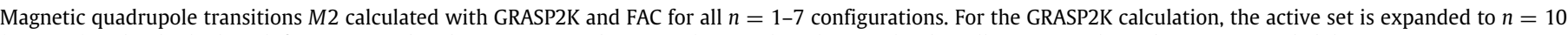
(GRASP3) and only the length form is considered. A comparison between the wavelengths, weighted oscillator strengths and transition probabilities is given.

\begin{tabular}{|c|c|c|c|c|c|c|c|}
\hline \multirow[t]{2}{*}{ Lower } & \multirow[t]{2}{*}{ Upper } & \multicolumn{3}{|l|}{ GRASP2K } & \multicolumn{3}{|l|}{ FAC } \\
\hline & & $\lambda(\AA)$ & $g f$ & $A\left(\mathrm{~s}^{-1}\right)$ & $\lambda(\AA)$ & $g f$ & $A\left(\mathrm{~s}^{-1}\right)$ \\
\hline $1 s 2 s^{3} S_{1}$ & $1 s 2 p^{3} P_{1}^{o}$ & $1.6053 \mathrm{E}+02$ & $1.033 \mathrm{E}-13$ & $8.911 \mathrm{E}-03$ & $1.5824 \mathrm{E}+02$ & $1.235 \mathrm{E}-13$ & $1.087 \mathrm{E}-02$ \\
\hline $1 s^{2}{ }^{1} S_{0}$ & $1 s 2 p^{3} P_{2}^{o}$ & $4.0520 \mathrm{E}-01$ & $3.150 \mathrm{E}-04$ & $2.559 \mathrm{E}+12$ & $4.0356 \mathrm{E}-01$ & $3.398 \mathrm{E}-04$ & $2.760 \mathrm{E}+12$ \\
\hline $1 s 2 s^{3} S_{1}$ & $1 s 2 p^{3} P_{2}^{o}$ & $2.6617 \mathrm{E}+01$ & $1.588 \mathrm{E}-08$ & $2.990 \mathrm{E}+04$ & $2.6489 \mathrm{E}+01$ & $1.614 \mathrm{E}-08$ & $3.043 \mathrm{E}+04$ \\
\hline $1 s 2 s{ }^{1} S_{0}$ & $1 s 2 p^{3} P_{2}^{o}$ & $3.2564 \mathrm{E}+01$ & $5.805 \mathrm{E}-09$ & $7.303 \mathrm{E}+03$ & $3.2508 \mathrm{E}+01$ & $5.823 \mathrm{E}-09$ & $7.289 \mathrm{E}+03$ \\
\hline $1 s 2 s^{3} S_{1}$ & $1 s 2 p^{1} P_{1}^{o}$ & $2.4759 \mathrm{E}+01$ & $1.985 \mathrm{E}-08$ & $7.200 \mathrm{E}+04$ & $2.4615 \mathrm{E}+01$ & $2.008 \mathrm{E}-08$ & $7.308 \mathrm{E}+04$ \\
\hline $1 s 2 p^{3} P_{1}^{o}$ & $1 s 3 s^{3} S_{1}$ & $2.2065 \mathrm{E}+00$ & $1.514 \mathrm{E}-09$ & $6.915 E+05$ & $2.1974 \mathrm{E}+00$ & $2.462 \mathrm{E}-09$ & $1.124 \mathrm{E}+06$ \\
\hline $1 s 2 p^{3} P_{2}^{o}$ & $1 s 3 s^{3} S_{1}$ & $2.3705 \mathrm{E}+00$ & $1.311 \mathrm{E}-06$ & $5.189 \mathrm{E}+08$ & $2.3604 \mathrm{E}+00$ & $1.277 \mathrm{E}-06$ & $5.054 \mathrm{E}+08$ \\
\hline $1 s 2 p{ }^{1} P_{1}^{o}$ & $1 s 3 s^{3} S_{1}$ & $2.3864 \mathrm{E}+00$ & $1.336 \mathrm{E}-06$ & $5.217 \mathrm{E}+08$ & $2.3765 \mathrm{E}+00$ & $1.249 \mathrm{E}-06$ & $4.877 \mathrm{E}+08$ \\
\hline $1 s 2 s^{3} S_{1}$ & $1 s 3 p^{3} P_{1}^{o}$ & $2.1686 \mathrm{E}+00$ & $1.372 \mathrm{E}-08$ & $6.484 \mathrm{E}+06$ & $2.1593 \mathrm{E}+00$ & $1.146 \mathrm{E}-08$ & $5.418 \mathrm{E}+06$ \\
\hline $1 s 3 s^{3} S_{1}$ & $1 s 3 p^{3} P_{1}^{o}$ & $5.8954 \mathrm{E}+02$ & $1.165 \mathrm{E}-14$ & $7.452 \mathrm{E}-05$ & $5.8484 \mathrm{E}+02$ & $1.282 \mathrm{E}-14$ & $8.264 \mathrm{E}-05$ \\
\hline $1 s 2 p^{3} P_{2}^{o}$ & $1 s 3 s^{1} S_{0}$ & $2.3603 \mathrm{E}+00$ & $8.223 \mathrm{E}-07$ & $9.845 \mathrm{E}+08$ & $2.3502 \mathrm{E}+00$ & $8.627 \mathrm{E}-07$ & $1.032 \mathrm{E}+09$ \\
\hline $1 s^{2}{ }^{1} S_{0}$ & $1 s 3 p^{3} P_{2}^{o}$ & $3.4470 \mathrm{E}-01$ & $8.419 \mathrm{E}-05$ & $9.448 \mathrm{E}+11$ & $3.4333 \mathrm{E}-01$ & $9.334 \mathrm{E}-05$ & $1.047 \mathrm{E}+12$ \\
\hline $1 s 2 s^{3} S_{1}$ & $1 s 3 p^{3} P_{2}^{o}$ & $2.1256 \mathrm{E}+00$ & $8.321 \mathrm{E}-06$ & $2.457 \mathrm{E}+09$ & $2.1165 \mathrm{E}+00$ & $8.544 \mathrm{E}-06$ & $2.522 \mathrm{E}+09$ \\
\hline $1 s 2 s{ }^{1} S_{0}$ & $1 s 3 p^{3} P_{2}^{o}$ & $2.1571 \mathrm{E}+00$ & $5.628 \mathrm{E}-06$ & $1.614 \mathrm{E}+09$ & $2.1483 \mathrm{E}+00$ & $5.447 \mathrm{E}-06$ & $1.561 \mathrm{E}+09$ \\
\hline $1 s 3 s^{3} S_{1}$ & $1 s 3 p^{3} P_{2}^{o}$ & $9.0741 \mathrm{E}+01$ & $2.451 \mathrm{E}-09$ & $3.972 \mathrm{E}+02$ & $9.0375 \mathrm{E}+01$ & $2.466 \mathrm{E}-09$ & $3.995 \mathrm{E}+02$ \\
\hline $1 s 3 s{ }^{1} S_{0}$ & $1 s 3 p^{3} P_{2}^{o}$ & $1.0861 \mathrm{E}+02$ & $9.552 \mathrm{E}-10$ & $1.080 \mathrm{E}+02$ & $1.0840 \mathrm{E}+02$ & $9.529 \mathrm{E}-10$ & $1.072 \mathrm{E}+02$ \\
\hline $1 s 2 p^{3} P_{1}^{o}$ & $1 s 3 d^{3} D_{2}$ & $2.1510 \mathrm{E}+00$ & $8.811 \mathrm{E}-07$ & $2.541 \mathrm{E}+08$ & $2.1422 \mathrm{E}+00$ & $9.246 \mathrm{E}-07$ & $2.665 \mathrm{E}+08$ \\
\hline $1 s 2 p^{3} P_{0}^{o}$ & $1 s 3 d^{3} D_{2}$ & $2.1533 \mathrm{E}+00$ & $5.689 \mathrm{E}-07$ & $1.637 \mathrm{E}+08$ & $2.1443 E+00$ & $5.905 \mathrm{E}-07$ & $1.698 \mathrm{E}+08$ \\
\hline $1 s 2 p^{3} P_{2}^{o}$ & $1 s 3 d^{3} D_{2}$ & $2.3065 \mathrm{E}+00$ & $1.618 \mathrm{E}-10$ & $4.057 \mathrm{E}+04$ & $2.2968 \mathrm{E}+00$ & $2.186 \mathrm{E}-10$ & $5.482 \mathrm{E}+04$ \\
\hline $1 s 2 p{ }^{1} P_{1}^{o}$ & $1 s 3 d^{3} D_{2}$ & $2.3216 \mathrm{E}+00$ & $2.587 \mathrm{E}-10$ & $6.403 \mathrm{E}+04$ & & & \\
\hline $1 s 3 p^{3} P_{1}^{o}$ & $1 s 3 d^{3} D_{2}$ & $1.0002 \mathrm{E}+02$ & $4.701 \mathrm{E}-11$ & $6.269 \mathrm{E}+00$ & $9.9770 \mathrm{E}+01$ & $4.770 \mathrm{E}-11$ & $6.338 \mathrm{E}+00$ \\
\hline $1 s 3 p^{3} P_{0}^{o}$ & $1 s 3 d^{3} D_{2}$ & $1.0130 \mathrm{E}+02$ & $2.911 \mathrm{E}-11$ & $3.784 \mathrm{E}+00$ & $1.0100 \mathrm{E}+02$ & $2.943 \mathrm{E}-11$ & $3.815 \mathrm{E}+00$ \\
\hline $1 s 3 p^{3} P_{2}^{o}$ & $1 s 3 d^{3} D_{2}$ & $1.4830 \mathrm{E}+03$ & $2.920 \mathrm{E}-18$ & $1.771 \mathrm{E}-09$ & & & \\
\hline $1 s 2 p^{3} P_{1}^{o}$ & $1 s 3 d^{3} D_{1}$ & $2.1505 \mathrm{E}+00$ & $8.705 \mathrm{E}-07$ & $4.185 \mathrm{E}+08$ & $2.1416 \mathrm{E}+00$ & $9.091 \mathrm{E}-07$ & $4.370 \mathrm{E}+08$ \\
\hline $1 s 2 p^{3} P_{2}^{0}$ & $1 s 3 d^{3} D_{1}$ & $2.3059 \mathrm{E}+00$ & $6.634 \mathrm{E}-13$ & $2.774 \mathrm{E}+02$ & & & \\
\hline $1 s 2 p{ }^{1} P_{1}^{o}$ & $1 s 3 d^{3} D_{1}$ & $2.3210 \mathrm{E}+00$ & $1.204 \mathrm{E}-09$ & $4.968 \mathrm{E}+05$ & $2.3114 \mathrm{E}+00$ & $1.079 \mathrm{E}-09$ & $4.453 \mathrm{E}+05$ \\
\hline $1 s 3 p^{3} P_{1}^{o}$ & $1 s 3 d^{3} D_{1}$ & $9.8828 \mathrm{E}+01$ & $4.804 \mathrm{E}-11$ & $1.094 \mathrm{E}+01$ & $9.8590 \mathrm{E}+01$ & $4.859 \mathrm{E}-11$ & $1.102 \mathrm{E}+01$ \\
\hline $1 s 3 p^{3} P_{2}^{o}$ & $1 s 3 d^{3} D_{1}$ & $1.2588 \mathrm{E}+03$ & $1.549 \mathrm{E}-18$ & $2.174 \mathrm{E}-09$ & & & \\
\hline $1 s 2 s^{3} S_{1}$ & $1 s 3 p^{1} P_{1}^{o}$ & $2.1220 \mathrm{E}+00$ & $8.175 \mathrm{E}-06$ & $4.037 \mathrm{E}+09$ & $2.1129 \mathrm{E}+00$ & $8.576 \mathrm{E}-06$ & $4.234 \mathrm{E}+09$ \\
\hline $1 s 3 s^{3} S_{1}$ & $1 s 3 p{ }^{1} P_{1}^{o}$ & $8.4608 \mathrm{E}+01$ & $3.031 \mathrm{E}-09$ & $9.414 \mathrm{E}+02$ & $8.4198 \mathrm{E}+01$ & $3.046 \mathrm{E}-09$ & $9.473 \mathrm{E}+02$ \\
\hline $1 s 3 d^{3} D_{2}$ & $1 s 3 p^{1} P_{1}^{o}$ & $8.0349 \mathrm{E}+03$ & $2.086 \mathrm{E}-20$ & $7.185 \mathrm{E}-13$ & & & \\
\hline $1 s 3 d^{3} D_{1}$ & $1 s 3 p^{1} P_{1}^{o}$ & $2.2947 \mathrm{E}+05$ & $3.151 \mathrm{E}-24$ & $1.331 \mathrm{E}-19$ & & & \\
\hline $1 s 2 p^{3} P_{1}^{o}$ & $1 s 3 d^{3} D_{3}$ & $2.1376 \mathrm{E}+00$ & $1.374 \mathrm{E}-05$ & $2.865 E+09$ & $2.1288 \mathrm{E}+00$ & $1.394 \mathrm{E}-05$ & $2.907 \mathrm{E}+09$ \\
\hline $1 s 2 p^{3} P_{2}^{o}$ & $1 s 3 d^{3} D_{3}$ & $2.2910 \mathrm{E}+00$ & $6.490 \mathrm{E}-05$ & $1.178 \mathrm{E}+10$ & $2.2814 \mathrm{E}+00$ & $6.717 \mathrm{E}-05$ & $1.219 \mathrm{E}+10$ \\
\hline $1 s 2 p^{1} P_{1}^{o}$ & $1 s 3 d^{3} D_{3}$ & $2.3059 \mathrm{E}+00$ & $1.173 \mathrm{E}-05$ & $2.101 \mathrm{E}+09$ & $2.2965 \mathrm{E}+00$ & $1.190 \mathrm{E}-05$ & $2.133 \mathrm{E}+09$ \\
\hline $1 s 3 p^{3} P_{1}^{o}$ & $1 s 3 d^{3} D_{3}$ & $7.7367 \mathrm{E}+01$ & $1.611 \mathrm{E}-09$ & $2.565 \mathrm{E}+02$ & $7.7185 \mathrm{E}+01$ & $1.597 \mathrm{E}-09$ & $2.533 \mathrm{E}+02$ \\
\hline $1 s 3 p^{3} P_{2}^{o}$ & $1 s 3 d^{3} D_{3}$ & $2.7769 \mathrm{E}+02$ & $1.724 \mathrm{E}-10$ & $2.130 \mathrm{E}+00$ & $2.7772 \mathrm{E}+02$ & $1.707 \mathrm{E}-10$ & $2.091 \mathrm{E}+00$ \\
\hline $1 s 3 p{ }^{1} P_{1}^{o}$ & $1 s 3 d^{3} D_{3}$ & $3.5684 \mathrm{E}+02$ & $1.466 \mathrm{E}-11$ & $1.097 \mathrm{E}-01$ & $3.5856 \mathrm{E}+02$ & $1.436 \mathrm{E}-11$ & $1.055 \mathrm{E}-01$ \\
\hline $1 s 2 p^{3} P_{1}^{o}$ & $1 s 3 d^{1} D_{2}$ & $2.1371 \mathrm{E}+00$ & $5.299 \mathrm{E}-06$ & $1.548 \mathrm{E}+09$ & $2.1283 \mathrm{E}+00$ & $5.541 \mathrm{E}-06$ & $1.618 \mathrm{E}+09$ \\
\hline $1 s 2 p^{3} P_{0}^{o}$ & $1 s 3 d^{1} D_{2}$ & $2.1393 \mathrm{E}+00$ & $6.338 \mathrm{E}-06$ & $1.847 \mathrm{E}+09$ & $2.1304 \mathrm{E}+00$ & $6.476 \mathrm{E}-06$ & $1.887 \mathrm{E}+09$ \\
\hline $1 s 2 p^{3} P_{2}^{o}$ & $1 s 3 d^{1} D_{2}$ & $2.2905 \mathrm{E}+00$ & $1.624 \mathrm{E}-05$ & $4.129 \mathrm{E}+09$ & $2.2809 \mathrm{E}+00$ & $1.680 \mathrm{E}-05$ & $4.273 \mathrm{E}+09$ \\
\hline $1 s 2 p{ }^{1} P_{1}^{o}$ & $1 s 3 d^{1} D_{2}$ & $2.3053 \mathrm{E}+00$ & $3.662 \mathrm{E}-05$ & $9.192 \mathrm{E}+09$ & $2.2959 \mathrm{E}+00$ & $3.746 \mathrm{E}-05$ & $9.401 \mathrm{E}+09$ \\
\hline $1 s 3 p^{3} P_{1}^{o}$ & $1 s 3 d^{1} D_{2}$ & $7.6708 \mathrm{E}+01$ & $6.269 \mathrm{E}-10$ & $1.421 \mathrm{E}+02$ & $7.6517 \mathrm{E}+01$ & $6.228 \mathrm{E}-10$ & $1.407 \mathrm{E}+02$ \\
\hline $1 s 3 p^{3} P_{0}^{o}$ & $1 s 3 d^{1} D_{2}$ & $7.7462 \mathrm{E}+01$ & $7.386 \mathrm{E}-10$ & $1.642 \mathrm{E}+02$ & $7.7242 \mathrm{E}+01$ & $7.336 \mathrm{E}-10$ & $1.626 \mathrm{E}+02$ \\
\hline $1 s 3 p^{3} P_{2}^{o}$ & $1 s 3 d^{1} D_{2}$ & $2.6938 \mathrm{E}+02$ & $4.725 \mathrm{E}-11$ & $8.687 \mathrm{E}-01$ & $2.6926 \mathrm{E}+02$ & $4.683 \mathrm{E}-11$ & $8.544 \mathrm{E}-01$ \\
\hline $1 s 3 p{ }^{1} P_{1}^{o}$ & $1 s 3 d^{1} D_{2}$ & $3.4323 \mathrm{E}+02$ & $5.187 \mathrm{E}-11$ & $5.874 \mathrm{E}-01$ & $3.4458 \mathrm{E}+02$ & $5.080 \mathrm{E}-11$ & $5.659 \mathrm{E}-01$ \\
\hline $1 s 2 p^{3} P_{1}^{o}$ & $1 s 4 s^{3} S_{1}$ & $1.6341 \mathrm{E}+00$ & $6.171 \mathrm{E}-10$ & $5.138 \mathrm{E}+05$ & $1.6274 \mathrm{E}+00$ & $9.120 \mathrm{E}-10$ & $7.592 \mathrm{E}+05$ \\
\hline $1 s 2 p^{3} P_{2}^{o}$ & $1 s 4 s^{3} S_{1}$ & $1.7223 \mathrm{E}+00$ & $5.190 \mathrm{E}-07$ & $3.890 \mathrm{E}+08$ & $1.7151 \mathrm{E}+00$ & $5.041 \mathrm{E}-07$ & $3.778 \mathrm{E}+08$ \\
\hline $1 s 2 p{ }^{1} P_{1}^{o}$ & $1 s 4 s^{3} S_{1}$ & $1.7308 \mathrm{E}+00$ & $5.278 \mathrm{E}-07$ & $3.918 \mathrm{E}+08$ & $1.7236 \mathrm{E}+00$ & $4.959 \mathrm{E}-07$ & $3.680 \mathrm{E}+08$ \\
\hline $1 s 3 p^{3} P_{1}^{o}$ & $1 s 4 s^{3} S_{1}$ & $6.3675 \mathrm{E}+00$ & $3.701 \mathrm{E}-10$ & $2.029 \mathrm{E}+04$ & $6.3413 \mathrm{E}+00$ & $5.844 \mathrm{E}-10$ & $3.204 \mathrm{E}+04$ \\
\hline $1 s 3 p^{3} P_{2}^{o}$ & $1 s 4 s^{3} S_{1}$ & $6.7695 \mathrm{E}+00$ & $3.592 \mathrm{E}-07$ & $1.743 \mathrm{E}+07$ & $6.7412 \mathrm{E}+00$ & $3.541 \mathrm{E}-07$ & $1.718 \mathrm{E}+07$ \\
\hline $1 s 3 p{ }^{1} P_{1}^{o}$ & $1 s 4 s^{3} S_{1}$ & $6.8063 \mathrm{E}+00$ & $3.654 \mathrm{E}-07$ & $1.754 \mathrm{E}+07$ & $6.7783 \mathrm{E}+00$ & $3.479 \mathrm{E}-07$ & $1.669 \mathrm{E}+07$ \\
\hline $1 s 2 s^{3} S_{1}$ & $1 s 4 p^{3} P_{1}^{o}$ & $1.6159 \mathrm{E}+00$ & $5.547 \mathrm{E}-09$ & $4.724 \mathrm{E}+06$ & $1.6090 \mathrm{E}+00$ & $5.114 \mathrm{E}-09$ & $4.355 E+06$ \\
\hline $1 s 3 s^{3} S_{1}$ & $1 s 4 p^{3} P_{1}^{o}$ & $6.2721 \mathrm{E}+00$ & $1.725 \mathrm{E}-09$ & $9.747 \mathrm{E}+04$ & $6.2463 \mathrm{E}+00$ & $1.366 \mathrm{E}-09$ & $7.720 \mathrm{E}+04$ \\
\hline $1 s 3 d^{3} D_{2}$ & $1 s 4 p^{3} P_{1}^{o}$ & $6.7686 \mathrm{E}+00$ & $4.387 \mathrm{E}-09$ & $2.129 \mathrm{E}+05$ & $6.7403 \mathrm{E}+00$ & $4.352 \mathrm{E}-09$ & $2.111 \mathrm{E}+05$ \\
\hline $1 s 3 d^{3} D_{1}$ & $1 s 4 p^{3} P_{1}^{o}$ & $6.7741 \mathrm{E}+00$ & $4.345 \mathrm{E}-09$ & $2.105 E+05$ & $6.7458 \mathrm{E}+00$ & $4.267 \mathrm{E}-09$ & $2.067 \mathrm{E}+05$ \\
\hline $1 s 3 d^{3} D_{3}$ & $1 s 4 p^{3} P_{1}^{o}$ & $6.9054 \mathrm{E}+00$ & $7.364 \mathrm{E}-08$ & $3.434 \mathrm{E}+06$ & $6.8762 \mathrm{E}+00$ & $7.351 \mathrm{E}-08$ & $3.427 \mathrm{E}+06$ \\
\hline $1 s 3 d d^{1} D_{2}$ & $1 s 4 p^{3} P_{1}^{o}$ & $6.9107 \mathrm{E}+00$ & $2.786 \mathrm{E}-08$ & $1.297 \mathrm{E}+06$ & $6.8816 \mathrm{E}+00$ & $2.620 \mathrm{E}-08$ & $1.219 \mathrm{E}+06$ \\
\hline $1 s 4 s^{3} S_{1}$ & $1 s 4 p^{3} P_{1}^{o}$ & $1.4439 \mathrm{E}+03$ & $2.589 \mathrm{E}-15$ & $2.761 \mathrm{E}-06$ & $1.4544 \mathrm{E}+03$ & $2.665 E-15$ & $2.778 \mathrm{E}-06$ \\
\hline $1 s 2 p^{3} P_{2}^{o}$ & $1 s 4 s^{1} S_{0}$ & $1.7202 \mathrm{E}+00$ & $3.276 \mathrm{E}-07$ & $7.385 \mathrm{E}+08$ & $1.7129 \mathrm{E}+00$ & $3.373 \mathrm{E}-07$ & $7.604 \mathrm{E}+08$ \\
\hline $1 s 3 p^{3} P_{2}^{o}$ & $1 s 4 s{ }^{1} S_{0}$ & $6.7361 \mathrm{E}+00$ & $2.278 \mathrm{E}-07$ & $3.349 \mathrm{E}+07$ & $6.7077 \mathrm{E}+00$ & $2.396 \mathrm{E}-07$ & $3.523 \mathrm{E}+07$ \\
\hline $1 s 3 d^{3} D_{2}$ & $1 s 4 p^{3} P_{0}^{o}$ & $6.7663 \mathrm{E}+00$ & $2.811 \mathrm{E}-09$ & $4.096 \mathrm{E}+05$ & $6.7381 \mathrm{E}+00$ & $2.782 \mathrm{E}-09$ & $4.052 \mathrm{E}+05$ \\
\hline $1 s 3 d{ }^{1} D_{2}$ & $1 s 4 p^{3} P_{0}^{o}$ & $6.9083 \mathrm{E}+00$ & $3.383 \mathrm{E}-08$ & $4.728 \mathrm{E}+06$ & $6.8793 \mathrm{E}+00$ & $3.327 \mathrm{E}-08$ & $4.650 \mathrm{E}+06$ \\
\hline $1 s^{2}{ }^{1} S_{0}$ & $1 s 4 p^{3} P_{2}^{o}$ & $3.2750 \mathrm{E}-01$ & $3.417 \mathrm{E}-05$ & $4.249 \mathrm{E}+11$ & $3.2620 \mathrm{E}-01$ & $3.824 \mathrm{E}-05$ & $4.754 \mathrm{E}+11$ \\
\hline $1 s 2 s^{3} S_{1}$ & $1 s 4 p^{3} P_{2}^{o}$ & $1.6057 \mathrm{E}+00$ & $3.719 \mathrm{E}-06$ & $1.924 \mathrm{E}+09$ & $1.5989 \mathrm{E}+00$ & $3.831 \mathrm{E}-06$ & $1.982 \mathrm{E}+09$ \\
\hline $1 s 2 s^{1} S_{0}$ & $1 s 4 p^{3} P_{2}^{o}$ & $1.6236 \mathrm{E}+00$ & $2.491 \mathrm{E}-06$ & $1.261 \mathrm{E}+09$ & $1.6170 \mathrm{E}+00$ & $2.469 \mathrm{E}-06$ & $1.249 \mathrm{E}+09$ \\
\hline $1 s 3 s^{3} S_{1}$ & $1 s 4 p^{3} P_{2}^{o}$ & $6.1216 \mathrm{E}+00$ & $1.070 \mathrm{E}-06$ & $3.810 \mathrm{E}+07$ & $6.0965 \mathrm{E}+00$ & $1.086 \mathrm{E}-06$ & $3.865 \mathrm{E}+07$ \\
\hline $1 s 3 s{ }^{1} S_{0}$ & $1 s 4 p^{3} P_{2}^{o}$ & $6.1903 \mathrm{E}+00$ & $7.330 \mathrm{E}-07$ & $2.552 \mathrm{E}+07$ & $6.1656 \mathrm{E}+00$ & $6.999 \mathrm{E}-07$ & $2.435 \mathrm{E}+07$ \\
\hline $1 s 3 d^{3} D_{2}$ & $1 s 4 p^{3} P_{2}^{o}$ & $6.5936 \mathrm{E}+00$ & $2.191 \mathrm{E}-13$ & $6.724 \mathrm{E}+00$ & & & \\
\hline
\end{tabular}


Table 6 (continued)

\begin{tabular}{|c|c|c|c|c|c|c|c|}
\hline \multirow[t]{2}{*}{ Lower } & \multirow[t]{2}{*}{ Upper } & \multicolumn{3}{|l|}{ GRASP2K } & \multicolumn{3}{|l|}{ FAC } \\
\hline & & $\lambda(\AA)$ & $g f$ & $A\left(\mathrm{~s}^{-1}\right)$ & $\lambda(\AA)$ & $g f$ & $A\left(\mathrm{~s}^{-1}\right)$ \\
\hline $1 s 3 d^{3} D_{1}$ & $1 s 4 p^{3} P_{2}^{o}$ & $6.5989 \mathrm{E}+00$ & $1.089 \mathrm{E}-12$ & $3.337 \mathrm{E}+01$ & & & \\
\hline $1 s 3 d^{3} D_{3}$ & $1 s 4 p^{3} P_{2}^{o}$ & $6.7234 \mathrm{E}+00$ & $2.581 \mathrm{E}-07$ & $7.616 \mathrm{E}+06$ & $6.6951 \mathrm{E}+00$ & $2.493 \mathrm{E}-07$ & $7.359 \mathrm{E}+06$ \\
\hline $1 s 3 d^{1} D_{2}$ & $1 s 4 p^{3} P_{2}^{o}$ & $6.7284 \mathrm{E}+00$ & $6.488 \mathrm{E}-08$ & $1.912 \mathrm{E}+06$ & $6.7001 \mathrm{E}+00$ & $6.220 \mathrm{E}-08$ & $1.832 \mathrm{E}+06$ \\
\hline $1 s 4 s^{3} S_{1}$ & $1 s 4 p^{3} P_{2}^{o}$ & $2.1675 E+02$ & $6.059 \mathrm{E}-10$ & $1.720 \mathrm{E}+01$ & $2.1631 \mathrm{E}+02$ & $6.045 \mathrm{E}-10$ & $1.709 \mathrm{E}+01$ \\
\hline $1 s 4 s^{1} S_{0}$ & $1 s 4 p^{3} P_{2}^{o}$ & $2.5764 \mathrm{E}+02$ & $2.410 \mathrm{E}-10$ & $4.843 \mathrm{E}+00$ & $2.5767 \mathrm{E}+02$ & $2.384 \mathrm{E}-10$ & $4.750 \mathrm{E}+00$ \\
\hline $1 s 2 p^{3} P_{1}^{o}$ & $1 s 4 d^{3} D_{2}$ & $1.6212 \mathrm{E}+00$ & $2.912 \mathrm{E}-07$ & $1.478 \mathrm{E}+08$ & $1.6144 \mathrm{E}+00$ & $3.095 \mathrm{E}-07$ & $1.571 \mathrm{E}+08$ \\
\hline $1 s 2 p^{3} P_{0}^{0}$ & $1 s 4 d^{3} D_{2}$ & $1.6224 \mathrm{E}+00$ & $1.846 \mathrm{E}-07$ & $9.355 \mathrm{E}+07$ & $1.6156 \mathrm{E}+00$ & $1.932 \mathrm{E}-07$ & $9.790 \mathrm{E}+07$ \\
\hline $1 s 2 p^{3} P_{2}^{o}$ & $1 s 4 d^{3} D_{2}$ & $1.7079 E+00$ & $1.219 \mathrm{E}-10$ & $5.573 \mathrm{E}+04$ & $1.7007 \mathrm{E}+00$ & $1.718 \mathrm{E}-10$ & $7.857 \mathrm{E}+04$ \\
\hline $1 s 2 p^{1} P_{1}^{o}$ & $1 s 4 d^{3} D_{2}$ & $1.7162 \mathrm{E}+00$ & $7.192 \mathrm{E}-12$ & $3.257 \mathrm{E}+03$ & & & \\
\hline $1 s 3 p^{3} P_{1}^{o}$ & $1 s 4 d^{3} D_{2}$ & $6.1751 \mathrm{E}+00$ & $9.219 \mathrm{E}-08$ & $3.225 \mathrm{E}+06$ & $6.1494 \mathrm{E}+00$ & $9.398 \mathrm{E}-08$ & $3.287 \mathrm{E}+06$ \\
\hline $1 s 3 p^{3} P_{0}^{0}$ & $1 s 4 d^{3} D_{2}$ & $6.1799 \mathrm{E}+00$ & $5.803 \mathrm{E}-08$ & $2.027 \mathrm{E}+06$ & $6.1541 \mathrm{E}+00$ & $5.875 \mathrm{E}-08$ & $2.051 \mathrm{E}+06$ \\
\hline $1 s 3 p^{3} P_{2}^{0}$ & $1 s 4 d^{3} D_{2}$ & $6.5523 E+00$ & $4.903 \mathrm{E}-11$ & $1.524 \mathrm{E}+03$ & $6.5248 \mathrm{E}+00$ & $5.664 \mathrm{E}-11$ & $1.760 \mathrm{E}+03$ \\
\hline $1 s 3 p{ }^{1} P_{1}^{o}$ & $1 s 4 d^{3} D_{2}$ & $6.5868 \mathrm{E}+00$ & $6.167 \mathrm{E}-13$ & $1.896 \mathrm{E}+01$ & & & \\
\hline $1 s 4 p^{3} P_{1}^{0}$ & $1 s 4 d^{3} D_{2}$ & $2.3795 \mathrm{E}+02$ & $1.520 \mathrm{E}-11$ & $3.582 \mathrm{E}-01$ & $2.3625 E+02$ & $1.552 \mathrm{E}-11$ & $3.679 \mathrm{E}-01$ \\
\hline $1 s 4 p^{3} P_{0}^{0}$ & $1 s 4 d^{3} D_{2}$ & $2.4086 \mathrm{E}+02$ & $9.221 \mathrm{E}-12$ & $2.120 \mathrm{E}-01$ & $2.3903 \mathrm{E}+02$ & $9.376 \mathrm{E}-12$ & $2.170 \mathrm{E}-01$ \\
\hline $1 s 4 p^{3} P_{2}^{0}$ & $1 s 4 d^{3} D_{2}$ & $3.5515 E+03$ & $2.231 \mathrm{E}-18$ & $2.359 \mathrm{E}-10$ & & & \\
\hline $1 s 2 p^{3} P_{1}^{o}$ & $1 s 4 d^{3} D_{1}$ & $1.6210 \mathrm{E}+00$ & $2.862 \mathrm{E}-07$ & $2.422 \mathrm{E}+08$ & $1.6143 \mathrm{E}+00$ & $3.012 \mathrm{E}-07$ & $2.548 \mathrm{E}+08$ \\
\hline $1 s 2 p^{3} P_{2}^{0}$ & $1 s 4 d^{3} D_{1}$ & $1.7078 \mathrm{E}+00$ & $3.653 \mathrm{E}-13$ & $2.785 \mathrm{E}+02$ & & & \\
\hline $1 s 2 p{ }^{1} P_{1}^{0}$ & $1 s 4 d^{3} D_{1}$ & $1.7161 \mathrm{E}+00$ & $3.609 \mathrm{E}-10$ & $2.724 \mathrm{E}+05$ & $1.7090 \mathrm{E}+00$ & $3.716 \mathrm{E}-10$ & $2.805 \mathrm{E}+05$ \\
\hline $1 s 3 p^{3} P_{1}^{o}$ & $1 s 4 d^{3} D_{1}$ & $6.1732 \mathrm{E}+00$ & $8.985 \mathrm{E}-08$ & $5.242 \mathrm{E}+06$ & $6.1476 \mathrm{E}+00$ & $9.158 \mathrm{E}-08$ & $5.342 \mathrm{E}+06$ \\
\hline $1 s 3 p^{3} P_{2}^{o}$ & $1 s 4 d^{3} D_{1}$ & $6.5502 \mathrm{E}+00$ & $5.443 \mathrm{E}-13$ & $2.821 \mathrm{E}+01$ & & & \\
\hline $1 s 3 p^{1} P_{1}^{o}$ & $1 s 4 d^{3} D_{1}$ & $6.5847 \mathrm{E}+00$ & $1.129 \mathrm{E}-10$ & $5.788 \mathrm{E}+03$ & $6.5574 \mathrm{E}+00$ & $8.882 \mathrm{E}-11$ & $4.553 \mathrm{E}+03$ \\
\hline $1 s 4 p^{3} P_{1}^{o}$ & $1 s 4 d^{3} D_{1}$ & $2.3523 \mathrm{E}+02$ & $1.537 \mathrm{E}-11$ & $6.175 \mathrm{E}-01$ & $2.3358 \mathrm{E}+02$ & $1.564 \mathrm{E}-11$ & $6.320 \mathrm{E}-01$ \\
\hline $1 s 4 p^{3} P_{2}^{o}$ & $1 s 4 d^{3} D_{1}$ & $3.0279 \mathrm{E}+03$ & $4.275 \mathrm{E}-19$ & $1.037 \mathrm{E}-10$ & & & \\
\hline $1 s 2 s^{3} s_{1}^{2}$ & $1 s 4 p{ }^{1} P_{1}^{o}$ & $1.6048 \mathrm{E}+00$ & $3.673 \mathrm{E}-06$ & $3.171 \mathrm{E}+09$ & $1.5980 \mathrm{E}+00$ & $3.832 \mathrm{E}-06$ & $3.308 \mathrm{E}+09$ \\
\hline $1 s 3 s^{3} S_{1}$ & $1 s 4 p{ }^{1} P_{1}^{o}$ & $6.1092 \mathrm{E}+00$ & $1.049 \mathrm{E}-06$ & $6.249 \mathrm{E}+07$ & $6.0840 \mathrm{E}+00$ & $1.091 \mathrm{E}-06$ & $6.499 \mathrm{E}+07$ \\
\hline $1 s 3 d^{3} D_{2}$ & $1 s 4 p{ }^{1} P_{1}^{o}$ & $6.5793 \mathrm{E}+00$ & $6.881 \mathrm{E}-13$ & $3.535 \mathrm{E}+01$ & & & \\
\hline $1 s 3 d^{3} D_{1}$ & $1 s 4 p{ }^{1} P_{1}^{0}$ & $6.5845 \mathrm{E}+00$ & $2.464 \mathrm{E}-13$ & $1.264 \mathrm{E}+01$ & & & \\
\hline $1 s 3 d^{3} D_{3}$ & $1 s 4 p{ }^{1} P_{1}^{o}$ & $6.7085 E+00$ & $4.497 \mathrm{E}-08$ & $2.222 \mathrm{E}+06$ & $6.6801 \mathrm{E}+00$ & $4.629 \mathrm{E}-08$ & $2.286 \mathrm{E}+06$ \\
\hline $1 s 3 d^{1} D_{2}$ & $1 s 4 p^{1} P_{1}^{o}$ & $6.7135 E+00$ & $1.426 \mathrm{E}-07$ & $7.036 \mathrm{E}+06$ & $6.6851 \mathrm{E}+00$ & $1.415 \mathrm{E}-07$ & $6.984 \mathrm{E}+06$ \\
\hline $1 s 4 s^{3} S_{1}$ & $1 s 4 p{ }^{1} P_{1}^{o}$ & $2.0225 \mathrm{E}+02$ & $7.466 \mathrm{E}-10$ & $4.058 \mathrm{E}+01$ & $2.0166 \mathrm{E}+02$ & $7.451 \mathrm{E}-10$ & $4.039 \mathrm{E}+01$ \\
\hline $1 s 4 d^{3} D_{2}$ & $1 s 4 p{ }^{1} P_{1}^{o}$ & $2.0339 \mathrm{E}+04$ & $7.155 \mathrm{E}-23$ & $3.846 \mathrm{E}-16$ & & & \\
\hline $1 s 4 d^{3} D_{1}$ & $1 s 4 p{ }^{1} P_{1}^{o}$ & $2.1218 \mathrm{E}+06$ & $1.608 \mathrm{E}-26$ & $7.940 \mathrm{E}-24$ & & & \\
\hline $1 s 2 p^{3} P_{1}^{o}$ & $1 s 4 d^{3} D_{3}$ & $1.6179 \mathrm{E}+00$ & $4.628 \mathrm{E}-06$ & $1.685 \mathrm{E}+09$ & $1.6112 \mathrm{E}+00$ & $4.723 E-06$ & $1.719 \mathrm{E}+09$ \\
\hline $1 s 2 p^{3} P_{2}^{o}$ & $1 s 4 d^{3} D_{3}$ & $1.7043 E+00$ & $2.069 \mathrm{E}-05$ & $6.788 \mathrm{E}+09$ & $1.6972 \mathrm{E}+00$ & $2.154 \mathrm{E}-05$ & $7.068 \mathrm{E}+09$ \\
\hline $1 s 2 p^{1} P_{1}^{o}$ & $1 s 4 d^{3} D_{3}$ & $1.7126 \mathrm{E}+00$ & $3.719 \mathrm{E}-06$ & $1.208 \mathrm{E}+09$ & $1.7055 \mathrm{E}+00$ & $3.855 \mathrm{E}-06$ & $1.252 \mathrm{E}+09$ \\
\hline $1 s 3 p^{3} P_{1}^{0}$ & $1 s 4 d^{3} D_{3}$ & $6.1283 \mathrm{E}+00$ & $1.363 \mathrm{E}-06$ & $3.459 \mathrm{E}+07$ & $6.1030 \mathrm{E}+00$ & $1.371 \mathrm{E}-06$ & $3.478 \mathrm{E}+07$ \\
\hline $1 s 3 p^{3} P_{2}^{o}$ & $1 s 4 d^{3} D_{3}$ & $6.4997 \mathrm{E}+00$ & $7.099 \mathrm{E}-06$ & $1.601 \mathrm{E}+08$ & $6.4725 E+00$ & $7.232 \mathrm{E}-06$ & $1.631 \mathrm{E}+08$ \\
\hline $1 s 3 p^{1} P_{1}^{o}$ & $1 s 4 d^{3} D_{3}$ & $6.5336 \mathrm{E}+00$ & $1.286 \mathrm{E}-06$ & $2.871 \mathrm{E}+07$ & $6.5067 \mathrm{E}+00$ & $1.277 \mathrm{E}-06$ & $2.850 \mathrm{E}+07$ \\
\hline $1 s 4 p^{3} P_{1}^{0}$ & $1 s 4 d^{3} D_{3}$ & $1.8386 \mathrm{E}+02$ & $5.115 \mathrm{E}-10$ & $1.442 \mathrm{E}+01$ & $1.8281 \mathrm{E}+02$ & $5.134 \mathrm{E}-10$ & $1.451 \mathrm{E}+01$ \\
\hline $1 s 4 p^{3} P_{2}^{0}$ & $1 s 4 d^{3} D_{3}$ & $6.5884 \mathrm{E}+02$ & $5.524 \mathrm{E}-11$ & $1.213 \mathrm{E}-01$ & $6.5162 \mathrm{E}+02$ & $5.648 \mathrm{E}-11$ & $1.256 \mathrm{E}-01$ \\
\hline $1 s 4 p^{1} P_{1}^{o}$ & $1 s 4 d^{3} D_{3}$ & $8.4240 \mathrm{E}+02$ & $4.763 \mathrm{E}-12$ & $6.396 \mathrm{E}-03$ & $8.3413 \mathrm{E}+02$ & $4.865 \mathrm{E}-12$ & $6.606 \mathrm{E}-03$ \\
\hline $1 s 2 s^{3} s_{1}$ & $1 s 4 f^{3} F_{3}^{o}$ & $1.6017 \mathrm{E}+00$ & $1.411 \mathrm{E}-10$ & $5.242 \mathrm{E}+04$ & & & \\
\hline $1 s 3 s^{3} S_{1}$ & $1 s 4 f^{3} F_{3}^{o}$ & $6.0645 \mathrm{E}+00$ & $6.814 \mathrm{E}-12$ & $1.765 \mathrm{E}+02$ & & & \\
\hline $1 s 3 d^{3} D_{2}$ & $1 s 4 f^{3} F_{3}^{o}$ & $6.5275 \mathrm{E}+00$ & $2.415 \mathrm{E}-06$ & $5.402 \mathrm{E}+07$ & $6.5002 \mathrm{E}+00$ & $2.450 \mathrm{E}-06$ & $5.479 \mathrm{E}+07$ \\
\hline $1 s 3 d^{3} D_{1}$ & $1 s 4 f^{3} F_{3}^{o}$ & $6.5326 \mathrm{E}+00$ & $4.080 \mathrm{E}-07$ & $9.111 \mathrm{E}+06$ & $6.5052 \mathrm{E}+00$ & $4.131 \mathrm{E}-07$ & $9.224 \mathrm{E}+06$ \\
\hline $1 s 3 d^{3} D_{3}$ & $1 s 4 f^{3} F_{3}^{o}$ & $6.6546 \mathrm{E}+00$ & $1.660 \mathrm{E}-11$ & $3.572 \mathrm{E}+02$ & & & \\
\hline $1 s 3 d^{1} D_{2}$ & $1 s 4 f^{3} F_{3}^{o}$ & $6.6595 \mathrm{E}+00$ & $2.267 \mathrm{E}-10$ & $4.871 \mathrm{E}+03$ & & & \\
\hline $1 s 4 s^{3} S_{1}$ & $1 s 4 f^{3} F_{3}^{o}$ & $1.6258 \mathrm{E}+02$ & $2.126 \mathrm{E}-19$ & $7.665 \mathrm{E}-09$ & $1.6211 \mathrm{E}+02$ & $1.540 \mathrm{E}-16$ & $5.538 \mathrm{E}-06$ \\
\hline $1 s 4 d^{3} D_{2}$ & $1 s 4 f^{3} F_{3}^{o}$ & $7.9649 \mathrm{E}+02$ & $3.345 \mathrm{E}-12$ & $5.024 \mathrm{E}-03$ & $8.0096 \mathrm{E}+02$ & $3.264 \mathrm{E}-12$ & $4.808 \mathrm{E}-03$ \\
\hline $1 s 4 d^{3} D_{1}$ & $1 s 4 f^{3} F_{3}^{o}$ & $8.2863 E+02$ & $5.020 \mathrm{E}-13$ & $6.967 \mathrm{E}-04$ & $8.3335 E+02$ & $4.900 \mathrm{E}-13$ & $6.666 \mathrm{E}-04$ \\
\hline $1 s 4 d^{3} D_{3}$ & $1 s 4 f^{3} F_{3}^{0}$ & $5.1914 \mathrm{E}+04$ & $8.399 \mathrm{E}-23$ & $2.970 \mathrm{E}-17$ & & & \\
\hline $1 s^{2}{ }^{1} S_{0}$ & $1 s 4 f^{3} F_{2}^{o}$ & $3.2740 \mathrm{E}-01$ & $1.149 \mathrm{E}-10$ & $1.430 \mathrm{E}+06$ & $3.2603 \mathrm{E}-01$ & $4.930 \mathrm{E}-12$ & $6.135 \mathrm{E}+04$ \\
\hline $1 s 2 s^{3} S_{1}$ & $1 s 4 f^{3} F_{2}^{o}$ & $1.6017 \mathrm{E}+00$ & $4.643 \mathrm{E}-11$ & $2.414 \mathrm{E}+04$ & $1.5949 \mathrm{E}+00$ & $4.969 \mathrm{E}-13$ & $2.583 \mathrm{E}+02$ \\
\hline $1 s 2 s{ }^{1} S_{0}$ & $1 s 4 f^{3} F_{2}^{o}$ & $1.6195 \mathrm{E}+00$ & $4.740 \mathrm{E}-11$ & $2.411 \mathrm{E}+04$ & $1.6129 \mathrm{E}+00$ & $3.203 E-13$ & $1.628 \mathrm{E}+02$ \\
\hline $1 s 3 s^{3} S_{1}$ & $1 s 4 f^{3} F_{2}^{o}$ & $6.0636 \mathrm{E}+00$ & $9.483 \mathrm{E}-12$ & $3.441 \mathrm{E}+02$ & & & \\
\hline $1 s 3 s{ }^{1} S_{0}$ & $1 s 4 f^{3} F_{2}^{o}$ & $6.1310 \mathrm{E}+00$ & $6.089 \mathrm{E}-12$ & $2.161 \mathrm{E}+02$ & & & \\
\hline $1 s 3 d^{3} D_{2}$ & $1 s 4 f^{3} F_{2}^{o}$ & $6.5264 \mathrm{E}+00$ & $6.054 \mathrm{E}-07$ & $1.896 \mathrm{E}+07$ & $6.4991 \mathrm{E}+00$ & $6.141 \mathrm{E}-07$ & $1.923 \mathrm{E}+07$ \\
\hline $1 s 3 d^{3} D_{1}$ & $1 s 4 f^{3} F_{2}^{o}$ & $6.5315 \mathrm{E}+00$ & $1.411 \mathrm{E}-06$ & $4.412 \mathrm{E}+07$ & $6.5041 \mathrm{E}+00$ & $1.429 \mathrm{E}-06$ & $4.469 \mathrm{E}+07$ \\
\hline $1 s 3 d^{3} D_{3}$ & $1 s 4 f^{3} F_{2}^{o}$ & $6.6535 E+00$ & $1.331 \mathrm{E}-14$ & $4.010 \mathrm{E}-01$ & & & \\
\hline $1 s 3 d^{1} D_{2}$ & $1 s 4 f^{3} F_{2}^{o}$ & $6.6584 \mathrm{E}+00$ & $6.185 \mathrm{E}-12$ & $1.861 \mathrm{E}+02$ & & & \\
\hline $1 s 4 s^{3} S_{1}$ & $1 s 4 f^{3} F_{2}^{o}$ & $1.6191 \mathrm{E}+02$ & $2.730 \mathrm{E}-16$ & $1.389 \mathrm{E}-05$ & $1.6143 \mathrm{E}+02$ & $9.377 \mathrm{E}-17$ & $4.760 \mathrm{E}-06$ \\
\hline $1 s 4 s^{1} S_{0}$ & $1 s 4 f^{3} F_{2}^{o}$ & $1.8368 \mathrm{E}+02$ & $2.477 \mathrm{E}-16$ & $9.794 \mathrm{E}-06$ & $1.8340 \mathrm{E}+02$ & $8.510 \mathrm{E}-17$ & $3.346 \mathrm{E}-06$ \\
\hline $1 s 4 d^{3} D_{2}$ & $1 s 4 f^{3} F_{2}^{o}$ & $7.8052 \mathrm{E}+02$ & $8.910 \mathrm{E}-13$ & $1.951 \mathrm{E}-03$ & $7.8473 \mathrm{E}+02$ & $8.697 \mathrm{E}-13$ & $1.868 \mathrm{E}-03$ \\
\hline $1 s 4 d^{3} D_{1}$ & $1 s 4 f^{3} F_{2}^{o}$ & $8.1135 E+02$ & $1.850 \mathrm{E}-12$ & $3.749 \mathrm{E}-03$ & $8.1580 \mathrm{E}+02$ & $1.806 \mathrm{E}-12$ & $3.590 \mathrm{E}-03$ \\
\hline $1 s 4 d^{3} D_{3}$ & $1 s 4 f^{3} F_{2}^{o}$ & $2.2242 \mathrm{E}+04$ & $1.812 \mathrm{E}-22$ & $4.886 \mathrm{E}-16$ & & & \\
\hline $1 s 2 p^{3} P_{1}^{o}$ & $1 s 4 d{ }^{1} D_{2}$ & $1.6178 \mathrm{E}+00$ & $1.786 \mathrm{E}-06$ & $9.103 \mathrm{E}+08$ & $1.6111 \mathrm{E}+00$ & $1.843 \mathrm{E}-06$ & $9.395 \mathrm{E}+08$ \\
\hline $1 s 2 p^{3} P_{0}^{o}$ & $1 s 4 d^{1} D_{2}$ & $1.6191 \mathrm{E}+00$ & $2.139 \mathrm{E}-06$ & $1.089 \mathrm{E}+09$ & $1.6123 \mathrm{E}+00$ & $2.188 \mathrm{E}-06$ & $1.113 \mathrm{E}+09$ \\
\hline $1 s 2 p^{3} P_{2}^{o}$ & $1 s 4 d{ }^{1} D_{2}$ & $1.7042 \mathrm{E}+00$ & $5.187 \mathrm{E}-06$ & $2.383 \mathrm{E}+09$ & $1.6970 \mathrm{E}+00$ & $5.388 \mathrm{E}-06$ & $2.474 \mathrm{E}+09$ \\
\hline $1 s 2 p^{1} P_{1}^{o}$ & $1 s 4 d^{1} D_{2}$ & $1.7124 \mathrm{E}+00$ & $1.163 \mathrm{E}-05$ & $5.291 \mathrm{E}+09$ & $1.7053 \mathrm{E}+00$ & $1.205 \mathrm{E}-05$ & $5.484 \mathrm{E}+09$ \\
\hline $1 s 3 p^{3} P_{1}^{o}$ & $1 s 4 d{ }^{1} D_{2}$ & $6.1264 \mathrm{E}+00$ & $5.148 \mathrm{E}-07$ & $1.830 \mathrm{E}+07$ & $6.1011 \mathrm{E}+00$ & $5.378 \mathrm{E}-07$ & $1.911 \mathrm{E}+07$ \\
\hline
\end{tabular}


Table 6 (continued)

\begin{tabular}{|c|c|c|c|c|c|c|c|}
\hline \multirow[t]{2}{*}{ Lower } & \multirow[t]{2}{*}{ Upper } & \multicolumn{3}{|l|}{ GRASP2K } & \multicolumn{3}{|l|}{ FAC } \\
\hline & & $\lambda(\AA)$ & $g f$ & $A\left(\mathrm{~s}^{-1}\right)$ & $\overline{\lambda(\AA)}$ & $g f$ & $A\left(\mathrm{~s}^{-1}\right)$ \\
\hline $1 s 3 p^{3} P_{0}^{o}$ & $1 s 4 d^{1} D_{2}$ & $6.1312 \mathrm{E}+00$ & $6.270 \mathrm{E}-07$ & $2.225 \mathrm{E}+07$ & $6.1056 \mathrm{E}+00$ & $6.363 \mathrm{E}-07$ & $2.257 \mathrm{E}+07$ \\
\hline $1 s 3 p^{3} P_{2}^{o}$ & $1 s 4 d^{1} D_{2}$ & $6.4976 \mathrm{E}+00$ & $1.773 \mathrm{E}-06$ & $5.603 \mathrm{E}+07$ & $6.4704 \mathrm{E}+00$ & $1.809 \mathrm{E}-06$ & $5.717 \mathrm{E}+07$ \\
\hline $1 s 3 p^{1} P_{1}^{o}$ & $1 s 4 d{ }^{1} D_{2}$ & $6.5315 \mathrm{E}+00$ & $4.042 \mathrm{E}-06$ & $1.264 \mathrm{E}+08$ & $6.5045 \mathrm{E}+00$ & $4.060 \mathrm{E}-06$ & $1.269 \mathrm{E}+08$ \\
\hline $1 s 4 p^{3} P_{1}^{o}$ & $1 s 4 d^{1} D_{2}$ & $1.8218 \mathrm{E}+02$ & $1.978 \mathrm{E}-10$ & $7.953 \mathrm{E}+00$ & $1.8111 \mathrm{E}+02$ & $1.990 \mathrm{E}-10$ & $8.026 \mathrm{E}+00$ \\
\hline $1 s 4 p^{3} P_{0}^{0}$ & $1 s 4 d{ }^{1} D_{2}$ & $1.8388 \mathrm{E}+02$ & $2.351 \mathrm{E}-10$ & $9.278 \mathrm{E}+00$ & $1.8274 \mathrm{E}+02$ & $2.365 \mathrm{E}-10$ & $9.368 \mathrm{E}+00$ \\
\hline $1 s 4 p^{3} P_{2}^{o}$ & $1 s 4 d^{1} D_{2}$ & $6.3767 \mathrm{E}+02$ & $1.524 \mathrm{E}-11$ & $5.000 \mathrm{E}-02$ & $6.3050 \mathrm{E}+02$ & $1.558 \mathrm{E}-11$ & $5.186 \mathrm{E}-02$ \\
\hline $1 s 4 p^{1} P_{1}^{o}$ & $1 s 4 d^{1} D_{2}$ & $8.0810 \mathrm{E}+02$ & $1.703 \mathrm{E}-11$ & $3.478 \mathrm{E}-02$ & $7.9983 \mathrm{E}+02$ & $1.737 \mathrm{E}-11$ & $3.591 \mathrm{E}-02$ \\
\hline $1 s 4 f^{3} F_{3}^{o}$ & $1 s 4 d^{1} D_{2}$ & $3.2125 \mathrm{E}+04$ & $6.900 \mathrm{E}-21$ & $8.919 \mathrm{E}-15$ & & & \\
\hline $1 s 4 f^{3} F_{2}^{o}$ & $1 s 4 d^{1} D_{2}$ & $1.8409 \mathrm{E}+05$ & $1.136 \mathrm{E}-24$ & $4.473 \mathrm{E}-20$ & & & \\
\hline $1 s 3 d^{3} D_{2}$ & $1 s 4 f^{3} F_{4}^{o}$ & $6.5015 \mathrm{E}+00$ & 4.667E-06 & $8.182 \mathrm{E}+07$ & $6.4744 \mathrm{E}+00$ & $4.702 \mathrm{E}-06$ & $8.243 E+07$ \\
\hline $1 s 3 d^{3} D_{3}$ & $1 s 4 f^{3} F_{4}^{o}$ & $6.6276 \mathrm{E}+00$ & $2.919 \mathrm{E}-05$ & $4.925 \mathrm{E}+08$ & $6.5997 \mathrm{E}+00$ & $2.959 \mathrm{E}-05$ & $4.992 \mathrm{E}+08$ \\
\hline $1 s 3 d{ }^{1} D_{2}$ & $1 s 4 f^{3} F_{4}^{o}$ & $6.6325 \mathrm{E}+00$ & $2.355 \mathrm{E}-06$ & $3.967 \mathrm{E}+07$ & $6.6046 \mathrm{E}+00$ & $2.385 \mathrm{E}-06$ & $4.019 \mathrm{E}+07$ \\
\hline $1 s 4 d^{3} D_{2}$ & $1 s 4 f^{3} F_{4}^{o}$ & $5.3564 \mathrm{E}+02$ & $2.134 \mathrm{E}-11$ & $5.513 \mathrm{E}-02$ & $5.3733 \mathrm{E}+02$ & $2.081 \mathrm{E}-11$ & $5.297 \mathrm{E}-02$ \\
\hline $1 s 4 d^{3} D_{3}$ & $1 s 4 f^{3} F_{4}^{o}$ & $1.5856 \mathrm{E}+03$ & $5.232 \mathrm{E}-12$ & $1.542 \mathrm{E}-03$ & $1.6034 \mathrm{E}+03$ & $5.007 \mathrm{E}-12$ & $1.431 \mathrm{E}-03$ \\
\hline $1 s 4 d{ }^{1} D_{2}$ & $1 s 4 f^{3} F_{4}^{o}$ & $1.7232 \mathrm{E}+03$ & $3.306 \mathrm{E}-13$ & $8.252 \mathrm{E}-05$ & $1.7474 \mathrm{E}+03$ & $3.143 E-13$ & $7.566 \mathrm{E}-05$ \\
\hline $1 s 2 s^{3} S_{1}$ & $1 s 4 f^{1} F_{3}^{o}$ & $1.6001 \mathrm{E}+00$ & $2.058 \mathrm{E}-13$ & $7.659 \mathrm{E}+01$ & & & \\
\hline $1 s 3 s^{3} S_{1}$ & $1 s 4 f^{1} F_{3}^{o}$ & $6.0414 \mathrm{E}+00$ & $5.284 \mathrm{E}-12$ & $1.380 \mathrm{E}+02$ & & & \\
\hline $1 s 3 d^{3} D_{2}$ & $1 s 4 f^{1} F_{3}^{o}$ & $6.5007 \mathrm{E}+00$ & $5.480 \mathrm{E}-07$ & $1.236 \mathrm{E}+07$ & $6.4736 \mathrm{E}+00$ & $5.559 \mathrm{E}-07$ & $1.253 \mathrm{E}+07$ \\
\hline $1 s 3 d^{3} D_{1}$ & $1 s 4 f^{1} F_{3}^{o}$ & $6.5058 \mathrm{E}+00$ & $3.118 \mathrm{E}-06$ & $7.020 \mathrm{E}+07$ & $6.4786 \mathrm{E}+00$ & $3.140 \mathrm{E}-06$ & $7.069 \mathrm{E}+07$ \\
\hline $1 s 3 d^{3} D_{3}$ & $1 s 4 f^{1} F_{3}^{o}$ & $6.6268 \mathrm{E}+00$ & $3.503 \mathrm{E}-06$ & $7.602 \mathrm{E}+07$ & $6.5989 \mathrm{E}+00$ & $3.552 \mathrm{E}-06$ & $7.708 \mathrm{E}+07$ \\
\hline $1 s 3 d{ }^{1} D_{2}$ & $1 s 4 f^{1} F_{3}^{o}$ & $6.6317 \mathrm{E}+00$ & $2.098 \mathrm{E}-05$ & $4.545 \mathrm{E}+08$ & $6.6038 \mathrm{E}+00$ & $2.124 \mathrm{E}-05$ & $4.602 \mathrm{E}+08$ \\
\hline $1 s 4 s^{3} S_{1}$ & $1 s 4 f^{1} F_{3}^{o}$ & $1.4747 \mathrm{E}+02$ & $1.639 \mathrm{E}-15$ & $7.182 \mathrm{E}-05$ & $1.4705 \mathrm{E}+02$ & $1.598 \mathrm{E}-15$ & $6.984 \mathrm{E}-05$ \\
\hline $1 s 4 d^{3} D_{2}$ & $1 s 4 f^{1} F_{3}^{o}$ & $5.3023 \mathrm{E}+02$ & $2.655 \mathrm{E}-12$ & $8.999 \mathrm{E}-03$ & $5.3185 \mathrm{E}+02$ & $2.605 \mathrm{E}-12$ & $8.701 \mathrm{E}-03$ \\
\hline $1 s 4 d^{3} D_{1}$ & $1 s 4 f^{1} F_{3}^{o}$ & $5.4428 \mathrm{E}+02$ & $1.364 \mathrm{E}-11$ & $4.388 \mathrm{E}-02$ & $5.4594 \mathrm{E}+02$ & $1.331 \mathrm{E}-11$ & $4.220 \mathrm{E}-02$ \\
\hline $1 s 4 d^{3} D_{3}$ & $1 s 4 f^{1} F_{3}^{o}$ & $1.5391 \mathrm{E}+03$ & $6.867 \mathrm{E}-13$ & $2.763 \mathrm{E}-04$ & $1.5556 \mathrm{E}+03$ & $6.579 \mathrm{E}-13$ & $2.568 \mathrm{E}-04$ \\
\hline $1 s 4 d{ }^{1} D_{2}$ & $1 s 4 f^{1} F_{3}^{o}$ & $1.6685 \mathrm{E}+03$ & $3.227 \mathrm{E}-12$ & $1.105 \mathrm{E}-03$ & $1.6908 \mathrm{E}+03$ & $3.068 \mathrm{E}-12$ & $1.014 \mathrm{E}-03$ \\
\hline $1 s 2 p^{3} P_{1}^{o}$ & $1 s 5 s^{3} s_{1}^{3}$ & $1.4605 \mathrm{E}+00$ & $3.050 \mathrm{E}-10$ & $3.179 \mathrm{E}+05$ & $1.4544 \mathrm{E}+00$ & $4.408 \mathrm{E}-10$ & $4.594 \mathrm{E}+05$ \\
\hline $1 s 2 p^{3} P_{2}^{0}$ & $1 s 5 s^{3} S_{1}$ & $1.5306 \mathrm{E}+00$ & $2.559 \mathrm{E}-07$ & $2.429 \mathrm{E}+08$ & $1.5241 \mathrm{E}+00$ & $2.484 \mathrm{E}-07$ & $2.357 \mathrm{E}+08$ \\
\hline $1 s 2 p{ }^{1} P_{1}^{o}$ & $1 s 5 s^{3} S_{1}$ & $1.5372 \mathrm{E}+00$ & $2.603 \mathrm{E}-07$ & $2.449 \mathrm{E}+08$ & $1.5308 \mathrm{E}+00$ & $2.448 \mathrm{E}-07$ & $2.302 \mathrm{E}+08$ \\
\hline $1 s 3 p^{3} P_{1}^{o}$ & $1 s 5 s^{3} S_{1}$ & $4.3516 \mathrm{E}+00$ & $1.805 \mathrm{E}-10$ & $2.119 \mathrm{E}+04$ & $4.3336 \mathrm{E}+00$ & $2.612 \mathrm{E}-10$ & $3.066 \mathrm{E}+04$ \\
\hline $1 s 3 p^{3} P_{2}^{0}$ & $1 s 5 s^{3} S_{1}$ & $4.5356 \mathrm{E}+00$ & $1.712 \mathrm{E}-07$ & $1.851 \mathrm{E}+07$ & $4.5167 \mathrm{E}+00$ & $1.689 \mathrm{E}-07$ & $1.825 \mathrm{E}+07$ \\
\hline $1 s 3 p^{1} P_{1}^{o}$ & $1 s 5 s^{3} s_{1}$ & $4.5521 \mathrm{E}+00$ & $1.739 \mathrm{E}-07$ & $1.866 \mathrm{E}+07$ & $4.5333 \mathrm{E}+00$ & $1.668 \mathrm{E}-07$ & $1.790 \mathrm{E}+07$ \\
\hline $1 s 4 p^{3} P_{1}^{o}$ & $1 s 5 s^{3} s_{1}$ & $1.3877 \mathrm{E}+01$ & $1.225 \mathrm{E}-10$ & $1.414 \mathrm{E}+03$ & $1.3818 \mathrm{E}+01$ & $1.886 \mathrm{E}-10$ & $2.178 \mathrm{E}+03$ \\
\hline $1 s 4 p^{3} P_{2}^{o}$ & $1 s 5 s^{3} s_{1}$ & $1.4675 \mathrm{E}+01$ & $1.220 \mathrm{E}-07$ & $1.260 \mathrm{E}+06$ & $1.4612 \mathrm{E}+01$ & $1.208 \mathrm{E}-07$ & $1.247 \mathrm{E}+06$ \\
\hline $1 s 4 p{ }^{1} P_{1}^{o}$ & $1 s 5 s^{3} S_{1}$ & $1.4747 \mathrm{E}+01$ & $1.241 \mathrm{E}-07$ & $1.268 \mathrm{E}+06$ & $1.4684 \mathrm{E}+01$ & $1.189 \mathrm{E}-07$ & $1.215 E+06$ \\
\hline $1 s 4 f^{3} F_{3}^{o}$ & $1 s 5 s^{3} S_{1}$ & $1.5014 \mathrm{E}+01$ & $7.117 \mathrm{E}-15$ & $7.019 \mathrm{E}-02$ & & & \\
\hline 1s $4 f^{3} F_{2}^{o}$ & $1 s 5 s^{3} S_{1}$ & $1.5020 \mathrm{E}+01$ & $3.744 \mathrm{E}-18$ & $3.690 \mathrm{E}-05$ & & & \\
\hline $1 s 4 f^{1} F_{3}^{o}$ & $1 s 5 s^{3} S_{1}$ & $1.5158 \mathrm{E}+01$ & $4.158 \mathrm{E}-14$ & $4.024 \mathrm{E}-01$ & & & \\
\hline $1 s 2 s^{3} S_{1}$ & $1 s 5 p^{3} P_{1}^{o}$ & $1.4466 \mathrm{E}+00$ & $2.751 \mathrm{E}-09$ & $2.923 \mathrm{E}+06$ & $1.4405 \mathrm{E}+00$ & $2.623 E-09$ & $2.787 \mathrm{E}+06$ \\
\hline $1 s 3 s^{3} s_{1}$ & $1 s 5 p^{3} P_{1}^{o}$ & $4.3132 \mathrm{E}+00$ & $9.001 \mathrm{E}-10$ & $1.076 \mathrm{E}+05$ & $4.2953 \mathrm{E}+00$ & $7.868 \mathrm{E}-10$ & $9.401 \mathrm{E}+04$ \\
\hline $1 s 3 d^{3} D_{2}$ & $1 s 5 p^{3} P_{1}^{o}$ & $4.5423 \mathrm{E}+00$ & $1.872 \mathrm{E}-09$ & $2.018 \mathrm{E}+05$ & $4.5233 \mathrm{E}+00$ & $1.852 \mathrm{E}-09$ & $1.996 \mathrm{E}+05$ \\
\hline $1 s 3 d^{3} D_{1}$ & $1 s 5 p^{3} P_{1}^{o}$ & $4.5448 \mathrm{E}+00$ & $1.860 \mathrm{E}-09$ & $2.002 \mathrm{E}+05$ & $4.5258 \mathrm{E}+00$ & $1.819 \mathrm{E}-09$ & $1.957 \mathrm{E}+05$ \\
\hline $1 s 3 d^{3} D_{3}$ & $1 s 5 p^{3} P_{1}^{o}$ & $4.6035 \mathrm{E}+00$ & $3.130 \mathrm{E}-08$ & $3.284 \mathrm{E}+06$ & $4.5841 \mathrm{E}+00$ & $3.112 \mathrm{E}-08$ & $3.264 \mathrm{E}+06$ \\
\hline $1 s 3 d^{1} D_{2}$ & $1 s 5 p^{3} P_{1}^{o}$ & $4.6059 \mathrm{E}+00$ & $1.184 \mathrm{E}-08$ & $1.241 \mathrm{E}+06$ & $4.5865 \mathrm{E}+00$ & $1.116 \mathrm{E}-08$ & $1.169 \mathrm{E}+06$ \\
\hline $1 s 4 s^{3} S_{1}$ & $1 s 5 p^{3} P_{1}^{o}$ & $1.3679 \mathrm{E}+01$ & $3.955 \mathrm{E}-10$ & $4.699 \mathrm{E}+03$ & $1.3623 \mathrm{E}+01$ & $3.048 \mathrm{E}-10$ & $3.621 \mathrm{E}+03$ \\
\hline $1 s 4 d^{3} D_{2}$ & $1 s 5 p^{3} P_{1}^{o}$ & $1.4661 \mathrm{E}+01$ & $2.272 \mathrm{E}-09$ & $2.350 \mathrm{E}+04$ & $1.4602 \mathrm{E}+01$ & $2.264 \mathrm{E}-09$ & $2.341 \mathrm{E}+04$ \\
\hline $1 s 4 d^{3} D_{1}$ & $1 s 5 p^{3} P_{1}^{o}$ & $1.4671 \mathrm{E}+01$ & $2.229 \mathrm{E}-09$ & $2.302 \mathrm{E}+04$ & $1.4612 \mathrm{E}+01$ & $2.198 \mathrm{E}-09$ & $2.269 \mathrm{E}+04$ \\
\hline $1 s 4 d^{3} D_{3}$ & $1 s 5 p^{3} P_{1}^{o}$ & $1.4932 \mathrm{E}+01$ & $3.752 \mathrm{E}-08$ & $3.742 \mathrm{E}+05$ & $1.4871 \mathrm{E}+01$ & $3.761 \mathrm{E}-08$ & $3.749 \mathrm{E}+05$ \\
\hline $1 s 4 d{ }^{1} D_{2}$ & $1 s 5 p^{3} P_{1}^{o}$ & $1.4943 \mathrm{E}+01$ & $1.413 \mathrm{E}-08$ & $1.408 \mathrm{E}+05$ & $1.4882 \mathrm{E}+01$ & $1.340 \mathrm{E}-08$ & $1.334 \mathrm{E}+05$ \\
\hline $1 s 5 s^{3} S_{1}$ & $1 s 5 p^{3} P_{1}^{o}$ & $2.8648 \mathrm{E}+03$ & $8.236 \mathrm{E}-16$ & $2.231 \mathrm{E}-07$ & $2.9004 \mathrm{E}+03$ & $8.265 E-16$ & $2.165 \mathrm{E}-07$ \\
\hline $1 s 2 p^{3} P_{2}^{o}$ & $1 s 5 s^{1} S_{0}$ & $1.5297 \mathrm{E}+00$ & $1.624 \mathrm{E}-07$ & $4.628 \mathrm{E}+08$ & $1.5233 \mathrm{E}+00$ & $1.659 \mathrm{E}-07$ & $4.728 \mathrm{E}+08$ \\
\hline $1 s 3 p^{3} P_{2}^{o}$ & $1 s 5 s^{1} S_{0}$ & $4.5281 \mathrm{E}+00$ & $1.095 \mathrm{E}-07$ & $3.561 \mathrm{E}+07$ & $4.5091 \mathrm{E}+00$ & $1.132 \mathrm{E}-07$ & $3.682 \mathrm{E}+07$ \\
\hline $1 s 4 p^{3} P_{2}^{o}$ & $1 s 5 s^{1} s_{0}$ & $1.4597 \mathrm{E}+01$ & $7.783 \mathrm{E}-08$ & $2.437 \mathrm{E}+06$ & $1.4533 \mathrm{E}+01$ & $8.188 \mathrm{E}-08$ & $2.563 \mathrm{E}+06$ \\
\hline $1 s 4 f^{3} F_{2}^{o}$ & $1 s 5 s{ }^{1} S_{0}$ & $1.4938 \mathrm{E}+01$ & $9.202 \mathrm{E}-15$ & $2.751 \mathrm{E}-01$ & & & \\
\hline $1 s 3 d^{3} D_{2}$ & $1 s 5 p^{3} P_{0}^{o}$ & $4.5418 \mathrm{E}+00$ & $1.202 \mathrm{E}-09$ & $3.888 \mathrm{E}+05$ & $4.5228 \mathrm{E}+00$ & $1.184 \mathrm{E}-09$ & $3.828 \mathrm{E}+05$ \\
\hline $1 s 3 d^{1} D_{2}$ & $1 s 5 p^{3} P_{0}^{o}$ & $4.6053 \mathrm{E}+00$ & $1.441 \mathrm{E}-08$ & $4.531 \mathrm{E}+06$ & $4.5860 \mathrm{E}+00$ & $1.410 \mathrm{E}-08$ & $4.436 \mathrm{E}+06$ \\
\hline $1 s 4 d^{3} D_{2}$ & $1 s 5 p^{3} P_{0}^{o}$ & $1.4656 \mathrm{E}+01$ & $1.425 \mathrm{E}-09$ & $4.426 \mathrm{E}+04$ & $1.4597 \mathrm{E}+01$ & $1.412 \mathrm{E}-09$ & $4.385 E+04$ \\
\hline $1 s 4 d{ }^{1} D_{2}$ & $1 s 5 p^{3} P_{0}^{o}$ & $1.4937 \mathrm{E}+01$ & $1.725 \mathrm{E}-08$ & $5.156 \mathrm{E}+05$ & $1.4877 \mathrm{E}+01$ & $1.705 \mathrm{E}-08$ & $5.096 \mathrm{E}+05$ \\
\hline $1 s^{2}{ }^{1} S_{0}$ & $1 s 5 p^{3} P_{2}^{0}$ & $3.2010 \mathrm{E}-01$ & $1.717 \mathrm{E}-05$ & $2.234 \mathrm{E}+11$ & $3.1883 \mathrm{E}-01$ & $1.929 \mathrm{E}-05$ & $2.510 \mathrm{E}+11$ \\
\hline $1 s 2 s^{3} S_{1}$ & $1 s 5 p^{3} P_{2}^{o}$ & $1.4424 \mathrm{E}+00$ & $1.922 \mathrm{E}-06$ & $1.232 \mathrm{E}+09$ & $1.4363 \mathrm{E}+00$ & $1.984 \mathrm{E}-06$ & $1.272 \mathrm{E}+09$ \\
\hline $1 s 2 s{ }^{1} S_{0}$ & $1 s 5 p^{3} P_{2}^{o}$ & $1.4568 \mathrm{E}+00$ & $1.283 \mathrm{E}-06$ & $8.066 \mathrm{E}+08$ & $1.4509 \mathrm{E}+00$ & $1.283 \mathrm{E}-06$ & $8.066 \mathrm{E}+08$ \\
\hline $1 s 3 s^{3} S_{1}$ & $1 s 5 p^{3} P_{2}^{o}$ & $4.2763 \mathrm{E}+00$ & $5.990 \mathrm{E}-07$ & $4.370 \mathrm{E}+07$ & $4.2586 \mathrm{E}+00$ & $6.082 \mathrm{E}-07$ & $4.435 \mathrm{E}+07$ \\
\hline $1 s 3 s{ }^{1} S_{0}$ & $1 s 5 p^{3} P_{2}^{o}$ & $4.3097 \mathrm{E}+00$ & $4.071 \mathrm{E}-07$ & $2.924 \mathrm{E}+07$ & $4.2923 \mathrm{E}+00$ & $3.960 \mathrm{E}-07$ & $2.843 E+07$ \\
\hline $1 s 3 d^{3} D_{2}$ & $1 s 5 p^{3} P_{2}^{o}$ & $4.5014 \mathrm{E}+00$ & $6.201 \mathrm{E}-14$ & $4.082 \mathrm{E}+00$ & & & \\
\hline $1 s 3 d^{3} D_{1}$ & $1 s 5 p^{3} P_{2}^{o}$ & $4.5039 \mathrm{E}+00$ & $5.894 \mathrm{E}-13$ & $3.876 \mathrm{E}+01$ & & & \\
\hline $1 s 3 d^{3} D_{3}$ & $1 s 5 p^{3} P_{2}^{o}$ & $4.5615 \mathrm{E}+00$ & $1.111 \mathrm{E}-07$ & $7.123 \mathrm{E}+06$ & $4.5423 \mathrm{E}+00$ & $1.073 \mathrm{E}-07$ & $6.882 \mathrm{E}+06$ \\
\hline $1 s 3 d^{1} D_{2}$ & $1 s 5 p^{3} P_{2}^{o}$ & $4.5638 \mathrm{E}+00$ & $2.802 \mathrm{E}-08$ & $1.794 \mathrm{E}+06$ & $4.5447 \mathrm{E}+00$ & $2.679 \mathrm{E}-08$ & $1.716 \mathrm{E}+06$ \\
\hline $1 s 4 s^{3} S_{1}$ & $1 s 5 p^{3} P_{2}^{o}$ & $1.3315 \mathrm{E}+01$ & $2.473 \mathrm{E}-07$ & $1.861 \mathrm{E}+06$ & $1.3261 \mathrm{E}+01$ & $2.502 \mathrm{E}-07$ & $1.882 \mathrm{E}+06$ \\
\hline $1 s 4 s^{1} S_{0}$ & $1 s 5 p^{3} P_{2}^{o}$ & $1.3446 \mathrm{E}+01$ & $1.705 \mathrm{E}-07$ & $1.258 \mathrm{E}+06$ & $1.3392 \mathrm{E}+01$ & $1.619 \mathrm{E}-07$ & $1.194 \mathrm{E}+06$ \\
\hline $1 s 4 d^{3} D_{2}$ & $1 s 5 p^{3} P_{2}^{o}$ & $1.4243 \mathrm{E}+01$ & $5.361 \mathrm{E}-13$ & $3.525 \mathrm{E}+00$ & & & \\
\hline $1 s 4 d^{3} D_{1}$ & $1 s 5 p^{3} P_{2}^{o}$ & $1.4253 \mathrm{E}+01$ & $3.646 \mathrm{E}-13$ & $2.394 \mathrm{E}+00$ & & & \\
\hline
\end{tabular}


Table 6 (continued)

\begin{tabular}{|c|c|c|c|c|c|c|c|}
\hline \multirow[t]{2}{*}{ Lower } & \multirow[t]{2}{*}{ Upper } & \multicolumn{3}{|l|}{ GRASP2K } & \multicolumn{3}{|l|}{ FAC } \\
\hline & & $\lambda(\AA)$ & $g f$ & $A\left(\mathrm{~s}^{-1}\right)$ & $\lambda(\AA)$ & $g f$ & $A\left(\mathrm{~s}^{-1}\right)$ \\
\hline $1 s 4 d^{3} D_{3}$ & $1 s 5 p^{3} P_{2}^{o}$ & $1.4499 \mathrm{E}+01$ & $1.363 \mathrm{E}-07$ & $8.649 \mathrm{E}+05$ & $1.4440 \mathrm{E}+01$ & $1.327 \mathrm{E}-07$ & $8.420 \mathrm{E}+05$ \\
\hline $1 s 4 d^{1} D_{2}$ & $1 s 5 p^{3} P_{2}^{o}$ & $1.4509 \mathrm{E}+01$ & $3.425 \mathrm{E}-08$ & $2.170 \mathrm{E}+05$ & $1.4450 \mathrm{E}+01$ & $3.310 \mathrm{E}-08$ & $2.097 \mathrm{E}+05$ \\
\hline $1 s 5 s^{3} S_{1}$ & $1 s 5 p^{3} P_{2}^{0}$ & $4.2565 \mathrm{E}+02$ & $2.014 \mathrm{E}-10$ & $1.483 \mathrm{E}+00$ & $4.2515 E+02$ & $2.001 \mathrm{E}-10$ & $1.464 \mathrm{E}+00$ \\
\hline $1 s 5 s{ }^{1} S_{0}$ & $1 s 5 p^{3} P_{2}^{o}$ & $5.0438 \mathrm{E}+02$ & $8.081 \mathrm{E}-11$ & $4.238 \mathrm{E}-01$ & $5.0484 \mathrm{E}+02$ & $7.970 \mathrm{E}-11$ & $4.136 \mathrm{E}-01$ \\
\hline $1 s 2 p^{3} P_{1}^{o}$ & $1 s 5 d^{3} D_{2}$ & $1.4552 \mathrm{E}+00$ & $1.344 \mathrm{E}-07$ & $8.464 \mathrm{E}+07$ & $1.4491 \mathrm{E}+00$ & $1.437 \mathrm{E}-07$ & $9.054 \mathrm{E}+07$ \\
\hline $1 s 2 p^{3} P_{0}^{0}$ & $1 s 5 d^{3} D_{2}$ & $1.4562 \mathrm{E}+00$ & $8.458 \mathrm{E}-08$ & $5.321 \mathrm{E}+07$ & $1.4501 \mathrm{E}+00$ & $8.881 \mathrm{E}-08$ & $5.586 \mathrm{E}+07$ \\
\hline $1 s 2 p^{3} P_{2}^{0}$ & $1 s 5 d^{3} D_{2}$ & $1.5247 \mathrm{E}+00$ & $7.189 \mathrm{E}-11$ & $4.125 \mathrm{E}+04$ & $1.5183 \mathrm{E}+00$ & $1.049 \mathrm{E}-10$ & $6.019 \mathrm{E}+04$ \\
\hline $1 s 2 p^{1} P_{1}^{o}$ & $1 s 5 d^{3} D_{2}$ & $1.5313 \mathrm{E}+00$ & $7.295 \mathrm{E}-15$ & $4.150 \mathrm{E}+00$ & & & \\
\hline $1 s 3 p^{3} P_{1}^{0}$ & $1 s 5 d^{3} D_{2}$ & $4.3049 \mathrm{E}+00$ & $4.655 \mathrm{E}-08$ & $3.351 \mathrm{E}+06$ & $4.2870 \mathrm{E}+00$ & $4.769 \mathrm{E}-08$ & $3.433 \mathrm{E}+06$ \\
\hline $1 s 3 p^{3} P_{0}^{0}$ & $1 s 5 d^{3} D_{2}$ & $4.3072 \mathrm{E}+00$ & $2.903 \mathrm{E}-08$ & $2.087 \mathrm{E}+06$ & $4.2892 \mathrm{E}+00$ & $2.951 \mathrm{E}-08$ & $2.121 \mathrm{E}+06$ \\
\hline $1 s 3 p^{3} P_{2}^{0}$ & $1 s 5 d^{3} D_{2}$ & $4.4849 \mathrm{E}+00$ & $3.092 \mathrm{E}-11$ & $2.051 \mathrm{E}+03$ & $4.4661 \mathrm{E}+00$ & $3.730 \mathrm{E}-11$ & $2.473 \mathrm{E}+03$ \\
\hline $1 s 3 p^{1} P_{1}^{o}$ & $1 s 5 d^{3} D_{2}$ & $4.5010 \mathrm{E}+00$ & $3.286 \mathrm{E}-13$ & $2.164 \mathrm{E}+01$ & & & \\
\hline $1 s 4 p^{3} P_{1}^{o}$ & $1 s 5 d^{3} D_{2}$ & $1.3413 E+01$ & $1.867 \mathrm{E}-08$ & $1.385 \mathrm{E}+05$ & $1.3354 \mathrm{E}+01$ & $1.890 \mathrm{E}-08$ & $1.401 \mathrm{E}+05$ \\
\hline $1 s 4 p^{3} P_{0}^{0}$ & $1 s 5 d^{3} D_{2}$ & $1.3422 \mathrm{E}+01$ & $1.165 \mathrm{E}-08$ & $8.628 \mathrm{E}+04$ & $1.3363 \mathrm{E}+01$ & $1.171 \mathrm{E}-08$ & $8.675 E+04$ \\
\hline $1 s 4 p^{3} P_{2}^{0}$ & $1 s 5 d^{3} D_{2}$ & $1.4158 \mathrm{E}+01$ & $1.382 \mathrm{E}-11$ & $9.197 \mathrm{E}+01$ & $1.4095 \mathrm{E}+01$ & $1.553 \mathrm{E}-11$ & $1.034 \mathrm{E}+02$ \\
\hline $1 s 4 p^{1} P_{1}^{o}$ & $1 s 5 d^{3} D_{2}$ & $1.4224 \mathrm{E}+01$ & $2.600 \mathrm{E}-13$ & $1.714 \mathrm{E}+00$ & & & \\
\hline $1 s 4 f^{3} F_{3}^{o}$ & $1 s 5 d^{3} D_{2}$ & $1.4473 \mathrm{E}+01$ & $7.457 \mathrm{E}-09$ & $4.750 \mathrm{E}+04$ & $1.4409 \mathrm{E}+01$ & $7.405 \mathrm{E}-09$ & $4.718 \mathrm{E}+04$ \\
\hline $1 s 4 f^{3} F_{2}^{o}$ & $1 s 5 d^{3} D_{2}$ & $1.4478 \mathrm{E}+01$ & $1.873 \mathrm{E}-09$ & $1.192 \mathrm{E}+04$ & $1.4414 \mathrm{E}+01$ & $1.853 \mathrm{E}-09$ & $1.179 \mathrm{E}+04$ \\
\hline $1 s 4 f^{3} F_{4}^{o}$ & $1 s 5 d^{3} D_{2}$ & $1.4602 \mathrm{E}+01$ & $1.516 \mathrm{E}-08$ & $9.483 \mathrm{E}+04$ & $1.4537 \mathrm{E}+01$ & $1.503 \mathrm{E}-08$ & $9.412 \mathrm{E}+04$ \\
\hline $1 s 4 f^{1} F_{3}^{o}$ & $1 s 5 d^{3} D_{2}$ & $1.4606 \mathrm{E}+01$ & $1.852 \mathrm{E}-09$ & $1.158 \mathrm{E}+04$ & $1.4541 \mathrm{E}+01$ & $1.832 \mathrm{E}-09$ & $1.146 \mathrm{E}+04$ \\
\hline $1 s 5 p^{3} P_{1}^{0}$ & $1 s 5 d^{3} D_{2}$ & $4.6651 \mathrm{E}+02$ & $5.572 \mathrm{E}-12$ & $3.415 \mathrm{E}-02$ & $4.6168 \mathrm{E}+02$ & $5.725 \mathrm{E}-12$ & $3.553 \mathrm{E}-02$ \\
\hline $1 s 5 p^{3} P_{0}^{0}$ & $1 s 5 d^{3} D_{2}$ & $4.7209 \mathrm{E}+02$ & $3.350 \mathrm{E}-12$ & $2.005 \mathrm{E}-02$ & $4.6698 \mathrm{E}+02$ & $3.426 \mathrm{E}-12$ & $2.078 \mathrm{E}-02$ \\
\hline $1 s 5 p^{3} P_{2}^{o}$ & $1 s 5 d^{3} D_{2}$ & $6.9791 \mathrm{E}+03$ & $1.076 \mathrm{E}-18$ & $2.947 \mathrm{E}-11$ & & & \\
\hline $1 s 2 s^{3} S_{1}^{2}$ & $1 s 5 p{ }^{1} P_{1}^{o}$ & $1.4421 \mathrm{E}+00$ & $1.903 \mathrm{E}-06$ & $2.034 \mathrm{E}+09$ & $1.4360 \mathrm{E}+00$ & $1.983 \mathrm{E}-06$ & $2.120 \mathrm{E}+09$ \\
\hline $1 s 3 s^{3} S_{1}$ & $1 s 5 p^{1} P_{1}^{o}$ & $4.2732 \mathrm{E}+00$ & $5.899 \mathrm{E}-07$ & $7.183 \mathrm{E}+07$ & $4.2555 E+00$ & $6.087 \mathrm{E}-07$ & $7.410 \mathrm{E}+07$ \\
\hline $1 s 3 d^{3} D_{2}$ & $1 s 5 p{ }^{1} P_{1}^{o}$ & $4.4980 \mathrm{E}+00$ & $3.519 \mathrm{E}-13$ & $3.867 \mathrm{E}+01$ & & & \\
\hline $1 s 3 d^{3} D_{1}$ & $1 s 5 p{ }^{1} P_{1}^{o}$ & $4.5005 \mathrm{E}+00$ & $4.503 \mathrm{E}-14$ & $4.944 \mathrm{E}+00$ & & & \\
\hline $1 s 3 d^{3} D_{3}$ & $1 s 5 p{ }^{1} P_{1}^{0}$ & $4.5580 \mathrm{E}+00$ & $1.937 \mathrm{E}-08$ & $2.073 \mathrm{E}+06$ & $4.5388 \mathrm{E}+00$ & $1.975 \mathrm{E}-08$ & $2.113 \mathrm{E}+06$ \\
\hline $1 s 3 d^{1} D_{2}$ & $1 s 5 p{ }^{1} P_{1}^{o}$ & $4.5603 \mathrm{E}+00$ & $6.171 \mathrm{E}-08$ & $6.598 \mathrm{E}+06$ & $4.5411 \mathrm{E}+00$ & $6.080 \mathrm{E}-08$ & $6.500 \mathrm{E}+06$ \\
\hline $1 s 4 s^{3} S_{1}$ & $1 s 5 p^{1} P_{1}^{o}$ & $1.3285 \mathrm{E}+01$ & $2.421 \mathrm{E}-07$ & $3.050 \mathrm{E}+06$ & $1.3231 \mathrm{E}+01$ & $2.516 \mathrm{E}-07$ & $3.168 \mathrm{E}+06$ \\
\hline $1 s 4 d^{3} D_{2}$ & $1 s 5 p^{1} P_{1}^{o}$ & $1.4209 \mathrm{E}+01$ & $7.511 \mathrm{E}-15$ & $8.271 \mathrm{E}-02$ & & & \\
\hline $1 s 4 d^{3} D_{1}$ & $1 s 5 p{ }^{1} P_{1}^{o}$ & $1.4219 \mathrm{E}+01$ & $3.306 \mathrm{E}-13$ & $3.636 \mathrm{E}+00$ & & & \\
\hline $1 s 4 d^{3} D_{3}$ & $1 s 5 p{ }^{1} P_{1}^{o}$ & $1.4463 \mathrm{E}+01$ & $2.382 \mathrm{E}-08$ & $2.532 \mathrm{E}+05$ & $1.4404 \mathrm{E}+01$ & $2.459 \mathrm{E}-08$ & $2.612 \mathrm{E}+05$ \\
\hline $1 s 4 d^{1} D_{2}$ & $1 s 5 p{ }^{1} P_{1}^{o}$ & $1.4474 \mathrm{E}+01$ & $7.561 \mathrm{E}-08$ & $8.025 \mathrm{E}+05$ & $1.4415 \mathrm{E}+01$ & $7.547 \mathrm{E}-08$ & $8.007 \mathrm{E}+05$ \\
\hline $1 s 5 s^{3} S_{1}$ & $1 s 5 p{ }^{1} P_{1}^{o}$ & $3.9729 \mathrm{E}+02$ & $2.478 \mathrm{E}-10$ & $3.491 \mathrm{E}+00$ & $3.9647 \mathrm{E}+02$ & $2.465 \mathrm{E}-10$ & $3.457 \mathrm{E}+00$ \\
\hline $1 s 5 d^{3} D_{2}$ & $1 s 5 p{ }^{1} P_{1}^{o}$ & $4.1006 \mathrm{E}+04$ & $1.343 \mathrm{E}-22$ & $1.776 \mathrm{E}-16$ & & & \\
\hline $1 s 2 p^{3} P_{1}^{o}$ & $1 s 5 d^{3} D_{1}$ & $1.4551 \mathrm{E}+00$ & $1.319 \mathrm{E}-07$ & $1.385 \mathrm{E}+08$ & $1.4491 \mathrm{E}+00$ & $1.392 \mathrm{E}-07$ & $1.461 \mathrm{E}+08$ \\
\hline $1 s 2 p^{3} P_{2}^{o}$ & $1 s 5 d^{3} D_{1}$ & $1.5247 \mathrm{E}+00$ & $2.190 \mathrm{E}-13$ & $2.095 \mathrm{E}+02$ & & & \\
\hline $1 s 2 p^{1} P_{1}^{o}$ & $1 s 5 d^{3} D_{1}$ & $1.5313 \mathrm{E}+00$ & $1.600 \mathrm{E}-10$ & $1.517 \mathrm{E}+05$ & $1.5249 \mathrm{E}+00$ & $1.733 \mathrm{E}-10$ & $1.643 \mathrm{E}+05$ \\
\hline $1 s 3 p^{3} P_{1}^{0}$ & $1 s 5 d^{3} D_{1}$ & $4.3044 \mathrm{E}+00$ & $4.527 \mathrm{E}-08$ & $5.432 \mathrm{E}+06$ & $4.2865 \mathrm{E}+00$ & $4.624 \mathrm{E}-08$ & $5.548 \mathrm{E}+06$ \\
\hline $1 s 3 p^{3} P_{2}^{0}$ & $1 s 5 d^{3} D_{1}$ & $4.4844 \mathrm{E}+00$ & $3.083 \mathrm{E}-13$ & $3.408 \mathrm{E}+01$ & & & \\
\hline $1 s 3 p^{1} P_{1}^{o}$ & $1 s 5 d^{3} D_{1}$ & $4.5005 \mathrm{E}+00$ & $5.280 \mathrm{E}-11$ & $5.796 \mathrm{E}+03$ & $4.4818 \mathrm{E}+00$ & $4.790 \mathrm{E}-11$ & $5.257 \mathrm{E}+03$ \\
\hline $1 s 4 p^{3} P_{1}^{0}$ & $1 s 5 d^{3} D_{1}$ & $1.3409 \mathrm{E}+01$ & $1.812 \mathrm{E}-08$ & $2.241 \mathrm{E}+05$ & $1.3350 \mathrm{E}+01$ & $1.835 \mathrm{E}-08$ & $2.270 \mathrm{E}+05$ \\
\hline $1 s 4 p^{3} P_{2}^{0}$ & $1 s 5 d^{3} D_{1}$ & $1.4153 \mathrm{E}+01$ & $2.043 \mathrm{E}-13$ & $2.268 \mathrm{E}+00$ & & & \\
\hline $1 s 4 p{ }^{1} P_{1}^{o}$ & $1 s 5 d^{3} D_{1}$ & $1.4219 \mathrm{E}+01$ & $2.169 \mathrm{E}-11$ & $2.385 \mathrm{E}+02$ & $1.4157 \mathrm{E}+01$ & $1.589 \mathrm{E}-11$ & $1.748 \mathrm{E}+02$ \\
\hline $1 s 4 f^{3} F_{3}^{o}$ & $1 s 5 d^{3} D_{1}$ & $1.4467 \mathrm{E}+01$ & $1.254 \mathrm{E}-09$ & $1.332 \mathrm{E}+04$ & $1.4404 \mathrm{E}+01$ & $1.253 \mathrm{E}-09$ & $1.332 \mathrm{E}+04$ \\
\hline $1 s 4 f^{3} F_{2}^{o}$ & $1 s 5 d^{3} D_{1}$ & $1.4473 \mathrm{E}+01$ & $4.348 \mathrm{E}-09$ & $4.616 \mathrm{E}+04$ & $1.4409 \mathrm{E}+01$ & $4.329 \mathrm{E}-09$ & $4.596 \mathrm{E}+04$ \\
\hline $1 s 4 f^{1} F_{3}^{o}$ & $1 s 5 d^{3} D_{1}$ & $1.4601 \mathrm{E}+01$ & $1.014 \mathrm{E}-08$ & $1.058 \mathrm{E}+05$ & $1.4536 \mathrm{E}+01$ & $1.009 \mathrm{E}-08$ & $1.053 \mathrm{E}+05$ \\
\hline $1 s 5 p^{3} P_{1}^{o}$ & $1 s 5 d^{3} D_{1}$ & $4.6125 \mathrm{E}+02$ & $5.605 \mathrm{E}-12$ & $5.858 \mathrm{E}-02$ & $4.5655 \mathrm{E}+02$ & $5.740 \mathrm{E}-12$ & $6.071 \mathrm{E}-02$ \\
\hline $1 s 5 p^{3} P_{2}^{o}$ & $1 s 5 d^{3} D_{1}$ & $5.9615 E+03$ & $1.448 \mathrm{E}-19$ & $9.058 \mathrm{E}-12$ & & & \\
\hline $1 s 5 p^{1} P_{1}^{o}$ & $1 s 5 d^{3} D_{1}$ & $1.4006 \mathrm{E}+07$ & $1.496 \mathrm{E}-28$ & $1.698 \mathrm{E}-27$ & & & \\
\hline $1 s 2 p^{3} P_{1}^{o}$ & $1 s 5 d^{3} D_{3}$ & $1.4539 \mathrm{E}+00$ & $2.153 \mathrm{E}-06$ & $9.704 \mathrm{E}+08$ & $1.4478 \mathrm{E}+00$ & $2.200 \mathrm{E}-06$ & $9.919 E+08$ \\
\hline $1 s 2 p^{3} P_{2}^{o}$ & $1 s 5 d^{3} D_{3}$ & $1.5233 E+00$ & $9.425 \mathrm{E}-06$ & $3.871 \mathrm{E}+09$ & $1.5168 \mathrm{E}+00$ & $9.852 \mathrm{E}-06$ & $4.045 \mathrm{E}+09$ \\
\hline $1 s 2 p^{1} P_{1}^{o}$ & $1 s 5 d^{3} D_{3}$ & $1.5298 \mathrm{E}+00$ & $1.691 \mathrm{E}-06$ & $6.884 \mathrm{E}+08$ & $1.5235 \mathrm{E}+00$ & $1.767 \mathrm{E}-06$ & $7.196 \mathrm{E}+08$ \\
\hline $1 s 3 p^{3} P_{1}^{0}$ & $1 s 5 d^{3} D_{3}$ & $4.2932 \mathrm{E}+00$ & $7.013 \mathrm{E}-07$ & $3.626 \mathrm{E}+07$ & $4.2754 \mathrm{E}+00$ & $7.111 \mathrm{E}-07$ & $3.676 \mathrm{E}+07$ \\
\hline $1 s 3 p^{3} P_{2}^{0}$ & $1 s 5 d^{3} D_{3}$ & $4.4722 \mathrm{E}+00$ & $3.457 \mathrm{E}-06$ & $1.647 \mathrm{E}+08$ & $4.4535 \mathrm{E}+00$ & $3.522 \mathrm{E}-06$ & $1.678 \mathrm{E}+08$ \\
\hline $1 s 3 p^{1} P_{1}^{o}$ & $1 s 5 d^{3} D_{3}$ & $4.4883 \mathrm{E}+00$ & $6.233 \mathrm{E}-07$ & $2.948 \mathrm{E}+07$ & $4.4696 \mathrm{E}+00$ & $6.279 \mathrm{E}-07$ & $2.969 \mathrm{E}+07$ \\
\hline $1 s 4 p^{3} P_{1}^{o}$ & $1 s 5 d^{3} D_{3}$ & $1.3300 \mathrm{E}+01$ & $2.701 \mathrm{E}-07$ & $1.455 \mathrm{E}+06$ & $1.3242 \mathrm{E}+01$ & $2.699 \mathrm{E}-07$ & $1.454 \mathrm{E}+06$ \\
\hline $1 s 4 p^{3} P_{2}^{o}$ & $1 s 5 d^{3} D_{3}$ & $1.4032 \mathrm{E}+01$ & $1.483 \mathrm{E}-06$ & $7.178 \mathrm{E}+06$ & $1.3970 \mathrm{E}+01$ & $1.506 \mathrm{E}-06$ & $7.291 \mathrm{E}+06$ \\
\hline $1 s 4 p^{1} P_{1}^{o}$ & $1 s 5 d^{3} D_{3}$ & $1.4097 \mathrm{E}+01$ & $2.694 \mathrm{E}-07$ & $1.292 \mathrm{E}+06$ & $1.4036 \mathrm{E}+01$ & $2.656 \mathrm{E}-07$ & $1.274 \mathrm{E}+06$ \\
\hline $1 s 4 f^{3} F_{3}^{o}$ & $1 s 5 d^{3} D_{3}$ & $1.4341 \mathrm{E}+01$ & $5.108 \mathrm{E}-14$ & $2.367 \mathrm{E}-01$ & & & \\
\hline $1 s 4 f^{3} F_{2}^{o}$ & $1 s 5 d^{3} D_{3}$ & $1.4347 \mathrm{E}+01$ & $1.695 \mathrm{E}-13$ & $7.849 \mathrm{E}-01$ & & & \\
\hline $1 s 4 f^{3} F_{4}^{o}$ & $1 s 5 d^{3} D_{3}$ & $1.4468 \mathrm{E}+01$ & $8.474 \mathrm{E}-08$ & $3.857 \mathrm{E}+05$ & $1.4405 \mathrm{E}+01$ & $8.385 \mathrm{E}-08$ & $3.818 \mathrm{E}+05$ \\
\hline $1 s 4 f^{1} F_{3}^{o}$ & $1 s 5 d^{3} D_{3}$ & $1.4472 \mathrm{E}+01$ & $1.019 \mathrm{E}-08$ & $4.636 \mathrm{E}+04$ & $1.4409 \mathrm{E}+01$ & $1.005 \mathrm{E}-08$ & $4.575 \mathrm{E}+04$ \\
\hline $1 s 5 p^{3} P_{1}^{o}$ & $1 s 5 d^{3} D_{3}$ & $3.6020 \mathrm{E}+02$ & $1.859 \mathrm{E}-10$ & $1.366 \mathrm{E}+00$ & $3.5728 \mathrm{E}+02$ & $1.880 \mathrm{E}-10$ & $1.392 \mathrm{E}+00$ \\
\hline $1 s 5 p^{3} P_{2}^{0}$ & $1 s 5 d^{3} D_{3}$ & $1.2888 \mathrm{E}+03$ & $2.022 \mathrm{E}-11$ & $1.160 \mathrm{E}-02$ & $1.2633 \mathrm{E}+03$ & $2.122 \mathrm{E}-11$ & $1.256 \mathrm{E}-02$ \\
\hline $1 s 5 p^{1} P_{1}^{o}$ & $1 s 5 d^{3} D_{3}$ & $1.6441 \mathrm{E}+03$ & $1.755 \mathrm{E}-12$ & $6.188 \mathrm{E}-04$ & $1.6091 \mathrm{E}+03$ & $1.854 \mathrm{E}-12$ & $6.766 \mathrm{E}-04$ \\
\hline $1 s 2 s^{3} S_{1}$ & $1 s 5 f^{3} F_{3}^{o}$ & $1.4408 \mathrm{E}+00$ & $9.780 \mathrm{E}-11$ & $4.489 E+04$ & & & \\
\hline $1 s 3 s^{3} S_{1}$ & $1 s 5 f^{3} F_{3}^{o}$ & $4.2620 \mathrm{E}+00$ & $6.114 \mathrm{E}-13$ & $3.207 \mathrm{E}+01$ & & & \\
\hline $1 s 3 d^{3} D_{2}$ & $1 s 5 f^{3} F_{3}^{o}$ & $4.4855 \mathrm{E}+00$ & $7.993 \mathrm{E}-07$ & $3.786 \mathrm{E}+07$ & $4.4667 \mathrm{E}+00$ & $8.162 \mathrm{E}-07$ & $3.865 \mathrm{E}+07$ \\
\hline $1 s 3 d^{3} D_{1}$ & $1 s 5 f^{3} F_{3}^{o}$ & $4.4880 \mathrm{E}+00$ & $1.349 \mathrm{E}-07$ & $6.380 \mathrm{E}+06$ & $4.4691 \mathrm{E}+00$ & $1.376 \mathrm{E}-07$ & $6.509 \mathrm{E}+06$ \\
\hline
\end{tabular}


Table 6 (continued)

\begin{tabular}{|c|c|c|c|c|c|c|c|}
\hline \multirow[t]{2}{*}{ Lower } & \multirow[t]{2}{*}{ Upper } & \multicolumn{3}{|l|}{ GRASP2K } & \multicolumn{3}{|l|}{ FAC } \\
\hline & & $\lambda(\AA)$ & $g f$ & $A\left(\mathrm{~s}^{-1}\right)$ & $\lambda(\AA)$ & $g f$ & $A\left(\mathrm{~s}^{-1}\right)$ \\
\hline $1 s 3 d^{3} D_{3}$ & $1 s 5 f^{3} F_{3}^{o}$ & $4.5452 \mathrm{E}+00$ & $4.800 \mathrm{E}-12$ & $2.214 \mathrm{E}+02$ & & & \\
\hline $1 s 3 d{ }^{1} D_{2}$ & $1 s 5 f^{3} F_{3}^{o}$ & $4.5475 \mathrm{E}+00$ & $6.705 \mathrm{E}-11$ & $3.089 \mathrm{E}+03$ & & & \\
\hline $1 s 4 s^{3} S_{1}$ & $1 s 5 f^{3} F_{3}^{o}$ & $1.3177 \mathrm{E}+01$ & $2.554 \mathrm{E}-13$ & $1.402 \mathrm{E}+00$ & & & \\
\hline $1 s 4 d^{3} D_{2}$ & $1 s 5 f^{3} F_{3}^{o}$ & $1.4085 \mathrm{E}+01$ & $4.505 \mathrm{E}-07$ & $2.164 \mathrm{E}+06$ & $1.4028 \mathrm{E}+01$ & $4.534 \mathrm{E}-07$ & $2.177 \mathrm{E}+06$ \\
\hline $1 s 4 d^{3} D_{1}$ & $1 s 5 f^{3} F_{3}^{o}$ & $1.4095 \mathrm{E}+01$ & $7.610 \mathrm{E}-08$ & $3.650 \mathrm{E}+05$ & $1.4038 \mathrm{E}+01$ & $7.640 \mathrm{E}-08$ & $3.663 \mathrm{E}+05$ \\
\hline $1 s 4 d^{3} D_{3}$ & $1 s 5 f^{3} F_{3}^{o}$ & $1.4335 \mathrm{E}+01$ & $2.763 \mathrm{E}-12$ & $1.281 \mathrm{E}+01$ & & & \\
\hline $1 s 4 d{ }^{1} D_{2}$ & $1 s 5 f^{3} F_{3}^{o}$ & $1.4345 \mathrm{E}+01$ & $5.743 \mathrm{E}-11$ & $2.659 \mathrm{E}+02$ & $1.4286 \mathrm{E}+01$ & $5.957 \mathrm{E}-11$ & $2.757 \mathrm{E}+02$ \\
\hline $1 s 5 s^{3} s_{1}$ & $1 s 5 f^{3} F_{3}^{o}$ & $3.1888 \mathrm{E}+02$ & $1.023 \mathrm{E}-17$ & $9.585 \mathrm{E}-08$ & $3.1776 \mathrm{E}+02$ & $8.216 \mathrm{E}-17$ & $7.688 \mathrm{E}-07$ \\
\hline $1 s 5 d^{3} D_{2}$ & $1 s 5 f^{3} F_{3}^{o}$ & $1.5543 \mathrm{E}+03$ & $1.612 \mathrm{E}-12$ & $6.359 \mathrm{E}-04$ & $1.5716 \mathrm{E}+03$ & $1.545 \mathrm{E}-12$ & $5.911 \mathrm{E}-04$ \\
\hline $1 s 5 d^{3} D_{1}$ & $1 s 5 f^{3} F_{3}^{o}$ & $1.6158 \mathrm{E}+03$ & $2.424 \mathrm{E}-13$ & $8.846 \mathrm{E}-05$ & $1.6341 \mathrm{E}+03$ & $2.321 \mathrm{E}-13$ & $8.213 \mathrm{E}-05$ \\
\hline $1 s 5 d^{3} D_{3}$ & $1 s 5 f^{3} F_{3}^{o}$ & $9.3195 \mathrm{E}+04$ & $4.604 \mathrm{E}-23$ & $5.051 \mathrm{E}-18$ & & & \\
\hline $1 s^{2}{ }^{1} S_{0}$ & $1 s 5 f^{3} F_{2}^{o}$ & $3.2000 \mathrm{E}-01$ & $9.916 \mathrm{E}-11$ & $1.291 \mathrm{E}+06$ & $3.1875 \mathrm{E}-01$ & $4.044 \mathrm{E}-12$ & $5.265 E+04$ \\
\hline $1 s 2 s^{3} S_{1}$ & $1 s 5 f^{3} F_{2}^{o}$ & $1.4408 \mathrm{E}+00$ & $3.316 \mathrm{E}-11$ & $2.131 \mathrm{E}+04$ & $1.4347 \mathrm{E}+00$ & $4.172 \mathrm{E}-13$ & $2.681 \mathrm{E}+02$ \\
\hline $1 s 2 s{ }^{1} S_{0}$ & $1 s 5 f^{3} F_{2}^{o}$ & $1.4551 \mathrm{E}+00$ & $3.223 \mathrm{E}-11$ & $2.030 \mathrm{E}+04$ & $1.4492 \mathrm{E}+00$ & $2.698 \mathrm{E}-13$ & $1.699 \mathrm{E}+02$ \\
\hline $1 s 3 s^{3} s_{1}$ & $1 s 5 f^{3} F_{2}^{o}$ & $4.2617 \mathrm{E}+00$ & $1.699 \mathrm{E}-12$ & $1.248 \mathrm{E}+02$ & & & \\
\hline $1 s 3 s^{1} S_{0}$ & $1 s 5 f^{3} F_{2}^{o}$ & $4.2949 \mathrm{E}+00$ & $1.182 \mathrm{E}-12$ & $8.548 \mathrm{E}+01$ & & & \\
\hline $1 s 3 d^{3} D_{2}$ & $1 s 5 f^{3} F_{2}^{o}$ & $4.4853 \mathrm{E}+00$ & $2.005 \mathrm{E}-07$ & $1.330 \mathrm{E}+07$ & $4.4664 \mathrm{E}+00$ & $2.044 \mathrm{E}-07$ & $1.355 \mathrm{E}+07$ \\
\hline $1 s 3 d^{3} D_{1}$ & $1 s 5 f^{3} F_{2}^{o}$ & $4.4877 \mathrm{E}+00$ & $4.668 \mathrm{E}-07$ & $3.092 \mathrm{E}+07$ & $4.4688 \mathrm{E}+00$ & $4.763 \mathrm{E}-07$ & $3.155 \mathrm{E}+07$ \\
\hline $1 s 3 d^{3} D_{3}$ & $1 s 5 f^{3} F_{2}^{o}$ & $4.5449 \mathrm{E}+00$ & $4.501 \mathrm{E}-15$ & $2.907 \mathrm{E}-01$ & & & \\
\hline $1 s 3 d^{1} D_{2}$ & $1 s 5 f^{3} F_{2}^{o}$ & $4.5472 \mathrm{E}+00$ & $1.914 \mathrm{E}-12$ & $1.235 \mathrm{E}+02$ & & & \\
\hline $1 s 4 s^{3} S_{1}$ & $1 s 5 f^{3} F_{2}^{o}$ & $1.3175 \mathrm{E}+01$ & $1.082 \mathrm{E}-12$ & $8.314 \mathrm{E}+00$ & & & \\
\hline $1 s 4 s^{1} S_{0}$ & $1 s 5 f^{3} F_{2}^{o}$ & $1.3303 \mathrm{E}+01$ & $5.809 \mathrm{E}-13$ & $4.379 \mathrm{E}+00$ & & & \\
\hline $1 s 4 d^{3} D_{2}$ & $1 s 5 f^{3} F_{2}^{o}$ & $1.4083 \mathrm{E}+01$ & $1.128 \mathrm{E}-07$ & $7.589 \mathrm{E}+05$ & $1.4025 \mathrm{E}+01$ & $1.136 \mathrm{E}-07$ & $7.641 \mathrm{E}+05$ \\
\hline $1 s 4 d^{3} D_{1}$ & $1 s 5 f^{3} F_{2}^{o}$ & $1.4092 \mathrm{E}+01$ & $2.633 \mathrm{E}-07$ & $1.768 \mathrm{E}+06$ & $1.4035 \mathrm{E}+01$ & $2.646 \mathrm{E}-07$ & $1.776 \mathrm{E}+06$ \\
\hline $1 s 4 d^{3} D_{3}$ & $1 s 5 f^{3} F_{2}^{o}$ & $1.4332 \mathrm{E}+01$ & $2.202 \mathrm{E}-14$ & $1.430 \mathrm{E}-01$ & & & \\
\hline $1 s 4 d{ }^{1} D_{2}$ & $1 s 5 f^{3} F_{2}^{o}$ & $1.4343 \mathrm{E}+01$ & $2.824 \mathrm{E}-12$ & $1.832 \mathrm{E}+01$ & & & \\
\hline $1 s 5 s^{3} S_{1}$ & $1 s 5 f^{3} F_{2}^{o}$ & $3.1755 \mathrm{E}+02$ & $1.065 \mathrm{E}-16$ & $1.409 \mathrm{E}-06$ & $3.1643 \mathrm{E}+02$ & $4.872 \mathrm{E}-17$ & $6.436 \mathrm{E}-07$ \\
\hline $1 s 5 s^{1} S_{0}$ & $1 s 5 f^{3} F_{2}^{o}$ & $3.5940 \mathrm{E}+02$ & $1.023 \mathrm{E}-16$ & $1.057 \mathrm{E}-06$ & $3.5855 \mathrm{E}+02$ & $4.660 \mathrm{E}-17$ & $4.795 \mathrm{E}-07$ \\
\hline $1 s 5 d^{3} D_{2}$ & $1 s 5 f^{3} F_{2}^{o}$ & $1.5232 \mathrm{E}+03$ & $4.293 \mathrm{E}-13$ & $2.469 \mathrm{E}-04$ & $1.5396 \mathrm{E}+03$ & $4.117 \mathrm{E}-13$ & $2.297 \mathrm{E}-04$ \\
\hline $1 s 5 d^{3} D_{1}$ & $1 s 5 f^{3} F_{2}^{o}$ & $1.5821 \mathrm{E}+03$ & $8.937 \mathrm{E}-13$ & $4.763 \mathrm{E}-04$ & $1.5996 \mathrm{E}+03$ & $8.566 \mathrm{E}-13$ & $4.428 \mathrm{E}-04$ \\
\hline $1 s 5 d^{3} D_{3}$ & $1 s 5 f^{3} F_{2}^{o}$ & $4.1865 \mathrm{E}+04$ & $1.183 \mathrm{E}-22$ & $9.007 \mathrm{E}-17$ & & & \\
\hline $1 s 2 p^{3} P_{1}^{o}$ & $1 s 5 d^{1} D_{2}$ & $1.4538 \mathrm{E}+00$ & $8.312 \mathrm{E}-07$ & $5.247 \mathrm{E}+08$ & $1.4478 \mathrm{E}+00$ & $8.539 E-07$ & $5.388 \mathrm{E}+08$ \\
\hline $1 s 2 p^{3} P_{0}^{o}$ & $1 s 5 d^{1} D_{2}$ & $1.4548 \mathrm{E}+00$ & $9.966 \mathrm{E}-07$ & $6.282 \mathrm{E}+08$ & $1.4487 \mathrm{E}+00$ & $1.018 \mathrm{E}-06$ & $6.420 \mathrm{E}+08$ \\
\hline $1 s 2 p^{3} P_{2}^{0}$ & $1 s 5 d{ }^{1} D_{2}$ & $1.5232 \mathrm{E}+00$ & $2.366 \mathrm{E}-06$ & $1.360 \mathrm{E}+09$ & $1.5168 \mathrm{E}+00$ & $2.463 \mathrm{E}-06$ & $1.416 \mathrm{E}+09$ \\
\hline $1 s 2 p{ }^{1} P_{1}^{o}$ & $1 s 5 d^{1} D_{2}$ & $1.5298 \mathrm{E}+00$ & $5.292 \mathrm{E}-06$ & $3.017 \mathrm{E}+09$ & $1.5234 \mathrm{E}+00$ & $5.518 \mathrm{E}-06$ & $3.145 \mathrm{E}+09$ \\
\hline $1 s 3 p^{3} P_{1}^{o}$ & $1 s 5 d^{1} D_{2}$ & $4.2927 \mathrm{E}+00$ & $2.650 \mathrm{E}-07$ & $1.918 \mathrm{E}+07$ & $4.2749 \mathrm{E}+00$ & $2.739 \mathrm{E}-07$ & $1.982 \mathrm{E}+07$ \\
\hline $1 s 3 p^{3} P_{0}^{o}$ & $1 s 5 d^{1} D_{2}$ & $4.2951 \mathrm{E}+00$ & $3.229 \mathrm{E}-07$ & $2.335 \mathrm{E}+07$ & $4.2771 \mathrm{E}+00$ & $3.287 \mathrm{E}-07$ & $2.377 \mathrm{E}+07$ \\
\hline $1 s 3 p^{3} P_{2}^{o}$ & $1 s 5 d^{1} D_{2}$ & $4.4717 \mathrm{E}+00$ & $8.646 \mathrm{E}-07$ & $5.768 \mathrm{E}+07$ & $4.4529 \mathrm{E}+00$ & $8.809 \mathrm{E}-07$ & $5.876 \mathrm{E}+07$ \\
\hline $1 s 3 p{ }^{1} P_{1}^{o}$ & $1 s 5 d^{1} D_{2}$ & $4.4877 \mathrm{E}+00$ & $1.961 \mathrm{E}-06$ & $1.299 \mathrm{E}+08$ & $4.4691 \mathrm{E}+00$ & $1.983 \mathrm{E}-06$ & $1.313 \mathrm{E}+08$ \\
\hline $1 s 4 p^{3} P_{1}^{o}$ & $1 s 5 d^{1} D_{2}$ & $1.3295 \mathrm{E}+01$ & $1.013 \mathrm{E}-07$ & $7.645 \mathrm{E}+05$ & $1.3238 \mathrm{E}+01$ & $1.057 \mathrm{E}-07$ & $7.981 \mathrm{E}+05$ \\
\hline $1 s 4 p^{3} P_{0}^{0}$ & $1 s 5 d^{1} D_{2}$ & $1.3304 \mathrm{E}+01$ & $1.241 \mathrm{E}-07$ & $9.356 \mathrm{E}+05$ & $1.3246 \mathrm{E}+01$ & $1.253 \mathrm{E}-07$ & $9.450 \mathrm{E}+05$ \\
\hline $1 s 4 p^{3} P_{2}^{o}$ & $1 s 5 d^{1} D_{2}$ & $1.4027 \mathrm{E}+01$ & $3.703 \mathrm{E}-07$ & $2.511 \mathrm{E}+06$ & $1.3965 \mathrm{E}+01$ & $3.769 \mathrm{E}-07$ & $2.556 \mathrm{E}+06$ \\
\hline $1 s 4 p{ }^{1} P_{1}^{o}$ & $1 s 5 d^{1} D_{2}$ & $1.4092 \mathrm{E}+01$ & $8.483 \mathrm{E}-07$ & $5.699 \mathrm{E}+06$ & $1.4031 \mathrm{E}+01$ & $8.480 \mathrm{E}-07$ & $5.698 \mathrm{E}+06$ \\
\hline $1 s 4 f^{3} F_{3}^{o}$ & $1 s 5 d^{1} D_{2}$ & $1.4336 \mathrm{E}+01$ & $1.005 \mathrm{E}-12$ & $6.522 \mathrm{E}+00$ & & & \\
\hline $1 s 4 f^{3} F_{2}^{o}$ & $1 s 5 d^{1} D_{2}$ & $1.4341 \mathrm{E}+01$ & $4.083 \mathrm{E}-16$ & $2.648 \mathrm{E}-03$ & & & \\
\hline $1 s 4 f^{3} F_{4}^{o}$ & $1 s 5 d^{1} D_{2}$ & $1.4463 \mathrm{E}+01$ & $6.852 \mathrm{E}-09$ & $4.371 \mathrm{E}+04$ & $1.4399 \mathrm{E}+01$ & $6.850 \mathrm{E}-09$ & $4.370 \mathrm{E}+04$ \\
\hline $1 s 4 f^{1} F_{3}^{o}$ & $1 s 5 d^{1} D_{2}$ & $1.4467 \mathrm{E}+01$ & $6.065 \mathrm{E}-08$ & $3.866 \mathrm{E}+05$ & $1.4403 \mathrm{E}+01$ & $6.025 \mathrm{E}-08$ & $3.842 \mathrm{E}+05$ \\
\hline $1 s 5 p^{3} P_{1}^{0}$ & $1 s 5 d^{1} D_{2}$ & $3.5679 \mathrm{E}+02$ & $7.174 \mathrm{E}-11$ & $7.518 \mathrm{E}-01$ & $3.5385 E+02$ & $7.273 \mathrm{E}-11$ & $7.683 \mathrm{E}-01$ \\
\hline $1 s 5 p^{3} P_{0}^{0}$ & $1 s 5 d^{1} D_{2}$ & $3.6004 \mathrm{E}+02$ & $8.559 \mathrm{E}-11$ & $8.808 \mathrm{E}-01$ & $3.5695 \mathrm{E}+02$ & $8.675 E-11$ & $9.006 \mathrm{E}-01$ \\
\hline $1 s 5 p^{3} P_{2}^{o}$ & $1 s 5 d^{1} D_{2}$ & $1.2461 \mathrm{E}+03$ & $5.596 \mathrm{E}-12$ & $4.807 \mathrm{E}-03$ & $1.2214 \mathrm{E}+03$ & $5.871 \mathrm{E}-12$ & $5.205 \mathrm{E}-03$ \\
\hline $1 s 5 p{ }^{1} P_{1}^{o}$ & $1 s 5 d^{1} D_{2}$ & $1.5753 \mathrm{E}+03$ & $6.301 \mathrm{E}-12$ & $3.387 \mathrm{E}-03$ & $1.5418 \mathrm{E}+03$ & $6.642 \mathrm{E}-12$ & $3.695 \mathrm{E}-03$ \\
\hline $1 s 5 f^{3} F_{3}^{o}$ & $1 s 5 d^{1} D_{2}$ & $6.3169 \mathrm{E}+04$ & $3.487 \mathrm{E}-21$ & $1.166 \mathrm{E}-15$ & & & \\
\hline $1 s 5 f^{3} F_{2}^{o}$ & $1 s 5 d^{1} D_{2}$ & $3.7392 \mathrm{E}+05$ & $6.620 \mathrm{E}-25$ & $6.316 \mathrm{E}-21$ & & & \\
\hline $1 s 3 d^{3} D_{2}$ & $1 s 5 f^{3} F_{4}^{o}$ & $4.4792 \mathrm{E}+00$ & $1.568 \mathrm{E}-06$ & $5.791 \mathrm{E}+07$ & $4.4604 \mathrm{E}+00$ & $1.578 \mathrm{E}-06$ & $5.829 \mathrm{E}+07$ \\
\hline $1 s 3 d^{3} D_{3}$ & $1 s 5 f^{3} F_{4}^{0}$ & $4.5387 \mathrm{E}+00$ & $9.572 \mathrm{E}-06$ & $3.444 \mathrm{E}+08$ & $4.5196 \mathrm{E}+00$ & $9.774 \mathrm{E}-06$ & $3.516 \mathrm{E}+08$ \\
\hline $1 s 3 d d^{1} D_{2}$ & $1 s 5 f^{3} F_{4}^{0}$ & $4.5410 \mathrm{E}+00$ & $7.718 \mathrm{E}-07$ & $2.774 \mathrm{E}+07$ & $4.5219 \mathrm{E}+00$ & $7.887 \mathrm{E}-07$ & $2.834 \mathrm{E}+07$ \\
\hline $1 s 4 d^{3} D_{2}$ & $1 s 5 f^{3} F_{4}^{o}$ & $1.4024 \mathrm{E}+01$ & $8.559 \mathrm{E}-07$ & $3.226 \mathrm{E}+06$ & $1.3967 \mathrm{E}+01$ & $8.610 \mathrm{E}-07$ & $3.243 \mathrm{E}+06$ \\
\hline $1 s 4 d^{3} D_{3}$ & $1 s 5 f^{3} F_{4}^{o}$ & $1.4271 \mathrm{E}+01$ & $5.505 \mathrm{E}-06$ & $2.003 \mathrm{E}+07$ & $1.4212 \mathrm{E}+01$ & $5.539 \mathrm{E}-06$ & $2.015 \mathrm{E}+07$ \\
\hline $1 s 4 d^{1} D_{2}$ & $1 s 5 f^{3} F_{4}^{o}$ & $1.4281 \mathrm{E}+01$ & $4.469 \mathrm{E}-07$ & $1.624 \mathrm{E}+06$ & $1.4223 \mathrm{E}+01$ & $4.490 \mathrm{E}-07$ & $1.631 \mathrm{E}+06$ \\
\hline $1 s 5 d^{3} D_{2}$ & $1 s 5 f^{3} F_{4}^{0}$ & $1.0450 \mathrm{E}+03$ & $1.024 \mathrm{E}-11$ & $6.949 \mathrm{E}-03$ & $1.0522 \mathrm{E}+03$ & $9.888 \mathrm{E}-12$ & $6.563 \mathrm{E}-03$ \\
\hline $1 s 5 d^{3} D_{3}$ & $1 s 5 f^{3} F_{4}^{o}$ & $3.0834 \mathrm{E}+03$ & $2.544 \mathrm{E}-12$ & $1.984 \mathrm{E}-04$ & $3.1501 E+03$ & $2.359 \mathrm{E}-12$ & $1.747 \mathrm{E}-04$ \\
\hline $1 s 5 d{ }^{1} D_{2}$ & $1 s 5 f^{3} F_{4}^{o}$ & $3.3584 \mathrm{E}+03$ & $1.601 \mathrm{E}-13$ & $1.052 \mathrm{E}-05$ & $3.4443 E+03$ & $1.470 \mathrm{E}-13$ & $9.108 \mathrm{E}-06$ \\
\hline $1 s 2 p^{3} P_{2}^{o}$ & $1 s 5 g^{3} G_{4}$ & $1.5225 \mathrm{E}+00$ & $2.015 \mathrm{E}-11$ & $6.442 \mathrm{E}+03$ & & & \\
\hline $1 s 3 p^{3} P_{2}^{o}$ & $1 s 5 g{ }^{3} G_{4}$ & $4.4657 \mathrm{E}+00$ & $2.464 \mathrm{E}-11$ & $9.157 \mathrm{E}+02$ & & & \\
\hline $1 s 4 p^{3} P_{2}^{o}$ & $1 s 5 g{ }^{3} G_{4}$ & $1.3968 \mathrm{E}+01$ & $1.156 \mathrm{E}-12$ & $4.392 \mathrm{E}+00$ & & & \\
\hline $1 s 4 f^{3} F_{3}^{o}$ & $1 s 5 g{ }^{3} G_{4}$ & $1.4275 \mathrm{E}+01$ & $2.706 \mathrm{E}-06$ & $9.841 \mathrm{E}+06$ & $1.4214 \mathrm{E}+01$ & $2.727 \mathrm{E}-06$ & $9.920 \mathrm{E}+06$ \\
\hline $1 s 4 f^{3} F_{2}^{o}$ & $1 s 5 g^{3} G_{4}$ & $1.4280 \mathrm{E}+01$ & $2.186 \mathrm{E}-07$ & $7.945 \mathrm{E}+05$ & $1.4219 \mathrm{E}+01$ & $2.202 \mathrm{E}-07$ & $8.004 \mathrm{E}+05$ \\
\hline $1 s 4 f^{3} F_{4}^{o}$ & $1 s 5 g^{3} G_{4}$ & $1.4400 \mathrm{E}+01$ & $4.154 \mathrm{E}-12$ & $1.485 \mathrm{E}+01$ & & & \\
\hline $1 s 4 f^{1} F_{3}^{o}$ & $1 s 5 g^{3} G_{4}$ & $1.4404 \mathrm{E}+01$ & $1.035 \mathrm{E}-11$ & $3.695 \mathrm{E}+01$ & & & \\
\hline $1 s 5 p^{3} P_{2}^{o}$ & $1 s 5 g{ }^{3} G_{4}$ & $9.0842 \mathrm{E}+02$ & $6.390 \mathrm{E}-20$ & $5.739 \mathrm{E}-11$ & & & \\
\hline $1 s 5 f^{3} F_{3}^{o}$ & $1 s 5 g{ }^{3} G_{4}$ & $3.1830 \mathrm{E}+03$ & $4.013 \mathrm{E}-13$ & $2.936 \mathrm{E}-05$ & $3.1753 \mathrm{E}+03$ & $4.002 \mathrm{E}-13$ & $2.917 \mathrm{E}-05$ \\
\hline
\end{tabular}


Table 6 (continued)

\begin{tabular}{|c|c|c|c|c|c|c|c|}
\hline \multirow[t]{2}{*}{ Lower } & \multirow[t]{2}{*}{ Upper } & \multicolumn{3}{|l|}{ GRASP2K } & \multicolumn{3}{|l|}{ FAC } \\
\hline & & $\lambda(\AA)$ & $g f$ & $A\left(\mathrm{~s}^{-1}\right)$ & $\lambda(\AA)$ & $g f$ & $A\left(\mathrm{~s}^{-1}\right)$ \\
\hline $1 s 5 f^{3} F_{2}^{o}$ & $1 s 5 g{ }^{3} G_{4}$ & $3.3221 \mathrm{E}+03$ & $2.853 \mathrm{E}-14$ & $1.916 \mathrm{E}-06$ & $3.3144 \mathrm{E}+03$ & $2.845 \mathrm{E}-14$ & $1.903 \mathrm{E}-06$ \\
\hline $1 s 5 f^{3} F_{4}^{o}$ & $1 s 5 g{ }^{3} G_{4}$ & $1.7265 \mathrm{E}+06$ & $3.911 \mathrm{E}-27$ & $9.725 \mathrm{E}-25$ & & & \\
\hline $1 s 2 p^{3} P_{1}^{o}$ & $1 s 5 g{ }^{3} G_{3}$ & $1.4532 \mathrm{E}+00$ & $1.974 \mathrm{E}-14$ & $8.909 E+00$ & $1.4471 \mathrm{E}+00$ & $1.086 \mathrm{E}-15$ & $4.903 \mathrm{E}-01$ \\
\hline $1 s 2 p^{3} P_{2}^{o}$ & $1 s 5 g{ }^{3} G_{3}$ & $1.5225 \mathrm{E}+00$ & $2.038 \mathrm{E}-12$ & $8.377 \mathrm{E}+02$ & $1.5161 \mathrm{E}+00$ & $4.521 \mathrm{E}-15$ & $1.858 \mathrm{E}+00$ \\
\hline $1 s 2 p^{1} P_{1}^{o}$ & $1 s 5 g{ }^{3} G_{3}$ & $1.5291 \mathrm{E}+00$ & $1.334 \mathrm{E}-11$ & $5.435 \mathrm{E}+03$ & & & \\
\hline $1 s 3 p^{3} P_{1}^{o}$ & $1 s 5 g{ }^{3} G_{3}$ & $4.2871 \mathrm{E}+00$ & $4.526 \mathrm{E}-14$ & $2.347 \mathrm{E}+00$ & $4.2694 \mathrm{E}+00$ & $3.515 \mathrm{E}-16$ & $1.822 \mathrm{E}-02$ \\
\hline $1 s 3 p^{3} P_{2}^{o}$ & $1 s 5 g{ }^{3} G_{3}$ & $4.4656 \mathrm{E}+00$ & $2.799 \mathrm{E}-12$ & $1.337 \mathrm{E}+02$ & $4.4470 \mathrm{E}+00$ & $1.621 \mathrm{E}-15$ & $7.745 \mathrm{E}-02$ \\
\hline $1 s 3 p{ }^{1} P_{1}^{o}$ & $1 s 5 g{ }^{3} G_{3}$ & $4.4816 \mathrm{E}+00$ & $1.566 \mathrm{E}-11$ & $7.431 \mathrm{E}+02$ & & & \\
\hline $1 s 4 p^{3} P_{1}^{o}$ & $1 s 5 g{ }^{3} G_{3}$ & $1.3242 \mathrm{E}+01$ & $1.450 \mathrm{E}-15$ & $7.879 \mathrm{E}-03$ & & & \\
\hline $1 s 4 p^{3} P_{2}^{0}$ & $1 s 5 g{ }^{3} G_{3}$ & $1.3967 \mathrm{E}+01$ & $7.954 \mathrm{E}-13$ & $3.886 \mathrm{E}+00$ & & & \\
\hline $1 s 4 p{ }^{1} P_{1}^{o}$ & $1 s 5 g{ }^{3} G_{3}$ & $1.4032 \mathrm{E}+01$ & $1.012 \mathrm{E}-12$ & $4.900 \mathrm{E}+00$ & & & \\
\hline $1 s 4 f^{3} F_{3}^{o}$ & $1 s 5 g{ }^{3} G_{3}$ & $1.4273 \mathrm{E}+01$ & $3.250 \mathrm{E}-07$ & $1.520 \mathrm{E}+06$ & $1.4213 \mathrm{E}+01$ & $3.276 \mathrm{E}-07$ & $1.532 \mathrm{E}+06$ \\
\hline $1 s 4 f^{3} F_{2}^{o}$ & $1 s 5 g{ }^{3} G_{3}$ & $1.4278 \mathrm{E}+01$ & $1.949 \mathrm{E}-06$ & $9.109 E+06$ & $1.4218 \mathrm{E}+01$ & $1.963 \mathrm{E}-06$ & $9.178 \mathrm{E}+06$ \\
\hline $1 s 4 f^{3} F_{4}^{o}$ & $1 s 5 g{ }^{3} G_{3}$ & $1.4399 \mathrm{E}+01$ & $1.054 \mathrm{E}-18$ & $4.843 \mathrm{E}-06$ & & & \\
\hline $1 s 4 f^{1} F_{3}^{o}$ & $1 s 5 g{ }^{3} G_{3}$ & $1.4403 \mathrm{E}+01$ & $1.506 \mathrm{E}-12$ & $6.918 \mathrm{E}+00$ & & & \\
\hline $1 s 5 p^{3} P_{1}^{o}$ & $1 s 5 g^{3} G_{3}$ & $3.2168 \mathrm{E}+02$ & $4.463 \mathrm{E}-20$ & $4.110 \mathrm{E}-10$ & & & \\
\hline $1 s 5 p^{3} P_{2}^{0}$ & $1 s 5 g{ }^{3} G_{3}$ & $9.0225 \mathrm{E}+02$ & $3.077 \mathrm{E}-18$ & $3.602 \mathrm{E}-09$ & & & \\
\hline $1 s 5 p{ }^{1} P_{1}^{o}$ & $1 s 5 g{ }^{3} G_{3}$ & $1.0631 \mathrm{E}+03$ & $3.176 \mathrm{E}-19$ & $2.678 \mathrm{E}-10$ & & & \\
\hline $1 s 5 f^{3} F_{3}^{o}$ & $1 s 5 g{ }^{3} G_{3}$ & $3.1085 E+03$ & $5.176 \mathrm{E}-14$ & $5.104 \mathrm{E}-06$ & $3.1008 \mathrm{E}+03$ & $5.161 \mathrm{E}-14$ & $5.071 \mathrm{E}-06$ \\
\hline $1 s 5 f^{3} F_{2}^{o}$ & $1 s 5 g{ }^{3} G_{3}$ & $3.2410 \mathrm{E}+03$ & $2.739 E-13$ & $2.485 \mathrm{E}-05$ & $3.2333 \mathrm{E}+03$ & $2.731 \mathrm{E}-13$ & $2.468 \mathrm{E}-05$ \\
\hline $1 s 5 f^{3} F_{4}^{o}$ & $1 s 5 g{ }^{3} G_{3}$ & $1.2333 \mathrm{E}+05$ & $1.693 \mathrm{E}-26$ & $1.061 \mathrm{E}-21$ & & & \\
\hline $1 s 2 s^{3} S_{1}^{4}$ & $1 s 5 f^{1} F_{3}^{o}$ & $1.4401 \mathrm{E}+00$ & $1.285 \mathrm{E}-13$ & $5.904 \mathrm{E}+01$ & & & \\
\hline $1 s 3 s^{3} S_{1}$ & $1 s 5 f^{1} F_{3}^{o}$ & $4.2561 \mathrm{E}+00$ & $2.007 \mathrm{E}-12$ & $1.056 \mathrm{E}+02$ & & & \\
\hline $1 s 3 d^{3} D_{2}$ & $1 s 5 f^{1} F_{3}^{o}$ & $4.4790 \mathrm{E}+00$ & $1.841 \mathrm{E}-07$ & $8.745 E+06$ & $4.4602 \mathrm{E}+00$ & $1.862 \mathrm{E}-07$ & $8.846 \mathrm{E}+06$ \\
\hline $1 s 3 d^{3} D_{1}$ & $1 s 5 f^{1} F_{3}^{o}$ & $4.4815 \mathrm{E}+00$ & $1.047 \mathrm{E}-06$ & $4.968 \mathrm{E}+07$ & $4.4626 \mathrm{E}+00$ & $1.054 \mathrm{E}-06$ & $5.003 \mathrm{E}+07$ \\
\hline $1 s 3 d^{3} D_{3}$ & $1 s 5 f^{1} F_{3}^{o}$ & $4.5385 \mathrm{E}+00$ & $1.150 \mathrm{E}-06$ & $5.319 \mathrm{E}+07$ & $4.5194 \mathrm{E}+00$ & $1.173 \mathrm{E}-06$ & $5.426 \mathrm{E}+07$ \\
\hline $1 s 3 d^{1} D_{2}$ & $1 s 5 f^{1} F_{3}^{o}$ & $4.5408 \mathrm{E}+00$ & $6.878 \mathrm{E}-06$ & $3.179 E+08$ & $4.5217 \mathrm{E}+00$ & $7.019 \mathrm{E}-06$ & $3.243 \mathrm{E}+08$ \\
\hline $1 s 4 s^{3} S_{1}$ & $1 s 5 f^{1} F_{3}^{o}$ & $1.3121 \mathrm{E}+01$ & $1.246 \mathrm{E}-12$ & $6.899 \mathrm{E}+00$ & & & \\
\hline $1 s 4 d^{3} D_{2}$ & $1 s 5 f^{1} F_{3}^{o}$ & $1.4022 \mathrm{E}+01$ & $1.031 \mathrm{E}-07$ & $4.999 \mathrm{E}+05$ & $1.3965 \mathrm{E}+01$ & $1.047 \mathrm{E}-07$ & $5.074 \mathrm{E}+05$ \\
\hline $1 s 4 d^{3} D_{1}$ & $1 s 5 f^{1} F_{3}^{o}$ & $1.4031 \mathrm{E}+01$ & $5.739 \mathrm{E}-07$ & $2.778 \mathrm{E}+06$ & $1.3974 \mathrm{E}+01$ & $5.770 \mathrm{E}-07$ & $2.791 \mathrm{E}+06$ \\
\hline $1 s 4 d^{3} D_{3}$ & $1 s 5 f^{1} F_{3}^{o}$ & $1.4269 \mathrm{E}+01$ & $6.604 \mathrm{E}-07$ & $3.091 \mathrm{E}+06$ & $1.4210 \mathrm{E}+01$ & $6.650 \mathrm{E}-07$ & $3.111 \mathrm{E}+06$ \\
\hline $1 s 4 d{ }^{1} D_{2}$ & $1 s 5 f^{1} F_{3}^{o}$ & $1.4279 \mathrm{E}+01$ & $3.956 \mathrm{E}-06$ & $1.849 \mathrm{E}+07$ & $1.4221 \mathrm{E}+01$ & $3.974 \mathrm{E}-06$ & $1.856 \mathrm{E}+07$ \\
\hline $1 s 5 s^{3} S_{1}$ & $1 s 5 f^{1} F_{3}^{o}$ & $2.8907 \mathrm{E}+02$ & $8.703 E-16$ & $9.925 \mathrm{E}-06$ & $2.8810 \mathrm{E}+02$ & $8.531 \mathrm{E}-16$ & $9.711 \mathrm{E}-06$ \\
\hline $1 s 5 d^{3} D_{2}$ & $1 s 5 f^{1} F_{3}^{o}$ & $1.0343 \mathrm{E}+03$ & $1.288 \mathrm{E}-12$ & $1.147 \mathrm{E}-03$ & $1.0413 \mathrm{E}+03$ & $1.252 \mathrm{E}-12$ & $1.091 \mathrm{E}-03$ \\
\hline $1 s 5 d^{3} D_{1}$ & $1 s 5 f^{1} F_{3}^{o}$ & $1.0612 \mathrm{E}+03$ & $6.565 \mathrm{E}-12$ & $5.555 \mathrm{E}-03$ & $1.0684 \mathrm{E}+03$ & $6.346 \mathrm{E}-12$ & $5.252 \mathrm{E}-03$ \\
\hline $1 s 5 d^{3} D_{3}$ & $1 s 5 f^{1} F_{3}^{o}$ & $2.9925 \mathrm{E}+03$ & $3.341 \mathrm{E}-13$ & $3.555 \mathrm{E}-05$ & $3.0547 \mathrm{E}+03$ & $3.104 \mathrm{E}-13$ & $3.143 \mathrm{E}-05$ \\
\hline $1 s 5 d^{1} D_{2}$ & $1 s 5 f^{1} F_{3}^{o}$ & $3.2509 \mathrm{E}+03$ & $1.560 \mathrm{E}-12$ & $1.406 \mathrm{E}-04$ & $3.3306 \mathrm{E}+03$ & $1.434 \mathrm{E}-12$ & $1.221 \mathrm{E}-04$ \\
\hline $1 s 5 g{ }^{3} G_{4}$ & $1 s 5 f^{1} F_{3}^{o}$ & $1.0795 \mathrm{E}+05$ & $4.552 \mathrm{E}-23$ & $3.723 \mathrm{E}-18$ & & & \\
\hline $1 s 5 g^{3} G_{3}$ & $1 s 5 f^{1} F_{3}^{o}$ & $5.7657 \mathrm{E}+05$ & $3.514 \mathrm{E}-26$ & $1.007 \mathrm{E}-22$ & & & \\
\hline $1 s 4 f^{3} F_{3}^{o}$ & $1 s 5 g^{3} G_{5}$ & $1.4237 \mathrm{E}+01$ & $1.884 \mathrm{E}-06$ & $5.637 \mathrm{E}+06$ & $1.4177 \mathrm{E}+01$ & $1.892 \mathrm{E}-06$ & $5.660 \mathrm{E}+06$ \\
\hline $1 s 4 f^{3} F_{4}^{o}$ & $1 s 5 g^{3} G_{5}$ & $1.4362 \mathrm{E}+01$ & $1.643 \mathrm{E}-05$ & $4.829 \mathrm{E}+07$ & $1.4301 \mathrm{E}+01$ & $1.656 \mathrm{E}-05$ & $4.868 \mathrm{E}+07$ \\
\hline $1 s 4 f^{1} F_{3}^{o}$ & $1 s 5 g{ }^{3} G_{5}$ & $1.4366 \mathrm{E}+01$ & $7.767 \mathrm{E}-07$ & $2.282 \mathrm{E}+06$ & $1.4305 \mathrm{E}+01$ & $7.827 \mathrm{E}-07$ & $2.300 \mathrm{E}+06$ \\
\hline $1 s 5 f^{3} F_{3}^{o}$ & $1 s 5 g{ }^{3} G_{5}$ & $1.9964 \mathrm{E}+03$ & $1.136 \mathrm{E}-12$ & $1.728 \mathrm{E}-04$ & $1.9918 \mathrm{E}+03$ & $1.127 \mathrm{E}-12$ & $1.708 \mathrm{E}-04$ \\
\hline $1 s 5 f^{3} F_{4}^{o}$ & $1 s 5 g{ }^{3} G_{5}$ & $5.3388 \mathrm{E}+03$ & $5.211 \mathrm{E}-13$ & $1.109 \mathrm{E}-05$ & $5.3203 \mathrm{E}+03$ & $5.207 \mathrm{E}-13$ & $1.106 \mathrm{E}-05$ \\
\hline $1 s 5 f^{1} F_{3}^{o}$ & $1 s 5 g{ }^{3} G_{5}$ & $5.6350 \mathrm{E}+03$ & $2.097 \mathrm{E}-14$ & $4.004 \mathrm{E}-07$ & $5.6164 \mathrm{E}+03$ & $2.094 \mathrm{E}-14$ & $3.992 \mathrm{E}-07$ \\
\hline $1 s 2 p^{3} P_{2}^{o}$ & $1 s 5 g{ }^{1} G_{4}$ & $1.5221 \mathrm{E}+00$ & $4.230 \mathrm{E}-15$ & $1.353 \mathrm{E}+00$ & & & \\
\hline $1 s 3 p^{3} P_{2}^{o}$ & $1 s 5 g{ }^{1} G_{4}$ & $4.4619 \mathrm{E}+00$ & $5.547 \mathrm{E}-15$ & $2.065 \mathrm{E}-01$ & & & \\
\hline $1 s 4 p^{3} P_{2}^{o}$ & $1 s 5 g{ }^{1} G_{4}$ & $1.3931 \mathrm{E}+01$ & $1.748 \mathrm{E}-13$ & $6.674 \mathrm{E}-01$ & & & \\
\hline $1 s 4 f^{3} F_{3}^{o}$ & $1 s 5 g{ }^{1} G_{4}$ & $1.4236 \mathrm{E}+01$ & $1.107 \mathrm{E}-07$ & $4.048 \mathrm{E}+05$ & $1.4175 \mathrm{E}+01$ & $1.111 \mathrm{E}-07$ & $4.065 \mathrm{E}+05$ \\
\hline $1 s 4 f^{3} F_{2}^{o}$ & $1 s 5 g{ }^{1} G_{4}$ & $1.4241 \mathrm{E}+01$ & $1.421 \mathrm{E}-06$ & $5.192 \mathrm{E}+06$ & $1.4181 \mathrm{E}+01$ & $1.426 \mathrm{E}-06$ & $5.211 \mathrm{E}+06$ \\
\hline $1 s 4 f^{3} F_{4}^{0}$ & $1 s 5 g{ }^{1} G_{4}$ & $1.4361 \mathrm{E}+01$ & $1.174 \mathrm{E}-06$ & $4.218 E+06$ & $1.4299 \mathrm{E}+01$ & $1.183 \mathrm{E}-06$ & $4.252 \mathrm{E}+06$ \\
\hline $1 s 4 f^{1} F_{3}^{0}$ & $1 s 5 g{ }^{1} G_{4}$ & $1.4364 \mathrm{E}+01$ & $1.291 \mathrm{E}-05$ & $4.637 \mathrm{E}+07$ & $1.4303 \mathrm{E}+01$ & $1.301 \mathrm{E}-05$ & $4.673 \mathrm{E}+07$ \\
\hline $1 s 5 p^{3} P_{2}^{o}$ & $1 s 5 g{ }^{1} G_{4}$ & $7.7310 \mathrm{E}+02$ & $3.149 \mathrm{E}-18$ & $3.904 \mathrm{E}-09$ & & & \\
\hline $1 s 5 f^{3} F_{3}^{o}$ & $1 s 5 g{ }^{1} G_{4}$ & $1.9730 \mathrm{E}+03$ & $6.937 \mathrm{E}-14$ & $1.321 \mathrm{E}-05$ & $1.9684 \mathrm{E}+03$ & $6.886 \mathrm{E}-14$ & $1.306 \mathrm{E}-05$ \\
\hline $1 s 5 f^{3} F_{2}^{o}$ & $1 s 5 g{ }^{1} G_{4}$ & $2.0256 \mathrm{E}+03$ & $8.205 E-13$ & $1.482 \mathrm{E}-04$ & $2.0210 \mathrm{E}+03$ & $8.142 \mathrm{E}-13$ & $1.464 \mathrm{E}-04$ \\
\hline $1 s 5 f^{3} F_{4}^{o}$ & $1 s 5 g{ }^{1} G_{4}$ & $5.1744 \mathrm{E}+03$ & $4.089 \mathrm{E}-14$ & $1.132 \mathrm{E}-06$ & $5.1563 \mathrm{E}+03$ & $4.086 \mathrm{E}-14$ & $1.129 \mathrm{E}-06$ \\
\hline $1 s 5 f^{1} F_{3}^{o}$ & $1 s 5 g{ }^{1} G_{4}$ & $5.4521 \mathrm{E}+03$ & $3.846 \mathrm{E}-13$ & $9.589 \mathrm{E}-06$ & $5.4339 \mathrm{E}+03$ & $3.841 \mathrm{E}-13$ & $9.561 \mathrm{E}-06$ \\
\hline $1 s 2 p^{3} P_{1}^{o}$ & $1 s 6 s^{3} s_{1}$ & $1.3813 \mathrm{E}+00$ & $1.649 \mathrm{E}-10$ & $1.922 \mathrm{E}+05$ & $1.3756 \mathrm{E}+00$ & $2.617 \mathrm{E}-10$ & $3.049 \mathrm{E}+05$ \\
\hline $1 s 2 p^{3} P_{2}^{o}$ & $1 s 6 s^{3} S_{1}$ & $1.4438 \mathrm{E}+00$ & $1.444 \mathrm{E}-07$ & $1.540 \mathrm{E}+08$ & $1.4378 \mathrm{E}+00$ & $1.405 \mathrm{E}-07$ & $1.498 \mathrm{E}+08$ \\
\hline $1 s 2 p{ }^{1} P_{1}^{o}$ & $1 s 6 s^{3} S_{1}$ & $1.4497 \mathrm{E}+00$ & $1.473 \mathrm{E}-07$ & $1.558 \mathrm{E}+08$ & $1.4438 \mathrm{E}+00$ & $1.385 \mathrm{E}-07$ & $1.465 \mathrm{E}+08$ \\
\hline $1 s 3 p^{3} P_{1}^{o}$ & $1 s 6 s^{3} S_{1}$ & $3.7169 \mathrm{E}+00$ & $1.013 \mathrm{E}-10$ & $1.630 \mathrm{E}+04$ & $3.7018 \mathrm{E}+00$ & $1.480 \mathrm{E}-10$ & $2.381 \mathrm{E}+04$ \\
\hline $1 s 3 p^{3} P_{2}^{o}$ & $1 s 6 s^{3} S_{1}$ & $3.8504 \mathrm{E}+00$ & $9.482 \mathrm{E}-08$ & $1.422 \mathrm{E}+07$ & $3.8346 \mathrm{E}+00$ & $9.350 \mathrm{E}-08$ & $1.401 \mathrm{E}+07$ \\
\hline $1 s 3 p^{1} P_{1}^{o}$ & $1 s 6 s^{3} S_{1}$ & $3.8623 \mathrm{E}+00$ & $9.621 \mathrm{E}-08$ & $1.434 \mathrm{E}+07$ & $3.8466 \mathrm{E}+00$ & $9.250 \mathrm{E}-08$ & $1.378 \mathrm{E}+07$ \\
\hline $1 s 4 p^{3} P_{1}^{0}$ & $1 s 6 s^{3} s_{1}$ & $8.9847 \mathrm{E}+00$ & $6.745 \mathrm{E}-11$ & $1.858 \mathrm{E}+03$ & $8.9484 \mathrm{E}+00$ & $9.833 \mathrm{E}-11$ & $2.707 \mathrm{E}+03$ \\
\hline $1 s 4 p^{3} P_{2}^{o}$ & $1 s 6 s^{3} S_{1}$ & $9.3128 \mathrm{E}+00$ & $6.550 \mathrm{E}-08$ & $1.679 \mathrm{E}+06$ & $9.2750 \mathrm{E}+00$ & $6.491 \mathrm{E}-08$ & $1.663 \mathrm{E}+06$ \\
\hline $1 s 4 p{ }^{1} P_{1}^{o}$ & $1 s 6 s^{3} S_{1}$ & $9.3416 \mathrm{E}+00$ & $6.643 \mathrm{E}-08$ & $1.693 \mathrm{E}+06$ & $9.3040 \mathrm{E}+00$ & $6.422 \mathrm{E}-08$ & $1.635 \mathrm{E}+06$ \\
\hline $1 s 4 f^{3} F_{3}^{o}$ & $1 s 6 s^{3} S_{1}$ & $9.4480 \mathrm{E}+00$ & $3.729 E-15$ & $9.287 \mathrm{E}-02$ & & & \\
\hline $1 s 4 f^{3} F_{2}^{o}$ & $1 s 6 s^{3} S_{1}$ & $9.4503 \mathrm{E}+00$ & $1.759 \mathrm{E}-16$ & $4.380 \mathrm{E}-03$ & & & \\
\hline $1 s 4 f^{1} F_{3}^{o}$ & $1 s 6 s^{3} S_{1}$ & $9.5047 \mathrm{E}+00$ & $1.805 \mathrm{E}-14$ & $4.441 \mathrm{E}-01$ & & & \\
\hline $1 s 5 p^{3} P_{1}^{o}$ & $1 s 6 s^{3} s_{1}$ & $2.5714 \mathrm{E}+01$ & $4.946 \mathrm{E}-11$ & $1.663 \mathrm{E}+02$ & $2.5617 \mathrm{E}+01$ & $7.675 \mathrm{E}-11$ & $2.578 \mathrm{E}+02$ \\
\hline $1 s 5 p^{3} P_{2}^{o}$ & $1 s 6 s^{3} S_{1}$ & $2.7109 \mathrm{E}+01$ & $4.962 \mathrm{E}-08$ & $1.501 \mathrm{E}+05$ & $2.7006 \mathrm{E}+01$ & $4.914 \mathrm{E}-08$ & $1.485 \mathrm{E}+05$ \\
\hline
\end{tabular}


Table 6 (continued)

\begin{tabular}{|c|c|c|c|c|c|c|c|}
\hline \multirow[t]{2}{*}{ Lower } & \multirow[t]{2}{*}{ Upper } & \multicolumn{3}{|l|}{ GRASP2K } & \multicolumn{3}{|l|}{ FAC } \\
\hline & & $\lambda(\AA)$ & $g f$ & $A\left(\mathrm{~s}^{-1}\right)$ & $\overline{\lambda(\AA)}$ & $g f$ & $A\left(\mathrm{~s}^{-1}\right)$ \\
\hline $1 s 5 p^{1} P_{1}^{o}$ & $1 s 6 s^{3} S_{1}$ & $2.7232 \mathrm{E}+01$ & $5.040 \mathrm{E}-08$ & $1.511 \mathrm{E}+05$ & $2.7131 \mathrm{E}+01$ & $4.840 \mathrm{E}-08$ & $1.449 \mathrm{E}+05$ \\
\hline $1 s 5 f^{3} F_{3}^{o}$ & $1 s 6 s^{3} S_{1}$ & $2.7699 \mathrm{E}+01$ & $4.652 \mathrm{E}-15$ & $1.348 \mathrm{E}-02$ & & & \\
\hline $1 s 5 f^{3} F_{2}^{o}$ & $1 s 6 s^{3} S_{1}$ & $2.7709 \mathrm{E}+01$ & $7.330 \mathrm{E}-17$ & $2.123 \mathrm{E}-04$ & & & \\
\hline $1 s 5 f^{1} F_{3}^{o}$ & $1 s 6 s^{3} S_{1}$ & $2.7950 \mathrm{E}+01$ & $3.169 \mathrm{E}-14$ & $9.021 \mathrm{E}-02$ & & & \\
\hline $1 s 2 s^{3} S_{1}^{3}$ & $1 s 6 p^{3} P_{1}^{o}$ & $1.3692 \mathrm{E}+00$ & $1.564 \mathrm{E}-09$ & $1.855 \mathrm{E}+06$ & $1.3634 \mathrm{E}+00$ & $2.513 E-09$ & $2.980 \mathrm{E}+06$ \\
\hline $1 s 3 s^{3} S_{1}$ & $1 s 6 p^{3} P_{1}^{o}$ & $3.6909 \mathrm{E}+00$ & $5.170 \mathrm{E}-10$ & $8.438 E+04$ & $3.6756 \mathrm{E}+00$ & $7.777 \mathrm{E}-10$ & $1.269 \mathrm{E}+05$ \\
\hline $1 s 3 d^{3} D_{2}$ & $1 s 6 p^{3} P_{1}^{o}$ & $3.8574 \mathrm{E}+00$ & $9.776 \mathrm{E}-10$ & $1.461 \mathrm{E}+05$ & $3.8413 \mathrm{E}+00$ & $9.678 \mathrm{E}-10$ & $1.445 \mathrm{E}+05$ \\
\hline $1 s 3 d^{3} D_{1}$ & $1 s 6 p^{3} P_{1}^{o}$ & $3.8592 \mathrm{E}+00$ & $9.778 \mathrm{E}-10$ & $1.460 \mathrm{E}+05$ & $3.8431 \mathrm{E}+00$ & $9.506 \mathrm{E}-10$ & $1.419 \mathrm{E}+05$ \\
\hline $1 s 3 d^{3} D_{3}$ & $1 s 6 p^{3} P_{1}^{o}$ & $3.9015 \mathrm{E}+00$ & $1.634 \mathrm{E}-08$ & $2.387 \mathrm{E}+06$ & $3.8850 \mathrm{E}+00$ & $1.597 \mathrm{E}-08$ & $2.333 \mathrm{E}+06$ \\
\hline $1 s 3 d{ }^{1} D_{2}$ & $1 s 6 p^{3} P_{1}^{o}$ & $3.9032 \mathrm{E}+00$ & $6.202 \mathrm{E}-09$ & $9.052 \mathrm{E}+05$ & $3.8868 \mathrm{E}+00$ & $6.120 \mathrm{E}-09$ & $8.930 \mathrm{E}+05$ \\
\hline $1 s 4 s^{3} S_{1}$ & $1 s 6 p^{3} P_{1}^{o}$ & $8.9132 \mathrm{E}+00$ & $2.368 \mathrm{E}-10$ & $6.628 \mathrm{E}+03$ & $8.8765 E+00$ & $3.358 \mathrm{E}-10$ & $9.397 \mathrm{E}+03$ \\
\hline $1 s 4 d^{3} D_{2}$ & $1 s 6 p^{3} P_{1}^{o}$ & $9.3198 \mathrm{E}+00$ & $1.118 \mathrm{E}-09$ & $2.863 \mathrm{E}+04$ & $9.2819 \mathrm{E}+00$ & $1.114 \mathrm{E}-09$ & $2.851 \mathrm{E}+04$ \\
\hline $1 s 4 d^{3} D_{1}$ & $1 s 6 p^{3} P_{1}^{o}$ & $9.3241 \mathrm{E}+00$ & $1.101 \mathrm{E}-09$ & $2.815 \mathrm{E}+04$ & $9.2861 \mathrm{E}+00$ & $1.082 \mathrm{E}-09$ & $2.767 \mathrm{E}+04$ \\
\hline $1 s 4 d^{3} D_{3}$ & $1 s 6 p^{3} P_{1}^{o}$ & $9.4285 \mathrm{E}+00$ & $1.839 \mathrm{E}-08$ & $4.600 \mathrm{E}+05$ & $9.3898 \mathrm{E}+00$ & $1.808 \mathrm{E}-08$ & $4.521 \mathrm{E}+05$ \\
\hline $1 s 4 d^{1} D_{2}$ & $1 s 6 p^{3} P_{1}^{o}$ & $9.4329 \mathrm{E}+00$ & $6.934 \mathrm{E}-09$ & $1.733 \mathrm{E}+05$ & $9.3943 E+00$ & $6.934 \mathrm{E}-09$ & $1.732 \mathrm{E}+05$ \\
\hline $1 s 5 s^{3} S_{1}$ & $1 s 6 p^{3} P_{1}^{o}$ & $2.5356 \mathrm{E}+01$ & $1.264 \mathrm{E}-10$ & $4.372 \mathrm{E}+02$ & $2.5254 \mathrm{E}+01$ & $1.596 \mathrm{E}-10$ & $5.519 \mathrm{E}+02$ \\
\hline $1 s 5 d^{3} D_{2}$ & $1 s 6 p^{3} P_{1}^{o}$ & $2.7067 \mathrm{E}+01$ & $1.122 \mathrm{E}-09$ & $3.406 \mathrm{E}+03$ & $2.6964 \mathrm{E}+01$ & $1.119 \mathrm{E}-09$ & $3.395 E+03$ \\
\hline $1 s 5 d^{3} D_{1}$ & $1 s 6 p^{3} P_{1}^{o}$ & $2.7084 \mathrm{E}+01$ & $1.096 \mathrm{E}-09$ & $3.323 E+03$ & $2.6981 \mathrm{E}+01$ & $1.082 \mathrm{E}-09$ & $3.276 \mathrm{E}+03$ \\
\hline $1 s 5 d^{3} D_{3}$ & $1 s 6 p^{3} P_{1}^{o}$ & $2.7538 \mathrm{E}+01$ & $1.837 \mathrm{E}-08$ & $5.387 \mathrm{E}+04$ & $2.7432 \mathrm{E}+01$ & $1.814 \mathrm{E}-08$ & $5.314 \mathrm{E}+04$ \\
\hline $1 s 5 d^{1} D_{2}$ & $1 s 6 p^{3} P_{1}^{o}$ & $2.7558 \mathrm{E}+01$ & $6.906 \mathrm{E}-09$ & $2.022 \mathrm{E}+04$ & $2.7452 \mathrm{E}+01$ & $6.892 \mathrm{E}-09$ & $2.016 \mathrm{E}+04$ \\
\hline $1 s 5 g^{3} G_{3}$ & $1 s 6 p^{3} P_{1}^{o}$ & $2.7793 \mathrm{E}+01$ & $9.899 \mathrm{E}-18$ & $2.850 \mathrm{E}-05$ & & & \\
\hline $1 s 6 s^{3} S_{1}$ & $1 s 6 p^{3} P_{1}^{o}$ & $4.9854 \mathrm{E}+03$ & $3.274 \mathrm{E}-16$ & $2.929 \mathrm{E}-08$ & $4.6146 \mathrm{E}+03$ & $7.235 \mathrm{E}-16$ & $7.490 \mathrm{E}-08$ \\
\hline $1 s 2 p^{3} P_{2}^{o}$ & $1 s 6 s^{1} s_{0}$ & $1.4434 \mathrm{E}+00$ & $9.229 \mathrm{E}-08$ & $2.955 \mathrm{E}+08$ & $1.4374 \mathrm{E}+00$ & $9.373 \mathrm{E}-08$ & $3.000 \mathrm{E}+08$ \\
\hline $1 s 3 p^{3} P_{2}^{o}$ & $1 s 6 s^{1} S_{0}$ & $3.8473 \mathrm{E}+00$ & $6.108 \mathrm{E}-08$ & $2.753 \mathrm{E}+07$ & $3.8320 \mathrm{E}+00$ & $6.246 \mathrm{E}-08$ & $2.813 \mathrm{E}+07$ \\
\hline $1 s 4 p^{3} P_{2}^{o}$ & $1 s 6 s^{1} S_{0}$ & $9.2946 \mathrm{E}+00$ & $4.218 \mathrm{E}-08$ & $3.257 \mathrm{E}+06$ & $9.2596 \mathrm{E}+00$ & $4.349 \mathrm{E}-08$ & $3.354 \mathrm{E}+06$ \\
\hline $1 s 4 f^{3} F_{2}^{o}$ & $1 s 6 s^{1} s_{0}$ & $9.4316 \mathrm{E}+00$ & $4.585 \mathrm{E}-15$ & $3.438 \mathrm{E}-01$ & & & \\
\hline $1 s 5 p^{3} P_{2}^{o}$ & $1 s 6 s^{1} s_{0}$ & $2.6955 \mathrm{E}+01$ & $3.177 \mathrm{E}-08$ & $2.917 \mathrm{E}+05$ & $2.6876 \mathrm{E}+01$ & $3.323 E-08$ & $3.043 E+05$ \\
\hline $1 s 5 f^{3} F_{2}^{o}$ & $1 s 6 s{ }^{1} S_{0}$ & $2.7549 \mathrm{E}+01$ & $6.717 \mathrm{E}-15$ & $5.903 \mathrm{E}-02$ & & & \\
\hline $1 s 3 d^{3} D_{2}$ & $1 s 6 p^{3} P_{0}^{o}$ & $3.8572 \mathrm{E}+00$ & $6.303 E-10$ & $2.826 \mathrm{E}+05$ & $3.8418 \mathrm{E}+00$ & $6.184 \mathrm{E}-10$ & $2.771 \mathrm{E}+05$ \\
\hline $1 s 3 d^{1} D_{2}$ & $1 s 6 p^{3} P_{0}^{o}$ & $3.9029 \mathrm{E}+00$ & $7.563 \mathrm{E}-09$ & $3.312 \mathrm{E}+06$ & $3.8873 \mathrm{E}+00$ & $7.362 \mathrm{E}-09$ & $3.222 \mathrm{E}+06$ \\
\hline $1 s 4 d^{3} D_{2}$ & $1 s 6 p^{3} P_{0}^{o}$ & $9.3186 \mathrm{E}+00$ & $7.033 \mathrm{E}-10$ & $5.403 E+04$ & $9.2852 \mathrm{E}+00$ & $6.944 \mathrm{E}-10$ & $5.327 \mathrm{E}+04$ \\
\hline $1 s 4 d^{1} D_{2}$ & $1 s 6 p^{3} P_{0}^{o}$ & $9.4317 \mathrm{E}+00$ & $8.476 \mathrm{E}-09$ & $6.355 \mathrm{E}+05$ & $9.3976 \mathrm{E}+00$ & $8.342 \mathrm{E}-09$ & $6.247 \mathrm{E}+05$ \\
\hline $1 s 5 d^{3} D_{2}$ & $1 s 6 p^{3} P_{0}^{o}$ & $2.7056 \mathrm{E}+01$ & $6.977 \mathrm{E}-10$ & $6.357 \mathrm{E}+03$ & $2.6991 \mathrm{E}+01$ & $6.888 \mathrm{E}-10$ & $6.253 \mathrm{E}+03$ \\
\hline $1 s 5 d^{1} D_{2}$ & $1 s 6 p^{3} P_{0}^{o}$ & $2.7547 \mathrm{E}+01$ & $8.449 \mathrm{E}-09$ & $7.426 \mathrm{E}+04$ & $2.7481 \mathrm{E}+01$ & $8.332 \mathrm{E}-09$ & $7.297 \mathrm{E}+04$ \\
\hline $1 s^{2}{ }^{1} S_{0}$ & $1 s 6 p^{3} P_{2}^{o}$ & $3.1630 \mathrm{E}-01$ & $9.827 \mathrm{E}-06$ & $1.310 \mathrm{E}+11$ & $3.1498 \mathrm{E}-01$ & $1.106 \mathrm{E}-05$ & $1.475 \mathrm{E}+11$ \\
\hline $1 s 2 s^{3} S_{1}$ & $1 s 6 p^{3} P_{2}^{o}$ & $1.3670 \mathrm{E}+00$ & $1.115 \mathrm{E}-06$ & $7.956 \mathrm{E}+08$ & $1.3612 \mathrm{E}+00$ & $1.152 \mathrm{E}-06$ & $8.224 \mathrm{E}+08$ \\
\hline $1 s 2 s{ }^{1} S_{0}$ & $1 s 6 p^{3} P_{2}^{o}$ & $1.3800 \mathrm{E}+00$ & $7.427 \mathrm{E}-07$ & $5.203 \mathrm{E}+08$ & $1.3743 \mathrm{E}+00$ & $7.463 \mathrm{E}-07$ & $5.226 \mathrm{E}+08$ \\
\hline $1 s 3 s^{3} S_{1}$ & $1 s 6 p^{3} P_{2}^{o}$ & $3.6753 \mathrm{E}+00$ & $3.545 \mathrm{E}-07$ & $3.501 \mathrm{E}+07$ & $3.6601 \mathrm{E}+00$ & $3.604 \mathrm{E}-07$ & $3.559 \mathrm{E}+07$ \\
\hline $1 s 3 s{ }^{1} S_{0}$ & $1 s 6 p^{3} P_{2}^{o}$ & $3.6999 \mathrm{E}+00$ & $2.408 \mathrm{E}-07$ & $2.347 \mathrm{E}+07$ & $3.6849 \mathrm{E}+00$ & $2.354 \mathrm{E}-07$ & $2.293 \mathrm{E}+07$ \\
\hline $1 s 3 d^{3} D_{2}$ & $1 s 6 p^{3} P_{2}^{o}$ & $3.8403 \mathrm{E}+00$ & $1.356 \mathrm{E}-14$ & $1.227 \mathrm{E}+00$ & & & \\
\hline $1 s 3 d^{3} D_{1}$ & $1 s 6 p^{3} P_{2}^{o}$ & $3.8421 \mathrm{E}+00$ & $3.557 \mathrm{E}-13$ & $3.214 \mathrm{E}+01$ & & & \\
\hline $1 s 3 d^{3} D_{3}$ & $1 s 6 p^{3} P_{2}^{o}$ & $3.8840 \mathrm{E}+00$ & $5.812 \mathrm{E}-08$ & $5.139 \mathrm{E}+06$ & $3.8677 \mathrm{E}+00$ & $5.623 \mathrm{E}-08$ & $4.972 \mathrm{E}+06$ \\
\hline $1 s 3 d d^{1} D_{2}$ & $1 s 6 p^{3} P_{2}^{0}$ & $3.8856 \mathrm{E}+00$ & $1.476 \mathrm{E}-08$ & $1.304 \mathrm{E}+06$ & $3.8694 \mathrm{E}+00$ & $1.403 \mathrm{E}-08$ & $1.240 \mathrm{E}+06$ \\
\hline $1 s 4 s^{3} S_{1}$ & $1 s 6 p^{3} P_{2}^{o}$ & $8.8224 \mathrm{E}+00$ & $1.571 \mathrm{E}-07$ & $2.692 \mathrm{E}+06$ & $8.7863 E+00$ & $1.589 \mathrm{E}-07$ & $2.723 \mathrm{E}+06$ \\
\hline $1 s 4 s^{1} S_{0}$ & $1 s 6 p^{3} P_{2}^{o}$ & $8.8798 \mathrm{E}+00$ & $1.078 \mathrm{E}-07$ & $1.824 \mathrm{E}+06$ & $8.8440 \mathrm{E}+00$ & $1.038 \mathrm{E}-07$ & $1.756 \mathrm{E}+06$ \\
\hline $1 s 4 d^{3} D_{2}$ & $1 s 6 p^{3} P_{2}^{o}$ & $9.2206 \mathrm{E}+00$ & $2.240 \mathrm{E}-13$ & $3.514 \mathrm{E}+00$ & & & \\
\hline $1 s 4 d^{3} D_{1}$ & $1 s 6 p^{3} P_{2}^{o}$ & $9.2248 \mathrm{E}+00$ & $2.274 \mathrm{E}-13$ & $3.564 \mathrm{E}+00$ & & & \\
\hline $1 s 4 d^{3} D_{3}$ & $1 s 6 p^{3} P_{2}^{o}$ & $9.3270 \mathrm{E}+00$ & $6.780 \mathrm{E}-08$ & $1.040 \mathrm{E}+06$ & $9.2888 \mathrm{E}+00$ & $6.619 E-08$ & $1.014 \mathrm{E}+06$ \\
\hline $1 s 4 d{ }^{1} D_{2}$ & $1 s 6 p^{3} P_{2}^{o}$ & $9.3314 \mathrm{E}+00$ & $1.709 E-08$ & $2.619 \mathrm{E}+05$ & $9.2933 \mathrm{E}+00$ & $1.652 \mathrm{E}-08$ & $2.530 \mathrm{E}+05$ \\
\hline $1 s 5 s^{3} S_{1}$ & $1 s 6 p^{3} P_{2}^{o}$ & $2.4635 \mathrm{E}+01$ & $7.930 \mathrm{E}-08$ & $1.743 \mathrm{E}+05$ & $2.4537 \mathrm{E}+01$ & $8.016 E-08$ & $1.761 \mathrm{E}+05$ \\
\hline $1 s 5 s^{1} S_{0}$ & $1 s 6 p^{3} P_{2}^{o}$ & $2.4860 \mathrm{E}+01$ & $5.498 \mathrm{E}-08$ & $1.187 \mathrm{E}+05$ & $2.4762 \mathrm{E}+01$ & $5.199 \mathrm{E}-08$ & $1.121 \mathrm{E}+05$ \\
\hline $1 s 5 d^{3} D_{2}$ & $1 s 6 p^{3} P_{2}^{o}$ & $2.6247 \mathrm{E}+01$ & $4.110 \mathrm{E}-13$ & $7.959 \mathrm{E}-01$ & & & \\
\hline $1 s 5 d^{3} D_{1}$ & $1 s 6 p^{3} P_{2}^{o}$ & $2.6264 \mathrm{E}+01$ & $1.397 \mathrm{E}-13$ & $2.703 \mathrm{E}-01$ & & & \\
\hline $1 s 5 d^{3} D_{3}$ & $1 s 6 p^{3} P_{2}^{o}$ & $2.6690 \mathrm{E}+01$ & $6.829 \mathrm{E}-08$ & $1.279 \mathrm{E}+05$ & $2.6588 \mathrm{E}+01$ & $6.672 \mathrm{E}-08$ & $1.248 \mathrm{E}+05$ \\
\hline $1 s 5 d{ }^{1} D_{2}$ & $1 s 6 p^{3} P_{2}^{o}$ & $2.6709 \mathrm{E}+01$ & $1.716 \mathrm{E}-08$ & $3.210 \mathrm{E}+04$ & $2.6607 \mathrm{E}+01$ & $1.664 \mathrm{E}-08$ & $3.110 \mathrm{E}+04$ \\
\hline $1 s 5 g^{3} G_{4}$ & $1 s 6 p^{3} P_{2}^{o}$ & $2.6923 \mathrm{E}+01$ & $3.604 \mathrm{E}-16$ & $6.634 \mathrm{E}-04$ & & & \\
\hline $1 s 5 g^{3} G_{3}$ & $1 s 6 p^{3} P_{2}^{o}$ & $2.6929 \mathrm{E}+01$ & $8.766 \mathrm{E}-16$ & $1.613 \mathrm{E}-03$ & & & \\
\hline $1 s 5 g{ }^{1} G_{4}$ & $1 s 6 p^{3} P_{2}^{o}$ & $2.7064 \mathrm{E}+01$ & $1.057 \mathrm{E}-15$ & $1.924 \mathrm{E}-03$ & & & \\
\hline $1 s 6 s^{3} S_{1}$ & $1 s 6 p^{3} P_{2}^{o}$ & $7.3822 \mathrm{E}+02$ & $8.147 \mathrm{E}-11$ & $1.994 \mathrm{E}-01$ & $7.2782 \mathrm{E}+02$ & $8.413 E-11$ & $2.100 \mathrm{E}-01$ \\
\hline $1 s 6 s^{1} S_{0}$ & $1 s 6 p^{3} P_{2}^{o}$ & $8.7342 \mathrm{E}+02$ & $3.282 \mathrm{E}-11$ & $5.739 \mathrm{E}-02$ & $8.3711 \mathrm{E}+02$ & $3.686 \mathrm{E}-11$ & $6.958 \mathrm{E}-02$ \\
\hline $1 s 2 p^{3} P_{1}^{o}$ & $1 s 6 d^{3} D_{2}$ & $1.3786 \mathrm{E}+00$ & $7.357 \mathrm{E}-08$ & $5.164 \mathrm{E}+07$ & $1.3729 \mathrm{E}+00$ & $8.090 \mathrm{E}-08$ & $5.677 \mathrm{E}+07$ \\
\hline $1 s 2 p^{3} P_{0}^{0}$ & $1 s 6 d^{3} D_{2}$ & $1.3795 \mathrm{E}+00$ & $4.617 \mathrm{E}-08$ & $3.237 \mathrm{E}+07$ & $1.3737 \mathrm{E}+00$ & $4.698 \mathrm{E}-08$ & $3.293 \mathrm{E}+07$ \\
\hline $1 s 2 p^{3} P_{2}^{o}$ & $1 s 6 d^{3} D_{2}$ & $1.4409 \mathrm{E}+00$ & $4.355 \mathrm{E}-11$ & $2.798 \mathrm{E}+04$ & $1.4348 \mathrm{E}+00$ & $1.874 \mathrm{E}-10$ & $1.204 \mathrm{E}+05$ \\
\hline $1 s 2 p^{1} P_{1}^{o}$ & $1 s 6 d^{3} D_{2}$ & $1.4467 \mathrm{E}+00$ & $3.892 \mathrm{E}-13$ & $2.481 \mathrm{E}+02$ & $1.4407 \mathrm{E}+00$ & $1.020 \mathrm{E}-10$ & $6.504 \mathrm{E}+04$ \\
\hline $1 s 3 p^{3} P_{1}^{o}$ & $1 s 6 d^{3} D_{2}$ & $3.6972 \mathrm{E}+00$ & $2.620 \mathrm{E}-08$ & $2.557 \mathrm{E}+06$ & $3.6817 \mathrm{E}+00$ & $2.754 \mathrm{E}-08$ & $2.688 \mathrm{E}+06$ \\
\hline $1 s 3 p^{3} P_{0}^{0}$ & $1 s 6 d^{3} D_{2}$ & $3.6989 \mathrm{E}+00$ & $1.627 \mathrm{E}-08$ & $1.586 \mathrm{E}+06$ & $3.6834 \mathrm{E}+00$ & $1.603 \mathrm{E}-08$ & $1.563 \mathrm{E}+06$ \\
\hline $1 s 3 p^{3} P_{2}^{o}$ & $1 s 6 d^{3} D_{2}$ & $3.8292 \mathrm{E}+00$ & $1.916 \mathrm{E}-11$ & $1.744 \mathrm{E}+03$ & $3.8130 \mathrm{E}+00$ & $6.803 \mathrm{E}-11$ & $6.189 \mathrm{E}+03$ \\
\hline $1 s 3 p{ }^{1} P_{1}^{o}$ & $1 s 6 d^{3} D_{2}$ & $3.8409 \mathrm{E}+00$ & $6.830 \mathrm{E}-13$ & $6.176 \mathrm{E}+01$ & $3.8249 \mathrm{E}+00$ & $4.396 \mathrm{E}-11$ & $3.974 \mathrm{E}+03$ \\
\hline $1 s 4 p^{3} P_{1}^{o}$ & $1 s 6 d^{3} D_{2}$ & $8.8702 \mathrm{E}+00$ & $1.140 \mathrm{E}-08$ & $1.933 \mathrm{E}+05$ & $8.8316 \mathrm{E}+00$ & $1.183 \mathrm{E}-08$ & $2.006 \mathrm{E}+05$ \\
\hline $1 s 4 p^{3} P_{0}^{o}$ & $1 s 6 d^{3} D_{2}$ & $8.8742 \mathrm{E}+00$ & $7.076 \mathrm{E}-09$ & $1.199 \mathrm{E}+05$ & $8.8354 \mathrm{E}+00$ & $6.901 \mathrm{E}-09$ & $1.169 \mathrm{E}+05$ \\
\hline $1 s 4 p^{3} P_{2}^{o}$ & $1 s 6 d^{3} D_{2}$ & $9.1898 \mathrm{E}+00$ & $9.056 \mathrm{E}-12$ & $1.431 \mathrm{E}+02$ & $9.1496 \mathrm{E}+00$ & $3.048 \mathrm{E}-11$ & $4.816 \mathrm{E}+02$ \\
\hline
\end{tabular}


Table 6 (continued)

\begin{tabular}{|c|c|c|c|c|c|c|c|}
\hline \multirow[t]{2}{*}{ Lower } & \multirow[t]{2}{*}{ Upper } & \multicolumn{3}{|l|}{ GRASP2K } & \multicolumn{3}{|l|}{ FAC } \\
\hline & & $\overline{\lambda(\AA)}$ & $g f$ & $A\left(\mathrm{~s}^{-1}\right)$ & $\overline{\lambda(\AA)}$ & $g f$ & $A\left(\mathrm{~s}^{-1}\right)$ \\
\hline $1 s 4 p^{1} P_{1}^{o}$ & $1 s 6 d^{3} D_{2}$ & $9.2178 \mathrm{E}+00$ & $4.459 \mathrm{E}-13$ & $7.001 \mathrm{E}+00$ & $9.1778 \mathrm{E}+00$ & $2.140 \mathrm{E}-11$ & $3.361 \mathrm{E}+02$ \\
\hline $1 s 4 f^{3} F_{3}^{o}$ & $1 s 6 d^{3} D_{2}$ & $9.3215 \mathrm{E}+00$ & $3.189 \mathrm{E}-09$ & $4.896 \mathrm{E}+04$ & $9.2809 \mathrm{E}+00$ & $3.158 \mathrm{E}-09$ & $4.849 \mathrm{E}+04$ \\
\hline $1 s 4 f^{3} F_{2}^{o}$ & $1 s 6 d^{3} D_{2}$ & $9.3237 \mathrm{E}+00$ & $8.011 \mathrm{E}-10$ & $1.229 \mathrm{E}+04$ & $9.2831 \mathrm{E}+00$ & $7.907 \mathrm{E}-10$ & $1.213 \mathrm{E}+04$ \\
\hline $1 s 4 f^{3} F_{4}^{o}$ & $1 s 6 d^{3} D_{2}$ & $9.3749 \mathrm{E}+00$ & $6.477 \mathrm{E}-09$ & $9.832 \mathrm{E}+04$ & $9.3339 \mathrm{E}+00$ & $6.370 \mathrm{E}-09$ & $9.671 \mathrm{E}+04$ \\
\hline $1 s 4 f^{1} F_{3}^{o}$ & $1 s 6 d^{3} D_{2}$ & $9.3766 \mathrm{E}+00$ & $7.961 \mathrm{E}-10$ & $1.208 \mathrm{E}+04$ & $9.3356 \mathrm{E}+00$ & $8.302 \mathrm{E}-10$ & $1.260 \mathrm{E}+04$ \\
\hline $1 s 5 p^{3} P_{1}^{o}$ & $1 s 6 d^{3} D_{2}$ & $2.4798 \mathrm{E}+01$ & $5.473 \mathrm{E}-09$ & $1.187 \mathrm{E}+04$ & $2.4683 \mathrm{E}+01$ & $5.652 \mathrm{E}-09$ & $1.227 \mathrm{E}+04$ \\
\hline $1 s 5 p^{3} P_{0}^{o}$ & $1 s 6 d^{3} D_{2}$ & $2.4813 \mathrm{E}+01$ & $3.400 \mathrm{E}-09$ & $7.368 \mathrm{E}+03$ & $2.4698 \mathrm{E}+01$ & $3.305 \mathrm{E}-09$ & $7.167 \mathrm{E}+03$ \\
\hline $1 s 5 p^{3} P_{2}^{o}$ & $1 s 6 d^{3} D_{2}$ & $2.6092 \mathrm{E}+01$ & $4.739 \mathrm{E}-12$ & $9.286 \mathrm{E}+00$ & $2.5970 \mathrm{E}+01$ & $1.516 \mathrm{E}-11$ & $2.974 \mathrm{E}+01$ \\
\hline $1 s 5 p^{1} P_{1}^{o}$ & $1 s 6 d^{3} D_{2}$ & $2.6207 \mathrm{E}+01$ & $2.750 \mathrm{E}-13$ & $5.341 \mathrm{E}-01$ & $2.6085 \mathrm{E}+01$ & $1.163 \mathrm{E}-11$ & $2.262 \mathrm{E}+01$ \\
\hline $1 s 5 f^{3} F_{3}^{o}$ & $1 s 6 d^{3} D_{2}$ & $2.6639 \mathrm{E}+01$ & $5.658 \mathrm{E}-09$ & $1.064 \mathrm{E}+04$ & $2.6517 \mathrm{E}+01$ & $5.635 \mathrm{E}-09$ & $1.060 \mathrm{E}+04$ \\
\hline $1 s 5 f^{3} F_{2}^{o}$ & $1 s 6 d^{3} D_{2}$ & $2.6648 \mathrm{E}+01$ & $1.421 \mathrm{E}-09$ & $2.669 \mathrm{E}+03$ & $2.6526 \mathrm{E}+01$ & $1.410 \mathrm{E}-09$ & $2.651 \mathrm{E}+03$ \\
\hline 1s $5 f^{3} F_{4}^{o}$ & $1 s 6 d^{3} D_{2}$ & $2.6863 \mathrm{E}+01$ & $1.142 \mathrm{E}-08$ & $2.112 \mathrm{E}+04$ & $2.6740 \mathrm{E}+01$ & $1.129 \mathrm{E}-08$ & $2.089 \mathrm{E}+04$ \\
\hline $1 s 5 f^{1} F_{3}^{o}$ & $1 s 6 d^{3} D_{2}$ & $2.6870 \mathrm{E}+01$ & $1.403 \mathrm{E}-09$ & $2.593 \mathrm{E}+03$ & $2.6747 \mathrm{E}+01$ & $1.470 \mathrm{E}-09$ & $2.718 \mathrm{E}+03$ \\
\hline $1 s 6 p^{3} P_{1}^{o}$ & $1 s 6 d^{3} D_{2}$ & $8.0869 \mathrm{E}+02$ & $2.361 \mathrm{E}-12$ & $4.815 \mathrm{E}-03$ & $7.9302 \mathrm{E}+02$ & $2.548 \mathrm{E}-12$ & $5.360 \mathrm{E}-03$ \\
\hline $1 s 6 p^{3} P_{0}^{o}$ & $1 s 6 d^{3} D_{2}$ & $8.1823 \mathrm{E}+02$ & $1.413 \mathrm{E}-12$ & $2.816 \mathrm{E}-03$ & $7.6994 \mathrm{E}+02$ & $1.621 \mathrm{E}-12$ & $3.618 \mathrm{E}-03$ \\
\hline $1 s 6 p^{3} P_{2}^{o}$ & $1 s 6 d^{3} D_{2}$ & $1.2115 \mathrm{E}+04$ & $5.193 \mathrm{E}-19$ & $4.720 \mathrm{E}-12$ & & & \\
\hline $1 s 2 s^{3} S_{1}$ & $1 s 6 p^{1} P_{1}^{o}$ & $1.3668 \mathrm{E}+00$ & $1.105 \mathrm{E}-06$ & $1.315 \mathrm{E}+09$ & $1.3611 \mathrm{E}+00$ & $1.149 \mathrm{E}-06$ & $1.368 \mathrm{E}+09$ \\
\hline $1 s 3 s^{3} S_{1}$ & $1 s 6 p^{1} P_{1}^{o}$ & $3.6740 \mathrm{E}+00$ & $3.498 \mathrm{E}-07$ & $5.762 \mathrm{E}+07$ & $3.6589 \mathrm{E}+00$ & $3.600 \mathrm{E}-07$ & $5.928 \mathrm{E}+07$ \\
\hline $1 s 3 d^{3} D_{2}$ & $1 s 6 p^{1} P_{1}^{o}$ & $3.8389 \mathrm{E}+00$ & $2.598 \mathrm{E}-13$ & $3.919 \mathrm{E}+01$ & & & \\
\hline $1 s 3 d^{3} D_{1}$ & $1 s 6 p^{1} P_{1}^{o}$ & $3.8407 \mathrm{E}+00$ & $1.055 \mathrm{E}-14$ & $1.590 \mathrm{E}+00$ & & & \\
\hline $1 s 3 d^{3} D_{3}$ & $1 s 6 p^{1} P_{1}^{o}$ & $3.8825 \mathrm{E}+00$ & $1.013 \mathrm{E}-08$ & $1.494 \mathrm{E}+06$ & $3.8664 \mathrm{E}+00$ & $1.059 \mathrm{E}-08$ & $1.562 \mathrm{E}+06$ \\
\hline $1 s 3 d^{1} D_{2}$ & $1 s 6 p^{1} P_{1}^{o}$ & $3.8842 \mathrm{E}+00$ & $3.253 \mathrm{E}-08$ & $4.794 \mathrm{E}+06$ & $3.8681 \mathrm{E}+00$ & $3.153 \mathrm{E}-08$ & $4.646 \mathrm{E}+06$ \\
\hline $1 s 4 s^{3} S_{1}$ & $1 s 6 p^{1} P_{1}^{o}$ & $8.8149 \mathrm{E}+00$ & $1.545 \mathrm{E}-07$ & $4.420 \mathrm{E}+06$ & $8.7796 \mathrm{E}+00$ & $1.589 \mathrm{E}-07$ & $4.545 \mathrm{E}+06$ \\
\hline $1 s 4 d^{3} D_{2}$ & $1 s 6 p^{1} P_{1}^{o}$ & $9.2125 \mathrm{E}+00$ & $1.128 \mathrm{E}-14$ & $2.955 \mathrm{E}-01$ & & & \\
\hline $1 s 4 d^{3} D_{1}$ & $1 s 6 p^{1} P_{1}^{o}$ & $9.2166 \mathrm{E}+00$ & $1.136 \mathrm{E}-13$ & $2.974 \mathrm{E}+00$ & & & \\
\hline $1 s 4 d^{3} D_{3}$ & $1 s 6 p^{1} P_{1}^{o}$ & $9.3186 \mathrm{E}+00$ & $1.187 \mathrm{E}-08$ & $3.038 \mathrm{E}+05$ & $9.2814 \mathrm{E}+00$ & $1.247 \mathrm{E}-08$ & $3.192 \mathrm{E}+05$ \\
\hline $1 s 4 d{ }^{1} D_{2}$ & $1 s 6 p^{1} P_{1}^{o}$ & $9.3230 \mathrm{E}+00$ & $3.780 \mathrm{E}-08$ & $9.670 \mathrm{E}+05$ & $9.2858 \mathrm{E}+00$ & $3.718 \mathrm{E}-08$ & $9.507 \mathrm{E}+05$ \\
\hline $1 s 5 s^{3} S_{1}$ & $1 s 6 p^{1} P_{1}^{o}$ & $2.4577 \mathrm{E}+01$ & $7.756 \mathrm{E}-08$ & $2.855 \mathrm{E}+05$ & $2.4485 \mathrm{E}+01$ & $8.050 \mathrm{E}-08$ & $2.960 \mathrm{E}+05$ \\
\hline $1 s 5 d^{3} D_{2}$ & $1 s 6 p^{1} P_{1}^{o}$ & $2.6180 \mathrm{E}+01$ & $9.174 \mathrm{E}-15$ & $2.976 \mathrm{E}-02$ & & & \\
\hline $1 s 5 d^{3} D_{1}$ & $1 s 6 p^{1} P_{1}^{o}$ & $2.6197 \mathrm{E}+01$ & $2.364 \mathrm{E}-13$ & $7.658 \mathrm{E}-01$ & & & \\
\hline $1 s 5 d^{3} D_{3}$ & $1 s 6 p^{1} P_{1}^{o}$ & $2.6621 \mathrm{E}+01$ & $1.196 \mathrm{E}-08$ & $3.753 \mathrm{E}+04$ & $2.6527 \mathrm{E}+01$ & $1.267 \mathrm{E}-08$ & $3.970 \mathrm{E}+04$ \\
\hline $1 s 5 d^{1} D_{2}$ & $1 s 6 p^{1} P_{1}^{o}$ & $2.6640 \mathrm{E}+01$ & $3.799 \mathrm{E}-08$ & $1.190 \mathrm{E}+05$ & $2.6546 \mathrm{E}+01$ & $3.758 \mathrm{E}-08$ & $1.175 \mathrm{E}+05$ \\
\hline $1 s 5 g{ }^{3} G_{3}$ & $1 s 6 p^{1} P_{1}^{o}$ & $2.6859 \mathrm{E}+01$ & $8.269 \mathrm{E}-18$ & $2.548 \mathrm{E}-05$ & & & \\
\hline $1 s 6 s^{3} S_{1}$ & $1 s 6 p^{1} P_{1}^{o}$ & $6.8918 \mathrm{E}+02$ & $1.001 \mathrm{E}-10$ & $4.688 \mathrm{E}-01$ & $6.8482 \mathrm{E}+02$ & $1.007 \mathrm{E}-10$ & $4.737 \mathrm{E}-01$ \\
\hline $1 s 6 d^{3} D_{2}$ & $1 s 6 p^{1} P_{1}^{o}$ & $7.2196 \mathrm{E}+04$ & $1.674 \mathrm{E}-22$ & $7.143 \mathrm{E}-17$ & & & \\
\hline $1 s 2 p^{3} P_{1}^{o}$ & $1 s 6 d^{3} D_{1}$ & $1.3786 \mathrm{E}+00$ & $7.220 \mathrm{E}-08$ & $8.447 \mathrm{E}+07$ & $1.3729 \mathrm{E}+00$ & $7.636 \mathrm{E}-08$ & $8.932 \mathrm{E}+07$ \\
\hline $1 s 2 p^{3} P_{2}^{o}$ & $1 s 6 d^{3} D_{1}$ & $1.4408 \mathrm{E}+00$ & $1.425 \mathrm{E}-13$ & $1.527 \mathrm{E}+02$ & & & \\
\hline $1 s 2 p^{1} P_{1}^{o}$ & $1 s 6 d^{3} D_{1}$ & $1.4467 \mathrm{E}+00$ & $8.401 \mathrm{E}-11$ & $8.924 \mathrm{E}+04$ & $1.4407 \mathrm{E}+00$ & $1.079 \mathrm{E}-10$ & $1.146 \mathrm{E}+05$ \\
\hline $1 s 3 p^{3} P_{1}^{o}$ & $1 s 6 d^{3} D_{1}$ & $3.6970 \mathrm{E}+00$ & $2.546 \mathrm{E}-08$ & $4.142 \mathrm{E}+06$ & $3.6817 \mathrm{E}+00$ & $2.602 \mathrm{E}-08$ & $4.232 \mathrm{E}+06$ \\
\hline $1 s 3 p^{3} P_{2}^{o}$ & $1 s 6 d^{3} D_{1}$ & $3.8290 \mathrm{E}+00$ & $1.910 \mathrm{E}-13$ & $2.896 \mathrm{E}+01$ & & & \\
\hline $1 s 3 p^{1} P_{1}^{o}$ & $1 s 6 d^{3} D_{1}$ & $3.8407 \mathrm{E}+00$ & $2.859 \mathrm{E}-11$ & $4.309 \mathrm{E}+03$ & $3.8249 \mathrm{E}+00$ & $3.310 \mathrm{E}-11$ & $4.989 \mathrm{E}+03$ \\
\hline $1 s 4 p^{3} P_{1}^{o}$ & $1 s 6 d^{3} D_{1}$ & $8.8691 \mathrm{E}+00$ & $1.105 \mathrm{E}-08$ & $3.125 \mathrm{E}+05$ & $8.8318 \mathrm{E}+00$ & $1.118 \mathrm{E}-08$ & $3.161 \mathrm{E}+05$ \\
\hline $1 s 4 p^{3} P_{2}^{0}$ & $1 s 6 d^{3} D_{1}$ & $9.1886 \mathrm{E}+00$ & $1.273 \mathrm{E}-13$ & $3.352 \mathrm{E}+00$ & & & \\
\hline $1 s 4 p^{1} P_{1}^{o}$ & $1 s 6 d^{3} D_{1}$ & $9.2166 \mathrm{E}+00$ & $1.238 \mathrm{E}-11$ & $3.240 \mathrm{E}+02$ & $9.1781 \mathrm{E}+00$ & $1.357 \mathrm{E}-11$ & $3.553 \mathrm{E}+02$ \\
\hline $1 s 4 f^{3} F_{3}^{o}$ & $1 s 6 d^{3} D_{1}$ & $9.3202 \mathrm{E}+00$ & $5.366 \mathrm{E}-10$ & $1.374 \mathrm{E}+04$ & $9.2811 \mathrm{E}+00$ & $5.341 \mathrm{E}-10$ & $1.367 \mathrm{E}+04$ \\
\hline $1 s 4 f^{3} F_{2}^{o}$ & $1 s 6 d^{3} D_{1}$ & $9.3225 \mathrm{E}+00$ & $1.860 \mathrm{E}-09$ & $4.759 \mathrm{E}+04$ & $9.2834 \mathrm{E}+00$ & $1.845 \mathrm{E}-09$ & $4.719 \mathrm{E}+04$ \\
\hline $1 s 4 f^{1} F_{3}^{o}$ & $1 s 6 d^{3} D_{1}$ & $9.3753 \mathrm{E}+00$ & $4.340 \mathrm{E}-09$ & $1.098 \mathrm{E}+05$ & $9.3359 \mathrm{E}+00$ & $4.305 \mathrm{E}-09$ & $1.088 \mathrm{E}+05$ \\
\hline $1 s 5 p^{3} P_{1}^{o}$ & $1 s 6 d^{3} D_{1}$ & $2.4789 \mathrm{E}+01$ & $5.299 \mathrm{E}-09$ & $1.917 \mathrm{E}+04$ & $2.4685 \mathrm{E}+01$ & $5.349 \mathrm{E}-09$ & $1.935 \mathrm{E}+04$ \\
\hline $1 s 5 p^{3} P_{2}^{o}$ & $1 s 6 d^{3} D_{1}$ & $2.6083 \mathrm{E}+01$ & $8.543 \mathrm{E}-14$ & $2.792 \mathrm{E}-01$ & & & \\
\hline $1 s 5 p^{1} P_{1}^{o}$ & $1 s 6 d^{3} D_{1}$ & $2.6197 \mathrm{E}+01$ & $6.135 \mathrm{E}-12$ & $1.988 \mathrm{E}+01$ & $2.6087 \mathrm{E}+01$ & $6.049 \mathrm{E}-12$ & $1.959 \mathrm{E}+01$ \\
\hline $1 s 5 f^{3} F_{3}^{o}$ & $1 s 6 d^{3} D_{1}$ & $2.6629 \mathrm{E}+01$ & $9.512 \mathrm{E}-10$ & $2.983 E+03$ & $2.6519 \mathrm{E}+01$ & $9.521 \mathrm{E}-10$ & $2.984 \mathrm{E}+03$ \\
\hline $1 s 5 f^{3} F_{2}^{o}$ & $1 s 6 d^{3} D_{1}$ & $2.6638 \mathrm{E}+01$ & $3.300 \mathrm{E}-09$ & $1.034 \mathrm{E}+04$ & $2.6529 \mathrm{E}+01$ & $3.290 \mathrm{E}-09$ & $1.030 \mathrm{E}+04$ \\
\hline $1 s 5 f^{1} F_{3}^{o}$ & $1 s 6 d^{3} D_{1}$ & $2.6860 \mathrm{E}+01$ & $7.654 \mathrm{E}-09$ & $2.359 \mathrm{E}+04$ & $2.6749 \mathrm{E}+01$ & $7.630 \mathrm{E}-09$ & $2.351 \mathrm{E}+04$ \\
\hline $1 s 6 p^{3} P_{1}^{o}$ & $1 s 6 d^{3} D_{1}$ & $7.9964 \mathrm{E}+02$ & $2.369 \mathrm{E}-12$ & $8.238 \mathrm{E}-03$ & $7.9517 \mathrm{E}+02$ & $2.385 \mathrm{E}-12$ & $8.315 \mathrm{E}-03$ \\
\hline $1 s 6 p^{3} P_{2}^{o}$ & $1 s 6 d^{3} D_{1}$ & $1.0358 \mathrm{E}+04$ & $5.839 \mathrm{E}-20$ & $1.210 \mathrm{E}-12$ & & & \\
\hline $1 s 6 p^{1} P_{1}^{o}$ & $1 s 6 d^{3} D_{1}$ & $6.7159 \mathrm{E}+06$ & $2.936 \mathrm{E}-27$ & $1.447 \mathrm{E}-25$ & & & \\
\hline $1 s 2 p^{3} P_{1}^{o}$ & $1 s 6 d^{3} D_{3}$ & $1.3779 \mathrm{E}+00$ & $1.185 \mathrm{E}-06$ & $5.945 \mathrm{E}+08$ & $1.3722 \mathrm{E}+00$ & $1.211 \mathrm{E}-06$ & $6.080 \mathrm{E}+08$ \\
\hline $1 s 2 p^{3} P_{2}^{o}$ & $1 s 6 d^{3} D_{3}$ & $1.4401 \mathrm{E}+00$ & $5.129 \mathrm{E}-06$ & $2.357 \mathrm{E}+09$ & $1.4340 \mathrm{E}+00$ & $5.376 \mathrm{E}-06$ & $2.470 \mathrm{E}+09$ \\
\hline $1 s 2 p^{1} P_{1}^{o}$ & $1 s 6 d^{3} D_{3}$ & $1.4460 \mathrm{E}+00$ & $9.200 \mathrm{E}-07$ & $4.193 \mathrm{E}+08$ & $1.4400 \mathrm{E}+00$ & $9.661 \mathrm{E}-07$ & $4.402 \mathrm{E}+08$ \\
\hline $1 s 3 p^{3} P_{1}^{o}$ & $1 s 6 d^{3} D_{3}$ & $3.6922 \mathrm{E}+00$ & $3.978 \mathrm{E}-07$ & $2.780 \mathrm{E}+07$ & $3.6768 \mathrm{E}+00$ & $4.047 \mathrm{E}-07$ & $2.829 \mathrm{E}+07$ \\
\hline $1 s 3 p^{3} P_{2}^{o}$ & $1 s 6 d^{3} D_{3}$ & $3.8238 \mathrm{E}+00$ & $1.918 \mathrm{E}-06$ & $1.250 \mathrm{E}+08$ & $3.8077 \mathrm{E}+00$ & $1.956 \mathrm{E}-06$ & $1.275 \mathrm{E}+08$ \\
\hline $1 s 3 p^{1} P_{1}^{o}$ & $1 s 6 d^{3} D_{3}$ & $3.8355 \mathrm{E}+00$ & $3.451 \mathrm{E}-07$ & $2.236 \mathrm{E}+07$ & $3.8195 \mathrm{E}+00$ & $3.499 \mathrm{E}-07$ & $2.266 \mathrm{E}+07$ \\
\hline $1 s 4 p^{3} P_{1}^{o}$ & $1 s 6 d^{3} D_{3}$ & $8.8415 \mathrm{E}+00$ & $1.680 \mathrm{E}-07$ & $2.047 \mathrm{E}+06$ & $8.8033 \mathrm{E}+00$ & $1.690 \mathrm{E}-07$ & $2.060 \mathrm{E}+06$ \\
\hline $1 s 4 p^{3} P_{2}^{o}$ & $1 s 6 d^{3} D_{3}$ & $9.1590 \mathrm{E}+00$ & $8.734 \mathrm{E}-07$ & $9.922 \mathrm{E}+06$ & $9.1192 \mathrm{E}+00$ & $8.851 \mathrm{E}-07$ & $1.005 \mathrm{E}+07$ \\
\hline $1 s 4 p^{1} P_{1}^{o}$ & $1 s 6 d^{3} D_{3}$ & $9.1868 \mathrm{E}+00$ & $1.579 \mathrm{E}-07$ & $1.783 \mathrm{E}+06$ & $9.1472 \mathrm{E}+00$ & $1.575 \mathrm{E}-07$ & $1.778 \mathrm{E}+06$ \\
\hline $1 s 4 f^{3} F_{3}^{o}$ & $1 s 6 d^{3} D_{3}$ & $9.2898 \mathrm{E}+00$ & $2.242 \mathrm{E}-14$ & $2.476 \mathrm{E}-01$ & & & \\
\hline $1 s 4 f^{3} F_{2}^{o}$ & $1 s 6 d^{3} D_{3}$ & $9.2920 \mathrm{E}+00$ & $9.727 \mathrm{E}-14$ & $1.074 \mathrm{E}+00$ & & & \\
\hline $1 s 4 f^{3} F_{4}^{o}$ & $1 s 6 d^{3} D_{3}$ & $9.3429 \mathrm{E}+00$ & $3.624 \mathrm{E}-08$ & $3.957 \mathrm{E}+05$ & $9.3023 E+00$ & $3.574 \mathrm{E}-08$ & $3.903 \mathrm{E}+05$ \\
\hline $1 s 4 f^{1} F_{3}^{o}$ & $1 s 6 d^{3} D_{3}$ & $9.3445 \mathrm{E}+00$ & $4.358 \mathrm{E}-09$ & $4.756 \mathrm{E}+04$ & $9.3039 \mathrm{E}+00$ & $4.287 \mathrm{E}-09$ & $4.679 \mathrm{E}+04$ \\
\hline $1 s 5 p^{3} P_{1}^{o}$ & $1 s 6 d^{3} D_{3}$ & $2.4575 \mathrm{E}+01$ & $7.820 \mathrm{E}-08$ & $1.234 \mathrm{E}+05$ & $2.4463 \mathrm{E}+01$ & $7.792 \mathrm{E}-08$ & $1.230 \mathrm{E}+05$ \\
\hline $1 s 5 p^{3} P_{2}^{o}$ & $1 s 6 d^{3} D_{3}$ & $2.5845 \mathrm{E}+01$ & $4.441 \mathrm{E}-07$ & $6.335 \mathrm{E}+05$ & $2.5726 \mathrm{E}+01$ & $4.508 \mathrm{E}-07$ & $6.436 \mathrm{E}+05$ \\
\hline
\end{tabular}


Table 6 (continued)

\begin{tabular}{|c|c|c|c|c|c|c|c|}
\hline \multirow[t]{2}{*}{ Lower } & \multirow[t]{2}{*}{ Upper } & \multicolumn{3}{|l|}{ GRASP2K } & \multicolumn{3}{|l|}{ FAC } \\
\hline & & $\lambda(\AA)$ & $g f$ & $A\left(\mathrm{~s}^{-1}\right)$ & $\overline{\lambda(\AA)}$ & $g f$ & $A\left(\mathrm{~s}^{-1}\right)$ \\
\hline $1 s 5 p^{1} P_{1}^{o}$ & $1 s 6 d^{3} D_{3}$ & $2.5958 \mathrm{E}+01$ & $8.083 \mathrm{E}-08$ & $1.143 \mathrm{E}+05$ & $2.5839 \mathrm{E}+01$ & $7.947 \mathrm{E}-08$ & $1.124 \mathrm{E}+05$ \\
\hline $1 s 5 f^{3} F_{3}^{o}$ & $1 s 6 d^{3} D_{3}$ & $2.6382 \mathrm{E}+01$ & $3.376 \mathrm{E}-14$ & $4.622 \mathrm{E}-02$ & & & \\
\hline $1 s 5 f^{3} F_{2}^{o}$ & $1 s 6 d^{3} D_{3}$ & $2.6391 \mathrm{E}+01$ & $8.504 \mathrm{E}-14$ & $1.163 \mathrm{E}-01$ & & & \\
\hline $1 s 5 f^{3} F_{4}^{o}$ & $1 s 6 d^{3} D_{3}$ & $2.6602 \mathrm{E}+01$ & $6.478 \mathrm{E}-08$ & $8.723 E+04$ & $2.6482 \mathrm{E}+01$ & $6.437 \mathrm{E}-08$ & $8.672 E+04$ \\
\hline $1 s 5 f^{1} F_{3}^{o}$ & $1 s 6 d^{3} D_{3}$ & $2.6609 \mathrm{E}+01$ & $7.788 \mathrm{E}-09$ & $1.048 \mathrm{E}+04$ & $2.6489 \mathrm{E}+01$ & $7.718 \mathrm{E}-09$ & $1.039 \mathrm{E}+04$ \\
\hline $1 s 6 p^{3} P_{1}^{0}$ & $1 s 6 d^{3} D_{3}$ & $6.2409 \mathrm{E}+02$ & $7.844 \mathrm{E}-11$ & $1.919 \mathrm{E}-01$ & $6.1530 \mathrm{E}+02$ & $7.917 \mathrm{E}-11$ & $1.975 \mathrm{E}-01$ \\
\hline $1 s 6 p^{3} P_{2}^{o}$ & $1 s 6 d^{3} D_{3}$ & $2.2306 \mathrm{E}+03$ & $8.574 \mathrm{E}-12$ & $1.642 \mathrm{E}-03$ & $2.1370 \mathrm{E}+03$ & $9.635 \mathrm{E}-12$ & $1.993 \mathrm{E}-03$ \\
\hline $1 s 6 p^{1} P_{1}^{o}$ & $1 s 6 d^{3} D_{3}$ & $2.8416 \mathrm{E}+03$ & $7.471 \mathrm{E}-13$ & $8.817 \mathrm{E}-05$ & $2.6199 \mathrm{E}+03$ & $9.643 E-13$ & $1.327 \mathrm{E}-04$ \\
\hline $1 s 2 s^{3} S_{1}$ & $1 s 6 f^{3} F_{3}^{o}$ & $1.3662 \mathrm{E}+00$ & $6.354 \mathrm{E}-11$ & $3.244 \mathrm{E}+04$ & & & \\
\hline $1 s 3 s^{3} S_{1}$ & $1 s 6 f^{3} F_{3}^{o}$ & $3.6691 \mathrm{E}+00$ & $3.991 \mathrm{E}-14$ & $2.825 \mathrm{E}+00$ & & & \\
\hline $1 s 3 d^{3} D_{2}$ & $1 s 6 f^{3} F_{3}^{o}$ & $3.8336 \mathrm{E}+00$ & $3.774 \mathrm{E}-07$ & $2.447 \mathrm{E}+07$ & $3.8174 \mathrm{E}+00$ & $3.877 \mathrm{E}-07$ & $2.513 \mathrm{E}+07$ \\
\hline $1 s 3 d^{3} D_{1}$ & $1 s 6 f^{3} F_{3}^{o}$ & $3.8354 \mathrm{E}+00$ & $6.366 \mathrm{E}-08$ & $4.124 \mathrm{E}+06$ & $3.8192 \mathrm{E}+00$ & $6.429 \mathrm{E}-08$ & $4.164 \mathrm{E}+06$ \\
\hline $1 s 3 d^{3} D_{3}$ & $1 s 6 f^{3} F_{3}^{o}$ & $3.8771 \mathrm{E}+00$ & $2.152 \mathrm{E}-12$ & $1.364 \mathrm{E}+02$ & & & \\
\hline $1 s 3 d d^{1} D_{2}$ & $1 s 6 f^{3} F_{3}^{o}$ & $3.8788 \mathrm{E}+00$ & $2.980 \mathrm{E}-11$ & $1.887 \mathrm{E}+03$ & & & \\
\hline $1 s 4 s^{3} S_{1}$ & $1 s 6 f^{3} F_{3}^{o}$ & $8.7872 \mathrm{E}+00$ & $1.208 \mathrm{E}-13$ & $1.491 \mathrm{E}+00$ & & & \\
\hline $1 s 4 d^{3} D_{2}$ & $1 s 6 f^{3} F_{3}^{o}$ & $9.1821 \mathrm{E}+00$ & $2.268 \mathrm{E}-07$ & $2.563 \mathrm{E}+06$ & $9.1440 \mathrm{E}+00$ & $2.296 \mathrm{E}-07$ & $2.595 \mathrm{E}+06$ \\
\hline $1 s 4 d^{3} D_{1}$ & $1 s 6 f^{3} F_{3}^{o}$ & $9.1863 \mathrm{E}+00$ & $3.827 \mathrm{E}-08$ & $4.321 \mathrm{E}+05$ & $9.1480 \mathrm{E}+00$ & $3.808 \mathrm{E}-08$ & $4.299 \mathrm{E}+05$ \\
\hline $1 s 4 d^{3} D_{3}$ & $1 s 6 f^{3} F_{3}^{o}$ & $9.2876 \mathrm{E}+00$ & $1.258 \mathrm{E}-12$ & $1.390 \mathrm{E}+01$ & & & \\
\hline $1 s 4 d d^{1} D_{2}$ & $1 s 6 f^{3} F_{3}^{o}$ & $9.2919 \mathrm{E}+00$ & $2.710 \mathrm{E}-11$ & $2.991 \mathrm{E}+02$ & & & \\
\hline $1 s 5 s^{3} S_{1}$ & $1 s 6 f^{3} F_{3}^{o}$ & $2.4362 \mathrm{E}+01$ & $5.695 \mathrm{E}-15$ & $9.143 \mathrm{E}-03$ & & & \\
\hline $1 s 5 d^{3} D_{2}$ & $1 s 6 f^{3} F_{3}^{o}$ & $2.5937 \mathrm{E}+01$ & $1.250 \mathrm{E}-07$ & $1.770 \mathrm{E}+05$ & $2.5831 \mathrm{E}+01$ & $1.257 \mathrm{E}-07$ & $1.780 \mathrm{E}+05$ \\
\hline $1 s 5 d^{3} D_{1}$ & $1 s 6 f^{3} F_{3}^{o}$ & $2.5954 \mathrm{E}+01$ & $2.111 \mathrm{E}-08$ & $2.987 \mathrm{E}+04$ & $2.5848 \mathrm{E}+01$ & $2.083 \mathrm{E}-08$ & $2.946 \mathrm{E}+04$ \\
\hline $1 s 5 d^{3} D_{3}$ & $1 s 6 f^{3} F_{3}^{o}$ & $2.6370 \mathrm{E}+01$ & $7.159 \mathrm{E}-13$ & $9.810 \mathrm{E}-01$ & & & \\
\hline $1 s 5 d{ }^{1} D_{2}$ & $1 s 6 f^{3} F_{3}^{o}$ & $2.6388 \mathrm{E}+01$ & $1.786 \mathrm{E}-11$ & $2.444 \mathrm{E}+01$ & & & \\
\hline $1 s 5 g{ }^{3} G_{4}$ & $1 s 6 f^{3} F_{3}^{o}$ & $2.6598 \mathrm{E}+01$ & $6.169 \mathrm{E}-09$ & $8.309 E+03$ & $2.6482 \mathrm{E}+01$ & $6.138 \mathrm{E}-09$ & $8.270 \mathrm{E}+03$ \\
\hline $1 s 5 g^{3} G_{3}$ & $1 s 6 f^{3} F_{3}^{o}$ & $2.6603 \mathrm{E}+01$ & $7.426 \mathrm{E}-10$ & $9.999 \mathrm{E}+02$ & $2.6487 \mathrm{E}+01$ & $7.367 \mathrm{E}-10$ & $9.922 \mathrm{E}+02$ \\
\hline $1 s 5 g^{3} G_{5}$ & $1 s 6 f^{3} F_{3}^{o}$ & $2.6730 \mathrm{E}+01$ & $4.453 \mathrm{E}-09$ & $5.938 \mathrm{E}+03$ & $2.6614 \mathrm{E}+01$ & $4.418 \mathrm{E}-09$ & $5.893 \mathrm{E}+03$ \\
\hline $1 s 5 g{ }^{1} G_{4}$ & $1 s 6 f^{3} F_{3}^{o}$ & $2.6735 \mathrm{E}+01$ & $2.634 \mathrm{E}-10$ & $3.512 \mathrm{E}+02$ & $2.6618 \mathrm{E}+01$ & $2.759 \mathrm{E}-10$ & $3.679 \mathrm{E}+02$ \\
\hline $1 s 6 s^{3} S_{1}$ & $1 s 6 f^{3} F_{3}^{o}$ & $5.5267 \mathrm{E}+02$ & $1.173 \mathrm{E}-17$ & $3.658 \mathrm{E}-08$ & & & \\
\hline $1 s 6 d^{3} D_{2}$ & $1 s 6 f^{3} F_{3}^{0}$ & $2.6863 \mathrm{E}+03$ & $7.605 \mathrm{E}-13$ & $1.004 \mathrm{E}-04$ & $2.7429 \mathrm{E}+03$ & $7.086 \mathrm{E}-13$ & $8.898 \mathrm{E}-05$ \\
\hline $1 s 6 d^{3} D_{1}$ & $1 s 6 f^{3} F_{3}^{o}$ & $2.7912 \mathrm{E}+03$ & $1.144 \mathrm{E}-13$ & $1.399 \mathrm{E}-05$ & $2.7175 \mathrm{E}+03$ & $1.210 \mathrm{E}-13$ & $1.548 \mathrm{E}-05$ \\
\hline $1 s 6 d^{3} D_{3}$ & $1 s 6 f^{3} F_{3}^{o}$ & $1.5394 \mathrm{E}+05$ & $2.306 \mathrm{E}-23$ & $9.272 \mathrm{E}-19$ & & & \\
\hline $1 s^{2}{ }^{1} S_{0}$ & $1 s 6 f^{3} F_{2}^{o}$ & $3.1620 \mathrm{E}-01$ & $6.848 \mathrm{E}-11$ & $9.134 \mathrm{E}+05$ & & & \\
\hline $1 s 2 s^{3} S_{1}$ & $1 s 6 f^{3} F_{2}^{o}$ & $1.3662 \mathrm{E}+00$ & $2.222 \mathrm{E}-11$ & $1.588 \mathrm{E}+04$ & & & \\
\hline $1 s 2 s{ }^{1} S_{0}$ & $1 s 6 f^{3} F_{2}^{o}$ & $1.3791 \mathrm{E}+00$ & $2.075 \mathrm{E}-11$ & $1.456 \mathrm{E}+04$ & & & \\
\hline $1 s 3 s^{3} S_{1}$ & $1 s 6 f^{3} F_{2}^{o}$ & $3.6690 \mathrm{E}+00$ & $4.287 \mathrm{E}-13$ & $4.249 \mathrm{E}+01$ & & & \\
\hline $1 s 3 s^{1} S_{0}$ & $1 s 6 f^{3} F_{2}^{o}$ & $3.6936 \mathrm{E}+00$ & $3.226 \mathrm{E}-13$ & $3.155 \mathrm{E}+01$ & & & \\
\hline $1 s 3 d^{3} D_{2}$ & $1 s 6 f^{3} F_{2}^{o}$ & $3.8335 \mathrm{E}+00$ & $9.471 \mathrm{E}-08$ & $8.598 E+06$ & $3.8174 \mathrm{E}+00$ & $9.688 \mathrm{E}-08$ & $8.794 \mathrm{E}+06$ \\
\hline $1 s 3 d^{3} D_{1}$ & $1 s 6 f^{3} F_{2}^{o}$ & $3.8353 \mathrm{E}+00$ & $2.205 \mathrm{E}-07$ & $2.000 \mathrm{E}+07$ & $3.8192 \mathrm{E}+00$ & $2.257 \mathrm{E}-07$ & $2.047 \mathrm{E}+07$ \\
\hline $1 s 3 d^{3} D_{3}$ & $1 s 6 f^{3} F_{2}^{o}$ & $3.8770 \mathrm{E}+00$ & $1.142 \mathrm{E}-18$ & $1.013 \mathrm{E}-04$ & & & \\
\hline $1 s 3 d d^{1} D_{2}$ & $1 s 6 f^{3} F_{2}^{o}$ & $3.8787 \mathrm{E}+00$ & $8.803 E-13$ & $7.806 \mathrm{E}+01$ & & & \\
\hline $1 s 4 s^{3} S_{1}$ & $1 s 6 f^{3} F_{2}^{o}$ & $8.7866 \mathrm{E}+00$ & $5.076 \mathrm{E}-13$ & $8.772 \mathrm{E}+00$ & & & \\
\hline $1 s 4 s^{1} S_{0}$ & $1 s 6 f^{3} F_{2}^{o}$ & $8.8435 \mathrm{E}+00$ & $3.256 \mathrm{E}-13$ & $5.554 \mathrm{E}+00$ & & & \\
\hline $1 s 4 d^{3} D_{2}$ & $1 s 6 f^{3} F_{2}^{o}$ & $9.1815 \mathrm{E}+00$ & $5.683 \mathrm{E}-08$ & $8.994 \mathrm{E}+05$ & $9.1440 \mathrm{E}+00$ & $5.738 \mathrm{E}-08$ & $9.077 \mathrm{E}+05$ \\
\hline $1 s 4 d^{3} D_{1}$ & $1 s 6 f^{3} F_{2}^{o}$ & $9.1856 \mathrm{E}+00$ & $1.325 \mathrm{E}-07$ & $2.095 \mathrm{E}+06$ & $9.1480 \mathrm{E}+00$ & $1.337 \mathrm{E}-07$ & $2.113 \mathrm{E}+06$ \\
\hline $1 s 4 d^{3} D_{3}$ & $1 s 6 f^{3} F_{2}^{o}$ & $9.2869 \mathrm{E}+00$ & $1.242 \mathrm{E}-14$ & $1.921 \mathrm{E}-01$ & & & \\
\hline $1 s 4 d{ }^{1} D_{2}$ & $1 s 6 f^{3} F_{2}^{o}$ & $9.2913 \mathrm{E}+00$ & $1.382 \mathrm{E}-12$ & $2.135 \mathrm{E}+01$ & & & \\
\hline $1 s 5 s^{3} S_{1}$ & $1 s 6 f^{3} F_{2}^{o}$ & $2.4358 \mathrm{E}+01$ & $1.928 \mathrm{E}-13$ & $4.335 \mathrm{E}-01$ & & & \\
\hline $1 s 5 s{ }^{1} S_{0}$ & $1 s 6 f^{3} F_{2}^{o}$ & $2.4577 \mathrm{E}+01$ & $9.292 \mathrm{E}-14$ & $2.052 \mathrm{E}-01$ & & & \\
\hline $1 s 5 d^{3} D_{2}$ & $1 s 6 f^{3} F_{2}^{o}$ & $2.5932 \mathrm{E}+01$ & $3.129 \mathrm{E}-08$ & $6.207 \mathrm{E}+04$ & $2.5831 \mathrm{E}+01$ & $3.141 \mathrm{E}-08$ & $6.227 \mathrm{E}+04$ \\
\hline $1 s 5 d^{3} D_{1}$ & $1 s 6 f^{3} F_{2}^{o}$ & $2.5948 \mathrm{E}+01$ & $7.306 \mathrm{E}-08$ & $1.447 \mathrm{E}+05$ & $2.5848 \mathrm{E}+01$ & $7.316 \mathrm{E}-08$ & $1.448 \mathrm{E}+05$ \\
\hline $1 s 5 d^{3} D_{3}$ & $1 s 6 f^{3} F_{2}^{o}$ & $2.6365 \mathrm{E}+01$ & $1.482 \mathrm{E}-14$ & $2.845 \mathrm{E}-02$ & & & \\
\hline $1 s 5 d{ }^{1} D_{2}$ & $1 s 6 f^{3} F_{2}^{o}$ & $2.6383 \mathrm{E}+01$ & $1.033 \mathrm{E}-12$ & $1.979 \mathrm{E}+00$ & & & \\
\hline $1 s 5 g^{3} G_{4}$ & $1 s 6 f^{3} F_{2}^{o}$ & $2.6592 \mathrm{E}+01$ & $4.970 \mathrm{E}-10$ & $9.377 \mathrm{E}+02$ & $2.6482 \mathrm{E}+01$ & $4.963 \mathrm{E}-10$ & $9.362 \mathrm{E}+02$ \\
\hline $1 s 5 g^{3} G_{3}$ & $1 s 6 f^{3} F_{2}^{o}$ & $2.6598 \mathrm{E}+01$ & $4.442 \mathrm{E}-09$ & $8.376 \mathrm{E}+03$ & $2.6488 \mathrm{E}+01$ & $4.420 \mathrm{E}-09$ & $8.334 \mathrm{E}+03$ \\
\hline $1 s 5 g{ }^{1} G_{4}$ & $1 s 6 f^{3} F_{2}^{o}$ & $2.6729 \mathrm{E}+01$ & $3.356 \mathrm{E}-09$ & $6.266 \mathrm{E}+03$ & $2.6618 \mathrm{E}+01$ & $3.343 \mathrm{E}-09$ & $6.241 \mathrm{E}+03$ \\
\hline $1 s 6 s^{3} S_{1}$ & $1 s 6 f^{3} F_{2}^{o}$ & $5.5035 \mathrm{E}+02$ & $4.304 \mathrm{E}-17$ & $1.896 \mathrm{E}-07$ & & & \\
\hline $1 s 6 s^{1} S_{0}$ & $1 s 6 f^{3} F_{2}^{o}$ & $6.2215 \mathrm{E}+02$ & $4.276 \mathrm{E}-17$ & $1.474 \mathrm{E}-07$ & & & \\
\hline $1 s 6 d^{3} D_{2}$ & $1 s 6 f^{3} F_{2}^{o}$ & $2.6325 \mathrm{E}+03$ & $2.025 \mathrm{E}-13$ & $3.898 \mathrm{E}-05$ & $2.7436 \mathrm{E}+03$ & $1.769 \mathrm{E}-13$ & $3.109 \mathrm{E}-05$ \\
\hline $1 s 6 d^{3} D_{1}$ & $1 s 6 f^{3} F_{2}^{o}$ & $2.7332 \mathrm{E}+03$ & $4.220 \mathrm{E}-13$ & $7.536 \mathrm{E}-05$ & $2.7182 \mathrm{E}+03$ & $4.245 \mathrm{E}-13$ & $7.601 \mathrm{E}-05$ \\
\hline $1 s 6 d^{3} D_{3}$ & $1 s 6 f^{3} F_{2}^{o}$ & $7.0900 \mathrm{E}+04$ & $6.510 \mathrm{E}-23$ & $1.728 \mathrm{E}-17$ & & & \\
\hline $1 s 2 p^{3} P_{1}^{o}$ & $1 s 6 d^{1} D_{2}$ & $1.3779 \mathrm{E}+00$ & $4.573 \mathrm{E}-07$ & $3.213 \mathrm{E}+08$ & $1.3722 \mathrm{E}+00$ & $4.669 \mathrm{E}-07$ & $3.280 \mathrm{E}+08$ \\
\hline $1 s 2 p^{3} P_{0}^{o}$ & $1 s 6 d^{1} D_{2}$ & $1.3788 \mathrm{E}+00$ & $5.490 \mathrm{E}-07$ & $3.852 \mathrm{E}+08$ & $1.3730 \mathrm{E}+00$ & $5.623 \mathrm{E}-07$ & $3.945 \mathrm{E}+08$ \\
\hline $1 s 2 p^{3} P_{2}^{0}$ & $1 s 6 d{ }^{1} D_{2}$ & $1.4401 \mathrm{E}+00$ & $1.289 \mathrm{E}-06$ & $8.293 \mathrm{E}+08$ & $1.4340 \mathrm{E}+00$ & $1.343 \mathrm{E}-06$ & $8.644 \mathrm{E}+08$ \\
\hline $1 s 2 p^{1} P_{1}^{o}$ & $1 s 6 d^{1} D_{2}$ & $1.4459 \mathrm{E}+00$ & $2.881 \mathrm{E}-06$ & $1.839 \mathrm{E}+09$ & $1.4399 \mathrm{E}+00$ & $3.012 \mathrm{E}-06$ & $1.921 \mathrm{E}+09$ \\
\hline $1 s 3 p^{3} P_{1}^{0}$ & $1 s 6 d^{1} D_{2}$ & $3.6920 \mathrm{E}+00$ & $1.505 \mathrm{E}-07$ & $1.472 \mathrm{E}+07$ & $3.6767 \mathrm{E}+00$ & $1.542 \mathrm{E}-07$ & $1.509 \mathrm{E}+07$ \\
\hline $1 s 3 p^{3} P_{0}^{0}$ & $1 s 6 d^{1} D_{2}$ & $3.6937 \mathrm{E}+00$ & $1.833 \mathrm{E}-07$ & $1.793 \mathrm{E}+07$ & $3.6783 \mathrm{E}+00$ & $1.874 \mathrm{E}-07$ & $1.832 \mathrm{E}+07$ \\
\hline $1 s 3 p^{3} P_{2}^{o}$ & $1 s 6 d{ }^{1} D_{2}$ & $3.8236 \mathrm{E}+00$ & $4.803 \mathrm{E}-07$ & $4.383 \mathrm{E}+07$ & $3.8077 \mathrm{E}+00$ & $4.891 \mathrm{E}-07$ & $4.462 \mathrm{E}+07$ \\
\hline $1 s 3 p{ }^{1} P_{1}^{o}$ & $1 s 6 d^{1} D_{2}$ & $3.8353 \mathrm{E}+00$ & $1.087 \mathrm{E}-06$ & $9.861 \mathrm{E}+07$ & $3.8195 E+00$ & $1.102 \mathrm{E}-06$ & $9.999 \mathrm{E}+07$ \\
\hline $1 s 4 p^{3} P_{1}^{o}$ & $1 s 6 d{ }^{1} D_{2}$ & $8.8403 E+00$ & $6.306 \mathrm{E}-08$ & $1.076 \mathrm{E}+06$ & $8.8028 \mathrm{E}+00$ & $6.473 \mathrm{E}-08$ & $1.104 \mathrm{E}+06$ \\
\hline $1 s 4 p^{3} P_{0}^{o}$ & $1 s 6 d{ }^{1} D_{2}$ & $8.8442 \mathrm{E}+00$ & $7.726 \mathrm{E}-08$ & $1.318 \mathrm{E}+06$ & $8.8066 \mathrm{E}+00$ & $7.837 \mathrm{E}-08$ & $1.336 \mathrm{E}+06$ \\
\hline
\end{tabular}


Table 6 (continued)

\begin{tabular}{|c|c|c|c|c|c|c|c|}
\hline \multirow[t]{2}{*}{ Lower } & \multirow[t]{2}{*}{ Upper } & \multicolumn{3}{|l|}{ GRASP2K } & \multicolumn{3}{|l|}{ FAC } \\
\hline & & $\lambda(\AA)$ & $g f$ & $A\left(\mathrm{~s}^{-1}\right)$ & $\lambda(\AA)$ & $g f$ & $A\left(\mathrm{~s}^{-1}\right)$ \\
\hline $1 s 4 p^{3} P_{2}^{o}$ & $1 s 6 d{ }^{1} D_{2}$ & $9.1577 \mathrm{E}+00$ & $2.183 \mathrm{E}-07$ & $3.473 E+06$ & $9.1187 \mathrm{E}+00$ & $2.212 \mathrm{E}-07$ & $3.520 \mathrm{E}+06$ \\
\hline $1 s 4 p^{1} P_{1}^{o}$ & $1 s 6 d{ }^{1} D_{2}$ & $9.1855 \mathrm{E}+00$ & $4.979 \mathrm{E}-07$ & $7.872 \mathrm{E}+06$ & $9.1467 \mathrm{E}+00$ & $4.996 \mathrm{E}-07$ & $7.900 \mathrm{E}+06$ \\
\hline $1 s 4 f^{3} F_{3}^{o}$ & $1 s 6 d^{1} D_{2}$ & $9.2884 \mathrm{E}+00$ & $4.634 \mathrm{E}-13$ & $7.165 E+00$ & & & \\
\hline $1 s 4 f^{3} F_{2}^{o}$ & $1 s 6 d^{1} D_{2}$ & $9.2907 \mathrm{E}+00$ & $4.578 \mathrm{E}-18$ & $7.075 \mathrm{E}-05$ & & & \\
\hline $1 s 4 f^{3} F_{4}^{o}$ & $1 s 6 d^{1} D_{2}$ & $9.3415 E+00$ & $2.936 \mathrm{E}-09$ & $4.489 \mathrm{E}+04$ & $9.3018 \mathrm{E}+00$ & $2.962 \mathrm{E}-09$ & $4.529 E+04$ \\
\hline $1 s 4 f^{1} F_{3}^{o}$ & $1 s 6 d{ }^{1} D_{2}$ & $9.3432 \mathrm{E}+00$ & $2.595 \mathrm{E}-08$ & $3.966 \mathrm{E}+05$ & $9.3035 \mathrm{E}+00$ & $2.562 \mathrm{E}-08$ & $3.915 E+05$ \\
\hline $1 s 5 p^{3} P_{1}^{0}$ & $1 s 6 d{ }^{1} D_{2}$ & $2.4566 \mathrm{E}+01$ & $2.924 \mathrm{E}-08$ & $6.463 \mathrm{E}+04$ & $2.4460 \mathrm{E}+01$ & $3.038 \mathrm{E}-08$ & $6.718 \mathrm{E}+04$ \\
\hline $1 s 5 p^{3} P_{0}^{o}$ & $1 s 6 d{ }^{1} D_{2}$ & $2.4581 \mathrm{E}+01$ & $3.593 \mathrm{E}-08$ & $7.934 \mathrm{E}+04$ & $2.4474 \mathrm{E}+01$ & $3.629 \mathrm{E}-08$ & $8.014 \mathrm{E}+04$ \\
\hline $1 s 5 p^{3} P_{2}^{o}$ & $1 s 6 d{ }^{1} D_{2}$ & $2.5835 \mathrm{E}+01$ & $1.108 \mathrm{E}-07$ & $2.215 E+05$ & $2.5723 \mathrm{E}+01$ & $1.127 \mathrm{E}-07$ & $2.254 \mathrm{E}+05$ \\
\hline $1 s 5 p^{1} P_{1}^{o}$ & $1 s 6 d{ }^{1} D_{2}$ & $2.5947 \mathrm{E}+01$ & $2.547 \mathrm{E}-07$ & $5.046 \mathrm{E}+05$ & $2.5836 \mathrm{E}+01$ & $2.540 \mathrm{E}-07$ & $5.034 \mathrm{E}+05$ \\
\hline $1 s 5 f^{3} F_{3}^{o}$ & $1 s 6 d{ }^{1} D_{2}$ & $2.6371 \mathrm{E}+01$ & $7.765 \mathrm{E}-13$ & $1.490 \mathrm{E}+00$ & & & \\
\hline $1 s 5 f^{3} F_{2}^{o}$ & $1 s 6 d{ }^{1} D_{2}$ & $2.6380 \mathrm{E}+01$ & $5.047 \mathrm{E}-15$ & $9.676 \mathrm{E}-03$ & & & \\
\hline $1 s 5 f^{3} F_{4}^{o}$ & $1 s 6 d^{1} D_{2}$ & $2.6591 \mathrm{E}+01$ & $5.248 \mathrm{E}-09$ & $9.902 E+03$ & $2.6478 \mathrm{E}+01$ & $5.337 \mathrm{E}-09$ & $1.006 \mathrm{E}+04$ \\
\hline $1 s 5 f^{1} F_{3}^{0}$ & $1 s 6 d^{1} D_{2}$ & $2.6598 \mathrm{E}+01$ & $4.638 \mathrm{E}-08$ & $8.747 E+04$ & $2.6485 \mathrm{E}+01$ & $4.613 \mathrm{E}-08$ & $8.700 \mathrm{E}+04$ \\
\hline $1 s 6 p^{3} P_{1}^{o}$ & $1 s 6 d^{1} D_{2}$ & $6.1807 \mathrm{E}+02$ & $3.022 \mathrm{E}-11$ & $1.055 \mathrm{E}-01$ & $6.1320 \mathrm{E}+02$ & $3.228 \mathrm{E}-11$ & $1.135 \mathrm{E}-01$ \\
\hline $1 s 6 p^{3} P_{0}^{0}$ & $1 s 6 d{ }^{1} D_{2}$ & $6.2363 E+02$ & $3.613 \mathrm{E}-11$ & $1.239 \mathrm{E}-01$ & $5.9930 \mathrm{E}+02$ & $4.033 \mathrm{E}-11$ & $1.485 \mathrm{E}-01$ \\
\hline $1 s 6 p^{3} P_{2}^{o}$ & $1 s 6 d{ }^{1} D_{2}$ & $2.1556 \mathrm{E}+03$ & $2.376 \mathrm{E}-12$ & $6.821 \mathrm{E}-04$ & $2.1118 \mathrm{E}+03$ & $2.495 \mathrm{E}-12$ & $7.402 \mathrm{E}-04$ \\
\hline $1 s 6 p^{1} P_{1}^{o}$ & $1 s 6 d^{1} D_{2}$ & $2.7210 \mathrm{E}+03$ & $2.688 \mathrm{E}-12$ & $4.843 \mathrm{E}-04$ & $2.5822 \mathrm{E}+03$ & $3.083 \mathrm{E}-12$ & $6.116 \mathrm{E}-04$ \\
\hline $1 s 6 f^{3} F_{3}^{o}$ & $1 s 6 d^{1} D_{2}$ & $1.0989 \mathrm{E}+05$ & $1.664 \mathrm{E}-21$ & $1.838 \mathrm{E}-16$ & & & \\
\hline $1 s 6 f^{3} F_{2}^{o}$ & $1 s 6 d^{1} D_{2}$ & $6.7056 \mathrm{E}+05$ & $3.229 \mathrm{E}-25$ & $9.582 \mathrm{E}-22$ & & & \\
\hline $1 s 3 d^{3} D_{2}$ & $1 s 6 f^{3} F_{4}^{o}$ & $3.8310 \mathrm{E}+00$ & $7.454 \mathrm{E}-07$ & $3.764 \mathrm{E}+07$ & $3.8148 \mathrm{E}+00$ & $7.494 \mathrm{E}-07$ & $3.784 \mathrm{E}+07$ \\
\hline $1 s 3 d^{3} D_{3}$ & $1 s 6 f^{3} F_{4}^{0}$ & $3.8744 \mathrm{E}+00$ & $4.504 \mathrm{E}-06$ & $2.224 \mathrm{E}+08$ & $3.8580 \mathrm{E}+00$ & $4.617 \mathrm{E}-06$ & $2.279 E+08$ \\
\hline $1 s 3 d{ }^{1} D_{2}$ & $1 s 6 f^{3} F_{4}^{o}$ & $3.8761 \mathrm{E}+00$ & $3.632 \mathrm{E}-07$ & $1.792 E+07$ & $3.8597 \mathrm{E}+00$ & $3.727 \mathrm{E}-07$ & $1.838 \mathrm{E}+07$ \\
\hline $1 s 4 d^{3} D_{2}$ & $1 s 6 f^{3} F_{4}^{0}$ & $9.1669 \mathrm{E}+00$ & $4.363 \mathrm{E}-07$ & $3.848 \mathrm{E}+06$ & $9.1288 \mathrm{E}+00$ & $4.400 \mathrm{E}-07$ & $3.879 E+06$ \\
\hline $1 s 4 d^{3} D_{3}$ & $1 s 6 f^{3} F_{4}^{0}$ & $9.2719 \mathrm{E}+00$ & $2.746 \mathrm{E}-06$ & $2.367 \mathrm{E}+07$ & $9.2331 \mathrm{E}+00$ & $2.774 \mathrm{E}-06$ & $2.391 \mathrm{E}+07$ \\
\hline $1 s 4 d^{1} D_{2}$ & $1 s 6 f^{3} F_{4}^{0}$ & $9.2763 \mathrm{E}+00$ & $2.228 \mathrm{E}-07$ & $1.919 \mathrm{E}+06$ & $9.2375 \mathrm{E}+00$ & $2.251 \mathrm{E}-07$ & $1.938 \mathrm{E}+06$ \\
\hline $1 s 5 d^{3} D_{2}$ & $1 s 6 f^{3} F_{4}^{o}$ & $2.5816 \mathrm{E}+01$ & $2.354 \mathrm{E}-07$ & $2.617 \mathrm{E}+05$ & $2.5711 \mathrm{E}+01$ & $2.362 \mathrm{E}-07$ & $2.625 E+05$ \\
\hline $1 s 5 d^{3} D_{3}$ & $1 s 6 f^{3} F_{4}^{0}$ & $2.6244 \mathrm{E}+01$ & $1.538 \mathrm{E}-06$ & $1.656 \mathrm{E}+06$ & $2.6136 \mathrm{E}+01$ & $1.544 \mathrm{E}-06$ & $1.661 \mathrm{E}+06$ \\
\hline $1 s 5 d{ }^{1} D_{2}$ & $1 s 6 f^{3} F_{4}^{o}$ & $2.6262 \mathrm{E}+01$ & $1.253 \mathrm{E}-07$ & $1.346 \mathrm{E}+05$ & $2.6155 \mathrm{E}+01$ & $1.254 \mathrm{E}-07$ & $1.347 \mathrm{E}+05$ \\
\hline $1 s 5 g^{3} G_{4}$ & $1 s 6 f^{3} F_{4}^{o}$ & $2.6470 \mathrm{E}+01$ & $8.803 \mathrm{E}-15$ & $9.312 \mathrm{E}-03$ & & & \\
\hline $1 s 5 g^{3} G_{3}$ & $1 s 6 f^{3} F_{4}^{0}$ & $2.6475 \mathrm{E}+01$ & $7.111 \mathrm{E}-16$ & $7.519 \mathrm{E}-04$ & & & \\
\hline $1 s 5 g^{3} G_{5}$ & $1 s 6 f^{3} F_{4}^{0}$ & $2.6601 \mathrm{E}+01$ & $3.646 \mathrm{E}-08$ & $3.819 E+04$ & $2.6486 \mathrm{E}+01$ & $3.625 \mathrm{E}-08$ & $3.797 E+04$ \\
\hline $1 s 5 g{ }^{1} G_{4}$ & $1 s 6 f^{3} F_{4}^{0}$ & $2.6606 \mathrm{E}+01$ & $2.610 \mathrm{E}-09$ & $2.732 E+03$ & $2.6491 \mathrm{E}+01$ & $2.588 \mathrm{E}-09$ & $2.710 \mathrm{E}+03$ \\
\hline $1 s 6 d^{3} D_{2}$ & $1 s 6 f^{3} F_{4}^{o}$ & $1.8056 \mathrm{E}+03$ & $4.817 \mathrm{E}-12$ & $1.095 \mathrm{E}-03$ & $1.8327 \mathrm{E}+03$ & $4.512 \mathrm{E}-12$ & $9.871 \mathrm{E}-04$ \\
\hline $1 s 6 d^{3} D_{3}$ & $1 s 6 f^{3} F_{4}^{o}$ & $5.3175 E+03$ & $1.207 \mathrm{E}-12$ & $3.163 \mathrm{E}-05$ & $5.5117 \mathrm{E}+03$ & $1.070 \mathrm{E}-12$ & $2.590 \mathrm{E}-05$ \\
\hline $1 s 6 d{ }^{1} D_{2}$ & $1 s 6 f^{3} F_{4}^{o}$ & $5.7984 \mathrm{E}+03$ & $7.578 \mathrm{E}-14$ & $1.670 \mathrm{E}-06$ & $5.6864 \mathrm{E}+03$ & $8.061 \mathrm{E}-14$ & $1.832 \mathrm{E}-06$ \\
\hline $1 s 2 p^{3} P_{2}^{o}$ & $1 s 6 g^{3} G_{4}$ & $1.4397 \mathrm{E}+00$ & $2.007 \mathrm{E}-11$ & $7.176 E+03$ & & & \\
\hline $1 s 3 p^{3} P_{2}^{o}$ & $1 s 6 g^{3} G_{4}$ & $3.8211 \mathrm{E}+00$ & $1.773 \mathrm{E}-11$ & $9.000 \mathrm{E}+02$ & & & \\
\hline $1 s 4 p^{3} P_{2}^{o}$ & $1 s 6 g^{3} G_{4}$ & $9.1432 \mathrm{E}+00$ & $1.834 \mathrm{E}-14$ & $1.626 \mathrm{E}-01$ & & & \\
\hline $1 s 4 f^{3} F_{3}^{o}$ & $1 s 6 g{ }^{3} G_{4}$ & $9.2736 \mathrm{E}+00$ & $8.732 \mathrm{E}-07$ & $7.525 E+06$ & $9.2341 \mathrm{E}+00$ & $8.851 \mathrm{E}-07$ & $7.628 \mathrm{E}+06$ \\
\hline $1 s 4 f^{3} F_{2}^{o}$ & $1 s 6 g{ }^{3} G_{4}$ & $9.2758 \mathrm{E}+00$ & $7.052 \mathrm{E}-08$ & $6.074 \mathrm{E}+05$ & $9.2363 \mathrm{E}+00$ & $7.076 \mathrm{E}-08$ & $6.095 \mathrm{E}+05$ \\
\hline $1 s 4 f^{3} F_{4}^{o}$ & $1 s 6 g^{3} G_{4}$ & $9.3264 \mathrm{E}+00$ & $1.314 \mathrm{E}-12$ & $1.120 \mathrm{E}+01$ & & & \\
\hline $1 s 4 f^{1} F_{3}^{o}$ & $1 s 6 g^{3} G_{4}$ & $9.3281 \mathrm{E}+00$ & $3.260 \mathrm{E}-12$ & $2.776 \mathrm{E}+01$ & & & \\
\hline $1 s 5 p^{3} P_{2}^{o}$ & $1 s 6 g^{3} G_{4}$ & $2.5720 \mathrm{E}+01$ & $1.495 \mathrm{E}-13$ & $1.675 \mathrm{E}-01$ & & & \\
\hline $1 s 5 f^{3} F_{3}^{o}$ & $1 s 6 g^{3} G_{4}$ & $2.6251 \mathrm{E}+01$ & $7.032 \mathrm{E}-07$ & $7.563 \mathrm{E}+05$ & $2.6139 \mathrm{E}+01$ & $7.069 \mathrm{E}-07$ & $7.603 \mathrm{E}+05$ \\
\hline $1 s 5 f^{3} F_{2}^{o}$ & $1 s 6 g^{3} G_{4}$ & $2.6260 \mathrm{E}+01$ & $5.684 \mathrm{E}-08$ & $6.109 E+04$ & $2.6148 \mathrm{E}+01$ & $5.650 \mathrm{E}-08$ & $6.072 \mathrm{E}+04$ \\
\hline $1 s 5 f^{3} F_{4}^{o}$ & $1 s 6 g{ }^{3} G_{4}$ & $2.6469 \mathrm{E}+01$ & $1.089 \mathrm{E}-12$ & $1.152 \mathrm{E}+00$ & & & \\
\hline $1 s 5 f^{1} F_{3}^{o}$ & $1 s 6 g{ }^{3} G_{4}$ & $2.6476 \mathrm{E}+01$ & $3.093 \mathrm{E}-12$ & $3.270 \mathrm{E}+00$ & & & \\
\hline $1 s 6 p^{3} P_{2}^{o}$ & $1 s 6 g^{3} G_{4}$ & $1.5705 \mathrm{E}+03$ & $1.699 \mathrm{E}-21$ & $5.106 \mathrm{E}-13$ & & & \\
\hline $1 s 6 f^{3} F_{3}^{o}$ & $1 s 6 g{ }^{3} G_{4}$ & $5.4970 \mathrm{E}+03$ & $2.497 \mathrm{E}-13$ & $6.123 E-06$ & $5.5303 \mathrm{E}+03$ & $2.427 \mathrm{E}-13$ & $5.831 \mathrm{E}-06$ \\
\hline $1 s 6 f^{3} F_{2}^{0}$ & $1 s 6 g{ }^{3} G_{4}$ & $5.7369 \mathrm{E}+03$ & $1.775 \mathrm{E}-14$ & $3.997 \mathrm{E}-07$ & $5.5276 \mathrm{E}+03$ & $1.944 \mathrm{E}-14$ & $4.676 \mathrm{E}-07$ \\
\hline $1 s 6 f^{3} F_{4}^{0}$ & $1 s 6 g{ }^{3} G_{4}$ & $2.7996 \mathrm{E}+06$ & $2.940 \mathrm{E}-27$ & $2.780 \mathrm{E}-25$ & & & \\
\hline $1 s 2 p^{3} P_{1}^{o}$ & $1 s 6 g{ }^{3} G_{3}$ & $1.3775 E+00$ & $8.809 \mathrm{E}-15$ & $4.423 E+00$ & & & \\
\hline $1 s 2 p^{3} P_{2}^{0}$ & $1 s 6 g{ }^{3} G_{3}$ & $1.4397 \mathrm{E}+00$ & $2.018 \mathrm{E}-12$ & $9.277 \mathrm{E}+02$ & & & \\
\hline $1 s 2 p^{1} P_{1}^{o}$ & $1 s 6 g{ }^{3} G_{3}$ & $1.4456 \mathrm{E}+00$ & $1.399 \mathrm{E}-11$ & $6.382 E+03$ & & & \\
\hline $1 s 3 p^{3} P_{1}^{o}$ & $1 s 6 g^{3} G_{3}$ & $3.6896 \mathrm{E}+00$ & $3.546 \mathrm{E}-14$ & $2.482 E+00$ & & & \\
\hline $1 s 3 p^{3} P_{2}^{0}$ & $1 s 6 g{ }^{3} G_{3}$ & $3.8210 \mathrm{E}+00$ & $2.034 \mathrm{E}-12$ & $1.327 \mathrm{E}+02$ & & & \\
\hline $1 s 3 p^{1} P_{1}^{o}$ & $1 s 6 g{ }^{3} G_{3}$ & $3.8327 \mathrm{E}+00$ & $1.119 \mathrm{E}-11$ & $7.261 \mathrm{E}+02$ & & & \\
\hline $1 s 4 p^{3} P_{1}^{o}$ & $1 s 6 g{ }^{3} G_{3}$ & $8.8264 \mathrm{E}+00$ & $1.545 \mathrm{E}-16$ & $1.889 \mathrm{E}-03$ & & & \\
\hline $1 s 4 p^{3} P_{2}^{o}$ & $1 s 6 g{ }^{3} G_{3}$ & $9.1429 \mathrm{E}+00$ & $6.432 \mathrm{E}-14$ & $7.331 \mathrm{E}-01$ & & & \\
\hline $1 s 4 p{ }^{1} P_{1}^{o}$ & $1 s 6 g{ }^{3} G_{3}$ & $9.1706 \mathrm{E}+00$ & $4.170 \mathrm{E}-16$ & $4.725 \mathrm{E}-03$ & & & \\
\hline $1 s 4 f^{3} F_{3}^{o}$ & $1 s 6 g{ }^{3} G_{3}$ & $9.2732 \mathrm{E}+00$ & $1.050 \mathrm{E}-07$ & $1.163 E+06$ & $9.2341 \mathrm{E}+00$ & $1.062 \mathrm{E}-07$ & $1.176 \mathrm{E}+06$ \\
\hline $1 s 4 f^{3} F_{2}^{o}$ & $1 s 6 g^{3} G_{3}$ & $9.2754 \mathrm{E}+00$ & $6.289 \mathrm{E}-07$ & $6.966 \mathrm{E}+06$ & $9.2363 \mathrm{E}+00$ & $6.368 \mathrm{E}-07$ & $7.053 \mathrm{E}+06$ \\
\hline $1 s 4 f^{3} F_{4}^{o}$ & $1 s 6 g^{3} G_{3}$ & $9.3261 \mathrm{E}+00$ & $1.641 \mathrm{E}-18$ & $1.798 \mathrm{E}-05$ & & & \\
\hline $1 s 4 f^{1} F_{3}^{o}$ & $1 s 6 g{ }^{3} G_{3}$ & $9.3277 \mathrm{E}+00$ & $4.791 \mathrm{E}-13$ & $5.247 \mathrm{E}+00$ & & & \\
\hline $1 s 5 p^{3} P_{1}^{o}$ & $1 s 6 g{ }^{3} G_{3}$ & $2.4459 \mathrm{E}+01$ & $1.101 \mathrm{E}-16$ & $1.754 \mathrm{E}-04$ & & & \\
\hline $1 s 5 p^{3} P_{2}^{o}$ & $1 s 6 g{ }^{3} G_{3}$ & $2.5717 \mathrm{E}+01$ & $2.230 \mathrm{E}-13$ & $3.213 \mathrm{E}-01$ & & & \\
\hline $1 s 5 p^{1} P_{1}^{0}$ & $1 s 6 g{ }^{3} G_{3}$ & $2.5829 \mathrm{E}+01$ & $1.653 \mathrm{E}-13$ & $2.361 \mathrm{E}-01$ & & & \\
\hline $1 s 5 f^{3} F_{3}^{o}$ & $1 s 6 g{ }^{3} G_{3}$ & $2.6248 \mathrm{E}+01$ & $8.445 \mathrm{E}-08$ & $1.168 \mathrm{E}+05$ & $2.6139 \mathrm{E}+01$ & $8.483 \mathrm{E}-08$ & $1.173 E+05$ \\
\hline $1 s 5 f^{3} F_{2}^{o}$ & $1 s 6 g{ }^{3} G_{3}$ & $2.6257 \mathrm{E}+01$ & $5.067 \mathrm{E}-07$ & $7.003 E+05$ & $2.6148 \mathrm{E}+01$ & $5.085 \mathrm{E}-07$ & $7.026 \mathrm{E}+05$ \\
\hline $1 s 5 f^{3} F_{4}^{o}$ & $1 s 6 g^{3} G_{3}$ & $2.6466 \mathrm{E}+01$ & $3.237 \mathrm{E}-17$ & $4.403 \mathrm{E}-05$ & & & \\
\hline
\end{tabular}


Table 6 (continued)

\begin{tabular}{|c|c|c|c|c|c|c|c|}
\hline \multirow[t]{2}{*}{ Lower } & \multirow[t]{2}{*}{ Upper } & \multicolumn{3}{|l|}{ GRASP2K } & \multicolumn{3}{|l|}{ FAC } \\
\hline & & $\lambda(\AA)$ & $g f$ & $A\left(\mathrm{~s}^{-1}\right)$ & $\overline{\lambda(\AA)}$ & $g f$ & $A\left(\mathrm{~s}^{-1}\right)$ \\
\hline $1 s 5 f^{1} F_{3}^{o}$ & $1 s 6 g^{3} G_{3}$ & $2.6473 \mathrm{E}+01$ & $3.503 E-13$ & $4.762 \mathrm{E}-01$ & & & \\
\hline $1 s 6 p^{3} P_{1}^{0}$ & $1 s 6 g^{3} G_{3}$ & $5.5707 \mathrm{E}+02$ & $5.442 \mathrm{E}-20$ & $1.671 \mathrm{E}-10$ & & & \\
\hline $1 s 6 p^{3} P_{2}^{o}$ & $1 s 6 g{ }^{3} G_{3}$ & $1.5598 \mathrm{E}+03$ & $2.160 \mathrm{E}-18$ & $8.458 \mathrm{E}-10$ & & & \\
\hline $1 s 6 p{ }^{1} P_{1}^{o}$ & $1 s 6 g^{3} G_{3}$ & $1.8359 \mathrm{E}+03$ & $1.466 \mathrm{E}-19$ & $4.146 \mathrm{E}-11$ & & & \\
\hline $1 s 6 f^{3} F_{3}^{o}$ & $1 s 6 g^{3} G_{3}$ & $5.3682 \mathrm{E}+03$ & $3.220 \mathrm{E}-14$ & $1.065 \mathrm{E}-06$ & $5.5303 \mathrm{E}+03$ & $2.912 \mathrm{E}-14$ & $8.997 \mathrm{E}-07$ \\
\hline $1 s 6 f^{3} F_{2}^{o}$ & $1 s 6 g^{3} G_{3}$ & $5.5968 \mathrm{E}+03$ & $1.704 \mathrm{E}-13$ & $5.185 \mathrm{E}-06$ & $5.5277 \mathrm{E}+03$ & $1.750 \mathrm{E}-13$ & $5.411 \mathrm{E}-06$ \\
\hline $1 s 6 f^{3} F_{4}^{o}$ & $1 s 6 g^{3} G_{3}$ & $2.1175 \mathrm{E}+05$ & $1.685 \mathrm{E}-26$ & $3.580 \mathrm{E}-22$ & & & \\
\hline $1 s 2 s^{3} S_{1}$ & $1 s 6 f^{1} F_{3}^{o}$ & $1.3658 \mathrm{E}+00$ & $9.089 \mathrm{E}-14$ & $4.643 \mathrm{E}+01$ & & & \\
\hline $1 s 3 s^{3} S_{1}$ & $1 s 6 f^{1} F_{3}^{o}$ & $3.6666 \mathrm{E}+00$ & $9.718 \mathrm{E}-13$ & $6.888 \mathrm{E}+01$ & & & \\
\hline $1 s 3 d^{3} D_{2}$ & $1 s 6 f^{1} F_{3}^{o}$ & $3.8309 \mathrm{E}+00$ & $8.752 \mathrm{E}-08$ & $5.683 \mathrm{E}+06$ & $3.8148 \mathrm{E}+00$ & $8.744 \mathrm{E}-08$ & $5.677 \mathrm{E}+06$ \\
\hline $1 s 3 d^{3} D_{1}$ & $1 s 6 f^{1} F_{3}^{o}$ & $3.8326 \mathrm{E}+00$ & $4.980 \mathrm{E}-07$ & $3.230 \mathrm{E}+07$ & $3.8165 E+00$ & $5.017 \mathrm{E}-07$ & $3.254 \mathrm{E}+07$ \\
\hline $1 s 3 d^{3} D_{3}$ & $1 s 6 f^{1} F_{3}^{o}$ & $3.8743 \mathrm{E}+00$ & $5.411 \mathrm{E}-07$ & $3.435 \mathrm{E}+07$ & $3.8580 \mathrm{E}+00$ & $5.541 \mathrm{E}-07$ & $3.517 \mathrm{E}+07$ \\
\hline $1 s 3 d{ }^{1} D_{2}$ & $1 s 6 f^{1} F_{3}^{o}$ & $3.8760 \mathrm{E}+00$ & $3.237 \mathrm{E}-06$ & $2.053 \mathrm{E}+08$ & $3.8597 \mathrm{E}+00$ & $3.316 \mathrm{E}-06$ & $2.103 E+08$ \\
\hline $1 s 4 s^{3} S_{1}$ & $1 s 6 f^{1} F_{3}^{o}$ & $8.7727 \mathrm{E}+00$ & $6.785 \mathrm{E}-13$ & $8.401 E+00$ & & & \\
\hline $1 s 4 d^{3} D_{2}$ & $1 s 6 f^{1} F_{3}^{o}$ & $9.1664 \mathrm{E}+00$ & $5.257 \mathrm{E}-08$ & $5.962 E+05$ & $9.1288 \mathrm{E}+00$ & $5.284 \mathrm{E}-08$ & $5.991 \mathrm{E}+05$ \\
\hline $1 s 4 d^{3} D_{1}$ & $1 s 6 f^{1} F_{3}^{o}$ & $9.1705 \mathrm{E}+00$ & $2.925 \mathrm{E}-07$ & $3.314 \mathrm{E}+06$ & $9.1329 \mathrm{E}+00$ & $2.954 \mathrm{E}-07$ & $3.347 \mathrm{E}+06$ \\
\hline $1 s 4 d^{3} D_{3}$ & $1 s 6 f^{1} F_{3}^{o}$ & $9.2714 \mathrm{E}+00$ & $3.296 \mathrm{E}-07$ & $3.654 \mathrm{E}+06$ & $9.2331 \mathrm{E}+00$ & $3.328 \mathrm{E}-07$ & $3.689 E+06$ \\
\hline $1 s 4 d^{1} D_{2}$ & $1 s 6 f^{1} F_{3}^{o}$ & $9.2758 \mathrm{E}+00$ & $1.973 \mathrm{E}-06$ & $2.185 \mathrm{E}+07$ & $9.2375 \mathrm{E}+00$ & $1.990 \mathrm{E}-06$ & $2.204 \mathrm{E}+07$ \\
\hline $1 s 5 s^{3} S_{1}$ & $1 s 6 f^{1} F_{3}^{o}$ & $2.4251 \mathrm{E}+01$ & $3.672 \mathrm{E}-13$ & $5.949 \mathrm{E}-01$ & & $1.030 \mathrm{C}$ & \\
\hline $1 s 5 d^{3} D_{2}$ & $1 s 6 f^{1} F_{3}^{o}$ & $2.5812 \mathrm{E}+01$ & $2.867 \mathrm{E}-08$ & $4.101 \mathrm{E}+04$ & $2.5711 \mathrm{E}+01$ & $2.878 \mathrm{E}-08$ & $4.113 \mathrm{E}+04$ \\
\hline $1 s 5 d^{3} D_{1}$ & $1 s 6 f^{1} F_{3}^{o}$ & $2.5828 \mathrm{E}+01$ & $1.581 \mathrm{E}-07$ & $2.258 \mathrm{E}+05$ & $2.5727 \mathrm{E}+01$ & $1.587 \mathrm{E}-07$ & $2.266 \mathrm{E}+05$ \\
\hline $1 s 5 d^{3} D_{3}$ & $1 s 6 f^{1} F_{3}^{o}$ & $2.6240 \mathrm{E}+01$ & $1.845 \mathrm{E}-07$ & $2.554 \mathrm{E}+05$ & $2.6136 \mathrm{E}+01$ & $1.853 \mathrm{E}-07$ & $2.562 \mathrm{E}+05$ \\
\hline $1 s 5 d{ }^{1} D_{2}$ & $1 s 6 f^{1} F_{3}^{o}$ & $2.6259 \mathrm{E}+01$ & $1.106 \mathrm{E}-06$ & $1.528 \mathrm{E}+06$ & $2.6155 \mathrm{E}+01$ & $1.107 \mathrm{E}-06$ & $1.529 \mathrm{E}+06$ \\
\hline $1 s 5 g^{3} G_{4}$ & $1 s 6 f^{1} F_{3}^{o}$ & $2.6466 \mathrm{E}+01$ & $2.632 \mathrm{E}-14$ & $3.580 \mathrm{E}-02$ & & & \\
\hline $1 s 5 g{ }^{3} G_{3}$ & $1 s 6 f^{1} F_{3}^{o}$ & $2.6471 \mathrm{E}+01$ & $3.695 \mathrm{E}-15$ & $5.025 \mathrm{E}-03$ & & & \\
\hline $1 s 5 g^{3} G_{5}$ & $1 s 6 f^{1} F_{3}^{o}$ & $2.6597 \mathrm{E}+01$ & $1.722 \mathrm{E}-09$ & $2.319 \mathrm{E}+03$ & $2.6486 \mathrm{E}+01$ & $1.729 E-09$ & $2.329 \mathrm{E}+03$ \\
\hline $1 s 5 g{ }^{1} G_{4}$ & $1 s 6 f^{1} F_{3}^{o}$ & $2.6601 \mathrm{E}+01$ & $2.865 \mathrm{E}-08$ & $3.857 \mathrm{E}+04$ & $2.6490 \mathrm{E}+01$ & $2.846 \mathrm{E}-08$ & $3.832 \mathrm{E}+04$ \\
\hline $1 s 6 s^{3} S_{1}$ & $1 s 6 f^{1} F_{3}^{o}$ & $5.0083 \mathrm{E}+02$ & $4.301 \mathrm{E}-16$ & $1.634 \mathrm{E}-06$ & & & \\
\hline $1 s 6 d^{3} D_{2}$ & $1 s 6 f^{1} F_{3}^{o}$ & $1.7871 \mathrm{E}+03$ & $6.094 \mathrm{E}-13$ & $1.818 \mathrm{E}-04$ & $1.8323 \mathrm{E}+03$ & $5.891 \mathrm{E}-13$ & $1.658 \mathrm{E}-04$ \\
\hline $1 s 6 d^{3} D_{1}$ & $1 s 6 f^{1} F_{3}^{o}$ & $1.8330 \mathrm{E}+03$ & $3.093 \mathrm{E}-12$ & $8.773 \mathrm{E}-04$ & $1.8209 \mathrm{E}+03$ & $3.120 \mathrm{E}-12$ & $8.892 \mathrm{E}-04$ \\
\hline $1 s 6 d^{3} D_{3}$ & $1 s 6 f^{1} F_{3}^{o}$ & $5.1604 \mathrm{E}+03$ & $1.585 \mathrm{E}-13$ & $5.671 \mathrm{E}-06$ & $5.5079 E+03$ & $1.287 \mathrm{E}-13$ & $4.010 \mathrm{E}-06$ \\
\hline $1 s 6 d^{1} D_{2}$ & $1 s 6 f^{1} F_{3}^{o}$ & $5.6121 \mathrm{E}+03$ & $7.375 \mathrm{E}-13$ & $2.231 \mathrm{E}-05$ & $5.6823 \mathrm{E}+03$ & $7.009 \mathrm{E}-13$ & $2.051 \mathrm{E}-05$ \\
\hline $1 s 6 g{ }^{3} G_{4}$ & $1 s 6 f^{1} F_{3}^{o}$ & $1.8628 \mathrm{E}+05$ & $3.059 \mathrm{E}-23$ & $8.401 \mathrm{E}-19$ & & & \\
\hline $1 s 6 g^{3} G_{3}$ & $1 s 6 f^{1} F_{3}^{o}$ & $9.9701 \mathrm{E}+05$ & $2.038 \mathrm{E}-26$ & $1.953 \mathrm{E}-23$ & & & \\
\hline $1 s 4 f^{3} F_{3}^{o}$ & $1 s 6 g^{3} G_{5}$ & $9.2643 \mathrm{E}+00$ & $6.142 \mathrm{E}-07$ & $4.339 \mathrm{E}+06$ & $9.2249 \mathrm{E}+00$ & $6.158 \mathrm{E}-07$ & $4.350 \mathrm{E}+06$ \\
\hline $1 s 4 f^{3} F_{4}^{o}$ & $1 s 6 g^{3} G_{5}$ & $9.3170 \mathrm{E}+00$ & $5.280 \mathrm{E}-06$ & $3.688 \mathrm{E}+07$ & $9.2773 \mathrm{E}+00$ & $5.351 \mathrm{E}-06$ & $3.738 \mathrm{E}+07$ \\
\hline $1 s 4 f^{1} F_{3}^{o}$ & $1 s 6 g^{3} G_{5}$ & $9.3187 \mathrm{E}+00$ & $2.496 \mathrm{E}-07$ & $1.743 \mathrm{E}+06$ & $9.2790 \mathrm{E}+00$ & $2.529 \mathrm{E}-07$ & $1.766 \mathrm{E}+06$ \\
\hline $1 s 5 f^{3} F_{3}^{o}$ & $1 s 6 g^{3} G_{5}$ & $2.6177 \mathrm{E}+01$ & $4.863 \mathrm{E}-07$ & $4.304 \mathrm{E}+05$ & $2.6065 E+01$ & $4.885 \mathrm{E}-07$ & $4.323 E+05$ \\
\hline $1 s 5 f^{3} F_{4}^{o}$ & $1 s 6 g^{3} G_{5}$ & $2.6394 \mathrm{E}+01$ & $4.285 \mathrm{E}-06$ & $3.730 \mathrm{E}+06$ & $2.6281 \mathrm{E}+01$ & $4.304 \mathrm{E}-06$ & $3.747 \mathrm{E}+06$ \\
\hline $1 s 5 f^{1} F_{3}^{o}$ & $1 s 6 g^{3} G_{5}$ & $2.6400 \mathrm{E}+01$ & $2.028 \mathrm{E}-07$ & $1.764 \mathrm{E}+05$ & $2.6287 \mathrm{E}+01$ & $2.035 \mathrm{E}-07$ & $1.771 \mathrm{E}+05$ \\
\hline $1 s 6 f^{3} F_{3}^{0}$ & $1 s 6 g^{3} G_{5}$ & $3.4474 \mathrm{E}+03$ & $7.054 \mathrm{E}-13$ & $3.599 \mathrm{E}-05$ & $3.4619 \mathrm{E}+03$ & $6.849 \mathrm{E}-13$ & $3.436 \mathrm{E}-05$ \\
\hline $1 s 6 f^{3} F_{4}^{o}$ & $1 s 6 g^{3} G_{5}$ & $9.2152 \mathrm{E}+03$ & $3.245 \mathrm{E}-13$ & $2.317 \mathrm{E}-06$ & $9.2775 E+03$ & $3.143 E-13$ & $2.195 \mathrm{E}-06$ \\
\hline $1 s 6 f^{1} F_{3}^{o}$ & $1 s 6 g^{3} G_{5}$ & $9.7284 \mathrm{E}+03$ & $1.305 \mathrm{E}-14$ & $8.363 \mathrm{E}-08$ & $9.2884 \mathrm{E}+03$ & $1.494 \mathrm{E}-14$ & $1.041 \mathrm{E}-07$ \\
\hline $1 s 3 d^{3} D_{3}$ & $1 s 6 h^{3} H_{5}^{o}$ & $3.8728 \mathrm{E}+00$ & $5.411 \mathrm{E}-12$ & $2.188 \mathrm{E}+02$ & & & \\
\hline $1 s 4 d^{3} D_{3}$ & $1 s 6 h^{3} H_{5}^{o}$ & $9.2626 \mathrm{E}+00$ & $5.290 \mathrm{E}-12$ & $3.739 \mathrm{E}+01$ & & & \\
\hline $1 s 5 d^{3} D_{3}$ & $1 s 6 h^{3} H_{5}^{o}$ & $2.6170 \mathrm{E}+01$ & $2.629 \mathrm{E}-13$ & $2.328 \mathrm{E}-01$ & & & \\
\hline $1 s 5 g^{3} G_{4}$ & $1 s 6 h^{3} H_{5}^{0}$ & $2.6394 \mathrm{E}+01$ & $2.528 \mathrm{E}-06$ & $2.200 \mathrm{E}+06$ & $2.6281 \mathrm{E}+01$ & $2.542 \mathrm{E}-06$ & $2.212 \mathrm{E}+06$ \\
\hline $1 s 5 g^{3} G_{3}$ & $1 s 6 h^{3} H_{5}^{0}$ & $2.6399 \mathrm{E}+01$ & $1.213 \mathrm{E}-07$ & $1.055 \mathrm{E}+05$ & $2.6286 \mathrm{E}+01$ & $1.209 \mathrm{E}-07$ & $1.052 \mathrm{E}+05$ \\
\hline $1 s 5 g^{3} G_{5}$ & $1 s 6 h^{3} H_{5}^{0}$ & $2.6525 \mathrm{E}+01$ & $1.198 \mathrm{E}-12$ & $1.032 \mathrm{E}+00$ & & & \\
\hline $1 s 5 g{ }^{1} G_{4}$ & $1 s 6 h^{3} H_{5}^{o}$ & $2.6529 \mathrm{E}+01$ & $2.458 \mathrm{E}-12$ & $2.118 \mathrm{E}+00$ & & & \\
\hline $1 s 6 d^{3} D_{3}$ & $1 s 6 h^{3} H_{5}^{o}$ & $3.3705 \mathrm{E}+03$ & $7.804 \mathrm{E}-21$ & $4.165 \mathrm{E}-13$ & & & \\
\hline $1 s 6 g^{3} G_{4}$ & $1 s 6 h^{3} H_{5}^{o}$ & $9.2359 \mathrm{E}+03$ & $7.177 \mathrm{E}-14$ & $5.102 \mathrm{E}-07$ & $9.2564 \mathrm{E}+03$ & $7.054 \mathrm{E}-14$ & $4.950 \mathrm{E}-07$ \\
\hline $1 s 6 g^{3} G_{3}$ & $1 s 6 h^{3} H_{5}^{0}$ & $9.6239 \mathrm{E}+03$ & $3.044 \mathrm{E}-15$ & $1.993 \mathrm{E}-08$ & $9.2564 \mathrm{E}+03$ & $3.359 \mathrm{E}-15$ & $2.357 \mathrm{E}-08$ \\
\hline $1 s 6 g^{3} G_{5}$ & $1 s 6 h^{3} H_{5}^{o}$ & $8.7873 \mathrm{E}+06$ & $3.968 \mathrm{E}-29$ & $3.115 \mathrm{E}-28$ & & & \\
\hline $1 s 3 d^{3} D_{2}$ & $1 s 6 h^{3} H_{4}^{0}$ & $3.8293 \mathrm{E}+00$ & $6.327 \mathrm{E}-16$ & $3.198 \mathrm{E}-02$ & & & \\
\hline $1 s 3 d^{3} D_{3}$ & $1 s 6 h^{3} H_{4}^{o}$ & $3.8727 \mathrm{E}+00$ & $3.323 \mathrm{E}-13$ & $1.642 \mathrm{E}+01$ & & & \\
\hline $1 s 3 d^{1} D_{2}$ & $1 s 6 h^{3} H_{4}^{o}$ & $3.8744 \mathrm{E}+00$ & $4.003 \mathrm{E}-12$ & $1.976 \mathrm{E}+02$ & & & \\
\hline $1 s 4 d^{3} D_{2}$ & $1 s 6 h^{3} H_{4}^{o}$ & $9.1575 \mathrm{E}+00$ & $7.295 \mathrm{E}-16$ & $6.448 \mathrm{E}-03$ & & & \\
\hline $1 s 4 d^{3} D_{3}$ & $1 s 6 h^{3} H_{4}^{0}$ & $9.2624 \mathrm{E}+00$ & $3.180 \mathrm{E}-13$ & $2.747 \mathrm{E}+00$ & & & \\
\hline $1 s 4 d^{1} D_{2}$ & $1 s 6 h^{3} H_{4}^{0}$ & $9.2667 \mathrm{E}+00$ & $3.921 \mathrm{E}-12$ & $3.384 \mathrm{E}+01$ & & & \\
\hline $1 s 5 d^{3} D_{2}$ & $1 s 6 h^{3} H_{4}^{o}$ & $2.5741 \mathrm{E}+01$ & $3.296 \mathrm{E}-17$ & $3.686 \mathrm{E}-05$ & & & \\
\hline $1 s 5 d^{3} D_{3}$ & $1 s 6 h^{3} H_{4}^{o}$ & $2.6168 \mathrm{E}+01$ & $2.500 \mathrm{E}-14$ & $2.706 \mathrm{E}-02$ & & & \\
\hline $1 s 5 d^{1} D_{2}$ & $1 s 6 h^{3} H_{4}^{0}$ & $2.6186 \mathrm{E}+01$ & $2.013 \mathrm{E}-13$ & $2.176 \mathrm{E}-01$ & & & \\
\hline $1 s 5 g^{3} G_{4}$ & $1 s 6 h^{3} H_{4}^{o}$ & $2.6392 \mathrm{E}+01$ & $1.806 \mathrm{E}-07$ & $1.922 \mathrm{E}+05$ & $2.6281 \mathrm{E}+01$ & $1.815 \mathrm{E}-07$ & $1.931 \mathrm{E}+05$ \\
\hline $1 s 5 g^{3} G_{3}$ & $1 s 6 h^{3} H_{4}^{o}$ & $2.6397 \mathrm{E}+01$ & $1.986 \mathrm{E}-06$ & $2.113 \mathrm{E}+06$ & $2.6286 \mathrm{E}+01$ & $1.996 \mathrm{E}-06$ & $2.123 \mathrm{E}+06$ \\
\hline $1 s 5 g^{3} G_{5}$ & $1 s 6 h^{3} H_{4}^{0}$ & $2.6523 \mathrm{E}+01$ & $4.039 \mathrm{E}-17$ & $4.255 \mathrm{E}-05$ & & & \\
\hline $1 s 5 g{ }^{1} G_{4}$ & $1 s 6 h^{3} H_{4}^{0}$ & $2.6527 \mathrm{E}+01$ & $6.353 \mathrm{E}-13$ & $6.691 \mathrm{E}-01$ & & & \\
\hline $1 s 6 d^{3} D_{2}$ & $1 s 6 h^{3} H_{4}^{o}$ & $1.5031 \mathrm{E}+03$ & $4.993 \mathrm{E}-24$ & $1.638 \mathrm{E}-15$ & & & \\
\hline $1 s 6 d^{3} D_{3}$ & $1 s 6 h^{3} H_{4}^{0}$ & $3.3388 \mathrm{E}+03$ & $3.254 \mathrm{E}-21$ & $2.163 \mathrm{E}-13$ & & & \\
\hline $1 s 6 d^{1} D_{2}$ & $1 s 6 h^{3} H_{4}^{0}$ & $3.5222 \mathrm{E}+03$ & $6.134 \mathrm{E}-21$ & $3.664 \mathrm{E}-13$ & & & \\
\hline $1 s 6 g^{3} G_{4}$ & $1 s 6 h^{3} H_{4}^{o}$ & $9.0013 \mathrm{E}+03$ & $5.541 \mathrm{E}-15$ & $5.068 \mathrm{E}-08$ & $9.2564 \mathrm{E}+03$ & $5.038 \mathrm{E}-15$ & $4.321 \mathrm{E}-08$ \\
\hline
\end{tabular}


Table 6 (continued)

\begin{tabular}{|c|c|c|c|c|c|c|c|}
\hline \multirow[t]{2}{*}{ Lower } & \multirow[t]{2}{*}{ Upper } & \multicolumn{3}{|l|}{ GRASP2K } & \multicolumn{3}{|l|}{ FAC } \\
\hline & & $\overline{\lambda(\AA)}$ & $g f$ & $A\left(\mathrm{~s}^{-1}\right)$ & $\lambda(\AA)$ & $g f$ & $A\left(\mathrm{~s}^{-1}\right)$ \\
\hline $1 s 6 g^{3} G_{3}$ & $1 s 6 h^{3} H_{4}^{o}$ & $9.3695 \mathrm{E}+03$ & $5.404 \mathrm{E}-14$ & $4.562 \mathrm{E}-07$ & $9.2564 \mathrm{E}+03$ & $5.542 \mathrm{E}-14$ & $4.753 \mathrm{E}-07$ \\
\hline $1 s 6 g{ }^{3} G_{5}$ & $1 s 6 h^{3} H_{4}^{o}$ & $3.4069 \mathrm{E}+05$ & $1.141 \mathrm{E}-30$ & $7.288 \mathrm{E}-27$ & & & \\
\hline $1 s 2 p^{3} P_{2}^{o}$ & $1 s 6 g{ }^{1} G_{4}$ & $1.4395 \mathrm{E}+00$ & $5.680 \mathrm{E}-15$ & $2.031 \mathrm{E}+00$ & & & \\
\hline $1 s 3 p^{3} P_{2}^{o}$ & $1 s 6 g{ }^{1} G_{4}$ & $3.8194 \mathrm{E}+00$ & $3.778 \mathrm{E}-15$ & $1.919 \mathrm{E}-01$ & & & \\
\hline $1 s 4 p^{3} P_{2}^{o}$ & $1 s 6 g{ }^{1} G_{4}$ & $9.1339 \mathrm{E}+00$ & $6.563 \mathrm{E}-14$ & $5.830 \mathrm{E}-01$ & & & \\
\hline $1 s 4 f^{3} F_{3}^{o}$ & $1 s 6 g{ }^{1} G_{4}$ & $9.2640 \mathrm{E}+00$ & $3.610 \mathrm{E}-08$ & $3.117 \mathrm{E}+05$ & $9.2249 \mathrm{E}+00$ & $3.550 \mathrm{E}-08$ & $3.065 \mathrm{E}+05$ \\
\hline $1 s 4 f^{3} F_{2}^{o}$ & $1 s 6 g{ }^{1} G_{4}$ & $9.2662 \mathrm{E}+00$ & $4.630 \mathrm{E}-07$ & $3.997 E+06$ & $9.2271 \mathrm{E}+00$ & $4.648 \mathrm{E}-07$ & $4.012 \mathrm{E}+06$ \\
\hline $1 s 4 f^{3} F_{4}^{o}$ & $1 s 6 g{ }^{1} G_{4}$ & $9.3167 \mathrm{E}+00$ & $3.774 \mathrm{E}-07$ & $3.222 \mathrm{E}+06$ & $9.2773 \mathrm{E}+00$ & $3.822 \mathrm{E}-07$ & $3.263 E+06$ \\
\hline $1 s 4 f^{1} F_{3}^{o}$ & $1 s 6 g{ }^{1} G_{4}$ & $9.3184 \mathrm{E}+00$ & $4.149 \mathrm{E}-06$ & $3.541 \mathrm{E}+07$ & $9.2790 \mathrm{E}+00$ & $4.203 \mathrm{E}-06$ & $3.587 \mathrm{E}+07$ \\
\hline $1 s 5 p^{3} P_{2}^{0}$ & $1 s 6 g{ }^{1} G_{4}$ & $2.5647 \mathrm{E}+01$ & $7.477 \mathrm{E}-14$ & $8.425 \mathrm{E}-02$ & & & \\
\hline $1 s 5 f^{3} F_{3}^{o}$ & $1 s 6 g{ }^{1} G_{4}$ & $2.6175 E+01$ & $2.866 \mathrm{E}-08$ & $3.100 \mathrm{E}+04$ & $2.6065 \mathrm{E}+01$ & $2.825 \mathrm{E}-08$ & $3.055 \mathrm{E}+04$ \\
\hline $1 s 5 f^{3} F_{2}^{o}$ & $1 s 6 g{ }^{1} G_{4}$ & $2.6184 \mathrm{E}+01$ & $3.669 \mathrm{E}-07$ & $3.966 \mathrm{E}+05$ & $2.6074 \mathrm{E}+01$ & $3.687 \mathrm{E}-07$ & $3.985 \mathrm{E}+05$ \\
\hline $1 s 5 f^{3} F_{4}^{o}$ & $1 s 6 g{ }^{1} G_{4}$ & $2.6391 \mathrm{E}+01$ & $3.061 \mathrm{E}-07$ & $3.257 \mathrm{E}+05$ & $2.6281 \mathrm{E}+01$ & $3.074 \mathrm{E}-07$ & $3.271 \mathrm{E}+05$ \\
\hline $1 s 5 f^{1} F_{3}^{o}$ & $1 s 6 g{ }^{1} G_{4}$ & $2.6398 \mathrm{E}+01$ & $3.368 \mathrm{E}-06$ & $3.582 \mathrm{E}+06$ & $2.6287 \mathrm{E}+01$ & $3.380 \mathrm{E}-06$ & $3.595 \mathrm{E}+06$ \\
\hline $1 s 6 p^{3} P_{2}^{o}$ & $1 s 6 g{ }^{1} G_{4}$ & $1.3363 E+03$ & $2.421 \mathrm{E}-18$ & $1.005 \mathrm{E}-09$ & & & \\
\hline $1 s 6 f^{3} F_{3}^{o}$ & $1 s 6 g{ }^{1} G_{4}$ & $3.4068 \mathrm{E}+03$ & $4.316 \mathrm{E}-14$ & $2.756 \mathrm{E}-06$ & $3.4619 \mathrm{E}+03$ & $4.205 E-14$ & $2.578 \mathrm{E}-06$ \\
\hline $1 s 6 f^{3} F_{2}^{o}$ & $1 s 6 g{ }^{1} G_{4}$ & $3.4975 E+03$ & $5.097 \mathrm{E}-13$ & $3.088 \mathrm{E}-05$ & $3.4608 \mathrm{E}+03$ & $5.197 \mathrm{E}-13$ & $3.188 \mathrm{E}-05$ \\
\hline $1 s 6 f^{3} F_{4}^{o}$ & $1 s 6 g{ }^{1} G_{4}$ & $8.9311 \mathrm{E}+03$ & $2.547 \mathrm{E}-14$ & $2.366 \mathrm{E}-07$ & $9.2773 \mathrm{E}+03$ & $2.245 \mathrm{E}-14$ & $1.917 \mathrm{E}-07$ \\
\hline $1 s 6 f^{1} F_{3}^{o}$ & $1 s 6 g{ }^{1} G_{4}$ & $9.4124 \mathrm{E}+03$ & $2.394 \mathrm{E}-13$ & $2.002 E-06$ & $9.2882 \mathrm{E}+03$ & $2.460 \mathrm{E}-13$ & $2.096 \mathrm{E}-06$ \\
\hline $1 s 6 h^{3} H_{5}^{o}$ & $1 s 6 g{ }^{1} G_{4}$ & $2.9962 \mathrm{E}+05$ & $2.062 \mathrm{E}-24$ & $1.703 \mathrm{E}-20$ & & & \\
\hline $1 s 6 h^{3} H_{4}^{0}$ & $1 s 6 g{ }^{1} G_{4}$ & $1.9372 \mathrm{E}+06$ & $1.973 \mathrm{E}-27$ & $3.896 \mathrm{E}-25$ & & & \\
\hline $1 s 5 g^{3} G_{4}$ & $1 s 6 h^{3} H_{6}^{o}$ & $2.6344 \mathrm{E}+01$ & $8.894 \mathrm{E}-07$ & $6.576 \mathrm{E}+05$ & $2.6232 \mathrm{E}+01$ & $8.917 \mathrm{E}-07$ & $6.593 \mathrm{E}+05$ \\
\hline $1 s 5 g^{3} G_{5}$ & $1 s 6 h^{3} H_{6}^{o}$ & $2.6474 \mathrm{E}+01$ & $1.048 \mathrm{E}-05$ & $7.674 \mathrm{E}+06$ & $2.6361 \mathrm{E}+01$ & $1.054 \mathrm{E}-05$ & $7.716 \mathrm{E}+06$ \\
\hline $1 s 5 g{ }^{1} G_{4}$ & $1 s 6 h^{3} H_{6}^{o}$ & $2.6478 \mathrm{E}+01$ & $3.306 \mathrm{E}-07$ & $2.420 \mathrm{E}+05$ & $2.6365 \mathrm{E}+01$ & $3.324 \mathrm{E}-07$ & $2.432 \mathrm{E}+05$ \\
\hline $1 s 6 g^{3} G_{4}$ & $1 s 6 h^{3} H_{6}^{0}$ & $5.5520 \mathrm{E}+03$ & $1.164 \mathrm{E}-13$ & $1.938 \mathrm{E}-06$ & $5.5614 \mathrm{E}+03$ & $1.139 \mathrm{E}-13$ & $1.874 \mathrm{E}-06$ \\
\hline $1 s 6 g^{3} G_{5}$ & $1 s 6 h^{3} H_{6}^{o}$ & $1.3897 \mathrm{E}+04$ & $8.779 \mathrm{E}-14$ & $2.332 \mathrm{E}-07$ & $1.3933 \mathrm{E}+04$ & $8.611 \mathrm{E}-14$ & $2.256 \mathrm{E}-07$ \\
\hline $1 s 6 g{ }^{1} G_{4}$ & $1 s 6 h^{3} H_{6}^{o}$ & $1.4598 \mathrm{E}+04$ & $2.390 \mathrm{E}-15$ & $5.755 \mathrm{E}-09$ & $1.3934 \mathrm{E}+04$ & $2.733 \mathrm{E}-15$ & $7.163 \mathrm{E}-09$ \\
\hline $1 s 3 d^{3} D_{3}$ & $1 s 6 h^{1} H_{5}^{o}$ & $3.8717 \mathrm{E}+00$ & $4.245 \mathrm{E}-16$ & $1.717 \mathrm{E}-02$ & & & \\
\hline $1 s 4 d^{3} D_{3}$ & $1 s 6 h{ }^{1} H_{5}^{o}$ & $9.2562 \mathrm{E}+00$ & $4.469 \mathrm{E}-16$ & $3.163 \mathrm{E}-03$ & & & \\
\hline $1 s 5 d^{3} D_{3}$ & $1 s 6 h^{1} H_{5}^{o}$ & $2.6119 \mathrm{E}+01$ & $2.181 \mathrm{E}-16$ & $1.939 \mathrm{E}-04$ & & & \\
\hline $1 s 5 g^{3} G_{4}$ & $1 s 6 h^{1} H_{5}^{o}$ & $2.6342 \mathrm{E}+01$ & $3.299 \mathrm{E}-08$ & $2.883 \mathrm{E}+04$ & $2.6232 \mathrm{E}+01$ & $3.216 \mathrm{E}-08$ & $2.810 \mathrm{E}+04$ \\
\hline $1 s 5 g^{3} G_{3}$ & $1 s 6 h{ }^{1} H_{5}^{o}$ & $2.6348 \mathrm{E}+01$ & $7.157 \mathrm{E}-07$ & $6.251 E+05$ & $2.6237 \mathrm{E}+01$ & $7.181 \mathrm{E}-07$ & $6.272 \mathrm{E}+05$ \\
\hline $1 s 5 g^{3} G_{5}$ & $1 s 6 h^{1} H_{5}^{o}$ & $2.6473 E+01$ & $4.992 \mathrm{E}-07$ & $4.320 \mathrm{E}+05$ & $2.6361 \mathrm{E}+01$ & $5.019 \mathrm{E}-07$ & $4.342 \mathrm{E}+05$ \\
\hline $1 s 5 g{ }^{1} G_{4}$ & $1 s 6 h^{1} H_{5}^{o}$ & $2.6477 \mathrm{E}+01$ & $8.653 \mathrm{E}-06$ & $7.485 E+06$ & $2.6365 \mathrm{E}+01$ & 8.697E-06 & $7.523 E+06$ \\
\hline $1 s 6 d^{3} D_{3}$ & $1 s 6 h{ }^{1} H_{5}^{o}$ & $2.6964 \mathrm{E}+03$ & $7.459 \mathrm{E}-22$ & $6.221 \mathrm{E}-14$ & & & \\
\hline $1 s 6 g^{3} G_{4}$ & $1 s 6 h{ }^{1} H_{5}^{o}$ & $5.4809 E+03$ & $4.490 \mathrm{E}-15$ & $9.064 \mathrm{E}-08$ & $5.5614 \mathrm{E}+03$ & $4.383 \mathrm{E}-15$ & $8.520 \mathrm{E}-08$ \\
\hline $1 s 6 g{ }^{3} G_{3}$ & $1 s 6 h^{1} H_{5}^{o}$ & $5.6153 \mathrm{E}+03$ & $9.058 \mathrm{E}-14$ & $1.742 \mathrm{E}-06$ & $5.5614 \mathrm{E}+03$ & $9.204 \mathrm{E}-14$ & $1.789 \mathrm{E}-06$ \\
\hline $1 s 6 g^{3} G_{5}$ & $1 s 6 h^{1} H_{5}^{o}$ & $1.3461 \mathrm{E}+04$ & $4.602 \mathrm{E}-15$ & $1.540 \mathrm{E}-08$ & $1.3933 \mathrm{E}+04$ & $4.100 \mathrm{E}-15$ & $1.269 \mathrm{E}-08$ \\
\hline $1 s 6 g{ }^{1} G_{4}$ & $1 s 6 h{ }^{1} H_{5}^{o}$ & $1.4116 \mathrm{E}+04$ & $6.916 \mathrm{E}-14$ & $2.105 \mathrm{E}-07$ & $1.3934 \mathrm{E}+04$ & $7.107 \mathrm{E}-14$ & $2.201 \mathrm{E}-07$ \\
\hline $1 s 2 p^{3} P_{1}^{o}$ & $1 s 7 s^{3} s_{1}$ & $1.3378 \mathrm{E}+00$ & $7.061 \mathrm{E}-11$ & $8.772 E+04$ & $1.3323 \mathrm{E}+00$ & $1.617 \mathrm{E}-10$ & $2.008 \mathrm{E}+05$ \\
\hline $1 s 2 p^{3} P_{2}^{o}$ & $1 s 7 s^{3} S_{1}$ & $1.3964 \mathrm{E}+00$ & $8.788 \mathrm{E}-08$ & $1.002 \mathrm{E}+08$ & $1.3905 \mathrm{E}+00$ & $8.717 \mathrm{E}-08$ & $9.938 \mathrm{E}+07$ \\
\hline $1 s 2 p^{1} P_{1}^{o}$ & $1 s 7 s^{3} S_{1}$ & $1.4019 \mathrm{E}+00$ & $9.183 \mathrm{E}-08$ & $1.039 \mathrm{E}+08$ & $1.3961 \mathrm{E}+00$ & $8.599 \mathrm{E}-08$ & $9.725 \mathrm{E}+07$ \\
\hline $1 s 3 p^{3} P_{1}^{o}$ & $1 s 7 s^{3} S_{1}$ & $3.4180 \mathrm{E}+00$ & $6.412 \mathrm{E}-11$ & $1.220 \mathrm{E}+04$ & $3.4039 \mathrm{E}+00$ & $8.987 \mathrm{E}-11$ & $1.710 \mathrm{E}+04$ \\
\hline $1 s 3 p^{3} P_{2}^{o}$ & $1 s 7 s^{3} S_{1}$ & $3.5305 E+00$ & $5.822 \mathrm{E}-08$ & $1.038 \mathrm{E}+07$ & $3.5159 \mathrm{E}+00$ & $5.726 \mathrm{E}-08$ & $1.021 \mathrm{E}+07$ \\
\hline $1 s 3 p^{1} P_{1}^{o}$ & $1 s 7 s^{3} S_{1}$ & $3.5405 E+00$ & $5.893 \mathrm{E}-08$ & $1.045 \mathrm{E}+07$ & $3.5260 \mathrm{E}+00$ & $5.669 \mathrm{E}-08$ & $1.005 \mathrm{E}+07$ \\
\hline $1 s 4 p^{3} P_{1}^{o}$ & $1 s 7 s^{3} s_{1}$ & $7.4166 \mathrm{E}+00$ & $4.198 \mathrm{E}-11$ & $1.697 \mathrm{E}+03$ & $7.3859 \mathrm{E}+00$ & $5.786 \mathrm{E}-11$ & $2.338 \mathrm{E}+03$ \\
\hline $1 s 4 p^{3} P_{2}^{o}$ & $1 s 7 s^{3} S_{1}$ & $7.6388 \mathrm{E}+00$ & $3.929 \mathrm{E}-08$ & $1.497 \mathrm{E}+06$ & $7.6070 \mathrm{E}+00$ & $3.893 \mathrm{E}-08$ & $1.483 \mathrm{E}+06$ \\
\hline $1 s 4 p{ }^{1} P_{1}^{o}$ & $1 s 7 s^{3} S_{1}$ & $7.6581 \mathrm{E}+00$ & $3.974 \mathrm{E}-08$ & $1.507 \mathrm{E}+06$ & $7.6265 \mathrm{E}+00$ & $3.858 \mathrm{E}-08$ & $1.462 \mathrm{E}+06$ \\
\hline $1 s 4 f^{3} F_{3}^{o}$ & $1 s 7 s^{3} S_{1}$ & $7.7295 \mathrm{E}+00$ & $3.020 \mathrm{E}-15$ & $1.124 \mathrm{E}-01$ & & & \\
\hline $1 s 4 f^{3} F_{2}^{o}$ & $1 s 7 s^{3} S_{1}$ & $7.7310 \mathrm{E}+00$ & $4.463 \mathrm{E}-16$ & $1.660 \mathrm{E}-02$ & & & \\
\hline $1 s 4 f^{1} F_{3}^{o}$ & $1 s 7 s^{3} S_{1}$ & $7.7674 \mathrm{E}+00$ & $9.051 \mathrm{E}-15$ & $3.336 \mathrm{E}-01$ & & & \\
\hline $1 s 5 p^{3} P_{1}^{0}$ & $1 s 7 s^{3} S_{1}$ & $1.6020 \mathrm{E}+01$ & $2.993 \mathrm{E}-11$ & $2.593 \mathrm{E}+02$ & $1.5955 \mathrm{E}+01$ & $4.220 \mathrm{E}-11$ & $3.655 \mathrm{E}+02$ \\
\hline $1 s 5 p^{3} P_{2}^{0}$ & $1 s 7 s^{3} S_{1}$ & $1.6551 \mathrm{E}+01$ & $2.882 \mathrm{E}-08$ & $2.340 \mathrm{E}+05$ & $1.6483 \mathrm{E}+01$ & $2.871 \mathrm{E}-08$ & $2.329 \mathrm{E}+05$ \\
\hline $1 s 5 p{ }^{1} P_{1}^{o}$ & $1 s 7 s^{3} S_{1}$ & $1.6597 \mathrm{E}+01$ & $2.919 \mathrm{E}-08$ & $2.356 \mathrm{E}+05$ & $1.6529 \mathrm{E}+01$ & $2.843 \mathrm{E}-08$ & $2.294 \mathrm{E}+05$ \\
\hline $1 s 5 f^{3} F_{3}^{o}$ & $1 s 7 s^{3} S_{1}$ & $1.6769 \mathrm{E}+01$ & $2.832 \mathrm{E}-15$ & $2.239 \mathrm{E}-02$ & & & \\
\hline $1 s 5 f^{3} F_{2}^{o}$ & $1 s 7 s^{3} S_{1}$ & $1.6773 \mathrm{E}+01$ & $2.941 \mathrm{E}-17$ & $2.324 \mathrm{E}-04$ & & & \\
\hline $1 s 5 f^{1} F_{3}^{o}$ & $1 s 7 s^{3} S_{1}$ & $1.6860 \mathrm{E}+01$ & $1.476 \mathrm{E}-14$ & $1.154 \mathrm{E}-01$ & & & \\
\hline $1 s 6 p^{3} P_{1}^{o}$ & $1 s 7 s^{3} S_{1}$ & $4.2860 \mathrm{E}+01$ & $2.300 \mathrm{E}-11$ & $2.784 \mathrm{E}+01$ & $4.2690 \mathrm{E}+01$ & $5.831 \mathrm{E}-11$ & $7.054 \mathrm{E}+01$ \\
\hline $1 s 6 p^{3} P_{2}^{o}$ & $1 s 7 s^{3} S_{1}$ & $4.5091 \mathrm{E}+01$ & $2.306 \mathrm{E}-08$ & $2.522 E+04$ & $4.4909 \mathrm{E}+01$ & $2.285 \mathrm{E}-08$ & $2.498 \mathrm{E}+04$ \\
\hline $1 s 6 p^{1} P_{1}^{o}$ & $1 s 7 s^{3} S_{1}$ & $4.5287 \mathrm{E}+01$ & $2.341 \mathrm{E}-08$ & $2.537 \mathrm{E}+04$ & $4.5084 \mathrm{E}+01$ & $2.254 \mathrm{E}-08$ & $2.444 \mathrm{E}+04$ \\
\hline $1 s 6 f^{3} F_{3}^{o}$ & $1 s 7 s^{3} S_{1}$ & $4.6035 \mathrm{E}+01$ & $2.643 \mathrm{E}-15$ & $2.773 \mathrm{E}-03$ & & & \\
\hline $1 s 6 f^{3} F_{2}^{o}$ & $1 s 7 s^{3} S_{1}$ & $4.6051 \mathrm{E}+01$ & $1.251 \mathrm{E}-16$ & $1.312 \mathrm{E}-04$ & & & \\
\hline $1 s 6 f^{1} F_{3}^{o}$ & $1 s 7 s^{3} S_{1}$ & $4.6435 \mathrm{E}+01$ & $1.966 \mathrm{E}-14$ & $2.027 \mathrm{E}-02$ & & & \\
\hline $1 s 2 s^{3} S_{1}$ & $1 s 7 p^{3} P_{1}^{o}$ & $1.3266 \mathrm{E}+00$ & $9.923 \mathrm{E}-10$ & $1.254 \mathrm{E}+06$ & $1.3210 \mathrm{E}+00$ & $1.573 \mathrm{E}-09$ & $1.987 \mathrm{E}+06$ \\
\hline $1 s 3 s^{3} S_{1}$ & $1 s 7 p^{3} P_{1}^{o}$ & $3.3968 \mathrm{E}+00$ & $3.292 \mathrm{E}-10$ & $6.343 E+04$ & $3.3827 \mathrm{E}+00$ & $4.928 \mathrm{E}-10$ & $9.495 \mathrm{E}+04$ \\
\hline $1 s 3 d^{3} D_{2}$ & $1 s 7 p^{3} P_{1}^{o}$ & $3.5373 E+00$ & $5.806 \mathrm{E}-10$ & $1.032 \mathrm{E}+05$ & $3.5225 \mathrm{E}+00$ & $5.732 \mathrm{E}-10$ & $1.018 \mathrm{E}+05$ \\
\hline $1 s 3 d^{3} D_{1}$ & $1 s 7 p^{3} P_{1}^{0}$ & $3.5388 \mathrm{E}+00$ & $5.924 \mathrm{E}-10$ & $1.052 E+05$ & $3.5240 \mathrm{E}+00$ & $5.632 \mathrm{E}-10$ & $9.998 \mathrm{E}+04$ \\
\hline $1 s 3 d^{3} D_{3}$ & $1 s 7 p^{3} P_{1}^{o}$ & $3.5743 \mathrm{E}+00$ & $9.720 \mathrm{E}-09$ & $1.692 \mathrm{E}+06$ & $3.5593 \mathrm{E}+00$ & $9.462 \mathrm{E}-09$ & $1.646 \mathrm{E}+06$ \\
\hline $1 s 3 d^{1} D_{2}$ & $1 s 7 p^{3} P_{1}^{o}$ & $3.5757 \mathrm{E}+00$ & $3.723 \mathrm{E}-09$ & $6.474 \mathrm{E}+05$ & $3.5607 \mathrm{E}+00$ & $3.624 \mathrm{E}-09$ & $6.301 \mathrm{E}+05$ \\
\hline $1 s 4 s^{3} S_{1}$ & $1 s 7 p^{3} P_{1}^{o}$ & $7.3716 \mathrm{E}+00$ & $1.514 \mathrm{E}-10$ & $6.195 E+03$ & $7.3412 \mathrm{E}+00$ & $2.184 \mathrm{E}-10$ & $8.937 \mathrm{E}+03$ \\
\hline $1 s 4 d^{3} D_{2}$ & $1 s 7 p^{3} P_{1}^{0}$ & $7.6476 \mathrm{E}+00$ & $6.420 \mathrm{E}-10$ & $2.441 \mathrm{E}+04$ & $7.6163 \mathrm{E}+00$ & $6.362 \mathrm{E}-10$ & $2.418 \mathrm{E}+04$ \\
\hline
\end{tabular}


Table 6 (continued)

\begin{tabular}{|c|c|c|c|c|c|c|c|}
\hline \multirow[t]{2}{*}{ Lower } & \multirow[t]{2}{*}{ Upper } & \multicolumn{3}{|l|}{ GRASP2K } & \multicolumn{3}{|l|}{ FAC } \\
\hline & & $\lambda(\AA)$ & $g f$ & $A\left(\mathrm{~s}^{-1}\right)$ & $\lambda(\AA)$ & $g f$ & $A\left(\mathrm{~s}^{-1}\right)$ \\
\hline $1 s 4 d^{3} D_{1}$ & $1 s 7 p^{3} P_{1}^{o}$ & $7.6504 \mathrm{E}+00$ & $6.386 \mathrm{E}-10$ & $2.426 \mathrm{E}+04$ & $7.6191 \mathrm{E}+00$ & $6.185 \mathrm{E}-10$ & $2.348 \mathrm{E}+04$ \\
\hline $1 s 4 d^{3} D_{3}$ & $1 s 7 p^{3} P_{1}^{o}$ & $7.7206 \mathrm{E}+00$ & $1.056 \mathrm{E}-08$ & $3.939 E+05$ & $7.6888 \mathrm{E}+00$ & $1.031 \mathrm{E}-08$ & $3.845 \mathrm{E}+05$ \\
\hline $1 s 4 d{ }^{1} D_{2}$ & $1 s 7 p^{3} P_{1}^{o}$ & $7.7236 \mathrm{E}+00$ & $4.009 \mathrm{E}-09$ & $1.494 \mathrm{E}+05$ & $7.6918 \mathrm{E}+00$ & $3.961 \mathrm{E}-09$ & $1.476 \mathrm{E}+05$ \\
\hline $1 s 5 s^{3} S_{1}$ & $1 s 7 p^{3} P_{1}^{o}$ & $1.5898 \mathrm{E}+01$ & $8.382 \mathrm{E}-11$ & $7.373 \mathrm{E}+02$ & $1.5833 \mathrm{E}+01$ & $1.150 \mathrm{E}-10$ & $1.011 \mathrm{E}+03$ \\
\hline $1 s 5 d^{3} D_{2}$ & $1 s 7 p^{3} P_{1}^{o}$ & $1.6554 \mathrm{E}+01$ & $6.141 \mathrm{E}-10$ & $4.983 \mathrm{E}+03$ & $1.6489 \mathrm{E}+01$ & $6.088 \mathrm{E}-10$ & $4.936 \mathrm{E}+03$ \\
\hline $1 s 5 d^{3} D_{1}$ & $1 s 7 p^{3} P_{1}^{o}$ & $1.6561 \mathrm{E}+01$ & $6.033 \mathrm{E}-10$ & $4.891 \mathrm{E}+03$ & $1.6495 \mathrm{E}+01$ & $5.889 \mathrm{E}-10$ & $4.771 \mathrm{E}+03$ \\
\hline $1 s 5 d^{3} D_{3}$ & $1 s 7 p^{3} P_{1}^{o}$ & $1.6729 \mathrm{E}+01$ & $1.001 \mathrm{E}-08$ & $7.953 \mathrm{E}+04$ & $1.6663 \mathrm{E}+01$ & $9.785 \mathrm{E}-09$ & $7.770 \mathrm{E}+04$ \\
\hline $1 s 5 d^{1} D_{2}$ & $1 s 7 p^{3} P_{1}^{o}$ & $1.6737 \mathrm{E}+01$ & $3.776 \mathrm{E}-09$ & $2.997 \mathrm{E}+04$ & $1.6670 \mathrm{E}+01$ & $3.758 \mathrm{E}-09$ & $2.981 \mathrm{E}+04$ \\
\hline $1 s 5 g^{3} G_{3}$ & $1 s 7 p^{3} P_{1}^{o}$ & $1.6823 \mathrm{E}+01$ & $5.398 \mathrm{E}-18$ & $4.241 \mathrm{E}-05$ & & & \\
\hline $1 s 6 s^{3} S_{1}$ & $1 s 7 p^{3} P_{1}^{o}$ & $4.2260 \mathrm{E}+01$ & $5.061 \mathrm{E}-11$ & $6.301 \mathrm{E}+01$ & $4.2057 \mathrm{E}+01$ & $6.306 \mathrm{E}-11$ & $7.859 \mathrm{E}+01$ \\
\hline $1 s 6 d^{3} D_{2}$ & $1 s 7 p^{3} P_{1}^{o}$ & $4.4993 \mathrm{E}+01$ & $5.884 \mathrm{E}-10$ & $6.463 E+02$ & $4.4844 \mathrm{E}+01$ & $5.978 \mathrm{E}-10$ & $6.553 \mathrm{E}+02$ \\
\hline $1 s 6 d^{3} D_{1}$ & $1 s 7 p^{3} P_{1}^{o}$ & $4.5021 \mathrm{E}+01$ & $5.741 \mathrm{E}-10$ & $6.298 \mathrm{E}+02$ & $4.4837 \mathrm{E}+01$ & $5.631 \mathrm{E}-10$ & $6.175 E+02$ \\
\hline $1 s 6 d^{3} D_{3}$ & $1 s 7 p^{3} P_{1}^{o}$ & $4.5746 \mathrm{E}+01$ & $9.580 \mathrm{E}-09$ & $1.018 \mathrm{E}+04$ & $4.5589 \mathrm{E}+01$ & $9.401 \mathrm{E}-09$ & $9.972 \mathrm{E}+03$ \\
\hline $1 s 6 d{ }^{1} D_{2}$ & $1 s 7 p^{3} P_{1}^{o}$ & $4.5778 \mathrm{E}+01$ & $3.602 \mathrm{E}-09$ & $3.821 \mathrm{E}+03$ & $4.5600 \mathrm{E}+01$ & $3.564 \mathrm{E}-09$ & $3.779 \mathrm{E}+03$ \\
\hline $1 s 6 g^{3} G_{3}$ & $1 s 7 p^{3} P_{1}^{o}$ & $4.6153 \mathrm{E}+01$ & $1.003 \mathrm{E}-17$ & $1.047 \mathrm{E}-05$ & & & \\
\hline $1 s 7 s^{3} S_{1}$ & $1 s 7 p^{3} P_{1}^{o}$ & $7.6504 \mathrm{E}+03$ & $1.709 \mathrm{E}-16$ & $6.493 \mathrm{E}-09$ & $7.3461 \mathrm{E}+03$ & $3.329 E-16$ & $1.360 \mathrm{E}-08$ \\
\hline $1 s 2 p^{3} P_{2}^{o}$ & $1 s 7 s^{1} S_{0}$ & $1.3961 \mathrm{E}+00$ & $5.700 \mathrm{E}-08$ & $1.951 \mathrm{E}+08$ & $1.3903 \mathrm{E}+00$ & $5.814 \mathrm{E}-08$ & $1.989 \mathrm{E}+08$ \\
\hline $1 s 3 p^{3} P_{2}^{o}$ & $1 s 7 s^{1} S_{0}$ & $3.5288 \mathrm{E}+00$ & $3.806 \mathrm{E}-08$ & $2.038 \mathrm{E}+07$ & $3.5145 \mathrm{E}+00$ & $3.822 \mathrm{E}-08$ & $2.046 \mathrm{E}+07$ \\
\hline $1 s 4 p^{3} P_{2}^{o}$ & $1 s 7 s^{1} S_{0}$ & $7.6311 \mathrm{E}+00$ & $2.568 \mathrm{E}-08$ & $2.941 \mathrm{E}+06$ & $7.6005 \mathrm{E}+00$ & $2.602 \mathrm{E}-08$ & $2.979 \mathrm{E}+06$ \\
\hline $1 s 4 f^{3} F_{2}^{o}$ & $1 s 7 s{ }^{1} S_{0}$ & $7.7232 \mathrm{E}+00$ & $2.042 \mathrm{E}-15$ & $2.284 \mathrm{E}-01$ & & & \\
\hline $1 s 5 p^{3} P_{2}^{o}$ & $1 s 7 s^{1} S_{0}$ & $1.6515 \mathrm{E}+01$ & $1.876 \mathrm{E}-08$ & $4.589 \mathrm{E}+05$ & $1.6452 \mathrm{E}+01$ & $1.924 \mathrm{E}-08$ & $4.702 \mathrm{E}+05$ \\
\hline $1 s 5 f^{3} F_{2}^{o}$ & $1 s 7 s^{1} S_{0}$ & $1.6736 \mathrm{E}+01$ & $3.426 \mathrm{E}-15$ & $8.160 \mathrm{E}-02$ & & & \\
\hline $1 s 6 p^{3} P_{2}^{o}$ & $1 s 7 s^{1} S_{0}$ & $4.4825 \mathrm{E}+01$ & $1.486 \mathrm{E}-08$ & $4.934 \mathrm{E}+04$ & $4.4685 \mathrm{E}+01$ & $1.546 \mathrm{E}-08$ & $5.123 \mathrm{E}+04$ \\
\hline $1 s 6 f^{3} F_{2}^{o}$ & $1 s 7 s^{1} S_{0}$ & $4.5774 \mathrm{E}+01$ & $4.081 \mathrm{E}-15$ & $1.299 \mathrm{E}-02$ & & & \\
\hline $1 s 3 d^{3} D_{2}$ & $1 s 7 p^{3} P_{0}^{o}$ & $3.5372 \mathrm{E}+00$ & $3.772 \mathrm{E}-10$ & $2.011 \mathrm{E}+05$ & $3.5228 \mathrm{E}+00$ & $3.663 \mathrm{E}-10$ & $1.952 \mathrm{E}+05$ \\
\hline $1 s 3 d d^{1} D_{2}$ & $1 s 7 p^{3} P_{0}^{o}$ & $3.5756 \mathrm{E}+00$ & $4.547 \mathrm{E}-09$ & $2.372 \mathrm{E}+06$ & $3.5610 \mathrm{E}+00$ & $4.361 \mathrm{E}-09$ & $2.274 \mathrm{E}+06$ \\
\hline $1 s 4 d^{3} D_{2}$ & $1 s 7 p^{3} P_{0}^{o}$ & $7.6470 \mathrm{E}+00$ & $4.064 \mathrm{E}-10$ & $4.636 \mathrm{E}+04$ & $7.6177 \mathrm{E}+00$ & $3.968 \mathrm{E}-10$ & $4.522 \mathrm{E}+04$ \\
\hline $1 s 4 d^{1} D_{2}$ & $1 s 7 p^{3} P_{0}^{o}$ & $7.7230 \mathrm{E}+00$ & $4.905 \mathrm{E}-09$ & $5.486 \mathrm{E}+05$ & $7.6932 \mathrm{E}+00$ & $4.762 \mathrm{E}-09$ & $5.322 \mathrm{E}+05$ \\
\hline $1 s 5 d^{3} D_{2}$ & $1 s 7 p^{3} P_{0}^{o}$ & $1.6552 \mathrm{E}+01$ & $3.834 \mathrm{E}-10$ & $9.335 \mathrm{E}+03$ & $1.6495 \mathrm{E}+01$ & $3.754 \mathrm{E}-10$ & $9.126 \mathrm{E}+03$ \\
\hline $1 s 5 d{ }^{1} D_{2}$ & $1 s 7 p^{3} P_{0}^{o}$ & $1.6734 \mathrm{E}+01$ & $4.624 \mathrm{E}-09$ & $1.101 \mathrm{E}+05$ & $1.6677 \mathrm{E}+01$ & $4.518 \mathrm{E}-09$ & $1.074 \mathrm{E}+05$ \\
\hline $1 s 6 d^{3} D_{2}$ & $1 s 7 p^{3} P_{0}^{o}$ & $4.4975 \mathrm{E}+01$ & $3.644 \mathrm{E}-10$ & $1.202 \mathrm{E}+03$ & $4.4891 \mathrm{E}+01$ & $3.446 \mathrm{E}-10$ & $1.131 \mathrm{E}+03$ \\
\hline $1 s 6 d{ }^{1} D_{2}$ & $1 s 7 p^{3} P_{0}^{o}$ & $4.5759 \mathrm{E}+01$ & $4.411 \mathrm{E}-09$ & $1.405 \mathrm{E}+04$ & $4.5649 \mathrm{E}+01$ & $4.337 \mathrm{E}-09$ & $1.376 \mathrm{E}+04$ \\
\hline $1 s^{2}{ }^{1} S_{0}$ & $1 s 7 p^{3} P_{2}^{o}$ & $3.1400 \mathrm{E}-01$ & $6.200 \mathrm{E}-06$ & $8.387 \mathrm{E}+10$ & $3.1270 \mathrm{E}-01$ & $6.930 \mathrm{E}-06$ & $9.375 \mathrm{E}+10$ \\
\hline $1 s 2 s^{3} S_{1}$ & $1 s 7 p^{3} P_{2}^{o}$ & $1.3253 \mathrm{E}+00$ & $7.079 \mathrm{E}-07$ & $5.377 \mathrm{E}+08$ & $1.3197 \mathrm{E}+00$ & $7.260 \mathrm{E}-07$ & $5.513 \mathrm{E}+08$ \\
\hline $1 s 2 s{ }^{1} S_{0}$ & $1 s 7 p^{3} P_{2}^{o}$ & $1.3374 \mathrm{E}+00$ & $4.714 \mathrm{E}-07$ & $3.516 \mathrm{E}+08$ & $1.3320 \mathrm{E}+00$ & $4.707 \mathrm{E}-07$ & $3.509 \mathrm{E}+08$ \\
\hline $1 s 3 s^{3} S_{1}$ & $1 s 7 p^{3} P_{2}^{o}$ & $3.3884 \mathrm{E}+00$ & $2.267 \mathrm{E}-07$ & $2.634 \mathrm{E}+07$ & $3.3744 \mathrm{E}+00$ & $2.291 \mathrm{E}-07$ & $2.661 \mathrm{E}+07$ \\
\hline $1 s 3 s^{1} S_{0}$ & $1 s 7 p^{3} P_{2}^{o}$ & $3.4094 \mathrm{E}+00$ & $1.548 \mathrm{E}-07$ & $1.776 \mathrm{E}+07$ & $3.3955 E+00$ & $1.499 \mathrm{E}-07$ & $1.720 \mathrm{E}+07$ \\
\hline $1 s 3 d^{3} D_{2}$ & $1 s 7 p^{3} P_{2}^{o}$ & $3.5282 \mathrm{E}+00$ & $1.202 \mathrm{E}-17$ & $1.288 \mathrm{E}-03$ & & & \\
\hline $1 s 3 d^{3} D_{1}$ & $1 s 7 p^{3} P_{2}^{o}$ & $3.5297 \mathrm{E}+00$ & $2.420 \mathrm{E}-13$ & $2.591 \mathrm{E}+01$ & & & \\
\hline $1 s 3 d^{3} D_{3}$ & $1 s 7 p^{3} P_{2}^{o}$ & $3.5650 \mathrm{E}+00$ & $3.454 \mathrm{E}-08$ & $3.626 \mathrm{E}+06$ & $3.5501 \mathrm{E}+00$ & $3.333 \mathrm{E}-08$ & $3.499 \mathrm{E}+06$ \\
\hline $1 s 3 d d^{1} D_{2}$ & $1 s 7 p^{3} P_{2}^{o}$ & $3.5665 \mathrm{E}+00$ & $8.900 E-09$ & $9.335 \mathrm{E}+05$ & $3.5515 \mathrm{E}+00$ & $8.324 \mathrm{E}-09$ & $8.730 \mathrm{E}+05$ \\
\hline $1 s 4 s^{3} S_{1}$ & $1 s 7 p^{3} P_{2}^{o}$ & $7.3324 \mathrm{E}+00$ & $1.020 \mathrm{E}-07$ & $2.530 \mathrm{E}+06$ & $7.3023 \mathrm{E}+00$ & $1.027 \mathrm{E}-07$ & $2.547 \mathrm{E}+06$ \\
\hline $1 s 4 s^{1} S_{0}$ & $1 s 7 p^{3} P_{2}^{o}$ & $7.3720 \mathrm{E}+00$ & $7.087 \mathrm{E}-08$ & $1.740 \mathrm{E}+06$ & $7.3421 \mathrm{E}+00$ & $6.736 \mathrm{E}-08$ & $1.652 \mathrm{E}+06$ \\
\hline $1 s 4 d^{3} D_{2}$ & $1 s 7 p^{3} P_{2}^{o}$ & $7.6054 \mathrm{E}+00$ & $7.859 \mathrm{E}-14$ & $1.813 \mathrm{E}+00$ & & & \\
\hline $1 s 4 d^{3} D_{1}$ & $1 s 7 p^{3} P_{2}^{o}$ & $7.6082 \mathrm{E}+00$ & $1.657 \mathrm{E}-13$ & $3.818 \mathrm{E}+00$ & & & \\
\hline $1 s 4 d^{3} D_{3}$ & $1 s 7 p^{3} P_{2}^{o}$ & $7.6776 \mathrm{E}+00$ & $3.901 \mathrm{E}-08$ & $8.828 \mathrm{E}+05$ & $7.6461 \mathrm{E}+00$ & $3.790 \mathrm{E}-08$ & $8.575 E+05$ \\
\hline $1 s 4 d{ }^{1} D_{2}$ & $1 s 7 p^{3} P_{2}^{o}$ & $7.6806 \mathrm{E}+00$ & $9.934 \mathrm{E}-09$ & $2.246 \mathrm{E}+05$ & $7.6491 \mathrm{E}+00$ & $9.464 \mathrm{E}-09$ & $2.139 \mathrm{E}+05$ \\
\hline $1 s 5 s^{3} S_{1}$ & $1 s 7 p^{3} P_{2}^{o}$ & $1.5717 \mathrm{E}+01$ & $5.498 \mathrm{E}-08$ & $2.969 \mathrm{E}+05$ & $1.5653 \mathrm{E}+01$ & $5.521 \mathrm{E}-08$ & $2.980 \mathrm{E}+05$ \\
\hline $1 s 5 s{ }^{1} S_{0}$ & $1 s 7 p^{3} P_{2}^{o}$ & $1.5808 \mathrm{E}+01$ & $3.839 \mathrm{E}-08$ & $2.049 \mathrm{E}+05$ & $1.5745 \mathrm{E}+01$ & $3.617 \mathrm{E}-08$ & $1.930 \mathrm{E}+05$ \\
\hline $1 s 5 d^{3} D_{2}$ & $1 s 7 p^{3} P_{2}^{o}$ & $1.6358 \mathrm{E}+01$ & $1.841 \mathrm{E}-13$ & $9.179 \mathrm{E}-01$ & & & \\
\hline $1 s 5 d^{3} D_{1}$ & $1 s 7 p^{3} P_{2}^{o}$ & $1.6364 \mathrm{E}+01$ & $1.037 \mathrm{E}-13$ & $5.164 \mathrm{E}-01$ & & & \\
\hline $1 s 5 d^{3} D_{3}$ & $1 s 7 p^{3} P_{2}^{o}$ & $1.6529 \mathrm{E}+01$ & $3.782 \mathrm{E}-08$ & $1.847 \mathrm{E}+05$ & $1.6463 \mathrm{E}+01$ & $3.680 \mathrm{E}-08$ & $1.796 \mathrm{E}+05$ \\
\hline $1 s 5 d^{1} D_{2}$ & $1 s 7 p^{3} P_{2}^{o}$ & $1.6536 \mathrm{E}+01$ & $9.557 \mathrm{E}-09$ & $4.663 \mathrm{E}+04$ & $1.6471 \mathrm{E}+01$ & $9.189 \mathrm{E}-09$ & $4.480 \mathrm{E}+04$ \\
\hline $1 s 5 g^{3} G_{4}$ & $1 s 7 p^{3} P_{2}^{o}$ & $1.6618 \mathrm{E}+01$ & $1.900 \mathrm{E}-16$ & $9.176 \mathrm{E}-04$ & & & \\
\hline $1 s 5 g^{3} G_{3}$ & $1 s 7 p^{3} P_{2}^{o}$ & $1.6620 \mathrm{E}+01$ & $4.014 \mathrm{E}-16$ & $1.939 \mathrm{E}-03$ & & & \\
\hline $1 s 5 g{ }^{1} G_{4}$ & $1 s 7 p^{3} P_{2}^{0}$ & $1.6671 \mathrm{E}+01$ & $5.026 \mathrm{E}-16$ & $2.413 \mathrm{E}-03$ & & & \\
\hline $1 s 6 s^{3} S_{1}$ & $1 s 7 p^{3} P_{2}^{o}$ & $4.1005 \mathrm{E}+01$ & $3.153 \mathrm{E}-08$ & $2.501 \mathrm{E}+04$ & $4.0810 \mathrm{E}+01$ & $3.175 \mathrm{E}-08$ & $2.522 \mathrm{E}+04$ \\
\hline $1 s 6 s{ }^{1} S_{0}$ & $1 s 7 p^{3} P_{2}^{o}$ & $4.1360 \mathrm{E}+01$ & $2.208 \mathrm{E}-08$ & $1.722 \mathrm{E}+04$ & $4.1111 \mathrm{E}+01$ & $2.071 \mathrm{E}-08$ & $1.620 \mathrm{E}+04$ \\
\hline $1 s 6 d^{3} D_{2}$ & $1 s 7 p^{3} P_{2}^{o}$ & $4.3572 \mathrm{E}+01$ & $2.575 \mathrm{E}-13$ & $1.809 \mathrm{E}-01$ & & & \\
\hline $1 s 6 d^{3} D_{1}$ & $1 s 7 p^{3} P_{2}^{o}$ & $4.3599 \mathrm{E}+01$ & $6.437 \mathrm{E}-14$ & $4.518 \mathrm{E}-02$ & & & \\
\hline $1 s 6 d^{3} D_{3}$ & $1 s 7 p^{3} P_{2}^{o}$ & $4.4278 \mathrm{E}+01$ & $3.619 \mathrm{E}-08$ & $2.463 \mathrm{E}+04$ & $4.4127 \mathrm{E}+01$ & $3.518 \mathrm{E}-08$ & $2.389 \mathrm{E}+04$ \\
\hline $1 s 6 d{ }^{1} D_{2}$ & $1 s 7 p^{3} P_{2}^{o}$ & $4.4308 \mathrm{E}+01$ & $9.105 \mathrm{E}-09$ & $6.187 \mathrm{E}+03$ & $4.4138 \mathrm{E}+01$ & $8.787 \mathrm{E}-09$ & $5.966 \mathrm{E}+03$ \\
\hline $1 s 6 g^{3} G_{4}$ & $1 s 7 p^{3} P_{2}^{o}$ & $4.4650 \mathrm{E}+01$ & $3.033 \mathrm{E}-16$ & $2.030 \mathrm{E}-04$ & & & \\
\hline $1 s 6 g{ }^{3} G_{3}$ & $1 s 7 p^{3} P_{2}^{o}$ & $4.4659 \mathrm{E}+01$ & $1.017 \mathrm{E}-15$ & $6.805 \mathrm{E}-04$ & & & \\
\hline $1 s 6 g{ }^{1} G_{4}$ & $1 s 7 p^{3} P_{2}^{o}$ & $4.4874 \mathrm{E}+01$ & $1.154 \mathrm{E}-15$ & $7.646 \mathrm{E}-04$ & & & \\
\hline $1 s 7 s^{3} S_{1}$ & $1 s 7 p^{3} P_{2}^{o}$ & $1.1691 \mathrm{E}+03$ & $3.824 \mathrm{E}-11$ & $3.733 \mathrm{E}-02$ & $1.1590 \mathrm{E}+03$ & $3.901 \mathrm{E}-11$ & $3.842 \mathrm{E}-02$ \\
\hline $1 s 7 s^{1} S_{0}$ & $1 s 7 p^{3} P_{2}^{o}$ & $1.3812 \mathrm{E}+03$ & $1.542 \mathrm{E}-11$ & $1.078 \mathrm{E}-02$ & $1.3316 \mathrm{E}+03$ & $1.714 \mathrm{E}-11$ & $1.279 \mathrm{E}-02$ \\
\hline $1 s 2 p^{3} P_{1}^{o}$ & $1 s 7 d^{3} D_{2}$ & $1.3362 \mathrm{E}+00$ & $4.478 \mathrm{E}-08$ & $3.345 \mathrm{E}+07$ & $1.3307 \mathrm{E}+00$ & $4.941 \mathrm{E}-08$ & $3.691 \mathrm{E}+07$ \\
\hline $1 s 2 p^{3} P_{0}^{o}$ & $1 s 7 d^{3} D_{2}$ & $1.3371 \mathrm{E}+00$ & $2.808 \mathrm{E}-08$ & $2.095 \mathrm{E}+07$ & $1.3315 \mathrm{E}+00$ & $2.860 \mathrm{E}-08$ & $2.133 \mathrm{E}+07$ \\
\hline $1 s 2 p^{3} P_{2}^{o}$ & $1 s 7 d^{3} D_{2}$ & $1.3946 \mathrm{E}+00$ & $2.722 \mathrm{E}-11$ & $1.867 \mathrm{E}+04$ & $1.3888 \mathrm{E}+00$ & $1.197 \mathrm{E}-10$ & $8.211 \mathrm{E}+04$ \\
\hline $1 s 2 p{ }^{1} P_{1}^{o}$ & $1 s 7 d^{3} D_{2}$ & $1.4001 \mathrm{E}+00$ & $5.100 \mathrm{E}-13$ & $3.471 \mathrm{E}+02$ & $1.3943 \mathrm{E}+00$ & $6.804 \mathrm{E}-11$ & $4.629 \mathrm{E}+04$ \\
\hline
\end{tabular}


Table 6 (continued)

\begin{tabular}{|c|c|c|c|c|c|c|c|}
\hline \multirow[t]{2}{*}{ Lower } & \multirow[t]{2}{*}{ Upper } & \multicolumn{3}{|l|}{ GRASP2K } & \multicolumn{3}{|l|}{ FAC } \\
\hline & & $\lambda(\AA)$ & $g f$ & $A\left(\mathrm{~s}^{-1}\right)$ & $\overline{\lambda(\AA)}$ & $g f$ & $A\left(\mathrm{~s}^{-1}\right)$ \\
\hline $1 s 3 p^{3} P_{1}^{o}$ & $1 s 7 d^{3} D_{2}$ & $3.4075 \mathrm{E}+00$ & $1.627 \mathrm{E}-08$ & $1.869 \mathrm{E}+06$ & $3.3932 \mathrm{E}+00$ & $1.702 \mathrm{E}-08$ & $1.956 \mathrm{E}+06$ \\
\hline $1 s 3 p^{3} P_{0}^{o}$ & $1 s 7 d^{3} D_{2}$ & $3.4089 \mathrm{E}+00$ & $1.009 \mathrm{E}-08$ & $1.158 \mathrm{E}+06$ & $3.3946 \mathrm{E}+00$ & $9.875 \mathrm{E}-09$ & $1.133 \mathrm{E}+06$ \\
\hline $1 s 3 p^{3} P_{2}^{o}$ & $1 s 7 d^{3} D_{2}$ & $3.5193 \mathrm{E}+00$ & $1.222 \mathrm{E}-11$ & $1.316 \mathrm{E}+03$ & $3.5045 \mathrm{E}+00$ & $4.385 \mathrm{E}-11$ & $4.723 E+03$ \\
\hline $1 s 3 p^{1} P_{1}^{o}$ & $1 s 7 d^{3} D_{2}$ & $3.5292 \mathrm{E}+00$ & $7.061 \mathrm{E}-13$ & $7.563 \mathrm{E}+01$ & $3.5145 \mathrm{E}+00$ & $2.920 \mathrm{E}-11$ & $3.127 \mathrm{E}+03$ \\
\hline $1 s 4 p^{3} P_{1}^{o}$ & $1 s 7 d^{3} D_{2}$ & $7.3673 \mathrm{E}+00$ & 7.197E-09 & $1.769 \mathrm{E}+05$ & $7.3356 \mathrm{E}+00$ & $7.478 \mathrm{E}-09$ & $1.838 \mathrm{E}+05$ \\
\hline $1 s 4 p^{3} P_{0}^{o}$ & $1 s 7 d^{3} D_{2}$ & $7.3700 \mathrm{E}+00$ & $4.458 \mathrm{E}-09$ & $1.095 \mathrm{E}+05$ & $7.3382 \mathrm{E}+00$ & $4.345 \mathrm{E}-09$ & $1.067 \mathrm{E}+05$ \\
\hline $1 s 4 p^{3} P_{2}^{o}$ & $1 s 7 d^{3} D_{2}$ & $7.5864 \mathrm{E}+00$ & $5.874 \mathrm{E}-12$ & $1.361 \mathrm{E}+02$ & $7.5537 \mathrm{E}+00$ & $2.000 \mathrm{E}-11$ & $4.636 \mathrm{E}+02$ \\
\hline $1 s 4 p^{1} P_{1}^{o}$ & $1 s 7 d^{3} D_{2}$ & $7.6055 \mathrm{E}+00$ & $4.053 \mathrm{E}-13$ & $9.348 \mathrm{E}+00$ & $7.5729 \mathrm{E}+00$ & $1.427 \mathrm{E}-11$ & $3.293 \mathrm{E}+02$ \\
\hline $1 s 4 f^{3} F_{3}^{o}$ & $1 s 7 d^{3} D_{2}$ & $7.6759 \mathrm{E}+00$ & $1.692 \mathrm{E}-09$ & $3.830 \mathrm{E}+04$ & $7.6429 \mathrm{E}+00$ & $1.661 \mathrm{E}-09$ & $3.762 \mathrm{E}+04$ \\
\hline $1 s 4 f^{3} F_{2}^{o}$ & $1 s 7 d^{3} D_{2}$ & $7.6774 \mathrm{E}+00$ & $4.251 \mathrm{E}-10$ & $9.622 \mathrm{E}+03$ & $7.6444 \mathrm{E}+00$ & $4.160 \mathrm{E}-10$ & $9.418 \mathrm{E}+03$ \\
\hline $1 s 4 f^{3} F_{4}^{o}$ & $1 s 7 d^{3} D_{2}$ & $7.7121 \mathrm{E}+00$ & $3.413 \mathrm{E}-09$ & $7.654 \mathrm{E}+04$ & $7.6789 \mathrm{E}+00$ & $3.354 \mathrm{E}-09$ & $7.524 \mathrm{E}+04$ \\
\hline $1 s 4 f^{1} F_{3}^{o}$ & $1 s 7 d^{3} D_{2}$ & $7.7133 \mathrm{E}+00$ & $4.204 \mathrm{E}-10$ & $9.426 \mathrm{E}+03$ & $7.6800 \mathrm{E}+00$ & $4.388 \mathrm{E}-10$ & $9.841 \mathrm{E}+03$ \\
\hline $1 s 5 p^{3} P_{1}^{o}$ & $1 s 7 d^{3} D_{2}$ & $1.5792 \mathrm{E}+01$ & $3.717 \mathrm{E}-09$ & $1.988 \mathrm{E}+04$ & $1.5722 \mathrm{E}+01$ & $3.848 \mathrm{E}-09$ & $2.059 \mathrm{E}+04$ \\
\hline $1 s 5 p^{3} P_{0}^{0}$ & $1 s 7 d^{3} D_{2}$ & $1.5798 \mathrm{E}+01$ & $2.303 \mathrm{E}-09$ & $1.231 \mathrm{E}+04$ & $1.5728 \mathrm{E}+01$ & $2.239 \mathrm{E}-09$ & $1.197 \mathrm{E}+04$ \\
\hline $1 s 5 p^{3} P_{2}^{o}$ & $1 s 7 d^{3} D_{2}$ & $1.6307 \mathrm{E}+01$ & $3.226 \mathrm{E}-12$ & $1.619 \mathrm{E}+01$ & $1.6234 \mathrm{E}+01$ & $1.060 \mathrm{E}-11$ & $5.324 \mathrm{E}+01$ \\
\hline $1 s 5 p^{1} P_{1}^{o}$ & $1 s 7 d^{3} D_{2}$ & $1.6352 \mathrm{E}+01$ & $2.514 \mathrm{E}-13$ & $1.255 \mathrm{E}+00$ & $1.6279 \mathrm{E}+01$ & $7.983 \mathrm{E}-12$ & $3.984 \mathrm{E}+01$ \\
\hline $1 s 5 f^{3} F_{3}^{o}$ & $1 s 7 d^{3} D_{2}$ & $1.6519 \mathrm{E}+01$ & $2.775 \mathrm{E}-09$ & $1.357 \mathrm{E}+04$ & $1.6446 \mathrm{E}+01$ & $2.756 \mathrm{E}-09$ & $1.347 \mathrm{E}+04$ \\
\hline $1 s 5 f^{3} F_{2}^{o}$ & $1 s 7 d^{3} D_{2}$ & $1.6522 \mathrm{E}+01$ & $6.968 \mathrm{E}-10$ & $3.405 E+03$ & $1.6450 \mathrm{E}+01$ & $6.900 \mathrm{E}-10$ & $3.373 E+03$ \\
\hline $1 s 5 f^{3} F_{4}^{o}$ & $1 s 7 d^{3} D_{2}$ & $1.6605 E+01$ & $5.591 \mathrm{E}-09$ & $2.705 E+04$ & $1.6532 \mathrm{E}+01$ & $5.516 \mathrm{E}-09$ & $2.669 \mathrm{E}+04$ \\
\hline $1 s 5 f^{1} F_{3}^{o}$ & $1 s 7 d^{3} D_{2}$ & $1.6607 \mathrm{E}+01$ & $6.887 \mathrm{E}-10$ & $3.331 E+03$ & $1.6535 \mathrm{E}+01$ & $7.220 \mathrm{E}-10$ & $3.493 \mathrm{E}+03$ \\
\hline $1 s 6 p^{3} P_{1}^{o}$ & $1 s 7 d^{3} D_{2}$ & $4.1263 \mathrm{E}+01$ & $2.028 \mathrm{E}-09$ & $1.589 E+03$ & $4.1063 \mathrm{E}+01$ & $2.093 \mathrm{E}-09$ & $1.641 \mathrm{E}+03$ \\
\hline $1 s 6 p^{3} P_{0}^{0}$ & $1 s 7 d^{3} D_{2}$ & $4.1288 \mathrm{E}+01$ & $1.257 \mathrm{E}-09$ & $9.836 \mathrm{E}+02$ & $4.1000 \mathrm{E}+01$ & $1.227 \mathrm{E}-09$ & $9.657 \mathrm{E}+02$ \\
\hline $1 s 6 p^{3} P_{2}^{o}$ & $1 s 7 d^{3} D_{2}$ & $4.3326 \mathrm{E}+01$ & $1.907 \mathrm{E}-12$ & $1.355 \mathrm{E}+00$ & $4.3112 \mathrm{E}+01$ & $6.034 \mathrm{E}-12$ & $4.294 \mathrm{E}+00$ \\
\hline $1 s 6 p^{1} P_{1}^{o}$ & $1 s 7 d^{3} D_{2}$ & $4.3508 \mathrm{E}+01$ & $1.635 \mathrm{E}-13$ & $1.152 \mathrm{E}-01$ & $4.3273 \mathrm{E}+01$ & $3.074 \mathrm{E}-12$ & $2.171 \mathrm{E}+00$ \\
\hline $1 s 6 f^{3} F_{3}^{o}$ & $1 s 7 d^{3} D_{2}$ & $4.4197 \mathrm{E}+01$ & $3.605 E-09$ & $2.462 E+03$ & $4.4000 \mathrm{E}+01$ & $3.604 \mathrm{E}-09$ & $2.462 \mathrm{E}+03$ \\
\hline $1 s 6 f^{3} F_{2}^{o}$ & $1 s 7 d^{3} D_{2}$ & $4.4212 \mathrm{E}+01$ & $9.051 \mathrm{E}-10$ & $6.177 \mathrm{E}+02$ & $4.4000 \mathrm{E}+01$ & $9.006 \mathrm{E}-10$ & $6.153 \mathrm{E}+02$ \\
\hline $1 s 6 f^{3} F_{4}^{o}$ & $1 s 7 d^{3} D_{2}$ & $4.4555 \mathrm{E}+01$ & $7.249 \mathrm{E}-09$ & $4.871 E+03$ & $4.4354 \mathrm{E}+01$ & $7.175 \mathrm{E}-09$ & $4.824 \mathrm{E}+03$ \\
\hline $1 s 6 f^{1} F_{3}^{o}$ & $1 s 7 d^{3} D_{2}$ & $4.4566 \mathrm{E}+01$ & $8.930 \mathrm{E}-10$ & $5.998 \mathrm{E}+02$ & $4.4354 \mathrm{E}+01$ & $9.294 \mathrm{E}-10$ & $6.249 \mathrm{E}+02$ \\
\hline $1 s 6 h^{3} H_{4}^{o}$ & $1 s 7 d^{3} D_{2}$ & $4.4777 \mathrm{E}+01$ & $1.788 \mathrm{E}-21$ & $1.190 \mathrm{E}-09$ & & & \\
\hline $1 s 7 p^{3} P_{1}^{o}$ & $1 s 7 d^{3} D_{2}$ & $1.2946 \mathrm{E}+03$ & $1.100 \mathrm{E}-12$ & $8.757 \mathrm{E}-04$ & $1.2624 \mathrm{E}+03$ & $1.212 \mathrm{E}-12$ & $1.006 \mathrm{E}-03$ \\
\hline $1 s 7 p^{3} P_{0}^{o}$ & $1 s 7 d^{3} D_{2}$ & $1.3100 \mathrm{E}+03$ & $6.563 \mathrm{E}-13$ & $5.102 \mathrm{E}-04$ & $1.2259 \mathrm{E}+03$ & $7.685 E-13$ & $6.764 \mathrm{E}-04$ \\
\hline $1 s 7 p^{3} P_{2}^{o}$ & $1 s 7 d^{3} D_{2}$ & $2.0924 \mathrm{E}+04$ & $2.094 \mathrm{E}-19$ & $6.381 \mathrm{E}-13$ & & & \\
\hline $1 s 2 p^{3} P_{1}^{o}$ & $1 s 7 d^{3} D_{1}$ & $1.3362 \mathrm{E}+00$ & $4.400 \mathrm{E}-08$ & $5.479 E+07$ & $1.3307 \mathrm{E}+00$ & $4.658 \mathrm{E}-08$ & $5.799 \mathrm{E}+07$ \\
\hline $1 s 2 p^{3} P_{2}^{o}$ & $1 s 7 d^{3} D_{1}$ & $1.3946 \mathrm{E}+00$ & $9.500 \mathrm{E}-14$ & $1.086 \mathrm{E}+02$ & & & \\
\hline $1 s 2 p^{1} P_{1}^{o}$ & $1 s 7 d^{3} D_{1}$ & $1.4001 \mathrm{E}+00$ & $4.968 \mathrm{E}-11$ & $5.634 \mathrm{E}+04$ & $1.3943 \mathrm{E}+00$ & $6.617 \mathrm{E}-11$ & $7.503 E+04$ \\
\hline $1 s 3 p^{3} P_{1}^{o}$ & $1 s 7 d^{3} D_{1}$ & $3.4074 \mathrm{E}+00$ & $1.582 \mathrm{E}-08$ & $3.030 \mathrm{E}+06$ & $3.3932 \mathrm{E}+00$ & $1.606 \mathrm{E}-08$ & $3.075 \mathrm{E}+06$ \\
\hline $1 s 3 p^{3} P_{2}^{o}$ & $1 s 7 d^{3} D_{1}$ & $3.5192 \mathrm{E}+00$ & $1.300 \mathrm{E}-13$ & $2.334 \mathrm{E}+01$ & & & \\
\hline $1 s 3 p^{1} P_{1}^{o}$ & $1 s 7 d^{3} D_{1}$ & $3.5291 \mathrm{E}+00$ & $1.664 \mathrm{E}-11$ & $2.970 \mathrm{E}+03$ & $3.5145 \mathrm{E}+00$ & $2.062 \mathrm{E}-11$ & $3.680 \mathrm{E}+03$ \\
\hline $1 s 4 p^{3} P_{1}^{o}$ & $1 s 7 d^{3} D_{1}$ & $7.3668 \mathrm{E}+00$ & $6.984 \mathrm{E}-09$ & $2.861 \mathrm{E}+05$ & $7.3357 \mathrm{E}+00$ & $7.059 \mathrm{E}-09$ & $2.892 \mathrm{E}+05$ \\
\hline $1 s 4 p^{3} P_{2}^{o}$ & $1 s 7 d^{3} D_{1}$ & $7.5859 \mathrm{E}+00$ & $8.791 \mathrm{E}-14$ & $3.396 \mathrm{E}+00$ & & & \\
\hline $1 s 4 p^{1} P_{1}^{o}$ & $1 s 7 d^{3} D_{1}$ & $7.6050 \mathrm{E}+00$ & $7.421 \mathrm{E}-12$ & $2.853 E+02$ & $7.5730 \mathrm{E}+00$ & $8.740 \mathrm{E}-12$ & $3.359 \mathrm{E}+02$ \\
\hline $1 s 4 f^{3} F_{3}^{o}$ & $1 s 7 d^{3} D_{1}$ & $7.6754 \mathrm{E}+00$ & $2.849 \mathrm{E}-10$ & $1.075 \mathrm{E}+04$ & $7.6430 \mathrm{E}+00$ & $2.810 \mathrm{E}-10$ & $1.060 \mathrm{E}+04$ \\
\hline $1 s 4 f^{3} F_{2}^{o}$ & $1 s 7 d^{3} D_{1}$ & $7.6769 \mathrm{E}+00$ & $9.882 \mathrm{E}-10$ & $3.728 \mathrm{E}+04$ & $7.6445 \mathrm{E}+00$ & $9.709 \mathrm{E}-10$ & $3.662 \mathrm{E}+04$ \\
\hline $1 s 4 f^{1} F_{3}^{o}$ & $1 s 7 d^{3} D_{1}$ & $7.7127 \mathrm{E}+00$ & $2.289 \mathrm{E}-09$ & $8.555 E+04$ & $7.6801 \mathrm{E}+00$ & $2.268 \mathrm{E}-09$ & $8.477 \mathrm{E}+04$ \\
\hline $1 s 5 p^{3} P_{1}^{o}$ & $1 s 7 d^{3} D_{1}$ & $1.5790 \mathrm{E}+01$ & $3.602 \mathrm{E}-09$ & $3.212 \mathrm{E}+04$ & $1.5722 \mathrm{E}+01$ & $3.635 \mathrm{E}-09$ & $3.241 \mathrm{E}+04$ \\
\hline $1 s 5 p^{3} P_{2}^{o}$ & $1 s 7 d^{3} D_{1}$ & $1.6305 E+01$ & $5.927 \mathrm{E}-14$ & $4.957 \mathrm{E}-01$ & & & \\
\hline $1 s 5 p{ }^{1} P_{1}^{o}$ & $1 s 7 d^{3} D_{1}$ & $1.6349 \mathrm{E}+01$ & $3.876 \mathrm{E}-12$ & $3.224 \mathrm{E}+01$ & $1.6280 \mathrm{E}+01$ & $4.371 \mathrm{E}-12$ & $3.635 \mathrm{E}+01$ \\
\hline $1 s 5 f^{3} F_{3}^{o}$ & $1 s 7 d^{3} D_{1}$ & $1.6516 \mathrm{E}+01$ & $4.669 \mathrm{E}-10$ & $3.806 \mathrm{E}+03$ & $1.6447 \mathrm{E}+01$ & $4.657 \mathrm{E}-10$ & $3.795 E+03$ \\
\hline $1 s 5 f^{3} F_{2}^{o}$ & $1 s 7 d^{3} D_{1}$ & $1.6520 \mathrm{E}+01$ & $1.620 \mathrm{E}-09$ & $1.320 \mathrm{E}+04$ & $1.6451 \mathrm{E}+01$ & $1.610 \mathrm{E}-09$ & $1.311 \mathrm{E}+04$ \\
\hline $1 s 5 f^{1} F_{3}^{o}$ & $1 s 7 d^{3} D_{1}$ & $1.6605 \mathrm{E}+01$ & $3.751 \mathrm{E}-09$ & $3.025 E+04$ & $1.6535 \mathrm{E}+01$ & $3.730 \mathrm{E}-09$ & $3.008 \mathrm{E}+04$ \\
\hline $1 s 6 p^{3} P_{1}^{o}$ & $1 s 7 d^{3} D_{1}$ & $4.1248 \mathrm{E}+01$ & $1.961 \mathrm{E}-09$ & $2.563 E+03$ & $4.1067 \mathrm{E}+01$ & $1.974 \mathrm{E}-09$ & $2.581 \mathrm{E}+03$ \\
\hline $1 s 6 p^{3} P_{2}^{0}$ & $1 s 7 d^{3} D_{1}$ & $4.3310 \mathrm{E}+01$ & $4.142 \mathrm{E}-14$ & $4.910 \mathrm{E}-02$ & & & \\
\hline $1 s 6 p{ }^{1} P_{1}^{o}$ & $1 s 7 d^{3} D_{1}$ & $4.3491 \mathrm{E}+01$ & $2.199 \mathrm{E}-12$ & $2.585 \mathrm{E}+00$ & $4.3277 \mathrm{E}+01$ & $3.706 \mathrm{E}-12$ & $4.363 \mathrm{E}+00$ \\
\hline $1 s 6 f^{3} F_{3}^{o}$ & $1 s 7 d^{3} D_{1}$ & $4.4180 \mathrm{E}+01$ & $6.062 \mathrm{E}-10$ & $6.905 E+02$ & $4.4005 \mathrm{E}+01$ & $5.983 \mathrm{E}-10$ & $6.812 \mathrm{E}+02$ \\
\hline $1 s 6 f^{3} F_{2}^{o}$ & $1 s 7 d^{3} D_{1}$ & $4.4195 \mathrm{E}+01$ & $2.104 \mathrm{E}-09$ & $2.395 E+03$ & $4.4004 \mathrm{E}+01$ & $2.101 \mathrm{E}-09$ & $2.392 \mathrm{E}+03$ \\
\hline $1 s 6 f^{1} F_{3}^{o}$ & $1 s 7 d^{3} D_{1}$ & $4.4549 \mathrm{E}+01$ & $4.862 \mathrm{E}-09$ & $5.447 \mathrm{E}+03$ & $4.4358 \mathrm{E}+01$ & $4.862 \mathrm{E}-09$ & $5.447 \mathrm{E}+03$ \\
\hline $1 s 7 p^{3} P_{1}^{o}$ & $1 s 7 d^{3} D_{1}$ & $1.2801 \mathrm{E}+03$ & $1.102 \mathrm{E}-12$ & $1.496 \mathrm{E}-03$ & $1.2659 \mathrm{E}+03$ & $1.132 \mathrm{E}-12$ & $1.558 \mathrm{E}-03$ \\
\hline $1 s 7 p^{3} P_{2}^{o}$ & $1 s 7 d^{3} D_{1}$ & $1.7684 \mathrm{E}+04$ & $2.108 \mathrm{E}-20$ & $1.499 \mathrm{E}-13$ & & & \\
\hline $1 s 2 s^{3} S_{1}^{2}$ & $1 s 7 p^{1} P_{1}^{o}$ & $1.3252 \mathrm{E}+00$ & $7.024 \mathrm{E}-07$ & $8.893 \mathrm{E}+08$ & $1.3196 \mathrm{E}+00$ & $7.245 \mathrm{E}-07$ & $9.173 \mathrm{E}+08$ \\
\hline $1 s 3 s^{3} S_{1}$ & $1 s 7 p^{1} P_{1}^{o}$ & $3.3877 \mathrm{E}+00$ & $2.240 \mathrm{E}-07$ & $4.340 \mathrm{E}+07$ & $3.3738 \mathrm{E}+00$ & $2.287 \mathrm{E}-07$ & $4.430 \mathrm{E}+07$ \\
\hline $1 s 3 d^{3} D_{2}$ & $1 s 7 p^{1} P_{1}^{o}$ & $3.5275 \mathrm{E}+00$ & $2.870 \mathrm{E}-13$ & $5.129 \mathrm{E}+01$ & & & \\
\hline $1 s 3 d^{3} D_{1}$ & $1 s 7 p^{1} P_{1}^{o}$ & $3.5290 \mathrm{E}+00$ & $3.245 \mathrm{E}-15$ & $5.794 \mathrm{E}-01$ & & & \\
\hline $1 s 3 d^{3} D_{3}$ & $1 s 7 p^{1} P_{1}^{o}$ & $3.5643 \mathrm{E}+00$ & $6.031 \mathrm{E}-09$ & $1.055 \mathrm{E}+06$ & $3.5494 \mathrm{E}+00$ & $6.273 \mathrm{E}-09$ & $1.097 \mathrm{E}+06$ \\
\hline $1 s 3 d{ }^{1} D_{2}$ & $1 s 7 p^{1} P_{1}^{o}$ & $3.5657 \mathrm{E}+00$ & $1.963 \mathrm{E}-08$ & $3.433 E+06$ & $3.5508 \mathrm{E}+00$ & $1.869 \mathrm{E}-08$ & $3.269 \mathrm{E}+06$ \\
\hline $1 s 4 s^{3} S_{1}$ & $1 s 7 p^{1} P_{1}^{o}$ & $7.3292 \mathrm{E}+00$ & $1.005 \mathrm{E}-07$ & $4.160 \mathrm{E}+06$ & $7.2994 \mathrm{E}+00$ & $1.026 \mathrm{E}-07$ & $4.245 E+06$ \\
\hline $1 s 4 d^{3} D_{2}$ & $1 s 7 p^{1} P_{1}^{o}$ & $7.6019 \mathrm{E}+00$ & $3.982 \mathrm{E}-14$ & $1.532 \mathrm{E}+00$ & & & \\
\hline $1 s 4 d^{3} D_{1}$ & $1 s 7 p^{1} P_{1}^{o}$ & $7.6047 \mathrm{E}+00$ & $4.575 \mathrm{E}-14$ & $1.759 \mathrm{E}+00$ & & & \\
\hline $1 s 4 d^{3} D_{3}$ & $1 s 7 p^{1} P_{1}^{o}$ & $7.6740 \mathrm{E}+00$ & $6.836 \mathrm{E}-09$ & $2.581 E+05$ & $7.6429 \mathrm{E}+00$ & $7.123 \mathrm{E}-09$ & $2.688 \mathrm{E}+05$ \\
\hline $1 s 4 d^{1} D_{2}$ & $1 s 7 p^{1} P_{1}^{o}$ & $7.6770 \mathrm{E}+00$ & $2.199 \mathrm{E}-08$ & $8.294 \mathrm{E}+05$ & $7.6459 \mathrm{E}+00$ & $2.128 \mathrm{E}-08$ & $8.026 \mathrm{E}+05$ \\
\hline $1 s 5 s^{3} S_{1}$ & $1 s 7 p^{1} P_{1}^{o}$ & $1.5702 \mathrm{E}+01$ & $5.403 \mathrm{E}-08$ & $4.873 E+05$ & $1.5640 \mathrm{E}+01$ & $5.523 \mathrm{E}-08$ & $4.978 \mathrm{E}+05$ \\
\hline $1 s 5 d^{3} D_{2}$ & $1 s 7 p^{1} P_{1}^{o}$ & $1.6342 \mathrm{E}+01$ & $9.126 \mathrm{E}-20$ & $7.599 \mathrm{E}-07$ & & & \\
\hline
\end{tabular}


Table 6 (continued)

\begin{tabular}{|c|c|c|c|c|c|c|c|}
\hline \multirow[t]{2}{*}{ Lower } & \multirow[t]{2}{*}{ Upper } & \multicolumn{3}{|l|}{ GRASP2K } & \multicolumn{3}{|l|}{ FAC } \\
\hline & & $\lambda(\AA)$ & $g f$ & $A\left(\mathrm{~s}^{-1}\right)$ & $\overline{\lambda(\AA)}$ & $g f$ & $A\left(\mathrm{~s}^{-1}\right)$ \\
\hline $1 s 5 d^{3} D_{1}$ & $1 s 7 p^{1} P_{1}^{o}$ & $1.6348 \mathrm{E}+01$ & $1.012 \mathrm{E}-13$ & $8.420 \mathrm{E}-01$ & & & \\
\hline $1 s 5 d^{3} D_{3}$ & $1 s 7 p^{1} P_{1}^{o}$ & $1.6512 \mathrm{E}+01$ & $6.645 \mathrm{E}-09$ & $5.418 \mathrm{E}+04$ & $1.6449 \mathrm{E}+01$ & $6.925 \mathrm{E}-09$ & $5.643 \mathrm{E}+04$ \\
\hline $1 s 5 d^{1} D_{2}$ & $1 s 7 p^{1} P_{1}^{0}$ & $1.6520 \mathrm{E}+01$ & $2.120 \mathrm{E}-08$ & $1.727 \mathrm{E}+05$ & $1.6456 \mathrm{E}+01$ & $2.069 \mathrm{E}-08$ & $1.684 \mathrm{E}+05$ \\
\hline $1 s 5 g{ }^{3} G_{3}$ & $1 s 7 p^{1} P_{1}^{o}$ & $1.6603 \mathrm{E}+01$ & $6.845 \mathrm{E}-18$ & $5.521 \mathrm{E}-05$ & & & \\
\hline $1 s 6 s^{3} s_{1}$ & $1 s 7 p^{1} P_{1}^{o}$ & $4.0902 \mathrm{E}+01$ & $3.083 E-08$ & $4.097 \mathrm{E}+04$ & $4.0720 \mathrm{E}+01$ & $3.189 E-08$ & $4.241 \mathrm{E}+04$ \\
\hline $1 s 6 d^{3} D_{2}$ & $1 s 7 p^{1} P_{1}^{o}$ & $4.3457 \mathrm{E}+01$ & $1.217 \mathrm{E}-14$ & $1.433 \mathrm{E}-02$ & & & \\
\hline $1 s 6 d^{3} D_{1}$ & $1 s 7 p^{1} P_{1}^{o}$ & $4.3483 \mathrm{E}+01$ & $1.543 \mathrm{E}-13$ & $1.814 \mathrm{E}-01$ & & & \\
\hline $1 s 6 d^{3} D_{3}$ & $1 s 7 p^{1} P_{1}^{o}$ & $4.4159 \mathrm{E}+01$ & $6.358 \mathrm{E}-09$ & $7.250 \mathrm{E}+03$ & $4.4022 \mathrm{E}+01$ & $6.675 \mathrm{E}-09$ & $7.593 \mathrm{E}+03$ \\
\hline $1 s 6 d^{1} D_{2}$ & $1 s 7 p^{1} P_{1}^{o}$ & $4.4189 \mathrm{E}+01$ & $2.019 \mathrm{E}-08$ & $2.299 \mathrm{E}+04$ & $4.4032 \mathrm{E}+01$ & $1.986 \mathrm{E}-08$ & $2.258 \mathrm{E}+04$ \\
\hline $1 s 6 g^{3} G_{3}$ & $1 s 7 p^{1} P_{1}^{o}$ & $4.4538 \mathrm{E}+01$ & $9.976 \mathrm{E}-19$ & $1.118 \mathrm{E}-06$ & & & \\
\hline $1 s 7 s^{3} S_{1}$ & $1 s 7 p^{1} P_{1}^{o}$ & $1.0915 \mathrm{E}+03$ & $4.698 \mathrm{E}-11$ & $8.768 \mathrm{E}-02$ & $1.0907 \mathrm{E}+03$ & $4.671 \mathrm{E}-11$ & $8.656 \mathrm{E}-02$ \\
\hline $1 s 7 d^{3} D_{2}$ & $1 s 7 p^{1} P_{1}^{o}$ & $7.6625 \mathrm{E}+04$ & $4.221 \mathrm{E}-22$ & $1.598 \mathrm{E}-16$ & & & \\
\hline $1 s 7 d^{3} D_{1}$ & $1 s 7 p^{1} P_{1}^{o}$ & $2.3287 \mathrm{E}+05$ & $1.361 \mathrm{E}-22$ & $5.579 \mathrm{E}-18$ & & & \\
\hline $1 s 2 p^{3} P_{1}^{o}$ & $1 s 7 d^{3} D_{3}$ & $1.3358 \mathrm{E}+00$ & $7.245 \mathrm{E}-07$ & $3.869 \mathrm{E}+08$ & $1.3303 \mathrm{E}+00$ & $7.408 \mathrm{E}-07$ & $3.955 \mathrm{E}+08$ \\
\hline $1 s 2 p^{3} P_{2}^{o}$ & $1 s 7 d^{3} D_{3}$ & $1.3942 \mathrm{E}+00$ & $3.102 \mathrm{E}-06$ & $1.520 \mathrm{E}+09$ & $1.3883 \mathrm{E}+00$ & $3.269 \mathrm{E}-06$ & $1.602 \mathrm{E}+09$ \\
\hline $1 s 2 p^{1} P_{1}^{o}$ & $1 s 7 d^{3} D_{3}$ & $1.3997 \mathrm{E}+00$ & $5.609 \mathrm{E}-07$ & $2.728 \mathrm{E}+08$ & $1.3939 \mathrm{E}+00$ & $5.880 \mathrm{E}-07$ & $2.859 \mathrm{E}+08$ \\
\hline $1 s 3 p^{3} P_{1}^{0}$ & $1 s 7 d^{3} D_{3}$ & $3.4048 \mathrm{E}+00$ & $2.467 \mathrm{E}-07$ & $2.028 \mathrm{E}+07$ & $3.3906 \mathrm{E}+00$ & $2.512 \mathrm{E}-07$ & $2.065 \mathrm{E}+07$ \\
\hline $1 s 3 p^{3} P_{2}^{0}$ & $1 s 7 d^{3} D_{3}$ & $3.5164 \mathrm{E}+00$ & $1.176 \mathrm{E}-06$ & $9.066 \mathrm{E}+07$ & $3.5016 \mathrm{E}+00$ & $1.199 \mathrm{E}-06$ & $9.241 \mathrm{E}+07$ \\
\hline $1 s 3 p^{1} P_{1}^{o}$ & $1 s 7 d^{3} D_{3}$ & $3.5263 \mathrm{E}+00$ & $2.112 \mathrm{E}-07$ & $1.619 \mathrm{E}+07$ & $3.5116 \mathrm{E}+00$ & $2.148 \mathrm{E}-07$ & $1.645 \mathrm{E}+07$ \\
\hline $1 s 4 p^{3} P_{1}^{o}$ & $1 s 7 d^{3} D_{3}$ & $7.3548 \mathrm{E}+00$ & $1.069 \mathrm{E}-07$ & $1.882 \mathrm{E}+06$ & $7.3233 \mathrm{E}+00$ & $1.078 \mathrm{E}-07$ & $1.899 \mathrm{E}+06$ \\
\hline $1 s 4 p^{3} P_{2}^{o}$ & $1 s 7 d^{3} D_{3}$ & $7.5732 \mathrm{E}+00$ & $5.437 \mathrm{E}-07$ & $9.033 \mathrm{E}+06$ & $7.5406 \mathrm{E}+00$ & $5.503 \mathrm{E}-07$ & $9.145 E+06$ \\
\hline $1 s 4 p{ }^{1} P_{1}^{o}$ & $1 s 7 d^{3} D_{3}$ & $7.5922 \mathrm{E}+00$ & $9.799 \mathrm{E}-08$ & $1.620 \mathrm{E}+06$ & $7.5597 \mathrm{E}+00$ & $9.827 \mathrm{E}-08$ & $1.624 \mathrm{E}+06$ \\
\hline $1 s 4 f^{3} F_{3}^{o}$ & $1 s 7 d^{3} D_{3}$ & $7.6624 \mathrm{E}+00$ & $1.187 \mathrm{E}-14$ & $1.927 \mathrm{E}-01$ & & & \\
\hline $1 s 4 f^{3} F_{2}^{o}$ & $1 s 7 d^{3} D_{3}$ & $7.6639 \mathrm{E}+00$ & $5.976 \mathrm{E}-14$ & $9.696 \mathrm{E}-01$ & & & \\
\hline $1 s 4 f^{3} F_{4}^{o}$ & $1 s 7 d^{3} D_{3}$ & $7.6985 \mathrm{E}+00$ & $1.906 \mathrm{E}-08$ & $3.064 \mathrm{E}+05$ & $7.6654 \mathrm{E}+00$ & $1.878 \mathrm{E}-08$ & $3.020 \mathrm{E}+05$ \\
\hline $1 s 4 f^{1} F_{3}^{o}$ & $1 s 7 d^{3} D_{3}$ & $7.6996 \mathrm{E}+00$ & $2.291 \mathrm{E}-09$ & $3.683 \mathrm{E}+04$ & $7.6665 \mathrm{E}+00$ & $2.252 \mathrm{E}-09$ & $3.621 \mathrm{E}+04$ \\
\hline $1 s 5 p^{3} P_{1}^{o}$ & $1 s 7 d^{3} D_{3}$ & $1.5735 \mathrm{E}+01$ & $5.408 \mathrm{E}-08$ & $2.082 \mathrm{E}+05$ & $1.5665 \mathrm{E}+01$ & $5.427 \mathrm{E}-08$ & $2.089 \mathrm{E}+05$ \\
\hline $1 s 5 p^{3} P_{2}^{o}$ & $1 s 7 d^{3} D_{3}$ & $1.6246 \mathrm{E}+01$ & $2.913 \mathrm{E}-07$ & $1.052 \mathrm{E}+06$ & $1.6174 \mathrm{E}+01$ & $2.949 \mathrm{E}-07$ & $1.065 \mathrm{E}+06$ \\
\hline $1 s 5 p{ }^{1} P_{1}^{o}$ & $1 s 7 d^{3} D_{3}$ & $1.6290 \mathrm{E}+01$ & $5.274 \mathrm{E}-08$ & $1.894 \mathrm{E}+05$ & $1.6219 \mathrm{E}+01$ & $5.244 \mathrm{E}-08$ & $1.883 \mathrm{E}+05$ \\
\hline $1 s 5 f^{3} F_{3}^{o}$ & $1 s 7 d^{3} D_{3}$ & $1.6456 \mathrm{E}+01$ & $1.679 \mathrm{E}-14$ & $5.907 \mathrm{E}-02$ & & & \\
\hline $1 s 5 f^{3} F_{2}^{o}$ & $1 s 7 d^{3} D_{3}$ & $1.6460 \mathrm{E}+01$ & $5.449 \mathrm{E}-14$ & $1.917 \mathrm{E}-01$ & & & \\
\hline $1 s 5 f^{3} F_{4}^{o}$ & $1 s 7 d^{3} D_{3}$ & $1.6542 \mathrm{E}+01$ & $3.177 \mathrm{E}-08$ & $1.106 \mathrm{E}+05$ & $1.6469 \mathrm{E}+01$ & $3.151 \mathrm{E}-08$ & $1.097 \mathrm{E}+05$ \\
\hline $1 s 5 f^{1} F_{3}^{o}$ & $1 s 7 d^{3} D_{3}$ & $1.6544 \mathrm{E}+01$ & $3.820 \mathrm{E}-09$ & $1.330 \mathrm{E}+04$ & $1.6472 \mathrm{E}+01$ & $3.779 \mathrm{E}-09$ & $1.316 \mathrm{E}+04$ \\
\hline $1 s 6 p^{3} P_{1}^{0}$ & $1 s 7 d^{3} D_{3}$ & $4.0875 \mathrm{E}+01$ & $2.875 \mathrm{E}-08$ & $1.639 \mathrm{E}+04$ & $4.0680 \mathrm{E}+01$ & $2.791 \mathrm{E}-08$ & $1.593 \mathrm{E}+04$ \\
\hline $1 s 6 p^{3} P_{2}^{o}$ & $1 s 7 d^{3} D_{3}$ & $4.2898 \mathrm{E}+01$ & $1.671 \mathrm{E}-07$ & $8.652 E+04$ & $4.2690 \mathrm{E}+01$ & $1.695 \mathrm{E}-07$ & $8.791 \mathrm{E}+04$ \\
\hline $1 s 6 p{ }^{1} P_{1}^{o}$ & $1 s 7 d^{3} D_{3}$ & $4.3077 \mathrm{E}+01$ & $3.045 \mathrm{E}-08$ & $1.563 \mathrm{E}+04$ & $4.2848 \mathrm{E}+01$ & $3.049 \mathrm{E}-08$ & $1.569 \mathrm{E}+04$ \\
\hline $1 s 6 f^{3} F_{3}^{o}$ & $1 s 7 d^{3} D_{3}$ & $4.3752 \mathrm{E}+01$ & $1.969 \mathrm{E}-14$ & $9.804 \mathrm{E}-03$ & & & \\
\hline $1 s 6 f^{3} F_{2}^{o}$ & $1 s 7 d^{3} D_{3}$ & $4.3767 \mathrm{E}+01$ & $4.018 \mathrm{E}-14$ & $1.999 \mathrm{E}-02$ & & & \\
\hline $1 s 6 f^{3} F_{4}^{o}$ & $1 s 7 d^{3} D_{3}$ & $4.4102 \mathrm{E}+01$ & $4.150 \mathrm{E}-08$ & $2.033 \mathrm{E}+04$ & $4.3907 \mathrm{E}+01$ & $4.131 \mathrm{E}-08$ & $2.024 \mathrm{E}+04$ \\
\hline $1 s 6 f^{1} F_{3}^{o}$ & $1 s 7 d^{3} D_{3}$ & $4.4114 \mathrm{E}+01$ & $4.989 \mathrm{E}-09$ & $2.443 \mathrm{E}+03$ & $4.3908 \mathrm{E}+01$ & $4.957 \mathrm{E}-09$ & $2.429 \mathrm{E}+03$ \\
\hline $1 s 6 h^{3} H_{5}^{o}$ & $1 s 7 d^{3} D_{3}$ & $4.4315 \mathrm{E}+01$ & $9.137 \mathrm{E}-18$ & $4.434 \mathrm{E}-06$ & & & \\
\hline $1 s 6 h^{3} H_{4}^{o}$ & $1 s 7 d^{3} D_{3}$ & $4.4320 \mathrm{E}+01$ & $2.233 \mathrm{E}-18$ & $1.083 \mathrm{E}-06$ & & & \\
\hline $1 s 6 h{ }^{1} H_{5}^{o}$ & $1 s 7 d^{3} D_{3}$ & $4.4461 \mathrm{E}+01$ & $1.723 \mathrm{E}-18$ & $8.305 \mathrm{E}-07$ & & & \\
\hline $1 s 7 p^{3} P_{1}^{o}$ & $1 s 7 d^{3} D_{3}$ & $9.9748 \mathrm{E}+02$ & $3.656 \mathrm{E}-11$ & $3.502 \mathrm{E}-02$ & $9.7908 \mathrm{E}+02$ & $3.761 \mathrm{E}-11$ & $3.707 \mathrm{E}-02$ \\
\hline $1 s 7 p^{3} P_{2}^{0}$ & $1 s 7 d^{3} D_{3}$ & $3.5985 \mathrm{E}+03$ & $3.897 \mathrm{E}-12$ & $2.868 \mathrm{E}-04$ & $3.3938 \mathrm{E}+03$ & $4.609 \mathrm{E}-12$ & $3.780 \mathrm{E}-04$ \\
\hline $1 s 7 p^{1} P_{1}^{o}$ & $1 s 7 d^{3} D_{3}$ & $4.6072 \mathrm{E}+03$ & $3.345 \mathrm{E}-13$ & $1.502 \mathrm{E}-05$ & $4.1560 \mathrm{E}+03$ & $4.627 \mathrm{E}-13$ & $2.531 \mathrm{E}-05$ \\
\hline $1 s 2 s^{3} S_{1}$ & $1 s 7 f^{3} F_{3}^{o}$ & $1.3248 \mathrm{E}+00$ & $4.372 \mathrm{E}-11$ & $2.374 \mathrm{E}+04$ & & & \\
\hline $1 s 3 s^{3} S_{1}$ & $1 s 7 f^{3} F_{3}^{o}$ & $3.3852 \mathrm{E}+00$ & $9.240 \mathrm{E}-15$ & $7.683 \mathrm{E}-01$ & & & \\
\hline $1 s 3 d^{3} D_{2}$ & $1 s 7 f^{3} F_{3}^{o}$ & $3.5247 \mathrm{E}+00$ & $2.120 \mathrm{E}-07$ & $1.626 \mathrm{E}+07$ & $3.5099 \mathrm{E}+00$ & $2.183 \mathrm{E}-07$ & $1.674 \mathrm{E}+07$ \\
\hline $1 s 3 d^{3} D_{1}$ & $1 s 7 f^{3} F_{3}^{o}$ & $3.5262 \mathrm{E}+00$ & $3.577 \mathrm{E}-08$ & $2.742 \mathrm{E}+06$ & $3.5113 \mathrm{E}+00$ & $3.619 \mathrm{E}-08$ & $2.773 \mathrm{E}+06$ \\
\hline $1 s 3 d^{3} D_{3}$ & $1 s 7 f^{3} F_{3}^{o}$ & $3.5615 \mathrm{E}+00$ & $1.257 \mathrm{E}-12$ & $9.441 \mathrm{E}+01$ & & & \\
\hline $1 s 3 d{ }^{1} D_{2}$ & $1 s 7 f^{3} F_{3}^{o}$ & $3.5629 \mathrm{E}+00$ & $1.636 \mathrm{E}-11$ & $1.228 \mathrm{E}+03$ & & & \\
\hline $1 s 4 s^{3} S_{1}$ & $1 s 7 f^{3} F_{3}^{o}$ & $7.3173 \mathrm{E}+00$ & $6.507 \mathrm{E}-14$ & $1.158 \mathrm{E}+00$ & & & \\
\hline $1 s 4 d^{3} D_{2}$ & $1 s 7 f^{3} F_{3}^{o}$ & $7.5891 \mathrm{E}+00$ & $1.297 \mathrm{E}-07$ & $2.146 \mathrm{E}+06$ & $7.5575 \mathrm{E}+00$ & $1.316 \mathrm{E}-07$ & $2.177 \mathrm{E}+06$ \\
\hline $1 s 4 d^{3} D_{1}$ & $1 s 7 f^{3} F_{3}^{o}$ & $7.5919 \mathrm{E}+00$ & $2.189 \mathrm{E}-08$ & $3.619 E+05$ & $7.5603 \mathrm{E}+00$ & $2.182 \mathrm{E}-08$ & $3.607 \mathrm{E}+05$ \\
\hline $1 s 4 d^{3} D_{3}$ & $1 s 7 f^{3} F_{3}^{o}$ & $7.6610 \mathrm{E}+00$ & $6.830 \mathrm{E}-13$ & $1.109 \mathrm{E}+01$ & & & \\
\hline $1 s 4 d^{1} D_{2}$ & $1 s 7 f^{3} F_{3}^{o}$ & $7.6640 \mathrm{E}+00$ & $1.512 \mathrm{E}-11$ & $2.452 \mathrm{E}+02$ & & & \\
\hline $1 s 5 s^{3} S_{1}$ & $1 s 7 f^{3} F_{3}^{o}$ & $1.5648 \mathrm{E}+01$ & $8.099 \mathrm{E}-15$ & $3.152 \mathrm{E}-02$ & & & \\
\hline $1 s 5 d^{3} D_{2}$ & $1 s 7 f^{3} F_{3}^{o}$ & $1.6283 \mathrm{E}+01$ & $7.588 \mathrm{E}-08$ & $2.727 \mathrm{E}+05$ & $1.6216 \mathrm{E}+01$ & $7.648 \mathrm{E}-08$ & $2.748 \mathrm{E}+05$ \\
\hline $1 s 5 d^{3} D_{1}$ & $1 s 7 f^{3} F_{3}^{o}$ & $1.6289 \mathrm{E}+01$ & $1.281 \mathrm{E}-08$ & $4.601 \mathrm{E}+04$ & $1.6222 \mathrm{E}+01$ & $1.268 \mathrm{E}-08$ & $4.552 \mathrm{E}+04$ \\
\hline $1 s 5 d^{3} D_{3}$ & $1 s 7 f^{3} F_{3}^{o}$ & $1.6452 \mathrm{E}+01$ & $4.024 \mathrm{E}-13$ & $1.417 \mathrm{E}+00$ & & & \\
\hline $1 s 5 d{ }^{1} D_{2}$ & $1 s 7 f^{3} F_{3}^{o}$ & $1.6459 \mathrm{E}+01$ & $1.044 \mathrm{E}-11$ & $3.671 \mathrm{E}+01$ & & & \\
\hline $1 s 5 g^{3} G_{4}$ & $1 s 7 f^{3} F_{3}^{o}$ & $1.6541 \mathrm{E}+01$ & $2.537 \mathrm{E}-09$ & $8.837 \mathrm{E}+03$ & $1.6470 \mathrm{E}+01$ & $2.522 \mathrm{E}-09$ & $8.786 \mathrm{E}+03$ \\
\hline $1 s 5 g^{3} G_{3}$ & $1 s 7 f^{3} F_{3}^{o}$ & $1.6543 \mathrm{E}+01$ & $3.069 \mathrm{E}-10$ & $1.069 \mathrm{E}+03$ & $1.6472 \mathrm{E}+01$ & $3.028 \mathrm{E}-10$ & $1.054 \mathrm{E}+03$ \\
\hline $1 s 5 g^{3} G_{5}$ & $1 s 7 f^{3} F_{3}^{o}$ & $1.6592 \mathrm{E}+01$ & $1.834 \mathrm{E}-09$ & $6.348 \mathrm{E}+03$ & $1.6521 \mathrm{E}+01$ & $1.818 \mathrm{E}-09$ & $6.295 E+03$ \\
\hline $1 s 5 g{ }^{1} G_{4}$ & $1 s 7 f^{3} F_{3}^{o}$ & $1.6593 \mathrm{E}+01$ & $1.091 \mathrm{E}-10$ & $3.777 \mathrm{E}+02$ & $1.6522 \mathrm{E}+01$ & $1.137 \mathrm{E}-10$ & $3.937 \mathrm{E}+02$ \\
\hline $1 s 6 s^{3} S_{1}$ & $1 s 7 f^{3} F_{3}^{o}$ & $4.0535 \mathrm{E}+01$ & $3.060 \mathrm{E}-16$ & $1.774 \mathrm{E}-04$ & & & \\
\hline $1 s 6 d^{3} D_{2}$ & $1 s 7 f^{3} F_{3}^{0}$ & $4.3043 \mathrm{E}+01$ & $4.447 \mathrm{E}-08$ & $2.287 \mathrm{E}+04$ & $4.2880 \mathrm{E}+01$ & $4.463 \mathrm{E}-08$ & $2.293 \mathrm{E}+04$ \\
\hline $1 s 6 d^{3} D_{1}$ & $1 s 7 f^{3} F_{3}^{o}$ & $4.3069 \mathrm{E}+01$ & $7.515 \mathrm{E}-09$ & $3.860 \mathrm{E}+03$ & $4.2873 E+01$ & $7.412 \mathrm{E}-09$ & $3.810 \mathrm{E}+03$ \\
\hline $1 s 6 d^{3} D_{3}$ & $1 s 7 f^{3} F_{3}^{o}$ & $4.3731 \mathrm{E}+01$ & $2.440 \mathrm{E}-13$ & $1.216 \mathrm{E}-01$ & & & \\
\hline $1 s 6 d^{1} D_{2}$ & $1 s 7 f^{3} F_{3}^{o}$ & $4.3761 \mathrm{E}+01$ & $6.745 \mathrm{E}-12$ & $3.356 \mathrm{E}+00$ & & & \\
\hline
\end{tabular}


Table 6 (continued)

\begin{tabular}{|c|c|c|c|c|c|c|c|}
\hline \multirow[t]{2}{*}{ Lower } & \multirow[t]{2}{*}{ Upper } & \multicolumn{3}{|l|}{ GRASP2K } & \multicolumn{3}{|l|}{ FAC } \\
\hline & & $\lambda(\AA)$ & $g f$ & $A\left(\mathrm{~s}^{-1}\right)$ & $\lambda(\AA)$ & $g f$ & $A\left(\mathrm{~s}^{-1}\right)$ \\
\hline $1 s 6 g{ }^{3} G_{4}$ & $1 s 7 f^{3} F_{3}^{o}$ & $4.4095 \mathrm{E}+01$ & $5.957 \mathrm{E}-09$ & $2.919 \mathrm{E}+03$ & $4.3907 \mathrm{E}+01$ & $5.943 \mathrm{E}-09$ & $2.912 \mathrm{E}+03$ \\
\hline $1 s 6 g{ }^{3} G_{3}$ & $1 s 7 f^{3} F_{3}^{o}$ & $4.4103 \mathrm{E}+01$ & $7.176 \mathrm{E}-10$ & $3.515 \mathrm{E}+02$ & $4.3907 \mathrm{E}+01$ & $7.132 \mathrm{E}-10$ & $3.495 \mathrm{E}+02$ \\
\hline $1 s 6 g^{3} G_{5}$ & $1 s 7 f^{3} F_{3}^{o}$ & $4.4306 \mathrm{E}+01$ & $4.285 \mathrm{E}-09$ & $2.080 \mathrm{E}+03$ & $4.4116 \mathrm{E}+01$ & $4.256 \mathrm{E}-09$ & $2.066 \mathrm{E}+03$ \\
\hline $1 s 6 g{ }^{1} G_{4}$ & $1 s 7 f^{3} F_{3}^{o}$ & $4.4313 \mathrm{E}+01$ & $2.540 \mathrm{E}-10$ & $1.233 \mathrm{E}+02$ & $4.4116 \mathrm{E}+01$ & $2.616 \mathrm{E}-10$ & $1.270 \mathrm{E}+02$ \\
\hline $1 s 7 s^{3} S_{1}$ & $1 s 7 f^{3} F_{3}^{o}$ & $8.7909 E+02$ & $9.255 \mathrm{E}-18$ & $1.141 \mathrm{E}-08$ & & & \\
\hline $1 s 7 d^{3} D_{2}$ & $1 s 7 f^{3} F_{3}^{o}$ & $4.2661 \mathrm{E}+03$ & $3.833 \mathrm{E}-13$ & $2.007 \mathrm{E}-05$ & $4.3591 \mathrm{E}+03$ & $3.562 \mathrm{E}-13$ & $1.771 \mathrm{E}-05$ \\
\hline $1 s 7 d^{3} D_{1}$ & $1 s 7 f^{3} F_{3}^{o}$ & $4.4317 \mathrm{E}+03$ & $5.768 \mathrm{E}-14$ & $2.799 \mathrm{E}-06$ & $4.3178 \mathrm{E}+03$ & $6.085 \mathrm{E}-14$ & $3.083 \mathrm{E}-06$ \\
\hline $1 s 7 d^{3} D_{3}$ & $1 s 7 f^{3} F_{3}^{o}$ & $2.3237 \mathrm{E}+05$ & $1.291 \mathrm{E}-23$ & $2.278 \mathrm{E}-19$ & & & \\
\hline $1 s^{2}{ }^{1} S_{0}$ & $1 s 7 f^{3} F_{2}^{o}$ & $3.1390 \mathrm{E}-01$ & $4.947 \mathrm{E}-11$ & $6.694 \mathrm{E}+05$ & & & \\
\hline $1 s 2 s^{3} S_{1}$ & $1 s 7 f^{3} F_{2}^{o}$ & $1.3248 \mathrm{E}+00$ & $1.507 \mathrm{E}-11$ & $1.146 \mathrm{E}+04$ & & & \\
\hline $1 s 2 s{ }^{1} S_{0}$ & $1 s 7 f^{3} F_{2}^{o}$ & $1.3369 \mathrm{E}+00$ & $1.400 \mathrm{E}-11$ & $1.045 \mathrm{E}+04$ & & & \\
\hline $1 s 3 s^{3} S_{1}$ & $1 s 7 f^{3} F_{2}^{o}$ & $3.3851 \mathrm{E}+00$ & $1.421 \mathrm{E}-13$ & $1.654 \mathrm{E}+01$ & & & \\
\hline $1 s 3 s{ }^{1} S_{0}$ & $1 s 7 f^{3} F_{2}^{o}$ & $3.4060 \mathrm{E}+00$ & $5.718 \mathrm{E}-14$ & $6.576 \mathrm{E}+00$ & & & \\
\hline $1 s 3 d^{3} D_{2}$ & $1 s 7 f^{3} F_{2}^{o}$ & $3.5247 \mathrm{E}+00$ & $5.320 \mathrm{E}-08$ & $5.713 E+06$ & $3.5099 \mathrm{E}+00$ & $5.455 \mathrm{E}-08$ & $5.857 \mathrm{E}+06$ \\
\hline $1 s 3 d^{3} D_{1}$ & $1 s 7 f^{3} F_{2}^{o}$ & $3.5262 \mathrm{E}+00$ & $1.239 \mathrm{E}-07$ & $1.330 \mathrm{E}+07$ & $3.5113 \mathrm{E}+00$ & $1.271 \mathrm{E}-07$ & $1.363 \mathrm{E}+07$ \\
\hline $1 s 3 d^{3} D_{3}$ & $1 s 7 f^{3} F_{2}^{o}$ & $3.5614 \mathrm{E}+00$ & $1.346 \mathrm{E}-14$ & $1.416 \mathrm{E}+00$ & & & \\
\hline $1 s 3 d^{1} D_{2}$ & $1 s 7 f^{3} F_{2}^{o}$ & $3.5628 \mathrm{E}+00$ & $5.049 \mathrm{E}-13$ & $5.306 \mathrm{E}+01$ & & & \\
\hline $1 s 4 s^{3} S_{1}$ & $1 s 7 f^{3} F_{2}^{o}$ & $7.3170 \mathrm{E}+00$ & $2.407 \mathrm{E}-13$ & $5.998 \mathrm{E}+00$ & & & \\
\hline $1 s 4 s^{1} S_{0}$ & $1 s 7 f^{3} F_{2}^{o}$ & $7.3564 \mathrm{E}+00$ & $1.604 \mathrm{E}-13$ & $3.954 \mathrm{E}+00$ & & & \\
\hline $1 s 4 d^{3} D_{2}$ & $1 s 7 f^{3} F_{2}^{o}$ & $7.5889 \mathrm{E}+00$ & $3.251 \mathrm{E}-08$ & $7.530 \mathrm{E}+05$ & $7.5575 \mathrm{E}+00$ & $3.288 \mathrm{E}-08$ & $7.616 \mathrm{E}+05$ \\
\hline $1 s 4 d^{3} D_{1}$ & $1 s 7 f^{3} F_{2}^{o}$ & $7.5917 \mathrm{E}+00$ & $7.584 \mathrm{E}-08$ & $1.755 \mathrm{E}+06$ & $7.5603 \mathrm{E}+00$ & $7.665 \mathrm{E}-08$ & $1.773 \mathrm{E}+06$ \\
\hline $1 s 4 d^{3} D_{3}$ & $1 s 7 f^{3} F_{2}^{o}$ & $7.6607 \mathrm{E}+00$ & $6.576 \mathrm{E}-15$ & $1.495 \mathrm{E}-01$ & & & \\
\hline $1 s 4 d{ }^{1} D_{2}$ & $1 s 7 f^{3} F_{2}^{o}$ & $7.6637 \mathrm{E}+00$ & $7.964 \mathrm{E}-13$ & $1.809 \mathrm{E}+01$ & & & \\
\hline $1 s 5 s^{3} S_{1}$ & $1 s 7 f^{3} F_{2}^{o}$ & $1.5646 \mathrm{E}+01$ & $1.174 \mathrm{E}-13$ & $6.400 \mathrm{E}-01$ & & & \\
\hline $1 s 5 s{ }^{1} S_{0}$ & $1 s 7 f^{3} F_{2}^{o}$ & $1.5737 \mathrm{E}+01$ & $7.104 \mathrm{E}-14$ & $3.827 \mathrm{E}-01$ & & & \\
\hline $1 s 5 d^{3} D_{2}$ & $1 s 7 f^{3} F_{2}^{o}$ & $1.6281 \mathrm{E}+01$ & $1.900 \mathrm{E}-08$ & $9.564 \mathrm{E}+04$ & $1.6216 \mathrm{E}+01$ & $1.911 \mathrm{E}-08$ & $9.613 \mathrm{E}+04$ \\
\hline $1 s 5 d^{3} D_{1}$ & $1 s 7 f^{3} F_{2}^{o}$ & $1.6288 \mathrm{E}+01$ & $4.437 \mathrm{E}-08$ & $2.231 E+05$ & $1.6222 \mathrm{E}+01$ & $4.453 \mathrm{E}-08$ & $2.238 \mathrm{E}+05$ \\
\hline $1 s 5 d^{3} D_{3}$ & $1 s 7 f^{3} F_{2}^{o}$ & $1.6451 \mathrm{E}+01$ & $9.948 \mathrm{E}-15$ & $4.904 \mathrm{E}-02$ & & & \\
\hline $1 s 5 d{ }^{1} D_{2}$ & $1 s 7 f^{3} F_{2}^{o}$ & $1.6458 \mathrm{E}+01$ & $6.222 \mathrm{E}-13$ & $3.064 \mathrm{E}+00$ & & & \\
\hline $1 s 5 g^{3} G_{4}$ & $1 s 7 f^{3} F_{2}^{o}$ & $1.6539 \mathrm{E}+01$ & $2.043 \mathrm{E}-10$ & $9.963 \mathrm{E}+02$ & $1.6470 \mathrm{E}+01$ & $2.039 \mathrm{E}-10$ & $9.947 \mathrm{E}+02$ \\
\hline $1 s 5 g^{3} G_{3}$ & $1 s 7 f^{3} F_{2}^{o}$ & $1.6541 \mathrm{E}+01$ & $1.835 \mathrm{E}-09$ & $8.949 E+03$ & $1.6472 \mathrm{E}+01$ & $1.817 \mathrm{E}-09$ & $8.858 \mathrm{E}+03$ \\
\hline $1 s 5 g{ }^{1} G_{4}$ & $1 s 7 f^{3} F_{2}^{o}$ & $1.6592 \mathrm{E}+01$ & $1.387 \mathrm{E}-09$ & $6.722 \mathrm{E}+03$ & $1.6522 \mathrm{E}+01$ & $1.376 \mathrm{E}-09$ & $6.669 \mathrm{E}+03$ \\
\hline $1 s 6 s^{3} S_{1}$ & $1 s 7 f^{3} F_{2}^{o}$ & $4.0528 \mathrm{E}+01$ & $4.682 \mathrm{E}-14$ & $3.803 \mathrm{E}-02$ & & & \\
\hline $1 s 6 s{ }^{1} S_{0}$ & $1 s 7 f^{3} F_{2}^{o}$ & $4.0875 E+01$ & $2.291 \mathrm{E}-14$ & $1.830 \mathrm{E}-02$ & & & \\
\hline $1 s 6 d^{3} D_{2}$ & $1 s 7 f^{3} F_{2}^{o}$ & $4.3034 \mathrm{E}+01$ & $1.113 \mathrm{E}-08$ & $8.018 E+03$ & $4.2880 \mathrm{E}+01$ & $1.115 \mathrm{E}-08$ & $8.022 \mathrm{E}+03$ \\
\hline $1 s 6 d^{3} D_{1}$ & $1 s 7 f^{3} F_{2}^{o}$ & $4.3060 \mathrm{E}+01$ & $2.601 \mathrm{E}-08$ & $1.871 \mathrm{E}+04$ & $4.2874 \mathrm{E}+01$ & $2.603 \mathrm{E}-08$ & $1.873 \mathrm{E}+04$ \\
\hline $1 s 6 d^{3} D_{3}$ & $1 s 7 f^{3} F_{2}^{o}$ & $4.3722 \mathrm{E}+01$ & $9.348 \mathrm{E}-15$ & $6.523 \mathrm{E}-03$ & & & \\
\hline $1 s 6 d{ }^{1} D_{2}$ & $1 s 7 f^{3} F_{2}^{o}$ & $4.3752 \mathrm{E}+01$ & $4.162 \mathrm{E}-13$ & $2.900 \mathrm{E}-01$ & & & \\
\hline $1 s 6 g^{3} G_{4}$ & $1 s 7 f^{3} F_{2}^{o}$ & $4.4085 E+01$ & $4.800 \mathrm{E}-10$ & $3.295 E+02$ & $4.3907 \mathrm{E}+01$ & $4.754 \mathrm{E}-10$ & $3.262 \mathrm{E}+02$ \\
\hline $1 s 6 g^{3} G_{3}$ & $1 s 7 f^{3} F_{2}^{o}$ & $4.4094 \mathrm{E}+01$ & $4.293 \mathrm{E}-09$ & $2.946 \mathrm{E}+03$ & $4.3907 \mathrm{E}+01$ & $4.279 \mathrm{E}-09$ & $2.936 \mathrm{E}+03$ \\
\hline $1 s 6 g{ }^{1} G_{4}$ & $1 s 7 f^{3} F_{2}^{o}$ & $4.4303 \mathrm{E}+01$ & $3.232 \mathrm{E}-09$ & $2.197 \mathrm{E}+03$ & $4.4116 \mathrm{E}+01$ & $3.227 \mathrm{E}-09$ & $2.193 \mathrm{E}+03$ \\
\hline $1 s 7 s^{3} s_{1}$ & $1 s 7 f^{3} F_{2}^{o}$ & $8.7540 \mathrm{E}+02$ & $1.929 \mathrm{E}-17$ & $3.359 \mathrm{E}-08$ & & & \\
\hline $1 s 7 s^{1} S_{0}$ & $1 s 7 f^{3} F_{2}^{o}$ & $9.8912 \mathrm{E}+02$ & $1.980 \mathrm{E}-17$ & $2.700 \mathrm{E}-08$ & & & \\
\hline $1 s 7 d^{3} D_{2}$ & $1 s 7 f^{3} F_{2}^{o}$ & $4.1806 \mathrm{E}+03$ & $1.020 \mathrm{E}-13$ & $7.789 \mathrm{E}-06$ & $4.3602 \mathrm{E}+03$ & $8.894 \mathrm{E}-14$ & $6.188 \mathrm{E}-06$ \\
\hline $1 s 7 d^{3} D_{1}$ & $1 s 7 f^{3} F_{2}^{o}$ & $4.3395 E+03$ & $2.128 \mathrm{E}-13$ & $1.508 \mathrm{E}-05$ & $4.3189 \mathrm{E}+03$ & $2.135 E-13$ & $1.514 \mathrm{E}-05$ \\
\hline $1 s 7 d^{3} D_{3}$ & $1 s 7 f^{3} F_{2}^{o}$ & $1.0992 \mathrm{E}+05$ & $3.776 \mathrm{E}-23$ & $4.169 \mathrm{E}-18$ & & & \\
\hline $1 s 2 p^{3} P_{1}^{o}$ & $1 s 7 d^{1} D_{2}$ & $1.3358 \mathrm{E}+00$ & $2.774 \mathrm{E}-07$ & $2.074 \mathrm{E}+08$ & $1.3303 \mathrm{E}+00$ & $2.850 \mathrm{E}-07$ & $2.130 \mathrm{E}+08$ \\
\hline $1 s 2 p^{3} P_{0}^{o}$ & $1 s 7 d^{1} D_{2}$ & $1.3367 \mathrm{E}+00$ & $3.356 \mathrm{E}-07$ & $2.506 \mathrm{E}+08$ & $1.3311 \mathrm{E}+00$ & $3.437 \mathrm{E}-07$ & $2.566 \mathrm{E}+08$ \\
\hline $1 s 2 p^{3} P_{2}^{0}$ & $1 s 7 d^{1} D_{2}$ & $1.3942 \mathrm{E}+00$ & $7.807 \mathrm{E}-07$ & $5.358 \mathrm{E}+08$ & $1.3883 \mathrm{E}+00$ & $8.173 E-07$ & $5.609 \mathrm{E}+08$ \\
\hline $1 s 2 p^{1} P_{1}^{o}$ & $1 s 7 d^{1} D_{2}$ & $1.3997 \mathrm{E}+00$ & $1.753 \mathrm{E}-06$ & $1.194 \mathrm{E}+09$ & $1.3939 \mathrm{E}+00$ & $1.832 \mathrm{E}-06$ & $1.247 \mathrm{E}+09$ \\
\hline $1 s 3 p^{3} P_{1}^{o}$ & $1 s 7 d^{1} D_{2}$ & $3.4047 \mathrm{E}+00$ & $9.352 \mathrm{E}-08$ & $1.076 \mathrm{E}+07$ & $3.3905 E+00$ & $9.546 \mathrm{E}-08$ & $1.098 \mathrm{E}+07$ \\
\hline $1 s 3 p^{3} P_{0}^{0}$ & $1 s 7 d^{1} D_{2}$ & $3.4061 \mathrm{E}+00$ & $1.139 \mathrm{E}-07$ & $1.310 \mathrm{E}+07$ & $3.3919 \mathrm{E}+00$ & $1.162 \mathrm{E}-07$ & $1.336 \mathrm{E}+07$ \\
\hline $1 s 3 p^{3} P_{2}^{o}$ & $1 s 7 d^{1} D_{2}$ & $3.5163 E+00$ & $2.950 \mathrm{E}-07$ & $3.183 \mathrm{E}+07$ & $3.5016 \mathrm{E}+00$ & $2.998 \mathrm{E}-07$ & $3.234 \mathrm{E}+07$ \\
\hline $1 s 3 p{ }^{1} P_{1}^{o}$ & $1 s 7 d^{1} D_{2}$ & $3.5262 \mathrm{E}+00$ & $6.667 \mathrm{E}-07$ & $7.153 \mathrm{E}+07$ & $3.5116 \mathrm{E}+00$ & $6.762 \mathrm{E}-07$ & $7.254 \mathrm{E}+07$ \\
\hline $1 s 4 p^{3} P_{1}^{o}$ & $1 s 7 d^{1} D_{2}$ & $7.3543 \mathrm{E}+00$ & $4.023 \mathrm{E}-08$ & $9.922 E+05$ & $7.3231 \mathrm{E}+00$ & $4.103 \mathrm{E}-08$ & $1.012 \mathrm{E}+06$ \\
\hline $1 s 4 p^{3} P_{0}^{o}$ & $1 s 7 d^{1} D_{2}$ & $7.3570 \mathrm{E}+00$ & $4.925 \mathrm{E}-08$ & $1.214 \mathrm{E}+06$ & $7.3257 \mathrm{E}+00$ & $4.994 \mathrm{E}-08$ & $1.230 \mathrm{E}+06$ \\
\hline $1 s 4 p^{3} P_{2}^{o}$ & $1 s 7 d^{1} D_{2}$ & $7.5726 \mathrm{E}+00$ & $1.361 \mathrm{E}-07$ & $3.167 E+06$ & $7.5404 \mathrm{E}+00$ & $1.375 \mathrm{E}-07$ & $3.200 \mathrm{E}+06$ \\
\hline $1 s 4 p^{1} P_{1}^{o}$ & $1 s 7 d^{1} D_{2}$ & $7.5916 \mathrm{E}+00$ & $3.096 \mathrm{E}-07$ & $7.166 \mathrm{E}+06$ & $7.5595 \mathrm{E}+00$ & $3.110 \mathrm{E}-07$ & $7.199 \mathrm{E}+06$ \\
\hline $1 s 4 f^{3} F_{3}^{o}$ & $1 s 7 d^{1} D_{2}$ & $7.6618 \mathrm{E}+00$ & $2.579 \mathrm{E}-13$ & $5.861 \mathrm{E}+00$ & & & \\
\hline $1 s 4 f^{3} F_{2}^{o}$ & $1 s 7 d^{1} D_{2}$ & $7.6633 \mathrm{E}+00$ & $1.012 \mathrm{E}-18$ & $2.299 \mathrm{E}-05$ & & & \\
\hline $1 s 4 f^{3} F_{4}^{o}$ & $1 s 7 d^{1} D_{2}$ & $7.6979 \mathrm{E}+00$ & $1.547 \mathrm{E}-09$ & $3.482 \mathrm{E}+04$ & $7.6651 \mathrm{E}+00$ & $1.558 \mathrm{E}-09$ & $3.507 \mathrm{E}+04$ \\
\hline $1 s 4 f^{1} F_{3}^{o}$ & $1 s 7 d^{1} D_{2}$ & $7.6990 \mathrm{E}+00$ & $1.365 \mathrm{E}-08$ & $3.073 E+05$ & $7.6663 \mathrm{E}+00$ & $1.346 \mathrm{E}-08$ & $3.029 \mathrm{E}+05$ \\
\hline $1 s 5 p^{3} P_{1}^{o}$ & $1 s 7 d^{1} D_{2}$ & $1.5732 \mathrm{E}+01$ & $2.027 \mathrm{E}-08$ & $1.093 \mathrm{E}+05$ & $1.5665 \mathrm{E}+01$ & $2.079 \mathrm{E}-08$ & $1.121 \mathrm{E}+05$ \\
\hline $1 s 5 p^{3} P_{0}^{o}$ & $1 s 7 d^{1} D_{2}$ & $1.5738 \mathrm{E}+01$ & $2.489 \mathrm{E}-08$ & $1.341 \mathrm{E}+05$ & $1.5671 \mathrm{E}+01$ & $2.518 \mathrm{E}-08$ & $1.356 \mathrm{E}+05$ \\
\hline $1 s 5 p^{3} P_{2}^{o}$ & $1 s 7 d^{1} D_{2}$ & $1.6243 \mathrm{E}+01$ & $7.285 \mathrm{E}-08$ & $3.683 E+05$ & $1.6173 \mathrm{E}+01$ & $7.372 \mathrm{E}-08$ & $3.728 \mathrm{E}+05$ \\
\hline $1 s 5 p^{1} P_{1}^{o}$ & $1 s 7 d^{1} D_{2}$ & $1.6288 \mathrm{E}+01$ & $1.665 \mathrm{E}-07$ & $8.375 E+05$ & $1.6218 \mathrm{E}+01$ & $1.667 \mathrm{E}-07$ & $8.385 E+05$ \\
\hline $1 s 5 f^{3} F_{3}^{o}$ & $1 s 7 d^{1} D_{2}$ & $1.6454 \mathrm{E}+01$ & $4.024 \mathrm{E}-13$ & $1.983 \mathrm{E}+00$ & & & \\
\hline $1 s 5 f^{3} F_{2}^{0}$ & $1 s 7 d^{1} D_{2}$ & $1.6457 \mathrm{E}+01$ & $1.812 \mathrm{E}-15$ & $8.923 \mathrm{E}-03$ & & & \\
\hline $1 s 5 f^{3} F_{4}^{o}$ & $1 s 7 d^{1} D_{2}$ & $1.6539 \mathrm{E}+01$ & $2.579 \mathrm{E}-09$ & $1.258 \mathrm{E}+04$ & $1.6468 \mathrm{E}+01$ & $2.613 \mathrm{E}-09$ & $1.274 \mathrm{E}+04$ \\
\hline $1 s 5 f^{1} F_{3}^{o}$ & $1 s 7 d^{1} D_{2}$ & $1.6542 \mathrm{E}+01$ & $2.277 \mathrm{E}-08$ & $1.110 \mathrm{E}+05$ & $1.6471 \mathrm{E}+01$ & $2.258 \mathrm{E}-08$ & $1.101 \mathrm{E}+05$ \\
\hline $1 s 6 p^{3} P_{1}^{o}$ & $1 s 7 d^{1} D_{2}$ & $4.0858 \mathrm{E}+01$ & $1.073 \mathrm{E}-08$ & $8.576 E+03$ & $4.0674 \mathrm{E}+01$ & $1.190 \mathrm{E}-08$ & $9.521 \mathrm{E}+03$ \\
\hline
\end{tabular}


Table 6 (continued)

\begin{tabular}{|c|c|c|c|c|c|c|c|}
\hline \multirow[t]{2}{*}{ Lower } & \multirow[t]{2}{*}{ Upper } & \multicolumn{3}{|l|}{ GRASP2K } & \multicolumn{3}{|l|}{ FAC } \\
\hline & & $\overline{\lambda(\AA)}$ & $g f$ & $A\left(\mathrm{~s}^{-1}\right)$ & $\lambda(\AA)$ & $g f$ & $A\left(\mathrm{~s}^{-1}\right)$ \\
\hline $1 s 6 p^{3} P_{0}^{o}$ & $1 s 7 d^{1} D_{2}$ & $4.0882 \mathrm{E}+01$ & $1.321 \mathrm{E}-08$ & $1.055 \mathrm{E}+04$ & $4.0612 \mathrm{E}+01$ & $1.340 \mathrm{E}-08$ & $1.074 \mathrm{E}+04$ \\
\hline $1 s 6 p^{3} P_{2}^{o}$ & $1 s 7 d^{1} D_{2}$ & $4.2880 \mathrm{E}+01$ & $4.171 \mathrm{E}-08$ & $3.026 \mathrm{E}+04$ & $4.2684 \mathrm{E}+01$ & $4.240 \mathrm{E}-08$ & $3.079 \mathrm{E}+04$ \\
\hline $1 s 6 p^{1} P_{1}^{o}$ & $1 s 7 d^{1} D_{2}$ & $4.3058 \mathrm{E}+01$ & $9.601 \mathrm{E}-08$ & $6.908 \mathrm{E}+04$ & $4.2841 \mathrm{E}+01$ & $9.514 \mathrm{E}-08$ & $6.856 \mathrm{E}+04$ \\
\hline $1 s 6 f^{3} F_{3}^{o}$ & $1 s 7 d^{1} D_{2}$ & $4.3733 \mathrm{E}+01$ & $5.031 \mathrm{E}-13$ & $3.509 \mathrm{E}-01$ & & & \\
\hline $1 s 6 f^{3} F_{2}^{o}$ & $1 s 7 d^{1} D_{2}$ & $4.3748 \mathrm{E}+01$ & $6.962 \mathrm{E}-15$ & $4.853 \mathrm{E}-03$ & & & \\
\hline $1 s 6 f^{3} F_{4}^{o}$ & $1 s 7 d^{1} D_{2}$ & $4.4083 \mathrm{E}+01$ & $3.368 \mathrm{E}-09$ & $2.312 \mathrm{E}+03$ & $4.3900 \mathrm{E}+01$ & $3.427 \mathrm{E}-09$ & $2.352 \mathrm{E}+03$ \\
\hline $1 s 6 f^{1} F_{3}^{o}$ & $1 s 7 d^{1} D_{2}$ & $4.4094 \mathrm{E}+01$ & $2.973 \mathrm{E}-08$ & $2.040 \mathrm{E}+04$ & $4.3901 \mathrm{E}+01$ & $2.963 \mathrm{E}-08$ & $2.033 \mathrm{E}+04$ \\
\hline $1 s 6 h^{3} H_{4}^{o}$ & $1 s 7 d^{1} D_{2}$ & $4.4301 \mathrm{E}+01$ & $4.583 \mathrm{E}-18$ & $3.115 \mathrm{E}-06$ & & & \\
\hline $1 s 7 p^{3} P_{1}^{o}$ & $1 s 7 d^{1} D_{2}$ & $9.8772 \mathrm{E}+02$ & $1.410 \mathrm{E}-11$ & $1.928 \mathrm{E}-02$ & $9.7564 \mathrm{E}+02$ & $1.532 \mathrm{E}-11$ & $2.128 \mathrm{E}-02$ \\
\hline $1 s 7 p^{3} P_{0}^{0}$ & $1 s 7 d^{1} D_{2}$ & $9.9663 \mathrm{E}+02$ & $1.685 \mathrm{E}-11$ & $2.263 \mathrm{E}-02$ & $9.5369 \mathrm{E}+02$ & $1.915 \mathrm{E}-11$ & $2.785 \mathrm{E}-02$ \\
\hline $1 s 7 p^{3} P_{2}^{o}$ & $1 s 7 d^{1} D_{2}$ & $3.4747 \mathrm{E}+03$ & $1.082 \mathrm{E}-12$ & $1.196 \mathrm{E}-04$ & $3.3529 E+03$ & $1.194 \mathrm{E}-12$ & $1.405 \mathrm{E}-04$ \\
\hline $1 s 7 p^{1} P_{1}^{o}$ & $1 s 7 d^{1} D_{2}$ & $4.4062 \mathrm{E}+03$ & $1.207 \mathrm{E}-12$ & $8.296 E-05$ & $4.0947 \mathrm{E}+03$ & $1.481 \mathrm{E}-12$ & $1.169 \mathrm{E}-04$ \\
\hline $1 s 7 f^{3} F_{3}^{o}$ & $1 s 7 d^{1} D_{2}$ & $1.7858 \mathrm{E}+05$ & $7.949 \mathrm{E}-22$ & $3.325 \mathrm{E}-17$ & & & \\
\hline $1 s 7 f^{3} F_{2}^{o}$ & $1 s 7 d^{1} D_{2}$ & $1.2412 \mathrm{E}+06$ & $1.105 \mathrm{E}-25$ & $9.570 \mathrm{E}-23$ & & & \\
\hline $1 s 3 d^{3} D_{2}$ & $1 s 7 f^{3} F_{4}^{o}$ & $3.5233 \mathrm{E}+00$ & $4.205 \mathrm{E}-07$ & $2.510 \mathrm{E}+07$ & $3.5085 \mathrm{E}+00$ & $4.222 \mathrm{E}-07$ & $2.520 \mathrm{E}+07$ \\
\hline $1 s 3 d^{3} D_{3}$ & $1 s 7 f^{3} F_{4}^{o}$ & $3.5600 \mathrm{E}+00$ & $2.526 \mathrm{E}-06$ & $1.477 \mathrm{E}+08$ & $3.5449 \mathrm{E}+00$ & $2.596 \mathrm{E}-06$ & $1.518 \mathrm{E}+08$ \\
\hline $1 s 3 d d^{1} D_{2}$ & $1 s 7 f^{3} F_{4}^{0}$ & $3.5614 \mathrm{E}+00$ & $2.038 \mathrm{E}-07$ & $1.191 \mathrm{E}+07$ & $3.5463 \mathrm{E}+00$ & $2.095 \mathrm{E}-07$ & $1.224 \mathrm{E}+07$ \\
\hline $1 s 4 d^{3} D_{2}$ & $1 s 7 f^{3} F_{4}^{0}$ & $7.5826 \mathrm{E}+00$ & $2.510 \mathrm{E}-07$ & $3.235 E+06$ & $7.5510 \mathrm{E}+00$ & $2.533 \mathrm{E}-07$ & $3.265 E+06$ \\
\hline $1 s 4 d^{3} D_{3}$ & $1 s 7 f^{3} F_{4}^{o}$ & $7.6543 \mathrm{E}+00$ & $1.564 \mathrm{E}-06$ & $1.979 \mathrm{E}+07$ & $7.6222 \mathrm{E}+00$ & $1.584 \mathrm{E}-06$ & $2.003 \mathrm{E}+07$ \\
\hline $1 s 4 d{ }^{1} D_{2}$ & $1 s 7 f^{3} F_{4}^{o}$ & $7.6573 \mathrm{E}+00$ & $1.270 \mathrm{E}-07$ & $1.605 E+06$ & $7.6252 \mathrm{E}+00$ & $1.286 \mathrm{E}-07$ & $1.625 \mathrm{E}+06$ \\
\hline $1 s 5 d^{3} D_{2}$ & $1 s 7 f^{3} F_{4}^{0}$ & $1.6252 \mathrm{E}+01$ & $1.445 \mathrm{E}-07$ & $4.054 \mathrm{E}+05$ & $1.6186 \mathrm{E}+01$ & $1.453 \mathrm{E}-07$ & $4.077 \mathrm{E}+05$ \\
\hline $1 s 5 d^{3} D_{3}$ & $1 s 7 f^{3} F_{4}^{0}$ & $1.6421 \mathrm{E}+01$ & $9.255 \mathrm{E}-07$ & $2.544 \mathrm{E}+06$ & $1.6353 \mathrm{E}+01$ & $9.309 \mathrm{E}-07$ & $2.558 \mathrm{E}+06$ \\
\hline $1 s 5 d{ }^{1} D_{2}$ & $1 s 7 f^{3} F_{4}^{o}$ & $1.6428 \mathrm{E}+01$ & $7.534 \mathrm{E}-08$ & $2.069 \mathrm{E}+05$ & $1.6360 \mathrm{E}+01$ & $7.572 \mathrm{E}-08$ & $2.078 \mathrm{E}+05$ \\
\hline $1 s 5 g^{3} G_{4}$ & $1 s 7 f^{3} F_{4}^{o}$ & $1.6509 \mathrm{E}+01$ & $3.180 \mathrm{E}-15$ & $8.647 \mathrm{E}-03$ & & & \\
\hline $1 s 5 g^{3} G_{3}$ & $1 s 7 f^{3} F_{4}^{0}$ & $1.6511 \mathrm{E}+01$ & $4.291 \mathrm{E}-16$ & $1.166 \mathrm{E}-03$ & & & \\
\hline $1 s 5 g^{3} G_{5}$ & $1 s 7 f^{3} F_{4}^{0}$ & $1.6560 \mathrm{E}+01$ & $1.498 \mathrm{E}-08$ & $4.050 \mathrm{E}+04$ & $1.6489 \mathrm{E}+01$ & $1.487 \mathrm{E}-08$ & $4.021 \mathrm{E}+04$ \\
\hline $1 s 5 g{ }^{1} G_{4}$ & $1 s 7 f^{3} F_{4}^{o}$ & $1.6562 \mathrm{E}+01$ & $1.077 \mathrm{E}-09$ & $2.909 E+03$ & $1.6491 \mathrm{E}+01$ & $1.062 \mathrm{E}-09$ & $2.871 \mathrm{E}+03$ \\
\hline $1 s 6 d^{3} D_{2}$ & $1 s 7 f^{3} F_{4}^{0}$ & $4.2832 \mathrm{E}+01$ & $8.329 \mathrm{E}-08$ & $3.365 \mathrm{E}+04$ & $4.2671 \mathrm{E}+01$ & $8.278 \mathrm{E}-08$ & $3.340 \mathrm{E}+04$ \\
\hline $1 s 6 d^{3} D_{3}$ & $1 s 7 f^{3} F_{4}^{o}$ & $4.3514 \mathrm{E}+01$ & $5.502 \mathrm{E}-07$ & $2.154 \mathrm{E}+05$ & $4.3345 \mathrm{E}+01$ & $5.513 \mathrm{E}-07$ & $2.156 \mathrm{E}+05$ \\
\hline $1 s 6 d^{1} D_{2}$ & $1 s 7 f^{3} F_{4}^{0}$ & $4.3544 \mathrm{E}+01$ & $4.488 \mathrm{E}-08$ & $1.754 \mathrm{E}+04$ & $4.3355 \mathrm{E}+01$ & $4.548 \mathrm{E}-08$ & $1.778 \mathrm{E}+04$ \\
\hline $1 s 6 g^{3} G_{4}$ & $1 s 7 f^{3} F_{4}^{o}$ & $4.3874 \mathrm{E}+01$ & $8.351 \mathrm{E}-15$ & $3.215 E-03$ & & & \\
\hline $1 s 6 g^{3} G_{3}$ & $1 s 7 f^{3} F_{4}^{0}$ & $4.3882 \mathrm{E}+01$ & $5.086 \mathrm{E}-16$ & $1.957 \mathrm{E}-04$ & & & \\
\hline $1 s 6 g^{3} G_{5}$ & $1 s 7 f^{3} F_{4}^{0}$ & $4.4083 \mathrm{E}+01$ & $3.532 \mathrm{E}-08$ & $1.347 \mathrm{E}+04$ & $4.3895 \mathrm{E}+01$ & $3.519 \mathrm{E}-08$ & $1.342 \mathrm{E}+04$ \\
\hline $1 s 6 g^{1} G_{4}$ & $1 s 7 f^{3} F_{4}^{0}$ & $4.4090 \mathrm{E}+01$ & $2.529 \mathrm{E}-09$ & $9.643 \mathrm{E}+02$ & $4.3895 \mathrm{E}+01$ & $2.514 \mathrm{E}-09$ & $9.588 \mathrm{E}+02$ \\
\hline $1 s 7 d^{3} D_{2}$ & $1 s 7 f^{3} F_{4}^{o}$ & $2.8680 \mathrm{E}+03$ & $2.422 \mathrm{E}-12$ & $2.182 \mathrm{E}-04$ & $2.9119 \mathrm{E}+03$ & $2.266 \mathrm{E}-12$ & $1.964 \mathrm{E}-04$ \\
\hline $1 s 7 d^{3} D_{3}$ & $1 s 7 f^{3} F_{4}^{0}$ & $8.4332 \mathrm{E}+03$ & $6.104 \mathrm{E}-13$ & $6.362 \mathrm{E}-06$ & $8.7593 \mathrm{E}+03$ & $5.382 \mathrm{E}-13$ & $5.155 \mathrm{E}-06$ \\
\hline $1 s 7 d^{1} D_{2}$ & $1 s 7 f^{3} F_{4}^{o}$ & $9.2016 \mathrm{E}+03$ & $3.828 \mathrm{E}-14$ & $3.351 \mathrm{E}-07$ & $9.0446 \mathrm{E}+03$ & $4.045 \mathrm{E}-14$ & $3.633 \mathrm{E}-07$ \\
\hline $1 s 2 p^{3} P_{2}^{o}$ & $1 s 7 g{ }^{3} G_{4}$ & $1.3940 \mathrm{E}+00$ & $1.685 \mathrm{E}-11$ & $6.427 \mathrm{E}+03$ & & & \\
\hline $1 s 3 p^{3} P_{2}^{o}$ & $1 s 7 g{ }^{3} G_{4}$ & $3.5149 \mathrm{E}+00$ & $1.323 \mathrm{E}-11$ & $7.934 \mathrm{E}+02$ & & & \\
\hline $1 s 4 p^{3} P_{2}^{o}$ & $1 s 7 g{ }^{3} G_{4}$ & $7.5664 \mathrm{E}+00$ & $2.232 \mathrm{E}-13$ & $2.889 \mathrm{E}+00$ & & & \\
\hline $1 s 4 f^{3} F_{3}^{o}$ & $1 s 7 g{ }^{3} G_{4}$ & $7.6554 \mathrm{E}+00$ & $4.102 \mathrm{E}-07$ & $5.187 \mathrm{E}+06$ & $7.6229 \mathrm{E}+00$ & $4.168 \mathrm{E}-07$ & $5.271 \mathrm{E}+06$ \\
\hline $1 s 4 f^{3} F_{2}^{o}$ & $1 s 7 g{ }^{3} G_{4}$ & $7.6569 \mathrm{E}+00$ & $3.311 \mathrm{E}-08$ & $4.186 \mathrm{E}+05$ & $7.6244 \mathrm{E}+00$ & $3.332 \mathrm{E}-08$ & $4.212 \mathrm{E}+05$ \\
\hline $1 s 4 f^{3} F_{4}^{o}$ & $1 s 7 g{ }^{3} G_{4}$ & $7.6914 \mathrm{E}+00$ & $6.083 \mathrm{E}-13$ & $7.620 \mathrm{E}+00$ & & & \\
\hline $1 s 4 f^{1} F_{3}^{o}$ & $1 s 7 g{ }^{3} G_{4}$ & $7.6926 \mathrm{E}+00$ & $1.492 \mathrm{E}-12$ & $1.869 \mathrm{E}+01$ & & & \\
\hline $1 s 5 p^{3} P_{2}^{o}$ & $1 s 7 g^{3} G_{4}$ & $1.6215 \mathrm{E}+01$ & $2.286 \mathrm{E}-14$ & $6.444 \mathrm{E}-02$ & & & \\
\hline $1 s 5 f^{3} F_{3}^{o}$ & $1 s 7 g{ }^{3} G_{4}$ & $1.6424 \mathrm{E}+01$ & $3.500 \mathrm{E}-07$ & $9.616 \mathrm{E}+05$ & $1.6354 \mathrm{E}+01$ & $3.528 \mathrm{E}-07$ & $9.693 \mathrm{E}+05$ \\
\hline $1 s 5 f^{3} F_{2}^{o}$ & $1 s 7 g{ }^{3} G_{4}$ & $1.6428 \mathrm{E}+01$ & $2.827 \mathrm{E}-08$ & $7.765 E+04$ & $1.6358 \mathrm{E}+01$ & $2.820 \mathrm{E}-08$ & $7.745 \mathrm{E}+04$ \\
\hline $1 s 5 f^{3} F_{4}^{0}$ & $1 s 7 g{ }^{3} G_{4}$ & $1.6509 \mathrm{E}+01$ & $5.308 \mathrm{E}-13$ & $1.443 \mathrm{E}+00$ & & & \\
\hline $1 s 5 f^{1} F_{3}^{o}$ & $1 s 7 g{ }^{3} G_{4}$ & $1.6512 \mathrm{E}+01$ & $1.496 \mathrm{E}-12$ & $4.068 \mathrm{E}+00$ & & & \\
\hline $1 s 6 p^{3} P_{2}^{o}$ & $1 s 7 g{ }^{3} G_{4}$ & $4.2681 \mathrm{E}+01$ & $1.962 \mathrm{E}-14$ & $7.981 \mathrm{E}-03$ & & & \\
\hline $1 s 6 f^{3} F_{3}^{o}$ & $1 s 7 g{ }^{3} G_{4}$ & $4.3526 \mathrm{E}+01$ & $2.388 \mathrm{E}-07$ & $9.340 \mathrm{E}+04$ & $4.3346 \mathrm{E}+01$ & $2.395 \mathrm{E}-07$ & $9.368 \mathrm{E}+04$ \\
\hline $1 s 6 f^{3} F_{2}^{0}$ & $1 s 7 g{ }^{3} G_{4}$ & $4.3540 \mathrm{E}+01$ & $1.930 \mathrm{E}-08$ & $7.546 \mathrm{E}+03$ & $4.3346 \mathrm{E}+01$ & $1.916 \mathrm{E}-08$ & $7.494 \mathrm{E}+03$ \\
\hline $1 s 6 f^{3} F_{4}^{o}$ & $1 s 7 g{ }^{3} G_{4}$ & $4.3873 \mathrm{E}+01$ & $3.710 \mathrm{E}-13$ & $1.429 \mathrm{E}-01$ & & & \\
\hline $1 s 6 f^{1} F_{3}^{o}$ & $1 s 7 g{ }^{3} G_{4}$ & $4.3884 \mathrm{E}+01$ & $1.132 \mathrm{E}-12$ & $4.356 \mathrm{E}-01$ & & & \\
\hline $1 s 6 h^{3} H_{5}^{o}$ & $1 s 7 g{ }^{3} G_{4}$ & $4.4083 \mathrm{E}+01$ & $4.476 \mathrm{E}-09$ & $1.707 \mathrm{E}+03$ & $4.3896 \mathrm{E}+01$ & $4.459 \mathrm{E}-09$ & $1.700 \mathrm{E}+03$ \\
\hline $1 s 6 h^{3} H_{4}^{o}$ & $1 s 7 g{ }^{3} G_{4}$ & $4.4088 \mathrm{E}+01$ & $3.202 \mathrm{E}-10$ & $1.221 \mathrm{E}+02$ & $4.3896 \mathrm{E}+01$ & $3.185 \mathrm{E}-10$ & $1.214 \mathrm{E}+02$ \\
\hline $1 s 6 h^{3} H_{6}^{o}$ & $1 s 7 g{ }^{3} G_{4}$ & $4.4223 \mathrm{E}+01$ & $1.616 \mathrm{E}-09$ & $6.123 \mathrm{E}+02$ & $4.4034 \mathrm{E}+01$ & $1.606 \mathrm{E}-09$ & $6.089 \mathrm{E}+02$ \\
\hline $1 s 6 h^{1} H_{5}^{o}$ & $1 s 7 g{ }^{3} G_{4}$ & $4.4227 \mathrm{E}+01$ & $6.000 \mathrm{E}-11$ & $2.273 \mathrm{E}+01$ & $4.4034 \mathrm{E}+01$ & $6.182 \mathrm{E}-11$ & $2.342 \mathrm{E}+01$ \\
\hline $1 s 7 p^{3} P_{2}^{o}$ & $1 s 7 g{ }^{3} G_{4}$ & $2.5209 \mathrm{E}+03$ & $4.451 \mathrm{E}-21$ & $5.191 \mathrm{E}-13$ & & & \\
\hline $1 s 7 f^{3} F_{3}^{o}$ & $1 s 7 g{ }^{3} G_{4}$ & $8.7347 \mathrm{E}+03$ & $1.399 \mathrm{E}-13$ & $1.359 \mathrm{E}-06$ & $8.7974 \mathrm{E}+03$ & $1.354 \mathrm{E}-13$ & $1.286 \mathrm{E}-06$ \\
\hline $1 s 7 f^{3} F_{2}^{o}$ & $1 s 7 g{ }^{3} G_{4}$ & $9.1165 E+03$ & $9.944 \mathrm{E}-15$ & $8.867 \mathrm{E}-08$ & $8.7927 \mathrm{E}+03$ & $1.085 \mathrm{E}-14$ & $1.031 \mathrm{E}-07$ \\
\hline $1 s 7 f^{3} F_{4}^{o}$ & $1 s 7 g{ }^{3} G_{4}$ & $4.7642 \mathrm{E}+06$ & $1.343 \mathrm{E}-27$ & $4.385 \mathrm{E}-26$ & & & \\
\hline $1 s 2 p^{3} P_{1}^{0}$ & $1 s 7 g{ }^{3} G_{3}$ & $1.3356 \mathrm{E}+00$ & $3.671 \mathrm{E}-14$ & $1.961 \mathrm{E}+01$ & & & \\
\hline $1 s 2 p^{3} P_{2}^{o}$ & $1 s 7 g{ }^{3} G_{3}$ & $1.3940 \mathrm{E}+00$ & $1.616 \mathrm{E}-12$ & $7.925 \mathrm{E}+02$ & & & \\
\hline $1 s 2 p^{1} P_{1}^{o}$ & $1 s 7 g{ }^{3} G_{3}$ & $1.3995 \mathrm{E}+00$ & $1.116 \mathrm{E}-11$ & $5.428 \mathrm{E}+03$ & & & \\
\hline $1 s 3 p^{3} P_{1}^{0}$ & $1 s 7 g{ }^{3} G_{3}$ & $3.4034 \mathrm{E}+00$ & $3.734 \mathrm{E}-14$ & $3.072 \mathrm{E}+00$ & & & \\
\hline $1 s 3 p^{3} P_{2}^{0}$ & $1 s 7 g{ }^{3} G_{3}$ & $3.5149 \mathrm{E}+00$ & $1.511 \mathrm{E}-12$ & $1.166 \mathrm{E}+02$ & & & \\
\hline $1 s 3 p{ }^{1} P_{1}^{o}$ & $1 s 7 g{ }^{3} G_{3}$ & $3.5248 \mathrm{E}+00$ & $7.982 \mathrm{E}-12$ & $6.122 \mathrm{E}+02$ & & & \\
\hline $1 s 4 p^{3} P_{1}^{o}$ & $1 s 7 g{ }^{3} G_{3}$ & $7.3482 \mathrm{E}+00$ & $8.284 \mathrm{E}-16$ & $1.462 \mathrm{E}-02$ & & & \\
\hline $1 s 4 p^{3} P_{2}^{0}$ & $1 s 7 g{ }^{3} G_{3}$ & $7.5662 \mathrm{E}+00$ & $2.075 \mathrm{E}-15$ & $3.453 \mathrm{E}-02$ & & & \\
\hline $1 s 4 p{ }^{1} P_{1}^{o}$ & $1 s 7 g{ }^{3} G_{3}$ & $7.5852 \mathrm{E}+00$ & $9.542 \mathrm{E}-14$ & $1.580 \mathrm{E}+00$ & & & \\
\hline
\end{tabular}


Table 6 (continued)

\begin{tabular}{|c|c|c|c|c|c|c|c|}
\hline \multirow[t]{2}{*}{ Lower } & \multirow[t]{2}{*}{ Upper } & \multicolumn{3}{|l|}{ GRASP2K } & \multicolumn{3}{|l|}{ FAC } \\
\hline & & $\lambda(\AA)$ & $g f$ & $A\left(\mathrm{~s}^{-1}\right)$ & $\lambda(\AA)$ & $g f$ & $A\left(\mathrm{~s}^{-1}\right)$ \\
\hline $1 s 4 f^{3} F_{3}^{o}$ & $1 s 7 g{ }^{3} G_{3}$ & $7.6553 \mathrm{E}+00$ & $4.932 \mathrm{E}-08$ & $8.020 \mathrm{E}+05$ & $7.6229 \mathrm{E}+00$ & $5.002 \mathrm{E}-08$ & $8.132 E+05$ \\
\hline $1 s 4 f^{3} F_{2}^{o}$ & $1 s 7 g{ }^{3} G_{3}$ & $7.6568 \mathrm{E}+00$ & $2.955 \mathrm{E}-07$ & $4.803 \mathrm{E}+06$ & $7.6244 \mathrm{E}+00$ & $2.999 E-07$ & $4.874 \mathrm{E}+06$ \\
\hline $1 s 4 f^{3} F_{4}^{o}$ & $1 s 7 g{ }^{3} G_{3}$ & $7.6913 \mathrm{E}+00$ & $1.523 \mathrm{E}-19$ & $2.453 \mathrm{E}-06$ & & & \\
\hline $1 s 4 f^{1} F_{3}^{o}$ & $1 s 7 g{ }^{3} G_{3}$ & $7.6924 \mathrm{E}+00$ & $2.237 \mathrm{E}-13$ & $3.603 \mathrm{E}+00$ & & & \\
\hline $1 s 5 p^{3} P_{1}^{o}$ & $1 s 7 g{ }^{3} G_{3}$ & $1.5705 \mathrm{E}+01$ & $1.628 \mathrm{E}-19$ & $6.288 \mathrm{E}-07$ & & & \\
\hline $1 s 5 p^{3} P_{2}^{o}$ & $1 s 7 g{ }^{3} G_{3}$ & $1.6214 \mathrm{E}+01$ & $8.575 \mathrm{E}-14$ & $3.108 \mathrm{E}-01$ & & & \\
\hline $1 s 5 p{ }^{1} P_{1}^{o}$ & $1 s 7 g{ }^{3} G_{3}$ & $1.6258 \mathrm{E}+01$ & $3.715 \mathrm{E}-14$ & $1.339 \mathrm{E}-01$ & & & \\
\hline $1 s 5 f^{3} F_{3}^{o}$ & $1 s 7 g{ }^{3} G_{3}$ & $1.6423 \mathrm{E}+01$ & $4.206 \mathrm{E}-08$ & $1.486 \mathrm{E}+05$ & $1.6354 \mathrm{E}+01$ & $4.233 \mathrm{E}-08$ & $1.495 \mathrm{E}+05$ \\
\hline $1 s 5 f^{3} F_{2}^{o}$ & $1 s 7 g{ }^{3} G_{3}$ & $1.6427 \mathrm{E}+01$ & $2.522 \mathrm{E}-07$ & $8.905 E+05$ & $1.6358 \mathrm{E}+01$ & $2.538 \mathrm{E}-07$ & $8.963 \mathrm{E}+05$ \\
\hline $1 s 5 f^{3} F_{4}^{o}$ & $1 s 7 g{ }^{3} G_{3}$ & $1.6508 \mathrm{E}+01$ & $1.692 \mathrm{E}-17$ & $5.915 \mathrm{E}-05$ & & & \\
\hline $1 s 5 f^{1} F_{3}^{o}$ & $1 s 7 g{ }^{3} G_{3}$ & $1.6511 \mathrm{E}+01$ & $1.720 \mathrm{E}-13$ & $6.013 \mathrm{E}-01$ & & & \\
\hline $1 s 6 p^{3} P_{1}^{o}$ & $1 s 7 g{ }^{3} G_{3}$ & $4.0673 \mathrm{E}+01$ & $3.156 \mathrm{E}-18$ & $1.818 \mathrm{E}-06$ & & & \\
\hline $1 s 6 p^{3} P_{2}^{o}$ & $1 s 7 g{ }^{3} G_{3}$ & $4.2676 \mathrm{E}+01$ & $7.118 \mathrm{E}-14$ & $3.724 \mathrm{E}-02$ & & & \\
\hline $1 s 6 p{ }^{1} P_{1}^{o}$ & $1 s 7 g{ }^{3} G_{3}$ & $4.2852 \mathrm{E}+01$ & $3.084 \mathrm{E}-14$ & $1.601 \mathrm{E}-02$ & & & \\
\hline $1 s 6 f^{3} F_{3}^{o}$ & $1 s 7 g{ }^{3} G_{3}$ & $4.3521 \mathrm{E}+01$ & $2.867 \mathrm{E}-08$ & $1.442 \mathrm{E}+04$ & $4.3346 \mathrm{E}+01$ & $2.874 \mathrm{E}-08$ & $1.445 \mathrm{E}+04$ \\
\hline $1 s 6 f^{3} F_{2}^{o}$ & $1 s 7 g{ }^{3} G_{3}$ & $4.3535 \mathrm{E}+01$ & $1.721 \mathrm{E}-07$ & $8.651 E+04$ & $4.3346 \mathrm{E}+01$ & $1.724 \mathrm{E}-07$ & $8.672 E+04$ \\
\hline $1 s 6 f^{3} F_{4}^{o}$ & $1 s 7 g{ }^{3} G_{3}$ & $4.3867 \mathrm{E}+01$ & $3.588 \mathrm{E}-17$ & $1.777 \mathrm{E}-05$ & & & \\
\hline $1 s 6 f^{1} F_{3}^{o}$ & $1 s 7 g{ }^{3} G_{3}$ & $4.3878 \mathrm{E}+01$ & $1.112 \mathrm{E}-13$ & $5.505 \mathrm{E}-02$ & & & \\
\hline $1 s 6 h^{3} H_{5}^{o}$ & $1 s 7 g{ }^{3} G_{3}$ & $4.4077 \mathrm{E}+01$ & $2.145 \mathrm{E}-10$ & $1.052 \mathrm{E}+02$ & $4.3896 \mathrm{E}+01$ & $2.123 E-10$ & $1.041 \mathrm{E}+02$ \\
\hline $1 s 6 h^{3} H_{4}^{o}$ & $1 s 7 g{ }^{3} G_{3}$ & $4.4083 \mathrm{E}+01$ & $3.516 \mathrm{E}-09$ & $1.724 \mathrm{E}+03$ & $4.3896 \mathrm{E}+01$ & $3.503 \mathrm{E}-09$ & $1.718 \mathrm{E}+03$ \\
\hline $1 s 6 h^{1} H_{5}^{o}$ & $1 s 7 g{ }^{3} G_{3}$ & $4.4222 \mathrm{E}+01$ & $1.299 \mathrm{E}-09$ & $6.331 \mathrm{E}+02$ & $4.4034 \mathrm{E}+01$ & $1.297 \mathrm{E}-09$ & $6.323 \mathrm{E}+02$ \\
\hline $1 s 7 p^{3} P_{1}^{o}$ & $1 s 7 g{ }^{3} G_{3}$ & $8.8963 \mathrm{E}+02$ & $4.277 \mathrm{E}-20$ & $5.149 \mathrm{E}-11$ & & & \\
\hline $1 s 7 p^{3} P_{2}^{o}$ & $1 s 7 g{ }^{3} G_{3}$ & $2.5035 \mathrm{E}+03$ & $1.266 \mathrm{E}-18$ & $1.925 \mathrm{E}-10$ & & & \\
\hline $1 s 7 p^{1} P_{1}^{o}$ & $1 s 7 g{ }^{3} G_{3}$ & $2.9534 \mathrm{E}+03$ & $5.714 \mathrm{E}-20$ & $6.242 \mathrm{E}-12$ & & & \\
\hline $1 s 7 f^{3} F_{3}^{o}$ & $1 s 7 g{ }^{3} G_{3}$ & $8.5297 \mathrm{E}+03$ & $1.804 \mathrm{E}-14$ & $2.362 \mathrm{E}-07$ & $8.7975 E+03$ & $1.625 \mathrm{E}-14$ & $1.984 \mathrm{E}-07$ \\
\hline $1 s 7 f^{3} F_{2}^{o}$ & $1 s 7 g{ }^{3} G_{3}$ & $8.8934 \mathrm{E}+03$ & $9.547 \mathrm{E}-14$ & $1.150 \mathrm{E}-06$ & $8.7928 \mathrm{E}+03$ & $9.768 \mathrm{E}-14$ & $1.193 \mathrm{E}-06$ \\
\hline $1 s 7 f^{3} F_{4}^{o}$ & $1 s 7 g{ }^{3} G_{3}$ & $3.3768 \mathrm{E}+05$ & $1.239 \mathrm{E}-26$ & $1.035 \mathrm{E}-22$ & & & \\
\hline $1 s 2 s^{3} s_{1}^{4}$ & $1 s 7 f^{1} F_{3}^{o}$ & $1.3246 \mathrm{E}+00$ & $2.065 \mathrm{E}-13$ & $1.121 \mathrm{E}+02$ & & & \\
\hline $1 s 3 s^{3} S_{1}$ & $1 s 7 f^{1} F_{3}^{o}$ & $3.3838 \mathrm{E}+00$ & $5.178 \mathrm{E}-13$ & $4.309 \mathrm{E}+01$ & & & \\
\hline $1 s 3 d^{3} D_{2}$ & $1 s 7 f^{1} F_{3}^{o}$ & $3.5233 \mathrm{E}+00$ & $4.933 \mathrm{E}-08$ & $3.787 \mathrm{E}+06$ & $3.5085 \mathrm{E}+00$ & $4.924 \mathrm{E}-08$ & $3.779 E+06$ \\
\hline $1 s 3 d^{3} D_{1}$ & $1 s 7 f^{1} F_{3}^{o}$ & $3.5247 \mathrm{E}+00$ & $2.810 \mathrm{E}-07$ & $2.155 \mathrm{E}+07$ & $3.5099 \mathrm{E}+00$ & $2.827 \mathrm{E}-07$ & $2.168 \mathrm{E}+07$ \\
\hline $1 s 3 d^{3} D_{3}$ & $1 s 7 f^{1} F_{3}^{o}$ & $3.5600 \mathrm{E}+00$ & $3.035 \mathrm{E}-07$ & $2.282 \mathrm{E}+07$ & $3.5449 \mathrm{E}+00$ & $3.115 \mathrm{E}-07$ & $2.342 \mathrm{E}+07$ \\
\hline $1 s 3 d^{1} D_{2}$ & $1 s 7 f^{1} F_{3}^{o}$ & $3.5614 \mathrm{E}+00$ & $1.816 \mathrm{E}-06$ & $1.364 \mathrm{E}+08$ & $3.5463 \mathrm{E}+00$ & $1.864 \mathrm{E}-06$ & $1.400 \mathrm{E}+08$ \\
\hline $1 s 4 s^{3} S_{1}$ & $1 s 7 f^{1} F_{3}^{o}$ & $7.3110 \mathrm{E}+00$ & $3.646 \mathrm{E}-13$ & $6.500 \mathrm{E}+00$ & & & \\
\hline $1 s 4 d^{3} D_{2}$ & $1 s 7 f^{1} F_{3}^{o}$ & $7.5823 \mathrm{E}+00$ & $3.022 \mathrm{E}-08$ & $5.008 \mathrm{E}+05$ & $7.5510 \mathrm{E}+00$ & $3.040 \mathrm{E}-08$ & $5.038 \mathrm{E}+05$ \\
\hline $1 s 4 d^{3} D_{1}$ & $1 s 7 f^{1} F_{3}^{o}$ & $7.5851 \mathrm{E}+00$ & $1.684 \mathrm{E}-07$ & $2.788 \mathrm{E}+06$ & $7.5538 \mathrm{E}+00$ & $1.701 \mathrm{E}-07$ & $2.817 \mathrm{E}+06$ \\
\hline $1 s 4 d^{3} D_{3}$ & $1 s 7 f^{1} F_{3}^{o}$ & $7.6541 \mathrm{E}+00$ & $1.878 \mathrm{E}-07$ & $3.055 \mathrm{E}+06$ & $7.6222 \mathrm{E}+00$ & $1.901 \mathrm{E}-07$ & $3.091 \mathrm{E}+06$ \\
\hline $1 s 4 d{ }^{1} D_{2}$ & $1 s 7 f^{1} F_{3}^{o}$ & $7.6570 \mathrm{E}+00$ & $1.125 \mathrm{E}-06$ & $1.828 \mathrm{E}+07$ & $7.6252 \mathrm{E}+00$ & $1.137 \mathrm{E}-06$ & $1.848 \mathrm{E}+07$ \\
\hline $1 s 5 s^{3} S_{1}$ & $1 s 7 f^{1} F_{3}^{o}$ & $1.5619 \mathrm{E}+01$ & $2.264 \mathrm{E}-13$ & $8.845 \mathrm{E}-01$ & & & \\
\hline $1 s 5 d^{3} D_{2}$ & $1 s 7 f^{1} F_{3}^{o}$ & $1.6251 \mathrm{E}+01$ & $1.759 \mathrm{E}-08$ & $6.347 \mathrm{E}+04$ & $1.6186 \mathrm{E}+01$ & $1.767 \mathrm{E}-08$ & $6.374 \mathrm{E}+04$ \\
\hline $1 s 5 d^{3} D_{1}$ & $1 s 7 f^{1} F_{3}^{o}$ & $1.6258 \mathrm{E}+01$ & $9.706 \mathrm{E}-08$ & $3.499 \mathrm{E}+05$ & $1.6192 \mathrm{E}+01$ & $9.776 \mathrm{E}-08$ & $3.523 \mathrm{E}+05$ \\
\hline $1 s 5 d^{3} D_{3}$ & $1 s 7 f^{1} F_{3}^{o}$ & $1.6420 \mathrm{E}+01$ & $1.111 \mathrm{E}-07$ & $3.925 \mathrm{E}+05$ & $1.6353 \mathrm{E}+01$ & $1.117 \mathrm{E}-07$ & $3.946 \mathrm{E}+05$ \\
\hline $1 s 5 d^{1} D_{2}$ & $1 s 7 f^{1} F_{3}^{o}$ & $1.6427 \mathrm{E}+01$ & $6.654 \mathrm{E}-07$ & $2.349 \mathrm{E}+06$ & $1.6360 \mathrm{E}+01$ & $6.680 \mathrm{E}-07$ & $2.357 \mathrm{E}+06$ \\
\hline $1 s 5 g^{3} G_{4}$ & $1 s 7 f^{1} F_{3}^{o}$ & $1.6508 \mathrm{E}+01$ & $8.835 \mathrm{E}-15$ & $3.089 \mathrm{E}-02$ & & & \\
\hline $1 s 5 g^{3} G_{3}$ & $1 s 7 f^{1} F_{3}^{o}$ & $1.6510 \mathrm{E}+01$ & $1.556 \mathrm{E}-15$ & $5.439 \mathrm{E}-03$ & & & \\
\hline $1 s 5 g^{3} G_{5}$ & $1 s 7 f^{1} F_{3}^{o}$ & $1.6559 \mathrm{E}+01$ & $7.076 \mathrm{E}-10$ & $2.459 \mathrm{E}+03$ & $1.6489 \mathrm{E}+01$ & $7.100 \mathrm{E}-10$ & $2.467 \mathrm{E}+03$ \\
\hline $1 s 5 g{ }^{1} G_{4}$ & $1 s 7 f^{1} F_{3}^{o}$ & $1.6561 \mathrm{E}+01$ & $1.182 \mathrm{E}-08$ & $4.106 \mathrm{E}+04$ & $1.6491 \mathrm{E}+01$ & $1.168 \mathrm{E}-08$ & $4.060 \mathrm{E}+04$ \\
\hline $1 s 6 s^{3} S_{1}$ & $1 s 7 f^{1} F_{3}^{o}$ & $4.0343 \mathrm{E}+01$ & $1.294 \mathrm{E}-13$ & $7.577 \mathrm{E}-02$ & & & \\
\hline $1 s 6 d^{3} D_{2}$ & $1 s 7 f^{1} F_{3}^{o}$ & $4.2826 \mathrm{E}+01$ & $1.020 \mathrm{E}-08$ & $5.300 \mathrm{E}+03$ & $4.2671 \mathrm{E}+01$ & $1.086 \mathrm{E}-08$ & $5.638 \mathrm{E}+03$ \\
\hline $1 s 6 d^{3} D_{1}$ & $1 s 7 f^{1} F_{3}^{o}$ & $4.2851 \mathrm{E}+01$ & $5.600 \mathrm{E}-08$ & $2.906 \mathrm{E}+04$ & $4.2665 \mathrm{E}+01$ & $5.622 \mathrm{E}-08$ & $2.918 \mathrm{E}+04$ \\
\hline $1 s 6 d^{3} D_{3}$ & $1 s 7 f^{1} F_{3}^{o}$ & $4.3507 \mathrm{E}+01$ & $6.599 E-08$ & $3.322 \mathrm{E}+04$ & $4.3345 \mathrm{E}+01$ & $6.615 E-08$ & $3.327 \mathrm{E}+04$ \\
\hline $1 s 6 d^{1} D_{2}$ & $1 s 7 f^{1} F_{3}^{o}$ & $4.3537 \mathrm{E}+01$ & $3.957 \mathrm{E}-07$ & $1.989 \mathrm{E}+05$ & $4.3355 \mathrm{E}+01$ & $3.952 \mathrm{E}-07$ & $1.986 \mathrm{E}+05$ \\
\hline $1 s 6 g^{3} G_{4}$ & $1 s 7 f^{1} F_{3}^{o}$ & $4.3867 \mathrm{E}+01$ & $2.568 \mathrm{E}-14$ & $1.272 \mathrm{E}-02$ & & & \\
\hline $1 s 6 g^{3} G_{3}$ & $1 s 7 f^{1} F_{3}^{o}$ & $4.3875 \mathrm{E}+01$ & $3.279 \mathrm{E}-15$ & $1.623 \mathrm{E}-03$ & & & \\
\hline $1 s 6 g^{3} G_{5}$ & $1 s 7 f^{1} F_{3}^{o}$ & $4.4076 \mathrm{E}+01$ & $1.668 \mathrm{E}-09$ & $8.183 E+02$ & $4.3895 \mathrm{E}+01$ & $1.679 \mathrm{E}-09$ & $8.235 E+02$ \\
\hline $1 s 6 g{ }^{1} G_{4}$ & $1 s 7 f^{1} F_{3}^{o}$ & $4.4083 \mathrm{E}+01$ & $2.777 \mathrm{E}-08$ & $1.362 \mathrm{E}+04$ & $4.3895 \mathrm{E}+01$ & $2.765 \mathrm{E}-08$ & $1.355 \mathrm{E}+04$ \\
\hline $1 s 7 s^{3} s_{1}$ & $1 s 7 f^{1} F_{3}^{o}$ & $7.9654 \mathrm{E}+02$ & $2.248 \mathrm{E}-16$ & $3.376 \mathrm{E}-07$ & & & \\
\hline $1 s 7 d^{3} D_{2}$ & $1 s 7 f^{1} F_{3}^{o}$ & $2.8385 \mathrm{E}+03$ & $3.075 E-13$ & $3.636 \mathrm{E}-05$ & $2.9112 \mathrm{E}+03$ & $2.970 \mathrm{E}-13$ & $3.312 \mathrm{E}-05$ \\
\hline $1 s 7 d^{3} D_{1}$ & $1 s 7 f^{1} F_{3}^{o}$ & $2.9109 \mathrm{E}+03$ & $1.557 \mathrm{E}-12$ & $1.751 \mathrm{E}-04$ & $2.8927 \mathrm{E}+03$ & $1.569 \mathrm{E}-12$ & $1.772 \mathrm{E}-04$ \\
\hline $1 s 7 d^{3} D_{3}$ & $1 s 7 f^{1} F_{3}^{o}$ & $8.1837 \mathrm{E}+03$ & $8.017 \mathrm{E}-14$ & $1.141 \mathrm{E}-06$ & $8.7525 E+03$ & $6.474 \mathrm{E}-14$ & $7.985 \mathrm{E}-07$ \\
\hline $1 s 7 d^{1} D_{2}$ & $1 s 7 f^{1} F_{3}^{o}$ & $8.9054 \mathrm{E}+03$ & $3.724 \mathrm{E}-13$ & $4.474 \mathrm{E}-06$ & $9.0373 \mathrm{E}+03$ & $3.515 \mathrm{E}-13$ & $4.066 \mathrm{E}-06$ \\
\hline $1 s 7 g^{3} G_{4}$ & $1 s 7 f^{1} F_{3}^{o}$ & $2.9370 \mathrm{E}+05$ & $1.846 \mathrm{E}-23$ & $2.039 \mathrm{E}-19$ & & & \\
\hline $1 s 7 g{ }^{3} G_{3}$ & $1 s 7 f^{1} F_{3}^{o}$ & $1.5307 \mathrm{E}+06$ & $1.214 \mathrm{E}-26$ & $4.936 \mathrm{E}-24$ & & & \\
\hline $1 s 4 f^{3} F_{3}^{o}$ & $1 s 7 g{ }^{3} G_{5}$ & $7.6514 \mathrm{E}+00$ & $2.899 \mathrm{E}-07$ & $3.003 \mathrm{E}+06$ & $7.6190 \mathrm{E}+00$ & $2.900 \mathrm{E}-07$ & $3.003 E+06$ \\
\hline $1 s 4 f^{3} F_{4}^{o}$ & $1 s 7 g{ }^{3} G_{5}$ & $7.6874 \mathrm{E}+00$ & $2.475 \mathrm{E}-06$ & $2.540 \mathrm{E}+07$ & $7.6547 \mathrm{E}+00$ & $2.516 \mathrm{E}-06$ & $2.582 \mathrm{E}+07$ \\
\hline $1 s 4 f^{1} F_{3}^{o}$ & $1 s 7 g{ }^{3} G_{5}$ & $7.6885 \mathrm{E}+00$ & $1.170 \mathrm{E}-07$ & $1.200 \mathrm{E}+06$ & $7.6558 \mathrm{E}+00$ & $1.189 \mathrm{E}-07$ & $1.220 \mathrm{E}+06$ \\
\hline $1 s 5 f^{3} F_{3}^{o}$ & $1 s 7 g{ }^{3} G_{5}$ & $1.6406 \mathrm{E}+01$ & $2.441 \mathrm{E}-07$ & $5.499 E+05$ & $1.6336 \mathrm{E}+01$ & $2.453 \mathrm{E}-07$ & $5.528 \mathrm{E}+05$ \\
\hline $1 s 5 f^{3} F_{4}^{o}$ & $1 s 7 g{ }^{3} G_{5}$ & $1.6491 \mathrm{E}+01$ & $2.124 \mathrm{E}-06$ & $4.737 \mathrm{E}+06$ & $1.6420 \mathrm{E}+01$ & $2.140 \mathrm{E}-06$ & $4.772 \mathrm{E}+06$ \\
\hline $1 s 5 f^{1} F_{3}^{o}$ & $1 s 7 g{ }^{3} G_{5}$ & $1.6493 \mathrm{E}+01$ & $1.005 \mathrm{E}-07$ & $2.240 \mathrm{E}+05$ & $1.6423 \mathrm{E}+01$ & $1.012 \mathrm{E}-07$ & $2.256 \mathrm{E}+05$ \\
\hline $1 s 6 f^{3} F_{3}^{o}$ & $1 s 7 g{ }^{3} G_{5}$ & $4.3397 \mathrm{E}+01$ & $1.644 \mathrm{E}-07$ & $5.294 \mathrm{E}+04$ & $4.3219 \mathrm{E}+01$ & $1.643 \mathrm{E}-07$ & $5.291 \mathrm{E}+04$ \\
\hline $1 s 6 f^{3} F_{4}^{o}$ & $1 s 7 g{ }^{3} G_{5}$ & $4.3742 \mathrm{E}+01$ & $1.459 \mathrm{E}-06$ & $4.624 \mathrm{E}+05$ & $4.3559 \mathrm{E}+01$ & $1.462 \mathrm{E}-06$ & $4.634 \mathrm{E}+05$ \\
\hline
\end{tabular}


Table 6 (continued)

\begin{tabular}{|c|c|c|c|c|c|c|c|}
\hline \multirow[t]{2}{*}{ Lower } & \multirow[t]{2}{*}{ Upper } & \multicolumn{3}{|l|}{ GRASP2K } & \multicolumn{3}{|l|}{ FAC } \\
\hline & & $\lambda(\AA)$ & $g f$ & $A\left(\mathrm{~s}^{-1}\right)$ & $\lambda(\AA)$ & $g f$ & $A\left(\mathrm{~s}^{-1}\right)$ \\
\hline $1 s 6 f^{1} F_{3}^{o}$ & $1 s 7 g^{3} G_{5}$ & $4.3753 \mathrm{E}+01$ & $6.906 \mathrm{E}-08$ & $2.188 \mathrm{E}+04$ & $4.3560 \mathrm{E}+01$ & $6.976 \mathrm{E}-08$ & $2.210 \mathrm{E}+04$ \\
\hline $1 s 6 h^{3} H_{5}^{o}$ & $1 s 7 g{ }^{3} G_{5}$ & $4.3951 \mathrm{E}+01$ & $2.044 \mathrm{E}-15$ & $6.415 \mathrm{E}-04$ & & & \\
\hline $1 s 6 h^{3} H_{4}^{o}$ & $1 s 7 g{ }^{3} G_{5}$ & $4.3956 \mathrm{E}+01$ & $4.299 \mathrm{E}-19$ & $1.349 \mathrm{E}-07$ & & & \\
\hline $1 s 6 h^{3} H_{6}^{o}$ & $1 s 7 g{ }^{3} G_{5}$ & $4.4090 \mathrm{E}+01$ & $1.830 \mathrm{E}-08$ & $5.710 \mathrm{E}+03$ & $4.3903 \mathrm{E}+01$ & $1.822 \mathrm{E}-08$ & $5.684 \mathrm{E}+03$ \\
\hline $1 s 6 h{ }^{1} H_{5}^{o}$ & $1 s 7 g{ }^{3} G_{5}$ & $4.4094 \mathrm{E}+01$ & $8.725 \mathrm{E}-10$ & $2.721 \mathrm{E}+02$ & $4.3903 \mathrm{E}+01$ & $8.677 \mathrm{E}-10$ & $2.706 \mathrm{E}+02$ \\
\hline $1 s 7 f^{3} F_{3}^{o}$ & $1 s 7 g{ }^{3} G_{5}$ & $5.4759 \mathrm{E}+03$ & $3.951 \mathrm{E}-13$ & $7.989 \mathrm{E}-06$ & $5.5025 \mathrm{E}+03$ & $3.830 \mathrm{E}-13$ & $7.606 \mathrm{E}-06$ \\
\hline $1 s 7 f^{3} F_{4}^{o}$ & $1 s 7 g{ }^{3} G_{5}$ & $1.4632 \mathrm{E}+04$ & $1.821 \mathrm{E}-13$ & $5.159 \mathrm{E}-07$ & $1.4765 \mathrm{E}+04$ & $1.751 \mathrm{E}-13$ & $4.831 \mathrm{E}-07$ \\
\hline $1 s 7 f^{1} F_{3}^{o}$ & $1 s 7 g{ }^{3} G_{5}$ & $1.5450 \mathrm{E}+04$ & $7.324 \mathrm{E}-15$ & $1.861 \mathrm{E}-08$ & $1.4784 \mathrm{E}+04$ & $8.325 E-15$ & $2.290 \mathrm{E}-08$ \\
\hline $1 s 3 d^{3} D_{3}$ & $1 s 7 h^{3} H_{5}^{o}$ & $3.5591 \mathrm{E}+00$ & $6.235 \mathrm{E}-12$ & $2.985 \mathrm{E}+02$ & & & \\
\hline $1 s 4 d^{3} D_{3}$ & $1 s 7 h^{3} H_{5}^{o}$ & $7.6503 \mathrm{E}+00$ & $3.513 \mathrm{E}-12$ & $3.640 \mathrm{E}+01$ & & & \\
\hline $1 s 5 d^{3} D_{3}$ & $1 s 7 h^{3} H_{5}^{o}$ & $1.6403 \mathrm{E}+01$ & $6.894 \mathrm{E}-14$ & $1.554 \mathrm{E}-01$ & & & \\
\hline $1 s 5 g^{3} G_{4}$ & $1 s 7 h^{3} H_{5}^{o}$ & $1.6491 \mathrm{E}+01$ & $7.809 \mathrm{E}-07$ & $1.741 \mathrm{E}+06$ & $1.6420 \mathrm{E}+01$ & $7.881 \mathrm{E}-07$ & $1.757 \mathrm{E}+06$ \\
\hline $1 s 5 g^{3} G_{3}$ & $1 s 7 h^{3} H_{5}^{0}$ & $1.6493 \mathrm{E}+01$ & $3.746 \mathrm{E}-08$ & $8.351 \mathrm{E}+04$ & $1.6423 \mathrm{E}+01$ & $3.751 \mathrm{E}-08$ & $8.363 E+04$ \\
\hline $1 s 5 g^{3} G_{5}$ & $1 s 7 h^{3} H_{5}^{o}$ & $1.6542 \mathrm{E}+01$ & $3.738 \mathrm{E}-13$ & $8.285 \mathrm{E}-01$ & & & \\
\hline $1 s 5 g{ }^{1} G_{4}$ & $1 s 7 h^{3} H_{5}^{0}$ & $1.6543 \mathrm{E}+01$ & $7.520 \mathrm{E}-13$ & $1.666 \mathrm{E}+00$ & & & \\
\hline $1 s 6 d^{3} D_{3}$ & $1 s 7 h^{3} H_{5}^{o}$ & $4.3385 \mathrm{E}+01$ & $6.788 \mathrm{E}-14$ & $2.187 \mathrm{E}-02$ & & & \\
\hline $1 s 6 g^{3} G_{4}$ & $1 s 7 h^{3} H_{5}^{o}$ & $4.3742 \mathrm{E}+01$ & $8.186 \mathrm{E}-07$ & $2.594 \mathrm{E}+05$ & $4.3559 \mathrm{E}+01$ & $8.213 \mathrm{E}-07$ & $2.602 \mathrm{E}+05$ \\
\hline $1 s 6 g^{3} G_{3}$ & $1 s 7 h^{3} H_{5}^{o}$ & $4.3751 \mathrm{E}+01$ & $3.929 \mathrm{E}-08$ & $1.245 \mathrm{E}+04$ & $4.3559 \mathrm{E}+01$ & $3.911 \mathrm{E}-08$ & $1.239 \mathrm{E}+04$ \\
\hline $1 s 6 g{ }^{3} G_{5}$ & $1 s 7 h^{3} H_{5}^{o}$ & $4.3950 \mathrm{E}+01$ & $3.908 \mathrm{E}-13$ & $1.227 \mathrm{E}-01$ & & & \\
\hline $1 s 6 g^{1} G_{4}$ & $1 s 7 h^{3} H_{5}^{o}$ & $4.3957 \mathrm{E}+01$ & $8.040 \mathrm{E}-13$ & $2.523 \mathrm{E}-01$ & & & \\
\hline $1 s 7 d^{3} D_{3}$ & $1 s 7 h^{3} H_{5}^{o}$ & $5.3474 \mathrm{E}+03$ & $4.485 \mathrm{E}-21$ & $9.510 \mathrm{E}-14$ & & & \\
\hline $1 s 7 g^{3} G_{4}$ & $1 s 7 h^{3} H_{5}^{o}$ & $1.4659 \mathrm{E}+04$ & $5.334 \mathrm{E}-14$ & $1.505 \mathrm{E}-07$ & $1.4689 \mathrm{E}+04$ & $5.242 \mathrm{E}-14$ & $1.460 \mathrm{E}-07$ \\
\hline $1 s 7 g^{3} G_{3}$ & $1 s 7 h^{3} H_{5}^{o}$ & $1.5275 \mathrm{E}+04$ & $2.262 \mathrm{E}-15$ & $5.879 \mathrm{E}-09$ & $1.4689 \mathrm{E}+04$ & $2.496 \mathrm{E}-15$ & $6.956 \mathrm{E}-09$ \\
\hline $1 s 7 g^{3} G_{5}$ & $1 s 7 h^{3} H_{5}^{o}$ & $1.1751 \mathrm{E}+07$ & $4.945 \mathrm{E}-29$ & $2.170 \mathrm{E}-28$ & & & \\
\hline $1 s 3 d^{3} D_{2}$ & $1 s 7 h^{3} H_{4}^{o}$ & $3.5224 \mathrm{E}+00$ & $9.274 \mathrm{E}-16$ & $5.539 \mathrm{E}-02$ & & & \\
\hline $1 s 3 d^{3} D_{3}$ & $1 s 7 h^{3} H_{4}^{0}$ & $3.5591 \mathrm{E}+00$ & $4.087 \mathrm{E}-13$ & $2.391 \mathrm{E}+01$ & & & \\
\hline $1 s 3 d^{1} D_{2}$ & $1 s 7 h^{3} H_{4}^{0}$ & $3.5605 \mathrm{E}+00$ & $4.517 \mathrm{E}-12$ & $2.641 \mathrm{E}+02$ & & & \\
\hline $1 s 4 d^{3} D_{2}$ & $1 s 7 h^{3} H_{4}^{o}$ & $7.5785 \mathrm{E}+00$ & $4.314 \mathrm{E}-16$ & $5.566 \mathrm{E}-03$ & & & \\
\hline $1 s 4 d^{3} D_{3}$ & $1 s 7 h^{3} H_{4}^{0}$ & $7.6502 \mathrm{E}+00$ & $2.093 \mathrm{E}-13$ & $2.650 \mathrm{E}+00$ & & & \\
\hline $1 s 4 d{ }^{1} D_{2}$ & $1 s 7 h^{3} H_{4}^{0}$ & $7.6531 \mathrm{E}+00$ & $2.617 \mathrm{E}-12$ & $3.312 \mathrm{E}+01$ & & & \\
\hline $1 s 5 d^{3} D_{2}$ & $1 s 7 h^{3} H_{4}^{o}$ & $1.6234 \mathrm{E}+01$ & $1.476 \mathrm{E}-17$ & $4.152 \mathrm{E}-05$ & & & \\
\hline $1 s 5 d^{3} D_{3}$ & $1 s 7 h^{3} H_{4}^{o}$ & $1.6402 \mathrm{E}+01$ & $2.182 \mathrm{E}-15$ & $6.010 \mathrm{E}-03$ & & & \\
\hline $1 s 5 d{ }^{1} D_{2}$ & $1 s 7 h^{3} H_{4}^{o}$ & $1.6409 \mathrm{E}+01$ & $4.943 \mathrm{E}-14$ & $1.361 \mathrm{E}-01$ & & & \\
\hline $1 s 5 g^{3} G_{4}$ & $1 s 7 h^{3} H_{4}^{0}$ & $1.6490 \mathrm{E}+01$ & $5.583 \mathrm{E}-08$ & $1.522 \mathrm{E}+05$ & $1.6420 \mathrm{E}+01$ & $5.629 \mathrm{E}-08$ & $1.534 \mathrm{E}+05$ \\
\hline $1 s 5 g^{3} G_{3}$ & $1 s 7 h^{3} H_{4}^{o}$ & $1.6492 \mathrm{E}+01$ & $6.137 \mathrm{E}-07$ & $1.672 \mathrm{E}+06$ & $1.6423 \mathrm{E}+01$ & $6.190 \mathrm{E}-07$ & $1.686 \mathrm{E}+06$ \\
\hline $1 s 5 g^{3} G_{5}$ & $1 s 7 h^{3} H_{4}^{0}$ & $1.6541 \mathrm{E}+01$ & $8.746 \mathrm{E}-16$ & $2.369 \mathrm{E}-03$ & & & \\
\hline $1 s 5 g^{1} G_{4}$ & $1 s 7 h^{3} H_{4}^{o}$ & $1.6543 \mathrm{E}+01$ & $1.942 \mathrm{E}-13$ & $5.259 \mathrm{E}-01$ & & & \\
\hline $1 s 6 d^{3} D_{2}$ & $1 s 7 h^{3} H_{4}^{o}$ & $4.2704 \mathrm{E}+01$ & $9.336 \mathrm{E}-18$ & $3.794 \mathrm{E}-06$ & & & \\
\hline $1 s 6 d^{3} D_{3}$ & $1 s 7 h^{3} H_{4}^{o}$ & $4.3381 \mathrm{E}+01$ & $8.044 \mathrm{E}-15$ & $3.168 \mathrm{E}-03$ & & & \\
\hline $1 s 6 d^{1} D_{2}$ & $1 s 7 h^{3} H_{4}^{o}$ & $4.3411 \mathrm{E}+01$ & $5.285 \mathrm{E}-14$ & $2.079 \mathrm{E}-02$ & & & \\
\hline $1 s 6 g^{3} G_{4}$ & $1 s 7 h^{3} H_{4}^{0}$ & $4.3739 \mathrm{E}+01$ & $5.849 \mathrm{E}-08$ & $2.266 \mathrm{E}+04$ & $4.3559 \mathrm{E}+01$ & $5.866 \mathrm{E}-08$ & $2.272 \mathrm{E}+04$ \\
\hline $1 s 6 g^{3} G_{3}$ & $1 s 7 h^{3} H_{4}^{o}$ & $4.3747 \mathrm{E}+01$ & $6.433 \mathrm{E}-07$ & $2.491 \mathrm{E}+05$ & $4.3559 \mathrm{E}+01$ & $6.453 \mathrm{E}-07$ & $2.499 \mathrm{E}+05$ \\
\hline $1 s 6 g^{3} G_{5}$ & $1 s 7 h^{3} H_{4}^{0}$ & $4.3947 \mathrm{E}+01$ & $9.550 \mathrm{E}-18$ & $3.665 \mathrm{E}-06$ & & & \\
\hline $1 s 6 g{ }^{1} G_{4}$ & $1 s 7 h^{3} H_{4}^{o}$ & $4.3954 \mathrm{E}+01$ & $2.072 \mathrm{E}-13$ & $7.949 \mathrm{E}-02$ & & & \\
\hline $1 s 7 d^{3} D_{2}$ & $1 s 7 h^{3} H_{4}^{o}$ & $2.3873 \mathrm{E}+03$ & $4.238 \mathrm{E}-24$ & $5.512 \mathrm{E}-16$ & & & \\
\hline $1 s 7 d^{3} D_{3}$ & $1 s 7 h^{3} H_{4}^{o}$ & $5.2970 \mathrm{E}+03$ & $2.811 \mathrm{E}-21$ & $7.426 \mathrm{E}-14$ & & & \\
\hline $1 s 7 d{ }^{1} D_{2}$ & $1 s 7 h^{3} H_{4}^{o}$ & $5.5903 \mathrm{E}+03$ & $3.729 \mathrm{E}-21$ & $8.844 \mathrm{E}-14$ & & & \\
\hline $1 s 7 g^{3} G_{4}$ & $1 s 7 h^{3} H_{4}^{0}$ & $1.4287 \mathrm{E}+04$ & $4.118 \mathrm{E}-15$ & $1.495 \mathrm{E}-08$ & $1.4689 \mathrm{E}+04$ & $3.744 \mathrm{E}-15$ & $1.275 \mathrm{E}-08$ \\
\hline $1 s 7 g^{3} G_{3}$ & $1 s 7 h^{3} H_{4}^{0}$ & $1.4871 \mathrm{E}+04$ & $4.016 \mathrm{E}-14$ & $1.346 \mathrm{E}-07$ & $1.4689 \mathrm{E}+04$ & $4.119 \mathrm{E}-14$ & $1.402 \mathrm{E}-07$ \\
\hline $1 s 7 g^{3} G_{5}$ & $1 s 7 h^{3} H_{4}^{o}$ & $5.3648 \mathrm{E}+05$ & $2.939 \mathrm{E}-30$ & $7.568 \mathrm{E}-27$ & & & \\
\hline $1 s 2 p^{3} P_{2}^{o}$ & $1 s 7 g{ }^{1} G_{4}$ & $1.3938 \mathrm{E}+00$ & $1.901 \mathrm{E}-14$ & $7.251 \mathrm{E}+00$ & & & \\
\hline $1 s 3 p^{3} P_{2}^{o}$ & $1 s 7 g{ }^{1} G_{4}$ & $3.5141 \mathrm{E}+00$ & $2.638 \mathrm{E}-15$ & $1.583 \mathrm{E}-01$ & & & \\
\hline $1 s 4 p^{3} P_{2}^{o}$ & $1 s 7 g{ }^{1} G_{4}$ & $7.5624 \mathrm{E}+00$ & $3.399 \mathrm{E}-14$ & $4.405 \mathrm{E}-01$ & & & \\
\hline $1 s 4 f^{3} F_{3}^{o}$ & $1 s 7 g{ }^{1} G_{4}$ & $7.6513 \mathrm{E}+00$ & $1.704 \mathrm{E}-08$ & $2.158 \mathrm{E}+05$ & $7.6190 \mathrm{E}+00$ & $1.671 \mathrm{E}-08$ & $2.116 \mathrm{E}+05$ \\
\hline $1 s 4 f^{3} F_{2}^{0}$ & $1 s 7 g{ }^{1} G_{4}$ & $7.6528 \mathrm{E}+00$ & $2.186 \mathrm{E}-07$ & $2.766 \mathrm{E}+06$ & $7.6205 E+00$ & $2.189 \mathrm{E}-07$ & $2.770 \mathrm{E}+06$ \\
\hline $1 s 4 f^{3} F_{4}^{o}$ & $1 s 7 g{ }^{1} G_{4}$ & $7.6873 \mathrm{E}+00$ & $1.770 \mathrm{E}-07$ & $2.220 \mathrm{E}+06$ & $7.6547 \mathrm{E}+00$ & $1.797 \mathrm{E}-07$ & $2.254 \mathrm{E}+06$ \\
\hline $1 s 4 f^{1} F_{3}^{o}$ & $1 s 7 g{ }^{1} G_{4}$ & $7.6884 \mathrm{E}+00$ & $1.945 \mathrm{E}-06$ & $2.439 \mathrm{E}+07$ & $7.6558 \mathrm{E}+00$ & $1.977 \mathrm{E}-06$ & $2.478 \mathrm{E}+07$ \\
\hline $1 s 5 p^{3} P_{2}^{o}$ & $1 s 7 g{ }^{1} G_{4}$ & $1.6196 \mathrm{E}+01$ & $4.008 \mathrm{E}-14$ & $1.132 \mathrm{E}-01$ & & & \\
\hline $1 s 5 f^{3} F_{3}^{o}$ & $1 s 7 g{ }^{1} G_{4}$ & $1.6405 \mathrm{E}+01$ & $1.439 \mathrm{E}-08$ & $3.963 \mathrm{E}+04$ & $1.6336 \mathrm{E}+01$ & $1.419 \mathrm{E}-08$ & $3.908 \mathrm{E}+04$ \\
\hline $1 s 5 f^{3} F_{2}^{o}$ & $1 s 7 g{ }^{1} G_{4}$ & $1.6409 \mathrm{E}+01$ & $1.841 \mathrm{E}-07$ & $5.068 \mathrm{E}+05$ & $1.6340 \mathrm{E}+01$ & $1.852 \mathrm{E}-07$ & $5.100 \mathrm{E}+05$ \\
\hline $1 s 5 f^{3} F_{4}^{o}$ & $1 s 7 g{ }^{1} G_{4}$ & $1.6490 \mathrm{E}+01$ & $1.518 \mathrm{E}-07$ & $4.138 \mathrm{E}+05$ & $1.6420 \mathrm{E}+01$ & $1.528 \mathrm{E}-07$ & $4.166 \mathrm{E}+05$ \\
\hline $1 s 5 f^{1} F_{3}^{o}$ & $1 s 7 g{ }^{1} G_{4}$ & $1.6493 \mathrm{E}+01$ & $1.670 \mathrm{E}-06$ & $4.549 \mathrm{E}+06$ & $1.6423 \mathrm{E}+01$ & $1.681 \mathrm{E}-06$ & $4.581 \mathrm{E}+06$ \\
\hline $1 s 6 p^{3} P_{2}^{o}$ & $1 s 7 g{ }^{1} G_{4}$ & $4.2553 \mathrm{E}+01$ & $3.141 \mathrm{E}-14$ & $1.286 \mathrm{E}-02$ & & & \\
\hline $1 s 6 f^{3} F_{3}^{o}$ & $1 s 7 g{ }^{1} G_{4}$ & $4.3393 \mathrm{E}+01$ & $9.707 \mathrm{E}-09$ & $3.821 \mathrm{E}+03$ & $4.3219 \mathrm{E}+01$ & $1.009 \mathrm{E}-08$ & $3.971 \mathrm{E}+03$ \\
\hline $1 s 6 f^{3} F_{2}^{o}$ & $1 s 7 g{ }^{1} G_{4}$ & $4.3407 \mathrm{E}+01$ & $1.241 \mathrm{E}-07$ & $4.880 \mathrm{E}+04$ & $4.3218 \mathrm{E}+01$ & $1.246 \mathrm{E}-07$ & $4.903 \mathrm{E}+04$ \\
\hline $1 s 6 f^{3} F_{4}^{o}$ & $1 s 7 g{ }^{1} G_{4}$ & $4.3738 \mathrm{E}+01$ & $1.042 \mathrm{E}-07$ & $4.037 \mathrm{E}+04$ & $4.3559 \mathrm{E}+01$ & $1.044 \mathrm{E}-07$ & $4.045 \mathrm{E}+04$ \\
\hline $1 s 6 f^{1} F_{3}^{o}$ & $1 s 7 g{ }^{1} G_{4}$ & $4.3749 \mathrm{E}+01$ & $1.147 \mathrm{E}-06$ & $4.441 \mathrm{E}+05$ & $4.3560 \mathrm{E}+01$ & $1.148 \mathrm{E}-06$ & $4.449 \mathrm{E}+05$ \\
\hline $1 s 6 h^{3} H_{5}^{o}$ & $1 s 7 g{ }^{1} G_{4}$ & $4.3947 \mathrm{E}+01$ & $4.178 \mathrm{E}-15$ & $1.603 \mathrm{E}-03$ & & & \\
\hline $1 s 6 h^{3} H_{4}^{o}$ & $1 s 7 g{ }^{1} G_{4}$ & $4.3952 \mathrm{E}+01$ & $1.102 \mathrm{E}-15$ & $4.227 \mathrm{E}-04$ & & & \\
\hline $1 s 6 h^{3} H_{6}^{0}$ & $1 s 7 g{ }^{1} G_{4}$ & $4.4086 \mathrm{E}+01$ & $5.767 \mathrm{E}-10$ & $2.199 \mathrm{E}+02$ & $4.3903 \mathrm{E}+01$ & $5.785 \mathrm{E}-10$ & $2.205 \mathrm{E}+02$ \\
\hline $1 s 6 h^{1} H_{5}^{o}$ & $1 s 7 g{ }^{1} G_{4}$ & $4.4090 \mathrm{E}+01$ & $1.511 \mathrm{E}-08$ & $5.759 \mathrm{E}+03$ & $4.3903 \mathrm{E}+01$ & $1.504 \mathrm{E}-08$ & $5.734 \mathrm{E}+03$ \\
\hline
\end{tabular}


Table 6 (continued)

\begin{tabular}{|c|c|c|c|c|c|c|c|}
\hline \multirow[t]{2}{*}{ Lower } & \multirow[t]{2}{*}{ Upper } & \multicolumn{3}{|l|}{ GRASP2K } & \multicolumn{3}{|l|}{ FAC } \\
\hline & & $\lambda(\AA)$ & $g f$ & $A\left(\mathrm{~s}^{-1}\right)$ & $\overline{\lambda(\AA)}$ & $g f$ & $A\left(\mathrm{~s}^{-1}\right)$ \\
\hline $1 s 7 p^{3} P_{2}^{o}$ & $1 s 7 g{ }^{1} G_{4}$ & $2.1414 \mathrm{E}+03$ & $1.517 \mathrm{E}-18$ & $2.452 \mathrm{E}-10$ & & & \\
\hline $1 s 7 f^{3} F_{3}^{o}$ & $1 s 7 g{ }^{1} G_{4}$ & $5.4114 \mathrm{E}+03$ & $2.420 \mathrm{E}-14$ & $6.124 \mathrm{E}-07$ & $5.5025 \mathrm{E}+03$ & $2.355 \mathrm{E}-14$ & $5.716 \mathrm{E}-07$ \\
\hline $1 s 7 f^{3} F_{2}^{o}$ & $1 s 7 g{ }^{1} G_{4}$ & $5.5556 \mathrm{E}+03$ & $2.854 \mathrm{E}-13$ & $6.854 \mathrm{E}-06$ & $5.5006 \mathrm{E}+03$ & $2.907 \mathrm{E}-13$ & $7.060 \mathrm{E}-06$ \\
\hline $1 s 7 f^{3} F_{4}^{o}$ & $1 s 7 g{ }^{1} G_{4}$ & $1.4181 \mathrm{E}+04$ & $1.429 \mathrm{E}-14$ & $5.268 \mathrm{E}-08$ & $1.4765 \mathrm{E}+04$ & $1.251 \mathrm{E}-14$ & $4.217 \mathrm{E}-08$ \\
\hline $1 s 7 f^{1} F_{3}^{o}$ & $1 s 7 g{ }^{1} G_{4}$ & $1.4947 \mathrm{E}+04$ & $1.343 \mathrm{E}-13$ & $4.455 \mathrm{E}-07$ & $1.4784 \mathrm{E}+04$ & $1.370 \mathrm{E}-13$ & $4.608 \mathrm{E}-07$ \\
\hline $1 s 7 h^{3} H_{5}^{o}$ & $1 s 7 g{ }^{1} G_{4}$ & $4.7824 \mathrm{E}+05$ & $1.517 \mathrm{E}-24$ & $4.917 \mathrm{E}-21$ & & & \\
\hline $1 s 7 h^{3} H_{4}^{o}$ & $1 s 7 g{ }^{1} G_{4}$ & $3.2051 \mathrm{E}+06$ & $1.293 \mathrm{E}-27$ & $9.331 \mathrm{E}-26$ & & & \\
\hline $1 s 5 g^{3} G_{4}$ & $1 s 7 h^{3} H_{6}^{o}$ & $1.6478 \mathrm{E}+01$ & $2.768 \mathrm{E}-07$ & $5.230 \mathrm{E}+05$ & $1.6408 \mathrm{E}+01$ & $2.770 \mathrm{E}-07$ & $5.234 \mathrm{E}+05$ \\
\hline $1 s 5 g{ }^{3} G_{5}$ & $1 s 7 h^{3} H_{6}^{o}$ & $1.6529 \mathrm{E}+01$ & $3.232 \mathrm{E}-06$ & $6.069 \mathrm{E}+06$ & $1.6459 \mathrm{E}+01$ & $3.261 \mathrm{E}-06$ & $6.125 E+06$ \\
\hline $1 s 5 g{ }^{1} G_{4}$ & $1 s 7 h^{3} H_{6}^{o}$ & $1.6531 \mathrm{E}+01$ & $1.019 \mathrm{E}-07$ & $1.913 \mathrm{E}+05$ & $1.6460 \mathrm{E}+01$ & $1.028 \mathrm{E}-07$ & $1.931 \mathrm{E}+05$ \\
\hline $1 s 6 g^{3} G_{4}$ & $1 s 7 h^{3} H_{6}^{0}$ & $4.3656 \mathrm{E}+01$ & $2.869 \mathrm{E}-07$ & $7.725 E+04$ & $4.3473 \mathrm{E}+01$ & $2.870 \mathrm{E}-07$ & $7.727 \mathrm{E}+04$ \\
\hline $1 s 6 g^{3} G_{5}$ & $1 s 7 h^{3} H_{6}^{o}$ & $4.3863 \mathrm{E}+01$ & $3.400 \mathrm{E}-06$ & $9.068 \mathrm{E}+05$ & $4.3678 \mathrm{E}+01$ & $3.410 \mathrm{E}-06$ & $9.095 \mathrm{E}+05$ \\
\hline $1 s 6 g{ }^{1} G_{4}$ & $1 s 7 h^{3} H_{6}^{o}$ & $4.3870 \mathrm{E}+01$ & $1.073 \mathrm{E}-07$ & $2.860 \mathrm{E}+04$ & $4.3678 \mathrm{E}+01$ & $1.082 \mathrm{E}-07$ & $2.887 \mathrm{E}+04$ \\
\hline $1 s 7 g{ }^{3} G_{4}$ & $1 s 7 h^{3} H_{6}^{o}$ & $8.8116 \mathrm{E}+03$ & $8.646 \mathrm{E}-14$ & $5.714 \mathrm{E}-07$ & $8.8256 \mathrm{E}+03$ & $8.470 \mathrm{E}-14$ & $5.532 \mathrm{E}-07$ \\
\hline $1 s 7 g^{3} G_{5}$ & $1 s 7 h^{3} H_{6}^{0}$ & $2.2048 \mathrm{E}+04$ & $6.532 \mathrm{E}-14$ & $6.895 \mathrm{E}-08$ & $2.2104 \mathrm{E}+04$ & $6.405 \mathrm{E}-14$ & $6.670 \mathrm{E}-08$ \\
\hline $1 s 7 g{ }^{1} G_{4}$ & $1 s 7 h^{3} H_{6}^{o}$ & $2.3159 \mathrm{E}+04$ & $1.778 \mathrm{E}-15$ & $1.701 \mathrm{E}-09$ & $2.2104 \mathrm{E}+04$ & $2.033 \mathrm{E}-15$ & $2.117 \mathrm{E}-09$ \\
\hline $1 s 4 f^{3} F_{4}^{o}$ & $1 s 7 i^{3} I_{6}{ }^{\circ}$ & $7.6847 \mathrm{E}+00$ & $1.806 \mathrm{E}-12$ & $1.569 \mathrm{E}+01$ & & & \\
\hline $1 s 5 f^{3} F_{4}^{o}$ & $1 s 7 i^{3} I_{6}$ & $1.6478 \mathrm{E}+01$ & $1.456 \mathrm{E}-12$ & $2.751 \mathrm{E}+00$ & & & \\
\hline $1 s 6 f^{3} F_{4}^{o}$ & $1 s 7 i^{3} I_{6}$ & $4.3655 \mathrm{E}+01$ & $6.343 \mathrm{E}-14$ & $1.708 \mathrm{E}-02$ & & & \\
\hline $1 s 6 h^{3} H_{5}^{o}$ & $1 s 7 i^{3} I_{6}$ & $4.3863 \mathrm{E}+01$ & $2.235 \mathrm{E}-06$ & $5.960 \mathrm{E}+05$ & $4.3678 \mathrm{E}+01$ & $2.243 \mathrm{E}-06$ & $5.983 \mathrm{E}+05$ \\
\hline $1 s 6 h^{3} H_{4}^{o}$ & $1 s 7 i^{3} I_{6}$ & $4.3869 \mathrm{E}+01$ & $7.139 \mathrm{E}-08$ & $1.903 \mathrm{E}+04$ & $4.3678 \mathrm{E}+01$ & $7.123 \mathrm{E}-08$ & $1.899 \mathrm{E}+04$ \\
\hline $1 s 6 h^{3} H_{6}^{0}$ & $1 s 7 i^{3} I_{6}$ & $4.4002 \mathrm{E}+01$ & $4.282 \mathrm{E}-13$ & $1.135 \mathrm{E}-01$ & & & \\
\hline $1 s 6 h^{1} H_{5}^{o}$ & $1 s 7 i^{3} I_{6}$ & $4.4006 \mathrm{E}+01$ & $9.095 \mathrm{E}-13$ & $2.410 \mathrm{E}-01$ & & & \\
\hline $1 s 7 f^{3} F_{4}^{0}$ & $1 s 7 i^{3} I_{6}$ & $8.7950 \mathrm{E}+03$ & $4.030 \mathrm{E}-22$ & $2.673 \mathrm{E}-15$ & & & \\
\hline $1 s 7 h^{3} H_{5}^{o}$ & $1 s 7 i^{3} I_{6}$ & $2.2088 \mathrm{E}+04$ & $1.687 \mathrm{E}-14$ & $1.774 \mathrm{E}-08$ & $2.2113 \mathrm{E}+04$ & $1.661 \mathrm{E}-14$ & $1.728 \mathrm{E}-08$ \\
\hline $1 s 7 h^{3} H_{4}^{o}$ & $1 s 7 i^{3} I_{6}$ & $2.2991 \mathrm{E}+04$ & $4.777 \mathrm{E}-16$ & $4.637 \mathrm{E}-10$ & $2.2113 \mathrm{E}+04$ & $5.275 \mathrm{E}-16$ & $5.488 \mathrm{E}-10$ \\
\hline $1 s 7 h^{3} H_{6}^{o}$ & $1 s 7 i^{3} I_{6}$ & $2.7778 \mathrm{E}+08$ & $1.588 \mathrm{E}-33$ & $1.036 \mathrm{E}-35$ & & & \\
\hline $1 s 4 f^{3} F_{3}^{o}$ & $1 s 7 i^{3} I_{5}$ & $7.6487 \mathrm{E}+00$ & $4.069 \mathrm{E}-17$ & $4.218 \mathrm{E}-04$ & & & \\
\hline $1 s 4 f^{3} F_{4}^{o}$ & $1 s 7 i^{3} I_{5}$ & $7.6846 \mathrm{E}+00$ & $6.867 \mathrm{E}-14$ & $7.052 \mathrm{E}-01$ & & & \\
\hline $1 s 4 f^{1} F_{3}^{o}$ & $1 s 7 i^{3} I_{5}$ & $7.6858 \mathrm{E}+00$ & $1.434 \mathrm{E}-12$ & $1.472 \mathrm{E}+01$ & & & \\
\hline 1s $5 f^{3} F_{3}^{o}$ & $1 s 7 i^{3} I_{5}$ & $1.6393 \mathrm{E}+01$ & $2.438 \mathrm{E}-17$ & $5.502 \mathrm{E}-05$ & & & \\
\hline $1 s 5 f^{3} F_{4}^{o}$ & $1 s 7 i^{3} I_{5}$ & $1.6478 \mathrm{E}+01$ & $5.569 \mathrm{E}-14$ & $1.244 \mathrm{E}-01$ & & & \\
\hline $1 s 5 f^{1} F_{3}^{o}$ & $1 s 7 i^{3} I_{5}$ & $1.6481 \mathrm{E}+01$ & $1.157 \mathrm{E}-12$ & $2.584 \mathrm{E}+00$ & & & \\
\hline $1 s 6 f^{3} F_{3}^{o}$ & $1 s 7 i^{3} I_{5}$ & $4.3310 \mathrm{E}+01$ & $6.496 \mathrm{E}-19$ & $2.100 \mathrm{E}-07$ & & & \\
\hline $1 s 6 f^{3} F_{4}^{o}$ & $1 s 7 i^{3} I_{5}$ & $4.3653 \mathrm{E}+01$ & $2.487 \mathrm{E}-15$ & $7.915 \mathrm{E}-04$ & & & \\
\hline $1 s 6 f^{1} F_{3}^{o}$ & $1 s 7 i^{3} I_{5}$ & $4.3664 \mathrm{E}+01$ & $5.058 \mathrm{E}-14$ & $1.609 \mathrm{E}-02$ & & & \\
\hline $1 s 6 h^{3} H_{5}^{o}$ & $1 s 7 i^{3} I_{5}$ & $4.3861 \mathrm{E}+01$ & $1.065 \mathrm{E}-07$ & $3.356 \mathrm{E}+04$ & $4.3678 \mathrm{E}+01$ & $1.068 \mathrm{E}-07$ & $3.367 \mathrm{E}+04$ \\
\hline $1 s 6 h^{3} H_{4}^{o}$ & $1 s 7 i^{3} I_{5}$ & $4.3866 \mathrm{E}+01$ & $1.845 \mathrm{E}-06$ & $5.814 \mathrm{E}+05$ & $4.3678 \mathrm{E}+01$ & $1.851 \mathrm{E}-06$ & $5.836 \mathrm{E}+05$ \\
\hline $1 s 6 h^{3} H_{6}^{0}$ & $1 s 7 i^{3} I_{5}$ & $4.4000 \mathrm{E}+01$ & $6.477 \mathrm{E}-17$ & $2.029 \mathrm{E}-05$ & & & \\
\hline $1 s 6 h{ }^{1} H_{5}^{o}$ & $1 s 7 i^{3} I_{5}$ & $4.4004 \mathrm{E}+01$ & $2.541 \mathrm{E}-13$ & $7.958 \mathrm{E}-02$ & & & \\
\hline $1 s 7 f^{3} F_{3}^{o}$ & $1 s 7 i^{3} I_{5}$ & $4.3626 \mathrm{E}+03$ & $1.645 \mathrm{E}-27$ & $5.241 \mathrm{E}-20$ & & & \\
\hline $1 s 7 f^{3} F_{4}^{o}$ & $1 s 7 i^{3} I_{5}$ & $8.6997 \mathrm{E}+03$ & $1.609 \mathrm{E}-23$ & $1.289 \mathrm{E}-16$ & & & \\
\hline $1 s 7 f^{1} F_{3}^{o}$ & $1 s 7 i^{3} I_{5}$ & $8.9822 \mathrm{E}+03$ & $3.033 \mathrm{E}-22$ & $2.280 \mathrm{E}-15$ & & & \\
\hline $1 s 7 h^{3} H_{5}^{o}$ & $1 s 7 i^{3} I_{5}$ & $2.1496 \mathrm{E}+04$ & $8.714 \mathrm{E}-16$ & $1.144 \mathrm{E}-09$ & $2.2113 \mathrm{E}+04$ & $7.913 E-16$ & $9.729 \mathrm{E}-10$ \\
\hline $1 s 7 h^{3} H_{4}^{0}$ & $1 s 7 i^{3} I_{5}$ & $2.2351 \mathrm{E}+04$ & $1.344 \mathrm{E}-14$ & $1.631 \mathrm{E}-08$ & $2.2113 \mathrm{E}+04$ & $1.371 \mathrm{E}-14$ & $1.686 \mathrm{E}-08$ \\
\hline $1 s 7 h^{3} H_{6}^{0}$ & $1 s 7 i^{3} I_{5}$ & $8.0038 \mathrm{E}+05$ & $8.707 \mathrm{E}-31$ & $8.242 \mathrm{E}-28$ & & & \\
\hline $1 s 3 d^{3} D_{3}$ & $1 s 7 h^{1} H_{5}^{o}$ & $3.5586 \mathrm{E}+00$ & $2.702 \mathrm{E}-16$ & $1.294 \mathrm{E}-02$ & & & \\
\hline $1 s 4 d^{3} D_{3}$ & $1 s 7 h^{1} H_{5}^{o}$ & $7.6476 \mathrm{E}+00$ & $2.941 \mathrm{E}-16$ & $3.049 \mathrm{E}-03$ & & & \\
\hline $1 s 5 d^{3} D_{3}$ & $1 s 7 h^{1} H_{5}^{o}$ & $1.6390 \mathrm{E}+01$ & $1.850 \mathrm{E}-16$ & $4.176 \mathrm{E}-04$ & & & \\
\hline $1 s 5 g^{3} G_{4}$ & $1 s 7 h^{1} H_{5}^{o}$ & $1.6478 \mathrm{E}+01$ & $1.027 \mathrm{E}-08$ & $2.293 E+04$ & $1.6408 \mathrm{E}+01$ & $9.994 \mathrm{E}-09$ & $2.231 \mathrm{E}+04$ \\
\hline $1 s 5 g^{3} G_{3}$ & $1 s 7 h^{1} H_{5}^{o}$ & $1.6480 \mathrm{E}+01$ & $2.227 \mathrm{E}-07$ & $4.972 E+05$ & $1.6410 \mathrm{E}+01$ & $2.231 \mathrm{E}-07$ & $4.981 \mathrm{E}+05$ \\
\hline $1 s 5 g^{3} G_{5}$ & $1 s 7 h^{1} H_{5}^{o}$ & $1.6529 \mathrm{E}+01$ & $1.539 \mathrm{E}-07$ & $3.417 \mathrm{E}+05$ & $1.6459 \mathrm{E}+01$ & $1.553 \mathrm{E}-07$ & $3.447 \mathrm{E}+05$ \\
\hline $1 s 5 g{ }^{1} G_{4}$ & $1 s 7 h^{1} H_{5}^{o}$ & $1.6530 \mathrm{E}+01$ & $2.668 \mathrm{E}-06$ & $5.920 \mathrm{E}+06$ & $1.6460 \mathrm{E}+01$ & $2.692 \mathrm{E}-06$ & $5.973 E+06$ \\
\hline $1 s 6 d^{3} D_{3}$ & $1 s 7 h^{1} H_{5}^{o}$ & $4.3297 \mathrm{E}+01$ & $1.613 \mathrm{E}-16$ & $5.219 \mathrm{E}-05$ & & & \\
\hline $1 s 6 g{ }^{3} G_{4}$ & $1 s 7 h^{1} H_{5}^{o}$ & $4.3653 \mathrm{E}+01$ & $1.064 \mathrm{E}-08$ & $3.387 E+03$ & $4.3473 \mathrm{E}+01$ & $1.104 \mathrm{E}-08$ & $3.512 \mathrm{E}+03$ \\
\hline $1 s 6 g{ }^{3} G_{3}$ & $1 s 7 h{ }^{1} H_{5}^{o}$ & $4.3661 \mathrm{E}+01$ & $2.309 \mathrm{E}-07$ & $7.345 \mathrm{E}+04$ & $4.3473 \mathrm{E}+01$ & $2.318 \mathrm{E}-07$ & $7.376 \mathrm{E}+04$ \\
\hline $1 s 6 g^{3} G_{5}$ & $1 s 7 h{ }^{1} H_{5}^{o}$ & $4.3860 \mathrm{E}+01$ & $1.619 \mathrm{E}-07$ & $5.104 \mathrm{E}+04$ & $4.3678 \mathrm{E}+01$ & $1.624 \mathrm{E}-07$ & $5.118 \mathrm{E}+04$ \\
\hline $1 s 6 g{ }^{1} G_{4}$ & $1 s 7 h^{1} H_{5}^{o}$ & $4.3867 \mathrm{E}+01$ & $2.807 \mathrm{E}-06$ & $8.845 E+05$ & $4.3678 \mathrm{E}+01$ & $2.815 \mathrm{E}-06$ & $8.872 \mathrm{E}+05$ \\
\hline $1 s 7 d^{3} D_{3}$ & $1 s 7 h^{1} H_{5}^{o}$ & $4.2781 \mathrm{E}+03$ & $7.935 \mathrm{E}-22$ & $2.629 \mathrm{E}-14$ & & & \\
\hline $1 s 7 g{ }^{3} G_{4}$ & $1 s 7 h{ }^{1} H_{5}^{o}$ & $8.6987 \mathrm{E}+03$ & $3.334 \mathrm{E}-15$ & $2.672 \mathrm{E}-08$ & $8.8256 \mathrm{E}+03$ & $3.259 \mathrm{E}-15$ & $2.515 \mathrm{E}-08$ \\
\hline $1 s 7 g{ }^{3} G_{3}$ & $1 s 7 h^{1} H_{5}^{o}$ & $8.9120 \mathrm{E}+03$ & $6.726 \mathrm{E}-14$ & $5.135 \mathrm{E}-07$ & $8.8255 \mathrm{E}+03$ & $6.841 \mathrm{E}-14$ & $5.281 \mathrm{E}-07$ \\
\hline $1 s 7 g{ }^{3} G_{5}$ & $1 s 7 h^{1} H_{5}^{o}$ & $2.1355 \mathrm{E}+04$ & $3.424 \mathrm{E}-15$ & $4.553 \mathrm{E}-09$ & $2.2104 \mathrm{E}+04$ & $3.050 \mathrm{E}-15$ & $3.753 \mathrm{E}-09$ \\
\hline $1 s 7 g{ }^{1} G_{4}$ & $1 s 7 h^{1} H_{5}^{o}$ & $2.2395 \mathrm{E}+04$ & $5.146 \mathrm{E}-14$ & $6.221 \mathrm{E}-08$ & $2.2104 \mathrm{E}+04$ & $5.287 \mathrm{E}-14$ & $6.506 \mathrm{E}-08$ \\
\hline $1 s 7 i^{3} I_{6}$ & $1 s 7 h{ }^{1} H_{5}^{o}$ & $6.8074 \mathrm{E}+05$ & $2.383 \mathrm{E}-25$ & $3.119 \mathrm{E}-22$ & & & \\
\hline $1 s 7 i^{3} I_{5}$ & $1 s 7 h^{1} H_{5}^{o}$ & $4.4823 \mathrm{E}+06$ & $2.314 \mathrm{E}-28$ & $6.985 \mathrm{E}-27$ & & & \\
\hline $1 s 6 h^{3} H_{5}^{o}$ & $1 s 7 i^{3} I_{7}$ & $4.3801 \mathrm{E}+01$ & $4.717 \mathrm{E}-07$ & $1.093 \mathrm{E}+05$ & $4.3617 \mathrm{E}+01$ & $4.715 \mathrm{E}-07$ & $1.092 \mathrm{E}+05$ \\
\hline $1 s 6 h^{3} H_{6}^{o}$ & $1 s 7 i^{3} I_{7}$ & $4.3940 \mathrm{E}+01$ & $7.252 \mathrm{E}-06$ & $1.670 \mathrm{E}+06$ & $4.3754 \mathrm{E}+01$ & $7.280 \mathrm{E}-06$ & $1.676 \mathrm{E}+06$ \\
\hline $1 s 6 h{ }^{1} H_{5}^{o}$ & $1 s 7 i^{3} I_{7}$ & $4.3944 \mathrm{E}+01$ & $1.639 \mathrm{E}-07$ & $3.775 E+04$ & $4.3754 \mathrm{E}+01$ & $1.654 \mathrm{E}-07$ & $3.810 \mathrm{E}+04$ \\
\hline $1 s 7 h^{3} H_{5}^{o}$ & $1 s 7 i^{3} I_{7}$ & $1.2900 \mathrm{E}+04$ & $1.788 \mathrm{E}-14$ & $4.778 \mathrm{E}-08$ & $1.2909 \mathrm{E}+04$ & $1.756 \mathrm{E}-14$ & $4.647 \mathrm{E}-08$ \\
\hline $1 s 7 h^{3} H_{6}^{0}$ & $1 s 7 i^{3} I_{7}$ & $3.1010 \mathrm{E}+04$ & $1.983 \mathrm{E}-14$ & $9.171 \mathrm{E}-09$ & $3.1022 \mathrm{E}+04$ & $1.957 \mathrm{E}-14$ & 8.967E-09 \\
\hline $1 s 7 h^{1} H_{5}^{o}$ & $1 s 7 i^{3} I_{7}$ & $3.2494 \mathrm{E}+04$ & $3.897 \mathrm{E}-16$ & $1.641 \mathrm{E}-10$ & $3.1022 \mathrm{E}+04$ & $4.448 \mathrm{E}-16$ & $2.038 \mathrm{E}-10$ \\
\hline
\end{tabular}


Table 6 (continued)

\begin{tabular}{|c|c|c|c|c|c|c|c|}
\hline \multirow[t]{2}{*}{ Lower } & \multirow[t]{2}{*}{ Upper } & \multicolumn{3}{|l|}{ GRASP2K } & \multicolumn{3}{|l|}{ FAC } \\
\hline & & $\overline{\lambda(\AA)}$ & $g f$ & $A\left(\mathrm{~s}^{-1}\right)$ & $\lambda(\AA)$ & $g f$ & $A\left(\mathrm{~s}^{-1}\right)$ \\
\hline $1 s 4 f^{3} F_{4}^{o}$ & $1 s 7 i{ }^{1} I_{6}$ & $7.6827 \mathrm{E}+00$ & $9.016 \mathrm{E}-17$ & $7.837 \mathrm{E}-04$ & & & \\
\hline $1 s 5 f^{3} F_{4}^{0}$ & $1 s 7 i^{1} I_{6}$ & $1.6469 \mathrm{E}+01$ & $7.167 \mathrm{E}-17$ & $1.356 \mathrm{E}-04$ & & & \\
\hline $1 s 6 f^{3} F_{4}^{o}$ & $1 s 7 i^{1} I_{6}$ & $4.3592 \mathrm{E}+01$ & $1.033 \mathrm{E}-18$ & $2.790 \mathrm{E}-07$ & & & \\
\hline $1 s 6 h^{3} H_{5}^{o}$ & $1 s 7 i{ }^{1} I_{6}$ & $4.3799 \mathrm{E}+01$ & $1.213 \mathrm{E}-08$ & $3.246 \mathrm{E}+03$ & $4.3617 \mathrm{E}+01$ & $1.257 \mathrm{E}-08$ & $3.362 E+03$ \\
\hline $1 s 6 h^{3} H_{4}^{o}$ & $1 s 7 i{ }^{1} I_{6}$ & $4.3805 \mathrm{E}+01$ & $3.950 \mathrm{E}-07$ & $1.056 \mathrm{E}+05$ & $4.3617 \mathrm{E}+01$ & $3.961 \mathrm{E}-07$ & $1.059 \mathrm{E}+05$ \\
\hline $1 s 6 h^{3} H_{6}^{0}$ & $1 s 7 i^{1} I_{6}$ & $4.3938 \mathrm{E}+01$ & $2.473 \mathrm{E}-07$ & $6.572 \mathrm{E}+04$ & $4.3754 \mathrm{E}+01$ & $2.481 \mathrm{E}-07$ & $6.595 \mathrm{E}+04$ \\
\hline $1 s 6 h^{1} H_{5}^{o}$ & $1 s 7 i{ }^{1} I_{6}$ & $4.3942 \mathrm{E}+01$ & $6.182 \mathrm{E}-06$ & $1.643 \mathrm{E}+06$ & $4.3754 \mathrm{E}+01$ & $6.204 \mathrm{E}-06$ & $1.648 \mathrm{E}+06$ \\
\hline $1 s 7 f^{3} F_{4}^{o}$ & $1 s 7 i^{1} I_{6}$ & $6.8024 \mathrm{E}+03$ & $6.449 \mathrm{E}-27$ & $7.151 \mathrm{E}-20$ & & & \\
\hline $1 s 7 h^{3} H_{5}^{o}$ & $1 s 7 i{ }^{1} I_{6}$ & $1.2726 \mathrm{E}+04$ & $4.791 \mathrm{E}-16$ & $1.518 \mathrm{E}-09$ & $1.2909 \mathrm{E}+04$ & $4.684 \mathrm{E}-16$ & $1.430 \mathrm{E}-09$ \\
\hline $1 s 7 h^{3} H_{4}^{o}$ & $1 s 7 i{ }^{1} I_{6}$ & $1.3020 \mathrm{E}+04$ & $1.456 \mathrm{E}-14$ & $4.408 \mathrm{E}-08$ & $1.2909 \mathrm{E}+04$ & $1.475 \mathrm{E}-14$ & $4.504 \mathrm{E}-08$ \\
\hline $1 s 7 h^{3} H_{6}^{0}$ & $1 s 7 i^{1} I_{6}$ & $3.0020 \mathrm{E}+04$ & $7.453 \mathrm{E}-16$ & $4.243 \mathrm{E}-10$ & $3.1022 \mathrm{E}+04$ & $6.673 \mathrm{E}-16$ & $3.527 \mathrm{E}-10$ \\
\hline $1 s 7 h^{1} H_{5}^{o}$ & $1 s 7 i^{1} I_{6}$ & $3.1409 \mathrm{E}+04$ & $1.627 \mathrm{E}-14$ & $8.462 \mathrm{E}-09$ & $3.1022 \mathrm{E}+04$ & $1.668 \mathrm{E}-14$ & $8.818 \mathrm{E}-09$ \\
\hline
\end{tabular}


Table 7

Calculated values using the GRASP2K code of the magnetic dipole $A$, electric quadrupole $B$ and Landé $g$-factors for all levels of interest. For this calculation, the active set expanded to $n=10$ (GRASP3) has been considered.

\begin{tabular}{|c|c|c|c|}
\hline Configuration & $A(\mathrm{MHz})$ & $B(\mathrm{MHz})$ & $g_{J}$ \\
\hline $1 s 2 s^{3} S_{1}$ & $1.329 E+07$ & $1.941 \mathrm{E}+00$ & $1.935167 \mathrm{E}+00$ \\
\hline $1 s 2 p^{3} P_{1}^{o}$ & $1.218 \mathrm{E}+07$ & $4.527 E+03$ & $1.285193 \mathrm{E}+00$ \\
\hline $1 s 2 p^{3} P_{2}^{o}$ & $5.965 E+06$ & $-6.466 \mathrm{E}+04$ & $1.458966 \mathrm{E}+00$ \\
\hline $1 s 2 p{ }^{1} P_{1}^{o}$ & $-5.636 \mathrm{E}+06$ & $-3.488 E+04$ & $1.150634 \mathrm{E}+00$ \\
\hline $1 s 3 s^{3} S_{1}$ & $1.218 \mathrm{E}+07$ & $1.709 \mathrm{E}+00$ & $1.942586 \mathrm{E}+00$ \\
\hline $1 s 3 p^{3} P_{1}^{o}$ & $1.184 \mathrm{E}+07$ & $1.265 \mathrm{E}+03$ & $1.291576 \mathrm{E}+00$ \\
\hline $1 s 3 p^{3} P_{2}^{o}$ & $5.890 \mathrm{E}+06$ & $-1.976 \mathrm{E}+04$ & $1.467393 \mathrm{E}+00$ \\
\hline $1 s 3 d^{3} D_{2}$ & $5.879 \mathrm{E}+06$ & $-3.874 \mathrm{E}+03$ & $1.067061 \mathrm{E}+00$ \\
\hline $1 s 3 d^{3} D_{1}$ & $-5.824 \mathrm{E}+06$ & $-1.941 E+03$ & $5.145892 \mathrm{E}-01$ \\
\hline $1 s 3 p{ }^{1} P_{1}^{o}$ & $-5.774 \mathrm{E}+06$ & $-1.065 E+04$ & $1.165553 \mathrm{E}+00$ \\
\hline $1 s 3 d^{3} D_{3}$ & $3.915 E+06$ & $-5.068 \mathrm{E}+03$ & $1.308971 \mathrm{E}+00$ \\
\hline $1 s 3 d{ }^{1} D_{2}$ & $-3.892 E+06$ & $-4.040 \mathrm{E}+03$ & $1.074253 \mathrm{E}+00$ \\
\hline $1 s 4 s^{3} S_{1}$ & $1.191 \mathrm{E}+07$ & $8.684 \mathrm{E}-01$ & $1.945151 \mathrm{E}+00$ \\
\hline $1 s 4 p^{3} P_{1}^{o}$ & $1.175 \mathrm{E}+07$ & $5.208 \mathrm{E}+02$ & $1.293822 \mathrm{E}+00$ \\
\hline $1 s 4 p^{3} P_{2}^{o}$ & $5.871 E+06$ & $-8.406 E+03$ & $1.470347 \mathrm{E}+00$ \\
\hline $1 s 4 d^{3} D_{2}$ & $5.867 E+06$ & $-1.672 E+03$ & $1.070326 \mathrm{E}+00$ \\
\hline $1 s 4 d^{3} D_{1}$ & $-5.843 \mathrm{E}+06$ & $-8.397 \mathrm{E}+02$ & $5.195030 \mathrm{E}-01$ \\
\hline $1 s 4 p^{1} P_{1}^{o}$ & $-5.809 E+06$ & $-4.532 E+03$ & $1.170777 \mathrm{E}+00$ \\
\hline $1 s 4 d^{3} D_{3}$ & $3.909 E+06$ & $-2.169 E+03$ & $1.312098 \mathrm{E}+00$ \\
\hline $1 s 4 f^{3} F_{3}^{o}$ & $3.908 E+06$ & $-7.731 \mathrm{E}+02$ & $1.025485 \mathrm{E}+00$ \\
\hline $1 s 4 f^{3} F_{2}^{0}$ & $-3.901 \mathrm{E}+06$ & $-6.174 \mathrm{E}+02$ & $6.782567 \mathrm{E}-01$ \\
\hline $1 s 4 d^{1} D_{2}$ & $-3.900 E+06$ & $-1.729 E+03$ & $1.078311 \mathrm{E}+00$ \\
\hline $1 s 4 f^{3} F_{4}^{o}$ & $2.930 \mathrm{E}+06$ & $-8.773 E+02$ & $1.232907 \mathrm{E}+00$ \\
\hline $1 s 4 f^{1} F_{3}^{o}$ & $-2.927 \mathrm{E}+06$ & $-7.820 \mathrm{E}+02$ & $1.044150 \mathrm{E}+00$ \\
\hline $1 s 5 s^{3} s_{1}^{3}$ & $1.181 \mathrm{E}+07$ & $4.676 \mathrm{E}-01$ & $1.946327 \mathrm{E}+00$ \\
\hline $1 s 5 p^{3} P_{1}^{o}$ & $1.172 \mathrm{E}+07$ & $2.630 \mathrm{E}+02$ & $1.294862 \mathrm{E}+00$ \\
\hline $1 s 5 p^{3} P_{2}^{0}$ & $5.865 \mathrm{E}+06$ & $-4.316 \mathrm{E}+03$ & $1.471711 \mathrm{E}+00$ \\
\hline $1 s 5 d^{3} D_{2}$ & $5.862 \mathrm{E}+06$ & $-8.637 \mathrm{E}+02$ & $1.071827 \mathrm{E}+00$ \\
\hline $1 s 5 p^{1} P_{1}^{o}$ & $-5.822 \mathrm{E}+06$ & $-2.327 \mathrm{E}+03$ & $1.173184 \mathrm{E}+00$ \\
\hline $1 s 5 d^{3} D_{1}$ & $-5.851 E+06$ & $-4.344 \mathrm{E}+02$ & $5.217790 \mathrm{E}-01$ \\
\hline $1 s 5 d^{3} D_{3}$ & $3.907 \mathrm{E}+06$ & $-1.116 \mathrm{E}+03$ & $1.313549 \mathrm{E}+00$ \\
\hline $1 s 5 f^{3} F_{3}^{o}$ & $3.906 E+06$ & $-3.993 E+02$ & $1.026955 \mathrm{E}+00$ \\
\hline $1 s 5 f^{3} F_{2}^{o}$ & $-3.903 E+06$ & $-3.188 \mathrm{E}+02$ & $6.802985 \mathrm{E}-01$ \\
\hline $1 s 5 d{ }^{1} D_{2}$ & $-3.902 E+06$ & $-8.908 E+02$ & $1.080205 E+00$ \\
\hline $1 s 5 f^{3} F_{4}^{o}$ & $2.930 \mathrm{E}+06$ & $-4.520 \mathrm{E}+02$ & $1.234400 \mathrm{E}+00$ \\
\hline $1 s 5 g^{3} G_{4}$ & $2.930 E+06$ & $-2.098 E+02$ & $1.011596 \mathrm{E}+00$ \\
\hline $1 s 5 g^{3} G_{3}$ & $-2.928 E+06$ & $-1.874 \mathrm{E}+02$ & $7.594756 \mathrm{E}-01$ \\
\hline $1 s 5 f^{1} F_{3}^{o}$ & $-2.928 E+06$ & $-4.029 E+02$ & $1.046058 \mathrm{E}+00$ \\
\hline $1 s 5 g^{3} G_{5}$ & $2.344 \mathrm{E}+06$ & $-2.262 E+02$ & $1.186952 \mathrm{E}+00$ \\
\hline $1 s 5 g{ }^{1} G_{4}$ & $-2.343 \mathrm{E}+06$ & $-2.112 \mathrm{E}+02$ & $1.029827 \mathrm{E}+00$ \\
\hline $1 s 6 s^{3} s_{1}$ & $1.177 \mathrm{E}+07$ & $2.710 \mathrm{E}-01$ & $1.946963 \mathrm{E}+00$ \\
\hline $1 s 6 p^{3} P_{1}^{o}$ & $1.171 \mathrm{E}+07$ & $1.507 \mathrm{E}+02$ & $1.295417 \mathrm{E}+00$ \\
\hline $1 s 6 p^{3} P_{2}^{o}$ & $5.862 \mathrm{E}+06$ & $-2.497 \mathrm{E}+03$ & $1.472450 \mathrm{E}+00$ \\
\hline $1 s 6 d^{3} D_{2}$ & $5.860 \mathrm{E}+06$ & $-5.019 E+02$ & $1.072640 \mathrm{E}+00$ \\
\hline $1 s 6 p{ }^{1} P_{1}^{o}$ & $-5.828 E+06$ & $-1.346 E+03$ & $1.174496 \mathrm{E}+00$ \\
\hline $1 s 6 d^{3} D_{1}$ & $-5.854 \mathrm{E}+06$ & $-2.527 \mathrm{E}+02$ & $5.230139 \mathrm{E}-01$ \\
\hline $1 s 6 d^{3} D_{3}$ & $3.907 E+06$ & $-6.475 E+02$ & $1.314338 \mathrm{E}+00$ \\
\hline $1 s 6 f^{3} F_{3}^{o}$ & $3.906 \mathrm{E}+06$ & $-2.320 \mathrm{E}+02$ & $1.027753 \mathrm{E}+00$ \\
\hline $1 s 6 f^{3} F_{2}^{o}$ & $-3.904 \mathrm{E}+06$ & $-1.853 \mathrm{E}+02$ & $6.814050 \mathrm{E}-01$ \\
\hline $1 s 6 d^{1} D_{2}$ & $-3.903 E+06$ & $-5.171 \mathrm{E}+02$ & $1.081237 \mathrm{E}+00$ \\
\hline $1 s 6 f^{3} F_{4}^{o}$ & $2.929 E+06$ & $-2.624 \mathrm{E}+02$ & $1.235214 \mathrm{E}+00$ \\
\hline $1 s 6 g^{3} G_{4}$ & $2.929 \mathrm{E}+06$ & $-1.220 \mathrm{E}+02$ & $1.012411 \mathrm{E}+00$ \\
\hline $1 s 6 g^{3} G_{3}$ & $-2.929 E+06$ & $-1.090 \mathrm{E}+02$ & $7.605229 \mathrm{E}-01$ \\
\hline $1 s 6 f^{1} F_{3}^{o}$ & $-2.928 \mathrm{E}+06$ & $-2.339 E+02$ & $1.047097 \mathrm{E}+00$ \\
\hline $1 s 6 g^{3} G_{5}$ & $2.344 \mathrm{E}+06$ & $-1.314 \mathrm{E}+02$ & $1.187784 \mathrm{E}+00$ \\
\hline $1 s 6 h^{3} H_{5}^{o}$ & $2.343 E+06$ & $-7.193 E+01$ & $1.005467 \mathrm{E}+00$ \\
\hline $1 s 6 h^{3} H_{4}^{o}$ & $-2.343 E+06$ & $-6.707 E+01$ & $8.080359 \mathrm{E}-01$ \\
\hline $1 s 6 g{ }^{1} G_{4}$ & $-2.343 \mathrm{E}+06$ & $-1.227 \mathrm{E}+02$ & $1.030843 \mathrm{E}+00$ \\
\hline $1 s 6 h^{3} H_{6}^{o}$ & $1.953 \mathrm{E}+06$ & $-7.560 \mathrm{E}+01$ & $1.156123 \mathrm{E}+00$ \\
\hline $1 s 6 h{ }^{1} H_{5}^{o}$ & $-1.952 E+06$ & $-7.210 \mathrm{E}+01$ & $1.021980 \mathrm{E}+00$ \\
\hline $1 s 7 s^{3} S_{1}$ & $1.175 E+07$ & $1.638 \mathrm{E}-01$ & $1.947346 \mathrm{E}+00$ \\
\hline $1 s 7 p^{3} P_{1}^{o}$ & $1.170 \mathrm{E}+07$ & $9.511 \mathrm{E}+01$ & $1.295852 \mathrm{E}+00$ \\
\hline $1 s 7 p^{3} P_{2}^{o}$ & $5.860 \mathrm{E}+06$ & $-1.576 E+03$ & $1.472878 \mathrm{E}+00$ \\
\hline $1 s 7 d^{3} D_{2}$ & $5.859 E+06$ & $-3.166 E+02$ & $1.073129 \mathrm{E}+00$ \\
\hline $1 s 7 d^{3} D_{1}$ & $-5.855 E+06$ & $-1.596 \mathrm{E}+02$ & $5.237575 \mathrm{E}-01$ \\
\hline $1 s 7 p{ }^{1} P_{1}^{o}$ & $-5.830 \mathrm{E}+06$ & $-8.507 \mathrm{E}+02$ & $1.175151 \mathrm{E}+00$ \\
\hline $1 s 7 d^{3} D_{3}$ & $3.906 \mathrm{E}+06$ & $-4.081 E+02$ & $1.314814 \mathrm{E}+00$ \\
\hline $1 s 7 f^{3} F_{3}^{o}$ & $3.906 \mathrm{E}+06$ & $-1.466 \mathrm{E}+02$ & $1.028235 \mathrm{E}+00$ \\
\hline $1 s 7 f^{3} F_{2}^{o}$ & $-3.904 \mathrm{E}+06$ & $-1.171 \mathrm{E}+02$ & $6.820742 \mathrm{E}-01$ \\
\hline $1 s 7 d^{1} D_{2}$ & $-3.904 \mathrm{E}+06$ & $-3.263 E+02$ & $1.081860 \mathrm{E}+00$ \\
\hline
\end{tabular}


Table 7 (continued)

\begin{tabular}{llll}
\hline Configuration & $A(\mathrm{MHz})$ & $B(\mathrm{MHz})$ & $g_{J}$ \\
\hline $1 s 7 f^{3} F_{4}^{o}$ & $2.929 \mathrm{E}+06$ & $-1.654 \mathrm{E}+02$ & $1.235703 \mathrm{E}+00$ \\
$1 s 7 g^{3} G_{4}$ & $2.929 \mathrm{E}+06$ & $-7.700 \mathrm{E}+01$ & $1.012903 \mathrm{E}+00$ \\
$1 s 7 g^{3} G_{3}$ & $-2.929 \mathrm{E}+06$ & $-6.881 \mathrm{E}+01$ & $7.611546 \mathrm{E}-01$ \\
$1 s 7 f^{1} F_{3}^{o}$ & $-2.928 \mathrm{E}+06$ & $-1.475 \mathrm{E}+02$ & $1.047721 \mathrm{E}+00$ \\
$1 s 7 g{ }^{3} G_{5}$ & $2.343 \mathrm{E}+06$ & $-8.289 \mathrm{E}+01$ & $1.188286 \mathrm{E}+00$ \\
$1 s 7 h^{3} H_{5}^{o}$ & $2.343 \mathrm{E}+06$ & $-4.542 \mathrm{E}+01$ & $1.005969 \mathrm{E}+00$ \\
$1 s 7 h^{3} H_{4}^{o}$ & $-2.343 \mathrm{E}+06$ & $-4.235 \mathrm{E}+01$ & $8.086497 \mathrm{E}-01$ \\
$1 s 7 g{ }^{1} G_{4}$ & $-2.343 \mathrm{E}+06$ & $-7.742 \mathrm{E}+01$ & $1.031456 \mathrm{E}+00$ \\
$1 s 7 h^{3} H_{6}^{o}$ & $1.953 \mathrm{E}+06$ & $-4.771 \mathrm{E}+01$ & $1.156632 \mathrm{E}+00$ \\
$1 s 7 i^{3} I_{6}$ & $1.953 \mathrm{E}+06$ & $-2.870 \mathrm{E}+01$ & $8.402692 \mathrm{E}-01$ \\
$1 s 7 i^{3} I_{5}$ & $-1.953 \mathrm{E}+06$ & $-2.743 \mathrm{E}+01$ & $1.022582 \mathrm{E}+00$ \\
$1 s 7 h^{1} H_{5}^{o}$ & $-1.952 \mathrm{E}+06$ & $-4.550 \mathrm{E}+01$ & $1.134057 \mathrm{E}+00$ \\
$1 s 7 i^{3} I_{7}$ & $1.674 \mathrm{E}+06$ & $-2.974 \mathrm{E}+01$ & $1.017106 \mathrm{E}+00$ \\
$1 s 7 i{ }^{1} I_{6}$ & $-1.674 \mathrm{E}+06$ & $-2.879 \mathrm{E}+01$ & \\
\hline
\end{tabular}


Table 8

Calculated values using the GRASP2K code of normal, specific mass shifts and electron densities at the nucleus for the all singly excited levels up to $n=7$ with increasing the active set to $n=10$ (GRASP3).

\begin{tabular}{|c|c|c|c|c|}
\hline Index & Configuration & $K_{N M S}$ & $K_{S M S}$ & Electron densities at the nucleus \\
\hline 1 & $1 s^{2}{ }^{1} S_{0}$ & $2.862115 \mathrm{E}+03$ & $8.372810 \mathrm{E}+00$ & $2.355815 \mathrm{E}+05$ \\
\hline 2 & $1 s 2 s^{3} S_{1}$ & $1.819580 \mathrm{E}+03$ & $6.544528 \mathrm{E}-01$ & $1.364262 \mathrm{E}+05$ \\
\hline 3 & $1 s 2 p^{3} P_{1}^{o}$ & $1.817000 \mathrm{E}+03$ & $-8.021940 \mathrm{E}+01$ & $1.201622 \mathrm{E}+05$ \\
\hline 4 & $1 s 2 p^{3} P_{0}^{o}$ & $1.815498 \mathrm{E}+03$ & $-1.953285 \mathrm{E}+02$ & $1.201173 \mathrm{E}+05$ \\
\hline 5 & $1 s 2 s^{1} S_{0}$ & $1.815192 \mathrm{E}+03$ & $1.618605 \mathrm{E}+00$ & $1.360149 \mathrm{E}+05$ \\
\hline 6 & $1 s 2 p^{3} P_{2}^{o}$ & $1.803659 \mathrm{E}+03$ & $-2.058238 \mathrm{E}+02$ & $1.196642 \mathrm{E}+05$ \\
\hline 7 & $1 s 2 p^{1} P_{1}^{o}$ & $1.802099 \mathrm{E}+03$ & $8.321168 \mathrm{E}+01$ & $1.198328 \mathrm{E}+05$ \\
\hline 8 & $1 s 3 s^{3} S_{1}$ & $1.613866 \mathrm{E}+03$ & $2.454228 \mathrm{E}-01$ & $1.247247 \mathrm{E}+05$ \\
\hline 9 & $1 s 3 p^{3} P_{1}^{o}$ & $1.613208 \mathrm{E}+03$ & $-1.706693 \mathrm{E}+01$ & $1.199436 \mathrm{E}+05$ \\
\hline 10 & $1 s 3 s^{1} S_{0}$ & $1.612703 \mathrm{E}+03$ & $5.420968 \mathrm{E}-01$ & $1.246202 \mathrm{E}+05$ \\
\hline 11 & $1 s 3 p^{3} P_{0}^{o}$ & $1.612808 \mathrm{E}+03$ & $-4.218003 \mathrm{E}+01$ & $1.199297 \mathrm{E}+05$ \\
\hline 12 & $1 s 3 p^{3} P_{2}^{o}$ & $1.609187 \mathrm{E}+03$ & $-4.894801 \mathrm{E}+01$ & $1.197682 \mathrm{E}+05$ \\
\hline 13 & $1 s 3 d^{3} D_{2}$ & $1.608843 \mathrm{E}+03$ & $3.867396 \mathrm{E}-02$ & $1.197815 \mathrm{E}+05$ \\
\hline 14 & $1 s 3 d^{3} D_{1}$ & $1.608690 \mathrm{E}+03$ & $1.545303 \mathrm{E}-01$ & $1.197794 \mathrm{E}+05$ \\
\hline 15 & $1 s 3 p^{1} P_{1}^{o}$ & $1.608726 \mathrm{E}+03$ & $1.988737 \mathrm{E}+01$ & $1.198104 \mathrm{E}+05$ \\
\hline 16 & $1 s 3 d^{3} D_{3}$ & $1.607560 \mathrm{E}+03$ & $6.261143 \mathrm{E}-02$ & $1.197794 \mathrm{E}+05$ \\
\hline 17 & $1 s 3 d^{1} D_{2}$ & $1.607466 \mathrm{E}+03$ & $2.896360 \mathrm{E}-03$ & $1.197827 \mathrm{E}+05$ \\
\hline 18 & $1 s 4 s^{3} S_{1}$ & $1.542191 \mathrm{E}+03$ & $1.159027 \mathrm{E}-01$ & $1.218552 \mathrm{E}+05$ \\
\hline 19 & $1 s 4 p^{3} P_{1}^{o}$ & $1.541967 \mathrm{E}+03$ & $-6.423856 \mathrm{E}+00$ & $1.198675 \mathrm{E}+05$ \\
\hline 20 & $1 s 4 s^{1} S_{0}$ & $1.541823 \mathrm{E}+03$ & $2.449015 \mathrm{E}-01$ & $1.218158 \mathrm{E}+05$ \\
\hline 21 & $1 s 4 p^{3} P_{0}^{o}$ & $1.541804 \mathrm{E}+03$ & $-1.595949 \mathrm{E}+01$ & $1.198612 \mathrm{E}+05$ \\
\hline 22 & $1 s 4 p^{3} P_{2}^{0}$ & $1.540267 \mathrm{E}+03$ & $-1.916717 \mathrm{E}+01$ & $1.197893 \mathrm{E}+05$ \\
\hline 23 & $1 s 4 d^{3} D_{2}$ & $1.540095 \mathrm{E}+03$ & $2.748062 \mathrm{E}-02$ & $1.197824 \mathrm{E}+05$ \\
\hline 24 & $1 s 4 d^{3} D_{1}$ & $1.540053 \mathrm{E}+03$ & $8.509648 \mathrm{E}-02$ & $1.197813 E+05$ \\
\hline 25 & $1 s 4 p^{1} P_{1}^{o}$ & $1.540076 \mathrm{E}+03$ & $7.809108 \mathrm{E}+00$ & $1.198064 \mathrm{E}+05$ \\
\hline 26 & $1 s 4 d^{3} D_{3}$ & $1.539548 \mathrm{E}+03$ & $4.557849 \mathrm{E}-02$ & $1.197813 \mathrm{E}+05$ \\
\hline 27 & $1 s 4 f^{3} F_{3}^{o}$ & $1.539594 \mathrm{E}+03$ & $1.470809 \mathrm{E}-02$ & $1.198033 \mathrm{E}+05$ \\
\hline 28 & $1 s 4 f^{3} F_{2}^{o}$ & $1.539566 \mathrm{E}+03$ & $2.242135 \mathrm{E}-02$ & $1.198053 \mathrm{E}+05$ \\
\hline 29 & $1 s 4 d^{1} D_{2}$ & $1.539534 \mathrm{E}+03$ & $8.086057 \mathrm{E}-03$ & $1.197830 \mathrm{E}+05$ \\
\hline 30 & $1 s 4 f^{3} F_{4}^{o}$ & $1.539324 \mathrm{E}+03$ & $6.452494 \mathrm{E}-03$ & $1.198035 E+05$ \\
\hline 31 & $1 s 4 f^{1} F_{3}^{o}$ & $1.539303 \mathrm{E}+03$ & $1.282208 \mathrm{E}-02$ & $1.198050 \mathrm{E}+05$ \\
\hline 32 & $1 s 5 s^{3} s_{1}^{3}$ & $1.509220 \mathrm{E}+03$ & $6.414105 \mathrm{E}-02$ & $1.208374 \mathrm{E}+05$ \\
\hline 33 & $1 s 5 p^{3} P_{1}^{o}$ & $1.509162 \mathrm{E}+03$ & $-3.114675 \mathrm{E}+00$ & $1.198376 \mathrm{E}+05$ \\
\hline 34 & $1 s 5 s^{1} s_{0}$ & $1.509195 \mathrm{E}+03$ & $1.322070 \mathrm{E}-01$ & $1.208210 \mathrm{E}+05$ \\
\hline 35 & $1 s 5 p^{3} P_{0}^{o}$ & $1.509081 \mathrm{E}+03$ & $-7.758204 \mathrm{E}+00$ & $1.198342 \mathrm{E}+05$ \\
\hline 36 & $1 s 5 p^{3} P_{2}^{o}$ & $1.508292 \mathrm{E}+03$ & $-9.475364 \mathrm{E}+00$ & $1.197965 \mathrm{E}+05$ \\
\hline 37 & $1 s 5 d^{3} D_{2}$ & $1.508180 \mathrm{E}+03$ & $1.819042 \mathrm{E}-02$ & $1.197829 E+05$ \\
\hline 38 & $1 s 5 p^{1} P_{1}^{o}$ & $1.508197 \mathrm{E}+03$ & $3.868506 \mathrm{E}+00$ & $1.198052 \mathrm{E}+05$ \\
\hline 39 & $1 s 5 d^{3} D_{1}$ & $1.508177 \mathrm{E}+03$ & $4.981599 \mathrm{E}-02$ & $1.197822 \mathrm{E}+05$ \\
\hline 40 & $1 s 5 d^{3} D_{3}$ & $1.507896 \mathrm{E}+03$ & $2.909923 \mathrm{E}-02$ & $1.197823 \mathrm{E}+05$ \\
\hline 41 & $1 s 5 f^{3} F_{3}^{o}$ & $1.507945 \mathrm{E}+03$ & $9.888449 \mathrm{E}-03$ & $1.198037 \mathrm{E}+05$ \\
\hline 42 & $1 s 5 f^{3} F_{2}^{o}$ & $1.507930 \mathrm{E}+03$ & 1.398999E-02 & $1.198047 \mathrm{E}+05$ \\
\hline 43 & $1 s 5 d^{1} D_{2}$ & $1.507911 \mathrm{E}+03$ & $7.316622 \mathrm{E}-03$ & $1.197832 \mathrm{E}+05$ \\
\hline 44 & $1 s 5 f^{3} F_{4}^{o}$ & $1.507807 \mathrm{E}+03$ & $5.878058 \mathrm{E}-03$ & $1.198038 \mathrm{E}+05$ \\
\hline 45 & $1 s 5 g^{3} G_{4}$ & $1.507772 \mathrm{E}+03$ & $6.925163 \mathrm{E}-03$ & $1.197834 \mathrm{E}+05$ \\
\hline 46 & $1 s 5 g^{3} G_{3}$ & $1.507765 \mathrm{E}+03$ & $9.936995 \mathrm{E}-03$ & $1.197835 \mathrm{E}+05$ \\
\hline 47 & $1 s 5 f^{1} F_{3}^{o}$ & $1.507796 \mathrm{E}+03$ & $8.879435 \mathrm{E}-03$ & $1.198046 \mathrm{E}+05$ \\
\hline 48 & $1 s 5 g^{3} G_{5}$ & $1.507690 \mathrm{E}+03$ & $3.848136 \mathrm{E}-03$ & $1.197834 \mathrm{E}+05$ \\
\hline 49 & $1 s 5 g{ }^{1} G_{4}$ & $1.507684 \mathrm{E}+03$ & $6.841721 \mathrm{E}-03$ & $1.197835 \mathrm{E}+05$ \\
\hline 50 & $1 s 6 s^{3} S_{1}$ & $1.491393 \mathrm{E}+03$ & $3.966973 \mathrm{E}-02$ & $1.203896 \mathrm{E}+05$ \\
\hline 51 & $1 s 6 p^{3} P_{1}^{o}$ & $1.491425 \mathrm{E}+03$ & $-1.747137 \mathrm{E}+00$ & $1.198237 \mathrm{E}+05$ \\
\hline 52 & $1 s 6 s^{1} S_{0}$ & $1.491595 \mathrm{E}+03$ & $8.044927 \mathrm{E}-02$ & $1.203841 \mathrm{E}+05$ \\
\hline 53 & $1 s 6 p^{3} P_{0}^{o}$ & $1.491378 \mathrm{E}+03$ & $-4.358955 E+00$ & $1.198217 \mathrm{E}+05$ \\
\hline 54 & $1 s 6 p^{3} P_{2}^{o}$ & $1.490923 \mathrm{E}+03$ & $-5.374441 \mathrm{E}+00$ & $1.197997 \mathrm{E}+05$ \\
\hline 55 & $1 s 6 d^{3} D_{2}$ & $1.490835 \mathrm{E}+03$ & $1.260114 \mathrm{E}-02$ & $1.197831 \mathrm{E}+05$ \\
\hline 56 & $1 s 6 p^{1} P_{1}^{o}$ & $1.490867 \mathrm{E}+03$ & $2.197926 \mathrm{E}+00$ & $1.198047 \mathrm{E}+05$ \\
\hline 57 & $1 s 6 d^{3} D_{1}$ & $1.490852 \mathrm{E}+03$ & $3.168686 \mathrm{E}-02$ & $1.197827 \mathrm{E}+05$ \\
\hline 58 & $1 s 6 d^{3} D_{3}$ & $1.490668 \mathrm{E}+03$ & $1.933123 \mathrm{E}-02$ & $1.197827 \mathrm{E}+05$ \\
\hline 59 & $1 s 6 f^{3} F_{3}^{o}$ & $1.490720 \mathrm{E}+03$ & $6.648316 \mathrm{E}-03$ & $1.198039 \mathrm{E}+05$ \\
\hline 60 & $1 s 6 f^{3} F_{2}^{0}$ & $1.490711 \mathrm{E}+03$ & $9.068110 \mathrm{E}-03$ & $1.198045 \mathrm{E}+05$ \\
\hline 61 & $1 s 6 d^{1} D_{2}$ & $1.490698 \mathrm{E}+03$ & $6.001879 \mathrm{E}-03$ & $1.197833 \mathrm{E}+05$ \\
\hline 62 & $1 s 6 f^{3} F_{4}^{o}$ & $1.490640 \mathrm{E}+03$ & $4.399590 \mathrm{E}-03$ & $1.198040 \mathrm{E}+05$ \\
\hline 63 & $1 s 6 g{ }^{3} G_{4}$ & $1.490603 \mathrm{E}+03$ & $4.841594 \mathrm{E}-03$ & $1.197834 \mathrm{E}+05$ \\
\hline 64 & $1 s 6 g^{3} G_{3}$ & $1.490603 \mathrm{E}+03$ & $6.624087 \mathrm{E}-03$ & $1.197835 \mathrm{E}+05$ \\
\hline 65 & $1 s 6 f^{1} F_{3}^{o}$ & $1.490634 \mathrm{E}+03$ & $6.033506 \mathrm{E}-03$ & $1.198044 \mathrm{E}+05$ \\
\hline 66 & $1 s 6 g^{3} G_{5}$ & $1.490556 \mathrm{E}+03$ & $2.987498 \mathrm{E}-03$ & $1.197834 \mathrm{E}+05$ \\
\hline 67 & $1 s 6 h^{3} H_{5}^{o}$ & $1.490594 \mathrm{E}+03$ & $2.961360 \mathrm{E}-03$ & $1.198041 \mathrm{E}+05$ \\
\hline 68 & $1 s 6 h^{3} H_{4}^{o}$ & $1.490591 \mathrm{E}+03$ & $3.804622 \mathrm{E}-03$ & $1.198043 \mathrm{E}+05$ \\
\hline 69 & $1 s 6 g{ }^{1} G_{4}$ & $1.490556 \mathrm{E}+03$ & $4.747536 \mathrm{E}-03$ & $1.197835 \mathrm{E}+05$ \\
\hline 70 & $1 s 6 h^{3} H_{6}^{o}$ & $1.490562 \mathrm{E}+03$ & $1.899926 \mathrm{E}-03$ & $1.198041 \mathrm{E}+05$ \\
\hline 71 & $1 s 6 h{ }^{1} H_{5}^{o}$ & $1.490560 \mathrm{E}+03$ & $2.752518 \mathrm{E}-03$ & $1.198043 \mathrm{E}+05$ \\
\hline
\end{tabular}


Table 8 (continued)

\begin{tabular}{|c|c|c|c|c|}
\hline Index & Configuration & $K_{N M S}$ & $K_{S M S}$ & Electron densities at the nucleus \\
\hline 72 & $1 s 7 s^{3} S_{1}$ & $1.480650 \mathrm{E}+03$ & $2.694334 \mathrm{E}-02$ & $1.201626 \mathrm{E}+05$ \\
\hline 73 & $1 s 7 p^{3} P_{1}^{o}$ & $1.481084 \mathrm{E}+03$ & $-1.087763 \mathrm{E}+00$ & $1.198166 \mathrm{E}+05$ \\
\hline 74 & $1 s 7 s^{1} S_{0}$ & $1.481167 \mathrm{E}+03$ & $5.410009 \mathrm{E}-02$ & $1.201644 \mathrm{E}+05$ \\
\hline 75 & $1 s 7 p^{3} P_{0}^{o}$ & $1.481058 \mathrm{E}+03$ & $-2.715643 \mathrm{E}+00$ & $1.198153 \mathrm{E}+05$ \\
\hline 76 & $1 s 7 p^{3} P_{2}^{o}$ & $1.480758 \mathrm{E}+03$ & $-3.364477 \mathrm{E}+00$ & $1.198013 \mathrm{E}+05$ \\
\hline 77 & $1 s 7 d^{3} D_{2}$ & $1.480376 \mathrm{E}+03$ & $9.571873 \mathrm{E}-03$ & $1.197832 \mathrm{E}+05$ \\
\hline 78 & $1 s 7 d^{3} D_{1}$ & $1.480415 \mathrm{E}+03$ & $2.200799 \mathrm{E}-02$ & $1.197830 \mathrm{E}+05$ \\
\hline 79 & $1 s 7 p^{1} P_{1}^{o}$ & $1.480741 \mathrm{E}+03$ & $1.381522 \mathrm{E}+00$ & $1.198045 \mathrm{E}+05$ \\
\hline 80 & $1 s 7 d^{3} D_{3}$ & $1.480267 \mathrm{E}+03$ & $1.390302 \mathrm{E}-02$ & $1.197830 \mathrm{E}+05$ \\
\hline 81 & $1 s 7 f^{3} F_{3}^{o}$ & $1.480333 \mathrm{E}+03$ & $4.632020 \mathrm{E}-03$ & $1.198040 \mathrm{E}+05$ \\
\hline 82 & $1 s 7 f^{3} F_{2}^{o}$ & $1.480328 \mathrm{E}+03$ & $6.156500 \mathrm{E}-03$ & $1.198044 \mathrm{E}+05$ \\
\hline 83 & $1 s 7 d^{1} D_{2}$ & $1.480318 \mathrm{E}+03$ & $5.311102 \mathrm{E}-03$ & $1.197834 \mathrm{E}+05$ \\
\hline 84 & $1 s 7 f^{3} F_{4}^{o}$ & $1.480278 \mathrm{E}+03$ & $3.356779 \mathrm{E}-03$ & $1.198040 \mathrm{E}+05$ \\
\hline 85 & $1 s 7 g^{3} G_{4}$ & $1.480235 \mathrm{E}+03$ & $3.714721 \mathrm{E}-03$ & $1.197834 \mathrm{E}+05$ \\
\hline 86 & $1 s 7 g{ }^{3} G_{3}$ & $1.480243 \mathrm{E}+03$ & $4.889674 \mathrm{E}-03$ & $1.197835 \mathrm{E}+05$ \\
\hline 87 & $1 s 7 f^{1} F_{3}^{o}$ & $1.480274 \mathrm{E}+03$ & $4.340171 \mathrm{E}-03$ & $1.198043 \mathrm{E}+05$ \\
\hline 88 & $1 s 7 g{ }^{3} G_{5}$ & $1.480206 \mathrm{E}+03$ & $2.491921 \mathrm{E}-03$ & $1.197834 \mathrm{E}+05$ \\
\hline 89 & $1 s 7 h^{3} H_{5}^{o}$ & $1.480247 \mathrm{E}+03$ & $2.300436 \mathrm{E}-03$ & $1.198041 \mathrm{E}+05$ \\
\hline 90 & $1 s 7 h^{3} H_{4}^{o}$ & $1.480245 \mathrm{E}+03$ & $2.822402 \mathrm{E}-03$ & $1.198042 \mathrm{E}+05$ \\
\hline 91 & $1 s 7 g{ }^{1} G_{4}$ & $1.480212 \mathrm{E}+03$ & $3.650499 \mathrm{E}-03$ & $1.197835 \mathrm{E}+05$ \\
\hline 92 & $1 s 7 h^{3} H_{6}^{o}$ & $1.480226 \mathrm{E}+03$ & $1.590809 \mathrm{E}-03$ & $1.198041 \mathrm{E}+05$ \\
\hline 93 & $1 s 7 i^{3} I_{6}$ & $1.480190 \mathrm{E}+03$ & $1.649752 \mathrm{E}-03$ & $1.197834 \mathrm{E}+05$ \\
\hline 94 & $1 s 7 i^{3} I_{5}$ & $1.480190 \mathrm{E}+03$ & $2.164438 \mathrm{E}-03$ & $1.197835 \mathrm{E}+05$ \\
\hline 95 & $1 s 7 h^{1} H_{5}^{o}$ & $1.480225 \mathrm{E}+03$ & $2.112037 \mathrm{E}-03$ & $1.198042 \mathrm{E}+05$ \\
\hline 96 & $1 s 7 i^{3} I_{7}$ & $1.480176 \mathrm{E}+03$ & $1.080355 \mathrm{E}-03$ & $1.197834 \mathrm{E}+05$ \\
\hline 97 & $1 s 7 i^{1} I_{6}$ & $1.480176 \mathrm{E}+03$ & $1.594008 \mathrm{E}-03$ & $1.197835 \mathrm{E}+05$ \\
\hline
\end{tabular}

